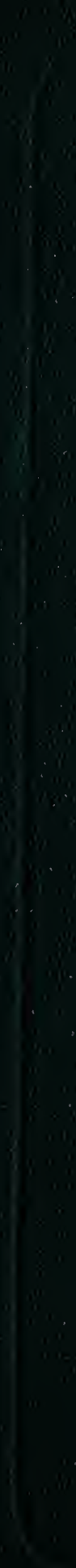



708.15 


\section{ELEMENTS}

OF

COMPARATIVE ANATOMY. 


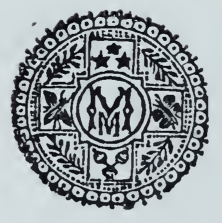




\section{ELEMENTS}

OF

\section{COMPARATIVE ANATOMY.}

$\mathrm{BY}$

\section{CARL GEGENBAUR,}

profissor of Anatomi and Director of the Anatomical Institute at Heidelberg.

TRANSLATED BY

F. JEFFREY BELL, B.A.,

Magdalem College, Oxford.

THE TRANSLATION REVISED AND A PREFACE WRITTEN BY

E. RAY LANKESTER, M.A., F.R.S.,

Fellow of Exetre College, Oxford, and Professor of Zoologi and Comparative Amatomy in University College, London.

LONDON :

MACMILLAN AND CO.

1878. 


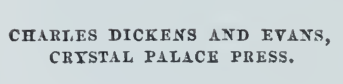




\section{PREFACE TO THE SECOND EDITION.}

Our knowledge has been so much increased in extent and exactness in almost every department of Comparative Anatomy since the time when I converted my "Grundzüge" into the first edition of this smaller manual-the "Grundriss"-that the publication of a second edition hardly seemed an easy task. Nevertheless, I gladly undertook it, for I had observed so much new evidence of the importance of the doctrine of development in anatomical enquiry. The road along which science may travel forward successfully seems indeed to be growing easier, yet the distance which we have made is but short in comparison with that which lies in front of us, and far beyond our view. Every question solved leads again to fresh problems, and renders unstable even what seemed to have taken a definite form. There are, therefore, great difficulties in giving such a comprehensive presentation of the subject as a text-book ought to supply. I have tried as much as possible to evade these difficulties where I have been unable to overcome thern. Much remains unaltered, because recent investigations appear to demand fundamental changes, the concrete expression of which cannot be immediately taken in hand.

I have somewhat modified the arrangement of the matter. I can hardly be blamed for separating the Brachiopoda from the Mollusca, and treating them as forming an independent phylum. Nor indeed is the change a real one, for even in my "Grundzüge" I drew especial attention to the great difference that obtained between them and the "other Mollusca." The Tunicata have 
been treated in the same way, but this does not require any apology at the present date.

By treating the subject more concisely I have been able to increase the real matter to a certain extent, without enlarging the size of the book. I have, of course, only dealt with what has seemed to me to be of capital importance; many, and even important, details have been omitted, owing to the limits imposed by the aim of the book.

I have endeavoured to correct some previous mistakes and to supply omissions. If any such have been retained, or have newly crept in, I shall be fairly judged, I know, by anatomists, who will remember the vast extent of our science and the object of this work. I hope that I have satisfied them, and if I have my toil is well repaid.

Heidelberg, November, 1877.

C. Gegenbaur. 


\section{PREFACE TO THE ENGLISH TRANSLATION.}

Iт is a great pleasure to me to be able to place in the hands of my pupils in Oxford and London an English translation of Professor Gegenbaur's "Grundriss der Vergleichenden Anatomie." I have to thank the energy and industry of Mr. JEFFrey BeLL, of Magdalen College, Oxford (now one of the staff of the British Museum), for the translation which he undertook and carried through at my request, when I found that my time was too fully occupied with other work to allow of my completing it myself within a sufficiently short period from the date of publication of the German work.

My share of the present work has therefore consisted in a careful revision of the MS. and proof-sheets, which has been by no means a mere formality, but enables me to give the assurance that the original work is faithfully rendered in the translation. The chapter on the Tunicata I took occasion to translate myself.

That Professor Gegenbaur's work will be of great service to those English students who do not already read German cannot be doubted. We have some excellent treatises in the English language on animal morphology, notably the Manuals of the Anatomy of Vertebrate and Invertebrate Animals, by Professor Huxler. But we do not possess any modern work on Comparative Anatomy, properly so-called; that is to say, a work in which the comparative method is put prominently forward as the guiding principle in the treatment of the results of anatomical investigation. The present work therefore appears to me to form a most important supplement to our existing treatises on the structure and classification of animals. It has, over and above this, a distinctive and weighty recommendation in that throughout and without reserve the Doctrine of Evolution appears as the living, moving investment of the dry bones of anatomical fact. Not only is the student thus taught to retain and accumulate his facts in relation to definite problems which are actually exercising the ingenuity of investigators, 
but he is encouraged, and to a certain extent trained, in the liealthy use of his speculative faculties; in fact the one great method by which new knowledge is attained, whether of little things or of big things-the method of observation (or experiment), directed by speculation-becomes the conscious and distinctive characteristic of his mental activity. Thus we may claim for the study of Comparative Anatomy, as set forth in the present work, the power of developing what is called "common sense" into the more precisely fixed "scientific habit" of mind.

I lave made no notes nor additions of any kind to the original text, with the exception of a few references to English works likely to be useful to the English student. These additions are indicated by brackets.

Whilst the work is thus presented to the reader precisely as its author designed that it should be, there can be no objection to the introduction in this place of a few remarks suggested by the fact that this English translation is intended for the use of English students, and that it is therefore desirable, in order to prevent confusion and perplexity, to point out certain statements of fact, or of interpretation of fact, in which Professor Gegenbaur differs widely from authorities usually followed in this country. I shall, moreover, refer to some recent additions to knowledge published since this work left Professor Gegenbaur's hands. It will be understood that the following paragraphs are intended as a supplement necessitated by the special objects of this translation, and are by no means to be regarded as conceived in the spirit of criticism or discussion, which would assuredly ill befit a writer who is making known to a new audience the teachings of a master to whom he is deeply indebted.

Nuclei of Cells.-In the first place, it seems necessary to notice that, whilst the last German edition of this work was in the press, very important additions to our knowledge of the nucleus of organic cells or plastids were being made. Though these investigations are not yet complete they tend to modify what is said concerning the nucieus on pages 15 and 16. The student is referred to an article by Mr. Priestley in the Quart. Journ. Microsc. Science, vol. xvi. (1876), for an account of the observations of Auerbach, Strasburger, Hertwig, and Van Bexedex, and to part iii. of the same Journal, vol xviii. (1878), for original observations on the same subject by Dr. Kuein.

Reproduction of Infusoria.-A most important modification in the current views as to the reproduction of the Infusoria has resulted from the same line of study as that just mentioned, when carried into the domain of unicellular organisms. O. Bütschli and EvGELMANe have shown that we are not at present in a position to assert that the process of colljugation in the Infusoria is followed by a production of spores (see $\S 70$ ). It results from their investigations that conjugation in the Infusoria is attended by a definite breaking-up of the nucleus and so-called nucleolus (paranucleus) of the conjugating individuals; but that the conjugating 
individuals separate, and after expelling portions of the broken-up nuclear structures (probably as effete products), proceed to re-form the nucleus, or nucleus and nucleolus characteristic of the species. The so-called $\Lambda$ cinetiform embryos appear to be parasites, the rod-like bodies occasionally observed in the nucleus are also parasites, whilst the striated structure and spindleshape exhibited by the nucleolus or paranucleus in such forms as Paramocium and Stylonichia at the period of conjugation, are simply due to changes in this body which are exactly paralleled in the nuclei of egg-cells and other tissue-elements of multicellular organisms, when those cells are about to divide by transverse fission. The process of conjugation in the Infusoria may be, and probably is, attended by an exchange of nuclear material between the conjugating individuals, and is so far comparable to sexual congress, but it results in a simple "rejuvenescence" of the conjugating individuals and not in a production of spores. Reproduction by fission and by the modification of fission, known as gemmation, has been accurately observed in Infusoria, but of the formation of "spores" in this group we are at present ignorant, in spite of all that has been written on the subject.

Origin of Male and Female Reproductive Elements from different Germ-layers.-In §95 Professor Gegenbaur has described the observations of ED. VAN BENEDEN on the development of the sexual products in Hydractinia, and has adopted his generalisation, so far at least as it applies to the Hydromedusæ. From more recent observations (Cramicinan, Zeitschr. für wiss. Zoologie, vol. xxx. p. 501, 1878) it appears that in other genera of hydroid polyps the same arrangement does not obtain. In Eudendrium ramosum the ova appear to develop from the ectoderm, and the sperm from the endoderm; in Tubularia mesembryanthemum both ova and sperm are ectodermal in origin according to Cramician; Van Beneden found the ova to be endodermal and the sperm ectodermal in Hydractinia, whilst KLeINenberg ascribes both to the ectoderm in Hydra.

Nervous System and Sensory Organs of Medusæ.-During the past year a considerable addition has been made to knowledge on these points, by the researches of the two Hermwigs ("Das Nervensystem und die Sinnesorgane der Medusen." Leipzig, 1877). It is no longer possible to deny the existence of differentiated nervous tissue in the Medusæ-the central organ having the form of a ring situated along the line of insertion of the velum in the Craspedota, and of a series of isolated ganglia, usually eight in number, placed on the edge of the disc in the Acraspeda. (See for an abstract of recent researches on this subject, Quart. Journal of Microsc. Science, vol. xviii. p. 340.)

Cirri and Elytra of Aphroditaceæ.-The statement in $\S 105$, that the elytra of the chrtopodous Worms, allied to Aphrodite, are formed by the metamorphosis of the dorsal cirri of the parapodia, appears to be contradicted 
by the fact, that in Sigalion the elytra and dorsal cirri exist side by side on the same segment.

Homologies of the Rami of the $\Lambda$ ppendages in Astacus.-The view taken by Professor Gegexbaur, as to the homologies of the parts of the appendages immediately following the mouth in Astacus, differs somewhat from that which is current in this country. In Fig. 122, p. 239, the mandible, two maxille, and three maxillipedes of the right side of Astacus fluviatilis are figured. This woodcut was kindly re-drawn for the English edition by the author, at my request, and gires a more complete outline of the parts in question, than does the older cut of the German edition. Throughout the series of appendages, three divisions are distinguished lyy the letters $a, c, c$. Taking the lowest figure first (the third maxillipedle) we find the endopodite marked $a$, the exopodite marked $c$, and the letter $d$ placed with the single epipodite (podobranchia, Huxuer) to its inner side, whilst the clouble arthrobranchia (HuxLEY) not forming part of the appendage proper, but a distinct respiratory development, is seen on its outer side. In the next figure (the second maxillipede), $a$ indicates endopodite, $c$ exopodite, and $d$ is placed close to the double arthrobranchia on its outer side, whilst the modified epipodite is seen to the inner side again, of this. In the figure of the first maxillipede, $a$ is placed near the foliaceous endopodite, which has a detached outstanding seginent, $c$ near the filamentous exopodite, and d near the broad epipodite. The same explanation of the lettering holds good for the next appendage, the second maxilla. In the next appendagethe first maxilla-the absence of the letters $c$ and $d$, indicates that the author regards the whole appendage as reduced to the representative of the foliaceous endopodite $a$ of the two inferior appendages-a view with which few will disagree. In the case of the mandible, however, Professor Gegenbaur marks the "palp" with the letter c-considering, therefore, the basal piece of this appendage to represent the endopodite, and the palp to represent the exopodite. The more usual opinion on this matter is that the mandible, together with its palp, corresponds to the simple foliaceous first maxilla. The jointed palp, mounted on its solid basal biting-piece, corresponds to the jointed endopodite $\boldsymbol{c}$ of the last maxillipede.

The question of the presence or the absence of a representative of the exopodite in the Decapod's mandible, is a matter of considerable importance in reference to possible comparisons between the gnathites of Crustacea and Tracheata. The actual development of the parts in question from the nauplius-form of appendage, must be the ultimate test of the homologies of their rami in the Crustacea.

Blood-corpuscles of the Mollusca. - The statement on p. 375, that "the form-elements of the blood are always colourless" in the MIollusca, is one which I may be allowed to correct, since I have published an account of the blood-corpuscles of Solen legumen (Proc. Royal Society, No. 140, 1873), which, besides colourless amœhoid forms, comprise a vast number of 
oval ones, deeply stained by hæmoglobin. The ummber of these corpuscles is so considerable as to give the blood of Solen legumen a bright bloodred colour.

I may add here that I have observed similar though larger corpuseles impregnated with hæmoglobin in the blood of species of Arca.

Homologies of the Arms of the Cephalopoda.-The view that the sucker-bearing arms of the cuttlefish are to be regarded as appendages of the head homologous with the tentacles on the head of Gasteropous (p. 326), is one which, it will be well for the student to remember, is not that usually taught. He should make himself acquainted with the older and the newer view, and the grounds on which they are based. Without entering into a discussion of the arguments which may be adduced in favour of this or of rival interpretations of the parts, it must suffice here briefly to mention that the arms of the Cephalopod (the development of which had been made known by Kölliker), were shown by Professor Huxler, five-and-twenty years ago, to correspond to the fore-part of the foot of the Gasteropoda, and the ganglion, from which they receive their nerve supply, was then considered as corresponding to the pedal (Morphology of the Cephalous Mollusca, Phil. Trans. 1853). This view was maintained in the earlier editions of Gegenbaur's work. It has been abandoned in the present edition, in deference to the statements of Mr. Jhering ("Vergleichende Anatomie des Nervensystems und Phylogenie der Molluscen, Leipzig," 1877). The whole of that author's work, both statement of fact and speculative superstructure, appears to me to call for very cautious treatment, involving the rejection of some of his principal conclusions.

Origin of the Limbs of Vertebrates.-Professor Gegenbaur is inclined to regard the skeleton of the limbs and limb-girdles of Vertebrata as derived from gill-arches and their branchial rays (\$ 357). The student is reminded that another possible derivation of these organs is from primitively continuous lateral fins-supported by cartilaginous rays, and comparable to the primitively continuous dorsal median fin. The specialisation and concentration of the lateral fin on each side in two regions, thoracic and pelvic, would be competent to give rise to the two pairs of fins, such as we find in the Elasmobranchs. Mr. Balfour ("Development of Elasmobranch Fishes," 1878 ) is led to adopt this view by the observation, that in the embryo dogfish the lateral fins have precisely the same mode of origin as has the dorsal median fin, arising "as special developments of a continuous ridge on each side, precisely like the ridges of epiblast, which form the rudiments of the unpaired fins." This view of the nature of the vertebrate limbs has been independently worked out with great care from the point of view of comparative anatomy, by Mr. J. K. Thacher (Median and Paired Fins, Transactions Connecticut Academy, vol. iii. 1877). In the important memoir just citer, Mr. Thachen shows very plausibly how the Elasmobranch fin, and not only the fin, but the supporting limb-girdle also, may have 
been derived from the gradual shifting, atrophy; hypertrophy, and concrescence of primitively similar cartilaginous rods, which formed a series on each side of the body, identical in character with the primitive median dorsal series. According to this view, the "archipterygium" of Professor" Gegendaudr is not antecedent to, but is derived from the type of fin found in Elasmobranchs. (See also on this subject, Huxley, On Ceratodus, Proc. Zool. Soc. vol. 1876, p. 24.)

Relation of the Malleus and Incus to the Mandibular and Hyoid Arches.-Investigations directed to the development of the skull led Professor Huxley some years since to adopt the conclusion of ReicherT and of Goodsir, that the small bones of the Mammals' tympanic cavity were derived from the upper ends of the anterior visceral arches. At first it appeared probable that the malleus and incus were both derived from the upper end of the cartilaginous mandibular arch, the lower part forming Meckel's cartilage. This led to the suggestion that the malleus corresponds to the articulare of the lower jaw of other Vertebrata, whilst the incus was considered to be the representative of the quadratum, since it articulates with the malleus just as the quadratum does with the articulare (Croonian Lecture "On the Theory of the Vertebrate Skull," Proc. Royal Society, vol. ix. p. 398).

Further investigation led Professor HuxLeY to a modification of his views. The embryological evidence is not quite complete, but the relations of the parts in question in the developing Frog, in certain Lizards, and in Mammalia, have led him to the conclusion ("Manual of Vertebrate Anatomy," p. 85,1871 ) that whilst the malleus is formed from the uppermost extremity of the mandibular arch, and therefore represents, not articulare, but quadratum, the incus is developed from the uppermost extremity of the second or hyoid arch, and corresponds to the hyomandibular of fishes. The stapes is also developed from the upper portion of the hyoid arch, just below the incus. The incus may therefore be spoken of as the supra-stapedial portion of the hyoid arch, and in certain Vertebrata it exists as a mere cartilaginous supra-stapedial rudiment.

These views in their later form have not been adopted by Professor Gegendaur. He observes ( $\$ 402$ ) that the lomologies of the ossicula a uditûs of the various classes of Vertebrata have not yet been satisfactorily determined. In $\S 352$ he maintains the earlier determination of the homology of the mammalian malleus with the articulare of other Vertebrates. Concerning the homologies of the incus and the stapes, he considers it advisable, in the present state of knowledge, to make no statement.

The student is advised of these differences of interpretation of structural fact, in order that he may the more carefully make himself acquainted from original sources with the details of development, relation to nerves, and other features of the parts under discussion.

Nomenclature of the Lobes of the Brain in Fishes.-In the earlier editions of the present work, Professor Gegendinur, led by the result 
of investigations carried out by his pupil Miknucho-MacLay ("Vergleich. Neurologie der Wirbelthiere," 1870), modified the current nomenclature of the lobes of the Fish's brain, so that the large bispherical part, which was usually considered as the mesencephalon in the Teleostei and Selachii, was assigned to the thalamencephalon-or second of the five cerebral segments-whilst the unpaired large projecting lobe, usually considered as the metencephalon (cerebellum, fourth segment), was identified with the mesencephalon of higher Vertebrates, and the cerebellum was considered as being represented by a small transverse plate, often overlapped by the folded mesencephalon, and usually of no larger size than the piece similarly identified in the frog. In the present edition Professor Gegenbaur has modified this system of nomenclature, and has returned to the older and usually accepted method of naming the parts of the Fish's brain. Thus in Fig. 281, $d$ marks the two spherical masses which were in former editions assigned to thalamencephalon, and are now, as is usual with other anatomists, designated mesencephalon, the expansion between them and $g$ being the reduced area of the thalamencephalon. The letter $b$ is now referred to as metencephalon (cerebellum): this was previously referred to as mesencephalon; the myelencephalon prosencephalon, and rhinencephala retain their names, which had not been affected by MACLAY's system.

Whilst Professor Gegenbaur has returned to the usual system of naming these parts, he still considers that the facts on which MacLAY's nomenclature was based possibly point to homologies other than those indicated by the names ; so that the Fish's cerebellum does not necessarily agree with that of higher Vertebrata. He remarks : "The mesencephalon is usually considered as being confluent with the thalamencephalon in Selachians; and a part which really represents it, so far at least as relations of position are concerned, is customarily called by the name 'cerebellum.' "

In translating the German terms, Vorderhirn, Zwischenhirn, Mittelhirn, Hinterhirn, and Nachirn, I have adopted Professor Huxley's equivalents, namely Prosencephalon, Thalamencephalon, Mesencephalon, Metencephalon, and Myelencephalon. In the edition of Quain and Sharpey's Anatomy, published in 1867, a similar but not identical series of terms was suggested. For the "primitiven Hirnschlitz," - the early strongly-marked sinking in of the cerebral roof which separates the prosencephalon from the thalamencephalon -we have no special term in use ; "primitive cerebral cleft" is the translation which has been adopted.

It is worth while pointing out to the student, in connection with this subject, and in fact in relation to the whole of the chapter on the Vertebrata, that Professor Gegendaur assumes some small amount of familiarity on the part of the reader with descriptive human anatomy ; reference to a manual treating of this subject, on the part of the student who has not previously mastered it, is indispensable.

Nomenclature of the Parts of the Digestive Tract.-The translation in the present work of the simple word "Darm," and its compounds 
Vorderdarm, Mitteldarm, Hinterdarm, Kopfdarm, has caused me some perplexity. It has been variously rendered in the translation by "gut," "enteron," "enteric tube," "alimentary canal," "digestive tract." The fact is that, whilst we lave no definite nomenclature at present in use in English which recognises the true morphology of the canal which commences with the mouth and ends with the anus, the nomenclature in use in Germany is of very doubtful advantage, since it has not a sound morphological basis, but is altogether superficial. "Darm," for which our readiest equivalent is "gut," is used indifferently for the whole or for any part of the physiological entity which reaches from oral to anal aperture. But the English word "gut" is associated rather with the hinder than with the foremost portion of this tract. It will probably be found most convenient to speak of the physiological whole as the "alimentary canal," or "digestive tube ;" and these terms I have endeavoured consistently to make use of in this sense, though sometimes the term "enteric tube" has been similarly applied.

The division of this tube or canal into pharynx, œsophagus, stomach, and intestine ; or, again, into fore-gut, mid-gut, and hind-gut (Vorderdarm, Mitteldarm, Hinterdarm, p. 48), is one based upon superficial adaptations of form, and does not admit of a comparison of the parts so designated in the various phyla of the Animal Kingdom. The pharynx and the oesophagus of the Vertebrata are developed from the endoderm of the embryo; the parts which receive the same names in the Mollusca and the Arthropoda are developed from the ectoderm. The hind-gut of the Vertebrate is endodermal in origin, ectodermal in the Arthropod, and partly endodermal partly ectodermal in the Mollusca. In fact there is no attempt to recognise the facts of embryology in the terminology applied to the alimentary canal.

Under these circumstances I have proposed (Quarterly Journ. Microse. Science, April, 1876, and "Notes on Embryology and Classification," London, 1877, p. 11), to distinguish the primitive digestive space which develops from the endoderm (in fact the gastrula-stomach) as the "enteron." The anterior passage leading into this from the mouth, and formed by an ingrowth of ectoderm, I have termed the "stomodæum," and the corresponding passage leading from the anus I similarly propose to call the "proctodæum." These three primary factors of the alimentary tract are most equally developed in the Arthropoda and some Mollusca. In Vertebrata the stomodrum is exceedingly small, if indeed its true homologue exists at all (excepting in the Tunicata). The proctodrum is also in them evanescent. The middle portion of the alimentary tract formed from the primitive enteron (archenteron), which does not entirely coincide with that part to which the term "Mitteldarm" is applied, does not in all the rarious animal phyla take up the whole of the primitive enteron. This, in fact, only occurs in some of the Coelenterata, which may therefore be said to possess in the adult condition an archenteron. In other groups the 
primitive enteric sac gives off the foundations for a variety of other structures, so that what is left of it as the central element of the alimentary canal is a changed and broken-up enteron, which may be called "metenteron" as opposed to the unchanged "archenteron."

It is to these three morphological factors then, the metenteron, the stomodæum, and the proctodrum, that we are called upon to assign the various adaptational swellings, constrictions, and outgrowths of the alimentary tract of higher animals.

These distinctions are not recognised in Professor Gegenbaur's work. It will be sufficient here to point out that the exact limit of stomodieum and of proctodrum in any particular case, can only be ascertained by direct observation of the process of development. The metenteron is that part of the alimentary canal with which the most important digestive glands are connected, such as the liver, and from its walls they are formed as outgrowths. The stomodreum gives rise to salivary glands, and usually to masticatory sacs (gizzards), but these latter may form also in the metenteron.

The proctodæum forms the cloacal chamber, where such exists, and always receives the openings of glands (such as the Malpighian filaments of insects) which are excretory rather than accessory to digestion.

These explanations will be sufficient to make clear to the reader the sense in which the words "enteron" and "enteric" have occasionally been employed in the translation.

Classification.-At the present day, naturalists liave learnt to recognise in their efforts after what was vaguely called the "natural" system of classification, an unconscious attempt to construct the pedigree of the animal world. The attempt has now become a conscious one. Necessarily classifications which aim at exhibiting the pedigree, vary from year to year with the increase in our knowledge. They also vary according to the importance attached by their authors to one or another class of facts as demonstrating blood-relationships. Probably no two zoologists of the present day would agree, within wide limits, as to the classification which comes nearest to expressing the pecligree. Accordingly it is by no means desirable that students should be taught to accept any one scheme of classification as finite. 'They should be taught to look upon these schemes as the condensed expression of an author's views-as the epitome of his teaching, facilitating the recollection and the comparison of conflicting solutions of the vast series of unsolved problems of morphology.

I propose here, for the convenience of the student, to place side by side the general outlines of the schemes of classification adopted by Professor Huxley in 1869 (No. I.), that adopted by Professor Gegenbaur in the present volume (No. II.), and that which I have made use of in my lectures during the past year (No. III.).

I have taken the older classification adopted by Professor HuxLer rather than that more recently put forward by him, because it is one with which 
my experience as teacher and examiner has shown me that Linglish students are thoroughly familiarised.

I.

SUB.KINGDOMS.

Protozos.

(Rhizopoda, Gregarinida, Radiolaria, Spongida.)

INFTSORIA.

Celentritata.

(Hydrozoa, Aclinozoa.)

Axvulö̈DA.

(Scolecida, Echinoderma.)

Axvulosa.

(Crustacea, Arachnida, Myriapoda, Insecta, Chætognatha, Annelida.)

Molduscö̈D.

(Polyzoa, Brachiopoda, Tunicata.)

Mollusca.

(Lamellibranchiata, Branchiogastropoda, Pulmo. gastropoda, Pteropoda, Cephalopoda.)

VERTEBRATA.

(Pisces,Amphibia, Reptilia, Aves, Mammalia.)
II.

PIYLA.

Protozoa.*

VERIES. $†$

FCHI NODERMI.

BRACHIOPODA. +

Arthropoda.

Mollusca.

Tuxicata.§

VERTEBRATA.
III.

PHYLA.

\section{Protozon.*}

Porifera.

Nemitophor.

Peatyhelia.||

Gepityrea.\|

ECHINOdERM.

Enteropaeusta, \|

NeMatoidea. \|

Chatognatha.\|

Appexdiculata.

Mollusca.***

VERTEBRATA. + †

Seeing that one of my chief objects in superintending the translation of the treatise to which these few pages are introductory, has been to be able

* The Protozoa in Nos. II. and III. include the same organisms as in No. I., excepting that the Infusoria are included in that phylum in Nos. II. and III., and that the Sponges are excluded, being in No. II. placed under the Colenterata, and in No. III. forming the phylum Porifera under the "grade" Coclentera, as shown in the genealogical tree on the adjacent page.

t The Vermes of No. II. include all the Annuloïda of No. I. excepting the Echinoderma, which are raised to the rank of an independent phylum. They also include the Annelida (Chætopoda, Hirudinea, and Gephyrea) from amongst the Annulosa of No. I. and the Polyzoa from amongst the Molluscoilda of the same series.

\$ The Brachiopoda, raised to the position of a distinct phylun in No. II., are placed among the Molluscoïda in No. I. and amongst the Mollusca in No. III.

$\S$ The Tunicata, considered as an independent phylum in No. II., are found amongst the Molluscoïda in No. I. and form a section of the Vertebrata in No. III.

II The Platyhelmia, Gephyrea, Enteropneusta, Nematoidea, and Chrotognatha form in No. III. a number of independent phyla. Together with the Polyzon (included in No. III. under the Mollusca), the Rotifera, and the Chætoporda, included under the Appendiculata, they constitute the series of phyla which are in No. II. massed together as "Vermes."

- The Appendiculata include animals with lateral locomotive appendages, and nsually a segmented body. The group is, excepting that it has the addition of the Rotifera, nearly co-extensive with the Annulosa of No. I.

** The phylum Mollusca in No. III. includes the Polyzoa and Brachiopoda, excluded from it in both No. I. and No. II.

t† The Vertebrate phylum in No. III. includes the Tnnicata, which it will be scen by reference to page 70 are already placed on the Vertebrate stem by Professor Gegenbaur. 
to place the work in the liands. of the students of my own classes, I need not apologise for adding here further details of the classification which I find it most convenient to adopt in teaching. I have arranged the chief phyla first of all in the form of a genealogical tree, and secondly in a series exhibiting their subdivisions into classes, etc. This classification is of course to a large extent only a modification and adaptation of systems already put forward by other naturalists.

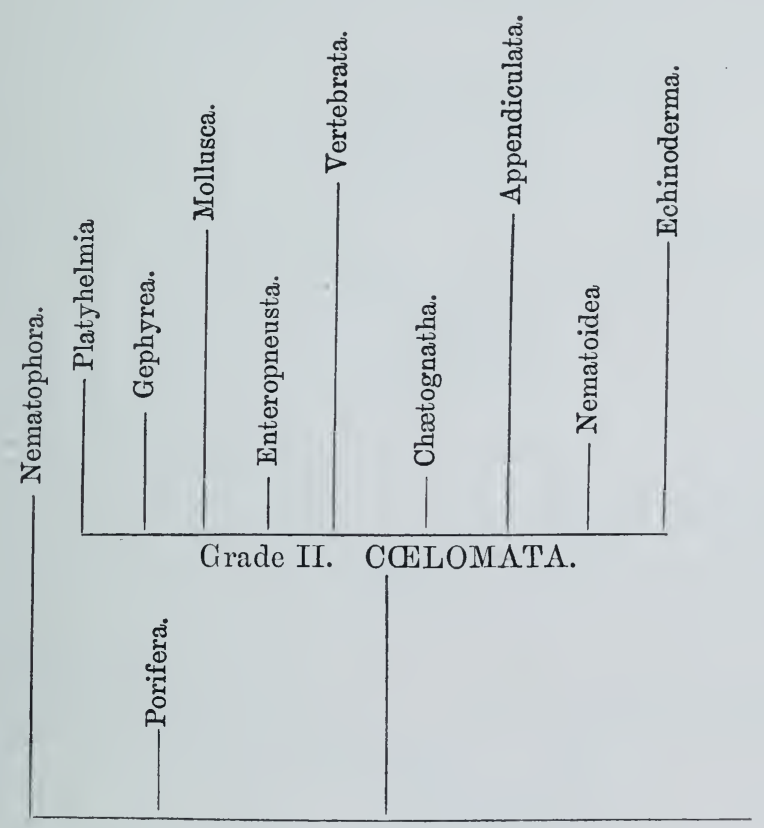

Grade I. CELLENTERA.

Grade II. ENTEROZOA.

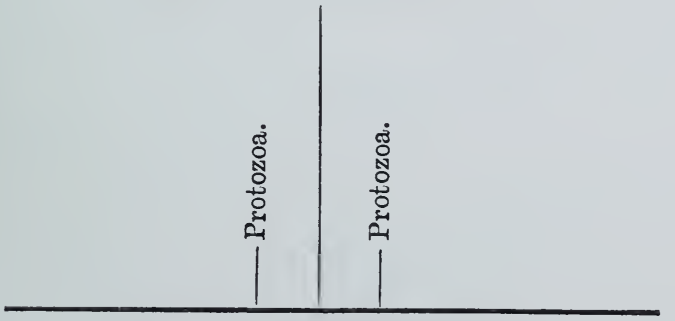

Grade I. PLASTIDOZOA. 
PROTOZOA.

GRADE A. GYMNOMYXA.

Class 1. Gymnomyxa.

GRADE B. CORTICATA.

Class 1. Lipostoma (Gregarinæ).

2. Suctoria.

3. Ciliata.

4. Flagellata.

5. Proboscidea (Noctiluca).

PORIFERA.

Class 1. Calcispongiæ.

2. Fibrospongiæ.

3. Myxospongiæ.

NEMATOPHORA.

Class 1. Hydromedusæ.

2. Scyphomedusæ.

3. Anthozoa.

4. Ctenophora.

ECHINODERMA

BRANCH. AMBULACRATA.

Class 1. Holothuridea.

2. Echinoidea.

3. Asteroidea.

BRANCH. TENTACULATA.

Class 1. Crinoidea.

2. Blastoidea.

3. Cystidea.

LATYHELMTA.

BRANCH. CILIATA.

Class 1. Planariæ.

2. Nemertina.

BRANCH. SUCTORIA.

Class 1. Trematoidea.

2. Cestoidea.

3. Hirudinea.

GEPHYREA.

Class 1. Echiuridæ.

2. Priapulidx.

3. Sipunculidæ.

4. Phoronidx. 
VERTEBRATA.

BRANCH. UROCHORDA (TUNICATA).

Class 1. Larvalia.

2. Saccata.

BRANCH. CEPHALOCHORDA.

Class. Leptocardia.

BRANCH. CRANIATA.

GRADE A. CICLOSTOMA.

Class. Cyclostoma.

GRADE B. GNATIIOSTONA.

Grade a. Heterodactyla branchiuta.

Class 1. Pisces.

2. Dipnoï.

Grade $\beta$. Pentadactyla branchiatu.

Class. Amphibia.

Grade $\gamma$. Pentadactyla lipotranchia.

$\left.\begin{array}{c}\text { Class 1. Reptilia. } \\ \text { 2. Aves. }\end{array}\right\}=$ Brauch. Monocoudyla.

3. Mammalia. = Branch. Amphicondyla.

APPENDICULATA.

BRANCH. CHETOPODA.

Class 1. Oligochæta.

Class 2. Polychæta。

BRANCH. ROTIEERA.

Class. (Orders only $)$

BRANCH. GNATHOPODA (ARTHROPODA):

GRADE A. IIALACOO'ODA.

Class. Peripatidea.

GRADE B. CONDYLOPODA.

Class 1. Crustacea.

2. Hexapoda.

3. Myriapoda.

4. Arachnida.*

* Following Prof. Ed. Van Beneden, I include Limulus, the Eurypterina, and Trilobites under the Arachnida as Branchiopulmonata. 
MOLLUSCA.

BRANCH. EUCEPHALA.

GRADE A. LIPOGLOSSA.

Class. Scolecomorpha (Neomenia).

GRADE B. ECIIINOGLOSSA.

Class 1. Gastropoda,*

2. Cephalopoda. $\dagger$

3. Scaphopoda.

BRANCH. LIPOCEPHALA.

Class 1. Tentaculibranchia (Poljzoa).

2. Spirobranchia (Brachiopoda).

3. Lamellibranchia.

The phyla Enteropneusta, Chætognatha, and Nematoidea, containing respectively the genus Balanoglossus, the genus Sagitta, and the various families of thread-worms, do not admit of subdivision into classes or orders.

* Includes the Chitons as a separate archaic grade "Amphomœa," the remaining Gastropoda, all of which are asymmetrical, being placed in a higher grade as "Cochlides."

+ Includes Siphonopoda (Cuttles and Nautilus) and Pteropoda.

E. RAY LANKESTER, Exeter College, Oxford.

September, 1878. 


\section{TABLE OF CONTEN'TS.}

Paragraph

1. 2. Introduction

Pago

3-10. Scope of Comparative Anatomy

\section{General Part.}

11. Structure of the animal body . . . . . . . 13

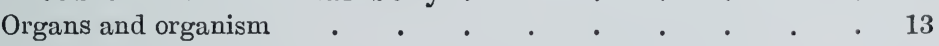

12. Differentiation . . . . . . . . . . . 14

13-15. Earliest stage of the animal organism. . . . . 1.5

The cell • • • • • . . . . . . . 15

16. Differentiation of the animal organism . . . . 18

17. Origin of the tissues . . . . . . . . . 20

18. 19. A. Vegetative tissues . . . . . . . . . 21

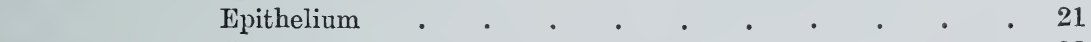

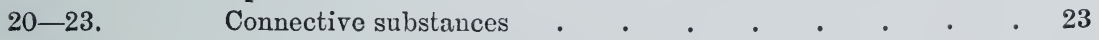

24. Form-elements of the nutrient fluid . . . . . . . . 29

25. B. Animal tissues . . . . . . . . . . 30

26. Muscular tissue . . . . . . . . . . 31

27. Nervous tissue . . . . . . . . . . 32

28. 29. Origin of the organs . . . . . . . . . 31

30. Systems of organs . . . . . . . . . . . . 37

a) Integument. . . . . . . . . . . 37

31. b) Skeleton . . . . . . . . . . . . 38

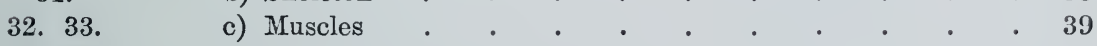

34. 35. $\quad$ d) Nervous system . . . . . . . . . . . 40

$36-38 . \quad$ e) Sensory organs . . . . . . . . . . . . . 42

$39 . \quad$ f) Respiratory organs of the integument (Dermal gills) . . 45

40. g) Excretory organs . . . . . . . . . 4 46

41. $\quad$ h) Alimentary Canal ․ . . . . . . . 47

42. Respiratory organs of the enteron . . . . . 49

43. 44. i) Vascular system . . . . . . . . . 50

45. $46 . \quad$ k) Reproductive organs . . . . . . . . . 52

47. Metamorphosis of the organs . . . . . . . 51

48. Development and degeneration . $\quad \cdot \quad \cdot \quad \cdot \quad \cdot \quad \cdot 54$ 
Paragraph

49. 50. Fundamental forms of the animal body

51. 52. Netamerism of the body . . . . . 61

53-55. Comparison of the organs . . . . . . . . . 63

56-58. Classification of the Animal Kingdom . . . . . 61

59. Bibliographical aids in Comparative $\Lambda$ natomy . . . . . 75

\section{Special Part.}

\section{First Section. Protozoa.}

60. General review of the group . . . . . . . 75 Bibliography . . . . . . . . . . . 77

61-70. Organisation of the Protozoa . . . . . . . . 57

Second Section. Cœlenterata (Zooplyta).

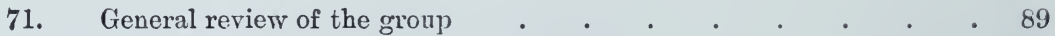

Bibliography . . . . . . . . . . . 90

72-78. Form of the body . . . . . . . . . . . 91

79. Appendages . . . . . . . . . . . 101

80. Integument . . . . . . . . . . . . 103

81. 82. Skeleton . . . . . . . . . . . 105

83. Muscular systen . . . . . . . . . . 108

81. Nervous system . . . . . . . . . . . . 108

85. Sensory organs . . . . . . . . . . . . . . 109

86-93. Alimentary canal . . . . . . . . . . . . 111

94-98. Generative organs . . . . . . . . . . 119

Third Section. Vermes.

99. General review of the group. . . . . . . . . 125

Bibliography . . . . . . . . . . . 127

100-103. Form of the body . . . . . . . . . 128

104. 105. Appendages . . . . . . . . . . . 132

$106 . \quad$ External gills . . . . . . . . . . . 135

107-111. Integument . . . . . . . . . . . 136

$112 . \quad$ Skeleton . . . . . . . . . . . . . . . 142

113. 114. Muscular system . . . . . . . . . . . . 142

115-121. Nervous system . . . . . . . . . . 145

122. 123. Sensory organs . . . . . . . . . . . . 152

124. 125. Visual organs . . . . . . . . . . 153

$126 . \quad$ Auditory organs . . . . . . . . . . 156

127-132. Alimentary canal . . . . . . . . . . 156

$133 . \quad$ Enteric branchix . . . . . . . . . 163

134. 135. Accessory organs of the alimentary canal. . . . . 164

136. Cœlom . . . . . . . . . . . 165 


\section{Fourth Section. Echinoderma}

157. General review of the group _ . . . . . . . 192

Bibliography . . . . . . . . . . . . 191

158-161. Form of the body . . . . . . . . . . . . 194

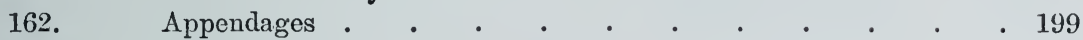

163-167. Integument and dermal skeleton . . . . . . . 200

168. Muscular system . . . . . . . . . . 207

169. Nervous system . . . . . . . . . . 208

170. Sensory organs . . . . . . . . . . 210

171-173. Alimentary canal . . . . . . . . . . 211

174. Organs appended to the alimentary canal . . . . . 215

175. Colom • • • • • • • . . . . . 216

176. Vascular system $\quad$. $\quad$. . . . . . . . . 217

Blood-vessels . . . . . . . . . . 217

177. 178. Water-vessels . . . . . . . . . , 219

$179 . \quad$ Excretory organs . . . . . . . . . . 224

180. 181. Generative organs . . . . . . . . . . 224

\section{Fifth Section. Arthropoda.}

182. General review of the group . . . . . 228

Bibliography . . . . . . . . . . . 232

183. Form of the body . . . . . . . . . . 234

184. Appendages . . . . . . . . . . . . . 237

185. Appendages of the Branchiata . . . , . . . 238

186. 187. Branchiæ . . . . . . . . . . 240

188-190. Appendages of the Tracheata . . . . . . . 243

191-193. Integument . . . . . . . . . . 248

194. Muscular system . . . . . . . . . . . 251

195-200. Nervous system . . . . . . . . . . 252

201. Sensory organs . . . . . . . . . . . . . . 260

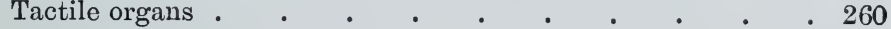

207

212.

gans

Visual organs

Alimentary canal

1) Appendages of the fore-gut . . . . . . 273

213.

2)

mid-gut

214.

215.

216-220.

Cœlon

hind-gut

221.

$222-225$.

Vascular system

$226-237$.

Excretory organs

Tracheæ . 
Sixth Section. Brachiopoda.

Paragraph

238. General review of the group

Page

Bibliography

239.

210.

$2+1$.

242.

213.

214.

2.15 .

216.

Form of the body

Integument, shell, and arms

Muscular sjstem

Nervous system and sensory organs

Alimentary canal

Colom and circulatory organs.

Excretory organs

Generative organs .

307

307

309

309

309

310

310

312

314

Seventh Section. Mollusca.

217. General review of the group

Bibliography

248-252. Form of the body .

253. 254. Appendages

255. 256. Integument .

257-259. Shell . .

$260-263$.

264.

Gills

265.

Internal skeleton

$266-269$

Muscular system

270.

271.

272. 273.

274.

$275-279$. 280.

281.

282.

283.

$281-288$.

289-292.

293-298.

Cœlom

Nervous system

Central organs and nerves of the body

Visceral nerves

Sensory organs

Tactile and olfactory organs

315

317

318

325

328

329

335

3.11

342

313

343

351

351

351

353

356

Auditory organs

Alimentary canal

Organs appended to the alimentary canal.

358

363

1) Appendages of the fore-gut

363

2) Appendages of the mid-gut

364

3) Appendages of the hind-gut

366

367

368

375

Excretory organs

380

Eighth Section. Tunicata.

299. General review of the group

Bibliography .

300. 301 .

302.

Form of the body.

303.

Integument

301.

Skeleton

305. 306 .

Muscular system

Nervous system 
Paragraph

307.

308.

$309-311$.

312.

313.

314.
Sensory organs

Page

Alimentary canal . . . . . . . . . . . . . . 398

Respiratory antechamber (Branchial cavity) . . . . 399

Enteron . . . . . . . . . . . 403

Vascular system . . . . . . . . . . 404

Generative organs . . . . . . . . . . 406

\section{Ninth Section. Vertebrata.}

315. General review of the group . . . . . . . . 408

Bibliography . . . . . . . . . . . 412

316. Form of the body . . . . . . . . . . . . 413

317. 318. Appendages . . . . . . . . . . . . . 414

319. 320. Integument . . . . . . . . . . . 417

321-323. Epidermal structures . . . . . . . . . . . . 419

324-326. Dermal skeleton . . . . . . . . . . 422

327. Internal skeleton . . . . . . . . . . 426

328-334. Vertebral column . . . . . . . . . 428

335-337. Ribs . . . . . . . . . . . . 438

338. Sternum . . . . . . . . . . . 442

339. 340. Cephalic skeleton . . . . . . . . . . . 444

341-352. Skull . . . . . . . . . . . . 447

353-356. Branchial skeleton . . . . . . . . . 468

357. Skeleton of the appendages . . . . . . . 472

358-360. Anterior appendages . . . . . . . . 474

Shoulder-girdle $. \quad . \quad . \quad . \quad . \quad . \quad . \quad . \quad .474$

361-365. Anterior extremity . . . . . . . . . 477

366. Posterior appendages . . . . . . . . 484

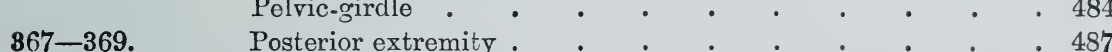

370. Muscular system . . . . . . . . . . . 491

371. Dermal muscles . . . . . . . . . . 492

372-377. Musculature of the skeleton . . . . . . . 493

378. Electric organs . . . . . . . . . . . . . 499

379. Nervous system . . . . . . . . . . 501

380. 381. A. Central organs of the nervous system . . . . . 503

382. 383.

384.

385.

386.

$387-392$.

393.

394. 395.

396.

$397-399$.

$400-403$.

404.

405.

a) Brain . . . . . . . . . 503

b) Spinal chord . . . . . . . . . . 511

c) Investments of the central nervous system . . . 512

B. Peripheral nervous system . . . . . . . 513

a) Spinal nerves . . . . . . . . . . 514

b) Cerebral nerves . . . . . . . . . 515

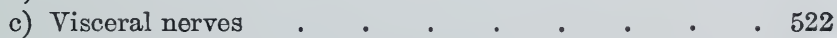

Sensory organs . . . . . . . . . . . 523

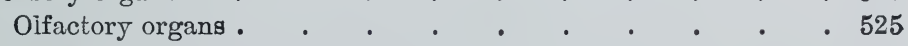

Visual organs . . . . . . . . . . . 50.527

Auditory organs . . . . . . . . . . . . 533

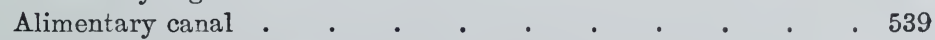

Respiratory antechamber (Cephalic enteron) . . . . 540

406-\$0Y. Branchiæ . . . . . . . . . . . 541 
Paragraph

410 .

411. 412. 413.

$414-416$.

417.

418.

419.

420.

421.

422.

423.

424.

425-427.

428.

429. 430 .

431-436.

$437-442$.

$443-446$.

$447-449$.

$450-458$.

\author{
Branchial clefts and palate of the Amniota
}

Page

Nasal cavity

Buccal cavity

Organs of the buccal cavity

Alimentary canal proper (Enteron of the trunk)

Fore-gut

Mid-gat

Hind-gut

Organs appended to the mid-gut

Mesentery

Pneumatic organs of the enteric tube

a) Air-bladder

b) Lungs

Cœlom

Vascular system

Heart and arterial system

Venous system .

Lymphatic system

Excretory organs .

Generative organs . 


\section{CORRIGENDA.}

Page 79, six lines from top, for "finer" read "firmer."

" 87, three lines from top, for "generation" read "gemmation."

" 88 , two lines from top, for " spiral " read "special."

" 88 , in the explanation of Fig. 30, for "broad" read "brood." 



\section{Introduction.}

\section{The Scope of Comparative Anatomy.}

$\S 1$.

THE department of science which has organic nature for the object of its investigations, breaks up into two great divisions, Botany and Zoology, corresponding to the two kingdoms of organised nature. The two disciplines together form the science of living natureBiology. They are closely connected with one another, in so far as the phænomena seen in both the animal and vegetable kingdoms rest on the same fundamental laws, and in so far as, notwithstanding the differences of their special arrangements, animals and plants have common beginnings, and are, in the economy of nature, closely interdependent.

In both of these disciplines several kinds of investigation are possible, and from these new disciplines arise. Putting aside the realm of Botany, let us follow out that of $\mathrm{Z}$ oolog $\mathrm{y}$ into its further subdivisions. The investigation of the functions of the animal body, or of its parts, the reduction of these functions to elementary processes, and the explanation of them by general laws, is the business of Physiology. The investigation of the material substratum of those functions, and accordingly of the phænomena of form of the body and its parts, as weil as the explanation of the phænomena of form by reference to function, is the business of Morphology. Physiology and Morphology have thus different duties, and their methods also are different; but for each it is necessary, although in different ways, to keep in view the other, as well as the common aim, which is indicated in the term Biology.

Morphology again is divided into Anatomy and Embryology; while the former has for the object of its investigations the adult organism, the latter has the growing organism as the object of its study. 
Anatomy may be divided into general and special Anatomy. General Anatomy has to do with the fundamental forms of animal organisms (Promorphology), and the morphological phænomena which arise from them. Special Anatomy takes for its object the organological composition of the animal body. Histology, or microscopic Anatomy, forms one of its branches, being the study of the elementary organs of the animal body. Embryology explains, by tracing their gradual development, the complications of the external and internal organisation, and, in fact, deduces them from simpler conditions. The changes in organisation can be followed out in the embryonic life of the individual, and also in the continuous series of organisms. The discipline ordinarily known as Embryology deals with the former; and as Ontogeny (or the development of the individual) is contrasted with Phylogeny (or the development of the phylum). As the latter includes the earlier and no longer existent conditions of animals, it also embraces Palæozoology. It is the history of the development of the series of organisms in their geological succession.

\section{$\S 2$.}

Since Anatomy has for its object the composition of organisms, it may be considered as the doctrine of structure, and is divided, according to the different points of view from which structure itself may be regarded, into several divisions. When the composition of the body itself, its forms, and the relations of the separate organs are taken as its scope, it is known as descriptive Anatomy, for it describes the objects examined, without drawing any further conclusions from them. Anatomical fact is the aim of the investigation, and empiricism satisfies this aim. Owing to its relations to medicine, and so to practical requirements, the descriptive Anatomy of the human organism, so far as it is restricted to a special series of facts, has become developed into a special branch, which, as Anthropotomy, is put side by side with the similarly descriptive Zootomy. The two differ only in their subject-matter, and not in their treatment of it, for both are analytical. In proportion as either abstains from drawing conclusions from its series of facts, and giving these the value of abstractions, is it wanting in the character of a science; for a science is constituted neither by an extensive range of observations, nor by the complication of the methods by means of which such observations are made. A critical appreciation of the scientific import of any branch of study has, therefore, little to do with the mechanical apparatus of investiga- 
tion, which has its value only in facilitating the discovery or demonstration of facts.

Anatomy assumes a very different character so soon as the knowledge of facts is only its means, and its aim the conclusions which can be drawn from an assemblage of such facts; the facts of individual phænomena being regarded not by themselves exclusively, but being brought into relation with one another. This happens when what is alike in the organisation of different organisms is made the object of search, and when the facts thus acquired are compared. Anatomy thus arrives at scientific results, and shapes the results of inductive inquiry into deductive conclusions. Thus it becomes Comparative Anatomy. Its method is synthetical. The analyses of Descriptive Anatomy (Anthropotomy as well as Zootomy) provide the basis for it; they are consequently not only not excluded from Comparative Anatomy, but are most closely embraced and logically permeated by it. The more careful the sifting of facts, the surer the basis of comparison. Empiricism is thus the first requisite, and abstraction is the second. Abstraction has no basis without pre-existing empiricism; and empiricism by itsel'f is, from the scientific point of view, a mere stepping-stone to real knowledge.

\section{$\S 3$.}

The task of Comparative Anatomy is the morphological explanation of the phænomena of form met with in the organisation of the animal body. Comparison is the method which serves for the performance of this task. It shows the way which scientific investigation has to go, and which it is necessary to know in order to avoid disjointed and fruitless labour. The comparative method seeks to test, in series of organisms, the morphological results of the observation of the organs of the body, places together similar characters, and separates the dissimilar from them. Thus it takes into consideration everything which can in any way be looked at as the result of anatomical observation: relation to other parts of the body, form, number, extent, structure, and texture. It thus collects series of stages for the several organs, in which the extremes may be so far different from one another as not to be recognised, but which are united to one another by numerous intermediate steps.

It is clear, in the first place, from the existence of various forms of one and the same organ, that the physiological value of an organ in different stages is not by any means the same, but that an organ, as its anatomical characters are modified, may come to have very different functions. The exclusive consideration of their physio- 
logical duties may thus bring organs which are morphologically connected into very different categories. Thence results the subordinate importance which we must assign to the physiological duties of an organ when we are engaged in an investigation in Comparative Anatomy. Physiological value is only to be regarded at all, and then in the second place only, when we are trying to make out the relations to the entire organism of those modifications which an organ may have undergone as compared with some other condition of the organ.

By this examination of anatomical facts, by means of comparison, Comparative Anatomy demonstrates the connection of entire series of organs. Within these series we find changes of the most varied range, sometimes slightly, sometimes widely extended; modifications which affect the size, number, form, and even the texture, of the parts of an organ, and which may even lead to changes in its situation. The review of such a series teaches us then to recognise a progress presented in those several successive stages, which the modifications of one and the same organ in different animals exhibit to us.

\section{$\S 4$.}

We ascribe the existence of a certain amount of similarity in the organisation of certain larger or smaller divisions of the animal kingdom to Transmission-a phænomenon which is exhibited in the passing on of its organisation by a given organism to its posterity. The descendants repeat the organisation of the parental organisms. This is an indubitable fact. Nevertheless now and again objections are raised either to the existence of 'Transmission or to its significance. The similarity of the organisation of the descendants and their ancestors is then ascribed, not to Transmission, but to certain physical forces acting during embryonic life. In reply, we may ask, how does it happen that in ancestor and descendant these forces are the same-viz. all those forces of tension, of pressure, and so on, from which it is sought to deduce the building up of the embryo? If, for example, a joint gets its ontogenetic development by the movement of the parts of the skeleton by means of muscular activity, a certain arrangement of the muscles is presupposed, and a perfectly definite structure of the muscles; and for these again, a perfectly definite number and arrangement of the morphological elements which make up a muscle. This being so we must ask, whence comes the definite arrangement of these parts? whence arises the similarity of arrangements in the ancestors and the descendants?

We find, in fact, that we must give full recognition to the exist- 
ence of the transmission of properties, and recognise in it a phænomenon of general prevalence, which may indeed present modifications of, but never exceptions to, certain definite laws. We may deduce it from the conditions involved in propagation, and thus explain it to a certain degree; for it is clear that portions of an organism, if they give rise to a new organism, will carry on to it the peculiarities which the primitive organism possessed. This is clearest in the lower organisms, which are propagated by mere division. Each portion forms at once an organism like the first. But from this there extends a continuous series of methods of propagation, up to those in which generative products come into action, which are quantitatively very different, although in all cases derived from the division of the parent organism.

The new organism in this case also represents in actual sub. stance the continuation of the ancestral, and will therefore possess qualities which agree with those of the latter.

The amount of similarity or agreement in the organisation of animals is very various. We recognise animals which differ from one another by slight points only; then those which are separated by considerable differences; and again others which, in external or internal organisation, present the greatest differences. Thus agreement, as well as variation, is found in interminable gradations. We call things which are more or less like to one another, "related;" and in like manner, when organisms exhibit likeness, we use that word to denote the reciprocal connection, but in this case we give to it its full meaning of blood-relationship.

We recognise similar organisms as related to one another, when we can explain the similarity of the organisation by common inheritance. But the degree of this similarity measures the degree of relationship which we can deduce from it. Relationship can be regarded as close when the differences are slight; while when the differences are great it must be regarded as more distant. We thus substitute for the conception of the agreement, or likeness, of the organisation, that of relationship, for we regard the agreements which obtain in the organisation of a collection of organisms as inherited peculiarities.

The doctrine of the Blood-relationship of Organisms or Phylogeny is based on the law of inheritance. Comparative anatomy thus reveals the relations of affinity within the various divisions of the Animal Kingdom by pointing out what is alike and what unlike. [A full account of this most important law of inheritance and its phænomena is to be found in Ḧ̈CKEL's luminous essay on the subject (Generelle Morphologie, vol. ii. p. 170).] 


\section{$\S 5$.}

By means of inheritance, characters are passed on to the organism, which are afterwards matured in the course of its individual development (Ontogeny). There is no such development in the simplest forms, inasmuch as the young, which arise by division of the maternal organism, only need increase in size to make them like the maternal organism. In this case, development is the same thing as growth, which is completely coextensive with it. The farther an organism is from a primitively simple condition, or the greater the sum of characters which have been inherited from its ancestors and transmitted to their descendants, the less simple is its ontogeny ; for during it a part at least of the characters which have been inherited from its ancestors are repeated, and are presented by the developing body in several successive stages. Ontogeny thus represents, to a certain degree, palæontological development, abbreviated or epitomised. The stages which are passed through by higher organisms in their ontogeny, correspond to stages which are maintained in others as the definitive organisation. These embryonic stages may accordingly be explained by comparing them with the mature stages of lower organisms, since we regard them as forms inherited from ancestors belonging to such lower stages. Regarded from this point of view, many of the so-called larvalstages, with their "provisional organs" - so named because they are transitory, and limited to the earlier stages of life only-are seen to be forms of great importance, and full of significance. Such organs, besides having physiological relations to the organism which possesses them, in consequence of which they are preserved as practical arrangements, and become heritable, can be recognised in lower grades of the existing series of animal forms, and thus reveal the phylogeny of the animal that possesses them. The "stadium larvatum" then, notwithstanding its name, often points out with great clearness the blood-relationship of an organism. At times these larval organs are not so well explained by transmission as by adaptation, and thus the estimation of their true meaning is made more difficult. The significance of these arrangements is more obvious in organisms which do not enter immediately into the "struggle for existence" in the external world, but are developed for a certain time within the coverings of the ovum, and so are less exposed to the moulding influences of the outer world. In these cases they are "provisional " arrangements, and may be with greater certainty regarded as having been transmitted, and consequently as repetitions of lower stages. The branchial clefts which appear in 
the embryos of the higher Vertebrata, but by-and-by disappear, are structures of this kind. Regarded alone they are inexplicable, for they neither lead to the formation of gills at any time, nor are they converted (with the exception of the anterior) into definitive organs of any other kind. Comparison shows us, however, that in a large division of lower Vertebrata these branchial clefts are important organs of respiration; and as we also know Vertebrata (Amphibia), in which the clefts function only for a time as respiratory organs, and close up later on, we are able to comprehend the branchial clefts of reptiles, birds, and mammals, as arrangements obtained by transmission from lower stages, which have lost their primitive function, and remain for a short time only-during fœtal life.

\section{$\S 6$.}

In the sum of the characters of the organisation, which inheritance passes on to an organism, we find, in consequence of what has been already pointed out, a greater or smaller number of arrangements, which pass on into the permanent adult stage of the organism without having any recognisable function in it. Such parts are, as a rule, seen in a more or less atrophied and rudimentary condition, which they often do not acquire until the ontogeny has run its course. In the early stages of the ontogeny they generally agree in completeness with the other arrangements which obtained in the ancestral form from which they are derived. These rudimentary organs commence to atrophy the earlier in proportion as they were inherited earlier, in a palæontological sense ; and, as a rule, disappear late when their origin is a relatively late one. The fullydeveloped form of the rudimentary organs is consequently to be found, in the former case, in widely separated species; in the latter, on the other hand, in species more closely allied. These organs are valuable objects, since phylogenetic relations can be very generally recognised by their aid. They show, too, how little physiological significance ought to be regarded in a morphological discussion; for in most of them a function is not to be made out at all, or, if it can be made out, is found to be quite different to the primitive one.

\section{$\S 7$.}

Comparative Anatomy forms part of Ontogeny, inasmuch as it treats of the phænomena of the organisation which appear in the course of the individual development of the animal; not only in relation to the complete stage of the organism, but in relation also to the definitive arrangements of other organisms. Comparative 
Anatomy explains the phrnomena of Ontogeny. Ontogeny by itself does not rise above the level of a descriptive discipline, and in proportion to the exactness of its investigation possesses a value as so much objective material. At the same time Ontogeny gains scientific importance by its connection with Comparative Anatomy. Its facts, which by themselves are incomprehensible, or are only teleologically explicable in a metaphysical sense, because restricted to the later events in the history of an organisation, are, by Comparative Anatomy, put in connection with the known phænomena of other organisms, and are thereby rendered explicable phylogenetically. The necessity of an exact knowledge of Comparative Anatomy for Ontogeny is sufficiently obvious. Just as little can the former separate itself from the latter : since from Ontog'eny Comparative Anatomy gains an insight into the lower stages of organisation. To the same extent, and in the same way as Ontogeny helps to form the basis of Phylogeny, does it render indispensable service to Comparative Anatomy.

A "Comparative Embryology" has sometimes been put in contrast with Comparative Anatomy; of course merely as a theoretical division of the scope of study. A "comparative" Ontogeny of this kind must, just as much as every individual ontogeny, have regard to the organisation of the fully-developed stage; and, in fact, without Comparative Anatomy it cannot lead to any scientific results.

\section{$\S 8$.}

The relations of every organism to the outer world in which it lives, from which it obtains material, and to which again it gives it up, cause the outer world to have an influence on the organism. This influence is practically seen in the changes of the organism, which depend further on a Variability which is inherent in it.

Variability comes under our observation as the capacity for adaptation, and in effect operates as a modifying and even metamorphosing agent upon the inherited organisation.

The organism is altered according to the conditions which influence it. The consequent Adaptations are to be regarded as gradual, but steadily progressive, changes in the organisation, which are striven after during the individual life of the organism, preserved by transmission in a series of generations, and further developed by means of natural selection. What has been gained by the ancestor becomes the heritage of the descendant. Adaptation and Transmission are thus alternately effective, the former representing the modifying, the latter the conservative principle. The endless variation of the phænomena of organisation is, we see, consequently 
due to Adaptation, just as we have seen that similarity is due to Transmission.

\section{$\S 9$.}

Adaptation is commenced by a change in the function of organs, so that the physiological relations of organs play the most important part in it. Since adaptation is merely the material expression of this change of function, the modification of the function as much as its expression is to be regarded as a gradual process. As a rule, therefore, Adaptation can be perceived by its results only in a long series of generations, while transmission can be recognised in every generation. Although Adaptation as a process cannot be directly observed, it is nevertheless possible to infer it with certainty by comparison. When, for example, we find a simple structure of the stomach in the Carnivorous Mammalia, and, on the other hand, a more complicated one in the Herbivora, and especially in those which take in large quantities of food, as, for example, the Ruminantia, we are entitled to consider the complication in the structure of these stomachs as a change caused by the food-as an Adaptation to the mode of nutrition; and when, further, the ontogeny of the Ruminantia shows us a form of stomach which is simple in the early stages of development, and is gradually converted into the more complicated condition, ontogeny confirms the supposition we have already gained by comparison. In many cases the influence of Adaptation on the organisation can be observed also directly; as, for example, in many Amphibia, where the branchiæ which are developed during the larval stage are retained in function for an extended period, if the opportunity of escaping from the water does not arrive; and in others, again, where the branchiæ undergo atrophy, as soon as their aquatic life has been exchanged for one on land, although their nearest allies live in the water, and always retain the branchir. In the one case we see development, and in the other atrophy, as phænomena resulting from Adaptation.

In Adaptation, the closest connection between the function and the structure of an organ is thus indicated. Physiological functions govern, in a certain sense, structure; and so far what is morphological is subordinated to what is physiological. This dependence of the form of an organ on its activity is seen in the most elementary way in the matter of size. When the function is increased, there is an increase in the size of the organ. The muscular system shows to what extent increase of activity affects size. Without exercise the muscles undergo degeneration, till they completely disappear. If they are kept in exercise, and if the demands on them are increased, they develop to a considerable size. The amount of 
development is in the closest connection with the amount of activity. But since, when a function ceases or diminishes, atrophy commences, we obtain, as a result of the process, rudimentary organs. They owe their origin to atrophy. Physiology alone, then, can give us the explanation of the origin of these organs; and thus again we are led to observe the great influence which it exerts on the study of Morphology.

\section{$\S 10$.}

An organ can be so much changed by the gradual modification of its function that it becomes, from the physiological point of view, a new one, and is then placed in quite another physiological category of organs. This fact is of considerable importance, for it helps to explain the appearance of new organs, and obviates the difficulty raised by the doctrine of evolution-viz. that a new organ cannot at once appear with its function completely developed; that it therefore cannot serve the organism in its first stages whilst it is gradually appearing; and that consequently the cause for its development can never come into operation. Every organ for which this objection has the appearance of justice can be shown to have made its first appearance with a significance differing from its later function. Thus, for example, the lungs of the Vertebrata did not arise simply as a respiratory organ, but had a predecessor among fishes breathing by gills, in the swim-bladder, which at first had no relation to respiration. Even where the lungs first assume the functions of a respiratory organ (Dipnoi, many Amphibia) they are not the sole organ of the kind, but share this function with the gills. The organ is therefore here caught, as it were, in the stage of conversion into a respiratory organ, and connects the exclusively respiratory lungs with the swim-bladder, which arose as an outgrowth of the enteric tube and was adapted to a hydrostatic function.

The earlier function of an organ which by adaptation is converted to new uses is generally a lower one, and less important for the organism, in comparison with the new function which is taken on, so that the organ thus rises to a higher grade. In other cases the value of the primary function is less, because it is shared by other similar organs. It is then quantitatively lower, for a share is taken by the other similar organs in discharging the total amount of the function necessary for the full activity of the organism. The atrophy of some of the organs which are of equal value raises the value of those that remain by causing their higher development. To these facts, as to their change of functions, the difference in the classification of organs, accordingly as we make use of a physiological or a morphological method, is due. 
GENERAL PART. 



\section{Structure of the Animal Body.}

\section{The Organs and the Organism.}

$\S 11$.

In the living body we observe a number of activities of its material substratum, by which the series of phænomena spoken of as life are conditioned. Underlying these, there are chemico-physical processes, which are accompanied by a continual degradation of the material, and consequent metastasis, or change in the arrangement of chemical elements. The body nourishes itself by replacing the material used up in metastasis by fresh matter, which is received from without; and this it assimilates, or makes like to the substances of which it is itself composed. The substances, partly taken in with the nutrient matter, partly produced by metastasis, which are of no further use in the organism, are passed out. Hence results the excretory function. If the quantity of matter assimilated is greater than that which is expelled, there is an increase in the size of the body: it grows. Thus it fulfils the first condition for the production of that material from which a new organism, like to itself, arises : and so reproduction is closely connected with nutrition.

The body is, in the first place, in relation to the exterior by its surface; this puts it in connection with the surrounding medium. Changes in the form of the surface of the body result in movements, and give rise to locomotion. The surface also is the medium by which it perceives the outer world, or has sensations.

The parts of the body which preside over these processes are the instruments by which life is carried on-organs. In virtue of their existence the body is an organism, and when we also include under the term "organisms" certain bodies in which no organs can be individually separated, it is because the virtual existence of organs in them is to be assumed from the mere fact that life is carried on in them. The term "organism" is therefore employed in this instance, not in an anatomical, but in a physiological sense. In the simplest condition of the organism, the vital phænomena take place in the homogeneous substance which forms the body, and which is the seat of all these processes equally. The body 
in this case represents, potentially, a collection of organs, which only appear, in fact, when the different functions are no longer performed by every part of the body. The condition which the lower organisms permanently exhibit in regard to this matter is possessed for a short time only by the more complicated.

\section{Differentiation.}

$\S 12$.

The complication of the organism arises from a process of division which transfers to separate parts the physiological activities of the primitively homogeneous body. What was previously accomplished by the whole body, is, subsequently to that process, carried out by particular portions. The work is then done either by a large number of parts, which are distinct from but similar to one another, or the separate parts acquire dissimilar shapes, and become different from one another. In the first case the division of la bour is quantitative, in the latter it is qualitative, and the separation of the different parts is in correspondence with a difference in function. According to the degree in which the separation or division originally set up in the primitively indifferent body is repeated in the organs derived from it, further complications arise, which present for our observation a step-by-step progression in development. Hence there arises a difference in the value of the organs, and it becomes necessary to distinguish their higher and lower conditions.

The distribution of work amongst a number of different organs leads to the perfecting of the operations of such organs. Each organ is enabled to develop in a definite direction, in correspondence with the particular function which is undertaken by it. The organism thus becomes more highly developed, as well as complicated. Division of labour leads to a perfecting of the whole organism. According as the division of labour involves only a few or many organs, a greater or less part of the organism is brought under the operation of this perfecting influence. The greater the importance, for the whole organism, of the organs affected, the more considerable is the perfecting accomplished in it through their modification. The functions which attach themselves to definite parts of the body bring about a difference in the development of those parts proportionate to their own difference; and thus it is that new parts and new organs arise, which are different from those already existing. The division of functions leads to the establishment of a difference, that is to a differentiation, of the parts. A part of the body which was formerly like the rest, and consequently not different from it-that is was indifferent-passes into the condition of being separate, becomes distinguishable, or different from the rest. And as this differentiation is connected with the division of labour, inasmuch as it is conditioned by it, it may be regarded as the product of it. Every physiological function can be again divided qualitatively into various sub-functions, by the locali- 
sation of which fresh organs again arise. 'Thus the principle of the division of labour is the cause of very great variation in the organisation, and all morphological phænomena are more or less closely connected with it, and with the differentiation which is due to it.

\section{First Stage of the Animal Organism.}

\section{The Cell.}

\section{$\S 13$.}

Living matter appears in its simplest form as an albuminous substance, known as Plasma, or Protoplasm, which, by the aid of our present optical instruments, seems to be homogeneous throughout. This substance occurs in the form of small lumps, in which condition we find the simplest organisms. In those simplest forms, where the protoplasm is homogeneous, and in which only a few granules at most are present as heterogeneous elements, there is no limitation of the lump to the exterior by distinct enveloping structures; but in organisms of little higher grade we find an envelope produced by a chemico-physical change in the most external layer of the protoplasm. Thus the protoplasm, which is endowed with all the phænomena of life, and even of movement, is enclosed by a more or less firm envelope, which forbids alterations in form, and is the cause of a definite shape being maintained. Such structures may be combined to form complex organisms, as is the case in many of the lower plants. This kind of form-element, or morphological unit, is known as the cytod, and is rightly distinguished from another more highly-developed form.

In this higher form there arises in the protoplasm a sharply marked-off dense structure, which is called the nucleus. It is the product of the first process of differentiation of the protoplasm, which no longer alone represents the living substance. In the nucleus a small body, the nucleolus, generally appears. The nucleus, unlike the protoplasm, is not contractile, or at any rate has not a large share of contractility; but it not only takes a part in most of the vital phrnomena of the surrounding protoplasm, but frequently gives evidence of being their regulator. Such corpuscles of protoplasm as are provided with a nucleus are called cells (cellulæ). These structures, like the cytods, may form independent organisms, which are then called "unicellular." When the cells form a complex by multiplication, we have a multicellular organism. The smallest parts of multicellular organisms, no longer separable into constituent pieces like to one another, are cells; and are therefore the form-elements of these organisms. The same remark applies to the cytods, or the simpler condition. While these, however, are rarely present, we find cells widely distributed in the Vegetable Kingdom, and as the sole form-elements in the Animal Kingdom. 


\section{$\S 14$.}

In the indifferent condition, that is as long as changes in a definite direction do not arise, leading to the formation of definite new structures, the cells of all animal organisms appear to have essentially the same character. In them we distinguish according to what has been remarked above: first, the protoplasm, which forms the principal mass of the body of the cell; and secondly, the cell-nucleus, surrounded by, and different from, the protoplasm, than which it is usually more dense. The share which this nucleus takes in many varied phænomena of the life of the cell, compels us to regard it as by no means a subordinate part of the cell-body. In addition to these parts of the cell, some persons recognise (formerly everyone did so) a membrane which differs from the protoplasm or contents of the cell, and envelopes it: from its presence arose the notion of the "vesicular form " of the cell and its name.

Although it cannot be denied that in many cells there are envelopes differentiated from the protoplasm, yet this condition is never found in the earliest life of the cell, but is always the result of an advanced change, and of the passage of the cell into a differentiated form.

Automatic movements of the protoplasm of the cell are such common manifestations of their life, that they are always definitely apparent as a property of all cells which are not highly differentiated, that is of cells in which the protoplasm is not metamorphosed. In free cells, and such as are not enveloped by firm membranes, this phænomenon of movement produces locomotion. Even in cells that are not free movement may be observed, consisting partly in a change in the form of the surface, partly in a change in the position of the granules in the protoplasm. That there are also properties resident in protoplasm which we may attribute to sensibility of a very low grade results from many experiments and observations.

We may further observe nutrition in the cell. At times, indeed, we can see the protoplasm ingesting food; in all cases the growth of the cell is an express indication of its nutrition. This phænomenon of growth, which may be seen in any indifferent cell, is expressed by the increase. in size of the protoplasmic body, owing to the assimilation of matter from without. The growth of the cell may be quite regular, increase in size obtaining in all directions, as is the case with all cells in their earliest stages; as long as this lasts the cell completely retains its spherical form, unless its movements or external influences modify it. Or growth may be unequal, and the cell become elongated by increase in size along one axis; or, again, it may become stellate by growing along sereral axes. Irregular increase of this kind is ordinarily accompanied by differentiation of the cell-substance, and is therefore the commencement of the conversion of the cells into tissue. 


\section{$\S 15$.}

Another phænomenon - that of reproduction-is a result of, and is indissolubly connected with, the growth of the cell; for multiplication is merely the extension of growth beyond the limits of individual cohesion. There are various modes of cell-multiplication; the simplest of these is a direct result of growth. A bud is formed by the cell-body growing out on one side. This gradually increases in size, and breaks off from the mother-cell, when it becomes a new free cell. The number of young cells which are budded off from a single cell is not always the same, also the part taken by the nucleus of the mother-cell in the process, varies. This mode of multiplication by gemmation passes imperceptibly into the more common mode of multiplication by fission. Gemmation is characterised by the difference in size which obtains at first between the cells that are formed and their mother-cell. If they break off at once they do not nearly equal it in size; if they delay their separation from the mother-cell they gradually get to equal it, and then the products of division are almost or altogether equal to one another, so that there is no possibility of distinguishing mother from daughter. It is evident that in proportion to the extent to which the products of division differ from one another in size, does division become more and more like gemmation; the whole difference therefore between fission and gemmation lies in the amount of protoplasm which is given over by the parent-cell to the one which arises from it. The difference is a quantitative one merely. Division commences by an enlargement of the nucleus; in some cases by a formation of fresh nuclei.

No form of reproduction other than multiplication by fission or by gemmation has been certainly observed in the animal-cell; a large number of the modes of cell-multiplication, which have been stated to obtain, such as the so-called endogenous cell-formation and similar processes, are merely forms of fission. As to free or spontaneous cell-formation, so much at least is certain, that it is not as common as was once supposed.

When the nucleus divides and the cell goes on growing without the protoplasm becoming marked off into separate portions corresponding to the nuclei, the structure which is formed cannot be any longer regarded as a single cell. But it is not a compound of cells either, for this would presuppose the existence of a number of separate cells. This condition has therefore been very rightly regarded as a special one, and called a Syncytium. Structures of this kind are found in nearly all groups of animals. The same result is obtained by the Concrescence of a number of separate cells, the protoplasm of which runs together into a continuous mass, in which there are of course a number of nuclei.

While the protoplasm in the above-mentioned series of phænomena undergoes no perceptible changes in constitution, a change 
in the protoplasm is essential to another kind of phænomena, to which we now pass. The substances contained in the protoplasm become separated from it, that is, are secreted from it. This process of secretion varies in character; it sometimes occurs within the protoplasmic body, and then substances differing in their chemicophysical properties from the protoplasm are formed within the cell. These substances may be distinguished by their constitution, such as fat, colouring matter, and so on; or by form, such as granules, drops, crystals. Sometimes secretion affects the surface of the protoplasm; and then the secreted substance may be fluid, in which case it will get separated and removed from the protoplasm; or it may be solid, in which case it will remain more or less intimately connected to the rest of the unaltered protoplasm. Substances which are different from the rest of the protoplasm, of a cell arise by chemicophysical changes of the whole surface, or of a part of it. We may regard these changes in the protoplasm as differentiations, for they are differentiated from matter which was previously in an indifferent condition within the protoplasm. In this way the structure which has been already alluded to as the cell-membrane, is formed at the periphery of the cell. But this same process leads to other arrangements also, which we shall have to examine more closely hereafter.

The series of vital processes exhibited by a single cell essentially agrees with those exhibited by any and every other organism, so that the cell itself is virtually an organism (Elementary organism).

\section{Differentiation of the Animal Organism.}

$\S 16$.

The simplest and lowest stage of the animal organism is represented by the earliest stage of its development, in which it is known as the egg. Except in some exceptional cases, which only prove the rule, and which need not be mentioned here, this egg is nothing more nor less than a cell. The

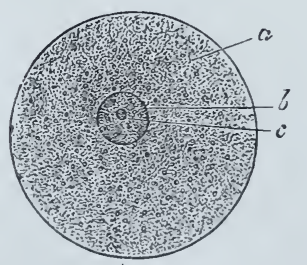

Fig. 1. Diagram of an Orum. a Granular protoplasm. $b$ Nucleus (Germinalvesicle). $c \mathrm{Nu}$. cleolus (Germinal spot). egg-cell does not differ from other cells in any essential points; it may increase in size, and special particles-yolk granulesmay appear in its protoplasm. Although the presence of these latter alters the character of egg-cells as indifferent cells, yet it does not destroy their character as cells, which is just as little affected by this as in other cells by the differentiation within their bodies of substances, such as chlorophyll granules, starch, pigment granules, \&c. This condition of the egg-cell, which on the whole 
is a simple one, agrees in character, although it may be for a time only, with many lower unicellular organisms (Protoplasta).

The egg-cell undergoes changes, which ordinarily commence after impregnation, and which are accompanied by changes in the nucleus (the so-called germinal vesicle). In its place, and in part from the material which formed it, two new nuclei arise, and
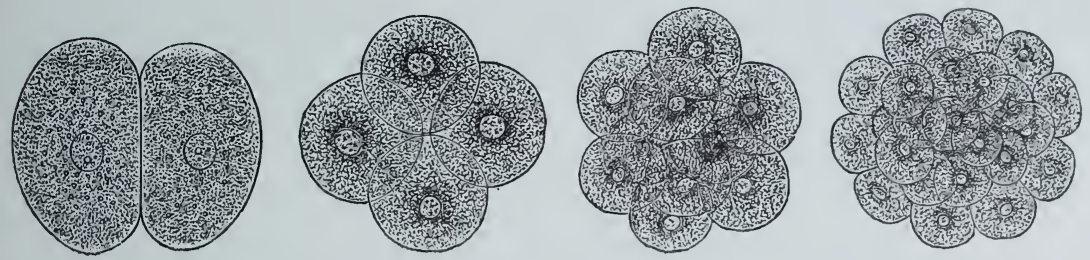

Figs. 2-5. Various stages of the so-called cleavage process (Division of the egg).

the egg-cell now begins to divide. Two cells thus arise, which are either like one another, or differ from one another, in size or in constitution. In both cases something fresh has arisen from the egg-cell, and in both there is a differentiation, for two parts have arisen from it. Four, eight, sixteen cells, and so on, are formed by continued division, although of course not always quite regularly, until at last a number of cells are formed. This process of the division of the egg-cell is known as the "segmentation of the yolk," and is a constant phænomenon, although it may present various modifications, which are always due to adaptation, and which may be so explained.

This is the first course of differentiation in the organism; in place of a single cell, a number of cells, similar to, or different from one another, arise. The functions of the organism, which were all performed previously by the egg-cell, are now performed by the separate cells. The division of the egg-cell must therefore be considered as leading to a division of its functions, although indeed this division is merely a quantitative one.

The various stages of this process of division have other relations also, for they appear to agree in character with the mature stage of many lower organisms (Protista), as for example the Volvocineæ and Catallacta, in the developmental history of which there is at one time an organism composed of a number of equi-formal cells. The animal organism, therefore, even in the commencement of its ontogeny, passes through several morphological stages, which are permanent among the Protista, and the process of segmentation of the ovum may be explained as a survival transmitted from early ancestors. Accordingly the teleological halo, with which it would necessarily be surrounded, were we limited to seeking its explanation exclusively in connection with the future organism which is to arise from this segmentation, is cleared away. The organism does not, however, get a specifically animal character from this formation of 
a number of cells; that character first makes its appearance in the course of further processes of differentiation.

These processes of differentiation consist in the more or less similar morphological elements (cells) which represent the organism, acquiring, in larger or smaller groups, distinct characters : in their being differentiated, and forming the rudiments (first stages) of organs, by taking a definite order and arrangement. These organs then are made up of cells, which form their tissues. We thus arrive at the essence of the architecture of organisms; we have tissues, which make up organs, and are themselves composed of form-elements-the cells.

\section{Origin of the Tissues.}

\section{$\S 17$.}

The cell, then, in those organisms which we regard as animals, constitutes the whole of the organism only for a time; that is, so long as it is an egg-cell. By division a multitude of cells is formed out of the egg-cell, and these form the rudiments of the animal. In later stages a part only of the material formed from the ovum retains the primitive character of the cells; the form and substance of most of the cells are altered, and therefore their physiological properties are altered; that is, new relations are established. The new cell-complexes formed from aggregates of similarly altered cells, and their derivatives, are the tissues. The process which leads to the formation of these tissues is essentially a differentiation. This, again, affords us an example of division of labour, for each differentiated aggregate of cells has to perform a definite function for the organism, which function was not the duty of a definite set of cells when the cells were indifferent, and indeed were performed in common with all others by one cell only (the egg-cell), in the earliest condition of the individual organism.

In all cases histological differentiation commences in the protoplasm of the primitive cell; the nucleus is less strikingly affected, but numerous changes may be seen to occur in it. When the chief part is played by a substance differentiated from the protoplasm, the nucleus becomes of but slight importance. According to the characters of their form-elements the tissues are divided into several large groups: these I call Epithelial tissues, tissues of the Connective Substance, Muscular and Nervous tissue. The first two form a lower group, which, as Vegetative tissues we may distinguish from the other two, which are the Animal tissues. The difference between the two groups lies in the quality of their differentiation; the products of the differentiation of the former having a more passive relation to the organism, while the products of the differentiation of the latter exhibit an independent activity in the carrying on of the life of the organism. The vegetative group, or tissues analogous to them, are, moreover, most widely distributed in 
the Vegetable Kingdom; whilst in that kingdom the animal tissues, which are the source of the arrangements characteristic of animals, are wanting. All other tissues, though often distinguished by name, are either not independent tissues at all, but only much more complicated structures formed of a variety of tissues, or are forms of tissue which should be ranged under one of the above-mentioned categories, or may be merely component parts of the tissues already named. We overlook the true conception of what a tissue is if we call structures which are made up of several tissues "compound tissues."

\section{A. Vegetative Tissues.}

\section{Epithelium.}

$\S 18$.

Cells placed side by side, and forming one or more layers which invest the surface of the body or the walls of internal spaces, are called epithelial. Epithelial tissue, then, consists simply of cells. It is distinguished from other tissues by the fact that the cells, at least so far as their arrangement is concerned, retain their primitive characters. Epithelium represents phylogenetically, and therefore, also, ontogenetically, the oldest form of tissue. The germinal layers which are the earliest organological products of the differentiation of the masses of cells which arise from the segmentation of the egg-cell, are layers of epithelial cells. Epithelial cells vary greatly in form, and are the starting-point of various organs. The protoplasm of epithelial cells very often loses its homogeneous character, owing to the differentiation of its outermost layer into a thickened membrane. In stratified epithelium this is best seen in the superficial layers, the absence of a membrane in the cells of the deeper layers being an indication of their younger condition.

Another differentiation is the development by the superficial layer of cells on the surface exposed to the exterior, or lining an internal cavity of the body, of fine processes, which are capable of movement; these processes, which vibrate during the life of the cell, are known as cilia. The hairs on these ciliated cells are sometimes in the form of a single flagellum, or occur in a group of many as cilia. In the former case the cell runs out into a fine process, and forms a flagellate cell; these are most common in the lower animals. Cilia are shown to be differentiated products, since their movements are not simply effected by the contractility which is already inherent in the protoplasm. In many of the lower or-

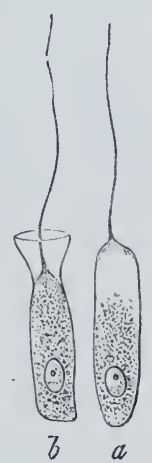

Fig. 6. Flagellate cells. $a$ of a Hydroid Polyp. $b$ of a Sponge (c o l l a $\mathrm{r}$ cell). ganisms cilia are formed for a time and are again drawn in and their substance fused with the protoplasm. This shows that they 
are differentiations of the protoplasm, and that their movements are due to the same cause as the movements of the protoplasm. This indication of their identity with protoplasm cannot be seen in the more differentiated forms of cilia.

Another differentiation may be seen on the outer surfaces of many epithelial cells. A membrane, instead of being formed by a change of the whole periphery of the superficial layer of protoplasm, may be formed on a definite portion of it only: in this case it is more highly developed, and may lead to a partial thickening of the outermost layer of protoplasm. In short, a layer of varying thickness of a substance different from, but as a rule still connected with, the protoplasm, forms on the outer face of each cell. Homogeneous membranes-cuticles-are formed by the further differentiation of the substance thus secreted in a layer from the protoplasm of the cells; that is by the part formed from each cell becoming more intimately connected with the layers formed by the cells around it than with its own cell. Where these layers are laid down irregularly and gradually undergo other changes, by means of which each fresh addition can be distinguished from the preceding one, they become laminated. The more the substance of which these cuticular structures are composed differs from the protoplasm of the cells which have deposited it, the more difficult is it to make out any passage of the protoplasm into it, and the more distinctly is the formation of cuticles seen to be a process of secretion. When the cuticle is not formed regularly on the surface of the separate cells, protoplasmic processes project from the secreting cell-layer into the secreted layer, which are then traversed by corresponding canals (pore-canals): these are ordinarily very fine. These cuticles differ greatly in consistency, and present every intermediate step between softness and extreme hardness. They are often converted into organs of support, when they are very firm; in which case they ordinarily consist of a substance known as "chitin." These chitinised cuticles are very common in the Invertebrata.

\section{$\S 19$.}

The secreting activity of the cells of large epithelial layers may give rise to liquid, or even to gaseous bodies. The epithelia thereupon enter into new relations to the economy of the organism; they no longer produce substances destined to build up the organism, but they present an intermediate step towards that condition of epithelial structures in which parts of the epithelium enter into the formation of a tissue of definite function-glandular tissue. As there is always a direct connection between the aggregation of cells which form the secreting organs, or glands, and the epithelium, which either persists permanently, as in the majority of glands, or which is at any rate present when they are first formed, this glandular tissue is seen to be nothing more than a modifi- 
cation of the epithelial tissue, due to its differentiation. Like it, glandular tissue always consists of cells. In the simplest stage individual cells in a layer of epithelium become secreting-cells, and function as gland-cells, by forming and secreting a substance such as is not produced by the other cells. In this way unicellular glands arise. They either retain their original position between the other cells of the layer or sink beneath the level of the epithelium, and open between the other cells by a fine duct formed by the membrane of the cell (Fig. 7). If the secreting surface be increased without the general epithelium taking any share in it, the sunken epithelium must increase in size, and so give rise to structures which are more or less separated off from the epithelium, such as pits, sacculi, or cæcal tubes; and these may be again complicated by fresh growths. The tissue lying: beneath the primitive epithelial layer forms envelopes for these pits as they grow; but it continues to have the same relation to them, however complicated in form the ramifications and similar proliferations of the epithelium may be, as it previously had to the simple even layer of epithelium.

Thus the gland in its simplest form appears as a depression of the epithelium into the subjacent tissue. In the more distinct forms of glands there is a further differentiation of the cells which form the gland. The constituent cells of the

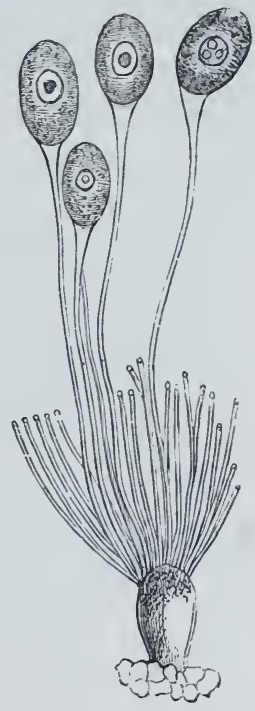

Fig. 7. Unicellular glands. Anterior salivary glands of the ant (after Stein). gland become separated into those which secrete and so represent true gland-cells, and those which connect the secreting portion of the gland with the still indifferent epithelial layer. These, in contradistinction to the secreting portion of the gland, form the epithelium of the duct.

\section{Connective Substances.}

$\S 20$.

The phænomenon which in epithelial tissue leads to the formation of homogeneous membranes may, by being extended over the whole periphery of every cell as well as by continued repetition, become of greater importance. Even in the epithelial tissue we often meet with a fine intermediate layer, the cement-substance. As the substance which is differentiated from the protoplasm of a number of cells gradually increases between the cells containing unaltered protoplasm, the cells become separated from one another, and a distinction is made between the cells which form and the intercellular substance which is formed. A number of very different 
tissues present this common character in their more intimate structure. They are called connective substances, as the majority of these tissues serve to unite other tissues to organs, or systems of organs.

The differences in these tissues are due partly to the character of their cells, partly to their relations with the intercellular substance, and partly to the chemico-physical constitution of the intercellular substance; but all these points are not equally well marked in every part of them. Whilst this latter circumstance allows us to recognise the passage of one of these tissues into the others, the fact that such passages do periodically take place under our observation, affords a more weighty reason for uniting them than the fact that we can detect common characters in their structure, although often hidden by various differences. The various tissues which belong to this group are: 1) cellular connective tissue, 2) gelatinous tissue, 3) fibrous connective tissue, 4) cartilaginous tissue, 5) osseous tissue.

\section{$\S 21$.}

Connective tissue is divided into the following varieties:

1) Cellular connective tissue (vesicular connective tissue) is

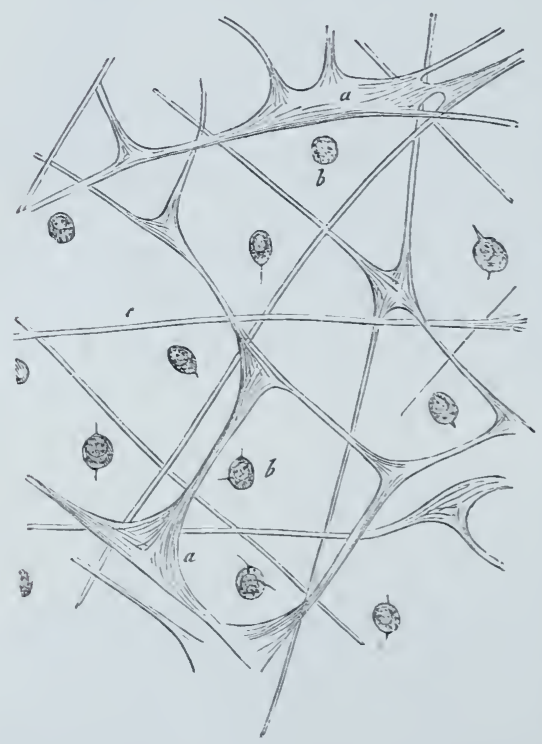

Fig. 8. From the gelatinous substance of the disc of Aurelia aurita, treated with iodised serum (after M. Schultze). $\times 500$. a Branched fibres in which no cells can be made out. $b$ Cells in the homogeneous gelatinous substance: the processes are largely retracted in this specimen. the simplest form. It is formed of rounded or elongated cells, which are separated by a small quantity of intercellular substance only. There are often vacuolated spaces in the cells, which are filled with a fluid. The intercellular substance often has the form of cell membranes, which serve to unite the juxtaposed cells to one another, and are common to neighbouring cells. In other cases again it is more largely present, without preponderating in quantity over the cells. The differentiation of the protoplasm of the intercellular substance varies in degree. This tissue is most widely found in the Arthropoda and Mollusca. In the Vertebrata it forms the chorda dorsalis, or notochord.

2) Gelatinous tissue (mucous tissue) is distinguished by the soft gelatinous character of the intercellular substance; it is ordinarily hyaline, and in it 
are placed either rounded and completely separated or filiform and branched cells, which are united to one another by their processes. Chords or tracts of cells also occur. In this way a fine network is formed, which traverses the gelatinous portion of the structure, the trabeculæ of which may become firmer by further differentiation, and may become broken up into fibrillæ. A similar fibrillation may affect the intercellular substance, in which case fibrous bands, in which there are no cells, can be made out. This tissue is found in many of the lower animals; in the umbrella of the Medusæ (Fig. 8), the integument of the Heteropoda, \&c.

3) Fibrous connective tissue may be regarded as afurther development of gelatinous tissue. Its morphological elements are elongated or branched cells, embedded in an intercellular substance formed of fibrous tracts and bundles. This substance is largely due to a differentiation of the walls of the cells, as is clear from its development. Development also reveals the fact that part of the protoplasm which sends off processes, is directly differentiated into fibrils and fibrous bundles; these are therefore distinct from the earlier formed, and more or less homogeneous intercellular substance. The thickness of the fibres and the direction they take vary greatly. The fibres, which are generally curved and undulating, sometimes run parallel to one another, sometimes anastomose; the cells and the cell-processes are, in their earlier stages, arranged in a manner corresponding to the subsequent arrangement of the fibres.

Fibrous connective tissue is distinguished as loose, or firm, according to the characters of its intercellular substance; the firmer sort is also known as "tendinous tissue," the fibrous bands of which are placed parallel to one another. In addition to the fibrillæ, which swell up when treated with acids and alkalies, there is another form of fibre, which is seen in the intercellular substance of fibrous connective tissue; this resists these agents more completely, and is called "elastic tissue," on account of its elasticity. It is, as may be seen from its relation to the intercellular substance, not an independent form of tissue, but merely a modification of connective tissue.

Inasmuch as a portion of the intercellular substance arises by subsequent differentiation of the protoplasm of the original cells, as was remarked above, the morphological elements which are present in fully-developed connective tissue represent the remains only of the primitive cells. According to the quantity of protoplasm used, and converted into fibrous structures, and so incorporated into the intercellular substance, the nucleus of the connective-tissue cells is surrounded by more or by less protoplasm, or the whole protoplasm disappears; the presence of isolated nuclei in the fibrous bands of connective tissue is an indication of this. Where the protoplasm still remains around its nucleus-where, that is to say, a cell, according to the conception given above, is present, this cell may undergo fresh changes, which are of so many 
kinds that connective tissue is richer than any other in the various phænomena of differentiation.

\section{$\S 22$.}

4) Cartilaginous tissue is characterised by cells lying in a firmer intercellular substance. Its cells do not, except in a few cases, possess distinct processes, or processes which can be easily made out; but are very nearly circular in form, or else oval or fusiform. The amount of intercellular substance varies in amount. It is distinguished from those forms of connective tissue which are formed of simple cells placed in a homogeneous intercellular substance, by its greater rigidity. Cartilaginous tissue is well adapted by the possession of this character to function as an organ of support. When the cells predominate, and there is but little intercellular substance, and when what there is is in the form of fine membranes, cartilage is seen to be directly allied to vesicular connective tissue. The protoplasm of these cells often takes on a definite arrangement, and forms bands which extend from the nucleus to the periphery, and unite together there. They are separated from one another by spaces which contain fluid (Fig. 9). In proportion as its intercellular substance is diminished, does this tissue differ more and more from ordinary cartilaginous tissue. In the

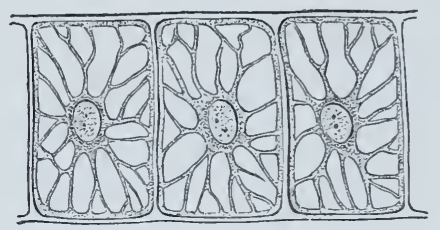

Fig. 9. Cartilage cells from the tentacle of a Medusa (Cunina). protoplasm of cells of this kind, which are found forming a sort of skeleton in the Medusæ, the phænomenon of streaming of the protoplasm may be seen.

If the intercellular substance increases, it either remains homogeneous (hyaline cartilage), or it undergoes further differentiations like those of connective tissue; but these differentiations do not much affect its relations to its cells. When the intercellular substance breaks up into fibres, we get fibrous cartilage; when elastic nets appear in it we get elastic cartilage. By gradual changes of the intercellular substance, as well as of the cells, cartilaginous tissue passes into fibrous connective tissue, and thus indicates its close connection with that form of tissue. The cells also become more specially modified in some cases by being elongated or producing radiating processes, which unite with those near them : as, for example, in many Selachii, or more developed still in many Cephalopoda. The intercellular substance then appears to be traversed by the processes from the cells (Fig. 10). The phænomenon, which in the cases just cited is greatly exaggerated, obtains also in ordinary hyaline cartilage, where the cells are apparently sharply marked off from one another; for the intercellular substance may be seen to be traversed by processes, although these are, of course, extremely fine.

The intercellular substance of cartilaginous tissue is always 
distinct from the protoplasm of the cartilage cells which lie in its cavities; but as it is differentiated from the protoplasm, it must, nevertheless, be regarded as a secreted product of the protoplasma layer secreted by a cell: often an intercellular substance may be seen in hyaline cartilage surrounding the cell like a capsule. This was formerly regarded as a cellmembrane belonging to the cell. As these "capsules" can often be shown to enclose groups of cells consisting of several generations, which have resulted from the fission of a single cell, the enclosed cells were looked upon as mother and daughter cells, \&c., and the phænomenon itself was regarded as a case of endogenous cellformation. As a fact, these "capsular systems" are merely the expression of secretions, not become homogeneous, and formed by several generations of cells which arose from one another. The perfectly gradual passage of cartilaginous tissue

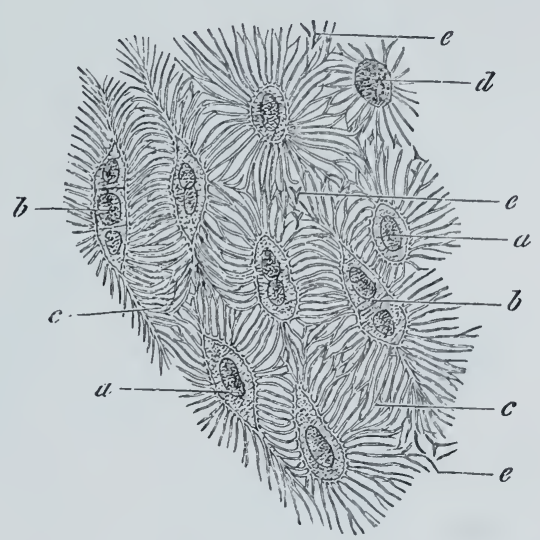

Fig. 10. Cartilage from a Cephalopod. $a$ Simple, $b$ Dividing cells. $c$ Canaliculi. $d$ An empty cartilage capsule with its pores. $e$ Transverse section of canaliculi (after M. Fürbringer). in which such capsules may be seen, into tissues where the intercellular substances is completely homogeneous, shows that we have here to do with different stages in the differentiation of one and the same secreted substance, which has arisen in the former case by an interrupted, and in the latter case by a regular, secreting activity of the cells. In virtue of the anastomoses of the processes of cartilage-cells, cartilaginous tissue comes very close to the next form of tissue, and it is only distinguished from it by the characters of its intercellular substance.

\section{$\S 23$.}

5) Osseous tissue. This, the firmest form of the connective substances, consists of an organic intercellular substance combined with lime-salts, in which there are cells with fine anastomosing processes; or it presents a ground-substance like that just mentioned, in which, however, there are no cells, but only cell-processes. These processes traverse it as fine canaliculi. There are therefore two structural phases of osseous tissue to be distinguished. Cells enter into the composition of the one, but in the other they simply send out fine processes into the pore-canals of its solid ground-substance.

The tissue containing bone-cells is the most common; it is 
found in the skeletal organs of all classes of the Vertebrata; whilst that form of osseous tissue with canaliculi only is found in the skeleton of many fishes, and as a general rule in the dental organs of all Vertebrata (dentine).

The development of osseous tissue explains the relations of the

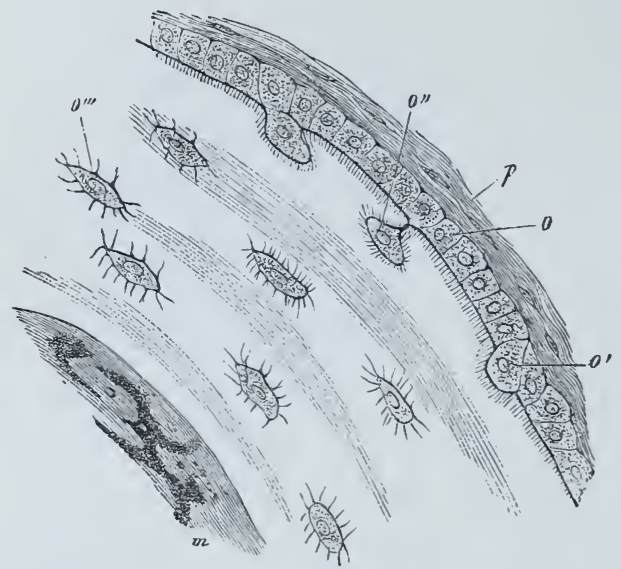

Fig. 11. Transverse section of the femur of Rana. o Osteoblast layer. $o^{\prime} o^{\prime \prime}$ Cells becoming bone-cells. $o^{\prime \prime \prime}$ A bone-cell. $p$ Periosteum. m Medullary cavity. intercellular substance to the cells. That form of it which contains cells may arise in two ways: either by the ossification of connective tissue, the cells in which become converted into bone-cells by the ossification of the intercellular substance, which becomes impregnated with calcareous salts, while the cells themselves become connected with one another by their processes, which traverse the pore-canals in the intercellular substance; or the same tissue is formed by apparently indifferent cells, which secrete a sclerogenous substance. This substance is laid down in stratified lamellæ, into which the secreting cells send fine protoplasmic processes (Fig. 11, o). The secretion of this substance is preceded by a change of part of the protoplasm of the cell. As soon as this is differentiated it does not belong any longer to the cell, and is therefore secreted from it. If some of the secreting cells $\left(o^{\prime} o^{\prime \prime}\right)$ cease to be active, while the cells near them do not cease to be so, the former gradually get to lie in a layer of intercellular substance, which finally surrounds them, and so converts them into bone-cells $\left(\mathrm{o}^{\prime \prime \prime}\right)$. The cells of the secreting layer (osteoblasts) are continuously connected by fine processes with those which are already enclosed (bone-cells). Thus each of the former is rendered capable of becoming a bone-cell.

The other form of osseous tissue is developed in a perfectly analogous manner, so far as its history is accurately known, through the development of dentine. In this case also a layer of cells secretes a substance, which hardens or is sclerogenous, and into this the cells at the same time send processes, which traverse porecanals. But the cells (odontoblasts), instead of gradually sinking into this extra-cellular substance, always remain outside it, and are connected with it by their processes only. The secreting substance is thus traversed by fine parallel canaliculi (the dentinal canals, so-called, since they were first made out in the dentine). This form of osseous tissue, notwithstanding its different appearance in later stages, 
is very closely allied to the former kind, for its intercellular substance also is secreted from cells-arises, that is, by the differentiation of a part of the protoplasm. The connection is still closer if we regard the earliest stage in the process. In both cases a homogeneous substance is secreted, which is hardened by calcareous compounds, and into this the cells, which form it, send their processes. If this process goes on in the same way as it began, so that a complete cell never passes into the secreted layers, it leads to the formation of that form of osseous tissue which is traversed by fine pore-canaliculi only, arranged for the most part in parallel lines. If some of the secreting cells gradually pass into the secreted substance, that substance becomes an intercellular substance containing bone-cells.

\section{Morphological Elements of the Nutrient Fluid.}

\section{$\S 24$.}

The cells, which are suspended in the nutrient fluid of the body, and which are its form-elements, are closely connected in origin with the connective tissue. If it is allowable to regard this fluid as an intercellular substance, then the whole of the nutrient fluid might be compared to a tissue, which would not differ from the other tissues of the connective series in any essential point other than its fluid condition. Even if we admit it to possess another function in consequence of this fluid condition, yet this function must be held as falling well within the category of vegetative functions. Apart from the importance of these considerations, the form-elements in question must be enumerated in the present place, for they take their earliest origin from the tissue which forms the walls for the vessels of the nutrient fluid. As far as its characters are known, a portion of the cells which form the mesoderm during the processes of division do not become connected with the rest, but remain isolated in the fluid which fills these canals or spaces, which fluid is known as blood. These form-elements then are the blood-cells. In the Invertebrata they appear, as a rule, in the form of completely indifferent cells, consisting of a nucleus (Fig. 12, n) and protoplasm, which latter exhibits amœboid movements. Among the Vertebrata these morphological elements persist as lymphcells in the Craniata, while in the bloodfluid proper there are elements which are derived from these forms, but are much altered. These latter have lost their amœboid character during differentiation, and

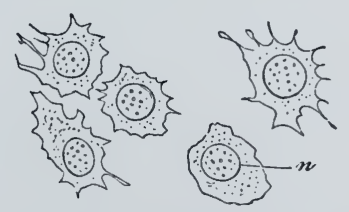

Fig. 12. Blood corpuscles of a Crustacean (Maja Squinado) with protoplas. mic processes. $n$ Nucleus. have the form of rounded or oval discs, the nucleus of which disappears in the Mammalia, though present in the rest of the Vertebrata. 


\section{в. The Animal Tissues.}

\section{$\S 25$.}

In the epithelial, as well as in connective tissues, the product of the differentiation of the protoplasm gives rise to phænomena which are limited to the sphere of vegetative operations. When a more highly contractile substance arises as a product of the division of the protoplasm, a new tissue is formed, which is known as contractile or muscular tissue. Its contractility, however, is not automatic, but dependent on stimuli, which come from the formelements of the nervous system. The contractile form-elements of the muscular tissue differ therefore essentially from the indifferent cell formed of protoplasm, although the latter also is contractile. They presuppose the existence of another, or nervous tissue, just as it on the other hand determines the existence of the muscular tissue. These intimate relations explain the causal relationship which these two tissues have to one another phylogenetically. The two kinds of elements are differentiated from a single neuro-muscular cell, which is in many Cœlenterata the representative of the two tissues. (Fig. 13.) This kind of cell corresponds to an indifferent stage of the animal

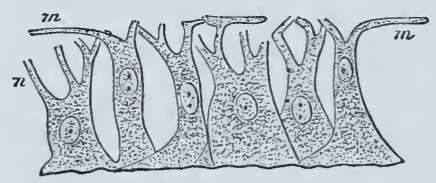

Fig. 13. Neuro-muscular cells of Hydra. $n$ Processes of the cells. $m$ Contractile fibres (after Kleinenberg). tissues, in which they have not yet become distinct tissues. The tissue which forms the starting-point of the differentiation is not a new structure. It is the outermost layer of the body, and consists of cells, which form an epithelium. The neuro-muscular tissue is therefore a differentiation from the epithelial tissue, and is thus connected with a more simple condition. Cells which are hardly at all different from other epithelial cells give off a band-like process at their base, which becomes connected to a layer of longitudinal fibres underlying the epithelium. While the epithelial cells of the outer layer of the body unite, when in their indifferent condition, a low grade of sensibility with a low grade of contractility, the sensibility, when the cells become more highly specialised, remains with them, and the contractility becomes assigned to a differentiated process of the protoplasm, which now appears as a distinct appendage of the cell. Thus commences that arrangement which, in the more highly differentiated stages, is expressed by the connection between ganglion-cells, nerve-fibres, and muscle-fibres. By supposing that the fibres, which in the earlier case appear to be merely processes of the cells, retain their nuclei, and that the products of the division of the nuclei of the cell gradually become fibres, and that further the neuro-muscular cell is no longer con- 
nected directly, but by means of a separate process with the fibre, which has at the same time itself also become independent, we can see how the more differentiated stage has been brought about. Nerves and muscles seem from this point of view to be the products of the separation of one and the same layer of tissue, which tissue we shall learn later on to know as the "ectoderm." And at the same time a physiological postulate is thus satisfied: for clearly it is impossible to imagine that nerve or muscle once came into existence with their elements totally distinct from one another, and that the connection between them, on which their functions depend, was the result of a later union.

\section{Muscular Tissue.}

\section{$\S 26$.}

The morphological elements of the muscular tissue are, so far as their more special characters are concerned, divisible into two groups. One consists of cells simple in form, the other of fibres derived from cell-aggregates, or from syncytia; the latter is indicated by the presence of numerous cell-nuclei. In either case the amount of protoplasm, which retains its indifferent character, is slight, and subordinate in importance as far as the function of the form-elements in question is concerned. Further differentiation of the contractile substance may in either case lead to the higher development of the fibre.

1) The so-called smooth muscular fibres, or contractile fibre-cells, constitute the first form. They are spindle-shaped cells, which are often greatly elongated, and then are band-like in form; in these cells either none of the indifferent protoplasm at all persists, or what does is to be found in the long axis, or at the periphery of the cell only. In all cases such remaining protoplasm surrounds the nucleus. The contractile substance is homogeneous and limited externally by a membrane, which is often difficult to demonstrate. The reaction of these muscle-fibres to nerve stimulation is slow.

Owing to differentiation of the contractile substance into singly and doubly refractive particles, the fibres gain the appearance of transverse striation; such is the origin of that variety of the tissue, which is known as transversely-striated muscular fibre. There are various intermediate forms between this kind of striated tissue, which consists of fibres derived each from a simple cell, and the other more homogeneous kind of fibrous muscular tissue.

2) The elementary parts of the other form of muscular tissue are formed by cell-aggregates (syncytia). They generally arise, as it seems, from the growth of one cell, the nucleus of which multiplies, so that they may be regarded as arising from the continuous but imperfect division of one cell. Their contractile substance either has a cylindrical shape, is limited externally by a homogeneous 
membrane (the sarcolemma), and contains several nuclei, with remnants of protoplasm along its axis ; or the contractile substance forms a solid cylinder, on the surface of which, and immediately below the sarcolemma, are the nuclei with the remains of the protoplasm.

Further, there are two varieties of this form of muscle-tissue in which the contractile substance is respectively more homogeneous or more heterogeneous. If more homogeneous the fibres resemble the so-called smooth fibrous cells, from which indeed they differ only by the fact that they do not correspond to a single cell, but to a multiple of cells, as is clear from the number of nuclei appertaining to the fibre. In the other condition, owing to the differentiation of the contractile substance, they resemble the second form of simple muscular fibres, and, like them, are transversely striped. These also correspond to a number of cells although they are derived from a single cell, and owe their elongation to its growth. The reaction to stimulus is, in transversely-striped fibres, rapid.

\section{Nervous Tissue.}

$\S 27$.

Nervous tissue appears (as has been already explained) at the same time as muscular tissue in the Animal Kingdom, and is distinguished by its functions, even in its lower conditions, from other tissues. It receives and passes on stimuli, converts them into sensations, and produces voluntary excitations. Two conditions are to be distinguished in the morphological characters of the elementary parts, nerve-fibres and nerve-cells. The former are mostly present in the peripheral portion of the nervous system, and are the conducting organs, while the latter form the central elements.

1) Nerve-fibres have not always the same relations, and their different conditions are to be regarded as stages of differentiation.

a) In their simplest form they are elongated homogeneous band-like bundles composed of fibres which are so slightly separated from one another that they appear to be merely striated. For the majority of Invertebrata the relation of nerve-trunks of this kind and their branches to the histological form-elements is not thoroughly made out; and even the question whether the numerous striations of the nerve-trunks are to be regarded as the indication of their being composed of separate fibres, is an open one. The presence of nuclei in their structures is the sole fact which points to their relation to cells. In other cases fibres united into bundles may be distinguished as individual elements of structure. The fibre consists of an apparently homogeneous substance which is limited superficially by a fine membrane, beneath which are the nuclei. Remains of protoplasm may be at times made out around the nucleus, which shows that the rest of the fibre is a differentiated substance. The 
structure of these nerve-fibres is therefore histologically of a similar grade to that of muscular-fibre, and the only difference between the two is in the quality of the differentiated protoplasm, which in one case gives rise to muscle, and in the other to nerve-substance. Such fibres are to be seen in the Invertebrata as well as in Amphioxus and the Cyclostomi. In the higher Vertebrata they are present only in the sympathetic nervous system.

b) Further differentiation gives rise to a second stage of the nerve-fibre. The nerve-substance, which lies beneath a more or less delicate envelope, is differentiated into a chord which traverses the axis of the fibre-the axis-cylinder-and into a fatty substance which surrounds it. The latter, known as the medullary cylinder (medullary sheath), gives a highly refractive contour to the nervefibre, and can be separated from the axis-cylinder only by artificial means. The homogeneous sheath which surrounds the medullary cylinder-the neurilemma-contains the nuclei which are the remains of the cells from which the fibre was formed. So far as is yet known this form obtains in the Gnathostomous Vertebrata only.

2) The other form-element of nervous tissue is represented by cells, which are called ganglion-cells, as they are principally present in the swellings (ganglia) of the nervous system. They form the central apparatus. Their substance is generally finely granular in character, with many other peculiarities which cannot be entered into more closely here. The nucleus, which as a rule is provided with distinct nucleoli, lies in the middle of the granular substance; this latter is often limited by an external membranous and firmer layer. A very complicated structure is ascribed to these cells, and is explained by every observer in essentially different ways, so that the questions involved appear to be still far from being settled.

The ganglion-cells possess processes by which they are connected partly to one another, and partly to nerve-fibres. They form therefore the points of origin of the nerve-fibres. It is not yet settled how ganglion-cells, which are devoid of processes, and therefore completely isolated, can be of any service. The fact is, that the belief in their existence grows less and less every day. The processes of the nerve-cells vary greatly in number, as well as in their relation to the fibres; the only point to be noted about them is that in the differentiated fibres it is the axis-cylinder which is continued into the substance of the cell, while the medullary cylinder ceases at some distance from it, or, rather, is no longer differentiated. The relations of the axis-cylinder to the substance of the cell appear to vary greatly, and are in many points a problem still.*

Solbrig, A., Ueb. d. fein. Structur der Nerronelemente der Gasteropoden. Leipzig, 1872. 


\section{Origin of the Organs.}

\section{$\S 28$.}

In section 13, the title of organs was given to those parts of the body which were entrusted with a definite function for the purposes of the organism, and which had a form in correspondence with this function. In this general sense every form-element is an organ, just as much as the parts, which are made up of formelements, and have a definite function, are organs. The conception of an organ is therefore a relative one. We must accordingly separate organs into those of a lower and those of a higher order. The former are represented by the morphological units or formelements-elementary organs-while the organs of a higher order are those which are made up of a number of elementary organscells, and their derivatives (tissues) - and which are set apart for a single function. There are but few of these organs of a higher order in the lowest stages of animal organisation, owing to the simplicity of the organism. But these few organs form the groundwork on which the gradual complication of the organism is raised up by continued differentiation, and in accordance with the principle of the division of labour. We may therefore call those simple organs of a higher order, from which complex organs are developed by differentiation, "primitive organs."

When we examine these primitive organs more closely, we find it convenient to associate them with the earliest processes of differentiation which take place in the organism, for they can be derived from them. A collection of smaller cells arises from the division of the egg-cell, and these have not all the same position. Some occupy the inner part of the organism, and others form a

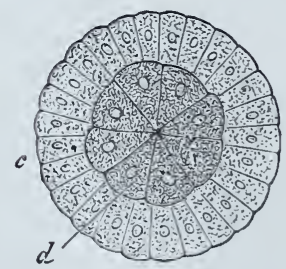

Fig. 14. Separation of the mass of cells formed by change of the yolk into a peripheral (c) and a central $(d)$ por. tion. layer which surrounds it, and at the same time forms the external boundary of the body (Fig. 14). If in this stage of development the taking in of food into the body commences, then the inner cell mass becomes converted into the limiting layer of the digestive cavity, and forms a primitive gut (enteron). In many observations the process of division into two layers is described as due to the invagination of a one-layered vesicle. In other cases it is represented as taking place differently, so that it is impossible to make out whether there is any phænomenon common to all cases, and, if so, how far it is common. Let us therefore turn to the results of the process, without making any generalisation about it. We now have an organism made up of two layers of cells. An outer one, or ectoderm, which forms the primitive integument, and an inner 
one, or endoderm, which surrounds a primitive enteric cavity. The two layers pass into one another at the oral opening which leads into the cavity. The two cell-layers, which form the body of such an organism, furnish the conditions under which it is possible for it to lead an independent animal existence. The outer one is the organ of support, and may be converted into an organ of locomotion if it gives rise to cilia, and may be the seat of respiratory functions also. In so far as it perceives the state of the surrounding medium it is an organ of sensation too. The inner layer is nutritive in function, produces changes in the food which is taken in, and allows what can be assimilated to pass into its cells; and these in their turn feed the outer layer of cells. What is useless is passed out again by the same opening as that by which it entered. As the functions of the two layers are different the special characters of the morphological elements which compose them are different also; we need only call attention now to the much greater size in most cases of the cells of the endoderm, as compared with the cells of the ectoderm.

This grade of organisation is to be seen in some of the lower divisions of the Animal Kingdom (Cœlenterata and Vermes), where it represents a lowly stage of development.

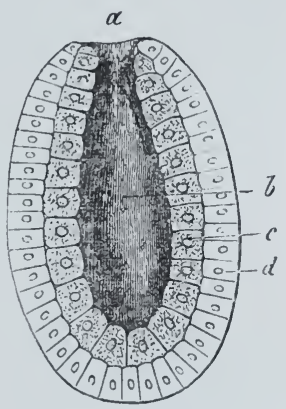

Fig. 15. Diagram to represent the first dif. ferentiation of the or. ganism into ectoderm and endoderm, and the formation of a digestive cavity. a Mouth. $b$ En. teric cavity. $c$ Endoderm. $d$ Ectoderm. (In transverse section.) Indications of it are to be seen even in the higher divisions. This form has been called the Gastrula, on account of the dominant development of the enteron. Starting from the hypothesis that forms agreeing with a Gastrula in all essential points were the precursors of all the higher forms of animal organisation, a Gastræa-form resembling the Gastrula has been regarded as the primitive ancestral form of all animals. This Gastræa theory is based, first, on the existence of independent animal forms which resemble the Gastræa; secondly, on the fact that the embryonic body which commences with a Gastrula, does not, in the lower divisions, rise very much above it, so that even apparently considerable complications of the organism can be traced back to the existence of these two layers of the body; thirdly, the presence of these two layers of cells, forming the ectoderm and endoderm, as a general, constant, and therefore regular phænomenon, even in the higher divisions of the Animal Kingdom, as well as their constant relation to the same functions, is a fact of the greatest importance for the hypothesis in question; indeed the occurrence of these layers as the so-called germinal layers, which make up the embryonic body, cannot be rightly understood without a reference to a hypothetical Gastræa-form. This hypothesis may therefore be regarded as justified. 
We recognise then the Gastræa as a fundamental form, and discover in the differentiation of two layers corresponding respectively to endoderm and ectoderm, which are present even in the highest grades of the Animal Kingdom, facts which point to such a Gastræa stage, and are due to it. But it must not be at all supposed that we have advanced farther than just on to the threshold of a knowledge of these relationships. The definitive explanation of many of the points which have considerable importance in this matter is still far distant, and but little light has yet fallen on even such apparently simple points as the origin of the Gastrula and its two layers. It is a question whether the form which precedes the Gastrula is a one-layered vesicle-that is, whether the two layers of the body are due to the endoderm being formed by invagination; or whether the endoderm is developed from a primitive internal cell-layer delamination. And again, whether the two conditions that have been observed are independent of or derivable from one another. Further investigations will have to settle all this, and our judgment must therefore be proportionately reserved until such investigations have been made.

\section{$\S 29$.}

The two layers of which the body of the lower animals is made up during their early stages, and which are, in the higher divisions, represented by the germ-layers-that is, the ectoderm and endoderm-give rise to an intermediate layer or mesoderm, in the formation of which the other two apparently take an equal share. But it is not yet definitely known what share each takes, since the earliest processes of the differentiation of the rudiments of the body still require much careful investigation, and, moreover, they do not present the same characters in all cases. These three layers appear directly after the segmentation of the ovum in the higher animal organisms, and are coincident with the first traces of histological differentiation. They represent the outline of the organism in the condition of a germ, and from this the whole organism by differentiation evolves itself.

These rudiments of the body exhibit great modifications in the higher divisions of the Animal Kingdom, and the stage which is represented by the Gastrula form is more difficult to make out in proportion as the differentiations through which the organism has to pass are more considerable; but the principal features can be easily recognised as identical in all cases. The outer germinal layer (deric layer or ectoderm) forms the outer limiting layer of the body, and the inner (lower) germinal layer (enteric layer, glandular layer, or endoderm) the foundation of the gut or enteron. The middle layer (mesoderm) afterwards arises between them.

As the ectoderm and endoderm are the first organs marked off in the course of development, the germinal layers are to be regarded as primitive organs, which have been transmitted from the earliest 
stages in the differentiation of the animal organism to later, and therefore higher stages, and which, following the law of the division of labour, give rise to series of new organs. We do not yet know enough of the details of the organological differentiation of the germinal layers to be able to give the history of every organ. However, the facts which are clearly established with regard to, at any rate, some divisions of the Animal Kingdom enable us to follow out the first steps in the process of differentiation. The organs which put the organism into relation with the outer world, such as organs of defence, of support, and of sensation, are principally derived from the ectoderm (hence called the sensory layer), also those of movement; while the endoderm principally provides the organs for the preservation of the individual and of the species (nutritive layer). As the origin of the mesoderm, out of which important organs are formed, is still very obscure, the relations of these organs to one or other of the two primitive germinal layers must be left an open question.

The primitive character of the organism more or less disappears as the rudiments of the body are formed out of the germinal layers, and as fresh organs which render the organism more complicated arise in it. Organs differentiated from the germ layers which act the part of primitive organs are reckoned as secondary organs. From these, tertiary organs are formed, and so on. The separate organs differentiated out of a primitive organ remain connected together, owing to the fact of these processes of separation being due to the division of a function, and of the separate functions being subordinated to the primary function, from the breaking up of which they took their origin. Combinations of organs are therefore formed, which are known as organic systems, on account of their morphological and physiological connection.

This connection does not always persist in the adult condition; and, in fact, organs primitively connected often become separated. This obtains chiefly in those organs which serve several purposes, for when the functions become independent the organs become so too. But even in these cases ontogeny indicates what was the primitive condition.

\section{Systems of Organs.}

a) Integument.

\section{$\S 30$.}

The ectoderm, as the outermost layer of the body, forms the simplest condition of the integument of animal organisms. In the lowest organisms (Protista) there is either no integument at all, the protoplasm which forms the body being protracted into everchanging processes (pseudopodia), or the integument is represented 
by the outermost layer of the protoplasm of a single cell, in which case we have the first example of a denser stratum of the cell becoming separable as a distinct envelope and covering for the rest of the organism. The ectoderm has the function of an organ of defence when its cells secrete a substance which invests, more or less perfectly, the surface of the body. This substance may harden and give rise to tests or shells, or form a continuous covering for the body, like the carapace of the Arthropoda.

When a mesoderm is formed, that part of it which becomes connected with the ectoderm takes on, in various ways, the functions of an organ of support. The calcareous deposits in the complicated integument of the Echinodermata are examples of this.

The activity of the ectoderm in producing firm organs which protect the body is seen also in the Vertebrata, where numerous and varied parts, which function as investing and protecting organs, are produced by it.

\section{b) Skeleton.}

$\S 31$.

In proportion as the various protective organs which are formed from the ectoderm increase in size or in strength, and at the same time become connected with internal organs, they attain the function of organs of support also. Such organs we designate as the skeleton. The combination of inorganic substances (chiefly calcareous salts) with an organic base plays an important part here. The supporting function of the integument gives rise to numerous adaptations. The combination of the functions of both protection and support is clearly a lower stage as compared with the formation of internal skeletons, which indicate a higher functional differentiation, and function exclusively as organs of support. Here, too, we meet with the most various conditions. 'The lowest forms, the first beginning of such internal skeletal organs, are solid deposits in the tissues, the separate pieces of which have no connection with one another. The growth and union of these deposits give rise to skeletal formations, which may be also regarded as excretions. Examples of them are found even in the Coelenterata. When a definite tissue, the properties of which specially fit it for the function of support, is brought into use, the internal skeleton assumes a higher degree of development. The differentiation of cartilage from the indifferent connective tissue is the first expression of this phænomenon. As low down as the Medusæ, among the Vermes and among the Mollusca, the employment of cartilaginous tissue for organs of support is commenced, and in the Vertebrata it attains to greater importance, until it is pushed aside by a second and more perfect skeletal tissue-the osseous. 
c) Muscles.

$\S 32$.

The locomotion of the body exhibits itself in its simplest phase as a change in the form of the body due to the contractility of its protoplasm. When these changes in form follow one another rapidly, and have all the same direction, the body either elongating or sending out processes which attaching themselves to some fixed point, are followed gradually by the rest of the semi-fluid body (Rhizopoda), locomotion is effected. The difference between this mode of locomotion and undefined change of form is seen to be merely one of degree. The contractility of protoplasm may produce changes in position even when it is invested by a differentiated, though soft, integument. This layer of integument will follow the movements of the body it invests. In such cases, and they are very common among the Protista, special organs of locomotion cannot be said to exist, for the cilia have other functions to perform for the organism in addition to locomotive ones; such, for example, as that of aiding in the ingestion of food.

Specific organs of locomotion make their first appearance when the contractile morphological elements known as muscle-fibres are differentiated; these, in the simplest case, form a muscular layer lying beneath the ectoderm.

The genesis of this earliest musculature of the body is due to a differentiation of the ectoderm (Hydroid polyps), the cells of which give off flattened processes, which form a continuous layer of contractile fibres.

Each individual ectoderm-cell concerned in the formation of this layer of fibres represents accordingly a sensory apparatus, which stands in direct continuity with a contractile apparatus. The cell is indeed replaced, when the musculature is differentiated, by groups of muscles which work so as to balance one another, and completely harmonise in their action (compare Sect. 31). We cannot yet say how far this process, which gives so deep an insight into the mode of differentiation of the tissues as well as of the organs, is repeated in the ontogeny of the higher forms of animals. In all divisions above the Colenterata we always find the separation between ectoderm and muscle complete. It may therefore be doubted whether a process of the kind described in the Hydroid polyps always accompanies the origin of the muscular system. But yet it is very probable that something of the kind does occur. Even though the process of differentiation in the higher organisms does not enable us to recognise these processes in their case, yet it is not to be assumed without further reason that the mode of origin of the muscular tissue was in them primitively different, for Ontogeny very seldom repeats phylogenetic processes in every detail. 


\section{$\S 33$.}

The earliest musculature of the body is closely related to the integument, from which it can with difficulty be separated. Since this is the case not in the Cœlenterata alone, we have here an instance in favour of an essentially equivalent origin for this part of the musculature in all cases. Together with the integument, it forms, on the appearance of a body-cavity, a "dermo-muscular tube," which encloses the other organs. The arrangement of the muscular fibre seldom presents much regularity till the body becomes jointed into separate parts, placed one behind the other (metameres); and with the development of organs of support the muscles become differentiated into separate groups. Collections of fibres form bundles, and these again make up larger complexes, muscles. The segmentation of the muscular system corresponds therefore to the segmentation of the body, and the separate segments differ in proportion to the difference in function of the metameres. The various kinds of movement which are produced by the crossing of the fibres of the dermo-muscular layer in different strata, are, where the muscular system is more highly differentiated, effected by groups of muscles acting in opposition to one another, and completely balancing one another in their action.

Locomotion by movement of the whole body is brought about by the dermo-muscular tube and the differentiations which arise from it; the whole integument, in the first instance, takes a share in this activity. A further differentiation arises from this state of things when special appendages are formed, as $\operatorname{limbs}$, on certain parts of the body. When the animal changes its place these act as the arms of a lever. They have the form of simple soft processes of the dermo-muscular tube (Ringed worms), or of jointed organs, which are supported by the integument (Arthropoda), or by means of internal skeletal structures (Vertebrata). The complication of the muscular system is in close connection with the development of supporting organs; and the two form a single locomotor system in which the skeleton plays the passive part.

\section{d) Nervous System.}

\section{$\S 34$.}

In the lowest conditions of animal organisation the protoplasm of the cells is the seat of sensation, as well as of movement; and this is permanently the case in the lowest organisms. As the muscular layer of the body becomes differentiated, the ectoderm becomes the principal organ of sensation. The differentiation of a nervous system is due to the further development of a portion of this layer 
as a sensory organ, for which reason such an organ must be at first placed superficially. This superficial position of the earliest rudiments of the nerve-centre has been already made out in so many forms that it may be regarded as a general phænomenon. As the sensory organ becomes differentiated from the ectoderm it sinks down into the body. The developing central organ is thus gradually covered over by other layers of the body. This arrangement, which is most peculiar, and by itself most unintelligible, is explained if we regard it as inherited from a more primitive stage, in which the nervous system was but slightly differentiated, and was represented by the whole cell-layer of the ectoderm, or by part of it. We must consider its gradual attainment of an internal position to be a process due to its continued differentiation, and consequent higher potentiality; the organ, which has become of greater value to the organism, gets hidden within the body.

With regard to the structural characters of the differentiated nervous system, the central organ, which is chiefly composed of ganglion-cells, is to be distinguished in the first place from the nerves, which pass to the terminal apparatus, and consist of fibrous elements (peripheral nervous system).

\section{$\S 35$.}

The earliest complications are due to the appearance of several parts (ganglia), in which are central form-elements connected with one another : the further development of these parts is very various. The ganglionic mass, which forms the central organ, is primitively dorsal, owing to the earliest separation of the central organs taking place from the dorsal ectoderm, as we have already seen. This dorsal nervous mass, which generally lies near the entrance to the alimentary canal, is differentiated into several parts, which are connected together by commissures; their fibres form an œsophageal ring.

In the animals built on a radiate plan the number of the ganglia is increased in correspondence with the radii ; the peripheral distribution of the nerves also follows these general structural relations exactly. The nervous system in bilaterally-symmetrical animals follows the bilateral arrangement. The more primitive form is represented by a superior ganglionic mass (cerebral ganglion). Other ganglia do not seem to be formed until the metameres are formed. We then are able to distinguish dorsal and ventral ganglia ; the latter may form ganglionic masses along a continuous longitudinal trunk, or a single subosophageal ganglion. The variations in size of these osophageal ganglia are in the closest connection with the nerves which pass off from them. When sensory organs are developed, the ganglion which sends off their nerves becomes of considerable size, while it seems to degenerate when they grow less. The supra-œsophag eal ganglia are the most important in this relation, for it is from 
them that the nerves of the higher sensory organs arise, which in position and direction have one widely-distributed arrangement.

From this form another is directly derived, determined apparently by the well-marked metamerism of the body. Whilst in unsegmented animals possessing an œsophageal ring, the ventral parts of the body are supplied by nerves which arise from the subœsophageal ganglia, we find that the number of ventral ganglia is increased when the body is broken up into parts lying one behind the other (metameres). A ventrally-placed series of ganglia is formed by the development of a separate pair of ganglia for each segment; and these, being united to each other by longitudinal commissures, form a ganglionic chain. The Ringed worms and the Arthropoda present us with this form. Further differentiation gives rise to all kinds of variations of this type.

In the first place, the size of the ganglia varies with the size of those parts of the body that have to be innervated; and in the second place, the ganglia of several segments of the ventral cord fuse into larger ganglionic masses.

Even when the central nervous system is entirely dorsal, as in the Vertebrata, it undergoes differentiations of this kind. When the most anterior part of the body is developed into a head, the most anterior part of the central nervous system is developed into a special region, the brain, which is marked off from the remainder of the medullary tube, or spinal cord, which remains more equal in size throughout. As differentiation advances, variously developed regions appear in the brain.

\section{e) Sensory Organs.}

\section{$\S 36$.}

The sensory organs inform the organism of the condition of the outer world. Protoplasm, in its indifferent condition, characteristic of the lowest organisms, reacts to various stimuli from without, and appears to be the seat of the lowest kind of sensation. When the surface of the body is not completely marked off from the inner portion of the organism (Rhizopoda) it is used as an organ of perception, of course of the very lowest grade; it functions therefore as a sensory organ of the lowest order. When the surface is more distinctly marked off, and a distinct outer layer of the body is established (Infusoria, Gregarinæ), we get a differentiation of great importance for sensory perception.

Although, indeed, particular parts of the surface in the Infusoria specially acquire the function of sensory organs, yet there is no ground for speaking of sensory "organs" in an anatomical sense, in this case, any more than there is in the still lower stages. Sensory organs only appear when a nervous system is marked off, for sensory organs are the end-organs of the sensitive nerves. 
Their presence therefore presupposes that form of differentiation which we treated of above, when speaking of the nervous system. Since ontogenetic facts point to the primitive segregation of the nervous system from the ectoderm, as being most probably a fundamental process, this same outermost layer of the body becomes also of the greatest importance in studying the origin of the sensory organs. Almost all the sensory organs are derived, directly or indirectly, from it; whence arises the permanent or temporary connection of these organs with the integument.

It is very unsafe to assert what are the functions of many of the sensory organs of the lower animals. This applies to all those organs which are not comprised amongst those which fall within the domain of our own judgment, on account of our possessing them or their homologues, in which case only is it possible that the connection between their structure and specific function can be estimated. Such outstanding organs have been classed together as organs of a sixth sense.

\section{$\S 37$.}

The sensory organs are divided into lower and higher. The former are commonly distributed over the integument, and are simple in structure. Compared with the higher they represent a more indifferent condition. Modified cells of the integument, which generally belong to the epidermis, connected on the one hand with a nerve fibre, and on the other provided with a process of varying shape, which is directed towards the surface of the body, are the most common examples of the lower sort. They are regarded as the organs of general tactile perception; but the physiological function of these organs, especially in aquatic animals, has not been definitely determined, and it is reasonable to suppose that many of them are the media of specific sensations, in which case they would resemble the higher organs of sense.

The significance of these arrangements is somewhat more certain when they are connected with special organs, such as movable processes of the integument and the like; they then appear to be tactile organs. It is still a question whether structures of this kind, especially in the lower divisions of the Animal Kingdom, are of use for perceptions other than tactile.

The higher sensory organs present themselves to us as special elaborations, with one special function and capable of response only to stimuli of one special kind; they are to be regarded as developed from the lower kind of sensory organs, and oftentimes still possess the essential structure of that lower kind. Organs of taste and smell can only be certainly distinguished in the higher divisions of the Animal Kingdom, and the function of the latter is certain only in those Vertebrata which live in the air; in the lower divisions it is still doubtful. Even in the case of the organs of taste the greatest caution is necessary as to their real import. The value of a sensory organ to the organism determines its being protected against 
external influences. This explains the foldings-in of the portion of the integument which is about to be differentiated into a sensory organ. It is for this reason that the higher sensory organs gradually sink beneath the level of the ectoderm as they are developing, and attain a favourable position for further development.

\section{$\S 38$.}

Vesicles filled with a fluid, on the walls of which a nerve ends, are regarded as auditory organs (otocysts). In its simplest form the vesicle is directly connected to the central nervous system, or the nerve passes from it to the vesicle. These vesicles almost always contain firm concretions or crystalline structures; and very often crystals of calcic carbonate. There are often hair-like prolongations of the end-organs in addition to them, which project into the lumen of the vesicle. This form of auditory organ, which obtains in the Invertebrata, is complicated in the Vertebrata by diverticula and outgrowths which form a labyrinth. New arrangements are produced in the form of organs for carrying and increasing the sound, which become attached to the auditory organ, although they primitively presided over other functions. Inasmuch as the labyrinth-vesicles of the Vertebrata are developed from the integument, the terminal organs of the auditory nerve which are differentiated in its walls are genetically connected with the terminal organs of the tactile nerves, which lie in the integument; they may therefore be regarded as a specific development of a lower sensory organ. The genetic relations of the simpler otocysts of most Invertebrata are as yet unknown, but all the more exact results point to the supposition that they arise by a differentiation of the ectoderm.

The optic organ also has a simple mode of origin. We exclude the pigment spots, which used to be often called eyes, and only recognise an eye where a nerve-ending of definite form can be detected, either under or on the surface of the body, acting as an organ for the perception of light. By the light-absorbing property of the pigment it is possible that indefinite sensations of light and shade may be produced, or other sensations altogether unlike that which we call "sight" may possibly be produced by the heat-rays alone of the light.

The function of pigment in the way just noticed is doubtful, but when it surrounds a part only of a rod-like nerve-ending, and that in such a way as to leave the outermost end free, and exposed alone to the influence of light, it has, clearly enough, a definite function. Optic organs of various degrees of complexity are formed by the union of a few or of many nerve-endings; the elements which are the medium of light-perception (rods) forming a convex or concave layer. Another complication is due to the addition of organs to refract the light (lenses); these, too, may have all kinds of relations, but they are always, either directly or indirectly, derived from the integument. In eyes in which the surface of the 
layer of rods is convex there are, as a rule, as many lenses as there are perceptive nerve-endings; when the layer of rods is concave, there is one lens only. By the addition of other arrangements to the nervous apparatus of the eye, by which its functional capacity is modified or increased, this organ becomes one of the most complicated of the animal economy. In most of the lower divisions the optic organ, even when fully developed, still retains its primitive relation to the ectoderm. In the higher divisions it is separated from it, and gets to lie, together with its perceptive apparatus, beneath the integument, or the perceptive apparatus is derived from the embryonic foundations of the nerve-centre.

The phænomena of differentiation may be seen even in what relates to the position of the optic organ, for the parts of the body which carry the eyes, as well as the number of the eyes, varies greatly in the lower divisions of Animals. Connected with this is the occurrence of a great number of eyes on the anterior part of the body, which goes to form the head, until, finally, the number of eyes on the part in question becomes limited to two. The different position occupied by visual organs forbids us to suppose that they have had a common hereditary origin, and is in favour of these heterotopic organs having been independently differentiated from an indifferent apparatus. On the other hand, that eyes which are connected to the cerebral ganglion, or the dorsal nerve-centre, have a common genetic relation, is not to be disputed.

\section{f) Respiratory Organs of the Integument.}

(Dermal Branchiæ.)

$\S 39$.

An important part is played by the integument, and therefore by the ectoderm, in the formation of respiratory organs. Before they appear the gas exchange is carried on probably by the whole surface of the body, and this mode of respiration obtains in many of the lower aquatic animals. A change of the surrounding medium is effected, partly by the movements of the body, and partly by special organs, for example cilia; thus fresh quantities of it are continually brought into contact with the respiratory surface. This is not, however, the only method of respiration in the lower animals, for the introduction of water into the interior of the body, in fact the bathing of the alimentary canal by water, is certainly not without significance in this direction, while it is of great importance as being the beginning of a long series of differentiations. Certain limited portions of the surface become more developed in this direction as the function becomes localised, and, in compensation for this limitation, acquire the form of blood-carrying processes, which are called branchiæ. In many cases they are differentiated 
from the appendages (Vermes, Crustacea). An increase of the surface, which is brought about in various ways, is the mode in which the further complication of branchiæ takes place; it is very frequently accompanied by a reduction in the number of separate branchial organs.

The importance of branchiæ to the body calls into existence various kinds of supporting arrangements for these organs, which, in their lowest condition, project freely from the surface of the body. Neighbouring parts of the integument being raised up into covering lamellæ, the branchiæ become hidden in cavities (branchial cavities), and the same tegumentary folds give rise to afferent and efferent canals for the water, which serves for respiration (Mollusca, higher Crustacea). In this way the development of respiratory organs may affect other parts of the integument, the direct relation of which to respiration had been lost for a very long time.

\section{g) Excretory Organs.}

\section{$\S 40$.}

Just as the gaseous excretory matters are eliminated from the organism by the respiratory organs, so too are there arrangements for eliminating the solid or fluid matters which have become useless to the organism. The whole surface of the ectoderm performs this function in the lower organisms ; in the higher forms of life, on the contrary, there are special organs, dermal glands, which have this function. Of those general arrangements which function as organs of secretion we have to do here with those special ones only which eliminate the excretory matters, and which are distinguished as excretory organs from those glands which secrete matters which are of use to the organism; these latter are either independent, or are united to definite systems of organs, of which they are, in that case, specialised parts.

The excretory nature of the products of secretion of those secreting organs, which are formed by the ectoderm, is least open to doubt, for the products are removed at once from the organism by the emptying of the gland.

Of the various kinds of organs which open on the surface of the body one sort attains to general importance. These are the kidneylike excreting organs, which eliminate the nitrogenous excreta from the body. These organs are distinctly derived from dermal glands, notwithstanding that in the Vermes, where they seem to have their most simple form, they penetrate deeply into the body; nor does the fact that in many cases (Annelida, Mollusca) the organ, which in other points also is much modified, opens into the bodycavity, and so connects it with the surrounding medium, and even serves in many groups (Mollusca) as a means for introducing water, 
affect the question of its origin. In other forms (Annulata) these organs, having a tubular form, assist in the generative functions, by serving as ducts for the generative products. The recurrence of this function for a portion of the primitive excretory apparatus (primitive kidney, archinephron) of the Vertebrata might be explained as an inheritance from a lower stage. How far such a view is justified is still uncertain. In any case a genetic point of contact between the primordial kidney of Vertebrata and the renal tubes of lower organisms, can only be looked for where the apparatus is, as in the Vertebrata, single on each side of the body.

\section{h) Alimentary Canal.}

\section{$\S 41$.}

The ingestion of nutrient matter into the body is, in some of the lowest organisms, effected by endosmotic processes, in which the surface of the body takes the principal share. In others solid nutriment is ingested, the soft protoplasm sending out pseudopodia, and embracing the nutrient matter which happens to come into the neighbourhood (Rhizopoda). The formation of a definite part of the surface of the body, serving for the ingestion of nutriment, is really a step towards organological differentiation (Infusoria); but this does not constitute an alimentary canal, which does not appear as a separate organ till the body is differentiated into cell-layers. The cell-layers, when they do appear-an inner and an outer-pass into one another at the margin of the orifice of entrance.

The inner layer, or endoderm, lining a space open to the exterior, forms the wall of a digestive cavity. In the simplest form, represented by the Gastrula, the endoderm is the sole wall of the primitive enteric cavity. The formation of a mesoderm gives rise to other layers external to this one. Of these the most important is a muscular layer, for by it the intestine is enabled to perform independent movements. The opening which leads into the enteric tube serves as a mouth for the ingestion of nutrient matters, as well as for an opening for the rejection of the undigested remains of the food (Cœlenterata, many V ermes). The appearance of an anal orifice produces a further separation of functions, and converts the blindly-ending gut or enteron into a tube open at both ends, the separate portions of which take on various functions, and so undergo different adaptations. The first portion, which is connected with the mouth, forms an œsophagus, which serves for the introduction of food; then follows the true digestive cavity, which is generally widened, or provided with cæcal sacs, and is generally called the stomach, though this name is not always applied to equivalent parts. The terminal part of the whole system serves for further alteration of the food, as well as for the excretion of the remnants 
by the anus. This differentiation of the digestive tube into several unequal parts is the most important complication which it undergoes; any further differentiations are subordinate to this. Three tracts are accordingly henceforward distinguished, as foregut, mid-gut, and hind-gut.

In addition to the varying and numerous changes in size which the different portions of the canal undergo, other arrangements, due either to special new functions, or mere expressions of further division of labour, arise in it. Organs for seizing and comminuting the food become attached to the mouth, or mark off a portion of the œsophagus (masticatory organs). In the stomach also there are sometimes masticatory organs of this kind. When they occur at the commencement of the csophagus, just behind the mouth, this part, which is frequently distinguished by its larger supply of muscles, is known as the pharynx.

The size of the cavity of the canal is increased by dilatations, or cæcal diverticula. Crops are formed in the course of the œsophagus, cæcal sacs on the stomach and on the rest of the intestine, which are variously complicated in number and arrangement. When the length of the alimentary canal is greater than that of the body, it is arranged in ascending and descending loops, or in coils, and so adapted to the size of the cavity in which it is contained. Both the quantity and quality of the food ingested is of the greatest importance as affecting all these relations of parts; and nowhere is the adaptation of the organ to its function-which results from the mode of life of the animal-more clearly seen than in the arrangements of the alimentary canal.

Secretory organs are generally connected with the alimentary canal, to aid in the process of digestion; their products dissolve, and act on the nutrient matter by chemical change. Glands of this kind are sometimes distributed over the whole canal, and sometimes distinguish certain portions of it ouly. In their simplest form they are not differentiated from the enteric wall, and in that case are not distinctly marked-off parts. Those marked off from the wall of the enteron are separated into two chief divisions. One of them comprises the glands which open in the buccal cavity, or its neighbourhood, and are distinguished as salivary glands. Another group is formed in the portion which serves for digestion, and is regarded as a bile-producing organ, a liver. It is right to note that the distinguishing of these organs by names which are applied to organs of higher organisms, physiologically better understood, is merely hypothetical, for nothing is known of the physiology of most of the organs of the lower animals. This holds especially for the epithelium of the gut, which generally appears coloured, and which is often called the "liver." This organ appears under the form of an epithelium, lining a part of the digestive carity in the Cœlenterata, in many Vermes, and even in Insects, till at last it becomes limited to definite cæcal appendages of the alimentary canal, and so attains to the lowest grade of independence. The liver presents 
itself from this point onwards either in the form of numerous follicles, which beset a large portion of the canal, or it forms a large group of glands, which open separately or together into the alimentary canal. The differentiation of the liver leads to a gradual separation of that organ from the digestive tube, so that finally it is merely connected to the canal by its ducts (higher Mollusca, Vertebrata).

\section{Respiratory Organs of the Enteron.}

\section{$\S 42$.}

The general differentiations of the primitive gut (archenteron), formed by the endoderm, which have been already mentioned, give rise, in obedience to the principle of division of labour, to organs which serve for the ingestion and digestion of nutrient matters; these do not confer any essentially new functions on the gut. But such a new function does appear when the gut acquires relations to respiration. It is not certain whether this function obtains in the primitive gut, although this is probable, for the endoderm is bathed by the surrounding medium, like the outer layer of the body, while the water which is taken in with the food may serve for respiration. The relation becomes much more definite when we note the regular streaming in of water into the rectum, which obtains in many Vermes and Mollusca. This phænomenon is an indication of the respiratory function of the gut, but has no bearing upon the formation of distinct respiratory organs, which are differentiated from the digestive tube.

Such a respiratory organ is brought into existence in the most anterior portion of the gut, by its walls being broken through by lateral pores; by special relations of these pores to the vascular system it acquires a respiratory significance. This arrangement, which already makes its appearance in the lower divisions, occurs again in the Vertebrata. Processes, known as branchiæ, in which the respiratory vascular network is distributed, arise on the walls of the clefts of this cavity. A region of the primitive digestive tube is thus converted into a special portion, a branchial cavity, at the hinder end of which the tube which serves exclusively for nutrition commences.

Another form of respiratory organ is developed from the wall of the gut, in the form of a diverticular outgrowth of the anterior portion of that organ. This appendage of the gut is filled with air, and in fishes has merely a hydrostatic function. As the relations of the circulation become changed it is gradually converted into a respiratory organ, and becomes the lungs; in the higher divisions of the Vertebrata new organs, namely, those for the production of a voice, are developed on the passages leading into it. 
i) Vascular System.

$\S 43$.

The substances prepared by the digestive process for the nourishment of the body are, in the lowest organisms which take in solid food, merely distributed from the digestive spaces into the protoplasm of the body. When a distinct digestive tube is formed, nutriment passes through its walls straight into the parenchyma of the body, so that the mesoderm and ectoderm, with the organs differentiated from them, are nourished by the endoderm. This is characteristic of the Colenterata and some groups of Vermes. In many others a dividing of the mesoderm occurs, which takes the form either of canalicular carities, or of a complete splitting of the mesoderm into an outer plate attached to the ectoderm, and an inner one attached to the endoderm. Between these dermal and gastric layers of the mesoderm is the body-cavity, or perientericcavity (cœlom), in which a fluid, to be regarded as the nutrient fluid, is collected. When morphological elements are found in this fluid, they are derived from the cells of the mesoderm. This fluid is not at first exclusively nutrient; it also subserves locomotion, by swelling out different parts of the body at the will of the animal. An important function of this kind is also played by the water, which in many cases is taken into the cœlom from the exterior.

The movement of the fluid in the general cavity of the cœlom is at first effected by the movements of the body. Contractions and expansions of the body-wall cause the fluid which is shut in by the dermo-muscular tube to continually change its position; this may be regarded as the lowest form of a circulation of the blood. In this case the passages have not special walls, nor are there any special arrangements for regulating the circulation.

This simple condition persists in many divisions in which the cœlom is developed (Bryozoa); in others canalicular cavities arise, which are arranged regularly, and have the form of vessels, and may undergo further complications. Their contents form the hæmal fluid or blood (Nemertines). When in addition to these vessels a perienteric-cavity is formed, the vascular system, which is partly enclosed in it, is either completely shut off from it (many Annelida), or is placed freely in communication with it at one or more points (Mollusca, Arthropoda, Vertebrata). In the latter case the vascular cavities must have arisen as portions of the body-cavity, while in the former case the body-cavity was not formed until after the vessels. The formation of the body-cavity is therefore, in the case exemplified by the Annelids, to be regarded as a secondary process; and the formation of a hollow space in the mesoderm has accordingly led to two different results successively; on the first occasion to the formation of blood vessels, on the second occasion to the formation of a body-cavity. 


\section{$\S 44$.}

Certain portions of the hollow cavitary system, which forms the hrmal passages, are converted into contractile vessels by the development of muscles in their walls. The earliest circulatory system arises by these producing by rhythmical action the regular in-and-out flow of the blood. But the direction of the stream of blood is not yet constant; it can be driven first to one side and then to the other. The portions of the vascular system which are distinguished by their greater contractility are sometimes extended over a large surface, and sometimes limited to shorter parts. 'They are the beginning of the formation of a heart.

The heart is therefore an organ differentiated from the bloodvascular passages, and in its simplest form is a portion of the vessels which is able to move its contents in two directions. It is only when valves appear at the ostia of the cardiac tube, that the direction of the flow is defined; the structure of the heart thus becomes complicated, and is further elaborated by being divided internally into separate portions (ventricles and auricles). Contractile organs of this kind often appear as the only differentiated parts of the blood-vascular system, formed from the cavity of the colom. The blood passes directly from the heart into lacunar portions of the colom, between the different organs, and from thence back again to the heart (Arthropoda), or there are definite vessels going off from the heart, which sometimes traverse the body in the place of the hollow cavity, or only partially replace the lacunar passage, in that they do not on their way back to the heart reach it as vessels, but into lacunar spaces. In this. case the cavity of the cœlom shows itself as a portion of the blood passage, which is only partly represented by true vessels (Mollusca). Where the vessels are completely developed and the heart differentiated, the vascular system is divided into three parts. That which leads from the heart and distributes the blood in the body is called the arterial, and its ressels arteries. The passage which takes the blood back to the centre of the circulation is formed by the veins, and the part of the passage which lies between the afferent and efferent vessels, forms a network of extremely fine canaliculi (capillaries). This intermediate portion is very frequently replaced by a lacunar system, in which case the greater number of the venous passages also have no special walls.

It is very often difficult to say what should be regarded as a vessel and what as a lacuna, and the distinction often depends on very unimportant points. It is not sufficient to say that the essential character of a vessel is the investment of a cavity by flattened elements derived from modified connective tissue, for these elements might just as fairly be regarded as the covering of the other organs which wall in these spaces; it is therefore questionable to call wide internal cavities, invested by such cells, "vessels." This cannot 
indeed be regarded as the sole criterion, and it should only have weight when considered together with the greater or less regularity of the lumen. But in examining this question we must bear in mind one thing; namely, that in all these cases we have to do with spaces which are walled in by connective substances, and that vessels are differentiations of these spaces, and therefore presuppose an indifferent condition. Between the two stages, the differentiated and the undifferentiated, there are all kinds of intermediate steps.

\section{k) Reproductive Organs.}

$$
\S 45 .
$$

The phrnomenon of the multiplication of the individual is primitively closely connected with nutrition. Not only is nutrition the cause of the growth and consequent increase in size of the body, but it gives rise to a condition in which the organism converts the excess of nutrient material brought to it into the means for producing a new individual. In the lower forms, as in elementary organisms, a process beginning with gemmation, and leading on to fission, is a very common phænomenon. The manner in which multiplication is effected varies with the amount of material which is used by an organism in producing a new organism.

The phænomena of multiplication by gemmation and spore-formation, which are so common in the lower divisions of the Invertebrata, have some relations to sexual differentiation, which indeed does occur among the Protista. It is derived from a stage in which two similar germ-cells fuse to form a new organism (Conjugation). As the two uniting cells become gradually dissimilar they become distinguished into eg g-cells and sperm-cells; these are the morphological elements of the sexual reproductive matter throughout the whole Animal Kingdom, notwithstanding their numerous modifications, which are seen most markedly in the seminal cells. While the ovum retains its most essential characters, and can be recognised as such in every division, the seminal cell very early undergoes considerable changes. Like other cells it gets a flagellate process, which may be greatly developed, while the cellbody and its nucleus are so reduced that they ordinarily form a structure of no great size. In this way filamentous structuresspermatozoa-are formed from the seminal cell. Sexual Reproduction, then, does not exhibit a real contrast to the asexual.

\section{$\S 46$.}

WVe do not exactly know in all cases what are the relations between the place where the reproductive matters are formed and the early rudiments of the body, but from what has been observed 
in certain Cœlenterata and Mollusca we may suppose that primitively the relations of the two are very different, for in these forms the ova are derived from the endoderm, and the sperm from the ectoderm. The endoderm is, therefore, the female, and the ectoderm the male germinal layer. But it is not yet known how far these relations obtain among the higher animals. As yet there are only uncertain indications, but these speak to a general agreement with the results already obtained.

The parts of the body which are set apart for the formation of the sexual products gradually take on the form of glands. This is a further step in differentiation, and is connected with the localisation of the function.

In the simplest cases the two kinds of generative products are formed in special parts of the body, which function as sexual organs (reproductive glands); but these parts are not at first distinguished by any special characters. The organs which produce the semen are called testes, and those which produce ova, ovaries. Going a step further, we find the reproductive glands still further differentiated. In their simplest condition the products of these organs merely break away from the spot where they are formed, and pass into the digestive sac, or into the body-cavity, or even directly to the exterior. Gradually, however, ducts, which are often very complicated in character, are added on : it is probable that these ducts are not primitively connected with the germinal glands. Where these ducts can be seen to have any relations to other organs, these appear to be excretory organs $(\$ 40)$ which have entered into the service of the genital organs, and have been altered so as to correspond to this function. It becomes a great question whether the excretory ducts of the reproductive matter are not in all cases excretory organs. Receptacles which serve for the collection of the sperm are formed on the outlet-tubes (seminal ducts) of the organs which produce the sperm; from the wall of these canals glands are differentiated, which secrete a fluid to be mixed with the sperm; finally, there are arrangements for passing the sperm into the system of the other sex (copulatory organs). The differentiations of the egg-forming organ are no less varied; the duct (oviduct) of the ovary is provided with dilatations, in which the ova get special envelopes, or are further developed. These portions of the oviduct are called the uterus. Special glands, Yolk glands, are formed from the ovary, and secrete a substance which is either taken up by the ovum or which merely forms an envelope for it. Appended organs receive the semen which is passed in copulation, and are known as receptacula seminis; lastly, other parts serve for the reception of the copulatory organ, or for the deposition or preservation of the ova.

The relation of the egg-forming and sperm-forming organs to one another varies greatly, and must be considered from the standpoint of differentiation. In the lower divisions organs of both kinds are united with one another, sometimes in such a way that one 
and the same gland produces both semen and ova (hermaphrodite gland). The ducts, also, are often more or less common to them both. But in other forms the genital organ is divided, the products of its two parts being different; testes and ovaries, that is, are present as separate organs, the excretory organs of which only are united more or less extensively; or each of them may have its separate orifice. All those animals which unite in themselves both kinds of reproductive organs are known as Hermaphrodites. A separation of sexes is apparently foreshadowed in various forms, by the alternating activity of the organs, at one time the eggforming and at another time the sperm-forming organ exercising its function.

The hermaphrodite stage is the lower, and the condition of distinct sexes has been derived from it. This change is due to the decrease in size of one or the other organ, so that hermaphroditism is the precursor of sexual differentiation. This differentiation, by the reduction of one kind of sexual apparatus, takes place at very different stages in the development of the organism, and often when the sexual organs have attained a very high degree of differentiation. In these cases ontogeny exhibits the two kinds of organs primitively united, and so causes the individual to be hermaphrodite at a certain stage in development.

The separation of the sexes affects the whole of the organism, for it produces a series of changes in each sex, which affect organs that had primitively little to do with the sexual function. Sexual differentiation is completed when the two kinds of organs are given over to different individuals. Thenceforward for reproduction, not only two different substances, semen and ova, and two different organs for producing them, are necessary, but also two individuals; these are distinguished as male and female.

\section{Changes in the Organs.}

\section{Development and Degeneration.}

\section{$\S 47$.}

The result of the continued differentiation of a given organ is a complication by which the organ is removed proportionately further from its primitive condition. As the primitive condition is the lower differentiation, it entails a per fecting corresponding to a higher condition. This is clear on the principle of division of labour, which is the cause of all differentiation (cf. \$ 12). In obedience to this law a function can be the more perfectly carried ont, the more exclusively the organ is related to that function. The more an organ is exercised for one function only, the more suitable are the conditions for its development in one direction, for there is no competition with 
other directions of development. A limb which is a gill too, that is which has both locomotive and respiratory functions, is of a lower grade than an arrangement resulting from a division of the two functions, in which a part separated off from the appendage represents a gill, and the rest an organ of locomotion. When the functions are united, locomotion is necessary for respiration, but when they are separated, they are independent of one another, and respiration is effected without the aid of locomotion, by the development of special organs for changing the water, these organs so far taking the place of locomotion. In both organs the independence which is necessary for their further development in one direction is gained.

The organs of the body are not always developed to the same extent. One or another often remains in a lower condition, and so retains its more lowly character in an otherwise highly differentiated organism. It is not therefore wise to draw any conclusions as to the extent of the differentiation of single organs from that of the organism itself; it is better to judge organs by comparing them with equivalent organs in other organisms.

The real factor in the development of an organ by differentiation must be sought for in the increased or modified function of the organ in the struggle for existence, that is in its adaptations to the external conditions of life. It is hence that transmission acquires its importance, since it not only causes a perpetuation of inherited characters, but is enabled to effect an elevation in those characters.

Degeneration or reduction is another constant phænomenon which is dependent on differentiation, inasmuch as it presupposes it. Its result is, in itself, the exact opposite to that of differentiation. For while differentiation is the cause of complications, reduction is the cause of simplifications of the organism, and is therefore the cause of organs or of organisms passing to a relatively lower stage. With regard, however, to the general organism, and its relation to other organisms, it produces the same effect as differentiation, for it leads to variety in form.

Reduction, like differentiation, varies in degree; it may affect separate portions of the body, or groups of organs, or finally the whole of the body. It is different, again, according as it affects the individual, the species, or the genus. In one case it may be seen to be a definite process, in another a condition, which can only be assigned its place as one of the several stages of such a process by the aid of a comparative series of allied forms. It may affect organs in two different ways. The affected organ may be independent of the general arrangements which obtain in the developed organism to which it belongs, and then reduction has but a transitory or provisional signification. Reductions of this kind during the course of the development produce simplifications, but as the differentiation which is going on in other parts may be producing new and higher organs, this reduction does not hold the organism back, but is a cause rather of the progress of differentiation in another direction. The reduction of parts which belong to certain 
developmental stages in the individual are examples of this kind of reduction (Larval organs). (Cf. § 5.)

The other kind of reduction affects organs which belong to the developed organism or its rudiments. It may affect the fully formed and completely functional organ as well as one just laid down, and in the primary state of differentiation. The process of reduction is seen therefore in various degrees of intensity. The process is often difficult to perceive amid the various other processes of differentiation which are affecting the rest of the organism when the organ affected is only just making its appearance: the further, however, differentiation has gone, the more striking must the process be.

The reduction of an organ is necessarily connected with its function, a change in which must be regarded as the cause of the reduction. Loss of function produces retrograde changes in an orgall, but of course neither process is a sudden one.

Although reduction is, on the whole, the cause of the simplification of an organ, and therefore of the organism also, it is not a phrnomenon which makes the organism absolutely lower in degree. Reduction may rather lead to a higher differentiation, as it does when larval organs are removed; it may give rise to higher forms even in whole series of organisms derived from one another, by facilitating: the higher- development of those not affected by it. In this case again reduction precedes differentiation. This is strikingly seen in the numerical relations of parts, which become individually more perfect as they diminish in number.

As reduction is a gradual process, the organs which are affected by it may be met with in various stages. These rudimentary organs are most significant indications of genetic relations, while they at the same time show us how an organ which has lost its primitive function, and which may even have no intelligible signification as regards the purposes of the organism, may persist for a very long time before it completely disappears. '(Cf. suprà, \& 6.)

Reduction may affect every organic system and be observable in every part of it. It is expressed in the form as well as in the size and number of the parts, and even in their histological characters. Its conditions are to be sought for, first of all, in the relations which alter the organism. According to the number of organs affected, reduction is more or less manifest in the organism as a whole.

Inasmuch as comparison everywhere reveals to us evidence of either progressive or of retrogressive change, we may regard the organism as a thing caught in the act of moving, as arrested in the midst of a career through the most diverse ranges of form. The changes of the various organs, and the phænomena which are observed in the elementary structure in the cell, are what make up this movement. 


\title{
Correlation of Organs.
}

\author{
$\S 48$.
}

The changes in the organism which are due to differentiation and reduction are the cause of a fresh series of phænomena in the factors which gave rise to them. From the conception that life is the harmonious expression of a collection of phænomena regularly conditioned, it follows that the activity of an organ cannot be regarded as really existing for itself alone.

Every kind of arrangement presupposes a series of other arrangements; every organ, therefore, must have intimate relations with the rest, and be more or less dependent on others. Every movement in a muscle presupposes the existence of a nerve; and both of these organs presuppose the existence of a nutrient system. In this way one function has an intimate connection with other apparently dissimilar functions. This relation, which was first definitely pointed out by Cuvier, and which is known as Correlation, shows us the road by which we can attain to a correct appreciation of animal organisation. By far the most important point is the conception of the organism as an individual whole, which is as much conditioned by its parts, as one part is conditioned by others. Correlation is a necessary result of this conception.

Not only the general arrangements of the organisation, but also its apparently more subordinate features, exhibit intimate relations with one another, and a change which affects one system of organs, simultaneously produces modifications in some of the other organs. These are adaptations to changes, which themselves are due to adaptations. They are, however, of a secondary character, while those which are of a primary character have their origin in the outer world.

Correlation may be divided into the more and the less remote; where less remote it is expressed in one system of organs or in other systems functionally connected with this; when more remote, in organs which are functionally less related to it. Physiological principles are essential in the investigation of correlation, and it is necessary, therefore, to know what are the functions of separate organs, or at least what their value is in the animal economy, in order to be able to recognise it. So, too, it is of importance to know what are the habits of the animal, for the original forces, on which the various relations of the organs depend, are due to them.

As the forces which cause changes in the organism either lie without the organism, or, as most of them, are to be sought for without it, they do not come within the scope of our work. Comparative Anatomy, therefore, is limited all round by a wide but uncultivated region, in which rich harvests may be gathered for biological science, whenever its treatment is taken in hand. 


\title{
Fundamental Forms of the Animal Body.
}

\author{
$\S 49$.
}

Owing to the infinite variety of the external characters of animal organisms, it is necessary to seek for fundamental forms to which this variety may be referred. We must also ascertain the conditions which influence and give rise to the most important modifications of these forms. These results may be obtained in different ways. We will choose the shortest by commencing with the lowest stage of the animal organism.

This is the stage which the Gastrula form presents to us; by its wide distribution this form will provide us with the characters which are best adapted for our purpose. An organism at this stage is some-

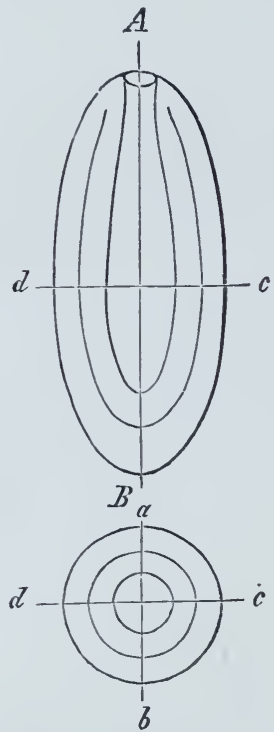

Fig. 16. Diagram of the axes of the body. $A B$ Primary axis. $a b$, $c$ d Secondary axes. The lower figure is a trans. verse section of the upper one, showing its two secondary axes. what spherical or oval in shape, and the mouth will be found at a point on the surface.

If we imagine an axis (Fig. 16, $A B$ ) drawn straight through the digestive cavity, the pole corresponding to the opening of the mouth represents the oral, and the opposite the aboral pole. This axis $(A B)$ is the primary axis of the body. In a body of a regular cylindrical or spheroidal shape we can imagine as many lines as we please drawn through the body perpendicular to this axis. (Secondary axes, $a b, c c$.) In this instance they are all equivalent. The secondary axes are in this case indifferent to one another, and are characteristic of a lower condition. The organism, either when moving freely in the water, or when fixed (by the aboral pole, of course), as it afterwards is, is differentiated by the development of a certain number of secondary axes, their development having relation to the maintenance of the balance of the body. We here, then, have to do with a statical cause. The development of the organism along its secondary axes takes place through the development of external appendages, tentacles and the like, or through differentiation of the enteric cavity, or through the laying down of other organs (such as the generative glands) in the direction of those axes. In consequence all the conceivable secondary axes are no longer equivalent. 'Those along which organs are differentiated are distinguished from the rest. They have passed, in fact, from the previously indifferent condition to a differentiated one. The socalled radiate fundamental-form of the body which is commonly found in the Colenterata, arises in this way, as may be seen by 
studying the relations of the axes to one another, as explained above (cf. Fig. 17). The importance of the mouth to the organism causes the differentiations which obtain around it to have a special value. These differentiations are developed as tentacles of various form, and cause the parts around the mouth to be much more varied in character than those at the aboral pole.

If the body grows in the direction of its primary axis, without becoming attached to the ground, the axes may acquire modified importance if locomotion in the direction of the animal's length be established. The primary axis will remain as before, but the secondary axes will necessarily differ according to the significance of the surfaces which they connect. When one and the same surface always touches the supporting object, it becomes the ventral
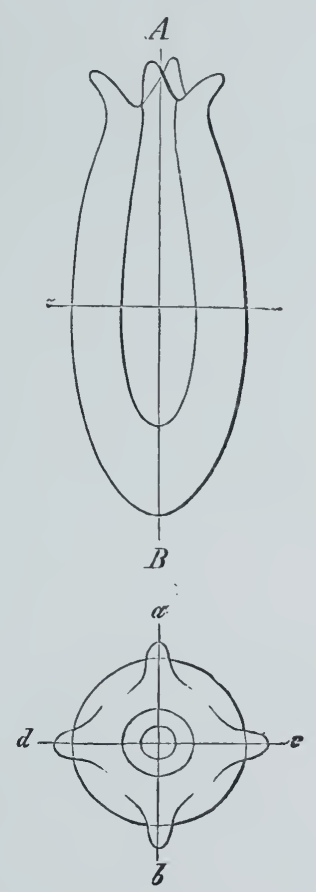

Fig. 17. Radiate fundamental form; letters as in Fig. 16. The anterior surface of the body is seen in the lower figure, and shows the appendages (tentacles) which are differentiated along the two transverse axes.
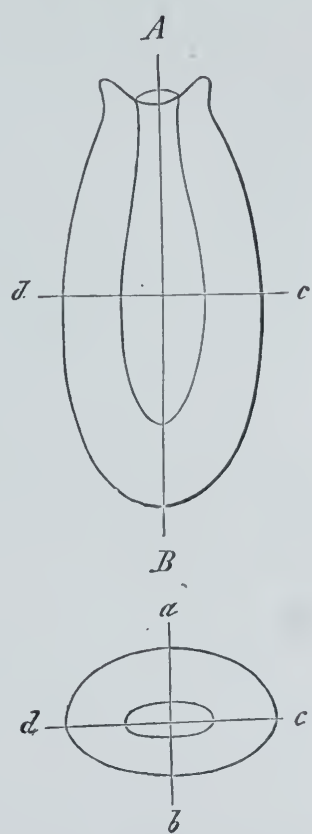

Fig. 18. Diagram to show the differentiation of the secondary axes. In the upper figure a cephalic portion is indicated by the development of a pair of dorsal tentacles. The lower is a transverse section of the upper figure, and the secondary axes are consequently seen in it. surface, and the opposite one becomes the dorsal. These trwo surfaces, the dorsal and the ventral, are placed under different conditions, and must therefore be differentiated in different ways, while the two sides, or-when the body is perfectly flattened out-the two lateral edges necessarily come to differ in cliaracter from the dorsal and ventral surfaces.

Such cases are instances of the development of only two inequivalent secondary axes. One comnects the ventral and dorsal surfaces, and is the dorso-ventral axis (Fig. 18, $a$ b), the other connects the sides $(c d)$ of the body, and is the transverse axis. The surfaces which correspond to the poles of the first or dorso-ventral axis are not, while those which correspond to the poles of the transverse axis are, equivalent. A primitive condition which has disappeared in the dorso-ventral axis in consequence of the differen- 
tiation of its surfaces, is retained in the case of the transverse axis. This, the second form, which can be derived from the Gastrula, and is ordinarily known as that of bilateral symmetry, first appears in the Vermes, and prevails in all the divisions above them.

When the secondary axes of the body retain their primitive indifferent character, we may imagine that there are as many similar pieces in the architectural composition of the body as there are possible secondary axes. But when the secondary axes become differentiated, the divisions of the body take on a definite numerical relation. They are known as antimeres. If two secondary axes become differentiated, and are like in character, we have four antimeres, for we can divide the body into four similar parts, along: these secondary axes. But when two unlike secondary axes are differentiated the body is only made up of two antimeres; two halves of the body, distinguished as right and left, are the parts corresponding to one another. In this way the eudipleural fundamental form is developed.

\section{$\S 50$.}

The differentiation which marks off the oral from the aboral pole, and which has been already mentioned, gives a higher significance to the former region of the body. This differentiation asserts itself in other forms, as in the primary radiate form, and in very various ways. It is not only the presence of the mouth, which favours the differentiation of organs around it, as organs for aiding in the prehension or ingestion of food, but the greater significance of the anterior end of the body in locomotion is also a cause of differentiation. This part takes the initiative. It has to show the way to the rest of the body, and often indeed, to lead it; it meets with a thousand foreign objects, which it has to examine, to follow, or to avoid. It is therefore exposed to external influences other than those which act on the opposite end of the body. The dignity of the relations of this region explains how it is that the mouth is not by any means always at the anterior end of the body, and that it much more frequently is placed close to or even altogether on the ventral surface, without the anterior end of the body being less highly developed. The high specialization of the anterior region is caused principally by the development of various kinds of sensory organs, and therefore of organs which put the organism into relation with the outer world; the region moreover often has various organs of defence connected with it, and with it is closely connected the development of the central nervous system. The whole region thus gets a higher value in comparison with the general organism, for it shelters and carries the organs which elevate and rule the latter. This anterior region of the body is therefore called the head, or chief portion. The differentiation of a head seems to depend primarily on the position of the mouth. This directs the course of movement, and to this, as a secondary cause, the anterior part of the body owes its 
various distinctions. The appearance of a head is at the same time a result which affects the whole body, for the body can now be divided into two portions at least, which differ in character.

\section{Metamerism of the Body.}

\section{$\S 51$.}

The planning out of the individual organism as a single structural entity is only characteristic of lower conditions of development, whether permanent, as in nearly all Cœlenterata and in the lower classes of Worms, or transitory, as in the higher divisions of the Animal Kingdom. Simultaneously with the growth of the body to a considerable length, we observe the beginning of the division of the organism into separate segments, following one on another, noticeable externally through the occurrence of separating constrictions, or through the regular distribution of appendicular structures or processes of the body, internally represented by the arrangement of the organs in the distinct successive compartments of the body. We term this segmentation of the body Metamerism; the separate segments are metameres. The metamerism which thus breaks up the body is only a further example of differentiation. From the primitive homogeneous indifferent body a heterogeneous, diversified body is developed, and the separate metameres differ from one another; not only are they something new in comparison with the earlier condition, but they are also-notwithstanding their resemblance one to another-different from one another, owing to the position which each occupies.

Metamerism is not in all cases, where it is perceptible, exhibited with equal clearness. Sometimes it is apparent in this or that organ, or system of organs, more than in another, and, again, in other organs may be altogether wanting. It is easy to recognise very various conditions of the commencement and of the incomplete carrying out of the process. Where we find it in fullest development it dominates the whole organism, and is exhibited in all organs ; so that each metamere possesses its individual system of organs, and particular systems of organs common to all metameres present a special differentiation of their structure in each metamere (ventral ganglion chain). In this manner the organism becomes built up of many component parts. And hereupon we have to take note of conditions in which independent importance is acquired by the metameres. Little by little a metamere, in virtue of the elaboration of its own set of organs, ceases to be dependent on the total organism, emancipates itself from the commonwealth, and gains the capability of leading a free existence. To this many phænomena are traceable, which are usually called gemmation (Worms). 


\section{$\S 52$.}

An efficient cause for metamerism may be sought, as has been above indicated, in the phænomena of growth. We can imagine a repetition of local outgrowths, resulting in practical advantage to the organism, taking place in particular systems of organs simultaneously with the elongation of the body. In this way the external metamerism may be brought into connection with the movement of the body, which was perhaps the earliest cause of this phænomenon. Many facts point to its being so. In any case there are numerous examples of the gradual elaboration of metamerism without all systems of organs being at once affected by it. Metamerism has, however, a less doubtful origin in its connection with gemmation, which is itself a kind of growth. It seems, indeed, in many cases, as if gemmation led to metamerism, in such a way that the metameres represent buds, which remain connected with the organism, and only in some cases attain to a higher stage of individual existence. Numerous instances of incomplete metamerism prevent us, however, from attributing a general significance to this process, and it cannot in any sense be regarded as the sole cause of metamerism.

Metamerism leads to perfection of the organism. By it the organism is enabled to get a larger number of organs, although, indeed, they are at first mere repetitions of one and the same arrangement. As the separate segments become more independent their action becomes more free, till at last the differentiation of a larger number of separate organs gives a larger scope for action. Differentiation, then, gains ground in every part, and alters the organs of the separate metameres in different ways, according as their functions become more various. By the development and reduction of metameric organs the inctameres get to differ in value, and become differentiated themselves; this differentiation is expressed externally by the difference in their size and form. This leads to the disappearance of the primitive similarity of the metameres. The amount, too, of their independence may be lessened, and a number of primitively separate metameres may gradually fuse into larger divisions. This gives rise to complexes of metameres, in which the fact of their being composed of separate units of the body is only suggested, and that often faintly"; sometimes a large, sometimes a small number of segments undergo concrescence. This, too, is on the whole a cause of differentiation of the organism, as the body consists in consequence of some independent and of some fused metameres. Finally, metamerism may altogether disappear, and the presence of separate organs alone indicate, and that often obscurely, the phenomenon which obtained in the primitive state. Every stage in metamerism is therefore a source of variation in the external and internal organisation of the organism. 


\section{Comparison of Organs.}

\section{$\S 53$.}

The variations in organisation among the various larger and smaller divisions of the Animal Kingdom are such as to lead us, at first sight, to perceive the points of difference rather than those of agreement. And this is more marked in proportion to the divergence between the particular divisions compared. It is, however, the business of Comparative Anatomy to follow out the changes in the organisation, and to discover what is "similar" in the changed and metamorphosed forms, however deeply hid it may be. An organ may be "similar" to another in one of two ways. Either in its functional relations, that is from a physiological point of view; or in its genetic and therefore anatomical relations, that is from a morphological point of view. These two relations of an organ must be kept well apart. The change of function in one and the same organ, as well as the similarity in arrangement of organs which are morphologically very different, compels us to ascribe a subordinate value to physiological relations, when we are comparing organs. The gills of a Fish, of a Crab, and of a Cephalopod, are organs of respiration, and have many structural points in common ; yet they are very different organs morphologically, as the relations of each of the three to the whole organism shows. By insisting on similarity of function, we bring together organs which are morphologically different, and so turn aside from the object of Comparative Anatomy. We distinguish, accordingly, physiological likeness, or Analogy, from morphological likeness, or Homology, and only consider the proof of the latter as our task.

The smaller the division to which the objects of comparison belong, the more obvious is the homology. Homology therefore corresponds to the hypothetical genetic relationship. In the more or the less clear homology, we have the expression of the more or less intimate degree of relationship. Blood-relationship becomes dubious exactly in proportion as the proof of homologies is uncertain. It is impossible therefore to say exactly how far homology extends throughout the Animal Kingdom. As a matter of fact, numerous investigations have discovered a larger number of homologous arrangements even in otherwise divergent groups, and have thereby extended the boundaries of homology further than was formerly thought possible.

In consequence of the existence of various possible modes of morphological agreement, homology is divided into two primary groups: General and Special Homology. 


\section{$\S 54$.}

I. General Homology is under consideration when an organ is referred to a category of organs, or when a single organ compared with another is taken merely as the representative of such a category. These categories always consist of several organs or parts present in the body. When we compare the body-segments of an Annelid, the vertebræ, or the appendages of an animal with one another, we lay the foundations of geneial homology. This again consists of sereral subdivisions, according to the kind of category which is made use of in the comparison.

1) Homotypy has reference to organs which are fellows to one another, such as the organs of the two sides of the body; the right kidney is homotypical with the left, and the right eye with the left eye, and so on. Whilst these examples may not show the necessity for the formation of this division, it should be noticed in addition that homotypical organs have not always the same characters. They are often so changed that their homotypy cannot be recognised, and has to be worked out.

2) Homodynamy (equivalent to the general homology of Owen, and partly also to his serial homology) subsists between parts of the body which are affected by a general morphological phænomenon serially expressed in the organism. Homodynamy is distinguished from the next subdivision by the fact that the parts in question are arranged along the long axis of the organism and define its type. The metameres therefore are homodynamous parts; as are the segments of the Arthropoda, the primitive vertebræe of the Vertebrates, etc.

3) Homonomy. This describes the relation to one another of those parts which are arranged along a transverse axis of the body, or in one segment only of its long axis. The rass of the pectoral and pelvic fins of fishes, the individual fingers and toes of the higher Vertebrata are homonomous structures.

Besides these there are other subdivisions of general homology distinguishable, which are however of very subordinate importance.

\section{$\$ 55$}

II. Special Homology, Homology in the restricted sense. This is the name we gire to the relations which obtain between two organs which have had a common origin, and which accordingly have also a common embrenic history. As exact proofs of genetic relations are necessary for the inrestigation of special homologies, this mode of comparison is generally limited in the lower dirisions of the Animal Kingdom to systems of organs; it is only in the Vertebrata that it is possible to extend this method to more minute features. Thus among the Termes or the Mollusca we can hardly indicate, with any certainty, particular parts of 
the alimentary canal as homologous; while in the Vertebrata we can confidently assert that even such unimportant structures as the cæca of the intestine from the Amphibia onwards are homologous. The homologies of the parts of the skeleton, which are the organs that have been investigated with the greatest exactness, are those which can be most definitely recognised. It is a large part of the main task of Comparative Anatomy to prove special homologies.

Special homology must be again separated into subdivisions, according as the organs dealt with are essentially unchanged in their morphological characters, or are altered by the addition $01^{\circ}$ removal of parts. I therefore distinguish-

1) Complete Homology, when the organ referred to is unchanged in position and connection, and is still perfect however much modified in form, size, and various other points. This kind of homology is generally found within the limits of small divisions, less often in larger ones. For example, the bones of the upper arm from the Amphibia to the Mammalia, the heart of the Amphibia and Reptilia, etc., exhibit complete homology.

2) Incomplete Homology. This consists herein, that an organ which is otherwise completely homologous with another, has other parts which are wanting in the latter added to it, or conversely, when an organ is wanting in some essential part in comparison with another organ. The heart of the Vertebrata may serve as an example. The organ is homologous throughout the division from the Cyclostomi onwards, but the homology is incomplete; for in Fishes a part, the venous sinus, which in the higher divisions is taken into the heart, and which in the Mammalia is absorbed into the right auricle, lies outside the heart. The homology between the heart of the Fish and of the Mammal is consequently incomplete owing to addition. In another case it may be incomplete owing to diminution. The reverse of the previous case may serve as an example, were it allowable to regard the heart of the fish as a reduced one. An example is presented by the pectoral fins of fishes. The skeleton of this organ in the Ganoidei or Teleostei is, owing to reduction, incompietely homologous with that of the Selachi. Parts have in this case disappeared which did primitively belong to the organ, just as in the former case parts, which although they were primitively present did not belong to the organ, were added to it.

\section{Systematic Classification of the Animal Kingdom.}

$\S 56$.

In the general organisation of every animal we recognișe a number of arrangements which it has in common with a greater or: less number of other animals. These relations are partly of a more 
general nature, affecting the position or arrangement of the most important systems of organs, and partly they affect the special development of individual organs; they extend to agreement in form, size, and number. The classifying spirit of man has formed definite conceptions of these relations of organisms to one another. All those individuals which agree in essential points he has called a species, and has united into a genus those species which resemble one another in a number of points; these again he has united into larger divisions, families, orders, and classes. Thus arose the zoological system, which, in so far as it unites what agree, and separates what differ, has come to be the expression of our general knowledge of the Animal Kingdom.

In this way the whole Animal Kingdom can be broken up into several large divisions, each of which differs from the rest by a number of special characteristics. The essential character may be recognised in all the subdivisions, and even under great individual variations. This has been called the "type." The type then means a collection of characteristics which are expressed in the organism, and which are predominant in a large division of the Animal Kingdom, and which are evident in the course of development as well as in the adult condition. Such larger divisions which differ from others in certain fundamental points of organisation are themselves called "types."

Within each type we note a variation in the characters of the divisions which make it up, and this often to such an extent that what is characteristic of the type appears to be lost in some forms. In this case it is always individual development which enables us to recognise the connection of these forms with the "type."

If we admit that similarity of organisation in different individuals is explicable by the fact that they have a common ancestor, and that therefore these similarities are due to affinity, we must regard less close similarities as due to a less close relationship. We therefore regard the individuals which belong to one species as more closely allied than are the representatives of different species, and within the limits of one species we shall again derive from common ancestors those individuals which are distinguished by special characters, and which we unite into a sub-species.

No one has any hesitation in recognising within the limits of small groups of individuals the phænomenon of the continuation of the peculiarities of a given organism into other individuals by means of transmission; indeed it is often possible to perceive, by direct observation, that descendants are like their ancestors. By extending this conception of affinity to a wider circle, and regarding what is common in organisation as due to a common descent, and what is divergent as due to adaptations, we take our stand on the theory of Descent (cf. $\S \S 4$ and 5). We consequently regard the large divisions known as "types," as phyla, or leading branches of the genealogical tree, and by so doing point to the cause which has determined their existence. 
Within one phylum a form of animal organisation is evolved along the most varied lines, which gradually lead from the simple to the more complex, and from the lower to the higher. The categories which we distinguish as species, genera, families, orders, and classes are due to continued differentiation. These subdivisions correspond to the ramifications of the branch, and in them the divergence of character is expressed.

The differences between the classes, orders, and so on, are so great that they do not seem to have any connecting links at all, but we must take into consideration the fact that in living forms we have before us only the final offshoots of developmental series of organisms; which have been ramified in very various ways, and which lived in earlier and often very far-distant periods, and which have gradually disappeared. The palæontological record proves this partly, though it may be but very slightly. In the strata of the earth remnants of forms which have disappeared, and which were the predecessors, and, in fact, the direct ancestors, of later living organisms, are preserved. Inasmuch as the living forms are but a small portion of the whole world of organisms, which has existed in the course of geological periods of development, we cannot expect that far-distant connections should be always evident, the intermediate steps determinable, and the genealogical connection made clear and indubitable. It is necessary to try and put the whole together out of fragments and to find lost traces of continuity. The most important part of the business of Comparative Anatomy is to find indications of genetic connection in the organisation of the Animal body.

Following out this conception we have to represent to ourselves a developmental series of organisms arising in each phylum from a primitive form, which has been, during geological development, differentiated into many branches and twigs, most of which have disappeared at different periods, while some, greatly changed though they may have been, have lived on until to-day. The general character which has remained in these various stages of differentiation, and has been transmitted, with modifications, from the stem-form, is what is typical in the organisation.

\section{$\S 57$.}

It is not always possible to prove, to the same extent, in all of the large divisions which are regarded as types, the common ancestry of the forms which belong to it. It is in fact very probable that several divisions have had a polyphyletic origin, in which case the organisms which belong to them must be united together for reasons other than genealogical. Such divisions cannot be regarded as phyla.

We meet with such relations in the lowest forms, in the boundary-territory between Animals and Plants. It is difficult to find a boundary line, for there are organisms which seem to belong to one as much as to the other Kingdom according to the phrenomena 
to which they give rise. The idea of a boundary line presupposes a rigidly-defined conception of Animal and Plant. The characteristic of the animal organism may be taken to be that differentiation affects the whole organism. This differentiation consists in its division into two layers, which have been already $(\$ 28)$ called ectoderm and endoderm, and from which the germinal layers of the higher divisions are derived. But the exclusion of all the lower organisms, which do not undergo this division from the Animal Kingdom, would put out of our scope many phænomena which are of great importance, if we would understand animal organisation. Although it might be best to regard this world of lower and very varied organisms as a special Kingdom placed between the Animal and the Vegetable, and containing the beginnings of both, that of the Protista, yet we, as our work embraces the connections between animals and these lowest organisms, must enter into a consideration of them. Tie therefore unite a number of those divisions of the Protista, which are more nearly related to animals than plants, as the Protozoa. As their genetic relations to one another are altogether unknown, the division which is formed by these organisms cannot be regarded as a "phylum." Nor is there a type common to them all. We therefore regard them as lower organisms, which are the nearest of the Protista to Animals, and we must compare them, not with the separate divisions of the higher animal organism, but with them all together. This compels us to unite the latter into a single group, which has been called the Metazoa.

The Protozoa and Metazoa are not so very sharply marked off from one another. Not a few of the Protozoa are known to be composed of a number of cells. It is the arrangement of cells in layers of definite physiological value which characterises the metazoic organism. 'This seems to take place very gradually, and at first the layers are incomplete. We find representatives of this in the parasitic Dicyemidre, which live in the so-called veinous appendages of the Cephalopoda, and deserve to be specially mentioned. A germinal cell gives rise to a number of cells by division, among which a single large cell becomes surrounded by a number of smaller cells, which form a continuous layer.

The central cell represents the endoderm, and is covered by the peripheral layer, which represents the ectoderm at all but one small spot. (Fig. 19.) The endodermal cell elongates considerably, and

Fig. 19. Fig. 20. Vermi-

Gastrula form embryo of stage of Dicyema typus Dicyema (after E. ran typus. Beneden). its protoplasm becomes differentiated in various ways. It forms the groundwork of the elongated body, and remains covered by the ectodermal cells, which also grow, without however multiplying very much; these give off fine cilia, and form the protective and locomotor organs of the body, while 
the endodermal cell in the axis of the body becomes an organ of nutrition, and takes on the function of reproduction, for the germs of young forms are found in it of two different types.

The organism of Dicyema is thus seen to be two-layered, and the two layers have different functions; the inner one is morphologically least differentiated, for it consists of a single cell. It is not quite certain whether this corresponds to a primitive condition or no, for the parasitic life of the Dicyemidæ may have been the cause of the reduction of a multicellular endoderm. But their development is quite easy to make out, and in it there is never more than one endodermal cell, so that the fact becomes more significant. Just as among the Protozoa the multicellular forms are allied by intermediate steps to the unicellular, from which they have been developed, so among the Metazoa does Dicyema exhibit to us the commencement of the separation of the body into cell-layers, and although the arrangement is not as perfect as it is in the rest, yet it is in the same direction as that which in them arrives at full expression.

Van Beneden, Ed., Recherches sur les Dicyemidæ. Bull. Acad. Belg. xli., xlii., 1876.

\section{$\S 58$.}

Passing over the Dicyemidæ, I recognise the following divisions of the Metazoa:

1. Colenterata.

2. Vermes.

3. Echinoderma.

4. Arthropoda.

5. Brachiopoda.

6. Mollusca.

7. 'Tunicata.

8. Vertebrata.

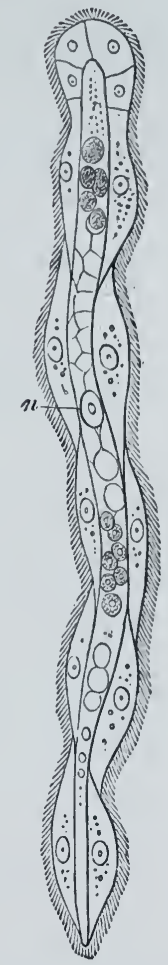

Fig. 21. Vermiform embryo of Dicyema typus. $n$ Nucleus of the endodermal cell (after E. van Bene. (len).

These divisions represent in a general way separate branches of the pedigree of animals, and each of them contains higher and lower forms in various proportion. But the degree and extent to which their organisation is developed is different in each of them. The divergence of organisation expressed in each division is indicated by their relations to one another, and it shows us how the lower forms of the higher phyla may have started from the lower phyla. These 
large divisions are therefore arranged in genealogical connection. The extent to which each division is separated from its fellows varies in each case. The relation of the various large divisions to one another is seen in the subjoined tree.

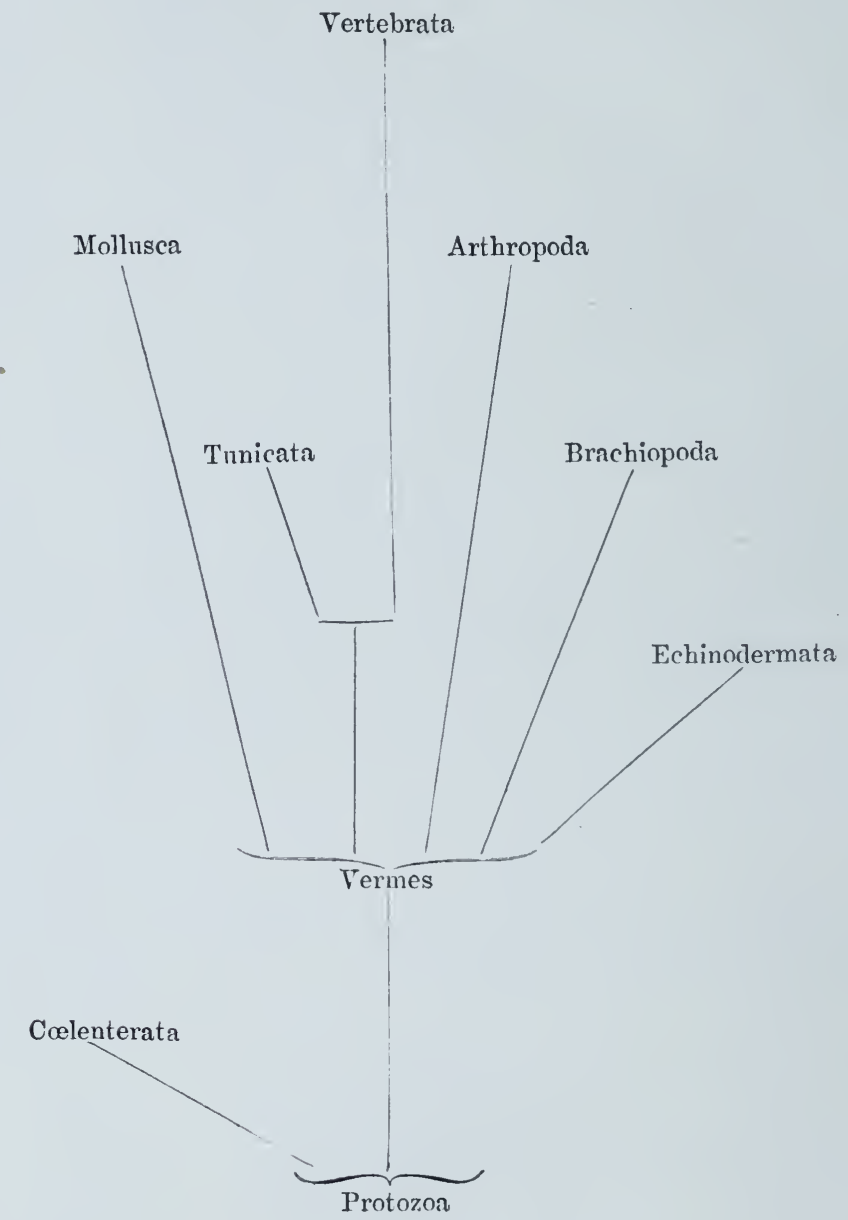

The more exact limitation of the separate divisions, as well as the reasons for the genealogical relations here merely indicated, will be found in the special chapters. 


\title{
Bibliographical Aids in Comparative Anatomy.
}

\author{
$\$ 59$.
}

The principal work which is to be recommended as a scientiflc guide to the whole of Morphology, and especially to the questions which I have, in the foregoing paragraphs, treated very concisely, and which should be carefully studied, is :

Ḧ̈скEL, F., Generelle Morphologie der Organismen. Allgemeine Grundzüge der Formenwissen. schaft, mechanisch hegriindet durch die von Cr. DARWIN reformirte Descendenztheorie. 2 Bde. Berlin, 1866.

\section{The following books also treat of Morphology philosophically:}

Lefckart, R., Die Morphologie und die Verwandtschaftsverhältnisse der wirbellosen Thiere. Braunschweig, 1818.

Carus, V., System der thierischen Morphologie. 1853.

Brons, Morphologische Studien iiber die Gestaltungsgesetze der Naturkörper. Leipzig und Heidelberg, 1858.

\section{a. Comprehensive Works on the whole subject of Comparative Anatomy:}

Currer, G., Leçons d'anatomie comparée recucillies et publiées par Dưréril et Duteryot. 5 vols. Paris, 1798-1805.

- Leçons, etc., recueillies et publiées par Dexúrí. Seconde édition. 8 vols. Paris, 1S35-46.

Mecket, J. F., System der vergleich. Anatomie. 6 Bde. Halle, 1821-33 (incomplete-sexual organs wanting).

MilNe-Edwards, H., Leçons sur la physiologie et l'anatomie comparée de l'homme et des animanx. T. I-XII. Paris, 1857-76. Unfinished.

Lexdig, F., Vom Bau des thierischen Körpers. I. Band. 1 Hälfte. Tühingen, 1861.

\section{b. Text-books and Manuals of Comparative Anatomy :}

Carus, C. G., Lehrbuch der Zootomie. Leipzig, 1818. Second edition, under the title of Lehrbuch der vergl. Zootomie. 2 Bde. Lcipzig, 1834.

WAGNer, R., Handbuch der vergleichenden Anatomie. 2 Bde. Leipzig, 1834. New edition, under the title of Lehrbuch der Zootomie. 2 Bde. Leipzig, 1813-18. (The second volume, containing the Anatomy of the Invertebrata, is by H. FreY and R. LEUCKART.)

V. Siebold and Stannius, Lehrbuch der vergleichenden Anatomie. 2 Bde. Berlin, 1815. Second edition under the title of Lehrbuch der Zootomie. Band I. Heft 1-2, containing the Anatomy of Fishes and Amphibia, is all that has as yet appeared. It will be continued.

Bergmann, C., and Ledckart, R., Anatomisch-physiologische Uebersicht des Thierreiches. Stuttgart, 1852.

Scнмгрт, O., Handbuch der vergl. Anatomie. 7e Auflage. Jena, 1876.

OwEs, R., Lectures on the comparative anatomy and physiology of the Invertebrate Animals. Second edition. London, 1855. - Of the Vertebratc Animals. P't. I. Fishes. London, 1816.

JoNes, RYMer, General Outline of the Organisation of the Animal Kingdom, and Manual of Comparative Anatomy. Fourth edition. London, 1871.

Harting, P., Leerboek van de Grondbeginselen der Dierkunde in haren geheelen Omvang. Deel I-III. Tiel, 1861-74 (deals also with Comparative Anatomy).

St. George Mrvart, Lessons in Elementary Anatomy. London, 1873. (Introduction to human anatomy.)

\section{c. Figures of the Structure of Animals :} Carts, C. G., and Отто, Erläuterungstafeln zur vergleichenden Anatomie. 8 Hefte. Leipzig

WAGNER, R., Icones zootomicæ, Handatlas zur vergl. Anatomie. Leipzig, 1841.

Sснмгрт, O., Handatlas der vergl. Anatomie. Jena, 1852.

Carus, V., Icones zootomicæ. Leipzig, 1857. First half (Invertebrata).

LEYdiG, F., Tafeln zur vergl, Anatomie. Erstes Heft. Tüihingen, 1861. 


\section{d. Comparative Histology :}

Lexdig, F., Lehrbuch der Histologie des Menschen und der Thiere. Frankfort, 1857.

\section{e. Ontogeny :}

Fostrr, M., and Balfour, T. M., The Elements of Embrjology. Part I. Macmillan and Co. London, 1874.

Kölliker, A., Entwickelungsgeschichte des Menschen u. der liöheren Thiere. 2e Auflage. 1 Hälfte. Leipzig, 1876.

In addition to these works, numerous monographs, treatises and essays, contained in the transactions of academies, and other learned societies, or Journals of Natural History, Zoology, and Anatomy, should be consulted.

「The English student should consult especially the "Journal of Anatomy" (Macmillan), dating from 1867, and the "Quarterly Journal of Microscopical Science" (Churchill), dating from 1853.-E. R. L.] 
SPECIAL PART. 



\section{First Section.}

\section{Protozoa.}

\section{General Review of the Group.}

$\S 60$.

I RECKON among these certain divisions of those organisms which, owing to the simplicity of their organisation, are examples of the lowest grades of living forms. The most essential character appears to be the absence of organs differentiated for the most important functions. The imperfect limitation of this division is due to this negative character ; in it, nothing that is "ty pical" of all its members, either in the relation of the body to its morphological elements, or in its organisation, can be made out. The want of any histological differentiation is a reason for considering the organisms enumerated in this group, in company with others, which we are wont to regard as lowly plants, as forms of life which stand between the Animal and Vegetable Kingdoms. It is on this consideration that the plan of uniting all the lower organisms which cannot be regarded as Animals or Plants into the Kingdom of the Protista is based. If we recognise this conception, it appears unnecessary to form a division of the "Protozoa." But the knowledge of the stages of organisation, which obtain in the Protista, are of such high value for the comprehension of animal organisms, that to pass them over completely would not be in accordance with the aim of this book. I have, therefore, retained in this place the division of the Protozoa, and place in it a number of forms which, by the simple conditions of their organisation, and by the low grade of their differentiation, are well adapted for giving an idea of the group.

First of all $I$ exclude from it those forms which have not a nucleus, that is, which do not pass beyond the cytod stage. The derelopment of a nucleus in the otherwise simple protoplasmic body 
of the organism is, when compared with the cytod stage, the expression of a considerable advance, and prevents us from uniting the forms that do possess it with those that do not, whatever other points of agreement there may be in their protoplasm; even though it is quite clear that in these cytod forms (Monera) we have the beginnings of the higher stages. These beginnings vary greatly, correspond to separate divisions of more developed forms, and make it probable that the latter have had a polyphyletic origin.

Of the groups of the Protista I regard the Rhizopoda, Gregarinæ, and Infusoria as Protozoa.

There is no permanent limitation of the protoplasmic body in the Rhizopoda; their protoplasm sends out varying processes. The lowest division is that of the Amœbidæ, the organism of which is of the grade of a cell. The body is formed by protoplasm and a nucleus; it is ordinarily naked, but it can temporarily surround itself with a capsule, or the capsule may be a permanent covering, open at one or two points. The organism communicates with the outer world by this opening, and by it can extend itself over its shell. If there are several nuclei present the organism forms a syncytium. The Foraminifera form the second division. It is very probable that they all have a nucleus; and these organisms, therefore, are also similar to a cell. But the formation of a covering, provided with numerous pores, and often considerably complicated, is an indication of a tendency to higher development. The Heliozoa form a small group more nearly allied to the next division. The Radiolaria, finally, are distinguished from all other Rhizopoda by the possession of a "central capsule" within the body. The central capsule contains a number of nuclear structures; these, indeed, render the Radiolaria similar to cells, but their body cannot be regarded as a cell. Another course of differentiation seems to have affected them. Further, in some the entire capsular protoplasm contains separate cells, which are regarded by many as structures not belonging to the organism (yellow cells). The development of various kinds of supporting organs gives a peculiar character to the Radiolaria. We can make out a large number of axes in the body, hy the aid of these skeletons.

The second division of Protozoa is formed by the Gregarinæ. An outer limiting portion surrounding the nucleus, that is a body of the grade of a cell, is wanting in the earliest stages only. They pass, therefore, through the Cytod-stage. In the mature organism an envelope, differentiated from the protoplasm within, can be made out; and they give indications of higher differentiations of the subjacent layer of protoplasm. An anterior portion is in many separated by a constriction from the cylindrical or band-like body.

The Infusoria form the third large division. I do not, as do many, include the Flagellata among' them. 'The body, formed of protoplasm, has its external form defined by the differentiation of a cortical layer. 'This cortical layer surrounds the more indifferent protoplasm, which may in many cases be seen to be rotating, and so 
calls to mind the streaming of the protoplasm in certain vegetable cells. A nucleus, which may be various in form, shows that the body of the Infusorian is equivalent to a cell. In some there are sereral nuclei. A higher degree of potentiality is expressed by the differentiation of various histological structures in the cortical layer. But this does not affect the character of the Infusoria as unicellular organisms, so long as they have only one nucleus, if we may suppose that the cell is no longer in its indifferent condition. In many forms there is a small nuclear structure, the nucleolus, in addition to the nucleus. The Infusoria are divided into the Suctoria (Acinita) and the Ciliata. The former have definitely arranged fine processes, capable of a small amount of movement, which serve in the ingestion of food. The Ciliata are distinguished by an investment of cilia; and subdivisions are formed according to the way in which these cilia are distributed over the body.

\section{Bibliography.}

Rhizopoda: Aunrbacı, C., Zeitschr. f. wiss. Zool. Bd. VIT.-Dusandin in Anm. sc. I. III. IV.-SchUltze, M., Ueber den Organismus der Polythalamien. Leipzig, 1854.- C.spenter, W., Researches on the Foraminifera. Phil. Tr. 1856, 1859. -The same, Introduction to the study of
the Foraminifera. London, 1862. (R. S.)-HuxLEx, TH. H., On Thalassicolla. Ann. nat. hist. 1851.-MüLLter, J., Abhandl. der Berliner Acad, 1858.-Ḧ̈́скEL, E., Die Radiolarien. Fine Monographie. Berlin, 1862. - Scru Lze, F. E., Rhizopodenstudien. Arch. f. mikr. anat. Bd. X-XII. -Hertwig, R., Arch. f. mikr. anat. Bd. X. Suppl.--The same, Zur. Histolog. der Radiolariell. Leipzig, 1876.

Gregarinæ: Stein, Ueher die Natur der Gregarinen. Arch. f. Anat. u. Phyl. 1818.-KöLLIKER, Beitr. z. Kenntniss niederer Thiere. Zeits. f. Zool. I.-LIEв EnкüHN, Evolut. des Grégarines. Acad. Roy. de Belgique. Mém. des Soc. étrangères. T. XXVI. Ed. vıN BENEDEN, Rech. sur l'évolut. des Grégarines. Bull. de l'Acad, royale de Belgique. 2me Sér. T. XXXI. Sur la Struct. des Grég. Ibidem. T. XXXIII.

Infusoria: EhrenberG, C. G., Die Infusionsthiere als vollkommenc Organismen. Leipzig, 1839.DuJardin, Hist. nat. des Infusoires. Paris, 1811.-Stein, Fr., Die Infusionsthierc auf ihrc Entwickelung untersucht. Leipzig, 18ăt. -The same, Der Organismus der Infusionstliere. I. II. Leipzig, 1859-66.-CLAPARÈDE, E., ct LACHMAN, Etudes sur les Infusoires et les Rhizopodes. Genève, 1858-61.-Engelmann, Th. W., Zur Naturgeschichte der Infusionsthicre. Leipzig, Zeitschr. f. Zool. XI.-Morphol. Jahrb. Bd. I.-Häcké, Z. Morphol. d. Infusol. Jen. Zeit-

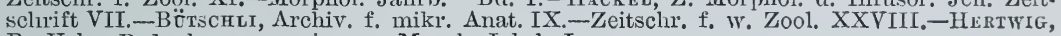
R., Ueber Podophrya g'emmipora. Morph. Jahrb. I.

\section{$\S 61$.}

As the body of the lowest organisms is formed of contractile protoplasm, which changes continually in form, there is no definite boundary to the body, nor any kind of differentiated integument. We see the bodies of most of those Protista, which are not provided with an envelope, alter in contour, just like the indifferent cells of higher organisms; processes of the protoplasm are extended from different points at different times, and the rest of the body flows after them. Thus, as the body moves, there is always a change in its surface, so that a particle of its substance, which at one moment is found in the interior, may at another moment enter into the formation of a process. The processes, Pseudopodia, have some- 
times the appearance of broad lobate prolongations (Fig. 22) separated from one another by shallow depressions; sometimes they

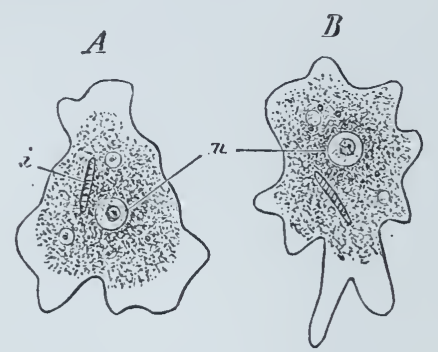

Fig. 22. An Amœeba figured at two different moments during moremeut. $\imath$ Nucleus. $i$ Ingested food. Some vacuoles may also be noted. flow out as delicate, sometimes as wedge-shaped, currents, which divide in all kinds of ways at their periphery, and so form ramified processes. These changes are always within definite limits of form in the various divisions, so that the form of the pseudopodia is the expression of the first differentiation of a definite morphological character of the protoplasm. The pseudopodia characterise the Rhizopoda, the superficial protoplasm of which is able to emit them in every form of this group (Fig. 23). Neighbouring pseudopodia can unite with one another at any point and in various numbers (Fig. 23, x), or may even become connected in a retiform manner. This character of the protoplasm is

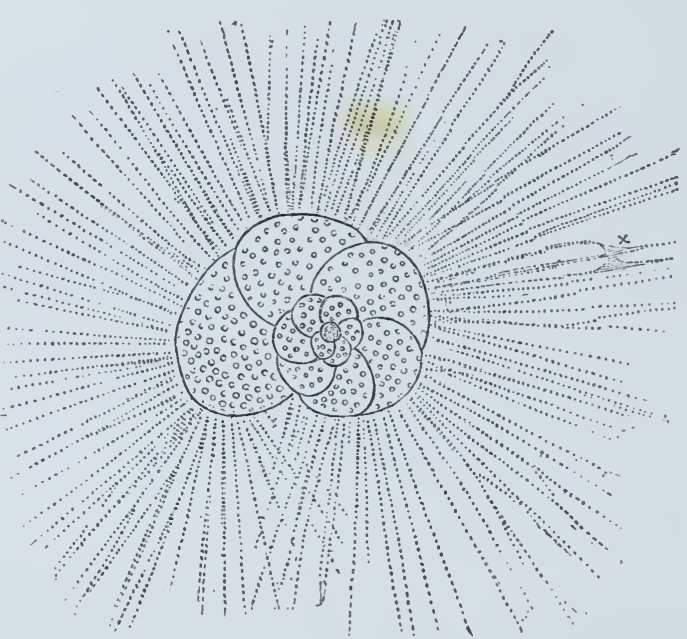

Fig. 23. A Foraminifer (Rotalia) with extended pseudopodia, which pass through the pores of the multiloculate shell. At $x$, several pseudopodia have united together. not affected by internal differentiation (skeletal organs, etc.). It is the expression of a stage in the lowest living material when it is not differentiated at its periphery.

When the outermost layer of the body is hardened, pseudopodia camnot be formed at every point. The chemicophysical changes in the peripheral parts lead to the formation of something unlike the rest of the protoplasm, which retains its indifferent character, and indeed still gives signs of its power of movement, but is restrained by the firmer cortical layer from any considerable extension of itself. 'This stage is found in the Gregarina; characters which obtain in many of the Amœbre exhibit intermediate steps towards it. A firm liomogeneous membrane sometimes delicately striated, extends in these forms over the whole body, which is formed by a single cell. It passes directly into the soft protoplasm, and appears to be a cuticle differentiated from it. Like all cuticles, it 
has no contractility; but it is extensible and elastic, and is thus able to follow the contractions and expansions of the protoplasm.

In addition to this separation of the cuticular layer there is also, in the Gregarinæ, a cortical layer separated off from the internal parts, which appears to be finer than the richly-granulated protoplasm; this is also found in the Infusoria.

\section{$\S 62$.}

With the separation of the body into an external cortical Iayer, and an internal parenchymatous substance, further metamorphoses of the cortical layer are connected. Of these the first that should be mentioned are the cilia which are widely distributed in the Infusoria. They appear to be direct but actively motile prolongations of the integument: if combined with a cuticle they traverse it. They either beset a limited part of the body only, as the so-called oral open-

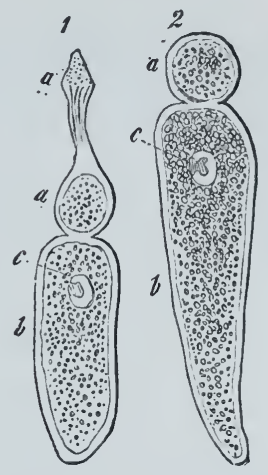

Fig. 24. Gregarina from the enteric canal of Opatrum sabulosum; $a$ is the younger stage, provided with a "proboscidiform" process. a Anterior portion. $b$ Posterior portion of the body. $c$ Nu. cleus. ing, or they are extended over larger tracts, or over the whole body often, with great regularity.

According to the definite distribution and arrangement of these cilia, the Infusoria have been subdivided into Holotricha, Heterotricha, Hypotricha, and Peritricha. It is clear that they are differentiations of the protoplasm, from what happens in some other groups of the Protista, where they form temporary structures only, and, like the pseudopodia, can be again withdrawn into the protoplasin of the rest of the body.

The flagella, as well as the undulating membranes, which are often formed in the region of the mouth of many Infusoria, are modifications of the cilia. The cilia appear sometimes to be modified in a peculiar way to form stiff processes, movable only at their point of attachment to the body (Stylonychia); sometimes, indeed, they are flattened and broadened.

The cilia, as well as the style-shaped processes, serve as locomotor organs, and show us that locomotion is connected with the integument, just as it was connected with the temporary external layer of the body, where pseudopodia were formed.

Another structure observed in the cutis of many Infusoria (e.g. Paramæcium), are firm rod-like bodies (Trichocysts), which, under certain influences, emit a fine stiff filament. These structures lie close to one another in the cortical layer, and in a direction perpendicular to the long axis of the body. They call to mind the stinging cells of the Coelenterata, but they are not to be regarded as the same things, for they are not formed from cells. 


\section{$\$ 63$.}

In the cortical layer of the body of the Gregarine, and of many Infusoria, there are indications of bands, or fibres, resembling muscles. In the Gregarinæ these structures are arranged circularly, or spirally, and form a layer just below the cuticle; it extends over a short part only of the "head," which, as a rulc, is separated from the body by a constriction, but it never passes into the wall of partition which separates this part from the body.

Among the Infusoria these contractile bands are principally known in the larger species of some genera (Spirostomum, Stentor, Prorodon, etc.). In others they are absent. They sometimes run spirally, sometimes longitudinally. They are also present in the Vorticellinæ, where they form spiral coils towards that end of the body which passes into the stalk. It is clear that these structures do not form the sole contractile system of the body, for those Infusoria which do not possess them are capable of executing powerful contractions. But that they are really contractile is shown by Spirostomum, in which the contractions of the body are not effected along its long axis but in the direction of the striated band, which describes several spiral turns. The contractile band, which runs in the interior of the stalk of the Vorticellinæ, is a differentiation from the protoplasm of the same kind; in Zoothammium it branches with the colony, in Carchesium it exists separately in each individual of the colony. Although these structures in many points resemble muscular fibres, and are physiologically the same, they cannot be compared with these histological elements from a morphological point of view, for neither cells nor the products of cells take any share in forming them. They are differentiations from the protoplasm of the organism, whilst in the tissues of the Metazoa they are formed by the differentiation of a whole lot of morphological elements. The whole contractile apparatus corresponds therefore to a muscular system in function only. The separate bands or stripes are merely analogous to muscles (myophana).

\section{$\$ 61$.}

The function of supporting organs of the body is performed in the Protozoa by firm structures, which either traverse the soft substance of the body as a framework, or invest it in the form of shells and tests. The latter serve as organs of defence in proportion to their size and firmness. All the structures here to be enumerated are either directly or indirectly differentiations of the protoplasm, which are formed either on the surface or in the parenchyma of the body. The more completely these secretions cover the body in the form of tests, the more do they interfere with its freedom of movement, unless there are compensating arrangements. Shells and internal supports are widely distributed in all divisions of the lower 
organisms; they vary greatly in complexity, and this complexity is sometimes in inverse proportion to that of the body. Simple shells, generally oval in form, and provided with an orifice, obtain in one division of the Amobre (Difflugia, Arcella). The shell is sometimes soft ; but sometimes it is more firm, and this firmness is increased by the addition of foreign bodies. They sometimes seem to be internal shells, owing to the extension of the protoplasm over them. The shells of the Foraminifera are more complicated in form, owing to the formation of new parts around a simple rounded test, which then form separate chambers communicating with one another by orifices, and

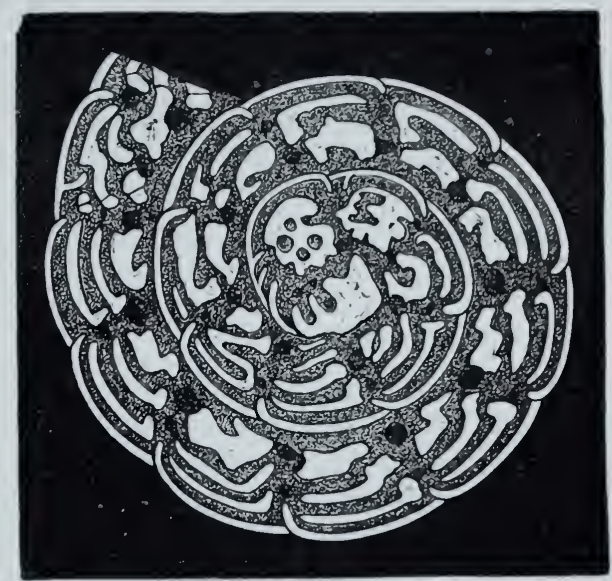

Fig. 25. Transverse section of a Foraminifer (Alveolina Quoii); the arrangement of the separate chambers in relation to one another can be seen (after W. Carpenter). with the exterior by pores

(Figs. 23, 25). These many-chambered shells become very firm by the addition of chalk, or, though more rarely, of silica (Polymorphina Nonionina); owing to the variations in the relative position, size, and mode of connection of the chambers, these structures vie in wealth of form with the more lightly constructed internal supports of the Radiolaria.

When the chambers are ranged along a straight line, rod-shaped shells, often swollen into beads, are formed; the separate portions of which, known as "chambers," may be all of the same size, or may increase in size from one end to the other (Nodosaridæ). A spiral arrangement of the chambers which lie in the same, or in different planes, gives rise to structures like those of the shells of the Nautilus (Fig. 23). Special modifications are due to the superposition of the spiral coils, the elongation or abbreviation of the spiral axis, and so on. The planorbis-like shells of the Milliolidæ, in which partial constrictions give the first sign of the formation of chambers, represent the simplest condition of these forms. By the unequal addition of fresh chambers the spiral form is completely effaced (Acervulinæ), and can only be seen in the earliest-formed chambers. These tests are usually confounded with external shells. But this only holds for a few. The shell seems rather to be an internal support, in those cases especially where the partition-walls of the so-called chambers are frequently broken through, and the pore-canals at the same time pass through the shell to the exterior, so that the protoplasm of the pseudopodia is able to cover the outside of the shell. When the partitions 
are merely represented by several separate columns, or lamellæ, which have wide openings between them (Fig. 25), and the cavity of the chamber itself is less than the various connections between two chambers, and where, finally, all the neighbouring chamber-spaces communicate with one another, and the whole "test" is thereby traversed by a cavitary system which communicates in all directions, then the character of an extermal shell is completely lost. And so, since the protoplasm is in all cases able to draw itself over the outer surface of the shell, the shells in the Foraminifera ought to be regarded as internal ones, and grouped accordingly with the skeletons of the Radiolaria.

\section{$\S 65$.}

The "central capsule" must be noted as an organ of support common to all the Radiolaria, although it is not very apparent. It is a capsular-closed organ, various in form, placed in the middle of the body, and formed of a membrane which closely resembles chitin. In addition to fat-globules and structures which are regarded as nuclei, it always contains a quantity of protoplasm, which is apparently continuous with the extra-capsular protoplasm by means of fine pore-canals. In most Radiolaria (not in Thalassicolla, Thalassolampe, and Collozoum) there is, in addition to this capsule, a

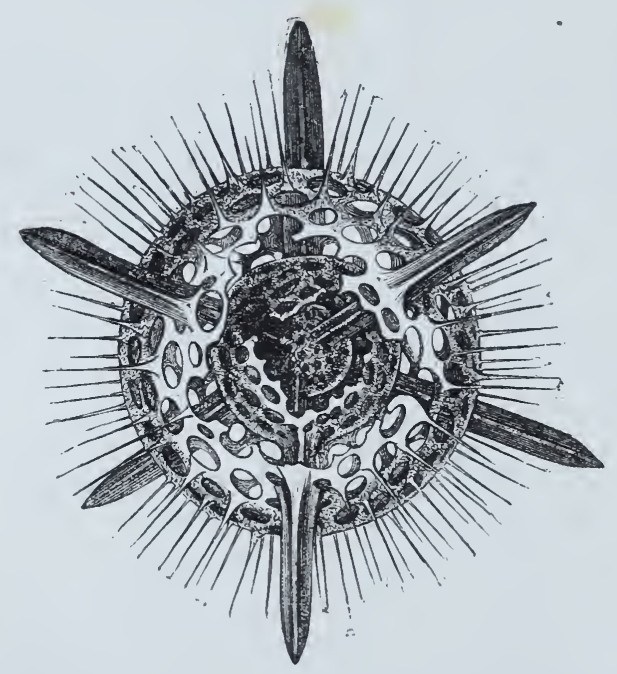

Fig. 26. Skeleton of a Radiolarian (Actinomma asteracanthion). Two concentrically-arranged fenestrated shells are broken through at one point, to show a third one (after E. Häckel). skelet on, ordinarily formed of silica, which traverses the capsule when most fully developed (Fig. 26). Several spicules then radiate from a common centre, and these may be connected with one another by means of a concentric highly fenestrated framework (Fig. 26). In some (Acanthometridæ) the organic base of the framework predominates, and is but slowly replaced by silica.

Separate spicular bits of silica, lying freely in the protoplasm outside the central capsule, are the earliest indications of thisfirm skeleton in the Collida and Polyzoa. In some they becomearranged in a radiate manner, withoutbeing firmly connected together. Circular skeletons, forming an open network, are formed by the radial spicula being connected together at equal distances by rods placed perpendicularly to them. When a very fine supporting reticulum is arranged around the radial spicula in 
a more irregular manner, the skeleton is sponge-like. The infinite variety of form is increased by discoidal and basket-like skeletons; or these skeletons may have a spiral arrangement. In this way a supporting organ of great complexity is formed, in which the soft parts of the body are embedded, and the separate pieces of which are developed in the protoplasm.

\section{$\S 66$.}

Compared with these internal organs of support in the Rhizopoda the tests of the Infusoria form a distinct series of arrangements, for they are only secretions from the surface of the body: they resemble the tests of the Arcellæ, mentioned above. The secreting: matrix is in thïs case an anatomically distinct part of the body. But this need not be regarded as a higher stage, for it is closely allied to the lowest, i.e. to the formation of a cell-membrane. Tests are principally developed in the fixed Infusoria. They are formed by the secretion of a substance, which is primitively soft, and which gradually hardens; this surrounds the body of the animal like a cup or an urn, except at one point, where it allows of communication with the exterior. These tests are distinguished from the merely cuticular structure, which tends to form a carapace, in that the differentiated layers attain greater firmness, and become separated from the greater part of their matrix surface. But the genesis of both structures is the same. It is identical with the formation of cysts, a process which is very common among the Infusoria, and by means of which the organism shuts itself off from the exterior for a time, so as to withstand unfavourable conditions (loss of water, and so on). The immovable stalk of Epistylis, and the external layer of the contractile stalk of the Vorticellinæ and Carchesinæ must be regarded as cuticular differentiations of this kind. The tests may be soft or hard, and membranous. Some are distinguished by the agglutination of foreig'n bodies-cemented grains of sand, and so on. The genera Vaginicola, Tintinnus, etc., have shells. Stentor has one in certain cases. Fenestrated shells have also been observed (Dictyocyrta). A carapace is formed from the firm hyaline cuticle in Stylonychia, Euplotes, Aspidisca, Spirochona, Coleps, etc.

\section{$\$ 67$.}

Organs for the prehension and alteration of food are wanting in the lowest organisms. In the Gregarinæ food is taken in by endosmotic processes at the surface, and solid nutritive matters do not reach the interior of the body. Where the body is not peripherally differentiated there is, on the other hand, a direct taking in of food, which may go on at any part of the body. This is the case in the Rhizopoda. In this case the nutritive matter is surrounded by the soft sulostance of the body, or it is embraced by the processes of the body-the pseudopodia. In both 
cases there is really one and the same phænomenon. Any place in the protoplasm can act as a digestive cavity by enveloping and absorbing nutritive matter, and at any neighbouring part of the surface the undigested substances can be again expelled. Even in Actinosphrrium solid food can be taken into the body; but in it the pseudopodia are not the direct agents, for they draw the prey to the body, and cause it to pass into the yielding parenchyma of the cortical layer at some suitable

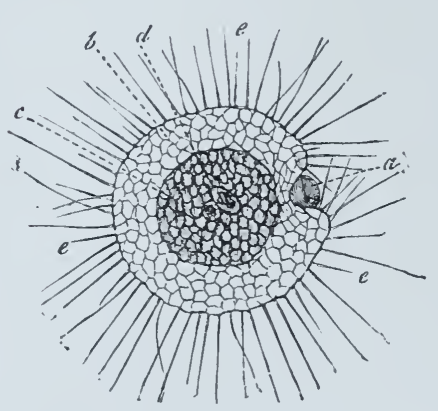

Fig. 27. Actinosphærium. a A morsel which has been taken in as food, and just pushed into the soft cortical layer $b$, by the animal. $c$ Central parenchyma of the body. a Some balls of food in it. $e$ Pseudopodia of the cortical layer. point (Fig. 27) ; thence it passes to the central substance of the body. In comparison with other forms Actinosphærium is characterised by taking its food directly into the more differentiated parts of the body, and not surrounding it by the amorphous protoplasm of the pseudopodia.

In the Infusoria the arrangement is more definite. They take in food in two different ways. In the Suctoria (Acinetinæ) there is no mouth; the radiate pseudopodia-like processes pass through the envelope of the body, and act as suckers (Fig. 30). They attach themselves by their sucker-like enlargements to the prey which has come within their reach (which consists of other Infusoria, etc.), and cause it to flow, as through a tube, into their body, where it fills the parenchyma in the form of drops. The presence of similar processes in the embryos of other Infusoria shows that this mode of nutrition is a very common one. A higher grade is represented in the other forms; in the Ciliata there are not only definitely organised parts for the reception of food, but also definite parts for the ejection of what is useless. An enteric tube is, however, wanting in all of them, and these differentiations are limited to the cortical layer of the body, so that the food passes beneath it into soft parenchyma, i.e. into the undifferentiated protoplasmic part of the body, in which there are no passages with special walls. Temporary spaces, which act as digestive cavities for the spherical food masses, are formed in it; these cavities are not permanent ones, as may be seen from their frequent disappearance when the protoplasm is in movement. In this point, therefore, they resemble the Rhizopoda; for a part of the digestive apparatus, that is those parts in which the food is digested, have no organological differentiation.

When the Ciliata have a mouth, it is either in the form of a simple cleft, which is in many cases only apparent when food is taken in; or it is not directly on the surface of the body, but at the bottom of a depression (vestibule), the form of which is very varied, and which at times contains the orifice of egestion also; the sur- 
rounding region (peristoma) has often also a special form. A tubular portion, or pharynx (Fig. 28, $\ell$ ) often passes from the moutl into the parenchyma of the body, and from it the ingested morsel finds its way into the soft substance of the latter.

The position and form of the mouth varies greatly. In many cases it can only be made out during the ingestion of food (as in Amplileptus, Loxophyllum), and disappears as soon as the morsel has passed into the parenchyma. There is sometimes an investment of cilia on the tubular pharynx (Paramæcium aurelia and bursaria); or an undulating membrane (Bursaria flava); or a covering of rod-shaped denticles, or fine longitudinal ridges.

Porodon, Chilodon, Nassula, etc., have an investment of small rods in the pharynx, arranged in eel-pot form. A regular thickening of its walls has been observed in Ervilia and Liosiphon.

The general presence of an anal opening is not by any means established. There is only in some few cases a permanent and dis-

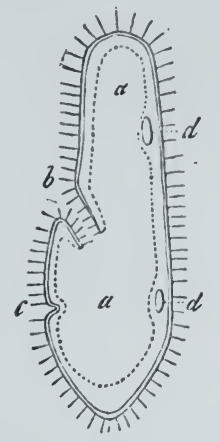

Fig. 28. Diagram of the aigestive carity of Paramæcium. a Bodyspace filled with soft pro. toplasm, into which the food is taken. $b$ Mouth. $c$ Anus. d Contractile vesicles (after Lach. mann). tinctly-marked opening; it can generally be distinguished during the expulsion of undigested food only. This anal spot is as a rule at the posterior end of the body, but is, on the whole, very changeable. It may even appear at the anterior end of the body; thus, in Stentor it lies near the mouth, and in the Vorticellina and Ophrydix in the vestibule. Taken on the whole it appears to consist more in the localisation of a function than in the development of an organ. The products of excretion pass through tho differentiated cortical layer of the body at a certain spot, without there being any special organisation of that spot.

\section{$\S 68$.}

In all Protozoa the outermost layer of the body has a respiratory significance, for it is by it alone that an exchange of gases with the surrounding medium can be effected. This relation must also be borne in mind in reference to the increase of surface, which is due to the pseudopodia. The cilia of the Infusoria are of importance in changing the water.

More definite respiratory arrangements are seen when, as in many Protozoa, water is taken into the body. Cavities, which are filled with a fluid, and which gradually contract and completely empty themselves, after having reached their maximum of distension, appear within the protoplasm; when empty they seem to disappear. These vacuoles, like the vacuoles in the cells of certain tissues, are partly variable structures, now appearing and now disappearing, and 
partly constant. When they are constant their function is increased, and they often expand and contract regularly and rhythmically, like the cardiac systole and diastole. Contractile vesicles of this kind are often seen in the Amobre (Difflugia and Arcella), and are very common among the Infusoria. They are also known as vacuoles. The fluid which collects in the vesicles is drawn from the parenchyma of the body and is returned to it, or passed out to the extcrior on the contraction of the vesicle. Fine communications with the exterior have been made out, so that the latter course is the probable one; but we need on this account conclude that water does not enter by the same passage.

In the Infusoria the vesicles lie in the cortical layer (Fig. 28, $d, 7$ ), g'enerally just under the delicate cuticle, and at definite points. If only one vesicle is present, it lies either anteriorly or posteriorly: if two, there is one near each end of the body. Trachelius ovum is remarkable for a large number of small vesicles. No special membranes can be made out on the wall of the vesicle nor in the canals which pass off from it. Like the vesicle the canals can only be made out while they are filling. The vesicle and canals contract alternately. In Paramæcium the canals enlarge at the commencement of the systole, and approach one another as the vesicle diminishes in size, so that they form a stellate figure at the moment when its systole is most complete and the vesicle has disappeared. While the vesicle is filling the canals look like small diverticula on it, and are not again fully distended until the diastole is complete. The number of canals, which is limited in $\mathrm{P}$. aurelia to eight or ten, is increased to thirty in Bursaria flava, and is much higher in Cyrtostomum lencas. In these forms the canals have a wave-like course, and ramify at their extremities. Canalicular tracts are formed by the fusion of several spaces filled with water into longer tracts, as in Stylonychia (St. mytilus), and they empty themselves into the contractile vesicle, by definite passages. The long canals of Spirostomum ambiguum, which also are visible for a time only, but which are longer than these, are like them, so that we can make out a continuous series from the first appearance of an apparently indifferent cavity to a definitely arranged system of tubes.

Another arrangement can be put beside this formation of indifferent vacuoles. When such spaces in the protoplasm increase in number they run together, and so give to the protoplasm the appearance of a network which traverses the interior of the body, which is filled with fluid (Trachelius ovum). These hollow spaces have then become perfectly different organs from the pulsating racuoles, and they may both exist at the same time.

\section{$\$ 69$.}

In correspondence witl their low grade of organisation the Protozoa have no sexual organs, and give but the faintest indica- 
tions of sexual differentiation. They always propagate, therefore, by modes which are called asexual, among which the chief part is played by fission and generation. The nucleus appears to be of great importance in all their modes of multiplication.

Spores have been observed to be formed within the organism in Rhizopoda. A larger or smaller part of the protoplasm of the body is used in forming them; when a larger portion is used this mode of multiplication is allied to that mode which is so common among the Protista, in which the whole body breaks up into a number of spores, and so multiplies by division. In the Radiolaria the contents of the central capsule are active in reproduction. The nuclei in it become surrounded with protoplasm, and form flagellate swarmspores.

The mode of reproduction is most exactly known in the Gregarinæ. As a rule multiplication commences by the concrescence of two individuals; this generally occurs very early, so that the two individuals, which form one body, the anterior end of one being attached to the posterior end of the other (Fig.29), go on growing for some time; or conjugation may only take place later, when the forms are mature. After this comes a condition of rest, accompanied by encystation, in which the two individuals form a rounded body, with a partition between them. Then the partition disappears, and the substance of the body, and also the nucleus, breaks up into an amorphous mass, from which numerous vesicles gradually arise. From these latter a number of germ corpuscles, called "Pseudonavicellæ," on account of their shape, are formed. These gradually fill the whole cyst, and each gives rise to a single very small organism, consisting of protoplasm solely, and this, being without a nucleus, corresponds to a cytod. Each of these structures moves about in an amœboid

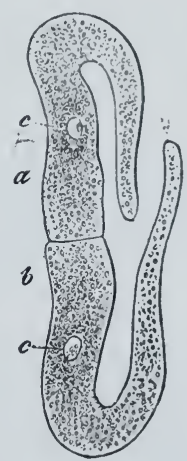

Fig. 29. $a b$ Two con. jugated individuals of Gregarina sænuridis. c Their nuclei. manner, and is gradually differentiated into a young Gregarina, after which a nucleus is differentiated in its interior, and it becomes limited externally by a cortical layer.

Although conjugation has no exclusive signification in bringing about these processes, as separate Gregarinæe are also able to pass through these reproductive processes in just the same way, yet it is not the less important. It points, at least in the cases where it exists, to the necessity of two individuals to bring about reproduction. It is, consequently, a phænomenon preliminary to sexual differentiation.

\section{$\S 70$.}

Conjugation also plays a part in the reproductive proceedings of Infusoria, for it is the first step in their multiplication. In this the 
nucleus is of considerable importance; it (Fig. 30, $u$ ) is a firm structure, sometimes provided with a spiral envelope, very various

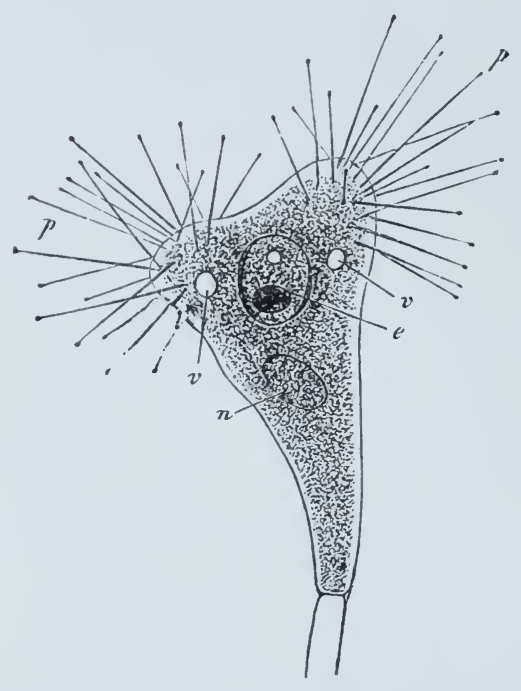

Fig. 30.-An Acineta with part of its stalk. $p$ P'seudopodia-like, but stiff, tentacles. $v$ Vacuoles. $n$ Nucleus. $e$ Aciliated young form lying in the so-called broad carity. in form. It lies in the cortical substance of the body, or is surrounded by a continuation of this substance, if it is more deeply sunk in the interior. It is sometimes oval or round, or it is flattened and curved (Vorticellinæ), or it is even greatly elongated and regularly constricted (Spirostomum). The importance of the nucleolus, which differs from the nucleus in nothing but its smaller size, is more obscure. The act of reproduction commences as a rule with the complete or partial fusion of two individuals, which may be of the same or of different sizes; this fact led to the mistaking of conjugation for stages of fission or gemmation. This concrescence gives the stimulus to changes in the appropriate parts. The nucleus becomes divided into a certain number of parts, around which the protoplasm is disposed. In this way a number of spores are formed, each of which becomes a new individual while within the mother-cell; and then gets an investment of cilia, and escapes to the exterior.

It is still a question as to the share which the nucleolus takes in this process; and the statement that in one group of the Ciliata it has the function of a sperm-forming organ, while the nucleus has the function of an ovary, requires to be confirmed. In any case this differentiation of a male organ is not a common phænomenon, but is limited to a very narrow circle. The nucleus, therefore, and the nucleus alone, is certainly known to take an active share in reproduction, and this share is of just the same character as that which it was seen above to have in spore formation, and as it has in gemmation, in which the nucleus of the bud has often been observed to arise from the previous gemmation of the nucleus of the mother-cell (Podophrya). Finally, multiplication by fission is very common, although conjugation was often confounded with this process at one time. 


\section{Second Section.}

\section{Cœlenterata (Zoophyta).}

\section{General Review.}

$\S 71$.

This division is the first of the Metazoa, or organisms which are undoubtedly animals. The embryonic body separates into two cell-layers-ectoderm and endoderm; which in many Sponges alone form the permanent body, though in many a mesoderm is developed. In the lower Acalephæ the formation of a mesoderm is incomplete; that is, the mesoderm is not an independent tissue as it is in all the higher Acalephæ. The most essential character of the animals which make up this division is the arrangement of the nutritive apparatus, a cavity sunk into the parencliyma of the body, and either divided into canals or extended into wider spaces. This digestive cavity, with its appended spaces, is invested by the endoderm, and in the lower forms is the sole representative of hollow organs in the body. When several individuals are united to form a colony, the canal system, which arises from the digestive cavity, is common to all of them, and is continued into the common substance of the colony or cœnenchyma. The primary axis alone can be made out in the body, and the secondary axes are indifferent, or if present appear to be equivalent.

I. Spongiæ.

Gastræades.*

Porifera.

Haliphysema, Gastrophysema.

Myxospongiæ.

* The Gastræades represent stages which are not permanent in the rest of the Spongiæ. 
Fibrospongiæ.

Ceraspongiæ.

Euspongia, Spongelia, Poterium.

Halichondriæ.

Axinella, Spongilla.

Corticata.

Thetya.

Hyalospongiæ.

Enplectella.

Calcispongiæ.

II. Acalephæ.

Ascon, Leucon, Sycon.

\section{Hydromedusæ.}

Hydriformes.

Hydra ;-Cordylophora ;-Hydractinia ;

- Coryne, Syncoryne, Eudendrium ;-

Tubularia, Corymorpha ; - Campante-

laria, Sertularia, Plumularia.
Medusif ormes.

Sarsia, Bougainvillea, Lizzia, Oceania ; -Eueope, Thaumantias;-Trachynema ; - Egina, Cunina;-Liriope, Geryonia, Equorea.

Siphonophora.

Velella, Porpita ;-Diphyes, Abyla;-Athorybia, Agalma, Phjsophora, Physalia.

2. Calycozoa.

Lucernaria.

3. Thecomedusæ.

Stephanoscyphus.

4. Medusæ (Discophora).

Charybdea, Pelagia, Aurelia, Rhizostoma, Cassiopeia.

๖. Anthozoa.

Tetractinia.

Cereanthus, Cyathophyllum.

Hexactinia.

Antipathes, Fungia, Madrepora, Astræa, Oculina, Caryophyllia.

Octactinia (Alcyonaria).

Alcyonium, Pennatula, Virgularia, Veretillum, Renilla, Gorgonia, Isis, Corallium, Tubipora.

6. Ctenophora.

Beroë, Сłclippe, Cestum, Eurhamphrea, Mnemia, Encharis.

\section{Bibliography.}

Spongiæe: GraNt, R. E., Observ, on the struct. and funct. of Sponges, Edinb. New Phil. Journal, 1826-32.-Lreberkiur, Beitr. z. Entw. der Spongillen. Areh. f. Anat. u. Physiol. 1856. Zur Anat. d. Spongillen, ibid.-The same, Z. Anat. d. Spongien, ibid. 1857, 1859, 1863. -Scrultze, M., Die Hyalonemen. Bonn, 1860.-Schurdt, O., Die Spongien des adriat. Meeres. Leipzig, 1862. Sinplement, 1864. Zweites Supplement, 1867. Drittes Supplement, 1868.-ClaUs, Ueber Euplectella Aspergillum. Marb. u. Leipzig, 1868.-HARTrvg, P., Sur le genre Poterium, Natuurkund. Verhandelingen. P. Il. St. 2. Utreeht, 1870.-Ḧ̈ckEL, Dic Kalksehwämme, eine Monographie. 3 Bde. Berlin, 1872.-The same: Die Physemarien. Jen. Zeitschr. Bd. X.Scrulze, F. E., Ueber Bau u. Éntw. v. Sycandra raphanes. Zeitschr. f. w. Zool. Bd. XT.-The same, Untersuch. uber d. Bau u. d. Entw. der Spongien (Halisarea), ibid. Bd. XXVIII.

Acalephæ: CAfolrxr, Memorie per scrvire alla storia dei polipi marini. Napoli, 1755. (German by sprengel. Nürnberg, 1813.) -Eschscholtz, System der Acalephen. Berlin, 1829.-Lrssox, Zoophytes acalèphes. l'aris, 1s 13. (Suite ì Buffon).-SARs, Fauna littoralis Norvegir I. 1816. 
-Frex u. Leuckirt, Beitrïge zur näheren Kenntniss wirbclloser Thiere. Braunschwcig, 1817. -HUxuer, On the anatomy and affinities of the family of the Medusæ. Phil. Tr. 1819.Ag.sssiz, L., Contributions to the nat. hist. of the Acalephr of N. Am. (Mem. of the Amer. Acad. of Aits and Sc. Cambridge, 1850).-The same, Contrib. to the nat. hist. of the United States of North America. Vol. III. IV. 1860-62.

Hydromedusæ: VAN Bendere, P., Mém. sur les Campanulaires de la côte d'Ostende. (Nouv. Mém. de l'Acad. royale de Bruxelles. T. XVII.) Reeherches sur l'embryogénie des Tubulaires (ibid.).-Kölliker, Die Schwimmpolypen von Messina. Leipzig, 1853.-Leuckart, R., Zur näheren Kenntniss der Siphonophoren von Nizza. Areh. f. Nat. 1851.-Grgenbatr, Beitr. zur näheren Kenntniss der Siphonophoren. Zeitschr. f. wiss. Zoologie. Bd. V.-Vogr, C., Sur les. Siphonophores de la mer de Niee. Mém. de l'inst. Genevois, 185̃ 1.-Claus, Ueber Physophora hydrostatiea. Zeitsehr. für w. Zoolog. Bd. X. Neue Beobachtungen. Ibid. Bd. XIII.-HӥскEL, E., Zur Entwickelungsgesch, der Siphonophoren. Natuurkund. Verhandelingen. P. I. St. 6, Utreeht, 1869.-Huxuex, Oceanie Hydrozoa. London, 1859. (R.S.) -Forbes, Ed., A monograph of the British naked-eyed Medusæ. London, 1818. (R.S.)-Ḧ̈ckE L, Dic Familie der Rtisselquallen. Jenaisehe Zeitschrift. Bd. I. II. (Also under the title : Beitr. zur Naturgeseh. d. Hydromedusen. I. 1865).-Scrulze, F. E., Ueber den Bau und die Entwickelung der Cordylophora lacustris. Leipzig, 1871.-The same, Uebcr d. Bau v. Syncoryne, etc. Leipzig, 1873.-Kuminenberg, N., Hydra. Leipzig, 1872.-Alluar, G. J., Monograph of the Gymnoblastic or tubularian Hydroids. P. I. and II. London, 1871-72. (R.S.) -The same, On the structure and develop. of Myriothela. Phil. Trans. Vol. 165.

Caly cozoa: Crark, H., Prodromus of the History, etc., of the order Lucernaria. Journal of Bost. Soc. of Nat. Hist. 1863.

Thecomedusæ: Alcrar, G. J., On the Structure and systemat. position of Steplianoseyphus mirabilis. Trans. Linn. Soc. Sec. ser. Vol. I. Zool.

Discophora: Ehrenserg, Ueber Aealephen des rothen Meeres und d. Organismus der Medusen der Ostsee. Abhandl, der Berl. Aead. 1835.-MILNe-Epwards, Ann. sc. nat. III. xvi.-WAGNER, R., Ueber den Bau der Pelagia noetiluca und über die Organisation der Medusen. Leipzig, 1811. -Ḧ̈скеL, E., Ueber die Crambessiden. Zeitschr. f. wiss. Zoolog. Bd. XIX.-Brandt, A., Ueber Rhizostoma Cuvieri. Mém. Acad. Inpl, des Sc. de St. Péter'sb. VII. Sér. T. XVI.-GreNachen, H., and Nolc, F. C., Beitr. z. Anatomie u. Systematik der Rhizostomecn. Abh. d. Senckenb. Gesellsch. Bd. X.

Anthozoa: Emrenberg, Die Corallenthiere des rothen Meeres. (Abh. d. Berl. Acad., 1832).Hollard, Monographie anatomique du genre actinia. Ann. sc. nat. III. xv.-HAIße, J., Mém. sur le genre Cereanthus. Ann. se. nat. IV. r.-LACAzE-Durmikrs, Hist. nat. du eorail. Paris, 1861. - The same, Mémoires sur les Antipathaires. Ann. sc. nat. V. Ir. IV. Développement des Coralliaires. Archives de Zoolog. exp. Vol. I. II.-KöLtiker, Die Pennatuliden. Abh. d. Senckenb. Gesellseh. Bd. VII._Erser, G., Bidr. tid kännedomen om Renilla. Kongl. Svensk. Vet. Handl. Bd. XIII.-r. KосH, G., Anat. d. Orgelcoralle. Jena, 1874.-Mosfiry, H. N. On the structure and relations of the Alcyonarian Heliopora cærulea, etc. Plil. 'Transac. Vol. 166, Pt. I.

Ctenophora: Wirc, Horæ tergestinæ. Leipzig, 1814-Mrnxr-Epwards, Anm. sc. nat. Sér. IV. Vol. VII.-Kowalevskx, A., Entw. des Rippenquallen. Mém. de l'Acarl. Imp. de St. Pétersh). VII. Sér. Tom. X.-Fou., H., Beitr. z. anatom. Entwickl. einiger Rippenquallen. Berlin, 1869, -Erarer, Th., Zoologische Studien auf Capri I. Ueber Beroe ovatus, 1873.

\section{Form of the Body.}

$\S 72$.

The forms of body of the two great divisions which make up the Colenterata are alike in the lowest stage ; in that stage, namely, which has been already (\$28) called the "Gastrula," on account of the development of an enteric cavity. This form represents a larval stage, in which an investment of cilia function as a locomotor organ, and which may fairly be regarded as the common fundamental form of the two chief divisions of the Zoophyta. In this form only one axis, the primary, can be made out; it extends from the oral to the aboral pole. The secondary axes are indifferent, for all the transverse axes drawn perpendicularly through the primary, at any angle whatsoever, are completely equivalent one with the other. This stage is permanent in all the Spongiæ; in the Acalephre it passes on into a condition which is characterised by differentiation of transverse axes.

Among the Spongire the Gastrula attains, when attached by its 
aboral pole, to a definite character of the most simple form in the Physemarix, and in Olynthus among the Ascones. In other Calcispongire, also, this simple form of body is retained, but more considerable changes in its internal characters obtain.

The most important changes in the form of the body are due to the formation of colonies. Colonies of the most varied form (cormi) are formed by budding or by incomplete division, the separate animals (persone) of which are connected with one another in very various ways, and may even partly or completely fuse with one another in different ways. When these stocks are fused they often have the appearance of single animals, and in proportion to the simplification of their external form is the complication of their internal organisation. The modification of the mouths of the colony affects their external form just as much as does this concrescence; the mouths may be collected into groups, or united as one, or they may completely disappear.

The great variety of form in this division, which is due to the causes here only indicated, may be still further modified by numerous adaptations, due to their position in space. Nowhere in the Animal Kingdom does the form of the body appear to be so changeable as in the Spongia, so that it is impossible to separate them into large divisions, to say nothing of species.

\section{$\$ 73$.}

In almost all the divisions of the Acalephr the body dereloped from the Gastræa-form is adapted to a sessile or fixed condition; the stomachal cavity which is formed when this development commences causes the organism to have, in all essential points, the same simple character as it has in the corresponding stage in the lifehistory of the Spongia. Processes, known as tentacles, are developed on the anterior region of the body-wall, which encloses the stomachal cavity; they present to us the earliest indication of a differentiation of the secondary axes, and therefore establish a well-marked distinction between these forms and the Spongiz.

The Hydroida or Hydroid-Polyps (Hydriformes) are the lowest of the Hydromedusæ.

Tentacles are placed in many cases irregularly on the parts of the body which surround the mouth (Coryne, Syncoryne, Cordylophora), or their number may be indefinite, even when the structures are limited to definite zones of the body, and encircle the inouth in the anterior region (Hydractinia, Eudendrium, Campanularia). As the number of tentacles varies we cannot suppose that the secondary axes are definitely differentiated. It is only in a few cases that they are definitely expressed by the position of the tentacles (Stauridium).

The free portion of the body, with its tentacles, becomes more independent of the rest of the body, which forms a stalk, while the aboral pole grows out into a stalk-like part, which carries the head, and is distinguished as the "capitellum " nl "hydranth." 
Colonies (cormi) are formed from single animals by gem mation. This may either occur at any point of the surface of the body (Hydra), and end by the bud breaking off, or it may take place in the stalklike part. The creeping cormi of the Syncorynidx, Hydractinia, etc., are formed by processes of the basal part, which give off new animals, attached here and there to it. When gemmation occurs in the free part of the stalk we get free, branched colonies, which become complicated in very various ways (Eudendrium, Campanularia), or become regularly branched (Sertularia, Plumularia).

The formation of colonies is almost always accompanied by the formation of a tubular investment, which is a secretion from the surface of the body, and which serves as a support for the whole trunk, as well as for its branches; it is also continued, in various degrees, on to the persons of the colony.

\section{$\S 74$.}

The process of gemmation in the Hydroid Polypes produces, in addition to the growth of the colony by freshly-formed similar individuals (persons), structures of quite a different kind, the most differentiated forms of which are developed into Medusæ.

The body of these buds is of a bell-shaped or discoid form (Fig. 32, $m$ ), and by its internal organisation, as well as by the tentacles, which arise from the edge of the bell, or disc, we are able to make out secondary axes, generally two in number, which cross the primary axis at right angles to one another, and are completely equivalent one with the other. A higher grade than that of the Hydroid-Polyps is expressed in this organisation. The animals move by contractions of the bell, the edge of which is produced into a membrane, the velum, which is also contractile. These Medusa-gemma always carry the organs of reproduction; from their ova Hydroid Polypes again arise. (Alternation of Generation.)

While gemmation of Medusæ intended for a free life distinguishes some Hydroid-Polyps (Fig. 31, $a-e$, Fig. 32, $a-c)$, in others the process stops at the formation of a Medusabud, the organisation of which does not quite attain that stage of development which conditions the free mode of life, and it remains therefore con-

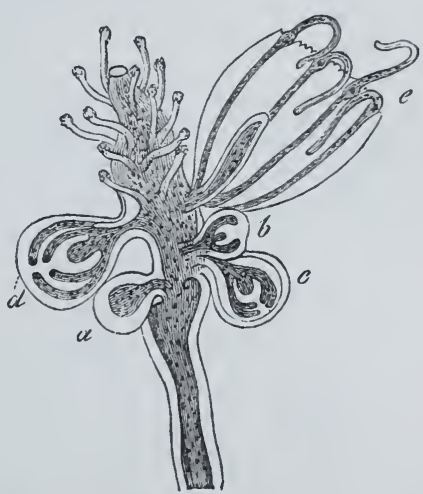

Fig. 31. Syncoryne with a numiber of budding Medusa on it at different stages $(a-e)$ of develop)ment (after Desor). nected with the colony. Nevertheless, the normal development of the sexual organs proceeds, and in fact these rudimentary Medusæ form "generative buds" (Gonophores), 
the products of which are developed in them in the same manner as are those of the free Medusæ.

With these are connected still simpler forms of buds, and the series ends with buds the structure of which has scarcely anything in common with a Medusa. But the series which leads to these is perfect, owing to numerous intermediate forms, so that external buds, merely containing generative products, and Medusæ of a relatively high organisation, which only become sexually mature some

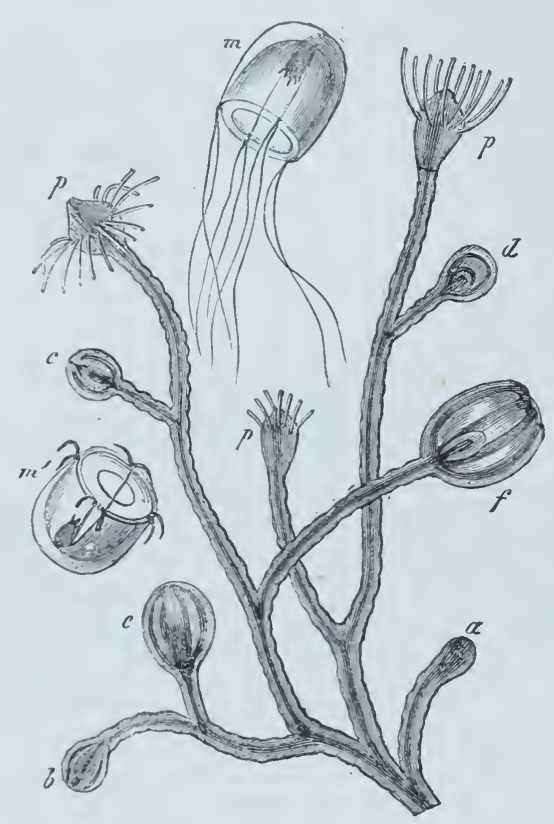

Fig. 32. A portion of a colony of a Hydroid. Polyp (Eudendrium ramosum) with bud. ding Medusac. $p$ p $p$ Nutritive persons. $a b c d$ ef Different stages in the differen. tiation of the budding Medusa. $m m$ Free Medusie in different positions. time after leaving the Hydroid stock, must be regarded as the widely-separateterminal points of one series.

This phænomenon is explained by the conception of a division of labour, in which the function of feeding the stock falls to the share of the individuals which remain sessile, while others which are broken off take on the duty of sexual reproduction. Those buds which will become free have a higher organisation, which has been gradually dereloped from the lower forms, and primitively resembled those that remain sessile. The separation from the stock may therefore be regarded as the primary cause of the differentiation of the sexual individuals in the medusoid direction, while the permanence of the sessile habit of the medusoid buds, in other cases, is accompanied by a degeneration of their medusoid organisation. But if this organisation, as we supposed above, has been obtained by a primitive freedom of life, the medusoid buds must necessarily be regarded not as arrested in an onward development, but rather as Medusa-buds in course of degeneration. A definite conclusion on the subject is not possible, on account of the fact that the several stages of degeneration might be precisely similar to those of development, and retrogressive metamorphoses have not been directly observed.

The gemmation of generative individuals, for such must the medusiform buds and their modifications be considered to be, takes place at different points. As the formation of a stock is a secondary process, the production of buds on the body of the single animalmust be 
the primitive mode. It is found on this region in all divisions of the Hydroid-Polyps. The stocks of the Corynidæ have buds distributed over the hydranth. They are frequently placed between the tentacles. In Pennaria they are found within the circlet of tentacles ; and on the same place in the Tubulariæ, where they are always placed in some quantity on a common stalk, forming groups like grapes, or ears of corn. Gemmation on the body of the Hydroida is, in many cases, accompanied by a degeneration. In many Campanularix, Hydractiniæ, and others, the proliferating individual gives up its share in the duty of feeding the stock, as is expressed by the diminished size of its tentacles and stomachal cavity. The animal stock is therefore composed of nutritive and proliferating persons, the latter again bearing the buds or generative persons. This is the way in which the Dimorphism of Persons arises in these stocks, and this passes into Polymorphism, in consequence of a number of the nutritive persons undergoing still further changes (Hydractinix).

The proliferating persons of a colony present various degrees of degeneration. In the most extreme case a portion only of the individual which bore the buds remains after they are developed (e.g. in many Campanulariæ). The complete degeneration of the proliferating person causes the gemmæ to arise from any part of the common stock, without any relation to a Hydroid person.

In the higher divisions of the Medusiformes, relations to the Hydroida are lost. Although there are many considerable complications in the mode of reproduction (see below, Generative Organs), yet, so far as is yet known, there is among the Trachynemidx, Aginidæ, and Geryonidæ no return to the hydroid form; and it is doubtful whether any such relation does obtain.

\section{$\S 75$.}

The division of labour, which in Hydroid-Polyps is essentially limited to the nutritive and generative functions of the persons united into a colony, is extended over a larger series in the Siphonophora; and we met therefore with great variety in the form of the component parts of the colony. Division of labour thus leads to Polymorphism of the persons. They all follow the medusiform type, which is developed to a greater or less extent. When it is distinctly developed, the fundamental form which shows itself in the medusa-buds of the Hydroid-Polyps predominates, so that it is clear that both groups have had a common origin. The Siphonophora, therefore, appear to be swimming Hydroid colonies, all the persons of which have passed into the medusa form ; a change which is complete in the generative persons only of the HydroidPolyps. The separate persons of the colony of the Siphonophora are developed on a common contractile stem, which in most forms represents the axis of the stock, and around which the persons which function as organs of the whole colony appear to be arranged. 
These are-

1) Locomotive Persons (Nectocalyces): these conform most completely to the Medusa-type, and are united together by twos

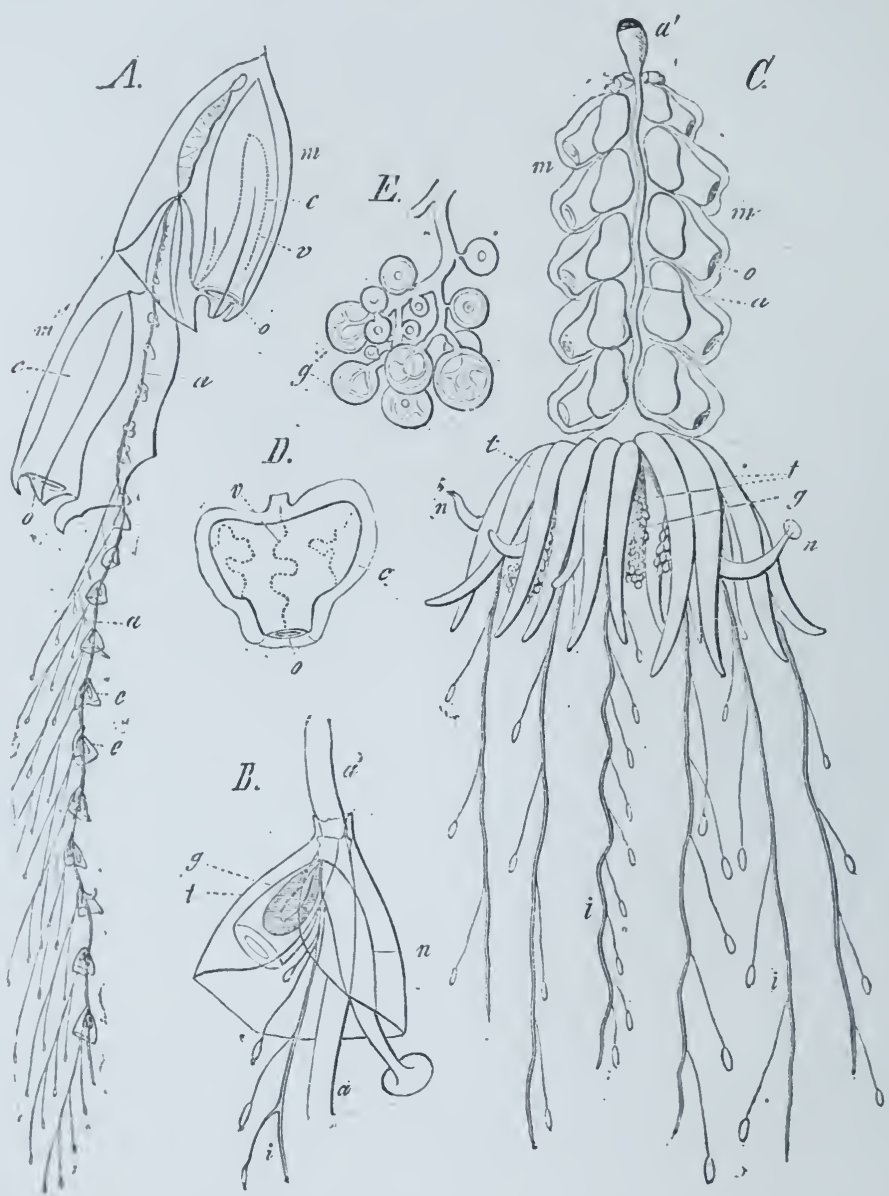

Fig. 33. Some colonies of Siphonophora. A Diphyes campanulata. B A group of appendages of the stem of the same Diphyes. C Physophora hydrostatica. A separate nectocalyx of it. E Cluster of female generative buds of Agalma Sarsii. I) "'Trunk or axis of the colony. (i) Air-bladder. $m$ Nectocalyx. $c$ Cavity in nectocaly $\mathrm{x}$, inrested by a contractile membrane. $v$ Canals in the walls of this carity. $\checkmark$ Opeuing of the nectocalrx. $t$ Bracts (in $c$ represented by tentacles). $n$ Stomach. $i$ Fishing-lines. $g$ Generative organs.

(Diphyidie), or', by a larger number, to form a swimming column (Physophoridie), which occupies the end of the stem (Fig. 33, $A C, m D)$, and which, therefore, takes the lead when the colony is moving, and is directed anteriorly.

2) Nutritive Persons are present in the sceond division of 
the stem, where they have the form of stomachal tubes (stomachs, sucking tubes) ( $B C$ n.) In some cases some of them are not fully developed, but form tubes closed at the end, which function as "tentacles."

3) Protective Persons (Hydrophyllia): in these one can often make out the Medusa-type quite clearly, but in other cases it is much less distinct, and they then have the appearance of hyaline, lamellar pieces, overlapping the persons described under 2, 4, and 5 .

4) Tentacular Persons: these form simple or elongated filaments (grappling-lines), which are arranged in tufts, are capable of great extension, and are provided with special urticating organs (Urticating batteries). The primitive Medusa form can be made out in a few only of these organs, and that faintly.

5) Generative Persons: as in the Hydroid-Polyps, these may be seen in various stages of development. Although it is in a very few cases only that they are metamorphosed into Medusæ that become free (Velella-Chrysosmitra), the medusiform type is very commonly well marked among them. They are generally found in racemose bunches, just as in the Tubulariæ.

The arrangement of these very variously differentiated persons of the stock of the Siphonophora differs in the different divisions, while the locomotor and the protecting persons are completely wanting in many genera. In general the arrangement or distribution of the polymorphous persons of the stock is observed to be very constant in genera and species; gemmation from the stock takes place on one side only, the arrangement of the buds all round the stock being due to its spiral twisting. This is the cause of the arrangement of the nectocalyces in two or more rows, as well as of the grouping of the other organs. Nutritive, generative, and tentacular individuals are generally placed together in groups, in such a way that there is one bract to a group. While in most Physophoridæe, these groups are very close to one another, they are set at greater distances from one another in the Diphyidæ (Fig. 33, $A B$ ), and each group is composed of a certain number of persons which, by breaking off from the stock, may become distinct individuals (Eudoxiæ).

The anterior end of the stem, which is distinguished by the presence of locomotor persons, becomes in many divisions particularly perfect, owing to the development of an air sac. This has the functions of a hydrostatic organ, and causes the anterior end of the body to be always directed upwards while the stock is at rest (Physophoridæ). ( $c$ á.) It has an opening to the exterior, which can be closed, and by which air has been observed to escape. The greater development of these bladders, which in most Physophoridæo are rather small, appears to cause a degeneration of the locomotor buds of the stock; and thus there seems to be a kind of compensating arrangement, through which, however, the power of the colony to move actively is diminished. Instead of swimming, it now is driven through the water. The locomotor persons are, for example, absent in Rhizophysa, in which the air sac is increased in size. By 
becoming greatly increased into a wide cavity, the air sac occupies the greatest part of the stem, and thus forms the largest portion of the colony, the separate pieces of which look like appendages placed on one side of the bladder. This character is greatly developed in the Physalidx, and is accompanied by the shortening of the stem. Another condition obtains in the Velellidæ, where the air sac is placed on the end of the greatly shortened trunk, and is developed by lateral extension into a disc, the cartilaginous firm walls of which divide the internal cavity into numerous chambers by forming walls of partition. In the earliest stages of development the air sac is simple in these forms also. In Porpita, the disc remains flat and circular; in Velella it is produced into a diagonal vertical crest, into which the air spaces of the disc are not continued. The concentrically-arranged chamber-spaces of the air sac in Velella are connected by apertures. They open to the exterior by a number of holes placed on the surface. In Porpita, fine air passages, in the form of canals, pass off from the inferior surface of the air sac, and enter into and branch in the portion of the stem, which carries the nutritive individuals.

\section{$\S 76$.}

The Thecomedusæ are polypoid Cœlenterata provided with a test, and are allied to the Hydriformes, although in organisation they resemble Medusæ; they are indeed, intermediate between these two groups, for they are representatives of forms which are closely

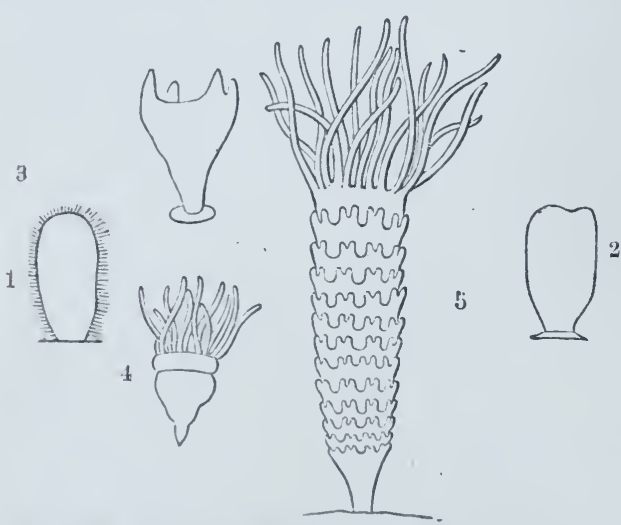

Fig. 34. Young stages of Aurelia aurita. 1 Planula-form, attaching itself. 2, 3 Passage into the Polyp-form. 4. Commencement of the formation of metameres. 5 Continued forma. tion of metameres (Strobila) and the differen. tiation of them (after M. Sars). are arranged by fours, so that four antimeres can be distinguished in the body. Medusæ are budded off from this polyp-form, but gemmation does not, as in the Hydroid-Polyps, take place at the side, but at the end. The terminal form (Scyphostoma) seems to be more highly organised than most of the HydroidPolyps ; it presents, indeed, points of connection with only a few of them (Corymorpha). It is developed, justlike the Hydroid-Polyps, from a planula, which is at first free, and which afterwards becomes fixed. But the fundamental form of the body resembles not only that of many HydroidPolyps, but their Medusa stage also, for two equivalent secondary axes cross the primary one. The organs allied to the larvæ of the
Discophora. This larval 
mouth-bearing portion of Scyphostoma is gradually nipped off from the rest of the body (Fig. 34, 4). As the body grows the new portions, which are formed towards the aboral pole, become separated metamerically (Strobila, Fig. 34, 5), and all are developed on a Medusa type. The polyp-body is thus divided into a number, often a large number, of Medusæ, which gradually break off (Ephyra form), and when they are free become more developed.

This process, which has been observed in Cephæa, Aurelia, and Cassiopeia, does not obtain in Pelagia, the ova of which are converted into swimming larvæ, which become young Medusæ, without passing through the polyp stage. The development of Pelagia is therefore compressed. into a fer stages, while in the others it is extended over a large series of forms, and is a more complete repetition of the palæontological development. The polypoid must be regarded as the initial stage, and was followed by the gradual metamorphosis of the polyp into a free Medusa. On this hypothesis, the strobilation of Scyphostoma and the consequent development of a number of Medusæ, appears to be a secondary process, which could only come about gradually, and after the whole polyp-body had ceased to be converted into a Medusa. It is clear, from the growth of the polyp, while it is passing into the Strobila, that an important part must be played by the nutritive relations of the Scyphostoma stage, in giving rise to the Strobila-form, or, in other words, in producing the Medusæ by gemmation; the whole phænomenon, therefore, appears to be causally related to the nutrition of the Scyphostoma. By the gemmation of Ephyræ, i.e. of young Discophora, from the body of the Strobila, an asexual mode of multiplication is interposed in the developmental process of tho Medusæ; and thus we have brought about one form of the so-called alternation of generation.

By the Scyphostoma form the Medusæ are closely related to the Calycozoa, which appear to be derived from them. The body, which is attached by a short stalk, is widened out like an umbrella, and agrees, as to its axes, with the Scyphostomr, and their descendants. In many points they also present relations with the Anthozoa. The Calycozoa, therefore, present us with a very important intermediate form, which has been continued on, with relatively few modifications, from the ancestral form common to several large divisions of the Acalephr.

\section{$\S 77$.}

In the Anthozoa the primitive form of the body is exactly the same as that of the other Cœlenterata; and even the earliest stages of the fixed planula present no essential differences. The appearance of tentacles and the subsequent internal differentiation give rise to various differences, the first of which affects the fundamental number of the secondary axes of the body. In some only four tentacles appear (Tetractinia), in others six (Hexactinia), and finally, 
in others, eight (Octactinia). In the first two divisions this number is not permanent, but the tentacles are soon increased in number, and there is a corresponding change in the internal organisation. A larger number of transverse axes can then be made out in the organism, but their fundamental number in most cases remains the same as at first. In the Octactinia, however, the first four transverse axes persist.

The body of the young animal is generally cylindrical, but this form is retained in a few divisions only (Cereanthus, Actinia). In the other forms colonies are built up, and this causes the external appearance to vary greatly. The stocks (Polypariæ) are formed cither by incomplete division, or by gemmation, or by both combined.

Longitudinal division aids in the formation of the colony to a varying extent. In many cases it is merely indicated by transverse growth, and does not lead to any division of the organism, as in many Fungia. In others, division affects the oral surface only, and. the internal parts remain continuous. When this process goes on for some time, colonies with a large number of orifices are formed, which are arranged in variously-curved rows, beset at their edges with tentacles (Mæandrina). Whilst flattened or extended racemose colonies are formed in this way, branched stocks are formed when division is combined with a considerable growth of the persons in a longitudinal direction; and these stocks may not only vary in size but also be branched in various ways. In the same way gemmation may be the cause of the formation of complicated colonies. In either case there is a portion of the body (cœnosarc, cœnenchyma) common to all the persons, and belonging to the common stock. The basal portion of the stocks of those Octactiniæ, which are not fixed but set loosely in the mud or sand, are developed from this cœnosarc and form a solid stalk-like portion of the stock, in which gemmation does not occur (Pennatulidr).

\section{$\S 78$.}

In the Ctenophora, which is the division differing most from the other Acalephr, the permanent form of the body is developed from a larva, which in all essential points is similar to that of the others. In the Ctenophora there are four secondary axes, perpendicular to the primary, and the most important organs are arranged conformably to these. The body therefore follows, generally, the radiate type, which is best developed in the Beroidæ. This eightrayed form is, however, derived from a four-rayed form, each radius having been split up into two. Each of the two radii which arise from a primitive radius are equivalent to the opposite radii of the same transverse axes. The development of the body follows the poles of one of the two transverse axes. The differentiation which arises in this way is clearly seen in the Cydippidæ; it is more 
distinct in the Mnemidæ, owing to the presence of lobate processes directed towards the oral pole, and most distinct in Cestum, where the form of the body has become that of a band, from its having grown in the direction of two similar interradii.

\section{Appendages.}

$\S 79$.

I comprise under the head of appendages those processes of the body which are known as tentacles; they are either altogether absent or are only just indicated in the Spongiæ, but in the Acalephæ they are widely distributed, and largely affect the external form of these organisms, in addition to which they are of great physiological importance to its general economy. Most of them are, like the wall of the body, contractile, but there are stiff forms which are not capable of much movement (Trachynemidæ). The tentacles are the seat of a large amount of sensibility, and function as sensory organs; in many cases they are organs of prehension; and, finally, they serve as organs of offence by means of the urticating cells which are attached to them.

The Hydroid-Polyps present the lowest condition; in many divisions of them the tentacles are scattered over the surface of the most anterior portion (or portion lying nearest the oral pole) of the body. In many they may be seen to be arranged more regularly, and in others they form a "circlet of tentacles" (Hydractinia, Eudendrium, Campanularia). This is generally placed at some distance from the mouth, and gives a higher importance to this part, which appears to be analogous to a head; and, indeed, the tentacular portion of the body (hydranth) of the Hydroida is called a "capitellum."

The development, in the Tubularia, of a second circlet of tentacles, which directly surrounds the mouth, is correlated with the higher differentiation of the whole body. The outer circlet of tentacles is moved to the edge of the hydranth, as this portion becomes flattened out into a disc. Oral and marginal tentacles can then be made out. The latter are greatly developed among the Hydromedusæ as well as among the Medusæ.

The marginal tentacles, or marginal filaments, which are generally greatly elongated filamentous appendages of the edge of the bell or disc in the Hydromedusæ, are always arranged in correspondence with the radii of the body. Where interradial tentacles are present, they generally follow the radial ones, even when there is a large number of them. Sometimes they are arranged in tufts (Lizzia), or are branched (Cladonema). In opposition to the increase in the number of tentacles, until it surpasses that of the rays, is the 
diminution of these structures. Saphenia has only two tentacles: in some forms, only one is developed (Stenstrupia). In the Trachynemidre also, the tentacles are arranged radially, and many, as the Eginidx, have interradial ones in addition. The attachment of the tentacles to the body is peculiar, as the tissue which supports them often sends a considerable process into the body. Reduction occurs liere also. Eginopsis has only two tentacles. In the Geryonidæ a change of tentacles takes place, the young animal having filaments which are not permanent (larval tentacles) and which are different in structure from the permanent ones.

The oral tentacles distributed among the Hydromedusæ likewise correspond in number to the radii of the body. They are sometimes simple, sometimes branched. They are not, however, always present, and are frequently replaced by outward growths of the edge of the mouth. They are generally wanting in the Trachynemidæ and Eginidæ.

Among the Siphonophora all the medusiform persons want the marginal filaments, which seem to be indicated merely as rudiments; as, for example, in the nematophorous enlargements of the protective persons. This want of an organ important in the economy of the colony is compensated for by the "tentacles" and the "grapplinglines," which can be shown to be modifications of medusiform persons $(\$ 75)$.

The marginal filaments are wanting in the divisions of the Rhizostomidx and Cyaneæ among the Discophora; they have four large groups of tentacles which arise from the lower surface of the umbrella, and which can be considered either as marginal filaments, or as oral tentacles. In others there are marginal filaments present, corresponding in number to the radii, and sometimes even interradial ones are present. Even in the Charabdeidæ, Charabdea has four tentacles carried by the arrow-shaped processes of the bell; in Tamoya (T. quadrumana) these are represented by the same number of tufts. The filaments are more numerous in the Pelagiæ, whilst the Aurelia are distinguished by a very large number of fime marginal ones. Oral tentacles are developed as fine fringing processes on the edges of the arms which surround the mouth. In the Rhizostomida they are distributed along the numerous grooves which carry the oral pores.

'Two kinds of marginal filaments may be observed in the Lucernaria ; in one division (L. cyathiformis) the filaments which beset the edge of the cup-sliaped body are just like those which are found in the Meclusie, but they may be seen to be broken up into eight groups; in the other (L. auricula) they form eight tufts placed on the ends of the four processes which project from the body.

The tentacles of the Anthozoa are different in the large groups of that class. Wight lamellar tentacles, indented or feathered in appearance, surround the month of the Octactinia. There is a much larger number of cylindrical tentacles in the Hexactinia. They surround 
the oral surface of the body, or are scattered over it; they are sometimes produced on to lobate processes also.

In the Ctenophora, processes of no great size are also occasionally present on the edge of the mouth in some families (Calymnidæ, Callianiridæ); and there are large elevated lobate extensions of the body, which we may regard as tentacular organs, although morphologically they are different structures. Besides these, some genera (Cydippidæ) have "grappling-lines," which resemble the marginal filaments in the Medusæ, and correspond in position with the poles of an interradial transverse axis: sometimes they are provided with secondary appendages.

\section{Integument.}

$\S 80$.

The most primitive characters of the integument of the Coclenterata are seen in the Spongir, where it is composed of the ectoderm, which is but slightly differentiated, and follows the various changes of form in the endoderm, which limits the nutritive cavity. The special characters consequent on this relation are referred to below ( $\$ 87)$. In the Physemariæe the cells of the ectoderm form a syncytium. In the Porifera they may be sometimes seen to form a thin layer (Halisarcina, Sycon).

Among the Acalephæ, the ectoderm undergoes differentiation very early, so that the most external layer of cells, or epidermis, which is distributed over the whole body, represents in most cases a portion only of the primitive ectodermal layer. The investment of cilia, which in the Spongiæ is limited to the earlier stages of development, not only persists in the Acalephæ during the socalled larval stages, when it has a locomotor function, but is frequently continued on into the later stages, when it is generally limited to separate parts, e.g. the tentacles.

As the body increases in size, the importance of the cilia, as locomotor organs, disappears. In one class only - the Ctenophorado they retain this function, and they are then increased in size. In the place of the general investment, as seen in the larva, structures resembling cilia are disposed in longitudinal rows, and by increasing in length and breadth become converted into movable swimming or rowing plates. The plates are attached to the body by their broader base; it is at this point only that contractility, dependent on the voluntary influence of the animal, is manifested; the rest and larger portion of the plate seems to be rigid. There are generally eight rows of these plates, which act as steering organs; but in many Ctenophora there are only four rows (Cestum). The urticating capsules (nemocysts) are special differentiations of the epithelial 
elements, which are found in all the Acalephæ, although they are not confined to them. They are firm capsules (Fig. 35, B), which are formed in the protoplasm of the

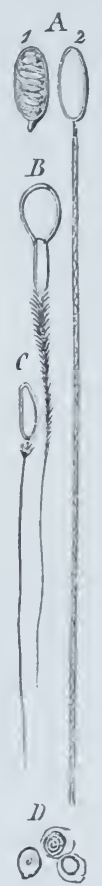

Fig. 35. Different forms of urticating capsules. $a$ Capsule of Corynactis; 1 With the filament spirally coiled; 2 Extended. BC Capsules of Siphonophora: the fila. ments extended and partly provided with hooks. $D$ Ur. ticating cells of Medusæ; filaments still rolled up; in one not yet differentiated, and the nucleus of the cell still visible.

of the stock (Tubularia, Eudendrium, Pennaria), and sometimes they are found even on the separate persons (Campanularia, Sertularia). This provides the soft polyp-stock with an organ of support, by which it is enabled to raise itself from the ground, as well as to attach itself. cell, and in which an elastic, spirally-coiled thread $(A)$ is found; this is generally emitted in the form of a stiff body, when the capsule is touched. These "stinging organs" are sometimes solitary, sometimes in groups, and at times they are very regularly arranged. They often become greatly complicated, as in the stinging knots of the Siphonophora, where they are often arranged in spiral bands. These "stinging batteries" develop on the surface, but are often provided with a special investment formed by a fold of the integument.

Although these structures are scattered over the whole surface of the body, and are not absent even from the endoderm and its products, yet many parts of the body are specially characterised by them; above all, the tentacles, or other processes of the body. The urticating capsules vary greatly in form, as does the filament in structure; and these differences are characteristic of the different divisions.

Further, the ectoderm has a secreting activity, by which tests, which more or less invest the body, are formed. They are very common among the Hydroid-Polyps, where they are formed of a firm substance allied to chitin, and are often provided with various sculpturings, flutings, spines, ridges, and so on. These tubular tests are especially found among the colonial Hydroid-Polyps; they are sometimes limited to the fixed portion of the common stock (H $\mathrm{H}_{\text {dractinia), }}$ sometimes continued on to the branches 


\section{Skeleton.}

\section{$\S 81$.}

In addition to the organs of support, which are formed by the above-mentioned tests, the Cœlenterata have various other forms of skeletons, which are differentiations of the mesoderm.

There are none in the Physemarix, which make up for the absence by taking up foreign bodies into their ectoderm; in the Porifera, some of which have no firm structures (Halisarcina), organs

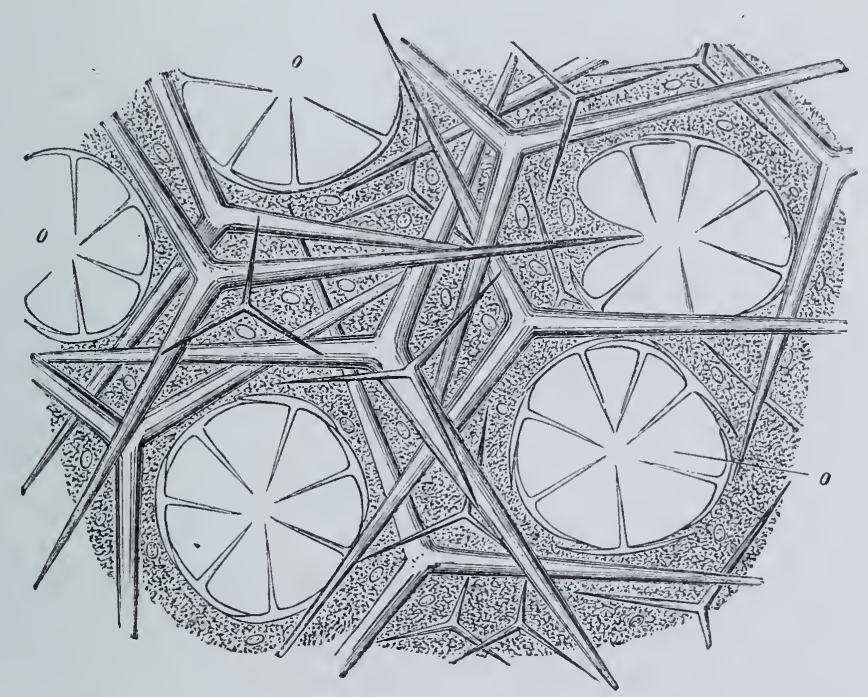

Fig. 36. A portion of the surface of the body of a Calcareous Sponge (Sycaltis perforata) to show the triradiate spicules. o Dermal ostia, each of them surrounded by a circlet of spicules (after Häckel).

of support are formed by firm needles (spicula), or softer fibres, which are placed in the mesoderm. The former are formed of chalk, or of silica; the sponges are known accordingly as Calcareous or Siliceous sponges. The spicules of the Calcispongiæ are simpler in character, for they are either acicular tri- or quadri-radiate; they present great regularity of distribution and arrangement, together with numerous modifications in individual characters. The above figure gives a representation of the spicula of a Calcareous Sponge. The hard structures, when formed of silica, are much more varied in form. In addition to the acicular structures, which are combined together in various ways to form multiradiate stars, there are various other kinds of firm parts, as, for example, the amphidiscs 
(Fig. 37, 2). The siliceous spicules are often greatly elongated, and form excessively delicate skeletons (Euplectella), or they form bulky structures which project as tufts of fila-

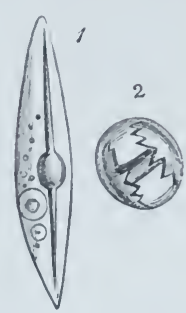

Fig. 37. 1 Cell with a siliceous spicule of Spongilla, 2 Ves. icle with an amphi. disc of Spongilla (after N. Lieber. kühn). ments far beyond the body (Hyalonema). Lastly, in the Fibrospongix, the skeleton of the body is formed by fibres united into a network, which consist of a substance allied to chitin.

In the Acalephr also the deposition of inorganic substances in the mesoderm leads to the formation of various kinds of skeletons. In the Anthozoa they generally have the form of colonies, and the hard substance is almost always formed of calcareous salts. These give rise to deposits (Fig. 45) of definite form (Fig. 38), which are scattered in the soft parts of the body; or to connected masses, which vary in form according to their mode of development. The calcareous bodies (spicula) always lie in the connective-tissue of the parenchyma, and are very varied in form. They have an organic basis, which retains the form of the spicula after the lime

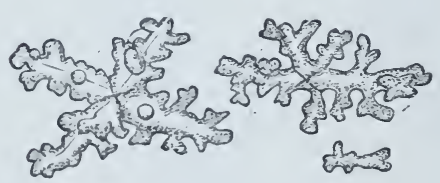

Fig. 38. Calcareous Spicules of Alcyonium. is remored. The connected skeletal parts are formed either by the union of spicula, which are connected together by a hard organic substance, as in Corallium, or by the direct calcification of a secreted horny substance, which lies in the axes of the cœnenchyma, and does not possess spicula. When the organic substances predominate the skeleton is horny, as it is in the Gorgonidæ and Antipathidæ. These axial skeletons are sometimes limited to the trunk of the colony, as in the Pennatulidæ, where they lie in the shaft of the stock, or they may be continued into all the branches of the stock. There is another form of skeleton, which resembles the axial; it is formed by the gradual calcification of the parenchyma of the body, in which process spicula sometimes take part. In this way the aboral portion of the whole body is more or less completely hardened. A proportionate forward growth of the body at its oral pole occurs at the same time, and the parts which are completely calcified represent the dead base. Skeletons of this kind form the calcareous supports of the Fungiæ, Astræidæ, Madrepores, and of Tubipora. The skeleton thus formed must be regarded as a continuation and development of the skeletons which are found in the Spongia.

\section{$\S 82$.}

Another kind of supporting organ is formed in the interior of the body by cuticular structures, or by differentiation of more resistent connectivo substances. The simplest example is again found in the 
Hydroid-Polyps, in which a homogeneous lamella appears between the ectoderm and endoderm; this functions as a supporting lamella to the softer tissues, which are attached to it. Oring to the formation of external tests, this structure has less importance as an organ of support in certain parts of the Hydroida : it is very thin in the parts where the tests are found, but it is much stronger in the free parts of the body, which are not sheltered in the test. We find a strong layer of supporting tissue in the wall of the body of the Tubularia, which belongs to the free portion or hydranth of the animal. This tissue consists of a homogeneous substance traversed by fibres, and embedded between the ectoderm and endoderm. This appears to be the first step in the formation of an arrangement, which is highly developed in the Medusæ, the so-called gelatinous disc, but in many of them (Meduse of Clavatella, and Eleutheria) is also only slightly developed.

The gelatinous disc is in the Hydromedusæe either completely homogeneous or is traversed by fibres, which extend from the ectoderm to the endoderm. It forms a disc, which is attached to the aboral surface of the body, and which determines the form of the body (Fig. 39, l); it may become modified into the form of a bell. The organs derived from the endoderm, which consist principally of the gastric apparatus, lie on the oral surface of the disc.

Although the gelatinous umbrella of the Discophora agrees in its external characters with that of the Hydromedusæ, it differs from it in some not unimportant characters. Its substance

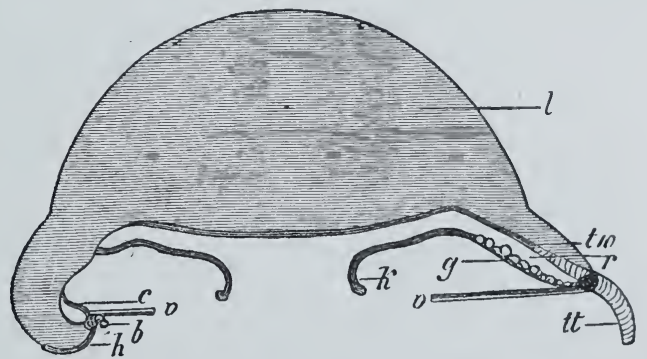

Fig. 39. Diagram of a vertical section through a growing Cunina rhododactyla; on the right side through a radial, on the left through an interradial vertical plane. $b$ Marginal vesicle. $c$ Raclial canal. $g$ Generative products. $h$ Mantlefold. $l$ Stomach. $l$ Gelatinous disc. $r$ Radial pouch. $t t$ Tentacle. $t w$ Base of tentacle. $v$ Velum (after E. Häckel). contains various morphological elements, which resemble those of gelatinous connective tissue, and it is continued on the oral surface over the so-called stomachal stalk. It surrounds, therefore, the greater part of the gastrovascular system.

Of less moment are the characters presented by the supporting structures of the tentacles of many Hydromedusæ. In both the Hydriformes and Medusæ (Trachynemidæ, Aginidæ) the axis of the tentacle is formed of a series of cells, the elements of which appear to be encapsuled by a more or less homogeneous membranous layer (cf. Fig. 9). The rows. of cells are thus to a certain extent rigid. A ring of much the same structure (annular cartilage) is found on the edge of the disc of the Geryonidæ. 


\section{Muscular System.}

\section{$\S 83$.}

Form-elements, referable to muscles, are not certainly known to exist among the Spongiæ; in the more exactly known Calcispongiæ they are certainly absent, and the protoplasm of the ecto- and endoderm performs all the movements of the animal.

A muscular layer is first marked off in the Hydromedusæ (Hydriformes), where the cells of the ectoderm possess band-like processes, which form a connected stratum beneath that layer of cells (cf. suprà, § 25). This layer is also continued on to the tentacles, but is wanting in those parts of the colony which are surrounded by a test. In some parts, as in the stem of the colony in the Siphonophora, it is greatly developed. In the Medusæ it is limited to the surface which carries the gastric apparatus, where it forms the "sub-umbrella." From the edge of the bell or disc it passes into a more or less broad membranous process, the velum, which consists essentially of muscular fibres; it is also continued on to the tentacular organs. The muscular system is more complicated in the Discophora, many of which also are provided with a velum (Aurelia). In all the Medusæ the form-elements of the muscles are finely striped transversely, but this is not the case in the same parts of the Hydriformes.

In the Ctenophora muscular bands have been observed running along the ciliated "ctenophores;" and there are muscular fibres in the interior of the gelatinous tissue of the body.

The muscular system appears to be best developed in the Anthozoa. Thus, in the Actiniæ, the attached base of the body is distinctly formed by muscles, and circular and longitudinal fibrous layers can be made out on the rest of the body, which are continued into the tentacles. In those Anthozoa that form stocks, the bodies of the separate animals appear to have circular and longitudinal muscles, whilst the soft conenchyma is also contractile, the network of canals of the gastrovascular system, which traverses it, being accompanied by muscular fibres.

\section{Nervous System.}

\section{$\S 84$.}

The Spongix are placed in the lowest grade of animal differentiation, owing to the absence in them of any arrangements which can be regarded as special organs of sensation. The Acalephæ are not far above them, for their lower forms likewise give no signs of distinct organs of this kind. Thus the cellular layer of the 
ectoderm, in the Hydroid-Polyps, is as yet an indifferentiated organ of sensation. Irritations of it produce movements of the fibres of the muscular layer connected with the cells (\$25); and it is only in the Medusæ that distinct parts can be recognised as belonging to a nervous system. These form a ring, which runs round the edge of the disc, and which is formed of a fibrous tissue, on which ganglionic swellings, formed of cellular elements, are placed at regular intervals. The ganglia correspond in position to the marginal bodies, which are to be considered as sensory organs, and send off fibres which pass partly to the tentacles, and partly accompany the radial canals. This nerve-ring, which is most accurately known in the Geryonidx, is supported on the annular cartilage, and lies between it and the circular canal at the edge of the disc. The swellings of the nerve-ring represent central organs, which are connected with one another by the fibrous portions. From experiments also in which the edge of the disc was divided, it seems clear that there is a central nervous system in it.

The nervous system of the Ctenophora is as yet not well known. As to the rest of the Acalephr, no organs of this kind are known with any certainty.

\section{Sensory Organs.}

$\S 85$.

Owing to the imperfection of our knowledge of the nervous system of the Colenterata, no definite opinion can be given as to the parts which are to be regarded as sensory organs. This remark refers as much to the arrangements which we regard as subserving: the sense of touch as to higher sensory organs. Special processes of the body appear to serve for the general tactile sense which is present in the integument, and these we have already spoken of as tentacles $(\S 79)$. Whether there are, on the other hand, special organs, must for the present remain undecided; although the presence of stiff setæ on the tentacles, and around the mouth, leads us to admit the existence of distinct organs of touch.

More differentiated organs, adapted for sensory perceptions, are found in the so-called "Marginal bodies," which are attached to the edge of the umbrella in the free Medusæ, and which are of two distinct kinds. The first have the appearance of vesicular structures, the second are collections of pigment provided with a transparent refracting body, similar to those organs which, in the higher animals, are seen to be the terminal organs of the optic nerves. The former, or marginal vesicles, are either embedded in the substance of the disc, or project freely at its edge. They consist of a homogeneous capsule, covered with epithelium, and enclose one or more concentrically striated concretions, or small crystals. The concretions are in close relation with the wall of the vesicle, being encased in a spherical outgrowth of it. As 
they do not lie in the free cavity of the vesicle they cannot be definitely regarded as similar to the auditory vesicles of other low animals; at the same time, it is not possible to give them exactly any other signification. It is clear that they represent sensory organs from their intimate connection with the nerve-ring; for a double fibrous band arises from the ganglion, which is placed below cach marginal vesicle, and surrounds the vesicle; after uniting: with it, the fibres pass into the spherical mass of cells, which contain the concretion (Geryonidx). This marginal vesicle is most common among the Eucopidæ, Trachynemidx, Geryonidx, and Eginidæ.

In Cunina crystals are present, so that its marginal vesicles form an intermediate step towards the similar structures in the Discophora. The marginal vesicle in the Discophora is always stalked (Fig. 40, $A B b$ ), and lies in a fissure, or a niche-like depression of the edge of the disc, covered with a lamellar umbrella-like process of it. A cavity (ampulla) forms a large part of the marginal body $(d)$, and is connected with the gastrovascular system by means of a canal, which passes into the stalk $(c)$; attached to this ampulla, and occupying the free edge of this marginal body, is a vesicle (e) filled with crystals, and resembling the similar one in the Eginidæ (Cunina). The most important difference between the two is therefore the absence in Cunina of the ampulla formed by the gastrovascular system.

Organs of another kind also are found in the Hydromedusæ. They appear to be in a relation of mutual exclusion to the marginal vesicles, as they appear in those families only (Oceanidæ) which have no vesicle. The first indication of them is the appearance of pigment spots on the base of the tentacle, which, as a rule, have no refractire media; in other cases, on the contrary, they are pro-

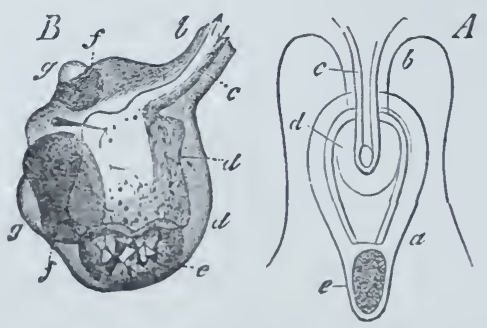

Fig. 40. Marginal body of Acraspedotro Medusæ: $A$ Of Pelagia noctiluca; $B$ Of Charybdea marsupialis. $a$ The free part of the marginal body placed between the marginal notches in the disc. $b$ Stalk. $c$ Canal in it. d Ampulla. $e$ Crystallino saccule. $f$ Pigment. $g$ Lens-like body. vided with structures which call to mind the crystalline cones of other lower animals. In the Discophora these ocelli are combined with the already-mentioned marginal bodies; they sometimes consist of pigment only, while in other forms the pigment appears as the investment of a highly refractive body (Fig. 40, B g).

In the Ctenophora, also, there are special sensory organs. The most important is a resicular structure, which is attached to the aboral pole of the body, and contains solid concretions, like the otoliths in the auditory vesicles of other lower animals. The functional importance of this organ is, however, not yet exactly 
kuown; just as uncertain is that of the two ciliated surfaces, on either side of this vesicle-the polar areas, which aro surrounded by short fringe-like processes.

\section{Alimentary Canal.}

\section{$\S 86$.}

With the separation of the body into an ectodermal and an endodermal layer, we get the lowest condition of the organs of nutrition; the endoderm investing a space which is open to the exterior, and which is the earliest distinct digestive cavity (stomach, enteron)-(cf. suprà, $\S 28$ ). This condition is simplest in the Gastrula form, and undergoes various differentiations in the two chief divisions of the Cœlenterata. The stomachal cavity, that is, does not remain a mere simple space, but grows out into various kinds of cavities, canals, pouches, and so on, which are either distributed irregularly in the organism, or are arranged in a definite way. As a rule, division of labour takes place at the same time, and only one definite part, or several such parts, functions as a digestive cavity, while the other spaces are used to distribute the nutrient fluid (chyme). But this gastric system has other functions too. There is no doubt that it also has a respiratory function, by distributing through the body the water that was taken in with the food; and for this purpose it has, especially in the Spongix, a much more extended surface than has the outside of the body. Finally, it has important relations to reproduction, for the generative elements are formed in its walls.

\section{$\S 87$.}

Among the Spongire the simplest form is limited to the early stages of development,'or is permanent as in the Gastræades. Almost the only complication in the Gastreades is the development of an arrangement for producing a current at the entrance of the simple enteric tube. In the Porifera there are various new complications. Temporary spaces appear in the endodermal layer, which break through to the exterior, so that in addition to its mouth (Fig. 41, $o)$, the enteric cavity is connected with the exterior by

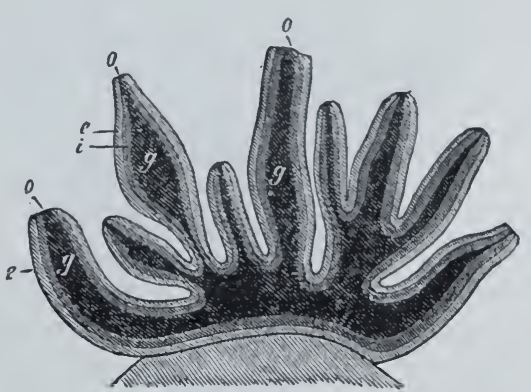

Fig. 41. An Ascon-stock of nine persons (individuals). Diagram. e Ectoderm. $i$ Endoderm. o Mouth. $g$ Enteric carity (after E. Häckel). pores at various points, which open and again close. The number of these pore-canals (dermo- 
gastric pores), which have consequently a dermal and gastric orifice, is generally very great; their number is dependent on that of the spaces which are bounded by the rays of the spicula (cf. Fig. 36, o). These characters are very distinct in the lowest forms of the Calcispongix, the Ascones (Olynthus).

'The development of diverticula of the enteric cavity gives rise to a second form; the diverticula are continued in to the correspondingly thickened ectoderm, where they form more or less branched canals; from these, again, fine canals, which are also branched, open into the dermal pores. The enteric cavity, as it becomes more and more divided into branched canals, loses its importance as a stomach, and at the same time its endodermal investment, which is now limited to the branched canals. But the endodermal layer does not extend over all of them, but is finally restricted to their diverticula, which

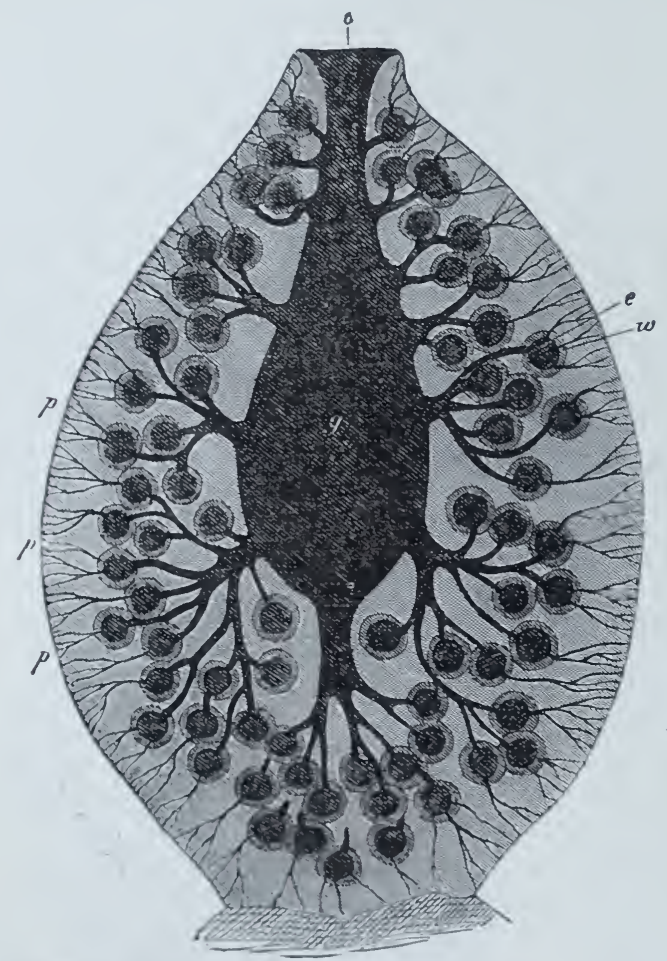

Fig. 12. Diagram of the gastric system of a Leucon (Dyssycus ananas), where branched canals are developed. o Mouth. g Euteric cavity. $p$ Dermal canals. $w$ Ciliated chambers. The difference between the ectoderm and endoderm is represented in the same way as in the previous figure (after E. Hiickel). stomachal cavity, which in their character's correspond to the simple Ascon form, but which generally communicate with the exterior are thus converted into the so-called ciliated chambers. Thus the function of the enteric tube passes more and more from its primitive locality into the additional spaces, which are gradually developed from it.

The subjoined figure (Fig. 42) represents the last stage in which the endoderm invests the ciliated chambers only (wi). Modifications of this form which obtains in the group of the Leucones, among the Calcispongia, are formed by the union of the branched canals and of the ciliated chambers one with another, whence arise retiform canal systems. The Siliceous and fibrous Sponges conform to this type.

A third form arises by the formation of closely adjoining canals, 
by dermal pores only. The primitive enteric cavity in them, as in the Leucones, where it loses its layer of flagellate cells (endoderm), also loses its nutritive functions, which are confined to the radial tubes. These latter seldom remain free, but generally unite either, in part or completely, by their walls with an important layer which surrounds the primary enteric cavity. A system of canals, which are only invested by ectoderm, is formed out of the space between the radial tubes when these only fuse in part. 'This form inay be seen in the Sycones, among the Calcareous Sponges.

Innumerable modifications, including individual varieties, are present within the range of one type-form. The primary enteric cavity is altered in character by the formation of diverticula, as well as of septa, or trabeculæ, and may even be completely atrophied, while the canal system arising from it is developed; this phænomenon (lipogastria) is not uncommon in the Fibrous and Siliceous Sponges. A similar atrophy may even affect the mouth (lipostomia) without affecting the stomach; in such a case the dermal pores take on the function of ingestive canals; or numerous small spaces, as in Euplectella, arise in the place of the mouth.

\section{$\S 88$.}

The form of the gastric system is greatly affected by the formation of stocks, a process due partly to the concrescence of free persons, and partly to budding. The union, according to the degree of its development, may then simply cause communication between the stomachal cavities, which persist for each person (Fig. 41), or lead to a complete union of the cavities; in which case the mouths too may undergo reduction, or become reduced to one, which likewise may disappear.

A special system of cavities (inter-canal system) also arises from the formation of stocks. This system is formed from the spaces which persist between the unconnected parts of the persons, or the anastomosing branches, of the body ; this, like the system mentioned above in the Sycones, is bounded by the ectoderm only, and is thus essentially distinguished from the gastric system. It is remarkable for considerable irregularities in its arrangement, and forms also wider spaces, which deceptively resemble a stomachal cavity in that they possess a mouth.

From all these arrangements a significant change of function in different parts is seen to accompany the change of form in the Spongix. The physiological activity of the digestive cavity is not only shared by the secondary canals which arise from it, but even passes away from it altogether, or is limited to separate portions of it, when consequently the stomach sinks, physiologically speaking, to a lower grade. On the other hand, an important function becomes localised by this change of the primitively subordinate portions of the canal system, and even the original surface of the body of the sponges gets a higher significance, in virtue of its serving as the lining of the inter-canal system. Everything distinctly 
shows that the organisation of the Spongiæ is not only very variable, but also that to understand it, it is absolutely necessary to distinguish clearly between the physiological and the morphological value of an organ.

\section{$\S 89$.}

In its earliest characters the formation of the enteric cavity of the Acalephre agrees with that of the Spongiæ, but in the matured condition there are peculiarities in the Acalephr, owing to the greater regularity in the arrangement of the system, which is developed out of a simple cavity. The mouth, the extent of which is often increased by the development of accessory parts in its neighbourhood, leads into the digestive cavity, and serves also as an opening for the excretion of undigested matters. The principal cavity seldom remains single, but grows out into secondary cavities, which have the character of pouches, or of canals, and which also, as a rule, correspond to physiological differences, since by them the chyme which is contained in them is distributed through the body of the person, and of the stock. These accessory spaces of the digestive cavity, included with the latter under the designation "gastrovascular system," undertake the function of a circulatory system, without being, morphologically, anything else than the differentiations of a primitive enteric cavity. The gastric system of the Acalephæ agrees therefore genetically with that of the Spongiæ, but is distinguished from it by the exhibition of a higher differentiation. This is seen in the difference between the accessory spaces and the central primary one, which forms the stomach, to which its functions are ordinarily limited, and which are not, as in the Spongix, handed over to the secondary spaces.

\section{$\S 90$.}

The simplest form of the gastrovascular system is found in the Hydroïda. In Hydra it forms a space traversing the long axis of the body, which commences with the mouth, in the middle of the circlet of tentacles, and is continued from the next portion, the stomach, which is capable of great extension, into the thinner portion of the body, where it is narrower. This space is also continued into the tentacles. In the Hydroid-Polyps which form colonies, the canal which arises from the stomach runs through the whole stock, and makes the gastrovascular system common to all the persons. In the stocks of the Siphonophora, some persons only are set apart for the ingestion of nutriment. Each corresponds in structure to the stomachal tube of a Medusa, and forms a tube capable of great extension, which is connected at its base with the general cavitary system of the stock. In this case, then, we must suppose that this sort of individuals has lost all the arrangements found in the body of a Medusa, with the exception of the stomach $(\$ 75)$.

The gastric system of the Medusa (Hydromedusæ as well as Discophora) presents numerous variations. It always occupies the 
concave portion of the gelatinous disc, and consists of a stomach placed in the middle of this cavity, and of hollow spaces which proceed from it. The stomach either lies directly beneath this surface, or is placed on a special stalk, which arises from it, and is often of considerable size. This free projection of an organ, which in other animals is hidden within the body, is explained by the differentiation of the stomach of Hydromedusæ from the most anterior portion of the body of the Hydroid-Polyps, so that it does not represent a single organ, but a complete portion of the body. The mouth is generally surrounded by tentacular organs, or pointed prolongations of the wall of the stomach; it seldom opens into a narrow portion resembling an œsophagus. In most Hydromedusæ the stomach is separated from the space that lies behind it by a ring which is developed at its base; by the contraction of this ring, the stomachal cavity can be shut off from the
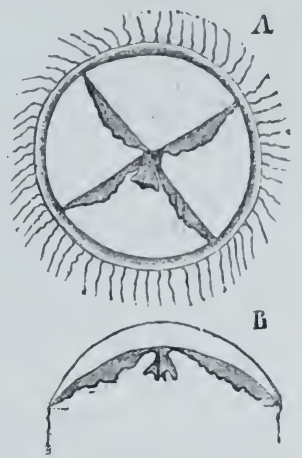

Fig. 43. A Thaumantias. $A$ From the lower surface. $B$ Seen in section. In the middle of the body is the stomach, from which the radial canals pass to the circular canal. rest of the gastrovascular system. The stomach varies greatly in form and size. It projects far beyond the edge of the bell-shaped umbrella in the Sarsiadæ. The hollow spaces which are distributed in the sub-umbrella arise from the base of the stomach, or from the space which lies behind it, and have the form of narrow canals, or of wide pouch-like diverticula. The narrow canals take a radial course (Fig. 43) to the edge of the umbrella; they are simple or regularly branched, and they open into the circular canal, which often sends processes into the marginal tentacles also. On their way to the margin, the radial canals may form diverticula, which are functionally connected with the generative apparatus (cf. §.96).

In the Aginidæ and Discophora the gastric cavity passes directly into the radial enlargements; these latter are derived from simpler canals. Narrower canals sometimes, indeed, alternate with wider spaces. The canals are branched (Fig. 44, gv), or form, as in

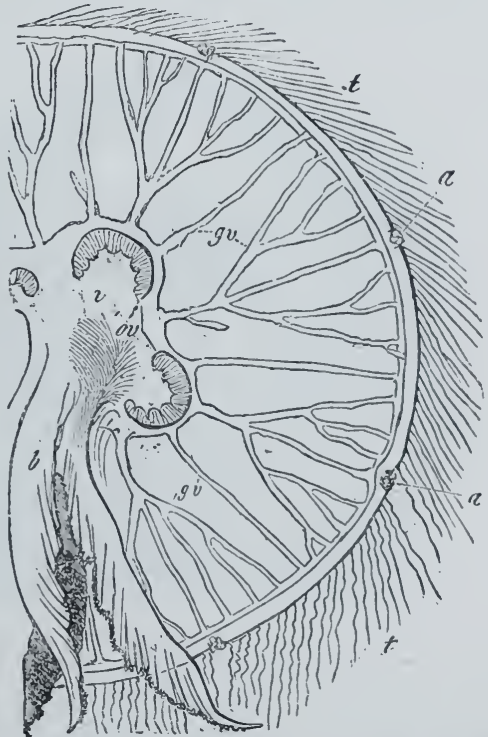

Fig. 44. Aurelia aurita. Half of the lower surface is seen. a Marginal bodies. $b$ Oral arms. $v$ Gastric carity. gr Canals of the gastrovascular system, which branch towards the edge and unite into a circular canal. ov Oraries. the Rhizostomidx, a peripheral network. As the gelatinous substance 
of the umbrella is continued into the wall of the stomach in the Discophora, the stomach is not very sharply marked off from the rest of the gastric system. Its wall is always continued into arm-like appendages, which, as a rule, project into folded membranes (oral arms); the mouth is placed between these. Division of these arms leads to further modifications, which give rise to greatly ramified appendages. In this case numerous grooves, which gradually unite, lead to the moutl, in correspondence with the form of the arms. In the Rhizostomidæ the mouth remains open during an early period of development only, and afterwards becomes closed by the gradual union of the "arms," which limit it, and in which the grooves form branched canals, which open at the ends of the ramifications of the arms by numerous fine pores (polystomia).

In the Lucernariæe the structural conditions of the gastrovascular system closely resemble those of the Medusæ. A stomachal tube, projecting from the concave surface of the umbrella, and produced into four angles, leads into a wide space, which is continued into four pouches, and may be elongated into four canals, which pass into the stalk. The four pouches correspond to widened radial canals, and are, as in the Medusæ, connected with one another at the edge of the umbrella, and so form a circular canal. In others this character is modified in such a way that the stomach is continued into the body, in a tubular form; and at its end, which projects into the stalk, gives rise to radial canals, which whilst becoming enlarged

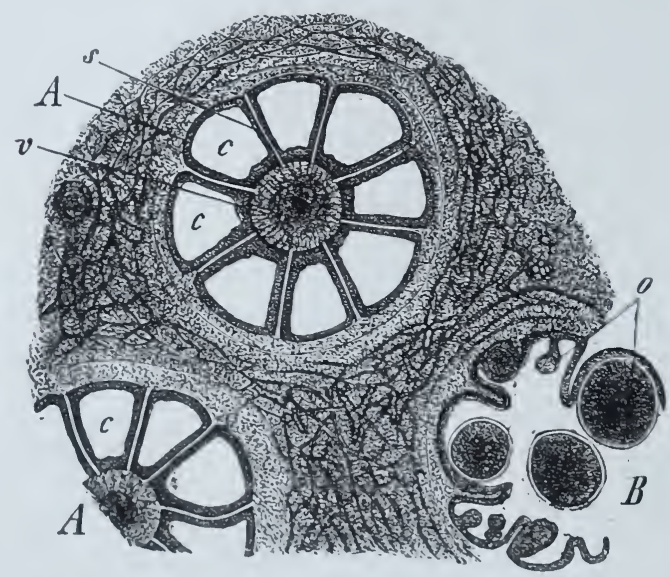

Fig. 45. Transverse section through a portion of the stock of Alc you ium, in which two individuals, $A A$, are cut through just below their junction with the conen. chyma, and a third, $B$, somewhat lower. $v$ Wall of wesophagus. c Radial canals (chambers of the body. cavity). s Septa. o Ova. Part of the conenchyma traversed by canals is seen to contain calcareous bodies. run outwards towards the margin of the disc. The gastrovascular system in the larvæ of the Discophora and in Scyphostoma is very similar in character.

\section{$\S 91$.}

The gastric system of the Anthozoa extends by means of an osophagus from the centre of the tentaclebearing surface of the body into the interior, where it opens into the digestive cavity. From this part canals passupwards along the sides of the cesophagus into the tentacles.

Owing to the wilth of the canals comnected witl the stomach, the intermediate tissue is reduced to a mere partition $(s)$, which extends in rays from the wall of the body to the wall of the 
œsophagus. The canals thus appear to be chambers $(c)$ attached to the oesophagus, which unite into a common central space the digestive cavity, or stomach $(B)$, and so communicate with the osopliagus. The number of these chambers is eight in the Octactiniæ, and varies in the other Anthozoa, but is arranged according to the same law of numbers, as is expressed in the other characters of their organisation, as for instance in the number of the tentacles. The septa of the gastrovascular system are usually continued for some distance along the wall of the digestive cavity, and terminate as elongated bands or pads. When, therefore, the stock is calcified interradial lamellæ are formed, passing inwards from the wall between the gastric lamellæ.

In the colonial Anthozoa, the central cavity is connected in each person by means of a canal system which traverses the cœenenchyma (Fig. 45), and thus every individual is directly connected with the rest. This canal system forms a network of tubes of various widtlis which distribute the nutritive fluid in the stock. At one point of the common trunk, in the stocks of the Octactiniæ, several canals are united into a wider space, from which an orifice leads to the exterior; this, probably, serves as a means of regulating the ingress and egress of the water which flows through the gastrovascular system (Pennatula, Renilla). A similar opening has been observed in Cereanthus; it corresponds to the pore of the Hydræ, and like it is placed at the aboral end of the body. These arrangements, which give to the gastric system the significance of a water vascular system, have, in many Anthozoa (Corals), the form of fine pores scattered over the surface of the body; they can only be perceived at the moment they are in function-that is, when expelling water. Similar pores are also found on the tips of the tentacles in many Actiniæ, etc. All these arrangements call to mind the dermal pores of the Spongix.

In the Pennatulidæ and Alcyonidæ (Sarcophyton) some, and at times many, persons in a colony are less well-developed, and seem to have lost the function of ingesting food. It is not known whether they have any share in the taking in of water.

\section{$\S 92$.}

In the Ctenophora, the nutrient cavitary system differs in details only. A stomach, which is very wide in the Beroïdæ, and narrower in the rest, is sunk in the body along its longitudinal axis; it passes into a space which is known as the "funnel," by means of a narrow canal, which can be closed by muscles. Radial canals (Fig. 46) pass out from the . funnel and run along the ciliated ribs or "ctenophores." The radial 
canals in the Beroïdæe and Callianiridæ pass into a circular canal at the oral pole. In the latter, two canals, which run along the sides of the wall of the stomach, and which come from the funnel, also enter the circular canal. In the Cydippidæ, these are very wide, and appear to form a common space around the stomach. Finally, two shorter canals, which do not pass directly from the funnel, but from the canals derived from it, run outwards and open by pores, which can be closed, at the sides of the polar areas (cf. p. 111). They are placed diagonally, and provide the gastrovascular system with a second means of communication with the surrounding water.

The form of the body leads to various modifications in the arrangement of this system of canals. The various groups of canals may be branched. Thus, in the Beroïdæ the radial canals form lateral branched diverticula, which are present, though they are not so large, in the other forms, and are in connection with the generative apparatus.

\section{$\S 93$.}

In some divisions of the Acalephre there are filamentous structures, which project into the central cavity of the gastrovascular system; they are called Gastric filaments (and, though less appropriately, mesenteric filaments). Theyare found for example in the Lucernaridæ and Discophora. In the latter they form tufts of filaments, placed in diverticula of this cavity, and execute vermiform movements. They hare similar characters in the Lucernaridæ, but are different in the Anthozoa. In the Anthozoa pad-like processes, richly provided with stinging cells, run along the free edge of each septum, turned towards the gastric cavity; they seldom become filamentous, and are sometimes limited to two of the septa (Tubipora). Nothing is known as to the function of these organs, which are differentiated very early.

Although glandular organs do not seem to be differentiated in the digestive cavity of the Colenterata, yet there is an arrangement which should be noted here; it may be regarded as an indication of a secreting system, perhaps analogous to the liver of higher animals. It is this, namely, that the epithelial investment of the stomach, which is present in many Colenterata, is distinguished by its peculiar colour. 'The pigmented cells are set longitudinally, and are generally placed on the projecting folds of the wall of the stomach in the Anthozoa; they are also developed in the Hydromedusæ, even in the polyp forms (e.g. Tubularia); in the Siphonophora they form distinct pad-like longitudinal rows at the base of the digestive cavity of the nutrient individuals. A network of "hepatic canals," attached to the single large stomach of the Velellidx, appears to be specially differentiated; it is found on the under surface of the disc. 


\section{Sexual Organs.}

\section{$\S 94$.}

Sexual differentiation is not the sole factor in reproduction among the Colenterata, for various forms of asexual multiplication (cf. suprà, §§ 73-77) obtain among them. Sexual products have been observed in most of them, but they are not formed in organs set apart; the function seems rather to be one which is being gradually localised. In the Spongix the endoderm is said to be the place where these products are formed, but in those Porifera which have a mesoderm, the differentiation appears to take place in it. The history of the ova is best known; they arise from cells in the mesoderm, but they are perhaps endodermal cells which have passed into it. In addition to what has been directly observed in this group we must bear in mind the characters which obtain in the Hydroid-Polyps (see below). The male elements have been less widely observed. The endoderm has been said to be the place where the seminal cells are formed, but masses of sperm have been observed in the mesoderm of Halisarca, together with a sexual differentiation of the stocks.

\section{$\S 95$.}

The place where the generative matters are formed-as a rule in the walls of the digestive cavity, or the spaces leading from it-is most exactly known in the Hydroîda among the Acalephæ. The material of the two kinds of generative products is, however, provided by different layers of the body : this fact deserves to be exactly described on account of its fundamental importance. The first, or indifferent stage, is represented by diverticula of the wall of the body, which have the form of buds, surrounding a prolongation of the gastric cavity, and formed by the ectoderm and endoderm. A number of

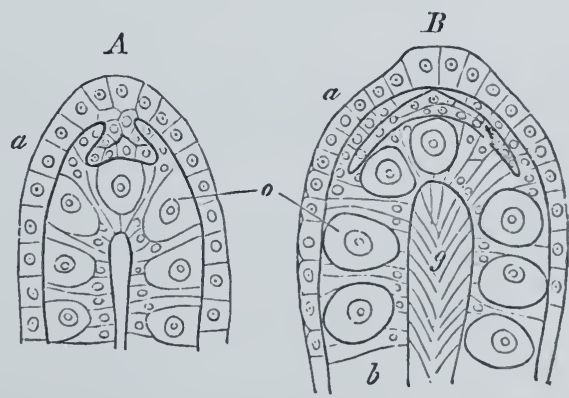

Fig. 47. Two female generative buds of $\amalg_{J}$ dractinia echinata. a Ectoderm. $b$ Endoderm. $g$ Gastric cavity. o Ovarian germs. In $A$ the ectoderm has begun to be pushed into the endoderm. In $B$ the invaginated portion has been constricted off from the rest of tho ectoderm (after Ed, van Beneden). the cells of the ectoderm $(a)$ of the growing bud (Fig. 47, $A B$ ) enlarge and become distinguished by their size from the other endodermal cells, which bound the gastric cavity $(g)$. These enlarged cells are pushed out towards the 
ectoderm, and are the ova (o). They gradually form a layer of cells placed apparently between the ectoderm and endoderm, and give to the whole bud the appearance of an ovary. While these processes of differentiation are going on in the endoderm, a growth of cells from the ectoderm at the tip of the bud is extending inwards $(A)$; as these cells become separated off from the ectoderm $(B)$, they form a thin lamella, which grows around the ovarian layer, but which has no further function except in another kind of bud.

In the male bud, in fact, the ectoderm has the same characters, but the endoderm does not undergo any change, and simply forms a layer of cells, investing the gastric cavity without being differentiated into ova. The depressed portion of the ectoderm being developed to a great size, forms by constriction a layer between the ectoderm and endoderm (Fig. 48, $A B C$ ), the cells of which give rise, later on, to the morphological elements of the sperm. In this

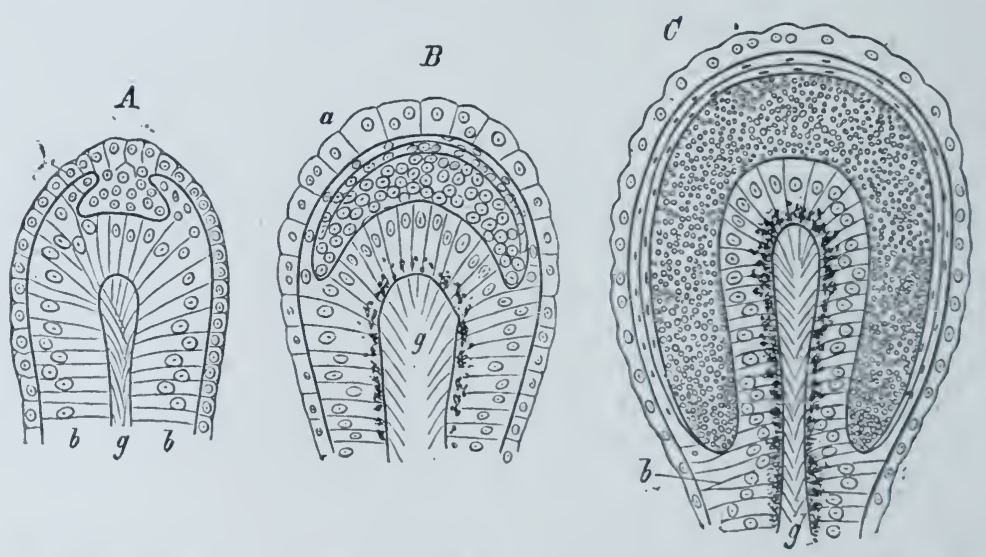

Fig. 48. Three male generative buds of Hzdractinia echinata. a Testes. Other letters as in Fig. 47 (after Ed, van Beneden).

way the male products of generation arise from the ectoderm, just as the female products are formed from the endoderm. The fact that even in the female buds the ectoderm is depressed, leads us to suppose that the buds were primitively hermaphrodite. It is not yet known how far the generative products lave separate origins in the rest of the Acalepha. The possibility of cellular elements having passed from one layer to another at a very early period of development may account for the fact that the endoderm appears to be the layer in which the products of both sexes are formed. Hydra appears to form an exception, for in it the generative products are formed in external bud-like organs, which are differentiations of the ectoderm. Among the Hydromedusa we not unfrequently meet witl a separation of the sexes, not only into different persons, but eren into different colonies; in the Siphonophora hermaphrodite colonies only are found as the rule, but there are exceptions to this. The generative products give rise to more or less considerable swell- 
ings in the parts of the body where they are formed, but as they are only present when the generative matters are being formed, they may be regarded as "temporary organs."

There are great peculiarities in the structural relations of the parts which enclose the generative products, but they are connected by a large number of intermediate stages. In those Hydroid colonies that give rise to free Medusæ (cf. \$ 74), the Medusæ carry the generative organs; the Medusæ form the generative animals of their proper Hydroid-Polyps, and elaborate the semen or ova in the walls of their stomach, or in the radial canals, or, lastly, sometimes in the circular canal. In some this production does not take place until a long time after they have broken away from the Hydroid colony; in others it happens earlier. The latter bring us to those in which the reproductive matters are formed while the Medusæ are still attached to the Hydroid stock. Next, then, comes that stage in which the Medusa is not only not broken off, but is not completely developed. All the organs which are of use in the full and independent mode of life-mouth, gastric cavity, tentacles, swimming-bell, \&c.-appear in a state of atrophy. We have in fact medusoid buds, in which the sexual products arise. In others the medusoid form is completely lost, and quite simple structures appear on the Hydroid stock in the form of generative capsules, into which, at the most, a process of the gastrovascular system still projects. Such are the structures described above in Hydractinia. These generative buds arise, like the medusoid buds, and the Medusæ themselves, sometimes on the common stock, sometimes on the body of the Polyps, and often only at certain parts of the latter, as, for example, between the outer and inner circlet of tentacles in the Tubularia. Where the proliferating Polyps are provided with a sheath, the generative buds are always enclosed by the same test as the Polyps themselves. Thus the phænomenon of the budding of Medusæ can be followed back to a stage in which the bud has the appearance of a mere generative organ of the hydroid stock.

The Siphonophora resemble the Hydroid-Polyps. The formation in them of sexual animals of the Medusa-type, with the simultaneous formation of other medusiform persons, helps to explain the phænomenon known in the Hydroid-Polyps as alternation of generation, as being a division of labour. In some of the Siphonophora the generative animals become free Medusæ, in the walls of whose stomachs the generative products are formed (Velella, Chrysomitra). Most of them have only medusiform buds, which are found in very various stages of degeneration (cf. Fig. 33, $B, E E$ ). The stomach of the Medusa becomes gradually represented by the generative organs only, and the Medusa-bell degenerates into a mere covering for them. Thus they occur arranged either singly (Diphyïdæ), or grouped into racemose tufts (Physophoridæ), which are placed on the stem of the stock, or, it may be, on definite persons belonging to it.

Ed. vay Beneden. De la distinction originelle du testicule et de l'ovaire. Bull. Acad. Belg. $2^{\text {me }}$ Sér. T. xxxvii. 5.-G. Kocr, Morph. Jahrb. Bd. ii. p. 83. 


\section{$\S 96$.}

In those Medusæ which have no longer any relation to Hydroids, the generative matters are formed in the wall of the gastrovascular system, just as in the Medusæ of the Hydroid-Polyps, and the Siphonophora. These matters are generally formed in the radial canals (Aquoridx), or in the pouch-like diverticula of the stomach (Aginidæ). When the canals are narrow the genitals form

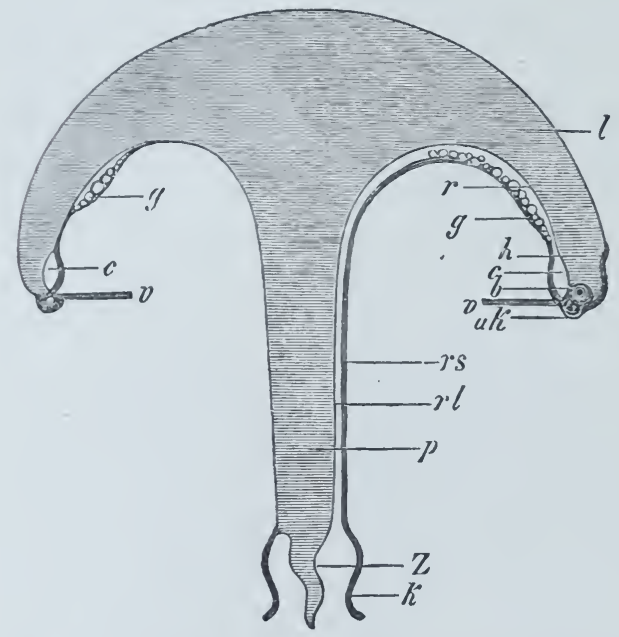

Fig. 49. Diagram of a radial rertical section through a sexually mature Gerjonid (Carmarina hastata); on the right it is taken through the whole length of a radial canal, and on the left throngh the lateral wings of a genital lamella, at an interradial plane. $b$ Marginal vesicles. $c$ Cir. cular vessel. g Generative products. $h$ Clasp of the mantle. $k$ Stomach. $l$ Gelatinous umbrella. $p$ Stomachal stalk. $r$ Radial canal. $s l$ Its inner; $r s$ Its outer wall. $u k$ Cartilaginous ring. $v$ Ve.

lum. Z Tongue-like process (after E. Häckel). freely projecting diverticula, which, when much developed, may have the form of ruff-like folds. The radial canals form lamellar enlargements in the Geryonidre, when the generative matters are developed. In all forms the lower wall of the canal, or that placed away from the umbrella, forms the genital region (Fig. 35, g). The germinal matters in some cases reach the exterior through the stomacil, and in other cases by a rupture of the tissue.

In the Discophora the generative organs have always much the same relations, and differ but little in position and form. They consist of four or eight frills, curved in a semilunar form, and arranged in a rosette on the inner surface of the umbrella (cf. suprà, Fig. 44, ov) ; the frills are formed by diverticula of the gastrorascular system. 'They are placed in depressions on the lower surface of the disc, or hang freely down from it, often in numerous folds.

The Iuccrnaria hare the generative organs in the form of eight radially-arranged longitudinal ridges, placed on that part of the body which corresponds to the sub-umbrella of the Medusa, where they form projections into the pouches of the gastrovascular system. 'They represent, therefore, an intermediate form between the Hydromedusæ and Discophora. 


\section{$\S 97$.}

The generative organs of the Anthozoa are very similar in character. They are connected with the gastric cavity, so that the generative matters pass to the exterior through the gullet. The septa of the gastric spaces, or their ridges, which project into the central stomachal cavity, generally function as organs of this kind. In the Alcyonarians the sexual products are formed at the free edge of these processes, either in the stomach or at some distance from it, at the base of the gastric space; two septa are sterile. They are distinguished by the presence of the ridges mentioned above, ( $(93)$, which project some way forwards. The other ridges do not always carry sexual products, for in many Alcyonarians they are found on four only, or only on two of them. In the Actinia the generative products are formed within the gastric ridges. The same thing happens in the Antipatharia (Gerardia). The Madreporinæ, also, may be mentioned here, for in them the generative products are developed in the ridges, which project far into the base of the gastric cavity, and they there form a special process on each of the two surfaces of the ridges (Astroides calycularis).

The sexes are usually separated, and in different persons, but there may be hermaphrodites also (Cerianthus). In the colonial forms both diœcious and monœcious conditions have been observed; in others these characters vary very greatly (Corallium rubrum). When the persons of a colony are dimorphic, those which are the more developed are at the same time those which are functionally sexual, while the others are sterile. But in some Pennatulidie the persons without tentacles are the only ones in which there are generative organs (Virgularia mirabilis).

\section{$\S 98$.}

In the Ctenophora the peripheral portion of the gastric system represents the genital region. Creal diverticula are developed from the sides of the canals which run parallel with the rows of natatory lamellæ; in these semen or ova are formed. One side of a canal is beset with ovarian follicles, and the other

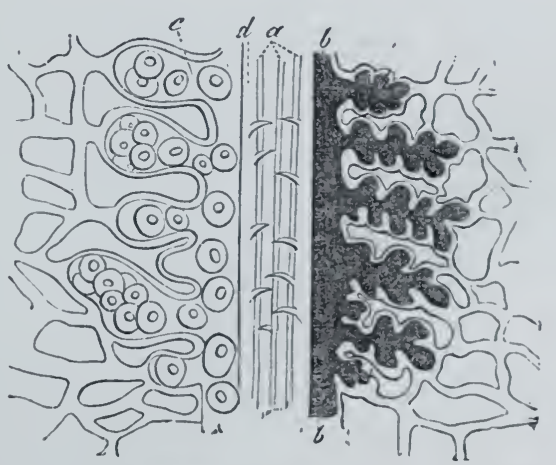

Fig. 50. Generative organs of Beroe rufescens; showing their relation to a tract of the radial canal. a Stripes running along the canal (Muscles?) $b$ Semen-producing side. $c$ Ovarian side, with eggs (after Will). with testicular lobules. Hermaphroditism repeats itself therefore in each of the radial segments of the body. The gastric system serves to carry the generative products to the exterior. This 
arrangement therefore is exactly the same as in some of the Anthozoa, and if we compare the body-substance between two radial canals with a septum of the Anthozoa, we find that the genital regions of both sexes are arranged in just the same way as in the hermaphrodite Anthozoa.

As a rule the ova of the Cœlenterata have no special coverings, and in many Spongix and Hydroïda (e.g. Hydra) they appear to change in form in consequence of amœboid movements. The seminal elements in the Acalephæ are formed by a small head with a movable appendage. 


\section{Third section.}

\section{Vermes.}

\section{General Review.}

$\S 99$.

In this division a large number of animal forms, which are more or less allied to one another, are put together; transverse axes are differentiated, whilst the longitudinal axis of the body is elaborated. In consequence of this an anterior and a posterior end can be made out, in addition to a dorsal and ventral surface. They differ markedly from the Colenterata in having two antimeres. The body is, or is not, divided into metameres; in the more simple forms the metameres are simple in character, in the higher divisions they undergo differentiation.

It is not certain whether this division does or does not represent a single phylum. 'The existence of a large number of small groups, represented merely by single forms, shows a considerable amount of divergence within the division; and this is still further exhibited by the fact that almost all the higher animal phyla can be brought into more or less close connection with forms of Vermes.

I arrange the various divisions of the Vermes in the following order. They might be considerably increased by the introduction of a large number of isolated genera; but a complete classification of such a kind is not part of our purpose here.

I. Platyhelminthes.

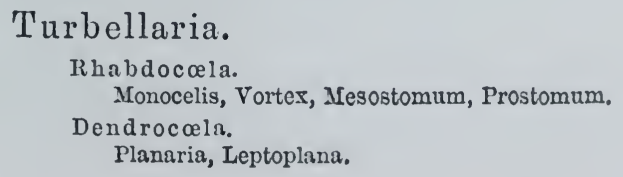


Trematoda.

Distoma, Monostomum, Tristoma, Polystomum, Aspidొgaster, Diplozoon, Gyrodactylus.

Cestoda.*

Caryophyllaus, Ligula, Bothryocephalus, Twuia, Tetrarhynchus.

Nemertina (Rhynchocola).

Pelagonemertes, Nemertes, Polia, Borlasia.

II. Nemathelminthes.

Nematodes.

Rhabditis, Dorylaimus, Strongylus, $\Delta$ scaris.

Gordiacea.

Gordius, Micrmis.

III. Chætognathi.†

Sagitta.

IV. Acanthocephali.

Echinorhynchus.

V. Bryozoa.

Phylactolæma.

Cristatella, Alcyonella, Lophopus, Plumatella.

Gymnolæma.

Crisia, Horncra, Alcyonidium, Flustra, Eschara.

VI. Rotatoria.

Hyclatina, Notommata, Brachionus, Mcliccrta, Floscularia.

VII. Enteropneusti.

Balanoglossus.

VIII. Gephyrea.\$

Inermes.

Sipunculus, Phascalosoma, Priapulus.

Chatiferi.

Echiuras, Boncllia.

IX. Annulata.\|

* Tho Cestoda are derired from a form common to them and to the Trematoda. The difference in organisation is due to their different kind of parasitic habits. There are several forms of which it is doubtful whether they belong to one or the other division (Amphiptyches).

+ The Chietognathi must not be regarded as allied to the Nemathelminthes because they are put next to them; tho same remark holds good for the Acanthocephali.

I Pedicellina and Lorosoma are genera allied to tho Bryozoa, and they might well be united with them into one division, but they must not be subordinated to them. fornis.

$\S$ In both divisions of tho Gephyrea there is a large number of very divergent

|| Tomonteris, Myzostoma, and Polygordius are special forms, allied to, but rery divergent from, the Annulata. The last mentioned unites the characters of the Nemertina and Nematodes with those of the Aumelides. 


\section{Hirudinea.*}

IIæmopis, Sanguisuga, Nephelis, Clepsine.

Annolides.

Oligochrta.

Scolcin

Lumbricus, Chætogastcr, Nais.

Haliscolecina.

Polyophthalmus, Capitella.

Chatopoda.

Vagantia.

Siphonostoma, Arcnicola, Glycera, Ncphtlyys, Phyllodoce, Alciopa, Syllis, Nercis, Eunice, Amphinome, Aphrodite, Polynoë.

Tubicolæ.

Amphitritc, Hcrmella, Terebclla, Sabclla, Scrpula, Branchiomma.

The position of the genera Neomenia and Chætoderma is not yet settled; but they must not be passed over, on account of the great importance of many points which have been made out in their organisation. Although they differ in not unimportant points from one another they are closely allied, and may be reckoned with the other divisions of the Vermes. I therefore unite them into a division, which I call that of the Solenogastres. It will not be possible to form any safe opinion as to their position till they are known more exactly; and for this knowledge, especially as regards their development, we must wait.

\section{Bibliography.}

V. BABR, Beiträge zur Kenntniss der niederen Thierc. N. A. Acad. Lcop. Carol. XIII. 1820., Dujardin, Histoire nat. des Helminthes. Paris, 1815. - VAN BBNEDEN, Mrémoiro sur les vers intestinaux. Paris, 1861.-Leuckart, R., Die mensehliehen Parasiten. Leipzig und Heidelberg. I. II. 1863-76.-CLAPARÈDE, Beobachtungen über Anatomie und Entwickelungsgeschiehto wirbelloser Thicre. Leipzig, 1863.

\section{ON THE SEPARATE CLASSES.}

Platyhelminthes: Degìs, Rccherches sur l'organisation ct les mœur's des Planaires. Amu. sc. nat. Sér. I. T. XV.; also Isis, 1830.-NoRDMrarv, A. v., Mierographischo Beitrüge zur Naturgesehichte der wirbeliosen Thiere. Erstes Heft. Berlin, 1832.-Quarrirages, A. de, Mémoiro sur quelques Planaires marines. Ann. sc. nat. Sér. III. T. IV.-The same, Sur la famillo des Némertines. Ib. T. VI.-Scнurdr, O., Die rhabdoeölen Strudelwürmer. Jena, 1818.-Tho same, Neue Beiträge zur Naturgeschichte der Würmer. Jcna, 1818.- The same, Ueber Rhabdocölen. Wiener Sitzungsbericht. Math. Naturw. Classe. Bd. IX. S. 23.-The same, Ueber Dendrocölen. Zeitsehr. f. w. Zoologie. X. XI.-VAN BBNBDEN, Les vers cestoides. Mémoires de l'Acadèmie de Bruxelles. XXV. 1850.-The same, Recherehes sur la faune littorale de Belgique, Turbellariés. Ib. XXII. 1860.-Levckart, R., MIesostomum Ehrenbergii. Arch. für Nat. 1852. S. 234. - ScrultzB, M., Bciträge zur Naturgesehichte der Turbellarien Greifswalcle. 1851. -The same, Ueber die Microstomeen. Arch. f. Nat., 1819. S. 250.-W WGENER, G., Die Entwickelung der Cestoden. N. A. L. C. T. XXIV. Suppl. 1851.-The same, Beiträge aur Entwiekelungsgeschichte der Eingeweidewürmer. Haarlem, 1857.-SrIBD A, Beitr. z. Anat. v. Bothryocephalus. Arch. f. Anat. u. Phys. 1861.-Soмmer and Lirdors, Beiträge z. Anat. d. Plattwürmer. Zeitsehr. f. wiss. Zool. 1872.-ScirseIDER, A., Ucber Platyhelminthen. Giessen, 1873

* I would place the genus Branchiobdella, which is counted as one of the Hirudinea with the Scoleina among the Annelides. There is nothing leech-like in the organisation of this worm except its sucker and jaws; and these parts are really structures which are due merely to its parasitic mode of life. 
-ZiLler, E., Uebcr Polyst. integer. z. f. w. Zool. XXII. p. 1. XXVII. p. 233.-The same, Ueber Leucoehlorid. paradox. Ib. XXIV. p. 564.-Soмmer, Ueber de Bau v. Tænia mediocanellata. Z. f. w. z. XXIV. p. 499.-MoseLEY, H. N., On the Anat. and Histol. of the Land Planarians of Ceylon. Trans. Royal Society. London, 1874.-M'Irrosh, C., Strueture of British Nemerteans. Edinb. Roy. Soe. XXV. II. p. 305.-Hubrechr, Unters üb. Nemertinen. Niederl. Areh. f. Zool. Bd. II.-GrafF, L., Zur Kenntniss der Turbellarien. Z. f. wiss. Zool. Bd. XXIV.

Nemathelminthes: Cloqurt, Anatomie des vers intestinaux. Paris, 1824.-EввRті, Untersuehungen über Nematorlen. Leipzig, 1863. - SchNEIDRR, Monographie der Nematoden. Berlin, 1866.-BAstiax, Monograph on the Anguillulidæ. Trans. Linn. Soe. vol. XXV. p. II. 1865-Grevicher, Zur Anat. der Gattung Gordius. Z. f. w. Z. XVIII. p. 322.-CLats, Uber Leptodera appendiculata. Marburg and Leipzig, 1869.-BüTscilı, Beitr. z. Kerıntn. der freilebcnden Nematoden. N. A. L. C. XXXVI.-The samc, Z. f. w. z. XXIV. p. 361.-The same, Abhandl. d. Senkenb. Ges. IX.

Chrotognatha: KRонк, Anatomiseh-physiol. Beolvachtungen iber die Sagitta bipunctata. Hamburg, 1811. - The same, Nachträgliehc Bemerkungen dazu. Arch. für Naturgesch. 1853.WILus, Observationes de Sagitta. Diss. Berol, 1816.

Bryozoa: VAN BENEDEx, Recherches sur l'anatomie, la physiologie et l'cmbryogénie des Bryozoaires. Mémoires de l'Acad. Royale de Belgique. 1815 et seq.-The same, Recherehes sur les Bryozoaires fluviatiles de Belgique. Ib. 1817. - The same and Druortier, Histoire naturelle des Polypes composés d'eau douce. Ib. 1850.-Allyax, A Monograph of the Fresh-water Polyzoa. London, 1856. (R.S.)-H. Niтsche, Beiträge zur Anat. und Entwickelungsgeschichte der phylaetolämen Süsswasserbryozoën. Arch. f. Anat. u. Phys. 1868. p. 465.- The same, Beit. z. Kenntn. d. Bryozoën. Zeitschr. f. wiss. Zool, XX. XXI. XXIV.-Vogr, C., Sur le Loxosoma. Arch, de Zool, Exp. Vol. V.

Rotatoria: EHRENBERG, Die Infusionthierchen, etc.-LExDiG, Zur Anat, u. Entwickelungsgeschichte der Lacinularia soeialis. Zeitschr. f. w. Zool. III. p. 452.-The same, Uber Bau und systemat. Stellung der Räderthiere. Ib. VI. p. 1.-HuXLEY, Quart. Journ. of Microsc. Sc. 1852.-CoHs, F., Zeitschr. f. w. Zool. VIII. p. 431. IX. p. 28t. XII. p. 197.

Enteropneusti: Kowalevsky, Mémoires de l'Académie de St. Pétersbourg. Sér. 7. T. X. No. 3.Agassiz, A., Mém. Amer. Acad. IX.

Gephyrea: Grube, Versuch einer Anatomie des Sipunculus nudus. Arch. f. A. u. Phys. 1837. p. 237.-Квон , Ueber Thalassema. Arch. f. Anat. u. Phys. 1812. - Qvatrefagrs, A. de, Mémoire sur l'Eehiure. Ann. sc. nat. 3 Sér. T. VII.-MüLLER, M., Observationes anatomiex de vermibus quibusdam maritimis. Berol. 1852. - SchMARDA, Zur' Naturgesehiehte der Adria. Wien. Denkschrift math. Naturw. Cl. Bd. 3. 1852,-LACAzE-Dithisks, H., Reeherehes sur la Bonellia. Ann. sc. nat. 4 Sér. T. X.-TheEL, H., Rech. sur les Phascolion. K. Vet. Acad. Handl. XIV.

Annulata : Audourn and Mruse-Edwards, Classifieation des Annelides et description des celles qui habitent les eôtes de la France. Ann. se. nat. 'T'. XXVII-XXX. 1832-33.-MrLXE-EDWARds' Artiele: Annelides, in Todd's Cyelopædia, I. 1835.-GRLBE, De Pleione earuneulata. Regiomonti, 1837. - The same, Zur Anatomie und Physiologie der Kiemenwürmer. Königsberg, 1838. - The same, Die Familien der Anneliden. Areh. für Naturgesch. 1850.-Qvatrefages, Etudes sur les types inférieures de l'embranchment des annélés. Ann. sc. nat. Sér. 3. Tomes X. XII. XIII. XIV. XVIII. 1828-52. (The results are given in the "Histoire nat. des Annélés" of the same Author.) - Lrxdig, Zur Anatomie von Pisicola geometriea. Zeitscher. für. w. Zoolog. I.Buснноцz, Beiträge zur Anat. der Gattung Enehytræus. Königsberger Physikal.-CEkonomisehe Sehriften. III. 1862.-CLAPARÈDE, Recherches anatomiques sur les Annélides, etc. Genève, 1861.-The same, Reeh. anat. sur les Oligoehétes. Geneve, 1862.-The same, Glanurcs zootomiques parmi les Annélides. Genève, 186t. -The same, Les Annélides Chétopodes du Golfe de Naples. Genève et Bâle, 1868. Supplement, 1870.-The same, Histol. Untersuchungen über $d$. Regenwurm. Zeitsch. f. wiss. Zool. XIX.-Perrier, E., Etudes sur l'organis, des Lombriciens terrestres. Arch, de Zool. III,-GrEeFF, A., Unters, über dic Alciopiden. N. Acta. Ac. Lcop. Carol. XXXIX.

Solenogastres: Tullberg, P., Neomenia, a new genus of invertebrate animals. Bihang till K. Svenska vet. acad. Handlinger III.-GRAfF, L., Anat. v. Chatoderma. nitid. Zeitsch, f. w. Zool. Bd. XXVI. -The same, Ueber Neomenia u. Chatoderma. Ib. Bd. XXVIII.

\section{Form of the Body.}

\section{$\$ 100$.}

The radiate form of body, which obtains in most Coelenterata, is never developed in the Vermes. It is replaced by the eudipleural form, which is generally known as that of bilateral symmetry (cf. suprà, p. 59). It predominates in all higher divisions of the Animal Kingdom. Although in some stages, as for example in the scolex-form of many Cestoda, this differentiation of the secondary 
axes is not expressed, so that similar characters to those which obtain in the Coelenterata can be made out, yet I do not regard this condition as one that has been inherited by the Cestoda, for they can only be derived from forms, which like the rest of the Platyhelminthes, possessed the original eudipleural form. Their condition, which depends on the equal development of the secondary axes, is at once explained by their loss of the power of locomotion, and by the attachment of the body by a point, which corresponds to one pole of the primary axis.

A head, which has a mouth placed as a rule somewhere on its ventral surface, can generally be distinguished at the oral pole. In most Platylelminthes the mouth is some distance from the head; in the Turbellaria, indeed, it is generally some way back on the ventral surface of the body. The aboral end of the body carries the anus; this, when present, has ordinarily a dorsal position.

In the fixed Vermes the form of the body undergoes considerable modifications. It is greatly influenced by the development of a covering, as in the Bryozoa. The aboral end of the body, by which the animal is attached, can no longer carry the anus, which is accordingly placed nearer to the anterior portion of the body, which is not enclosed by the cell.

\section{$\S 101$.}

Another phænomenon which is first seen among Vermes is the segmentation of the body. Already in the Rotatoria the hinder portion of the body is adapted to locomotion by being broken up into a number of segments. In this we see an indication of a condition which becomes very important in the higher divisions. In the Cestoda it is further developed. A differentiation is occasioned by the growth of the body in the direction of its primary axis. 'T'he anterior and posterior parts of the body no longer enclose the same organs. Thus in the Caryophyllæi the hinder portion of the body alone contains the generative organs. In Ligula this hinder portion of the body is considerably devcloped by the great repetition of the generative organs. In the Treniade a very large series of these generative organs are developed in the hinder end of the body, and each corresponding area forms a joint, which is gradually marked off on the outer surface, and has the rela-
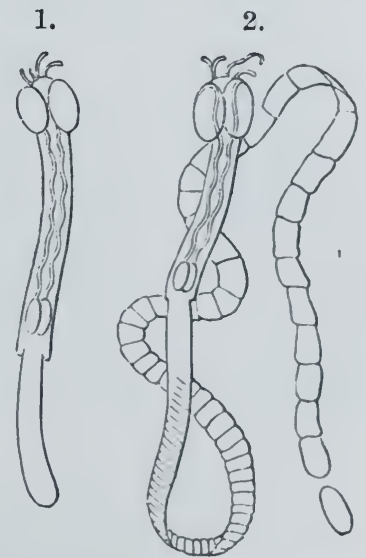

Fig. 51. 1. Tæunia (Tet. rarhynchus); asexual form (nurse). 2. The same in the joint-forming stage (strobila), in which the last joints (pro. glottids) are breaking off, one by one (after P.J.van Benerlen). tions of a metamere to the other joints (Fig. 51). In this way the Trnia-chain is formed, the last metameres of which (the so-called proglottids) break off at a certain 
stage of development, and form more or less independent individuals. This process represents therefore a process of gemmation, the product of which is the Tænia-chain; each separate joint is a metamere as compared with the general organism of the chain, but it is also to be regarded as a separate person, since it is capable of an independent existence, the slight duration of which is explained by its structure, which is adapted to a parasitic habit. The metamerism of the body, seen in the Cestoda, may be derived from a process of gemmation, and gemmation itsclf is correlated with the elongation of the body. It is an intermediate stage between the two phænomena; and there is, therefore, no well-defined antagonism between them. Where metamerism is faintly expressed, it becomes more and more nearly a case of simple elongation.

In many divisions we may find examples of this incomplete metamerism. It is indicated in various systems of organs in the Nemertina. In the Gephyrea also, metamerism is not a general phænomenon, for several systems of organs are not affected by it. On the other hand, it obtains generally in the Amnulata, and gives to the organism a multifid appearance. In them it is not unfrequently associated with a process of distinct gemmation. In the embryonic body there are generally fewer metameres than in the adult. The freshly-developed segments are formed in front of the last one. The elaboration of particular metameres gives rise to a large number of modifications. Such also result when a number of metameres undergo concrescence, and the primitive arrangement is only indicated by certain systems of organs; this gives rise to conditions which it is difficult to distinguish from those in which metamerism is just commencing. When metameres are developed the organism bccomes one of a higher grade of organisation, although indeed this is not the only path towards such a stage, for we meet with differentiations of other kinds which lead to higher conditions. The more definite differentiation of the ventral surface owing to the devclopment of a groove, as in the Solenogastres (in Chætoderma this is found in the posterior region of the body only) is an example of this; it represents the first stage in the formation of that pedal surface of the body which is seen in the lowest Mollusca.

\section{$\S 102$.}

There are various other modifications in certain smaller divisions, which are to be attributed to adaptations to changed external conditions of life; this is especially the case in the entoparasitic Platyhelminthes. "I'le "cystic form" must be regarded as the most important of these modifications; this, which is intercalated into the developmental history of the Cestoda, is, in its phylogenetic history, just as certainly due to the organism having entered into relations which at first were strange and abnormal to it, as is the general parasitism itself referable to habits, which were only secondarily acquired. The phylogenetic history of the cystic form 
is based on the notion that certain abnormal external conditions of life gradually became normal, in consequence of the adaptation of the organism to them, and that it did not arise by a simple antagonism to the worm's primitive ontogenesis, which now includes this cystic form as a normal part of its cycle. What has happened is this- that the process of adaptation has seized upon and exaggerated a normal inherited phase of the worm's ontogeny, and in virtue of the continuation of conditions favourable to the appearance of this exaggerated phase, its appearance has become a normal phænomenon. The variations of the cystic form are all readily deducible from the first developmental stage of the Cestoda. The embryo is generally provided with three pairs of hooks,

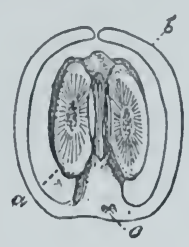

Fig. 52. Young Trnia, with head pushed in. $a$ Head. $b$ Envelope. $c$ The six embryonic hooks, remaining at one point of the envelope (after V. Sicbold).

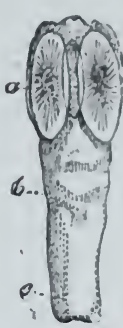

Fig. 53. $\quad \mathrm{Tl}_{\mathrm{ic}}$ sime Trenia, with head protrudec?. Letters as in Fig. 52 (after V. Sicbold). and a cestoid head may be observed to be differentiated within it (Fig. 52, a); when fully developed this is pushed out, so that the envelope, which at first was external, becomes the portion of the body below the head (Fig. 53, b). In the Cysticercus-form the embryo grows into a vesicle filled with fluid, from the walls of which the head is budded out. When the head is protracted, the vesicle forms a terminal appendage of the body (Fig. 54).

When a number of buds are formed on the wall of the vesicle, in which protractile heads are differentiated, we have the Coenurus-form. When the buds break off into the interior of the vesicle, and there form new vesicles, on the walls of which the same budding process goes on, leading to the formation of systems of vesicles, placed one within the other, and when the youngest of these can again bud off tænia-heads on its inner wall, we get the Echinococcus-form.

Notwithstanding the various characters of their final products, these processes of gemmation may be derived from a common ground-form. They are

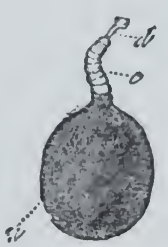

Fig. 5!. Cysticorcus cellu. losic; head pro. truded (nat. size). $a$ The caudal resicle, filled with fluid. $c$ The an. terior part of the body. $d$ The head (after V.Sicbold). byno means unparalleled among the Platyhelminthes, for in not a few an asexual multiplication occurs, which is very similar to these in many points. It is very common among the Trematoda, where the embryo gives rise to an asexual stage known as the "sporocyst." The body-parenchyma of this sporocyst becomes differentiated, generally into similar structures, in which in their turn the larvæ known as "Cercaria" "are produced, and these are developed into the sexually mature form. The variation in the forms of the separate generations seems to be due, in a general 
way, to the degenerations correlated with their parasitic habit, and in special points to their relations to their different hosts. Parasitisin, in fact, determines the whole phrenomenon in question, which is spoken of as "alternation of generations," but by no means replained by that phrase.

\section{$\S 103$.}

Gemmation is a common process among the Bryozoa also, where it leads to the formation of colonies. As in other Vermes and Coclenterata, the buds are developed from the wall of the body. Accordingly as the bud remains at the side of, and on the same level with, its mother, or grows at one end and raises itself from the ground, flattened or upwardly-growing ramified cormi are formed. At the edge of the flattened colony the youngest buds often form the rudiments of several individuals (persons), which are by-and-by separated from one another. We observe in the case of derelopment by gemmation as also in derelopment from the ovum, that the anterior portion of the body, which carries the crown of tentacles, develops inside the hinder portion of the body, which forms the cell round the animal. The proposal has therefore, though without reason, been made to regard the two portions as separate "individuals." All the persons of a Bryozoan colony are not equally well developed. In many, the parts belonging to the cells and muscles only are developed; these give rise to the so-called Avicularia, which function as prehensile organs for the colony. The Vibracularia, which are long spike-shaped structures, continually moving, are parts further modified. Iastly, some persons may serve only for the reception of ova, and form the so-called marsupial capsules. 'Thus we meet lere again with a polymorphism, which is due to a division of the pliysiological work of the colony.

\section{Appendages.}

$\S 104$.

The appendages have the form of actively mobile processes of the body, which inay be used for the most varied purposes, according to their relation to it, and their more special line of derelopment. As low down as the T'urbellaria processes are found on the portion of the body which represents the head. In many Planarians lateral lobate processes are developed as tentacles, or feelers; in others the dorsal surface of the body is distinguished by similar processes ('Thysanozoon).

While the parasitic mode of life of the 'l'rematoda, Cestoda, and many Nemathelminthes causes structures of this kind to disappear from them altogether, such structures are largely developed in the free-living Amnulata, and prove to us the great influence of the outer 
world on the organism. They are especially developed in the Chretopoda, the cephalic region of which is provided with contractile processes, either at the sides or in the middle line (Fig. $55, t t^{\prime}$ ). 'These processes are simple, or are further differentiated by segmentation, or even distinguished by the possession of secondary processes. By adaptation to the most varied conditions of life they are converted into very various structures, and serve for all kinds of functions.

In the tubicolous Chretopoda, where the cephalic region is that portion of the body which comes into closest relation with the surrounding medium, the tentacles are converted into an important apparatus. They form tufts of contractile filaments on the cephalic lobes, where they are arranged in one or more rows (Terebella [cf. infrà, Fig. 79, $t$, Hermella); or they may be converted into strong plume-like structures (branchial tentacles) by the development of an internal support (cartilage), and be beset with secondary branches; these, in addition to their respiratory function, may also aid in the movement of the whole apparatus when seizing food (Serpulacca). In some, these bran-

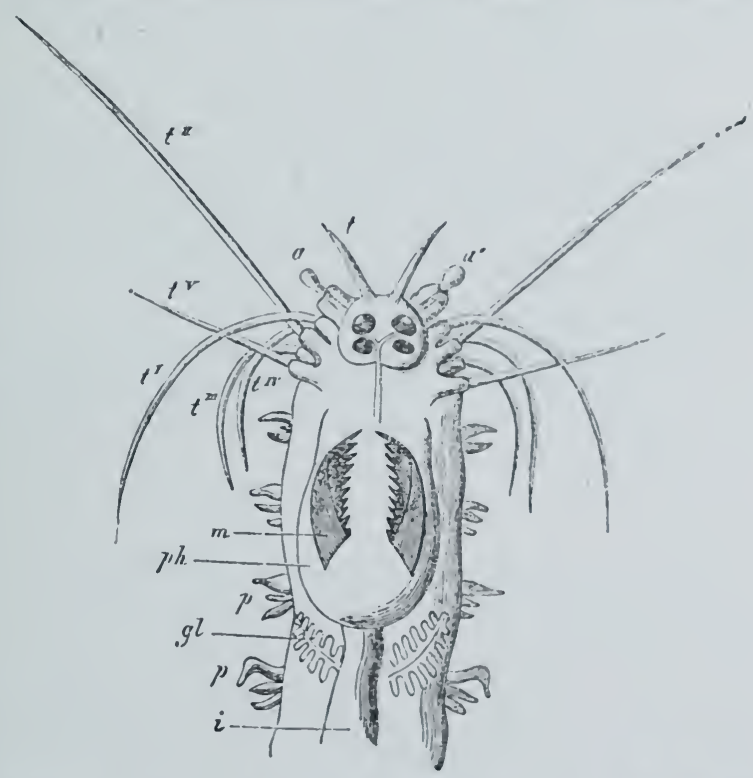

Fig. 55. Head of Nereis Dumerilii. $a a^{\prime}$ Tentacles. $t t^{i} t^{I I} t^{I I I} t^{I V} t^{V}$ Feclers. $p$ Parapodia. ph Pharyox. m Jaws. $i$ CEsophagus. gl Glands (after Claparede). chial tentacles are arranged in two groups, and resemble an open fan. In Siphonostoma they form short simple filaments, with two longer delicate feelers. In others the base of the two halves of the tuft, which are separated on the dorsal line, is drawn out into a spirally-coiled ridge, on which the separate filaments are arranged (Sabcllida). When optic organs are formed on the separate filaments of the branchial tufts, the tentacles acquire new and important relations (Branchiomma).

Sorne of the branchial filaments undergo other kinds of changes. In some Sabellida one or two of the primitively similar branchial tentacles (Protula) lose their respiratory function; in others they are converted into club-shaped organs, one of which is largely developed, and serves as an operculum to close the tube in which the animal 
lives. In Filigrana the stalk of the operculum retains some of its primitive characters by being feathered. But this feathered arrangement may be lost (Serpula), and then the operculum, during its derelopment, passes through stages which are permanent in other forms. A calcified layer is often secreted in this apparatus, which owes its origin to adaptation; it covers the free flattened end like a disc. In some cases the widened opercular stalk takes up the ova, and functions as a brooding pouch (Spirorbis spirillum). Thus we find one and the same organ passing through a series of the most varied relations, far removed from its original significance, and caused by certain external relations. In addition to the feelers there are special tentacles in the Chætopoda, which are shorter, but contractile (Fig. 55, a).

The tentacles of the Bryozoa are structures of this kind; they have the form of filamentous ciliated and contractile processes of a discoid or lobate extension of the oral end of the integument (lophophore). The discoid form of lophophore, in which the mouth is placed in the centre, is the most common. In the other case, the lophophore is drawn out into two processes, so as to have a horseshoe shape (Fig. 60, B br).

In Pedicellina and Loxosoma, the tentacles, which beset the edge of a discoid surface, which carries both mouth and anus, are simpler in character; they are not hollow internally, like the tentacles of the other Bryozoa.

\section{$\S 105$.}

Another group of appendages is represented by the locomotor processes developed in Chrtopoda, which are lateral processes of the metameres of the body, the foot-stumps or parapodia (Figs. $55,56, p)$. They are always arranged in pairs, of which there may be one or two on each segment. When there are two, one pair occupies the dorsal, and the other the ventral portion of the side of the body. They carry setæ, and often also filamentous appendages (cirri), which vary greatly in form, and may be larger than the parapodia, or may even take the place of these appendages, when the latter are atrophied. The dorsal and ventral appendages of either side are sometimes closely approximated; there are all kinds of intermediate steps between this stage, and that in which they are completely fused (Syllidx). Such a fused appendage occupies the side of the body, and carries the secondary appendages (setæ and cirri), which, in others, are distributed to the dorsal and ventral parapodia. The cirri appear to be atrophied in the Tubicolæ, where they cannot have any physiological significance, owing to the body occupying a tube, which has sometimes the form of a shell.

The parapodia are developed in very various degrees, and are complicated by their relation to groups of setæ. A metamorphosis is effected by a widening of the ends of the separate, or of fused parapodia, or rather of their cirri, to form swimming-plates (Phyllodocidæ). 'The elytra are special appendages of the parapodia 
formed by the metamorphosis of their dorsal cirri; they are scalelike lamellæ, which lie on one another on the dorsal surface, and alternate with short processes (Aphroditidæ). Although the parapodia of the Annelida, which function as locomotor organs, appear to be the rudiments of the appendages, which are more perfectly developed in the Arthropoda, they are not independent, for they have no special muscular apparatus of their own, like the appendages of the Arthropoda, and they are principally set in action by the general movement of the metameres to which they belong.

\section{External Branchiæ.}

$\S 106$.

The appendages on the head, as well as those on the metameres, of the Chrtopoda undergo various changes in adaptation to the respiratory function. Although in most Vermes this is performed by the general surface of the body, in the Chætopoda it is confined to definite parts, which are thereby converted into branchiæ, as may be seen from their relation to the vascular system, and from other points in their structure.

The cephalic tentacles are the first to enter into these respiratory relations $(\$ 104)$. In some (Pectinaria, Terebella) these structures contain a perienteric fluid, and are not distinctly branchiæ. They are more definitely branchial in the Pheruseidx (Siphonostoma). In the Sabellidæ they are still further differentiated in the manner described above, and the separate gill-filaments are beset with secondary pinnules, by which their surface is further increased in size.

Just as gills are formed by the special development of the cephalic tentacles, so, too, gills are formed from the appendages of the separate segments of the body, by the modifications of the cirri which are attached to the parapodia, or by the formation of special appendages. When simplest the cirri are not altered in character, but enclose a continuation of the cœlom, so that the perienteric fluid can enter into them. The presence of cilia on the cirri is also of importance for their respiratory functions. The exchange of gases is promoted by the walls of the cirri becoming considerably thinner at certain points. As a rule it is the dorsal appendages which are developed in this way. The so-called elytra of the Aphroditidæ also belong to this series of processes. They communicate freely with the cœlom. Cirri become more definitely related to the respiratory function by the continuation of the blood-vascular system into them. They then form branchiæ. These either retain the condition of simple processes-sometimes they are lamellar in form-or are variously branched. In Cirratulus they are greatly elongated single filaments. The branched form includes the more delicate branchiæ, which are either comb-like 
(Eunicidx, Fig. 56, A 13), or are arborescent (Fig. 82, br) (e. g. in the Amphinomer). As a dorsal cirrus is frequently present in addition to these branchiæ, they appear to be independent organs;

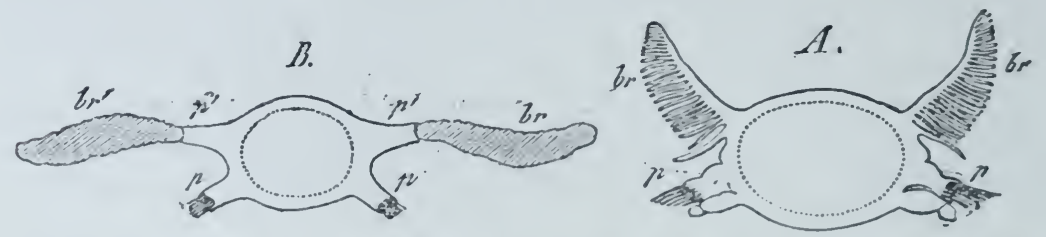

Fig. 56. Diagrams of vertical sections of Annulata, showing the appendages. $A$ Section of Eunice. $B$ of Myrianida. $p$ Nemopodium. $p$ 'Notopodium. Ur Brauchix. br' Cirri.

this is the more probable since they frequently are separated from the parapodia, and arise directly from the dorsal surface.

They are distributed in varying number over the body. They are sometimes found on all the segments of it, but they are generally less abundant at the tail (Eunice sanguinea, Amphinome). Sometimes they are limited to a number of segments and become. gradually rudimentary (Arenicola, Hermella). In the tubicolous forms, the mode of life leads to the development of the anterior, and the disappearance of the posterior gills. On the three anterior segments of the Terebellidxe there are branched branchial tufts (Fig. 79, $6 r$ ), in Pectinaria two comb-like branchix, and in Branchiosabella and Sabellides simple filiform appendages at the same point.

In other divisions, also, of the Vermes the respiratory function is assigned to processes of the body. This is true of the tentacles of the Bryozoa. In the Gephyrea there are special developments as respiratory processes; in Sternaspis the hinder end of the body carries vascular appendages. Finally, even in the Hirudinea there are lamellar extensions of the integument arranged metamerically (Branchellion).

\section{Integument.}

$\S 107$.

The integument of the Termes, which is separated off from the ectoderm, is closely mited to the muscular system, by which it is continued into the parenchyma of the body when a coelom is wanting. This obtains in most of the Platyhelminths (Flat-worms) and Hirndinea. Where the collom is present, the integument, with the muscles, forms a dermo-muscular tube, as in the Acanthocephali, Gephyrea, and most Annulata.

If we separate the dermo-muscular tube into its two constituen $t$ 
parts, we find the muscular portion to be, as a rule, the larger ; and the layer which corresponds to the true integument to be proportionately feebly developed.

The proper integument is formed, as a rule, of a layer of cells, the elements of which are often so slightly separated that they form a syncytium. 'This layer corresponds to an epidermis. In the 'Turbellaria it is everywhere provided with cilia. In many the cilia are placed on an apparently homogeneous layer, which resembles a cuticle. But the cilia must be regarded as processes of the cells. Even in those forms, such as the Cestoda, in which there are no cilia in the adult, there is an investment of cilia during the embryonic stages. The embryos of the 'Trematoda also have it. In many Annelids there are ciliated spots at various parts of the body, or large tracts may be clothed with cilia.

'The part that this investment of cilia plays in locomotion is best seen in the smallest forms. In the young state it is generally the sole organ of locomotion. By the growth of processes of the body the cilia-bearing surface is increased, and so the cilia become of greater importance in locomotion. 'This is their character in the larva of the Gephyrea and of most Annelids. The cilia are arranged on ridge-like processes, which surround certain tracts of the surface of the body, as lines or circlets of cilia; the arrangement of these is generally characteristic of the various divisions. One or more circlets of cilia surround the body; and by these the larva of the Chrotopoda are divided into mesotrochal, telotrochal, and polytrochal forms. Even if the surface of the body bears other cilia also, those of the circlets are more powerfully developed, and their action essentially aids in more rapid locomotion. Of these circlets of cilia (Fig. $57, C D v$ ) one is more remarkable than the rest, it appears
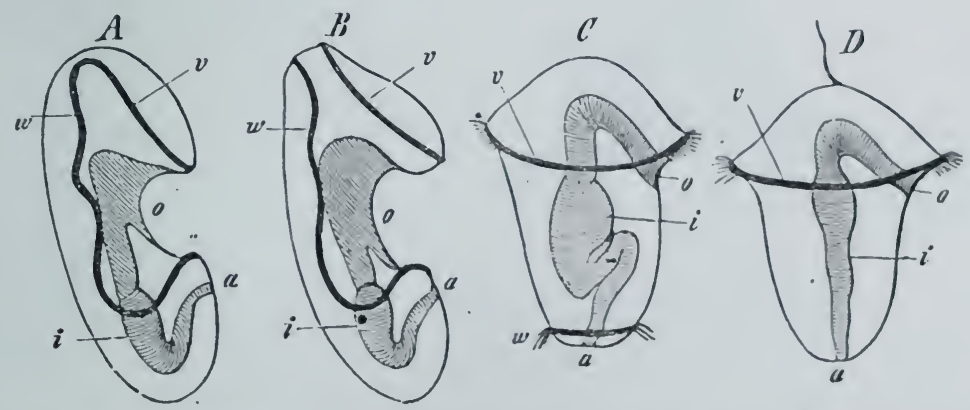

Fig. 57. Arrangement of the ciliated bands in the larr of the Echinoderma, $A B$, and of the Vermes, $C D$. $v$ Anterior; $v$ Posterior circlet of cilia. o Mouth. $i$ Enteric canal. a Anus.

in the very earliest stage, and divides the body into an anterior and a posterior portion. The former represents the upper part of the future head of the worm, while from the other portion the whole of the rest of the body is developed. The primitive circlet of cilin 
persists in one division of the Vermes, the Rotatoria. While the posterior portion is differentiated into a more or less jointed body, the anterior part, which carries long cilia on a discoid thickening, is developed into a special organ, which is characteristic of this division. This wheel-organ-so-called from the movement of its cilia-varies greatly in character. It may be either permanently simple, and retain its primitive state, or it may be broadened out into lobate processes ('Tubicolaria), or form tentacular prolongations (Stephanoceros), which frequently have a locomotor function in the larval stages only, and in the later fixed mode of life are used to bring food to the animal by means of the currents produced by the action of the cilia. In the Bryozoa, also, a circlet of cilia precedes the development of the tentacles, which are budded out internally to it. Owing to the position of the mouth, this circlet of tentacles does not resemble the more common form; but it nevertheless has close relations with the arrangements in some divisions, e.g. the Gephyrea, the larvæ of which have a circlet of cilia surrounding the oral region. Further, in Polygordius, which except in this point resembles the Nematodes, there is a circlet of cilia; so that we recognise in this circlet of cilia an arrangement which may have been transmitted

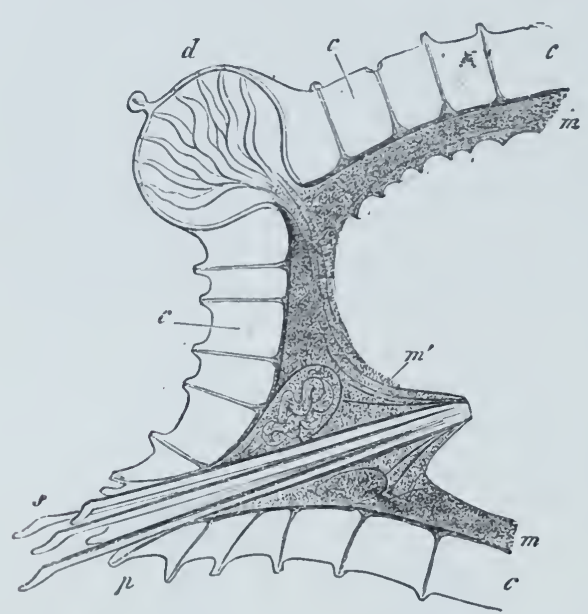

Fig. 58. Vertical section through the integument of an Annelid (Sphrodorum). c Thick cuticular layer with wide pore-canals. $m$ Muscular layer. $m$ 'Muscles for the tuft of setre, $s$, which occupies the ventral parapodium, $p$, while the dorsal one, $d$, is represented by a swelling, which contains glandular tubes. from an ancestral form common to many divisions of the Vermes.

\section{$\S 108$.}

When cilia are absent the epidermic layer is covered by a cuticle, which varies greatly in character, and is a product of the secretion of the epidermic cells. This cuticle is a thin or even a soft layer in the Trematoda and Cestoda among the Platyhelminthes. It has the same character in the Annelida, but in them it may be very greatly dereloped (Fig. 58, c). It is also present in the Acanthocephali. When this layer is thickened pore-canals may be seen in it. In the class of the Nemathelminthes it is very greatly developed, and is thicker than the subjacent matrix. Very often several layers, differing from one another, can be made out in it; they are formed of a substance which appears to be closely allied to chitin. When separate portions of the cuticular investment are very firm, a kind of dermal skeleton is formed in the Annulata, which is 
morphologically the same as the chitinous carapace of the Arthropoda, although it is not so hard.

The dermal carapace of the Rotatoria resembles completely the chitinous skeleton of the Arthropoda. Although it may not become as strong, yet the rigidity of tho most anterior segment, as well as of the succeeding ones, which are comnected together by softer intermediate pieces, gives to it the character of a true skeleton, which serves for the origin of muscles.

The cells of the Bryozoa are also cuticular structures; they are sometimes gelatinous (Lophopus crystallinus), soft and flexible; sometimes, owing to calcareous deposits, they are much harder. The latter kind are found in most of the Gymnolæmata. They differ from the tubes of many Rotatoria, and of tubicolous Annelids, by their close connection with the body; whereas in Rotatoria and Annelids the tubes are formed by a secretion which is detached from the surface of the body. But the fact that in many Rotatoria the body-wall loses its connection with the hinder portion of the tube, shows that there is no well-defined boundary between these structures. We see, in fact, that there are intermediate steps between typical cuticular structures and other secretions, which are ordinarily, though wrongly, grouped in contrast with them.

The firm cell of the Bryozoon is not developed over the whole body. It only surrounds the hinder portion, and is continued into a more delicate chitinous layer, which invests the anterior tentaclebearing portion, but is frequently wanting. This difference in the differentiation of the integument leads to a difference in the power of movement of these two regions of the body, and allows the anterior portion to be retracted, and to hide itself and its crown of tentacles in the hinder or cell-bearing portion. There are various differentiations of the cell, which, more or less, bring this relation of the parts to a state of perfection.

\section{$\S 109$.}

Those special structures, the aciculi, setæ, hooks, and so on, which often play an important part in the economy of the animal, must be regarded as differentiations of the integument, of the class of cuticular formations. The structures in question are most extraordinarily varied in character, and may be divided into two groups, according to their relations to the surface of the body. In the first group they have the character of simple elevations of the integument. A thicker cuticular layer is formed on papilliform processes, which may take the form of a wart, or when elongated, of a hair or seta. Even when it is very firm, it is still only apparently an independent structure, for it is nothing more than a modification of the cuticle, into which it passes at its base. The firm papillæ and aciculi which are found in the skin of many Trematoda, and sometimes variously extended over the anterior region of the body, are organs of this kind. So too are the fine and closely approximated aciculi, which cover the body of the Solenogastres as far as the 
ventral groove; the aciculi of the Echinorhynchi, and lastly, the hooks of the Cestoda, which are in many arranged in a crown (Figs. 59, 60), or placed in the wall of four protractile tubes

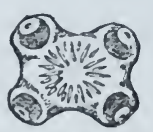

Fig. 59. Head of 'Tænia cœnurus (vesicle-form: Cœenu. rus cerebralis seen from in front). The four suckers and the crown of hooks in the milst of them can be seen.

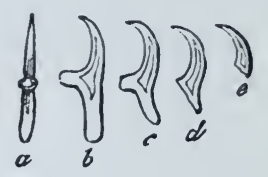

Fig. 60. $a b c d e$ Different hooks from the crown of hooks of the same form. Representing stages of derelopment (after V. Siebold). (Tetrarhynchus). These begin as thickenings of the cuticle, but they form an intermediate step towards the second group, for as they become chitinised they sink down into tho matrix, and still deeper.

In the second group the setæ or aciculi no longer arise on the surface, but in special depressions, which may be very well compared with glands. The secretion is formed from one or more cells, and gets a definite form as it becomes gradually chitinised; varying in different regions of the body. As a rule setæ are first formed when metameres are. These structures vary greatly in size and form, and are very different in the various genera and species. With the exception of the Hirudinea they are found in all the Annulata. 'They are almost always arranged in tufts (cf. Fig. 58, s), two or four of which are connected with the parapodia of each metamere. They function partly as locomotor organs, working like oars in the swimming forms (Vagantes); when they are metamorplosed into hooks they may serve as seizing or clenching organs (Tubicolæ). They are best developed in the Aphroditidæ, where some of the finer setw form a felted layer, which covers the back and elytra.

The "rod-like bodies" in the integument of the Turbellaria are special structures, as are the similar structures in the Annelides; in many cases they call to mind the "urticating capsules of the Acalephæ."

\section{$\S 110$.}

An organ, the function of which is still somewhat uncertain, belongs to the category of differentiations of the integument; this is the so-called proboscis of the Nemertina. It forms a tube, which is enclosed in a special sheath, placed above the enteron, and is often coiled; this tube opens in the anterior part of the body above the mouth, whence it can be protruded by eversion. Several divisions can be made out in this tube, one of which has stylets at its base-generally a larger stylet in the middle, and at eacl side several smaller ones in special pouches, which are sometimes regarded as reserve stylets, and sometimes as structures of a supernumerary character. The portion of the tube behind the stylets is glandular in character, and is provided with an excretory duct, which is placed close to the stylet. A muscle which arises 
from the body-wall is attached to the blind end of the tube; it is to be regarded as a retractor. In many Nemertina (Lineus, Nemertes, etc.) the stylets are absent.

In some the tube is small (Polia involuta), and so far resembles the structures in other Platyhelminthes, which may perhaps be regarded as the first stage towards the lighly differentiated proboscis of the Nemertina. Such aro the stylets present at the anterior end of the body of the Cercarix, which serve for boring, and are placed either on the surface, or at the bottom of a deep follicular depression. Lateral stylets having the same relation to the median one as in the proboscis of the Nemertina are often observed; so that we may conclude that primitively there was the same organisation in this respect in a large division of the Platyhelminthes. Even in certain Nemathelminthes we find similar arrangements, so that we have here to do with a wide distribution of similar characters. In some this arrangement is only found in the young stages, and disappears in the adult organism (Trematoda); in other's it not only persists, but is connected with important differentiations (Nemertina).

\section{$\S 111$.}

The integument of the Vermes attains to a higher position through the differentiation of glands as special organs of secretion. Organs of this kind have been recognised in nearly all the divisions of the Vermes, and are very common among the Annulata. In most cases they appear to be unicellular, and sometimes lic immediately beneath the integument, and sometimes, when a distinct cœlom is wanting, in the deeper parts of the body.

Among the Platyhelminthes, unicellular dermal glands are known in the Trematoda. They are generally placed in groups in the anterior part of the body, and in the hinder part also, where they are connected with the suckers. The glands are greatly developed in the Hirudinea, and especially in the Blood-leeches, where thicy are scattered in the parenchyma of the body, and open on to the skin by long ducts. They appear to be developed in relation to the generative function. Unicellular glands are also present in the integument of the Scoleina, where they are placed between the cells of the matrix. In many cases the glands take up a deeper position, and their ducts, only, pass between the epidermic cells.

In the Gephyrea tubular glands also are connected with the integument, and tubes are also found in the Annclides (Fig. 58, $九$ ). A glandular layer is developed on one portion of the body of the Lumbricida, as a clitellus; but this organ does not appear to be so simple in structure, for the tubes are invested by a special epithelium, and are sometimes lobate in form. Glandular tubes, contaming masses of rod-shaped bodies, are very common among the Chrotopoda (Spio, Aricia). In the Nemertina there are also glands which secrete a viscous fluid. In many cases the secretion of the dermal glands is used to form an investment for the ova. 


\section{Skeleton.}

\section{$\S 112$.}

When somewhat firmer than usual the integument in many divisions of the Vermes plays an important part as an organ of support; these relations have been already referred to. Organs which possess this function, without any subsidiary relations, are more worthy of consideration. Supporting organs of this kind are seen in the cartilaginous pieces in the cephalic segment of a number of tubicolous Annelides; from these pieces processes pass out and ramify, as fine bands, in the feather-like plates. This is to be regarded as the formation of an internal skeleton, but it presents analogies only to other similar arrangements.

This also holds for the branchial skeleton of the Enteropneusti, which is made up of a lattice-work of homogeneous rods (cuticular structures). Its arrangement and development call to mind the branchial skeleton of the lowest Vertebrata (Amphioxus), but it cannot be said to have any very close relations to it.

\section{Muscular System.}

$\S 113$.

The muscular system of the Vermes is connected with the integument, and forms in most of them the largest part of the covering of the internal organs. In some it is only slightly developed. The general arrangement of the fibres follows one of several types, which may be thus characterised:

1) Circular, longitudinal, and radial fibres form a connected mass of muscle, in which the two former are separated into layers, and are traversed by the radial fibres. The circular fibres form an outer and an inner layer, between which the longitudinal fibres are placed. The radial fibres run from the interior of the body to the surface. At the lateral edges of the body they pass directly from the dorsal to the ventral surface. This arrangement of the muscles is found in the Platyhelminthes and Hirudinea. In addition to these muscles there are fibres which run obliquely ; but they are not present in the Nemathelminthes nor in the Turbellaria rhabdocola.

2) 'The longitudinal fibrous layer is alone present. This is the case in the Nematodes, Chætognathi, and in Polygordius. The longitudinal muscles are distributed in various ways. The muscular fibres either pass directly below the epidermal layer (matrix of the cuticle) in the form of flat bands, the broad sides of which are approximated, or they have their edges approximated, and their 
surfaces therefore directed inwards and outwards. In either case the muscles are grouped in particular ways. They aro separated into two lateral masses by a dorsal and median line, formed by other tissues; and these masses consist of fibres lying directly on one another (Gordius, Trichocephalus). In the majority of Nemathelminthes there is a further differentiation, duo to the interposition of other organs at each side of the dermo-muscular tube. This lateral line (Fig. 61, $A r$ ) is, in very many Nematodes,
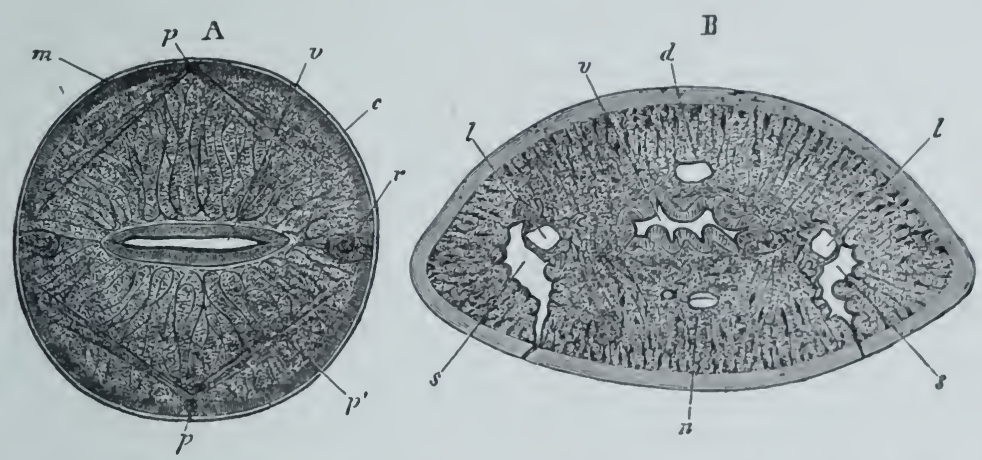

Fig. 61. Transverse section of Ascaris lumbricoides, $A$, and of Hirudo, $B$ $c$ Cuticular layer. $m$ Muscular layer. $r$ Lateral line with the excretory organ. $p p$ Upper and lower median line. $p^{\prime}$ Oblique fibres. $v$ Enteron. $d$ Dorsal. $l$ Lateral vascular trunk. $s$ Vesicle of the excretory organ. $n$ Ventral nerve-chord.

enlarged into a lateral tract, which is more or less developed; it is present in the Chætognathi also.

3) The muscular system of the body consists of a layer of external circular, and internal longitudinal fibres. Neither are separated into distinct tracts in the Gephyrea, and Acanthocephali, although in the former the separate longitudinal or transverse muscular bands are frequently placed at some distance from ono another. On the other hand, the Annelides, owing to the arrangement of the longitudinal fibres into two dorsal and two ventral layers, have a distinct lateral field or groove; the longitudinal layer is the thicker. A layer of transverse fibres, generally represented by distinct bundles, passes from the ventral median line to the lateral grooves.

In addition to these muscles, which are present throughout the body, there are also separate muscles for special organs. We need only mention here the muscles which move the bundles of setæ, and which are probably nothing more than fibres separated from the muscular mass, which extends over the whole body.

The suckers found in the Trematoda, Cestoda, and Hirudinea, are special differentiations of the dermo-muscular tube, which agree with one another in all the essential points of their structure. 


\section{$\S 114$.}

The muscular system of the Bryozoa consists of an external layer of circular, and an internal layer of longitudinal fibres (Phylactolæma). The circular layer is frequently separated into distinct bands. The muscles which connect the protractile portion of the body with the cell are the best developed. When the walls of the cell are very strong the circular bands are separated (Flustra), and form bundles which pass from the side walls of the cell to its superior free edge. Some of these are inserted into the portion of the cell, which functions as an operculum. When longitudinal muscles are present, some of the muscular fibres are separated off behind the invaginated portion of the body, and pass inwards to the duplicature of the body-wall, whence most of them are produced on to the base of the tentacles. They form the retractors of the anterior part of the body (parieto-vaginal muscles).

The Vermes differ considerably from one another in the structure of the form-elements of their muscular system. The muscular fibres are more or less elongated structures, which as a rule are the product of a single cell, even where they are very long, as may be inferred from the presence of a single nucleus. The lower forms of the Platyhelminthes have pale fibres often difficult to make out, which may be branched. In the higher Platyhelminthes they form tubes, the contractile substance forming a hollow cylinder, which contains indifferent protoplasm and the nucleus. The contractile portion of the fibres sometimes presents a fibrillar striation. This is seen in the Hirudinea, Acanthocephali, and Gephyrea. In the last two of these divisions the fibres of each layer form a network.

Among the Nemathelminthes the simplest condition is seen in Gordius. The muscular fibres are broad thin bands, with their surfaces applied to one another. In other's, special differentiations of the fibres may be seen forming rhomboidal plates, which are frequently continued into elongated fibres. The contractile substance is fibrillated and striated, and lies on the outer side of the fibres, while the portion of the fibre directed towards the cœlom is formed of protoplasm, which remains indifferent, and encloses a nucleus. With this are allied the special metamorphoses of the fibres into canalicular, or -flattened cylindrical forms. Each fibre has a very deep groove; this it either retains for its whole length, or it becomes cylindrical towards its ends ; its open part being always directed towards the body-cavity. 'The walls consist of contractile substance, broken up into fibrilla. Protoplasm fills the small space of the groove, and a delicate membrane is produced from the edges into a pouch-shaped organ, which projects from each muscular fibre into the body-cavity, the greater part of which is filled up by these pouch-like appendages of the muscular fibres (Ascaris lumbricoides, Fig. 61,1$)$. From the pouches, oblique fibres run to the median lines; they often have a fibrillar character, and hare been 
regarded as nerves. In some parts they exist as distinctly muscular fibrillæ. If the pouch is not developed, these fibres are attached to processes of the muscular fibres, which often become converted into tubes flattened at the sides. Both these conditions are found not only in the same genera, but even in the same individual, where they gradually pass into one another. In the last-mentioned form of the muscle-cells, a large number of fibres are generally placed closely side by side in the muscle-tube. 'The muscular fibres of the Chrotognathi are distinctly striated transversely; there are traces of such striation in many other Vermes.

\section{Nervous System.}

\section{$\S 115$.}

The close relation between the nervous system and the general organisation is shown by the general arrangement of this system. The centres and peripheral parts are simple when the body is not divided into metameres; while, when the body is segmented, metamerism is exhibited with the greatest regularity in the central organs of the nervous system. In all worms the most important central organs of the nervous system are placed in the anterior part of the body, and generally near the commencement of the alimentary canal. A development of the nervous tissue from the ectoderm has been made out in several divisions at least. The central organ above the foregut is the most primitive portion of the nervous system, whatever modifications it may present. When a head is separated off it lies in it, and always innervates the sensory organs that are developed in the head; it varies in the degree of its development with these organs. Nervetrunks, radiating thence to the periphery of the body, appear in various degrees of elaboration, in proportion to the extent of the area of their distribution. Two different conditions may be developed from this arrangement. The first consists in the ventral connection of the superior central organs. This gives rise to an œsophageal nerve-ring. The second is distinguished by the development of two longitudinal trunks, which approach one another

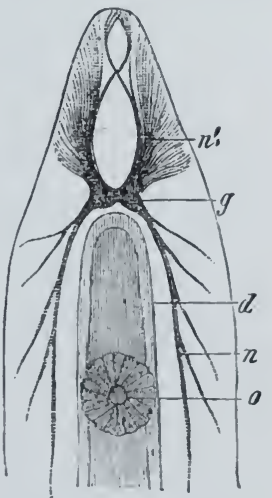

Fig. 62. Anterior portion of the body of Mesostom nu E h ren bergii.

$g$ Cerebral ganglia. $n$ Lateral nerres. $n$ ' Nerres to the anterior end of the body. d Enteron. o Mouth surrounded by a sucker (after L. Graff). on the ventral surface, and have central elements placed in them.

The primitive form of the nervous system is retained in most of the Platyhelminthes, since they possess two large ganglionic masses, connected by a transverse commissure in the anterior region of the body. These "cerebral ganglia" (Fig. 62, g), with the two 
longitudinal nerve-trunks $(n)$ which pass out from them, form the principal portion of the nervous system; from it finer branches pass off to the sensory organs of the integument $\left(n^{\prime}\right)$, to the dermomuscular tube, and to the internal organs. The longitudinal trunks pass along the lateral edges of the body, and are placed closer together, or are more widely separated from one another, according to the breadth of the body. In the dendrocœlous Turbellaria, as well as in many Trematoda, these lateral longitudinal trunks are only slightly developed, so that it is difficult to separate them from the other nerves, which arise from the cerebral ganglia, although they are not unfrequently distinguished from the other nerves by their larger size.

The Rotatoria come nearest to the Platyhelminthes. The central organ is a ganglionic mass lying on, but never surrounding, the œsophagus. In some it is distinctly separated into two lateral halves. The peripheral nerves arise from this cerebrum; and as they are not collected into longitudinal trunks, the simplest form, which is most like to that of the Turbellaria, obtains in this group.

The nervous system of Pedicellina appears to be of this low grade, for it is placed on the stomach, and does not form an œsophageal ring. It is not quite certain whether or no the ganglionic masses, which lie on the osophagus in Echinoderes, are separated from one another. If there is a dorsal commissure the arrangement would be similar to that in the lower Platyhelminthes.

The nervous system of the Bryozoa is more highly developed; its single central mass is a simple ganglionic swelling, lying between the mouth and anus, and sending out, in addition to large branches for the tentacles, two nerves which form a ring around the commencement of the œsophagus. Where the nervous system is most exactly known, as in Alcyonella, there is no doubt about the œsophageal ring. From the lateral part of the central nerve-mass a lobate process goes to the lophophore, and, like the rest of the œsophageal ring, gives off nerves to the tentacles.

In addition to this nerrous system in each individual, a colonial nervous system has been recognised in the stock, but it is not quite certain that this system does exist.

\section{$\$ 116$.}

The nerrous system of the Nemathelminthes appears to be arranged in a special manner, so far as the facts about it are agreed upon. It consists of a central organ placed on the œsophagus, and surrounding it as a ring, from which nerves radiate forwards, as well as backwards. This distribution of the nerves corresponds to the arrangement of the ganglionic cells of the œsophageal ring. The nerves which run forward from it may be separated into six fibrous bundles. Two run in the middle of the lateral tracts, and four in the direction of the secondary median lines. Ganglionic cells lie both on their origin and their course. The nerves which pass back- 
wards consist of a dorsal and a ventral trunk, which pass along the corresponding median lines. In addition to these, two chords arise from the ventral portion of the osophageal ring, and, converging posteriorly, unite to form a mass of ganglionic cells (G. cephalicum). The median nerves run along the whole length of the body. Both send fibres into the matrix of the integument. It is clear that this arrangement is a modification, speaking generally, of the simple conditions of the nervous system of other Vermes; but it is so peculiar that any special comparison is altogether impossible.

The same holds for the nervous system of the Acanthocephali. A small "ganglion" placed at the base of the sheath of the proboscis gives off branches anteriorly as well as posteriorly. Its relation to the dorsal central organ of other Vermes is obscure, as it is placed between the bundles of the ventral retractors of the sheath of the proboscis.

\section{$\S 117$.}

In the second form of the nervous system two longitudinal trunks are predominant ; these arise from the cerebral ganglia, and pass backwards. This arrangement is first seen in the Nemertina, and is directly related to what obtains in the Turbellaria, in which there are often two greatly developed longitudinal nerves passing backwards. The size of these two peripheral longitudinal trunks is dependent on the length of the body. As there are ganglionic cells in them, they are not exclusively peripheral organs. The cerebrum, too, in the Nemertina is more largely developed, for several large segments can be made out in each of the two ganglia. The commissure between the two halves is traversed by the organ

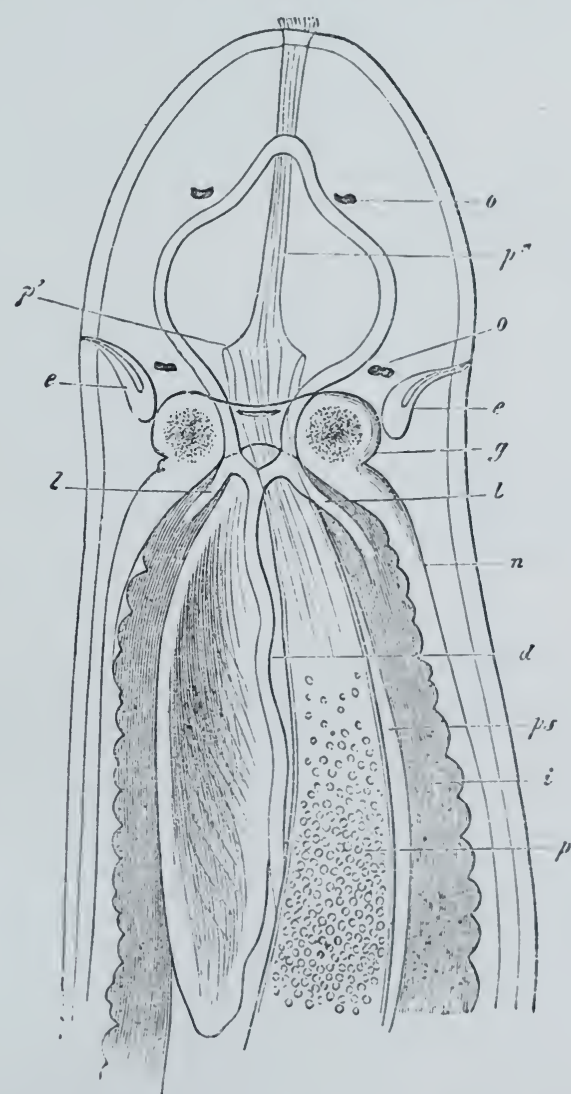

Fig. 63. Head of a Nemertine (Omma. toplea alba). $g$ Central nervous system. $n$ Lateral trunks. o Eye-spot. $p p^{\prime} p^{\prime \prime}$ Pro. boscis. $p s$ Its sheath. $i$ Enteron. $e$ Lateral organ. d Dorsal vascular trunk. l Lateral vascular trunk (after Carm. M'Intosh). which we have already described above as the proboscis. Although in most of them the longitudinal trunks (Fig. $63, n$ ) run exactly along the lateral edge of 
the body (embedded within the muscular layers), in others (Erstedia) they approach one another ventrally, and are distinguished by swellings at the points where nerve-branches are given off. This is an anticipation of the future development of ventral ganglia, the elements of which are already present in the longitudinal trunks. The ventral approximation of the longitudinal trunks shows us how the central system got its rentral position, which becomes further developed by the formation of ganglia. But the ventral approximation of the longitudinal trunks, by surrounding the œesophagus, leads to the formation of an osophageal nerre-ring, when the rentral longitudinal trunks meet. It is an open question whether the osophageal ring of the Bryozoa, and of the Nemathelminthes arose in such a way as this, and we would only just remark that even if they had a similar origin it does not follow that there is any connection between them and the cesophageal nerve-ring of other worms. For in the Nemertina we see that the origin of this arrangement is due to the two peripheral longitudinal trunks, which are not present in the other case.

In the Nemertina the osophageal ring is not closed; in the Annulata it is closed by transverse connections between the primitive longitudinal trunks. These have gained a central signification, owing to the large number of ganglion cells in them; the longitudinal trunks now appear to commence as commissures, which connect the primitive dorsal nerve-centre (cerebrum) abore the œsophagus with the ventral one, which is formed from the longitudinal trunks.

\section{$\S 118$.}

The cerebral ganglionic mass is not always present in the Gephyrea. It is well marked in Sipunculus and Sternaspis, and in the former it is divided into two parts. In Bonellia and Priapulus, however, only fibrous elements surround the œsophagus, so that in comparison with the other two a change has occurred, in which the central elements must be supposed to have become degenerated, or to have taken on a ventral position. The consequent arrangement corresponds to a great development of the commissure, which was formerly present between the two halves of the superior ganglia. Instead of the two rentral longitudinal trunks there is a single nerve-chord, in which the fusion of two trunks is only a matter of inference. This ventral chord generally lies within the cœlom, but in some it is placed outside the muscular layer, just below the integument (Priapulus).

As a rule, there are no collections of ganglion cells into special swellings, or expressions of metamerism; it is only in Echiurus that they are present, and in it they are but feebly dereloped; in other cases (Sipunculus, Sternaspis) there is a terminal thickening, of the chord which gives off fine filaments.

The ventral chord gives off filaments on either side, which are frequently irregular in origin; they are the peripheral nerres. 
Similar nerves are given off from the œsophageal ring to the alimentary canal.

The concrescence of two separate structures in the ventral chord of the Gephyrea is the reverse of the permanent separation of the two halves of the ventral chord, which obtains in other divisions of the Annulata. It would not, however, be safe to regard these stages as lower ones, until observation shall have shown, which it has not done yet, whether or no they are preceded by an earlier stage, like that in the Gephyrea. The connection of two separate ventral chords by means of transverse commissures would be more easily explicable if the ventral chord were previously single.

The nervous system of Sagitta has a peculiar character. Lateral commissures from the cerebral ganglion in the head, pass backwards and downwards to the ventral surface of the body, and pass into a large ventral ganglion, which lies just below the integument; and gives off peripheral nerves to all sides.

\section{$\S 119$.}

A higher grade of differentiation is seen in the nervous system of the Hirudinea and Annelides. The cerebral ganglia are connected by commissures with a ventral chord, and so far these groups resemble the Gephyrea. In many Annelids the two halves of the ventral chord are homogeneous, and only indicate their metameric character by giving off nerves. In most, however, there are centralform-elements regularly distributed along it. The ventral chord then appears to be broken up into separate ganglia, which are connected with one another by longitudinal commissures. Kach ganglion, again, is broken up more or less regularly into two halves, which are connected together by transverse commissures. The two ventral chords then form a chain of ventral ganglia (Fig. 61). In many Hirudinea the longitudinal chords of the ventral medulla are, during the early stages, separated from one another. Later on they are placed very close to one another, and almost seem to be a single chord. In this case, therefore, the separation of the chords must be regarded as the more primitive condition. The longitudinal chords are still closer in the Scoleina, and in the Nereidæ, Amphinomidæ, and Eunicer among the Chrto-

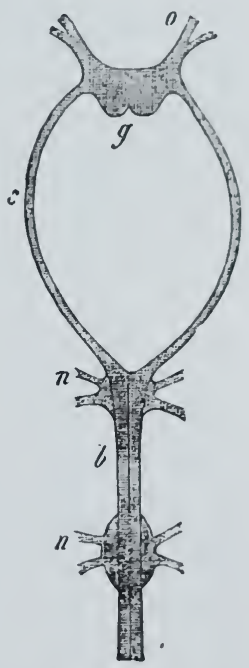

Fig. 61. Anterior portion of the ner. vous system of Capitella capilata. g Cerebral ganglion. o Optic nerves. $c$ Esophageal commissure. $b$ Ventral chord, with two ganglia. $n$ Nerves passing off from them (after Claparède). poda; but in all these cases there is not a real fusion, but only a close approximation, which appears to be still closer on account of the connective tissue investing the two nerve-chords.

In the tubicolous Annelids the ganglia-bearing longitudinal trunks are separate; in the Serpulidæe especially, the lateral portions 
of the ganglionic chain are widely separated from each other anteriorly. In the Sabellidæe and Hermellide the chords are closer together, and indeed in the anterior portion of the ventral nervechord the transverse commissures are much shorter than in the posterior. Finally, the Terebellidæ close the series, for in them the transverse commissures between the ganglia are distinct in the posterior portion only, while in the anterior portion the ganglia of either side are completely fused.

With regard to the ganglia, the perfection and greater development of the cerebral ganglia in Hirudinea and Annelides must be mentioned as contrasting with the lower Vermes. The two halves are very seldom united into a single mass; in Enchytræus, this condition appears to be due to degeneration. A breaking-up into distinct lobate segments, of which the Nemertina present a simple case, may give rise to a great diversity in form. 'The lobes frequently have the form of rounded projections, at times almost pedunculated. The cerebral ganglia are then complexes of smaller ganglia.

In the ganglia of the ventral chord also, remarkable differentiations may arise, partly from an increase in size, and partly from concrescence. In the Hirudinea, the first ganglion is generally very large, and always larger than the rest; it corresponds to a large number of separate ganglia united with one another, as may be seen from the segments which compose it, as well as from the nervebranches which arise from it. A similar condition is found at the end of the ventral chord, where the larger ganglion present, and innervating the sucker, is produced by the concrescence of several primitive ganglia (seven in Clepsine), which corresponds to as many metameres as form the sucker. This phænomenon of the approximation (by shortening of the longitudinal commissures) of separate ganglia obtains also in the Scoleina, but here the independence of the parts is often clearly recognisable, owing to the presence of separate transverse commissures. The Hermellidie among the Chætopoda are an example of this, for in them the first seven ganglia on each side are in direct contact with one another. 'The length of the commissures, as well as the number of ganglia, is directly connected with metamerism. In the Lumbricidie with small rings they are very close together, so that the whole ventral chord presents a compact series of swelling's and constrictions. The ganglia in Clymene and Cirratulus are still closer together.

Orving to this close association of the ganglionic structures of the ventral cliord, it has been supposed to be analogous to the spinal chord of the Vertebrata. And the rentral ganglionic chain has therefore been called the "ventral medulla." Although we may allow the analogy, there is no reason at all for supposing that there is any homology. Position, development, and structure forbid such a supposition. As to structure, let it be here noted that the ganglion cells in the ventral chord are in the periphery of the ganglia, while the inner part is essentially occupied by fibrous bands. 


\section{$\S 120$.}

The cerebral ganglia give off, chiefly, the nerves of the higher senses, and are developed in different degrees, according to the perfection of these latter. The tentacular nerves, and those of the organs of sight, deserve to be particularly noticed (Fig. 64, o).

'The nerves which rise from the ventral chain go off, as a rule, from the ganglionic swellings; in many divisions, indeed, there is an apparent origin from the longitudinal commissures, but the nerve may then be always referred to the nearest anterior ganglion. This happens in the Scoleina, in the Siphonostomæ, in Aphrodite, as well as in the Nereidx, and others. Very often the lateral branches of the ventral medulla form small ganglia, which are generally placed at the base of the parapodia, and from which finer nerve-twigs take their origin (e.g. in the Nereidæ). These ganglia are often connected together by longitudinal commissures, and thus there arises a separate part of the nervous system which is co-ordinated with the ventral nerve-chord (Pleione).

The visceral nerves are differentiated in the same way. In the lower divisions of the Vermes nerves pass from the superior single ganglion to the alimentary canal. This has been observed in the Turbellaria as well as in the Trematoda. In the Annelides not only are these nerves more developed, but they become independent to a certain degree, owing to the deposition of ganglia in them. We divide the apparatus, which has in this way become a special system of visceral nerves, into an anterior and a posterior portion. The former is distributed over the oral region, and is specially developed in the Chrotopoda which are provided with a protractile proboscis (Phyllodoce, Glycera, etc.). The posterior portion, which is less developed, passes on to the enteric tube. In the Hirudinea there is an azygos enteric nerve; in the Lumbricidæ a nerve is continued from the œsophageal commissure, on either side, to the ganglia placed on the enteron; these ganglia have been observed to vary in number. These two portions of the visceral nervous system, notwithstanding their distribution in parts which are physiologically connected, must be kept apart, for the anterior portion is distributed in parts, which are movable at will, while the latter alone correspond to a true enteric nervous system, and in view of their physiological relation can be called a sympathetic nervous system.

Leydig, Ueber d. Nerrensystem der Anneliden, Arch. f. Anat. Ph. 1862.

Hermanx, E., Des Centralnervensystem von Hirudo medicinalis. Munich, 1875.

$\S 121$.

The nervous system in the Solenogastres differs in several points from the forms which have been already mentioned as obtaining in the Vermes. The cerebral ganglion, which in Chætoderma 
is composed of four lobes, gives off four nerve-trunks, which pass backwards. Two of them have a central, and two a lateral course. They unite in a ganglion near the end of the body. In Neomenia there is a considerable complication. The cerebrum gives off a commissure, which surrounds the osophagus, and of these also a commissural chord on either side, each of which passes to a ganglion at the side of the œsophagus ; from each ganglia a lateral nerve-trunk is given off. The lateral nerves unite in a terminal ganglion (branchial ganglion). A commissure passes from each of the lateral ganglia to a ventral ganglion, which gives off a ventral nervetrunk, which is connected with its fellow of the opposite side by a number of transverse commissures. If the lateral and inferior pair of ganglia be regarded as parts separated off from the cerebrum, this form would be seen to approximate very closely to Chrtoderma, and the only difference would lie in the osophageal commissure, in the transverse commissures of the ventral trunks, and in the exclusion of the latter from any share in the terminal ganglion. In any case we have in Neomenia a further development of the simple characters of Chrtoderma. This is not the place to indicate further points of comparison, for as yet we are only beginning to obtain any exact knowledge as to the structure of these animals.

\section{Sensory Organs.}

\section{Tactile Organs.}

$\S 122$.

The sensory organs of the Vermes are of a high grade of differentiation. The organs of tactile sensation appear in the form of fine modifications in the structure of the integument with which the peripheral nervous system enters into connection. Of this kind are the true tactile organs, while the coarser arrangements, such as the processes of the integument, are only bearers of them. The essential part of these organs consists in the connection between the sensitive nerve-fibres and the modified cells of the integument; these cells, as a rule, project beyond the surface of the integument, as stiff setiform processes (tactile setæ, or rods). These arrang'ements are most exactly known in the Rotatoria and Annelida, but they have been recognised in other divisions.

Tactile setre are widely distributed among the Turbellaria and Nemertina, where they are sometimes found orer the whole body, and sometimes are richly developed on the head. 'They are found on the tentacles of the Bryozoa; and are widely distributed on the cephalic segment in the Lumbricidie, and in the Chrtopoda. 'They appear in the Chatopoda on the true tentacles and antenne, as also 
on those appendages of the parapodia, which are known as cirri, as well as on the structures which are formed from modifications of these cirri (cf. $\$ 106$ ). The appendages just mentioned are provided with a large number of end-organs of sensitive nerves, and thus become complicated tactile organs, which are of a somewhat high grade, on account of their power of movement.

A special complication of the tactile rods obtains in some Hirudinea, where groups of these structures are embedded in the base of cup-shaped organs. There is a large number of such organs in the head, and they are scattered over the hinder rings of the body. The arrangement of the sensory parts in depressions of the surface of the body justifies us in supposing that we have here to do, not with a special tactile organ, but with a sensory organ of general character.

The tactile papillæ are less differentiated than the tactile rods or setæ. They are developed in places where the body is covered by a stronger cuticular layer, and are conical or wart-shaped elevations of the cuticular layer, which are traversed by a pore-canal. We find these tactile papillie in the Nematodes, where they are grouped in a regular manner, some near the oral, and some round the genital orifice.

\section{$\S 123$.}

Very little is certainly known as to their function, but organs which may be regarded as sensory are formed by parts of the body which either carry cilia, or have their epithelium distinguished by some other peculiarity; such are the cephalic pits of many Nemertina, and the similar parts in Polygordius. The clefts at the side of the head lead into a narrow ciliated canal, which is connected, either directly or by means of a fibrous chord, with the cerebral ganglion. Perhaps the apparatus presented by the proboscis of Balanoglossus may be regarded as an organ of this kind. It is uncertain whether these organs serve for the perception of the conditions of the surrounding medium, and possess a function analogous to that of olfactory organs.

\section{Visual Organs.}

$\S 124$.

The visual organs of the Vermes offer numerous examples of the gradual evolution of an organ from an indifferent condition. In many of the lower Vermes, Turbellaria, Trematoda, Nemertina, and Rotatoria we often find, at the place where other forms have distinctly developed eyes, pigment-spots only, which are arranged symmetrically, and either placed directly on the brain, or close to it. Nothing is known as to the mode of termination of nerves in these organs, so that it is uncertain whether such "eje-spots" should be regarded as organs for the perception of light. 
We can be more certain about function when the pigment-spot is merely a covering for the special end-organs of the sensitve nerve. 'These end-organs have the form of specially modified cells, which traverse the pigment either singly or in groups; judging from what obtains in cases where the optic organs are more exactly known, we may confidently say that these structures are in direct connection with nerves. They are the so-called crystalline rods, or crystalline cones.

Eyes of this kind are common enough in the 'Turbellaria, among the Platyhelminthes (species of Mesostomum and Vortex); they are as a rule found in pairs on the upper surface of the head. Many marine Planaria have a larger number of regularly-arranged, well-defined, pigment-spots on this part, some of which surround a crystalline body. These eyes frequently appear in the early stages of the embryo as pigment-spots; this is also their condition in many Trematode larvæ, although, in many, distinct crystalline bodies may also be made out (Amphistoma subclavatum, Monostomum mutabile). In the entoparasitic forms of this division the visual organs disappear, while they are persistent in many of the ectoparasitic forms (Dactylogyrus). They are also persistent in Polystomum. They are absent in all stages of the Cestoda, unless, indeed, we are to regard the red pigment-spots, which in some lie behind the suckers, as rudiments of such organs.

In the Nemertina, where eye-spots are frequently present, true eyes have been observed in but few cases (Polia coronata, Nemertes antonina). Eye-spots and true eyes of simple form are found on their œesophageal ring, in the free living Nematodes (Enoplus), while they are absent in nearly all the parasitic forms; here, therefore, degeneration of sensory organs goes hand in hand with parasitic habit.

The visual organs in the Rotatoria are placed immediately on the cerebrum. There is one crystalline rod to each of two connected pigment-spots; or there is only a single visual organ with one crystalline rod. Others have a pigment-spot and nothing else.

The complex pair of eyes in Sagitta is distinguished by a large number of radially-arranged crystalline cones, and with these are found characters which remind us of what obtains in the Annulata.

\section{$\S 125$.}

Among the Annulata the optic organs of the Hirudinea occupy the lowest position. The eyes, which are present in many, lie, as in the Platyhelminthes, on the surface of the cephalic portion of the body, and are, as in them, generally arranged symmetrically and in large numbers. In their structure they agree in so remarkable a manner with the cup-shaped structures mentioned in speaking of the tactile organs, that in them a condition appears to exist, in which a specific sensory organ is evolved from the indifferent organs of sensation, which are found in the integument. 
Among the Annelides we find the eyes of the Chatopoda generally hidden beneath the integument, and placed on the cerebral ganglion in one or two pairs : a single cye is seldom present. Generally one pair is considerably developed, and the second often redueed to a pigment-spot. Where these visual organs are specially developed they stand out on the surface of the integument (Syllidic, Nereidic, Fig. $65, a)$; and may attain to a highly complicated structure. 'This is the case in the Alciopre, the pelagic mode of life in which is in connection with the high grade of development of this sensory organ. This influence of the mode of life is also seen in their nearest allies, the Phyllodoceida, which live at the bottom of the sea, and have rudimentary or very simple eyes. The spherical bulb (Fig. 66) presents this, the

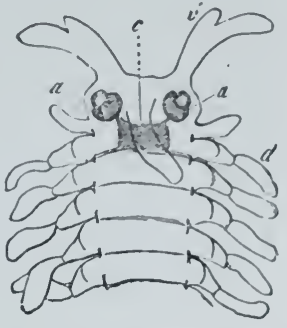

Fig. 65. IIcad and most anterior segments of a Myrianida. a Eyes. $b$ Tentacles.

c Unpaired cephalic tentacle. d. Cirri. highest degrec of development in the Alciopidio only. The integument $(c)$ covers the anterior, strongly-curved segment, immediately behind which there is a spherical lens (7). The hinder segment, the innermost layer of which forms the layer of rods $(b)$, surrounds a homogeneous vitreous body $(h)$. A layer of pigment $(p)$ separates the layer of rods from the parts of the retina which lie more to the exterior; outside all these is the expansion of fibres of the optic nerve $\left(o^{\prime}\right)$. While in the simpler forms of eye the terminal organs of the nerve lie in the integument, they are here pressed together into a concave layer. Influential in the development of this arrangement is the multiplication of the perceptive elements, and the formation of refracting media. Just as the eyes are completely wanting in the majority of the Scoleina which live in the dark, so also these organs undergo degeneration in the Tubicola among' the Chætopoda. The eyes which are present in the larvæ, and even in later stages, disappear,

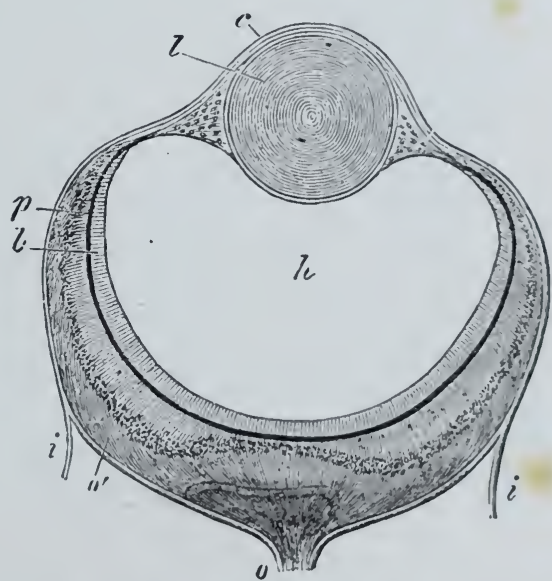

Fig. 66. Eyc of an Alciopid (Neophanta celox) (after Greefi). $i$ Integument, cover. ing the anterior segment of the bulb, $c$. $l$ Lens. $h$ Vitreous body. o Optic nerve. $o^{\prime}$ Expansion of the optic nerve. $p$ Layer of pigment. $b$ Layer of rods. or are represented by mere pigment-spots, when they enter upon the fixed mode of life.

The development of visual organs on the branchial tufts of the head is an adaptation of another kind, which is seen in certain 
Sabellidæ (Branchiomma); in them the eyes are either placed in large numbers on the pinnate branches of the branchial filaments, or at their ends only. In other Annelides there is a similar change in position as compared with the primitive one. In many there are eyes at the posterior end of the body, as well as on the cephalic segment; and finally, in the genus Polyophthalmus there is a pair of eyes on each metamere, in addition to those on the head. We here find an arrangement which is not only of importance as bearing on the estimation of the metameres, but is also a proof that visual organs may be developed at points which in other forms only carry sensory organs of a lower kind.

\section{Auditory Organs.}

$\S 126$.

We consider as auditory organs in the Vermes organs which, as in the Coelenterata, consist of a vesicular capsule, in which there is a firm, large concretion, or a number of smaller ones. The wall of the capsule is frequently invested with cilia, as may be seen from the trembling movements of the auditory stones (otoliths). The difficulty of making out the nerve-branches in the lower Vermes-in which, indeed, these organs are most largely distributed-has generally caused the connection of the auditory vesicles with the nervous system to be missed.

These auditory vesicles are generally unpaired in the Turbellaria, in species of Monocelis, Convoluta, Proporus, Derostomum. 'They generally lie close to the cerebral ganglia, and are found as a rule in those genera which are devoid of eyes or eye-spots. In the Nemertina they have only been observed in some cases (Erstedia). In the rest of the Platyhelminthes these auditory vesicles are not, apparently, present, and they are also wanting in the Nematodes.

Only in the Annelida do they appear again, where they are paired, and as a rule placed at the sides of the brain (Alciopidæ Arenicola, Fabricia, Amphiglena, etc.).

\section{Alimentary Canal.}

\section{$\S 127$.}

The alimentary canal of the Vermes forms a tube, which is either embedded in the parenchyma of the body, or, when a coelom is present, in it; it has a general adaptation to the form of the body. The mouth lies, as a rule, at the anterior end of the body, and is always placed on the ventral surface. Where an anus is present, 
it is placed, as a rule, at the hinder part of the body, and is sometimes ventral and sometimes dorsal.

A differentiation of the enteric tube into several functionally different portions can always be made out; accessory organs for the prehension of food are also often present at the entrance into the digestive cavity. The three portions, which are here present for the first time, are distinguished as fore-, mid-, and hind-gut; the last is absent when there is no anus.

The primitive form of enteron agrees with the characters which are seen in the Gastrulaform (\$28). It makes its appearance in all in the embryonic commencement of the organism as a cæcal cavity, with but slight complications, which opens on the surface at one point only; it persists in this form in the lower Vermes. This opening serves for the ingestion of food, and also for the ejection of its undigested remains; it is mouth and anus at the same time. This arrangement is very common among the Platyhelminthes, being the only condition of the alimentary canal among the Trematoda, and the dominant one among the Turbellaria. In the rhabdocœlous Turbellaria the alimentary canal is distinctly marked in its anterior portion only, and has the form of a simple blind tube extending through the body. The simple mouth varies in position; it may be in the anterior portion of the body, or towards the middle of the ventral surface, and lastly, even in the posterior portion; it leads into a muscular pharynx, which is seldom absent (Schizostomeæ), and which is, in many cases, protractile. This is the portion of the alimentary tract, which, under many modifications, can be most clearly traced in most divisions of the Vermes.

\section{$\S 128$.}

In the dendrocolous Turbellaria the gut is adapted to the broad form of the body.

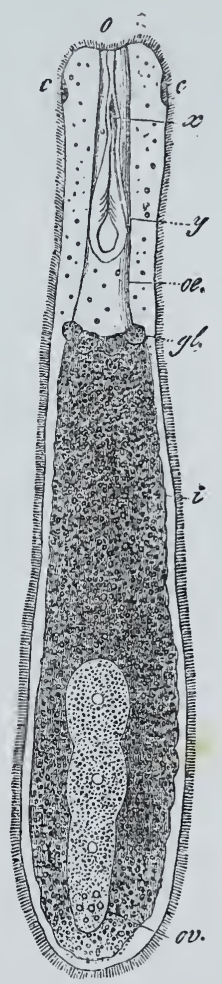

Fig. 67. Prorhynchus fluviatilis. o Mouth. oe Esophagus, protrac. tile like a proboscis. $i$ Enteron. gl Glands opening into the enteron. $c$ Ciliated pits. $x$ Spike in the organ above the œsophagus, which ends cæcally at $y$. ov Ovary, in which there are, in the anterior parts, ova at various stages of development. The mouth is (Fig. 68, o) placed ventrally, and often near the middle. The muscular pharynx $(p)$ is often metamorphosed into a proboscidiform organ, which is capable of great enlargement in size, and is cylindrical or drawn out into lobes. It leads into an enteric cavity $(v)$, which occupies the middle of the flat body, and which is broken up into numerous branches, which pass towards 
the edge of the body; an elegant mesh-work may be formed by the connections of these with one another (Thysanozoon). Owing

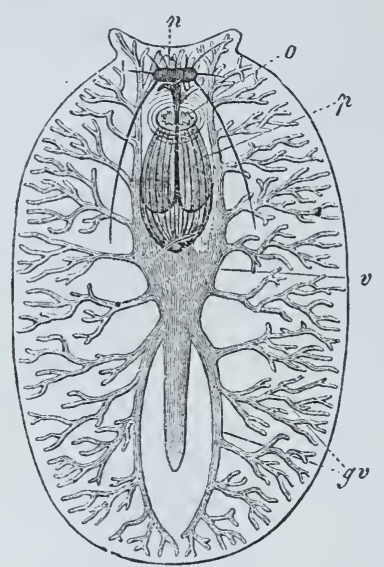

Fig. 68. Digestire apparatus of Eurylepta sanguinolenta. o Mouth. $p$ Pharynx. $v$ Stomach. gv Ramifications of the digestive cavity. $n$ Nerve ganglion (brain) (after Quatrefages).

velopment (Redia-form). to the free communication that these branches have with the central cavity, the chyme is distributed in the body, and so the enteric canal takes on the function of a vascular system. The land Planarians are remarkable, inasmuch as their enteric tube consists anteriorly of a median canal, while it is divided posteriorly. $\mathrm{Nu}$ merous and regular transverse processes pass off from both divisions of it.

The enteric tube is branched in many of the Trematoda. The gut commences by a mouth, which is generally placed in the anterior region of the body, and, as a rule, has the surrounding parts metamorphosed into a sucker (Fig. 69, $s$ ); this is followed by a muscular pharynx (b), from which the enteron proper is given off. This is, when most simple, a cæcal sac (Aspidogaster, Gasterostomum), and corresponds to a low grade of development; this is very common among the Trematoda at certain stages of their dedivides into two branches, which pass backwards, and either give off greatly ramified branches into the body

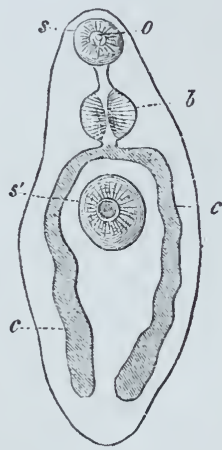

Fig. 69. Alimentary canal of Distoma flarescens. - o Mouth surrounded by a sucker, $s . s^{\prime}$ Ventral sucker. 6 Muscular portion of the œso. phagus or pharynx. $c$ Bifurcated enteric tube.
(Distoma hepaticum), or form simple cæcal sacs (c) (Distoma flavescens, D. lanceolatum). The two branches may unite again and form an arrangement like that which obtains in some Planarix. It is clear, from the homogeneity of its structure, as well as from its contents, that even in the Trematoda this branching of the gut is merely an enlargement of the tract in the body, and not the formation of heteronomous segments. The texture of the wall is in correspondence with tho low stage of this form of enteron, for only the epithelial investment is independent, being bounded, exteriorly, by the tissue of the parenchyma of the bodyconnective tissue.

Complete degeneration of the gut is clearly due to adaptations to definite modes of life, in which the food passes through the integument by endosmosis. This phænomenon, brought about by parasitism, 
attains its highest derelopment in the sporocyst forms of the Trematoda. Finally, the absence of an enteric canal is the rule among the Cestoda, where the enteron is not present for a time even. The enteron is altogether wanting in the Acanthocephali, and for the same reason-namely, parasitism.

Among the Platyhelminthes there are forms which are distinguished by the possession of an anus, and which may be contrasted with those which indicate their lower condition by not possessing one. Such are the Microstomex among the Turbellaria rhabdocœla, and the Nemertina, the enteric tube of which has pretty much the same form throughout, and which begins by an elongated ventral mouth, which lies behind the central nervous system. In Malacobdella the mouth is placed at the anterior end of the body. A muscular, but generally feebly-developed, pharynx, leads into the intestinal tube, which is provided with a large number of lateral diverticula. This fills the greater part of the body-cavity, to the walls of which it is attached by muscular fibres. The lateral diverticula of the enteric tube are sometimes regularly arranged, and are the first indications of metamerism. 'This is best seen in Pelagonemertes; and so far this form calls to mind the dendrocolous Turbellaria.

\section{$\S 129$.}

In the Nemathelminthes all three portions of the alimentary tube are generally present. In correspondence with the form of the body, it forms a long: tube, which traverses the body, beginning by a mouth in the centre of the anterior end of the body, and ending by a ventrally-placed anus, which is more or less near the caudal end. The most anterior portion (œsophagus) forms a narrow canal, the walls of which pass gradually backwards into a thick-walled pharynx (Fig. 70). This is distinctly marked off from the rest, and is distinguished by a musculature, which enables it to act as a sucking organ. The layer of chitin, which invests the tract

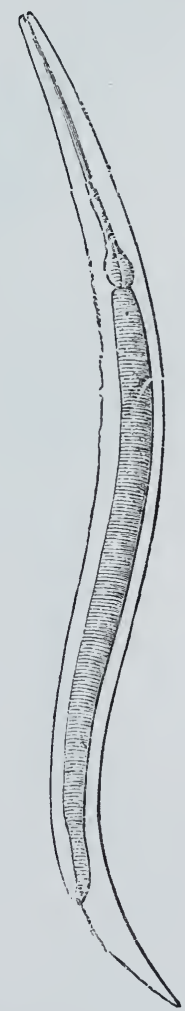

Fig. 70. Alimentary canal of a Neinatode (Dia. gram). from the mouth to this portion, not unfrequently forms ridges or tooth-like organs. The mid-gut (chyle-stomach), which succeeds the pharynx, is, as a rule, the largest part; it has simple walls, often formed by a single layer of cells, which in some (Heterakis vesicularis, Oxyuris vermicularis) is provided in places with a muscular covering of annular fibre. A cuticular layer generally lies outside the epithelium; an internal cuticle, which is traversed by pore-canals, appears to be present also. In many, the mid-gut forms a cacal diverticulum in its anterior portion. This portion 
of the intestine is attached by laterally-directed fibrous chords to the body-wall, as a rule, along the lateral lines. The hind-gut, which arises from the mid-gut, is the shortest portion of the whole canal, and is distinguished from the part in front of it by its diminished breadth.

In the Gordiacea the enteric canal is present in the entoparasitic

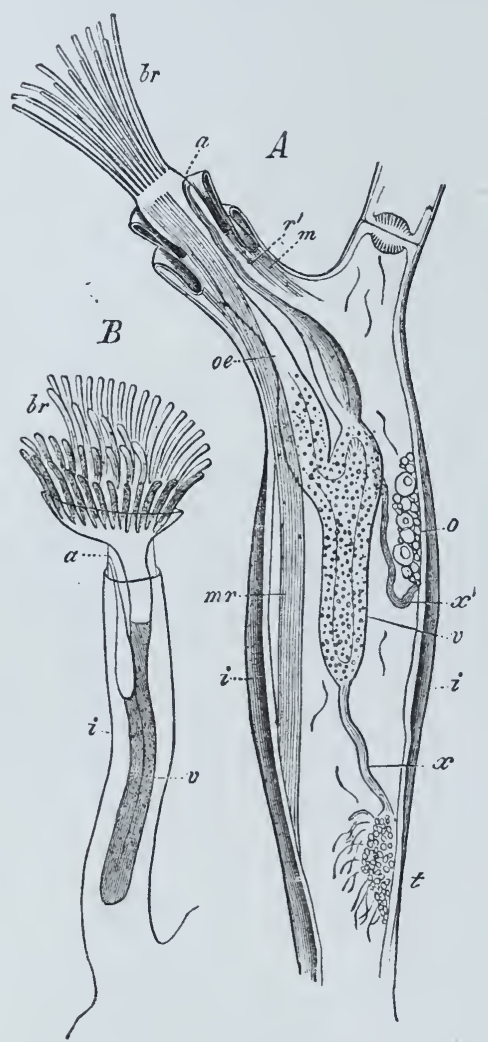

Fig. 71. Organisation of Bryozoa. A Paludicella Ehrenbergii. B Plu. matella fruticosa. br Tentacular branchiæ. oe Esophagus (fore-gut). $v$ Stomach. $v$ Hind-gut. a Anus. $i$ Covering of the body (ccll). $x$ Posterior; $x^{\prime}$ Anterior chord, at the insertion of which into the body the generative products are dereloped. $t$ Testes. $o$ Ovary. $m$ Retractor muscles of the anterior portion of the cell. $m r$ Prin. cipal retractor muscle (after $\Lambda$ llman).

it (Bowerbankia, Vesicularia). larval stages only, and undergoes retrogressive metamorphosis when the sexual organs are developed. In Gordius even the mouth disappears. The organism, when it is free, uses up the material which it ingested by its enteron during the earlier stages, in forming generative products, after it has given up its parasitic habit, and the ingestion of food.

The enteric canal of the Chætognathi resembles in many points that of the Nemathelminthes, but the enteron is connected to the body-wall in a different way, namely along its dorsal and ventral median lines. Setiform hooks, arranged in rows at the sides of the mouth, serve as organs of prehension.

\section{$\S 130$.}

Although the digestive organs of the Bryozoa are sharply marked off into the three primitive divisions, they are exceedingly simple in character. The mouth, which is surrounded by the tentacles, or placed in the centre of the lobate process which carries them, is in one division (Phylactolæmata) overhung by a movable process- the epistom. Thence it passes straight back to an œsophageal portion (Fig. 71, ve), which in some is widened out or even converted into a gizzard by the development of denticular processes in one part of The second portion is separated from the fore-gut, which is invested with cilia, by a constriction $(v)$, and forms the mid-gut. It functions as a stomach, and forms a cacum, which generally descends some way down into the cœlom. 
The hind-gut follows a constriction of the somewhat more deeplyplaced pyloric portion, and, ascending by the side of the fore-gut, is continued into an anus $(B a)$, which is placed close to the mouth, but always below and outside the circlet of tentacles. The hind-gut is sometimes widened (Flustra).

The ciliated tentacles function as accessory organs of nutrition, food being brought to the fixed animals with the changing water.

In the Pedicellinæ the same parts can be made out as in the true Bryozoa, but the stomach has no cæcum.

The enteric canal of the Rotatoria exhibits sometimes agreement with lower conditions, for it may consist of foreand mid-gut only, the hind-gut being absent (species of Notommata), while on the other hand it is more highly developed, owing to the differentiation of masticatory organs in the most anterior portion. These are formed by chitinous structures, placed at the sides and opposite to one another; they are provided with tooth-like processes, etc. (Fig. 43, $m)$. The fore-gut commences with the mouth, which lies below the ciliated velum, and is distinguished by its lesser width from the mid-gut (ordinarily called the "stomach"). Where a hind-gut is continued on from the mid-gut, it turns to the dorsal surface of the body, to open into a cavity common to the openings of the excretory and sexual systems - the cloaca-a peculiarity not found in other divisions of Vermes.

\section{$\S 131$.}

In the Gephyrea the three divisions of the enteric canal are, as a rule, distinct

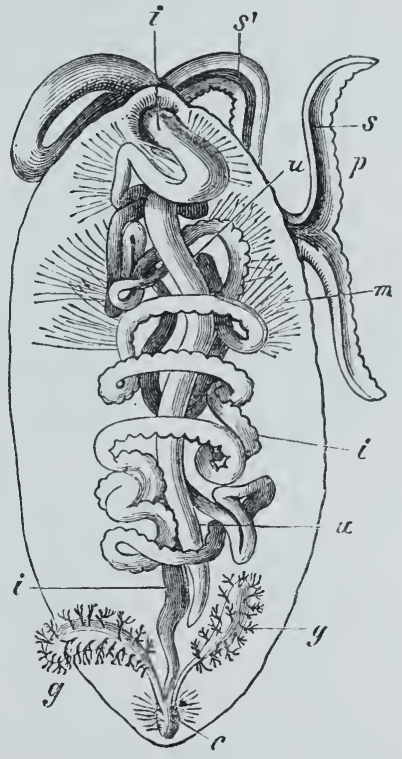

Fig. 72. Enteric canal of Bonellia. The proboscis of the animal is coiled, so that the whole of it cannot be seen. $p$ Front of the proboscis. $s s^{\prime}$ Groove of the proboscis. $i i$ Enteric canal. $m$ Mesenteric filaments : they are only drawn in the anterior region. $g \mathrm{Ex}$. cretory organs. $c$ Cloaca. $u$ Uterus (after Lacaze-Duthiers). in the earlier stages only; in some, however, for a longer time (Priapulus); while in others, as the enteric tube elongates, the separation becomes less noticeable. The enteron then forms a tube, which is generally a good deal longer than the body, and which does not vary much in diameter. It is either disposed in several partially-coiled longitudinal loops, when the anus is on the dorsal surface of the animal (Sipunculus, Phascolosoma); or the enteron (Fig. 72, $i$ ) passes to the posterior end of the body, in numerous shorter coils, without forming large longitudinal loops, and ends in the anus, which is placed there (Echiurus, Bonellia). The latter agree with the majority of the other Vermes in the aboral 
position of the anus, while the Sipunculidre seem to differ more from them. But this position of the anus is really only a further

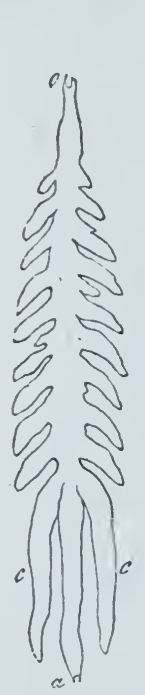

Fig. 73. Enteric canal of Sanguisuga. o Esophagus. $c$ Posterior pair of cæca. $a$ Anus.

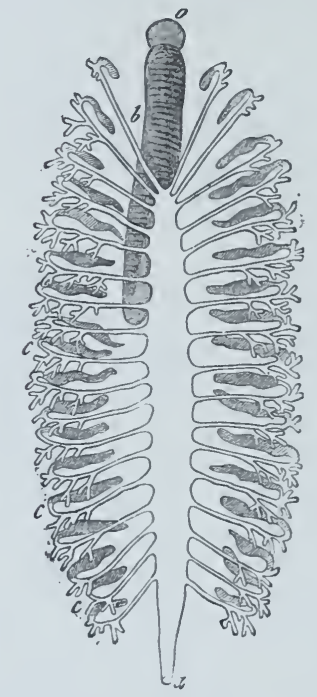

Fig. 74. Enteric canal of Aphrodite. o Anterior portion. 6 Middle (muscular) portion of the fore-gut. $c$ Branched cxcal appendages of the mid-gut. a Anus. development of that dorsal position which it has in many of the Vermes, and does not in any way affect the homology of their enteron with that of the other members of this group.

\section{$\S 132$.}

The metamerism of the body in the Annulata affects the enteric tube; but there are various other differentiations in it, which are due to adaptations to special modes of life. Here again the enteric canal begins as a cæcal invagination. The aproctous condition, which persists in most of the Platyhelminthes, is passed through by these forms at an early stage in development. The entrance to the fore-grut is most variously differentiated in the Hirudinea. In some the protractile œsophagus is greatly complicated, in others its entrance is armed with chitinous ridges, which are the first signs of jaws. But in most the mid-gut is beset by pouch-like diverticula (Fig. 73), which are branched in Clepsine; the last two of these

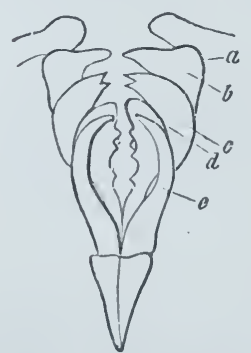

Fig. 75. Maxillary apparatus of a Eunicea (Lysidice). $a-c$ Pairs of jaws (after Milne-Edwards). diverticula sometimes form longer cæcal tubes $(c)$ on the narrow hind-gut, which extends to the end of the body (Clepsine, Hrmopis). These are the only crea of the gut of Aulacostomum. In others the cæca are merely indicated by constrictions. In all cases these arraugements correspond to the metamerism expressed also in the nervechord.

In nearly all Annelids the fore-gut is separated into several, often very different, portions. A median portion is distinguishable by its more powerful muscular investment, and is separated from the mid-gut by a tract of varying length. Among the Scoleina, this portion, which is known as the "muscular stomach," is very greatly developed (Lumbricus). It forms the end of the fore-gut. It is placed more towards the middle of the latter in most Chretopoda, and is frequently provided with denticles, which work on one another like 
jaws. There is sometimes only one pair of these jaws (Fig. 55, m), and sometimes there are several, which differ from one another in particular characters, and form a complicated apparatus (Fig. 75). This portion is very greatly developed in the Aphroditeidx. It can be protruded, as in many other voracious Annelids (Phyllodoce, Glycera, etc.), so that the anterior portion is everted and forms the outer surface of the "proboscis." This protrusible portion is sometimes very long.

The whole arrangement undergoes atrophy in the Tubicolæ, to which Arenicola is an intermediate step. The third division of the fore-gut is feebly developed in the Scoleina, and more so in the Chætopoda, where it is often seen to be provided with a pair of cæca (Syllis, Arenicola).

The mid-gut forms the largest, and also the most uniform, portion of the whole enteric tube. It generally has a perfectly straight course, and is seldom disposed in coils or loops. It is not only attached by the muscular lamellæ from the body-wall, or by the separate fibres from the edges of the metameres, but is also divided into separate, and often diverticulate, portions, which correspond to the metameres. In the family of the Aphroditeidx, as in the Hirudinea, diverticula of this kind are developed into larger appendages, which may exhibit numerous branches (Fig. 74, c).

The hind-gut is generally a short portion, and is of some size in the Tubicola and in Arenicola only; it seldom has a median enlargement, and generally extends to the anal opening from the mid-gut, without being sharply marked off from it.

Myzostoma agrees with the Annelides in the character of its intestinal tube. The fore-gut is represented by a long protractile proboscis, which leads into a widened mid-gut, from which a narrower hind-gut leads to the anus. Branched crea are distributed through the body from either side of the mid-gut.

\section{Enteric Branchiæ.}

$\S 133$.

The development of the respiratory function of the enteric tube leads to special arrangements in it, which are greatly developed in Balanoglossus. The anterior portion of its enteric tube is separated by two lateral processes into two semi-canals, which lie one above the other, and communicate freely by means of the longitudinal opening which lies between the two laterally outstanding processes. The semicanal, which I regard as the lower one, leads directly to the commencement of that portion of the enteron which functions exclusively as a nutritive canal. The investment of cilia drives the particles of food into it; it has a nutritive function. The semi-canal, however, which is placed dorsally, has a respiratory function. There is a branchial support in its walls in the form of a delicate framework of chitinous 
lamellæ, covered with epithelium. There are clefts between the branchial arches and the several lamellæ which go to form them; these lead, on each side, to a series of spiracles, by which they open on to the surface of the body. A network of vessels is spread over the branchial framework. The water taken in by the mouth streams through the superior respiratory semi-canal into this branchial apparatus, and passes again to the exterior by the rows of spiracles.

The hind-gut of many Annelids may be seen to take in water, and this may be connected with a respiratory function of this division of the intestine. No special organs of respiration have been observed to be developed in this portion. It is not yet certain whether the structures which are found in the hind-gut of Neomenia are really branchiæ. More exact anatomical knowledge of the protractile branchiæ of Chrtoderma is necessary before we can say what is their morphological signification.

\section{Accessory Organs of the Alimentary Canal.}

\section{$\S 134$.}

The enteric canal of the Vermes has glandular organs of various kinds connected with it, which are to be regarded as differentiations of the enteric wall, that is to say of the endoderm. Single cells, or groups of cells, acquire a different character from their neighbours, and so give rise to special organs, which are of different degrees of individuality, according as their position is on the enteric wall, or without it; in the latter case they are connected with the lumen of the intestine by ducts. They may further be more exactly classified, according to their relation to the separate divisions of the enteron.

In the fore-gut, close behind the muscular pharynx, small groups of unicellular glands open in the rhabdocolous Turbellaria. In the Trematoda similar groups of cells are placed at the anterior end of the body, opening near the mouth, and are regarded as pharyngeal glands. Glandular organs have been observed in the so-called pharynx of the Nematodes, and distinct glandular cells in the oral region.

Among the Annulata the histology of the Hirudinea, especially, has been carefully investigated; in them, a large number of unicellular glands open into the proboscis, when there is one, or into the jaws, when they are present. In the Annelides a pair of lobate glandular tubes are found in the last division of the fore-gut, just behind its muscular portion in the Nereids and others provided with pharyngeal jaws (cf. Fig. 55, $g l$ ); they are modifications of the simpler tubes of the Syllidx. The Rotatoria are provided with glandular appendages at the same spot. It is usual to call these glands, which have however various kinds of functional relations, "salivary glands." 


\section{$\S 135$.}

The glandular organs connected with the mid-gut are ordinarily regarded as hepatic, or as a "liver." We must be careful not to attribute anything more than the value of a convenient distinction to these names. Separate glands are almost always absent from the mid-gut of the Vermes, but the epithelium is generally found to be different from the epithelia of the other divisions of the enteron, so that a secretory function is not improbable. This is indicated by the granular character of the cells in many cases, and by the difference in the coloration of the cell-contents. The latter point is probably more important than the former, for the former may be due to the absorbing function of the enteric epithelium. The mid-gut of the Bryozoa is distinguished by this character, and even in the Rotatoria the epithelial layer of this region may be seen to be differentiated. This character is more highly developed in the Platyhelminthes (Planarix, many Trematoda), in which the stomachal branches (Fig. 68) are the chief seat of this peculiarity ; these branches, therefore, may be regarded as secreting appendages. Independent glands are still more distinct in the lateral appendages of the mid-gut of Aphrodite (Fig. 74), which are developed by the gradual narrowing and lengthening of the simpler enteric appendages found in the allies of this genus. Finally, we must mention here the tubular enteric appendages of Balanoglossus, which beset the whole dorsal surface of the enteric canal beyond the respiratory segment, and are grouped in agreement with the segments of the body.

\section{Cœlom.}

\section{$\S 136$.}

The Colom of the Vermes is the earliest differentiation of a hollow cavitary system placed between the enteric tube and the integument, and leading to the formation of a vascular system, and takes its origin in a cleavage of the mesoderm. The food taken in by the enteron is no longer distributed in the organism, as it is in the Cœlenterata, by continual imbibition into the tissues from the wall of the enteron, but the nutrient fluid is collected into a perienteric space, where it may enter into relation with organs differentiated from the enteric canal, and from the integument.

In a large number of Vermes this perienteric space (Cœlom) is either altogether absent, or only rudimentarily present. This is the case in most of the Platyhelminthes and Nemathelminthes, as well as in some others, such as Pedicellina. In the land Planarians two cavities traversed by a reticulum of connective tissue extend along the body; they are largely broken up anteriorly. They are to be regarded as indications of a cœlom of this kind. The cœlom is 
well developed in the Rotatoria and in most Annulata. It forms a continuous, and generally a very wide, cavity in the Bryozoa and in the Gephyrea. The cœlom of the Annulata is arranged in correlation with the metamerism of the body. Partitions (dissepiments) extend from the wall of the body to the enteric tube, and form a series of separate chambers, each of which corresponds to one segment of the gut, etc.; these characters have been already pointed out in the Nemertina. When the dissepiments are reduced to single chords the chambers are more or less completely fused. In many cases this causes the separate chambers to disappear, either along certain tracts, which are generally placed in the anterior region, or along the whole length of the body; and a single body-space is formed, which is, generally, still traversed by remnants of the dissepiments, in the form of filaments or fibrous bands. These fibres keep the enteron in its place, especially when it is coiled (cf. Bonellia, Fig. 72, $\mathrm{m}$ ).

The perienteric fluid is generally quite clear, and in most Vermes contains form-elements, of which there are sometimes a large quantity. When there is communication between the vascular system and the colom, the contents of the two spaces are similar in character. The movement of the fluid is dependent on the action of the body-wall, so that in many, locomotion of the body produces at the same time a circulation of the nutrient fluid: and thus is established the lowest form of circulation.

The colom communicates with the surrounding medium, the water, by means of various arrangements. The excretory apparatus, with its internal orifices (cf. $\$ 142$ ), is onc, but special openings are also known. For example, there is an opening of this kind in the Bryozoa, which serves also as the passage for the generative products, and in the Rotatoria, where the orifice is generally drawn out into a tube (siphon: cf. Fig. 81, s). Similar orifices have been observed to be present in the Amnelides.

\section{Vascular System.}

$\S 137$.

The differentiation of hollow spaces in the mesoderm is the first beginning of the formation of a complicated system of canals; which gradually acquire special walls and become blood-vessels. The earliest main trunks form long canals, which are first visible in the Nemertina. Two (Fig. 76, 77 ) of the three chief trunks take a lateral course; the third is dorsal and median. In the cephalic region the lateral vessel forms several coils, which surround the cerebrum, and are connected with the dorsal vessel as well as with one another more anteriorly. At the posterior end of the body all three ressels are more simply connected. In some genera other vessels are connected with these three; fire transverse vessels connect the dorsal 
and the lateral ones at regular distances. In this way the whole arrangement is in a way segmented, and corresponds to the metamerism indicated in other organs.

The canal system which ramifies through the integument of the Acanthocephali, and is also united with the canals of the lemnisci (p. 174), must not be regarded as a similar system of vessels. It is uncertain to what morphological group of organs they belong.

\section{$\S 138$.}

The vascular system of the Annulata resembles that of the Nemertina in all essential points. In almost all there are longitudinal trunks, which have a dorsal and ventral, or even a lateral, course; these are connected with one another by transverse anastomoses, and pass into one another in the anterior as well as in the posterior region. The dorsal longitudinal vessel, which runs above the enteron, is the most constant in character; it is always contractile, and the current of blood in it is driven from behind forwards. It corresponds to the dorsal median vessel of the Nemertina, while the two lateral vessels of the latter may correspond to the ventral vessel of the Annulata. These vessels are not closed in all Annulata, but are connected with wider spaces, which represent a body-cavity. The vascular system is not completely differentiated in these forms. The body-cavity remains in direct connection with the vascular system in the Hirudinea, owing to the fact that organs which formerly lay in the cœlom are now enclosed in hæmal spaces. There are usually three such sinuses. A median one, representing the principal portion of the cœlom, embraces, in Clepsine and Piscicola, the alimentary canal and the ventral medulla, and perhaps also a portion of the dorsal vessel, except where this has, as in Piscicola, a special sinus for itself. Two pulsating lateral vessels (Fig. $61, B l$ ) partly communicate with the median sinus, and are partly connected to each other by transverse

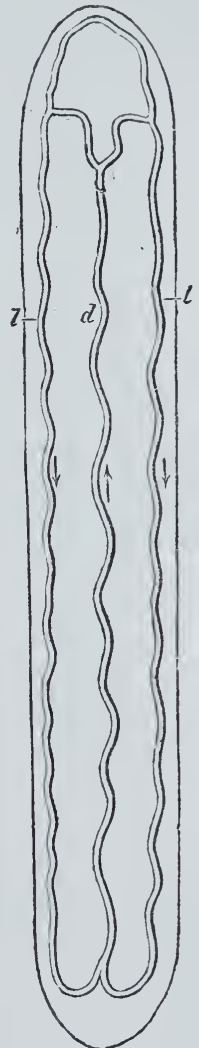

Fig. 76. Diagram of the vascular system of the Nemertina. d Dorsal longitudinal trunk. $\quad l l$ Lateral vessels. The arrows indicate the direction of the stream of blood. anastomoses. In Hirudo and its allies, the median sinus is, at first, only present in the region of the head, where it surrounds the œsophageal ring. It has only a ventral development in the rest of the body, where it encloses the ventral medulla (Fig. 61, $B$ n). This disappearance of the great sinus is due to the development of a fine vascular network in its place, and, like it, is connected with the transverse vessels, which unite the longitudinal vessels with 
one another. New longitudinal trunks are formed from the ressels distributed to the enteron. While in these forms a complex system is produced by the combination of the primitive median trunks with a system of canals formed from lacunæe of the cœlom, the whole vascular system may be made simpler by the disappearance of these median trunks. This is the case in Nephelis, where there is a wide median sinus, and two lateral vessels.

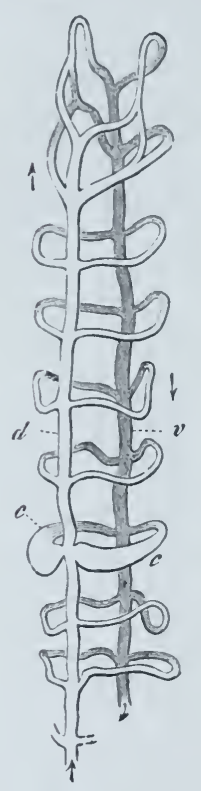

Fig. 77. Anterior portion of the blood vascular system of Sænuris variegata. $d$ Dorsal res. sel. $v$ Tentral vessel. $c$ Heart-like enlarge. ment of a trausverse anastomosis. The arrows indicate the direction of the current of blood.

This form of vascular apparatus, developed out of a lacunar system, is limited to the Hirudinea, for in the Annelides the vascular system is almost always shut off from the cœlom. Where it is not so, we have to do, not with further development, as is the case in the differentiation of the cœlom of the Hirudinea, but with degeneration.

The dorsal vessel lies, as a rule, immediately upon the enteric canal, and often appears to be embedded in the same glandular layer. In addition to anterior and posterior connecting vessels, there are lateral vessels which correspond with the metameres. They are divided into those which directly surround the enteron, and form a capillary network, often a highly-ramified one, in its walls (visceral vessels), and into those which project into the cœlom, and run either along its walls or its appendages (parietal vessels). In the Scoleina the arrangement is generally the same throughout the whole body. In many cases the transverse vessels, as well as the dorsal longitudinal trunks, are pulsatile, and one or more pairs are considerably widened (Fig. $77, c$ ). In this differentiation of a portion of the vascular system we see the beginning of the development of a central organ of the circulation-a heart. The ventral vessel is very seldom contractile. Fresh complications in structure are due to the development of fine vascular networks, such as, in Lumbricus for example, are distributed as capillaries through the body. Branchiobdella is allied in the cliaracters of its vascular system to the simpler conditions found in the Scoleina.

\section{$\S 139$.}

The development of the respiratory organs is of influence in producing changes in the distribution and differentiation of the blood vascular system. In the Scoleina there are no distinct organs of this kind, and either the whole surface of the body, or, by the introduction of water into it, the colom, acquires a respiratory functiou. Consequently we do not see any great variations of the 
vascular system in various portions of the body, and it is only in some which live in the mud of fresh water, and in which the hinder part of the body takes a special share in respiration, that the parietal vascular coils present any very great development (Lumbriculus).

Among the Chætopoda also these simpler relations obtain, but the greater differentiation of the head, and of the fore-gut, is followed by some changes in the vascular system. When branchiæ are present the parietal vascular system is continued into them; in the simplest case a loop of the vessel passes into the appendage, which has the function of a gill. Here we have the commencement of the gradual separation of an arterial and a venous portion.

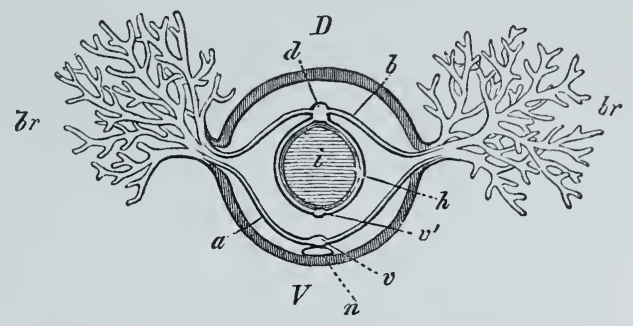

Fig. 78. Diagrammatic transverse section through the hinder half of the body of Arenicola, to show the arrangement of the vessels. $D$ Dorsal; $V$ Ventral side. $n$ Ventral medulla. $i$ Enteric cavity. or Branchiæ. $v$ Ventral vascular trunk. $a b$ Branchial vessels. d Dorsal vascular trunk. $h$ Branch surrounding the enteric canal. $v^{\prime}$ Visceral ventral vessel.

This arrangement is re-

peated when the branchiæ are distributed over a large number of metameres, as in Eunice and Arenicola. The dorsal trunk gives off, in addition to the vessels for the enteron, others which pass to the laterally-placed branchiæ; from each of these a vessel passes to the ventral trunk (Fig. 78). The same characters obtain in the Hermellidæ, where the branchiæ have but a single central cavity, and where, therefore, there can be no anatomical separation of the efferent and afferent blood. In Arenicola these characters obtain in the hinder half only of the body. In the anterior half one branchial vessel passes to the chief ventral trunk, and the other to a visceral ventral vessel.

When the respiratory appendages are limited to a smaller portion of the body, as is the case in the Tubicolæ, there is a corresponding increase in the difference between the development of various vascular regions. Thus in the Terebellidæ (Fig. 79) the dorsal vessel $(v d)$, above the muscular pharynx, is widened out into a large tube, which supplies the branchiæ $\left(b r^{\circ}\right)$, and so functions as a "branchial heart." Afferent vessels pass from the branchiæ to the ventral vessel. In many the function of a central organ is taken on by transverse anastomoses (Scoleina). A vessel of this kind, which passes from the ventral to the dorsal vessel, is found even in the Terebellidx, where it functions as a part of the cardiac division of the dorsal vessel. In Arenicola this vessel is continuous with two very wide transverse vessels, which pass to the ventral trunk.

The arrangement of blood-vessels is constant enough when they are not richly distributed; but this is not the case in those divisions in which the enteric-wall and the body-wall are largely provided with 
vascular ramifications. A breaking-up such as affects the parietal transverse anastomoses in the gills, may affect also the longitudinal

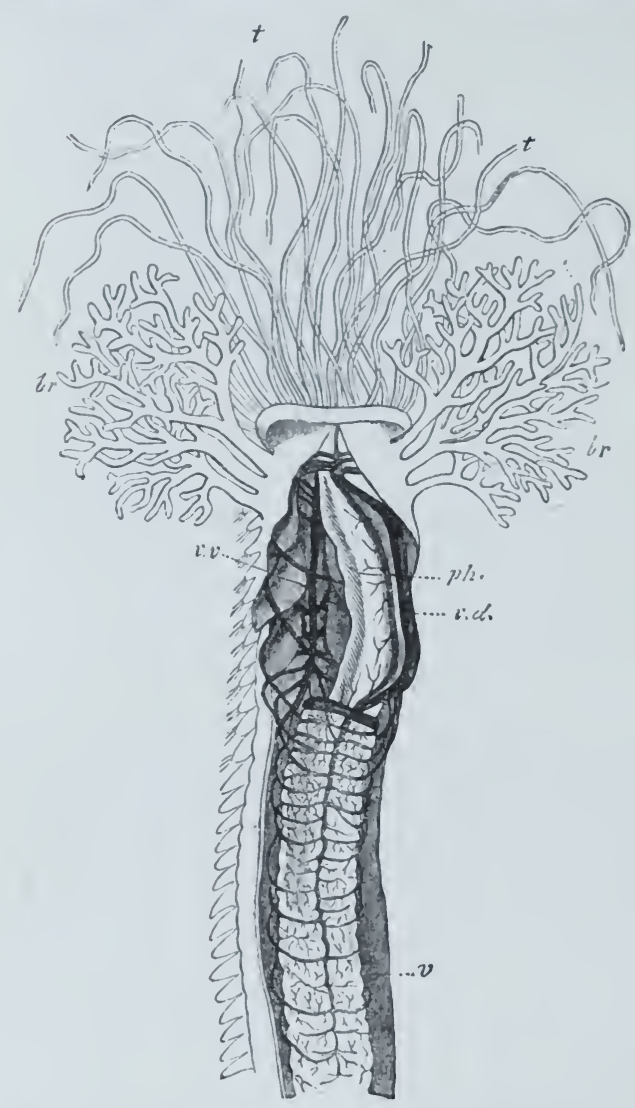

Fig. 79. Vascular system of Terebella nebu. losa; opened from the dorsal surface. $t$ Tentacles (not fully drawn). br Three pairs of branchix. ph Muscular portion of the fore-gut (pharynx). $v$ Enteron. vd Dorsal ressel. $v v$ Ventral vessel (after Milne-Edwards). trunks, which then take the form of a continuous network of vessels, from which new passages are developed. The phrnomena, which gire rise to a collateral circulation, must also furnish us with the explanation of these relations. 'Thus in Polyophthalmus the median trunk is broken up along the course of the mid-gut. 'Two dorsal and two ventral trunks arise from the anterior and posterior simple median ressels in the Hermellidx; in Eunice the ventral, and in Nephthys the dorsal, pair are present.

In some the vascular system is atrophied (Glrcera, Capitella).

A combination of the types of the vascular srstem, which obtain in the Amnelides and the Nemertines, can be made out in Balanoglossus. This consists in the presence of both median and lateral longitudinal trunks, but their visceral branches partly function as branchial vessels, and so give rise to an arrangement which is very different from that of the majority of Termes.

$$
\text { \$ } 140 .
$$

The vascular system of the Gephyrea presents characters which, not only in their relations to the circulatory system of other Vermes, but even when we compare their various conditions with ono another, are by no means easy to understand; at the same time there are many important lacune in our knowledge of the anatomical facts. 'T'he most important of these is the question whether' there is any connection between the cavitios of the rascular system 
and the cœlom; the only character which points to the existence of one is the nature of the perienteric fluid.

The essential arrangement of the course of the circulation is determined by the presence of two longitudinal trunks, which correspond to the principal trunks already noted in the Annelides. The ventral one runs along the wall of the body, while the dorsal one is attached to the enteric canal, and accompanies it along its coils and loops. The blood-current has the same direction as in the dorsal and ventral vessels of the Annelides.

The two vessels are at their simplest in the young stages of the Sipunculidx. They appear to be connected with one another around the mouth, where they also communicate with the cavities of the tentacles. At the posterior end of the body a number of actively contractile cæca are connected with the dorsal vessel. In Sternaspis these cæca have a special significance, for they are divided into two tufts which project to the exterior and serve as branchiæ. In the Sipunculidæ similar, but internal, appendages are distributed along the whole dorsal vessel. The dorsal vessel is coiled in Sternaspis, Bonellia, and Echiurus. Where the tentacles are absent it is continued into the ventral vessel by a vascular loop which surrounds the mouth, and which is sometimes broken up into finer vessels. Owing to the formation in the Bonellix of a large proboscis out of the greatly elongated upper lip, the anterior portion of the vascular system is very much increased in length. The dorsal vessel is continued to the end of the proboscis, and divided into two branches, which pass along its edges and again meet in the body below the mouth. In Echiurus this arrangement is wanting, as is the proboscis. The ventral vessel formed from the union of the two vascular loops in Echiurus and Sternaspis, passes backwards, giving off numerous lateral branches as it does so. In Bonellia it divides shortly after it becomes a single vessel behind the mouth; but later on it again becomes single. It gives off visceral vessels in Echiurus and in Bonellia; these, of which there is a large number in Echiurus, run in the mesentery. In Echiurus the most anterior of these vessels forms a considerable enlargement on the enteron, whence a ventral enteric vessel, and two anastomoses, which embrace the enteric tube, are given off to the dorsal vessel. This is clearly a mode of connection of the dorsal and ventral vessels similar to that which is repeated several times in the segments of an Annelid. In these forms the arrangement is limited to, or greatly developed at, one point. The variations from the Annelid type are caused by the distance of the alimentary canal from the ventral median line, in consequence of which the anastomosis is not paired, but arises as a single vessel from the ventral trunk. In Bonellia further changes are noticeable. The transverse anastomosis to the dorsal trunk running along the intestine is developed, on either side, into a large tube, from which the dorsal vessel appears to arise anteriorly, since its posterior portion is either wanting, or has become very small in comparison with the widened anterior part. In this 
character also distant relations to what obtains in the Annelides are expressed. The most important difference consists in the limitation of the transverse anastomoses, which surround the intestines, to one only, which is consequently very much modified. Here, then, again the characters exhibited are in correspondence with a rudimentary metamerism. More or less extended portions of the vessels serve as organs for the movement of the blood, and are very different in various genera.

\section{$\S 141$.}

The nutritive fluid forms the contents of the colom, as well as of the vascular system; its form-elements are generally slightly differentiated cells. When the vascular system is marked off from the colom its contents are known as blood. The form-elements in many Amnelids are colourless. In many Nemertina the blood-cells have a red colour (Borlasia) ; the fluid is coloured in many Annelids, and this colour is occasionally green, but more frequently red. In several cases the form-elements contain the colouring matter. 'The plasma also may be definitely coloured, as, for example, in the Lumbricidæ. When the vascular system is separate, the contents of the body-cavity are generally indifferent; we then find a perivisceral fluid (known also as chyle) in addition to the blood; this is always uncoloured. When the vascular system is atrophied, the fluid filling the cœlom is frequently of a red colour (Glyceridæ), like the blood of other forms.

\section{Excretory Organs.}

\section{$\$ 142$.}

It is quite uncertain what is the functional significance of a very large number of organs comprised under this head; of others, however, it is certain that their secretion is essentially similar to that of the renal organs of higher animals. But they all have a number of common relations to the organism, which are of weight, although the organs are so differently connected that it is impossible to completely prove their homology.

In its more developed forms the excretory apparatus is a system of simple or branched canals, which opens to the exterior on the surface of the body, and is provided with internal orifices when a colom is distinctly differentiated; when it is not, the ends of the tubes, or the finest branches of the canals, are closed. When the body is not segmented there is a single pair of these organs; when metameres are formed this system is segmented. 'The indifferent condition of the excretory organs is represented by a pair of crecal tubes, differentiated from the integument, and therefore derived from the ectoderm. Organs of this kind, which open behind the head, are known in the Nemertina, but the canal system said to 
be continued from them requires to be more closely examined. The relations of the canals, which are known as the water vascular system, are more exactly known in most Platylhelminthes. They have not been observed in the land Planarians. In the Trematoda, and many Turbellaria, two excretory canals, one on each side, ramify in the body; the chief trunks giving off fine branches which traverse its parenchyma (Fig. 80, $A B$ ). Long cilia are
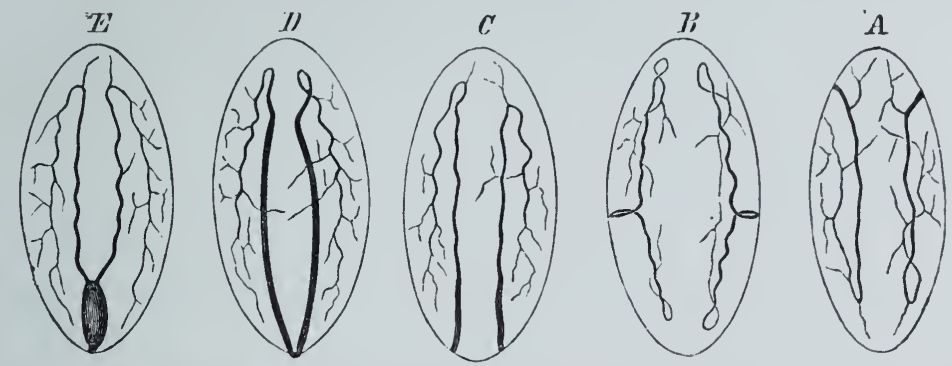

Fig. 80. Diagram of the excretory organs of Platyhelminthes, showing the different forms, which may be derived from one another.

distributed on the walls of the fine canals. The principal trunks, which are as a rule somewhat enlarged, still open in many forms in the anterior region of the body (Fig. 80, A) (Tristoma papillosum). The orifice (porus excretorius) is most commonly placed towards the posterior region $(D)$, where the two vascular trunks approximate and unite at a common orifice. In this way a terminal tract, common to both canals, is formed. This tract, which is generally enlarged, has the form of a contractile vesicle (E). Vesicles of this kind may be formed even on trunks which open separately. They form a third division of the apparatus.

In the Cestoda, the fusion of the excretory canals into a single porus excretorius, placed at the end of the scolex-body, which in the other Platyhelminthes is only an acquired character, has apparently become typical. A contractile vesicle generally forms the point of union. There are generally a larger number of principal trunks- that is four, six, or eight-which either unite with one another by loops in the head, or merely curve round it, and passing backwards branch again; in this case their more special characters are similar to what are found in other Platyhelminthes. When the scolex-form is divided into metameres, the terminal portion of this canal system is apportioned to the oldest proglottid, and the succeeding proglottides contain portions of the canals. At the termination of the metameres the longitudinal trunks are, in many forms, united by a circular canal. As the proglottides break off, a new porus excretorius must be formed every time.

That part of these organs, which is formed of the finest canals, contains a clear fluid only. In the Tape-Worms, on the other hand, there are calcareous concretions at the enlarged points, which 
appear to be excretory products. In the Trematoda these concretions are collected into the large trunks, which contract and drive them into the terminal vesicle, whence they are evacuated by the porus excretorius.

An anastomosis of the finest ramifications of the canals may be often made out in the Cestoda, as well as in the Trematoda (Distoma dimorphum), and may even affect the larger vessels, which either become connected in a simple manner (into a ring in Distoma rhachiæum, and into regularly succeeding transverse canals in many Cestoda); or become converted into a rich meshwork, in which the chief trunks disappear.

The excretory organs in the Nemathelminthes, which again may be derived from a cacal tube, are simpler in character. They form tubes or canals, which pass along the body embedded in the lateral arex (Fig. $61, A v$ ). Near the fore-g'ut the canals of either side bend towards one another, and unite into a common portion of varying length, which opens to the exterior by a pore in the ventral line. Sometimes these canals are coiled, and they vary greatly in the way in which they are connected with the pore. In the Gordiacea

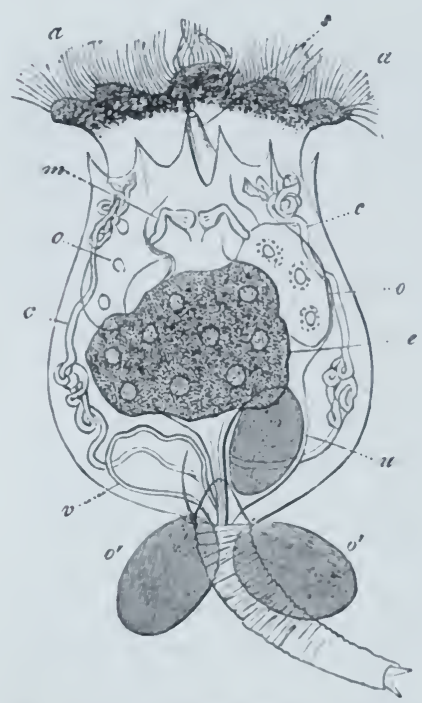

Fig. 81. Organisation of a Brachionus. a Ciliated cephalic disc. s Siphon. m Masticatory organs. $e$ Glandular layer in the stomach. o Ovary. $u$ Uterus, containing an egg. o' Eggs, attached to the root of the tail. e Excretory canals. $v$ Contractilo terminal resicle. this apparatus appear's to be rudimentary, for in Mermis it is simply represented by a row of cells, and Gordius, in which there are no lateral tracts, has no distinct organ of this kind.

It is doubtful whether the organs found in the anterior region of the body in the Acanthocephali, which are known as "lemnisci," belong to the excretory system or not. 'They form two longish lamella, without a lumen, which are processes of the body-wall, and like it are provided with branches of canals, between which dark granular masses occur.

\section{$\$ 143$.}

When a colom is formed, the characters of the excretory organs are so far altered that the canals communicate with it by internal ciliated orifices. This new condition must be regarded as nothing more than a modification of the blindly-ending canal system, as it has been already described in the Platyhelminthes. Internal openings have been obsersed in the larra of Trematoda. They characterise also the excretory canal system of the Rotatoria, which is disposed in just the same way as in the Trematoda. 'The canal system, which lies in the 
cœlom, or projects into it from the wall of the body, is composed of two trunks (Fig. 81, c), which in many forms open by lateral branches into the colom (species of Notommata). The two principal canals, which are often much coiled, either unite at the cloaca, and open by it to the exterior, or they pass into a contractile vesicle $(v)$, which must be regarded as a differentiation of the common terminal portion of the two canals. The internal openings, as well as the lumen of the two chief trunks, are here and there provided with flagelliform cilia, which produce a trembling movement. The walls themselves may be seen to be glandular in character, either along the whole length of the canal, or in certain portions only of it. In this last point a further development of the simpler characters found in the Platyhelminthes ought very probably to be recognised, which points to a closer relationship with the Annelida.

Echinoderes also possesses two coiled excretory tubes, but they appear to open separately in the anterior region of the body.

\section{$\$ 144$.}

In the Gephyrea we have to distinguish two kinds of excretory organs.

The first of these is an indication of the comnection between the Gephyrea, and lower stages, for it is correlated with the undeveloped-or if developed, developed only externallymetamerism of the body. These organs are formed by tubes which open into the termination of the enteric canal (Fig. 72, g), and are provided-at any rate in the cases where they are most exactly known (Bonellia) - with numerous ciliated funnels, which open into the colom (Fig. 82, a). In other cases the branches with internal openings seem to be wanting (Echiurus), and in others, again, the degeneration is complete. As similar characters may be seen in the Echinoderma, the form of excretory organ, which obtains in the Gephyrea, appears to be common to a large circle, and referable to a single typical form, whence it has been transmitted to the Echinoderma, as well as to the Gephyrea. Variety in the function of this organ may be inferred from its structure. The excretory

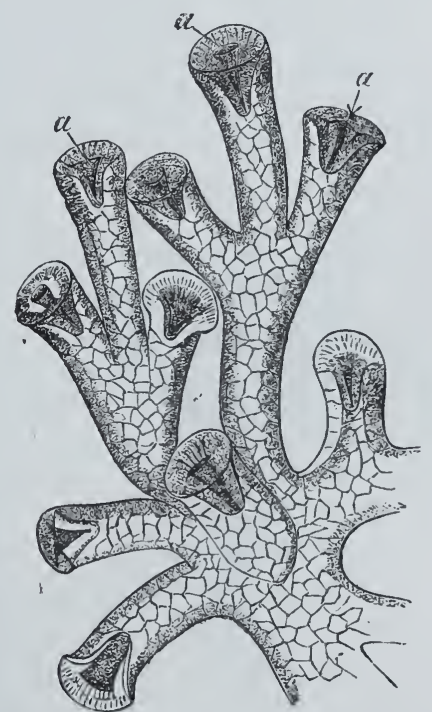

Fig. 82. Portion of a branch of the excretory organ of Bonellia viridis. a Ciliated openings (after Lacaze-Duthiers). function is certain only in Bonellia, where the walls of its branches have a glandular character.

The other form consists of paired tubes, opening on the ventral 
surface, which may be derived from the paired organs of similar form in the Annelides. There is either a single pair (Sipunculus), or a few pairs (Thalassema, Sternaspis, Echiurus), in correspondence with the slight development of metamerism. Internal openings into the body-cavity lie close to the insertion of the tubes into the body-wall, and in several are of service in the generative function, acting as the ducts for the generative products. The greater part of the tube, that is the cxeal portion, which lies behind the internal orifice, appears to hare an excretory function in the Sipunculida, and as a rule, is distinguished by its brown colour. In others the whole tube serves merely as a duct for the generative system. In most Gephyrea these organs hare each and all similar functions, but in some cases there is a division of labour (Sternaspis), the posterior pair of tubes being in connection with the generative, and the anterior with the excretory, function; so that the difference of function which appears in different genera, is here expressed in the same individual.

\section{$\$ 14.5$.}

So far as the structure of the excretory organs is concerned, ferw fresh characters appear in the Annulata. The organs correspond to the metamerism of the body, for they are regularly distributed on either side of almost every one of its segments. 'They have therefore, though with but little reason, been called "segmental organs;" a name which is just as suitable for many other organs. Each of them consists of a closely-coiled or loop-like canal (looped canals),

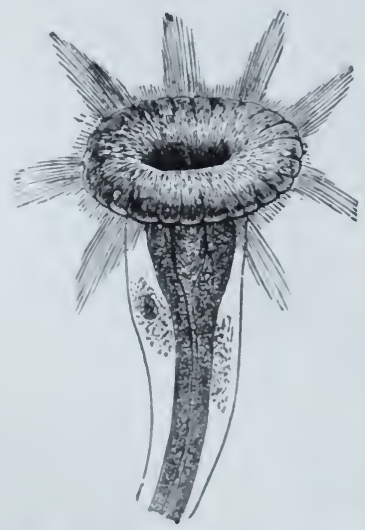

Fig. 83. Internal opening of a looped canal of $\mathrm{Br}$ a $\mathrm{n}$. chiobdolla. which has an internalopening, often peculiar in form and always ciliated, and which at the other end opens on to the surface of the body. 'This canal is sometimes similar' in character throughout its whole length, or but slightly differentiated; frequently several segments may be made out in it, which generally correspond with those already described in the Platylelminthes and Rotatoria. The innermost portion, on which the opening into the coclom is placed, is ordinarily the longest; it is distinguished by its fumnel-like or rosetteshaped orifice (Fig. 83). In the next portion the walls may be seen to be glandular in structure. 'The last portion, which is frequently widened, is provided with a layer of muscle; it almost always opens on to the rentral surface. These organs are no more purely excretory in function than they are in other Vermes, for we not unfrequently find them entrusted with other functions. 
In the Hirudinea these organs are preceded, in the embryonic stage, by three pairs of looped canals, which are not connected with those formed at a later stage; they are found in the posterior half of the ventral surface. In structure they are similar to, but simpler than the permanent canals, and disappear after these are developed. 'This most important fact shows that the looped canals of the Annulata cannot be regarded as the direct homologues of the excretory organs of the lower Vermes; at the same time arises the question: Are the looped canals of those Annulata, which show no signs of primitive organs of this kind, comparable to the permanent looped canals of the Hirudinea, or only to the primitive ones?

In their more special characters there is great variety among the Hirudinea, the canals in one division having no inner opening. Instead of this they begin with a closed portion, of the form of a loop, which consists of numerous canals, united with one another into a labyrinth (Hirudo). From these looped organs a single canal is given off, which opens by a vesicular enlargement on the surface of the body (v. suprà, Fig. 61, B). In others (Clepsine, Nephelis) the labyrinthine portion is present, but it has an internal opening, which projects into the lateral blood sinuses of the body.

The division of the Limicolæ is remarkable among the Scoleina, because of the two different conditions of its looped canals. Usually the canal is much-coiled, and the greater part of it is embedded in a common mass of cells, and has much the same calibre throughout its course. The canals always break through the dissepiment in front of them by the end which carries the internal orifice, so that each pair of looped canals is connected with two segments of the body. The portion which leads to the exterior lies in one, the internal orifice in the other. This form, which is distributed in much the same way through the greater number of the segments, is wanting in the tracts occupied by the generative system. In place of the simple looped canals we there find organs of greater complexity, and proportionately much more developed; in structure they resemble the others, but they function as organs for the excretion of the sperm; looped canals are converted into seminal ducts. The same thing happens in Branchiobdella.

In the Lumbricidie there are none of these physiological changes. But the apparatus is greatly complicated by the marked development of its separate divisions, as well as by the arrangement of the loops. Each canal forms several ascending and descending loops, closely connected with one another, and surrounded by a rich network of blood-vessels. The different portions have different significations, The innermost portion carries the funnel-like opening (Fig. 84, $a$ ), and is followed by a part $(b b b)$ which has transparent walls, and is provided with cilia at various points. After forming several loops, this portion passes, by a change in its walls, into another portion, the lumen of which is widened $(d)$, and surrounded by cells with finely granular contents. This portion also has a looped course $(d)$, and passes into a wider part, which is provided with muscular 
walls $(c)$; this, after a simple course, passes to the body-wall $(c)$, where it finds its opening:

In the Chretopoda simple forms of looped canals are most

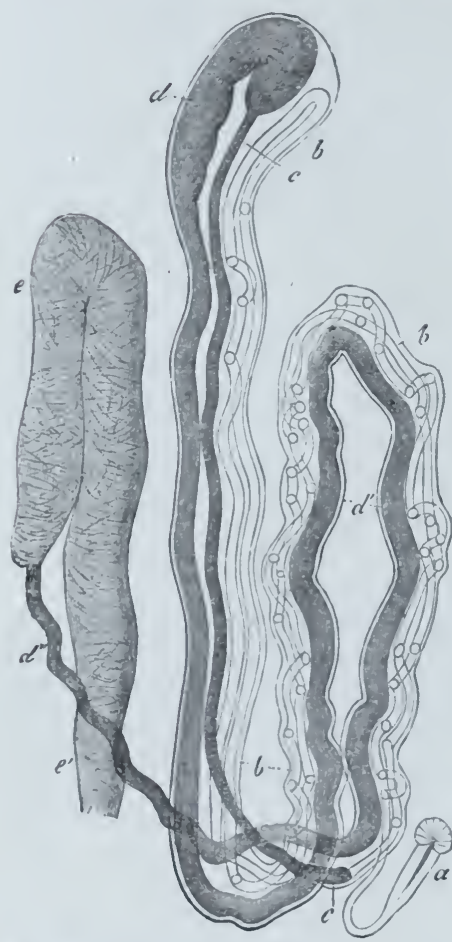

Fig. 8t. $\Lambda$ looped canal of $\mathrm{Lnm}$. bricus-not too highly magnified. $a$ Internal opening. $b b b$ Clear portion of the canal, arranged in two double loops. c c Narrower portion with glandular walls. $d$ Widened portion, which becomes narrower at $d^{\prime}$, and at $d^{\prime \prime}$ is continued into the muscular portion, e. $e^{\prime}$ External opening. common, the separate canals in them forming sometimes coiled bodies, and sometimes presenting a very few coils. The funnel-like internal opening, which has been recognised in many, has in somo (Alciopa) just the same relations to the septa of the coelom as it has in the Scoleina. In many of them the relation to the generative system can be similarly recognised.

In addition to the more secondary relations which the looped canals of the Annelides have to tho generative system, either at certain points only, or for a greater distance, their relation to the excretion, as well as to the introduction or expulsion, of water must be borne in mind. That these organs have a close relation to the function of excretion is shown by the glandular investment of their walls, and the glands which open directly into them. 'They, in fact, resemble the chief truuks of the excretory organs of the Trematoda. A relation between the perienteric fluid and the surrounding medium, either by the outflow of the former, or the entrance of the latter, is rendered possible by the internal opening of the looped canal. From the direction of the ciliary movemont in the canals, and at their internal orifices, which is in nearly all cases towards the exterior, it is probable that solids also may be moved in this direction. Further investigation, however, is necessary in order to confirm this supposition.

\section{Generative Organs.}

$\$ 116$.

Wo meet with a larger number of intermediato steps in the sexual differentiation of the Vermes than in that of any other division. The lowest stages are hermaphrodite, but this arrange- 
ment is not unfrequently connected with great complications in comparison with which the arrangements seen in diocious Vermes are very simple.

The simplest state is seen in the Bryozoa, the generative pro-ducts of which are developed either on the inner face of the bodywall from simple aggregations of cells, which give rise to seminal elements or to ova; or they arise on a chord which extends from the enteric canal to the inner wall of the body (funiculus) (Fig. 71, n). The mature generative products pass into the cœlom, and are thence passed out into the surrounding water by the orifice of communication mentioned above. The two sexes are ordinarily united in the same individual, the male and female germinal glands being separate.

In all phylactolomatous fresh-water Bryozoa, special bodies, (statoblasts) formed of an aggregation of cells, are developed in the body-wall, at the points where the ova are formed; these break off, just like the ova, and form free-living buds. Various differentiations give rise to complicated shell-structures around them.

\section{\& 147.}

Hermaphroditism obtains also in the Platyhelminthes generally ('Lurbellaria, Trematoda, Cestoda). The two groups of sexual organs are, as a rule, united at a common orifice, being otherwise separated from one another, and embedded in the parenchyma of the body. The secreting glands (testis and ovary) are generally small and simple in character. The excretory duct and the glandular organs connected with them, as well as the diverticula, or pouch-like appendages of the former, which act as places for the development of the fertilised ovum, or as receptacles for the semen, take by far the greatest share in the complication of the apparatus.

As to the male organs, the testes vary in number, and are generally indistinctly marked-off spots for the formation of the semen, which reaches the common duct by narrow seminal ducts. A widened portion of the former has the function of a seminal vesicle, and its end is converted into a protractile organ, which serves as a penis.

The ovary forms the most important part of the female organs. An organ, generally widely branched, is connected with its ducts - the yelk-gland: in the lobules of this gland cells are produced. The cells of this gland are used in building up the embryo, a number of them together with the egg-cell forming the egg. The origin of the yelkgland is probably to be found in the division of labour of a primitively very large ovary, a portion only of which has continued as ovary, while the cells of the other parts have ceased to be ovarian germs, but becoming surrounded by the products of the fission of the egg-cells, are taken into the future body of the embryo. The oviducts and the ducts of the yelk-gland unite in a canal of varying length, which, according to the number of eggs to be developed, is 
either extraordinarily long, or short and simple, or provided with diverticula. These cavities are known as uteri ; for in them the egg is not only enclosed in its shell, but as a rule passes through the early stages of embryonic development. A diverticulum of the female excretory duct, which has generally the form of a stalked vesicle, receives the sperm during copulation. In some cases there is a second diverticulum also ; it serves apparently for the reception of the male organ (Bursa copulatrix).

The most important complications of this system are seen in the parasitic Platyhelminthes. The preservation of the species is here subject to innumerable difficulties, owing to the animal living in different hosts at different stages of development, and to the wanderings which this mode of life entails; consequently a large number of ova have to be produced, and the certainty of fecundation insured.

\section{$\$ 148$.}

The more special characters of this generative system exhibit

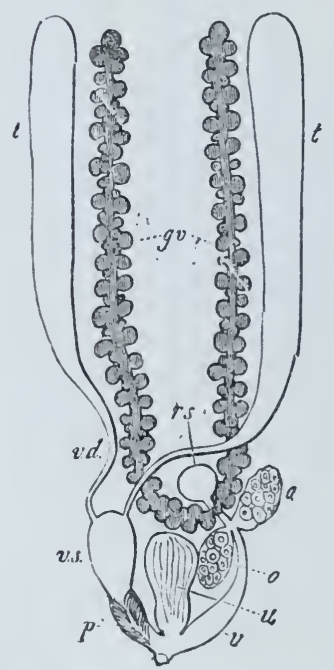

Fig. 85. Generative system of $\mathrm{V}$ ortex viridis. $t t$ Testes. $v d$ Vasa deferentia. is Se. minal vesicle, $p$ Protractile organ of copulation. oo Ovaries. go Yolk-glauds. r's Receptaculum seminis. $v$ Vagina. $\iota$ Uterus (after M. Schultze). The copulatory organ is, as a rule, protractile, or can be everted, rhereupon certain spines or hook's which lie on its inner surface, 
in its retracted state come to lie on its outer surface. Most Platyhelminthes, except the Planaria, have the penis thus armed; it appears to be connected with a more intimate copulation.

\section{$\S 149$.}

There are greater variations in the female apparatus. The ovaries are, as a rule, one or two elongated tubes of no great size (Fig. $85, o ; 87, o v)$, in which the ovarian germs are formed. Where a single oviduct is present it becomes connected with accessory parts as it passes to the generative pore, and varies in length. Several such may unite together and form a common oviduct. In most Rhabdocœla, as in the Cestoda (Fig. 87, odl) and Trematoda, the duct is single, though the ovaries are

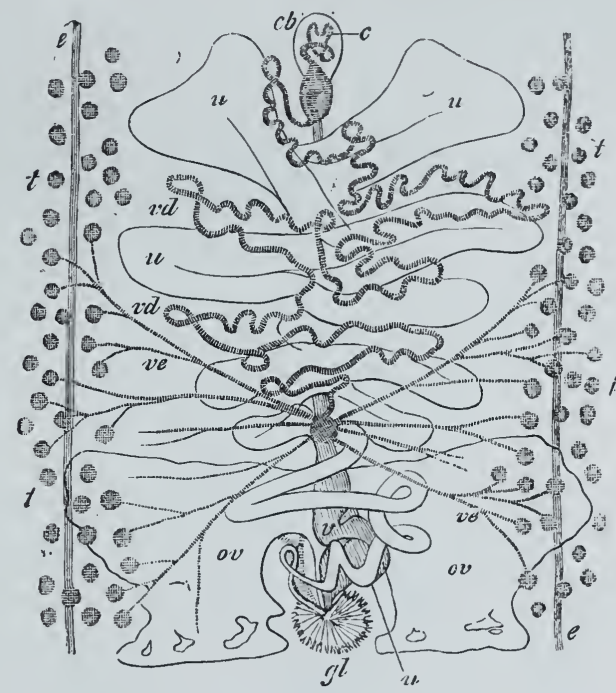

Fig. 86. Male apparatus, with parts of the female, of Bothryocephalus latus (after Landois and Sommer). a Testicular follicles: part only are represented. ve Their excretory ducts. $v d$ Vas deferens. $c$ Cirrus. $c l$ Bag of cirrus. Other letters as in Fig. 87. double. It is shortest in

the Rhabdocœla, where, as in most of the Cestoda, it has an enlarged portion, which is clearly a receptaculum seminis. This organ appears as a unilateral diverticulum of the oviduct, and gradually becomes distinct. It is still more well-marked when it is attached to the base, or along the course of the oviduct (Fig. 85, $r s$ ), in the form of a stalked appendage. The Planarians have a double oviduct; as a rule, a short portion only is common to both ducts, and functions as uterus or vagina. The oviducts are of some length in the land Planarians, the ovaries of which lie in the most anterior parts of the body. They may be provided with short lateral branches along their course, which open into lacunar spaces of the cœlom (Bipalium). This peculiar character raises the question as to whether these ciliated oviducts are parts of another system of organs; for there is no reason for supposing that ovarian tubes have degenerated so as to form these backwardly-directed lateral branches. Their open mouths forbid us to suppose that there has been any such process. These mouths, indeed, point to an excretory organ having here entered into the service of the generative function.

When yelk-glands are connected with the ovary they appear as two or more arborescent, ramified, or lobate organs (Fig. 73, gv), and are often widely spread out through the parenchyma of the 
body (Fig. 87, $l$ ). Their excretory ducts come together from all sides and form with the oviduct a single common portion $\left(l^{\prime}\right)$.

Special portions of the oviduct function as a Uterus, by which name parts, very different morphologically, are known. In general

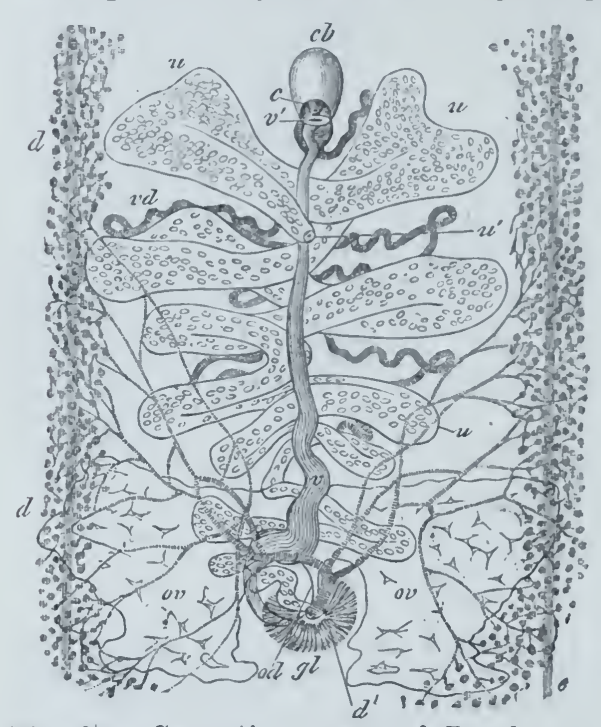

Fig. 87. Generative organs of Bothryo cephalus latus (after Landois and Sommer). Female portion of the system. $v$ Vaginal canal. $v^{\prime}$ Its mouth. $u$ Uterus (with ora). $u^{\prime}$ Its mouth. ov Orary. od Oriduct. gl Shell. glands. I Yelk-glands (part only is shown).

d' Duct of yelk-gland. $e$ Vascular trunks.

three different kinds of such uterine organs can be made out in connection with the oviduct. In the first the oviduct itself is used for this purpose; and then it is not only widened, but also greatly elongated, so that it has the form of a coiled tube, which traverses the body several times. This arrangement is found in the Trematoda, and also among the Cestoda (Trianophorus, Ligula, Bothryocephalus) (Fig. 87, u). A second form is represented by lateral diverticula, or pouch-like appendages on the courso of the oviduct; this is found in a fow Rhabdocola and, in a more complicated form, in most 'TapeWorms. In the 'Truniada a tube passes from the duct, near the opening of the yelk-gland, along the middle line of a sexually-mature proglottis, and forms, according to the quantity of ora in it, a number of arborescent branches. Finally, a third kind is formed by appendages, which are found only on the end of the oviduct, or rather in the vestibule common to the organs of both sexes, and close to the genital pore. This occurs in most Turbellaria (Fig. 85, $\iota$ ) ; in the Rhabdocœla there are, as a rule, two such nterine pouches, which are considerably distended, and which may be branched, if they have to serve for the reception of a large number of ova. In the Dendrocola there is either only one such uterus, opening into the vestibule, which in them is greatly distended; or it is altogether wanting, when the two oviducts take on its function (Leptoplana). The size and the number of the ora, which become mature and get their envelope at one time, is always in close connection with the condition of the organ which acts as nterus.

$\Lambda$ terminal portion of the oviduct is likewise frequently differentiated into a special canal, known as the "vagina;" in some cases this is further provided with an appendage which has the function of a "bursa copulatrix." 
A large number of unicellular glands are attached to the point where the ducts of the yelk-gland and the oviduct unite, in Trematoda (Distoma, Polystomum, Amphistoma) and Cestoda (Bothryocephalus, Tænia). This group of glands is known as the shell-gland, and its secretion serves to form the investment of the ova (Fig. 87, gl). In addition there is in the Bothryocephali and many Trematoda a special canal at this point, which opens either into the sinus genitalis, as in the former, or on the dorsal surface of the body, as in Distoma hepaticum: it evidently functions as a vagina, as it has been found full of sperm (Fig. 87, v). This second communication between the female organs and the exterior makes it possible for impregnation to take place without interfering with the gradual evacuation and deposition of the ova. In this double opening of the female organs we may perceive an indication of the primitive double nature of the whole system.

The influence of a change in the external conditions of life upon the genital apparatus in the case of Polystomum (P. integerrimum) furnishes an instructive example of the capacity for adaptation of an organ in full function, and therefore presumably mature. The change is one of abode, and results in an increased production of generative matters, and the correlative appearance of new parts in the generative apparatus.

\section{$\S 150$.}

We know as yet very little as to the way in which the hermaphrodite apparatus acts in copulation. In many cases there are arrangements favourable to self-impregnation.

The genital pores differ in position in the various divisions of the Platyhelminthes. In most cases the generative organs open in the ventral median line, sometimes very far forwards, just behind the oral sucker, as in many Trematoda (Distoma, Gyrodactylus, etc.), and sometimes nearer the hinder end of the body (Turbellaria), or even quite at that end (Distoma macrostomum). Among the Cestoda also the ventral pasition is not uncommon (Ligula, Bothryocephalus); in most cases the genital pore is a flattened depression on the lateral edge of the proglottides, placed alternately on one and the other lateral edge. The fact, that in some Cestoda (Tænia elliptica, T. cucumerina) there are two symmetrically-disposed generative systems in each proglottid, is important as bearing on the meaning of this want of symmetry, which obtains even in some Trematoda (Tristoma). The unilateral condition may be regarded as the remains of a primitively double arrangement; it was, then, only gradually that the system of one side got to predominate over the other, and led to that relation of parts which is now the most widely distributed-the unilateral development of the generative system.

There is, with but few exceptions, only a single genital pore in the rhabdocœlous Turbellaria, the male and female organs both leading to it. In the dendrocœlous forms the orifices become 
separated, owing to the development of a vestibule. In most of the marine Planaria they are completely separated, and there is a double genital pore, the male one lying in front of the female. In most of the Trematoda, too, the openings of the genital organs are distinct, although placed close to one another. The same arrangement is seen in the Cestoda. Even in those cases where the bag of the cirrus and the vagina open into a genital pore, this latter is only a flat pit, walled in by the integument. In other cases the two pores open directly on to the surface, though close together. Or we have the noteworthy provision of a second female opening, with a vaginal cluct, as already described. Finally, the two apertures may be still more separated, the male organ opening on the lateral edge, and the female on the surface of the proglottid.

The two kinds of organs are sometimes unequally developed in one and the same individual; in the Rhabdocœla especially the sexes are separated, the two organs being unequal in different individuals; in one the female, and in another the male organs are most developed, while the organs of the other sex remain rudimentary (Convoluta). These important examples show us how, by the continued atrophy of one organ, diøcious forms are derived from hermaphrodite organisms. T'he process here observed in statu nascenti is complete in other

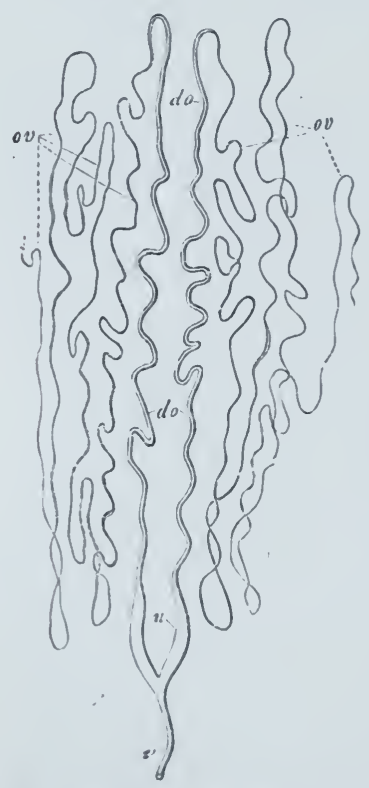

Fig. 88. Female generative orgaus of $\Lambda$ scaris $l u m b r i$. coides. on Ovaries. do ()viduct. u Uterus. "Vagina.

Turbellaria. The Microstomee have the sexes separate, as hare also some Planarie and Trematoda. The generative system is simplified in the Nemertina, which are almost always diœcious. The various divisions of the excretory ducts and of the accessory organs are absent. The testes and ovaries are the only parts which are distinctly recognisable. In some (Prorhynchus) these organs occur singly in each individual (Fig. 67,ov), and so call to mind the rhabdocœlous 'Turbellaria. In others, however, there is a large number of follicles on either side of the enteric canal; they have no direct connection with one another, and being arranged regularly in pairs along the body are evidence of metamerism.

\section{$\S 151$.}

In the Nematodes hermaphroditism is a rare exception. As a rule the sexes are separate. Both kinds of organs consist of tubes cmbedded in the colom and opening on to the surface. The organs of the fernale system are very generally paired. This is less common in the case of the male organs. The double opening observed in some few cases also speaks to the primitircly 
double character of these parts. But this would also be the result if the two tubes were separated from one another in adaptation to the elongated form of the body. The blind terminal portion of the generative tubes serves as an ovary or testis, the remaining portion as an efferent organ, adapted to different arrangements in different parts, and variously differentiated.

The male generative tube is a simple one, opening on the ventral face of the hind-gut; in the larger species it is several times coiled. It is only by its epithelial investment that the terminal portion, which is generally of some length, is distinguished from the excretory duct, and seen to be a testis; a widened portion, or seminal vesicle of the ductus ejaculatorius, is sometimes added to the duct. Two fine, and sometimes very long, chitinous rods (spicula) are developed in the cloacal portion of the hind-gut, and serve as copulatory organs.

The female generative tubes are, as a rule, double, and are either separate, except at their orifice, or have their terminal portions united into a common tract. The tubes are more or less coiled, according to their length. The terminal portion is to be regarded as an ovary (Fig. 88, ov), from which a portion which is ordinarily wider leads into a canal known as the uterus $(u)$, and this opens by a narrow vagina. The female generative pore is always ventral in position, in front of the anus, and generally about the middle of the long axis of the body. The increase in number of the female generative tubes to five, with the atrophy of one of the two primitive ones, gives rise to changes in the form of the apparatus: this is more marked when particular regions are specially differentiated. The same thing happens to the male organs. In some cases the terminal portion of the ovary functions as a yelk-gland (Leptodera).

Mermis, at any rate, among the Gordiacea, resembles the other Nematodes in the characters of its generative organs. In Gordius the excretory ducts of the paired germ-glands are, in both sexes, united with the hind-gut. This happens in male Nematodes only.

In the Chætognathi (Sagitta) the arrangement is somewhat different; owing to their hermaphroditism, and the way in which the organs are dis-

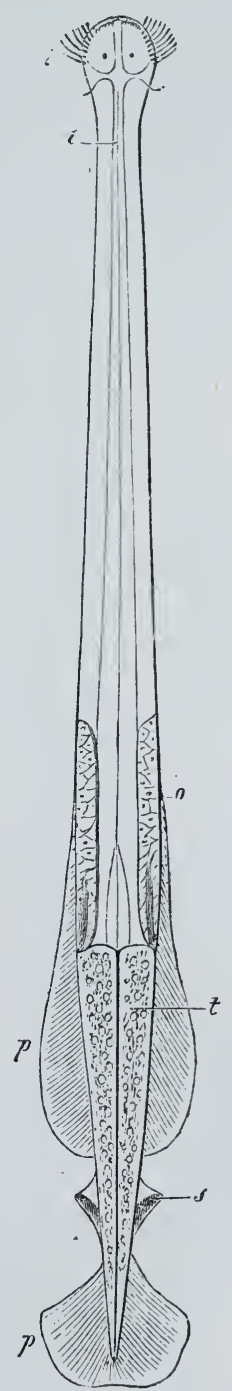

Fig. 89. A Sagitta with its generative organs. $o$ Ovary. $t$ Testes. $s$ Seminal vesicles. $i$ Enteron. $p$ Fins. posed, we cannot compare them very closely with other divisions. 'The male and female generative glands lie at the sides of the posterior ends of the body; the testes lie behind the ovaries, and 
occupy the extreme end of the body. Each opens into a short excretory luct, which is directed forwards, and is continued for some distance on the surface of the body; it is

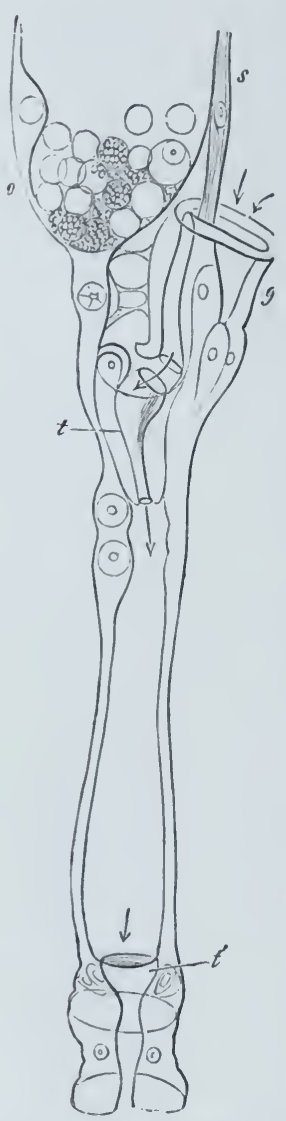

Fig. 90. Posterior portion of the female generative organs of Echinorhynchus. o Orary. s Ligamentum suspensorium. g) Bell-shaped organ. $t$ Funnel. $t^{\prime}$ Terminal portion of the oviduct. The arrows indicato the course taken by the ova, as they pass to the exterior (after Greeff). often found distended with spermatozoa, and so functions as a seminal vesicle. According to the extent to which their contents are developed the ovaries project more or less into the cœlom. They pass from before backwards, and open to the exterior by a short and projecting tube, with which a receptaculum seminis, placed beside the ovary, is connected.

\section{$\S 152$.}

The generative organs of the Acanthocepliali are very peculiar, a higher stage of development being implied by the separation in them of the sexes. A chord (ligamentum suspensorium) traversing the ancnterous colom carries seminal organs in the male, and ovarian organs in the fomale. The testes are two rounded glands, which lie one above the other; a vas deferens passes off from each of them to the posterior end of the body, where it opens, in company with a number of tubular glands, into the copulatory organ. This consists of a sucker-like structure, in the centre of which lies a conical processthe true penis. This apparatus can be protracted and retracted. In copulation it embraces the similarly-shaped posterior end of the female's body. The ova are dereloped in an orary ( $\rho)$ which accompanies the chord-like axis (Fig.90,s), and is either placed on, or is partly enclosed by, it. The ova escape into the coelom, and are taken up by the mouth of a wide bell-shaped organ $(g)$, which projects inwards from the hinder end of the body, and leads into the short uterus, which opens externally by a narrow vagina.

\section{$\S 153$.}

In the arrangement of their generative organs the Hirudinea closely resemble the Platyhelminthes, and especially the 'Trematoda and Turbellaria dendrocœla. This does not apply only to their hermaphroditism, but to the donble character of their germ-glands, which are ordinarily arranged symmetrically; and also to the position of the orifice of the whole system, which is placed in the ventral median line. The position of the male genital pore in front of the female is a repetition of the 
arrangement which obtains in the marine Planarians. There is always a larger number of germ-glands ( $5-12$ pairs : $t)$ for the male organ (Fig. 91); they correspond to a number of metameres, are rounded bodies, and are arranged serially on either side. A duct leads from each to a laterally-placed vas deferens $(v d)$, which widens internally, and becomes much coiled in front of the first pair of testes $(v s)$. From this coiled portion a terminal piece, accompanied by its fellow of the opposite side, passes to the genital pore. A large number of glandular tubes $(g)$ join the united efferent ducts, and not unfrequently form, as in the Planariæ, a large mass of acini (Clepsine). Either the two terminal tracts of the vas deferens function as copulatory organs, and, together with a portion of the gland which surrounds them, project from the body in the form of a vesicle (Clepsine, Piscicola); or there is a special copulatory organ, which receives the ends of the seminal vesicle. In this case (Sanguisuga, Hæmopis, etc.) the portion formed by the union of the two seminal ducts is developed into a strong muscular organ $(p)$, the thinner end of which forms a short penis. As in the Planariæ and Trematoda, this lies in a penial pouch, which opens at the genital pore, and from which it can be protruded during copulation.

The female organs, also, of the Hirudinea resemble in many points the organs of several Platyhelminthes (marine Planariæ). The ovaries, which in the latter are scattered through the body, are

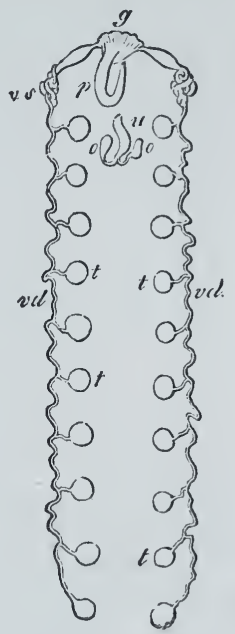

Fig. 91. Generative organs of a Leech. $t$ Testes. vd Vas deferens commune. vs Coiled portion of the seminal duct, analogous to a seminalvesicle. $p$ Penis. $g$ Glands. $o$ Ovaries. $u \mathrm{Va}$ gina. here formed by two rounded, tubular, or lobate organs (o), which lie near the middle line of the body, just behind the male efferent organs. In some they open, merely by a short oviduct, at the female genital pore (Leeches with a proboscis). In others the efferent ducts are separated. The narrow oviducts give rise to a longer common tract (Hirudo). The common duct, the several coils of which are held together by a glandular layer, then widens into a vagina at the terminal portion of the efferent duct $(u)$.

\section{$\S 154$.}

In the Scoleina the organs lie in the anterior metameres, generally occupying the tract between the eighth and fifteenth. Two different types of the sexual apparatus are to be distinguished. One is well marked in the Terricolæ, and is characterised by the independence of the efferent organs. The male part of the system in the Lumbricidæe is formed by two pairs of testes, which are connected with wide sacs, in which the elements of the semen are further developed. 
Each pair of testes has a seminal resicle of this kind (Fig. 92, $s^{\prime} s^{\prime \prime}$ ), which is placed across the middle line, and is further provided with

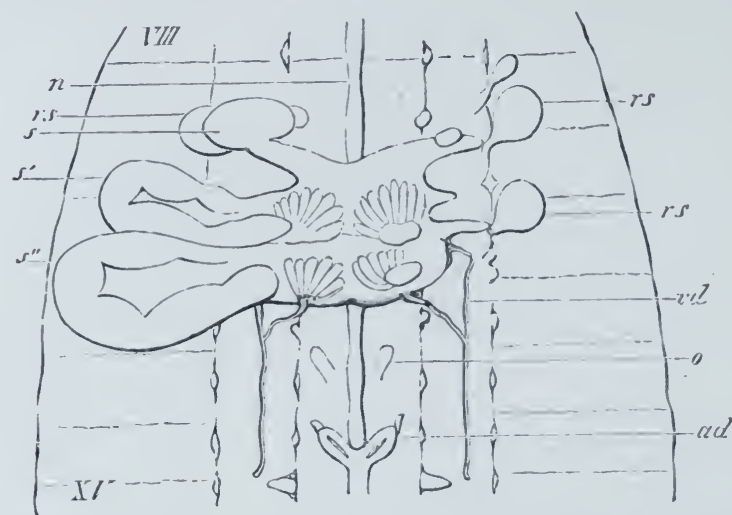

Fig. 92. Generative organs of the Earth-Worm. The portion of the body which contains these organs is opened from above, and the walls are laid out on either side. Segments VIII.-XV. are figured. $n$ Ventral ganglionic chain. $s s^{\prime} s^{\prime \prime}$ Diverticula of the testes. $v d$ Their efferent ducts. o Orary. ad Ovarian ducts. rs Receptaculum seminis (after Hering). lateral diverticula. In each of these, two funnel-shaped organs are placed, which are continued laterally into the seminal ducts. The two seminal ducts of either side unite into a common duct $(v(l)$, which extends backwards, and opens laterally on the ventral surface. In the same metamere there are two protractile copulatory organs, developed from modifications of the setigerous follicles.

The ovaries $(o)$ are the smallest organs of the female portion of the generative system. They lie behind the second pair of testes and on each side of the ventral nerve-chord. Behind them two oviducts $(a d)$ are attached to a dissepiment, and begin by two wide abdominal ostia; they pass to the exterior by a short canal placed in the segment in front of the openings of the male organs. Sereral pairs (generally two) of seminal pouches (receptacula seminis) (rs) are also present. They are large, rounded organs, which lie near the testes, and open by a short duct, without having any close relations to the male apparatus. The paired character of the genital pores, the position of the female one in front of the male, and, finally, the connection between the testes of either side, form an arrangement which, so far as is yet known, has not its like among living allied forms.

Even in the Limicolæe the organisation is different. The two kinds of generative organs, which are liere also present in one individual, have no proper efferent ducts. We may suppose that the system of oviducts, seminal ducts, and seminal vesicles, which is found in the Lumbricidre is not dereloped in them, so that there are only ovaries, testes, and receptacula seminis. So far as we can see at present, some of the looped canals (cf. p. 17 7 ), which in the Lumbricida have nothing to do with the generative system, here form the efferent ducts for the reproductive matters, and mudergo changes in correspondence with this function. Parts of the dissepiments function as germ-glands, on which the generative products when undeveloped generally form paired saccular diver- 
ticula which project far into the cavity of the cœlom, and often extend through several segments. As a rule there are several (as many as four) testes in different metameres. There is generally only one pair of ovaries. As these laterally-placed organs, just like the testes, force their products, when a large quantity is developed, through several metameres, they seem to surround the unpaired testes (Tubifex). When the generative matters break off from their point of origin they float in the cœlom. In some (Enchytræus) a number of ovarian germs break off together in a mass, of which one ovum only comes to maturity.

The efferent ducts for the semen are formed from the alreadymentioned looped canals, a pair of which are, as a rule, converted to this purpose: these generally undergo modifications in size. The funnel-like internal orifice, just as in the case of ordinary looped canals, lies in the next preceding segment. The canal continued from it, which is generally distinguished by its large supply of cilia, passes, after several coils, to the terminal portion, which opens to the exterior; a large lobed glandular organ is attached to it. The terminal portion anteriorly to its orifice forms an ampulla, into which the tube is invaginated for some distance, and from which it may be protracted, so that it serves as a copulatory organ. The excretory ducts of the ova are either special oviducts, similarly derived from mocified looped canals, or they are functionally connected with the seminal ducts. In this case the enlarged terminal portion of the latter consists of a double tube, the internal portion is the continuation of the seminal duct, and the external portion which surrounds it, functions as an oviduct. Branchiobdella also conforms to this type.

\section{$\S 155$.}

The generative system of the Chætopoda is very much like that of the lower division of the Scoleina. Only in a few, however, does hermaphroditism persist, and sexual separation has become the rule coincidently with a more free habit of life. The generative products arise in the walls of the body-cavity, as is also the case in the Gephyrea. As a rule, the places at which the ova or the sperm is developed are merely distinguished by the presence of these products (Fig. 93, o); they have no special characters, and are therefore distinguishable orily at the time when they are functionally active. They are developed at the same points in the same genera or species;

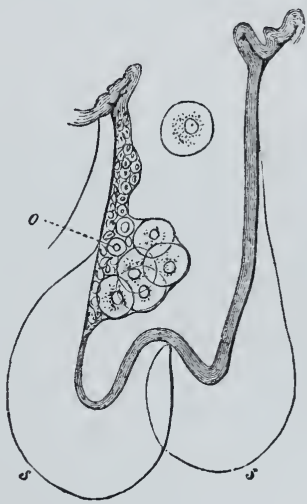

Fig. 93. A parapodium of T o m opteris. ss Scale-like formations of the integument, which arise from two processes, homologous with the ventral and dorsal parapodia of other Annelids. o Ovary : a collection of cells from which the ova are developed. thus, for example, in Eunice they lie at the sides of the ventral nerve-chord. In a few cases they are limited to a small number of 
segments, as in the Scoleina. The generative products formed on the wall of the body break off when they become mature; or they become free whilst still unripe, and float in the colom (Fig. 93), in which they further develop. The looped canals here also serve as efferent ducts for both male and female generative matters; but this matter needs more careful investigation. Even in the Gephyrea, as has been remarked above $(\$ 141)$, the organs which in them are the homologues of the looped canals aid in the generative function, and present still more remarkable modifications; which require to be more closely examined.

A special place must be assigned to the generative organs of the Rotatoria. They have nothing' in common with those of Chrtopoda save their diocious character, and they are distinguished from them, as from all Ammulata, by the fact that they are not metamerically repeated. The sexes differ not only in the characters of their reproductive organs, but also by the rest of their organisation. The males are not only smaller in size, but several of their systems of organs are atrophied, especially the enteric canal. The testis consists of a single tube, which opens at the hinder end of the body ; accessory glandular tubes are sometimes connected with it. In the female, the flattened ovary is ventral in position, and opens into the cloaca by means of a short oviduct. Parts of the duct are widened and serve as receptacles for the egg's; it thus forms a uterus, and in some species the eggrs are developed into embryos before they leave it.

\section{$\$ 1 \check{6} 6$.}

The generative products have the same form in most divisions of the Vermes. The egg is represented by a more or less modified cell. In the Nemathelminthes, the ova are formed in a peculiar manner; they are budded off from a common nucleated chord of protoplasin, which forms the contents of the tubular ovary. $\Lambda$ large number of eggs are produced at one time, while the rest of the protoplasm forms the axis of the chord (the rhachis), and is surrounded by wedge-shaped ovarian buds. The same thing happens in the Hirudinea; the ovaries of Hremopis contain a coiled filament, which corresponds to this rhachis; and on this the ovarian germs are budded. The ova are connected with the filamont by a thin envelope, which is drawn out into a stalk. In Nephelis there is no chord, and the ovarian germs form groups of cells. In all forms which have a yelk-gland it is not the egg-cell only which forms the material for the development of the embryo, but this is supplemented by the products of the yelk-gland-yelkcells (cf. \$147). The structure, therefore, which appear's to be the egg consists in them of a complex of cells, one only of which has retained the value of an egg-cell. In almost all cases they are surrounded by an investment, which varies greatly in character. In some it is a layer of albumen only, in others this layer is surrounded by a hard shell. 
The form-elements of the sperm are generally rounded or elongated bodies, from which a fine movable flagellum projects. The Nematodes again are peculiar in this matter; their seminal elements, like the ova, being budded off from a rhachis. The cells thus formed increase in size, and form cell-like bodies, which perform amœboid movements, but do not develop a flagellum.

In many Annulata the seminal filaments are united into masses of definite form (spermatophores) in special parts of the male efferent ducts; these are passed as such into the female apparatus. Many Scoleina have spermatophores of this kind, which are merely formed by agglutinated seminal filaments (Tubifex). In the Hirudinea the spermatophores are provided with a special covering. 


\section{Fourth Section.}

\section{Echinoderma.}

\section{General Review.}

\section{$\S 157$.}

The Echinoderma form a strictly-defined and independent group, owing to the well-marked development of a special trpe. It is distinguished from the Coclenterata by the differentiation of the enteric canal and formation of a perienteric cavity (colom). The calcification of the integumentary layer (perisome), which encloses this body-cavity, and the radiate arrangement of the body, which consists of more than two antimeres, affords a fairly sharp boundary between it and the higher divisions. This difference between the fully-developed form of the Echinoderma and that of the other types does not obtain in the larval stages, so that in these we can make out genetic relations with the other types. These are the more to be insisted on, as the actinoid type of the Echinoderma was regarded as a reason for uniting them and the Coclenterata into a large group - that of the Radiata ; a union which on close examination cannot be justified. Such a union is inconsistent with the close relationship between them and the Vermes, especially the Annelides and Gephyrea. The observation that the internal as well as the external organisation of the Echinoderma gives evidence of metamerism, has further justified their separation from the Coclenterata, and has given rise to the hypothesis propounded by Häckel, which regards the Echinoderma as derived from colonies of worm-like organisms.

The larval form of the Lichinoderma agrees exactly with the larva of the Vermes. Here, as in many of the latter, a new organism is built up within the body of the larva. But radial gemmation takes place, and a number of individuals are produced, and thereupon the phrenomenon enters upon the well-known series of changes. Gradually the 
radially disposed buds separate from one another to a certain extent, without however ever becoming completely separated; so that a number of organs, or separate portions of organic systems, are common to them all. In this way the budding individuals remain connected as a single organism, and losing their individuality, sink to the level of mere parts only of a body (antimera).

This hypothesis of the derivation of the Echinoderma from the Vermes leads us to classify the former above the latter, for we suppose that the Vermes gave the starting-point for the development of the Echinoderma.

The separate divisions of the Echinoderma (Star-fishes) are thus arranged :

\section{Asteroïda (Sea-stars).*}

\section{Asterida.}

Asteracanthion, Solaster, Astropecten, Luidia.

Brisingida.

Brisinga.

Ophiurida.

Ophioderma, Ophiolepis, Ophiothrix, Ophiocoma.

Euryalida.

Astrophyton.

II. Crinoïda (Lily-stars).

Brachiata.

Pentacrinus, Comatula.

III. Echinö̈da (Sea-urchins).

Demosticha.

Echinothurida.t

Calveria, Phormosoma.

Cidarida. Cidaris.

Echinida.

Echinus, Echinometra.

Petalosticha.

spatangida.

Spatangus.

Clypeastrida.

Clypeaster, Laganum, Scutella.

IV. Holothuroïda (Sea-cucumbers).

Eupodia.

Apodia.

Holothuria, Molpadia, Pentacta, Psolus, Cuvieria.

Synapta, Chirodota.

* The Asteroïda must be put first, as including the oldest Echinoderma, and as being the nearest in organisation to the hypothetical stem form. Among the fossil forms of this group we find conditions which are allied to those of the next class (Crinoïda).

+ This most important family presents points of connection with the Asterida; notably in the possession of a movable dermal skeleton. 


\section{Bibliography.}

TIEDEMANx, Anatomie der Röhrenholothurie, des pomeranzenfarbigen Seesternes und Steinsceigels. Landshut, 1816.-SHARPEY, Art. Echinodermata in ToDD's Crclopædia. II.-M ÜLler, J., Anatomische Studien über dic Echinoderma. Arch. f. Anat. u. Phrs. 1850. -The same, Die Erzeugung von Schnecken in Holothurien. Bcrlin, 1852.-The same, Ueber den Bau der Echinodermer. Abhandl. d. Berl. Aead. 1853. - The same, Sieben Abhandlungen über die Larven und Metamorphosen der Echinodermen. Abhandl. d. Bcrl. Acad. 1819-55.-SARs, M., Oversigt of Norges Fichinodermer. Christiania, 1S61.-GRERFF, Sitzungsber, d. Gcsellseh. z. Beförd. d. Naturw. zu Marburg, 1871-76.-Teuscrer, R., Beitr. z. Anat. d. Echinodermen. Jen. Zeitsehr. Bd. X.

Asteroïda: ForBes, Ed., A History of British Starfishes. Iondon, 1841.-MüLler, J., and Trosch BL, System der Asteriden. Braunsehweig, 1812.-AGASsIz, A., Embrrology of the Starfish. Contrib. to the nat. hist. of the U.S. Cambridge, 186-1.-Hoffraxs, C. K., z. Anat. d. Asteriden. Niederl. Arclı. f. Zoolog. Bd. II.-SAns, G. O., On some remarkable forms of Animal Life, II. Christiania, 1875.-LAYGe, Wilm., z. Anat. d. Aster. u. Ophiur. Morpli. Jahrib. Bd. II.

Crinoida: Müller, J., Ueber den Bau des Pentacrinus caput medusæ. Abhanill. d. Berl. Acad., 1844. - Tномrox, W., On the Embrtogeny of Antedon rosaccus. Phil. Trans, 1 65, II.Carpexter, W. B., Researches on structure, ete, of Antedon rosaceus. Phil. Trans. Ivff.SARS, M., Mémoire pour servir à la connaissanee des Crinoïdes vivants. Christiania, 1stj8. PERRIRr, Ed, Rech. s. l'Anat. ete. des bras de la Comatula rosacer. Arch. de 'Zoolog. expériment. T. II.-Götrf, Vergl. Entrickelungsgesch. d. Comatula rosacea. Arch. f. mikr. Anat. Bd. XII.-Levwig, H., Morph. Stud. (Crinoideen). Zeitschr. f. wiss. Zool. Bd. XXVIII.

Echinoida: VAlexir, Anatomie du genre Echinus, 1841.-Hoffrars, C. K., Anat. d. Echinen u. Spatangen. Nicderl. Arch. f. Zool. Bd. I.-AG.sssiz, A., Revision of the Echini, P. 1-4. Cambridge, U.'S. 1872-73.-Lovér, S., Etudes sur les Echinoidées. Kongl. Svenska V'ct. Acad. Handl. Bd. XI.-Wrinle Thossor, On the Echinodea, ete. Phil. Trans. 1874, P. II.

Holothuroïa: Qdatrefagrs, Anatomie der Synapta Durcrnar. Ann. se. nat. II. xviii.DANIELSEN, C., og KoREN, J., Bidrag til Holothuriarun udviklings-historic in Fauna littoralis Norvegiæ. II. Bergen, 1856.-BAUr, Beiträge zar Naturgeschichte der Synapta digitata. N. A L. C. XXXI.-Sexper, C., Reisen in den Philippinen. Bd. II. 1. Holothurien.-SeLExKA, Z. Entw. d. Holothur. Zcitehr. f. T. Zool. Bd. XXVII.

General Form of the Body.

$\S 158$.

'The morphological relations of the various divisions of the Echinoderma to one another, and to the lower forms, are best understood by a reference to their development. 'The larva, which arises from the ovum, has only two antimera (bilateral symmetry)
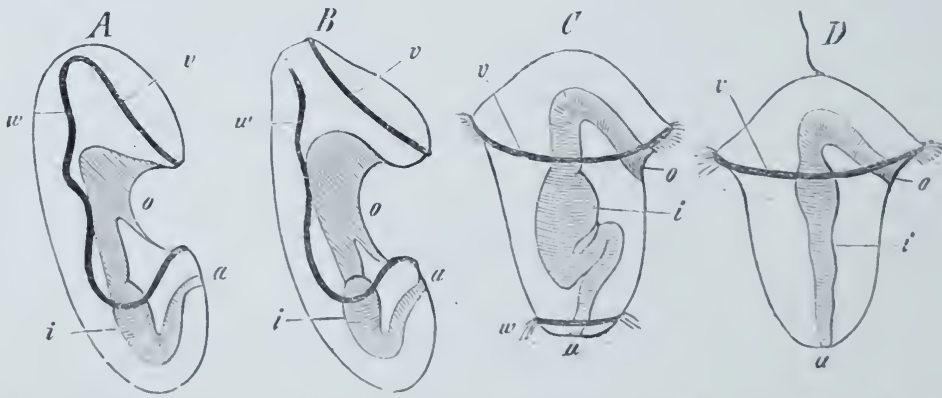

Fig. 94. Larral forms seen from the side. A Larva of a Holothurian. Is Larva of a Starfish (Bipinnaria.type). C D Vermian larva. o Nouth. $i$ Stomach. a Anus. $v$ Prooral band of cilia, which is independent in $B, C$, and $D$, but in $A$ surrounds an oral tract.

and agrees in all essential points with the larra of the Annelides. A ciliated band either surrounds the oral surface, which carries the mouth (cf. Fig. 94, A), or is dirided into two circlets, one of which bounds the preoral, and the other the postoral region $(B)$. The 
latter larval form is seen in the Asteroilda, the former in the Holothuroïda. These forms also obtain in the larvæ of other Echinoderma, but in the Ophiurida and Echinoïda a number of processes are developed, on to which the band of cilia is continued. In a few cases (where development takes place within the maternal organism) the larval form is passed over, and the Echinoderm appears without this intermediate stage. The resemblance which exists between the larval form of even very different divisions points to the common origin of the phylum; which origin, indeed, was from forms which were not of the radiate type. This important point is ignored by those who try to derive the Echinoderma from the Colenterata; they regard the Echinoderma as forming an exception, which they are unable to explain.

The rudiments of the Echinoderm body appear round the enteron of the larva. In the Asteroïda five or more parts are budded off from a common rudiment; these are the future "arms" or "rays" of the starfish (Fig. 95, A). The free end of the ray appears at first to be independent, and the other end remains in connection with the general mass. This corresponds to the anterior part, and the free end of the ray to the posterior part of a worm's body. As the rudiment of each arm grows, joints (metameres) appear in it between the base and the tip. There is a certain amount of independent organisation in each arm of a starfish ; its organs, such as the intestine, nervous, and vascular systems, and

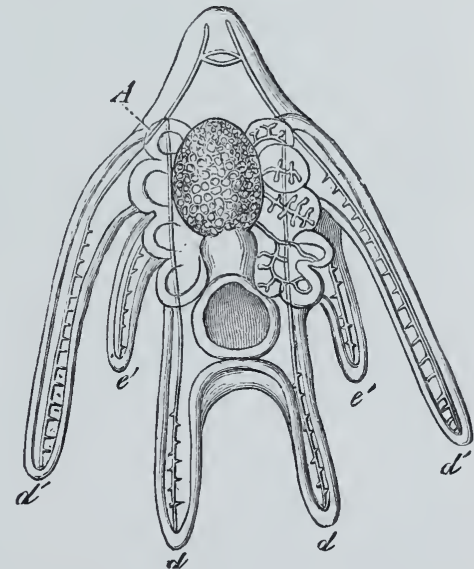

Fig. 95. Larva of an Ophiurid (Pluteus form). $a$ Rudiment of the Echinoderm with budding arms. $d d^{\prime} e^{\prime}$ Processes of the larval body with their framework of lattice-like rods (after J. Müller). also the sexual organs, have exactly the same position as the homologous organs of an Annulate worm. If, then, we compare each of the budding arms with a worm-like organism, we must regard the starfish developed by this process of gemmation as corresponding to a multiple of such organisms; and, further, we must recognise in this phrnomenon the same process of gemmation as that which takes place in other lower animals; for example, in the compound Ascidians. It is a process in which several separate animals are simultaneously budded off; the process does not go on till these animals are completely separated, hut stops in such a way as to keep them connected together as an individual of a higher order.

It is not hard to see how, owing to the incompleteness of their separation, the products of gemmation are not only connected externally, but have a certain number of internal organs connected, and so permanently common to the whole organism. 


\section{$\$ 159$.}

Thus, then, an organism is developed, the antimeres of which present themselves as the radially-arranged "arms ;" each of these arms has primitively the value of one person. By the concrescence of these persons an individual of a higher order, an animal colony, is formed. In the Asteroïda the number of arms is not definitely fixed; in some there is a large (1+ in Solaster), in others (Asteracanthion) a smaller number (4). In most the number is limited to five, and the typical number, which predominates in other Echinoderma, is fixed at this. The number of arms in Brisinga varies from 9-12. The point at which all the arms are connected forms the common body in the Asteroida ; it carries the mouth. This lies on the ventral surface, which is oral, and has an aboral surface opposed to it. In the arms the ventral surface is distinguished by the possession of rows of expansive and movable processes-ambulacral feet; these form an "ambulacrum" in each arm, and are attached to a depression, which runs along the arm (ambulacral groove). They correspond in character with the metameric arrangement of the arms, which is expressed in other parts. There are four rows in Asteracanthion, and two in most of the other forms. It is uncertain whether these structures are allied to the parapodia of Vermes or not. The ventral surface is also known as the ambulacral. There is no difference in the extent of the ambulacral and antambulacral (dor'sal) surfaces.

The radii or arms vary greatly in the extent to which they are independent of the common body; in not a ferw forms they show a tendency to shorten so as to form a body disc, and so, at the same time, the indication of the primitively individual character of the
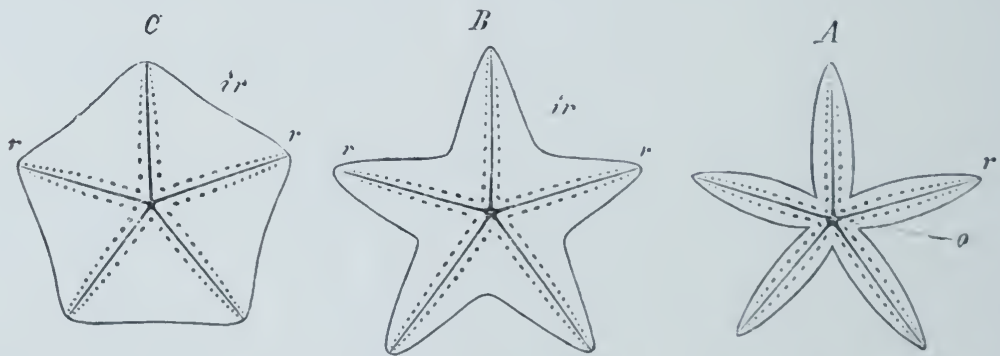

Fig. 96. Three forms of Starfishes, $A B C$, in which concrescence and consequent loss of individuality in the arms is being gradually effected. All three aro figured from the oral, or ambulacral, surface of the body. The ambulacra are represented by rows of dots. o Mouth. $r$ Radii (Arms). ir Interradii.

arms is lost (Oreaster, Pteraster, Asteriscus, etc.). A comparison of the three forms of Sea-stars (Fig. 96, $A B C$ ) here figured will make this quite clear. 


\section{$\S 160$.}

The arrangements in other Echinoderma are derived from the form which obtains in the Asteroïda; and that in two divergent lines. In both, although in different ways, it leads to a greater centralisation of the organism. Along one line the arms are more largely developed, and at the same time gradually lose their relations to the internal organs. Along the other the arms pass completely into the common body. The number of rays appears to be always five. The first arrangement is seen in Brisinga and the Ophiurida, where the body is separated into a discoid central portion (Fig. 97, s),

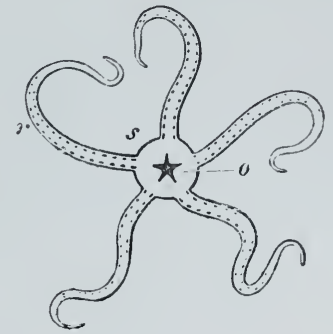

Fig. 97. Diagram of the body. form of an Ophiurid. o Mouth. $s$ Body disc. $r$ Arms. and the projecting, but sharply separated, arms $(v)$. The arms take but a small share in the formation of the body-cavity, which is almost exclusively limited to the body disc. The ambulacral groove is wanting in the Ophiurida, but the ambulacra still extend along the arms.

The arms of the Euryalida are greatly developed, being repeatedly divided in a dichotomous fashion. $\mathrm{A}$ shallow groove is continued into the divisions. The Crinoïda, which in earlier periods were widely distributed, and very rich in forms, but which are now represented by few genera, have lost the power of free locomotion, and passed into the fixed condition. In the division Brachiata, which includes the extant forms, a stalk, often of great size, jointed, and complicated by branchings and appended structures (Fig. 98), is developed on the antambulacral portion of the cup-like body. This serves as an organ of attachment. The arms are not always limited to five, but there is often a larger number of them; they are considerably developed, being divided, or provided with secondary appendages. These appendages, which are attached

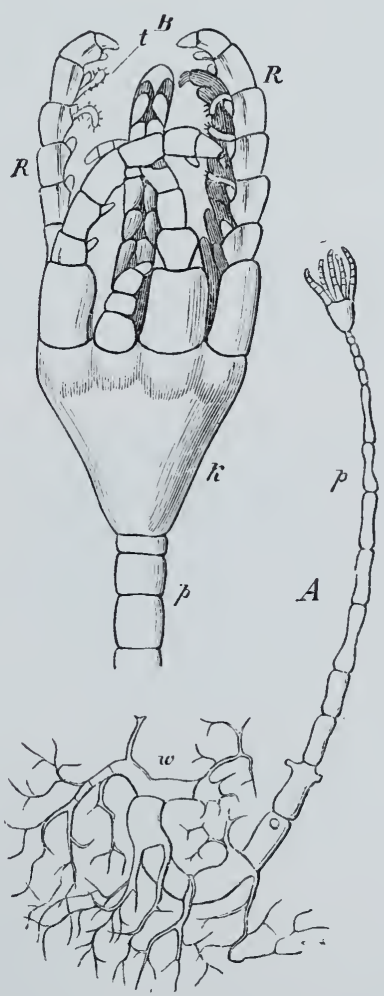

Fig. 98. Rhizocrinus lofoten. sis. A A young specimen. $B$ Cup $(k)$, with seven arms, of a larger specimen. $p$ Stalk. $v$ Roots. $R$ Arms. $t$ Tentacles (after M. Sars). to the arms and have the form of feathery plates, are known as pinnulæ. The ambulacral groove 
extends on to the arms, and gives passage to the tentacula-like feet. In some the fixed condition obtains in the young only, and later on in life the arm-bearing body breaks off from its stalk (Antedon, Comatula).

\section{$\$ 161$.}

The other series of modifications of the form of the body leads to the Echinoïda. The arms have altogether disappeared as independent parts. In the more or less cone-shaped body of the true SeaUrchins (Desmosticha), the ambulacra extend over the greater part of the surface. The ambulacral tracts form five bands, which extend from the oral (Fig. 99, $A$ o) to the opposite pole $(B a)$; these are separated by as many tracts devoid of suckers (Interambulacra).
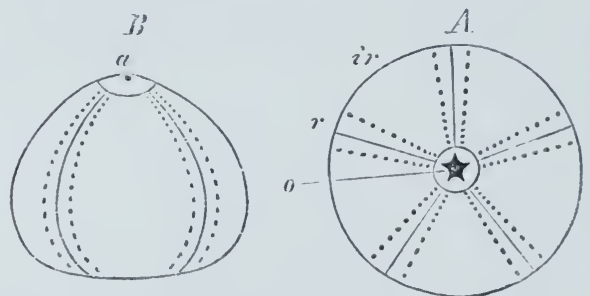

Fig. 99. Diagrammatic figure of a Sea-Urchin. $A$ From the oral surface. $B$ A lateral riew. The ambulacin are represented by rows of dots. $r$ Radii. ir Interradii. o Month. a Anus. The latter is surrounded by the antambulacral surface. (cf. Fig. 96, c), then the decrease in size of the antambulacral surface, and the consequent increase in that of the ambulacral, will give us the Urchin-form.

This arrangement is modified in the Petalosticha, partly by a change in the relations of the mouth and anus, partly by modification of the ambulacral tracts. The diminution in size of these tracts is of importance. They form a five-leaved rosette on the dorsal surface, and from these leaf-onds signs of a continuation of the tracts as far as the mouth can be still followed out in the Clypeastrida.

The indications of the development of the Echinoderm body from a multiple of individuals are less apparent in the Holothuroïda than in the Echinoïda. But the sausage-shaped body can be derived from the arrangement seen in most of the regular Sea-Urchins, by imagining the body to be pulled out. 'The oral and aboral poles in each correspond, the former being distinguished by the oral, the latter by the anal orifice. 'The antambulacral surface has altogether disappeared. In the true Holothuroïda (Eupodia) the ambulacral and interambulacral tracts extend alternately from the month to the anus. Some of the ambulacral tracts may however undergo an increase, and the other's a decrease in size, according to the varying necessities of function. 'Thus the three ambulacral tracts on that surface which 
functions as ventral, are retained in Psolus, while the other two which belong to the portion of the surface of the body, which functions as the dorsal, are atrophied. In Cuvieria this modification of parts is extended so as to result in the disappearance of the three ventral ambulacra.

A complete degeneration of the ambulacra takes place in the Synaptæ, and the radiate organisation, which is implied by the division into ambulacra, is thus lost; they have followed out the line of modification indicated in those Asteroïda, where the rays begin to lose the organs belonging to them, as if in preparation for a centralised organisation.

\section{Appendages.}

\section{$\S 162$.}

The organs appended to the integument, which may be regarded. as appendages, are not so various as in the Vermes. Of such parts the sucking or ambulacral feet must be placed foremost, for they form the most common arrangement, and belong to the typical Echinoderm organisation, and are evidently derived from an ancestor common to the whole group. They are tubular, and generally cylindrical processes of the body-wall, which not only agree in being arranged in rows (in accordance with the metamerism of the rays), but also in their most essential points of structure, with the parapodia of the Annelides: on the whole they are more simple in character.
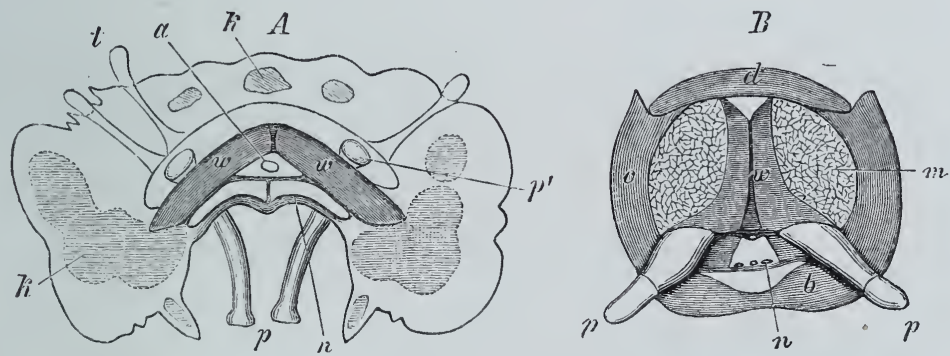

Fig. 100. Diagram of the cross section of an arm, $A$, of Asteracanthion rubens; $B$, of Ophiura texturata. $p$ Ambulacral feet. $p^{\prime}$ Ampullæ. $t$ Dermal tentacles. $n$ Nerve-chords. $w$ Ambulacral plates. $m$ Muscles. a Ambulacral vein. $b$ Ventral plate. $c$ Lateral plates. ¿ Dorsal plate. $k$ Calcified portion of the integument (after Wilh. Lange).

(Fig. 100). As they have always much the same structure they vary but little in function.

The free end of these tubular structures $(p)$ is either flattened out, or provided with a sucker-like termination (Echinoïda); or it has a conical tip, or is rounded off (many Asteroïda); sometimes it is provided with a rounded head. Others are provided with lateral indentations or secondary processes (Ophiurida and Crinoïda); these 
are intermediate towards those forms which have lost their locomotor function, and have the form of ambulacral branchire or tentacles (tentacular organs).

When they are filled with fluid the feet swell, and are thereby erected and capable of protrusion. The extent to which they are protruded depends on the length of the firm appendages of the integument, so that the sucker feet are longest in the Echinoilda with their long spicules. The end becomes fixed when the sucker is protruded, so that the foot is now able, when it contracts, to draw the body of the animal towards the point of attachment; this mode of locomotion is often effected with great rapidity by the Echinoïda. A whole group of suckers takes part in the movement, and by working together display considerable energy. The distribution of these structures has been referred to in the previous paragraphs; their relation to the vascular system will be spoken of when that system is dealt with.

In the Crinoïda the circumoral suckers acquire the function of tentacles; this is in many other cases combined with the locomotor functions. But we get also independent tentacular structures, allied in origin to these, namely, the tentacles in the neighbourhood of the oral aperture of Holothuroïda (cf. Fig. 113, T). They are sometimes pinnate, sometimes branched, and, as a rule, can be completely drawn in. In many Synaptre they carry suckers (S. Duvernea). 'Their internal cavity is in communication with the same vascular system, as are the ambulacral feet.

The so-called dermal branchix, tentacles which are distributed over the antambulacral (dorsal) surface of the body in the Starfishes (Fig. 100, t), differ from these; in the Echinoïda they form five pairs of contractile tree-like organs near the mouth. They communicate with the body-cavity, and are merely protrusions of the integument.

\section{Integument and Dermal Skeleton.}

$\$ 163$.

In the Echinoderma the same dermo-muscular layer is present as in the Vermes, but the integument is more sharply marked off from the musculature. This latter forms, for the most part, a layer which limits the body-cavity, and is covered externally by the integument. Its special characters are due to the fact that the locomotor power of the body is more or less determined by the deposition of chalk in that layer, which goes to form, in conjunction with the muscular layer, the "perisome."

The same structure exists in the larva; but in it this exoskeleton is never of any great size, but is merely a firm support formed by a large number of rod-like processes. T'lie ciliated bands extend on to the processes, which they fringe, and form, in a more or less complicated manner, the locomotor apparatus of the larra (ride Fig. 95, 
$\left.d l^{\prime} e\right)$. The whole body is covered with cilia at a period before that when they are arranged on the ridge-like projections from the ciliated band; but this general ciliation is only found during the most indifferent condition of the larva.

The cilia are retained even later on, on many parts of the soft dermal layer which invests the calcareous skeleton: as, for example, in the ciliated tracts, which reach to the mouth in the Spatangidæe (semitæ). On other parts, such as the dermal branchiæ (cf. suprà), ciliation appears to be correlated with the respiratory function of the integument, in which the ambulacral feet may also have a share.

The extent to which the integument is calcified varies greatly. Sometimes the calcareous particles are united with one another into larger pieces, and form plates which are movably or immovably connected together : this arrangement either extends over the whole body, or is confined to definite tracts of its surface. In other cases the calcareous particles are scattered, and allow of great variations in the form of the body. In this case a large number of the characteristic features of the Echinoderm disappear from other parts of its organisation; so that the disappearance of a calcified integument is a departure from the type, and the general phænomenon of a scanty deposit of calcareous matters is not to be regarded as an early, but as a final stage in the series of forms.

Calcification converts the integument into an organ of support for the body, or dermal skeleton; in many cases this sends out processes into the interior of the body. These give rise to calcified structures, which form an internal skeleton and combine with the external. The whole thickness of the perisome is not affected by the process of calcification. A thin non-calcified layer of tissue is always found on the inner, as well as on the outer surface; on the latter, however, this layer disappears at an early period from some parts, so that the calcified parts are exposed; this happens, for instance, on the spine-like structures, as well as on other processes of the calcareous skeleton.

The lime-salts are always deposited in a regular manner in the integumentary layer. They form delicate frameworks or retiform structures (Fig. 101), in the spaces

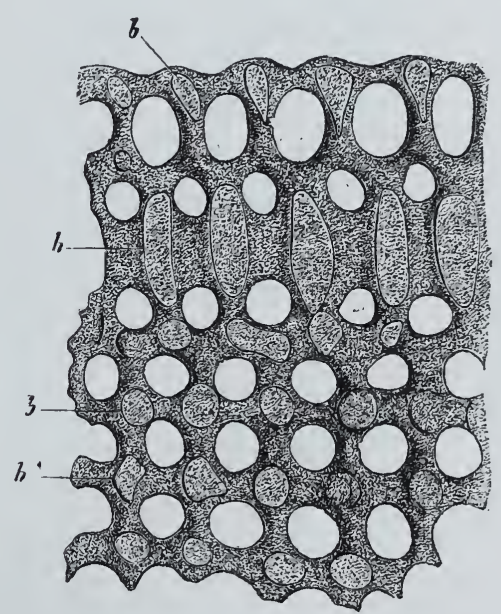

Fig. 101. View of a calcareous net. work of a plate of the dermal skeleton of an Echinid (Cidaris). $b$ Trabeculae cut through. These were directly per. pendicular to the horizontal network (somewhat highly magnified).

of which soft organic substance persists. The most solid skeletal parts are thus traversed by soft structures; when the calcareous 
skeleton is merely represented by separate and microscopic deposits, these are generally definite in shape, and characteristic of genera and species.

The calcareous skeleton of the larva forms an organ of support, which is generally made up by a framework of delicately attached, and often perforated rods. They are ordinarily found in the larval Echinoïda and Ophiurida; there are also calcareous bodies in the larve of the Holothuroïda. 'The presence of a calcareous skeleton in the larva is clearly an instance of an arrangement which is common to the group; but it must not be forgotten that this larval skeleton corresponds to the form of the larva, and not to that of the adult Echinoderm; none of it passes permanently into the adult form. 'There is, in fact, a repeated chang'e of the calcareous skeleton in the Holothuroïda.

\section{$\S 164$.}

As regards the special characters of the dermal skeleton, the presence of pieces, movably connected with one another, on the

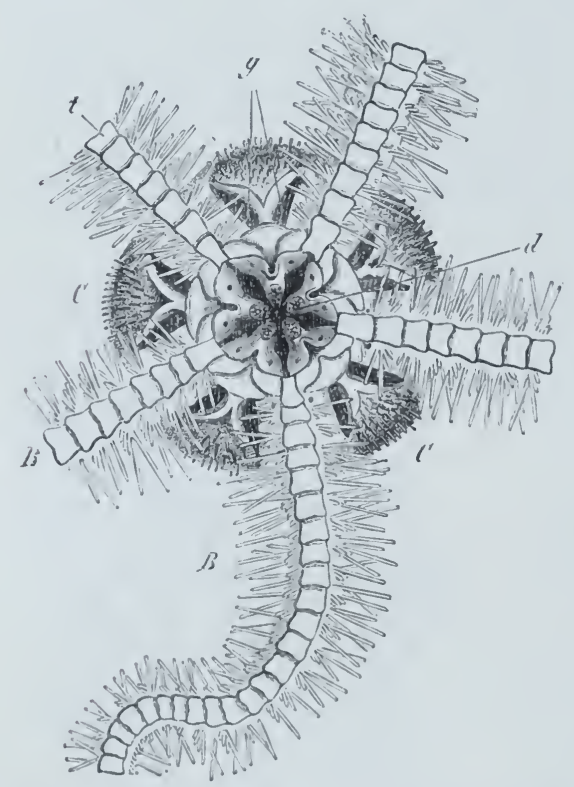

Fig. 102. Bocly disc of an Ophiurid (() p hiothrix fragilis); seen from the oral surface; the bases of the arms beset with spicules may be seen (magnified). C) Body disc. $B$ Arms. $t$ Calcareous plates, which cover the canal which corresponds to the tentacular groove of the Asteroilda. g Genital clefts. \&l Masticatory plates. cuticle. At the side it passes into a layer of cells, which is placed much deeper. At the lateral edges of the groove the skeleton is ambulacral surface of the arms, is characteristic of the Asteroïda. 'Transversely - placed pairs of calcareous pieces, which gradually diminish in size, are found from the mouth as far as the tip of the arm (Fig. 100, $A v$ ); they form the floor of a groove-the tentacular groove. The separate pieces form a jointed series by their articular attachments, and the suckers pass out between the solid joints $(p)$. These calcareous pieces are consequently known as ambulacral plates. But as special soft parts (ambulacral canal and nerres) are also embedded in this groore, the jointed segments do not appear to be purely dermoskeletal parts. The ambulacral groove is covered by the integument, which is continued laterally on to the ambulacral plates. It consists largely of a layer of long cylindrical cells, covered by a
layer of cells, which is placed menterer. 
continuous with the dermal skeleton, which covers the back of the arms ; at this point there are frequently found one or more longitudinal rows of plates or scutes. These structures may be replaced by knobs, and are sometimes continued on to the integument of the antambulacral surface of the body; or the integument is distinguished by retiform deposits of calcareous matter and smaller tubercles, separated by the non-calcified parts of the perisome. Brisinga resembles the Asteroïda in the structure of its arms; that is, they have an ambulacral groove.

Larger flat plates, marginal plates, form the edge of the arms; these are often distinguished by spicules and other processes.

The structure of the integument of the Ophiurida resembles that of the Asteroïda. There is seldom any great development of calcareous plates on the antambulacral surface; they are, as a rule, found near the base of the arm only in these forms. The ambulacral or ventral integument is also provided with plates around the mouth (Fig. 102). But several parts of the firm skeleton of the arms are different to those of the same parts in the Asteroïda. The pieces homologous with the ambulacral plates of the latter form a closelyset series (vertebral pieces, Fig. 100, B w), which almost completely fill up the arm, and only leave a narrow canal on the dorsal, and a groove for the nerves and other organs on the ventral surface. The coelcm is therefore continued into the arms as a narrow canal only. Instead of the soft covering of the ambulacral groove, which the Asteroïda possess, there is a series of firm calcareous scutes (Fig. 100, $B$ b) in the Ophiurida; other lateral processes of various kinds are added on to them.

In the Euryalida also the leathery investment of the body covers in a skeletal structure, formed of vertebral calcareous plates attached to one another. This, as in the Ophiurida and Asteroïda, belongs to the oral surface of the body; the plates are continued from the edges along the radii, and to the finest ramifications of them. In them, too, this skeleton forms the floor of the ambulacral groove. On the aboral surface the body-disc is enclosed in a dermis, merely impregnated with calcareous granules, which passes on to the arms, and covers them as far as the edge of the ventral groove.

There is a larger number of knob-like and spicular processes in the integument, which may vary very greatly in character. A special form is very common among the Asteroïda; namely, bundies of movable spicules attached to a common stalk (paxillæ). The pedicellariæ are described in $\$ 166$.

\section{$\S 165$.}

This dermal skeleton is modified in the Crinoïda. The dorsal integument is drawn out into a stalk, to the end of which the animal is attached. The skeleton of the stalk is formed of calcareous plates, which lie regularly one over the other, and are connected with flattened basal pieces, to which other calcareous plates, 
which form the boundaries of the body, are attached. In the young stages of the Comatulie, a simple knob-like piece (centro-dorsal) unites the skeleton of the stalk with the body. Radial jointed pieces, which are continued into the joints of the arms, are attached to the central piece. The ambulacral groove extends along the dichotomous branches of the arms (Pentacrinus), as well as along: the lateral appendages (pinnulæ of Comatula) which are set alternately on either side of the arms. The groove becomes united with the groove of the next arm, and passes along the ventral surface of the cup-shaped body as far as the mouth. Deposits of calcareous plates are embedded in all parts of the portion of the integument which remains soft and covers over the skeleton.

\section{$\$ 166$.}

The differences in the dermal skeleton of the Echinoïda, and the consequent changes in the form of their body, as compared with the Asteroïda, are chiefly due to the calcification of the oral (ventral) perisome, that is of the portion which covers the ambulacral groove and the soft parts which lie in it, and which is permanently soft in the Asteroïda. In the place of the articulated joints, there are plates which are calcified externally, and are connected with the body in various ways.

In the Desmosticha the portion which corresponds to the dorsal,

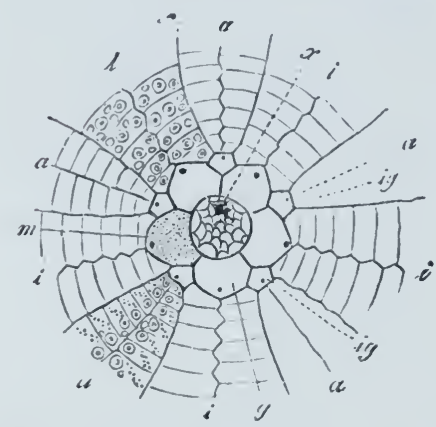

Fig. 103. Apical pole of an Fich inn s. a Ambulacral arex. i Interambulacral arex. g Genital plates. ig Intergenital plates. $m \mathrm{~A}$ genital plate with the characters of a madreporic plate. $x$ Anus placed in the apical area surrounded by the genital plates. The knols of the plates are figured in only ono ambulacral and one interambulacral area; in the former the pores also, which are ounitted from the other four, are indicated. perisome of the Starfishes, which covers over the ambulacral groove on the ventral surface of the arms. The rows of non-perforated 
plates, which lie between the ambulacral tracts (interambulacral areæ, $i$ ), are homologous with the marginal plates of the arms in the Asteroïda. There are two rows of interambulacral, as well as of ambulacral plates. The Echinoïda of earlier periods had a larger number of these rows-forms with three, five, and even seven rows in one interambulacral area are known.

These pieces are connected with one another in different ways. In many Eichinoïda, as in the Asteroïda, the calcareous plates of the perisorne are movably connected together, and so allow of changes in the form of the body. In the family of the Echinothurida the plates of the perisome are movably connected with one another, so that the body is able to change its form. The ambulacral as well as the interambulacral plates project over one another like the tiles of a roof in the middle of each area, and the interambulacral ones are separated laterally from one another by a narrow intermediate space. Owing to the thinness of these plates the soft parts of the perisome are of more importance than they are in other families of the Echinoïda. These plates are also continued with slight modifications on to the area around the mouth, while in the rest of the Desmosticha this portion is more sharply marked off from the rest. So far the Echinothurida approximate to indifferent conditions, and form an intermediate step between the other Desmosticha, and the hypothetical forms which can be derived from the Asteroïda. This point of view is further justified by the fact that a firm fascia extends along each ambulacral area on the inner face of the shell, and separates the parts (nerves, vessels, ampullæ) which lie on the ambulacrum from the cœlom. It forms a process, which is attached to either side of an ambulacral groove, and projects some way into the cœlom ; this process is, moreover, fenestrated by fine pores. This arrangement corresponds to the skeleton of the ambulacral groove in the Asterida, which is calcified in this portion in them; while the same portion in these forms, in which the perisome corresponding to the ambulacral groove of the Asterida is formed by calcareous plates, remains soft.

Several important modifications of the regular form of the dermal skeleton of the Echinoïda, which are not directly comparable with the arrangements found in the Asteroïda, are developed; these are accompanied by the disappearance of the rest of the primitive dorsal perisome, and by the passage of the radiate into other forms. The ambulacral areæ no longer extend regularly from the mouth to the back; in the Spatangidæ and Clypeastridæ they are limited to a five-leaved rosette (ambulacra petaloïdea) placed on the dorsal surface. At the same time the number of plates, which is very large in the regular Echinoïda, is diminished; so that there is a smaller number of larger plates.

The internal skeleton, which in the Asteroïda is formed by the skeleton of the ambulacral groove, is represented in the Echinoidda by processes of the ambulacral plates. These processes, which are well developed in Cidaris, for example, embrace the nerves as well as the 
ambulacral canal, and so point to an affinity with this skeleton. The skeleton of the masticatory apparatus in the Echinoïda and Clypeastrida is to be regarded as an independent development; it surrounds the commencement of the enteron, and consists of a number of calcareous blocks comnected together like a scaffold.

Spinc-like processes are comnected with the integument in the Echinoïda, as well as in the Asteroïda, but they

1

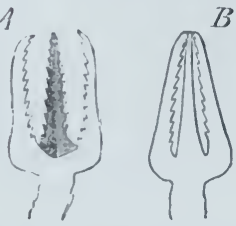

Fig. 104. Pedicellarix of $\mathrm{Ech}$ in us saxat i 1 i s. A A Pedicel. laria with its pincer. arms oven; $B$ With them closed (after Erdl). are more independent owing to their power of movement. They are articulated to protuberances of the calcareous plates, and are provided with a special system of muscles. The spines vary greatly in form and size; sometimes they are as fine as hairs (Spatangidæ); sometimes club-like structures (Acrocladia); sometimes they are long rods (Cidaris).

The Pedicellaria are also dermal organs of a peculiar character, which are found in the Asteroïda, as well as in the Echinoïda. They consist of a stalk-like muscular process of the integument, which is supported at its end by a fine calcareous skeleton; it terminates in two or three pincer-like valves, which are movable on one another. These too are provided with a calcareous skeleton. In the Echinoïda the three-valved, and in the Asterida the two-valved forms predominate. Brisinga resembles the Asterida in this point. They are scattered over the whole body; but in the Asterida they are principally found at the base of the spicules, and in the Echinoilda in the perisome around the mouth.

These bodies may be regarded as spines modified in such a way that the incompletely calcified stalk of the pedicellaria corresponds to the stalk of the paxilla of the Asterida; the tuft of spinules
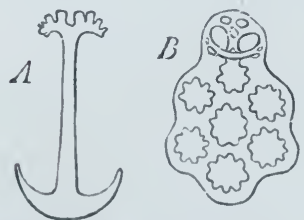

Fig. 10j. A Calcareous anchor. $B$ Calcareous plate, which serves to attach the former; from the integument of $\mathrm{Sy}$. uapta lappa (after J. Mïller). on the latter being represented by the arms of the pedicellaria, which are moved by muscles, just as are the spines of the Echinoïda. The four-valved pedicellaria of Calveria fenestrata are intermediate between the more common pedicellaria and the paxilla, for each of the valves, which is provided with a long stalk, is continued into a broad lamella, bent over at its edges.

\section{$\$ 167$.}

In the Holothuroïda the integument has no longer any dermo-skeletal significance. The calcareous plates of the other Echinoderma are represented by disconnected deposits of lime in the firm dermal layer.

The calcaroous deposits of the skin are definite, and often very regular in form; and these forms are characteristic both in Symapta 
and Holothuriæ. They sometimes form larger firm pieces, such as the scutiform structures, which cover the dorsal or antambulacral surface in Cuvieria, and which though much smaller are widely distributed in the dermis of Echinocucumis.

In the Holothuriæ the leathery layer of connective tissue becomes very strong indeed, but it is quite soft in the Synaptix. In these latter, however, there are calcareous bodies deposited, which are frequently definite in form; such as the calcareous rotulæ of the Chirodotæ, or the fenestrated plates (Fig. 105, B) into which the bases of the anchor-shaped hooks $(A)$ are inserted. These latter project from the integument, and give to the skin of the Synaptre its great power of attachment.

The Holothuroïda also possess an internal skeleton proceeding from their dermal one. It consists of a calcareous ring surrounding the gullet, which serves as a point of insertion for the muscles of the body, and as a support for the other organs. In the Holothurix it consists of 10, and in the Synaptæ of 12-15 separate pieces. In the former five larger pieces alternate with as many smaller ones, and are more or less movably connected with one another. They are homologous with the processes, which project inwards from the oral edge of the shell in the Echinoïda. Like those processes, they are provided in the Synaptre with pores, which give passage to the nerves and ambulacral canals; in the Holothuria these organs pass out through forked processes.

\section{Muscular System.}

$\S 168$.

In the Echinoderma, as in the Vermes, the muscular system is connected with the integument, and the structures derived from it. The arrangement of it is essentially dependent on the development of the dermal skeleton, so that it is only developed into a system of body muscles in those cases, where the body is able, thanks either to the articulations of its separate firm parts (Asteroïda and Crinoïda), or to the presence of disconnected calcareous deposits in its integument (Holothuroïda), to chang"e its form.

In the Asteroïda and Crinoïda the musculature of the arms, like the arms themselves, is jointed; it fills up the interspaces between the solid parts. In the Crinoïda, where the skeletal parts of the arms are connected together by elastic tissue, these muscles lie on the ambulacral or ventral surface of the body, and serve principally to bend them, while the elastic tissue between the joints straightens them again. The same arrangement obtains in the pinnulæ of the Crinoïda.

This system of muscles is rudimentary in the Echinoïda, where the perisome is hardened into a firm "shell" consisting of immovably 
connected pieces; in them the only separate muscles on the shell are those which move the spicules or spicular processes; those too which are found in the interior of the body serve only to move definite organs, as for example the muscles of the masticatory apparatus of the Sea-Urchin. In the Spatangida, however, the shell is movable at one point.

Very different relations obtain in the Holothuroïda, where the absence of large skeletal pieces leads to a proportionate derelopment of the muscular system. Its connection with the integument is very definite. 'There is a circular layer of muscle beneath the connective tissue of the skin; within this are fire longitudinal bands of muscle, which are sometimes divided (Fig. 113, $m$ ) and are separated by intermediate spaces of varying breadth; these bands are inserted anteriorly into the already described calcarcous ring $(R)$. They aro comnected through the five pieces which are pierced so as to gire passage to the nerves and ambulacral vessels. The circular layer is continuous only in the Synaptre; in the Holothuria it is broken through at the radii, so that it really consists of interradial transverse fibrous tracts only.

\section{Nervous System.}

$\S 169$.

The principal parts of the nervous system of the Echinoderma consist of a number of trunks, corresponding with the antimeres of the body; they are placed ventrally and have a radial direction, and are also connected by commissures around the osophagus. 'These commissures are formed by each of the nerve-trunks, which accompanies the ambulacral vessels, being divided into two halves near the mouth; these pass to cach side, and become connected with the corresponding halves of the neighbouring nerre-trunks, which go to meet them. In this way a ring is formed, which surrounds the gullet, which, however, on account of its mode of formation, must not be compared with the osophageal ring of the Vermes. Each of the radial trunks corresponds rather to the ventral ganglionic chain, or ventral medulla of the Annulata; and the commissures therefore between sereral such trunks are connections between the ventral medulla, which owe their origin to the concrescence of several incompletely separated persons.

As to their more special characters; the position of the radial nerves immediately below the well-dereloped epithelial layer of the ambulacral groove (Fig. 100, A n) in the Asteroïda and Comatula is an important point; for it indicates that the relations between the nervous system and the ectoderm are almost direct. Perhaps this position is due to the mode of derelopment of the radial nerres, and possibly we here meet with a very low condition, in which differentiation is not completed. The fact that princesses of the epithelial 
form-elements enter into the formation of these nervous tracts, and have the function of a supporting tissue, points to this conclusion. In the Asterida each radial nerve consists of two bands thickened in the middle, in which cellular and fibrous elements are equally distributed. At the ends of the arms the radial nerves form a large swelling, which is connected with the optic organs placed there.

In Comatula this nervous band has the same characters. It is accompanied by a blood-vessel, which is placed in the middle of it, and, being pushed into it from above, divides it into two halves. Branches are given off in regular order to the pinnula. In the Ophiurida the radial nerve-trunks (Fig. 100, $B$ n) are placed in a space, covered over by the ventral plates $(b)$, and supported by a layer which, by its continuation into the ambulacral feet, is shown to belong to the integument. In many, however (Ophiura texturata), the nerves themselves are considerably differentiated. They each consist of two nerve-chords, in which masses of ganglionic cells are deposited; these correspond to the metameres of the arms. The longitudinal trunks are connected by transverse commissures with these ganglia, from which peripheral nerves are given off. Each radial nerve therefore represents a ventral ganglionic chain.

The connection between the nervous system and the integument, although at first sight this is implied only by the position of one on the other, is an important aid to the comprehension of the relations of the skeleton. For when this arrangement obtains the ambulacral groove cannot be calcified; this can only happen when the nervous system becomes more independent.

In the Echinoïda provided with a masticatory apparatus, the nerve-pentagon is intimately connected with it. In Echinus (Fig. 106) the nerve-pentagon lies above the floor of the oral cavity, between the esophagus and the tips of the ossicles of the masticatory apparatus, and is attached by five pairs of bands in this position. The nerve-trunks $(c)$ pass from the angles of the pentagon to the spaces between the pieces of the pyramid; and extend thence over the oral integument to the ambulacral areæ. They are greatly widened in the middle

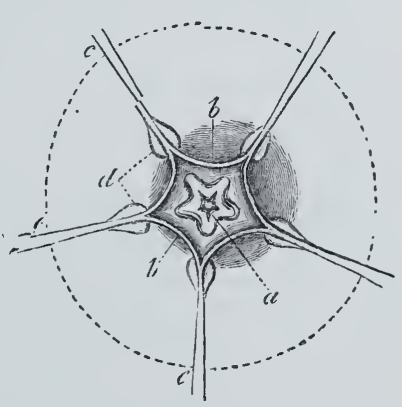

Fig. 106. Nerrous system of Echinus lividus; the masti. catory apparatus is removed. $a$ Esophagus cut through transversely. $b$ The commissures of the nerve-trunks, forming a pentagonal œsophageal ring. $c$ The nerve-trunks passing to the radii. $d$ Bands which hold together the tips of the pyramids of the masticatory apparatus (after Krohn). of their course, and divided into two lateral halves by a median groove. The lateral branches from the principal trunks accompany the branches of the ambulacral vessels. The nervous system has the same arrangement in the Spatangidæ, but in them the oral ring forms a pentagon with unequal angles.

The nervous ring of the Holothuroïda lies just in front of and a 
little inside the calcareous ring; anteriorly it is limited by the oral integument (Fig. 113, n). As it is larger than any of the five nerves (ii) which it gives off, which is not the case in the nervous ring of the Asteroïda and Echinoïda, it has more distinctly the significance of a central organ; in this point some analogies may be made out between it and the ganglionic œsophageal ring of other animals. But it is clear that it has no real homology whaterer with any ring of this kind in consequence of the origin of the œesophageal ring in the Echinoderma, already described when we were treating of the Asteroïda. The peripheral nerve-trunks pass out by openings in the five larger pieces of the calcareous ring, and extend, becoming broader as they go along the outer side of the bands of longitudinal muscles to the hinder end of the body; near the cloaca they again diminish in breadth; they give off fine branches along their course. Each radial nerve-trunk may be divided into two layers, which are separated from one another by a layer of connective tissue. A vessel accompanies the radial nerres; this is separated by a wall of partition from the ambulacral vessels, which lie still more to the interior. The oral ring gives off tentacular nerves, in addition to these radial trunks.

\section{Sensory Organs.}

\section{$\$ 170$.}

Definite portions of the integument have, in this group also, a special signinicance as tactile parts. The tentacles, as well as the sucker comnected with the water-vascular system, may be reckoned as tactile organs, and the former become greatly developed, and so of greater importance, when the ambulacral system is reduced, as it is in the Holothurorda (Apodia).

Five pairs of vesicles, which lic on the roots of the radial trunks in the Synaptidx, are said to be auditory organs, but their sensory function is as doubtful as is that of the so-called eye specks in the same genus.

Visual organs are exactly known in the Asterida only; in all other Echinoderma mere collections of pigment are regarded as ejes or " eye-spots." The cyes of the sea-stars are placed at the tip of each arm, which is ordinarily bent up, and so turned towards the light; they оссиру a pad-like elevation of the end of the ambulacral groove, the epithelial layer of which is formed of long cylindrical cells, and is very thick at this point. The rod-shaped cells contain pigment. The eyes lie on separate points of the "optic-pad." A funnel-like cavity covered by the cuticle has its walls bounded by rod-like cells, which are inclined from the periphery to the funnel; in this way their ends form the wall of the funnel. A transparent body projects from the pigmented part of the cells into the cavity of the funnel, and so fills up the greater part of its lumen. 
As this apparatus lies on the terminal ganglionic swelling of the radial nerves, and the cells give off fine processes to this ganglion, the two parts may be regarded as connected at this point (Asteracanthion rubens). Each eye which consists of a complex of cells is a differentiation of the epithelial layer, and resembles therefore the optic organs of other Invertebrata.

\section{Alimentary Canal.}

\section{$\S 171$.}

The alimentary canal, which varies greatly in character in the adult Echinoderma, has a simpler predecessor in the primitive enteron of the larval form, which is similar in all Echinoderma. Of course this does not refer to those forms in which there is no larval stage, and where the development is compressed.

The first rudiment of the enteron is formed by the in-growth of the cell-layer, which invests the body of the young larva. This gives rise to a cæcal tube, which is pushed down into the body, and the wall of this tube forms the endoderm, while the outer cell-layer represents the ectoderm. The organism is in fact a Gastrula. The entrance into the rudimentary enteron is regarded as the primitive mouth. A second invagination soon grows from the other side of the body towards the blind end of the enteron; this unites with the enteron, becomes hollow, and so forms a continuous tube with the part first formed. The parts formed last are the mouth and the osophagus, which is connected with it, and the part formed first is the mid-and the hind-gut. The orifice which becomes later the anus, and the portion of the enteron connected with it, are consequently the parts first formed.

The larval intestinal canal is formed of three portions (cf. Fig. 94, $A B)$. A wide oral opening leads into a contractile tube lying in the long axis of the body; this is the pharynx or cesophagus. Then follows a wider part, the mid-gut or stomach, which is continued into a narrow and retort-shaped tube, which is the hind-gut and leads to the anus. These three portions correspond exactly to the primitive divisions of the canal, which are distinguishable in nearly all Vermes. The mouth and anus are at first on different surfaces of the body of the larva. As the body is differentiated, especially by the development of the ciliated band, they apparently come to lie on one and the same surface, the so-called anterior side. It is, however, quite clear that the ciliated band distinctly divides two surfaces of the body; a decreased oral, and an increased anal surface turned towards the former.

But before the enteron is fully developed by becoming connected with the fore-grut, a portion of it, which forms a closed vesicle, is constricted off. Two pieces are then separated off from this vesicle, 
or two new vesicles are formed from the sides of the enteric cæcal tube. In this way three different bodies are differentiated from the enteron. The two-paired vesicles, which lie at the sides of the enteron, represent the commencement of the cœlom; the third vesicle bccomes connected with the dorsal ectoderm and opens on to it ; this is the commencement of the water-vascular system. This apparatus, like the lining cell-layer of the cœlom, takes its origin therefore from the enteron, and from that portion of it, which is without doubt its hinder part, although it appears first of all and grows inwards, from what is later on the anus. This arrangement appears to indicate that there are arrangements in the watcr-vascular system, as well as in the colom (for the two are connected together), which are phylogenetically connected with the terminal division of the enteron ; in this case this tract of the enteron is not homologous with the enterou of a Gastrula, but corresponds à priori to a hind-gut, the early development of which is clearly due to the complicated character of the organs about to be differentiated from it. These organs are those which are especially necessary to the organism. I consider therefore that the first formed rudiment of the enteron is not a Gastræa-enteron, and that its orifice is not the primitive mouth, but that they are respectively the true hind-gut and anus. The median division of the enteron which is divided from the bind-gut is morphologically a part of it. The differentiation of the abovementioned organs out of the hind-gut points to stages in which organs were connected with the hind-gut in much the same way as they are in many Gephyrea. But as yet it is impossible to prove directly that such structures have been passed on to the Echinoderma; and it is better to regard these remarkable processes as presenting us with a problem which has still to be solved.

When the body of the Echinoderm is formed in and partly from the larva, the enteron of the larva does not completely pass into it. The perisome, when formed, first grows round its middle part, and in the sea-stars takes up this only with the hind-gut. In the Echinoïda the anus also appears to be formed anew. The larval enteron is retained most completcly in the mature stage of the Holothuroïda.

The fully-developed enteron is found to hang, in the mature Echinoderm, in a colom, which is often wide, and undergoes various changes during its differentiation, which are generally correlated with the characters of the perisome. As a rule the mouth always retains its position in the middle of the ventral surface of the body.

\section{$\$ 172$.}

The mouth in the sea-stars has a radiate form, owing to the projection into it of interradial processes; hard papilla and spicules are formed by the perisome, and function as masticatory organs. 'They are specially developed in the Ophiurida, where they generally 
form several rows one above the other (Fig. 102, $d$ ). In these therefore the dermal skeleton forms the organs for the comminution of the food. A short, wide osophagus follows the mouth; this is continued into a wide mid-gut (stomach), which occupies the middle of the body. In the Ophiurida and many Asterida (Astropecten,

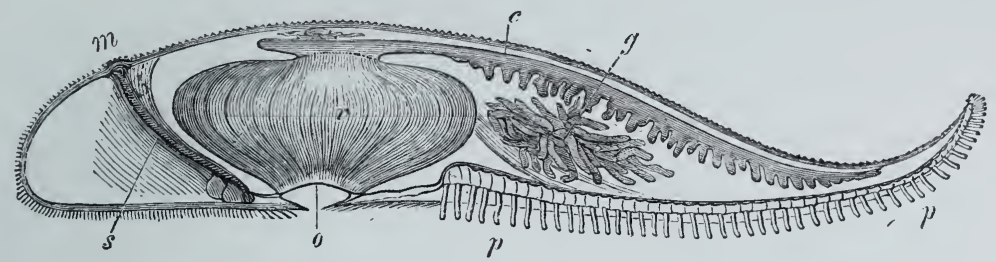

Fig. 107. Transverse section through the arm and disc of S olaster endeca. The radial and the interradial portions are figured on opposite sides. o Mouth. $v$ Stomachal cavity. $c$ Radial cæca. $g$ Genital gland. $m$ Madreporic plate. $s$ Stone. canal with its so-called heart. $p$ Ambulacral feet (after G. O. Sars).

Luidia) the stomach is always a blind sac, as it is also in Brisinga. But in all Asteroïda it is provided with diverticula or cæcal saccular appendages, which are indicated in the Ophiurida by radial constrictions. The gastric cæca of the Asterida extend in pairs into the arms; they spring from the stomach, and have the form of thin-walled tubes, closely beset with lateral appendages (Figs. $107, c ; 108, h)$, which as a rule are united by pairs into one canal before they open into the stomach. This tract represents an unpaired portion of the enteron belonging to each antimere (arm) of the Asteroilda, while the cæcal tubes form a paired portion. In Astropecten aurantiacus these tubes arise separately from the stomach. The unpaired portion in each arm has therefore disappeared in this form, and with it the primitive condition. In most

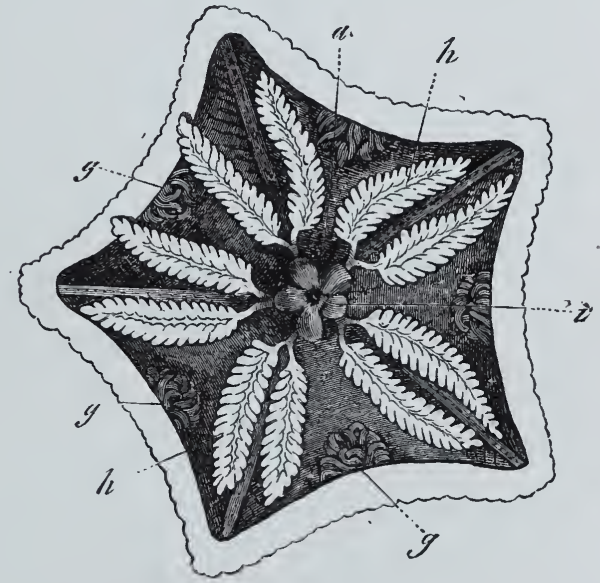

Fig. 108. Asteriscus verruculatus: opened on the dorsal surface. a Anus. $i$ Rosette-shaped enlarged enteron (stomach). $h$ Tubular radial appendages of the enteron. $g$ Genital glands. of the Asterida the short hind-gut is continued from the stomach to the anus, which is placed on the dorsal surface.

The enteric tube of the Crinoïda (Comatula) is modified; it describes a spiral coil, and its narrower short terminal portion passes into a tubular and projecting anus, which is placed interradially near the mouth. This coiled arrangement, which is apparently very anomalous, 
may be also seen in young Asteroïda; in them it is only seen for a time during their development, but in the Crinoilda it is continued as a permanent condition.

The enteron is attached to the body-wall by radial fibres. The radial cæca of the Asteroïda are attached to their body-wall by a special peritoneal fold, which extends along each cxeum.

\section{$\S 173$.}

In the Echinoïda the mouth is similarly provided with Mastica tory Organs, but they are removed from the outer surface and placed in the colom. 'They there form an apparatus, which, in the Clypeastrida, consists of five pairs of triangular calcareous pieces, but in the Echinothurida, Cidarida, and Echinida is much more complicated. Five pieces directed towards one another carry a tooth-like point, and are united with several others into a complex organ known as the "Lantern of Aristotle;" the œsophagus traverses it. The enteric tube always describes several coils. The narrow fore-gut passes into a wider portion, which forms the longest part of the canal. It has sometimes faintly-indicated diverticula (Echinida), sometimes veritable cæca (Clypeastrida), which (as in Laganum) project into the carity of body which is marked off by the supporting pillars of the calcareous shell. In these forms "mesenteric fibres" extend to the body-wall for the whole length of the coiled intestine.

In the Holothuroïda the enteric tube, which is longer than the body, forms a double loop, while in the Synaptre (with the exception of the Chirodote) it extends straight through the body-cavity, and is provided with numerous diverticula. A muscular portion of the enteron which succeeds the œsophagus is to be regarded as a special differentiation; it appears to function as a muscular stomach (Synaptr). 'This character is also seen in the Asteroïda, where the œsophagus has in the same way a stronger muscular wall than the rest of the intestine. The portion of the intestine behind the muscular part in the Holothuroïda may thus correspond to the stomacl of the Asteroïda. The end of the canal is widened out in the Holothuroïda; but this only corresponds to the hind-gut of the Asteroïda, although it is called a cloaca; it has two or more arborescent organs opening into it.

A sieve-like fenestrated lamella fastens the canal to the bodywall. This mesentery is simpler in the Synaptre which have a straight canal, while in Chirodota it is separated into three parts, in correspondence with the extent of the enteric loop; each part is connected with an interradial portion of the body-wall. 


\section{Appendages of the Alimentary Canal.}

$\S 174$.

The above-mentioned radial cæca of the Asteroïda might be regarded as organs differentiated from the primitive enteron, were it not that they must be regarded differently from a phylogenetic point of view. I consider that only certain other interradial cæca are to be regarded as appendages of this kind; they present very various degrees of development. In the aproctous Asterida they are absent, or are reduced to two (Astropecten), while in the others they are often very greatly developed. Archaster has fivesuch cæcalsacs, divided at their ends, and in Culcita this division is carried still further, so that each branch forms a racemose tube. In this way these appendages acquire the form of glands, and exhibit relationship with a structure which is very common in the Holothuroïda.

The structure in question is connected with the terminal portion of the alimentary canal, known as the "cloaca," and as a rule consists of two chief trunks, with short

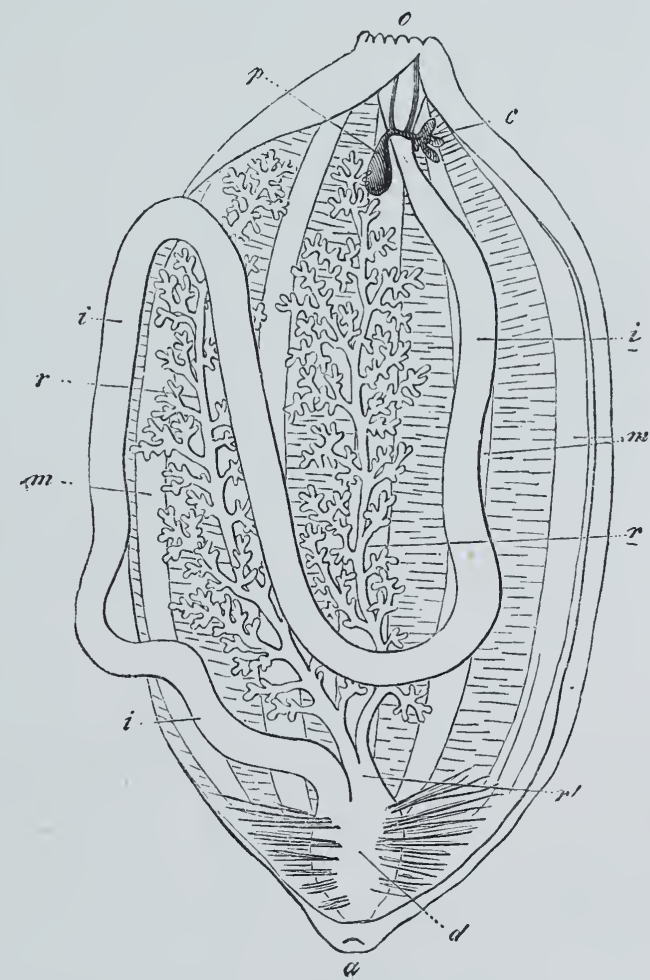

Fig. 109. Enteric canal and tree-like organs of a Holothurian. o Mouth. $i$ Enteric tube. $d$ Cloaca. a Anus. $c$ Branched stone-canal. $p$ Polian vesicle. $r r$ Tree-like organs. $r^{\prime}$ Connection between them, at the opening into the cloaca. $m$ Longitudinal muscular layer of the body. branches, which extend forwards throughout the whole length of the body-cavity (Fig. 109, $r$ ), and are provided with a large number of ramified cæcal tubes. Although the function of these organs, which were formerly known as "lungs," and considered to be internal respiratory organs, is different from that of the interradial cæcal tubes of the enteron of the Asterida, yet they are exactly the same morphologically, and are a further development of the simpler tubes of the Asteridi. 
The function of these organs is not at all certain. The view that they are respiratory organs is opposed to the fact that only one of them has any connection with the network of blood-vessels, while the other is merely attached to the body-wall, and projects into the body-cavity. However, the fact that water is taken up by these organs, and is again expelled, chiefly by the aid of the strong: muscular wall of the hind-gut, is of importance.

In some Apodia (Molpadia borealis) they are only provided here and there with branched cæca, while in others the number of caca is increased. Thus in M. chilensis not only is one of the trees divided, but the rectum also bears a number of smaller trees. The organ is divided five times in some Lisarmatidx. 'They are simpler in character in Echinocucumis (E. typicus), where they form long fine tubes, provided with one short branch only.

The tree-like organs of the Holothurize are absent in the Synaptre, but there is an arrangement, which as yet is only very incompletely understood; this consists of canals, which are placed along the insertion of the mesentery and open into the coelom by funnel-like ciliated orifices (Chirodota pellucida).

Glandular organs are also present on the rectum of many Holothuriæ in addition to the tree-like organs. These-the Cuvierian organs-either form unbranched crecal tubes, which are inserted singly or in thick tufts (Bohadschia, etc.), or they form racemose organs (Molpadia), or, finally, filamentous canals, beset with lobate tufts of glands, and arranged in a whorl (Pentacta and Muelleria). They secrete a substance which forms fine sticky filaments, which may serve as organs of defence.

\section{$\mathrm{C} œ 10 \mathrm{~m}$.}

\section{$\S 175$.}

The development of the cœlom from a vesicular structure cut off from the earliest rudiments of the enteron ( $\$ 171)$ gives to this cavity a different signification to that which it has in other divisions, where the cœlom is not formed from any part of the rudiments of the enteron. The importance of this point must not be overlooked. But it may well be supposed that the water-vascular system, which is developed in just the same way, formed an apparatus which primitively formed part of the cœlom, and was connected with the hind-gut.

'The two colomatic tubes nipped off from the enteron gradually increase in size, and by becoming attached in part to the enteron, and in part to the body-wall, form the more or less spacious cavity of the colom. The mesenteric filaments or bands which pass from the perisome to the enteron are to be regarded as the remains of the walls of these primitive structures.

As the radiate Echinoderm body becomes developed, the cœlom 
passes into the rays. Thus in the Asterida and in Brisinga it extends through the arms. The same thing happens in the Crinoïda, but in them the canals are narrower. In each arm it can be divided into three parts, which are connected with special divisions of the cœlom of the disc. This latter portion is separated into several divisions by connective bands, which here and there form membranous tracts; they communicate with one another at certain points, and at other points pass into the canals. In the Echinoïda and Holothuroïda, where the organism is more concentrated, the colom is more simple. In the former, however, the mesenteric filaments, and still more the calcified pillars and columns, which traverse the cœlom of the Clypeastridæ, remind us of divisions into separate parts; several such spaces are also marked off in the cœlom of the Holothuroïda. In the Asterida and Echinida, as well as in the Holothuroïda, the parietal and visceral tracts of the colom have been observed to be provided with cilia. The contents of the coelom appear to be the same in character as the blood, so that in it we have to recognise a portion of the blood-cavity. In some cases communications with the exterior have been definitely observed (Crinoïda); as also communications with the water-vascular system (Crinoïda, Holothuroïda). The first set are due to numerous canaliculi, which traverse the interradii of the perisome, and open by the so-called calycine pores.

\section{Vascular System.}

Blood-Vessels. $\S 176$.

The nutrient fluid in the Echinoderma is a clear, or slightly opalescent fluid, which is seldom thick or even coloured, and which is very probably mixed with the water which is taken in from the exterior. The form-elements in this fluid are simple cells.

The blood-cavity is formed in the first place by a special system of canals, but also by the cœlom, which is probably connected. with a third cavitary system, the system of so-called water-vessels. Owing to the uncertainty of our knowledge of this Vascular System, that is, of its mutual relations and connections, it is as yet impossible to make any generalisation which will hold for all the divisions; although indeed remarkable progress has lately been made in our knowledge of this part of the anatomy of the Echinoderma. But from the similarity of construction of these canals and spaces we may suppose that a connection between them does really exist.

The close association of the hæmal system and the nerve-tracts may, however, be regarded as a general arrangement. A bloodvascular trunk accompanies each radial nerve-trunk, and is continued 
into a circular canal, which surrounds the mouth. The radial vascular trunk corresponds to the ventral vessel of the Vermes, which has a similar relation to the ventral medulla. A tube which passes from the oral ring to the stone-canal (for which see below) was formerly regarded as the heart, but this organ cannot be regrarded as such. The same remark applies to the similar structure in the Echinoïda. We have therefore still to search for a heart as the central organ of the blood-vascular system. The enteric vessels form a second division of the blood-vascular system.

In the Echinoîda the nerves lie within the radial blood-vascular trunks; in the Crinoïda and Holothuroïda they lie on their outer side, and the same is the case in the Asterida and Ophiurida. The circular vessel surrounding the mouth in the Asterida and Crinoïda and in the Spatangidæ among the Echinoïda, where it has the form of a wide sinus, is described as having the same relations to the nervetract; although in Echinus there is said to be a blood-ressel placed farther from the mouth, and above the masticatory apparatus which surrounds the osophagus. It is probable that this separation of the blood-vessel from the nerve-ring is due to the development of the masticatory apparatus. In the Holothuroïda the adoral bloodvascular ring is connected with the nerve-ring, but is placed inside it, and nearer the mouth. It may break up into a plexus. The aboral vascular ring found in the Asterida and Echinida does not appear to liave so much morphological importance, as it is confined to a few divisions. Other vessels, which surround the generative glands, and there form wide sinus-like spaces, pass into it, in addition to the vessels from the perisome. In Comatula also a ressel, which forms a covering around the genital chord, is continued into the arms and pinnulæ. In the Asteroidda and Crinoïda the vessels of the enteric canal are not independent. In Comatula they form a network, with wide meshes, in the cœlom; this is connected with the oral vascular ring. A bundle of vessels passes from this network, along the axis of the cup to the centrodorsal plate, forming a special organ widened out into five chambers, the importance of which is not known.

The enteric vessels in the Echinoïda and Holothuroïda are more independent. A dorsal and a ventral vessel can be distinguished, which have just the same characters, as the same vessels in the Vermes (cf. \$ 138). In Echinus the dorsal vessel is double, for in addition to the one which runs directly on the enteron there is one a little way from it, which gives off branches to the former one, as well as to the enteron. In the Spatangide the rentral vessel has been observed to communicate with the water-vascular ring. The enteric vascular trunks of the Holothuroïda are enlarged in the middle of their course, and the dorsal ressel passes into retia mirabilia. 
Water-Vessels.

$\S 177$.

In describing the ambulacra ( $\$ 160)$, mention was made of a "water-vascular system," which took in water from the exterior, and carried it to the ambulacral organs, which it put into the condition of erection. Other organs in addition to the structures which take part in locomotion are filled by this system of canals ; and these wo have already spoken of as modifications of the ambulacral feet. The probability of this system of canals being a portion of the bloodvascular system has been already pointed out. Communications have been noticed at several points; and in some cases openings into the cœlom also have been distinctly observed. It is, however, not yet certain how far these vessels have been formed from other organs. In any case we must still regard the water-vascular system as being independent, especially as its development shows that it is so, and as an important division of the system (stone-canal, etc.) arises as a structure, which is primitively quite independent of the circulatory system.

In the larvæ of the Echinoderma the water-vascular system is formed by a differentiation from the earliest rudiment of the enteron; as it gets nipped off, it forms a transparent tube, ciliated internally, and connected with the integument on the back of the larva, where it soon opens by a pore. When in this condition the organ has a close resemblance to the excretory organs in the larvæ of many Vermes (Sipunculidæ), so that from this point of view it does not seem improbable that the watervascular system has been differentiated from a primitive excretory apparatus.

This tube, with the other rudiments of the Echinoderm (Fig.110, A), becomes gradually surrounded by the perisome; it then changes its form by becoming metamorphosed into a five-leaved rosette (i). The portion which still continues

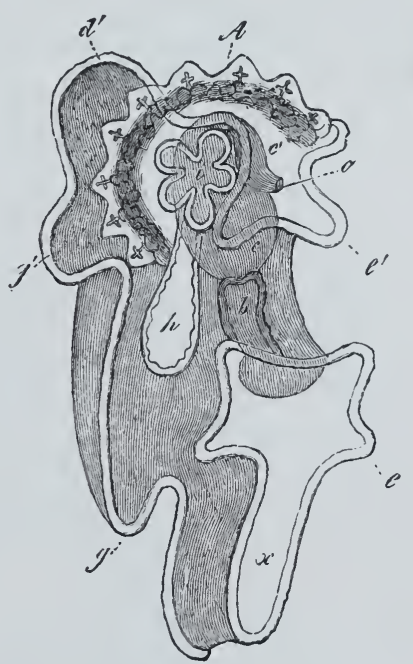

Fig. 110. Larva of an Asterid (Bipinnaria) with budding Echinoderm. $e e^{\prime} d^{\prime} g g^{\prime}$ Processes of the body. 6 Mouth. $o$ Anus of the larva. A Body of the embryonic Echinoderm. $h$ Ciliated tube. $i$ Ambulacral rosette (Rudiments of the watervessels) (after J. Müller). to open to the exterior by the dorsal pore gradually changes its position, and gets to lie on the ventral surface of the Echinoderm; each leaf of the rosette is now developed into an elongated canal with lateral diverticula; it is like a pinnate leaf, and forms the rudiments of the ambulacral portion of the water-vessels. In the 
Holothuroïda the similarly rosette-shaped rudiment forms the oral tentacles, which have therefore an indubitable relation to the ambulacral system (\$ 162). The succeeding processes which are of any importance affect the central portion of the rosette, in which the canals of the five leaves have their common orifice. This is converted into a circular canal, which continues to form the central portion of the apparatus; the canals in the leaves of the rosette grow out radially, and extend whilst the number of their lateral branches increases, over the ambulacra, which get larger at the same time.

The adult stage may be directly derived from these arrangements, formed during the development of the Echinoderm body. A branched vascular system (Fig. 111) has finally developed from the prinitive tube, and has its ends directly connected with the suckers $(p)$ and other such processes. The radial trunks of this system communicate with the circular canal $(c)$, and this again is in connection with

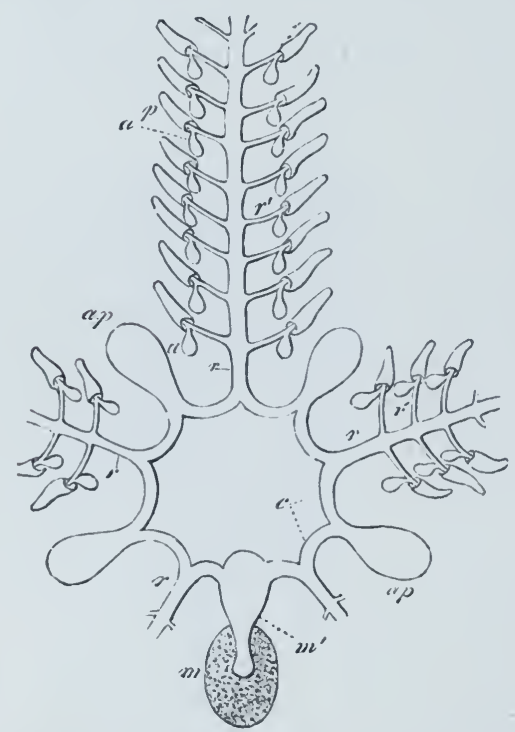

Fig. 111. Diagrammatic representation of the water-vascular system of a Starfish. $c$ Circular canal. ap Polian vesicles. $m$ Madreporic plate. $m^{\prime}$ 'Stone. canal. $r$ Radially-arranged principal trunks (Ambulacral canals). $r^{\prime}$ Lateral branches. $p$ Suckers. a Their ampullw (part only of the ambulacral canals and their appeudages are figured). the surrounding medium. TVe have already mentioned the fact that in Spatangus the water-rascular ring around the mouth is connected with an enteric vessel; and inasmuch as the contents of the two systems of canals are similar, it is very probable that they do not only communicate, but are also to be regarded as parts of one structure.

The connection with the cxterior is of a special nature, and is effected in various ways. As the Echinoderm is being differentiated in the larva, that portion of the rudimentary water-vascular system which is taken into the body of the Echinoderm remains connected with the perisome at one spot, where a porous calcareous platethe madreporic plate $(m)$-is developed; this plate communicates with the lumen of that portion of the canal which is comnected with it. 'The duct $\left(m^{\prime}\right)$ leading from the madreporic plate to the circular canal, which also is a portion of the primitive water-vascular system, has ordinarily calcareous substances deposited in its walls, and is therefore called the stone-canal; its walls form a complicated cavitary system. Water passes into the stone-canal by the cribriform madreporic plate, and thence to the circular ressel. It has also connections with the colom. 
The portion corresponding to the stone-canal is not always connected with the perisome. In the Holothuroilda the connection is broken close to the dorsal pore of the larva; the latter disappears, and the stone-canal hangs freely in the body-cavity, whence it takes up water by a very complicated and porous terminal apparatus.

There are furtler complications of the water-vascular system, due to the formation of contractile diverticula of the water-canals projecting into the body-cavity; these must be mentioned in addition to the arrangement, just sketched. These diverticula vary greatly in character; on the circular canal they form large pearshaped vesicles (Polian vesicles) $(a p)$; where the ambulacral canals pass into the sucking feet they form small ampullæ $(a)$, which always project into the body-cavity, and which may be regarded as enlargements or diverticula of the branches of the ambulacral canals. They are cavernous in structure. Both these kinds of organs serve as receptacles for the fluid passing into the canals, and owe their structure to their adaptation to the function of this vascular system; that is to say, when the suckers are drawn in, their ampullæ are always filled, and when the suckers are protruded the contents of the ampullæ swell them out. What the ampullæ are for the separate suckers, the Polian vesicles of the circular canal are for the whole system of canals; that is, they allow of a much more rapid action of the ambulacral structures, whether these are pushed out or drawn in, than would be possible if the quantity of fluid needed for the erection of each separate sucker must be first taken in either by the stone-canal or the madreporic plate. This activity of the ampullæ of the suckers and of the Polian vesicles of the circular canal is due to the contractility of their walls, in which a muscular layer has been made out. The distribution of the fluid is also regulated by muscular fibres, which here and there enclose the canals. In addition to this the ciliated epithelium which is found throughout the water-vascular system serves to distribute and continually change the water, and so without a doubt render it efficient as an organ of respiration.

\section{$\S 178$.}

The arrangement already sketched in a general way applies most completely to the Asteroilda. In them the stone-canal is always inserted into a madreporic plate, which, as a rule, is placed interradially on the dorsal surface of the body. In some cases there are several (2-5) madreporic plates, and the number of stone-

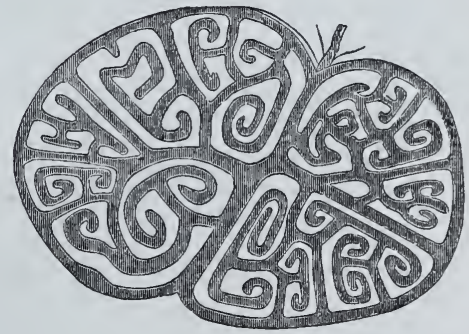

Fig. 112. Transverse section through the Stone-canal of Astropecten aurantiacus (after $\mathrm{R}$. Teuscher). canals is then also proportionately increased; this condition, however, does not remain constant in the species of the same genus. It is to be regarded as the more primitive one; it is important 
therefore to know the earliest rudiments of this arrangement. The stone-canal always runs close to the heart-like tube. Calcareous bodies are deposited in it and form a fine network; they do not differ from those found in the perisome. They are arranged in rings; internally to them there is a longitudinal ridge from which arise two coiled and thinner lamellæ, similarly calcified. The cavities which commence at the fine pores of the madreporic plate pass between these lamellæ. The ambulacral canals (Fig. 100, $A$ a) extend along the skeleton of the arms, embedded in the ambulacral groove, and give off branches to the feet which arise between the lateral processes of the segments of the ambulacral skeleton; the ampulla of the feet pass inwards through the clefts between the calcified segments, and so come to lie within the arms $(a p)$. At the points where the ampulla are comnected with the ambulacral feet there are valves, which shut when the ampulla contract (Asteracanthion rubens). The number of Polian vesicles varies; they are sometimes increased in number, and form racemose tufts (Astropecten aurantiacus), or they may be altogether wanting.

In the Ophiurida the stone-canal is inserted into a plate surrounding the mouth; but this plate is not formed in the same way as the madreporic plate, but so that the stone-canal takes up fluid from the body-cavity only. At the circular canal the stone-canal widcus out into an ampulla, and is attached to an interradial portion. Polian vessels are not always present. The suckers have no ampulle.

In the Crinoïda the ambulacral water-vascular trunk runs below the radial blood-vessel, and sends branches into the tentacles of the arms, as well as of the pinnulæ (Fig. 115, w). 'The radial trunks meet in a circular oral canal, which sends off short canaliculi, with open mouths, into the cœlom. They take the place of the stonecanal, which is not present. As there are no ampulle or Polian vesicles either, the water-vascular system is in the Crinoïda of a lower grade than in the other divisions.

The Echinoïda are allied to the Asteroïda. The madreporic plate always lies at the aboral pole; it is either formed by one of the genital plates (Fig. 103, m), or by several of these, or an interradial plate is converted into the madreporic plate, or it is formed by a special plate (Clypeastrida). The stone-canal is sometimes soft (Echinus) and sometimes provided with firm walls (Cidaris). The circular canal, provided with five Polian vesicles (these are absent in the Spatangida), lies in the Echinida at the base of the masticatory apparatus, and gives off its ambulacral canals downwards, whence they racliate out to the ambulacra. On the inner side of the shell, and ruming along each of the ambulacral aree, are the branches of the ambulacral canals, which are distributed to the pores of the calcareous plates, and supply the suckers or their equivalents which arise at this point; and give origin to transversely-placed ampullar enlargements (Fig. 116, $\iota)$.

In the Holothuroilda, owing to the scparation of the comnecting piece, which later on functions as the stone-canal, from the perisome of the larva, which passes into the substance of the Echinoderm, 
these parts have a different relation to that which they have in the rest of the Echinoderma. The walls of the stone-canal, which hangs freely into the cœlom, are rnore or less calcified; when they are more so they form a firm capsule. The porous parts of the canal are usually distinguished by calcification, and so repeat within the body the arrangements of a madreporic plate. The ends of each branch carry a porous piece when the stone-canal is broken up into branches; this repetition of parts leads to the formation of racemose structures, which are only functionally similar to a number of madreporic plates grouped around the stone-canal. The stonecanals vary in number, as well as in arrangement. Often only one is present; in other cases, and notably in the Synaptr, there are several arranged around the circular canal. The number too of the Polian vesicles (Fig. 113, p), which are present in these forms, varies; in Holothuria and Molpadia there is one, in Synapta Beselii about fifty, and in Cladolabes about a hundred.

The canals from the circular canal $(C)$ run forwards inside the calcareous ring $(R)$, and give off branches to the oral tentacles $\left(T^{\prime}\right)$; a cæcal elongated tube, corresponding to the ampullæ of the suckers, is

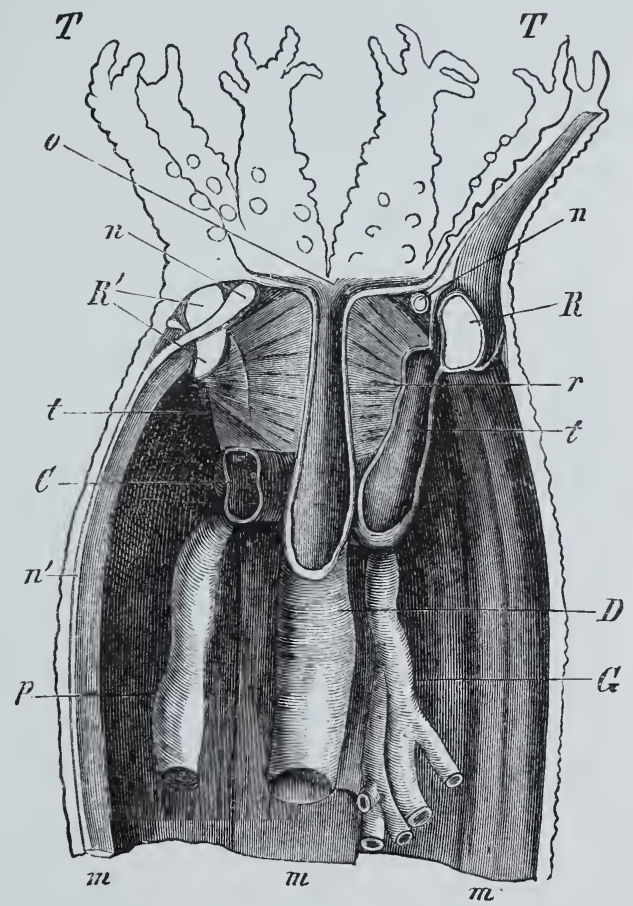

Fig. 113 Longitudinal section through the anterior part of the body of Synapta digitata. $R R^{\prime}$ Calcareous ring. $r$ Muscles passing from it to the œsophagus. o Mouth. $D$ Enteric tube. $C$ Circular canal. $t$ Canals to the tentacles $T$. $p$ Polian vesicles. $n$ Nerve-ring. $n^{\prime}$ Radial nerve-trunk, passing through the calcareous ring $R^{\prime}$. $m$ Bands of longitudinal muscles. $G$ Ducts of the generative organs (after Baur).

connected with each of them. These tubes are of some size in the Holothurida, and lie on the outer side of the calcareous ring; they are only feebly developed in the Synaptidæ. The radial trunks going to the ambulacra are placed, in Holothuria, in the bundles of longitudinal muscles, which are thus divided into two halves. In Cucumaria they are placed on the outer side of these muscles. The branches of these canals, as in other forms, go to the feet. When the feet are atrophied the vascular branches, which go to them, are atrophied also; but the principal trunks appear to persist even in the Apodia, for they have been observed in Synapta, although, indeed, diminished in size. 


\section{Excretory Organs.}

$\S 179$.

The arrangements commonly found among the Anuulata (looped canals, or nephridia) are not found in the Echinoderma, but there are signs that these organs, or organs of the same type at any rate, are not entirely foreign to the organisation of the Echinoderma. For in the Holothuroïda two canals have been obserred to run in the wall of the body; and these are beset with infundibular organs, which open into the cœlom (Chirodota pellucida). In the Synapte also there are organs which correspond to the internal mouths of the looped canals of Vermes, but these are not connected with canals. Finally, in the Crinoïda ciliated organs have been made out in the dorsal canal of the arms, which is a continuation of the cœlom. It cannot be definitely asserted that these structures are all of the same kind, but from the characters of those first mentioned it is probable that they have relatious to an excretory apparatus. We can as yet only suggest that a fundamental relation of the sâme kind holds for the ambulacral water-vascular system. Anyhow the arrangement of this system in the body does not justify us in regarding inquiries in this direction as barren ones, for the excretory organ of many Mollusca (Nudibranchiata) has the form of a richly-branched system of canals; and the communication of the water-vascular system with the exterior, as well as with the blood-vessels (or, what is the same thing, with the colom), can hardly point to its being anything else than part of an excretory apparatus.

\section{Generative Organs.}

\section{$\$ 180$.}

The asexual methods of reproduction so common among Vermes do not obtain in the Echinoderma, except in so far that the animal itself is the product of gemmation. An indication of this mode of reproduction is, however, retained in the Asteroïda-in the regeneration of lost antimeres (arms).

Almost all Echinoderma-there are but few exceptions-have the sexes separate, and their organs arranged conformably with the radiate type. The male and female organs are both very simple in character, and can only be distinguished easily when the generative products are mature, the ovaries being generally distinguished by the brighter coloration of the eggs, which are yellow or red, whilst the testicular tubes are almost always white. The form-elements of the sperm are very generally filamentous structures provided with a small head. The generative 
system is simple in structure, the excretory ducts aro not complicated, and there is no intromittent organ, so that the surrounding water is the medium of communication in impregnation. On the whole there is a great resemblance to the corresponding structures found in Vermes.

The lowest stages, both as to number, arrangement, and as to the more special characters of the organs, are seen in the Asteroïda. The testes or ovaries are tubular or lobate glandular canals, which in some forms are arranged in two rows, and disposed conformably with the metamerism of the arms (Ophidiaster, Archaster). In others there are only two groups in each arm, which may extend along the whole cavity of the arm (this is the case even in Brisinga), or may be limited to the interradial space merely (Fig. 108, g). A comparison of these characters shows us, that there is a gradual reduction in the number of the generative glands, which corresponds with the gradual centralisation of the organism, which we have already seen to occur in the Asteroïda. In the aproctous forms the tubes have no excretory orifices, and the generative products are passed into the bodycavity. We do not yet know how they reach the exterior. In other Asteroïda the generative glands open to the exterior by special plates, distinguished by their fine pores (cribriform plates), and placed in the dorsal interradii; or they have a single duct with a narrow orifice (Pteraster). Each organ is surrounded by a blood sinus, which envelopes the separate lobes and lobules. The generative products pass into this sinus and are not evacuated direetly.

The structure and arrangement of the generative organs of the Ophiurida

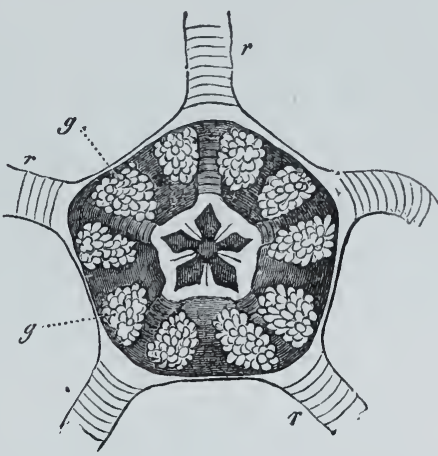

Fig. 114. Generative organs of an Ophiurid (Ophioderma longicauda). The dorsal integument and the digestive organs have been removed. $r$ Arms. g Ovarian acini.

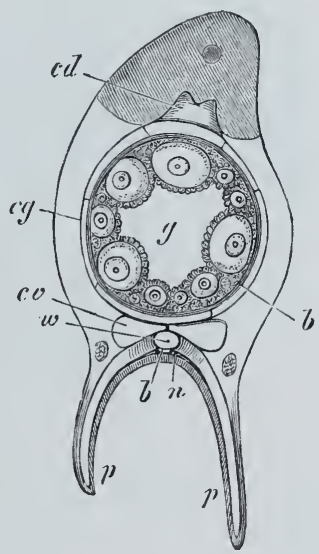

Fig. 115. Transverse section through the pinnula of a sexually mature Comatula (Antedon Esch. richti). The dorsal surface is turned upwards, and the ventral downwards. $p$ Tentacle. $g$ Lumen of the genital chord. $w$ Watervessel, with lateral branches into the tentacles. $n$ Nerve-chord. $b$ Blood-vessel on it. $c g$ Canal around the genital chord. $c d$ Dorsal, $c v$ Ventral canal. All three com. municating with the coelom (after H. Ludwig). is the same as in the Asteroïda. There is only one example of hermaphroditism (Ophiura squamata). The generative glands (Fig. $114,9)$ are arranged by pairs in each interradial space, and on the 
dorsal side; their products appear to pass into the cœlom, whence they reach the exterior by the narrow pores placed in the interradii of the ventral surface (Fig. 102, g). In the ovoviviparous Ophiurida this cleft may be seen to adapt itself to the necessary size. A chordlike structure placed in each arm, and ramifying into the pinnulæ, forms the generative organs of Comatula. This genital chord is surrounded by the blood-vessels just as are the branched tubes of the Asterida. In the arms it remains sterile, and its products are developed within the pinnulæ from the walls of the chord. The sperm passes out by the pores already described.

\section{$\S 181$.}

The generative glands, which in the Asteroïda are arranged by pairs in each radius, are converted into unpaired structures in the

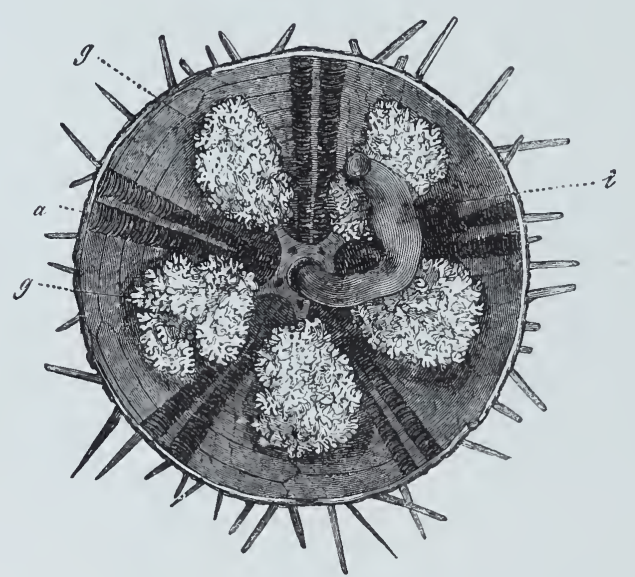

Fig. 116. Generative organs of an Echinus. Rather more than the ventral half of the shell has been removed. a Ampullæ of the ambulacra. $i$ Hind-gut. $g$ Orarian acini. Echinoïda; this implies further centralisation. Their relation to the primitive condition can only be made out from their interradial arrangement, so that each organ may be regarded as formed of two radial ones. They form greatly ramified glands, which generally project far into the colom (Fig. 116, g), and open at the genital plates (Fig. 103, g). In the Spatangidx one of the five genital glands, which are the typical set for the Echinoïda, is atrophied, and at the same time the genital plate, which was the madreporic plate also, becomes the madreporic plate only.

In the Holothuroïda they are still more reduced. The testis or ovary forms a tuft of richly-branched tubes, which unite at a common duct (Fig. 113, G); this opens near the mouth, and ordinarily between the tentacles. This indicates a relation to the radii; the organs, which in other forms are broken up, are here united into one, and the higher grade of organisation, already seen in the Echinoilda, is still present, as is shown by the presence of an excretory duct.

In the Synaptre the organs are hermaphrodite, and formed on the Holothurian type. 'The various tubular glands are united at a common excretory duct, which opens to the exterior above the 
calcareous ring. In each tube (in S. digitata) the sperm is developed on the inner surface, while the ova are formed beneath it, and when fully developed form a longitudinal band which projects into the lumen of the tube. There is a common duct for both sets of products. If this condition must necessarily be regarded as a lower one, from which the ordinary diccious arrangements have been derived, we find an interesting phænomenon in the Synaptæ; for while their generative gland has retained its primitive structure and primitive function, great changes have taken place, for the glands have been reduced in number and degree of complication, and have only one excretory duct for the whole apparatus. 


\section{Fifth Section.}

\section{Arthropoda.}

\section{General Review of the Group.}

$\$ 182$.

In this division of the Animal Kingdom the body consists of a number of metameres, and this number is generally definite for the various groups. As a rule the metameres are not all differentiated to the same extent; this is not implied merely by variations in external form and size, but also in the characters of the internal organs of the body. A number of metameres may be united to form larger segments, in which the separate metameres lose their individuality. These larger segments sometimes retain indications of this kind of composition; but these indications are sometimes lost, or only apparent in the early stages of development. This state of things results in a desegmentation of the body.

The movable appendages of the body form another characteristic of the whole group; these appendages are almost always segmented. In this point therefore, as in the metameric character, they have some resemblance to the Annulata among the Vermes. We do not know what are the forms that ally the two groups, and it is not certain that the two chief groups of the Arthropoda form a common phylum. There are many reasons for regarding the Branchiata and 'l'racheata as being distinct stem-forms. As in the Annulata the nervous system consists of an œsophageal ring connected with a ventral ganglionic chain; while the central organ of the circulatory apparatus is here, also, dorsal in position. In the Vermes organs are repeated in each segment, but in the Arthropoda they are common to the whole of the body; and even when the metameres are similar externally, the internal organisation often gives signs of the more confined dominion over the whole body now possessed by meta- 
merism, as compared, that is, with that which it exercised in the Annulata.

I give the following review of the Classification of the Arthropoda :

\section{A. Branchiata.}

\section{Crustacea*).}

a) Entomostraca.

1. Cirripedia.

Balanus, Coronula, Lepas.

Rhizocephala.

Sacculina, Peltogaster.

2. Copepoda.

Cyclops, Cyclopsina, Corycrus, Sapphirina.

Siphonostomat).

Caligus, Ergasilus, Dichelestium, Chondracanthus,

3. Ostracoda $)$. Achtheres, Lernæa, Lernæocern, Penella.

4. Branchiopoda§).

Cypris, Cspridina.

Cladocera.

Daphnia, Sida, Polyphemus, Evadne.

Phyllopoda.

Branchipus, Apus, Limnadia.

b) Malacostraca\|). 1. Thoracostraca (Podophthalma).
Schizopoda.

Carida $\uparrow$ ).

Mysis, Euphausia, Thysanopus.

Crangon, Alphens, Palæmon, Hippolste, Peneus. Decapoda.

Macrura.

Astacus, Palinurus, Galathea, Pagurus.

Brachyura.

Carcinus, Maja, Hyas, Dromia, Dorippe.

Cumace a $\left.{ }^{* * *}\right)$.

Cuma.

* The appendages are complete in each segment of the body, although they often undergo adaptive modifications. They either function directly as respiratory organs, or the latter are very closely counected with them.

+ A large number of families are brought together into this special subdivision, owing to the more or less complete parasitism they exhibit; they may be distingnished from free-living Copepoda, but must be considered as derived from them. The Rhizocephala bear the same relation to the Cirripedia.

* They show signs of affinity with the developmental stages of the Cirripedia, by the possession of a mantle-fold, which forms the two-valved shell.

$\S$ This division seems to be the most direct continuation of the Nauplius-form, from which stage it is derived by the mere formation of metameres, while the appen. dages themselves undergo only slight modifications.

II These forms, which are allied to the preceding division by the Nauplius stage, which is seen in Peneus and Euphausia, represent on the whole a further development of the Crustacean organisation. latter.

If Connect the Schizopoda and Decapoda: sometimes they are classified with the

** They correspond to the lower developmental stages of the Decapoda, for they closely resemble the Schizopoda in their form of body. The eyes have no movable stalk, and so far they approximate to the Arthrostraca. 


\author{
b) Malacostraca (continued). \\ Stomapoda. \\ Squilla. \\ Tanaïda $a^{*}$. \\ Tanais. \\ 2. Arthrostraca (Hedriophthalma). \\ Amphipoda. \\ Gammarus, Orchestia, H5peria, Phronyma. \\ Læmodipoda. \\ Is opoda. \\ Caprella, Cyamus. \\ Bopyrus, CJmothoa, Sphæroma, Oniscus, Asellus, \\ Idothea.
}

II. Pœcilop odat).

Limulus.

B. Protracheata + ).

C. Tracheata.

Peripatus.

I. Arachnida.

Autarachnæ§).

Arthrogastres.

Galeodea.

Galeodes.

Scorpionea.

Scorpio.

Phrynida.

Telyphonus, Phrrnus.

Pseudoscorpionea.

Chelifer.

Opilionea.

Phalangium, Opilio.

Aranea.

Salticus, Thomisus, Argyroneta, Tegenaria, Mrgale. Acarina\|l).

Acarus, Argus, Ixodes, Gamasus, Atax, Thrombidium.

* These form a division which is allied partly to the Thoracostraca, and partly to the Arthrostraca, and which seems to hare remained more like the primitive form of the Malacostraca.

+ These are connected with the, palæontologically, very old, and completely lost division of the Trilobita, through the fossil Belinuridæ. There are many characters in their structure and development which point to their being distinct from the Crustacea.

\# More exact investigations into the organisation of Peripatus show that this animal, which as jet has been generally placed with the Vermes, is the representative of a special class of Arthropoda, which must be placed before the Tracheata; a much lower stage is seen in it than in any of the larger divisions of the Tracheata. A form seems to be here retained, which separated from the Tracheate phylum, before it broke up into its sereral branches.

$\S$ The true Arachnida vary considerably in the characters of their body-segments, and in the larger sections formed by the fusion of a number of segments. We regard those forms in which there are several such sections, in which it is still possible to see that they are composed of metameres, as the least altered and most similar to the primitive form. The Cyphophthalmidæ (Gibocellum) and the Chernetidæ form small divisions, which belong to the Arthrogastres; the former are allied to the Opilionea, and the latter to the Pseudoscorpionea.

II There seems to be no doubt that degeneration is present in these, and is indi. cated by the parasitism which obtains in most of the families; it leads in the family of the Linguatulida to a very remarkable change in the form of the body. 
Autarachnæ (continued).

Linguatulina.

Pentastomum.

Pseudarachnæ*).

Tardigrada.

Macrobiotus.

Pycnogonida.

Pycnogonum, Nymphon.

II. Myriapoda.

Chilopoda.

Scolopendra, Lithobius.

Chilognatha.

Polydesmus, Julus, Glomeris.

III. Insecta (Hexapoda).

1. Aptera $\dagger$ ).

Collembola.

Symnthurus, Podura.

Thysanura.

Campodea, Lepisma, Machilis.

2. Pterygota.

Pseudoneuroptera.

Amphibiotica.

Ephemera, Chloë, Perla, Libellula, Agrion, Eschna.

Corrodentia.

Psocina.

Psocus, Troctes.

Embida.

Embia.

Thysanopoda.

Thrips.

Termitida.

Neuroptera.

Termes.

Planipennia.

Panorpina.

Panorpa, Bittacus.

Sialida.

Rhaphidia, Sialis.

Hemerobida.

Hemerobius, Chrysopa, Myrmeleon.

Trichoptera.

Phryganida.

Phryganea, Iimnophilus.

Strepsiptera.

Stylops, Xenos.

* Very divergent forms are found in both divisions of the Pseudarachnæ, and have really very little in common except their divergence from the Autarachnæ. It is not quite certain that the Tardigrada have any relations to the Tracheata.

- The two groups united in the division of the Aptera are removed from all other Insects, owing to the difference in their organisation; we cannot therefore place them with any one order. Though they have much in common with the Pseudo. neuroptera, it is because the latter are so low. Their want of wings must be regarded as a primitive condition, as compared with the adaptative wingless condition, examples of which may be seen in nearly all orders of the Pterygota. 


\section{Pterygota (continued).}

Orthoptera.

Ulonata.

Cursoria.

Blatta, Mantis.

Saltatoria.

Gryllus, Gryllotalpa, Acridium, Locusta.

Labidura.

Forficula.

\section{Coleoptera.}

Carabus, H5drophilus, Silpha, Lucanus, Melolontha, Scarabæus,

Tenebrio, Meloë, Chrysomela, Coccionella, Lampyris, Ełater, Bostrichus, Curculio.

Hrmenoptera.

Formica, Bombus, Apis, Vespa, Sphex, Sirex, Tenthredo, Ichneumon, Cynips.

Hemiptera.

Homoptera.

Cicadina.

Tettigonia, Cercopis, Fulgora, Cicada.

Phythophthires.

Aphis, Chermes, Coccus.

Heteroptera.

Notonecta, Nepa, Hydrometra, Reduvius, Cimex, Capsus, Ljgæus, Pentatoma.

Pediculina*).

Pediculus, Phthirius.

Diptera.

Nemocera.

Tipula, Simulia, Chironomus, Corethra, Culex.

Brachycera.

Estrus, Musca, Tachina, Sjrphus, Bombylius, Tabanus.

Pupipara $\dagger$ ).

Melophagus, Hippobosca.

Aphaniptera + ).

Pulex.

Lepidoptera.

Heterocera.

Pterophorus, Tinea, Tortrix, Geometra, Psjche, Noctua, Cossus, Bombyx, Sphinx, Smerynthus, Zjgæna.

Rhopalocera.

Hesperia, Pieris, Vanessa, Colias, Papilio.

\section{Bibliography.}

\section{Branchiata.}

Crustacea : Müluer, O. F., Entomostraca, 1785.-Jrkire, Histoire des Monocles, 1820._Mrusr. EDWARDS, Hist. nat. des Crustacés. III. Vol. 1831-10.-The same, "Crustacea" in the Cyclopædia of Anatomy. Vol. I.-Rıтнке, De Bopyro, et Nereide Comm. Rigæ et Dorpati, 1837.--ZADDACH, De Apodis cancriformis anatome, 1841.-GREBE, Bemerkungen über die Phyllopoclen. Arch. f. Nat. 1853.-Lerdig, Ueber Argulus foliaceus. Zeitschr. f. wiss. Zool. Bd, II. Ueber Artemia salina und Branchipus stagnalis. Ibid. Bd. III.-The same, Naturgeschichte der Daphniden. Tübingen, 1860.-D.tiwis, A Monograph of the Subclass

* Forms degenerated by a parasitic habit.

+ These too show the influence of the parasitic habit.

\& They have undergone modification owing to a parasitic habit. 
Cirripedia. I. II., 1851, 1853.-ZENkER; W., Anatomisch-systemat. Studien über die Krebsthiere. Arch. f. Nat. 1851. - VAN BENEDEN, Recherches sur la faune littorale de Belgique. Crustacés, Acad. Bruxelles, 1861.-Cuaus, Die frei lebenden Copepoden. Leipzig, 1863.The same, Ueber den Bau und die Entw. parasitischer Crustaceen. Cassel, 1858.-The same, Beiträge zur Kenntniss đer Entomostraken. Marburg, 1860.-The same, Ueber einige Schizopoden, Zeitschr, f, wiss. Zool. XIII-The same, Beobacht, üb. Lernæocera, etc. Marburg u. Leipzig, 1868. - The same, Beitrïge zur Kenntniss der Ostracoden. Marburg, 1868. Die Metamorphose der Squilliden. Gött. 1871. - The same, Zur Kenntn. d. Baues u. d. Entwickl, von Branchipus stagn. u. Apus cancriform. Gött. 1873. Zur Kenntn. d. Baues u. d. Entw. von Branchipus u. Apus: K. Gesellsch. d. Wiss. z. Gött. Bd. VIII.-The same, Z. Erforsch. d. genealog. Grundlage des Crustaceen-Systems. Wien, 1876.-MüLLER, Fr., Für Darwin. Leipzig, 1864.

Pœcilopoda: VAN DER HoEver, Rech. sur l'hist. nat. et l'anatomie des Limules. Leyden, 1838.-Packard, A. S., The devclopment of Limulus. Mem. Boston Soc. Nat. Hist. Vol. II. -Owex, R., On the Anatomy of the American King-Crab. Trans. Linn. Society. Vol. XXVIII.--Mrlne-Edwands, A., Rech. sur l'anat. des Limules. Ann. Sc. Nat. V. Sér. Tome XVII.

Protracheata: Grube, Ueber d. Bau v. Peripatus Edwardsii. Arch. f. Anat. 1853.SAEngER, N., Perip. cap. und Perip. Leuckarti. Verhandl. der ersten russ. Naturforscher versamml. zu Moskan, 1869 (russ.).-Moseley, H. N., On the Structure and Development of Peripatus capensis. Phil. Trans. London, 187٪. P. II.

\section{Tracheata.}

Arachnida: Trevirayus, G. R., Ueber den inneren Bau der Arachniden. Nürnberg, 1812. -Dugìs, Recherches sur l'ordre Acariens. Ann. sc. nat. II. I. Ir. 1834.-The same, Sur les Aranéides. Ibid. II. vi. 1856.-DoY Ère, Sur les Tardigrades. Ann. sc. nat. II. x. 1810.Tulk (Opilioniden). Ann. nat. hist. 1843. Fror. Not. Bd. 30.-NewPort, On the nervous and circulatory system in Myriapoda and macrourous Arachnida. Philos. Trans. 1843.Quatrefages, Organisation des Pycnogonides. Ann. Sc. nat. III. Iv. 1845. - VAN BenedeN (Linguatula), Acad. Bruxelles, 1819.-Levckart, Bau und Entwickelungsgesch. d. Pentas. tomen. Lcipzig u. Heidelberg, 1860.-Durour, L. Hist. anatomique et physiologique des Scorpions. Acad. d. sciences (Savants étrangers) XIV.-The same, Anat. physiol. et hist. nat. des Galéodes. Acad. des sciences (Savants étrangers) XVII.-KITTARY, Anat. Unters. v. Galéodes. Bull. de la soc. imp. des Naturalistes de Moscou, 1818; and in Frorikp's zoolog. Tagesberichte Nr. 108.-Stecrer, A., Anatom. u. Hist. über Gibocellum. Arch. f. Nat. 1876.

Myriapoda: Treviranus, G. R. (Scolopendra und Julus), Vermischte Schriften. II. Bremen, 1817.-Dufour, L., Recherches anatomiques sur le Lithobius fortificatus et le Scutigera lineata. Ann. sc. nat. II. 1821.-MüLLER, J., Zur Anat. der Scolopendra morsitans. Isis, 1829. p. 519.-BRANDT, Beiträge zur Kenntniss des inneren Baues von Glomeris marginata. A. A. Ph. 1837.- Jones, R., "Myriapoda" in the Cyciopædia of anatomy and physiology. Vol. III. 1812.-NEwPORT, On the organs of Reproduction and the development of the Myriapoda. Phil. Trans. 1811.-The same, On the structure, relations, and development of the nervous and circulatory system in Myriapoda and macrourous Arachnida. Phil. Trans. 1843.

Insecta : RÉAUMUR, Mémoires pour servir à l'histoire des Insects. 1731-42. Paris. 6 vols.Swamrerdar, Bibel der Natur. 1752. -LyonbT, Traité anatomique de la Chenille qui ronge le bois de saule. La Haye, 1762.-Strauss-Durckreim, Considérations sur l'anatomie comparée des animaux articulés, auxquelles on a joint l'anatomie descriptive du Melolontha vulgaris. 1828.-BURMEISTER, Handbuch der Entomologie. Bd. I. Berlin, 1833.-NewPort, "Insecta" in the Cyclopædia of anatomy and physiology. Vol. II. 1839.-Dufour, L., Recherches anatomiques et physiologiques sur les Hémiptères. Mém. Acad. des sc. (Sav. étrangers) IV. 1833.-The same, Sur les Orthoptères, les Hymenontères et les Neuroptères. Ibid. VII. 1841.-The same, Sur les Diptères. Ibid. XI. 1851.-PICTET, Recherches pour servir à l'hist. et à l'anatomie des Phryganides. Genève, 1831.-Lrucrart, Die Fortpflanzung u. Entwick. der Pupiparen. Halle, 185்8.-Brauer, Fr, Z. Anat. d. Neuropteren in Schrift. d.zool.-bot. Vereins z. Wien.-Works by Lokw in various entomological journals.-Lerpra's numerous researches into the more intimate structure of Insects. - WeIssmas, Die Entwickelung der Dipteren. Leipzig, 1861.-Liv dors, L., Anat. d. Hundeflohes. N. Acta Acad. L. C. Vol. XXXIII. - The same, Anatomie der Bettwanze. Zeitschr. für w. Zool. Bd. XVIII. XIX.Lowne, B. Th., Anat. and Phys. of the Blow-Fly. London, 1870.-Mülter, Fr., Z. n. Kenntniss d. Termiten. Jen. Zeitschr. Bd. X.-Kowalevskr, A., Embryol. Studien an Würmern und Arthropoden. Mém. Acad. St. Pétersbourg. I. XVI. No. 12.-Tullberg, 'T. Sveriges Podurider. Kongl. Vet. Acad. Handl. T. X.-Luввоск, J., Monogr. of the Collembola and Thysanura. London, 1873. Ray Soc.-Merer, P., Anat. v. Pyrrhocoris apterus. Arch. f. Anat. u. Phjs. 1875.-The same, Ueber Ontogenie u. Phylogenie der Insecten. Jen. Zeitschr. Bd. X. 


\section{Form of the Body.}

\section{\& 183.}

Among the Crustacea the simplest stage of the Arthropod body

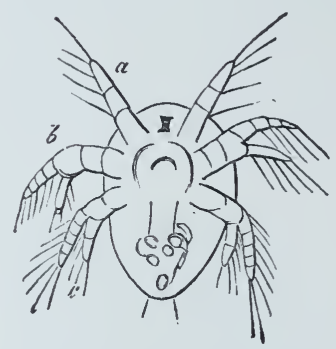

Fig. 117. Nauplius of a Copepod (Cyclops). $a b c$ Appendages. is seen in the Nauplius-form (Fig. 117). The unsegmented body carries several pairs of appendages. The body only becomes segmented by a gradual process of gemmation, which has many points of resemblance to the process which brings about metamerism in most of the Annulata. The most anterior portion of the body of the Nauplius which carries the first appendages forms the cephalic segment; the posterior part becomes the last metamere ; new metameres are formed between these two, on which appendages similarly sprout. In this way an organism composed of a larger number of metameres is gradually formed (Fig. 118); the complication of which, so far as it is due to metamerism, is the product

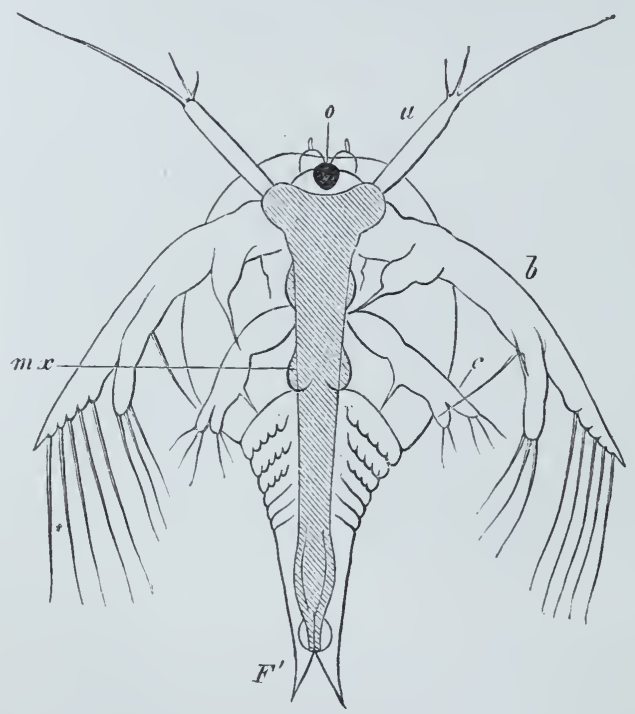

Fig. 118. Larva of Branchipus staguais. (second stage). $a b c$ Appendages. mx Rudiments of the maxillæ. $F^{\prime}$ Caudal fork. o Eye (after C. Claus). of a gradual process. This development of the form of the body predominates in the Entomostraca; it corresponds, probably enough, to the phylogeny of these Crustacea, which therefore may be referred back to an unsegmented condition. In the Malacostraca this process of the formation of new metameres can be made out in a few cases only, and the rudimentary body ordinarily consists at the very first of a larger number of metameres. The consecutive formation of metameres is here compressed; and the same happens in the Pœecilopoda and in most Tracheata. Although this might seem to remove any doubts as to the common origin of the Arthropoda, it is not of so much weight 
as are the arguments against such a supposition, which are afforded us by the differences presented by them in many points of their organisation. At present therefore we can only with safety regard the Crustacea as having a monophyletic origin; this is indicated by the common Nauplius stage. This stage, which is seen in all the Entomostraca, obtains in some cases only among the Malacostraca, whence we may conclude that this latter division of the Crustacea has a later point of origin than have the Entomostraca.

The body of the Arthropoda, which is formed into metameres either by gradual gemmation, or by the immediate differentiation of the embryo, gradually loses, to a greater or less extent, the primitive similarity of its segments. Great changes in external form are brought about by the development of some, and the degeneration of other, or, lastly, by the concrescence of a number of, metameres. As a general rule the metameres are similar in the early larval stages, and so indicate relationship to forms in which the metameres are still indifferent. The more compact portions of the body, which are formed by a fusion of the metameres, bear indications of their mode of origin in the appendages which they carry.

The most anterior metameres are those which most completely undergo concrescence. In this way a portion which carries the mouth and the higher seinsory organs, and especially the eyes and tentacles, is formed; this is the head. In the Myriapoda, many Crustacea, and Insect-larvæ, it is the only portion which is formed by the concrescence of a number of metameres. Owing to this concrescence of the metameres the appendages are approximated to the mouth, where they aid in taking up food, and are converted into mouth-organs. Other differentiations play various parts in the different divisions. In the Crustacea a number of metameres behind the head unite with it to form a cephalothorax. The other metameres are again often divided into two groups, inasmuch as those behind the cephalothorax sometimes differ from the most posterior ones. They thus form an abdomen and a post-abdomen. The segments of the abdomen fuse in the Poecilopoda, where the postabdomen is represented by the caudal spine.

Special arrangements for the protection of the appendages are formed by the folding and extension of the integument. Thus, in the Decapoda, the dermal skeleton of the cephalothorax grows out at the sides, covers the gills, and forms on either side a special cavity - the branchial cavity-which communicates with the surrounding medium. (Cf. $\$ 187$.

Such developments from the dermal skeleton, belonging to several primitive body-segments, may extend over the other divisions of the body, and form a "shell" for them. The earliest form of this is seen in the shield-like, enlarged, cephalothorax of the Phyllopoda (Apus) among the Branchiopoda. When the two halves of this structure are further developed we get a bivalve shell (Fig. 124, d) (Limnadia). In the Cladocera also a portion of the dorsal integument is converted into a shell, which covers the whole of the 
posterior portion of the body, and in the Ostracoda, as in many Phyllopoda, the two halves of this structure are movably connected with one another on the dorsal surface. In them the valves of the shell extend also over the anterior portion of the body, and so enclose the whole animal.

The very peculiar modifications of the integument in the Cirripedia are structures of this kind. The fold, which in the Ostracoda is formed into a bivalve shell, is seen in an early stage in the Cirripedia. When the animal becomes attached by its antennæ, the dorsal portion of the integument is converted into a wide sack or

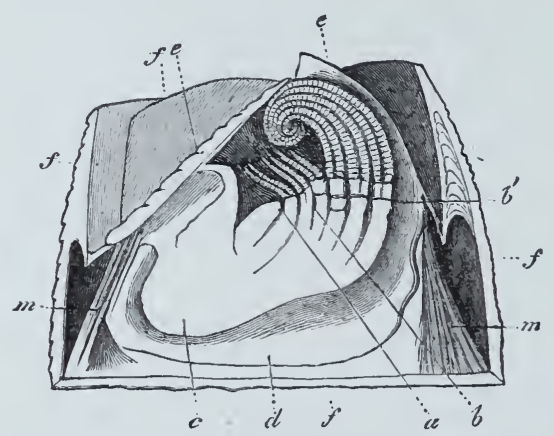

Fig. 119. Transverse section of a Balanus. $a$ Mouth of the animal. $b b^{\prime}$ Append. ages conrerted into tendril-like structures. $c$ Head of the animal. d Mantle-like en. velope. $e$ e Movable ralres which close the shell. $f f$ External shells. $m$ Miuscles (after Darwin). mantle (Fig. 119, $d$ ef) which encloses the body; the sack and the enclosed body are united to one another in the cephalic region only. The portion of this sack, which carries the primitive point of attachment, either remains soft and grows out into a stalk-like organ (Lepadidr), or is converted into a broad basal surface (Balanidæ). In many Cirripedes the whole mantle remains soft (Alepas). But most of them acquire firm calcified shell-pieces, which are developed in the outer lamella of the mantle. The rest of the body, with the post-abdomen, which is beset with tendril-like feet, is enveloped in this mantle, which has the form of a shell, and is in connection with the surrounding medium by means of a cleft, which can be closed at will.

In the Rhizocephala this mantle-like envelope forms a tube, which is smooth externally, or a disc, which is marked off into symmetrical lobes. A narrow orifice, like that which leads into the mantle cavity of the Cirripedia, leads into a space which corresponds to this mantle cavity, and functions as a marsupial pouch. In the Cirripedia part of the typical crustacean body, with its appendages, is enveloped by the mantle, and permanently retains its structure; but in the Rhizocephala the whole surface of the jointed body becomes converted into the mantle.

Another phænomenon, due to the mode of parasitism, is correlated with this atrophy of the body; numerous tubules are developed by the part of the head which is sunk into the body of the host; these tubules, which partly anastomose into retiform plexuses, extend to the enteric canal of the host, and surround long tracts of it. Thus an apparatus is formed which extracts nutrient fluid directly from the enteron of the host, and carries it to the parasite. Many other examples of the degenerating influence of the parasitic habit may be observed; as, for example, the varied forms of the Siphonostoma. 
Peripatus has a simple form of body very similar to that of the Annulata.

Among the Tracheata the most indifferent condition is found in the Myriapoda, where the metameres are similar and separate. The body of the Arachnida is very variously differentiated. The Galeodea possess the largest number of segments. A head is separated by three thoracic metameres from the succeeding and scparate abdomen, which is made up of separate metameres. In the Scorpions, however, the cephalic and thoracic metameres are united into one portion, and a post-abdomen is differentiated from the abdomen. 'The abdomen is more sharply marked off from the cephalothorax in the Phrynida; the same happens in the Aranea, but they differ in the fact that the abdominal segments have undergone more complete concrescence. In the Acarina the metameres have altogether lost their independence.

The more richly segmented body of the Insecta presents less variety in the distribution of the metameres in different portions. In addition to the head, which is formed of several (3) metameres, there are ordinarily three thoracic segments (Pro- Mesoand Meta-thorax), which are either indifferent, as in the Thysanura and many Pseudoneuroptera, and are only distinguished by the organs appended to them; or they all form a portion which is as sharply marked off from the head as from the abdomen (Neuroptera, Hymenoptera, Diptera, Lepidoptera); or the first thoracic segment only is specially modified, while the second and third are closely attached to the abdomen; this arrangement is indicated in the Orthoptera (Saltatoria), and well marked in the Coleoptera.

The characters of the abdomen are affected by its relations to the thorax already noted. Its segments are always independent, and the terminal ones, several of which are converted into parts of the generative apparatus, are often atrophied.

\section{Appendages.}

$\S 184$.

The appendages of the Arthropoda are paired, jointed structures, which are attached to the metameres, and can be separated into dorsal and ventral appendages. These structures are foreshadowed by the parapodia which are found in the higher Annulata. In the Arthropoda these processes are more highly differentiated, for they become jointed (Fig. 120, p), and differ greatly in form, in correspondence with their different functions; their

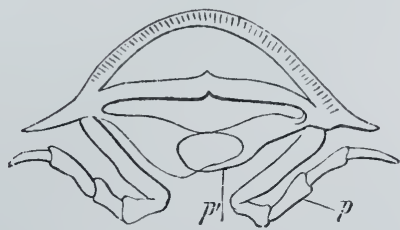

Fig. 120. Transverse section of a Wood-louse. $p \mathrm{~A}$ pair of feet. $p^{\prime} \mathrm{Ab}$. dominal appendages, which form a thoracic cavity (after Lereboullet). lower stage of similarity to one another is only to be seen in their earliest rudiments. 
The serial similarity, which accompanies the lower grade of structure of the parapodia in Annelids, is also seen in the lower types of the Arthropoda, as for example in Peripatus, the Myriapoda, and in many Crustacea (Phyllopoda, etc.). In Peripatus the appendages retain their lowest condition, and resemble the parapodia of Vermes; their resemblance indeed to the appendages of the Tracheata is merely due to the possession of a movable terminal segment, which carries a pair of claws. Two phænomena, which may be recognised in these appendages of the Arthropoda, tend to convert the multifid Annelid-like organism into a more compact one.

The first of these is the metamorphosis of the appendages into a series of different structures having the most varied functions. As the appendages come to differ in function they change in form, and become adapted to their new duties.

The second phænomenon is the limitation of the number of the appendages, which obtains in the ligher divisions, concurrently with the greater development of heteronomous metameres, or with the formation of larger divisions of the body by the fusion of its separate groups of metameres.

\section{Appendages of the Branchiata.}

\section{$\S 185$.}

The appendages are seen at their simplest in the Nauplius form of the Crustacea. At first two, and afterwards three pairs of jointed appendages appear in the unsegmented body. They all function as locomotor organs (swimming-feet), and are beset with

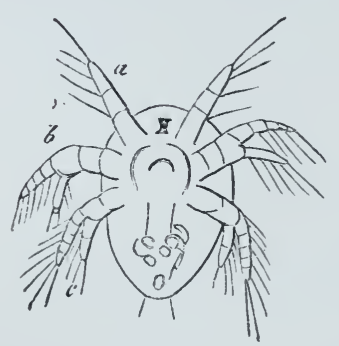

Fig. 121. Nauplius of a Copepod (Cyclops). a $b c$ Appendages. setre, which are often arranged in large bundles. The first pair of these appendages (Fig. 121, $a$ ) is simple, the second and third pairs forked, and this forked character obtains in all the succeeding appendages of the Crustacea. The first two pairs are distinguished from the third pair, and from those which appear later on, by their connection with the nerves, which arise from the supracsophageal ganglion, while the third and all succeeding pairs are supplied by the lower ganglia. With this is connected a difference in function, for the two anterior pairs are ordinarily developed into antennæ. In many Copepoda they still function as locomotor organs; they do so most completely in the Ostracoda. In the Cladocera also the second antenna is still developed as a swimming organ, and this stage is retained by the Phyllopoda during a very long period of development. This justifies us in reckoning the dorsal outgrowths (the first two pairs) as strictly belonging to the 
category of appendages even on functional grounds. In the Malacostraca the two pairs of antennæ have no relation to locomotion, as may indeed be always seen from their form. The hinder pair (Fig. 123, at') is ordinarily placed beside, and often is larger than, the anterior pair $(a t)$. (Cf. also Fig. 128, $a^{\prime} a^{\prime \prime}$.)

All the other appendages are ventral in position. When the metameres begin to be formed they make their appearance following on the first pair of swimming-feet, found in the Nauplius; one pair is distributed to each segment. Like this swimming-foot, and like the second pair of antennæ they have two terminal branches; these are not as a rule differentiated to an equal extent, for one branch is greatly developed and forms the principal part of the appendage, while the other forms an appendage to it. It sometimes, however, has relations to the respiratory function, and is then largely developed. The appendages have very different functions, and are so metamorphosed as to adapt themselves to these functions.

Such of these more anterior ventral appendages as lie near the mouth are converted into mouth-organs, and either form jaws and jaws only, or foot-jaws. The relation between this arrangement and the concrescence seen in the cephalothorax, has been already referred to. A few pairs only are converted into gnathites in the Branchiopoda, and the remaining appendages, of which there is a very large number in most of the Phyllopoda, have very much the same characters as swimming-feet. The same thing liappens in the Ostracoda, Copepoda and Cirripedia. In the last-mentioned group the posterior appendages are converted into the characteristic cirri (Fig. 119, $b b^{\prime}$ ). The metamorphosis of the appendages is seen most completely in the Malacostraca ; let us examine more closely the arrangement seen in one of the Decapoda. We here find six pairs of appendages converted into gnathites, in the more anterior of which the form of the

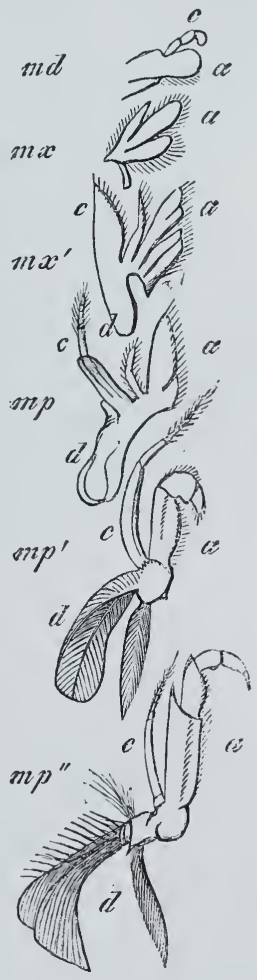

Fig. 122. Gnathites of Astacus fluvia. tilis. md Mandible. $m x$ First; $m x^{\prime}$ Second maxilla. $m p m p^{\prime} m p^{\prime \prime}$ Foot-jaws. a Endopodite. $c$ Exopodite. d Epipodite. Phyllopod foot is retained almost unmodified. A pair of strong jaws (Fig. 122, $m d$ ), are succeeded by two pairs of maxillæ $\left(m x m x^{\prime}\right)$, and these by three pairs of maxillipedes ( $m p$ $\left.m p^{\prime} m p^{\prime \prime}\right)$. The latter gradually pass into the locomotor appendages; of which there are five pairs (Fig. 123, $P^{\mathrm{x}}-P^{5}$ ), which are attached to the cephalothorax, and together with the maxillipedes and maxillæ indicate the number of metameres of which it is built up. The terminal joint of most of the ambulatory feet forms a scissorshaped termination, owing to the great size of the penultimate 
joint, which projects over the ultimate one; this arrangement is

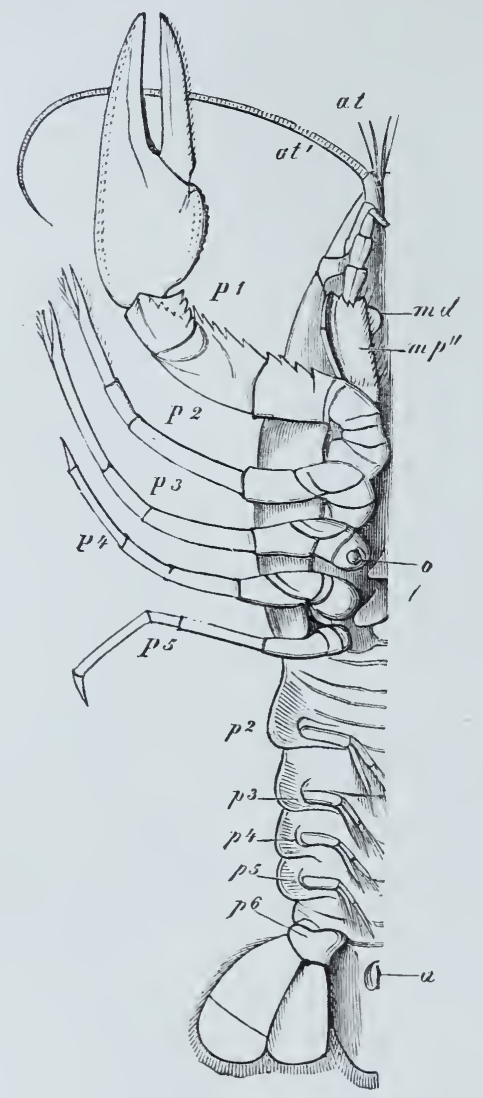

Fig. 123. Appendages of Astacus fluviatilis, seen from the ventral surface. at Anterior; at' Posterior antenna. $m d^{\prime}$ Mandibular piece. $m p^{\prime \prime}$ Third maxilliped, which covers over the other gnathites. $P^{x}-P^{5}$ Ambulatory-feet. $p^{2}-p^{5}$ Swimming-feet of the abdomen. $p^{6}$ Telson. $a$ Anus. - Orifice of the oviduct in the basal. joint of the third ambulatory foot. generally most marked on the first pair of feet, where it serves as an organ of offence. The ambulatory feet, as well as the maxillipedes, have branchial tufts appended to them.

A number of feet on the abdomen are converted into delicate swimming-feet; the first of them functions in the male as a copulatory organ; in the female it is reduced in size, while the other four $\left(p^{2}-p^{5}\right)$ carry the ova. The last pair of appendages are the most modified, for they $\left(p^{6}\right)$ form together with the last segment of the body a powerful caudal fin, of which they are the sides.

The other divisions of the Malacostraca present more or less marked deviations from this arrangement in the number of their gnathites or of the appendages, which are converted into locomotor organs and adapted to these functions. Thus, for example, in the wood-louse four appendages are converted into gnathites, the next eight are ambulatory, and the last four form respiratory plates.

The connection between respiration and locomotion, as implied by the conversion of appendages into branchial lamellæ, or in the differentiation of gills of various form on the appendages, is a most important phænomenon (see Branchiæ).

Branchiæ.

\section{$\$ 186$.}

The teudency to longitudinal division which affects the appendages of the Crustacea adapts these structures to the respiratory as well as to the locomotor function, by spreading out their joints. When the integument at certain points gets thinner it gires rise to 
arrangements by which the exchange of gases between the blood circulating within the appendages, and the surrounding medium is more easily effected, and causes either the whole appendage or a fork of it to become a respiratory organ.

A further differentiation on the same lines, leads to an increase in the number of the respiratory lamellæ of an appendage, or to the formation of filamentous structures; the reason for all these changes is a necessity for increasing the surface. These organs are branchiæ. The connection between the branchiæ and the appendages as seen in the Vermes is clearly a foreshadowing of the still more developed arrangement in the Crustacea, which has here become typical. But it is, of course, more than doubtful whether it has been directly derived from the Vermes.

The gradual development of gills may be followed out step by step through the Crustacea; the functions of respiration and of locomotion are often so closely united that it is difficult to say whether certain forms of these appendages should be regarded as gills, or as feet, or as both combined. The conversion of locomotor into respiratory organs can not unfrequently be made out in the successive appendages of one and the same individual. The branchiferous metameres vary greatly, so that we may say that the appendages of each segment can form gills, or supports for them by developing branchial organs from one of their two primitive branches. The number and special structure, as well as the position, of these respiratory organs varies.

Where the feet themselves become gills, they have the form of broad thin lamellæ (Fig. 124, $A$ br), the surfaces of which are adapted to effect the exchange between the blood that is circulating in them and the surrounding water. Organs of this kind are common among the Branchiopoda, in which, as a rule, a large number of feet become gills, while, in addition to these, peculiar pouch-like appendages may be seen
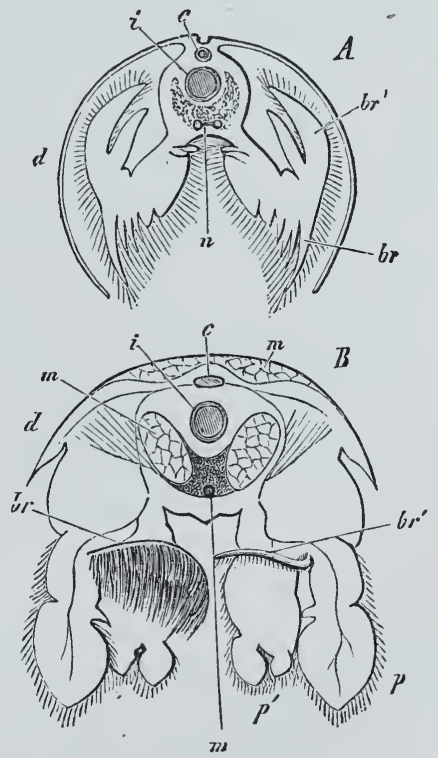

Fig. 124. Sections of Crustacea. $A$ of a Phyllopod (Limnetis) (after Grube). $B$ of Squilla (after Milne-Edwards). $c$ Heart. $i$ Enteron. $n$ Ventral medulla. br Branchix. d Fold of the dorsal integument, which forms a shell in $A$. to be specially entrusted with the respiratory function. The abdominal feet of the Isopoda form branchial lamellæ. In the Amphipoda the gills are tubular appendages of the thoracic segments, which are ordinarily attached to the basal-joints of the feet. In the Stomapoda the original form 
gives rise to another arrangement-the five pairs of abdominal swimming-feet bear at their base a mesially-directed tuft of branched branchial filaments $(B \quad r)$.

From the Schizopoda to the Decapoda we meet with a continuous series leading from the simplest relations to the most complicated ones. Distinct gills are not unfrequently wanting in the former (Mysidæ), or the gills have the form of ramified additions to the appendages of the cephalo-thorax floating freely outwards (Thysanopoda). A fold is gradually developed, from the dermal skeleton of the cephalo-thorax which forms a plate covering in a lateral space above the thoracic feet (p. 235). In this space the gills are placed; it becomes a laterally-placed closed branchial cavity (Decapoda), which is in connection with the surrounding medium by means of a cleft left between the free edge of this lamella, and the basal portion of the feet. As the covering lamella of the branchial cavity becomes more closely approximated to the ventral surface of the body, the primitively simple longitudinal cleft, which gave entrance to the water, becomes divided into two portions, and so gives rise to a larger posterior, and a smaller more anteriorly placed opening, by which the water which has entered by the larger opening, passes back to the exterior, after it has bathed the gills. The gills may separate themselves somewhat from the base of the feet, and arise from the wall of the branchial cavity, but even then they often correspond in number to the appendages. In most of the Decapoda, however, the number of gills is greatly increased, the most anterior of the ambulatory feet being provided with several gills, while some of the maxillipedes also share in this arrangement. The respiratory appendages are more distinctly differentiated in the Pocilopoda, where the anterior appendages have no appended organs, while the five pairs of feet attached to the abdomen are converted into broad plates, and carry a large number of branchial lamellæ.

\section{$\S 187$.}

A more rapid exchange of water around the branchial organs is effected in various ways. Most simply, when the appendages themselves function as gills, or when the gills, notwithstanding their being special organs, are attached to the swimming-feet. The action of the appendages produces a continual change of water around the organs, and puts respiration and locomotion into direct relation with one another. The appendages of the Branchiopoda and the swimming-feet of the Stomapoda may be cited as examples of this arrangement. In others the exchange of water is effected by a special covering-plate of the branchiæ which is formed from the modified abdominal feet; this is the case in the Pœcilopoda and the Isopoda. The water can be renovated, even when the animal is at rest, by the continual movement of this covering-plate.

The formation of a branchial cavity leads to the differentiation of new arrangements, by which the exchange of water is effected. In 
the Decapoda provided with branchial cavities there are special churning organs (flagella) on either side (Fig. 125, $f$ ), which rearh over the whole of the gills in the form of thin flat processes, and are attached to the base of a maxilliped, by which they are kept constantly moving (Brachyura).

The lamelli of the integument, which in many Entomostraca carry shells, must be regarded as having a respiratory significance. This relation to respiration is intelligible when we note that a considerable amount of blood passes through these lamellæ of the mantle, and that the thinness of the walls of these organs presents a condition well adapted for the exchange of the gases; and that, further, the movements of the appendages effect a considerable exchange of water within the mantle-chamber. When the pallial lamellæ become more extended (Limnadiaceæ) they become of greater importance for respiration, and this importance must increase in proportion to the reduction in number of the appendages, which lose their respiratory significance as less blood passes through them (Ostracoda, Daphnida).

In these cases, however, the

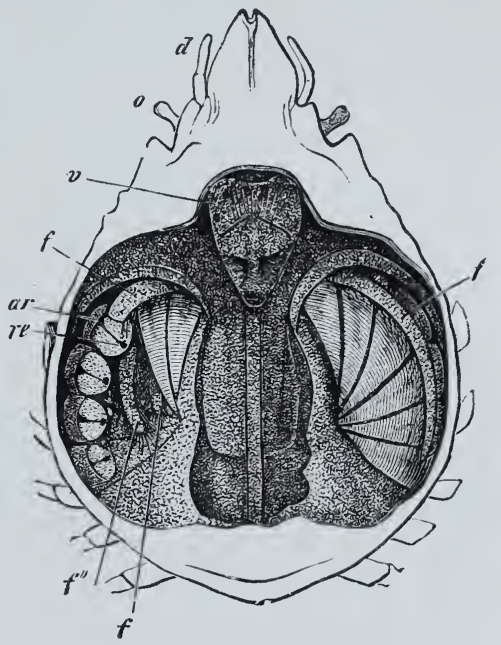

Fig. 125. Branchix of a Brachyurous Decapod. The dorsal integument has been removed from the greater part of the cephalothorax. In the middle is seen the cœlom with the intestine arising from the masticatory stomach $v$; the branchial cavities are placed at the sides and laid open; on the right are the branchize ar. ranged in six lamellar rows; on the left four of them have been cut away, as well as the flagellum $f$, so as to display the churning apparatus $f^{\prime} f^{\prime \prime}$, below the gills. o Eyes. $d$ Antennæ. ar A single gill cut short at re.

mantle does not become specially organised into a branchial organ, as it does in the Cirripedia. In the Balanidæ folded lamellæ, which have been regarded as gills, appear on the inner surface of the mantle-cavity, between its side-wall and base.

Appendages of the Tracheata.

\section{$\S 188$.}

The appendages of the Tracheata are distinguished from those of the Crustacea by the absence of the terminal bifurcation, so that they are composed of a single series of joints. In Peripatus these joints are but feebly differentiated. The terminal portion only which carries a claw has any large amount of independence. 
All the Tracheata have a single pair of antennæ; in this point the Pocilopoda among the Branchiata resemble them. In the Pœcilopoda and in the Arachnida these antennæe are converted into mouth-organs; in the Scorpions and Spiders they are known as cheliceræ. Notwithstanding these relations these structures are homologous with the antennæe of the Myriapoda and Insecta, for, like them, they are innervated from the supra-œsophageal ganglion. They are developed in very various ways among the Insecta, in adaptation to the various functions of the sensory organs connected with them.

The ventral appendages are disposed symmetrically, and in Peripatus all but two pairs retain this relation; in the Myriapoda most of the appendages are similar in character, but in all the other forms these appendages, so far as they persist, take on various forms in accordance with their variations in function. The appendages of the anterior metameres become mouth-organs; those of the posterior metameres are converted into feet, and those on the last are often completely atrophied, and often are not even formed in rudiment. On the whole the number of appendages is much less than in the Crustacea; within the several classes they are always definite in number, and the number of gnathites as well as of feet is constant. In Peripatus the first two pairs form mouth-organs; the first of these is surrounded by the sides of the wall of the mouth, and the second pair only lie close to the mouth. In the Arachnida there is only a single pair of these mouth-organs. In the Aranea it forms the base of a many-jointed palp, which correspond to the chelæ of the Scorpionea, and to the powerful hooked chele of the Phrynida. In the Mites the pieces of either side are united into a grooved lower lip, in which the stylet-shaped jaw-parts are placed. The four remaining pairs of appendages persist in all Arachnida as feet; the first of these is flagellate in form in the Phrynida.

There are three pairs of gnathites in the Myriapoda; the first pair is generally developed into a strong jaw ; in the Chilognatha the second and third pairs are converted into a kind of lower lip, and are said to be represented by a single pair in the embryo; in which case this order has only got two pairs of gnathites. In the Chilopoda, however, the second and third pairs are much more independent, and the first pair of feet is also associated with the mouthorgans. The other appendages of the body have all pretty much the same form; in the Chilognatha there are two pairs on each metamere. The last pair frequently loses its locomotor function, and forms an appendage which we shall again meet with in a modified form in the Insecta.

The appendages are not therefore separated into mouth-organs and locomotor appendages in the same way in all Tracheata; they vary, but not so much as they do in the Crustacea. The development of the guathites, or rather their differentiation from locomotor appendages, is correlated with the development of the head, that is to say, the head is formed as a result of this differentiation. 


\section{$\S 189$.}

Three pairs of the primitively similar ventral appendages are in the Insecta converted into mouth-organs; the same number are formed into feet. The former, arranged round the mouth, may at first have well served to seize and hold food, just as we see the maxillæ of the Crab do at the present time. In such a stage as this the food is seized as well as comminuted. The first pair form the mandibles, and become parts of the mouth, in the form of a single joint. The second and third pairs are many-jointed. But the basal joint only, or a few of those succeeding it, which are the nearest to the mouth, serve to comminute the food; these parts are correspondingly metamorphosed. They form the maxillæ, and the remaining portion of the appendage looks like a jointed addition to it, and functions as a tactile organ (palp); in this way two organs, which work in different ways, are differentiated from one appendage.

The most indifferent form of gnathite is found in the Aptera; in the Collembola they are sunk

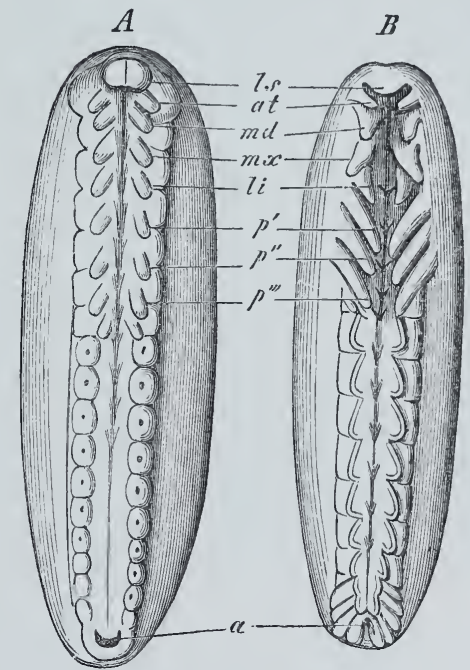

Fig. 126. Developmental stages of Hydrophilus piceus. $A$ An earlicr; $B$ A later stage. $l s$ Upper lip (labrum). at Antennæ and first pair of gnathites (Mandibles). mo Second pair (Maxilla). $\quad l i$ Third pair (Labium). $p^{\prime} p^{\prime \prime}$ $p^{\prime \prime \prime}$ Feet (after Kowalevsky). into the buccal cavity, and in the Thysanura they are but feebly developed. In the former when the parts of the mouth are in active use they can be protracted and drawn in again; and thus the mouth is adapted both to biting and sucking, though, of course, to a very small extent. This indifferent condition of the organisation is developed along two distinct lines in the Pterygota.

When the mandibles are well developed they have the form of cutting organs, which work on one another; the two pairs of maxillæ also become cutting organs, and carry palps at the same time. This condition is permanent in the Pseudoneuroptera, Neuroptera, and Orthoptera, although indeed points of similarity to the more indifferent stage may be seen in the first of these; and also the second pair of maxillæ begins to show signs of fusion. When these gnathites are fused in the middle line the so-called labium is formed; its palps then become articulated to it as labial palps, and indicate the more primitive condition of the organ. The mouth-organs of the Coleoptera are thus metamorphosed.

These parts undergo more remarkable modifications when they 
are adapted to another mode of ingesting food, namely, by sucking. In the Hymenoptera, in which these organs can function either as sucking or as cutting organs, the parts are of pretty much the same form as in the mandibulate Insecta, but the maxillæ are considerably elongated, as is the labium and its palps. A process, the tongue (lingua), is developed on the surface of the labium turned towards the mouth, and this has two lateral appendages, or secondary tongues (paraglossa), at its base. In many these appendages are as much developed as the tongue itself.

The mouth parts can be derived from cutting organs even in those insects that are purely suctorial. The Hemiptera and the Diptera have the mandibles and maxillæ converted into setæ; in many Diptera the maxillary setæ are rudimentary. The labium forms a sheath for these setæ, which is firm and jointed in the Hemiptera, and soft in most Diptera; this sheath still carries the labial palps, or rudiments of them. There is a rudimentary tongue on the short upper lip (labrum), which is wanting in the Hemiptera. The mouth-organs of the Lepidoptera are differentiated in a different way. In them the maxillæ are grooved, and united into a tube, which forms a spirally-coiled proboscis, which is ordinarily of some length; there are small maxillary palps at its base, which

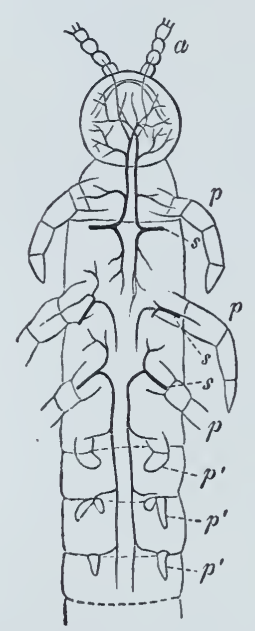

Fig. 127. Anterior half of the body of Campodea fragilis. $a$ Antennæ. $p$ Feet. $p^{\prime}$ Rudiments of feet. $s$ Stigma (after J. A. Palmen). The paired processes also of the terminal metaappendages. labium, which are generally large.

While gnathites are found on the metameres which fuse to form the head, the succeeding appendages form feet, or locomotor organs for the next three, or thoracic, metameres. The jointing observable in them is of the same character in all, and shows that they have had a common origin; more marked differences can only be made out in their terminal segments, which are more accessible to adaptation. Certain peculiarities in them are due to their varied modes of adaptation to modified requirements.

Although the number of three is constant for the feet, in many Insects a larger number can be made out in the embryo; whence we may conclude that they have been derived from forms with a larger number of feet. In the Thysanura the rudiments of these appendages are retained (Fig. 127, $p^{\prime}$ ), even on the abdominal metameres (Campodea). 'The locomotor processes in many Insect larvæ (Lepidoptera and Tenthredinæ) may be derived from these rudimentary appendages. are covered by the palps of the rudimentary 


\section{$\S 190$.}

Besides antennæ, dorsal appendages occur in no Tracheata except the Insects. They are altogether absent in the Thysanura and Collembola. As they are only present on the post-cephalic metameres they are innervated, like all the ventral appendages, from the ventral chord. They cannot be said to have any relations to the branchiæ of the Crustacea, or to be derived from the dorsal parapodia of the Annelides, and they may therefore be fairly regarded as independent structures.

The dorsal appendages have the form of lamellæ or filamentous processes of the metameres, which are sometimes grouped into tufts in the aquatic larvæ of the Ephemerida, Perlida, Phryganida, etc. These appendages have a respiratory function ; and as tracheæ pass into them, they are known as tracheal gills. They are widely distributed over the body, not only on the dorsal, but also on the ventral surface; and are therefore examples of processes in an indifferent condition, of which the dorsal ones placed at definite points acquire a typical significance. The lamellar widely-distributed forms move in such a way as to be of great importance in changing the water, just like the respiratory appendages of the Phyllopoda; but they cannot be said to have any locomotor functions.

The wings must be regarded as homologous with the lamellar tracheal gills, for they do not only agree with them in origin, but also in their connection with the body, and in structure. In being limited to the second and third thoracic segments they point to a reduction in the number of the tracheal gills. It is quite clear that we must suppose that the wings did not arise as such, but were developed from organs which had another function, such as the tracheal gills; I mean to say that such a supposition is necessary, for we cannot imagine that the wings functioned as such in the lower stages of their development, and that they could have been developed by having such a function.

But if the real cause of the development of these dorsal appendages into wings cannot be found in the locomotor function, it must be sought for in another. And this is that of respiration, which would be well served by increase of surface. Every increase of surface increases the respiratory value of the organ, and so leads towards its future function. It is no objection to this hypothesis to say that ontogenetically the wings are formed and developed later than the tracheal gills of other metameres, for these modified tracheal gills can only function when the unmodified respiratory ones have lost their function.

In many cases the appendicular character of the wings is indicated by segmentation, but this can only be regarded as a secondary adaptation. It obtains in the movable second pair of wings in the Coleoptera and Forficulidæ, where the first pair have been converted into elytra. 
In the Pseudoneuroptera both pairs of wings are very similar in character. In the other four-winged orders they have undergone considerable differentiations. In addition to the differences in size, which in the Hymenoptera and Lepidoptera are generally due to the increased size of the first pair, there are differences of structure, which imply a difference in function. In the Orthoptera the first pair is often merely a covering for the second pair; this is very distinct in the Beetles where the second pair is often rudimentary. The elytra have then become organs of defence for the subjacent abdomen. In the Hemiptera there is a similar differentiation. The Diptera have the anterior pair only, the hinder pair being: rudimentary, and represented by the so-called balancers (halteres). On the other hand, the Strepsiptera possess the hinder pair of wings, or those attached to the third thoracic segment, only.

\section{Integument.}

$\S 191$.

The integument of the Arthropoda is more independent and less connected with the muscular system than in the preceding divisions; it can always be divided into two layers. The cuticular layer, secreted by a layer of cells which is often much modified, extends over the whole surface of the body just as it does in many Vermes; it is also continued into the openings of the internal organs. Owing to its strength it forms the most important part of the integument, but it varies greatly in thickness and firmness. It is soft and flexible between the segments of the body, where these parts are movably commected with one another, but it is generally stronger on the metameres themselves, and on the appendages. Its physical characters vary widely; there are all kinds of transitions between the soft investment of the body of the Spiders and most Insect larvæ, and the firm carapace which covers the body of most of the Crustacea, the Myriapoda, Scorpions, and such Insects as the Cockroach. The variations in its firmness depend not merely on the thickness of the cuticle, but on the chitinisation of its layers. Eren thick layers, when freshly formed, are soft, and only become resistent when chemical change occurs. To increase the firmness of this chitinous carapace calcareous salts are deposited in it, in many Crustacea, and even in the Myriapoda. When this cuticle has become firm it limits the growth of the body; in these cases therefore so long as growth is going on the cuticle is periodically cast (Ecdysis).

In consequence of its mode of origin the cuticular layer is everywhere distinctly laminated. As a rule it is traversed by pore-canals, into which processes of the subjacent matrix are continued. The relatively thin "matrix" of the cuticular layer is homologous with 
the epidermis of other animals. Although in many cases it contains pigment (Crustacea), it is as a rule colourless, the coloration of the Arthropoda being generally due to the deposition of pigment in the outer chitinous covering. Below this epithelial layer, which is also distinguished as the "hypoderm," there is a layer of connective tissue, but this is, as a rule, slightly developed in comparison with the cuticular layer, and the matrix.

\section{$\S 192$.}

When the secreted chitinous layers are very firm they take on a new function, and form a dermal skeleton, which is not only an organ of protection for the organs placed in the body-cavity, but is also an organ of support, and provides points of origin and insertion for the muscles of the body. This relation holds too for the appendages of the body, the integument of which likewise serves as a skeleton for them.

The formation of large unequal regions of the body produces changes in the dermal skeleton by giving rise to differentiations in it. These are formed by internal processes and continuations of the dermal skeleton, which are principally found in those segments which carry oral or locomotor organs. These processes are very greatly developed on the cephalothorax of the higher Crustacea. But they are not wanting in the other classes. They are found chiefly in the head and thorax in many orders of the Insecta (Coleoptera, Hymenoptera, Orthoptera), where they form a complicated structure known as the "endothorax." These parts often form an organ of support for the nervous system. They are also important as increasing the internal surface of that portion of the dermal skeleton which gives origin to the muscles, and, in more individualised structures, they are closely connected with the differentiation of the musculature.

The shells, too, which are formed from the chitinous investment of the mantle-folds of many Branchiopoda and Ostracoda, are of importance as skeletal structures; so too are the shells of the Cirripedia. However different they may be in form or size they have always the same arrangement. Two pairs of ridges or plates surround the entrance into the mantle-cavity and form a movable operculum. The shell-pieces developed in the Balanidæ, but which are rudimentary only in the Lepadidæ, gives rise in the former to a continuous rigid cell (Fig. 119, $f f$ ), the only movable part of which is the operculum $(e)$ which covers over the entrance into the mantlecavity.

\section{$\S 193$.}

Continuations or processes of the integument take on all kinds of forms, such as spines, setæ, or hair-like structures; these are modified in innumerable ways in the Crustacea, Arachnida, and Insecta; they are sometimes attached closely and immovably to the chitinous 
carapace, of which they form outgrowths, as, for example, the setæ in certain parts of the body of the Crustacea, the hairs of the Spiders, Caterpillars, etc. ; sometimes they are more developed and only loosely attached to the body, like the scales of Butterflies, which are also present in other divisions, e. g. in the Thysanura. In any case the chitinous investment of the process is continuous with the rest of the integument. In movable appendages of this kind the chitinous layer is softer at the point of attachment, while the cuticle does not change in character on the stiff processes. Integumentary structures, such as denticles and ridges, are also converted into organs of voice in many Insecta (Grasshopper, Cicadæ).

There are glandular organs in the integument, which are derived from modifications of the epidermal layer. They are not so common in the Crustacea as in the Insecta. The secreting portion of the gland consists of one cell merely, or of a few; and its duct is principally formed by the pore-canals of the cuticular layer (cf. Fig. 7, p. 23).

The dermal glands are largely developed in certain parts of the body in those Insects that form wax. In the Aphides, and still more in some Hymenoptera, groups of dermal glands are converted into wax-secreting organs. The spinning glands of Spiders are further differentiations of dermal glands. Glands which lie in the abdomen and open by several pairs of papillæ placed behind the anus (spinning papillæ) produce a secretion, which hardens into a chitinous filament when exposed to the air, and so forms the thread of the Spider's web. An apparatus, which is only functionally similar to this is found in Peripatus. Two groups of ramified tubes pass into an excretory duct on either side; this duct, which is locally widened out, opens at the base of the second post-oral appendage. The secretion is of a sticky character and rapidly hardens. Morphologically, these organs appear to lead to those which are found in the larvæ of many Insecta, which, therefore, may be regarded as having had a common origin with them. In the larvæ of the Lepidoptera, and many Coleoptera and Hymenoptera, there is a pair of long and frequently coiled glandular tubes placed beside the enteron, the fine ducts of which have a common opening on the labium. Their secretion forms the silk-threads of the web of these larvæ. These "spinning vessels" are most highly developed before they pass into the quiescent pupa stage (Serictariæ); after the web is made they become atrophied.

Lastly, other glands seem from their secretion to be poison glands, e.g. those which open on the claws of the spiders, and on the caudal spine of the Scorpion; these add to the variety of differentiations which the glandular system of the integument may undergo. 


\section{Muscular System.}

\section{$\S 194$.}

The muscular system of the Arthropoda is not homogeneous in character, like the circular or longitudinal layers in the dermomuscular tube of the Vermes. It is more differentiated, and we find separate bundles composed of a varying number of transversely-striped muscular fibres. Peripatus is the only exception to this, and its muscular system, owing to the absence of transverse striæ from the muscular elements, rather resembles that of the Vermes. The dermo-muscular tube is however generally converted into a complex of separate muscles, which together form a muscular system. As the skeleton of the Arthropoda is an external one, the muscles arise from and are inserted into the inner face of the hollow cylinder, or portions of a cylinder, which are formed by the segments of the body as well as by those of the appendages. This development of a dermal skeleton is to be regarded also as an important factor in the differentiation of the muscular system, in so far as separate muscles can only be formed when they have a firm point of origin and of insertion. In the number as well as in the varied arrangement of the separate muscles, the muscular system presents a high grade of development, which always corresponds to the varying significance and development of the metameres. It differs from the muscular system of the Annelides in correspondence with the difference expressed by the homonomy of their metameres, as compared with the heteronomous ones of the Arthropoda.

When the metameres are similar the muscles of the metameres are also similar, and when the separate metameres are unequally developed, either by the fusion of a small, or a larger number into larger divisions of the body, or by atrophy, there is a corresponding want of similarity in the arrangement of the muscles of those parts. The development of the appendages greatly influences the development of the muscular system, and when the metameres which carry appendages are greatly enlarged in comparison with the rest, the muscular system takes a large share in the increase.

The numerical relation and arrangement of the muscles often undergo considerable alterations in those Arthropoda that undergo metamorphosis. This applies as much to progressive as to retrograde metamorphosis. In the former the change leads to a differentiation into unequal groups; in the latter to an atrophy of very large parts of the system; this obtains in the parasitic Crustacea and in the fixed forms. 


\section{Nervous System.}

\section{$\S 195$.}

The nervous system of the Arthropoda resembles that of the Annelides, with which it completely agrees in its fundamental characters. A ganglion placed above the osophagus is the cephalic ganglion or cerebrum; two commissures from it embrace the œsophagus, and form a nervous œsophageal ring, by being connected with a ventral ganglion. From this inferior ganglion a series of ganglia, connected by long commissures, extend along the ventral internal surface of the body, forming the ventral ganglionic chain. The greater size of the cephalic ganglion, compared with that of the ventral ganglia, has been already seen in many of the Annulata; in the Arthropoda it is ordinarily still more distinct; this condition may be partly explained by its relations to the more highly developed organs of sense, if we recognise in the dorsal œsophageal ganglion something similar to the brain of the Vertebrata. Led by an idea of this kind, some have compared even the ventral ganglia, or ventral medulla, with the dorsal medulla of the Vertebrata, and have striven to carry the comparison still further; these attempts ignore the complete difference between the type of structure of the Arthropoda and of the Vertebrata.

The increase in size of the cerebrum is, as has been above indicated, in direct connection with the development of the higher sensory organs, especially that of sight; and its modifications are in great part dependent on these. The ventral chain of ganglia also undergoes essential. modifications, although it is not always possible to make out in them a regular dependence on the metameric condition of the body. The repetition of similar metameres gives rise to similarity of character in the ganglia of the ventral chord, and to a regular series of them. Where some metameres are especially developed, their ganglia also are considerably increased in size, while where there is concrescence of the metameres an approximation of several groups of ganglia can also be noted; this not unfrequently leads to a complete fusion into several larger ganglia, or the formation of a single large ventral ganglionic mass.

The ganglia of the ventral ganglionic chain are primitirely paired, and connected by a transverse commissure, as in the Annulata. By the shortening of these transverse commissures, an approximation, and finally a more complete fusion, takes place.

The peripheral nervous system arises from the ganglia of the central system, that is, of the cerebrum and ventral chain, which are distinguished by the possession of ganglionic cells. The nerves either arise directly from the ganglionic portions, or run for some distance along the longitudinal commissures, and then pass off from them.

The higher sensory nerves arise, as a rule, from the cerebral ganglion. This holds especially for the nerves of the eyes and antennæ. 
In addition to the nerves, set apart for the muscular system and the integument, there are also nerves for the viscera; of these the enteric nerves are most exactly known. They partly agree with the characters which obtain in the Annelides. As separate ganglia are embedded in their course, they constitute to a certain extent an independent nervous system, which is called the "stomato-gastric nervous system." Another special system of nerves, which obtains especially in the Insecta, arises from the ganglia of the ventral medulla, and is known as the sympathetic nervous system.

\section{$\S 196$.}

The nervous system of the Crustacea presents us with a large number of examples of the phænomena described in the foreg'oing paragraph. The relation between the size of the cerebrum and the development of the optic organs is seen in the Thoracostraca, and in the large-eyed Hyperida (Phronima) among the Arthrostraca, where the optic nerves arise from distinct lobes; these lobes are also distinct in the Wood-lice. The separation of the cerebral mass into various groups of ganglia generally implies a higher differentiation. With this ought to be compared those degenerations which affect the cerebrum when the optic organs are reduced or altogether lost; when this happens the antennæ also generally disappear. Conditions of this kind are found in the parasitic Copepoda, and in the Cirripedia (Fig. $129, B g s)$, and in consequence of it the cerebrum is sometimes repre-

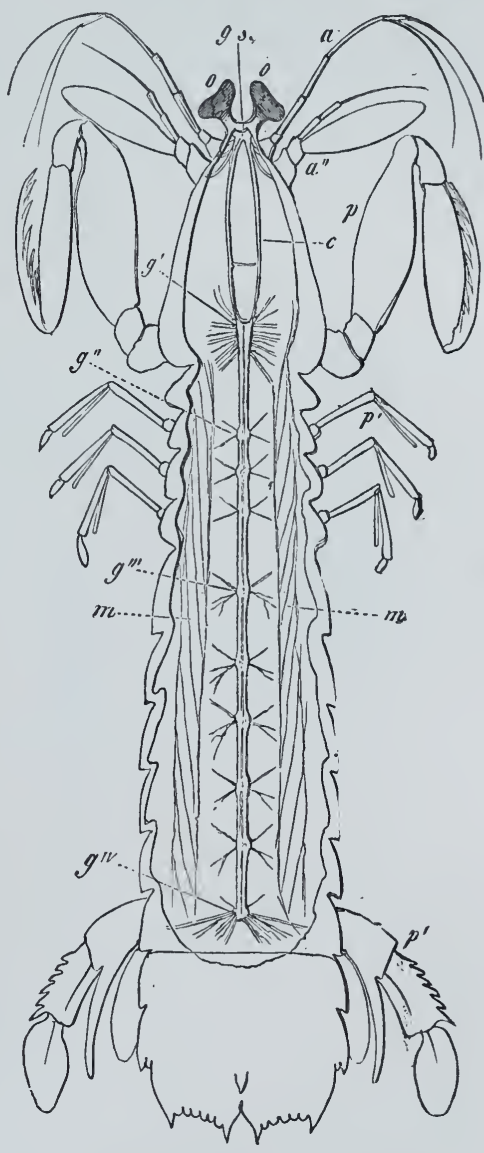

Fig. 128. Nervous system of Squilla. O Eyes. $a^{\prime}$ First ; $a^{\prime \prime}$ Second pair of antennæ. $p$ Claws, provided with prehensile terminal joints. $p^{\prime}$ Rowing-feet; the last pair of the foot-like appendages contribute to form a caudal-fin. $m$ Muscles. gs Supra-œsophageal ganglion. $c$ Commissural chords. g/ Thoracic ganglia. $g^{\prime \prime} g^{\prime \prime \prime} g^{\prime V}$ Abdominal ganglia. sented by nothing but a commissure.

The most anterior of the ventral ganglia is connected to the cerebrum by a shorter or longer commissure. The length of this chord appears to depend on the position of the mouth with regard to the cerebral ganglia (that is really to the eyes and antennæ). In the Malacostraca the length is very considerable (Fig.128, $c$; Fig. 129, A), 
as also in many lower Crustacea, e. g. the Cirripedia (Fig. 129, B c); in others, again, it is so much shortened that the cerebral and ventral ganglia form a single nervous mass, pierced by the œsophagus (e. g. in Corycæïdæ).

The ganglia of the ventral chain seem to be most regularly distributed to the separate metameres in the Phyllopoda, which, in this respect, retain most completely the primitive relations of parts.

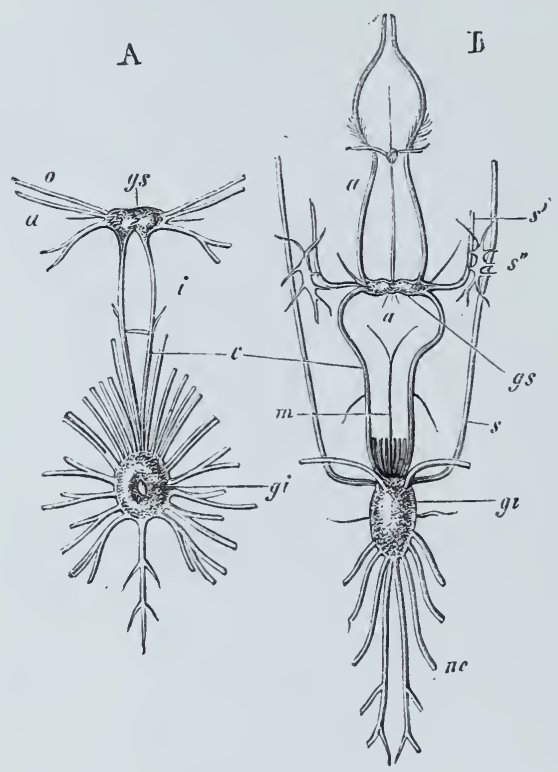

Fig. 129. A Nervous system of a Crab (Carcinus mænas). gs Cerebral ganglia. $o$ Optic ; a Antennary nerve. $C$ Esophageal commissure. $i$ Transverse connection of the commissure. gi Fused ventral medulla (after Milne-Edwards). $B$ Nervous system of a Cirripede (Coronula diadema). gs $c$ gi as in $A$. a Antennary nerves which are distributed in the mantle. Between them is the "optic ganglion," connected with the cerebrum. $m$ Nerre to the stomach. $s$ Visceral nerve, which unites with a second visceral nerve from the œsophageal ring to form a plexus $s^{\prime \prime}$ (after Darwin). thoracic ganglia seems to be very common; while the 6 smaller ganglia of the abdomen still correspond exactly to the metameres. Further fusion is seen in the thoracic ganglia of some Macrura (Palinurus); in Pagurus the ganglia of the abdomen are represented by one only, in correlation with the shortening of this region. So too in the Brachyura all the ganglia of the ventral chain are fused into a single one (Fig. 129, A gi).

Reductions of this kind obtain, also, in other divisions of the composed of a large number of pairs of ganglia (about 60 in Apus), which follow one another, gradually losing their transverse as well as the longitudinal commissures; in the Daphnida the ganglia have the same charasters, although they are less in number, in correspondence with the smaller number of the metameres.

Among the Thoracostraca, the ganglia of the ventral chord also are, for the most part, distinct, but in correspondence with the concrescence of the anterior metameres into a more or less extended cephalothorax, the anterior ganglionic masses are fused; this is either more or is less distinctly expressed. Thus in the Stomapoda (Fig. 128), the ganglia which innervate the anterior buccal, as well as the prehensile feet $(p)$, form a larger complex $\left(g^{\mathrm{T}}\right)$; a series of independent ganglia, extending as far as the candal segment, is connected with this $\left(g^{\mathrm{II}} \mathrm{g}^{\mathrm{III}} \mathrm{g}^{\mathrm{IV}}\right)$. In the Decapoda Macrura, likewise, concrescence of the 6 cephalo-

The ventral chord is in them 
Crustacea, and these also are often explicable as being adaptations to changes in the form of the body. We find this concentration in the Copepoda, where the Calanidæ possess a ventral chain, formed of ganglia, whilst in the Corycæïdæ this is concentrated into a mass fused with the cerebrum. So too, among the Cirripedia, the Lepadidx have a series of 4-5 ganglia in their ventral chord; in the Balanidæ this is represented by a single ganglionic mass (Fig. 129, $B, g i)$. Similar phænomena are seen in the Arthrostraca, but as a rule they have a large number of ganglia (10-12 in the Amphipoda, $7-13$ in the Isopoda).

\section{$\S 197$.}

In the Protracheata the nervous system remains in a lower condition. A highly developed and closely united pair of cerebral ganglia surround the mouth, and give off lateral nerve-chords downwards. They are closely approximated below the oesophagus, and then pass ventrally to the hinder end of the body, diverging somewhat in their course. These nerve-chords are united at their ends. Along their whole length they are connected by fine transverse commissures (in Peripatus Edwardsii); the most anterior of these are the most distinct. There are no swellings on the ventral chord, and the ganglionic cells are equally distributed in it. This arrangement is that of the more indifferent condition of the ventral ganglionic chain; the chain is formed by the separation of the ganglionic cells in the longitudinal trunks into separate parts, corresponding with the metameres.

Since in the Branchiata the ventral ganglia are always differentiated, the arrangement in Peripatus is a lower one, and so far justifies us in regarding the Tracheata as an independent phylum.

\section{$\S 198$.}

The nervous system of the Myriapoda indicates a well-marked step forward, for there is a ventral chord which traverses the whole length of the body with scarcely any variation in character, provided with ganglia so arranged as to correspond exactly with the metameres. The first ganglion which supplies the gnathites sometimes indicates distinctly that it is composed of a number of ganglia. The succeeding ones differ in size according to the extent to which their appendages are developed; they are arranged at regular distances from one another, and in the Diplopoda succeed one another in pairs. When the longitudinal commissures are shortened they form closely applied swellings (Julidæ). An approximation of this kind tending to concrescence is ordinarily seen in the last ganglia, even when the others are distinctly separated. These ganglia correspond in number to the metameres, so that there may be even as many as 140 (Geophilus). In these arrangements we see a condition which is most like the typical form of the higher Tracheata. 
Among the Arachnida the ventral ganglia are very often reduced and fused. They are all characterised by the close connection between the cerebral ganglia, and the ventral chord, owing to the extreme shortness of the commissures.

The nervous system is most richly segmented in the Scorpions.

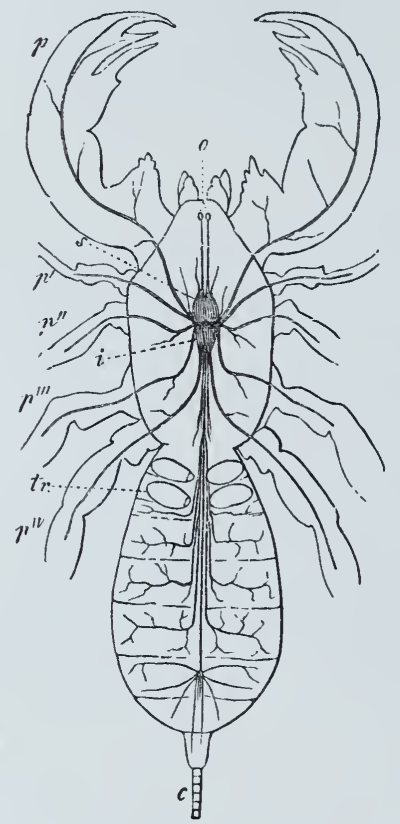

Fig. 130. Nervous system of Thelyphonus caudatus. $s$ Cerebral ganglion. $i$ Ventral ganglion. o Eyes. $p$ Palpi. $p^{\mathrm{I}}-p^{\mathrm{IV}}$ Feet. $\operatorname{tr}$ Lungs. $c$ Tail-like appendage of the body (after Blanchard).

The feebly developed cephalic ganglion gives off two short commissures to the ventral chain, which consists of 8 ganglia. The first of these is remarkable for its size, and appears to be homologous with the single large ganglion in the cephalothorax of the Spiders. As in them it is the point of origin of the pedal nerves, and must therefore be considered as composed of several ganglia. The three succeeding ganglia are also placed in the cephalothorax, and the four last, which are widely separated from one another, are found in the segments of the tail.

In the Galeodea and Phrynida, as in the Aranea, the ganglionic chain is replaced by a large ventral ganglion, which (Fig. 130, $i$ ) is, especially in the Spiders, of a radiate form, and gives off the nerves for the ventral appendages, and also two which run into the abdomen; in the Galeodea branched nerve-trunks are sent to the segments of the abdomen.

In all these divisions the cerebral ganglion, which is generally distinctly paired, and in the Galeodea (Fig. 130,s) especially large, gives off nerves for the eyes; close by the optic nerves those for the cheliceræ arise in the Spider, and thus we see that these organs are metamorphosed antennæ.

A complete concentration of the central portions of the nervous system is seen in the Acarina, where the cerebral ganglia, which are often feebly developed, may be replaced by a commissure. The rentral medulla, which is large and forms a single mass, exhibits numerous traces of segmentation in the arrangement of the ganglionic cells and fibrous elements; it gives off nerves all round.

The simple character of the nervous system in the Pycnogonida is due to the decrease in number of their ganglia, owing to a reduction of the segments of the body; their cerebrum is connected by short commissures with the ventral medulla, which is formed of four pairs of ganglia. 


\section{$\S 199$.}

In the Insecta, we find a form which corresponds to the primitively homonomous segmentation of the body at the commencement of the process of development; and all succeeding arrangements of the nervous system are developed from it. The ventral chord traverses, as a rule, the whole length of the body, the ganglia being separated by equal distances from one another, so that the last ganglion lies in the last segment of the body. This character corresponds to the equivalence of the metameres, which obtains at these stages, and points to its transmission from a lower stage, such as that which we find permanently in the
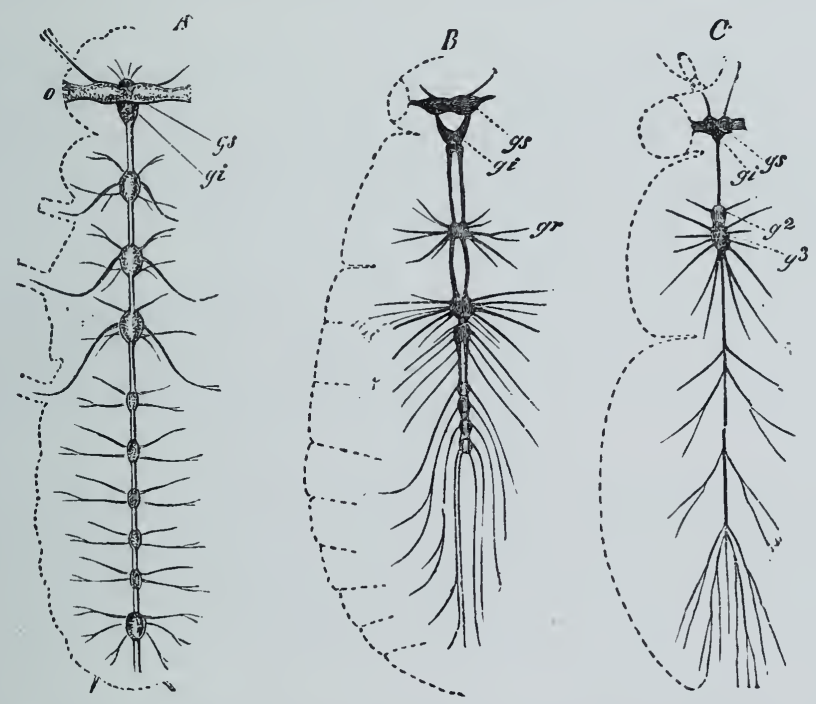

Fig. 131. Nervous system of Insecta. A Of Termes (after Lespès). $B$ Of a Beetle (Dytiscus). $C$ of a Fly (nfter Blanchard). gs Supra-oesophageal ganglion (Cerebral ganglion). gi Sub-œsophageal ganglion. $g r g^{2} g^{3}$ Fuscd ganglia of the ventral chord. o Eyes.

Myriapoda. It is only when the Insect passes out of its larval condition to the perfect one that changes appear. The development of some metameres, the intimate fusion of others to form larger portions of the body, the greater development of the appendages, which persist on some metameres only, with the consequent increase of the muscular supply, as well as many subordinate arrangements, must be considered as affecting the changes which take place in the nervous system. The decrease in number of the ganglia, by the shortening of the longitudinal commissures, and the consequent fusion of separate ganglia, produces a shortening of the whole ventral chord. Owing to the independent character of the head of the Insecta, in comparison with the other regions, the first ganglion of 
the ventral chord, which is primitively composed of three, remains embedded in the head (sub-csophageal ganglion), and takes no share in the concrescence which affects the other ganglia ; it is only in a fer cases - in Insecta degraded by parasitism - that a union of this ganglion with the rest of the ventral chord takes place.

'The cerebral ganglion (Fig. 131, $A B C, g s$ ) is almost always distinctly divided into two halves, each of which is again composed of several smaller masses of ganglia, which are often complicated in structure. The ganglia of the ventral chord are primitively paired, and often become closely united. On the other hand, the longitudinal commissures remain double, even when they are closely applied to one another. There is also a separation of the ventral chord into a superior and an inferior portion which corresponds to a physiological differentiation.

The first ganglion of the ventral chord (G. infraœsophageum) sands off fibres for the organs of the mouth. The three succeeding t'oracic ganglia principally give off nerves to the appendages, feet, and wing's; they are consequently of some size. On the other hand, the succeeding ganglia are, as a rule, small, the last alone being an exception to this, for it is of a larger size in correspondence with its relations to the generative system.

Even in the Aptera there is a fair amount of rariation, for 11 ventral ganglia (Lepisma) can be made out in the Thysanura, while there are only $3-4$ in the Collembola. The last portion of the ventral chord seems to form a complex of ganglia in many (Orchesella, Achorutes).

As to the Pterygota, the chief point is that of all the orders the least amount of metamorphosis is seen in the Pseudoneuroptera. In them the ventral medulla traverses the whole length of the body, and there are 5-9 abdominal ganglia in addition to the three thoracic (Fig. 131, A). The Orthoptera, which have 5-7 abdominal ganglia, resemble them in this.

There are great variations in the Coleoptera. In some the ventral chord extends as far as the end of the abdomen, sometimes possessing 8 separate ganglia (e.g. in Cerambycidæ, Carabidæ, etc.); in others again the 3 ganglia of the thoracic portion are only represented by two, the second and third being fused, whilst the abdominal ganglia are also connected into one mass, which immediately succeeds the preceding ganglion (Curculionida and Lamellicornix). In other families there are connecting links of various kinds between these conditions which represent the extremes. In the Hymenoptera we generally find the thoracic ganglia reduced to two, while the abdominal part of the ventral chord has frequently $\breve{\partial}$ or 6 separate ganglia. 'These are in many, however, reduced to 4 or 3 , and even to one. The abdominal part of the ventral chord is, in the Hemiptera, placed in the thorax, and is represented by a ganglionic mass connected with the thoracic ganglia, which are also simple, by a commissure of varying length. The nerves for the abdomen consequently take a longer course, and form two longitudinal trunks 
which arise from the last ganglion. A similar difference in the number of the ganglia of the ventral chord obtains in the Diptera, where the most primitive characters are seen in Pulex : 3 thoracic and 7-8 abdominal ganglia. In others there is generally a considerable reduction by the fusion of the thoracic, or of the abdominal ganglia, or of both (Fig. 131, C). With this is connected the complete fusion of the ventral chord into one somewhat long knot, in the parasitic Pupipara. We find the same characters in the Strepsiptera. As to the Lepidoptera there is more uniformity in them, a constant number of ganglia being found in the larva, while when it is metamorphosed into the butterfly the mode of fusion appears to be essentially the same in all.

\section{$\S 200$.}

The visceral nervous system of the Arthropoda shows signs of some common characters, together with great variation in particular points. Among the Crustacea nerve-filaments pass from the œsophageal commissure to the enteron, or a nerve passes to the enteric canal from the ventral chord. (In Astacus from the last ganglion also.)

Even in the Arachnida nerves are given off partly from the cerebrum, and partly from the ventral ganglion to the enteron; in the Opilionea the posterior ones are provided with a large number of ganglia.

In the Insecta and Myriapoda the breaking up of the visceral nervous system into several portions has been more generally made ont; we will therefore cxamine the arrangement of it more closely. One part forms the so-called paired system, which consists of two branches running backwards from the cerebral ganglion to the sides of the œsophagus; these form a simple

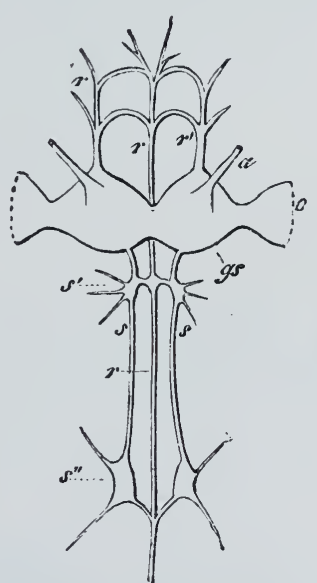

Fig. 132. Supra-œesophageal ganglion and visceral nervous system of one of the Lepidoptera (Bombyx Mori). g s Supra-œsophageal ganglion (Cerebruin). a Antennary nerve. o Optic nerve. $r$ Azygos trunk of visceral nervons system. $r^{\prime}$ Its roots arising from the supra-œsophageal ganglion. $s$ Paired nerve with its ganglionic enlargements $s^{\prime} s^{\prime \prime}$ (after Brandt). chain of ganglia (Fig. 132, $s^{\prime} s^{\prime \prime}$ ) on either side. The number of these ganglia varies, and it is often difficult, on account of their plexus-like connection with the unpaired system, to determine to which system they belong. The unpaired system $\left(r r^{\prime}\right)$ arises in a ganglion which lies in front of the cerebrum, and is more or less connected with it. From this ganglion a thicker nerve $(r)$ passes backwards over the œsophagus to the stomach, and forms a plexus with the branches of the paired system; from this plexus the neighbouring parts, especially those of the digestive system, are innervated. In many Insects this nerve (N. recurrens) forms a single ganglion (Coleoptera and Orthoptera), in others several (Lepidoptera) 
There is yet another system of nerve-branches in connection with this plexus; it is principally applied to the large branches of the tracher, and the muscles of the stigmata. This arrangement is brought about by a nerve-filament, which runs on the surface of the ventral chain, and is divided into two fork-like branches in front of each ganglion (Nervi transversi accessorii). The branches receive nerve-twigs from the upper chord of the ventral chain, and pass partly outwards to the branches of the trachere and the muscles of the stigmata, and partly backwards, where they unite in the middle line, and at the next ganglion repeat this arrangement.

\section{Sensory Organs.}

\section{Tactile Organs.}

\section{$\S 201$.}

The sensory organs of the Arthropoda are, for the most part, allied to those of the Vermes. All but a few indicate a connection of

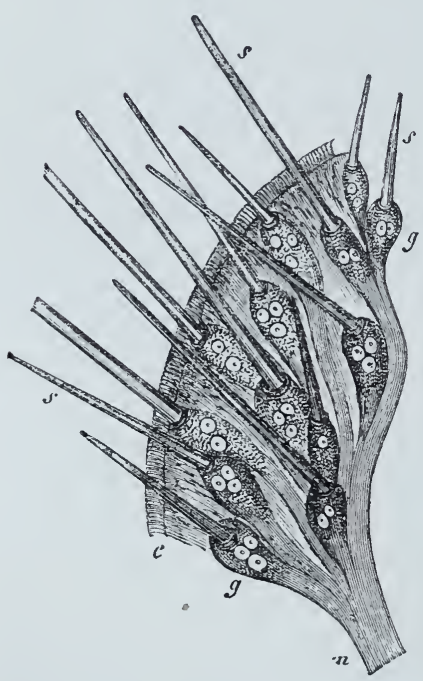

Fig. 133. Norve-ending with tactile rods, from the proboscis of a Fly (Musca). n Nerve. $g$ Ganglionic swelling. $s$ Tactile rods. $c$ Fine hairs of the cuticle (after Leydig). this kind, and these few are to be regarded as arrangements which are developed in this division only. The carapace-like covering of the body of most Arthropoda requires special organs to produce the sensation of touch ; the form-elements of these are connected with ganglionic cells, and form rod-like nerve-endings. These ganglionic cells are generally structures which are derived from the ectoderm, and the whole apparatus not unfrequently retains its primitive position.

These end organs, which are found in the most different parts of the body, form indifferent sensory organs, which in certain parts take on the form of tactile organs (cf. Fig. 133). Organs of this kind are mostly found on the appendages, where they present rodshaped projecting ends.

In the division of the Crustacea these tactile rods have been recognised in many forms, and that not only on the antennæ, especially in the lower Crustacea, but even on other appendages of the body. In the Myriapoda and Insceta there are tactile rods on the antennæ, and in the latter they are also found on the tarsal joints of the feet. 
In addition to these tactile rods special organs resembling them are found on the antennæ of the Crustacea and Insecta; they are sometimes of considerable size, and are innervated in the same way as are the tactile rods. In the Crustacea they are formed only on the inner (anterior) pair of antennæ. In the Insecta they are much shorter, and are conical in form. Their position, in addition to the circumstance that they are less long than the indifferent bristles, or are placed in depressions, makes it probable that these organs have another function, and it is very easy to suppose that they are organs of smell, or at least of a sensation very much like it. In this case the antennæ, by the differentiation of special nerve-endings, have more than one function, and do not merely preside over the sense of touch.

\section{Auditory Organs.}

\section{$\S 202$.}

Auditory organs are not widely known in the Arthropoda, no sign of them having been seen in the Myriapoda and Arachnida; on the other hand, in some divisions of the Crustacea and Insecta, organs may be made out, which appear to be adapted for the sensation of sound.

There are two principal forms of the organ, which are exactly correlated with the medium in which the animal lives. One form is found in the Crustacea, and consists of a saccular space, formed by an inpushing of the integument; it sometimes remains open and is sometimes closed. These auditory vesicles lie, in most of the higher Crustacea, in the basal joint of the internal antennæ. Thus in Leucifer, Sergestes, and other Malacostraca, as also in the Arthrostraca (Hyperida), a pair of these organs may be found in front of the cerebrum. As secondary structures they may also be found on other parts of the body. Thus in the Mysidæ, they lie in the two inner lamellæ of the fan of the tail. There are firm structures, otoliths, in the auditory vesicles, which, when the vesicles are closed (in Mysis and Hippolyta), consist of a concretion, which is held fast by fine, regularly-arranged hairs. When they are open, as they are very commonly among the Decapoda, and also in Tanais, the orifice is greatly complicated. The place of the otoliths is here taken by grains of sand brought in from the exterior ; these are regularly attached by special hairs, which arise from the wall of the auditory vesicle. They are like the other hairs of the integument, but are distinguished from them by not having their shaft directly connected with the floor of the vesicle, most of them standing on a fine membranous process, to which endings of nerves pass. In this they agree with the rod-like processes which carry the otoliths in the Mysidæ, to which nerves likewise pass. The auditory nerve in these forms, in which the auditory vesicle is embedded in the internal 
antennx, is a branch of the internal antennary nerve. Both structures thus represent the end organs of nerves, which are set in vibration by the shaking of the firm body (Otolith) which they carry, and thus produce an excitation of the nerre.

The general character of this remarkable system shows us how the auditory organs arise from a differentiation of an indifferent sensory organ connected with the integument. The auditory hairs are only modifications of other "hairs" of the integument which contain nerve-endings, and just like those which may appear on the free parts of the body (tactile rods). The formation of the unclosed auditory vesicle, or auditory pit, represents therefore a second stage in this differentiation; and the change into a closed vesicle is a further stage of this phrnomenon.

Hensex, Zeitschr. f. wiss. Zool. XIII.

\section{$\$ 203$.}

The other form of auditory organ is found in the Insecta. It is principally in the Orthoptera, which are also provided with vocal organs, that an organ for receiving the waves of sound can be made out. The ordinary ar'angement is a tympanic membrane, stretched on a firm chitinous ring, one surface being directed to the exterior, the other to the interior. On its inmer side there is a tracheal vesicle, and on this, or between it and the "tympanum," there is a ganglionic nervous enlargement, from which specially modified nerve-endings, having the form of small club-shaped rods, arise between fine filaments. The tympanum, as well as the tracheal resicles, serves as an organ for conducting sound. The organs of perception are represented by the nerve-endings, which are regularly arranged. In the Acridida the organ lies in the metathorax, just above the base of the third pair of legs, and receives its nerve from the third thoracic ganglion. The Locustida and the Achetida have the organ embedded in the tibia of the two anterior legs. In the former a tympanum lies on either side of this leg, either superficially, or at the bottom of a cavity, which opens anteriorly by a single orifice. Two tracheal branches occupy the space between the two tympana, one of which carries the ridge-shaped nervous endorgan. This auditory ridge is, in Locusta, formed by a series of cells which grow smaller towards one end; each of these contains a "rod" of proportional size. The tympanum in the Achetida lies on the outer side of the tibia of the anterior leg.

Other organs, the nature of which is less definitely settled, are allied to these, as by their general structure they represent auditory organs; the presence of the same pencil-shaped body in the terminations of nerves justifies us in at least ranking these organs with the auditory, while, further, such a relationship is implied by the ganglionic outspreading of the proper nerve along a tracheal branch. The ends of the nerves are directed towards the integument, the 
chitinous layer of which is always provided with a number of closelyapplied groups of pore-canals instead of a tympanum. Organs of this kind have now been recognised at the root of the posterior wing of the Coleoptera, as well as on the base of the halteres of the Diptera.

The two forms of auditory organs in the Arthropoda are indeed very different from one another in the details of their arrangements, but there is, nevertheless, a connection, for in both cases the chitinogenous cellular layer gives rise to parts which carry the special endorgans; in the Crustacea these are connected with processes of the integument, the auditory hairs; while in the Insecta they are converted into the small pencils, and are consequently differentiated in another direction; they remain within the dermal skeleton, and have no relations to the processes of it. No homology can be made out between these organs, owing to the diversity of their position, and from the fact that more complicated organs are derived from an elementary arrangement, which is distributed generally in the integument.

Leydig, Arch. f. Anat. u. Phys. 1855.--Graber, V., Die tympanalen Sinnesap. parate der Orthopteren. Denkschr. d. Wiener Acad. M. N. Cl. Bd. XXXVI.

\section{Visual Organs.}

\section{$\S 204$.}

In the visual organs of the Arthropoda we meet with points of resemblance to certain forms of eye found in the Vermes; to those, namely, in which a number of end-organs of the optic nerves are placed directly beneath the integument (Sagitta, Hirudinea, etc.). But they have no close affinity to the more developed eyes of the Annelides, which are distinguished by the possession of a separate lens $(\$ 125)$. In Arthropoda, as in other forms, the integument is the spot at which the eye is differentiated; its mode of composition out of the elements of the integument will be understood by a reference to the subjacent diagram; although, of course, this does not represent the simplest condition. The cuticular layer of the integument forms a biconvex thickening over the eye (l); this forms a refracting but also a defensive organ, and functions therefore as a cornealens. 'The eye, which is formed from the hypodermic layer (h), lies behind this lens. Around it

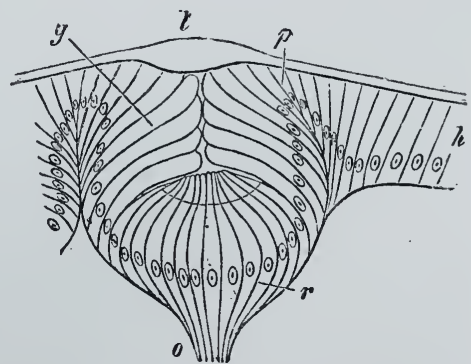

Fig. 134. Section throngh the simple eye of a young Dytiscus larva (after Grenacher). the hypodermic cells elongate and change their position; they become pigment cells $(p)$. 'The optic cup, into which project transparent 
cells $(g)$, which at first are placed beside the pigment cells, comes next. These cells represent a vitreous body. Lastly, there are the cells $(r)$ which form a kind of retina; these are connected with the optic nerve (c), but converge towards the exterior, and so towards the posterior surface of the lens, where they undergo various kinds of differentiation. The vitreous body, pigment cells, and "retina" are therefore clearly continuous with the ectodermal layer (hypoderm), and are differentiations of it, just as the cornea-lens was formed from the cuticular layer, which again can be derived from the hypoderm. The elements which compose the eye undergo various differentiations. A special structure, the "rod," is generally differentiated in the anterior end of the retinal cells. When a number of these cells are united into a single apparatus the rods also become united, and form a special structure, the "rhabdom," in the long axis of a group of combined retinal cells. The retinal cells, which give rise to the rhabdom, constitute a "retinula." The cells in the vitreous body in front of the retinal cells may also undergo great modifications. Each group separates off a transparent highly refractive substance, which forms the so-called "crystalline cone;" the apex of this is turned towards the rhabdom, and its base towards the integument, i.e. to the cornea-lens.

Owing to the different development of the various parts, and to the various ways in which they are combined, the optic organ of Arthropoda becomes very varied in character. Muscular fibres sometimes enter into the composition of the eye, and appear to form a focussing apparatus.

These eyes belong to the head. The optic nerve arises from the cerebral ganglion. In all divisions the eyes may undergo degeneration, and even completely disappear. Eyes are but seldom developed on other parts of the body, as they often are in the Annelides, so that the presence of eye-like organs on the thorax and abdomen of the Schizopod genus Euphausia is a rare exception.

\section{$\$ 205$.}

The simplest eyes, although indeed their structure is not yet exactly known, are found in the Entomostraca. Each eye appears to possess one crystalline cone only, which is sunk into a mass of pigment, and is generally separated from the integument. Two such cyes, which are generally placed immediately on the cerebrum, are characteristic of the Nauplius form of the Entomostraca. There are two eyes, connected in the middle line, placed close to one another, and fused into one organ by the connecting pigment; when they are not placed on the cerebrum itself, they are carried on a median process of it. The Cirripedia and Rhizocephala have eyes during their larval condition; the latter lose them later on. In many freeliving Copepoda the eye is more or less distinctly divided into two. In that case there is another and larger eye on each side, in addition to the larval eyc. Each of these is generally provided with a 
crystalline cone of some size, in front of which a corresponding portion of the cuticular layer of the integument forms a lens-like organ (Corycæidæ): The presence of several crystalline cones in each eye forms an intermediate step to the more complicated form of eye. As the integument, which is found over the simple pair of eyes, becomes thickened into two facets corresponding with the crystalline cones, these structures resemble in their mode of formation the cornea-lenses.

In addition to the median eye, which is sometimes represented by a mere speck of pigment, the Cladocera and Phyllopoda possess two compound eyes, from which it may be concluded that the median one, which corresponds to the eye of the Nauplius, is a special structure, which does not become developed into the permanent eye. This "larval eye" is probably a transmitted arrangement.

By their power of movement, and their position immediately below the chitinous carapace, the eyes of the Branchiopoda form an intermediate step towards those in which the chitinous carapace takes a more direct share in forming the optic organ. Further, the position of the eye, on a stalk-like process (Artemia and Branchipus), presents a point of affinity to the podophthalmate Malacostraca.

Two types of eye are derived from these conditions, which are very common in the higher divisions of the Crustacea, and in the Tracheata. According as the elements of the retina, which form the perceptive apparatus, unite into a compact simple organ, or as these organs are themselves part of a more complicated structure, the optic organs are distinguished as simple eyes (Stemmata, Ocelli), or as compound eyes. The cuticular layer of the integument takes a greater or less share in the formation of this apparatus.

The simple eye (Fig. 134) is commonly found in the larval forms of the Insecta, and there are generally a number of them on either side of the head. In the Thysanura this form appears to persist. The chitinous layer forms a cornea-lens over each eye. In many Insects we find these simple eyes in company with the compound ones, between which they are placed, generally in twos or threes, and on the frontal surface. They are distinguished from the compound eyes by being composed of a large number of retinal elements, which are covered by a simple cornea-lens.

In the Myriapoda the eyes, which are placed in one or two rows, vary in number (4-8). It seems as if we had the larval stage in the eye of insects permanently represented, but we have no exact knowledge on the subject.

In the Arachnida the arrangement is much the same. There are numerous peculiarities both in the arrangement and number of their eyes. Two large eyes are, in the Scorpions, approximated to one another, and on each side there is a group (2-5) of smaller eyes. In the Spiders and Phrynida there are, as a rule, 8, more seldom 6, symmetrically distributed on the anterior part of the cephalothorax; they generally vary in size; in the Opilionida there are only 3 or 4 in the same place, the largest of which are placed on 
an elevation of the cephalothorax. In the Pycnogonida 4 eyes occupy a similar position. On the other hand they are reduced to 2 in many Mites, and so also in the Tardigrada; in many parasitic Mites they have completely disappeared. The principal point in their structure is the presence of a cornea-lens, which is ordinarily very large in each eye; behind this is a lajer of cells, which represents the vitreous body, and to this the retina is attached. In the Aranea the retina is formed in two ways, the eyes directed anteriorly differing in structure from those which are turned upwards. That is to say, the retinal cells of the former surround a small longitudinally bisected rod at their anterior end (Epeira).

\section{$\$ 206$.}

The compound eye is characterised by the above-mentioned fusion ( $\$ 204)$ of a number (7-4) of retinal cells into a structure which surrounds the rhabdom-the "retinula" (Fig. 135, $C r$ ). The eye is made up of these retinula, each of which is enveloped in pigment. The multifid crystalline cone lies in front of the retinula. Two of these structures are represented in Fig. $C$. The crystalline cones may be made out in front of the retinulæ, and behind the cornea-lenses $(c)$. The whole arrangement is easy to understand, when we derive it from the simple eye. A reduction of the retinal elements of the simple eye gives rise to the retinula, and a compound eye is formed by the gradual concrescence of a number of simple eyes. Most of the Crustacea have eyes of this kind. In the Cladocera the movable eye (Fig. 136, oc) lies in a cavity roofed over by the integument. In the Lrmodipoda also the cuticular layer of the integument seems to take no part in forming the eye. On the other hand in the Phil-

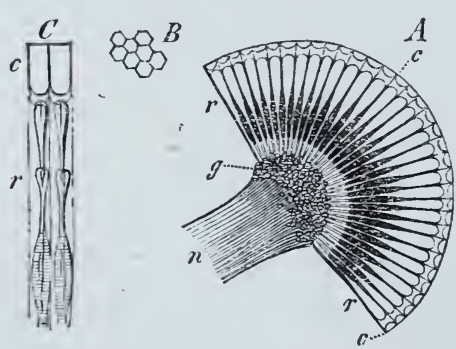

Fig. 135. A Diagrammatic section through a compound Arthropod. eye. $n$ Optic nerve. $g$ Its ganglionic sircllings. $r$ Retinulx. $c$ Facetcd cuticular laycr, each facet of which forms a cornea-lens. $B$ A few cuticular facets, seen from the surfacc. $C$ 'Two retinule, $r$, with their cornca-lenses, $c$. lopoda we meet with a faceting of the inner surface of the cuticle covering the eye, the facets corresponding to the crystalline cones. In the Isopoda the compound eje still consists of a number of simple eyes, distinctly separated from one another. When a number of these structures, which form the endorgans of an optic nerve, are closely connected together, a process, convextowards the outerface, is formed, the size of which is dependent on the number of the "retinulae" (Fig. 135). The chitinous covering of the whole eye is either smooth on its surface, and only forms on its inner face curves, which correspond to each of the crystalline cones, or it forms convexities for cach of the separate crystalline cones, or eren 
marks off each separate area $(B)$. Crustacea and of the Insecta.)

(Faceted eye of the higher

The number of the elements which make up one of these eyes, as well as their size, and the form taken by the several parts, may undergo various modifications. The crystalline cones are generally present in this kind of eye in the Crustacea, but in many Insecta the cells (crystalline cells), which in other eyes are differentiated into crystalline cones, persist without forming crystalline cones. Lastly, in many, the primitive condition of the retinula, in which the separate cells, each with its rod, may still be made out, persists ('Tipulidæ). The projecting character of the eye, owing to its curvature, may lead to a stage in which the eye is stalked. When still more developed this stalk may be movable (Podophthalmata).

Grenacher, H., Untersuchungen über das Arthropodenauge. Beilageheft zu den klin. Monatsbl. fur Augenheilkunde. XV. Jahrgang.

\section{Alimentary Canal.}

$\S 207$.

The enteric canal of the Arthropoda is differentiated in much the same way as in Vermes. The endoderm encloses what yolk-material is not used up in the early stages of differentiation, and this is absorbed during the gradual processes of further development. The mouth and anus, and the connected parts of the enteron, are not developed according to any general rule. When the enteric walls are completely differentiated the nutrient canal forms a tube, which traverses the whole length of the cœlom, and is but seldom adapted to the metameres of the body ; it begins by a mouth, which is placed on the ventral surface, and extends to an anus, which, as a rule, is placed in the last metamere. The external chitinous covering of the body extends into the fore- and hind-gut; in the mid-gut, which is formed from the endoderm, it is replaced by a soft cuticle. The appendages (\$189), converted into masticatory and other organs, are grouped round the mouth, and a process, which is formed from the integument, joins them as an upper lip.

\section{$\S 208$.}

The enteric canal of the Crustacea is distinguished by the straightness of its course, and the slight extent to which its divisions are complicated. The mouth, which is ventral, is often placed some way back, so that the fore-gut, which arises from it, runs forward at first, and then turns backwards at a sharp angle. The terminal portion of the ordinarily narrow fore-gut is known as the pharynx or œsophagus; it is generally widened and distinctly marked off from the succeeding mid-gut; in many forms a wedge-like process 
of this pharynx projects into the mid-gut. The walls of this portion are, as a rule, stronger, and its inner surface is often distinguished by the possession of a firm chitinous framework, which is provided with tooth-like processes turned towards one another, and moved by muscles; these ridges, spicules, or setæ, are derived from the chitinous membrane, which invests this portion. They form an apparatus which serves for the comminution of the ingesta; and hence this portion is known as the masticatory stomach. As a rule it is of considerable size, and is regular in form, owing to its firm framework. It is most largely developed in the Decapoda (Fig. 143, v). In the Entomostraca it is small, or not developed at all, while in the Isopoda among the Arthrostraca the small masticatory stomach is provided with a fairly complex framework, of which there are indications also in the Amphipoda (Gammarus).

The mid-gut (Fig. 136 i) forms by far the longest portion of the

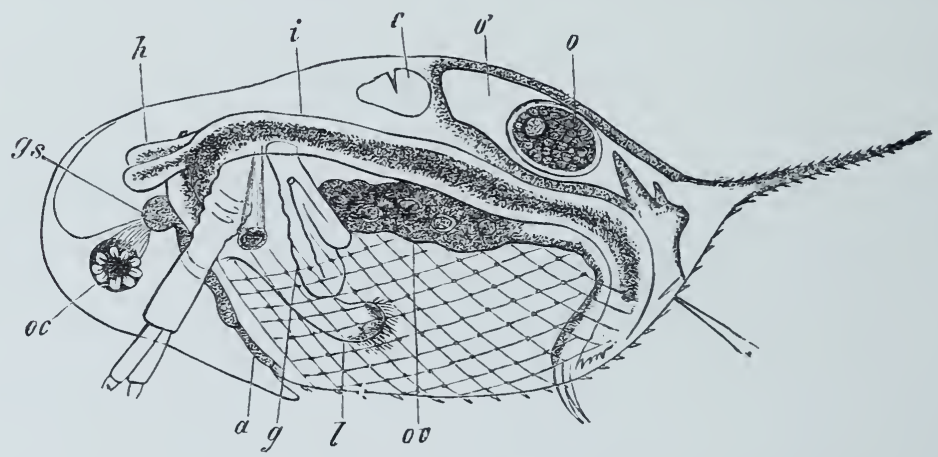

Fig. 136. Organisation of a Daphnia. a Tactile antenna. gs Cerebrum. oc Eye $i$ Enteric canal (mid-gut). $h$ Cæcal tubes at its commencement. g Shell gland. c Heart. $l$ Labrum. ov Ovary. o An Egg in the brood-space $\left(o^{\prime}\right)$ formed between the body and the mantle (after Leydig).

enteric tube, and varies greatly in width, and in the formation of crcal diverticula. In many cases it has the same calibre all along; in others it is widened somewhat anteriorly, or mesially ("Chylestomach"); or the widened portion extends over the whole of the mid-gut ("Chyle-intestine" of the Isopoda).

There are crecal diverticula at the commencement of the midgut, in all orders of the Crustacea. They arise as paired, and seldom as unpaired, crea. In the Copepoda they are found only in a few genera; in the Branchiopoda they are more common, and form either a single pair of short cxcal tubes (Fig. 136, h) (Daphnida), or are more richly branched (Argulus, Hedessa), or arise from the enteron in greater numbers, and are differentiated at their ends into glandular organs (Apus). We find that the same phænomenon of metamorphosis of the enteric cæca into secreting organs obtains in the Malacostraca, and at exactly the same spot. 
They become organs which will be considered when we come to speak of the appendages of the midgut.

The hind-gut forms the shortest, and generally a narrower portion of the enteric tract. It is seldom widened mesially, and in a few only is it provided with cæcal appendages.

The function of the enteric canal is not, in all Crustacea, limited to digestion. In some (Astacus, Limnadia, Daphnia) an almost rhythmical taking-in and drivingout of water may be observed in the hindgut, so that this portion appears to have a respiratory function also.

In many lower Crustacea the enteric canal undergoes degeneration. It disappears in the degenerate males of the parasitic Copepoda, and in some Cirripedia and most Rhizocephala, where nutrition is effected by other means (cf. suprà, p. 236).

\section{$\S 209$.}

The three divisions of the enteric tube are distinctly marked off in the Protracheata; the mid-gut, which is distinguished by its width, forms the largest part of it.

The enteric tube of the Arachnida is divided into a larger number of segments, except in the degenerate forms. The narrow fore-gut (Fig. 137, oe) leads into a mid-gut, which is generally elongated, and has its more anterior portion $(v)$ frayed out into lateral cacal sacs. These are absent in the Phrynida and Scorpionea. In the Aranea there are five pairs of them $\left(v^{\prime}\right)$, which extend to the base of the legs and palps. In the Galeodea four pairs, the last two of which are bifurcated, extend into the appendages (feet, claws, and palps); in the Pycnogonida these cæca extend almost to the end of the appendages (Fig. 138, Z). The presence of these parts greatly increases the internal cavity of the stomach.

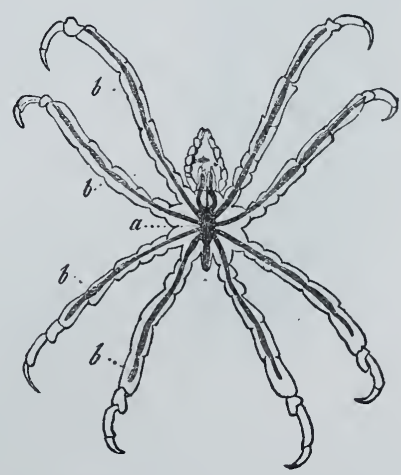

Fig. 133. Digestive organs of Ammothoë pycnogonoides. $a$ Stomach. $b$ Cæca (after Quatre. fages).

In the Acarina these creal sacs are confined to the body; there are generally eight of them, but any diminution in number is 
compensated for by the branching of the cæca. The Opilionida are provided with a much greater number (about thirty), and a median pair is provided with secondary appendages.

The portion of the mid-gut behind the stomach varies in length; when it is long it is widened out towards its end, and is separated

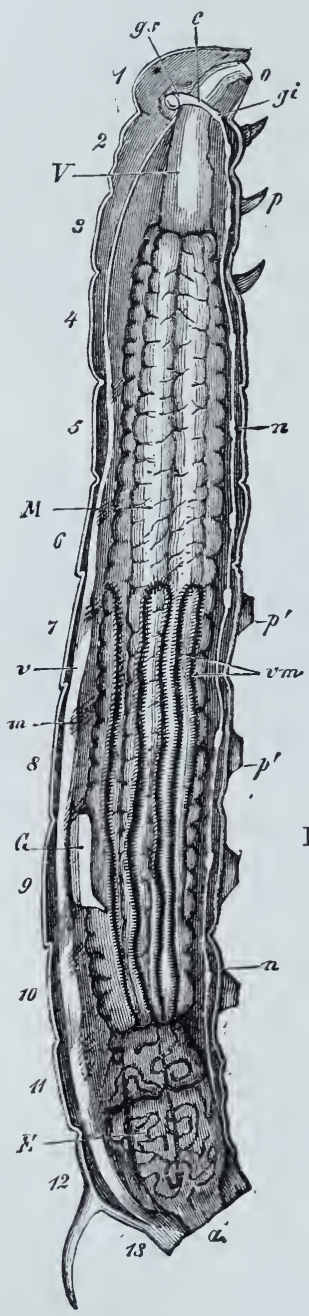

Fig. 139. Larva of a Lepidopterous Insect (Sphinx ligustri); seen from the side.

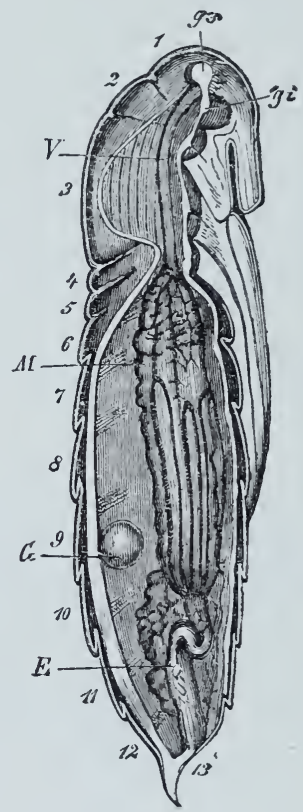

Fig. 110. Pupa of the same Insect.

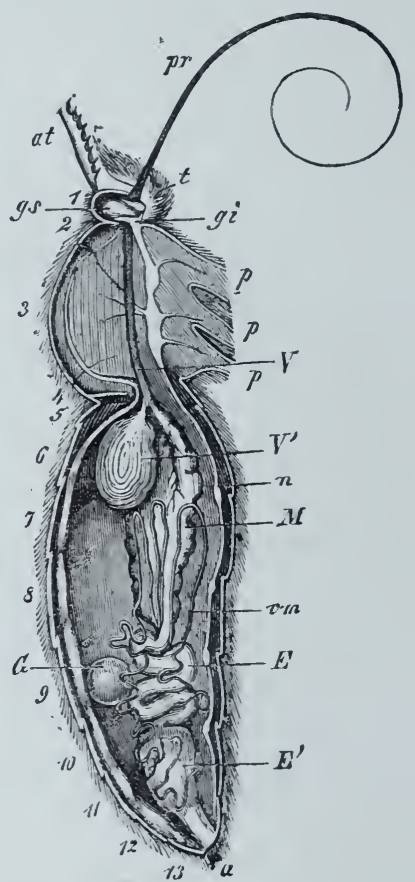

Fig. 111. Its imago. $i$ Head. 2, 3, 4 Thoracic segments. 5-13 Abdominal segments. $V$ Fore-gut. II Mid-gut. $E$ Hind-gut. gs Cerebral ganglion. gi Sub-œsophageal ganglion. $u$ Ventral ganglion. $v m$ Malpighian vessels. C Heart. G Generative organs (after Newport).

off from the hind-gut by a constriction; this latter is almost always widened, it is pretty long in the Scorpionea, but shorter in Galeodes, where it has a crecal sac. In the Aranea also, and in the Acarina, the hind-gut (Fig. 137, r) is of a considerable width.

\section{$\S 210$.}

The Myriapoda and Insecta agree in the mode of arrangement of the chief tracts of their digestive system; which at the same time closely resembles what obtains in Peripatus. Of the three divisions of the digestive tube the fore-gut only prepares the food, and the mid-gut has the principal share in digesting it. As a rule it forms the longest 
portion, and at the same time undergoes the largest amount of differentiation.

When most simple the enteric tube traverses the cœlom in a straight line; in this point the Myriapoda resemble the lower stages of the Insecta. In the Myriapoda the hind-gut is seldem very long; when it is, it is looped. A portion of the mid-gut still more rarely takes part in forming this loop (Glomeris).

'T'he simple character found in most larvæ does not persist in most divisions of the Insecta; in most the separate portions get to vary greatly in character, and these changes are, as a rule, correlated with the appearance of the imago-stage. They are really due to the great differences in the relations of the animal to extermal conditions, which now commences.

The mode of life seems to be of the greatest influence on the gencral configuration of the alimentary canal, and there is often a greater length of tube in the phytophagous insects-as, indeed, frequently happens elsewhere in the Animal Kingdom - than in those which live on animal matters. The character of the food is also another cause which comes into consideration; we find, that is, simpler characters in the intestines of those insects which live on fluids, while there is greater complication of structure in those which subsist on solid food.

These characters are seen most strikingly on comparing the digestive tube of an insect larva with that of a developed insect; we see, for example, a caterpillar (Fig. 139) provided with a wide tube running straight through the body. This arrangement is adapted to the enormous amount of food which is taken every day; while the butterfly, which takes in only a little food, and that fluid, has a canal which is longer indeed, but much more delicate (Fig. 141).

Further, the difference between the enteric canal of the perfect insect and that of its larva depends on a change in the relations of the various divisions of it; while, in the larval stage, the mid-gut is ordinarily the largest portion, it becomes gradually shorter, and the hind-gut is proportionately increased in length. Thus the enteric tube ceases to be a straight one. The increase in length of the separate portions produces curvatures of the tube, which is now longer than the whole body-cavity, and these may lead to the formation of various coils in it. These affect the mid-and hindguts, while the fore-gut most completely retains its primitive course (cf. Figs. 139-141).

Fresh differentiations in the various portions are connected with these, so that the boundaries between them often disappear. The mid- is distinguished from the fore-gut by its glandular character, and where the latter has appendages, or diverticula, they serve for the reception and the further comminution of the food; in the latter case they have the characters of a gizzard. Finally, the hind-gut is characterised by the Malpighian vessels which open into it.

Platent, F., Rech. sur la phénom. de la digest. et sur la structure de l'appareil digest. chez les Myriapodes. Mém. Acad. Belg. XLII. 


\section{$\S 211$.}

The gut in the Thysanura, and in most Pseudoneuroptera and Neuroptera, is most simple in character, and least different from that of the larval form; some of these (Panorpa) hare an enlargement at the end of the fore-gut; this is the masticatory stomach. The Orthoptera are distinguished by the possession of a stomach of this kind (Fig. 142, $A v$ ), which has long rows of chitinous plates on its inner surface. It is also found in the Coleoptera (Carabidæ, Cicindelæ, Dytiscidæ, etc.), where it carries setae and ridge-like projections. Many Hymenoptera (Formica, Cynips) also possess it, as do the larva of the Diptera.

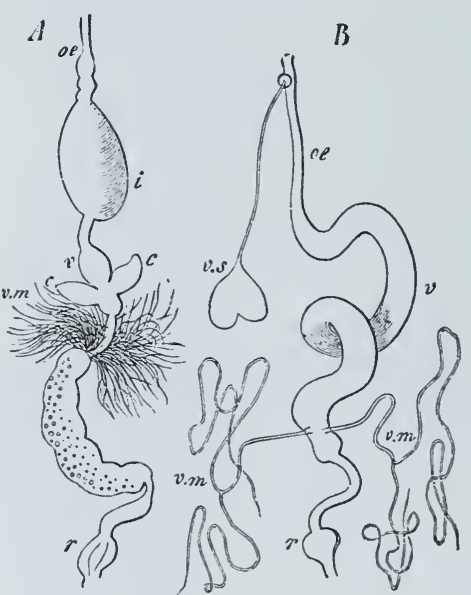

Fig. 142. A Digestive canal of the Ficld-cricket. $B$ Of a Fly. oe Esophagus. $i$ Its crop-like enlargement. $v$. Stomach. $c$ Its appendages. $r$ Hindgut. vm Malpighian canals.

Another differentiation seen in the fore-gut of many insects (Hemiptera), which is always short, is an enlargement which may be present all round, or on one side only. When it surrounds the œsophagus it serves as a crop (ingluvies) (i); this is the case in many Coleoptera and in the Orthoptera. This dilatation of the fore-gut is found in the Hymenoptera (Wasps and Bees), but in them it functions as a sucking organ, and leads towards the formation of the Sucking stomach, which is found in other insects. It also forms a vesicular thin-walled appendage, which is attached on the course of, or to the end of the fore-gut; in the Lepidoptera this opens directly (Fig. 141, $V^{\prime}$ ), and in the Diptera by means of a stalk of varying length (Fig. 142, $B, v \varepsilon$ ). In the Hymenoptera also an independent and stalked sucking stomach is formed (Crabro). In the Hemiptera it appears to be replaced by an enlargement of the fore-gut, which is frequently multi-sacculate (Bugs).

The mid-gut (" chyle-stomach") is no less varied in character. In many Coleoptera it is provided with short tubes, either along its whole length or in separate segments ; these are known as "glands." At its commencement we sometimes, and especially in the Orthoptera, find creal prolongations; as we do also in some families of the Diptera. In the latter it is ordinarily disposed in coils (Fig. 142, Bv), in correspondence with its great length. The same occurs also in the long mid-gut of some Coleoptera (e.g. Melolontha), of Bees and Wasps, and of many Hemiptera; in these forms further divisions are differentiated in it. 
In some cases the mid-gut ends blindly and has no connection with a hind-gut. 'This happens in the larva of Bees and Wasps, of the Ichneumonida, and of many Diptera, etc.

'The hind-gut in the Insecta generally forms the shortest portion of the straight alimentary canal. It is often divided into two portions, the second of which is widened out ("rectum") (Fig. 142, $A B$ r). In the Coleoptera (as in Dytiscus) its narrower and anterior portion appears to be of considerable length, as in many Orthoptera, where a larger number of divisions, varying in width, can be made out; it is longest in the Cicadæ: in all of these forms it is disposed in coils. As in many the Malpighian vessels, which belong to the hind-gut, open very far forwards, it appears as if part of the last division of the hind-gut had passed into the mid-gut, although, indeed, this rather seems to indicate that the mid-gut has been reduced in length.

The widened terminal portion of this part of the enteric canal is distinguished in a large number of Insects by papilliform ridges, which project into it: in these we find many tracheal branches. This portion in many aquatic larvæ of the Libellulidæ has numerous lamellæ arranged in longitudinal series, and provided with closelypacked tracheal ramifications. The lamellæ act as organs of respiration during the in-and-out flow of water, which is caused by the opening and closing of the anus (cf. Fig. 150, $B C$ ). There are many intermediate forms between these tracheal gills, and the papilliform processes of the hind-gut (in the larvæ of the Phryganeidæ), so that these organs can be seen to be homologous.

Plateau, F., Rech. sur les Phénomìnes de la digest. chez les Insectes. Mém. Acad. Belg. XLI.

\section{Organs appended to the Digestive Canal.}

\section{1) Appendages of the Fore-Gut.}

\section{$\S 212$.}

Glandular organs are differentiated in the various portions of the alimentary canal of the Arthropoda. The salivary glands, which open into the fore-gut, are but slightly developed in the Crustacea. Groups of unicellular glands have been made out in some divisions. On the other hand, we find them more widely distributed in the Tracheata, where they may have various functions; but as yet we have but very little exact information as to how they open. The great point which is undetermined is, how far they open into the mouth itself, or are connected with the fore-gut. But the glands which open into the gnathites of Peripatus (\$193) must be mentioned here. Among the Arachnida, the Scorpions have two 
pairs of lobed glands; in the Galeodea they are partly represented by coiled tubes, and in the Aranea, likewise, these organs do not appear to be wanting. The salivary glands are highly developed in Mites, where there are several pairs of them, which vary in structure; the secretion of them is probably partly used as poison.

In the Myriapoda, there are simple tubular (Julus), or lobed (Lithobius), or eren racemose glands (Scolopendra), which are probably salivary.

In the Insecta the salivary glands are very variously developed as to number, form, and histological structure. It is therefore certain that they also vary greatly in function. It is in a fer only, as the Ephemerida, Libellulida, and Aphides, that they appear to be completely wanting, or only feebly developed, as in the Myrmeleonida and Sialida. In others, they are long coiled tubes, or lobed or greatly-branched organs, which frequently accompany the enteric canal for a certain distance. There are often two, and not unfrequently three pairs of them, which vary greatly in structure. As to their external form and their distribution in the different groups of the Insecta-they have the form of a pair of long tubes in the Coleoptera, Diptera, and Lepidoptera; in the order of the Hemiptera and Orthoptera branched, racemose, or lobed forms predominate, as also in many Coleoptera.

\section{2) Appendages of the Mid-gut.}

\section{$\S 213$.}

Another group of glandular organs is differentiated from the midgut. They are regarded as a liver. According to their points of connection with the enteron, two different organs must be here distinguished from one another. One of them is connected with the most anterior portion, and has the form of simple or branched tubes, which, when more largely developed, form a compound glandular apparatus (cf. $\S 208$ ). The ends of these tubes seem to be secretory organs, but their ducts, owing to their wide lumen, form cavities of the enteron. The organ is, therefore, not yet completely differentiated from the enteron. This arrangement is seen in the Branchiopoda, and, among them, most markedly in the Phyllopoda; some have a simple or branched cæcal tube on either side (Fig. 136, $h$ ); in others it is converted into a liver (Limnadia, Apus), the greater part of which is placed in the cephalic shield. The Cirripedia have similar organs. In the Arthrostraca these cæcal tubes (Fig. 143, $A$ h) are long organs, which pass backwards and vary in number. They are not branched, but this is compensated for by their increased length. They have the same form in the Schizopoda, but in most of the 'Thoracostraca, as in all the Decapoda, they are formed by a paired mass of glandular matter, which fills up the cephalothorax and is 
grouped in tufts. Inasmuch as in the larvæ of the Decapoda they are mere diverticula of the wall of the enteron, it is clear that they are only more developed stages of those simpler tubes, which are found in many Entomostraca.

A second form of this hepatic organ is distinguished from the former by the larger number of its separate glands, and by the more backward position of its opening. into the mid-gut. Indications of this are even found in the Copepoda in the form of several succeeding diverticula of the mid-gut. They are more developed in some

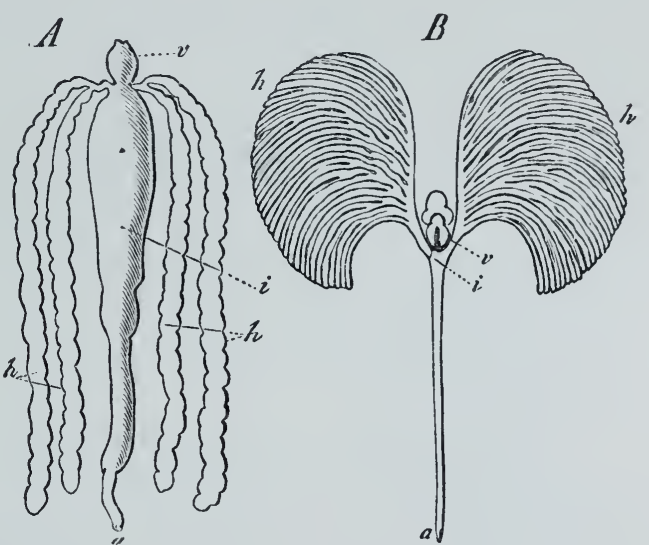

Fig. 143. Enteric canal and liver of Crustacea. $A$ Of Oniscus. $B$ Of a larval Palinurus (Phyl. losoma). $v$ Masticatory stomach. $i$ Chyle-stomach. a Anus. h Hepatic tubes. Isopoda (Bopyrus), where they beset the whole of the mid-gut in the form of paired and branched tufts of glands. In the Stomapoda also we find a larger number (10 pairs) of lobate tufts of glands placed all along the mid-gut.

The two forms cannot be directly derived from one another, for those parts which carry glands in the former groups do not do so in the others. It is possible that both kinds of organs were united in a common stem-form. If so, we can imagine the whole mid-gut beset with cæca, from which two sets of glands were developed; in one the most anterior pair were developed, and in the other suppressed, whilst the hinder pair was more or less largely developed. These hinder glands distinguish the mid-gut of the Pocilopoda, where they have the form of two pairs of branched tufts.

Among the Tracheata we find similar differentiations of the wall of the enteron in the Arachnida; in which division we must regard them as acquired organs only. The anterior pair are not, however, always developed into glandular organs, but persist as more or less wide pouches or tubes; these have been already fully described as stomachal crea ( $\$ 209)$. In the Opilionida they are exclusively glandular in character. In the Scorpionea and Aranea separate tufts of glands open into the hinder portion of the mid-gut. The Aranea have two or three (Fig. 137, $h$ ), the Scorpionea five pairs.

These appendages are not found in the Myriapoda or Insecta, and the absence of diverticula from this portion of the enteron during development shows that any diverticula, which appear in it, have merely a secondary significance. 


\section{3) Appendages of the Hind-gut.}

\section{$\S 214$.}

The glands differentiated off with the hind-gut, which is itself generally short, do not secrete anything, which is of importance in digestion or absorption. Their secretions are rather of the excretory series. And as we have chemical evidence to show that these matters resemble the urinary excretions of the Vertebrata, we may regard these bodies as being excretory organs, without thereby prejudicing any relations, which they may have, in some cases, to other functions.

In the Crustacea we sometimes meet with cæcal organs on the hind-gut, as for example in the larvæ of the Copepoda, but we cannot safely form any opinion as to their significance. It is probable that the concretions found in the walls of their entera are of an excretory character.

Excretory glandular organs are very generally found in the Tracheata; they arise as diverticula of the enteron, and have the form of long canals, which may be simple or branched, and which are often arranged in several coils or loops on the enteric canal; they open into the terminal widened portion of the enteric canal, and almost always behind the mid-gut. They are known as Malpighian vessels, or, from their function, as urinary canals. As they are formed at the same time as that portion of the hind-gut, which in the embryo is developed from the ectoderm, it is not improbable that they primitively opened on to the surface of the body, or were derived from organs which did so. In all divisions there are two chief canals, as is often seen at the point where a large number of canals open and unite; this number may therefore be regarded as a primitive character.

Among the Arachnida they are, in the Scorpionea, simple canals, which run between the lobes of the liver; one pair of them is branched. The urinary canals of the Aranea are much branched, and united into a plexus; they unite into two common excretory ducts (Fig. 137,e), by which they open into the wide hind-gut, or its crecal sac. In the Opilionida they form two long highly-coiled canals; in the Acarina, where they are sometimes provided with branches, they are of a similar form.

An equally small number of simple urinary vessels is found in the Myriapoda; the Julida have one and the Scolopendrida two pairs. They are allied, not only by their number and simplicity of structure, but also by their arrangement on the alimentary canal, to the corresponding organs of many Insect larvæ.

The greatest variation in number, arrangement, and special structure obtains in the urinary vessels of the Insecta. They are wanting in the Collembola, among the Aptera, and in many Thysanura 
(Campodea), but four are present in Lepisma. 'The function of the urinary canals is notably increased in the Insecta, which have a perfect metamorphosis, during their larval stage, as is shown not only by the great development of these organs (Fig. 139, vm), but also by the quantity of urine which is collected in the hind-gut during their pupal stage. This phænomenon corresponds exactly to that period in which the most intense plastic activity is going on in the organism, in connection with the development of the perfect body. It is clear that the function of the Malpighian canals of the Insecta is not to be found exclusively in the excretion of urine; but that an older hypothesis, according to which they were regarded as organs for the excretion of bile also, is not altogether without justification, is seen from the fact that portions of this canal have a different structure, while the secretion in these portions is different also.

The brownish-yellow, or light-yellow coloration of the urinary canals is due to the substances deposited in the cells of the canal-wall, and is more intense when secretion is more active. Most of the Diptera (Fig. 142, $B \mathrm{vm}$ ) and Hemiptera have two urinary canals, which are connected with one another; there are six canals in the Lepidoptera, in many Nocturnal flies, and in several Pseudoneuroptera (Termites); there are four to six in the Coleoptera;

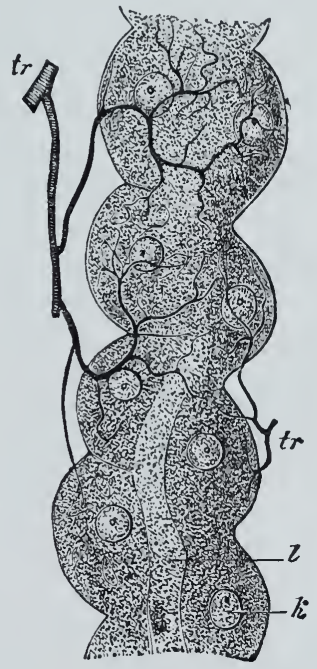

Fig. 144. Portion of a Mal. pighian vessel of Musca vomitoria. $t r$ Trachea. $l$ Lumen. $k$ Nucleus. the Hymenoptera are distinguished by a large number of short urinary canals, and indeed hundreds have been found in them, and in many Orthoptera (Fig. 142, $A \mathrm{vm}$ ). As a rule they are seldom branched; but we often meet with loop-like connections between their ends. They open at apparently very different points, according to the length of the hind-gut. They open very far forwards in the Cicadæ, Diptera, and Lepidoptera. In the Hymenoptera they open just behind the mid-gut.

Where the canals unite to form a common excretory duct there is a circular layer of muscles (Gryllotalpa). Separate circular fibres are very seldom found in the secretory canals (Brachinus).

\section{Cœlom.}

$\S 215$.

As the embryonic body is differentiated, a cavity, the cœlom, is formed in the endoderm between the enteron and the wall of the 
body, just as in the higher Vermes; it is found in all the Arthropoda. There are no signs in the Arthropoda of the dissepiments found in the Annulata in correlation with the metamerism of their bodies. This shows that their relationship to the Annulata is, to say the least, a very distant one. In all cases the colom forms a portion of the blood-vascular system, so that the perienteric fluid found in many Vermes as a fluid different from the blood is represented in the Arthropoda by the blood itself.

In most Arthropoda some of the form-elements of the mesoderm, which are not applied to the ectoderm-to form the wall of the body-or to the endoderm to form the wall of the enteron-persist as a number of cells, which are not apportioned to any definite organ. These masses of cells remain in various parts of the cœlom, and are often found, just like other connective substance in the Arthropoda, between the separate organs embedded in the cœlom.

Sometimes all of these cells remain indifferent, and form, by uniting with one another, cords or networks. As a rule, however, they are differentiated. Drops of fat are formed in them, which either fill up the cells in a regular manner, or run together into larger drops; in consequence of this these cells are comprised under the name of fat-bodies. This fat is sometimes variously coloured (yellow or red). Cells of this kind, containing fat-drops, have been observed in the Crustacea, and especially in the Entomostraca, where they are sometimes very large in comparison with the size of the animal's body, in which they are distributed in a constant and regular manner. This justifies us in supposing that they have some hydrostatic significance.

Deposits of this kind are most largely developed in the Insecta, where the fat-body, especially in the larval stages, consists of large cells, connected to one another by processes, and filling up a large part of the colom. We must not suppose, however, that these cells contain nothing but fat. This tissue is that in which the material, which is partly used up in the pupa stage, is deposited; in consequence of this not much of it is found in the adult Insect. The cells vary greatly in their mode of connection. This may be close, so that the fat-body forms lamellæ, or connected lobes, which are connected with branches of the tracheal system; or the cells are loosely connected together. In the most extreme case the cells may lie separately in the cœlom, where they must not be confounded with the much smaller and more indifferent blood-cells.

The cells of the fat-body in the Tracheata also serve for the deposition of excretory matters, which are known to be urates. These form crystalline concretions, or larger spheres of the form of small granules, which call to mind the renal concretions of the Mollusca. Among the Arachnida they are found in the Mites; they are found in the Myriapoda (Julus, Polydesmus, Glomeris), and are very common in the Insecta. They do not seem to be altogether absent from the Crustacea, for similar concretions have been observed in Asellus. 
The luminous organs of the Lampyridæ are special modifications of the fat-body. They are formed of plates of cells, to which a large number of tracheal and nerve branches are sent. On their inner side they are covered by other cells, which are not luminous, but which are loaded with a large number of urinary concretions. The superficial position of the luminous plates shows that they belong to the epidermal layer (hypoderm).

The regularity of the cœlom, along the long axis of the body, is modified by the muscular system. Where this is greatly developed (as in the cephalothorax of the Crustacea and Arachnida, and in the thoracic metameres of the Insecta) only a small space is left for the cœlom. The processes, too, of the chitinous skeleton are the cause of variations in it; chiefly by forming smaller cavities, as they especially do in the Crustacea. In the Insecta a subneural cavity is formed by muscles, which in some of them are inserted into the chain of ventral ganglia. In others similar muscles run horizontally from one side to the other of the abdomen, and so likewise mark off a portion of the colom.

\section{Vascular System.}

$\S 216$.

I'his system of organs, which in the Vermes had become highly developed, seems to be less so in many of the Arthropoda, chiefly because the cœlom generally forms a portion of the vascular system. There is, therefore, no difference between the blood and a perienteric fluid.

As a rule, a dorsally-placed vascular trunk is alone developed to any great extent; this functions as a heart, and seems to be homologous with the dorsal vascular trunk of the Vermes, parts of which may also function as hearts. But it seems to differ by not being connected with the enteron. The blood is either driven to the anterior, or to both ends of the body, by the cardiac tube. This dorsal cardiac tube of the Arthropoda is not, however, provided with afferent vessels, and the blood which passes into it does so by narrow venous ostia; so that, although a peripheral system of vessels may in some divisions be formed from continuations or ramifications of the arterial vessels, or by the differentiation of vascular canals from portions of the cœlom, yet close to the heart a sinus is formed from a portion of this latter. This "pericardial sinus" appears, therefore, to be a portion of the colom; and the slight development of the vessels which obtains in many Arthropoda is not to be regarded as a degeneration from a more perfect stage, but as 'a low stage, which is correlated with a less amount of development. It is still an open question whether, and how, this simpler form of the vascular system is connected with the arrangements which are found in Vermes. 
The localisation of the respiratory function leads to complication of the vessels : even where the vessels are not provided with separate walls the blood always flows in a definite and constant direction.

The blood-fluid of the Arthropoda is, as a rule, colourless; in a few Insects only it has a greenish, or red colour, owing to the coloration of the plasma. The formed constituents of the blood are indifferent colourless cells, very variable in form and size. They are wanting in many (lower Crustacea). The blood-cells of Insects are often distinguished by the large number of fine fat-molecules in them; but these must not be confounded with the cells of the fatty bodies, which are often also free.

\section{$\S 217$.}

The simplest form of a circulatory apparatus in the Brauchiata is that of a short tubular heart (cf. Fig. 136, c, of Daphnia), which is placed above the enteric canal in the anterior part of the body, takes in the blood by two lateral orifices, and drives it by a short anterior vessel to the cephalic organs, and especially to the cerebral ganglia. 'The blood is distributed in regular currents throughout the body, and, passing by the parts which serve chiefly as respiratory organs, again reaches the heart, which it enters by the slit-like orifices in it. This form of circulatory organ characterises the Copepoda and Cladocera; but it is also seen in the larval stages of the higher orders, and even in the developmental stages of the Decapoda, where it is but slightly modified. The simple forms cannot therefore be regarded as being degenerated from more complicated forms. The circulation is purely lacunar, and there are no vessels of any kind except those added on to an anterior artery, which is seldom much branched. In many Copepoda (Corycæidæ), and in the Cirripedia, there are no circulatory organs.

'The heart of the Phyllopoda is more developed. It has the form of a longer tube, made up by several such simple hearts as those of the Daphnida, and possessing a number of venous ostia (as many as 20 pairs in Artemia). The cardiac tube, that is, is divided into scparate chambers, which do not, however, exactly correspond to the metameres, of which there are more than one to each chamber. This segmentation seems, therefore, to be an independent one, and is, perhaps, to be regarded as a later arrangement. An arterial trunk is given off from the most anterior end only. This passes the blood on to the lacunar tract of the colom.

The heart of the Arthrostraca extends longitudinally along a large portion of the body in the Amphipoda and Isopoda; in the former it is placed in the metameres behind the head, and in the latter some way farther back. There may be an anterior vessel only, or it may give off a posterior one also. 'The former only is branched, and the branches are confined to the cephalic region. 'The number' of the ostia varies greatly in the Amphipoda (Phronima has 3 , Caprella 5 , Gammarus 7 pairs). 
The larvæ of the Thoracostraca have a simple cardiac tube with two lateral ostia only, which so far resembles the arrangement described above. A more complicated form is gradually derived from this, which is developed along two lines. One of these is seen in the Stomapoda, where the heart is elongated, and an anterior and a posterior arterial trunk alone given off, although the number of venous ostia is increased. As the anterior artery alone is branched, and the posterior has a widely-open mouth, the arrangement which obtains in the Arthrostraca is again repeated in these forms. Later on, not only do the anterior and posterior arteries form a larger number of branches, but a larger number of lateral arterial trunks are given off from the heart itself.

The second type is seen in the Schizopoda and Decapodia. Although the heart is provided with several pair of ostia it is more concentrated in form, and the lumen can no longer be seen to be divided into successive chambers. The primitive segmentation has given way to a more compact arrangement. This character is seen even in the arrangement of its several clefts, for they do not now follow by regular pairs, but are grouped in a different manner. The heart of the larvæ, however, arises as a thin-walled tube with only cne pair of clefts, and is continued forwards and backwards into a simple vascular trunk. The anterior one divides into thrce branches, which arise directly from the heart when the trunk shortens; the posterior trunk remains single. The heart is elongated for a time only, or passes at once into a more compact form. In both the Schizopoda and Decapoda it is placed in the posterior portion of the cephalothorax.

New portions are formed in the arterial system, while the whole venous portion is represented by lacunæ. The vascular system of the Schizopoda (Mysis) remains at this stage; the Decapoda pass through the different Schizopod-stages during their development. In the mature forms of the Macrourous Decapoda we find the muscular cardiac tube (Fig. 145, c) surrounded by a well-developed pericardial sinus ( $p c)$, from which the blood passes into the heart by three pairs of symmetrically-arranged clefts. Three anterior arterial trunks, and one posterior trunk arise from the heart. The anterior median one (av) runs to the cerebrum and eyes (o), without branching much; the two lateral trunks $(a a)$ give off branches to the generative organs, liver, and antennæ. The arterial trunk from the hinder end of the heart divides into two branches, which lie one over the other, and which may even arise separately from the heart. The dorsal one (ap), which is bifurcated in the Brachyura, supplies the muscles of the back and tail. The ventral branch $(a)$ takes a downward course at once, and divides into an anterior and a posterior trunk, both of which give off most of their branches to the appendages. There may be two smaller trunks in addition to the posterior median one. The highly-developed capillary system gradually passes into afferent canals (veins), which are at first collected into several trunks on the ventral side, and are then united $(v)$ into a 
large ventral sinus, placed at the base of the gills (in the so-called sternal canal). Each gill $\left(b r^{\circ}\right)$ receives a vessel from this sinus

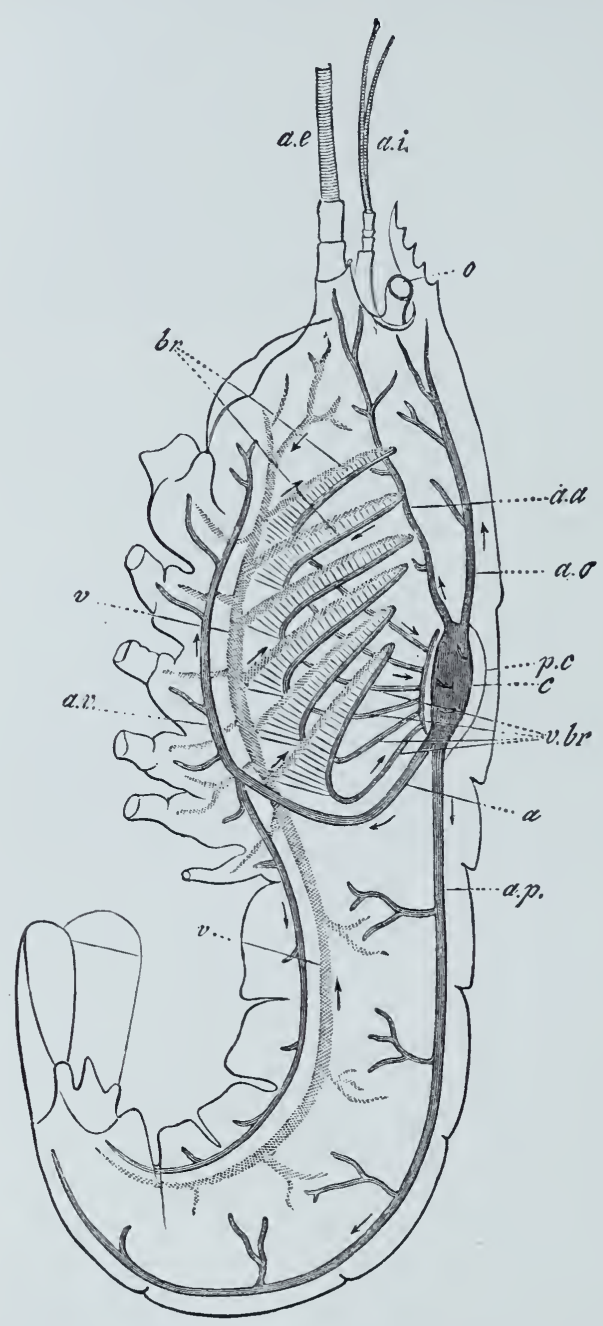

Fig. 145.' Diagram of the circulatory system of a Lobster. o Eye. ae Lateral antennæ. ai Mcsial antennæ. $b r$ Branchiæ. $c$ Heart. pc Pericardium. ao Median anterior aorta. a Hepatic artery. ap Posterior artery of the body. a Trunk of the ventral artery. ar Anterior ventral artery. $v$ Ventral renous sinus. $v$ br Branchial reins. The arrows indicate the direction of the current of blood.

blood-vessels are more perfectly developed, while when the respirntory organs are distributed over the whole body the arteries are less well developed. The Protracheata appear to resemble them in this.

(branchial artery). From the gills the blood passes into the branchial veins $(v b r)$, six or seven of which arise on either side of the pericardial sinus, into which they often open by funnelshaped mouths.

The valves of the venous ostia are to be regarded as special differentiations of the heart, which aid in dividing it into separate chambers when it is elongated.

The circulatory system of the Poecilopoda combines several of these different stages; their elongated heart lies in a pericardial sinus, from which it receives blood by seven pairs of ostia; it does not ouly give off arterial trunks anteriorly and posteriorly, but from the sides also, as do the Stomapoda.

\section{$\S 218$.}

The circulatory organs of the Tracheata very much resemble the elongated multi - camerate hearts found in some Crustacea; and the differences are due rather to the degree to which the vascular system which passes from the heart is developed. This again is affected by its relations to the respiratory organs, for when they are limited to a small space the 
So far as is yet known the circulatory system of Peripatus is represented by a "dorsal vessel," just as in the Insecta, so that here we find the simplest characters as compared with the other Tracheata. In the middle line of the ventral surface of the dorsal vessel there is a row of clefts, and it appears to agree with that of the Myriapoda in extending along the whole body, while in the Insecta it is limited to the abdomen. In Insecta it is attached to the wall of the body, and sometimes even to the tracheæ (in the larvæ of the Muscidæ) by the "alæ cordis" (Fig. 146, $\mathrm{m}$ ). In the larvæ it is divided into separate chambers, which are often not very distinct on the outside, and which have, owing partly to the arrangement of these muscles, and partly to the position of the cleft-like venous ostia, a metameric signification. The variation in the number of these chambers is not very great; in most there are eight of them; there are very seldom more, more frequently less than this. But these numbers still require a much more exact examination. The blood which is taken into the cardiac tube by the ostia is driven forwards by the systole of the chambers, and so passes from chamber to chamber, and from the most anterior of these into the aorta, where the pouch-like folds of the edges of the ostia function as valves, and prevent it from returning to the heart.

The aorta (Fig. 146, a) is a direct continuation of the heart, which, as compared with the Myriapoda, has disappeared from the thoracic metameres. It runs straight forwards to the cerebrum, but its more intimate relations after this are not exactly known. It is uncertain whether the branching of its anterior end, which is seen in some Insects, is a general phænomenon. In any case the blood very soon passes through

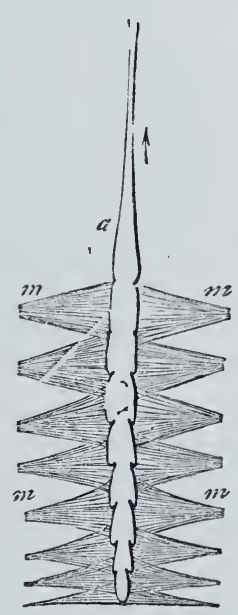

Fig. 146. Heart of Melolontha. a Artery arising from the most anterior cham. ber. $m$ Ala cordis (after Burmeister). a lacunar passage between the separate organs into regular currents; this may be easily observed in transparent insect larvæ; it is again collected into the venous ostia, which lie near the entrance into the heart. The separate passages in this tract are sometimes so sharply marked off, that in the appendages, for instance, vascular spaces appear to be formed.

As the alæ cordis do not end directly on the wall of the heart but in special cells on it, and at the same time unite to form a network surrounding the heart, a cavity resembling a pericardial sinus is formed below them.

\section{$\S 219$.}

The regular extension of the heart of the Myriapoda through out the whole length of the body, and the considerable increase in the number of its chambers, shows that there is in them a closer 
connection between the external segmentation of the body, and its internal organisation. Herein we may recognise a lower stage. The chambers (Fig. 147, $K$ ) are again separated from one another by

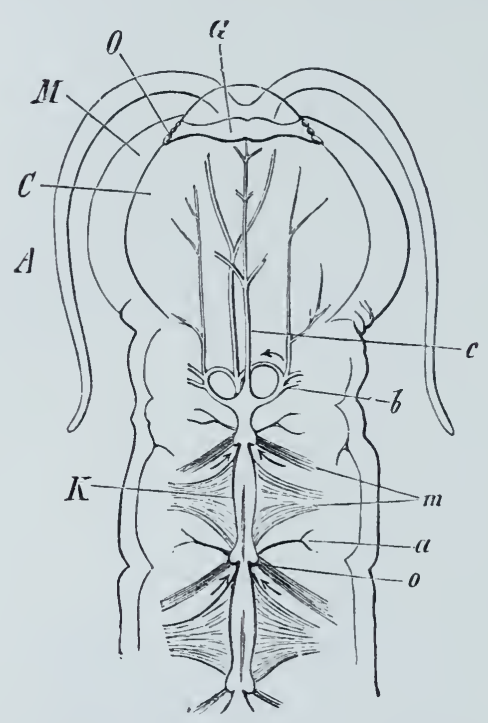

Fig. 117. Head and two segments of the body of Scolopendra, with the most anterior portion of the blood. vascular system. $C$ Head. G Supraosophageal ganglion (Cerebrum). $O$ Eyes. $I I$ Mandibles. A Antennæ. $K$ Chambers of the Heart. $m$ Ala cordis. o Venous ostia. a Lateral arteries. $b$ Arterial arches. $c$ Cephalic artery (after Newport). valves, placed at each venous ostium $(o)$, and are attached by large alæ cordis $(m)$. Paired arterial trunks, which are especially well developed in the Scolopendridæ, are sent off from each chamber to its proper metameres. As compared with the Insecta, a higher degree of development is implied by this arrangement. These arteries arise at about the level of the venous ostia. In the Julidæ they are double, for each chamber is composed of two, which were primitively separate. Three trunks are given off from the most anterior chamber; the median one (c) supplies the cephalic segments, and the two lateral ones $(b)$ surround the œsophagus. Where they unite, a larger trunk is formed, which lies on the ventral nerve-chord; just as in the Scorpionea, this runs as far as the last ganglion of the ventral chain, and gives off numerous branches.

\section{$\$ 220$.}

We find that the Scorpionea among the Arachnida are provided with the most complicated circulatory apparatus. The heart, surrounded by a pericardial sinus, is considerably elongated in correlation with the form of their body; it is divided into 8 chambers, which are held fast by lateral muscles (ala cordis). A pair of dorsal clefts (renous ostia) leads into each chamber; these clefts may be closed by valves which project into the interior. Arterial vessels are given off from the anterior as well as from the posterior end of the heart, of which they are direct prolongations; the anterior one, the aorta, enters the cephalothorax, while the hinder one runs to the tail. In addition to these a number of lateral arteries are given off close to the venous ostia, and are distributed to the neighbouring organs. Two of the numerous branches given off by the aorta form a vascular ring around the osophagus, whence an artery runs back (arteria supraspinalis) on, and as far as the end of, the ventral nerve-chord; this artery gives off a large number of branches. The venous blood is collected into a receptacle which lies directly on the ventral surface, just as in the higher Crustacea; from this it is carried to the 
respiratory organs. Before the blood from them gets to the heart, it passes into the pericardial sinus.

In the other Arachnida the many-chambered cardiac tube is reduced, and resembles in character that of the Insecta. It always lies in the abdomen; in the Aranca and Opilionida it is provided with three pairs of ostia, by which it is divided into chambers. From the most anterior of these an artery is continued into the cephalothorax : in Lycosa this is divided into two trunks (Fig. 148), each of which gives off branches for the eyes and appendages. The hindermost chamber
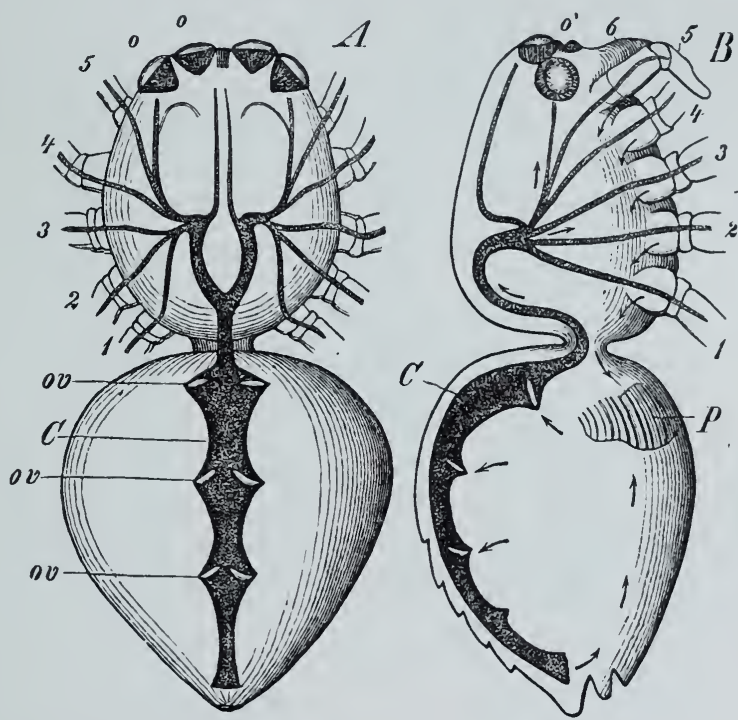

Fig. 148. Circulatory organs of Lycosa. A Seen from above; $B$ From the side. $o$ Eyes. 1,2,3,4,5,6 Appendages. $P$ Lungs. $C$ Heart. ov Venous ostia of the heart. The arrows indicate the direction of the blood (after Claparède). opens at the end

of the abdomen, and the blood-current from it corresponds to that which is distributed by the caudal artery in the Scorpionea. Since there is no pericardial sinus the blood passes to the respiratory organs, and from them to the heart, by lacunar passages only.

Among the Pycnogonida this apparatus is limited to a threechambered heart, into which two pairs of ostia open; in the Acarina no heart at all seems to be developed.

\section{Excretory Organs.}

\section{$\S 221$.}

The apparatus, which in the Vermes is formed of looped canals, is found in a modified form in the Crustacea. One of the organs, which represent it, consists of a coiled tube placed below the integument of the head, and opening at the base of the second (outer) pair of antennæ. In the Entomostraca, this organ is confined to the larva, in which it has been made out in most divisions. It may perhaps be retained in the Cirripedia as the so-called "cement glands," which lie in the stalk of the Lepadida, and open at its 
lower end; and which, in the Balanidæ, are converted into a special complex of glands. This organ persists in the Thoracostraca, and is known as the "green gland" in the Crayfish.

A second organ of this kind is also found in the Entomostraca, but is absent in the higher Crustacea. It lies in the mantle-like fold of the integument, where it forms a transparent looped canal, which opens below the mantle (cf. Fig. 136, g). Owing to its position below the shell it is known as the shell-gland. Its inner end is blind.

There are, therefore, two kinds of excretory glandular organs in the Crustacea, but it is doubtful whether they are homodynamous. The second organ may be homologous with the loop-like excretory organ of the Vermes, and be therefore derived from a common stemform, while it has lost its metameric signification.

These organs-the functional relations of which cannot as yet be definitely adjudged, and of which the green gland alone is distinctly similar to a renal excretory organ-are not found in the Tracheata. In them the function of excretion is performed by organs, which have been described anatomically among the appendages of the hind-gut ( $\$ 214)$, under the name of urinary canals, or Malpighian vessels.

\section{Tracheæ.}

$\S 222$.

The colom of the Protracheata and Tracheata is traversed by an aërating system of tubes, which, so far as is yet known, is derived from tegumentary organs. The characters of these organs, in Peripatus, is of the greatest significance as to this point; irregularly distributed tufts of fine tubes, filled with air, are distributed on the inner surface of the body-wall, and also on the oviducts and on the fore- and hind-gut.

The arrangements in the Tracheata are different from this, for, in them, the tracheæ are regularly arranged and symmetrically distributed. They consist of an outer layer of connective tissue (Fig. 149, a), the interior of which is covered by a chitinous layer continuous with the external integument. Its elasticity is almost altogether due to its chitinous layer, and when the trachea is more elastic it is because this layer is thicker; in this case it has the form of a spiral filament, projecting into the lumen of the trachea. At certain points the tracher form saccular enlargements, and at them this spiral arrangement of the thickenings disappears; that is to say, it is only deposited at certain disconnected parts. This chitinous layer and its spiral ridges do not form a specific arrangement, for the ducts of many glands have a very similar structure in the Tracheata.

The external orifices (stigmata) of the tracher are regularly arranged in pairs on either side of the body; there is not always the 
same number of them, and in some cases they are found on every segment of the body. Each stigma is an ellipse-shaped cleft, surrounded by a circular thickening of the external chitinous skeleton, which can be opened or closed by valves. Special muscles close these clefts. Each trunk from the stigma is lost, sooner or later, in a tuft of smaller branches, from which finer branches arise, which surround the organs. The way in which these branch, as well as the length and strength of the branches, varies greatly. Separate tracheal trunks may unite with one another, and form a system of tubes which passes longitudinally along, or transversely across the body, and from which finer ramifications are given off.

Owing to the distribution of these tracheæ throughout the body there is a great difference between the respiratory characters of the Tracheata and of the Branchiata. The medium which is to be respired is distributed through the whole organism; it is not only the bloodfluid which everywhere bathes the

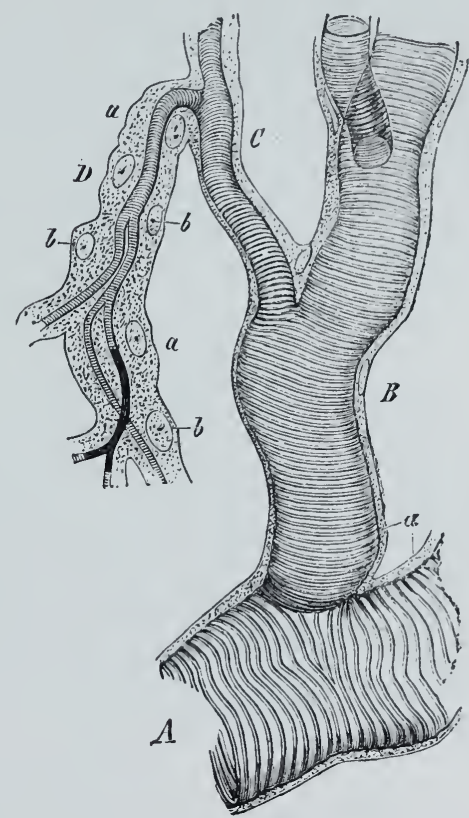

Fig. 149. Piece of a trachea of a Caterpillar, with its branches, $B C D$. $a$ Epithelial-like cellular layer. $b$ Nuclei. tracher that can exchange its gases, but in the tissues themselves respiration can be effected, for the tracheæ are distributed to them, and, indeed, may even come into relation with their form-elements (cf. Fig. 144, tr). But this does not apply to all cases, for, when the tracheæ are reduced, the respiratory regions are diminished in number and extent; in this way the diffuse respiration is localised. In these cases, as when there are branchiæ, the blood has to seek out the respiratory organs. In this way the characters of the tracher influence the circulation. In addition to their respiratory function, the system of tubes filled with air serves to diminish the specific gravity of the body, and is just as important in this relation to Insects during their aquatic stages, as to those Insects which rejoice in wings, and which are able by special arrangements to increase or diminish the amount of air in their tracheal system.

\section{$\S 223$.}

The arrangement of the tracheal system varies considerably, but all its forms may be derived from that simpler one mentioned above, in which there is a pair of tufted branched trachere in each metamere. 
These organs appear to be distributed in a metameric fashion even in the cephalic segments, for the rudiments of tracheæ formed by the ectoderm have been observed in many Insects in these metameres during their development. None of these rudiments are retained in any living 'Tracheate, owing to the development of the head. In the succeeding metameres, also, the number of trachex may be diminished, for in some cases at any rate the tracheal trunks have been observed to atrophy.

In the Myriapoda the trachea are ordinarily similar in character throughout the whole body, however different they may be in the various orders. The stigmata, which are either placed on the ventral surface, or more to the sides, and in some indeed on the dorsal surface (Scutigera), lead into tracheal trunks, which are distributed in correspondence with the number of metameres. They are most simply arranged in Julus. A tuft of trachere passes from each stigma to the viscera, without branching at all. In Glomeris, however, the tracheæ do branch; and in the Chilopoda they form longitudinal and transverse anastomoses, so that they get to be arranged in very much the same way as in many Insects.

Among the Insecta some Aptera appear to have lost their trachea. They are almost altogether wanting in the Collembola, two prothoracic tracheæ only having been observed in Smynthurus. Among the Thysanura, Campodea has as many as three pairs (Fig. 150),

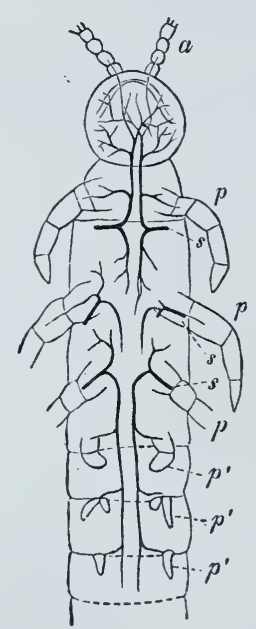

Fig. 150. Anterior half of Campodea fragilis. $s$ Stig. mata (after Palmén). belonging to the mesothoracic, metathoracic, and first abdominal segment respectively. The absence of anastomoses shows that they are of a lower grade of development than that found permanently in other insects. There are generally 10 pairs of stigmata. This is the highest number known in the imago stage of the Pterygota, but in some larvæ there are 11 stigmata, the first abdominal metamere being sometimes provided with a stigma, although as a rule it is altogether absent. There are never any stigmata in the last two metameres. The number of these stigmata, and of the tracheal trunks which arise from them, is not, however, always complete. The number varies greatly, pairs of stigmata undergoing degeneration at one point or another, so that only 2 or 3 of them remain open. In the imago they generally lie in the softer membrane, which connects the segments of the body, and they are sometimes placed so much on the dorsal surface of the abdomen that they are covered by the wings (Coleoptera). The number and arrangement of the trachere in the imago stage is not the same as in the pupæ or larvæ. The differences in the external conditions which obtain in the two stages define the arrangements of this system of respiratory tubes. The development of transverse and longitudinal 
anastomoses provides for the equal distribution of the respiratory medium. When the number of stigmata is reduced the longitudinal trunks become of great physiological importance, for they give off tracheal branches to those portions of the body in which there are no stigmata. The above-mentioned tracheal vesicles may be developed on the principal trunks, as well as on their branches and twigs; their development is correlated with the development of the power of flight. A very large number of them may be found in the Coleoptera (Lamellicornes); in the Lepidoptera, Hymenoptera, and Diptera they are not so numerous, but are larger; in the Diptera they are sometimes represented by a large pair of vesicles, which almost fills up the abdomen.

As the tracheal system is developed in correlation with aërial respiration, and therefore with a non-aquatic habitat, the modifications which are caused by the aquatic habitat of the larval or adult stages of many Insects, must be regarded as secondary arrangements. They are adapted to their altered mode of life. Thus in the larvæ of many Diptera there is but a single pair of stigmata, which are placed in the hinder end of the body (Corethra). A still further adaptation in the aquatic Hemiptera (Nepa, Ranatra) is the respiratory tube, which projects from the abdomen.

\section{$\S 224$.}

When Insects are most completely adapted to an aquatic habitat, all the stigmata, and the tracheal trunks from them, are atrophied. This leads to the formation of the closed tracheal system, which distinguishes the larvæ of the Pseudoneuroptera. The longitudinal trunks, which are also present in the open tracheal system, form the chief part of the apparatus. They give off branches to the viscera (enteron), as well as to the wall of the body. In both parts they give rise to the development of organs, in which the exchange of gases is effected. The relations between this closed tracheal system and the open one are explained by the presence of chords, which connect the longitudinal trunks with the body-wall, and are inserted at the very points where stigmata are found later on. The chords therefore appear to be obliterated tracheal trunks. And this view is confirmed by the fact that when the larvæ undergoes ecdysis, the intima of a portion of the tracheal system is also cast off by means of some of these chords and is found in the exuviæ (Ephemerida, Perlida). Part of these chords are cast off again in the last moult, and form an open tracheal system by giving rise to a stigma at their point of junction with the skin.

The tracheæ, which branch in the integument, allow of a dermal respiration during the closed condition of the apparatus (many Perlida). This determines the development of superficial growths, which lead to the formations of processes, in which a large number of tracher ramify (Tracheal gills, cf. $\$ 190$ ). These organs 
form tufts, or lamellæ, which beset the abdomen in the Ephemerida and Perlida (Fig. 151, A), or even form a tuft on the thorax (Perlida). Vague dermal respiration is here localised in definite organs. There is a larger surface in the tufted form, but this is compensated for, in the lamellar form, by the movements of the lamellæ, and the consequent

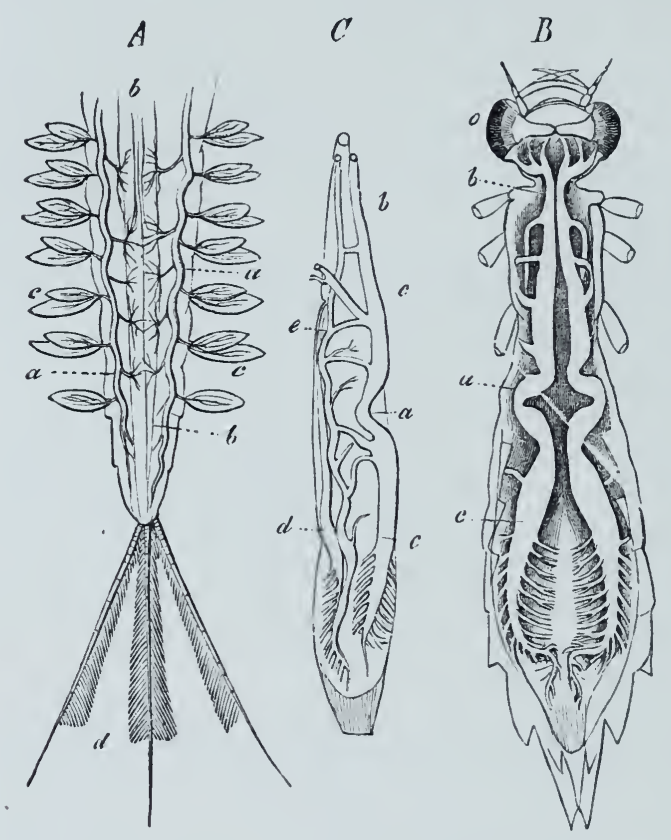

Fig. 151. A Hinder portion of the body of the larva of Ephemera vulgata. a Longitudinal tracheal trunks. $b$ Enteric canal. $c$ Tracheal gills. $d$ Feathered appendage to the tail. B Larva of Asschna grandis. $a$ Superior longitudinal tracheal trunks. $b$ Their anterior end. $c$ Posterior portion, branching on the hind-gut. o Eyes. The middle figure represents the enteric canal of the same larra, seen from the side. $d$ Inferior lateral tracheal trunk. $e$ Communication with the upper trunk. $a b c$ as in $B$ (after Suckow). increase of rapidity in exchanging the water. When tracheal branches are developed in the hind gut, this region becomes respiratory in function. Respiration seems to be partly effected in the same region in the larve of the Ephemerida and Perlida, although there are no special organs developed; for they have been observed to take water into the hind-gut. This function is more evident when the surface is increased in extent, as it is in the larvæ of the Libellulidæ, by the development of a large number of lamella, arranged in longitudinal rows. Two longitudinal trunks (Fig. 151, $B$ a) give off branches at their hinder end $(c)$ to the hind-gut, and form a close plexus of tracheæ in its lamellæ. These internal tracheal gills are constantly bathed with water, owing to the movements of a valvular arrangement at the anus. In these forms therefore the hind-gut functions as a respiratory organ, just as it does in many other divisions.

Palmen, J. A., Zur Morphologie des Tracheensystems. Helsingfors, 1877.

\section{$\S 225$.}

Among the Arachnida the tracheal system of the Galeodea most nearly resembles that of the Insecta, the separate tracher being 
united by lateral longitudinal trunks. As however they have only three pairs of stigmata they indicate their affinity to the other divisions of the Arachnida. A remarkable peculiarity is found in their tracheal system; a tracheal trunk arises from a stigma and speedily breaks up into a large number of short lamella-like flattened branches, which lie on one another like the leaves of a book; owing to this arrangement the whole organ is confined to a small space. These leaf-like tracher are known as "Lungs." Four pairs of them open on to the ventral surface of the abdomen in the Scorpionea. There are two pairs in the Phrynida and Mygalida. In the rest of the Aranea one pair only is developed, the stigmata of which lie in the anterior portion, and on the ventral face of, the abdomen. In some Aranea a second pair of stigmata, placed just behind the first, lead into tracher which end by two chief tubes, at the end of which are extremely fine tubules (Argyroneta, Dysdera, Segestria). In others this pair of stigmata is fused, and lies in front of the spinningwarts. Four tubes generally pass from the stigmatic cavity; these are either branched (Thomisus), or end simply (Tegeneria, Clubiona, Lycosa, Epeira). In the absence of branches and of anastomoses, the leaf-like tracheæ resemble the lowest stage of the tracher, and represent a special development of them.

The Opilionida, the tracheæ of which are distinguished by the large number of their branches, have only one pair of stigmata. The number is also reduced in the Acarina, in many of which (e.g. Sarcoptes) there is no tracheal system at all, as is the case also in the Linguatulida and Pycnogonida.

\section{Generative Organs.}

\section{$\S 226$.}

Reproduction in the Arthropoda is effected solely by the generative system; all the modes of reproduction in them, which are called asexual (phænomena of Parthenogenesis, and of alternation of generation), are due to sexual differentiation.

The disposition of the generative organs in different individuals, which obtains in some divisions only of the Vermes, is the rule in the Arthropoda; in a few only is the hermaphrodite structure retained. In many, sexual differentiation extends even to the outer parts of the body, to its size and form.

The germ-glands are always distinct organs, which are never distributed in a metameric manner; they are either single, or there is but one pair of them. We cannot yet say whether this is due to the generative system having been transmitted from animals in which it was single.

In the general arrangement of the organs there are many constant and very remarkable points. The typical form of the apparatus is 
evidently a compact germ-gland (Fig. 152, a), from which an efferent duct $(b)$ passes off on each side. We find this arrangement

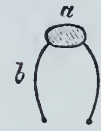

A

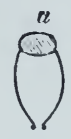

73

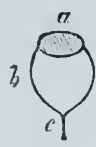

$C$

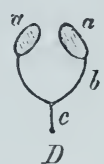

$D$

Fig. 152. Diagrams of the characters of the generative system in the Arthropoda. a Germ-glands. $b c$ Efferent duct. The approximation of the orifices leads to the formation of a common orifice, and this to the formation of an unpaired portion of the duct (c). In many Arachnida this azygos tract is connected with a circular part, more or less of which is formed by the germ-gland $(C)$. The ring is then formed by an inherited (primary) stage-the single germ-gland-and by an acquired (secondary) stage-the fused part of the efferent ducts. While the generative glands of the Crustacea form the lowest grade of this series, the Insecta appear to form the highest, for, owing to the bilateral separation of the germ-gland, and the terminal fusion of the efferent ducts, and the formation of a common unpaired portion, they are the farthest removed from the lowest stage $(D)$. The germ-glands, as well as the efferent dincts, may undergo various differentiations: especially the ducts, of which sometimes the paired and sometimes the unpaired portion is affected. Except in the fixed Cirripedia, impregnation is effected by copulation. In correspondence with this there is a space formed near to, or at some distance from, the terminal portion of the female efferent duct, from a diverticulum of a division of it; this (receptaculum seminis) serves to take up the sperm, and may be converted into appendices of a more independent character. Lastly, there is a bursa copulatrix for the reception of the penis.

The organs which serve to protect the eggs after they have passed out of the body are of many kinds. Some of the appendages, in the Crustacea especially, are often metamorphosed in this direction. Even whole regions of the body may be converted into marsupial pouches. Much of the difference between the male and female individuals is due to these relations to the care of the young. Finally, the number of eggs produced is an important element in modifying all parts of the female apparatus; for not only the enlargement of the efferent spaces, but the various changes of all the accessory organs, and, again, the increased size of the female are all due to the production of a large number of eggs.

The organs necessary for copulation, as well as the differentiations which affect the efferent ducts, lead to complications of the male apparatus. When the protractile end of the efferent ducts does not serve in copulation, there are special copulatory organs, which are 
partly formed from the appendages (Crustacea), or by them and whole metameres (Insecta). The appendages have further many other relations to the generative apparatus, for they serve as organs for seizing and holding the female, and are modified accordingly. The generative system is here seen to be so correlated with other parts, as to be of great importance in determining the form of the whole organism.

\section{$\$ 227$.}

Among the Crustacea we meet with hermaphroditism in some of the Cirripedia. The testes and ovaries are greatly ramified tubes, which can only be distinguished externally by their position in the body. In the Lepadidæ the ovaries are placed in the stalk formed by a diverticulum of the mantle; they give off an oviduct to the mantle-cavity on either side. In the Balanidxe they are embedded in the mantle. In both families the male reproductive glands are disposed around the intestinal tract, and unite at each side into a vas deferens, which rums alongside the hind-gut, and opens with its fellow of the other side at the end of the postabdomen.

In the other, or diocious Crustacea, the organs of bot? sexes are arranged in very much the same manner. There are two different forms of the generative apparatus according as the germ-gland is paired

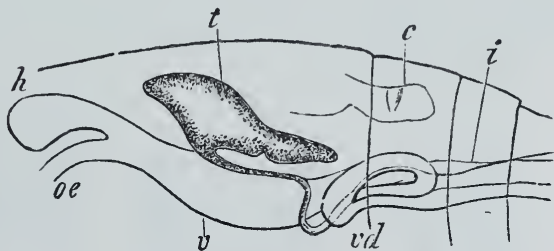

Fig. 153. Gut and male generative appa. ratus of Pleuroma. Side view. oe Foregut. $v$ Mid-gut. $h$ Unpaired cæcal-sac. $i$ Hind-gut. $c$ Heart. $t$ Testis. vd Coiled vas deferens (after Claus). or unpaired. But these are connected with one another by forms in which the two germ-glands are united into an organ which is externally single.

We meet with unpaired germ-glands in the free-living Copepoda. The ovary or testis (Fig. 153, $t$ ) lies in the middle line above the mid-gut $(v)$. The ovary gives off an oviduct on either side, which either takes a simple course backwards, or forms in its terminal portion several coils, which function as a uterus (parasitic Copepoda); or it may be beset along its whole course by a large number of diverticuia (Fig. 154, B), which hold the eggs (Corycæidæ). The short terminal portion has either glandular walls, or is provided with a special cement gland. An enlargement of the terminal portion functions as a receptaculum seminis, and in many cases, as for example in the Siphonostoma, may form a special tract, providing a special orifice for the reception of the sperm. In many Siphonostoma the ovary is double; but the two ovaries are often placed close together. We find the same in the male Copepoda, when the free-living forms have a simple testis, and the Corycæidæ have one divided into two halves, which pass on either side into a special vas deferens. In many families the right seminal duct is 
atrophied. The many-coiled end of the duct serves as a seminal vesicle (Fig. 153, $v d$ ), in which the spermatophores are formed.

In the Branchiopoda the germ-glands are separate tubes which lie beside the enteric canal. In the Cladocera, where they are directly continuous with the scarcely separable efferent duct, they are simple; the duct of both male and female organs opens near the end of the body. The Phyllopoda resemble them in this. Sometimes the testes or ovaries occupy the hinder part only of the coelom, while the duct is given off from their anterior end (Artemia, Branchipus) and bends backwards, or they begin farther forwards, and give off the excretory duct at, or near, their posterior end (Holopedium). In the former an enlargement of the oviduct serves as the uterus, while a swelling on the seminal duct forms the seminal vesicle. This simpler form of the generative organs is modified in the Phyllopoda, owing to the enlargement of the germinal glands. The ovary of Limnadia is beset with short pouch-like diverticula, which form a lobate gland of a larger size in Apus, owing to the larger number of branches in it. This organ also serves as a uterus for the ripe eggs. The testis has the same morphological characters.
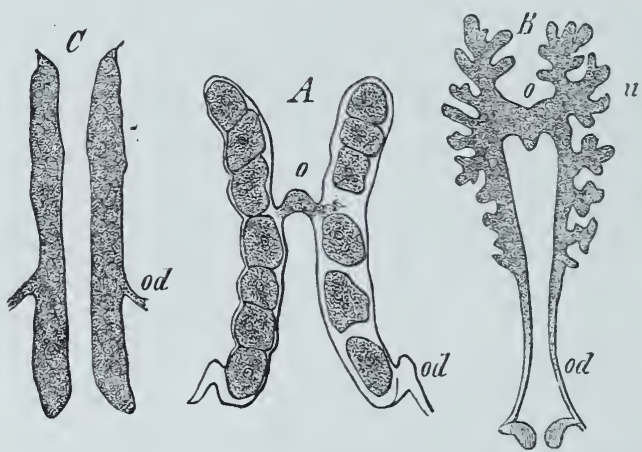

Fig. 154. Female generative organs in Crustacea. $A$ Of Iysis. $B$ Of Sapphirina. $C$ Of Oniscus. o Ovary. od Oviduct. $u$ Uterus.

Among the Arthrostraca the generative organs are generally double, each side provided with a separate orifice. In the Amphipoda the female organs consist of simple tubes which open as a rule at the base of the fifth thoracic segment. In the Isopoda (Fig. 154, C) the tube ends blindly in front and behind, and the efferent duct arises on the course of it. The ends of the tubes are to be regarded as true germ-glands, while the rest or largest portion resembles an oviduct or uterus. The male organs are similar, but in the Isopoda they are peculiar in character, sereral testicular tubes (Fig. 155, B) uniting into a special portion, from which a narrower and much-coiled excretory duct arises. This has either an orifice of its own, or is united with its fellow of the other side in front of the orifice.

\section{$\S 228$.}

Among the Thoracostraca the Schizopoda (Mysis) are provided with the simpler kind of generative organs. The female organs (Fig. 154, A) consist of an unpaired germ-gland (o), to the sides of which oviducts are attached, and these are widened ont anteriorly into 
a cæcal uterus which is continuous with the duct. At their hinder end they send off a short duct $(o d)$ to the generative pore. The organs of either side are united in the same way in the testis. This is formed of a double row of glandular follicles, which unite into a coiled canal, which forms the simple excretory duct and opens at the base of the last pair of feet.

The generative organs of the Decapoda resemble those of Mysis, by being similarly connected in the middle line; they appear to ke further developed by various differentiations. The female organs are formed by two long tubes which run forwards and backwards, and are united transversely with one another; these tubes function partly as germinal glands, but chiefly as oviduct and uterus. In the Crayfish the two anterior divisions have the form of short lobes, while the two hinder ones are fused into an unpaired piece. On each side a short duct passes to the genital pore, which in the Caridina has the same position as in the Schizopoda; in the Macrura it is placed on the basal joints of the third pair of feet, but in the Brachyura on

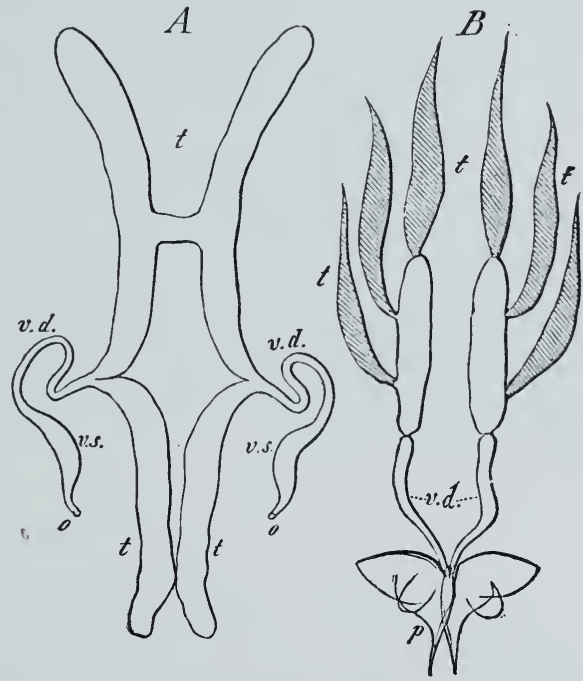

Fig. 155. Male generative organs. $A$ of Homarus. $B$ Of Oniscus. $t t$ Testes. vd Vas deferens. vs Seminal vesicle. o Its orifice. $p$ Copulatory organs. the segment of the body, which carries this pair. 'The Brachyura are also distinguished by a pouch-like enlargement of the oviduct (seminal pouch). In the male apparatus the testes are formed by two much-coiled tubes, which are transversely connected with one another in front, and which, like the female organs, lie for the most part in the cephalothorax; in Pagurus only are they placed in the abdomen. In the latter they give off two long and closely-coiled but gradually-widening ducts. Herein they agree with most of the Decapoda, but they are distinguished from them partly by the increased size of the lobes formed by the coils of the seminal canal, and partly also by the formation of the unpaired piece, which unites the glands of either side. The germinal glands are more completely united in Astacus. A vas deferens with long coils passes on each side to the outer generative orifice, which is placed, as a rule, on the basal joint of the last pair of feet; in the Brachyura, however, it is found at the end of a penis, which is formed by a metamorphosed appendage. The opening of the male apparatus only is then the same 
as in the Schizopoda, while the female orifice is placed farther forwards.

In the generative apparatus of the Stomapoda the testis commences as a fine unpaired tube in the middle line of the caudal fin; it is continued forwards into a paired tract, from which a muchcoiled vas deferens arises. Each of these passes to a penis, which springs from the coxa of the last thoracic foot. An unpaired gland, which begins in the cephalothorax, opens at the same point. The ovary passes backwards as an azygos, and afterwards as a paired, gland, as far as the cephalothorax. Each gives off an oviduct in the third thoracic segment, which opens at the base of a pouch placed in the middle line. The Decapod-type prevails in them, though modified in the female by the approximation of the orifices.

In the Pocilopoda we see a combination of the two forms represented in the Crustacea. One form is followed in the median connection of the organs of either side, and the other in the large number of germ-sacs formed by the fine terminal branches of the network, which makes up the generative organs. The wider tracts serve as efferent passages, which are considerably widened in the female so as to collect the eggs, and are continued into the efferent duct on each side.

\section{$\S 229$.}

A lower stage is seen in the female apparatus of the Protracheata. The ovary is a body divided into two halves by a septum, and sends off a paired oviduct; this passes forwards as a coiled tube, and then bends round to a widened portion, which functions as a uterus. These canals are continued backwards, and only unite to form a common short vagina near the generative pore.

In the male apparatus the testes are completely separated from one another; each of them is provided with a glandular appendage, and is continued into a long looped vas deferens. A common ductus ejaculatorius, which also opens at the hinder end of the body, is formed by the union of the two efferent ducts.

\section{$\$ 230$.}

The two kinds of generative glands in the Arachnida are, as a rule, unpaired; when paired they are connected transversely, and open either by one or two ducts anteriorly, and on the ventral surface. In addition to accessory glandular organs, or special enlargements of the excretory ducts serving for the storage and reception of the sperm-masses or ova, there are external organs which carry the sexual products outwards, and which are called penes or vaginæ according to the sex. The male organs repeat with slight variations the type of the female. The union of the genital glands of either side and the azygos portion of the apparatus which 
is formed in consequence of it, calls to mind the similar relations of parts in the Branchiata, and notably in the Pœcilopoda.

The ovaries in the Scorpionea are formed of three long tubes which bend towards, and pass into, one another at their hinder ends, while they are also connected with one another by four transverse anastomoses; in the walls of these tubes, which often form tubular. diverticula, the ova are formed. The segmented character of the organ is implied by the transverse connections, which form four wide meshes on either side, for these segments have exactly the same position as those of the abdomen. Spindle-shaped and widened oviducts are continued on from the two outer longitudinal tubes, which function as receptacula seminis for the sperm which they receive; they open at the base of the abdomen.

The testes also of the Scorpionea are a pair of winding canals, united by transverse commissures. Their double character is implied by the presence of two tubes on either side. The vas deferens of each testis opens to the exterior, after uniting with its fellow of the opposite side at just the same point as that occupied by the generative orifice of the female. In addition to the vas deferens there are accessory organs on either side, which as a rule have the form of two pairs of cæcal tubes, which vary in length and function partly as glands, and partly as seminal vesicles.

The separation of the germinal glands of either side is complete in the Galeodea and male Aranea. The ovaries are two tubes, on the outer surface of which the ova are developed; in the Spiders they are developed on stalked processes. In some (Segestria, Oletera) the ovaries are represented by a closed ring. A vaginal canal, which is sometimes widened out (Galeodes), is formed from the union of the two ovarian tubes, which serve to carry the ova outwards; this canal has one or two seminal vesicles at its termination. These are found also in the Aranea, where they often open independently in front of the orifice of the vagina. The male organs in the Galeodea may be derived from those of the Scorpionea, by supposing that the transverse anastomoses between the longitudinal trunks have disappeared. Finally, in the Aranea, these longitudinal tubes are reduced to two.

Bertkau, Ueber d. Generat.-Apparatus, Arazeiden. Arch. f. Nat. 1875.

\section{\& 231.}

In the Opilionida and Acarina the circular form of germinal gland is the dominant and general arrangement; it is derived from the transverse connection of the ovaries, which is seen in the Scorpionea. The unpaired stage of the germinal gland, which is to be regarded as the more primitive one, is implied by this arrangement. This circular form is most perfect in the Opilionida (Fig. $156, B$ o). Just as in the Aranea and Scorpionea the ova are formed in stalked diverticula on the surface of the ring, whence they pass 
into the cavity of the ovarian tubes, and from it to the efferent duct, which is provided with a considerable enlargement (u) (uterus). A narrow coiled continuation of this leads to the protractile ovipositor
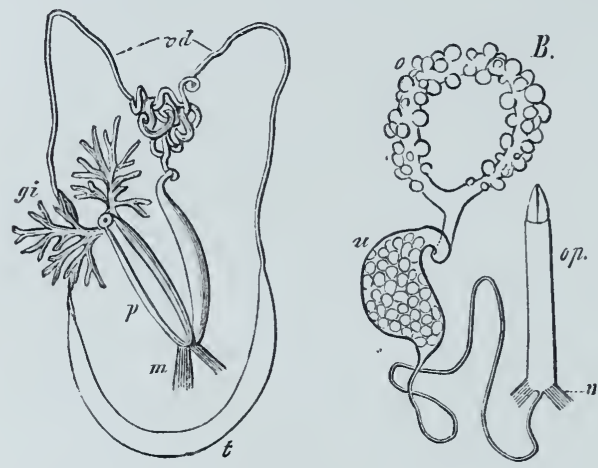

Fig. 156. Generative organs of Phalangium opilio. A Male organs. $t$ Testes. vd Vas deferens. $p$ Penis. $m$ Retractors of penis. gi Appended glands (after Krohn). B Female organs. o Orary. $u$ Uterus. op Ovipositor. $m$ Retractors of oripositor.

$(o p)$. In the male there is a circular canal, a portion only of which forms the testis, instead of the ovarian ring (Fig. 156, a $t$ ); the two ends of the testis pass into the efferent duct $(v d)$ which completes the ring. These unite into a closely-coiled portion, from which a widened canal or seminal vesicle arises - an organ similar to the ovipositor, and, like it, protractile; the penis is attached to it. Two large tufts of accessory glands (gi) are connected with the ends of the penis.

The circular form of germinal gland is still retained, in its completeness, in many of the Acarina. In the female apparatus the greater part of the ring is converted into the efferent organ, owing to the limitation of the ova-producing part to a small division of it. This is most marked in Pentastomum, where the ovary is attached to a circular canal. The ovary is here differentiated from the canal. The part of the ring, which forms the efferent ducts and passes into the single portion, is often widened out into a uterus; or the uterus is formed by the unpaired portion alone. This is the case in Pentastomum, the uterus of which forms a coiled canal of some length. The unpaired portion of the efferent duct is generally much shortened in the male, and the two parts of the rings connected with it are widened out into seminal vesicles. Appended glands are comnected with the unpaired portion in both sexes. The great differences in the distribution of the functions of parts of this canal lead to the separation of the ring into two genital tubes, the middle of the germproducing portion of the ring becoming sterile. The two halves of the ring are then distributed to the sides, although in some cases they are still connected by a canal, or by indifferent tissue; this gives rise to organs which are only united at their orifices, or along: an unpaired portion connected with them (Ixodes).

The hermaphrodite generative organs of the Tardigrada are altogether unlike these arrangements. They consist of an unpaired ovary and two testes which lie beside the enteron: their efferent ducts pass into a receptaculum seminis, and open, generally provided with special glands, into the cloaca. 
The arrangements in the Pycnogonida are just as peculiar ; their generative products are formed on the wall of the cœlom, and are passed out by special orifices (which are sometimes found on all, and sometimes on only one pair, of the feet). This character reminds us of the lower arrangements seen in the Annulata.

The conversion of appendages into copulatory organs, which obtains in the Crustacea, is seen in the Aranea only from among the Arachnida; in the males of this order the palpi are organs of a complicated structure, which convey the sperm to the female generative orifice.

\section{\&232.}

The generative organs of the Myriapoda are, in their form and arrangements, most similar to those of the Arachnida, and, as in these forms, they sometimes open far forwards on the body, namely, on the third segment of it. The generative organs of the Scolopendridæe are placed in the linder end of the body. In the females the generative glands are either simple externally and form an elongated tube, on the inner surface of which the ova form projections (Julidæ, Scolopendridæ, and Glomeridæ), or they are double (Craspedosoma), in which case they are united at their anterior ends, while the oviducts open separately. In the Scolopendridæe the simple ovarian tube is, as a rule, continued on by a simple oviduct; but the double character of these organs is implied in the development of ova on both sides of the ovarian tube.

The accessory organs are formed by two pairs of bodies, which sometimes open into the oviduct, but ordinarily directly into the genital orifice; they partly form cement glands, and

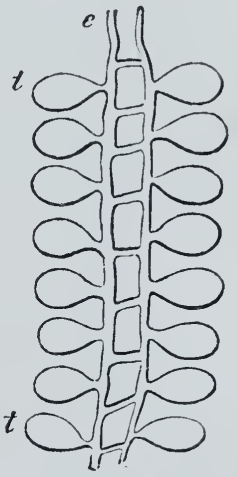

Fig. 157. Male generative organs of Julus. $t$ Testicular follicles. $e$ Efferent duct (after Stein). partly receptacula seminis.

The male organs also are often double in their efferent ducts and accessory parts only. However, many Glomeridæ and Julidæe are provided with a double testicular tube, which passes into a common vas deferens, and seems to be mnited into a single organ owing to the large number of its transverse connections (Fig. 157). When there is only one testicular tube, it is beset with separate follicles. The vas deferens is occasionally single (some Scolopendridæ); but as a rule it is divided into two branches, which either open on a short papilla (Julidæ, Glomeridæ), or are connected together and continued into a short penis placed at the hinder end of the body (Scolopendridæ). The last division of the efferent duct is provided with enlargements or diverticula, which serve to collect the sperm. Several pairs of glands are inserted into it just in front of its orifice. As to the general character of the generative organs, they are unmistakably approximated to the Crustacea by the possession 
of separate orifices, and resemble the Arachnida in forming an annular portion.

StelN, F., De Myriapodum part. geuital. Berol. $18 \pm 1$.

\section{$\$ 233$.}

Notwithstanding the great variations of more subordinate characters, the generative organs of the Insecta present on the whole a well-marked uniformity of structure. The organs and their accessory parts almost always lie in the abdomen, and generally open below, or in front of, the anus. The eighth abdominal segment generally seems to carry the genital orifice. In the Strepsiptera only is the female generative orifice placed some way forwards. The germinal glands are, as a rule, disposed in pairs, and retain this condition, although there are indications of the primitively single arrangement, or of a connection between the germ-glands of either side, as in the Arachnida and Myriapoda. Each germ-gland is composed of a varying number of equal parts, which are generally tubular in form, grouped into tufts, and united at an efferent duct. The ducts of the two germglands seldom have separate orifices. They are almost always united for a certain distance, and receive, before they unite, accessory organs, formed by the differentiation of a portion of their walls. In the females these organs appended to the ducts are sometimes pouch-like, or vesicular portions, which either serve for the reception of the male copulatory organ (bursa copulatrix), or as glandular organs of various kinds (cement glands), and also as a store-house for the sperm (receptaculum seminis). In the male, the paired-glands of the efferent ducts are greatly developed. In addition to them there are other parts which function as seminal vesicles (vesiculæ seminales).

External organs, which are generally formed by the metamorphosis of the terminal metameres and their appendages, are connected with the end of the genital duct; in the males these form copulatory organs, and in the female vary in form (as ovipositors).

\section{$\S 234$.}

In the female apparatus the complex of ovarian tubes, which is generally regarded as an "ovary," undergoes the most considerable modifications.

The relations which these tubes have to the formation of ova is somewhat different to those which they have in other Arthropoda. Each separate ovarian tube (Fig. 158) gradually widens at one end, where it is inserted into the oviduct; the opposite end is generally slender, and is often, indeed, continued into a fine filamentous process. When there are a large number of ovarian tubes, the free ends are directed towards a centre and connected together. The ora are 
formed in these terminal filaments, the cell-masses of which represent ovarian germs; these, while undergoing continual differentiations, gradually make their way out of the ovarian tube. The ovum is a true cell at the place where it is formed, but on its way through the ovarian tube it increases in size, so that we find the largest eggs farthest from the germinal region, and nearest the oviduct, while behind them there is a continual series of smaller and younger formations up to the above-mentioned blind end of the ovarian tube. The separate eggs cause the ovarian tube to appear to be divided into segments or chambers. The gradual descent of the egg is not only correlated with its growth, but with various changes also in the substance of the yolk; each egg is provided, especially in the last segment of the tube, with an external cuticular investment, the so-called chorion; this is formed by the epithelial layer of the ovarian tube.

As each eg'g passes into the so-called oviduct, a portion of the ovarian tube is degenerated, and so the egg next in front is brought close to the oviduct. The differentiation of the egg is accompanied by the growth of the thin end of the ovarian tube, which is made up for by its shortening at the other end. In

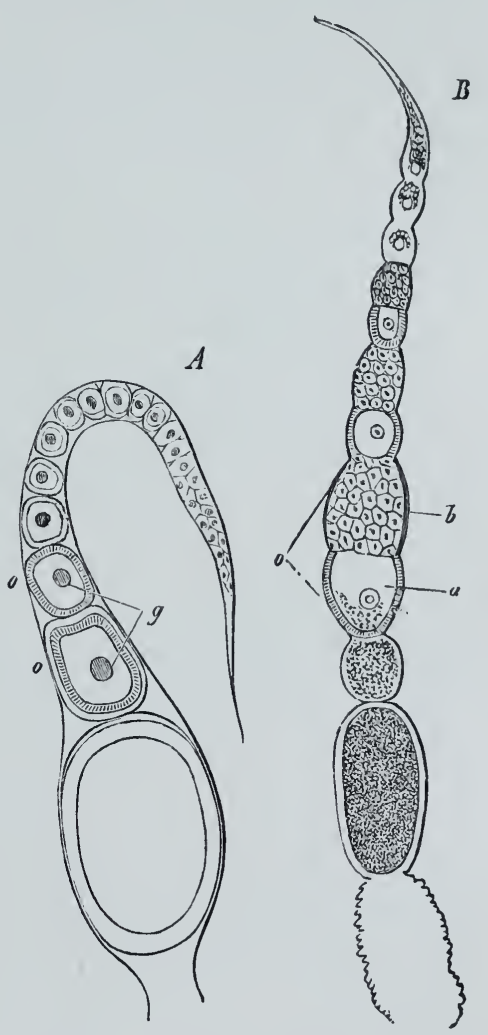

Fig. 158. A Ovarian tube of the Flea. o Ovum. $g$ Germinal vesicle. $B$ Ovarian tube of a Beetle (Carabus violaceus). $o$ Ovarian segment, divided into two portions, of which the ovarian cell is marked $a$, and the vitellogenous layer $b$. The ovum of the last segment has been ex. pelled; the walls of the ovarian tube are collapsed (after Lubbock). many Insects a group of cells is differentiated with each egg, in addition to the epithelial layer surrounding it; this vitellogenous layer occupies the portion $(b)$ of the chamber $(a)$ behind the egg-cell (Fig. 158, $B$ a), but is gradually used up by the latter, as it grows. An ovarian tube, or a collection of such tubes, does not therefore correspond merely to a germproducing reproductive gland, but is an organ entrusted with a much larger series of functions, and its blind end only is analogons to an ovary.

The length of the ovarian tube depends on the number of egrgs in it. The smallest number of chambers is found in most of the 
Diptera, where not unfrequently there is only one (Fig. 160, o), though more commonly two or three. In many Coleoptera and Hemiptera also the number of chambers is small. The ovarian tubes are longer in most of the Hemiptera and Hymenoptera; the largest number of chambers obtains in the Neuroptera and Orthoptera, and lastly in the Lepidoptera, where there are four ovarian tubes made up of a large number of chambers, which look like a string of pearls.

The arrangement of the ovarian tubes on the so-called oviduct also varies very greatly. They are sometimes united into tufts, sometimes broken up into groups, and sometimes arranged in rows.

The so-called pseudova have been distinguished from the egg's (ova); these structures are partly characterised by the absence of the germinal spot, like the products of the female generative glands in certain generations of the Aphides and Coccidæ. As the organs resemble those in which true egg-cells are formed, and as the same individual is able to produce pseudova and ova at different times, it is best not to regard the gulf between these two products of the ovary as a very wide one. These structures are links in a chain of phænomena, which are very common among Insects; the chain begins with the arrangement known as parthenogenesis, and extends to an apparent alternation of generations. The whole phænomenon depends on the emancipation of the ovum from the influence of the male reproductive elements. The simplest case is that in which there is no anatomical difference between the eg'gs, some of which are developed without previous fertilisation, while the rest require to be impregnated. The parthenogenesis of Bees, Wasps, and many other Insects is of this kind. The arrangement in which the same individual no longer produces these egg's at one and the same time is a further differentiation; the emancipated ovarian products are then, as a rule, differently formed (pseudova). Still more peculiar is the formation of these eggs in different individuals, when whole generations can do without the influence of the semen on the reproductive elements, and at the same time fall to a lower grade of organisation (Aphides). These structures, finally, may be formed in an earlier stage in the development of the animal, and from the still indifferent germinal gland; this arrangement, just like the rest, with which it is directly allied, is derivable from sexual differentiation (Cecidomyia).

\section{$\S 235$.}

The two, ordinarily short, oviducts seldom open separately into a depression of the integument (Ephemerida). As a rule this depression is further developed into a common efferent duct (Fig. 159, ov), the vagina; with this accessory organs, receptaculum seminis (Fig. 159, rss) and bursa copulatrix $(b c)$, are connected. The seminal receptacle is seldom absent; it is formed of a stalked and sometimes much-coiled vesicle. The receptacle is often a propor- 
tionately wider and coiled cæcal tube, which is sometimes provided with an appended gland.

The bursa copulatrix is another organ, which is directly connected with the vagina; it is a wide cæcal-sac (Fig. 159, bc), which looks like a diverticulum of the wall of the vagina. This organ is found in some orders only, and even in them it is not generally present. The bursa copulatrix of the Coleoptera appears to be the most independent, and not unfrequently is of a considerable size; in them it is generally connected to the vagina by a canal. In the Lepidoptera also it opens into the vagina by a narrow duct; but it is remarkable from the fact that it

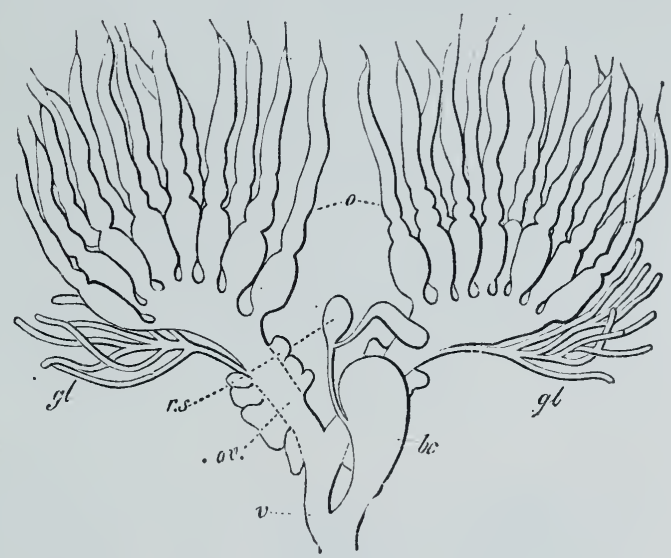

Fig. 159. Female generative organs of Hydrobius fuscipes. o Ovarian tubes. ov Oviduct, beset with glandular appendages. $g l$ Tubular glands. $v$ Vagina. bc Bursa eopulatrix. rs Receptaculum seminis (after Stein). has another efferent duct in addition to this one, which it sends off below the female generative pore, where it opens separately. In the Lepidoptera fertilisation is effected by means of this canal, the spermatozoa passing into the receptaculum seminis from the bursa copulatrix by the above-mentioned duct, which connects it with the vagina. The openings of the two parts into the vagina are opposite to one another.

The accessory glandular organs of the vagina either consist of a pair of simple canals which generally form long loops (Fig. 160, gl) (Lepidoptera, many Diptera), or of short cæcal tubes (Bugs). In others they are greatly ramified (Ichneumonidæ and Tenthredinidæ). The secretion of these cementglands serve to attach the eggs when laid, and at times to unite

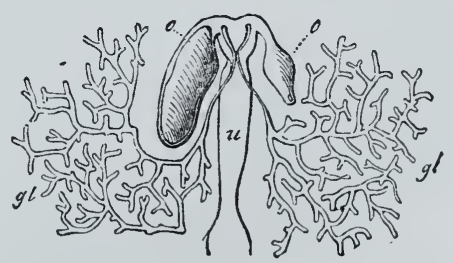

Fig. 160. Female generative organs of Mallophagus. o Ovarian tube. $u$ Uterus. gl Glands (after R. Leuckart). them into masses.

As a rule some portions of the integument, which have the form of valves, are connected with the female genital pore; the markings on these valves are always exactly adapted to the male copulatory organ ; sometimes they are arranged like nippers, and consist of processes which work laterally on one another. 


\section{$\S 236$.}

The male generative organs of Insects very often repeat in their development the forms of the female organs, so that even the separate divisions of both sets of glands not unfrequently correspond. The testes, which are always paired, and seldom fused into one organ, are composed of cacal tubes, just like the ovaries; they also vary in number and size, and are connected with one another in all kinds of ways (Figs. 161, 162, t). The testes of either side are often united in the Lepidoptera. The Diptera and Strepsiptera, as well as many Neuroptera, have two simple, long, and always separate testicular tubes. In many Coleoptera, also, each testis is a long, closely-coiled cæcal tube, surrounded by a special membrane. The testes of most insects are made up of a number of tubes. Thus each testis, in most of the Hemiptera, consists

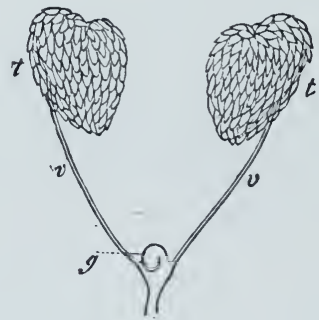

Fig. 161. Testes and efferent ducts of $\Lambda$ cheta campestris. $t$ Testes. $v$ Tas deferens. $g$ Seminal vesicle.

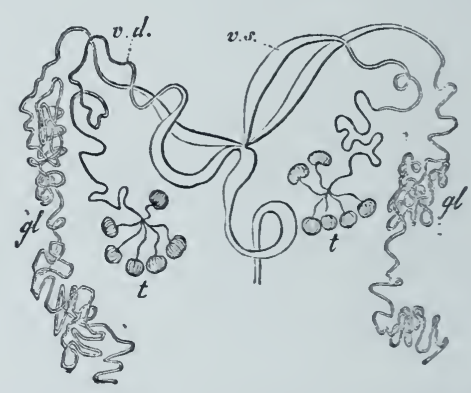

Fig. 162. Male generative organs of Melo. lontha vulgaris. $t$ Testes. $v^{\bullet} d$ Vas deferens. $\imath \cdot s$ Its widened portion. gl Coiled appended gland.

either of several tubes connected together to form a fan-like organ, or of a large number of separate tubes; this form of testis is also found in a large number of the Coleoptera. The testes of most Orthoptera consist of closely-applied tubes, which thus form a single mass, or of rounded vesicles grouped in a racemose fashion ; similar structures are also found in the Hymenoptera.

The efferent ducts of the separate testicular tubes are united into seminal ducts, and these, on each side, into a vas deferens (Fig. 161, v; Fig. 162, vd), which, when the tubes are closely united, passes directly from them. The two seminal ducts are, as a rule, not very long, but in some cases they are considerably elongated, and the widened portions of the coiled canals then function as seminal reserroirs (Fig. 162, vs). A common efferent duct (ductus ejaculatorius) is given off from their point of union; this, too, varies greatly in length, and also serves in part as a reservoir for the sperm.

The accessory glandular organs are as a rule paired, and like those of the female apparatus are either long coiled canals (Fig. 162, gl), 
or shorter and grouped into tufts, or branched tubes; they are attached to the efferent ducts at various points.

The male copulatory organs of the Insecta resemble the female ones, and are made up of chitinised ridges and valve-like arrangements, which vary greatly in form, and surround the generative orifice. They are divided into those which serve only for external copulation, and into others which are comparable to a penis, and are capable of intromission. The latter are formed by a tube, which is either attached externally, or is protrusible ; the ductus ejaculatorius is continuous with it, and it often carries pincer-like organs at its end. In the Coleoptera this copulatory organ is enclosed by a thick-walled chitinous capsule placed in the abdomen, which is often of considerable size, and is provided with a special muscular apparatus to protract it, and draw it in again.

\section{$\S 237$.}

The seminal elements of the Crustacea, though very variable in form, always agree in being motionless; the seminal filaments of the Cirripedia are an exception to this. Although the seminal elements are filamentous in the Isopoda, Amphipoda, and Ostracoda, they are incapable of movement; in the last-mentioned group they are extraordinarily long. Among the Schizopoda, in Mysis, at least, they are filamentous, and are bent at one end so as to form a hook. Cell-like bodies are the most common forms; owing to the presence of processes they present various peculiarities, the most notable of which is the radiate form of the semen of the Decapoda ("radiate cells"). The seminal filaments also of many Arachnida and Myriapoda appear to be incapable of movement, although in the former they are motile, when within the female generative organs.

In the Insecta the form-elements of the sperm are movable filaments, which are generally drawn out into a process at either end. The union of these filaments into tufts is an arrangement peculiar to them, as is also the union of the cells in two rows to form a rod-like structure which resembles in character a spermatophore (Orthoptera). 


\title{
Sixth Section.
}

\section{Brachiopoda.}

\section{General Review of the Group.}

\author{
$\S 238$.
}

The Brachiopoda, which by most authors were formerly placed with the Mollusca, with which they have little in common save a shell, which is quite different from the one found in the Mollusca, form a small and well-defined division, which is derived from the phylum of the Vermes. Among the Vermes the Chrtopoda, which are highly differentiated forms, appear to have several points of affinity with them; but nothing more, for there are such striking peculiarities even in the most important systems of organs, that it would be going too far to base any definite phylogenetic assertion on these relations. In any case the whole organism of the Brachiopoda, as compared with the Chætopoda and Annelides, is completely metamorphosed, and genetic relations can only be made out in a few rudiments.

This eminently isolated position of the Brachiopoda at the present time corresponds to the slight variations seen in the extant forms, as well as to the fact that we have here to do with a group of animals which was richly developed in earlier periods. Some genera are found as early as the Silurian epoch. But as the palæontological evidence is not sufficient to justify us in associating them with the Vermes, it is better to treat them separately than to unite them with those forms.*

We distinguish two orders only.

1) Ecardines.

Lingula, Orbicula, Crania.

2) Testicardines.

Terebratula, Argiope, Waldheimia, Thecidium.

* I recognised their affinity to the Vermes in my "Grundzuge," II. Aufl. 


\section{Bibliography.}

Owen, R., On the anatomy of the Brachiopoda. Transact. Zoolog. Soc. Vol. I. 1835.-Vogr, C., Anatomie der Lingula anatina. Denkschr. der schweiz. Gesellsch. für die gesammte Naturwissensch. Bd. VII. 1812. - HuxLex, Ann. and Mag. Nat. Hist. 1851. -Gritiolet, Journal de Conchiliologie, 1857-60.-Hancock, A., Phil. Transact. 1858.-LACaze-Duthiers, Sur la Thecidie. Ann. sc. nat. IV. xv.-Monse, E., On the systematic position of the Brachiopoda. Proceed. of Boston Soc. of Nat. Hist. Vol. XV.-The same, Embryology of Terebratulina. Mem. of Bost. Soc. Vol. II.-KowalEysky, Beobacht, tiber die Entwickelung der Brachiopoden. Moskau, 1874. (Russian.)

\section{Form of the Body.}

\section{$\S 239$.}

It is necessary to go back to embryonic stages to understand the characteristics which distinguish the form of the body in the Brachiopoda. In it, and at an early period, we meet with a stage in which the previously undivided body is separated into three (in Thecidium four) metameres; this discloses the Annulate type. A terminal circlet of cilia is predominant among the cilia formed all over the body in Terebratula, as is the case also in many Annelid larvæ. Bundles of setæ (Fig. 163, d) appear on the middle segment, which can be moved just as in the Chrtopoda, while the first metamere (cephalic segment) is converted into an umbrella-like enlargement over the mouth; this is surrounded by long cilia (Argiope). In this point also we can make out an affinity with Vermian larvæ (Actinotrocha).

While the larva becomes attached by the last metamere, two elevations are formed from the middle one, which enclose the first, and take on the form of two mantle-folds. The two shells, which can be distinguished as dorsal and ventral, are developed from this, and reach to the stalk formed from the last metamere. Owing to their position on the body, the shells are clearly quite unlike those of the Mollusca; this formation of the shells is a peculiarity, which marks off the Brachiopoda. And further this formation is probably the real cause of the cessation of the development of metameres, which

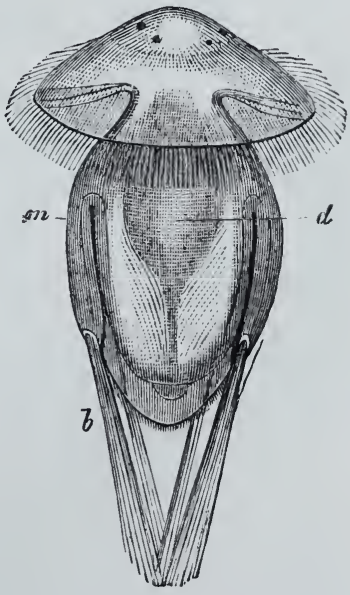

Fig. 163. Larva of Argiope. $m$ Mantle. $b$ Bundles of seta. d Enteron (after Kowalevsky). is also connected with the fixed position of the animal. Another peculiarity, the development of arms, is explained by the mode of life. 
A small number of ciliated tentacular processes are developed in the larvæ at the sides of the mouth. In the mature stage of the animal they are generally arranged on a stalk, which can be rolled up in a spiral fashion; there is a large number of them, and they are placed on either side of the mouth. When these arms are rolled up they are placed in the anterior portion of the mantle-cavity (Fig. 166, l); they appear to be erected by injection of blood. Owing to the great development of these arms, and of the mantlefolds, the rest of the body is reduced to a small size; and even the organs which, in other cases, lie in the cœlom, may be embedded in the folds of the mantle (pallial cavity). The mantle acquires a respiratory significance when the inner lamellæ of its folds are increased in surface by the development of ridges; it then functions as a gill (Lingula).

The tentacular processes around the mouth call to mind the tentacles of the Bryozoa, which may also be arranged on armlike structures (lophophore); but they can no more be compared with these structures than with the branchial tufts of the Tubicolæ.

Finally, the stalk is, in the older forms (Lingula), a long portion of the body, which passes out between the two shells, and is movable, while in the 'Testicardines it is short and largely chitinised.

\section{Integument, Shell, and Arms.}

\section{$\S 24.0$.}

As the two shells cover all the body except the stalk, the parts of the surface of the body are only free within the pallial cavity, and are only exposed when the shell is open. From the fact that muscles are connected with the integument, we may suppose that there is a dermo-muscular tube. Calcareous spicules, which distinguish the integument, are found in the mantle, as well as in the arms. They are sometimes branched, but may be stellate or form a network. The setre, which beset the edge of the mantle in various ways in various genera, are of more significance. Like the setæ of the Chrtopoda they are formed in glandular depressions of the integument, and, like them, are cuticular formations. They are generally simple structures, which terminate by a fine point, and indicate by their transverse striation that they have been gradually secreted.

In the Ecardines the two valves of the shell have pretty much the same form. But in the Testicardines the dorsal and ventral valves are distinctly differentiated. Towards the stalk they are united by a kind of clasp. The ventral valve also is drawn out into a beak-like process, the punctured end of which serves as a passage for the stalk. A skeleton, which projects inwards, is developed 
from the dorsal valve (Fig. 164,c). It serves as a support for the arms.

When first differentiated the shell is a soft chitinous layer, which later on becomes calcified. The valves are traversed by pore-canals, which are filled up by villous processes of the mantle. Between these the firm substance of the shell is seen to be composed of prismatic bodies, which can be made out even when the shell is first laid down; they are set obliquely to the edge of the shell.

Owing to the great increase in surface of the spirally-coiled arms of the Brachiopoda, thanks to their investment of tentacles, they form organs well adapted for the respiratory function. In the first place the tentacular filaments are suitably arranged for respiration. They are in communication with the blood-sinuses which pass along the arms. In their functional relation they may therefore be regarded as gills. The two arms are connected with one another at their bases, which are directed towards the middle line. A fold above the mouth extends on either side on to the arms, and aids in marking off a groove, which extends from the arms to the mouth. The tentacles or cirri, which are arranged in two rows, and closely approximated, rise up on the other edge of this groove; they extend to the end of the arms.

\section{Muscular System.}

\section{$\S 241$.}

Besides the muscles of the dermo-muscular tube, such as those of the mouth and of the arms, we find in the Brachiopoda a number of independent muscles, which traverse the cœlom, and serve to open and close, as well as to turn the shell (cf. Fig. 164). According to their function they traverse the colom in different directions, and arise from, as well as insert

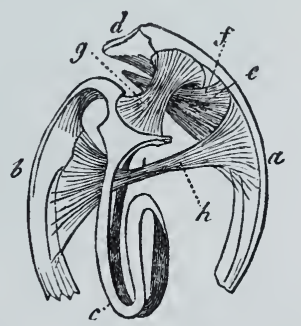

Fig. 16t. Muscular system of Terebratula. $a b$ The two halves of the shell. $c$ Support for the arms. $d$ Stalk. $e f$ $g h$ Muscles for opening and closing the shell (after Owen). themselves into, the valves of the shell, so that they may be regarded as differentiations of the dermo-muscular tube, which were formed when these valves were formed.

\section{Nervous System and Sensory Organs.}

The nervous system is very peculiar; it alone would justify us in giving the Brachiopoda an independent position. It is made up 
of masses of ganglia, which lie near the œesophag'us (Fig. 165, $九$ ). A larger ganglion lies transversely across and below the osophagus, or rather, owing to the downward bend of the œesophagus, behind it (in the Terebratulida). Two nerve-trunks pass off from it towards the posterior region, and break up into nerves for the stalk; they are provided with swellings $\left(n^{\prime}\right)$. The nerves for the ventral lamella of the

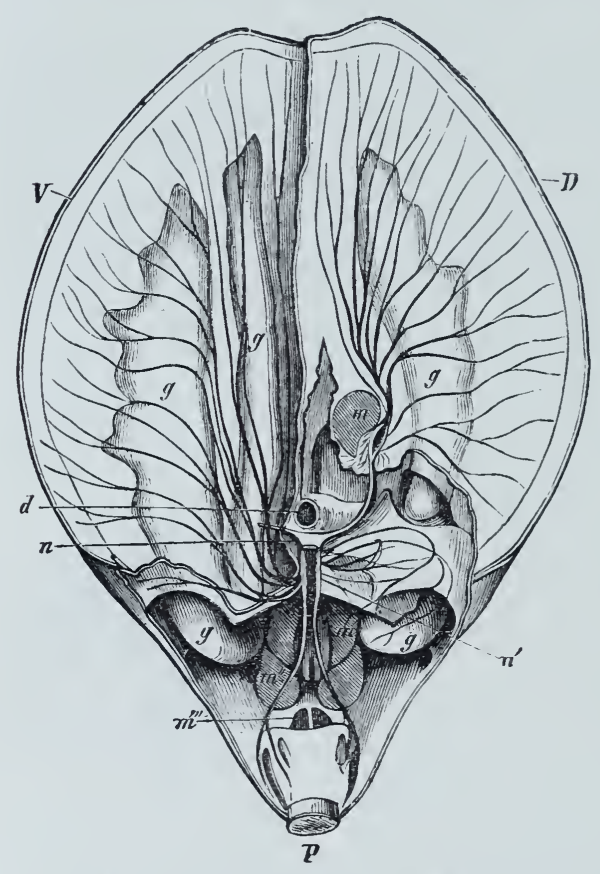

Fig. 16ã. Nervous system of Waldheimia, from the dorsal surface. The dorsal valve has been remored, as well as the left half of the dorsal mantle, $D$. $V$ Left half of the ventral lamella of the mantle. $P$ Stalk. $d$ Esophagus, cut through. (A pair of ganglia, which lie in front of the osophagus, and which are connected with the ganglion (n) by fine filaments, are not figured.) $n$ Anterior. $n$ ' Posterior œsopha. geal ganglion. $g g$ Generative organs. $m$ Occlusarmuscle. $m^{\prime}$ Divaricator. $m^{\prime \prime}$ Ventral adjustor. in' Accessory Divaricator (after A. Hancock).

mantle are given off from the swellings on these trunks. From the large ganglion, however, a nervetrunk is given off, on each side, to the dorsal lamella of the mantle, as well as a nerve to the arms. Two fine filaments surround the œsophagus and pass into a small ganglion, placed in front of, and therefore on the dorsal side of, the œsophagus; this is connected with the other ganglion by a commissure. In this way an œsophageal ring is formed, and the only question is whether the small upper ganglia represent central ganglia or not. If so, then there is this peculiarity, that the nerves for the arms arise from the ventral ganglia, and we can hardly regard the arms themselves as homologous with the tentacles of Vermes, even if it could be shown that the ganglionic parts in the œsophageal ring had altered their position. The ventral ganglionic mass must apparently be compared with a shortened ventral ganglionic chain; but to make a safe comparison we must have more exact information as to the facts.

The slight development of the superior ganglia is correlated with the absence of higher sensory organs, and this absence appears to be an acquired condition, for the four pigment spots, found on the first segment in the larva, point to the existence of visual organs (Fig. 163), and lead us to suppose that there were eyes in the ancestral forms. The two vesicles found in another larval form similarly point to the previous existence of auditory organs. 


\section{Alimentary Canal.}

$\S 243$.

In the Brachiopoda the enteric tube commences by the mouth, which is placed in the mantle-cavity between the two arms; thence, and without any accessory organs, it passes, generally in the form of a short canal, into the widened mid-gut (Fig. 166, $\left.d^{\prime}\right)$; this is generally known as the stomach. The portion which arises from this passes into an enteric loop in Lingula, which turns towards the right side, and opens at the anus into the mantle-cavity. This last portion of the enteron is rudimentary in the Testicardines, and generally terminates by a cæcal-sac which turns towards the ventral valve of the shell; from this a solid chord, which is perhaps the obliterated remnant of the enteron, is sometimes continued on. The end is sometimes widened out into a bulb.

A special peculiarity in the Brachiopoda is the way in which the enteron is attached. A lamella extends from the mid-gut to the wall of the body; this is the gastro-parietal band, which thus forms a kind of partition in the colom. It might be regarded as a dissepiment, formed in connection with the already noted metamerism of the body. This view of its meaning is, when we compare it with the arrangements seen in the Annelides, confirmed by its relations to the excretory organs. A second lamella, the ileoparietal band, is similarly attached to the hind-gut.

All the structures worthy of note which are differentiated from the enteric wall are to be found in the mid-gut. They have the form of branched tubes, which in many open into, or behind, the enlargement of the enteron, already spoken of as the stomach, by several pores (Crania), and in others are united into several (four) efferent ducts (Lingula). They are more largely developed in the Testicardines, where they are arranged in two lateral groups of glands, which surround the stomach, and are generally connected with it by a number of ducts on either side (Fig. 166, $h^{\prime}$ ).

\section{Cœlom and Circulatory Organs.}

\section{$\S 244$.}

The cœlom is divided by the organs which are embedded in it and by the muscles which traverse it, into several continuous spaces which are connected with the vascular system, and so form hæmal passages. These are also continued, as sinuses, into the lamella of the mantle, and into the arms; in the former they break up peripherally, and so come to be regularly arranged. The vascular apparatus ramifies in these spaces. The chief point as to their 
general arrangement is that the large trunks run dorsally along the enteron; in this they may be seen to resemble the arrangements which are found in the Vermes. But the special points in this system of organs require further investigation.

A saccular organ lying above the stomach is regarded as the heart; this receives a vascular trunk which runs from in front above the œsophagus, and gives off lateral branches. The former is regarded as an afferent vessel (vein). It seems to collect the blood from lacunæ, which are placed around the enteric canal. Two lateral vessels given off from the heart are united in the Testicardines (Waldheimia) for a short distance. In the Ecardines (Lingula) they are not given off till later from a median longitudinal trunk which passes backwards on the enteron. Two arterial trunks, which have been called aortæ, soon divide into two branches, one of which passes forwards and the other backwards. The anterior one represents the dorsal pallial artery, which divides into a median and a lateral branch, and supplies the mantle and the organs which lie in it. Smaller arteries are given off from the lateral branch to the lacunæ of the mantle; they pass to the edge of it, and then open after having divided several times. The hinder branch of the aorta also divides into two arteries. One runs along the middle line, and forms, with its fellow of the other side, an arterial trunk which passes to the stalk. The other artery turns forwards, and again divides into two branches in the ventral lobes of the mantle where it ramifies, in just the same way as the dorsal pallial artery. On the two pairs of pallial arteries there is a pouch-like appendage, or accessory heart. The blood seems to pass from the ends of the arteries into wider lacunæ which are placed in the mantle, as well as between the viscera, and around the muscles; and these are connected with a complicated system of canals, which traverses the arms, and is divided into an efferent and an afferent portion.

As the mantle is a secondary structure, its blood-vessels may be regarded as being so also. The pallial arteries are, therefore, of little importance, and the large trunks which accompany the enteron become of greater morphological significance. The heart appears to be a unilateral enlargement of the longitudinal trunk, as are also the accessory hearts of the pallial arteries.

\section{Excretory Organs.}

\section{$\S 24.5$.}

The excretory organs found in the Vermes, and adapted to the presence of a colom, are also found in the Brachiopoda, where they have essentially the same characters. Like the looped canals of the Annelides, these organs have an internal and an external orifice, so that I have no hesitation in regarding these structures as homologous, 
even though their function be modified. There are either one or two pair's of them. When there are four, two of them belong to the so-called dorsal, and two to the ventral half (Rhynchonella); this points to the presence of two metameres, which have disappeared in this portion of the body. The dorsal ones are absent in Lingula and the Terebratulida. The canals, which generally open to the exterior near the base of the arms, open into the coelom, after having taken a bent course, by funnel-like enlargements (Fig. 166, r), which are distinguished by their radially-arranged folds. This orifice passes through the ileoparietal band, and is thus directed

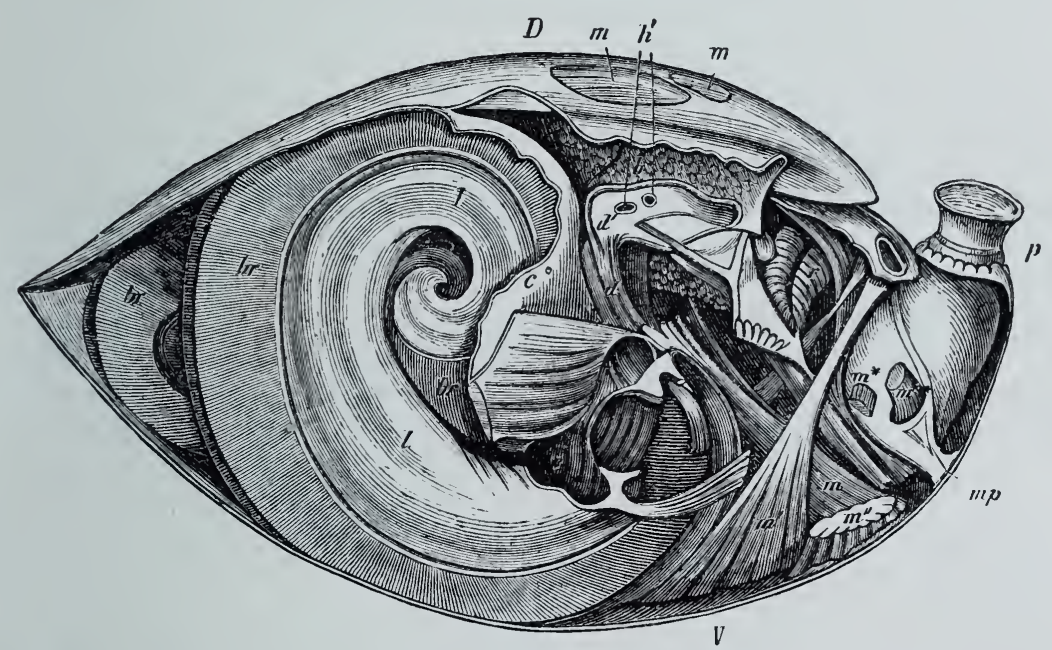

Fig. 166. Lateral view of the organisation of Waldheimia anstralis. D Dorsal, $V$ Ventral surface. $P$ Stalk. $l l$ Spirally-coiled arms. $\quad b r$ Branchial filaments. $c$ Anterior wall of the perivisceral cavity. $d$ CEsophagus. $d^{\prime}$ Mid-gut. $h$ Liver. $h^{\prime}$ Its openings into the mid-gut. $r$ Internal orifice of the right oviduct (some folds only of the left oviduct can be seen). $e$ Brachial canal. $m m^{\prime} m^{\prime \prime} m$ * Muscles to move the valves of the shell (after A. Hancock).

towards the pericardial cavity. The ileoparietal band resembles therefore, in its relation to the internal orifice, a dissepiment of the Vermes (cf. supra, §243).

Although the walls of these canals appear to be glandular in character, owing to the possession of projections, villous processes, or folds, we know nothing of their function, save that they have a relation to the generative organs; so that they appear to form an oviduct, and have indeed been hitherto regarded as being such. And as the looped-canals serve as parts of the generative apparatus in the Gephyrea and Annelides, it is not to be wondered at that they have the same relations in the Brachiopoda; but this does not exclude the possibility of their having an excretory function also. 


\title{
Generative Organs.
}

\author{
$\S 246$.
}

In some of the Brachiopoda the arrangement of the generative organs is hermaphrodite, so that the separation of the sexes seems to be an exception ('Thecidium). The organs merely consist of germinal glands, in which the sperm and ova are formed. In the hermaphrodite form there are four, and in Thecidium two, glandular masses. In the Ecardines they lie in the colom, partly surrounding the enteron and the muscles; in the Testicardines they form rounded masses in the cavity of the two lamellæ of the mantle (continuations of the cœlom) (Fig. 165, g); in either case they call to mind the character of the generative organs of the Gephyrea, and of the Annelides. In the dioecious forms they are ovaries in one, and testes in the other. It is not known what relation there is between the ovarian and seminal regions in the monœcious forms. The generative products escape into the colom.

The excretory organs act as the efferent ducts of the generative glands, so that here too a primitively unconnected apparatus functions as an oviduct, or as a seminal duct, according to the sex. 


\section{Seventh Section.}

\section{Mollusca.}

\section{General Review of the Group.}

$\S 247$.

I'he general characters of the body, and of its various systems of organs, distinctly define the phylum of the Mollusca. Owing to the absence of any distinctly-marked external metamerism, the body appears to be more compact than in the Arthropoda or Annulata among Vermes; indications of metamerism may, however, be made out in various organs. 'The supracesophageal position of the central nervous system, and its connection with lower-lying ganglia, or with commissures surrounding the pharynx, when taken in connection with the position of the heart, which is always dorsal, are the definitely typical characters of this division; to which we may add that in most forms shells are developed from the dorsal surface.

The complete disappearance of their primitive metamerism, and the gaps that there are between the classes here united together, are completely explained by the early appearance (palæontologically speaking) of most of the classes of the Mollusca; while the forms still living are seen to be an exceedingly small part of the phylum, which was rich in forms, but which has been continued on in a relatively small number of divisions. As yet we know very little of the phylogeny of the Mollusca, but their metameric arrangements, as indicated by their internal organisation, point to their affinity to segmented organisms, the nearest allies of which were some of the Vermes.

Although we can classify the various orders as higher and lower, yet all the systems of organs have not been developed to the same extent, so that we are able to find distinct proofs of the affinity between every single division and lower forms. 
I submit the following sketch of the classification of the group, and would remark that many of the older views took note of variations, which would still further separate the divisions, and especially the smaller ones, of the group.

\section{Placophora.}

II. Conchifera.*

Chiton, Crsptochiton.

\section{Lamellibranchiata.}

Asiphonia.

Ostrea, Anomia, Pecten, Mytilus, Arca, Anodouta, Unio.

Siphoniata.

Chama, Cardium, Csclas, Venus, Tellina, Mactra, Solen, Pholas, Teredo.

$$
\text { Scaphopoda. }{ }_{\text {Dentalium. }}
$$

\section{Gastropoda. $\ddagger$}

Prosobranchiata.

Chiastoneura.

Zeugobranchia.

Fissurella, Haliotis.

Anisobranchia.

Patella, Trochus, Littorina, Cjclostoma, Rissoa, Paludina, Turritella.

Orthoneura.

Nerita, Janthina, Valvata, Sigaretus, Marsenia, Crpraea, Cerithium, Strombus, Pterocera, Dolium, Cassis, Tritonium, Voluta, Harpa, Buccinum, Nassa, Purpura, Murex.

Heteropoda.

Atlanta, Carinaria, Pterotrachea.

Opisthobranchiata.

Tectibranchiata.

Bulla, Aplysia, Pleurobranchus.

Nudibranchiata.

Tritonia, Polycera, Aeolidia, P'yllirhoẻ, Doris, Phyllidia, Sacoglossa. Pleurophyllidia.

Pulmonata.|

Elysia, Limapontia, Placobranchus.

* What led me most to unite all the Mollusca, with the exception of the Chitonidæ, into one great division, to which I have given the name Conchifera, was the consideration that we must recognise the great significance of the shell as affecting the whole organisation of these animals. But although the Placophora are thereby sharply marked off from the rest, I do not see that there is any sufficient reason for removing them altogether from the Molluscan phylum, for it is possible to make ont in them many points in which they agree with, and are consequently allied to, the Conchifera. I regard the Placophora as the remnant of a division, the forms of which were allied to the Solenogastres (p. 12 7 ) on the one hand, and on the other were the predecessors of the Conchifera.

+ The Scaphopoda form a division which is allied to the Lamellibranchiata as well as to the Gastropoda; but they must not be regarded as a mere intermediate link.

\# The Zeugobranchia are, in many points, the oldest of the Gastropoda.

$\S$ I regard the Heteropoda as an order which has branched off from the Prosobranchiata, and is closely allied to the Orthoneura; but which has dereloped special characters which are not equal in value to those of the Orthoneura.

II The organisation of the two divisions of the Pulmonata does not seem to me to be so markedly divergent as to make them of equal value with the two other orders of the Gastropoda. We cannot as yet form a definite opinion as to many of the genera, c.g. Onchidium, of the Nephropnensta. 


\author{
Gastropoda (continued). \\ Branchiopneusta. \\ Lymnæus, Planorlbis, Auricula. \\ Nephropneusta. \\ Helix, Bulimus, Clausilia, Limax, Arion.
}

Pteropoda.*

Thecosomata.

Hyalea, Cleodora, Chreseis, Cymbulia.

Gymnosomata.

Clio, Pneumodermon.

\title{
Cephalopoda. $\dagger$ \\ Tetrabranchiata. \\ Nautilus.
}

Dibranchiata.

Decapoda.

Spirula, Sepia, Sepiola, Loligo.

Octopoda.

Octopus, Tremoctopus, Eledone, Argonauta.

\section{Bibliography.}

Cuvier, Mémoires pour servir à l'histoire et à l'anatomie des Mollusques. Paris, 1817.-VAN BENEREN, Exercices zootomiques. Fasc. I. II. Bruxclles, 1839.-Quoy and GaImard, Voyage de l'Astrolabe. Zijologie.-Delu e ChisJ E, Descrizione e notomia degli animali invertebrati della Sicilia citeriure. Napoli, 1841-44.-Souley ex, Voyage de la Bonite. Zoolog. T. II. Paris, 1852.Ledскаят, R., Zoolog. Úntersuch. III. Giessen, 1851.-Gegenbaur, C., Unters. üb. Pteropoden u. Heteropoden. Leipzig, 1855.-Krohs, A., Beitr. z. Entw. d. Pteropoden u. Heteropoden. Leipzig, 1860.-Lankester, Rıy, Contrib. to the develop. of the Mollusca. Philosoph. Transac. 1875. - V. Jherivg, Vergleichende Anatomie des Nervensystems u. Phylogenie der Mollusken. Leipzig, 1877.

Placophora:., V. Mrpdendorfs, Anat. v. Chiton. Mém. Acad. de St. Pétersbourg. VI. vi. 1819.LovéN, S., Öfvers. K. Vet. Acad. Förhand. Stockholm, 1355. (Arch. f. Nat. 1856.)

Lamellibranchiata : PoLr, Testacen utriusque Siciliæ eorumque historia et anatome. III. Tom 1791-1795. - Bojanus, Ueber die Athem- und Kreislaufwerkzeuge der zweischaligen Muscheln. Isis, 1819, 1820, 1827.-DeshaYes, Art. Conchifera in Todd's Encyclopædia, Vol. I. 1836.-Garner, On the anatomy of the lamellibranchiate Conchifera. Transact. Zoolog. Soc. London. Vol. II. 1841.-Quatrefages, Anatomie von Teredo. Ann. des sc. nat. III. XI.-Lovén, S., Bidrag till kännedomen om utvecklingen of Moll. acephala. Kongl. Vetensk. Acad. Handl. Stockholm, 1850.-Keв ER, Beiträge zur Anatomie und Physiologie der Weichthiere. 1851.-Davarne, C., Su1" la générat, des Huîtres. Paris, 1853.-V. Hessling, Die Perlmuscheln. Leipzig, 1859.-Lacaze. Duthiers, Anatomie von Anomia. Ann. sc. nat. IV. II.-L. Vaillant, Sur la fam. de 'Tridacnides. Ann. sc. nat. V. Iv.-Sabatier, A., Etudes sur la moule commune. Mém. de l'Acad. des Sc. de Montpellier. 1877.

Scaphopoda : LAcıze-Durniers, Hist. nat. organis. développement, etc. du Dentale. Paris, 1858.

Gastropoda: Nordrans, Monographie des Tergipes Edwardsii. Mém. de l'Acad. Impérialc de St. Fétersbourg. IV. 1813.-ALDER and HANсоск, Monograph of the British Nudibranchiate Mollusca. Ray Soc. I.-VII. 1815-55.-HANcock and Eubletos, On the Anatomy of Eolis. Ann. and Mag. of Nat. His. XV. 1815.-The same, On the Anatomy of Doris. Phil. Trans. 1852 Pt. II.-HАлсоск, Anatomy of Doridopsis. Trans. Linn. Soc. XXY.-LEYdig, Ueber Paludina vivipara. Zeitschr. f. wiss. Zool. II.-HuxLer, On the morphology of cephalous Mollusca. Phil. Trans. 1853.-BERGH, Bidrag til en Monographi of Marseniaderne. Kongl. dansk. Vidensk. Selsk. Skrifter. 1853.-The same, Anatomisk Undersögelse of Fiona atlantica. Vidensk. Meddelelser for 1857.- The samc, Anatomisk Bidrag til Kundskab om Aeolidierne. Danske Videnskab. Selskabs. Skrifter. 1861.-The same, Bidrag til en Monographi of Pleurophyllidierne. Naturhist. Tidsskrift. 3 Räkke. 4 Bind. 1866.-The same, Bidrag til en Monographi of Phyllidierne, ebend. 5 Bind. 1869.-The same, Malacolog. Untersuch. Heft. I. -X. Wicsbad. 1870-76. -ClaparÈdE, Anatomie und Entwickelungsgesch. der Neritina fluviatilis. Arch. f. Anat. 1857. -The same, Beiträge zur Anat, des Cyclostoma elegans. ibid. 1858.-LACAzE-Dutri IE Rs, Anatomie du Pleurobranche. Ann. nat. sc. IV. xI.-The same, Anat. et l'Embryogénie des Vermets. Ann. sc. nat. IV. Xiri,-Liwson, Anat. etc. of Limax maximus. Quart. Journal of Micr. Sc. 1863.-Fol, H., Sur le développement des Hétéropodes et des Gastéropodes pulmonées. Comptesrendus. T'. LXXXI. Nos. 11 et 13. Archives de zoologie. T. V.

* In many points of their organisation the Pteropodia indicate a relationship to the Cephalopoda, but this can only be regarded as a very distant one.

+ Most of the oldest fossil forms probably belonged to the Tetrabranchiata, which are represented by only one extant genus; at the same time these fossil forms varied greatly in character. 
Pteropoda : Esсниіснт, Ueber d. Clione borealis. Kopenhagen, 1838.-For, H., Sur le développement des Pteropodes. Archives de zoologie. T. IV.

Cephalopoda: Graxt, On Loligopsis. Trans. Zool. Soc. 1835.-Owex, Memoir on the Pearly Nautilus. London, 1832.-The same, Article on Cephalopoda in Todd's Cyclopædia.. I. 1836.Valencibnsis, Nouvelles recherches sur le Nautile flambé. Archives du Museum. 1811.Petrrs, Anatomie der Sepiola. Arch. f. Anat. 1812.-Köllik вr, Entwickelungsgesch, der Cephalopoden. Zürich, 1814.-VAN DRR HoEVBN, Bijdragen tot de Ontleedkundige Kennis aangaande Nautilus pompilius, Amsterdam, 1856.-GrenAcher, Zur Entwick. d. Cephalopod. Zeitschrift f. wiss, Zoolog. Bi. XXIV. p. 419.-For, H., Note s. l. développement des Mollusques ptéropodes et céphalopodes. Arch. de zool. T. III.-BовRBTSKx, Untersuch. über die Entrickelung der Cephalopoden. Nachr. d. k. Gesellsch. der Freunde d. Naturkenntniss etc. zu Moskau. Bd. XXIV. (Russian.) [RAY Lixkester, Development of Cephalopoda, Quart. Journ, Mic. Sci. 1875.]

\section{Form of the Body.}

$\S 248$.

The general form of the body of the Mollusca must be regarded as one so much altered by the relative positions of many organs, owing to the formation of shells, that it has only been possible to recognise a ground-form, which shall represent the common origin, by comparing the earlier larval stages with several mature forms. The Placophora have a worm-like larva, and a similar kind of external metamerism is indicated by the number of circlets of cilia seen in the Gymnosomatous Pteropoda. The relations thus implied are retained by the Placophora in their mature condition, at least in the dorsal portion of the body. This is separated from the ventral portion by a groove, and so defines two regions, which are found also, under the form of "mantle" and "foot," although much changed, in the Conchifera. The differentiation of a gutter-like ventral surface in the Solenogastres (cf. p. 130), as has been already explained, points to the Mollusca having genetic relations to these worms; this supposition is confirmed by the characters of the nervous system.

The Lamellibranchiata and Gastropoda, as well as the thecosomatous Pteropoda, develop a well-marked circlet of cilia in the region, which, later on, corresponds to the head; this circlet is afterwards carried on a special, symmetrical, and lobed process-the Velum. The primitive significance of this circlet is clearly shown by its presence in otherwise divergent divisions, and is even still more important from the fact that we can recognise in this organ the circlet of cilia which surrounds the same part of the body in many Vermes (cf. $\$ 107$ ). The velum of the Mollusca may therefore be regarded as an organ inherited from a lower stage.

Below the velum the rudiment of the opening into the enteric cavity is formed. As in the Placophora, the formation of a dorsal shell in the Lamellibranchiata does not prevent the enteric tube from being continued to the aboral pole of the body; for in the Placophora the shell, as well as the man tle which carries it, is adapted to the whole body, and in the latter it is principally developed at the sides. We are able, therefore, to distinguish a primary axis, which extends from the oral to the anal pole, and which is crossed by two 
secondary and variously-differentiated axes-the dorso-ventral and the transverse. The body is therefore of the original eudipleural form, which is the dominant one in the Vermes and Arthropoda.

These relations are different in the Gastropoda, where the dorsal cup-like shell gradually encloses the greater part of the body, and leaves a small portion only of the surface of the body exposed in addition to the head and foot. So that while in the previous case the shell was adapted to the body, in this case the soft parts of the body are adapted to the single shell. The body, therefore, becomes asymmetrical, and the aboral pole no longer carries the anus, which becomes lateral in position in consequence of the flexure of the enteron; this flexure is due to the formation of the shell. All of the many variations from the symmetrical ground-form, which are seen in the Gastropod-body, may be regarded as due to this.

The primitive similarity in the form of the body, due to the possession of a shell, undergoes great modifications even among the Gastropoda; the Veliger stage is not always developed, and has never yet been observed in the Cephalopoda. But even in this class the form of the body, and the disposition of its viscera, may be seen to be, in all forms, due to the possession of a shell.

\section{$\S 249$.}

The velum has different functions in different divisions. In the Lamellibranchiata, where it functions for some time as a locomotor organ, but where it is never independent and soon atrophied, its function is not very great. This may, perhaps, be correlated with the rudimentary character of the future head, and this, again, with the rapid disappearance of the free mode of life in this division (Acephala).

T'wo folds, however, which are given off laterally from the dorsal surface, become considerably developed and form a mantle; they surround the body, and excrete the shell, which corresponds with the lamellæ of the mantle in form and size.

A space, which functions as the respiratory cavity, is developed between the edges of the mantle; branchiæ are developed from the body-wall, and project into it (Fig. 167, $A b r)$. In a few Lamellibranchs (Asiphonia) this entrance into the mantle-cavity is a cleft of some size, by which water passes in and out, and so carries in nutriment and removes excreta. In most, the two edges of the mantle grow together, and so shut off, more or less completely, the cavity which surrounds the gills, and cause 
the two streams of water to enter and escape from it with greater regularity.

The least amount of concrescence which is observed, gives rise to an anterior larger, and a posterior smaller, orifice (Mytilidæ). The former serves as an outlet for the foot, and as the orifice of entrance for the food, while the latter, in correspondence with its position, is the orifice of exit for the fœcal matters, and of the water that has served for respiration. In the Chamacee there are also two large openings behind the anterior and larger cleft, through which the foot is protruded, and which serve respectively for the entrance and exit of water; this is an arrangement which attains a higher grade of development in a large division of the Lamellibranchiata (Siphoniata). That part of the mantle which surrounds these orifices forms an elongated tube (siphon), which undergoes other modifications in addition to its concrescence. The respiratory tubes may sometimes be formed by

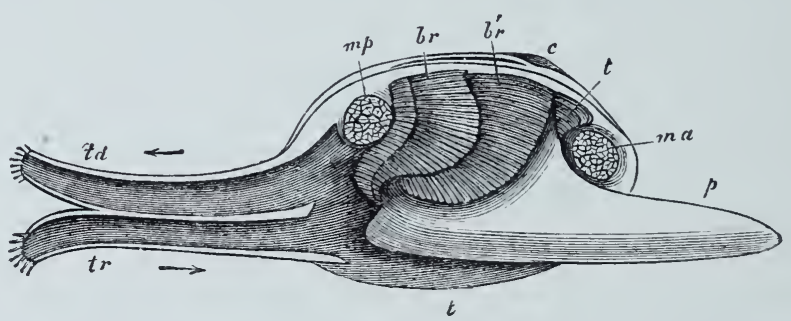

Fig. 168. Lateral view of the mantle-cavity of a Mactra; the right mantle-lamella has been remored. or $b r^{\prime}$ Branchiæ. $t$ Tentacle. ta tr Siphons. ma Anterior, $m p$ Posterior adductor. p Foot. $c$ Unbo.

separate portions of the mantle; or there may be a respiratory tube, which is single externally, and is only divided internally into two canals by a partition; or the two conditions may be combined (Fig. $168, t r$ ta); or, finally, two completely separate tubes may be developed: an upper one, the inner orifice of which is opposite the

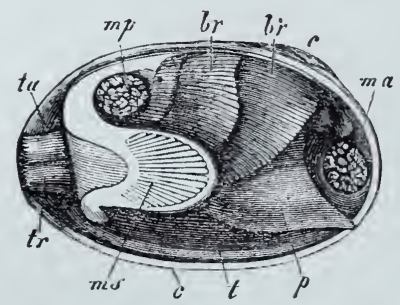

Fig. 169. The same animal with its foot and siphons re. tracted. ms Siphonal muscle. anus, and serves for the exit of the water, and a lower one, by which the water passes in. The investment of cilia causes the two streams to pass regularly in and out. Through these forms we are led up to those in which the respiratory cavity is most completely closed, and the pallial tubes most developed. This is accompanied by a diminution in the size of the cleft in the mantle through which the foot is protruded. This has become much narrower, and is placed some way from the respiratory tubes, so that the greater part of the edges of the mantle have grown together, in consequence of which the body of the animal is sac-like (Boring Mollusca). The orifice of passage for the foot is now placed at the anterior end, and the 
two respiratory tubes in the opposite region of the body. 'They are continued into special divisions of the mantle-cavity, owing to the division of the latter into an upper smaller, and a lower larger cavity, which are separated by a partition. The water is brought to the lower one by the efferent tube, and passes through the gills; streaming through the orifices in them, into the branchial plates or the intrabranchial cavity, and so into the upper division of the mantle-cavity, into which the anus also opens.

The edge of the mantle is often the seat of special differentiations, which are generally of the form of tentacular processes, and are sometimes of a fair size.

The second differentiation in the body of the Lamellibranchiata affects the ventral surface, which is differentiated even in the Placophora; it becomes flattened, and serves as the organ by which the animal creeps along. It is formed by the development of a muscular foot, which is more or less separated from the rest of the body (Fig. 167, $A p$ ), and which can be protruded from the cleft in the mantle, sometimes to a considerable extent. It is then hatchet or club-shaped, and functions as a locomotor organ. The two lateral surfaces of the foot are ordinarily produced into a median edge, but in some it is flat and sole-shaped, as in Chitons.

Many Lamellibranchs live under conditions in which this organ is not required, and it is then atrophied, as in the fixed Oysters and Anomiæ, and Scallops; in the latter, locomotion is effected by the action of the mantle and its shells.

The Scaphopoda are forms allied to the Lamellibranchiata, but intermediate between them and the Gastropoda. The body, which is enclosed by a shell, is provided with a mantle-cavity, from which a trifid foot can be protruded. A part which carries the mouth is headlike in form, but is really more of a proboscis, for it does not contain the nerve-centres, and is, moreover, enclosed in the mantle-cavity.

\section{$\S 250$.}

The velum is largest in the Gastropoda and the thecosomatous Pteropoda, and is absent in those only in which the earliest larval stages are not free (Land Snails). It has the form of a large, and frequently symmetrically lobate, organ (Fig. 170, $A B C v$ ), which in some is retained for a longer time, and so enables the body to continue swimming about (Macgillivraya). The development of this organ, which in its lower stages is merely represented by a circlet of cilia, appears to be correlated with the development of a shell, for when this is developed the cilia are less widely distributed. The cephalic portion of the body is alone free; and it compensates for the absence of other locomotor organs by the great development of its cilia, and of its ciliated margin. The velum increases in size, and undergoes great complications of form, in proportion to the increase in the weight of the body due to its shell. 
The size of this velum is correlated with the differentiation of the head, from the upper surface of which it is developed; it is in some Pteropoda only that the head, once formed, undergoes any considerable atrophy.

Just as in the Lamellibranchiata, the mantle rises up in the form of a fold of the body-wall, which covers over the dorsal surface and forms the shell on its outer side. As this dorsal area of the body-which is surrounded by the mantle-fold, and the shell, which is being developed into its house-continues to bulge out, it gradually forms a blind sac, which soon contains the greater part of the viscera (visceral sac); in this way the viscera come under the direct protection of the shell. As development goes on, the mantlefold becomes less intimately connected with the body, and gives rise, inferiorly, to a wider cavity, in which the growing gills are contained, and which is homologous with the branchial cavity of the Lamellibranchiata (Fig. 167, $A B$ ). This development of a fold of the integument into the mantle, and the consequent appearance of a
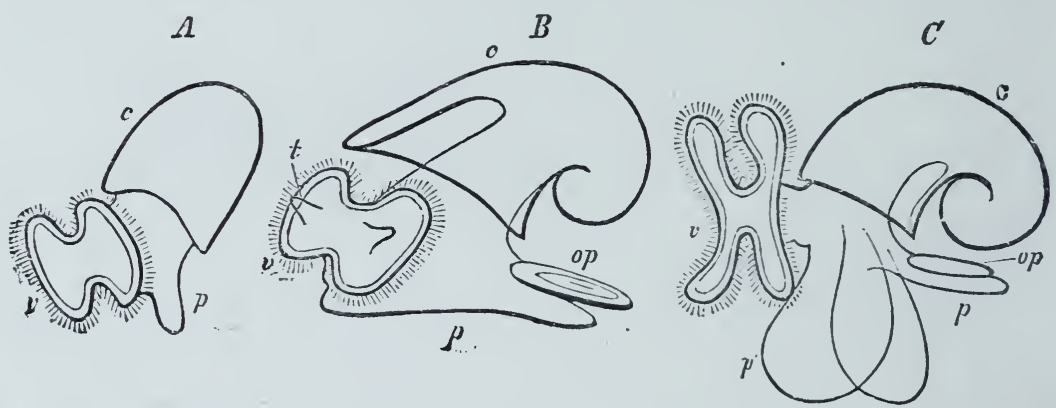

Fig. 170. Larvæ: $A$ Of a Gastropod; $B$ Later stage. $C$ Of a Pteropod (Cymbulia). $v$ Velum. $c$ Shell. $p$ Foot. op Operculum. $t$ Tentacles.

subjacent space, the branchial cavity-which looks like an invagination from the exterior-undergoes modifications, which are largely due to the formation of the shell. In consequence of the mantle growing unequally on either side, and not equally, as in the Lamellibranchiata, and from the fact that it is principally developed at one point in connection with the development of the shell, the branchial cavity comes to be a single cavity, placed in the same region. This region is either beneath the hinder portion of the mantle, as in the Pteropoda (Fig. 170, C), or beneath the anterior portion, as in most of the Gastropoda $(B)$. The want of symmetry, which is due to the coiling of the shell, causes the branchial cavity of most Gastropoda to lie on one side; this is an adaptation to the larger amount of space which is afforded by the lateral portion of the shell. The production of the unilateral and asymmetrical branchial cavity from a paired and symmetrical space is proved by numerous facts; so that we are led to think that the asymmetry of the shell is probably a secondary arrangement.

A number of degenerate and more perfect arrangements have 
their common origin in this disposition of parts. The latter are principally seen as differentiations of the edge of the mantle, and are connected with the function of the branchial cavity. Part of the edge of the mantle is produced into a groove, which serves to bring: in water, and which may be converted into a tube by the folding over of its two edges. We meet with a siphon of this kind, though in all stages of gradual differentiation, in a large number of aquatic Gastropoda (Buccinum, Dolium, Harpa, Tritonium, Murex, etc.). A second siphon, formed in the same way, but smaller in size, is generally found at the opposite end of the branchial cavity; it serves to carry the water out from it. Various other kinds of processes, or tentacular appendages, lead to fresh complications in its structure (e.g. in Strombus, Pterocera).

When the shell undergoes atrophy the mantle generally does so too. This is mostly the case in the division of the Opisthobranchiata, some of which have a more or less rudimentary shell ; while in others, when adult, there is no shell at all. As all these forms had a shelled larval stage, the atrophy of the shell must have been brought about during their ontogenetic development; and it follows that those Opisthobranchiata, which are naked in their later stages, were derived from forms that had shells. The larval shell and its accompanying mantle-fold, even though feebly developed, are therefore rudimentary organs, which prove that the naked Opisthobranchiata had the same origin as the rest of the Gastropoda. Where these rudimentary shells are retained by the adult animal they must even then be regarded as degenerate parts, and not as developing shells; for here again a comparison with the larval forms shows that the shell had a much greater significance than have the rudimentary structures found in the adult stage of these organisms. It is of great importance also as explaining the position of the anus and of the genital orifice, which can be due to nothing but a former greater development of the shell.

Within smaller divisions also we meet with series of degenerating parts, as for example in the Heteropoda, where Atlanta has a well-developed shell and mantle, while in Carinaria they are both rudimentary, and in Pterotrachea completely lost. A similar series is observable in the Nephropneusta.

\section{$\S 251$.}

The varying extent to which the Foot is developed is of importance as affecting the form of the body. In the larvæ of the Pteropoda and Gastropoda it has always very much the form of a short, conical, somewhat-flattened process, placed below the mouth (Fig. 170, $A p$ ). On the hinder, or dorsal surface, a shelly secretion is formed, which serves as an operculum for the orifice of the shell. Owing to its increase in size, especially in the aboral direction, the foot of most Gastropoda comes to have a broad lower face, which is 
the reason of its being called a foot (Fig. 171, B). Sometimes, however, it is elongated, and, at others, discoid in form. In most of the Gastropoda the foot is only sharply marked off along its plantar margin. In many of the lower Prosobranchiata (Haliotis) the

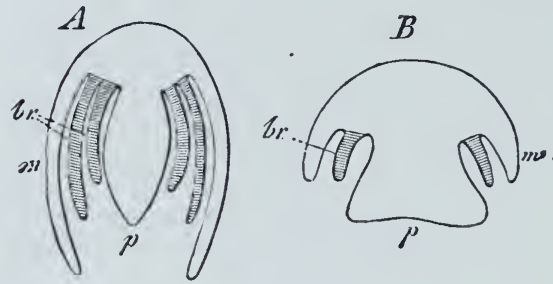

Fig. 171. Diagram of the relations of the mantle and foot; vertical section. $A$ In Lamellibranchiata. $B$ In Cephalopora. $m$ Mantle. $p$ Foot. or Branchire.

surface of the body above the foot is drawn out into an encircling edge (epipodium), which is distinguished from the mantle by surrounding the head. The foot of the Heteropoda is differentiated into a more independent organ, which springs from the ventral surface of the animal, and. forms a vertical fin. The body is continued in front of, as well as behind, the foot. This arrangement is very different from the primitive one; the body has no longer a flat surface, although the end of the foot in Atlanta still carries an operculum. The structure of the muscular sole of the Gastropod foot is retained in rudiment as a sucker-like organ, which in the Pterotracheæ is found in the males only. And we are reminded by this that even

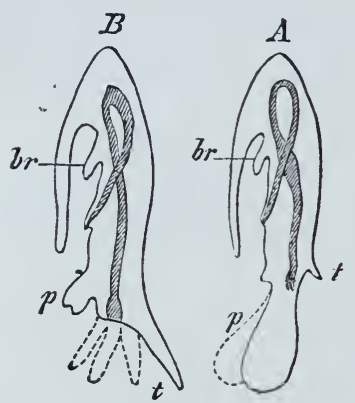

Fig. 172. Diagram of the rela. tions of the mantle. $A$ In Pteropoda. $B$ In Cephalo. poda. $p$ Foot. $b r$ Branchiæ. $t$ Tentacles. when fully developed the foot of the Gastropod functions as a sucker, for the animal is able to attach itself by it.

The modifications undergone by the foot in the Pteropoda are still more significant. The foot, which, in the earliest larval stages, is formed in just the same way as in the Gastropoda, gires rise, in the Cymbulidæ and Hyaleidæ, to a median and two lateral pieces (cf. Fig. $170, C p p)$. In the Hyaleidæ the median portion is feebly developed, while the lateral lobes become large fins, which embrace the rudimentary head, just like wings; in the Cymbulidæ the median piece is also well developed. It either fuses at its base only (Cymbulia), or along: its whole length (Tiedemannia), with the two lateral lobes; in this way the large fins of these animals are produced.

\section{$\S 252$.}

The greater development of the head in the Cephalopoda is an important peculiarity as affecting the form of the body, while the mantle acquires the same relations as it has in the Thecosomatous Pteropoda, from which therefore they may be derived. The cavity, 
arched over by a fold of the mantle, occupies the hinder part of the back, and so forms that region which is ordinarily known as the ventral surface. To make these relations clear we must imagine the animal placed in such a position that the aboral end points upwards, and the head forwards and downwards (cf. Fig. 172). All the body above the head would then correspond to the dorsum of the Gastropoda. The mantle is sometimes separated from the head by a circular groove (Sepia); sometimes this fold of the mantle is directly continuous with the integument of the head at the sides of the neck (Octopus), so that the mantle forms a fold above the branchial cavity only. Lateral processes of this mantle function as locomotor organs (fins); in the Sepiadx they are generally small, and extended along its whole length; in the Loliginida they are broader, but are limited to the aboral end of the body.

The formation of the mantle-cavity and the position of the anus lead us to the conclusion that this arrangement is due to the primitive possession of a shell which covered the whole mantle; and, indeed, the shelled Cephalopoda are by far the older forms, while the remarkable variations seen in the characters of their shells lead us to think that this structure had a very ancient origin.

An organ which has the same position as the foot of the Gymnosomatous Pteropoda-the funnel-corresponds to the foot of the Gastropoda. In Nautilus it is formed of two lamellæ, which arise from the ventral surface below the head, and which form a tube by being rolled over one another; this tube projects from the mantle-cavity (Fig. 175, $i$ ). In the Dibranchiata this organ cannot be seen to be composed of two lateral parts, except in the embryo; they take their origin in the space between the mantle and the rudiments of the arms. By growing together and gradually fusing they form a tube which is similar to the one formed in Nautilus, except that it is closed. The mantle, which is also muscular, is attached to the periphery of the funnel; this effects powerful contractions, and so drives out the water which has entered the mantle-cavity between the funnel and the edge of the mantle; and the animal is driven in an aboral direction by the force produced by the expulsion of this stream. The organ retains, therefore, its primitive locomotor function.

\section{Appendages.}

$\S 253$.

In the Mollusca the development of a cephalic region is closely connected with the differentiation of processes, which I regard as appendages, inasmuch as they are homologous with the antennæe and tentacles of Arthropoda and Vermes, and when more highly differentiated are able to undertake the duties of appendages. 
These structures, which are known as tentacles, are absent in the Placophora and in the Scaphopoda; the processes which are found around the mouth in the latter group being structures of a special kind, and not appendages as here limited.

In the Lamellibranchiata, lobate appendages (Fig. 168, $t$ ) (the socalled labial palps) are attached to the altogether rudimentary head; they may be homologous with the more highly-developed tentacles, which distinguish the cephalic region of the Gastropoda. As in many Platyhelminthes they are, when simplest, short processes of the body, but they undergo great differentiations. In the Prosobranchiata they are generally limited to two, and are formed from the surface which is surrounded by the velum (cf. Fig. 170, $B t$ ). In many forms the eye is placed at the base of the tentacle, which may be developed into a special process. The same happens in other forms, where the optic organ is placed on an optic stalk differentiated from the tentacles, and which, when more independent, may give rise to four tentacles, as in Helix, Limax, etc. These are invaginated when they are retracted, and are so far more highly developed. Many Opisthobranchiata are distinguished by the possession of a pair of tentacles, which are greatly dereloped (Fig. 177, $t t)$, but in addition to them there are other tentacular cephalic appendages, which characterise the various subdivisions merely, according to the way in which they are arranged, and according to the number present.

They have undergone degeneration in the Thecosomatous Pteropoda, for in these forms the tentacles are either completely absent or are rudimentary (Chreseis). The development of the parts of the foot which in them are converted into fins, does away with the necessity for the development of the cephalic tentacles, and explains why they are absent, just as, on the other hand, the distance of the fins from the head in the Gymnosomatous forms is the cause of the development of their tentacles. In these latter they have all kinds of forms, and one or more pairs of processes (Cephaloconi) are present in addition to the superior tentacles; these lead up to the tentacles of the Cephalopoda. In Pneumodermon, indeed, two of these bodies are beset with suckers.

\section{$\S 254$.}

In the Cephalopoda the large number of tentacles, which are arranged in rows on either side, and spring from lobate processes, distinguish the head of the Tetrabranchiata. In the Dibranchiata, where they form arms, there is a smaller number of them, but they are larger. The Loliginidæ, Sepiadæ, and Spirulidæ have ten arms. Two of them, which are longer, and in other points different from the rest, are placed outside the circle around the mouth, which is formed by the other eight; and as they spring from pouches which are arranged in pits at the side of the head, they must be distinguished from the inner series; so that these inner ones are always eight in number in 
all Dibranchiata. The arms of the Octopoda, like the similar ones in the Decapoda, are connected together at their bases by a web, excepting the pair which are nearest to the sides of the funnel. This connecting membrane extends farther in some Octopoda; sometimes over a few of the arms only (four in Tremoctopus), or over them all (Histioteuthis, more completely in Cirroteuthis), and is continued right up to the tips of the arms.

The suckers are special structures found on the arms of the Cephalopoda; they generally beset the oral surface of the arms in two rows (one in Eledone), and not unfrequently they are carried on stalks. Their free edge has often a cuticular thickening which has the form of a chitinous ring, and is sometimes toothed. Where a particular tooth is largely developed the sucker disappears, and is replaced by a hook (Onychoteuthis).

In many Cephalopoda certain of these arms are peculiarly altered by functioning as copulatory organs; even in Naqutilus the tentacles perform this function. It is not always the same arm that is thus metamorphosed; as a rule it is one of those which belong to the so-called ventral side of the animal. The mode of metamorphosis varies greatly in the different divisions; it may merely consist in the alteration of a part of the base of the arm (Sepia), or a more or less large number of the suckers may be altered, or the tip of the affected arm may be provided with a spoon-like hollow process (Octopus, Eledone).

The highest grade of this adaptive metamorphosis is seen when the arm becomes greatly increased in size, as well as different in organisation inter-

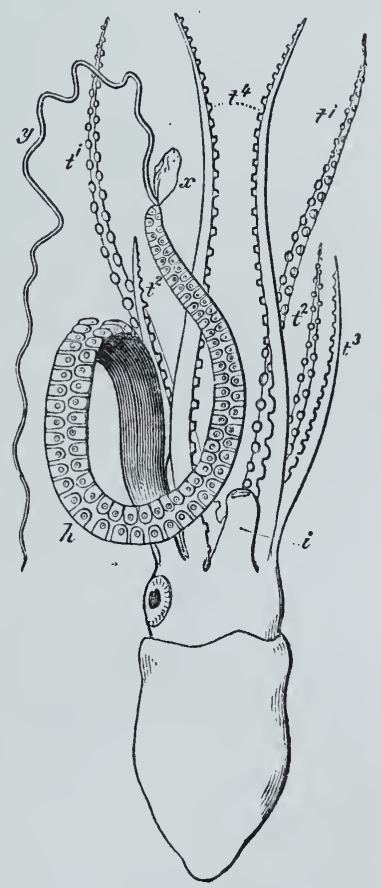

Fig. 173. Male of Tremoc. topus carenæ. $\quad t^{\mathrm{T}}$ Superior; $t^{2}$ Second pair of arms. $t^{3}$ Third left arm. $t^{4}$ Inferior pair of arms. $h$ Hectocotylus. $x$ Its terminal vesicle. $y$ Filamen. tous appendage, set free from the terminal vesicle. $i$ Funnel. nally (Argonauta, Tremoctopus). This "hectocotylised arm" is not developed, as are the others, by a process of free gemmation, but it is formed in a vesicle, from which it is not set loose till it is mature. The greatly-coiled flagelliform end of the arm (Fig. 173,y), which is not set free till the time of copulation, has a similar covering. This appendage, with its investing membrane $(x)$, corresponds to the modified end of the arm in Eledone and Octopus. The more highly differentiated copulatory arms may continue to live within the mantlecavity of the female for some time after they are broken off; this 
is the reason why these separate arms were formerly regarded as parasitic organisms (Hectocotylus).

Steesstrup, J. J., Hectocotyldannelsen. Kongl. Dansk. Vid. Selsk. Skrifter. V. R. $4 \mathrm{Bd}$.

\section{Integument.}

\section{$\S 255$.}

The body of the Mollusca is covered by a soft dermal layer, which is, as a rule, so closely interwoven with the subjacent muscles as to form a kind of dermo-muscular tube, just as in the Vermes. The locomotor organs are formed by the great development of the musculature in certain regions of the body, and by the consequent differentiation of some parts of the dermo-muscular tube.

In most divisions of the Mollusca there is an investment of cilia during the larval stages, which later on extends over the whole, or some parts of the body. The cilia in the velar circlet $(\$ 248)$ are those that are most markedly developed. The rest are chiefly found in the respiratory organs. In the Cephalopoda even, nearly the whole surface of the germinal disc (save the gills) is covered with cilia during development; later on a ciliated epithelium may be found on the yolk-sac also.

The integument is easily distinguishable into an epidermis and cutis. In many Heteropoda (Carinaria, Pterotrachea) the latter is specially modified; a strong transparent layer of connectire tissue preventing any great amount of change in the form of the body. In the rest of the Mollusca the body is generally prevented by the shell, which is developed from the integument, from undergoing any great changes in form.

The coloration of the body is due to the deposits of pigment in the integument. The most remarkable structures concerned in coloration are those which are found in many Pteropoda, and in all Cephalopoda-the "chromatophores." They are rounded cells, which are placed at various depths in the integument; they are filled with granular pigment, and are provided with radial muscular fibres at their periphery; when these fibres contract the cell broadens out, and the pigmented contents are thereby distributed in such a way that they become easily visible to the eye, as large, stellate, and often branched spots. Plate-like elements are found deposited in a layer, which is sometimes differentiated, and these give a silvery appearance to many parts of the body (spangled layer). The varying character of these several layers produces that play of colour which we admire in the skin of the living Cuttlefish.

Other deposits are found in the integument, such as those formed of carbonate of calcium, which are common in the Gastropoda; these are either simple granules, or larger rounded concretions, or they may be rod-shaped, denticulate, or even branched; there is often a 
large number of them, so that they may form a veritable calcareous network, as in Doris, Polycera, etc., the various species of which are distinguished by the special manner in which the various calcareous rods are grouped or arranged, as well as by the way in which they are formed.

\section{$\S 256$.}

The glands are differentiations of the epidermis; they partly resemble the structures found in Vermes (unicellular glands). Whell simplest, these organs are modifications of epidermal cells, which are placed between other cells, but are distinguished from them by having finely-granulated contents and a mouth (goblet-cells). They are found in the Lamellibranchiata as well as in the Gastropoda. In the Cephalopoda they are more commonly arranged in groups, and their blind ends extend below the level of the epidermis. In the Gastropoda-and especially in the terrestrial Pulmonata-they are found to be placed still deeper in the integument. These glands are variously modified in different parts of the body. Those found at the edge of the mantle in the shelled Gastropoda are examples; they secrete a fluid in which calcareous salts are dissolved, while others secrete colouring matters.

In Aplysia the dermal glands secrete a dark-red fluid. In Murex and Purpura a layer of epithelium, which is placed between the gills and the hind-gut, and in the mantle-carity, functions as a gland; this layer is formed by large superficially-ciliated cells. Their secretion gives rise to the substance known as Tyrian purple.

Some Opisthobranchiata (Eolidiæ) are characterised by the possession of urticating cells, which are placed on the ends of their dorsal papillæ.

The Byssus-gland of the Lamellibranchiata is a more independent glandular organ of the integument; when it is formed the foot undergoes certain modifications; it becomes, that is, a tongucshaped process grooved on its ventral surface. The groove passes to a depression at the base of the foot, at the bottom of which there is a gland which secretes the so-called "byssus." Pecten, Lima, Arca, 'I'ridacna, Malleus, Avicula, Mytilus have an organ of this kind; but it may be considered as generally present, for it is found for a time in the embryos of the Naiades and of Cyclas. Some divisions of the Gastropoda (Helicinæ, Limacinæ) have also a gland in their foot, which opens anteriorly and below the mouth. A large number of other kinds of glandular organs are also developed from the integument.

\section{Shells.}

\section{$\S 257$.}

The tegumentary investment is of special importance, for it secretes firm substances, which are laid down in layers, and which 
produce the varied forms of test and shell which characterise the Molluscan phylum. The hard structures, therefore, of this division differ essentially from those which are found in other classes of animals, by the way in which they are developed. They are products secreted from the body, and deposited on its exterior, and are of great importance as organs for the support and defence of the organisms to which they belong. They, just as much as other differentiations from the integument, imply that the outer dermal layer has a secretory activity. Although the outer layers of these structures often appear to be-as is especially the case in large shells-distinct from the organism, the shells always do form a part of it, and are at many points directly and closely connected with it; as, for example, at the insertion of the muscles into the shells.

The presence of calcified spicules in the integument of the Placophora calls to mind the relations which obtain in the Solenogastres (p. 139). The spicules arise in follicles, and do not reach the surface till they are larger in size, when they form slender and closely-approximated fine processes, or thicker bodies, which are distributed over the mantle. Eight large calcified plates are also arranged transversely on it, and form a series of skeletal parts, the arrangement of which implies that the body is arranged in a metameric fashion. As in Cryptochiton they are covered by the mantle, there is some reason for supposing that they were developed within it, and that they resemble the spicules. The plates would then be structures of the same kind, which had been greatly developed, while the spicules would be parts which had not enlarged laterally, but only vertically. This relation between the presence of a mantle and the formation of firm organs, which, when largely developed, form shells, is typical of all the other Mollusca; and the two kinds of organs are always intimately connected. Instead, however, of the dorsal plates being developed, as in Chiton, the formation is continuous, so that it gives rise to a single shell. The shell, therefore, just as much as the mantle-which we have seen to be homologous throughout the series of Mollusca-must be regarded as an organ, which is widely-distributed because inherited, great as may be the adaptive modifications which it has undergone.

The multifid shell was not replaced by the undivided one by any new process, but by the development of one part, for we cannot imagine that the shell, which, as an organ investing a large part of the body, is one of so great functional importance, appeared all at once. But, if the shell was at first an inconsiderable organ, it could not have attained to that perfection of function which would have been the cause of its being transmitted as a useful arrangement. We must therefore suppose that the structure, which later on formed the shell, was primitively one of several similar organs, and that it gradually got the better of the rest. This gradual development of the shell is the only mode which is intelligible, while at the same time it connects together the multifid shell of the Placophora and the single shell of the Conchifera. 


\section{$\S 258$.}

The earliest rudiment of the shell appears at the aboral end of the embryo, at a spot which is distinguished by the growth of its ectoderm. A viscid substance is secreted in the gland-like invagination which appears at this point (Fig. 174, s). This substance gradually fills up the invagination and reaches the surface, where it is hardened as soon as it comes in contact with the water $\left(s^{\prime}\right)$. When the invagination disappears its edges remain as a raised ridge, and so form the rudiment of the mantle, which is therefore very closely connected with the formation of the shell. This arrangement, which has been made out in the larger divisions of the Conchifera, points to the common origin of this group, while it also affords an explanation of the cause of the different ways in which their shells are formed. When this invagination disappears the shell becomes external,

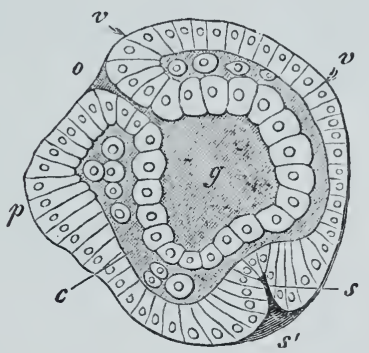

Fig. 174. Embryo of a Heteropod, transverse section. o Mouth. $v$ Velum. $g$ Enteric cavity. $p$ Foot. $s$ Shell-gland (after H. Fol). and then the edge of the mantle either remains below it or more or less covers it. T'he latter arrangement shows how the external shells are connected with the internal ones, which are formed when the invagination does not disappear, but becomes still further developed in the manner already indicated. The shell is then secreted from the inner face of the walls of this organ, and it varies greatly in character in various divisions, just as do the external shells.

When simplest, all the lamellæ of the shell are similar in character; in many, when in their lowest conditions, it is perforated by pore-canals. The simple condition is complicated by the appearance of layers of prisms set obliquely or perpendicularly to the lamellæ.

The shell increases in surface at its free edge, the deposits occurring in layers, and at the side of the mantle; superficially they have the appearance of concentric rings. The shell becomes thicker internally by being supplied from the outer surface of the mantle. These varying modes of formation give rise to variations in the structure of the formed shell, the inner portion of which often consists of a large number of superjacent folded layers, the presence of which is the cause of the nacreous appearance of the shell (motherof-pearl). These layers are covered by the external more complicated and compound layers, which are formed by the edge of the mantle. The horny covering (periostracum) of many shells is due to the same part.

The shell of the Lamellibranchiata, like the mantle, is developed on either side of the body, but it is not calcified in the middle line, so that it forms two valves, which are connected with one another 
along the median line by means of the uncalcified portion of the shell. The "hinge" is formed at the point where the two valves pass into one another; the non-calcified chitinous substance which connects the two shells forms the ligament. Its layers pass into those of the shells; and the two valves are seen to be merely parts of a structure which is rudimentarily single, and later on becomes so again, and is homologous with the shells of the rest of the Mollusca. Near the ligament the valves form alternating and interlocking processes (cardinal teeth) which serve to close the shell more perfectly.

The shells of the Gastropoda are most markedly distinguished from those of the Lamellibranchiata by the absence of any uncalcified portion. They are not unfrequently internal.

This internal position is generally found in those Tectibranchiata that have a rudimentary shell, and in some Pulmonata. In the latter (Helicinæ) the shell very soon becomes external, while in others (Limacinæ) it remains rudimentary, and placed within the mantle. Sometimes it merely consists of a few calcareous concretions.

The various stages of atrophy are also to be seen in the shells of some other divisions, as, for example, in the Heteropoda, in which the rudimentary shell of Carinaria is intermediate between the shell of Atlanta, which covers in the whole body, and that of the Pterotrachex, where it is altogether absent. But these latter have a temporary shell during their larval life, which covers in the whole body, just as it does in the Opisthobranchiata, which are also shell-less in later life. Its general presence points to its being a common heritage of all the Gastropoda, some divisions of which lose it early. The Thecosomatous Pteropoda resemble the Gastropoda in forming a shell.

The animal does not always occupy the whole of the shell. Many Gastropoda withdraw themselves from the end of their shell as growth proceeds; and the end is then shut off by a layer of shellsubstance. The same thing happens in some Pteropoda (Chreseïs), and indicates the commencement of that arrangement which is so much more distinctly marked in the Cephalopoda.

The substance of the shell, which is a product of excretion from the mantle, varies very greatly, from the soft structures of some to the firm solid parts which form the shells of most Prosobranchiata. The soft form of shell consists of organic substance merely. Shells become firmer and horny in character when impregnated with calcareous salts; and when the inorganic substance forms the greater part of the shell we get strong coverings.

The simple condition of the cup-shaped embryonic shells persists in some Gastropoda, and by growing regularly gets to have a more or' less flattened or conical shape (e.g. Patella); in most, however, it becomes spiral by growing out unequally, and these spiral forms may again undergo all kinds of modifications. As the embryonic shells serve to shelter the whole body, even in those which lose them later on, we must look for the typical form in them; from it all the other 
forms of shell have branched off. Derived from these we find on the one hand those which are more highly developed, and on the other those rudimentary forms which have the character of degenerate shells.

\section{$\S 259$.}

The simpler shells of the Cephalopoda must also be regarded as rudimentary structures, and not as early forms; as derived, in fact, from the more complicated and perfect forms, even if their geological succession did not indicate that the shell has undergone gradual

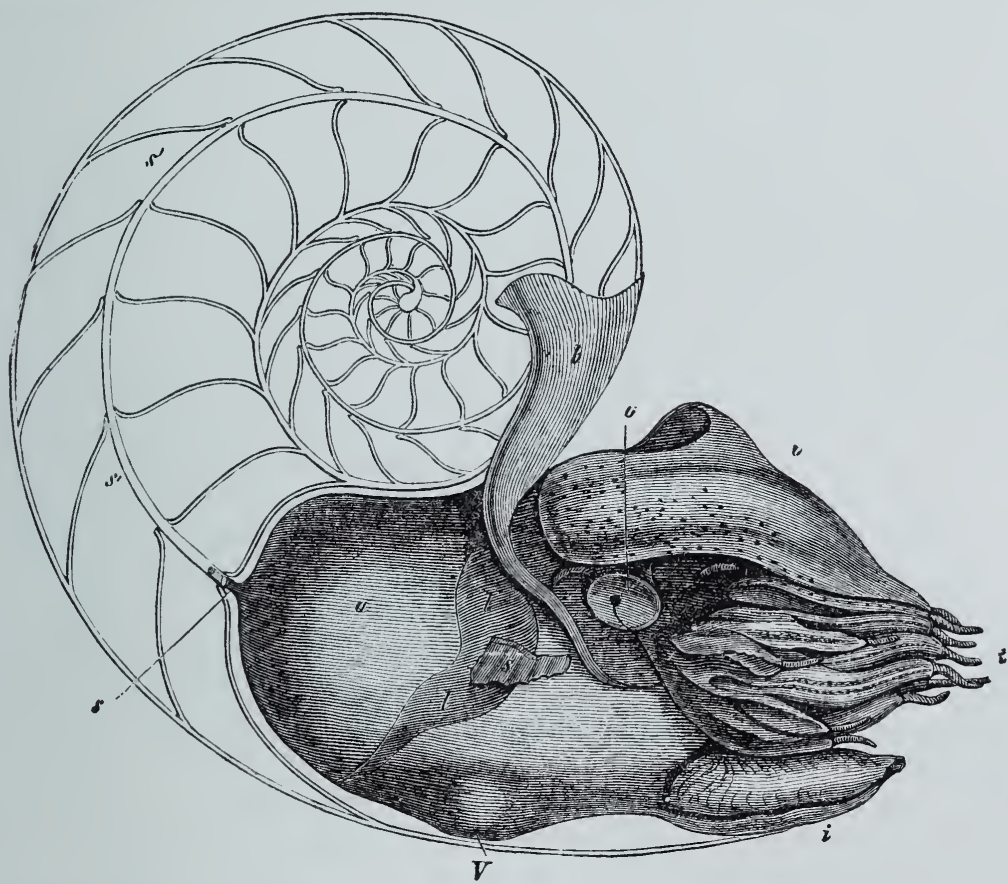

Fig. 175. Nautilus; median section of a shell. $i$ Funnel. $t$ Tentacles. $v$ Cephalic lobes. o Eye. $b$ Dorsal lobes of the mantle. $l l$ Connections between the shell and the mantle. s Part of the shell still connected with the right pallial muscle. a Mantle. $s$ Siphon. $s^{\prime}$ Siphonal canal of the shell (after Owen).

reduction. Their structural characters, as well as their relations to the body, that is, to that portion of the dorsal integument which represents the "mantle," are further instances of the arrangements which we have already described. We either meet with straight (in extinct families only) or coiled shells, which are formed by the mantle, and either completely enclose the animal, or are rudimentary and contained within the mantle; these latter have lost their significance as shells and only form internal organs of support.

The well-developed shells of the Cephalopoda, as seen in the fossil Ammonites and Orthoceratites, and in the extant Nautilus, 
differ somewhat in structure from those of the Gastropoda and Pteropoda. They are divided into successive chambers, the most anterior of which is alone occupied by the animal, although the hinder ones are closely connected with it by means of a tubular prolongation (siphon), which is given off by the animal, and traverses the partitions between the chambers. The animal (cf. Fig. 175) occupies therefore the last-formed or youngest chamber only. The separate chambers correspond to an equal number of stages in its growth; as each segment of the shell was formed the animal left the one before occupied; and as a partition was formed a new chamber was developed. The arrangement, which was merely indicated, and rarely seen, in the Gastropoda and Pteropoda, has become typical in the Cephalopoda. The straight shells of the fossil Orthoceratites, and those of the Ammonites, which are coiled in one plane, as also the shells of the Nautilidæ, are formed on this type. In these last a lobe of the mantle (Fig. 175, $b$ ) extends from the dorsal side of the animal over a portion of the shell, the greater thickness of which appears to be partly due to it. The shell of Spirula is completely covered in by the mantle, and is similar in character to that of Nautilus, except that its coils are not in contact with one another; the shells of the fossil Belemnites are intermediate between those perfect ones which are only enclosed by the mantle and those which are placed within it. On account of this the remnants of shells, which were in all probability largely internal, are of great morphological value. In them the chambers are found in a small conical portion only-the so-called phragmocone. The separate chambers, which form the parts of the phragmocone, look like horizontal sections of a cone superimposed on one another; they, too, are connected with one another by means of a siphon. The whole phragmocone is surrounded by thickened layers; but these are not distributed equally over it, but form a strong solid process (rostrum) behind its apex. The broadened lamella-like portion of the thickened layers, which extends forwards over the base of the phragmocone, is known as the "pro-ostracum." The phragmocone is the homologue of the chambered shell of the other Cephalopoda, while the projecting lamella-the so-called pro-ostracum-is a continuation of the wall of the most anterior chamber, and the massive rostrum, which is generally the best preserved portion of the whole shell, is derived from the simple thickened layers, which are formed from that part of the mantle which is turned over the shell.

The so-called "os sepiæ" of the Sepiadæ is a flattened shell which is completely hidden in the mantle; its posterior tip, however, often projects, and so calls to mind the shell of the Belemnites. It consists of several layers, which are rich in organic substances, and are separated from one another by layers of calcareous deposits in such a way that it appears to be made up of superimposed lamellæ. The outermost lamella, which is turned towards the dorsal surface of the animal, is pretty firm; it passes directly into the posterior end 
of the shell, and forms the groundwork for the lamellar deposits, which often become very thick on the inner face of the shell, the curve of which is slight. These shells may be directly derived from those of the Belemnites, especially if we take into consideration those shells which have a free projecting tip, like the shell of S. Orbigniana. The solid tip corresponds to the rostrum of the Belemnites, while the alveolar cavities of these latter, and the pro-ostracum, which is continued on from their dorsal surface, is homologous with the rest of the shell of the Sepiadæ. The partitions which form the chambers of the phragmocone in the alveoli of the Belemnites are represented by the flat or slightly concave lamellæ of the shell of the Sepiadæ. The layers succeed one another directly, instead of forming separate chambers. In this way the complicated shell of the Belemnites, when reduced, may be easily seen to be represented in a lower condition of the shells of the Sepiadæ. The shell of the Loliginidr is still more reduced; it is merely formed by an elongated flexible horny-blade (calamus), which is placed in the dorsal region of the mantle. An outwardly projecting carina extends along the middle line. This rudimentary shell corresponds to the external, curved, and more highly organic portion of the shell of the Sepiadx, and is therefore homologous with the horny-blade of a Belemnite shell. Finally, in the genus Octopus, where the mantle is not separated from the head in the region of the neck, we find a pair of thin plates, embedded in the dorsal integument; these are the last traces of a shell formed by the mantle, and are in all respects comparable to those described as existing in the Gastropoda. Inasmuch as the shell is, even in the Cephalopoda, formed in the earliest stage by an invagination of the mantle (Sepia), the internal and external shells are closely allied; and at the same time we may see that they are connected with the shells of other Mollusca.

The shell of Argonauta is to be regarded as altogether different from all these shells, which are intelligible when closely compared; it is not secreted by the mantle, but by a pair of arms, which do not lay down lamellar deposits. In the Gastropoda we met with a special arrangement by which the so-called "operculum ". was formed; we found this on the dorsal surface of the end of the foot in many Prosobranchiata, where it served to shut in the animal, when it was retracted into its shell. The question now arises, may not this structure be also derived from one plate of the Placophora?

[E. Ray Lankester, Developt. of the Pond Snail. Quarterly Journ. Microsc. Sci. 1874.]

\section{Branchiæ.}

$\S 260$.

The kind of respiratory organs-branchiæ-which obtains in the Mollusca is in correlation with their aquatic habitat; these are always differentiations of the integument, and have consequently a 
primitively superficial position, although they become covered over by folds of other regions of the integument (mantle), and so come to be placed in a special cavity - the branchial cavity.

The function of respiration is part of the duty of the integument, but it does not seem to be always localised in homologous regions, so that we cannot regard all the organs which appear to be gills as morphologically identical. As a rule the gills of the Mollusca are processes which are placed at the sides of the body, and when least metaniorphosed arise between the foot and the mantle (cf. Fig. 167, $A B b r$ ). They vary very greatly, not only as to the extent of the body which they occupy, but also in the way in which they are connected with different parts. In the Placophora they merely form a series of folds or lamellæ, which encircle the body

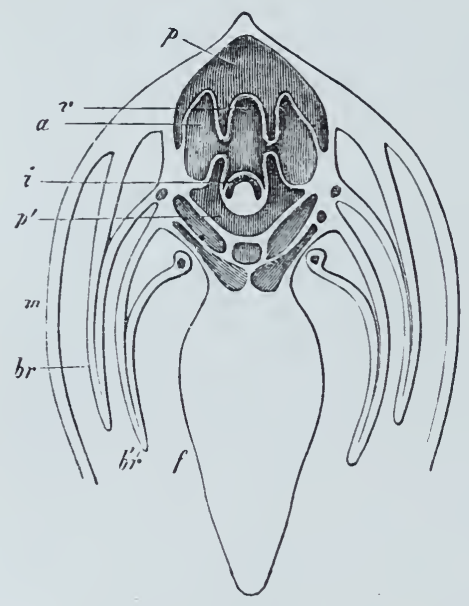

Fig. 176. Vertical section through an Anodouta. $m$ Mantle. $b r$ Onter; $b r^{\prime}$ Inner gill-lamella. $f$ Foot. $\imath$ Ven. tricle. a Auricle. $p p^{\prime}$ Pericardial cavity. $i$ Enteric canal. between the foot and the mantle, and appear to be formed from the epipodium (epipodial gills).

In the Lamellibranchiata they form lamellar organs, which project between the mantle and the visceral sac, which ends with the foot, into the cavity enclosed on either side by the mantle (Fig. 176, $\left.b r^{\circ} b r^{\prime}\right)$. Their free edge is directed towards the ventral surface.

Almost all the Lamellibranchiata have two pairs of these gills on either side, an inner pair, which are placed mediad, and an outer pair at the sides of these. The former are often the larger. Except in Anomia, where there are a large number of other adaptive modifications, the gills are arranged symmetrically. Each gill-lamella is developed from a row of processes which bud out close to one another; in many forms these processes remain separate from one another, and form separate branchial filaments, parallel with each other (Mrtilus, Avicula, Arca, Pectunculus, Pecten, Spondylus). In most, however, the gills lose this embryonic condition, owing to the concrescence of the gill-filaments. Owing to this union of the flattened filaments or lamellæ, which have their surfaces directed towards one another, a gill-plate is formed; this is either effected by the mere adhesion of the filaments, or by concrescence; in the latter case pad-like projections appear on each of the gill-filaments at regular distances from one another, and these unite together. As fine clefts are left between these junctions, by which the water passes through the gills, each plate forms a kind of lattice-work. These filaments are not simple prolongations, but loops, so that they enclose a space (intrabranchial space); when the gill- 
filaments grow together this space traverses the whole of the gillplate, and communicates with the exterior by means of the clefts between the filaments. The water which enters by these clefts is collected into a canal at the point where the plate is attached; and is carried by it to the hinder end of the body.

There are chitinous rods in each of the gill-filaments, which form a special organ of support.

The surface of the whole gill is covered by ciliated epithelium. Rows of large cilia extend along the ridge-like projections of the gills; between these there are finer closely-packed cilia, and the two together keep up a continual stream of water. There is a groove at the free edge of each gill-plate; this is formed by depressions on each of the gill-lamellæ and is invested by longer cilia; it leads to the mouth, and so produces a current of water, which is well adapted to bring in nutriment.

Great modifications of this system are brought about by the fusion of the gills of the two sides; this, which obtains posteriorly to the foot, is either effected by the direct junction of the free edges, or by the development of a special membrane, which unites the gills of either side together. This fusion is best seen in the falciform curved gill-plates of Anomia, where the whole branchial apparatus is separated from the greatly-reduced visceral sac, and is no longer lateral in position.

[R. Holman PECK, The structure of the Lamellibranch gill. Quart. Journal Microsc. Sci. 1876.]

B onnet, R., Der Bau u. die Circulationsverhältnisse der Acephalenkieme. IIorphol. Jahrb. III.

\section{$\S 261$.}

The branchial apparatus of the Gastropoda, though greatly varied in details, is arranged in very much the same way as in the Lamellibranchiata; that is to say, it is typically made up by lamellæe, or by more distinctly cylindrical processes, which are arranged parallel to one another. These project from the surface of the body, and are, therefore, bathed by the surrounding medium, the water, while a current of blood passes along them internally. This similarity is still more marked by their position relatively to the mantle, for they have just the same relation to it as have the gills of the Lamellibranchiata. As compared with these latter they are less numerous and more confined; their structure is comparatively much simpler. The epipodial gill is arranged circularly in the Placophora, as it is also in Patella; but in other Patellidæ (Lottia) the two pinnate branchiæ are more distinctly dorsal, so that they seem to be different from the epipodial gills. Fissurella and Emarginula also have their two gills placed in the anterior region and below the mantle. In Haliotis also, they are distinctly paired, but they are placed more to the left hand of the animal. They are also characteristically modified in the Zeugobranchia. In the Anisobranchia the left gill is smaller, and the right one more largely developed; this arrangement, which is 
distinctly allied to the arrangement in the Zeugobranchia, is adapted to the asymmetry of the branchial cavity, which, again, is dependent on the characters of the shell. The smaller gill is generally approximated to the other, and becomes asymmetrical in position; in some Prosobranchiata it disappears altogether (Janthina, Neritaceæ, Heteropoda).

The right gill is generally developed on one side only, so that it is semi-pinnate, owing to the disappearance of the second row of lamellæ. Although, as a general rule, we find the lamellar structure to be the most common, a few (Calyptræa, Crepidula) have filamentous gills, and so call to mind the primitive form of the Lamellibranch gill.

The gills become modified, and may disappear altogether when the mantle and the branchial cavity are atrophied. This happens in various divisions; thus, in the Heteropoda, among the Prosobranchiata, the gill of Carinaria is not covered over by the mantle; in Pterotrachea, where there is no mantle at all, the gill is quite free, while in Firoloïdes, the gill, as well as the mantle, disappears.

Among the Opisthobranchiata the characters of the gills are equally dependent on the condition of the mantle. There is a gill on either side, between the mantle and the foot (Pleurophyllidia), or there is only a single gill in the gill-chamber, or, finally, the gill is only partly covered over by the mantle (Tectibranchiata). When

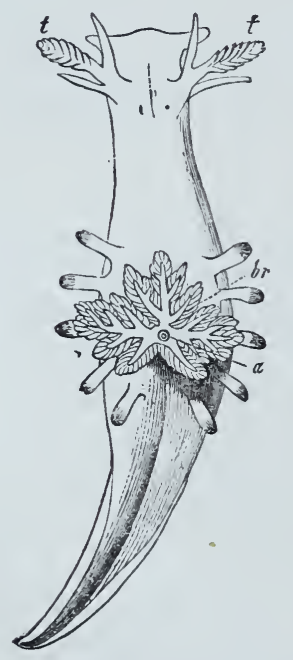

Fig, 177. Ancula (Polycera) cristata; dorsal view. a Anus. br Branchiæ. $t$ Tentacles (after Alder and Hancock). the shell and mantle disappear, gill-like structures may be developed on the dorsal surface of the body, as in some of the Nudibranchiata.

Lamellar, or tufted and branched appendages, are sometimes developed in the anal region (Doris), sometimes in rows over the whole body (Tritonia, Scyllæa). If we are right in regarding the possession of a shell by the larvæ of all the Opisthobranchiata as a fact, which proves conclusively that these Gastropoda are derived from shelled forms, and if we must suppose that the primitive position of the gills was within the mantle-cavity, then we must regard the arrangement of the gills in the Dorididx as having been inherited in its essential features from this condition, for we must remember that the anus also is placed in the mantle-cavity. There are many intermediate steps between this and the more general distribution of gills over the back of the body; and further, these gills, howsoever modified, and howsoever peculiar their form, are never anything more than mere processes of the integument. Their varied external form is due to their superficial position, which is due to the loss of the mantle which invested them ; and it is because of its absence that they 
lose their apparently specific structure, and get to be more and more like the surrounding integument, of which they form processes varying greatly in character. Their relations to the circulatory apparatus are of great significance as bearing on this view, for they so far agree completely in character with the true gills. Lastly, when most differentiated, the gills are seen to be distributed over the whole of the dorsal region of the body, where they form one or more rows of papillæ, or villous processes on either side; these again may be branched (Aolidiæ). The loss of the shell is the cause of the wider distribution of the gills, just as, on the other hand, the formation and development of this organ of defence was the cause of the gills being more limited in extent.

These gills are atrophied in many Opisthobranchiata, when the whole of the integument takes on the respiratory function (Phyllirhoë, Elysia, Pontolimax).

\section{$\S 262$.}

Another arrangement of the respiratory apparatus, which is a modification of the one first described, is due to the development of the respiratory canal-system in the walls of the mantle-cavity. In many of the branchiate Gastropoda this network of canals extends beyond the gills into the neighbouring parts of the branchial cavity, which are thereby enabled to take part in the respiratory function. In this way the mantle-cavity is adapted to taking in air, and becomes a lung. An organ of this kind-which is not at all adapted for those Molluses that are so organised as to be fit for an aquatic life -is found in various forms, which belong to very different divisions, and it is to be regarded as due to a change in their mode of life. A lung is present, in addition to a gill, in Ampullaria; in this animal it forms a sac, which is placed by the side of the gill, and is provided with a contractile orifice. In the terrestrial genus, Cyclostoma, the gill has disappeared altogether.

There is a lung in Onchidium, but it is also a renal organ. A similar cavity is found close to, and has the same orifice as, the renal organ in the Helicinæ and Limacinæ; this functions as a lung. In the Lymnæidæ and Planorbidæ, however, the mantle-cavity itself is adapted to the reception of air. But in these forms the abranchiate mantle-cavity also serves as a water-breathing organ, for many Lymnæidæ are known to live always in deep water.

\section{$\S 263$.}

In the Gymnosomatous Pteropoda either the whole of the integument (Clio) serves as an organ of respiration, or processes are developed from its surface which function as gills (Pneumodermon). In the thecosomatous forms only do we meet with plaited folds (Hyalea), which are placed in the mantle-cavity (Fig. 171, $A$ br), and so far resemble the arrangements which obtain in the rest of the Mollusca; their position is the same as that of the gills in the 
Cephalopoda. In these latter the gills arise in just the same waybetween the mantle and the foot (Fig. 171, B br) - as they do permanently in many Gastropoda. When, however, the mantle is developed they sink downwards, and are then placed in a mantlecavity, which, as compared with the Gastropoda, appears to open on the hinder surface. The gills are arranged symmetrically in all of

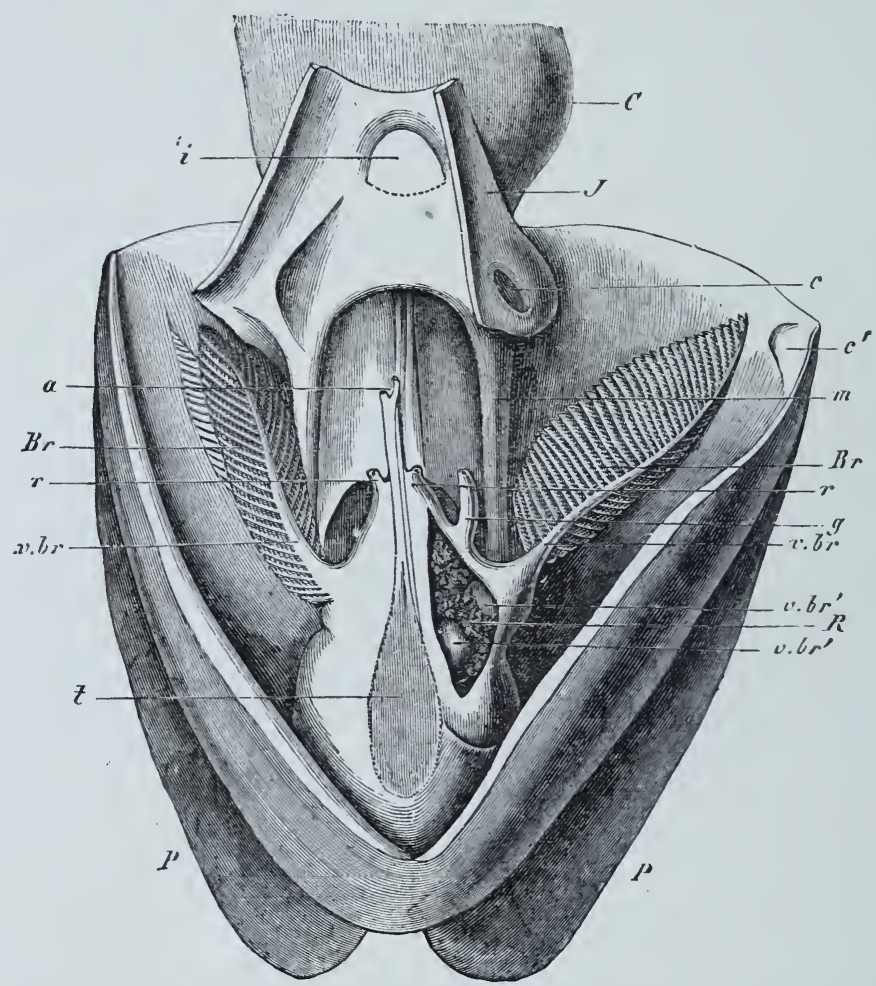

Fig. 178. Mantle-cavity and funnel of Sepia officinalis. The mantle-cavity has been opened by an incision along the middle line. In it is seen the visceral sac projecting, while posteriorly to it two muscular branches are given off $(\mathrm{m})$ to the funnel and head. $B r$ Branchix. $v b r$ Branchial vein. $v b r^{\prime}$ Its bulbous enlargement. $t$ Ink-bag. $r$ Orifice of the excretory organ, opened on the right side, and displaying at $R$ the venous appendage. $g$ Genital papilla. $a$ Anus. $J$ Funnel, opened by an incision along the middle line. $i$ Tongue-shaped organ. $c$ Depression for the process at the edge of the mantle (pallial hinge) $c^{\prime} . \quad C$ Head. $P$ Fins.

them; there are two pairs in the Nautilus, but only one in all the other extant Cephalopoda.

As a rule, each gill is pyramidal in form, with the apex directed towards the side, and its base towards the middle line (Fig. 178, $B r$ ). It either consists of closely-approximated lamellæ, which gradually increase in number at the tip (Nautilus, and most Loliginidæ), or of several much-coiled dermal folds, which arise between the two branchial vessels which pass to the edge of the mantle (Octopoda). 
In this group the mechanism of respiration is combined with the locomotion of the animal. Each time that the muscles of the edge of the mantle relax, water passes into the branchial cavity by its orifice, that is, at each side of the funnel; after it has bathed the gills it is driven out again by the contractions of the mantle. At this moment the cleft of the branchial cavity is closed, so that the water cannot get out except by the funnel, and this serves not only as the passage by which the water reaches the exterior, but also takes an active share in driving it out.

\section{Internal Skeleton.}

$\S 264$.

In most Mollusca the absence of an internal skeleton is compensated by the shells and tests described in $\$ 258$; for these serve as supports for the internal parts.

Independent internal organs of support are, however, found in the Gastropoda. Two, or sometimes four, small plates of cartilage are found in the head of these animals; they are surrounded by the muscles of the pharynx, and are more or less closely connected with one another. 'They form the supporting apparatus of the radula and the parts connected with it, and also afford points to which some of the pharyngeal muscles, and especially those of the radula, are inserted.

Cartilaginous organs of support are much more highly developed in the Cepha-

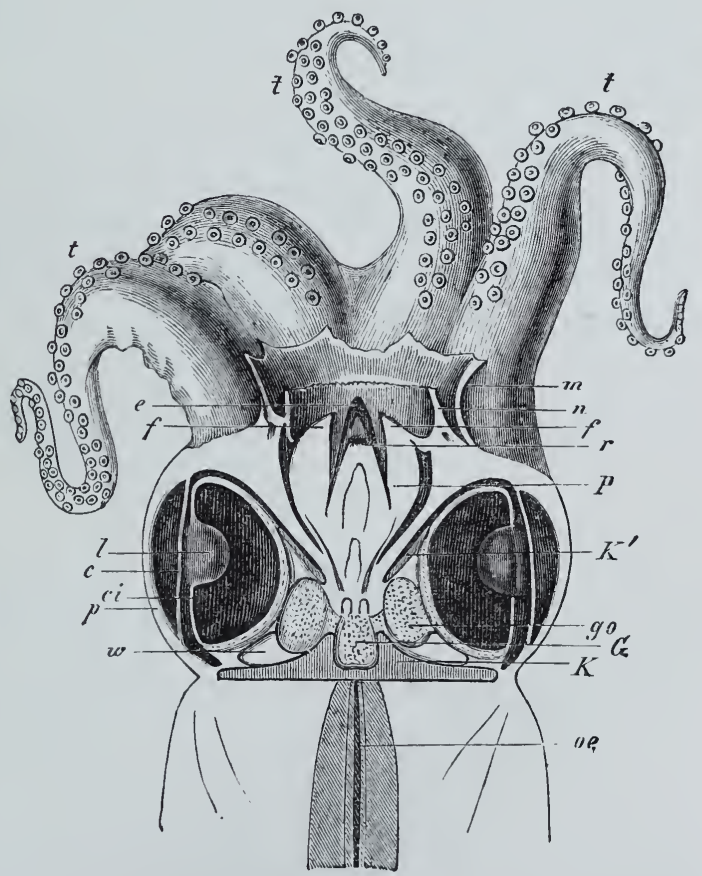

Fig. 179. Section through the head of Sepia offici. nalis. $K K^{\prime}$ Cephalic cartilages. $G$ Cerebrum. go G anglion of the optic nerve. $v$ White body. $l$ Lens. ci Ciliary body. $e$ Cornea. $p$ Eyelid. $P$ Buccal mass. $m$ External, $n$ Internal labial membrane. ef Jaws. $r$ Radula. oe Eso. phagus. $t$ Arms.

lopoda. The most important one lies in the head, where it serves as an investment of the nerve-centres, a support for the optic 
and auditory organs, as well as the point of origin of a large number of muscles. In Nautilus this cephalic cartilage is formed of two pieces, united along the middle line, and drawn out into anterior as well as posterior processes; these surround the commencement of the œsophagus. In the Dibranchiata the cephalic cartilage is much better developed. It consists of a median portion, which is traversed by the œsophagus (Fig. 179, K), and of two lateral processes, which are sometimes mere flat enlargements, in which case accessory cartilaginous plates are added on to them to form the orbits; at other times they are more highly developed, are then continuous with superior processes $\left(K^{\prime}\right)$, and completely enclose the orbits. The central nervous system $(G)$ is placed on that portion of the cephalic cartilage which is traversed by the œsophagus.

The Dibranchiata are provided with additional cartilaginous skeletal pieces. A dorsal cartilage is the most common. In the Sepiadæ this forms a semi-lunar piece, which lies in the anterior dorsal region of the mantle, and is continued into two small lateral cornua; in Octopus, where there is no median pieces, we find the cornua only.

There is a cartilaginous plate in the neck also, in addition to two cartilages at the base of the funnel-the hinge-cartilages. These are less constantly present than those which lie at the base of the fins, and which are found in all Dibranchiata provided with fins, for they serve as the point of attachment for the muscles of these organs.

\section{Muscular System.}

\section{$\S 265$.}

We can understand how it is that separate groups of muscles are so feebly developed, if we bear in mind that there is a dermomuscular tube united with the integument and external organs of support; and that these, notwithstanding the great modifications which they undergo, have very much the same character in all cases. With this may be correlated the absence of internal organs of support in the lower divisions, and their relatively slight development in the higher ones. The muscular system is made up of bandlike fibres, which, not unfrequently, give indications of their greater differentiation by the possession of transverse striæ.

In the Lamellibranchiata the adductor muscles, which pass either transversely or obliquely through the body from one valve to the other, are those that are best developed. There are either two of them, which form bundles separated by some distance from one another, one anterior (Fig. 167, $m a$ ), and the other posterior $(m p)$, as in Unio or Anodonta; or there is but one muscle, which corresponds to the hinder one of the Dimyaria, and occupies the middle of 
the shell (Pecten, Ostrea). Special muscles, which are interwoven with the integument, serve as retractors of the foot; these arise from the dorsal portion of the shell, and are sometimes broken up into several pairs. These retractors are also found again in the shelled Gastropoda. They generally form a single, but sometimes a double muscle, which arises from the base of the shell, and which passes to the anterior regions of the body, increasing in size as it does so. It supplies the foot as well as the head, and the anterior region of the digestive tube (pharynx); while further it gives off special bundles to the other protractile regions, that is, to the tentacles and copulatory organ. The muscle which arises from the columella of the shell, and accompanies it, is known as the columella muscle. In the Heteropoda it has a wide origin in the carinate foot. In the Pteropoda it spreads out into the fins given off from the foot. In addition to these muscles other bundles are given off to the viscera.

The muscular system of the Cephalopoda is much more differentiated, in correlation with the formation of an internal skeleton. Two powerful retractors are attached to the cephalic cartilage in Nautilus; these arise from the sides of the shell-chamber occupied by the animal. In those Decapoda that have an internal shell these muscles take their origin from the wall of the outer wall of the shell; and in the Octopoda, from a cartilage found at that spot. Two branches are given off from these two muscles, which pass to the funnel. Another and much larger pair of muscles arises in the neck of the animal, and broadens out towards the ventral surface, where they pass into the funnel. The muscles in the mantle are also arrangeed in separate layers, as are also the fin-muscles. Lastly, there is the greatly-developed muscular system of the arms, which partly arises from the cephalic cartilage, and surrounds a canal which passes along the axis of the arm.

\section{Nervous System.}

Central Organs and Nerves of the Body.

$\S 266$.

This system of organs also has points in which it resembles that of the Vermes. The whole of the central apparatus, that is, is divided into a superior ganglionic mass, which lies above the commencement of the œsophagus, the supra-œsophageal or cerebral ganglia, and a ventral mass which is connected with the other by commissures, and forms the inferior or pedal ganglia. They are both paired. The earliest rudiment of the cerebral ganglia is seen as a differentiation of the ectoderm, the form-elements of which grow inwards, and are accompanied by the rudiments of the eye (Gastropoda). The relations between the cerebral ganglia, and the higher sensory 
organs which are placed in the head, prove that these ganglia are homologous with the cerebral ganglia of Vermes (and of Arthropoda). The pedal ganglia may also be derived from a more simple condition, for in many of the lower Mollusca we find them replaced by an arrangement which corresponds to the ventral chord of the Annulata. Longitudinal trunks are given off from the pedal ganglia, and are distributed along the foot; since they are connected together by transverse chords, they are arranged in the same manner as a ventral nerve-chain.

Although there may be nothing really fresh in this arrangement of the nervous system, inasmuch as the two ventral (or pedal) ganglia must be regarded as a concentrated nervous mass, which is broken up in lower forms, and constitutes a ventral ganglionic chain; yet the greatly-developed visceral ganglia form an arrangement which is nothing like so well marked in the Vermes as it is here. In the Mollusca the visceral ganglia are not only of importance as forming a part of the general nervous system, but they may also fuse with the cerebral ganglia, owing to the gradual shortening of their commissures. New, and primitively peripherally-placed parts, are thereby added on to these central organs; and it becomes a matter of doubt whether or no these ganglia, which formerly belonged to the visceral nervous system, should still be regarded as belonging to it. 'These parts of the nervous system which supply the viscera (heart, branchial apparatus, and generative organs) are the cause of great complications of the whole system; owing to the way in which they vary in number in different divisions, they make comparison very difficult,

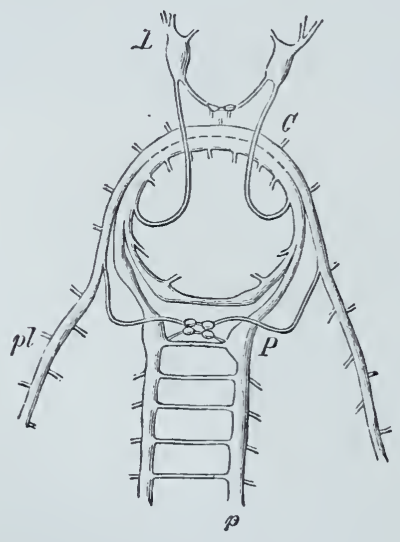

Fig. 180. Nerrous system of Chiton cinerens. $C$ Cerebral nerve-chord. $P$ Pedal ganglia. pl Pallial nerres. $p$ Pedal nerves. $B$ Buccal ganglia (after H. . Jhering). as indeed also do the great modifications in position undergone by the primitive ganglia, in consequence of the abbreviation or elongation of their commissures.

The nerrous system of the Placophora is one of the lowest found. A nervous band formed of two chords (Fig. $180, C$ ) surrounds the oesophagus, but there are no superior enlargements on it; this is probably due to the absence of eyes and tentacles. The inner of the two chords is continued separately below the œesophagus; part meets its fellow of the other side in the subpharyngeal ganglion and part passes on to a pedal ganglion $(P)$. Each of these bilateral ganglia gives off a thick nervetrunk, which passes backwards, and which, like the ganglia themselves, is connected with the trunk of the opposite side by transverse anastomoses set at regular distances; nerves are given off to the foot from corresponding points. The outer chord, 
extending over the osophagus, passes along the sides of the body internally to the branchiæ, and forms the pallial nerve-trunk $(p l)$. There is also a group of four small ganglia below the œsophagus (sublingual ganglia). The two trunks of the pedal nerves are thicker than the commissures which pass to them from the nervous band; they must consequently be regarded as central parts of the system. 'They seem to be the longitudinal trunks of a "ventral medulla," in which the ganglionic cells are not definitely distributed into distinct groups any more than they are in the Gephyrea. The structure of this chord requires, however, to be further investigated. The principal point in the arrangement of the nervous system in the Placophora is that we may recognise in it just the same relation of parts as in the Solenogastres, and specially in Chrtoderma (\$121).

\section{$\S 267$.}

The relatively feeble development of the cerebral ganglia in the Lamellibranchiata is due to the absence of a head, and its related sensory organs. These ganglia (Fig. 181, a) are often placed so much to the side that there is a long commissure between them (Lucina, Panopæa, Anodonta, Unio, Mytilus, Arca, Cardium, Pholas, etc.). A few smaller branches only are given off in addition to the large visceral commissures. There are two pedal ganglia instead of the pedal nervetrunks, the nerves from which are distributed in the ventral portion of the body, and especially in the foot. They are placed at the root of the foot, and are sometimes deeply imbedded in it. The commissural chords vary in length in proportion to the development of the foot, and the distance between it and the anterior parts of the body. When the foot is feebly developed, or when it is produced very

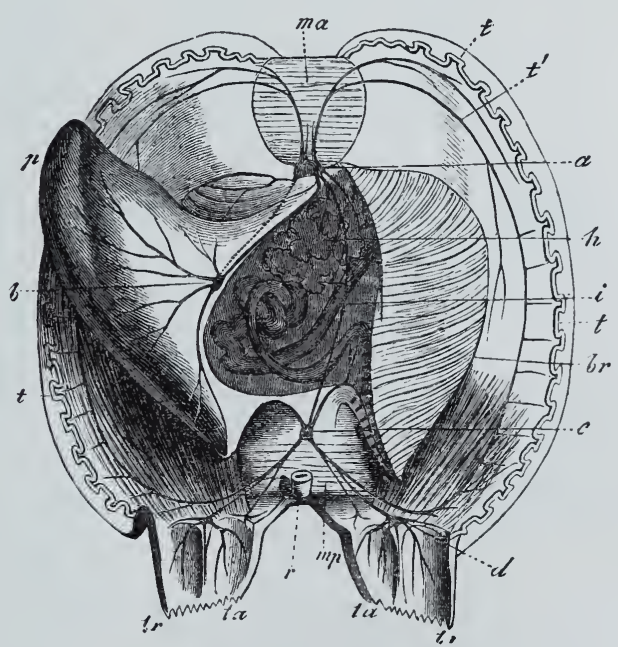

Fig. 181. Nervous system of Cytherea Chione. a Supra-œsophageal (cerebral) gauglion. $b$ Pedal ganglia. $c$ Visceral ganglion. d Ganglia of the respiratory tubes (siphonal ganglia). ma Anterior, $m p$ Posterior adductor of the shell. $p$ Foot. $t$ Edge of mantle. $t^{\prime}$ Nerve to edge of mantle. br Branchiæ. $i$ Enteric canal. $h$ Liver. $r$ Hind-gut. $t r$ Respiratory siphon. ta Cloacal siphon (after Duvernoy). far forwards, the cerebral and pedal ganglia may be markedly approximated (Solen, Mactra). They may indeed come to be directly approximated, as in Pecten (Fig. 182, c), where the smaller pedal ganglia are placed between 
the cerebral ganglia ( $($ ), which are connected together by a deeplycurved commissure. The extent to which the pedal ganglia are developed is dependent on the development of the foot. As a rule they are intimately connected, though always separate. The peripheral nerves of the cerebral ganglia are principally distributed to those parts of the body which lie nearest the mouth, while they also give off branches to the mantle. In some forms these pallial nerves (Fig. 181, $t^{\prime}$ ) have the appearance of two large trunks, in which case they are connected with other nerves given off from the visceral ganglia at the edge of the mantle; and the comnected trunks either form a single larger marginal nerve, or a regular plexus of nerves. The

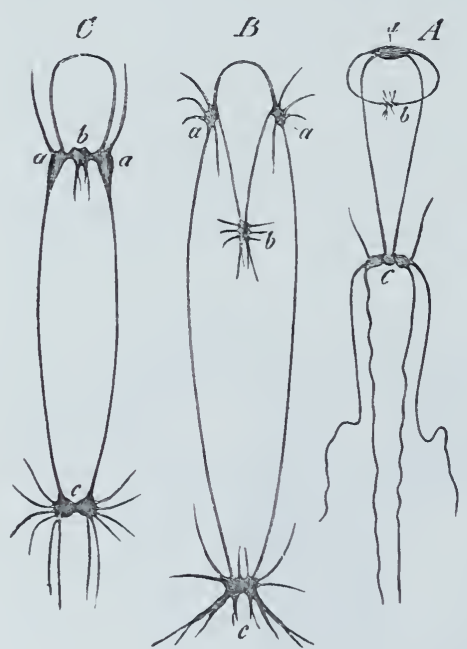

Fig. 182. Nervous system of the Lamellibranchiata. $A$ Of Teredo. $B$ Of Anodonta. $C$ of Pecten. $a$ Supra-œsophageal (cerebral) ganglia. $\checkmark$ Infra-œesophageal (pedal) ganglia. $c$ Branchial or visceral ganglia. visceral ganglion is often the largest of the whole nervous system. It lies behind the posterior adductor (Fig. 181, c; Fig. 182, c), and is connected with the cerebral ganglia by long commissures. We may regard this ganglion as consisting of two halves connected together by a short transverse commissure, which become more or less approximated, and finally form a single quadrangular knot, according as the bilateral gills of these animals are free or fused together. This fact points to the relation between this ganglion and the gills, which indeed is made still clearer by the large nerve-trunks which are given off from it, and which innervate the branchiæ. In addition to the branches which go to the neighbouring portions of the mantle, there are two other large nerves which pass to the edge of the mantle in many Lamellibranchiata, and take part in forming the above-mentioned plexus.

When siphons are developed, large nerves are given off from the visceral ganglia, which do not only ramify all along the respiratory tubes, but also go to form a special ganglion, which is placed at the base of the siphons (Fig. 181, d) (Solen, Mactra, Mya, Lutraria, Cytherea, etc.). Very little is known about the nerves which pass from the visceral ganglia to the various organs of the body. Such nerves have been observed in Pinna and Anomia, as well as in Arca and Solen, where they arise either from the ganglion or the commissures. It is a difficult matter to compare the chords which pass to the visceral ganglion (cerebro-visceral commissure) with the pallial nerves of the Placophora, because of their relation to a ganglion; on the 
other hand, the same nerves in Chætoderma ( $\$ 121)$ are connected with a terminal ganglion.

The nerrous system of the Scaphopoda is almost exactly similar to that of the Lamellibranchiata.

Duvernoy, Sur le système nerv. des Moll. acéphales. Mém. Acad. des Sc. Paris. T. XXIII.

\section{$\S 268$.}

Owing to the distinctness of the head, and the development in it of several sensory organs, which are often very highly differentiated, the nervous system of the Gastropoda is distinguished broadly from that of the preceding divisions by the larger size of its cerebral ganglia. These are connected with the ventral parts of the system by a commissure; in the lowest of the Prosobranchiata-the Zeugobranchia-the nervous system has many points of similarity with that of the Placophora. For instance, a rudiment of a ventral ganglionic chain can be made out in Fissurella (Fig. 183) and Haliotis, inasmuch as the nerves from the pedal ganglia - pedal nerves - are united by transverse commissures. The pallial nerves are not given off directly from the cerebral ganglia, but run below the pedal nerves, and appear to arise from a nervous mass common to themselves and these latter. The epipodium is supplied by the branches of the pallial nerves $(p l)$. Double commissures pass from the cerebral ganglia to the ganglionic mass, which gives off these nerves; one of them is connected with the pedal nervetrunks, and the other with the pallial nerves, that is, with the visceral nerves given off from the same point. In the rest of the Proso-

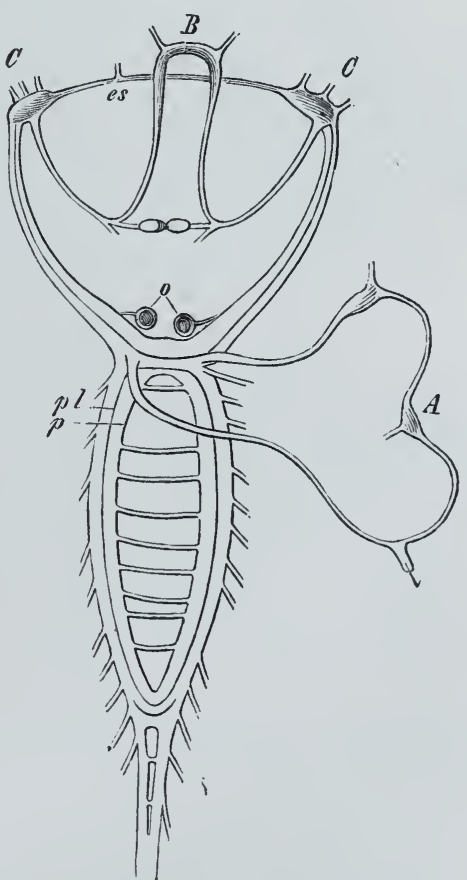

Fig. 183. Nervous system of Fissurella. $C$ Cerebral ganglia. $c s$ Their commissure. $p$ Pedal nerves. $p l$ Pallial nerves. A Visceral ganglion. $B$ Buccal ganglia (after H. v. Jhering). branchiata there are no indications whatever of any ventral ganglionic chain. There are pedal ganglia in which the ganglionic elements, distributed along the nerve-trunks of Haliotis and Fissurella, are distinctly concentrated. At the same time, the relations of these nerves must be more exactly investigated. A ganglion seems to be given off from the secondary pedal ganglia, and to pass laterally into the commissures, and so to be as much 
comnected with the cerebral as with the secondary pedal ganglia. It gives off the pallial nerves; these undergo degeneration in proportion to the extent to which other nerves are given off from the same commissural ganglia.

In some of the Prosobranchiata some of these nerves are remarkable on account of the course that they take. They are present in Haliotis, where they pass off from the common pedal ganglionic mass (the pallio-pedal ganglia). In other cases they are given off from the commissural ganglia (Fig. 184, Co). A nerve is given off on

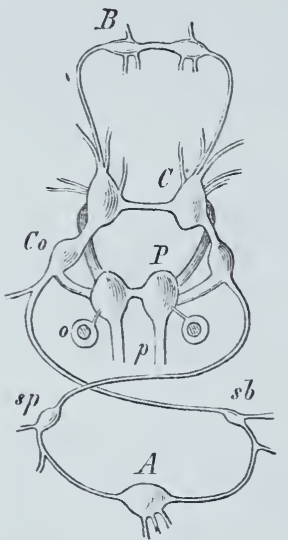

Fig. 181. Nervous system of Paludina vivipara. $C$ Cerebral, $P$ Pedal, $C_{5}$ Commissural gan. glia. $B$ Buccal ganglia. A Abdominal ganglion. $s p$ Supra. sb Subintes. tinal ganglion. $p$ Pedal nerves. o Otocyst (after

II. .. Jhering). the right side which passes above the visceral mass to a ganglion, which supplies the bodywall (supraintestinal ganglion) $(s p)$. From the left commissural ganglion a nerve passes below the viscera to a subintestinal ganglion $(s b)$, which, like the former, is connected by a commissure with an abdominal ganglion $(A)$.

The two nerves, therefore, which are given off from the commissural ganglia cross over one another; this peculiarity - namely, that the right nerve passes to the left, and the left to the right side-makes it difficult to explain the genesis of these nerves. It is probably due to changes in position, which have not affected the internal parts only, for the ganglia on these nerves give off branches to the bodywall. Although a large number of the Prosobranchiata are distinguished by this crossing of the nerres (Chiastoneura), it does not obtain in another division, in which the commissure to the abdominal or visceral ganglion takes a straight course backwards (Orthoneura), except when the ganglion appears to be fused with the right commissural ganglion (Nerita). The commissural are generally separated from the pedal ganglia, and, in the Heteropoda, are widely so (Carinaria), and in this case the commissures are proportionately elongated. The same thing happens also to the cerebro-pedal commissures in the Heteropoda.

There is a commissure between the pedal ganglia in the Tectibranchiata, which are consequently pushed more to the sides of the body. The primitive visceral ganglia are also placed at the sides, or between the pedal and cerebral ganglia (Umbrella, Gasteropteron); there may be commissural ganglia present, just as there are typically in the Prosobranchiata, which send off connecting chords to one or to a pair of ganglia which supply the gills; the ganglia appear to correspond to the above-mentioned abdominal ganglion (Aplysia, Acera).

The pedal ganglia are still more widely separated from one another in the Nudibranchiata, and are thereby approximated to 
the cerebral (Fig. 185); the visceral ganglia also get to be connected with these latter owing to the abbreviation and complete disappearance of the commissures between them (Alolidia). A dorsal plexus of ganglia, which lies on the pharynx, is formed by the more intimate fusion of these ganglia; in each half of this plexus the various ganglia which compose it can be more or less distinctly made out; several commissures are given off, which surround the pharynx (Doris, Tritonia). This connection of the ganglia of either side may lead at last to the complete fusion of the ganglionic masses of either side into a single one, and in correlation with this fusion the previously separate commissures may be represented by one only ('Tethys). This appears to be a lower stage, but it must not be regarded as an early arrangement, but as the final one in a series of differentiations, exactly similar to what we found to obtain in the Arthropoda. In the same way as the nerves, which pass off from the single nervous mass, indicate that it is made up of separate portions, the circum-cesophageal commissures prove that the ventral ganglia have become more dorsal in position.

The nervous system of the Branchiopneusta is similar in many points to that of the Tectibranchiata, and similar relations can also be made out in the Nephropneusta. Both divisions are characterised by the development of their cerebral ganglia into several portions, which can be distinguished as such even from the exterior.

\section{$\S 269$.}

The genetic relations of the nervous system of the Pteropoda to that of the

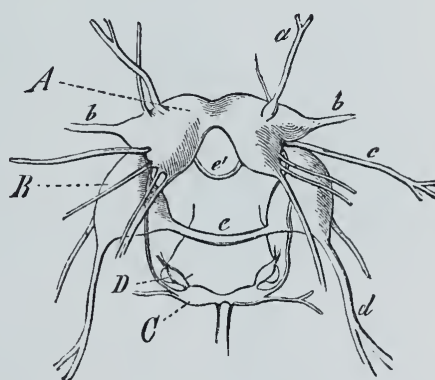

Fig. 185. Central nervous system of one of the Alolidiæ (Fiona atlantica). A Supra-œsopliageal mass, formed by the anterior cerebral, and posterior visceral or branchial ganglia. $B$ Pedal gan. glia. C Buccal ganglia. $D$ Gastrocesophageal ganglia. a Nerve to the superior (hinder) tentacles. $b$ Nerve to the inferior tentacles. $c$ Nerve to the generative organs. d Pedal nerves. e Commissure of the pedal ganglia. $e^{\prime}$ Commissure of the visceral ganglia (after $R$. Bergh).

Gastropoda may be seen in the Gymnosomata; the Thecosomata differ in the length of their cerebral commissures. The cerebral ganglia either retain their lateral position or approach the pedal ganglia with which the visceral ganglia are also fused. The central ganglionic mass lies therefore below the pharynx. The arrangement in the Gymnosomata is more primitive; the cerebral ganglia are placed superiorly, and visceral ganglia are evidently present. The pedal ganglia aiways innervate the fins formed from the foot. In the Gymnosomata the cerebral ganglia give off considerable nerves to the tentacles, at the base of which they form ganglia. The ganglia of either side are connected with one another by commissures (Clio).

The three ganglionic masses already pointed out in the Mollusca 
are also found in the Cephalopoda; but they are more closely approximated in consequence of the shortening of their commissures. They form a ring around the œsophagus. In the Dibranchiata this ring is enclosed by the cephalic cartilage in such a way that the peripheral nerves pass out through foramina in it.

In Nautilus, the upper part of the œsophageal ring is formed by a transversely disposed nervous mass (Fig. 186, a a); the nerves

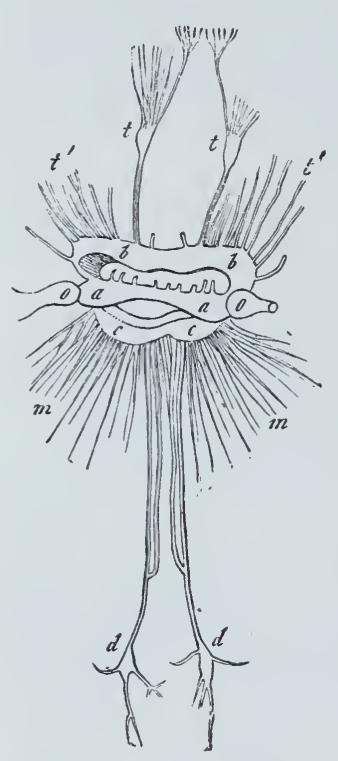

Fig. 186. Nervous system of Nautilus pompilius. $a$ Superior, $b$ Inferior ganglionic masses of the œesophangeal ring. $c$ Visceral ganglia. $d$ Genital ganglia. $m$ Pallial nerves. $t t^{\prime}$ Ten. tacular nerves (after Owen). of the higher sensory organs are given off at the sides. It corresponds to the cerebral ganglia, but these also extend some distance ventrally $(b b)$, and give off, in that region, nerves which pass to the tentacles $\left(t t^{\prime}\right)$. The ventral segment only of this ring is homologous with the pedal ganglion, as it gives off the nerves for the funnel. A second ventral mass $(c c)$ is attached to the lower nervous mass, which, as we have already said, is partly formed from the cerebral ganglia; this corresponds to the visceral ganglia, and gives off nerves for the mantle $(m)$, in addition to two small trunks which accompany the vena cava, and pass to the gills and vascular system. Each of these two nerves forms a ganglion $(d)$, which, again, supplies the generative apparatus.

In the Dibranchiata the nervous system is much more concentrated. The cerebral ganglionic masses are placed much more to the sides and ventral surface, so that they unite in the middle line in front of the part which corresponds to the pedal ganglia. The visceral are completely fused with the pedal ganglia, and the only remnant of the primitive independence of these ganglia is a narrow point through which the pedal artery passes; the separate character of these ganglia is much more distinct in the Tetrabranchiata. That portion of the cerebral ganglionic masses which still remains above the œsophagus is differentiated into several portions. The part which has reached the ventral surface gives off the nerves for the arms, and these unite to form a ganglion at their base. The pallial nerves are given off from the visceral masses, and each of these nerves forms a large ganglion (ganglion stellatum).

Compared with the rest of the Mollusca, the Cephalopoda have much more highly differentiated central organs from a histological point of view ; this is due to their greater size. In all parts of the nerve-centres it is possible to distinguish an outer gray layer, formed 
of ganglionic cells, from the inner white medullary substance, which is formed principally of fibres.

Chéron, Rech. p. servir à l'histoire du système nerveux des Céphalopodes dibranchiaux. Ann. sc. nat. V. Sér. T. V.

Owsjannikow und Kowalevsky, Ueber das Centralnervensystem und das Gehörorgan der Cephalopoden. Mém. Acad, de St. Pétersbourg. VII. Sér. T. XI.

\section{Visceral Nerves.}

\section{$\S 270$.}

Owing to the relations between the visceral nervous system and the central nerve-organs, we were obliged to consider parts of this visceral system while dealing with the central one; and we had to do with an example of the difference in the significance of the central organ, when the ganglia which belong to the peripheral parts become a part of it. In addition, however, to this hinder part of the visceral nervous system which is united to the nerve-centres, and which is principally distributed to the circulatory and excretory organs, as well as to the genitalia and gills, there is another portion which innervates the digestive canal.

In the Lamellibranchiata fine filaments arise from the cerebral ganglion and surround the mouth; these are the earliest signs of a portion of the nervous system, which is still more differentiated in the Gastropoda. The development of complicated mouth-organs appears to be correlated with the development of this system. Two nerves arise from the cerebral ganglion and pass to ganglia, which are placed on the buccal mass, and are connected together by a commissure. These buccal ganglia (Fig. 183, B ; Fig. 184, $B$ ) supply the organs of the mouth, and give off nerves to the gut. The commissures differ a good deal in character. As a rule the ganglia do not fuse. The same arrangement is found in the Pteropoda; and in Nautilus, among the Cephalopoda, the two buccal ganglia are connected at their side with pharyngeal ganglia, and are connected by commissural chords with the cerebral ones. There is but one buccal ganglion in the Dibranchiata, and behind it there is a large suprapharyngeal ganglion (Sepia).

The nerves given off from the buccal ganglia have various small ganglia on their enteric branches.

\section{Sensory Organs.}

\section{Tactile and Olfactory Organs.}

\section{$\S 271$.}

The sensory organs of the Mollusca are very similar to those of the Vermes. All parts of the body, with the exception of the hard 
ones, are capable of feeling when touched; the anatomical arrangements for this sense are found in various parts of the body, through which they are more or less widely distributed; they have the form of fine setiform prolongations from cells, which can be seen to be connected with nerves. These structures are most well marked on those parts of the body which function especially as tactile organs ; they are generally supplied with nerves of some size, and form the tentacles.

They are very common on the edge of the mantle in the Lamellibranchiata, where they are either found all over it, in which case they are arranged in several rows (as in Mactra, Lima, Pecten, etc.), or sometimes they are confined to certain regions; they are not unfrequently found on the siphons, and in either case they serve to watch over the particles that get into the mantle-cavity with the water. They are highly contractile, and are supplied with filaments from the marginal nerves of the mantle.

The prccesses found on the epipodium, and on the edge of the mantle in many Gastropoda, as well as the dorsal cirri of the Nudibranchiata, may function as organs of this kind.

It is doubtful whether the pair of lobes at the sides of mouth of the Lamellibranchiata are organs with this function, but on the other hand we find a very large number of tactile organs of this kind on the cephalic tentacles which are so commonly present in the Gastropoda. The tracts, which carry the nervous end-organs, are very often specially differentiated in these animals.

Although there is not much difficulty in the view that the abovementioned organs function in the perception of tactile impressions, it is almost impossible to say what is the physiological duty of a number of other organs, which are clearly sensory, and are connected with the integument. These enlargements are generally formed by ciliated regions to which a nerve passes, and at which it often forms enlargements. It is doubtful what part of the surrounding medinm acts on these organs, and we have to make a somewhat far-fetched analogy to be able to regard them as olfactory organs.

In the Gastropoda they are found near the respiratory organs, and in the Heteropoda I found them very widely distributed in this region. The same I found to be the case in the Pteropoda. In the Gymnosomatous forms of this division a ciliated organ of this kind is placed superficially, and close to the gills; in Pneumodermon it is wheel-shaped. In the shelled forms it is a transverse ridge, which is placed in that region of the mantle-cavity by which the water passes to the branchire.

In the Opisthobranchiata the hinder pair of tentacles (rhinophor) appear to have the function of an olfactory organ; in correlation with this function they vary greatly in character, and the surface of these tentacles may be seen to be increased by the formation of ridges and various other arrangements. They seem to be in all cases ciliated. If we reflect on the fact that respiration is largely carried on by organs which arise from the back of the animal, we shall see 
that the tentacles, when functioning as olfactory organs, have just the same relations as the respiratory ones; and with this we may correlate the great distance backwards at which the tentacles are sometimes found.

In the Cephalopoda the olfactory organs are more definite in character. Behind the eyes we find two small pits, or papillæ, which are level with the surface; these are ciliated. The processes of the more deeply-placed olfactory cells stand up between the ciliated ones; they are supplied by a nerve which arises close to the optio nerve.

\section{Visual Organs.}

\section{$\S 272$.}

Visual organs are found in all Mollusca that are endowed with the power of active movement. On the other hand they are always atrophied in the fixed forms, although they are present during the larval stages. This is also the case in the Placophora, where the larvæ indicate by the possession of a pair of pigment spots that they are provided with eyes, which are atrophied later on.

Structures of this kind are found on the nerve-centre, and in the head of the Lamellibranchiata during the larval stages; they are even provided with a refractive body, but they undergo degeneration later on. The same thing happens in the Scaphopoda.

It is different with the organs, which are ordinarily found on the edge of the mantle, in the higher divisions of many Lamellibranchiata. They are carried on special optic-stalks (Arca, Pectunculus, Tellina, Pinna, etc.) ; in many cases (Pecten, Spondylus) they were even observed by the older investigators, owing to the emeraldgreen colour of the tapetum, which is found at the base of the eye. Although these organs have many peculiarities of structure, they agree in all essential points with the optic organs of the rest of the Mollusca. They are supplied with nerves by the small trunks which pass along the edge of the mantle. These organs vary greatly in the extent to which they are developed, and are sometimes replaced by mere pigment spots. This arrangement must be regarded from that point of view, on which we have already insisted, when we pointed out that sensory organs might be differentiated from simple nerve-endings at any point of the integument; these eyes, therefore, on the edge of the mantle, can only be compared with the optic organs which are found on the head, from a physiological point of view ; morphologically, they are special structures, and are, like the similar organs found in Vermes, due to adaptation.

We never find more than one pair of cephalic eyes in the Gastropoda. They are often replaced by mere spots, placed on the supra-œsophageal ganglion, and disappear altogether when the animal loses its power of free movement (Vermetus). When it is 
simplest the eye lies beneath the integument (as in many Opisthobranchiata). In others it is embedded in the dermo-muscular tube, and retains its superficial position, while at the same time au elongation of the optic nerve is developed. This sub-integumentary position must be regarded as a secondary one, for in the Mollusca, just as in the Vermes, the integument takes part in the formation of the eye. The eye-bearing region of the body is ordinarily found to be the base of the tentacle (Prosobranchiata), which may be converted into a special eye-stalk (ommatophor). Or the eye may rest on a process formed from the tentacle (Strombus, Pterocera), or this process may become separated from the tentacle, and become independent. Owing to the possession of this optic-stalk the eye is capable of movement; in the Heteropoda this is effected by the muscles that are attached to the wide capsule which encloses the bulb of the eye (Fig. 187, o). Thanks to these muscles the bulb is enabled to vary its position; in

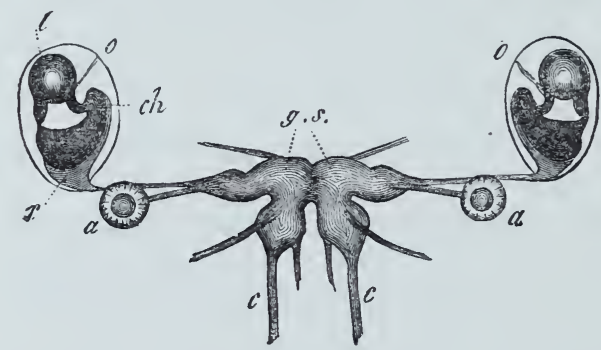

Fig. 187. Upper part of the nervous system, with the sensory organs of Pterotrachea. gs Cerebral ganglia (Cerebrum). $c$ Commissures. o Optic capsule. $l$ Lens. ch Pigment layer. $r$ Ganglionic enlargement of the optic rerre. a Auditory organ. form, the bulb is generally rounded or oval; in the Heteropoda it is very peculiar (Fig. 187).

The bulb has a thin outer covering, which passes anteriorly into the cornea (pellucida), which is formed from the integument. In the posterior portion the optic nerve widens out, and is generally provided with a ganglionic enlargement (r). Internally to it we find the retina, with the end organs of the optic nerve; these constitute a layer of rods which are turned towards the cavity of the eye, and are separated by a layer of pigment from the external layer of the retina. Behind the cornea there is a lens, which either fills up the cavity of the eye, or has a gelatinous substance posteriorly to it, which represents a vitreous body.

The sensory layer is formed from the ectoderm, and the lens also is an integumentary structure, inasmuch as it is developed from a cell, which gradually secretes the substance of the lens in stratified layers.

\section{$\S 273$.}

The eye of the Cephalopoda resembles, in many points, the optic organ of the Gastropoda. We know that it is gradually differentiated from the ectoderm. In Nautilus, each bulb is carried on a kind of optic stalk, and forms a lateral projection (vide suprà, Fig. 175, o); this is indicated in some of the Dibranchiata, but in them the bulb may be supported by processes of the cephalic cartilage, and lie in 
a kind of orbital cavity. In Nautilus the optic capsule is continuous with the stalk; in the Dibranchiata it is placed in the cartilaginous orbits, where it encloses the ganglionic enlargement of the optic nerve (Fig. 188, go), which is represented in Nautilus by a layer which extends over a larger portion of the bulb. Anteriorly, the optic capsule forms a thin investment $(C)$, which is known as the cornea; and the refractive media of the bulb are placed behind it. This cornea is absent from the eye of Nautilus, as is the lens. In front of this cornea the optic capsule is therefore directly continuous with a membrane which is connected with the integument of the optic stalk, and which has a pupil-like orifice which leads into the interior of the bulb.

In the Dibranchiata this direct communication between the internal cavity of the bulb and the surrounding medium is broken by a lens $(L)$; as, however, in many forms (Loli gopsis, Histiotheutis, etc.), the transparent portion of the optic capsule is alto-

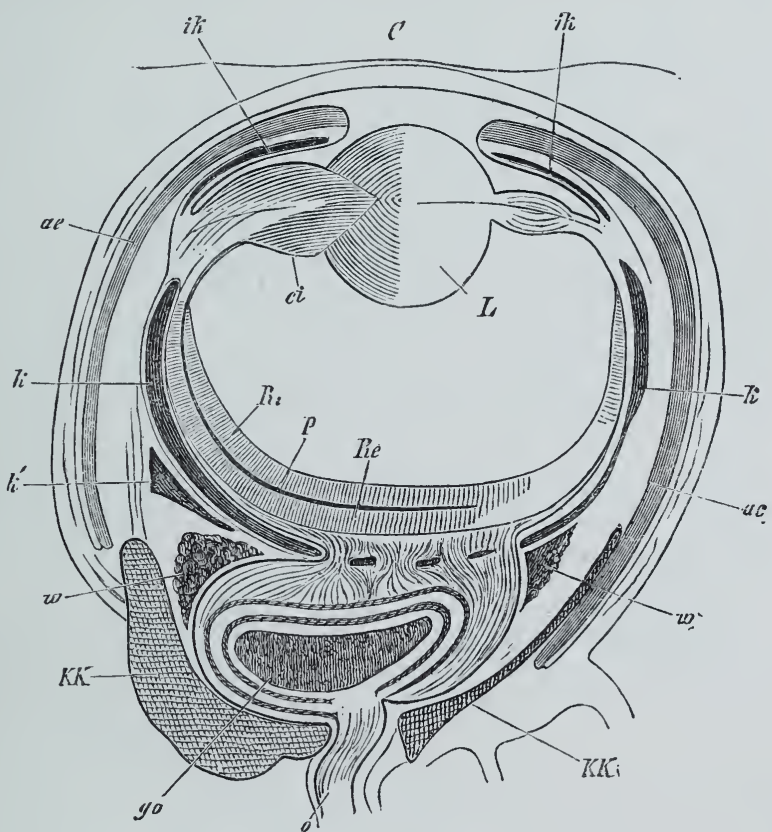

Fig. 188. Horizontal section of the eye of Sepia (Diagram. matic). $K K$ Cephalic cartilages. $C$ Cornea. $L$ 'Lens. ci Ciliary body of the lens. Ri Internal layer of the retina. Re External layer. $P$ Layer of pigment. O Optic nerve. go Ganglion. 7v Pupillary cartilage. ik Cartilage of the iris. $w$ White body. ae Argentea externa (after Hensen). gether absent or is perforated (Sepia, Loligo, Octopus), the anterior surface of the bulb enclosed in the capsule is bathed by water. This space, which communicates with the exterior, is not only continued through the optic cleft as far as the lens, but also extends more or less around the bulb.

In many the integument is thrown into folds around the cornea; these folds form "eye-lids," and are either limited in position or extend around a larger portion of the bulb, when they form a defensive organ for the eye by the possession of occlusor muscles.

The base of the bulb is formed by a cartilaginous capsule (Fig. 188, 7); around the pupil this becomes converted into the 
cartilage of the iris (iti). Outside and behind this optic capsule is the ganglion of the optic nerve, in the periphery of which there is a whitish organ $(w)$ which projects more or less forwards. Behind this there is a longitudinal layer of muscles, and lastly, a silvery membrane (argentea externa) (ae), which reaches as far as the edge of the pupil, and invests the bulb on its inner face, or the one turned towards the abore-mentioned cavity. Internally to it there is the argentea interna. The bundles of nerves which arise from the ganglion $(g 0)$ behind the cartilaginous capsule, pass to the retina by a large number of pores in it; the retina lies within the cartilaginous capsule, and is continued forwards as far as the edge of an organ which carries the lens. This retina is formed of essentially the same layers as the same portion of the eye of the Gastropoda ; an internal (Ri) portion, which contains the perceptive apparatus, is separated from the external part $(R e)$ by a layer of pigment $(P)$. A layer of connective tissue extends inwards to the lens $(L)$ from the layer of muscular fibres; this completely separates the eye into two parts, an anterior smaller and a posterior larger one; these unite to form an oval body, the long axis of which corresponds to the optic axis. There are epithelial thickenings on the anterior, as well as on the posterior, surface of this layer of connective tissue; they unite to form a system of lamellæ, which invests the edge of the lens, and is known as the "ciliary body" (ci) (corpus epitheliale). 'The cavity behind the lens is filled up by a fluid.

Hexsex, Zeitschr. f. wiss. Zool. Bd. XV.

[LANkester, E. Ray, Develt. of Cephalopoda. Quart. Journ. Microsc. Sci. 1875.]

\section{Auditory Organs.}

\section{$\S 274$.}

It is possible, apparently, to derive the organs, which seem to be auditory in the Mollusca, from the vesicles or otocysts which we met

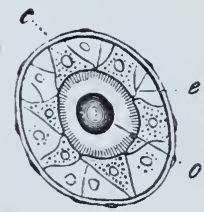

Fig. 189. Auditory organ of Cyclas. c Auditory capsule. $e$ Ciliated epithe. lial cells. $o$ Otolith (after Leydig.) with in the Vermes. They contain otoliths, and their inner wall is provided with nervous end-organs, which are formed by modified epithelial cells. These cells are derived from the ectoderm, for the otocyst itself is formed from that layer; this has been observed in the Gastropoda. What we know of the development of the ear in the Cephalopoda agrees with this statement.

The auditory nerve is generally given off from the cerebral ganglion. In its most primitive form the auditory vesicle is attached to this ganglion, and it is only when it is separated from it that we are able to make out a true auditory nerve. The otocyst, however, varies greatly in position; thus, it may be attached to the pedal ganglion; but eren then the auditory nerve can always be 
followed up to the cerebral ganglion; it sometimes passes along the cerebro-pedal commissure. This change in position is sometimes associated with a change in the position of the cerebral ganglia themselves.

In the Lamellibranchiata and Scaphopoda the auditory vesicles are attached to the pedal ganglion; they are either close to, or at some distance from it (Naiades), or are even placed more deeply in the substance of the foot (Cythera). In the Gastropoda the otocysts vary greatly in position; but the primitive position-that is, close to the cerebral ganglia-is the most common one; in the Heteropoda, and many Opisthobranchiata, they are always placed close to these ganglia.

The otoliths are either numerous, and consist of small crystalline structures, which form an otoconia, or there is but a single spherical otolith, which is derived from one cell of the rudimentary wall of the auditory resicle, and which forms a concentrically-striated concretion. Dentalium, the lower forms of the Lamellibranchiata, and Gastropoda, and also all the Pteropoda, possess otoconia. In the larval stages, however, of these Mollusca, the otoconia first appear, and then, later on, the spherical otolith; this apparently disappears afterwards. But when the adult form is provided with an otolith, an otolith is found in the larva, and is never preceded by the otoconia.

We have not as yet any connected statements about the structure of the end-organs in the otocyst. The most important fact that we do know is that part of the ciliated epithelium is represented by cells with fine rod-like processes, which appear to be true auditory fibres. They form the auditory organ, and are connected with the nerves, while the ciliated cells, which are grouped in tufts, form an accommodating arrangement which acts on the otoliths.

We have a permanent proof that the otocyst is developed from the ectoderm in the Cephalopoda in the presence of a fine canal, which, in many of them, leads from the auditory vesicles to the surface of the body. In Nautilus the otocysts lie on the cephalic cartilage; in the Dibranchiata they are enclosed by it. It is possible, therefore, to distinguish a membranous and a cartilaginous labyrinth, analogous to the similar parts found in the case of the Vertebrata.

In the Octopoda the auditory vesicles are simple in form; in the Decapoda they are complicated by the formation of diverticula and processes. At the same time they are more closely connected with the cartilage, whereas, in the Octopoda, they lie somewhat freely in their cavity. The otolith, which is placed in a watery fluid, varies in form-it may be flattened or rounded, or may be broken up into smaller acicular pieces. The terminations of the auditory nerves either form the "auditory plate," which is a thickened portion of the epithelium, from which the cells send hair-like processes (auditory hairs) (Sepia); or an "auditory crest," which generally takes 
a curved direction, and which is likewise covered by modified epithelium.

Lacaze-Duthiers, Otocystes des Mollusques. Arch. de Zoologie. I. p. 97.RANke, J., Das Gehörorgan etc. bei Pterotrachea. Zeitschr. f. wiss. Zool. XXV. Suppl.-V. Jhering, Die Gehörwerkzeuge der Mollusken. Erlangen, 1876.-Siмroth, Ueber die Sinnesorgane nuserer einheim. Weichthiere. Zeitschr. f. w. Zool. Bd. XXVI.

\section{Alimentary Canal.}

\section{$\S 275$.}

Owing to the complete separation of the wall of the body from that of the enteric canal in the Mollusca, the latter canal comes to be embedded in a cœlom. It forms coils or loops in this cavity, as it is always longer than it; at the same time the characters of its anal opening are well worthy of note. We find, indeed, that it is only in the Placophora and Lamellibranchiata that it traverses the body in such a way that the aboral and anal ends of the body are the same. In the Scaphopoda, Gastropoda, Pteropoda, and Cephalopoda it always ends at some distance from the aboral end of the body; it is looped or coiled. If we suppose that the gut was primitively arranged in a symmetrical manner, and that the anus was placed in the aboral region, and that, therefore, this change in the position of the anus was acquired afterwards, then we must also suppose that this arrangement obtained at a very far-distant period, inasmuch as there are no signs of it in the development of the indi-

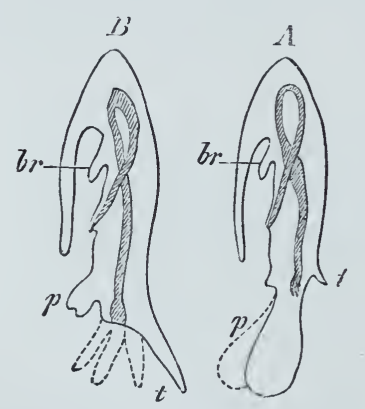

Fig. 190. Diagram of the relations of the enteric canal. $A$ In Pteropoda. $B$ In Cephalopoda. $p$ Foot. $t$ Arms or Tentacles. br Branchix. vidual. The real cause of this change in position must be sought for in the universal possession of a shell. The development of the dorsal mantle and its shell, together with that asymmetrical mode of development of both of these structures which obtains in most Mollusca, makes it easy to see how they affected the organism. We must distinguish two modes of development. In the first, the dorsal development of a part of the body which was protected by the shell, increased the space available for the growing gut, which formed more or less complicated loops or coils. At first, of course, this only enabled it to depart from its straight course. In the second, the development of a mantle-cavity, the appearance of which was correlated with that of the mantle and shell, affected the position of the gut. When the mantle-cavity is developed in the hinder region of the body, as it is in the Thecosomatous Pteropoda and the Cephalopoda (Fig. 190), the position of 
the anus is, relatively speaking, least altered. It may still lie more or less in the middle line. When the branchial cavity is more anterior in position, and asymmetrical -as it is in most of the shelled Gastropoda-the anus approaches it, inasmuch as its function is here least interfered with.

The enteric tube is divided into separate portions in just the same way as in Vermes.

Although embryological inquiries have not yet arrived at any very definite results, this much seems certain: the mid-gut is derived from the endoderm, and the fore-gut from the ectoderm. The hind-gut is laid down with the mid-gut, and has therefore the same history as it has.

\section{$\S 276$.}

As to the characters of the various divisions, we find that in the Placophora the digestive tube is coiled several times; but the anus still retains its aboral position, inasmuch as the above-mentioned causes, which lead to a change in position, do not obtain in this group.

The Lamellibranchiata likewise retain the most primitive relation of parts. The mouth, which is placed in the Dimyaria between the foot and the anterior adductor, is continued into a short portion which functions as the œesophagus; this passes into a widened portion, or stomach. The efferent ducts of the liver open into this gastric mid-gut. In many Lamellibranchiata this stomach is remarkable for the possession of a cæcal diverticulum, which is often of some size, and can be shut off by a valve; this is placed in the pyloric region. In many forms we meet with a peculiar structure in the cæca, or, when they are absent, in the enteric canal itself; this, which is known as the "crystalline style," is to be regarded as a secretion from the enteric epithelium. The hind-gut, which forms by far the largest portion of the whole tract, makes one or more coils, and then passes towards the back of the animal ; as a rule it is of the same calibre throughout, but it is sometimes differentiated into narrower and wider tracts. It is closely surrounded by the other organs (liver, generative glands) of the visceral sac; its terminal portion passes underneath the middle-line of the shell as far as the hinder margin of the body; in a large number of Lamellibranchiata it traverses, as it does so, the pericardium and heart (Fig. 176, i), and then ends, behind the posterior adductor, at the anus; this is placed on a process at the aboral end of the body, which projects freely into the mantle-cavity (Fig. 181, r). Here again the position of the anus is correlated with the characters of the shell, which is in the form of two lateral valves.

\section{$\S 277$.}

In all Mollusca, except the Lamellibranchiata, part of the fore. gut is differentiated into the so-called pharynx or buccal mass; this is generally a large organ, the structure of which is well adapted 
to its function; this function is to seize and comminute the food of the animal. The apparatus, which forms the chief portion of this arrangement, is a cuticular membrane which rises up from the lorver wall; on it there are small teeth or hooks, which are directed backwards and arranged in transverse rows. The teeth vary very greatly in arrangement (Fig. 191, $a$ b $\odot$ ), form, and number; they are not

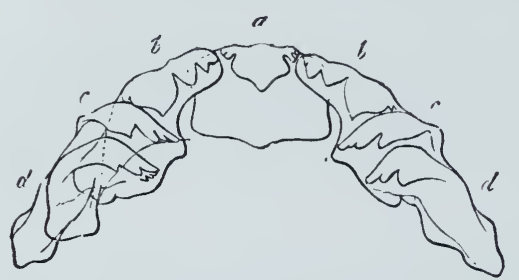

Fig. 191. A row of denticles from the radula of Littorina littorea. a Median.

$b c d$ Lateral denticles (after Gray).

only different in the larger divisions, but also in the orders and families, and even in the species, so that the genetic affinities of the groups is indicated by the form of these parts; and for this reason they have been much used in classification. As a rule they consist of a median longitudinal row $(a)$, with which symmetrically-arranged lateral denticles are connected. The organ formed by the whole of these hooklets is known as the radula. In many (Turbo, Patella) it projects some way into the cœlom, and is enclosed by the sac-like sheath formed by a diverticulum of the wall of the osophagus; it may even be longer than the body itself. This organ may become very broad, and reach to the sides of the pharynx. In the Heteropoda it is so far more highly developed that the outermost uncini of the transverse rows may not only be very long, but also be articulated in such a manner as to be movable. When, therefore, the radula is protruded, these teeth are erected, and when it is drawn back they come together like pincers, and so act as seizing organs.

'There are special muscles for moving the radula which, with the cartilage on the wall of the pharynx (p. 361), help to increase the size of this organ (Fig. 200, B). The size of the radula is therefore closely correlated with that of the buccal mass. This organ is found in all divisions of the Mollusca excepting the Lamellibranchiata, although in some (Thecosomatous Pteropoda) it is feebly developed. In rare cases the radula and pharynx are altogether absent (Tethys). The radula is relatively small in the Cephalopoda (Fig. 192, $C r$ ), where the oral opening is marked by the presence of strong jaws. These are two strong pieces (Fig. 192, C) which resemble the beak of a parrot, and are provided with sharp edges; the lower one $\left(m^{\prime}\right)$ projects in front of the upper one $(\mathrm{m})$. The soft edges of the lips cover the bases only of these jaws (Fig. 179, $m m^{\prime}$ ).

In the Gastropoda also the wall of the mouth has a firm investment, which forms a kind of jaw. In the Nephropneusta there is a single semi-lunar piece which is beset by tootled ridges at its free edge. In many of the Branchiopneusta lateral pieces are added on to this unpaired one, which work horizontally on one another. These paired jaws are also present in the Prosobranchiata, but are best developed in the Opisthobranchiata. 
The mouth of the Gastropoda is surrounded by the lips, which meet in front of the entrance into the pharynx. These lips are folds of the integument, which can be retracted or protruded with the pharynx. In some of the Prosobranchiata this arrangement is carried so far that the fold, which, as we have seen, ordinarily forms the lips, gives rise to a more or less "elongated sheath, in which a proboscis, within which again is the pharynx, lies freely. When this proboscis is protruded, the inner wall of the sheath, which encloses it, is gradually everted (Dolium, Cassis, Conus, Voluta, Buccinum, Harpa, Murex, etc.). It is clear then that this, the most anterior portion of the intestinal tract, may be very highly specialised.

\section{$\S 278$.}

Behind the pharynx in the Gastropoda the fore - gut
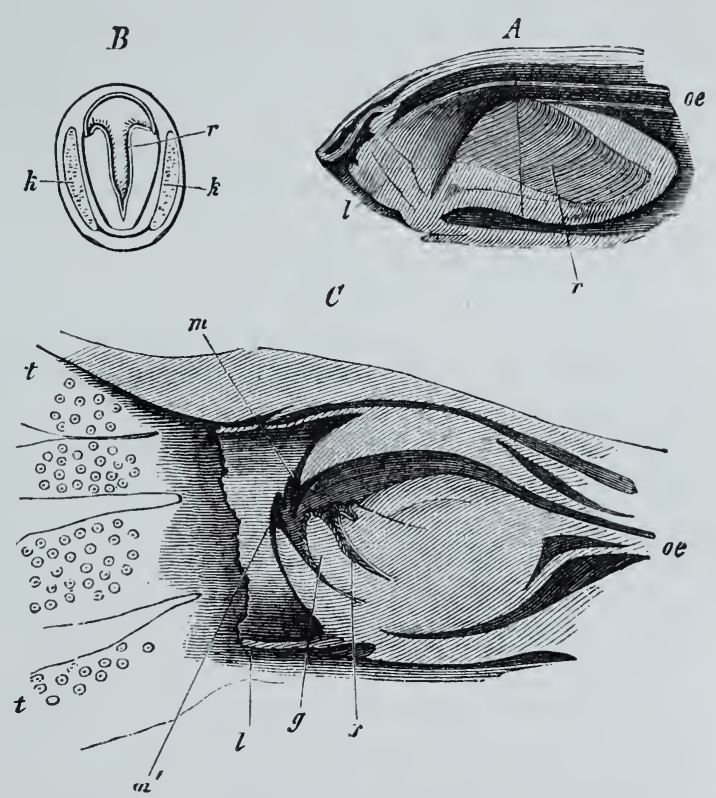

Fig. 192. A Pharynx of a Gastropod (Pleurobranchus); vertical longitudinal section. $B$ Transverse section of the same, taken along the vertical line drawn in $A$. oe Esophagus. $l$ Lips. $r$ Radula. 7. Cartilage. C Pharynx of a Cephalopod (Loligo); vertical longitudinal section. $t$ Arms. $m$ Upper, $m^{\prime}$ Lower jaw-piece. g Tongue. $r$ Radula. oe Eisophagus. forms the osophagus, and part of it widens out into a stomach; this is succeeded by the mid-gut, which often traverses the visceral sac in the form of a single loop; it passes on to the hind-gut, from which it is indistinctly separated.

The canal is modified in character by the enlargement of various parts of the œsophagus; in this way a special portion is formed which functions as a crop. This is either spindle-shaped, as it is in many of the Prosobranchiata (it is very long in the Heteropoda), or it forms a unilateral diverticulum, which may develop into a cæcal appendage (Lymnæus, Planorbis, Buccinum). Parts of the fore-gut may also widen out into stomachal enlargements, which are separated off from the neighbouring tracts by constrictions. These form divisions, set one behind the other.

This separation distinctly corresponds to a division of function ; this is also shown by the varying characters of the cuticular structures of the different portions. For example, in Aplysia, one portion 
is beset with pyramidal pieces of cartilaginous consistency, and another is provided with firm horny hooks. Similar kinds of hooks are found in the simple stomach of Tritonia; in Scyllæa there is a broad girdle of sharply-pointed plates in the same region, while in the stomach of those Pteropoda in which the mouth-parts are rudimentary, there are strong radulæ. The presence of these organs shows that these portions of the gut merely function in preparing the food for digestion.

The widened mid-gut is no less modified, both in form and in the differentiation of its various parts. In many it is faintly distinguishable. In others it forms a stomachal cæcum, so that the cardiac and pyloric ends get to lie close together; this is the more common form. The stomach may be broken up into several divisions. For example, the cardiac and pyloric portions are often separated by a longitudinal fold, which projects into the stomach (Littorina).

Of the peculiarities of the rest of the enteric tube, the frequent enlargement of the hind-gut must be mentioned. In many Nudibranchs the whole gut undergoes more marked modifications (Aolidiæ); in proportion as the liver takes on its functions it becomes more or less degenerated, the increased size of the liver compensating for the great abbreviation of the gut (vide infrà, p. 365).

In many of the Gastropoda there are glands connected with the anus; these are sometimes of some size (Murex, Purpura); but it is not yet known what their significance is.

The anus is lateral or dorsal in position according to the size of the shell and the development of the mantle-cavity. When the shell is absent, and a mantle-cavity also, the anus may be on the dorsal surface, and even in the middle line of that surface, as it is in some of the Nudibranchiata (Doris) (Fig. 200,a). In others it retains the lateral position which it acquired owing to the earlier possession of a shell (Aolidia).

\section{$\S 279$.}

In the Cephalopoda the pharynx (Fig. 199, $p /$ ) is continued into a narrow ossophagus, which, after passing through the cephalic cartilage, is either continued, without any change of diameter, into the stomach (Loliginidæ), or it is provided somewhere along its tract with a crop-like enlargement, which is often of considerable size (Nautilus, Octopoda). A stomach (Fig. 193, v) is formed by an oval or rounded enlargement, which is sometimes (Nautilus, Octopus) provided with strong muscular walls. On each side of the stomach there is a layer of muscles, which are arranged in a radial manner, and in the centre of which there is a tendinous plate; this is especially noticeable in the Nautilus.

The pylorus, which is placed close to the cardia, leads into the 
mid-gut, which is provided with a cæecum; this portion also has its inner wall thrown into longitudinal folds for the first part of its length. It generally takes a straight course (it is slightly coiled in Nautilus and the Octopoda only) forwards, to a short hind-gut (Fig. 193, i), which opens to the exterior at the base of the funnel. In many Cephalopoda there are two or three valves or valvular processes around the anus, the muscles in which are remarkably well developed.

The cæca (Fig. 193, c) at the commencement of the mid-gut vary not only in external form, but also in the characters of their internal surface. The cæcum is either rounded (Nautilus, Rossia, Loligopsis) or elongated; in the latter case it is often spirally coiled (Sepia, Octopus). When the cæecum is very long, it is often coiled several times $(e e)$. The inner surface is either thrown into processes, which are arranged in a lamellar fashion (Nautilus), or these processes may form circular folds arranged in conformation with the spiral form of the cæcum. Two of the largest folds receive the efferent ducts of the liver, and project so far into the lumen of the enteron as to form at times a valve-like apparatus which shuts off the cæcum. It is probable that this cæcum has a secretory function, as in those forms (Loligo vulgaris)

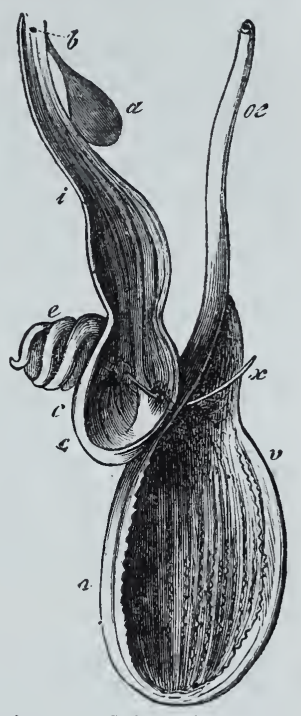

Fig.193. Digestiveapparatus of Loligo sagit. tata. oe Eisophagus. $v$ The stomach, opened longitudinally. $x$ A probe passed through pylorus. $c$ Commencement of the crecum. $e e$ Its spiral portion. $i$ Hind-gut. a Ink-bag. $b$ Its opening into the rectum (after Home). in which the folds are absent a large number of glands are to be found in its walls.

\section{Organs appended to the Enteric Canal.}

\section{1) Appendages of the fore-gut.}

\section{$\S 280$.}

Of those glandular organs which are connected with the enteric canal, the salivary are found ouly in those forms in which the pharynx is developed; it is possible therefore to make out a certain connection between these structures. In the Gastropoda they are always placed on each side of the fore-gut, and open into the pharynx. Sometimes they form short cæcal tubes (Pteropoda), which are sometimes hidden in the very substance of the pharynx 
(many Opisthobranchiata). When they are more highly developed their duct is elongated, so that the secreting portion comes to lie some way further back; sometimes it is placed on the œsophagus, and at others on the stomach itself. In this case the glands are rounded, elongated, and generally flattened tubes (Prosobranchiata, many Pulmonata), which may be broken up into several smaller parts, or have the form of ramified organs; the glands found on the stomach of Pleurobranchus are examples of this latter kind. We not unfrequently meet with two pairs, the efferent ducts of which are either separate all along their course, or the ducts of the hinder pair unite with one another. Even when there is but a single pair of glands they may be often observed to fuse into one mass, the double nature of which is shown by the presence of two efferent ducts. The salivary glands of many Prosobranchiata are differentiated functionally (Dolium, Cassis, Cassidaria, Tritonium), for part of the gland secretes free sulphuric acid. The glands of some Opisthobranchiata (Pleurobranchus, Doris) are differentiated in the same way.

Among the Cephalopoda, Nautilus is provided with a paired glandular mass, which is placed inside the pharynx. These glands are also present in many Dibranchiata (Octopus, Eledone), as are others, which are short and lie just behind the pharynx; these have an efferent duct which penetrates the wall of the pharynx, and unites with its fellow of the opposite side immediately in the orifice of the duct (Fig. 199, gls s). In addition, there are glands behind these which lie at the sides of the œsophagus, and behind the point at which it penetrates the cephalic cartilage. They are either simple or lobate; as a rule their efferent ducts unite into a single one, intermally to the cephalic cartilage, and this duct opens into the pharyngeal cavity in front of the lingual ridge (Fig. 199, gls i).

Pdicceri, P., Gli organi e la secretione dell' acido solforico nei Gasteropodi. Atti della R. Accad. delle Sc. fisiche. Napoli. Vol. III.

\section{2) Appendages of the Mid-gut.}

\section{$\S 281$.}

Appended organs are very generally found on the mid-gut of the Mollusca ; they represent the "liver," and are differentiations of the wall of the enteron, from which they are developed, under the form of diverticula, the primary origin of which is the sacculation of the endoderm.

In the Lammellibranchiata the liver is a gland which surrounds the stomach and a large portion of the rest of the enteron. It forms numerous acini, which are connected together into large lobes, and open at various points either into the stomach, or into the succeeding division of the enteron. 
In the Placophora it forms a pair of symmetrically disposed branched tubes.

In the Gastropoda this gland is no less well developed. In the shelled forms it occupies the largest portion of the visceral sac within the shell; it is always made up of a number of large lobes, and embraces more or less of the enteron. The bile-ducts from the lobes either open separately or together into the first portion of the mid-gut, and sometimes also into the stomach-like enlargement. The number, as well as the relative size of the separate portions of the liver, varies greatly. As a rule, however, when the liver increases in size it becomes less complex, while the smaller the lobes the more numerous they are.

This mode of arrangement along a large portion of the enteric canal leads to certain changes in this portion of the enteron in one division of the Opisthobranchiata. The ducts of the several lobes of the liver widen out, and so form diverticula of the stomach; when, therefore, there are a large number of hepatic tubes opening into the stomach, its inner surface has a reticular appearance (Duris, Doridopsis). Owing to this change, which is easily explicable by a reference to the origin of the liver, the glandular portion of this organ becomes apparently a mere covering for these irregular diverticula.

This is indeed the origin of that arrangement of the digestive system in the Aolidize and others, to which we have already called attention ( $\$ 278$ ). The liver has the form of wide cæcal appendages, which arise from the midgut (Fig. 194, m), or so-called stomach. 'l'hey are either directly connected, when the appendages open at once into the mid-gut, or indirectly, when they still form wide diverticula of it (Fig. 194); and these, too, may be due to certain

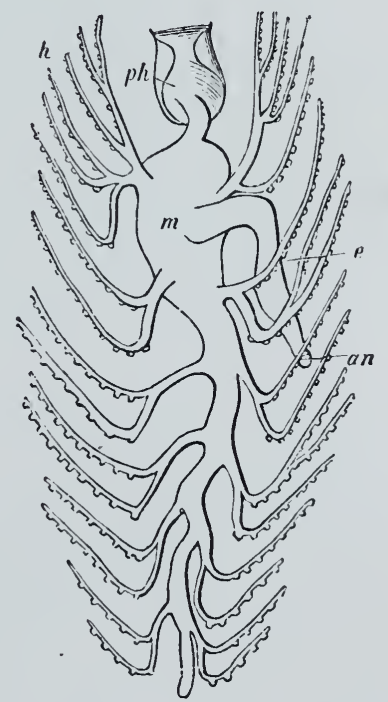

Fig. 194. Enteric canal of Aolidia papillosa. ph Pha. rynx. $m$ Mid-gut, with its hepatic appendages, $(h)$ all of which are not figured. $e$ Hind. gut. an Anus (after Alder and Hancock). changes in a part of the liver. These appendages traverse the cœlom and send blind processes into the dorsal cirri, when such are present. These processes are more or less branched, according to the number present, and they may, further, anastomose with one another. The size, just as much as the number and general form of the enteric appendages, may vary. Sometimes they are mere diverticula of the enteron, and may communicate with it by wide openings, and even be large enough to take in masses of food; or, again, they may form narrow canals which do not take any direct share in the reception of the food. There are intermediate stages 
between these extreme forms. The fact that these enteric cæca always have a glandular investment is of importance as bearing on their real character. These branches, therefore, are not merely physiological equivalents of a liver, but are to be regarded as modifications of a true liver, which have come to take a share in increasing: the enteric canal, owing to the enlargement of the lumen of their canals. The same organ, which in other Gastropoda has the appearance of a liver, becomes a part of the digestive tract in the Eolidire, and retains its primitive significance in its walls only, or, it may be, in parts only of these walls. The sliare taken by the cavities derived from the enteron in the functions of this tract explains how it is that the mesial enteric tube is so short. In other divisions also of the Opisthobranchiata the liver has the form of wide tubes-as in Phyllirhoë, Limapontia, etc. In all these structures the liver appear's to be degenerated, and there is no early stage of differentiation; this is because the Æolidiæ are derived from shelled Gastropoda.

In the Pteropoda the liver is broken up into a large number of small cæcal tubes. In Pnemmodermon these tubes are collected together into branched groups, and the wide orifices of their ducts are scattered on the wall of the stomach, and give it an almost sieve-like appearance. In the rest of the Pteropoda acini of simpler character beset a portion of the enteron; they form a well-defined mass, which is traversed by this tube (Fig. 201, $h$ ).

The liver of the Cephalopoda is always a large, and, frequently, a compact gland; in Nautilus it consists of four loosely connected lobes, each of which gives off an efferent duct. In the Dibranchiata, there are only two lobes, and these are either distinctly separated (Sepia), or only partly connected together (Rossia). In Sepiola and Argonauta the two lobes are more intimately connected; in the Loliginidæe and Octopoda they form a single mass, which is traversed by the œesophagus. In no case does the liver give off more than two efferent ducts, which point to the primitive existence of two lobes, and which, just as in Nautilus, always open into the end of the cæcum.

There are peculiar glandular lobules on the hepatic ducts at the openings into the cæcum, and also in the liver itself; these differ in structure from the acini of the liver. The glands at either of these spots have been regarded as pancreatic glands, but it must be noted that they have no close affinity to the organs of that narne found in the Vertebrata. In the Gastropoda also (Aplysia, Doris) special glands have been observed in the neighbourhood of the liver.

\section{3) Appendages of the Hind-gut.}

\section{$\S 282$.}

Various glandular organs belonging to this division are found in the Gastropoda only, where their significance has not been made 
out. In the Cephalopoda, the Dibranchiata are ordinarily provided with an ink-bag, which is an organ of this kind; in many (Loliginidæ) this opens together with the hind-gut, and may, therefore, represent a structure which has been developed from it, although, indeed, in other Cephalopoda, it opens below or behind the anus. It forms an elongated sac, the contractile walls of which form internallyprojecting lamellæ (Fig. 193, a), and which secretes the well-known black fluid.

\section{Cœlom.}

$\S 283$.

A colom appears very early in the differentiation of the Molluscan body. The complications which affect the cœlom, owing to the coils of the enteric canal which are embedded in it, and the appended organs which are developed from its walls, are further increased by the formation of other organs, and, especially, of the generative system; the cavity is thereby broken up into a number of segments varying in size. As a rule, the cœlom is continued into the processes of the body, as, for example, into the mantle-flaps of the Lamellibranch, and the mantle of the Gastropoda. Less important appendages of the body are also frequently connected with the coelom.

As a rule, the vascular system is freely connected with the cœlom, which therefore forms a portion of the hæmal system. This arrangement is more or less well marked; and more or less wide spaces are shut off from the colom, according to the degree to which the vascular system is developed. When the wider spaces of the cœlom are connected with the vascular system, these portions of the liæmal system form lacunæ; when these spaces are broken up, either by the organs which are embedded in them, or by the bands of tissue which connect together the walls of these organs, they become converted into narrow, and often into vascular canals, which, moreover, are frequently arranged in a regular manner. In the Lamellibranchiata and Gastropoda there are all kinds of stages of this kind; in the Cephalopoda the blood-vascular system is very complete, and true lacunar spaces are, for the most part, confined to the visceral sac. By means of the excretory organs ( $\$ 289$ ), the cœlom, as in many Vermes, communicates with the surrounding medium. The water is thereby enabled to mix with the blood. In addition to the communications which the cœlom has with the exterior by means of the excretory organs, it is able to communicate with it directly, owing to the presence of pores in the foot of the Lamellibranchiata and Gastropoda; the fluid is able to escape from the coelom through these pores. This has been definitely made out both in the Lamellibranchiata (Mactra, Cardium, Solen) and in the Gastropoda (Pyrula). This fluid is of especial importance in locomotion, inasmuch as the animal is enabled to swell out its 
body by taking in the water. By its aid, retracted parts can be protruded, limp parts erected, and the whole musculature of the body-wall, and especially that of the foot, is thus made very important. The power of protruding certain parts, which have been withdrawn into the shell, and especially the foot, depends on these relations, which are definitely known to exist in the case of the Lamellibranchiata and Gastropoda, as also in that of the Pteropoda. As yet it is not definitely known whether water is taken into the hremal system in the Cephalopoda.

\section{Vascular System.}

$\S 284$.

In all the Mollusca, excepting the Scaphopoda, the rascular system is arranged in almost exactly the same way, so far as all essential points are concerned. These are, first, the presence of a dorsal longitudinal trunk, a portion of which is developed into a central organ (ventricle); secondly, transverse vessels are connected with the longitudinal one, which, when lateral gills are present, carry the blood from them to the heart, and are further differentiated into organs of circulation by becoming auricles for the rentricle. This dorsal development of the chief portions of the circulatory system is a point in which they resemble the Vermes (cf. p. 168).

The symmetrical arrangement of the auricles in Mollusca which are in other points markedly divergent, shows us that this is a peculiarity which lies at the very bottom of their organisation;
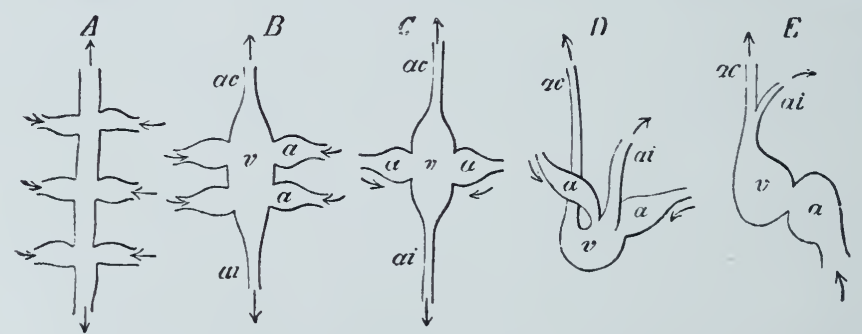

Fig. 195. Diagram to show the relations of the Circulatory Centres in the Mollusca. A Part of the dorsal vascular trunk and transverse trunks of a Worm. $B$ Heart and auricles of Nautilus, $C$ of a Lamellibranch or of Loligo, $D$ of Octopus. E Heart and auricle of a Gastropod. $v$ Ventricle. a Auricle. ac Arteria cephalica. ai Arteria abdominalis. The arrows show the direction of the current of blood.

while the presence of $t w 0$ pairs of auricles, which open behind one another into the rentricle, in the tetrabranchiate Cephalopoda, points to a metameric arrangement of the vascular system, just as much as does the presence of several transverse trunks in the 
Annulate Vermes. These vessels still retain so much of their primitive character that they are not called auricles, but branchial veins.

This homology of the two pairs of auricles with two transverse trunks of a dorsal vessel (Fig. 195, $A$ and $B$ ) points to a primitive condition, which, while it is characteristic of the Nautilidæ, agrees also with the palæontological relations which these Cephalopoda have to the rest of the extant members of their group. The presence of but one pair of auricles appears, on the other hand, to be a degeneration (Placophora, Dibranchiate Cephalopoda, Lamellibranchiata, and many Gastropoda), which corresponds to the reduction of the gills. The key, therefore, to the comprehension of the mode of formation of the ventricle and auricles in the Mollusca is to compare them with more indifferent organs. When part of the dorsal vessel is converted into the ventricle, the parts that are continued from it form arterial trunks, and these, when they retain their primitive course, are distinguished as anterior and posterior aortæ (aorta cephalica, et aorta intestinalis sive abdominalis). In some of the Cephalopoda, the Octopoda $(D)$, we meet with an important change in position, for the trunk of the dorsal vessel has made a loop-like turn, so that the two arterial portions ( $a c$ and $a i$ ) run in the same direction for a certain distance. The points at which they arise from the ventricle are thereby approximated. The circulatory system of those Gastropoda, which are characterised by the fact that only one arterial trunk is given off from the ventricle, may be derived from a similar relation of parts $(E)$. This single arterial trunk divides into two branches, which are distributed in just the same regions as are the two arterial trunks ( $a c$ and $a i$ ), which are given off from either end of the ventricle in the Cephalopoda and Lamellibranchiata. It may, therefore, be regarded as developed from the two arterial trunks, which were primitively disposed along a single axis. The final reduction of the auricles to one is due to a reduction in the number of the gills, and is correlated with the union of the anterior and posterior arterial trunks.

The auricles and ventricle appear, therefore, to have been derived from different portions of a primitive vascular apparatus, and point to a metameric arrangement; taken in conjunction with the signs of metamerism exhibited in the nervous system (p. 344), they lead us to suppose that the ancestors of the Molluscan phylum were segmented organisms.

\section{$\S 285$.}

The heart, in the Placophora and Lamellibranchiata (Fig. 196, v), lies in the middle line of the body, just below the back, and is surrounded by a pericardium; it receives blood from two lateral auricles $(a)$ while the above-mentioned vascular trunks arise from it anteriorly and posteriorly. In the Placophora the heart is placed a good way back, so that the anterior arterial trunk is of a considerable 
length. In most of the Lamellibranchiata the heart is divided into two limbs, which embrace the hind-gut (i), and which give off the

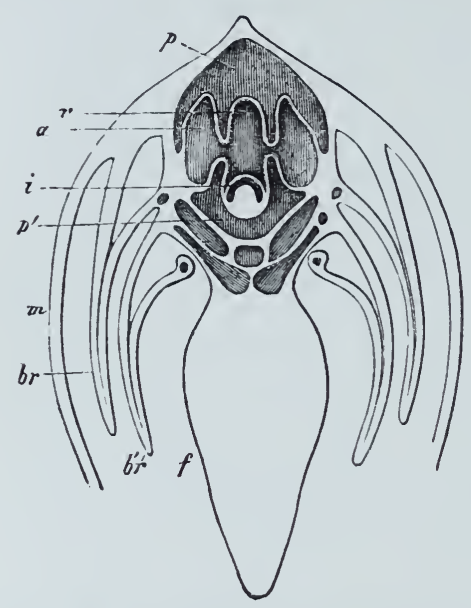

Fig. 196. Vertical section through an Anodonta. $v$ Ventricle. a Auricles. $p p^{\prime}$ Pericardial cavity. $i$ Hind.gut. in Mantle. $b r b r^{\prime}$ Branchiæ. $f$ Foot. aorta after they unite. In Arca this passage of the hind-gut through the heart leads to the formation of two ventricles, which are represented by two completely separate chambers, each of which is provided with an auricle. Each ventricle gives off an aorta, which unites with its fellow of the opposite side before it gives off any branches, so that there is a single aorta all the same. The same holds for the posterior arterial trunk.

The anterior arterial trunk passes as far as the region of the mouth, where it gives off branches and opens into wide hæmal spaces. The posterior arterial trunk, the length of which is dependent on the development of the hinder portions of the mantle, which represent the siphons, also passes into hæmal spaces or lacunæ.

Spaces of this kind, which are essentially marked off by connective tissue, ramify not only in the mantle, but also between the viscera. They may be distinguished into larger or smaller receptacles for blood, according to their width; while, furtlier, they take the place of a capillary and of a venous system. Larger sinuses are regularly present at the base of the gills, and a median azygos one-which collects together the renous spaces in the foot - extends between the two adductor muscles. All of these hæmal spaces are connected together, and form in different parts a more or less wide mesh-work. The two lateral spaces also communicate with the organs of Bojanus $(\$ 290)$.

If we follow out the course which is taken by the blood sent from - the arteries to the periphery, although, indeed, many points about it have not been definitely made out, we find that some of it goes to the mantle, and some to the visceral sac. Thence some of it passes into the branchial sinuses, and from these either directly into the gills, or to the glands of Bojanus, before it passes to the respiratory organs. The latter course is that which is taken by most of the blood. Since, however, the hæmal spaces at the base of the gills are in direct communication with the auricles of the heart, partalthough a small part-of the blood is able to return to the heart without going to the gills. The blood from the mantle also comes to the heart, and passes at once into the auricles; but this cannot be regarded as absolutely venous blood, on account of the respiratory function of the flaps of the mantle. As all the blood which comes 
from the gills is received into the auricles, all of the blood finally returns, by various ways, to the ventricle.

The character of the circulation in the glands of Bojanus is worthy of remark. These excretory organs are placed in the way of the blood which is going to the gills, and which, therefore, is venous; and herein we get a kind of primitive portal circulation.

\section{$\S 286$.}

In the Gastropoda, the heart, which is here also enclosed in a pericardium, is often provided with two lateral auricles (Haliotis, Fissurella, Nerita). Trochus also has two auricles, but the left one

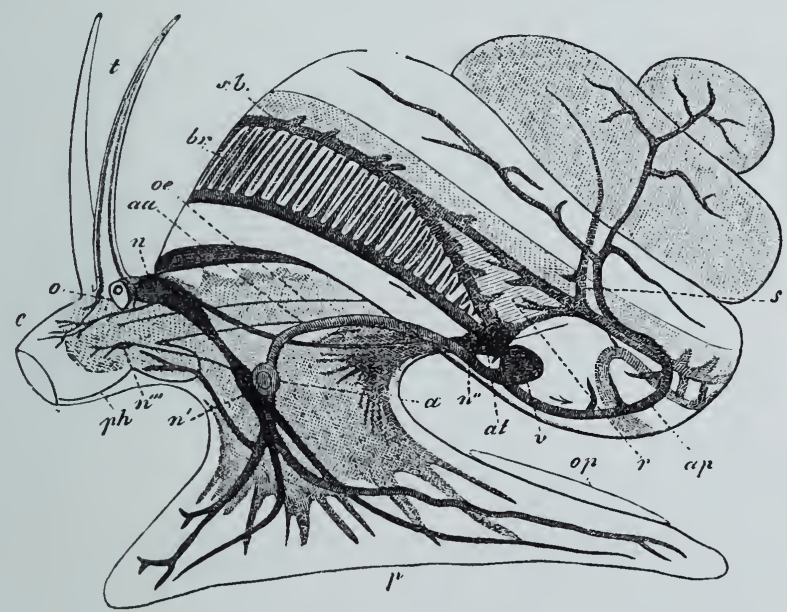

Fig. 197. Organisation of Paludina vivipara. $c$ Head. $t$ Tentacles. $p$ Foot. op Operculum. o Eye. a Auditory organ. $n$ Cerebral ganglion. $n^{\prime}$ Pedal ganglion. $n^{\prime \prime}$ Branchial ganglion. $n^{\prime \prime \prime}$ Buccal ganglion. (The commissure from the cerebral ganglion is not figured.) ph Pharynx. $\infty$ Esophagus. br Branchiæ. $r$ Renal organs. $\delta$ Venous sinus. $S v$ Venous sinus at the base of the branchiæ. $f$ Branchial artery. at Auricle of the heart. $v$ Ventricle. ap Posterior artery (visceral artery). aa Anterior artery (after Leydig).

is somewhat reduced; in the rest of the Gastropoda, as also in the Pteropoda, there is but one auricle (Fig. 197,at). The dorsal position of the heart is modified by the asymmetrical development of the visceral sac; it is always placed close to the respiratory organs, and the thin-walled auricle is always directed towards them. It is placed in front of them in the Prosobranchiata, and behind them in the Opisthobranchiata. It has in many Gastropoda the same relation to the rectum as it has in the Lamellibranchiata (Turbo, Nerita, Neritina); in some, indeed, the ventricle is divided (Haliotis, Fissurella, Emarginula).

An aorta arises from the ventricle and gives off a visceral artery which passes backwards $(a p)$, while the trunk is continued on as an aorta cephalica $(a a)$. This latter passes directly to the anterior 
parts of the body, and generally gives off a large branch to the foot; this sometimes looks as if it were a continuation of the chief trunk. In addition to this, it gives off on its course branches which pass to the stomach, salivary glands, and so on; it either ends simply, or after several ramifications, in the region of the pharynx. In the Pteropoda it is more widely distributed, for in them the pedal branch has the characters of a continuation of the chief trunk, and in the head it divides into two large terminal branches which ramify largely in the fins. The visceral artery, which corresponds to the posterior artery of the Lamellibranchiata, is but slightly broken up in the Pteropoda and lower Gastropoda. Like the cephalic artery, it loses itself in larger hæmal spaces. In most of the Prosobranchiata it is greatly developed, and much broken up $(a p)$; this is the case also in many of the Tectibranchiata (Pleurobranchus), but in others it is replaced by several smaller arteries (Aplysia). The visceral arteries of the Nudibranchiata are branches of the principal trunk (Doris).

The afferent vessels differ according to the number, form, and disposition of the respiratory organs. In many of the Nudibranchiata the blood is collected from the body-cavity near the auricle. In others, which have distinct respiratory organs, there are definite canals, or even vessels with special walls, which convey the blood

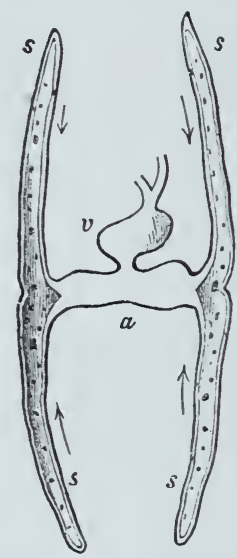

Fig. 198. Part of the circulatory or. gans of Tritonia. $s$ Venous sinuses laid open. The wall is perforated by the openings of the branchial veins. $\vartheta$ Ventricle.

from the venous passages to the respiratory organs. When these are most simple in character, the blood passes to the auricle without going through branchial veins. This is the case, too, in many of the Pteropoda. When the gills are more highly developed the returning blood is collected into special venous trunks, which open, either separately or together, into the auricle. These branchial veins are always so arranged as to be adapted to the size as well as to the position of the branchiæ. In many of the Nudibranchiata (Eolidia, Scyllæa, Tritonia), large vessels pass off from the gills and unite together to form larger trunks, which give rise to a median or two lateral branchial venous trunks, connected with the auricle of the heart. When the branchiæ are scattered over a larger portion of the surface of the body, this afferent system of branchial vessels is well developed, but when they are limited in extent it is reduced in size (Doris, Polycera). Tritonia is an example of the former arrangement (Fig. 198), for in it two lateral branchial venous trunks (ss) pass by means of a transverse trunk to the heart. The transverse canal forms a kind of double auricle (a), although indeed it has only one opening into the ventricle $(v)$.

The paths by which the blood goes to the branchiæ are always more or less lacunar. In many of the Opisthobranchiata the blood 
from the colom is collected into canals, which run in the integument; and thence it is distributed to the gills. All the blood, however, does not pass to them, for some is returned to the heart after having passed through the integument.

In Helix and Limax the hæmal spaces, which pass into the wall of their branchial cavity, and which form a system of canals which carries blood to the respiratory organs, are developed in a vascular manner. They break up into a rich network in the integument, and this network gives off a number of large trunks with distinct walls, which unite to form a "pulmonary vein;" and this vein passes into the auricle. The network of pulmonary vessels may be regarded as a large blood sinus, which extends into the walls of the lungs, and which is broken through at various points by islets of firm substance.

\section{$\$ 287$.}

The heart of the Cephalopoda is placed at the base of the visceral sac, and is formed of a rounded or transversely - oval ventricle (Fig. 195, BC ; Fig. 199, c), which receives blood from as many branchial veins as there are

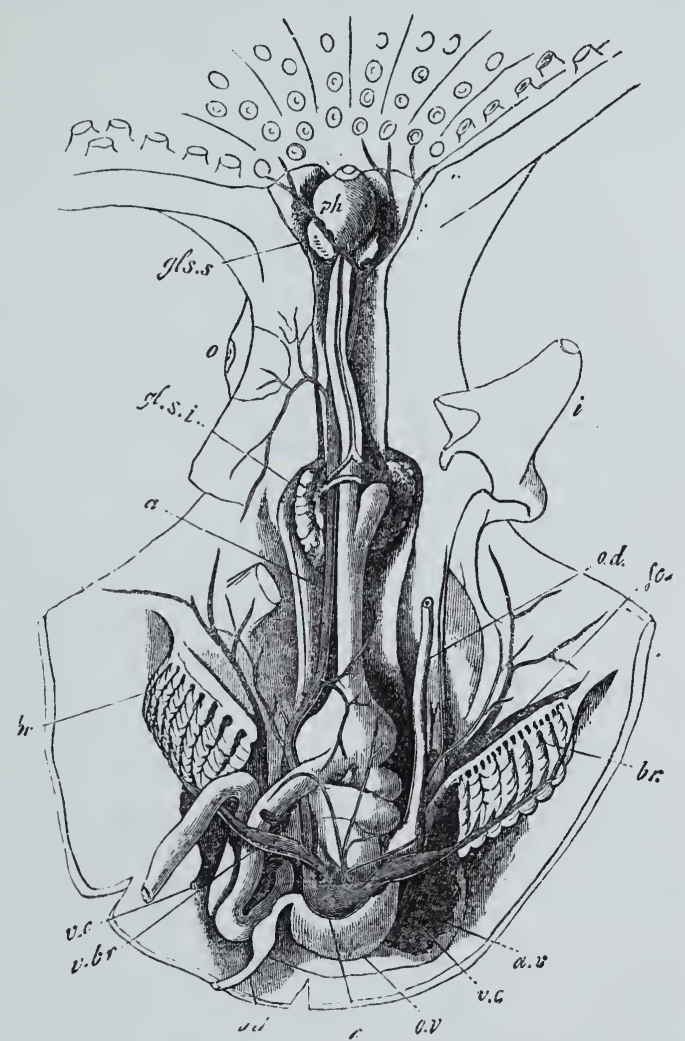

Fig. 199. Auatomy of Octopus. Mantle-cavity and visceral sac, opened from the ventral surface. $p h$ Pharynx. gls $s$ Superior salivary glands. $g l s i$ Inferior salivary glands. o Eye. $i$ Funnel. br Branchiæ. ov Ovary. od Oviduct. $c$ Heart. $v$ br Branchial veins. a Arteria cephalica. $v c$ Venæ cavæ. av Venous appendages (after Milne-Edwards.) branchiæ. 'That is to say, in Nautilus there are four, and in the rest of the Cephalopoda two branchial veins opening into the ventricle. These veins are generally considerably widened out in front of their opening into the ventricle (Fig. 199, v br), and this enlargement is homologous with the auricle in the Gastropoda and Lamellibranchiata. Two arterial trunks always arise from the heart; one, the larger, passes straight forwards; this is the arteria cephalica (Fig. 199, a); at some distance from it a smaller trunk, the arteria abdominalis $\left(a^{\prime}\right)$ is given 
off, and this generally passes backwards. This, which is the general mode of arrangement, shows distinctly how the Cephalopoda agree with the two other classes, while it points to their having a close affinity to those Mollusca which are distinguished by the possession of two auricles.

The cephalic artery first of all gives off large branches to the mantle, and some to the intestinal tract and to the fumnel; when it reaches the head it gives off the optic arteries and supplies the oral regions, and divides into larger branches, the number of which depends on that of the arms. In some of the Cephalopoda the brachial arteries spring from a circular vessel, which is developed around the commencement of the œsophagus. The abdominal artery is more varied in character; in some of the Sepiadre (Fig 202, $a^{\prime}$ ) and Loliginidæ, it arises opposite to the cephalic artery, and has, therefore, the same relations as the visceral artery in the Lamellibranchiata; but, in the Octopoda, it arises from the anterior region of the heart, close to the aorta (Fig. 199); it very soon breaks up into several branches for the enteric tube and generative organs. In the Loliginidæ, however, it gives off two additional branches for the fins; in Ommastrephes a special enlargement (which is perhaps an accessory organ of circulation) may be observed on these vessels.

All over the body the terminal branches of the arteries communicate with the veins by means of a well-developed system of capillaries. In the greater part of the body, at any rate, this system takes the place of the lacunar blood-passages which were found in the other Mollusca, and of which it seems to be a differentiation.

The venous roots from the capillaries are collected into larger trunks, which have sometimes the characters of true veins, and are sometimes widened out into large spaces, so that they are intermediate between true vessels and mere lacunæ. Of the more special characters of the venous system we have to note that the brachial veins are united into a circular sinus, placed in the head; this is supplied also by neighbouring smaller venous trunks, while it gives off a large hæmal canal (vena cephalica, or great vena cava) (Fig. 202, vc), which passes backwards to the branchial region. At the gills it breaks up into two (Dibranchiata) or four (Tetrabranchiata) venous trunks; these take up the other veins which come from the mantle and viscera $\left(v c^{\prime \prime}\right)$ and pass to the base of the gills. In the Dibranchiata the branchial arteries acquire a muscular investment and form a contractile portion, or branchial heart (Fig. 202, vc), which pulsates rapidly, and acts as an accessory organ of the circulation. Special appendages are attached to the branchial artery in front of these branchial hearts; these, which are diverticula of the walls of the vessels, are bathed by the venous blood, which passes into the branchix, in just the same way as are the organs of Bojanus in the Lamellibranchiata (vide Excretory Organs, \$ 289).

Although the venous blood-receptacles of the Cephalopoda which we have described may be regarded as a venous system, provided with closed walls, true blood lacunæ are not absent. They are distributed in just the same way as in the other classes of the Mollusca. 
The colom is such a blood-space; all the organs in it are bathed by venous blood. Various veins open into it, and it is also connected by two canals with the large vena cava (vena cephalica).

Milde-Edwards et Valenciennes, Nouv. obs. sur la constit. de l'appareil de la circulation chez les Moll. Mém. Acad. des Sc. T. XX. Milne-Edwards, Voyage en Sicile. T. I.

\section{$\S 288$.}

The blood fluid of the Mollusca is, as a rule, colourless, and often has a bluish or opalescent appearance. In many Cephalopoda, however, it is violet or green, and in some Gastropoda (Planorbis) the blood is red-the plasma being coloured.

The form-elements of the blood are always colourless, and have the character of indifferent cells, which give off all kinds of pseudopodia-like processes during their amoboid movements; this has been observed in the Lamellibranchiata and Gastropoda.

A rounded organ extends alongside the branchial arteries of the Cephalopoda; its function is not known, but it may perhaps be an organ which is of importance in the development of the formelements of the blood.

[Lankester, E. RAY, Distribution of Hæmoglobin in the Animal Kingdom (red corpuscles of Arca and Solen). Proc. Royal Society, 1873.]

\section{Excretory Organs.}

\section{$\S 289$.}

In addition to the various organs which have been already noted as present in the integument, and which serve as excretory organs, there are others which open on to the surface of the body, and which are much more important from this point of view.

In the Placophora there is a glandular organ which is placed close to the anus; but it is not certain that it is comparable to the excretory organ of the Conchifera. Its internal orifices have not been observed. For the present, therefore, we must regard this organ as not belonging to the same series as the excretory organs of the rest of the Mollusca.

These typical excretory organs of the Mollusca are homologous with the organs found throughout the Vermes, and there called renal; which organs form the looped canals (nephridia) of the Annulata. In the Mollusca also they commence by an external orifice, and open into the cœlom after a longer or shorter course. The internal opening is distinguished by special arrangements; most commonly, perhaps, indeed, generally, by an investment of cilia, whereby they call to mind the ciliated funnels of the looped canals of Vermes. Owing to the presence of these organs, the internal cavity of the body communicates with the surrounding medium. They are able, therefore, to bring water into the body, while, like their homologues among the Vermes, they may preside 
over other functions also. Thus they may have relations to the generative organs, as is clearly the case in some of the Lamellibranchiata. There is good reason also for thinking that the efferent ducts for the generative products in the Cephalopoda are derived from these excretory organs. They have not, therefore, any exclusive relation to excretion. When they are excretory in function, the walls of the canals, which are otherwise simple, undergo a certain amount of metamorphosis, and may then be seen to have a glandular structure. In these cases they may be regarded as "kidneys," on account of the chemical constitution of their products. In this case, if examined under the microscope, they are seen to be provided with secreting cells, the contents of which are formed by granular or concentrically striated concretions, similar to those which are of such importance in the renal secretions of other groups of animals.

Where the internal orifice has been observed, it has been seen to lead into the pericardial sinus, through the wall of which the duct passes. If it be true that the excretory organ is derived from a looped canal, it is highly probable that the wall of this pericardial sinus is derived from a dissepiment, such as those which carry the openings of the looped canals in the Annelides. Many facts, however, are wanting to confirm this supposition, and particularly those which would explain how the change in the position of this dissepiment has been brought about.

\section{$\S 290$.}

In the Lamellibranchiata the excretory organ is known as the Organ of Bojanus; it is always paired, although sometimes it is fused into one mass along the middle line; it lies on the dorsal side of the body, close to the base of the gills. Its substance is made up of a yellowish or brownish coloured spongy tissue, the interspaces in which often run together, and generally form a large central cavity. From this cavity a pore, on either side, leads into the pericardium, while another leads into the efferent duct. The latter either lies close to the genital pore, or is confluent with it, or, lastly, the generative organs open into the organ of Bojanus, so that the generative products are passed out to the exterior through it (Pecten, Lima, Spondylus). Arca and Pinna have the efferent ducts united; Cardium, Chama, Mactra, Pectunculus, Anodonta, Unio, etc., have the orifices of the excretory and generative organs separate. The walls, which rise up in folds, or the meshwork-like tissue of the organ, is thickly invested with secreting cells, which secrete the already-mentioned concretions, in many of which the characteristic excretion-uric acid-has, of course, not yet been observed. As to its relations to the vascular system, see p. 370 .

The Scaphopoda resemble the Lamellibranchiata in the possession of a paired excretory organ.

\section{$\S 291$.}

In the Gastropoda the excretory organ varies still more in character. A paired excretory organ-the predecessor of the per- 
manent kidney-has been made out in the Pulmonata. In adult animals the organ is, as a rule, unilateral. This double rudiment points to its being the same as the paired organ of the Lamellibranchiata. The recent discovery of a paired excretory organ in Haliotis, Fissurella, and Patella, in which forms the left organ is more or less rudimentary, seems to be decisive on the matter. 'T'he degeneration of one of the two organs appears to be correlated with the degeneration of other paired organs, as, for example, the gills. So far as exact observations show, it opens by one pore into the pericardial sinus, and by the other to the exterior. In the majority of the Gastropoda, uric acid has been detected in the organ. This is especially true of the Pulmonata, in which the kidney, which is placed between the heart and pulmonary veins, can be easily recognised on account of its whitish or yellowish colour. It is lamellar or spongy in structure, and the lamellæ, or bands which make it up, are covered by large secreting cells, in which firm concretions of various forms can be made out. The long efferent duct, which commences some way back, opens into the pulmonary cavity, which in Helix appears to be a widened terminal portion of this duct.

In the Prosobranchiata the kidney lies between the gills and the heart; it has the same position in some of the Opisthobranchiata. As a rule ar. efferent duct passes forwards and accompanies the hindgut, below which, and often not far behind the anus, we find its orifice.

In many of the Opisthobranchiata it appears to have lost its excretory function (as in Polycera), or the excretion is fluid. In this case the kidney (as in Phyllirhoë, Actæon, etc.) has the form of a long transparent tube, which extends some way back behind the heart along the middle line of the body, and is placed close to the

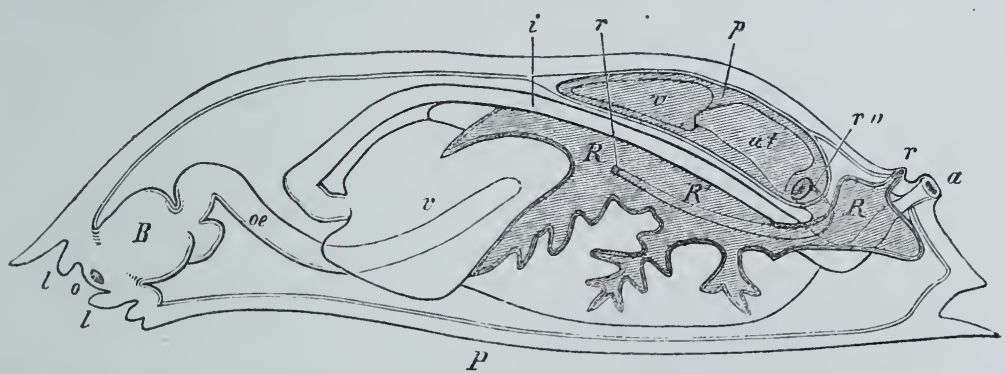

Fig. 200. Diagram of Doris, to show the excretory organ $R$. o Mouth. $l$ Lips. $B$ Buccal mass. $\infty$ Esophagus. $v$ Stomach. $i$ Intestine. a Anus. at Auricle. $v$ Ventricle (after A. Hancock).

back. It opens into the pericardial sinus by a ciliated orifice, and by a contractile one on to the surface of the body.

In many of the Opisthobranchiata a greatly-developed cæcal-sac is given off by this organ, which gives off secondary diverticula (Fig. 200, R), and so gradually forms a ramified tube. Structures of this kind, which resemble a ramified gland, have been observed in Doris, Dendronotus, Scyllæa, etc. A canal $\left(R^{\prime}\right)$ is continued from the 
pericardial orifice $\left(r^{\prime \prime}\right)$ into the interior of the tube, where it opens $\left(r^{\prime}\right)$, so that it only communicates with the exterior in a roundabout manner.

In the Thecosomatous Pteropoda, and also in the Heteropoda, the

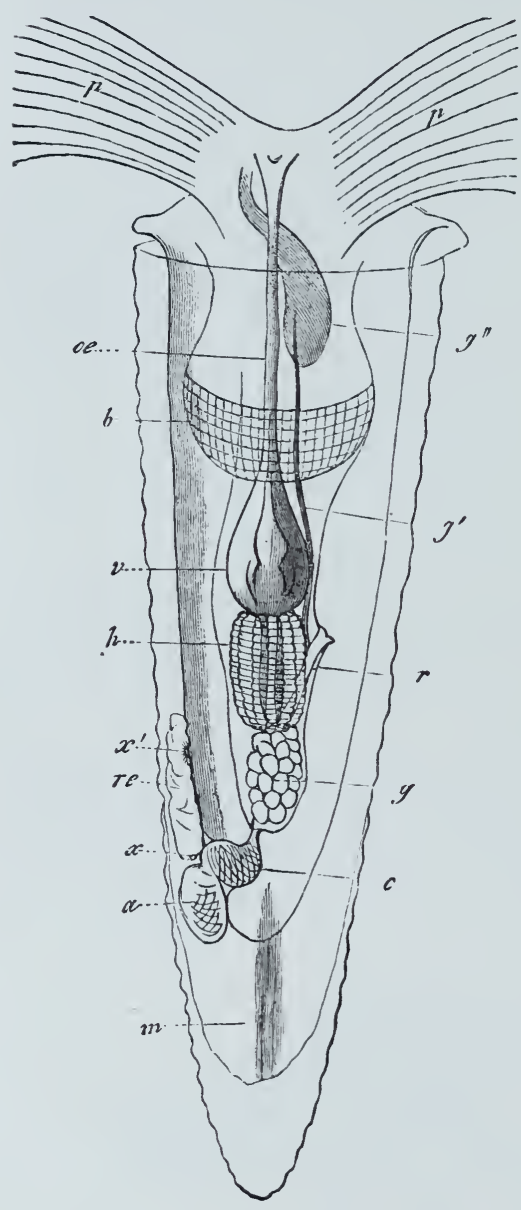

Fig. 201. Organisation of Chreseis. rp The fins. o Esophagus. $v$ Stomach. $r$ Hind-gut. $h$ Liver. a Auricle. $c$ Ven. tricle. re Kidney. $x$ Its internal orifice. $x^{\prime}$ External orifice. $b$ Ciliated organ. $g$ Hermaphrodite gland. $g^{\prime}$ Hermaphro: dite duct. $g^{\prime \prime}$ Penial pouch. $m$ Points to the position of the retractor muscle. organ which is regarded as the kidney, because of the similarity between its two orifices and those of the Prosobranchiata, is also remarkable for its spongy character. In Carinaria, among the Heteropoda, it is provided with a distinct investment of secreting cells; in all the rest there is a clear cellular layer instead. The framework of the kidney is stiff; but in Atlanta and the Firolidx it is contractile, and performs energetic and spasmodic contractions. In the Thecosomatous Pteropoda also, the kidney can act in this way, e.g. in Chreseis (Fig. 201, $r^{*}$ ).

As the glandular nature of this organ is doubtful when the secreting cells do not contain concretions, greater weight may be laid on its relations to the ingestion of water, which has been best observed in these cases. The movements of the organ are not limited to merely opening and closing its external orifice, but they also drive the water inwards and mix it with the blood which is returning to the respiratory organs from the general circulation; this organ is always placed in the course taken by this current. Although the ingestion of water by the excretory organ has not been directly observed except in these dirisions, there is no reason for concluding that it does not also obtain in the rest of the aquatic Gastropoda. It is in the Nephropneusta only that the parts concerned have other relations, but even in them the kidney has the same relations to the system of blood-canals, for thefluid of the blood may be obserred to pass out by the renal orifice. 


\section{$\S 292$.}

The great variations in the more special characters of the excretory organ, which are seen in the Gastropoda, prevent our being astonished at the fact that this same organ is otherwise modified in the Cephalopoda. All the Cephalopoda possess in their visceral mass closed sacs, which open into the mantle-cavity. As the efferent ducts for the generative products resemble the excretory canals in having that part of them which surrounds the germ-glands conmected with the cœlom, it is probable that these efferent ducts have been derived from primitive excretory organs; in this case the Cephalopoda must have liad a larger number of these organs, of which some alone have retained their primitive significance. Four of these organs are found in Nautilus, and two in the Dibranchiata. Their orifice is sometimes placed on a papilliform process (Fig. 178, r). The large branchial vascular trunks project into these sacs, and their walls, therefore, are irregular in form. So far, however, as these vessels project into the sacs, their walls must be regarded as belonging to the sac. In connection with the branchial arteries the wall of

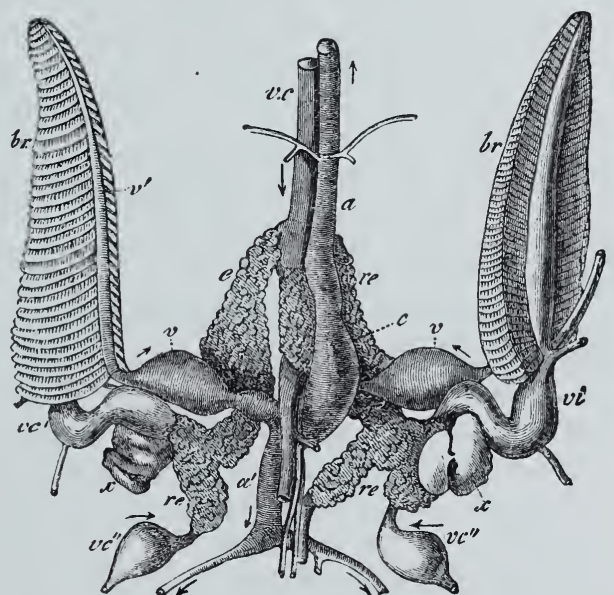

Fig. 202. Circulatory and excretory organs of Sepia. $b r$ Branchiæ. $c$ Heart. a Anterior artery of the body (aorta). $a^{\prime}$ Posterior artery. $v$ Enlargements of the branchial veins, representing the auricles of the heart. $v^{\prime}$ Branchial vein, running alongside the branchix. $v c$ Anterior large vena cava. $v c^{\prime}$ Branchial arteries (branches of the vena cava). $v c^{\prime \prime}$ Posterior venæ cavæ. re Spongy appendages of the branches of the venæ cavæ. $x$ Their diverticula. The arrows indicate the direction of the blood. current (after J. Hunter.) each sac presents numerous ramified appendages, which project into the lumen of the sacs (cf. Fig. 178, $R$; Fig. 202, re); they are formed by cæcal diverticula of the vessel, and a superjacent glandular investment. In Nautilus these appendages of the four venous trunks are clothed by tubular glands which open into the connected sac. It is still doubtful what we should regard the appendages on the other blood vessels, which project into the pericardial sinus, as being. As this sinus communicates with the mantle-cavity, perhaps they also represent excretory organs. In the Dibranchiata the venous appendages appear to have a somewhat different structure. Concretions of phosphate of calcium appear to be the principal products of this apparatus; in the Sepiada this organ (Fig. 201) is very large, and 
extends even on to the smaller roots of these veins. Owing to this arrangement, this secreting apparatus has relations to the venous current of blood which passes to the gills; it has therefore the same relation as the excretory organ of the Lamellibranchiata and Cephalophora.

It is less certainly proved that the sacs which contain the excretory venous appendages have any internal communications. While some authors assert that there is such a connection with the bloodvascular system, and especially with the pericardial sinus, others deny it. These organs still, therefore, require much elucidation.

Haxcock, A., On the Structure and Homologies of the Renal Organ in the Nudibranchiate Mollusca. Transact. Linnean Soc. Vol, XXIV.

\section{Generative Organs.}

\section{$\S 293$.}

Reproduction is never effected in the Mollusca by any of those asexual modes which we saw in the Arthropoda to be ultimately due to sexual differentiation. It is exclusively dependent on the complete activity of both sets of generative organs. In several divisions the two organs have been seen to arise from different layers of the embryo; the male having been found to have relations to the ectoderm, and the female to the endoderm. These organs are fairly distinct in the different classes of the Mollusca, so that it is only possible to derive them from a ground-form common to the whole group, by looking for this form at a very low stage of differentiation.

In the Placophora there is an unpaired germ-gland, from which paired efferent ducts lead to the laterally and posteriorly placed genital pores. Owing to the presence of separate efferent ducts, the arrangement is of a higher grade than it is in the Lamellibranchiata. In most, the sexes appear to be distinct.

In the Lamellibranchiata, individuals in which the two sexes are united are only found in a few diverse genera, or even in isolated species; they probably represent the remnant of an arrangement which was formerly found in the whole class. In the Oysters we find an intermediate step torards a separation of the sexes, inasmuch as these organs are not active at the same time in the same individual; but the male and female organs are alternately so. The g'erm-glands are paired and placed at the sides; they also open separately. They generally occupy a large portion of the coelom, and are often intimately connected with other organs.

Gradational differences can be made out in the relations of the two kinds of germ-glands in hermaphrodite forms, which indicate the lines along which the separation of the sexes has been effected. In some (e.g. Ostrea) the germ-gland is a hermaphrodite organ in the fullest sense of the word. The follicles which produce 
the ova, and those which produce the semen, are united together, and the efferent ducts are common to both sets of products. In Pecten also (P. varius) the latter arrangement obtains, but the germgland itself is differentiated into a male and a female portion. The former is placed in front and above, the latter behind and below. When, finally, as in other forms, the separate germ-glands have ducts which open separately, they are more highly differentiated (Pandora). In many genera hermaphroditism predominates in some species, while others have the sexes separate (Cardium).

The efferent ducts of the germ-glands are feebly developed, and the glandular lobules are often placed close to their common orifice. There are, therefore, no accessory organs of any kind. They open in various ways on either side. The genital canal is sometimes united with the excretory organ, so that it looks like a differentiation from it, and the generative products are passed out through it (e.g. Pecten, Lima, Spondylus); the genital canal is sometimes united with the orifice only of this organ (e.g. Arca, Mytilus, Pinna); lastly, it sometimes opens by a proper papilla (e.g. in Ostrea, Unio, Anodonta, Mactra, Chama).

From these facts it is clear that the excretory apparatus is of great importance in aiding in the formation of the efferent ducts of the generative products. The genital canal, inasmuch as it opens into the excretory organ, appears to be a differentiation of the latter, which is extended to the germ-glands in which the generative products are developed; and the gradual separation of the genital canal from the excretory organ implies a further stage of differentiation, which leads to a complete separation of the genital canal, and consequently of the generative organs, from the excretory organ. This arrangement, which is seen in all the higher Mollusca, must therefore be derived from a primitive functional connection between the generative and excretory organs; this relation is, later on, simply indicated in a faint manner by the approximation of the external openings of these organs.

The Lamellibranchiata, by showing the lines along which the differentiation of the efferent ducts of the generative organs has been effected, are not, so far as these relations are concerned, so very distant from the Vermes or the Brachiopoda. Some of the Vermes present us with relations identical with those of the Lamellibranchs; while others, having the efferent apparatus greatly, and apparently specially, complicated (Platyhelminthes), make it at first sight more difficult to give any explanation of this matter.

\section{$\S 294$.}

The generative organs of the Gastropoda and Pteropoda are in many ways more highly differentiated. Although there is a "hermaphrodite gland," as in the Lamellibranchiata, which is found in a larger number of forms, the apparatus is considerably complicated, and, as a rule, has copulatory organs connected with it. The 
generative gland is, moreover, always unpaired, and is placed, and opens, asymmetrically, so that, as compared with the Lamellibranchiata, it must have undergone unilateral degeneration.

The hermaphrodite gland varies greatly in character. It is
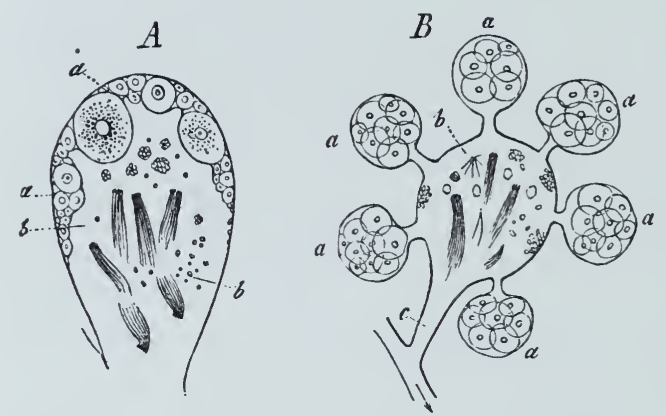

Fig. 203. Follicles of the hermaphrodite glands of Gastropoda. A Of Helix hortensis. The ova $(a a)$ are developed on the wall of the follicle; and the seminal masses $(b)$ internally. $B$ Of A eolidia. The seminal portion $(b)$ of a follicle is beset peripherally by ovarian saccules $(a)$. c Com. mon efferent duct. another give rise to seminal filaments. This double production does not seem to take place simultaneously, as a rule, so that the same lobule or the same gland produces ova at one time, and sperm at another.

A differentiation may be made out in the lobules when the ovarian portions form diverticula $(B, a)$; in this case they are grouped in a rosette around the seminal portion $(b)$, and have the appearance of secondary acini. The variations in the forms of the hermaphrodite gland are due to the way in which the separate lobules are united together; thus each lobule may have its proper duct, and the whole gland look like a greatly-ramified organ (Opisthobranchiata); or the acini may open in a row on one side of a duct, as in some Pteropoda (Cymbulia, Tiedemannia); or they may be grouped into racemose or lobate masses of glands, of which there may be a number (Phyllirhoë), or they may form a single, more or less compact, gland (some Pteropoda, as Pneumodermon, Hyalea; most of the Opisthobranchiata and Pulmonata).

\section{$\S 295$.}

The efferent ducts may be arranged in one or other of these modes in the hermaphrodite Gastropoda.

1) There is a cornmon efferent duct for the semen and ova, which represents, therefore, a vas deferens and oviduct, and carries both sets of products from the hermaphrodite gland to the generative orifice. A cæcal diverticulum forms the uterus, and also serves for 
the reception of the copulatory organ. At the generative orifice, the semen either passes directly into the subjacent and eversible copulatory organ, or, when this is some distance from the orifice, it is carried to it by a ciliated groove. This arrangement is found in some Opisthobranchiata, and in all the Thecosomatous Pteropoda.

2) The efferent duct of the hermaphrodite gland is common for a short distance only; it then divides, and each canal goes on its own way to the generative orifice. At the point of division it may be connected with secondary organs, or undergo a simpler kind of differentiation as expressed by modifications of the calibre of the canals. This may also happen to the common tube anteriorly to its bifurcation. In the Opisthobranchiata it is often widened out for a considerable distance, and so serves as a receptacle for the generative products which are about to be passed out. In the Nephropneusta (Fig. 204) the common efferent duct is divided into two portions. The upper one (ve) from the hermaphrodite gland $(z)$ is simple, while the lower is divided into two cavities for some distance; the narrower of these, which accompanies the wider one in the form of a half-groove, serves as a duct for the sperm, while the wider one ( $\iota$ ) belongs to the female apparatus. At its upper end this latter has an albuminiparous gland $(E d)$ attached to it, and in the Helicinæ is beset with diverticula, in which the ova obtain their envelope. As the other canal is not completely shut off from this uterus $(u)$, the separation of the ducts is not complete. It is only at the termination of the uterus that the vas deferens is continued on as an independent canal $(v d)$, which passes to the eversible penis $(p)$, which here forms a portion of the efferent ducts. The more distal portion of the canal secretes a substance which unites the seminal masses into a seminal rope

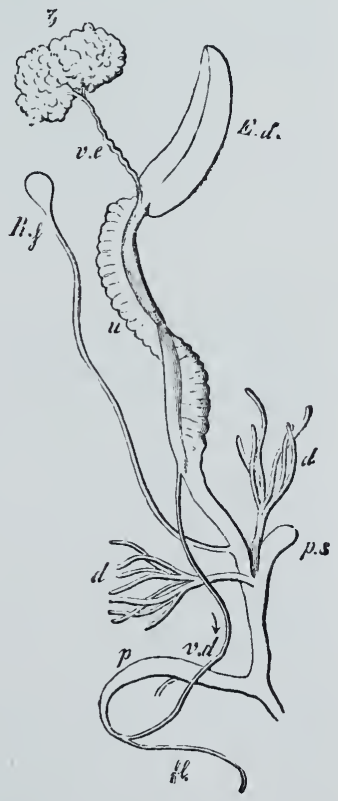

Fig. 204. Generative ap. paratus of Helix hor. tensis. $z$ Hermaphrodite gland. ve Hermaphrodite duct. $u$ Uterus. Ed Al. buminiparous gland. $d d$ Accessory glands. pc Dartsac. Rs Receptaculum seminis. $v d$ Seminal duct. $p$ Penis. $f l$ Flagelliform appendage. (Spermatophor). The uterus, finally, is continued into a terminal portion, which is known as the "vagina;" this extends as far as the common generative orifice, and may have a number of appendages $(p s, d)$ on its sides. In addition to a receptaculum seminis $(R s)$, the Helicinæ have a group of larger glandular tubes $(d)$, which are connected to a thick-walled tube $(p s)$. This latter appendage is eversible, and contains a pointed calcareous concretion (spiculum amoris), which appears to be moulded in its internal cavity. 
In the other hermaphrodite forms the two ducts are, as a rule, separated earlier, and the common canal undergoes merely unimportant modifications. The separate canals are modified in various ways, the vas deferens is very long in most of the Opisthobranchiata, and is consequently arranged in a number of coils. It is frequently united with a superiorly attached, and occasionally wider, gland, before it reaches the copulatory organ. The oviduct is not so long, and is but seldom much widened out. At the end, however, of the female efferent apparatus there are a number of accessory organs. The two sets of efferent ducts either open into a common space (generative cloaca), which is generally placed on the right side of, and towards the anterior region of the body, or the two canals open into a shallow depression, or they may open separately from one another, and on the surface of the body.

\section{$\S 296$.}

The appendages of the generative apparatus may be divided into those which belong to the male, and those which belong to the female systems. The receptaculum seminis is one of the most important of the female organs. It is a rounded or pear-shaped vesicle, inserted by a hollow stalk into the vagina, and receives the semen at the time of copulation (Fig. 204, Rs.) Sometimes there are two of these appendages (Pleurobranchus), which may be placed at some distance from the vagina, and on the narrower oviduct (Doris). In the Pteropoda and Opisthobranchiata the vagina is provided with a wide diverticulum, the glandular walls of which are set in folds, and which functions as a uterus. A special glandular organ, which resembles in arrangement the albuminiparous gland, opens into it. When this organ is absent, the wall of the uterus appears to take on its function. Lastly, we must mention the copulatory pouch of the Pteropoda, which appears to be a diverticulum of the vagina, and receives the penis during copulation (Hyalea).

The male apparatus, also, is provided with organs of this kind, which, in their simplest form, are widened parts or cæcal structures for the collection of the sperm. That elongation of the vas deferens, which we have already mentioned, is physiologically an organ of this kind. Similar arrangements are found in both the Gastropoda and the Pteropoda. The glandular organs attached to the vas deferens are also organs of this series; they are ordinarily known as prostatic glands.

Lastly, the male apparatus is connected with a copulatory organ, which is either the modified and eversible end of the seminal duct, which when not in use projects into the cœlom, or it is a special organ, which has no direct connection with the vas deferens, and which, when not in use, forms a retracted tube. The organ is either connected with the generative orifice, as in many Nudibranchs, or is separated from it. In the Tectibranchiata (Aplysia, Bulla, Bullea, etc.), the penis opens at a great distance from the common 
generative orifice; a ciliated groove conducts the semen, which passes out from the generative orifice, to the copulatory organ.

Among the Pteropoda, Pneumodermon has the penis merely represented by a papilla placed within the generative orifice; while in the Thecosomata there is an eversible organ below the opening of the vagina.

\section{$\S 297$.}

In many hermaphrodite Gastropoda the germ-glands may be seen to alternate in function, so that at one time they represent a male, and at another a female organ. This fact is an indication of that separation of the sexes which is completed in most of the Prosobranchiata.

Notwithstanding the sexes being separate, we find the lowest conditions in the Prosobranchiata, for in many the apparatus is merely represented by the germ-glands. They repeat, therefore, the same characters as those which we met with in the Lamellibranchiata. There are no efferent ducts in Haliotis and Patella. As in many of the Lamellibranchiata, the germ-gland appears to pass out its secretion through the excretory organ. In Fissurella this arrangement is still more definite in character, owing to the connection between the efferent duct and the excretory organ.

The generative organs of the two sexes have very much the same general characters, so that it is often only possible to distinguish them microscopically by the presence of the copulatory organ in the male. The male and female germ-glands are, like the hermaphrodite glands of many hermaphrodite Gastropoda, hidden in, or placed close to, the liver.

In the female organs an oviduct, which is ordinarily coiled, arises from the ovary, and turns towards the hind-gut, where it widens out and forms a uterus. A short vagina is given off from this, which passes to the generative orifice, which is placed close to the anus. Accessory organs are rare in the diøcious Gastropoda. Where they are best known they consist of an elongated spermatheca (receptaculum), which opens into the end of the saccular uterus, and which has the duct of an albuminiparous gland connected with it(Paludina). In the Heteropoda the seminal receptacle only is present, and it is either attached to the end of the uterus (Atlanta), or united to the vagina in front of this organ (Pterotrachea).

In the male organs, the duct (vas deferens) is either quite simple or is provided, on its way to the penis, with a swelling, which functions as a seminal vesicle. The end of the vas deferens opens on the surface, and on the right side, of the body. There is no copulatory organ in Haliotis, Patella, or Trochus. Where it is present it is formed by a process of the dermo-muscular tube, and is a massive broad body, which is frequently curved at its tip; it is placed on the right side of the body, or on the head, at the base of the right tentacle, or at times (Heteropoda) ciose to the anus. A ciliated semi-canal, which often extends for some distance on the surface of 
the body, is attached to this organ; it may be directly continuous with the copulatory organ, and forms a groove which reaches to it (Dolium, Harpa, Strombus), or it may traverse the penis in the form of a canal (Buccinum, Littorina, Paludina).

\section{$\$ 298$.}

The sexes are quite separate in all the Cephalopoda. The male and female organs greatly resemble one another in their general mode of arrangement; the most essential point in which is that

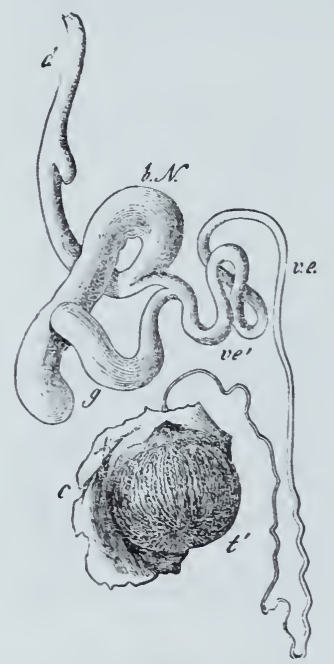

Fig. 205. Male generative organs of Octopus. $t^{\prime}$ Testis. $c$ Its capsule. ie Efferent duct. ' ${ }^{\prime} e^{\prime} \mathrm{En}$. largement, which serres as a seminal resicle. $g$ Ac. cessory glands. bN Need. ham's Pouch (after Carus and D'Alton). the germ-glands are not directly continuous with their efferent ducts. This fact is of importance, as it seems to point to the use of an organ which primitively did not belong to the generative apparatus. In any case, this arrangement is altogether different to that seen in the germglands of the Gastropoda and Pteropoda, where the secreting portions of the glands gradually pass into the efferent ducts (cf. suprà, \$ 292). In the Tetrabranchiata the efferent ducts are not perfectly continuous. The oviduct and sperm-duct both lead into a wider cavity, from which these ducts start again.

Of the female organs, the ovary is formed by a lobate gland, which is invested by a special sac, with which it is connected at one point only. The duct (oviduct) is, as a rule, single. In the Octopoda and in Loligo sagittata it is double (Fig. 199, od od), a fact which points to its having been primitively double; in the rest-as also in Nautilus-this double character has disappeared owing to the atrophy of one of the oviducts. The oviduct is attached to the covering of the ovary, so that the ova only reach the duct by passing: through the space enclosed by this covering: The oviduct ordinarily opens at the commencement of the funnel; it only opens some way back in the branchial cavity in those forms in which the male has a copulatory arm; hectocotylism is the cause therefore of a functional adaptation. At one point the oviduct has a pad-like circular investment of glands, formed by tubes set radially to the axis of the oviduct (Octopoda). In Nautilus, there is a larger number of these glands, and they extend almost as far as the opening of the duct. Where these glandular organs are absent their place is taken by a secreting apparatus placed close to the opening;

A pair of so-called "nidamental glands" are the accessory organs of the female apparatus; they consist of elongated lamellar 
tubes, which are placed in the anterior region of the animal ; their short efferent ducts open beside the generative orifice. Their secretion appears to cement the ova together; and these, in most Cephalopoda, are united into racemose groups. In front of the nidamental glands there is a pair of smaller glandular organs, which consist of closely-coiled tubes, and have the same function as the other set of glands.

A similar capsule (Fig. 205, c) to that found around the ovary encloses the testis $\left(t^{\prime}\right)$; this organ is made up of a number of branched cæcal tubes united together. These tubes are likewise attached to the wall of the capsule, so that in this case also the germinal matters first pass into the capsule, and then into the vas deferens, which is continuous with it. This duct is a much-coiled and gradually-widening canal $(v e)$, so that it forms a seminal vesicle. Glands are embedded in the walls of its widened portion, and in many cases part of the wall is converted into a larger glandular organ, so that this part has yet another function. One or two other distinct glandular appendages $(g)$ are found in various Octopoda. All these glandular differentiations of the wall of the vas deferens secrete matter which is mixed with the sperm, and which is used to form the special seminal ropes. At the end of the glandular portion, or after uniting with these ducts, the seminal duct is considerably enlarged, or pushed out on one side (Sepia, Loligo); this modification may also be converted into a considerable appendage $(b N)$ (Octopus). These "pouches of Needham" serve as receptacles for the seminal ropes or spermatophors, which are formed in the glandular portion of the seminal duct. The rest of the duct is continued, with but little change, into a papilliform process, which is placed on the left side in the mantle-cavity (Fig. 178, g), or it opens to the exterior at the base of such a papilla. We have already described the way in which single arms become functionally connected with the generative apparatus in many Cephalopoda (\$254).

The formation of spermatophors, which is a comparatively rare thing in the Gastropoda and in the other divisions, is the rule in all the Cephalopoda, and is in them most complete. As a rule, one of these seminal ropes forms a long cylindrical structure, on which several envelopes may be distinguished. The contents of the tube are only partly seminal, for in each spermatophor there is a special substance which occupies the hinder portion, and which we may call the exploding mass. The sperm is invested in a tubular manner by a special coat, and is placed in the anterior division of the spermatophor. Behind it is the anterior flattened end of a long: and spirally-coiled band, which extends through a large portion of the spermatophor, and is continuous at its hinder end with the outer coats. The substance of this spiral band is the exploding mass. When it comes in contact with the water, the spiral band immediately begins to elongate, and drives before it, and to the front end of the spermatophor, that part which encloses the semen. 


\section{Eighth Section.}

\section{Tunicata.}

\section{General Review.}

\section{$\S 299$.}

In conceding to the division of the Tunicata, formerly by general consent reckoned among the Mollusca, the rank of a special phylum of the Animal Kingdom, we do no more than assign its legitimate value to the very peculiar organisation of these animals. Their peculiarities separate them not only from all the classes of the Mollusca, but also from all the other animal phyla, althongh it must be admitted that they present certain distant affinities to some Vermes (the Enteropneusti), and that equally close relationships are readily to be observed between them and the lowest Vertebrata. We shall return to the subject of these relationships in treating of the Vertebrata; here it is only necessary to remark that the absence of a clearly-marked metamerism of the body forbids the intimate association of the two groups, although indications of the formation of metameres in certain regions of the body may be pointed out in some Tunicata.

In the position of the most important organs and their primitive relations we find the most obvious indications of Vertebrate affinity. Thus we have the enteron, with its foremost division adapted to the functions of a respiratory organ. A movable appendage of the body, present in the adult state in only one division, but in others in the larval stage, contains an organ of support, which exhibits the closest resemblance to the primitive axial skeleton of the Vertebrata. A further general characteristic of the group is seen in the hyaline body-covering, which often attains a considerable development, and is known as the "mantle." 


\section{The following divisions are recognised:}

Copelata.*

(Appendiculariæ).

Oikopleura, Fritillaria.

Acopa.

1) Ascidiæ.

Simplices.

Cynthia, Phallusia, Molgula.

Sociales.

Clavellina.

Compositæ.

2) Luciæ.

Amarrœcium, Botryllus.

Pyrosoma.

3) Cyclomyaria.

4) Thaliadæ.

Doliolu
Salpa.

\section{Bibliography.}

Huxlex, Observations on the Structure of Salpa and Pyrosoma. Transact. Royal Soc. Lond. 1851.VoGr, C., Recherches sur les animaux inférieures de la Méditerranée. II. Mémoires de l'Institut de Genève. 1852.-GANis, Zeitschr. f. w. Zool. Bd. XXV.

Copelata: GegrnbuUr, Zeitschr. f. w. Zool. VI.-Huxuer, Further observations, etc. Quarterly Journal of Microscop. Sc. 1856.-For, H., Etudes sur les Appendiculaires. Mém. Soc. de physique et d'hist. nat. de Genève. XXI.

Ascidiz: Savignx, Mémoire sur les animaux sans vertèbres. II. Paris, 1816.-Scralck, De ascidiarum structura. Halae, 1814.-MrLNe-Edwards, Observations sur les ascidies composées. Paris, 1841.-Eschricht, Videnskab. Selsk. Abhandl. IX. 1842.-VAN Beneden, Mémoire sur l'embryogénie, l'anatomie, et la physiologie des Ascidies simples. Mém. de l'Académie Rojale de Belgique. T. XX. 1846.-Meтsch Niк OFF, E., Ueber die Larven und Knospen von Botryllus. Bull. Acad. des Sc. de St. Pétersbourg. T. VI.-KRoHN, A., Fortpflanzung von Botryllus. Archiv f. Naturgesch. 1869.-Kowalevsky, Entw. d. einf. Ascid. Mém. Acad. imp. de St. Pétersb. VII. Ser. X.-The same, Ueber die Knospung der Ascidien. Arch. f. mikr. Anat. X.-KupFrer, C., Z. Entw, d. einf. Ascidien. Arch. f. mikr. Anat. VIII.-Hertwig, R., Zur Kenntnis des Baues der Ascidien. Jenaische Zeitschr. VII-GIARD, A., Rech. sur les Asc. comp. Arch. de Zool. I. -Lacaze-Duthiers, H. dx, Les Asc. simples des côtes de France. Arch. d. Żool. III.

Luciz: Kowalevskx, A., Entw. von Pyrosoma. Arch. f. mikr. Anat. XI.-Parcri, P., Gli organi luminosi dei Pirosomi. Atti del R. Accad. delle Sc. fis. e math. Napoli. V.

Cyclomyaria: Krons, Ueber die Gattung Doliolum. Arch. Nat. 1852.-Gegenbaur, Doliolum. Zeitschr. f. wiss. Zool. 1S5̃4.-Kefersteiv u. EHLERs, Zoolog. Beiträge. Leipz. 1861.

Thaliadæ : Esciricir, Undersogelser over Salperme. Kjöbenhavn, 1841.-SARS, Fauna litoralis Norvegiæ. I.-KroHn, A., Ann. sc. nat. III. vi.-MüLLER, H., Zeitschr. f. w. Zool. IV.SıLexsky, Embryonal. Entw. d. Salpen. Z. f. wiss. Zool. XXVII. - The same, Ueber die Knospung der Salpen. Morphol. Jahrb. III.

* These two classes were formerly separated according to whether they had or had not a propelling tail, as the names of the classes showed; I have retained the nomen. clature without giving an importance to this character, which does not belong to it; the larvæ of many Acopa have this directive organ. A much greater difference between the two divisions is to be found in the characters of their spiracles. In the Copelata these open to the exterior. In the Acopa they open into a cavity, which is formed from a part of the rudimentary spiracle of the Copelata. This is the most essential characteristic. 


\title{
Form of the Body.
}

\author{
$\S 300$.
}

The form of the body of the Tunicata undergoes in the various divisions such remarkable modifications, that the resulting forms compared in their extreme conditions of development appear to have no relationship whatever to one another. In the Copelata, the lowest Tunicata at present known, the body exhibits two divisions, of which one encloses the most important organs, whilst the other has the form of a very wide and long movable appendix-the propelling tail. The anterior division of the body contains the intestinal tract together with its respiratory portion. The gut opens to the exterior by the ventrally-placed anus. Two slits break through the body-wall from the respiratory cavity. On the whole a bilateral symmetry predominates, and accordingly two antimeres are recognisable. The tail portion of the body in the Copelata, attached ventrally to the fore-portion, projects at an angle from the latter, and thus has the appearance of a simple appendage. How the earlier stages explain this condition must be described below.

A form coming near to this condition is possessed by the larra of the Ascidians, in which the tail is simply a prolongation of the aboral end of the body, and so far appears to present us with a more primitive condition. A similar process is found also in the young of Doliolum, whence it may be supposed that all Tunicata have descended from a common ancestor provided with such a tail-like region of the body. In Doliolum, as the power of swimming becomes associated with modifications of the respiratory cavity, the propelling tail dwindles. In the Ascidians free locomotion is lost when the propelling organ atrophies. The complete animal takes on a fixed mode of life. Whilst a greater complication of the structure of the organism is acquired the external appearance becomes simplified. The tube-like body exhibits two openings, approximated to one another. The orifice of entrance corresponds to that of the Copelata. The second opening leads into a chamber having the function of a cloaca, which has arisen from a development in connection with the primitive respiratory slits. This condition of parts holds good also for the higher divisions of the Tunicata, amongst which the Cyclo. myaria and Thaliada appear as swimming forms, moving themselves by the action of the walls of the body. Their body, which is on the whole of a cylindrical form, has its incurrent orifice at one pole of the long axis, and the orifice leading from the cloaca at the other or aboral pole.

\section{$\S 301$.}

Complications of the external form of the Tunicata appear in connection with the very common process among them of a sexual 
reproduction which leads to the production of Colonies or Stocks. Gemmation is the fundamental process in this phænomenon. It predominates in the Acopa. In many Ascidia a new individual buds from the body of the adult animal, the parent sending forth a runner (stolo) which is composed of form-elements belonging both to ectoderm and endoderm. From this there is gradually differentiated an organism identical with the parent animal. In this way colonies of Ascidians (Ascidiæ sociales) are produced. In others this proceeding appears with its successive steps compressed at a very early period of life, and accordingly there buds forth from the embryonic body of one Ascidian a second (Didemnum). Thus we get two persons closely united to one another. From this case, it is easy to derive those conditions in which the sessile young individual gives rise by budding to a number of persons (Botryllus). In these several generations, all produced by budding, follow one another. From the first a bud grows out, which, like Didemnum, gives rise by budding to two persons, and from these in like manner four arise. When the mother-animal dies down, the eight budlings are left in communication with one another by means of the cloaca, and form a rosette-shaped group. Such and similar processes give rise to the colonies which we find among the Ascidix compositæ. The combination of the scattered groups takes place through the agency of a tissue belonging to the integument which in those persons which live isolated lives is known as the mantle (outer mantle).

By means of a peculiar method of grouping, the persons of the Luciæ form cylindrical colonies. The wall of the hollow cylinder is formed by the ascidian-like persons and their common investment. On the outer surface of the cylinder are found the entranceorifices ; opposite to them, and opening into the internal cavity, are the excurrent orifices. The multiplication of the persons of the colony is carried on by budding. The formation of new colonies is provided for by the sexual reproduction. From the egg arises an embryo, from which at once four persons bud forth. They remain invested by the mantle of the first, and constitute as soon as they are born a new colony.

Since the persons which develop from an egg never in the com. pound Ascidians acquire sexual organs, and since these organs on the contrary appear in those persons which arise by gemmation, we have here an example of the phrenomenon known as Altermation of Generations.

What is performed in the Ascidiæ by means of offshoots starting from the surface of the body, is carried out in the Cyclomyaria and Thaliadæ by a special organ-the germ-stock or stolo prolifer. It exists also in the Luciæ, but has there a more limited functional activity. In the Cyclomyaria it appears as a process springing usually from the dorsal surface of the body in the neighbourhood of the excurrent orifice; in the Salpæ and in the Pyrosomæ it has a ventral origin, and agrees with the condition found in Cyclomyaria only at the first, for, instead of budding out externally, it takes up a 
position, varying in different cases, within a cavity generally in the neighbourhood of the intestine. In its relation to the formation of buds the stolon of the Salpæ also behaves differently from that of Doliolum. In the latter, bud-generations sprout from the stolon; they are arranged in rows, are often dimorphic, and remain attached to the stolon by short processes. In the Salpæ similar buds arise by outgrowth from the stolon, but each bud encloses within its base half of the whole area of the stolon; so that when two rows of such buds are formed the material of the stolon itself is transferred to the body of the buds. The maturity of the chain-like connected series of young secondary buds (into which the primary bud divides) results, in consequence of this disposition, in a separation (Fig. 206,n) from the parent of the portion of the stolon concerned in its production.

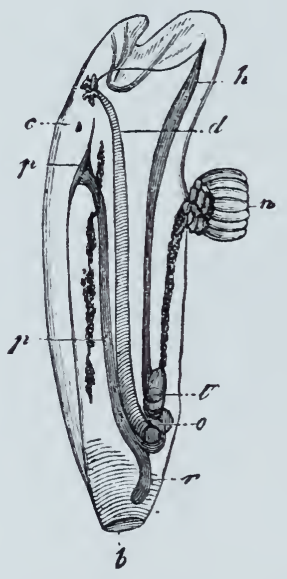

Fig. 206. Asexual form of Salpa pinnata (solitary form). $n$ Em. bryo-chain escaping to the ex. terior.

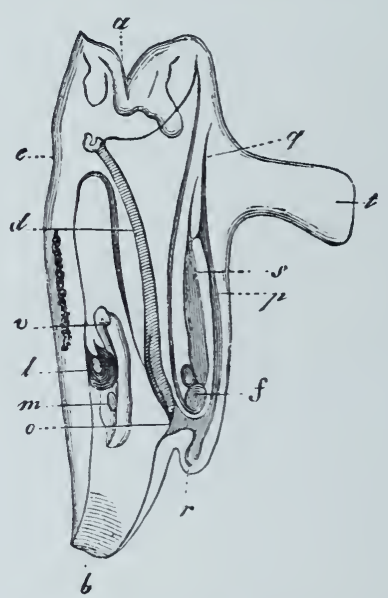

Fig. 207. Sexual form of Salpa pinnata (chain form). $t$ Connecting bar. a Incurrent orifice. $b$ Excurrent orifice. $c$ Ganglion. $d$ Gills. $f$ Heart. $h$ Ventral groore. $r$ Hepatic pouch. $\quad v \operatorname{lm}$ Embryo with embryonic organs (both figures after C. Vogt).

This arrangement brings about an "alternation of generations," seeing that the forms provided with a stolon are always permanently devoid of sexual organs. On comparing this- series of phænomena with that occurring in the Ascidiæ, we find, just as in the Ascidiæ, proliferous outgrowths, namely, the stolons. The outgrowth in question is here confined to a definite part of the body. In Pyrosoma a proliferous stolon is present, sunk in the mantle, and from every such stolon only a single bud is developed; side by side with this are developed sexual organs. Accordingly it is not possible to suggest that the stolon belongs to the sexual apparatus. In the Salpæ and in Doliolum, contrary to what we find in Pyrosoma, the proliferous stolons give rise to copious generations of buds. In this case, however, the absence of a sexual apparatus is the accompani- 
ment of the richer gemmation, and we must regard the sexual apparatus as having been atrophied. This atrophy of the sexual apparatus is referable to the increased reproductive activity of the stolon. In the Salpæ the progeny of the sexless generation are invariably sexually complete, and so we have an example of pure "alternatio generationis," whilst in Doliolum the asexual reproduction is only played out after a numerous series of stolon-bearing generations have succeeded one another. In this manner the condition of the Cyclomyaria approaches more closely to that of the original Ascidian-gemmation, and this both in the external position of the stolon and in the mode of connection of the buds with the stolon. The internal stolon of the Salpæ, on the contrary, is widely separated from the primitive arrangement, not only by its position, but also by the assumption of the material of the stolon by the buds.

\section{Integument.}

\$ 302 .

The investment of the body of the Tunicata consists in its primitive condition of a cell-layer formed from the ectoderm. It persists in this condition in the Copelata, in which it appears to represent, at any rate to a large extent, the body-wall. 'The flattened cells here form but a single layer. This simple arrangement gives place, in the higher division, to a complication; the primitive arrangement only occurring in a transient form in the earlier stages of development.

A layer of material secreted from the cells of the ectoderm forms a covering enclosing the body, and known as the "mantle." This condition is not altogether a new one, for in many Copelata a forerumner of the mantle is to be found. In them the cells in the neighbourhood of the incurrent orifice attain considerable dimensions, and secrete a slimy but consistent substance, which forms in large quantities, and gradually enclosing the body more or less completely, assumes the appearance of a basin-like structure of relatively large dimensions. It was described by earlier naturalists as the "house," and functions as a protective organ for the body (Oikopleura).

The secretory activity, which is here confined to a particular region of the body-surface, is spread in the other Tunicata over the whole superficies. Its products constitute the outer mantle, which in its essential character belongs to the category of cuticular structures. In consequence of the migration into it of formelements from the ectoderm, the resulting tissue assumes the characters of the connective substances. The primarily homogeneous layer becomes converted into an intercellular substance. The cells scattered in it present a great variety of characters. Often the mantle thus formed attains a preponderancy over all other organs, and serves, in consequence of its rigidity, as a supporting 
organ for the enclosed parts. The consistency of this covering varies from the softness of jelly to the hardness of cartilage. It is generally of glass-like transparency, and in the Ascidians often variously coloured. Complications of the structure of the mantle are brought about in many Ascidians (Phallusia) by the penetration into it of great numbers of blood-vessels. Very remarkable is the development of the mantle into two valves, movable on one another like the shells of the Lamellibranchiata, and which are able to open and shut as do the latter (Chevreulius). In the colony-forming kinds, this mantle-layer is common to all the persons, enclosing them together.

The formation of this mantle leads to a suppression of the differentiation of all other tegumentary organs; we have nevertheless to notice in the Copelata the formation of numerous other organs of this category-uniceliular glands, hair-like processes, etc.

A mass of cells is also developed from the ectoderm in the Pyrosomæ, and occupies a position in the neighbourhood of the incurrent orifice; it is the paired luminiferous organ of these animals.

Hertwig, O., Ueber den Bau und die Entwickelung des Tunicatenmantels. Jenaische Zeitschr. Bd. VII.

\section{Skeleton.}

\section{$\S 303$.}

In most 'Tunicata, the mantle, in virtue of its rigidity, functions us the supporting organ of the otherwise soft body. Besides this we meet with a peculiar organ of the greatest morphological importance. In the tail-like propeller of the Appendiculariæ there exists an axial organ which extends forward as far as the fore-body. It is made up of cells, which secrete a fairly resistent chord consisting of homogeneous substance enclosed in a continuous sheath, the remains of the cells being found at a later period still resting upon the chord. This chord acts by means of its elasticity, bringing the propellingtail back to its previous position after it has been bent by the contraction of its muscles. Such an axial organ (Fig. 208, ch) is retained by all those Tunicata-larva which possess a mobile propelling tail, both Ascidians and Cyclomyaria. It disappears with the tail. Its relations of position show it to be homologous with the chorda dorsalis of the Vertebrata, and we may therefore designate this structure also as chorda.

\section{Muscular System.}

$\S 304$.

The disposition of the musculature presents very different features in the various Tunicata. The Copelata, for instance, 
possess a complete muscular layer with longitudinal fibres, on the propulsory tail only. It can be divided into a dorsal and a ventral band of varying breadth, covering in the chorda from above and below. Muscles are entirely absent from the anterior division of the body which contains the viscera.

In the Ascidians the musculature forms a sac lying beneath the ectoderm, and is separable in Cynthia into several layers according to the course of the fibres. In others the muscular layer is feebler, composed of intercrossing bands (A. compositæ). The Pyrosomæ have no muscles excepting around the excurrent and incurrent orifices of the body. The musculature of the Cyclomyaria is broken up into isolated annular bands; and in the Salpæ also it forms hoops, which are, however, here and there joined to one another. This hoop-like formation arises from the differentiation of a primitively continuous muscular layer. Gaps arising in this become gradually larger until the breaking up of the layer into separate hoops is brought about. At the incurrent and excurrent opening of the Ascidians the musculature has also an annular disposition and has the characters of a sphincter.

The form-elements of the musculature are transversely striated.

\section{Nervous System.}

$$
\S 305 .
$$

The central apparatus of this system occupies a dorsal position in all Tunicata, and proceeds from a differentiation of the ectoderm, as has been ascertained from the study of the development of Ascidæ and of Salpæ. In the general relations of the parts there are

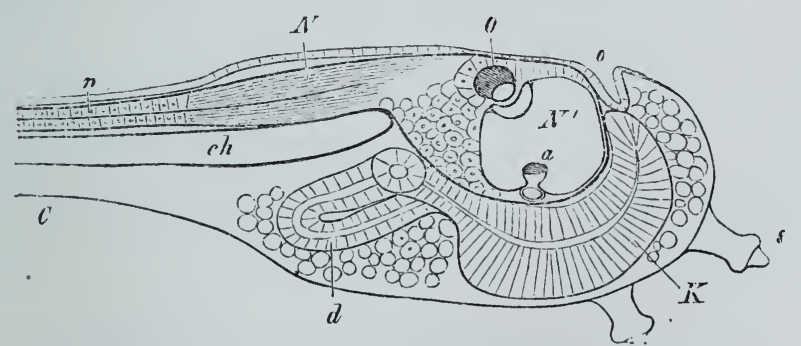

Fig. 208. Ascidian embryo with only a part of the tail $C . \quad N$ Nerve-centre, forming a cavity in front $N^{\prime}$, produced behind into a nerve-cord $n$. O Eye. a Auditory organ. $K$ Embryonic foundation of the respiratory chamber. $d$ Ditto of the digestive canal. $o$ Ditto of the mouth. ch Chorda (after Kupffer).

agreements with what we find in the lower Worms. The in-sinking of the ectoderm forms a tube which remains open for a time, and then becomes pinched off from the surface-layer and extends itself in Ascidian larvæ (Molgula) as a chord reaching throughout the length of the tail (Fig. 208, n). A central canal perforates the chord, and 
can be traced into the anterior larger mass $(N)$. A division of this anterior mass into three consecutive lobes, produced by an unequal thickening of the wall of the tube, the foremost of which is in Ascidiæ and Salpæ in close connection with the origin of the visual organ, is also to be made out in the Copelata, in which the fundamental structure sketched out in the Ascidian larvæ undergoes a further and permanent complication. In them we find the nervous system made up of an anterior elongated ganglion (Fig. 209, n) which exhibits three dilatations (App. flagellum), and passing backwards in the form of a chord $\left(n^{\prime}\right)$ to the base of the tail, is continued along that organ to its extreme point. At the root of the tail a ganglionic swelling is formed on the chord, and two others follow after this (A. furcata). The first appears to be the more constant. This apparatus, in its rudimentary condition a continuous one, as appears from the Ascidians' tadpole, must be regarded as the central-organ of the nervous system. It is noteworthy that in the Copelata, too, it encloses a canal which extends from the foremost ganglion to the ganglion at the root of the tail. The central elementary parts (the nerrecells) are not, however, equally distributed, but form the ganglia only, to which the rest of the chord serves as commissure. 'The continuation of the nerve-chord in the tail lies to the left of the chorda, if we consider the two surfaces of the tail (as its relation to the rest of the body requires), as dorsal and ventral respectively. This asymmetry is in the Ascidian larvæ either developed late or not at all, so that as the primitive condition we are justified in assuming a median dorsal nerve-chord. The form of the nerve-centre thus determined is one which is in the highest degree noteworthy, since it is not presented in any other division of invertebrate animals, in all of which the prolongation of the central organ takes place in a ventral position.

Peripheral nerves pass out from the anterior ganglion, branching latcrally around the incurrent orifice of the branchial chamber. Others pass backwards to the spiracula. In the tail, nerves pass out from the ganglia, as too in the Ascidian larvæ there are nervebranches on the caudal portion of the nerve-centre; terminally the chord gradually breaks up into a number of branches.

\section{$\S 306$.}

The atrophy of the tail, or its complete absence, brings about in connection with the further elaboration of the fore-end of the body by the development of the branchial slits, a considerable change in the nervous centre. In the Ascidians the caudal division of the nerve-centre disappears, and in Pyrosoma and Salpa the embryonic foundation of the organ is limited to the anterior portion which is accordingly more volumiuous in proportion. The three vesicular divisions which still appear as embryonic rudiments in the Salpæ give place to a single ganglion-mass. The Ascidians hare this 
body located between the incurrent and the excurrent orifices (Fig. 210,n), and it occupies the same position indicating the dorsal region in all Acopa. Whilst in Ascidians the peripheral nerves are given off more abundantly from the fore and hinder divisions of the often elongated ganglion, we find in the Cyclomyaria and Pyrosoma nerve-branches given off laterally also, and in the Salpæ the ganglion gives off numerous nerves all around in a radial manner.

The arrangement of the whole apparatus is very widely different from that of the higher Worms, Arthropods and Mollusca, and it is only among the Vertebrata that a comparable condition is to be found. To this subject we shall have to return hereafter.

\section{Organs of Sense.}

\section{$\S 307$.}

As sensory apparatuses of an indifferent nature, possibly serving a tactile function, certain cells are described in the integument of many Tunicata (Salpa), and appear to be nerve-endings. From such cells filamentous processes stretch towards the surface, as for instance in the denticulations of the incurrent orifice in Doliolum, and in the margin of the same orifice in Salpæ. The processes found in many Ascidians around both orifices of the body are no doubt provided with similar organs.

A more differentiated organ of sense is the so-called ciliated groove, which is formed on that surface of the ganglion which is turned towards the branchialchamber (Salpæe and Pyrosoma), and in all Tunicata, even when it takes up a position in front of the ganglion, retains a connection with the nerve-centre, which it gains at a very early period of development. By the upgrowth of the margin of this groove turned towards the branchial chamber, a variety of forms, some stalked, are brought into existence, whilst dilatations of the groove give rise to modifications of another kind. The signification of this organ, clothed as it is with flagellate cells, must be that of an Olfactory organ, or at any rate of an organ, the function of which is to test the quality of the water coming into the branchial chamber.

With more certainty can Visual organs be distinguished. They have been observed in the larvæ of Ascidians as well as in Pyrosoma and Salpæ. They take their origin in the anterior vesicular enlargement of the central nervous system (Fig. 208, $N^{\prime}$ ), and in fact, on the dorsal portion of the wall of this vesicle. A dark mass of pigment sunk in the wall carries on its surface a hemispherical refractive body, over which a second is stretched. In the neighbourhood of the pigment-mass, the cells are radially directed towards it, and accordingly represent a division of the central nervous system belonging to the eye. Probably processes of these 
radial cells are sunk in the mass of pigment directed against the light-breaking body perched upon it. In Pyrosoma the visual organ appears as a division of the ganglion, a pigment-clothed outgrowth. On one spot of this, free from pigment, is placed a multi-lamellar refractive apparatus.

Although also sessile on the ganglion, the eye of the Salpre seems to be more conspicuously elevated, and at the same time divided into several portions. Whether we have here the same type as in the Ascidian larvæ is not yet ascertained.

Auditory organs are known in the Copelata, Cyclomyaria, and many Ascidian larvæ. In the first named, a vesicle furnished with an otolith is affixed to the left side of the anterior ganglion, and has on its inner wall fine hairs which appear to hold the otolitl. Also on the left side, but at a greater distance from the ganglion, is a similar vesicle to be found in one generation of the Cyclomyaria. A nerve passes from the ganglion to the vesicle. In the Ascidian larvæ there is also in the same chamber of the ganglion which surrounds the rudimentary eye an otolith, which is supported by fine hairs (Fig. 208, a).

M. Ussoff, Beitr. z. Kenntniss der Organisation der Mantelthiere. Bericht der K. Ges. der Freunde der Naturforschung. Moskan, 1876 (Russ.).

\section{Alimentary Canal.}

$\S 308$.

This system of organs forms the part of the body which is the most peculiar in the Tunicate phylum, and enables one to draw a sharp line between that phylum and most of the other divisions of the Animal Kingdom. Its peculiarity consists in the elaboration of the anterior portion of the gut into a respiratory organ, similar to that which we saw among the Worms in the Enteropneusti. The water taken in not only brings nutrient matters, but serves also for respiration, finding its exit through special openings (spiracula) which are cut in the wall of this division of the gut. As a consequence, peculiar arrangements are brought into existence which have the function of directing the food particles entering the respiratory chamber to the commencement of the proper alimentary canal. The embryonic foundation of the entire gut proceeds from the entoderm, which, however, only corresponds to the rudiment of the respiratory portion to begin with, and from this, as a secondary process, the proper digestive tract buds forth. We shall therefore treat these two divisions of the primitively uniform alimentary canal separately from one another; the more so since considerable modifications which the respiratory chamber undergoes, bring about in their turn modifications of the form of the body. 


\section{Respiratory Chamber (Branchial Sac).}

$$
\S 309 \text {. }
$$

The simplest condition-that found in the Copelata-must serve as the starting-point. The incurrent orifice (Fig. 209, o) homologous with a mouth, occupies the foremost region of the body, and leads into a rapidly widening space, triangular in section $\left(k_{i}\right)$. The broad ventral surface is posteriorly somewhat expanded so that it forms two lateral cornua. These gradually narrow each into a tube-like continuation which breaks through the body-wall on the ventral surface $\left(k^{\prime}\right)$ and forms a branchial aperture (spiraculum). The dorsal continuation of the respiratory chamber is continued

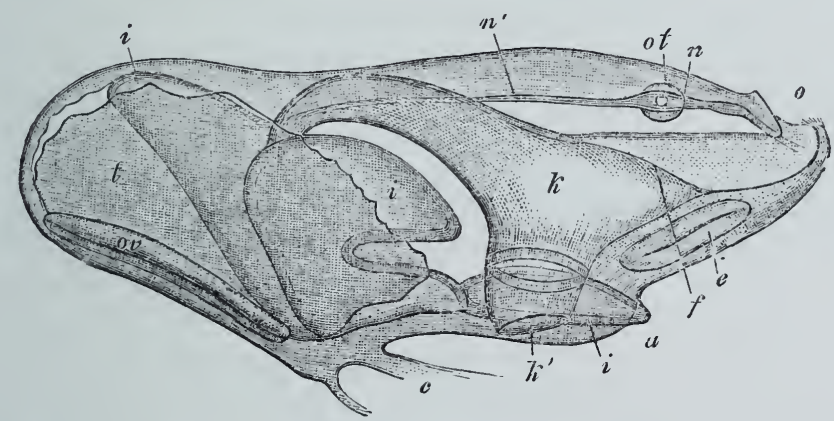

Fig. 209. An Appendicularia seen laterally. $n$ Nerve-centre. $n$ 'Nerve-chord. ot Otocyst. o Incurrent orifice. $l$. Respiratory chamber. $e$ Ventral groove. $f$ Ciliate tract. $i$ Digestive canal. a Anus. $k^{\prime}$ Spiracle. $t$ Testis. ov Ovary. $c$ Root of the tail (after H. Fol).

without any sharp line of demarcation into the beginning of the proper digestive canal $(i)$.

The two branchial apertures serving for the ejection of the water are cylindrical tubes, each of which takes its origin as an outgrowth of the wall of the respiratory chamber, into which an insinking of the outer body-wall pushes its way. The tube is beset with a ring of ciliated cells, which excite a streaming of the water directed at will now from the mouth through the respiratory chamber and the spiracula to the exterior, now in the reverse direction from without through the spiracula into the branchial pharynx towards the mouth. Mouth and spiraculum act here both as incurrent and excurrent orifices for the water.

On the ventral surface of the respiratory chamber is found a deep groove, the ventral groove (e), open by small slits to the respiratory chamber. In front two bands of cilia $(f)$ stretch from this groove towards the dorsal surface, embracing the entrance to the respiratory chamber. These structures are all connected with the taking in of food. 


\section{$\S 310$.}

The rudiment of the branchial chamber or branchial-gut gives rise in the Acopa to very highly elaborated differentiations which are in harmony with the condition presented by the Copelata. In the latter, two outgrowing sacs are formed which are not placed in communication with the exterior, until each is met by an ingrowth of the ectoderm; and so, too, in the Ascidians, two lateral sacs arise by a pinching off of the branchial gut. They communicate for a time with this their point of origin, but subsequently separate from it, and grow up around the walls of this part of the gut dorsally, until they meet and unite with one another. As a result we have a cavity formed around the branchial chamber by the lumen of these united sacs, the peribranchial space (perithoracic chamber of authors). An insinking of the surface of the body approaches the point of union of the two halves of the peribranchial space, and forms, when it has finally broken through, a communication to the exterior, the excurrent orifice. On the ventral face the separation of the two spaces persists. During the concrescence of the two sacs which grow up round the branchial chamber, and of the superficial insinking, the anal aperture is gradually brought into the area of this space. This region then constitutes the cloaca (Fig. 210, cl). In the

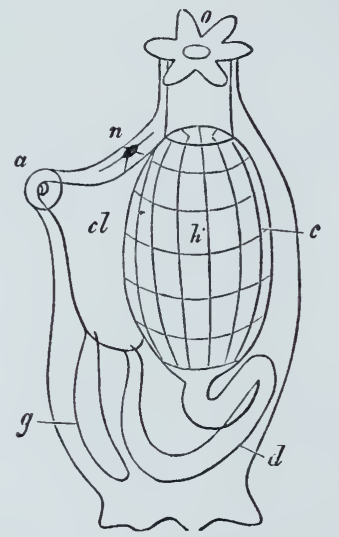

Fig. 210. Diagram of an Ascidian. o Incurrent orifice. $i$ Respiratory chamber. $c$ Ventral groove. $n$ Ganglion. $d$ Digestive canal. cl Cloaca. g Generative gland. walls of the branchial chamber there now arise clefts leading into the peribranchial space; in fact, branchial slits, which consequently have quite a different importance from that of the two primary spiracula.

Gradually, the entire wall of the respiratory chamber breaks up into a lattice. work, the fine slits of which, arranged in rows, are beset with cilia. In the rods of the lattice-work blood-channels are excavated. Water passes through the slits into the peribranchial spaces formed by the outgrowth of the above-mentioned sacs, whence it is conducted to the cloaca, and thence to the common excurrent aperture.

In the compound Ascidians the excurrent apertures of a number of individuals are united to form a common cavity, so that each group of individuals possesses a single excurrent aperture placed in the centre and surrounded by a number of incurrent apertures. The entrance into the respiratory chamber is, particularly in the Ascidians, surrounded by tentacular organs, which are partly in the form of external processes, partly placed at a distance from the orifice, and are pointed towards it. 'The lattice-work of the gills affords an endless variety in the arrangement of its component rods, and in the form and number of 
its rows of slits; as well as outgrowths of diverse kinds, which are sometimes ridge-like, sometimes papilliform, and give rise to numerous complications by the formation of anastomosing processes. The most remarkable are the tongue-like appendages ("languets") found in Ascidians and in Pyrosoma, which form a long row along the dorsal surface. Opposite to them lies the "ventral groove," already mentioned above.

The branchial chamber of the Ascidians appears, from what has just been stated, to be, in respect of the structure of its walls, a very different organ from that of the Appendiculariæ, and must have been formed only after a long series of modifications.

The same is essentially true for the other Acopa. The Pyrosomæ, in other respects very closely allied to the Ascidians, present an aboral position of the cloacal opening in conjunction with the arrangement of the several persons forming a colony around the walls of a hollow cylinder. The persons arranged in the wall of such a cylinder are placed with their incurrent orifices on the outer surface, whilst the cloacæ open into the cavity of the cylinder, the orifice of which serves as the common outlet of all the cloacæ.

In the Cyclomyaria the body, which in its mature form is tubshaped, has a wide internal cavity. The gill, which traverses this cavity obliquely, and is formed by a membrane perforated by a pair of clefts, divides the internal cavity into an anterior and a posterior division. The anterior is the branchial chamber, into which the incurrent orifice leads ; the posterior space, into which the mass of the viscera, covered by the body-wall, protrudes, is the cloaca, and corresponds to the chamber formed by concrescence around the primitive branchial chamber in the Ascidians. The Salpæ have a similar disposition of parts. The gill is, however, in them more completely detached from the wall of the respiratory chamber, and forms a rafter stretching: obliquely from the dorsal wall of the respiratory chamber in front, to the ventral wall behind, on each side of which the respiratory chamber is widely open to the hinder space representing the cloaca. The excurrent orifice proceeding from this has a more dorsal position, and is not unfrequently drawn out into a tube-like form (Fig. 212, $b$ ). In consequence of the reduction, in this case, of the gills to the rafter-like septum, there is no formation of actual gill-slits, and the water taken into the branchial chamber streams laterally on each side of the median branchial septum into the cloacal chamber.

The taking of water into, and its ejection from, the body, has in both Cyclomyaria and Thaliadæ a close connection with locomotion. This function is here in fact connected with respiration, and the position of the incurrent and excurrent apertures are consequently of importance.

The water taken in in front is, after it has passed the respiratory chamber, driven on towards the aborally-placed excurrent aperture by the action of the muscular rings of the body-wall, and the stream thus expelled works as a vis a terg 0 , and moves the body forwards by jerks. 
A phænomenon worthy of the greatest attention appears in the Acopa in the disposition of the branchial slits, and is in the Salpæ alone obliterated by the peculiarities of their organisation. This is the disposition of the slits in the manner of metameric structures. In Doliolum they form two rows of transverse clefts, and in Pyrosoma and Ascidians their transverse arrangement is also perceptible, although in the latter several or many slits occur in a cross series. Although this disposition is exhibited merely in a part of the gut, yet we are able to recognise in it a condition which supports the interpretation of it as an instance of metamerism. Metamerism exhibits itself here in fact without the participation of the entire organism, and it is not difficult to understand how, under certain conditions, other parts of the body might take part in it.

\section{$\S 311$.}

The close relationship among themselves of all divisions of the Tunicata is further exhibited in the existence of peculiar organs belonging to the respiratory chamber which are connected with the nutritive functions of the animal. These organs are the ventral groove and the ciliated tracts. The ventral groove (hypobranchial groove) (Fig. 211, Bn), also called Endostyle, is a groove lying in the ventral median line of the branchial chamber's wall, possessing up-standing margins (*) (ventral folds), and terminating
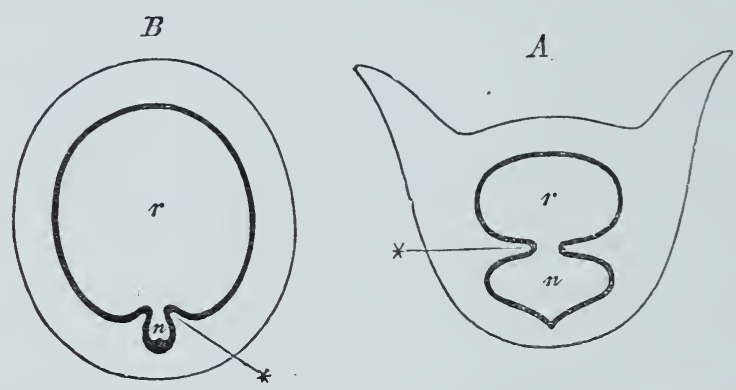

Fig. 211. Diagram to exhibit the relations of the respiratory chamber to the ventral groove. $A$ in Balanoglossus. $B$ in Tunicata. $r$ Respiratory chamber, $n$ Ventral groove. * Ventral folds.

at its anterior as well as at its posterior extremity in a blind dilatation.

The sides of the groove, which is at first in the Salpæ very broad, but later becomes narrow as in the other Tunicata, do not sink equally below the surface in all parts, but form in various regions a variety of prominences which may be described as longitudinal ridges parallel with the groove. Between these are more or less deeply-cut fissures, so that the contour of the groove in cross-section is described by a much contorted line. The epithelium of the respiratory chamber exhibits even at the free edge of the groove 
remarkable modifications. The cells form up-standing longitudinal ridges. At the bottom of the groove, between the two most deeply embedded ridges, there are cells provided with long vibratile filaments which may even project into the respiratory cavity. The Copelata possesses the simplest arrangement of this organ. In many there is only a single cellular ridge. Two are recorded in Doliolum. Three occur together, with other complications, in the Ascidiæ and Salpæ. The margins of the groove as a rule lie in close apposition, so that the groove is closed except at one part near its foremost extremity. At this spot commence the ciliate tracts which embrace the entrance to the respiratory chamber. They are clefts beset with ciliabearing cells which take a dorsal course and lead either to the œsophagus (Copelata) or to the neighbourhood of the great ganglion, taking a spiral turn on the way (Doliolum), or end in a ciliated groove (Salpæ). A similar division of the foremost section of the intestinal tract was noted in the Enteropneusti (Fig. 211, A). Two longitudinal folds $(*)$ separate this region into a respiratory $(r)$ and an alimentary $(n)$ portion. The latter seems to be strictly comparable with the ventral groove of the Tunicata, which in its early stage of development is also of very considerable dimensions relatively.

The function of the ventral groove is that of a glandular organ. The cellular ridges secrete a slimy substance which is carried forwards to the open part of the ventral groove by the cilia placed along its floor, and from thence is further moved along the ciliate tracts. At the same time the mucous masses project into the lumen of the respiratory chamber, arrest the nutrient particles taken in with the water, and become formed with these particles into a string which passes into the œsophagus. Since too the free margins of the ventral groove are beset with cilia, and a ciliate tract can be traced as far as the œsophagus, the mucus also which passes out of the slit of the groove is caught up by the cilia, and directed, together with the nutrient particles sticking to it, towards the œsophagus. Briefly, the ventral groove secretes mucus, which is destined to catch the nutrient matters suspended in the water, and to be carried with them by the ciliate tracts to the œsophagus. The entire arrangement has therefore a nutrient signification.

For, H., Ueber die Schleimdrüse, etc. der Tunicaten. Morph. Jahrb. I. p. 223.

\section{Digestive portion of the Enteron.}

\section{$\S 312$.}

At the bottom of the foremost section of the entire enteron or tractus intestinalis, namely, of that section which is modified as a respiratory chamber, commences the tract which serves exclusively the functions of nutrition. As a rule several divisions of it may be distinguished by differences in its breadth. An anterior, narrow section 
forms an œsophagus, which has a funnel-like commencement in the Copelata. A second, generally wider, section may be regarded as stomach, and corresponds to a mid-gut. In Ascidians it is broken up into numerous smaller chambers by a number of folds and sagittate up-growths of the wall; in Copelata it has a cæcal appendage. Cæcal structures are also found on the stomachs of many Salpæ. The section thus constituted is usually of considerable length in the Ascidians, and returns on itself in the form of a loop, from one arm of which the hind-gut proceeds. These two sections of the canal are quite short in the Copelata and also in the Cyclomyaria, where, as in the Ascidians, they are but little differentiated from one another. In many Ascidians, the single or double loop of the alimentary canal has a position at the side of the respiratory chamber in the outgrowth of the body-cavity which there surrounds the respiratory sac; others have the digestive tract confined entirely to the region behind the respiratory chamber, the various forms of which appear to determine this relation.

The Salpæ lave their digestive tract, together with its appendages, compacted into a mass (the nucleus).

As accessory organs of the digestive tube we have, besides the outgrowths already mentioned, other glandular tubes in all the higher divisions. These tubes open into the section of the canal which serves as stomach. There can be no doubt that they furnish a secretion adapted to the purposes of digestion. In form and arrangement they exhibit many variations. Sometimes they form net-like anastomoses.

Chandelon, Th., Rech. sur une annexe du tube dig. des Tuniciers. Bull. Acad. Be!g. XXXIX.

\section{Vascular System.}

\section{$\S 313$.}

In the arrangements of their circulatory organs, the two great divisions of the Tunicata differ from one another. In the Copelata a heart only is known, and that is absent from one genus. It consists of a short sac with its ends attached to two cells, whilst its delicate walls present two longitudinal slits placed on opposite sides to one another. The circulation of the blood is provided for by the pulsations of this pouch; in fact, without the existence of vessels, a distinct direction is given to the movement of the blood in the body-carity which can be recognised. In the Acopa a vascalar system in connection with the heart is present, which in certain parts has a lacunar character. It appears then that a portion of the primitive body-cavity is adapted to the purposes of a bloodcarrying system.

In the Ascidians the elongated heart lies in the neighbourhood of the digestive organs, and bending inwards at each end gives rise 
to a vessel. One of these two vessels taking a ventral course breaks up into a net-work which is distributed over the branchial latticework, whilst the other passes to the intestine and to the generative organs and branches out upon them. The same main-vessel sends also a branch to the mantle and twigs to the wall of the coelom (body-cavity). The blood distributed in this set of vessels is collected again into a longitudinal trunk lying on the dorsal side of the branchial sac, which also receives vessels from the intestine and from the generative organs. Whether these dispositions which have been observed in the solitary Ascidians have a more general distribution is not yet ascertained.

In the Salpæ the short, thin-walled heart (Fig. 212, c), generally having the form of a tube, constricted at intervals, is in connection with a large vascular canal $(v)$, which runs along the ventral surface, and also at the opposite end the heart is directly continuous with a large vessel; the latter, in those forms which possess a so-called nucleus (vi), breaks up at once into a reticular system, which is distributed in this body, and represents the intestinal vessels of the Ascidians. In other Salpæ (those without nucleus) it appears to divide into many branches, which run towards the dorsal surface and end in a longitudinal canal. This dorsal vessel $\left(v^{\prime}\right)$ is placed in communication with the rentral stem by a number of transverse canals $(v)$, which freely anastomose with one another. There exists a further direct

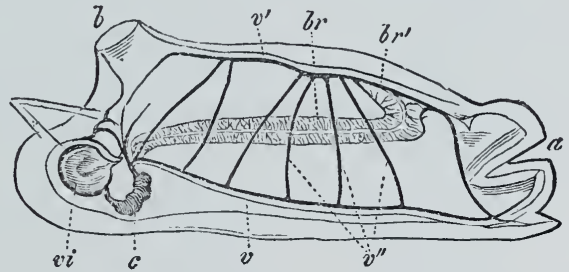

Fig. 212. Circulatory system of Salpa maxima. $a$ Incurrent orifice. $b$ Excurrent orifice. $b r$ Branchial septum. $b r^{\prime}$ Attach. ment of the gills. vi Visceral mass (nucleus). $c$ Heart. $v$ Ventral vascular stem. $v^{\prime}$ Dorsal vascular stem. $v^{\prime \prime}$ Communicating transverse stems. (The finer ramifications of the ressels are not represented.) (After Milne-Edwards.)

communication between the anterior portion of the dorsal vessel and the hinder vessel proceeding from the heart, through a number of vessels which run through the gills and break up there.

The most important peculiarity of the vascular system of the Tunicata is assuredly the existence of the two longitudinal stems which pass along the branchial sac, and which farther on meet on the intestine.

Let us suppose, starting from the Ascidians, the intestine to be continued in the direction of the long axis of its anterior division, the branchial sac, so that the anus came to be placed at the aboral pole of the body, then we should find the arrangement of the vascular apparatus to be similar to that of many Worms, even in respect of the detail that the branches of both longitudinal trunks are divisible into visceral (to the branchial sac and intestine) and parietal series (to the body-wall).

The heart belongs to the ventral longitudinal stem. It is in fact a differentiated portion of that stem. In this 
fact we have a special divergence from the arrangement obtaining: in all other invertebrate animals, for in them the central organ of the circulation is invariably a specialisation of a part of the dorsal vascular stem. Nevertheless there can be no mistake about the connection with that of the Worms in the disposition of the whole apparatus.

Peculiar to all Tunicates is the alternating direction of the blood-stream set in motion by the heart. In consequence of this change of direction, it is not possible in them to speak of an arterial and a venous division of the vascular system. When the heart has completed a certain number of pulsations in one direction,

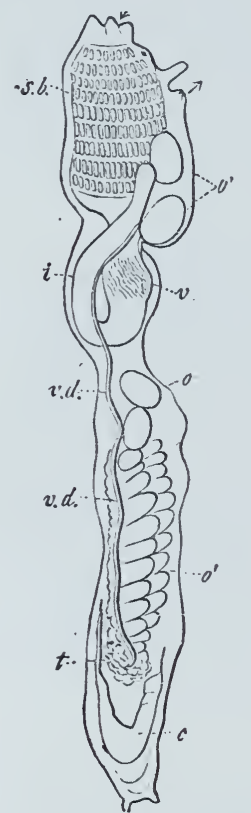

Fig. 213. Organisation of an Ascidian (Amarœcium pro. liferum). sb Bran. chial sac. $v$ Stomach. $i$ Intestine. $c$ Heart. $t$ Testis. vid Efferent duct of the testis. o Orary. o' Eggs in the body - cavity. The arrows indicate the direction of the stream of water at the orifices of the body (after Milne.

Edwards). suddenly a moment of quiescence occurs, and then the peristaltic movements of the cardiac tube begin again, but in the opposite direction. This condition of indifference is a further obstacle to the close assimilation of the vascular system of the Tunicata to that of any one of the other great divisions; at the same time, it calls to mind a similar phænomenon of the reversal of the bloodstream in the Gephyræa (Phoronis).

Organs of excretion have as yet been recognised in the Tunicata to a limited extent. In many Ascidians (Molgula, A. conchilega, complanata) a tubular organ is found in the neighbourhood of the branchial chamber, or placed farther back in the body, which exhibits, besides other cells, some containing concretions. In one species the murexide reaction has been obtained. No openings have been discovered in the organ, so that the arrangement appears to represent that condition in which excretionary matters are separated in the organism, and form concretions which are not removed to the exterior.

\section{Sexual Organs.}

\section{$\S 314$.}

Only one division of the Tunicata is provided with sexual organs universally - the Copelata. In the others, in consequence of the elaborate asexual reproduction, a large proportion of individuals are devoid of sexual organs; the absence of which is explained by the production of germs, a modification of the process of multiplication by budding (cf. p. 391).

The hermaphrodite arrangement, common among Tunicata, is often found at a very low stage of development. The Appen= 
dicularix have no efferent ducts for their sexual glands, which are sometimes paired and sometimes single. In the Acopa the sexual elements are discharged into the cloaca. The male organ has the form of a sperm-producing cæcum, which in Doliolum, and also in many Ascidians, retains this simple form; whilst in Pyrosoma it acquires a rosette-like shape, and in most Ascidians, and also in the Salpæ, is produced into branches, and forms a kind of multilobular gland. In many Ascidians (Molgula) the testes surround each of the two ovaries as a number of separate glands, and open to the exterior with separate efferent ducts. The ovaries too have often a multifid character, at least in many Ascidians; in others they are only represented by a group of eggs of different stages of development, each of which is enclosed in a kind of capsule. In many only a few such eggs, joined together by a common stalk, are present; and in the Salpæe and Pyrosoma we have only a single egg, the stalk of which exists in the early stages of growth, but gradually shortens. The development of the sexual products occurs here at different periods for the two sexes ; the male organ only reaches its maturity after the egg has commenced to develop as an embryo.

The presence of an efferent duct for the sexual products appear's to depend upon the greater or less distance of the sexual glands from the clcaca. The whole apparatus, however, requires a very much more careful investigation than it has yet received. 


\section{Ninth Section.}

\section{Vertebrata.}

\section{General Review of the Group.}

\section{$\$ 315$.}

The most essential characters of the Vertebrata are the possession of a skeleton traversing the longitudinal axis of the body, and the division of the body into a number of metameres (primitive vertebræ). They differ from the Tunicata, with which alone of all the divisions of the Invertebrata they have any well-marked relations, by this metamerism. They have more distant relations to the Vermes, but then this group obviously has relations to most of the other phyla.

The axial skeleton separates a dorsal from a ventral division of the body. The former contains the central nervous system, the latter the digestive canal, which is continued on from a respiratory chamber, and which, with the organs differentiated from it, is embedded in a cœlom. Two regions therefore can be made out, which extend along the body; the upper one is neural, and the lower enteric; the chief trunks of the system of canals for the nutrient fluid belong to the latter region.

The various divisions are set in order in the following review :

A. Acrania.

\section{Leptocardii.}

B. Craniota.

Amphioxus.

I. Cyclostomata.*

Mrinoïdea. Bdellostoma, Myxine.

Petromyzontes. Petromjzon.

* The Cyclostomata should be placed apart from all the rest of the Craniota, for their whole organisation shows that they branched off rery early from the Craniota. 
II. Gnathostomata.

a) Anamnia.

1) Pisces.

Selachii.

Squali.

Hexanchus, Heptanchus, Acanthias, Scymnus, Galeus, Scyllium, Rajæ. Squatina.

Holocephali.

Raja, Torpedo, Trygon.

Dipnö̈.

Chimæra.

Monopneumones.

Ceratodus.

Dipneumones.

Ganoïdei.*

Protopterus, Lepidosiren.

Sturiones.

Acipenser, Spatularia.

Polypterini.

Polypterus.

Lepidosteini.

Lepidosteus.

Amiadini.

Amia.

Teleostei.

Physostomi.

Abdominales.

Clupea, Salmo, Esox, Cyprinus, Silurus, Mormyrus.

Apodes.

Muræna, Conger, Gymnotus.

Physoclysti.

Anacanthini.

Gadus, Pleuronectes.

Pharyngognathi.

Belone, Hemirhamphus, Chromis, Labrus.

Acanthopteri.

Perca, Labrax, Trigla, Scropæna, Anabas, Mugil, Scomber, Zeus, Trachypterus, Gobius, Cyclopterus, Blennius, Lophius.

Plectognathi.

Ostracion, Diodon, Orthagoriscus.

Lophobranchii.

2) Amphibia.†

Syngnathus, Hippocampus.

Urodela.

Perennibranchiata.

Siredon, Menobranchus, Proteus.

Caducibranchiata.

* I regard each of these divisions of the Ganoïdei as highly independent. They represent the last shoots of very divergent series of forms, of which that of the Polypterini has many points of relationship to the Dipnoï; the Amiadæ, on the other hand, are the nearest allies of the Teleostei (Clupeïdæ). It would, perhaps, be best to separate them completely from the Ganoïdei. The Sturiones show the greatest resemblance to the Selachii.

I must regard the Selachii as being nearest to the ancestral form of the Gnathosto. matous Vertebrata. The Holocephali, as well as the Dipnoï and Ganoïdei, appear to have branched off from them, while the Teleostei again are a branching off from the Ganoïdei.

† The living Amphibia form only a very small group, which in many parts indicate considerable retrogression; but few fossil forms can be safely placed in it. The palæontological records of the Amphibian phylum are fragmentary in the highest degree. There are many reasons for placing the Archegosauria with them, but yet there are many points in which these forms resemble the Reptilia. 
a) Anamnia (continued).

Derotremata.

Cryptobranchus, Menopoma.

Salamandrina.

Anura.

Triton, Salamandra.

Pelobates, Bombinator, Hyla, Ceratophrys, Rana, Bufo.

Gymnophiona.

Cœecilia.

b) Amniota.

1) Sauropsida.

1. Reptilia.*

Chelonii.

Sphargis, Trionyx, Chelonia, Chelys, Chelydra, Emys, Testudo. Saurii.

Ascalabota.

Platydactylus, Hemidactylus.

Rhjnchocephala.

Sphenodon.

Lacertina.

Iguana, Calotes, Draco, Phrynosoma, Uromastix, Lacerta, Ameiva.

Monitores.

Monitor, Psammosaurus.

Scincoïdea.

Scincus, Seps, Anguis.

Chalcidea (Ptychopleura).

Chalcis, Zonurus.

Chamæleonida.

Chamæleo.

Amphisbænida (Annulata). Ophidii.t

Amphisbæna, Lepidosternum.

Eurystomata.

Python, Boa, Coluber, Tropidonotus, Dryophis, Dipsas, Hydrophis, Crotalus, Trigonocephalus, Vipera.

Stenostomata.

Typhlops, Uropeltis.

Crocodilini.

Alligator, Crocodilus, Ramphostoma.

2. Aves. $t$

Ratitæ.

Struthio, Dromæus, Aptersx.

Carinatæ.

Gallinaceæ.

Megopodius, Penelope, Crax, C'rypturus, Lagopus, Tetrao, Pavo, Numida, Gallus, Phasianus.

* The various divisions of this class appear to be the very divergent terminal twigs of a branch of the Vertebrata, which was in former times largely represented. Many of the fossil divisions which are ascribed to the Reptilia, such as the Enaliosauria, apparently, however, branched off from the Vertebrate phylum before the Amphibia. In another group of fossil Saurii we find forms intermediate between the Reptilia and Birds; and that most markedly in the characters of the skeleton of the foot. These are the Ornithoscelida. By uniting Reptiles and Birds into one division of the Sauropsida, as $H u x l e y$ has done, we put these relations in their proper light.

+ The Ophidii form a division nearest to that of the Saurii, and derived from it, and, with it, equivalent to the Chelonii or Crocodilini; and they were thus put together by Stannius as Streptostylica.

\# The class of Birds which arose from reptilian forms is one which is divided by the most important points in its organisation into groups which diverge rery slightly from one another, for the characters of these subdivisions present distinguishing points of very slight importance in comparison with those of the other divisions of the groups of the Vertebrata. Through the Saururi (Archæopteryx) they are directly connectcd with the Ornithoscelida. 
b) Amniota (continued).

Columbæ.

Columba.

Grallatores.

Otis, Dicholophus, Grus, Ardea, Ciconia, Vanellus, Charadrius, Scolopax, Fulica, Gallinula, Rallus.

Natatores (Palmipedes).

Procellaria, Sterna, Larus, Phaëton, Plotus, Pelecanus, Carbo, Anser, Anas, Cygnus, Phœnicopterus, Mormon, Uria, Alca, Aptenodytes.

Passeres (Insessores).

Fringilla, Alauda, Turdus, Sylvia, Motacilla, Parus, Muscicapa, Lanius, Sturnus, Corvus, Hirundo, Certhia, Trochilus,

Picides. Upupa, Merops, Coracias, Alcedo, Buceros.

Picus, Yunx.

Psittacides.

Psittacus, Strygops, Nestor.

Rapaces.

Gypogeranus, Falco, Buteo, Aquila, Gypaëtus, Vultur, Cathartes,

2) Mammalia. Harpyia, Surnia, Strix.

Ornithodelphia (Monotremata).

Ornithorhynchus, Echidna.

Didelphia* (Marsupialia).

Botanophaga.

Halmaturus, Dendrolagus, Phascolomys, Phascolarctus, Fhalangista.

Zoophaga.

Perameles, Dasyurus, Thylacinus, Didelphys, Chironectes.

Monodelphia (Placentalia).

Edentata.t

Myrmecophaga, Manis, Chlamydophorus, Dasypus, Bradypus.

Ungulata.

Artiodactyla.

Sus, Dicotyles, Moschus, Camelopardalis, Cervus, Antilope, Tylopoda. Capra, Ovis, Bos.

Camelus, Auchenia.

Perissodactyla،

Sirenia.

Tapirus, Rhinoceros, Equus.

Prosimii.

Manatus, Halicore.

Stenops, Lemur, Otolicnus, Tarsius, Galeopithecus, Chiromys.

Rodentia.

Sciurus, Arctomys; Mus, Hypudæus, Cricetus, Georhychus, Spalax, Pedetes, Dipus, Lagostomus, Myopotamus, Castor,

Proboscidea. §

Hystrix, Cœlogenys, Cavia, Lagomys, Lepus،

Elephas.

Lamnungia.\|

* I regard the division of the Marsupialia as equivalent to the monodelphous Mammalia, for not only are there found in it representatives of most of the orders of Monodelphia, but, further, there are in the MLonodelphia many indications which point to their having arisen from a didelphous form. The Marsupialia, or uniting with them the Monotremata, the Implacentalia, consequently represent the ancestors of the Placentalia.

+ The great variations which the relations of the placenta present in various Edentata weaken somewhat the value of the placental classification, although the various orders are generally distinguished by the similar characters of their placenta.

\$ The Prosimii form a stem-group, in some divisions of which peculiarities are retained which are found in various other of the following orders. Thus there are characters which we meet with in Insectivora, Rodentia, Carnivora, and Primates.

$\S$ and $\|$ The Proboscidea and Lamnungia are representatives of orders which it is very difficult to associate with the rest. They have genetic affinities to the Rodentia; Hyrax has also relations to the Ungulata. 
b) Amniota (continued).

Fera.

Hyrax.

Carnivora.

Felis, Hyæna, Proteles, Canis, Herpestes, Viverra, Lutra, Mustela, Meles, Nasua, Procyon, Ursus.

Pinnipedia.

Phoca, Otaria, Trichechus.

Cetacea.*

Delphinus, Physeter, Balænoptera, Balæna.

Insectivora.

Chrysochloris, Talpa, Sorex, Myogale, Erinaceus.

Chiroptera.

Pteropus, Rhinolophus, Glossophaga, Vespertilio, Vesperugo. Primates.

Hapale, Callithrix; Ateles, Mrcetes, Cebus; Cynocephalus, Inuus, Cercopithecus; Troglodytes, Hylobates, Pithecus; Homo.

\section{Bibliography.}

Owks, R., On the Anatomy of Vertebrates. Vols. I.-III. London, 1866-68.-HuxLer, T. H., A Manual of the Anatomy of Vertebrated Animals. London, 1871.

Leptocardii : MüLler, J., Ueber den Bau und die Lebenserscheinungen des Branchiostoma lubricum. Abhandl. d. Berl. Acad. 1814.-Goodsir, Transact. Royal Soc. of Edinburgh. T. XV. I. -Quatrefages, Ann. sc. nat. III. IV.-KownLevskx, A., Entwickl. des Amphioxus. Mém. Acad. des Sc. de St. Pétersbourg. Sér. VII. T. XI.-RoLpH, Unters, über d. Bau d. Amphioxus. Morphol. Jahrb. II.

Cyclostomata: MüLler, J., Vergl. Anatomie der Myxinoïden. Abhandl. d. Berl. Acad. 1835-45.The same, Ueber den Bau und die Grenzen der Ganoïden. Ibid. 1816.-RAтнкE, Bemerkungen über den inneren Bau der Pricke. Danzig, 1825.-The same. Ueher den Bau des Querders. Beitr. z. Gesch. der Thierwelt. IV. Halle, 1827.-LavgerHass, Unters. über Petromyzon Planeri. Freib, 1873.

Pisces: Monro, A., The structure and physiology of Fishes. Edinburgh, 1785.-Cevirr et VALENciBNNES, Hist. nat. des poissons. XXII. vols. 1828-18.-AGAssiz, Recherches sur les poissons fossiles. 5 vols. av. Atlas. 1833-13.-AGASsiz et VoGt, Anatomie des Salmones. Neufchâtel, 1815.-Lerdig, Beiträge zur mikroskop. Anatomie und Entwickelungsgeschichte der Rochen und Haie. Leipzig, 1852.-OwEs, Description of Lepidosiren annectens. Transact. Linn. Soc. XVIII.-Bischoff, Lepidosiren paradoxa. Leipzig, 1810.-Hrre, Lepidos. parad. Abhandl. der böhm. Ges. d. Wiss. 1815.-Peters, Lepirlosiren. Arch. f. Anat. u. Phys. 1845.Günther, Ceratodus. Phil. Trans. 1871.-Balfovr, F. M., Development of Elasmobranch Fishes. Journal of Anat. Vols. X. XI.

Amphibia : Curier, in Recueil d'observations de Zoologie et d'Anat. comp. I. 1805.-Rescovi et Configliacri, Del Proteo anguineo di Laurenti monografia. Pavia, 1818.-The same, Hist. naturelle, développement et metamorphose de la Salamandre terrestre. Pavie, 1854.-MüLLER, J., Beiträge zur Anatomie der Amphibien. Zeitschr. f. Physiol. IV. 1832.-Dugìs, Recherches sur l’ostéologie et la myologie des Batraciens. Paris, 1831.-MAYer, Zur Anatomie der Amphibien. Analecten f. rergleichende Anatomie. Bonn, 1835.-CALori, Sulla Anatomia del Axolotl. Mem. della A ccademia delle sc. dell' istituto di Bologna. III. 1851.- RAth K F. (Cœcilia annulata). Arch. f. Anatom. u. Phss. 1852. p. 331.-LrIDIG, Untersuchungen über Fische und Reptilien. Berlin, 1853.-Vaillant, L. (Siren lacertina). Ann. sc. nat. IV. Xrimi.-Fischer, J. G., Perennibranchiaten u. Derotremen. Hamburg, 1864.-Hrrtu, J., Cryptobranchus japonicus. Vindobonæ, 1865.- VAN DER HOETEN, Ontleed-en deerkundige Bijdragen tot de Kenniss von Menobranchus. Lejden, 1867.-Wiedersheir, R., Salamandra perspicillata, etc. Genua, 1875.-Göttr, Entwickelungsgeschichte der Unke. 'Leipzig, 1875.

Reptilia: Bojanos, Anatomia testudinis europææ. Vilnæ, 1819.-Drméril et Bibrox, Erpétologie générale. Paris, 1834-54.-Duvernor (Serpens). Ann. sc. nat. I. xxx.-Rathke, Entwickelungsgesch. der Natter. Königsberg, 1837. - The same, Entwick. der Schildkröten. Braunschw. 1818.-The same, Ucber die Entwick, und den Körperbau der Krokodile. Braunschw. 1866.('Alori (Uromastix). Mem. della Accad. delle sc. dell'ist. di Bologna. III. II. 1863.-Güхтпrr, (Hatteria), Phil. Tr. 1867. II.-Lrxpig, Die in Deutschland lebenden Arten der Sauricr. Tübingen, 1872.-Wiedersieir, R., Z. Anat. v. Phyllodactylus europ. Morph. Jahrb. I.

Aves: Trederrans, Anatomie und Naturgesch. der Vögel. Heidelberg, 1810-14.-Owrs, On the anatomy of the southern apteryx. Transact. Zool. Soc. Vols. II. III.-The same, Art. Aves in

* The Cetacea are apparently of the same phylum as the Pinnipedia, which phylum is derived from the Carnivora; this is shown by certain fossil forms (Zeuglodon). But the peculiarities of the Cetacean organisation are too great for them to be put under the Fera; they form the end of a series. 
Todd's Cyclopædia. I.-MrLve-EDwards, AtrH., Rech. sur les ossemens fossiles des oiseaux Paris, 1866.-ALIx, E., Appareil locomoteur des oiseaux. Paris, 1874.

Mammalia: Mrскеx, J. Fr., Ornithorhynchi paradoxi descriptio anatomica. Lips. 1826.-Vrolik (Dendrolagus), Verhandel. d. Kon. Acad. Amsterd. V.-GurLt, Handb. d. vergl. Anat. der Haussäugethiere. 4 Aufl. Berlin, 1860.-FrAN CK, L., Anatomie der Hausthiere. Stuttgart, 1871. -Brand (Lama), Mém. Acad. St. Pétersbourg, 1811.-Owen (Giraffe), 'Transact. Zool. Soc. II.-The same (Rhinoceros), Transact. Zool. Soc. IV. Ir.-Mrlxe-Edwards, AlPH. (Moschiæd), Ann. sc. nat. V. II.-MURIE, J. (Manatus). Tr. Zool. Soc. VIII.-CAMPER, Observations sur la structure intime et le squelette de Cétacées. Paris, 1820.-RApr, Die Cetaceen. Stuttgart u. Tübingen, 1837.-VrouIK, W., Natuur-en ontleedkund. Beschouwing van d. Hyperoodon. Haarlem, 1848 . Eschricht, Untersuch. über die nordischen Walthiere. Leipzig, 1819.-MURIE, J. (Globiocephalus, Otaria, Trichechus), Tr. Zool. Soc. VII. VIII.-RıPP, Anatom. Untersuchungen über die Edentaten. 2 Aufl. Tübingen, 1852.-OweN (Myrmecophaga jubata), Tr. Zool. Soc. IV. Hrrte (Chlamydophorus truncatus), Denkschr. d. Wien. Acad. IX. 1855.-Povchet, G., Mém. sur le grand Fourmilier. Paris, 1874.-Pallas, Nov. spec. quadrup. e glirium ordine. Erlangen, 1778. - Camper, Descript. anat. d'un Eléphant mâle. Paris, 1802.-Burmeister, Beiträge z. nähern Kentniss der Gattung Tarsius. Berlin, 1816.-VAN DER HoEves (Stenops), Verhand. d. Acad. Amst. VIII.-Owes, Monograph on the Aye-Aye. London, 1863.-Petres (Chiromys), Abhandl. d. Berliner Acad. 1865.-Murie, J. (Iemuridæ), Tr. Zool. Soc. VII.-Txson, Anatomy of a Pygmy. London, Sec. edit. 1751.-Vrolik, Rech. d'anat. comp. sur le Chimpanse. Amsterdam, 1841.-Duvernor, G, L., Caract. anat. des grands singes. Archives du Museum. VIII.FLower, Osteology of the Mammalia. London, 1870.-TuRNRR, W., Lectures on comp. anat. of the Placenta. Edinburgh, 1876,-For human anatomy, reference must be made to the manuals on the subject.

\section{Form of the Body.}

$\S 316$.

The external metamerism disappears, but dorsal and ventral surfaces can generally be distinguished; the entrance into the nutrient canal is placed near the anterior pole of the long axis of the body, and on the ventral surface; the anus is also ventral, but is at some distance from the aboral pole. Three great regions can be made out even in the body of the lowest divisions. The anterior one contains a respiratory chamber formed from the nutrient canal, and is consequently distinguished by clefts in the sides of the body-wall. It carries the higher sensory organs, and in the Craniota gives rise, by concrescence and differentiation, to a head.

The second portion, which in Amphioxus is not sharply marked off on the dorsal surface from the preceding one, forms the trunk, which encloses the cœlom and its contents; this is only marked off from the last or caudal portion of the body by the anus; so that from the outside there is not much difference between them.

We have already met with these divisions in the Tunicata. In the Ascidian larvæ (cf. Fig. 208), the most anterior one, which later on forms the principal portion of the body, contains the rudiments of the respiratory cavity, and the portion of the nervous system which contains the sensory organs. Connected with this is a faintly separated tract with the enteric tube, and this passes almost directly into the caudal portion. The earliest characters of the embryonic head, or of its equivalent in all Vertebrata, point to its being, phylogenetically, the most ancient portion of the body, and serve as a finger-post to the path of development of the Vertebrate body.

With the development of the head and of the organs differentiated in and on it, the body of the Vertebrata acquires characters, which, externally, separate it well off from the Invertebrata; the value of these is clear when we take note of the large number 
of metameres that have been suppressed in the course of this differentiation. Further differentiations are brought about by the development of paired appendages. The hinder ones in the Gnathostomata separate the trunk and tail more distinctly from one another, as do the anterior ones in the case of the head and trunk.

When the anterior appendages are separated from the head, as they are even in the Selachii among the Fishes, a cervical region is differentiated from the trunk, and connects it with the head. We meet with this arrangement in the Amphibia. The trunk is affected by further differentiations, and in the Amniota is separated into cervical, thoracic, and lumbar regions.

The caudal portion of the body undergoes a gradual metamorphosis. In the Fishes it is scarcely marked off; in the Amphibia (Urodela) and Reptilia (Saurii, Crocodilini) it is separated from the trunk by the hinder pair of appendages, and by its diminished bulk. Although it is atrophied in Birds, it is in the Mammals only that it acquires the character of an appendage of the body, in consequence of its great decrease in bulk, even though it may still be of some length.

\section{Appendages.}

\section{$\S 317$.}

We must divide the appendages which are given off from the body of the Vertebrata, and which function principally as locomotor organs, into paired and unpaired parts. The unpaired ones are developed from a vertical membrane-a continuation of the integument-which extends from the head to the anus. When firm structures and special muscles are developed in this membrane, this purely dermal process is converted into a fin. This organ either retains

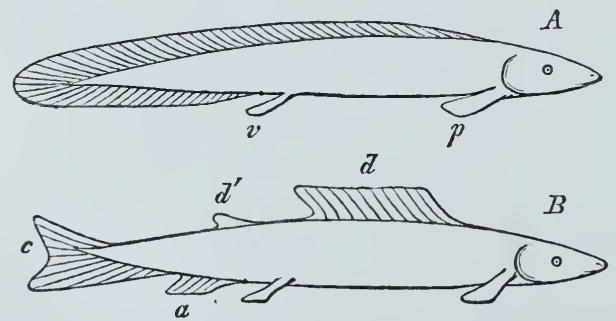

Fig. 214. Diagram of the unpaired fins. $A$ Primi. tive stage. $B$ Differentiated stage. i Dorsal fin ( $d^{\prime}$ Fatty fin). $c$ Caudal; a Anal; $p$ Thoracic; $v$ Ventral fins. its primitive continuity of arrangement(Fig.214, $A)$, or by the atrophy of some, and by the increased development of the remaining portions, is broken up into several parts. These are distinguished according to their position as dorsa], eaudal, and anal fins (Fig. 214, $B d c a$ ). They function chiefly as directing organs; the caudal fin alone has any higher locomotor significance in so far as the caudal portion of the body plays an important part in locomotion. These organs, which are commonly found in Fishes, are also seen in the early stages of development in the Amphibia, in some of which 
(many Urodela) they are indeed permanently present, but supporting organs are not developed in them.

In the Reptilia indications of the vertical dermal fringe can sometimes be just made out, but in most it is altogether absent, as it is also in the higher classes; the vertical fin-like structures seen in many of the Cetacea must be regarded as organs which have been acquired by that order independently. The same remark applies to the horizontal caudal fin of these Mammals.

\section{$\S 318$.}

Unlike the arrangement seen in many divisions of the Invertebrata, where paired appendages are found on all, or at least on a large number of metameres, they are-and, so far as we know, without one exception-confined to an anterior and a posterior pair in the Vertebrata.

They appear to be homodynamous organs, which gradually get to vary greatly in form in correlation with their great variety of function. They are probably derived from metamorphosed respiratory appendages of the head (branchial arches and rays), so that they are not absolutely new arrangements.

They are absent in the Acrania and Cyclostomata, but are generally present in the Gnathostomata. Although in some divisions of these latter the appendages are atrophied, this atrophy is in every case a secondary arrangement, which presupposes the fully-developed condition. This is proved by the various stages of atrophy, which may be seen in the appendages and in the parts of which they are composed.

In the lower condition seen in Fishes the appendages appear each to form a single whole, undivided by external jointing into a number of parts, while their increased surface is of importance as bearing on the directing function of the organ. The anterior and posterior appendages, which are here known as thoracic and ventral fins, have essentially the same structure, although the thoracic fins are, as a rule, much larger, in consequence of their position in the larger part of the body. Their more powerful structure may be also explained by the fact that they take the initiative, and are consequently of greater functional importance than the hinder appendages.

Owing to their similar mode of aquatic progression the appendages of the fossil Enaliosaurii, as shown by the remains of their skeletons, resembled the fins of Fishes-at any rate in the absence of any transverse segmentation.

Among the Amphibia we find the appendages transversely segmented, for now several parts are sharply marked off from one another. In the fore-limb we divide these into upper arm, forearm, and hand; to which the thigh, leg, and foot correspond in the hindlimb. This division is correlated with the greater elongation of the two first segments, which stand, in relation to one another, as the arms of a lever, and are therefore set at an angle to one another.

In addition to the differentiation herein implied, the terminal 
division undergoes differentiation; a number, generally not more than five, terminal joints can be distinguished as fingers and toes. As a part of the body which generally projects towards the exterior is more under modifying influences than any other part, we find a large number of adaptations in them; few parts of the body present so many metamorphoses as these terminal parts of the appendagesthe hand and foot.

The primitive union of the fingers or of the toes into a swimming-plate, represented by the hand and foot, is retained in the natatory membrane of many Reptiles, in the hind-limbs of many Birds, and also in a number of Mammals, where it is always connected with the adaptation of these appendages to the function of a

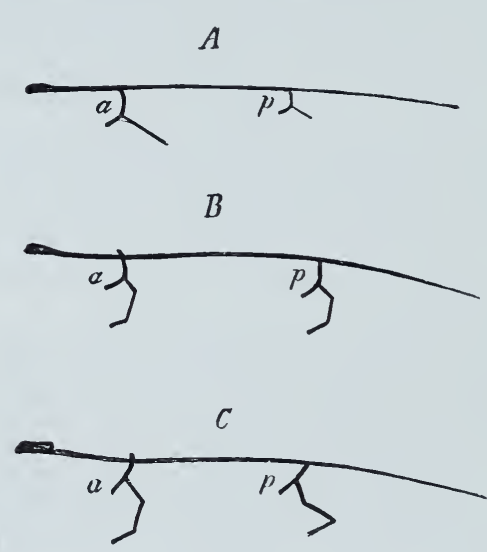
swimming organ.

The angulation of the limb attained to,in connection with terrestrial locomotion, and which is also advantageous in aquatic locomotion, becomes gradually different in the case of the two extremities; the difference corresponds to the functions performed by the anterior and posterior extremities when moving about on land.

These relations are distinctly seen even in the Amphibia $(B)$; but the difference in position between the upper and forearm, and thigh and leg, is not well marked. The upper arm and

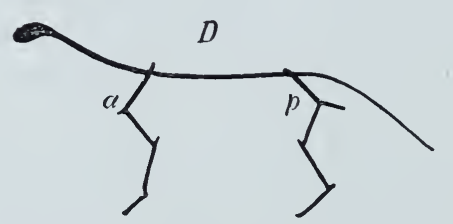

Fig. 215. Diagram to show the differen. tiation and alteration in the direction of the axes of the limbs in the Vertebrata. $A$ Fish. $B$ Amphibian (the side-riew, which one has been obliged to give so as to compare it with the rest, gives to this and to the next figure the appearance of the body being raised up). C Reptile. $D$ Mrammal. $a$ Shoulder girdle. $p$ Pelric girdle. thigh are turned outwards to almost the same extent. There is a greater difference between them in the Reptilia ( $C$ ), and this is still more marked in the Mammalia, where the planes in which the angles of the limbs of either side are set are parallel to the vertical median plane of the body. This gives greater independence to the limbs, which have now become supports for the body, as they raise it up from the ground. Together with this change in the aspect of the planes, in which the angle formed by the extremity lies, the angles between the equivalent portions in each limb in the Mammalia cease altogether to agree with one another $(D)$; in fact they point in an opposite direction in the case of the fore and hind limbs respectively. The angle between the upper and forearm is open 
towards the anterior, and that between the thigh and leg towards the posterior.

In addition to these general modifications of the appendages there are other changes, which are confined to smaller divisions, and are explicable by special variations in physiological activity. When the hind-limbs are greatly developed, they perform the more complicated function of a springing organ, as in frogs; or they may be converted into the chief organs of support for the body, in such a way that the fore-limbs, so far at least as terrestrial locomotion is concerned, may get to play a subordinate part, or even lose this function altogether. This arrangement obtains in Birds, where it has been attained to through a large number of intermediate steps, which have been made out in fossil Reptiles; the fore-limbs in the Carinatæ have taken on the function of a flying organ.

\section{Integument.}

$\S 319$.

In the primitive stage in the Vertebrata the investment of the body has the character of a cellular layer, the external germinal layer-the ectoderm. At a further stage in development this cellular layer is connected with a layer of connective tissue derived from the mesoderm, and the two together form the integument of the Vertebrata, and take an equal share in the formation and further development of various organs.

There are two layers in this integument (cutis), as might be inferred from its mode of origin : a superficial epidermis which is homologous with the epithelial structures in the Invertebrata, and which is directly derived from the ectoderm, and a deeper-lying layer of connective tissue, the corium, the deepest, and looser, layer of which forms the sub-integumentary tissue. The corium is strengthened by the formation of plexuses between its fibrous bands. The blood-vessels, and nerves of the skin, together with various sensory organs, are scattered in it, as are also glandular organs.

The corium is frequently pigmented. It varies greatly in thickness, and in microscopic structure. One of the more notable variations is a lamellar striation seen in Fishes, Amphibia, and Reptiles, where perpendicular fibrous bands divide the layers into partitions. Among the special structures are the wart-like elevations seen on its surface, which vary from low hillocks to long conical processes. These dermal papillæ give rise, in different divisions of the Vertebrata, to a number of variously complicated organs.

Contractile form-elements (smooth muscular fibres) are also found in the corium of Birds and Mammals. Another modification, which obtains in the cutis, is due to a change in texture; parts of it are ossified into hard structures, and bony plates are developed in 
the dermis, which vary greatly in form, and unite to form a dermal skeleton. Lastly, there are glandular organs connected with the cutis, which are developed from the epidermis, and are therefore to be regarded as epidermal organs.

\section{$\S 320$.}

In Amphioxus the epidermis is a single layer ; in other forms it is made up of a number of layers of cells, which invest the corium, with its elevations and depressions. Even in the Vertebrata we find a ciliated epithelium as a heritage from a lower condition; but it is limited to the embryonic stages in Fishes, and to certain parts of the body in the larval stages of the Amphibia. The lower layers of the epidermis, which lie closer to the corium, are seen to be younger, and these replace the effete portions of the superficial layers. The cells of the epidermis vary greatly in consistency, form, and mode of connection. Pigmented cells are not unfrequently found between the rest. They are sometimes able to produce a change of colour by the movement of their protoplasm (chromatophores); this has been observed in Fishes and Amphibia. In the aquatic Anamnia the whole of the epidermis is less consistent, and, owing to the softness of its elements, the whole layer is often gelatinous; so much so, indeed, that it was for a long time considered to be a mucous layer secreted by glands.

There is another arrangement which forms a contrast to the condition of the epidermis in the Anamnia; this is brought about by the cornification of the cells which is first seen in the Amphibia, and is commonly found in the Amniota. The cells form resisting plates or fibres, which give rise to firm structures becoming overlapped by, and being more or less marked off from, one another. The process of cornification never affects any but the superficial layers, the deeper-lying parts being always indifferent. When the cornified layers increase in thickness, various kinds of plates, knobs, and scale-like structures (Reptilia) are developed. The corium takes part in the formation of these structures, for it is almost always provided with elevations which correspond to those epidermal formations, which are developed from enlarged papillæ. The scales of the Saurii and Ophidii are therefore processes of the whole cutis. In Birds this cornified covering is retained in some parts only of the body; as beaks on the jaws, and as scales, plates, knobs, and so on, on the feet. Larger horny plates are connected with a bony dermal skeleton in the Chelonii, and in some families of the Edentata among Mammals. The cornification of the epidermis, which obtains in some divisions or in still smaller groups, cannot be directly referred to the organisation of the Reptilia ; it is rather due to adaptations to definite external conditions. However, we do meet with horny epidermal structures in many parts of the body, which must be regarded as acquired arrangements in consequence of their wide distribution and constancy of character. These are the nails 
and claws found at the ends of the limbs. There are indications of these in the Amphibia themselves (Salamander); they are generally present in Reptiles and Birds ; nails of this kind are not unfrequently retained even on some of the fingers of the bird's hand, which is used as an organ of flight. They are much larger in many Mammals, where they form hoofs.

\section{Epidermal Structures.}

\section{$\S 321$.}

Other differentiations beside the cornified structures already mentioned affect the epidermis. The most important of these are feathers and hairs; and that on account of their distribution in the two higher classes of the Vertebrata, and of their peculiar appearance. It is usual to regard them as organs very closely allied, as they have many points in common. They seem, nevertheless, to be divergent structures. The earliest rudiment either of a feather or of a hair is a thickening of the epidermis; that is to say, it forms a knob-like thickening (Fig. 216, A), into which there grows a papilla from the cutis. This process is small in the case of the hair, but larger in that of the feather. They resemble those elevations which are found in the Reptilia. The first sign of the feather is the growth of the knobs into papilliform processes ( $B C$, feather processes), which are made up of an outer epidermal layer $(C e)$, and a subjacent papilla. The arrangement also of these first rudiments of the feathers in definite areas (feather-tracts, pterylia) is much the same as that of the

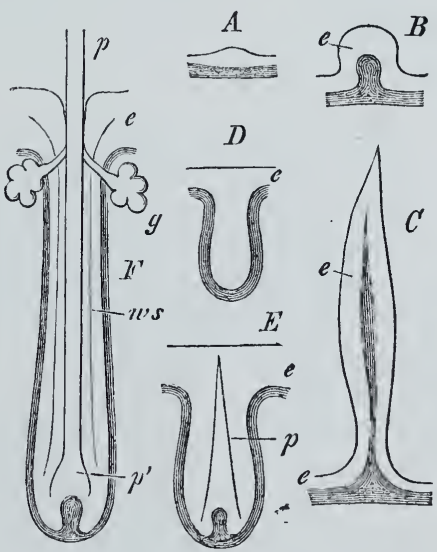

Fig. 216. Diagrams of the earliest rudiments of feather and hair. In section. A Thickening of the epidermis. $B$ Uprising of a papilla. $C$ Process of the feather. $D$ Depression of the epidermis. $E$ Differentiation of the rudiment of the hair. $F$ Hair. follicle and hair. $e$ Epidermis. $p$ Hair. $p^{\prime}$ Root of the hair. ws Root-sheath. $g$ Sebaceous glands. The corium is represented by the dark lines. scales in Reptiles. The feather, therefore, is, in this simple condition, a mere process of the epidermis and subjacent cutis. The depression of the embryonic feather which carries the cuticular papilla, and the consequent formation of a "feather follicle," is a later phænomenon, as is also the differentiation of the feather into rachis and vexillum. This differentiation does not obtain until the feather sheath is protruded, which sheath is itself an epidermal layer derived from the earliest rudiment. 
Various changes affect the morphological characters of the feather, after the development of its shaft and vexillum; but these are beyond the scope of this book.

A follicle is not formed till somewhat late in the course of the development of the feather; it then contains that portion of the rachis which is known as the "quill," and the vascular papilla which is continued into it; the hair, however, is characterised by the early appearance of the follicle. In this case then the papilliform thickening of the epidermis is a very early stage, and one of short duration; for the hair is not formed in this first elevation, but in a follicle which dips down from the epidermis into the cutis (Fig. 216, DE), and at the base of this follicle the cutis-papilla $\left(F^{\prime}\right)$ grows up. The shaft of the hair $(F v s)$ is differentiated from the invaginated epidermis by the cornification of its cells, while other cellular parts of the follicle form the root-sheaths.

The various forms of hairs, whether woolly or contour hairs, setre, or spines, are merely modifications of one and the same early condition.

\section{$\S 322$.}

The glands differentiated from the epidermis are, when simplest, modifications of single cells, the protoplasm of which is differentiated into fine granules, which are passed out to the exterior. These mucous cells (goblet-cells), which are placed among the other epidermal cells, form unicellular glands (Fishes). They are found also in the Amphibia, but in them there are, in addition, more complicated glandular organs. These latter have the form of flaskshaped tubes, which are scattered over the integument; several forms of these may be distinguished. In many cases they get to be very large, and form knob-like projections, which give the integument a roughened or wart-like appearance (Toads, Salamander). Sometimes a large number of integumentary glands are collected together, and characterise certain regions of the body (parotid).

The integumentary glands are less widely distributed in the Reptilia. In the Saurii the so-called "crural pores" lead into glands, which look like compound tubes, and which secrete cells which harden and fill up the lumen of the glands. In Birds the number of integumentary glands is still less. A number of glands unite to form the anal glands (glandula uropygii), which are especially large in the Natatores; their secretion serves to oil the feathers. In the Mammalia they are divided into two distinct groups; sweat glands and sebaceous glands, which are frequently connected with the hairfollicles. These two sets of glands are more easily distinguished by their anatomical characters than by the quality of their secretion, which is exactly known in a few cases only, although indeed the same form of gland may have a different function in different regions. The simpler tubes, which are coiled at their ends, are called sweat-glands, while the sebaceous glands are generally lobate. A number of them are often connected with one hair-follicle, and may become so greatly developed in relation to it, that the hair-follicle 
looks like an appendage of the gland. The sebaceous glands undergo the most various modifications in form, size, and number, as well as in the quality of their secretion. Both sets of glands frequently secrete odorous matters of various kinds, which play an important part in the economy of the animal. Glands of this kind are developed in the most diverse regions of the surface of the body in many orders of the Mammalia.

\section{$\S 323$.}

The most important modification of the integu. mentary glands in all Mammals is the development. of milk-secreting glands, which enter into relation to the reproductive function. They are regularly, and, as a rule, symmetrically arranged on the ventral surface of the body. Each " mammary gland" consists of a complex of separate glandular tubes, the ducts of which are either quite separate or united together.

In the Monotremata these organs differ but little from the other kinds of integumentary glands. Each of the two organs here present is made up of a group of tubes, which pass separately through the skin. The area on which theyopen is merely distinguished by the $a b$ sence of hairs, and in Ornithorhynchus is on the same level as the surrounding integument. In Echidna it is placed in a pouch-like depression (mammary pouch), which appears to serve as a receptacle for the young.

In the rest of the Mammalia nipples are present; these are special arrangements which were gradually developed by the process of sucking, and which afford the young a more suitable connection with themammary apparatus, while at

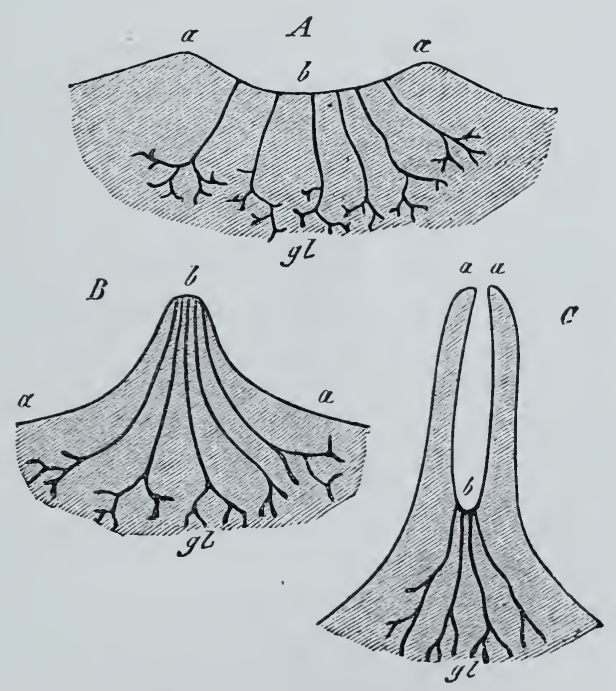

Fig. 217. Diagram of the development of the nipple; vertical section. $A$ Indifferent stage; glandular area flat. $B$ Elevation of the glandular area with the nipple. $C$ Elevation of the periphery of the glandular area into the pseudonipple. $a$ Periphery of the glandular area. $b$ Glan. dular area. gl Glands. the same time they make each complex of milk-glands distinguishable externally.

There are two very different conditions of the perfected nipple. They are both preceded by a similarly indifferent stage (Fig. 217, A), in which a nearly flat glandular area (b) has a number 
of glands at its base which grow into the corium; this area is marked off from the surrounding integument by a circular elevation (a). This arrangement corresponds to the mammary pouch in Echidna. In most of the Mammalia this arrangement is not a permanent one; it soon becomes level again, and the glandular area has then its central part $(B)$, in which the glands open, raised up into a papilla or nipple; at the tip of which a number of gland-ducts always open.

In the other arrangement the mammary pouch is persistent. Owing to the continued elevation of the periphery of the gland $(a)$ the glandular area is more and more depressed, the edge of the mammary pouch is developed into a pseudo-nipple, from the tip of which a single canal passes to the glandular area $(C)$.

This arrangement has been observed in some of the Ungulata. Intermediate stages between the two arrangements can be made out in the Marsupialia (Halmaturus) and Rodentia (Murina). The number of mammary glands which are distinguished by their nipples varies in different divisions. They generally correspond to the average number, or to the maximum number of young produced at one birth. They vary even within the limits of the same order; and they also vary in position. As a rule they form two rows, which, when there is a large number present, extend from the inguinal to the pectoral region (Carnivora, Suina). In many of the Didelphia they are arranged in a circle on the abdomen. When the number is not so large, they either occupy an abdominal position, as in many Didelphia, or they are only found in the lumbar region (Perissodactyla, Ruminantia, Cetacea), or, finally, they are limited to the pectoral region (Elephant, Sirenia, many Prosimii, Chiroptera, and Primates). When more than one pair is present some glands are sometimes aborted, so that there are rudimentary organs present, together with well-developed and functionally active ones; and these may be recognised by their rudimentary nipples. In a similar way the whole apparatus is atrophied in the male.

The most important adaptation of the integument to the function performed by the mammary glands, is the formation of the folds of integument found in the Marsupialia; these form a sac, the marsupium, which encloses the mammiferous region of the abdomen. The extent of its development appears to vary inversely with the extent to which the young are developed at birth.

\section{Dermal Skeleton.}

$\S 324$.

The function of the integument as a defensive organ for the body is increased in value by the formation of hard structures. When these parts are of some size they give rise to a dermal 
skeleton. In many cases we know but little as to the development of these structures, but they may all be reckoned among osseous

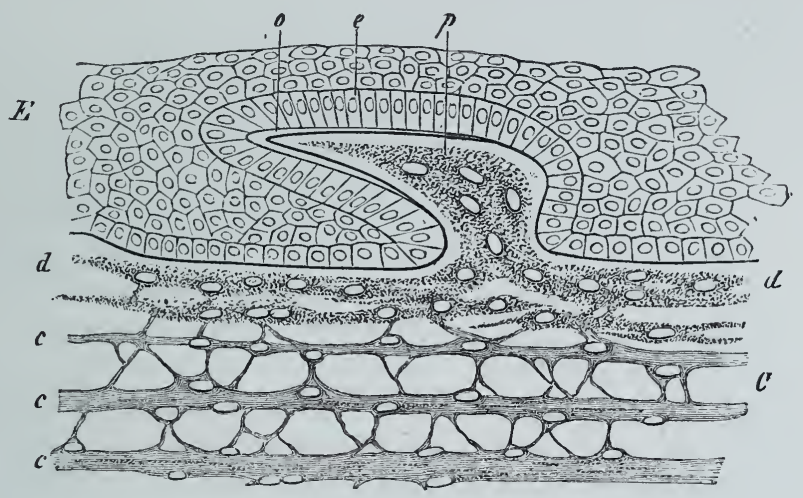

Fig. 218. Vertical section through the skin of an Embryonic Shark. C Corium. $c, c, c$ Layers of the corium. d Uppermost layer. $p$ Papilla. $E$ Epidermis. $e$ Its layer of columnar cells. o Enamel layer.

formations, to which indeed they completely correspond in the higher divisions.

The dermal denticles (placoid scales), which are distributed over the whole of the integument in the Selachii, may be regarded as the structures from which the various forms have been derived. In them we distinguish a basis, which is inserted into the corium, and is ordinarily rhomboidal in shape, and a portion, which stands out from it, and which ordinarily has its apex directed obliquely. This is covered over by the epidermis. In some parts, as, for example, on the head, they often have a bombous surface, and are set irregularly; while on the trunk they are generally set in perfectly regular and obliquely running rows (Fig.219). The y are developed on papillæ of the corium (Fig.218, p), which are covered over by a layer developed from the epidermis: this secretes an enamel-like substance on the projecting portion of the papilla, while the body of the papilla is ossified from the

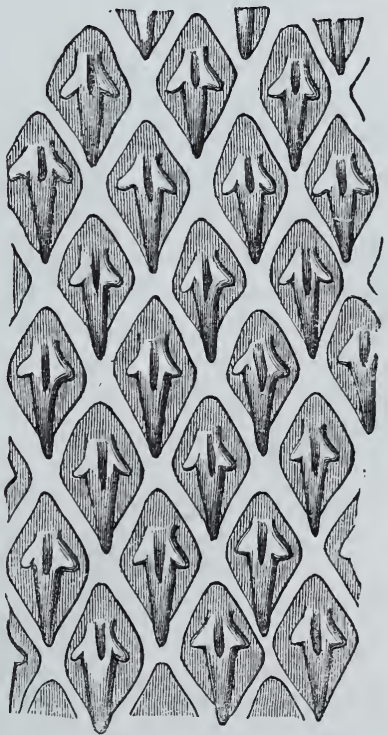

Fig. 219. Dermal denticles of Centrophorus calceus (a littlo magnified).

tip downwards. The epidermis and corium, therefore, both share in the formation of these structures. There is a central cavity in the 
papilla, whence fine branched canals radiate out to the surface. The placoid scale has therefore the structure of dentine, is covered by enamel, and is continued at its base into a plate formed of osseous tissue; as they agree with teeth in structure they may be spoken of as dermal denticles. In the Rays these structures have altogether disappeared (Electric Ray), or are replaced by larger structures, which are grouped together in the form of spines or larger bony teeth, or are separate from one another (Spiny Rays).

The dermal denticles of the Shark are very generally converted into larger bony plates in the Ganoïdei; in the Rhombifera they have not only the same arrangement on the body, but have essentially the same minute structure. In the Sturiones larger bony plates alternate with smaller ones. They generally retain exactly the rhomb-form, which is lost in the rest of the Ganoildei (the Cyclifera). The common flat and thin scales of the Teleostei follow on here. They appear to differ in many points from the ganoid scales, and represent an offshoot from the type, which obtains in the Ganoïdei, and which may be derived from the Selachii; this offshoot is characterised by its variety of form.

In many Teleostei the scales undergo complete degeneration. On the other hand they give rise to parts which differ somewhat from scales, and which are formed by the fusion of the dermal denticles; such are the plates and spines of the Plectognathi, where the plates may becorne more firmly united together and form a connected carapace (Ostracion, Lophobranchii).

Parts which are likewise formed from the concrescence of dermal denticles are found in the integument which covers the appendages of the Ganoïdei and Teleostei. To compensate for the atrophy of the internal or primary skeleton of the limbs, these bony plates form a number of rays, which often branch dichotomously at their ends, and unite to form an organ of support for the fins (secondary skeleton of the fin). The ray which occupies the anterior edge of the fin is frequently massive, or gives rise to a strong spiny ray, which may be connected with the internal skeleton. This ray may not only be larger than the rest of the rays, but it may even, as in certain Siluroids, represent the whole of the pectoral fin.

Hertwig, O., Ueber d. Bau n. die Entw. der Placoïdschuppen u. der Zähne der Selachier. Jen. Zeitschr. Bd. VIII.-The same, Ueber das Hautskelet der Fische. Morph. Jahrb. II.

\section{$\S 325$.}

The ossifications of the integument are of special importance in those regions of the body where parts of the internal skeleton come to the surface. 'These ossifications are developed in just the same way as the bony plates on other regions of the surface of the body, and may likewise be derived from the indifferent stage represented by the dermal denticles. Although the various kinds of dermal bones which are found on the trunk have an importance which is limited to 
the fishes, there are others which are of more importance; these are the bony plates which are definitely arranged, and constantly present, on the head, where they form the earliest rudiments of the bony skull, or, at first, of the roof of the skull (cf. Fig. 220). These dermal bones are inherited by all Vertebrata that are pro. vided with a bony skull, and are connected with other ossifications, which do not appear till later, in the cartilaginous skull. This arrangement is first seen in the Sturiones. There are a number of smaller bony plates in addition to the large ones, but most of these have no general significance. On account of these relations to the internal skeleton, their more special characters will be expounded when we come to treat of it. Other skeletal parts besides the bones of the skull are derived from ossifications of the integument; the clavicle, for example, has a similar origin.

Lastly, there is another category of bones which are likewise derived from placoid scales; the bones around the mouth have been recognised as having their origin in tooth-bearing plates derived from fused placoid scales.

\section{$\S 326$.}

We meet with dermal bones in the higher classes; in the Amphibia, and also in the fossil Archegosaurii, in which there were dermal bones in the form of scutiform plates. We find only scattered dermal bones in a rudimentary form in extant Amphibia. In Ceratophrys there is an osseous shield in the skin of the back; in

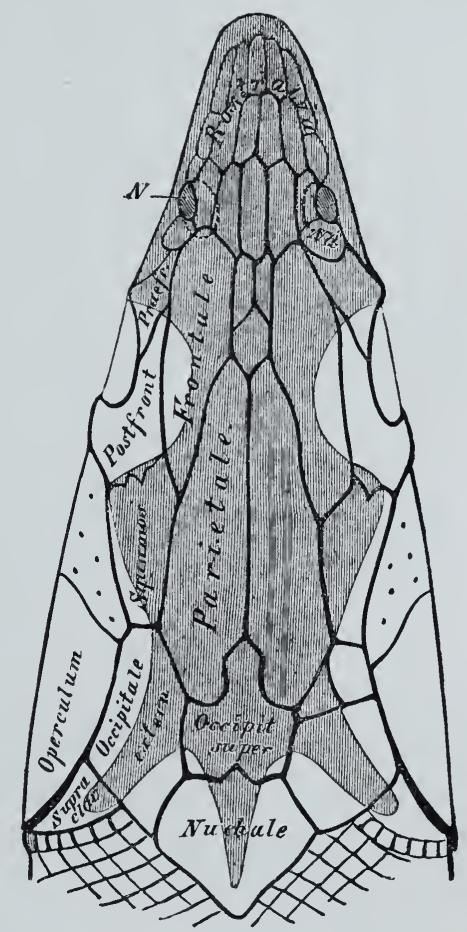

Fig. 220. Head of Acipenser sturio; seen from above, to show the osseous plates covering the cartilaginous cranium, which is shaded dark. Brachycephalus there are three which are united to several vertebræ. The bony scales which are pretty generally found in the Cœciliæ, and which are set in pouch-shaped depressions, do not apparently belong to this set of structures.

They are more common in the Reptilia, which so far approach the old Amphibian phylum. In the fossil Teleosaurii, as in the living Crocodilini, there are dermal bones distributed over the whole integument, which form a kind of carapace; in the Scincoïdea we generally meet with interlocking bony plates in the integument. Similar kinds of dermal ossifications in the Chelonii form a special, though well-developed form of, dermal skeleton, in consequence 
of their connection with the internal skeletal parts. They not only form a dorsal shield on the dorsal surface, but a ventral one on the ventral surface (plastron). In the dorsal shield we can make out a median row of bones, which are fused with the spines of the vertebræ, and project from them. At the sides there are larger plates, which are fused with rib-like processes, and in addition to these there are special marginal plates around the edge of the shield. These are wanting in Trionyx. Four paired pieces and one unpaired piece can be made out, as a rule, in the plastron. All of these parts are variously developed in different families of the Chelonii.

Although the dermal bones of the Reptilia may probably be rightly regarded as derived from the bony carapace of Fishes, we must regard the ossifications which are found in the Edentata as independent arrangements, which have had their origin in fresh adaptive modifications.

\section{Internal Skeleton.}

$\S 327$.

The internal skeleton is of greater morphological importance than the skeletal structures formed from the integument; it is connected, on the one hand, with arrangements which are found in the Inver-

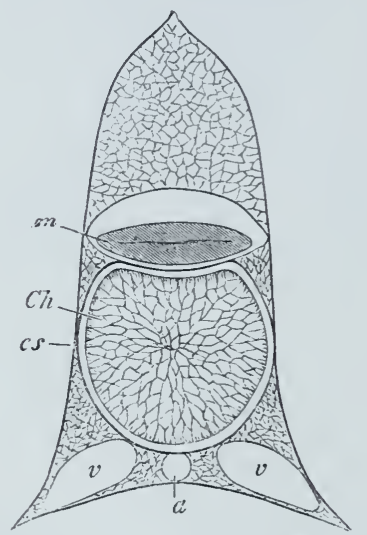

Fig. 221 a. Section through the vertebral column of $\mathrm{Am}$. mocœtes. Ch Chorda. cs Chordal sheath. m Spinal chord. a Aorta. $v$ Veins. tebrata, and on the other, and by a long series of very varied arrangements, it can be followed out through all divisions of the Vertebrata.

At first the internal skeleton has the form of a rod-like structure which traverses the whole length of the body, and is, when simplest, made up of indifferent cells, and surrounded by a cuticular structure which is formed from a secretion of these cells. This primitive organ of support is the chorda dorsalis or notochord; we have already met with it in the Tunicata (cf. §303). The investment formed by it is the chordal sheath $(c s)$.

The earliest rudiment of the noto. chord is placed just below the central nervous system; it has not always the same relations to the germ-layers, although it must be derived either directly or indirectly from the mesoderm. The compact, and, in all cases, primitively unjointed condition of the notochord speaks to its having been inherited from an unjointed condition of the organism, and this is what might be supposed from its early appearance in the embryo. 
The notochord has always the same topographical relations to the most important of the other organs. Above it, is the central nervous system, and below it, the respiratory and nutrient apparatus. Processes are given off from the connective tissue surrounding the chord which enclose the so-called dorsal and ventral cavities; these processes pass into the musculature of the body, which is thereby broken up into a number of segments, set one behind the other. In Amphioxus these segments are so far asymmetrical that they are found alternately on either side.

The low condition of the axial skeleton, which is represented by the chord, is permanent in the Leptocardii, where it merely presents special histological modifications. In all the rest of the Vertebrata the chord is the sole axial skeleton in the carliest stages of development only; new differentiations appear, and it becomes of less physiological importance. These differentiations affect the notochord as well as the tissue which surrounds it, and which has been called the "skeletogenous layer" or "skeletogenous tissue," on account of its relations to the future skeleton. The cells of the cliord form a tissue resembling cartilage, and the sheath becomes a more independent portion-it forms a supporting organ-owing to the thickening of its layers. Cartilaginous tissue forms around the chord (Fig. 221, $b$ li), and that segmentation of the axial skeleton into separate segments, the so-called vertebræ, which was before merely indicated, now becomes obvious. This segmentation of the axial skeleton is an expression of the metamerism of the whole body; a series of these vertebræ make up the vertebral column. In each vertebra we call that por-

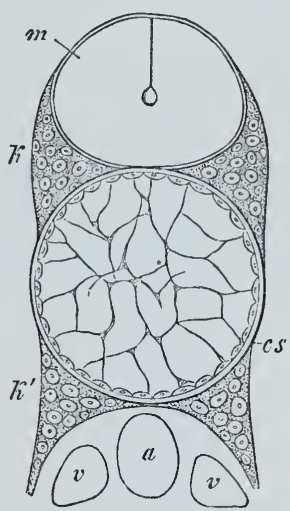

Fig. 221 b. Section through the spinal column of a young Salmon. Ch Chorda. cs Chordal sheath. $m$ Spinal chord. $k$ Supe. rior, $k^{\prime}$ Inferior arch (in rudiment). a Aorta. $v$ Veins. tion which surrounds the notochord the cent$\mathrm{r} u \mathrm{~m}$, and the outgrowing portions which enclose the dorsal and ventral cavities of the body, and which are given off directly or indirectly from the centrum, the arches. These again are distinguished as upper or lower arches, according to their relations to these two cavities.

As the axial skeleton becomes segmented, a well-defined portion in the most anterior segment forms the primitive Cranium in the Craniota.

An inferior system of arches, which encloses the most anterior portion of the intestinal tract, which functions as a respiratory organ, is distinguished as the branchial or visceral skeleton. The cranium and visceral skeleton make up the most anterior portion of the whole skeleton - the skeleton of the head. The other skeletal structures which are connected with it are represented by the more 
or less homogeneous vertebral column, which extends to the caudal end of the body. The upper arches remain in close connection with the centra. Movable girder-like pieces are, however, separated off from the lower arches in the region which encloses the colom; these are the ribs.

Lastly, there are the skeletal portions of the appendages which are connected by special organs-the pectoral and pelvic girdles-with the skeleton of the trunk.

The cartilaginous stage of the primitive skeleton is found in all of the higher divisions, but in them it has no function after a short time, for it is gradually replaced by osseous tissue, whereby the skeletal parts come to have a greater physiological importance. In correlation with this we note a greater differentiation in morphological points. Even in the osseous skeleton, however, the cartilage is of great importance. A modified form of cartilage, which is characterised by the deposit of calcareous matter in it, is also of importance. This form is not only antecedent to the ossification of the parts of the skeleton, which are laid down in cartilage, but-as is seen in that superficial calcification of the cartilaginous skeleton of the lower Gnathostomata-is also sometimes a permanent arrangement.

\section{Vertebral Column.}

\section{$\S 328$.}

The separation of the rachis into skull and vertebral column is not completely effected in Amphioxus; the whole axial skeleton is represented by the notochord. In the Craniota they begin to be separated. The lowest characters of the spinal column obtain in the Cyclostomata, where the more highly-developed notochord, with its sheath, forms the chief portion of it. Around the sheath there is cartilaginous tissue, which is continued into lateral ridges as well as into the wall of the dorsal canal. This tissue has its origin in the continuous differentiation of the skeletogenous layer, and must not be confounded with the cartilages, which ordinarily form the vertebral segments. Speaking exactly, therefore, the spinal column is not here separated into distinct vertebræ; of which, indeed, there are indications only in Petromyzon, where cartilaginous pieces, which correspond to the superior arches, are enclosed in the wall of the more anterior division of the dorsal canal. We meet also with indications of inferior arches.

The notochord also retains its primitive characters in Chimæra and the Dipnoï. In the Chimæræ circular calcifications of the large chordal sheath point to a segmentation of the notochordal tube, but they are more numerous than the primitive vertebræ, which are merely represented by the arches on the chordal sheath. In the most anterior region they grow round the chord and give rise by fusion to a larger undivided piece. In the Dipnoï a strong tube, 
derived from the skeletogenous layer, is developed around the primitive sheath, and on this the cartilaginous and superficially ossified arches are set.

The axial skeleton of the Selachii is much more highly developed. The rudiments of the superior and inferior cartilaginous arches appear around the notochord; these grow around it and so form cartilaginous circular centra. That part of the cartilage which encloses the chord is marked off from the peripheral part, which is continued into the arches, and the former represents, just as in the Dipnoï, a kind of cartilaginous sheath (skeletogenous chordal sheath), which is deposited on the cuticular sheath.

The vertebral column of the Selachii varies greatly in structure according to the mode of growth of the notochord and its skeletogenous sheath.

A

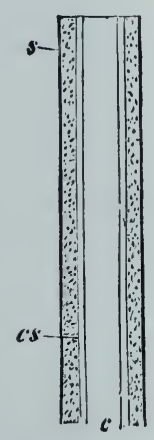

B

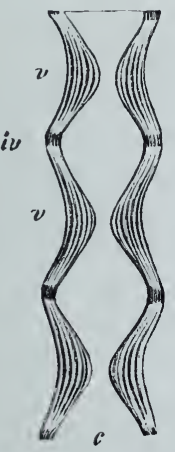

D

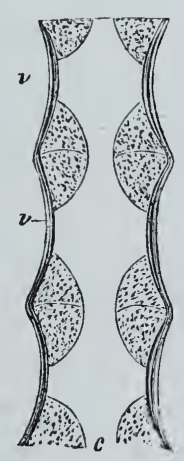

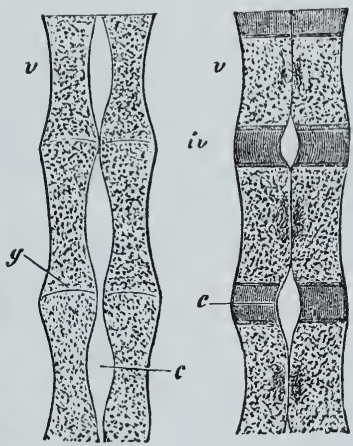

Fig. 222. Diagram of the changes produced in the notochord by the skeletogenous layer (longitudinal sections). $\quad c$ Chorda. cs Chordal sheath. $s$ Skeletogenous layer. $v$ Bodies of the vertebræ. $i v$ Intervertebral portion. $g$ Intervertebral joint. $A$ The chordal tube, when all its parts are equally well developed (Fishes). $B$ Intervertebral growth of the chorda. Formation of amphicolons vertebræ (Fishes). $C$ In. tervertebral constriction of the chorda by cartilage; while the rest of the chorda is retained in the vertebræ (Amphibia). D Intervertebral constriction of the chorda (Reptilia, Aves). $E$ Vertebral constriction of the chorda, where part of the intervertebral portion is retained (Mammalia).

The cartilage sometimes forms a cylindrical tube, in which the vertebræ are merely represented by the arches and circular parts of the skeletogenous sheath. The notochord is sometimes dereloped between the vertebræ (Fig. 222, $B$ ), and retains its earlier size at the points where the vertebra $(v)$ and arches were first laid down around it. This arrangement gives rise to biconcave (amphicœlous) vertebræ $(B)$, the depressions in which are filled up by the intervertebral chord. This is the way in which the vertebræ of nearly all other fishes are formed.

\section{$\S 329$.}

In the Ganoïdei the vertebral column, when simplest in organisa. tion, resembles that of the Selachii. Just as in the Selachii and 
Chimæræ special cartilages are intercalated, which aid the superior arches, which are connected with the bodies of the vertebræ, in closing the vertebral canal.

In the Sturiones the skeletogenous sheath forms a considerable tube, and the separation of the column into vertebre is only indicated by the superjacent arches. The vertebral column of the other Ganoïdei is sharply marked off from this, its lowest form. Amia resembles the Teleostei. A small portion of cartilage is retained at the point where the arches are connected with the centra of the vertebræ; but this is absent in Polypterus, so that in it the arches and the centra are united together by bone.

Lepidosteus is the most divergent form, for in it the cartilage

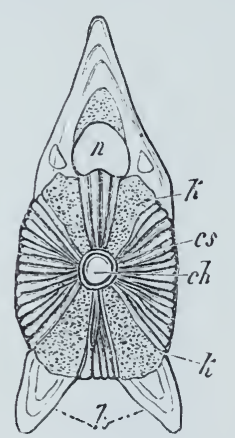

Fig. 223. Vertical section through the middle of a vertebra of Esox lucius. ch Notochord. cs Chordal sheath. $k k^{\prime}$ Cartilaginous cross. 7. Corresponds to the upper, and $h^{\prime}$ to the lower arches (in rudiment). $h$ Osseous transverse process. $n$ Spinal canal.

a vertical section becomes constricted between the vertebræ. In the cartilage which forms the constrictions, an intervertebral articular cavity is formed, so that the opisthocœlous vertebræ articulate with one another. So far they resemble the Amphibia, but, later on, the remnant of the vertebral portion of the notochord disappears, and a bony centrum is developed, which is connected, and continuous, with the upper arches.

The vertebral column of the Teleostei is characterised by the reduction of the cartilaginous rudiment. This reduction may be seen to be gradual, and may indeed be seen in one and the same vertebral column in certain stages of development; where, that is, the cartilage may be seen to diminish in quantity as we go from before backwards. As a rule, four cartilaginous pieces, belonging to the superior and inferior arches (Fig. $\left.221 \mathrm{~b}, 7_{i} \mathrm{i}^{\prime}\right)$, may be seen around the chord, and these take a certain share in the formation of the arches. They very rarely form complete superior arches. When osseous substance is developed, these cartilages are generally retained in the middle of the centrum, so that on making. a ver an obliquely set cross (cf. ig. $223, k)$, the arms of which are directed towards the bony arches. The notochord is always well developed between the vertebre, so that the centra are amphicolous.

\section{$\S 330$.}

The vertebral column of Fishes can only be divided into two regions, the body and the tail. They are distinguished from each other by the characters of the inferior processes of the vertebræ, while the upper arches are connected with the vertebræ in the same manner throughout; and are generally distinguished by the possession of median (spinous) processes. In the region of the 
trunk, the lower arches are divided into ribs, and supporting transverse processes (parapophyses). In the tail of the Selachii and Ganoildei they are continuously connected with the centrum, and run out into spinous processes, just like the upper arches.

In the Teleostei the costiferous transverse processes gradually converge, in the caudal region, and form inferior arches, which are not homologous with those of the Selachii and Ganoïdei, although they also form spinous processes.

In the Chimæræ, Dipnoï, and many Teleostei, the caudal portion of the vertebral column ends by gradually diminishing in size, but in most fishes it presents great modifications, which are correlated with the development of the caudal fin. 'These modifications first affect the lower arches, which, in the Sharks, form spinous processes, which are greatly widened out at their ends, and with which the caudal fin, which is most developed in its ventral region, is connected. ' In many Sharks, and still more in the Sturiones, this candal skeleton is differentiated in an unequal fashion. The inferior spinous processes are more largely developed; this is correlated with the degeneration of the superior spinous processes, and of the superior arches of the terminal caudal vertebræ; this of course produces an up-turning of the caudal end of the vertebral column; and in this way the inferior lobes of the caudal fin of the shark get to be terminal in position.

In the Teleostei this up-turn-

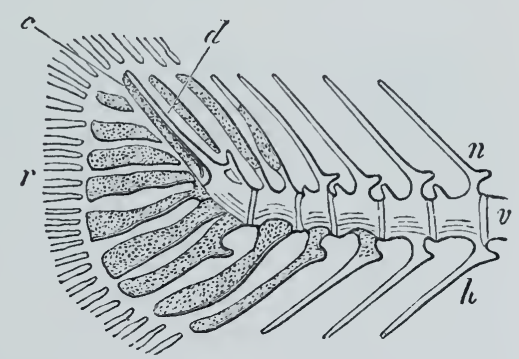

Fig. 224. End of the caudal portion of the vertebral column of a young $\mathrm{C}_{y}$ prinoid. $v$ Centrum. $n$ Superior; $h$ Inferior arches (the cartilaginous parts are dotted). $c$ End of the notochord. $d$ Covering bony lamella. $r$ Bony rays of the caudal fin. ing affects also the axial portion of the vertebral column. As a number of the terminal centra of this column are generally fused together, and, like their upper arches, feebly or not at all developed, while their inferior arches still persist, the up-turning must be the more marked in proportion as the inferior arches become more numerous and larger than the superior ones. This condition (Fig. 224) is carried still farther by the atrophy of a large number of vertebræ, so that nothing remains of them but their inferior arches (Physostomi).

Finally, the vertebræ completely disappear, and the remains of the inferior arches of the caudal region are connected, in the form of vertical plates, with a single vertebra which represents the end of the vertebral column; a style-shaped process (urostyle) of the column is directed upwards, and contains the end of the notochord (Acanthopteri).

Supporting organs, formed from the integument, are connected with the parts thus formed by the vertebral column, and they 
are continued into the caudal fin. In the Selachii the fin-rays are formed by the so-called horny filaments, and in the Ganoïdei and Teleostei by ossifications.

Like the caudal fin, the other unpaired ones have their supporting organs formed partly by the axial skeleton and partly by the integument. In the Selachii jointed pieces of cartilage pass from the spinous processes into these fins, and gradually acquire an independent significance. In the Ganoïdei and Teleostei they become distinct bony pieces, which are known as "supports for the finrays;" these are quite separate from the spinous processes. They are comnected with the fin-rays ; these are jointed structures, which are sometimes made up of separate bony plates, and are sometimes represented by solid bony rods (spinous rays).

\section{$\S 331$.}

In the Amphibian vertebræ the cartilaginous rudiment of the arches grows around the notochord, and forms constrictions in it by means of interrertebral enlargements (Fig. 222, C). In many of them the notochord is destroyed at these points. In the Anura the notochord remains persistent in the middle of the centrum; the only exception to this rule is to be found in those forms in which the centrum is developed on the surface of the notochord (Hyla, Bombinator, Pelobates, etc.); when the articulating cavities are developed, the articulating ends of the centra are developed from the intervertebral cartilage. These intervertebral articulations are incomplete in most of the Urodela, where we find the articulating processes, derived from the centra at every stage of development.

In the rest of the Urodela the intervertebral cartilage is only feebly developed, so that the notochord is but slightly or not at all constricted by it. It persists all along the vertebral column, and is alternately constricted and widened out in Menobranchus, Siredon, and Menopoma. In the latter the cartilage takes a markedly small share in the formation of the vertebra; indeed, a serjes, in which the intervertebral cartilage may be seen to undergo gradual degeneration, can be followed out from the Salamandrina up to Proteus. In proportion to this degeneration the vertebra is formed by deposits of bony layers, which may even be laid down directly on the chordal sheath itself.

No separate rudiments of superior and inferior arches can be seen in the trunk; they seem to have been fused into a common mass of cartilage. Henceforward, therefore, that arrangement which we saw in the Fishes disappears, and the rudiment of the cartilaginous vertebra is formed of a single piece early in life.

The shortening of the hinder end of the rertebral column in the Anura is the cause of the derelopment of a small number of vertebræ. When the tail disappears; a long dagger-shaped bony piece, 
which is ordinarily known as the urostyle (Fig. 225, c), is formed from the rudiments of a few vertebræ; counting this then, no more than ten vertebral segments can be made out. There are many more in the Urodela; Amphiuma has as many as 100 ; Menopoma, 48; Salamandra, 42 ; and the Cœciliæ, about 230.

The transverse processes $(t r)$ are small in the Salamandrina; the anterior ones are generally divided into two segments; in the Anura they are larger, but not divided. The superior spinous processes are always rudimentary. Articulations between the arches of the vertebræ are very common, and are effected by the formation of paired articular processes.

The connection between the pelvic girdle and the vertebral column does not only more distinctly mark off the caudal portion from the region of the trunk, but a sacral portion is thus represented by a vertebra, which is generally distinguished (and especially in Pipa) by the size of its transverse processes.

Gegenbaur, Unters. über die Wirbelsäule der Amphibien. Leipzig, 1861.

\section{$\S 332$.}

The rudiments of the vertebral column are

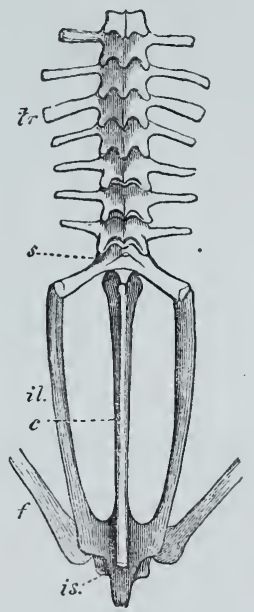

Fig. 225. Vertebral column and pel. vis of the Frog. tr Transverse processes. $s$ Sacral vertebra. $c$ Urostyle. il Ilium. is Ischium. $f$ Femur. developed around the chorda dorsalis of the Sauropsida, as of the Amphibia. Arches, which enclose the spinal canal, are given off by the cartilaginous centra. The notochord is also constricted between the vertebræ (cf. Fig. 222, D), but the whole of it eventually disappears (except in the Ascalobota). The continuous rudiment is separated into centra in just the same way as in the anourous Amphibia; in the Saurii and Ophidii, the centra are procœlous. In the Crocodilini and Aves the cartilaginous portions of the rudiment, which lie between the centra in the cervical region, are converted into a special intervertebral apparatus.

Articular processes extend from the superior arches to the vertebra next in front and behind. They are greatly developed in the cervical region of the Chelonii. The superior spinous processes vary in size, especially in the dorsal region; in the Crocodilini and many Saurii they are present on the caudal vertebræ. Transverse processes are either given off from the centrum itself, or quite close to it. They are greatly developed in the dorsal and caudal region of the Crocodile, but much more so in the Chelonii, where they are surrounded by the bony plates of the dorsal shield, which have been developed in the integument. They are seen to be divided into an upper and a lower portion in the Ophidii. In the 
Reptilia and Aves ribs are found along the whole of the dorsal portion of the vertebral column, and are absent only in the Chelonii.

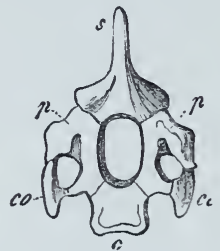

Fig. 226. Cervical vertebra of Vultur cinereus. $c$ Centrum. $p$ Arch. $s$ Spinous processes. co Rudimen. tary rib.

The movable cervical ribs of the Reptilia unite with the vertebre in the Aves (Fig. 226, $c o)$, and the two together bound a foramen transversarium.

In the caudal region of the column of the Saurii, Chelonii, and Crocodilini we meet with inferior arches, which are always attached between two centra, and take part in the formation of a caudal canal. They are rudimentary in Birds. The inferior processes of the Ophidii, Saurii, and Aves, which are given off directly from the centra, are quite different from these.

On comparing the vertebral column of the Reptilia and Aves with that of the Amphibia we can see that it is divided into a larger number of regions. A cervical and a lumbar

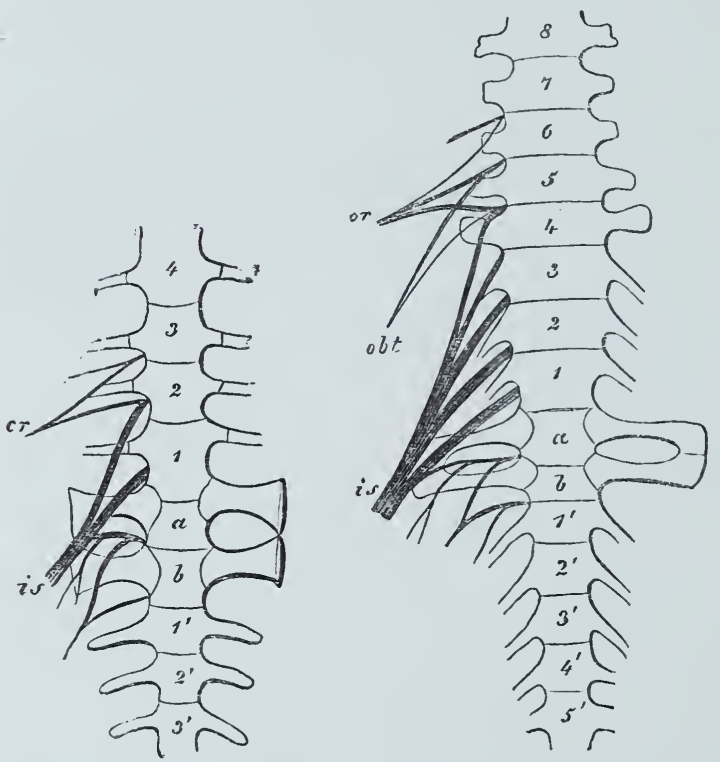

Fig. 227. Sacral portion of the vertebral column of a Reptile, with the adjacent pre. and post-sacral vertebræ.

Both of these diagrammatic figures are drawn as if from the ventral surface, and show the nerrous plexus on the left. In both the figures $a$ is the first sacral vertebra, $b$ the second sacral rertebra. . . . 1, 2, 3, 4 Presacral. $1^{\prime}, 2^{\prime}, 3^{\prime}, 4^{\prime}$ .... Post-sacral (or candal) vertebræ. region are more distinctly marked off, owing to the connection between a number of ribs and a sternum. The lumbar region contains the pre-sacral group of vertebræ, which have only short ribs; it is distinct in the Saurii and Crocodilini. The absence of any sternal connections in the Ophidii is the cause of there being no difference between the thoracic and cervical regions in these forms, as well as of the impossibility of distinguishing a lumbar one. In the Chelonii also the vertebræ of the trunk are similar in character throughout. These regions are not, however, very sharply marked off from one another, inasmuch as in the Saurii 
and Crocodilini, as well as in Aves, the last ribs of the cervical region differ but little in length from the succeeding ones, which are connected with the sternum. The same applies to the lumbar region in the Saurii; in Birds this region is united to the true sacral portion. The sacral portion of the column is increased in size, inasmuch as in Reptiles there is, at the least, a second vertebra (Fig. 227, $a b$ ) in addition to the one found in the Amphibia. These vertebræ attain firmer connections, and are completely fused in Birds, where a large number of pre-sacral and post-sacral vertebræ are attached to the primitive sacral vertebræ (Fig. 228, $(a b)$, and the whole set is united to the ilium. In the so-called sacrum of Birds, thoracic as well as lumbar and caudal vertebræ may be recognised; in the Struthiones the whole number is as great as twenty-three. The two true sacral vertebræ are very distinct in the Gallinæ, many Natatores, and in birds of prey.

The caudal region is the one which varies most in character; in the Chelonii and Aves it is considerably reduced.

In Carinate Birds, the caudal vertebræ are not only reduced in number, but four to six of them, which were separate in the embryo, are fused together; and the bone thus formed, which is of some size, and is ordinarily known as the "ploughshare-shaped bone," forms the terminal portion of the vertebral column; it is generally produced into an upright plate, in adaptation to its relations to the rectrices feathers.

\section{$\S 333$.}

In the Mammalia the cartilaginous rudiment of the vertebral column grows around the chorda dorsalis, and is constricted at points, each of which corresponds to a centrum; the notochord is therefore retained for some time between the vertebræ (Fig. 222, E). The intervertebral circular disc is developed out of the surrounding tissue, and the remnant of the notochord is preserved in it, under the form of the "gelatinous nucleus." The cartilage is continued from the centra into the superior arches. Independent ossifications appear in the centrum as well as in the arches, and the bony pieces thus formed do not fuse until growth is finished.

On most vertebræ the arches develop spinous processes. In the long-necked Ungulatæ (Giraffe, Camel, Horse), they are not found on the cervical vertebræ, but are greatly developed on the dorsal ones. This holds also for the Cetacea, where the caudal region is also of great size. As a rule articular processes are developed; they have undergone degeneration in the Cetacea only. We are in the habit of calling all kinds of structures transverse processes, whether they spring from the arches or from the centra. These processes are simpler in the cervical and thoracic regions. In the former they are somewhat complicated by being fused with rudimentary ribs, which unite with them and aid in the formation of a foramen transversarium. They likewise carry ribs in the thorax, and here they 
are attached on their ventral surface. They may, however, also carry terminal ribs, as in the posterior thoracic vertebræ of the Cetacea. As the thoracic pass into the lumbar vertebræ the transverse process is very commonly differentiated into three special processes. The mamillary processes form knobs, which are sometimes of great size, and which are directed forwards; they may get to be placed at the base of the anterior articular processes. The accessory processes are directed backwards and upwards; and a third process is lateral, though often directed downwards; these form the transverse processes (lateral processes) of the lumbar vertebræ.

The various divisions of the vertebral column are more sharply differentiated from one another in Mammals than in Birds and Reptiles. 'The cervical region is especially so ; it is distinguished by the constant possession of seven vertebræ, which are more definitely marked off from the thoracic region, owing to the fact that their rudimentary ribs rarely increase in size as they approach the thoracic ribs. The increase in number of the cervical vertebræ in Bradypus to eight, or nine, is explained by the fact that the thoracic vertebra pass into the cervical region, while the diminution to six in Cholœpus and the American Manatee is similarly explained by the complete development of the rib of the seventh cervical vertebra.

The lumbar region is distinguished by the absence of movable ribs. In the sacral region one vertebra only carries the ilium, as a rule; but a second one has frequently the same relations. It is more rarely that a third vertebra is connected with the ilium. The fusion of these vertebræ with one another, and with one or more caudal vertebræ, gives rise to the formation of a compact region, which is known as the "Os sacrum;" in this we must distinguish the true sacral vertebræ from the false ones which are developed from the caudal vertebræ. In the Edentata the number of the sacral vertebræ is increased by the union of the ischium with the caudal vertebræ. In this way the sacral region gets to extend over eight or nine vertebræ.

In the Mammalia also the caudal region of the vertebral column is the most variable of all regions; in most divisions it may be either greatly developed, or may undergo considerable reduction. Thus in the Simiæ the number of vertebræ may be as high as thirty, while in some again it may sink below the number, which has been retained by man.

In this point the last portion differs from the most anterior or cervical region; while the intermediate portion is, as regards its numerical relations, less constant than the cervical, but at the same time less variable than the caudal portion of the vertebral column. The number of dorso-lumbar vertebræ is very high in some of the half-apes (23-24 in Lemur); in Cholœpus it is 27; in the Perissodactyla, 24. It is highest in Hyrax (29). It is lower in all the other divisions.

Within the larger divisions the common ancestry of the different genera is implied by the great constancy of the whole number of the 
dorso-lumbar vertebræ. In the Marsupialia and Artiodactyla this is always 19 ; the same number, or 20, predominates in most Rodents, Carnivora (21 in Paradoxurus and Procyon), and the majority of Primates. In some of the latter, however, it falls to 17 or 18, in which number they agree with most of the Chiroptera.

The variations within the dorso-lumbar region depend on the ribs, for when they undergo degeneration the lumbar vertebræ are increased in number.

\section{$\S 334$.}

Varied as are the differentiations undergone by the vertebræ, the extreme conditions are usually connected by intermediate forms. It is in the first two vertebræ only that an arrangement obtains which is limited to them, and them only; and this arrangement is due to the way in which the skull is connected with, and moves on, the spinal column.

In Fishes we sometimes meet with peculiar arrangements in the way in which the skull and first vertebra are connected together; in the Rays there are articular facets, as there are also in the Telecstei, where they are placed on lateral processes. Another set of modifications commences in the Amphibia. The first cervical vertebra is circular, as it generally has no transverse processes, which are only present when it is fused with the succeeding vertebra (Pipa). This first vertebra is known as the atlas. In the Reptilia the centrum of the atlas is separated from its arch, and is placed in front of the centrum of the second, which is distinguished as the axis, and it is more closely connected with the centrum of the axis than with its own arch. Owing to this arrangement a special piece is developed, which connects the two sides of the arch on the ventral surface. In the Crocodilini the arch is also closed by bone on its dorsal surface. In the Ophidii, the part which corresponds to the centrum of the atlas fuses with the second cervical vertebra, and forms its odontoid process. The same arrangement obtains in Birds, where the ventral connection of the arch becomes very large indeed as compared to this "odontoid process."

The arrangement seen in the Reptilia is represented in an embryonic stage in Mammalia. In the Monotremata it lasts some time longer than in the rest. In the Marsupialia, however, it is often a permanent condition, owing to the separation of the centrum of the atlas from the axis. In other cases the centrum of the atlas passes completely into the odontoid process of the axis. The inferior union of the arches is, in the Marsupialia, merely represented by a ligament, or there may be a distinct bone in its place. In the Monodelphia there is a bony ventral clasp between the two halves of the arch. 


\section{Ribs.}

\section{$\S 335$.}

T'he name of ribs is given to those parts of the skeleton which have been developed from the inferior arches of the vertebræ, and which are either permanently, or transitorily, articulated to the vertebral column. As a rule they clasp round and enclose a subvertebral cavity. This cavity is divisible into two portions, which differ in size, and in the organs which they contain. The anterior one is the cœlom. The posterior one is continued into the tail, and forms the narrow caudal canal, which is sometimes divided horizontally into two parts. These relations may be seen in Fishes, where also the division of the body into regions is in its most indifferent condition.

A comparison of the contents of the two tracts of the subvertebral cavity will explain their difference in size. While in the caudal canal there is nothing but blood-vessels, or, at most, parts of the kidneys, which are always organs that vary but little in size, great variations in volume can be made out in the organs of the cœlom, and these variations may be often seen to be due to a regular succession of states, in which the organs are being filled or emptied. The arrangements which can be made out in the lower arches are correlated with this characteristic. They are seen to be direct processes of the vertebra in the caudal region, and are immovable. In the abdominal region, however, in adaptation to the varying size of the cavity they enclose, they are segmented off from the vertebræ, and are more or less movably articulated to the centra or its processes (transverse processes).

The ribs are therefore regarded as differentiations of the inferior system of arches; a number of arches, varying according to the extent of the cœlom, have been converted into the more free form of rib. This view, which explains the origin of the ribs, proves to us that the inferior arches, which resemble the ribs, but which do not now enclose the cœlom, are not primitive structures, but that they once were ribs; this necessarily presupposes that the cœlom once extended much farther back.

\section{$\S 336$.}

As we have already treated of the indifferent lower arches when speaking of the vertebral column, we have now only to deal with the ribs and the organs derived from them. They are not completely absent in any of the Vertebrata, save the Leptocardii, Cyclostomata, and the Chimæræ. In all the rest they are either in a rudimentary or in a well-developed condition; in the latter case they may become comnected together on the rentral surface, and a special skeletal piece, the sternum, is then developed. 
All the trunk vertebræ may carry ribs. In Fishes they generally extend as far as the caudal region, without any change in form. They never become united below, for when they are connected with other parts of the skeleton, these parts belong to the dermal skeleton (Clupeidæ). They are rudimentary in the Selachii, and are ordinarily represented by short pieces of cartilage only; they are larger in the Sturiones (Acipenser). They are not placed on the exact boundary of the colom, but at a certain depth in the muscles; but this does not affect the explanation that we have given of the origin of the ribs.

The ribs are better developed in those Ganoids that have a bony skeleton. In the caudal portion of the vertebral column, as in the Selachii and Sturiones, they gradually pass into the lower arches, which are at first connected with the centra in just the same way as are the true ribs in front of them.

In the Osseous Fishes the ribs vary very greatly in character. They are frequently rudimentary or completely absent (Lophobranchii, Gymnodonta, etc.). It is easy to understand, from what has been said above as to the origin of the lower arches in the Teleostei, that these lower arches may carry ribs (Fig. $229, C)$. In a few divisions of the Physostomi the most anterior ribs undergo changes, for bones connected with the air-bladder are developed from them to form a chain

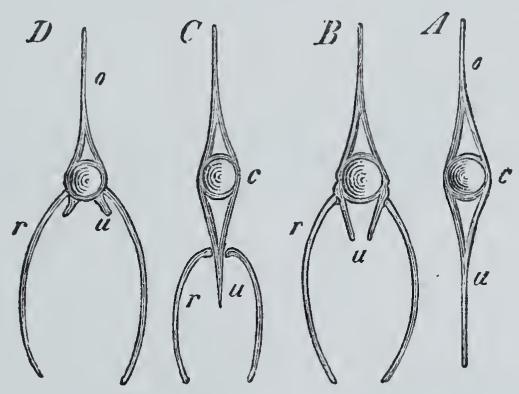

Fig. 229. Differences in the arrange. ment of the ribs and transverse processes in the Teleostei. $c$ Centrum. o Upper arches. $u$ Transverse processes. $r$ Ribs. which extends to the auditory organ (Cyprinoids). In Polypterus, rib-like structures are placed between the dorsal and ventral muscles, on the side of the trunk, and these extend to the integument. They are found also in Amia and the Physostomi, where they are sometimes so large that they have been regarded as the true ribs. As a rule they are bifurcated at their origin.

Among the Amphibia the most completely developed ribs are found in the Gymnophiona, where they are found on all but the first and last vertebra. In the Urodela they are rudimentary, and form short pieces, which are movably attached by two processes; posteriorly they are more simple in form. Just as in the Selachii, they extend into the muscles. The transverse process of the sacral vertebra also carries a rudimentary rib, by which it is connected with the pelvis. In the Anura, likewise, they are rudimentary or completely absent. 


\section{$\S 337$.}

Among the Reptilia, the Chelonii resemble the anourous Amphibia; there are no ribs in the cervical region of the vertebral column, and it is doubtful whether the rib-like processes, which in the trunk are connected with dermal bones, are not really transverse processes. In the rest of the Reptilia, ribs are found on nearly all the trunk vertebræ. In the Saurii and Ophidii there are no ribs to the atlas, and in the former there are none on the second cervical vertebra either. In the Saurii, some of the ribs of the trunk are connected with a sternum, and so make a great difference between the various costiferous portions of the vertebral column; in the Ophidii, however, the ribs, from the second cervical vertebra as far as the end of the trunk, have pretty much the same characters. They are all characterised by their free articulation with the vertebral column.

In the Saurii and Crocodilini, each rib connected with the sternum

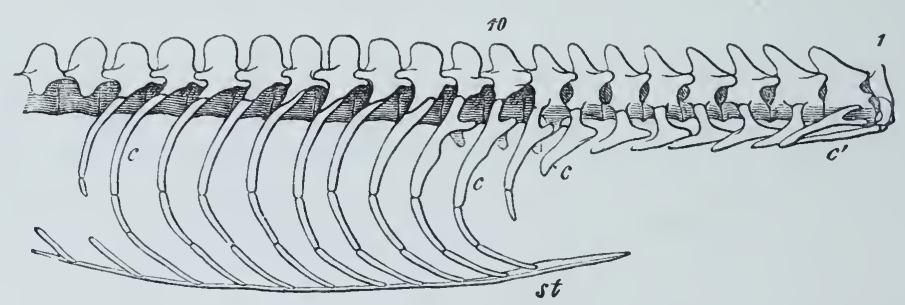

Fig. 230. Cervical and thoracic vertebra of Crocodilus. c Ribs. $c^{\prime}$ Rib of the atlas. st Sternum.

is always divided into several portions; of these the upper, or vertebral, is, as a rule, the only portion that is completely ossified. The sternal end ordinarily remains cartilaginous, and but few ribs are directly attached to the sternum. Not unfrequently a large number are connected with a cartilaginous arch which is attached to its hinder end. The more posterior cervical ribs, even, may be divided into two segments, and so form an intermediate stage towards the arrangement seen in the thoracic ribs.

In Birds, the union between the rudimentary cervical ribs and the vertebral column leads to most of them being completely fused with it; on the other hand, the hinder ones are less firmly connected, and their mode of union is intermediate in character between that of those in front of them, and of those thoracic ribs which reach to the sternum. As in the Saurii, these latter are not numerous, and are similarly divided into a vertebral and a sternal piece (os sternocostale). The vertebral pieces are distinguished by backwardly directed processes (processus uncinati), which are applied to the body of the succeeding rib, and so increase the strength of the thorax. This arrangement obtains also in some Saurii (Sphenodon) and in the 
Crocodilini. These processes are not laid down in cartilage, but are secondary ossifications. The lumbar portion of the vertebral column, which is fused with the sacrum, has no ribs in Birds, but distinct rudiments may be seen on the true sacral vertebræ, so that even here the ilium is not united directly to the vertebræ, but to the rudimentary ribs that are attached to them. Similar rudiments may be made out in the Crocodilini. When the caudal region is well developed, the same structures as those which have been already alluded to as rudimentary ribs may be seen to enclose the caudal canal.

With regard to the connection between the ribs and the vertebræ; in the Saurii, Crocodilini, and Aves, there are generally two points of attachment, for the rib is articulated to the centrum by a capitulum $(\beta)$, and to the transverse process by a tubercle $(a)$. Tho posterior ribs gradually get to have only one point of attachment.

In the Mammalia the cervical ribs have passed completely into the vertebræ, and it is only now and again that we meet with a free rib. The thoracic ribs, which vary in number, are divided into the two abovementioned pieces, and so show that ossification has not equally affected the whole rib, but that a sternal portion remains in its cartilaginous condition. When this portion is ossified (Edentata, Cetacea) it forms a separate piece; in Ornithorhynchus, it is again divided in the case of the last five ribs; this happens also in Manis.

The anterior ribs alone reach to the sternum. The hinder ones are either

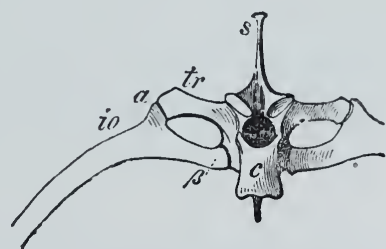

Fig. 231. Thoracic vertebra of Buteo vulgaris. $c$ Centrum of the vertebra. $s$ Superior spinous process. $t r$ Transverse process. io Rib. a Tuberculum.

$\beta$ Capitulum. connected with the sternal end of the one next in front, or project freely, and so get to resemble the rudimentary forms, to which class of ribs the hindermost ones in the Cetacea do belong; these are not even connected with the vertebral column. In the lumbar region the ribs are fused with the transverse processes. It is not certain whether the transverse process itself does not represent the rib. 'The rudiments of the ribs are much more distinct in the first two or three sacral vertebræ, where, as in the lower classes, they are the means of connection between the sacral vertebræ and the ilium. In this region they have the form of ventral pieces attached to the transverse processes. Finally, in long-tailed Mammals, the rudiments of ribs having the form of inferior arches occur. The double mode of union, which is generally seen in the case of the cervical ribs, obtains also in the thoracic ribs, but it is single in the case of the hinder ones. 


\section{Sternum.}

$\S 338$.

The Sternum forms the ventral portion of the framework of arches, which is formed by the ribs. It is developed from a rudi-

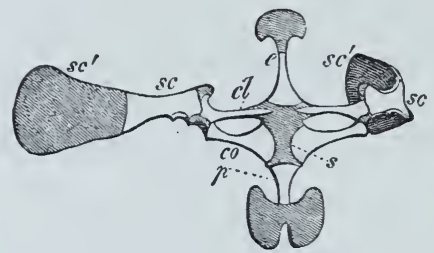

Fig. 232. Sternum and Shouldergirdle of Rana temporaria. $p$ Body of the Sternum. $s c$ Scapula. $s c^{\prime}$ Suprascapular. co Coracoid fused in the middle line with its fellow of the opposite side $(s)$. cl. Clavicle. e Episternum. The cartilaginous parts are shaded. represents the rudiment of the sternum; and the preservation of this rudiment is explained by the fact that it is connected with the shoulder-girdle. Thus in the Salamandrina it has the form of a

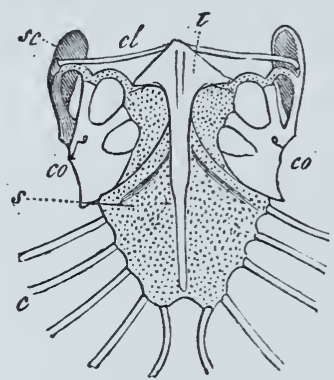

Fig. 233. Sternum and Shoulder-girdle of Uromastix spinipes. $s$ Sternal plate, which supports at its sides several pairs of ribs, and is provided posteriorly with two processes. sc Scapula. co Coracoid. $c l$ Clavicle. $t$ Episternum. The cartilaginous portions of the sternum and coracoids are dotted. ment similar to that of the ribs, as a band of cartilage, which connects together the proper ribs on each side. It appears, therefore, as a paired portion of the skeleton, and its later characters are due to its fusion along the middle line. We first meet with it in the Amphibia. We must therefore suppose that in these forms there was once a stage, in which the ribs were united by a sternum. Of this stage nothing has been retained, in addition to the rudiments of the ribs, except the part which broad thin plate of cartilage, in which there are deep grooves for the attachment of the coracoids. In the Anura (Fig. 232, $p$ ) it is placed at the hinder edge of the coracoids (co) which are united together in the middle line, and forms a partly ossified appendage to the shoulder-girdle, the binder end of which persists as a broad plate of cartilage.

The sternal plate of the Saurii and Crocodilini resembles the broader form of Amphibian sternum. It is generally rhomboidal in form, and has the same relations to the shoulder-girdle (Fig. $233, s)$. As a rule but few pairs of ribs are connected with the sternum, which frequently retains its cartilaginous condition (Fig. 233, s); at its hinder edge it gives off one or two processes, which also receive ribs. The paired condition of this second portion of the sternum must be regarded as a continuation of the embryonic arrangement.

The sternum of Birds is always ossified; it represents the more highly developed sternal plate of Reptiles, but the hinder portion is no longer developed. It has, moreover, but few pairs (no more than 
six) of ribs connected with it. In the Ratita it forms a broad piece of bone, which is very convex anteriorly. In the Carinatæ, however, it is distinguished by a projecting keel (Fig. 234, crs) on the anterior and convex face of the sternum, which serves to increase the surface from which the muscles take their origin. The form of the sternum is therefore correlated with the development of the muscles, while the size of the sternum and of its keel corresponds to the power of flight of which its possessor is capable. The hinder end often presents paired foramina, which are closed by membrane (Birds of Prey and Water Birds) ; when the periphery of these foramina is broken through towards the hinder edge of the sternum, clefts are formed, between

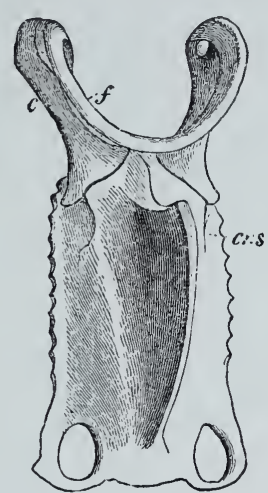

Fig. 234. Sternum of Buteo vulgaris (seen a little from one side). crs Crista sterni. $f$ Furcula. $c$ Coracoid.

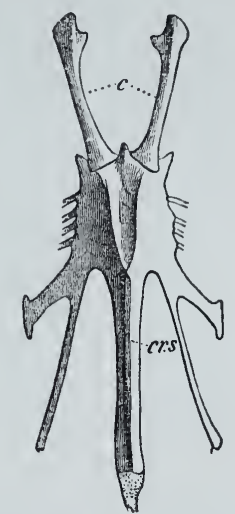

Fig. 235. Sternum of Numida meleagris (seen from in front). crs Crista sterni. $c$ Coracoid. which the so-called abdominal processes project (Fig. 235). The sternum of Birds has very much the same relations as in Reptiles, owing to its connection with the shoulder-girdle.

The sternum of Mammals is distinguished from that of the preceding classes by the large number of segments that are separately ossified in it. It is made up of bones, which are placed one behind the other, although they were all laid down in a continuous cartilage. They are not unfrequently developed from paired centres of ossification, and frequently fuse into one piece.

The form of the sternum is affected by its relations to the shoulder-girdle. The anterior piece is connected with the clavicles, and is distinguished by

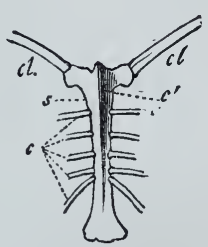

Fig. 236. Sternum of Vespertilio murina. $s$ Sternum. $c^{\prime}$ Crest. $c l$ Clavicle. $c$ Ribs.

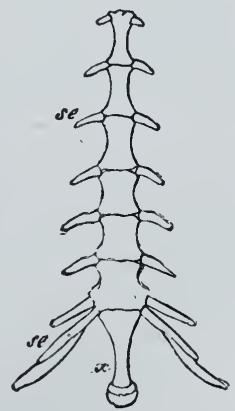

Fig. 237. Sternum of Cervus capreolus. se Costal cartilages. $x$ Xiphioid process. its greater breadth; it forms the manubrium. A ridge-like process (Fig. 236, $c^{\prime}$ ) is developed on the anterior face of this segment in aërial Mammalia ; this is physiologically similar to the keel of Birds. When the clavicles are absent, the anterior end of the sternum is generally much diminished in size. The hinder end is always drawn out into a median piece, which frequently remains cartilaginous (Fig. 237, x) (xiphioid process). 
A special piece, the episternum, is very commonly present in comnection with the sternum; this has one of two forms according to the way in which it is developed and connected.

In one the episternum is merely represented by bony structures, which are placed on the ventral face of the sternum. Thus, in the Reptilia, it has the appearance of a cross, or of a T-shaped bone (Fig. 233, $t$ ), the two arms of which support the clavicles, while the median piece is attached to, or even fused with, the sternum (Ascalabota). In the Crocodilini the transverse piece of the episternum and the clavicles are both wanting; as is the whole episternum in the Chamæleonidæ; nor is it present in Birds.

The second type of these structures is made up of skeletal parts, which are preformed in cartilage, and which lie in front of the sternum. There is a structure of this kind in the anourous Amphibia (Fig. 232, e), where it is represented by a bone, which is

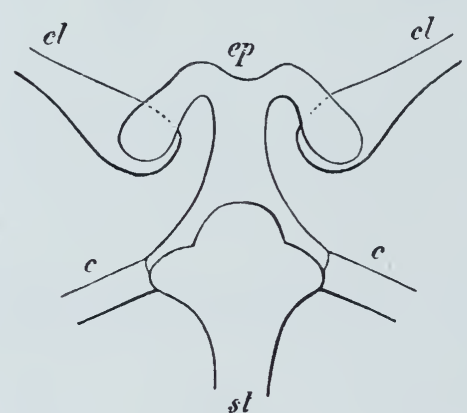

Fig. 238. Episternum, with the parts connected to it, of a young Opossum. st Anterior end of the sternum (ossified). ep Episternum (cartilaginous).

$c l$ Clavicle. $c$ The first two ribs.

or form bony parts, which are attached to the sternal end of the clavicle (Rodentia, Insectirora, Edentata). In the Primates these episternal structures form the intermediate cartilages of the articulation of the sternum with the clavicle.

Gegrinbaur, C., Ueber die episternalen Skelettheile und ihr Vorkommen bei den Säugethieren und beim Menschen. Jen. Zeitschr. I.-PARKER, W. K., Structure and development of the Shoulder-girdle and Sternum. Ray Soc. 1868.

\section{Cephalic Skeleton.}

$\S 339$.

The indifferent stage in which the head is found in the Acrania makes it impossible to distinguish any distinct cephalic skeleton. It is, however, true that the head of the Craniota is not an absolutely 
new structure, and to the same extent the cephalic skeleton is not new. If the anterior respiratory portion of the body in Amphioxus corresponds potentially to the head of the Craniota, then the skeletal parts in it must be potentially homologous with a cephalic skeleton. This applies to that region of the notochord, and to the tissue derived from it, which encloses the anterior portion of the central nervous system, as well as to the framework of the respiratory cavity.

In the Craniota this anterior portion of the body is different on the dorsal, as well as on the ventral, surface from what is behind it; owing to the changes which take place in its functional importance, in consequence of its relations to a large number of other organs, it gets to differ in many special points, owing to the existence of which it can be distinguished as the head; and it consequently becomes of greater importance than the rest of the body. It stands in relation to the entrance of the alimentary canal ; it contains the most important of the sensory organs; and affords a shelter to that portion of the central nervous system which is developed into the brain. These relations are, moreover, the cause of the metamorphosis which affects it.

In the skeleton of the head we may distinguish-1) the Skull, and 2) the Branchial skeleton.

1) The Skull (cranium) is the name given to that part which is a continuation of the dorsal column, and is connected with the axial skeleton. It has many things in common with the former, for it corresponds to a number of vertebral centra and upper arches. This similarity is not only implied in its texture, but also in its structural relations, and in its relations to the central and peripheral nervous system. The chorda dorsalis is continued into the basal portion of the cranium, where it may either remain permanently, or disappear after a short time. With increased development of the higher sensory organs the cranium becomes of more importance. A hinder segment encloses on either side the auditory organ, and may be distinguished as the auditory capsule. Going forward, there is, on either side, a depression which affords shelter for the eyes (orbit), while in the most anterior part there are cavities for the reception of the olfactory organ. The primitive condition of this cranium is cartilaginous ; it forms the "primordial cranium."

2) With this cartilaginous skull there is connected, at the commencement of the alimentary canal, a system of arches, which is likewise primitively cartilaginous; these branchial arches are, on the whole, similar to the ribs of the vertebral column, but at the same time they are not altogether homodynamous with ribs. The various arches diffe: in form, but they all give indications of having been primitively similar. Their difference in form is due to a differentiation which has been brought about by adaptation to different conditions. 


\section{$\S 340$.}

The relations between the cephalic skeleton and the vertebral column were, in past times, the cause of attempts to show that the former was made up of various segments resembling vertebræ; from this point of view the cephalic skeleton seemed to be merely a modification of the vertebral column. It was indeed believed that the characters of the various segments of the osseous cranium would be of aid in this comparison; as a fact, however, these are of very uncertain value, as in dealing with them we have to do with a condition which has already been much modified. In other words, the cephalic bones which have been said to belong to three, four, or five so-called "cranial vertebræ," have had very different origins, and are for the most part structures which primitively did not belong to the cranium.

The examination of the primordial cranium of the lower Vertebrata, especially with reference to the nerves which pass out from it, shows that, although indications of its primitive composition out of metameres, homodynamous with vertebræ, can be made out, as might be supposed indeed, yet this metamerism of the cranium is not the same as that segmentation which is faintly indicated by the bony cranium.

The view here adopted is chiefly based on the following points:

1) It can be shown that the arches of the branchial skeleton form inferior arches which belong to the cranium.

2) It follows that a general agreement may be made out between the visceral arches and the inferior arches of the vertebral column.

3) The cranium is comparable to a portion of the vertebral column, which contains, at least, as many vertebral segments as there are branchial arches.

4) In the cranium itself there are a number of important points in which it resembles the vertebral column.

a) The chorda dorsalis which forms the base-work of the vertebral column passes through the cranium in just the same way as it passes through the vertebral column.

7) All the nerves which pass out in this segment are homodynamous with the spinal nerves.

c) The differences seen in the cranium, as compared with the vertebral column, may be explained as adaptations to certain conditions, which are external to the cranium; that is, as acquired arrangements. They lead us, therefore, to presuppose a condition, in which the cranium had not yet acquired these peculiarities, and when therefore it did not differ very greatly from the vertebral column.

5) The differentiation of the cranium appears therefore to be due to the concrescence of a number of vertebræ: such 
concrescence may also obtain in the vertebral column. Modifications in the region, which thus became continuous, were brought about partly by influences which affected it directly from without, and partly by influences which affected it from within (the development of the brain).

6) Inasmuch as those nerves only can be seen to have any likeness to spinal nerves which are found in that division of the cranium which is traversed by the notochord, it follows that this portion only can be derived from vertebræ; the branchial skeleton also belongs to this portion. We must therefore distinguish between this portion of the cranium which is vertebral, and the anterior, or evertebral, portion, which does not exhibit any relations to the vertebræ. This latter is rather a secondary formation, although it has had its origin in the vertebral portion.

T'he number of vertebræ, which enter into the composition of the cranium, are, so far as we yet know, nine at least. But this is by no means saying that the number may not have been much larger. Such a supposition is borne out by a large number of facts which are known to us with regard to the distribution and mode of origin of the nerves in the Selachii ; and these point also to the atrophy of visceral arches. The arrangement in Amphioxus is just as much to the point, for in it a large number of branchial arches are persistent. All that portion of the primitive spinal column (notochord and perichordal tissue), which extends along the branchial framework, of Amphioxus would then be homologous with that portion of the axial skeleton which, in the Craniota, has passed into the cranium.

Gegenbaur, C., Untersuchungen zur vergl. Anat. der Wirbelthiere. III. Das Kopfskelet der Selachier als Grundlage zur Beurtheilung der Genese des Kopfskeletes der Wirbelthiere. Leipzig, 1872.

\section{Skull.}

$\S 341$.

The skulls of the Craniota are divided into two very different groups. In the one the above-mentioned internal branchial skeleton is well developed, and its most anterior segments take on the form of maxillary organs, and affect the form of the cranium by being either directly, or indirectly, connected with it. This arrangement obtains in the Gnathostomata. The other form is represented in the Cyclostomata, in which it is possible to make out indications of the same arrangements in the cephalic skeleton as those which are seen in the Gnathostomata; but the differences are so great, that it is impossible to compare all the parts with anything like exactness.

The notochord is continued into the base of a capsule which encloses the brain, and which is, in comparison with other parts of the skeleton which are to be regarded as belonging to the skull, of a remarkably small size. In Petromyzon two enlargements which 
contain the auditory organ (auditory capsules) $(f)$ are attached to the sides of this capsule (Fig. 239, $l$ ), and below them two divergent

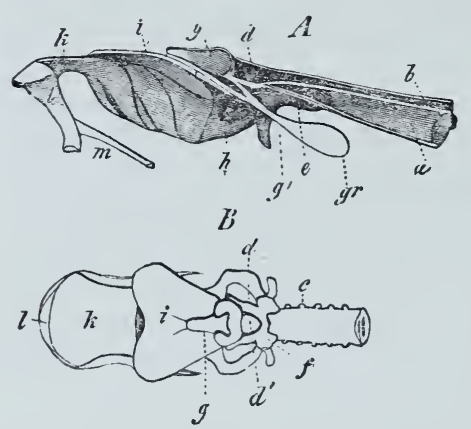

Fig. 239. Skull and commencement of the vertebral column of Petromyzon marinus. A Median section. $B$ Seen from above. a Notochord. $b$ Spinal caual. $c$ Rudiments of the vertebral arches. $d$ Cartilaginous cranial cavity. $d^{\prime}$ Membranous por. tion of the cranial cavity. $e$ Basis cranii. $f$ Auditory capsule. $g$ Nasal capsule. $g^{\prime}$ Palato-nasal passage. $g r$ Its blind end. $h$ Continuation of the bony palate. $i$ Posterior cover. ing-plate of the mouth. $k$ Anterior covering-plate. $l$ Labial ring. $m$ Its appendage (after J. Miiller).

forms the lower jaw, and is always freely movable (Gnathostomata).

This arch is differentiated into two pieces, which function as jaws; an upper one, the palato-quadrate, and a lower one, the

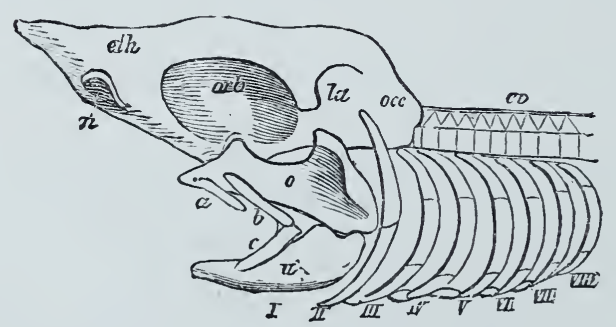

Fig. 240. Skull and visceral skeleton of a S elachian (Diagram). occ Occipital region. la Wall of the labyrinth. eth Ethmoidal region. $n$ Nasal pit. $a$ First, $b c$ Second labial cartilage. o Superior, $n$ Inferior portion of the mandibular arch $I$. II Hy. oid arch. III-VIII (1-6) Branchial arches. cartilaginous lower jaw. The palato-quadrate is articulated with the base of the skull; but it is enlarged horizontally backwards, and is connected with the second arch also, the upper portion of which is movably articulated with the skull. The hyoid forms the lower portion of this second arch. As this upper portion of the second arch is often greatly developed, it gets the appearance of a supporting apparatus for the two primitive jaw parts, which are developed from the first arch, and is known as the hyomandibular. The rudiments of other 
arches are embedded in the upper and lower lip, in front of the mandibular arch ; they are the labial cartilages.

The parts, therefore, which are derived from the branchial skeleton and enter into closer relation with the skull are these:

1) The two labial cartilages (Fig. 240, $a$ and $b c$ ) ; the anterior one consists of one, the posterior of two pieces.

2) The mandibular arch $(I)$, which consists of a superior piecepalato-quadrate $(o)$, and of an inferior piece-lower jaw $(u)$.

3) The hyoid arch $(I I)$; of this the upper portion only (hyomandibular) has any intimate relations to the skull.

We find various cartilaginous rods attached to all the arches, except the labial cartilages; these are directed backwards, and afford support for the branchial pouches; they are known as branchial rays. They undergo various modifications; they are diminished in number on the palato-quadrate, but may be seen in the wall of the spiracular cleft, which represents a rudimentary branchial pouch (spiracular cartilage).

We shall treat of these portions of the branchial skeleton with the skull; the other arches $(I I I-V I I I)$ will be considered later on (\$ 353).

The arrangement we have described may be seen in the cephalic skeleton of the Selachii. All its parts are formed of cartilage, which is, as a rule, covered over by a thin calcified layer, which however is never ossified. In the cartilaginous cranial capsule the most anterior portion is formed by the ethmoidal region. On its inferior surface there is a nasal pit $(n)$ on either side. The cranial cartilage is frequently continued forwards, and between these pits, into a process (rostrum). The succeeding portion, with its cavities, forms the orbits, which is overhung by cartilage above and behind. The next portion $(l a)$ is the broadest; at the sides this encloses the auditory labyrinth, and is continuous at its hinder surface with the last of the primary regions (occ); in some Sharks (Notidani) this hindermost region is continuous with the vertebral column.

Teeth are developed in the mucous membrane which covers the palato-quadrate, as well as in that which covers the lower jaw; and this explains why these cartilages are so well developed. The hyomandibular is attached posteriorly to the palato-quadrate, and is either directly continuous with the lower part of the hyoid arch (Notidani), or is more freely attached to it. The hyomandibular is consequently greatly developed in the Sharks, and is converted into a kind of suspensorium, by gradually gaining a closer connection with the lower jaw. Finally the hyoid portion ceases to appear to be a continuation of the hyomandibular; in the Rays, indeed, it loses all connection with it, and the hyomandibular is then merely the support of the jaws.

The skull of the Chimæræ is very different to this, for in it there is a continuous connection between the palato-quadrate portion of the first arch and the cartilaginous cranium. The mandibular portion, which is articulated with a process of the cranium, is the 
only part of the first arch which is movable. The partly ossified skull of the Dipnoï has just the same characters.

\section{$\S 342$.}

The cartilaginous cranium is most completely retained in the Sturiones (Fig. 241); in the rest of the Ganoïdei it is partly so ; in the Teleostei, and especially in Salmo and Esox, it is more or less so. In most forms the ethmoidal portion remains cartilaginous. Henceforward, and all through the Vertebrate phylum, a cartilaginous cranium can be made out in the earliest rudiments of the skull; howsoever modified, it may be derived from the primitive condition, and may therefore be regarded as a remnant of that condition.

The degenerations which affect this primordial cranium are chiefly due to the ossifications which take place in it. Bony parts, which perform the function of organs of support and protection, better than cartilage does, take its place; and the development of these bones,

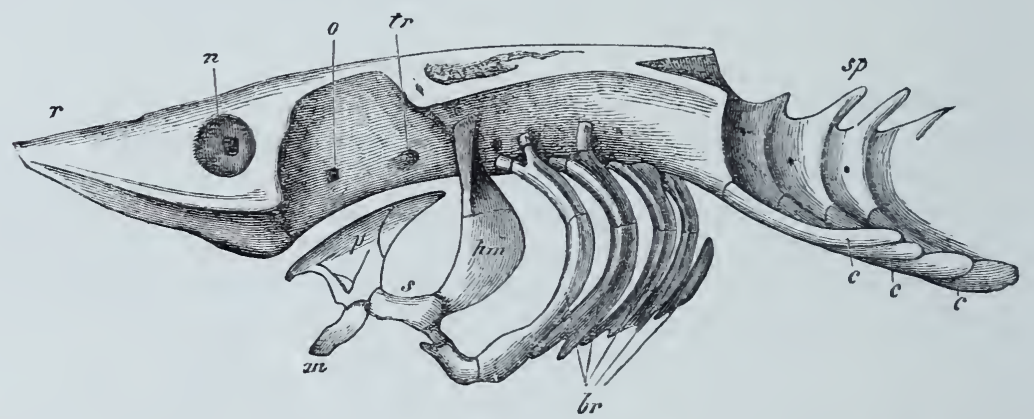

Fig. 241. Cephalic skeleton of Acipenser sturio; after the removal of the corering bones. $r$ Rostrum. $n$ Nasal cavity. o Optic foramen. $t r$ Trigeminal foramen. $s p$ Spinous processes of the anterior portion of the vertebral column, which is fused with the cranium. $p$ Palato-quadrate. $m$ Mandible. Hm. Hyomandibular. $s$ Sym. plectic. $b r$ Branchial arches. $r$ Ribs.

which enter into connection with the cartilaginous cranium, is clearly the cause of the degeneration of the cartilaginous tissue. A higher and more complete arrangement has ousted the lower one.

Bony pieces become connected with the cartilaginous pieces of the branchial skeleton, as well as with the cartilaginous cranium; in this way the whole cephalic skeleton gradually passes from a cartilaginous into an osseous condition. The osseous elements which go to make it up are almost all derived from the dermal structures, which we met with as dermal denticles in the Selachii. Some of these bones appear on the outer surface of the cartilaginous cranium, and form the covering bones of the skull (cf. suprà, §325). They are plates which are formed in the integument, and owe their origin to the growth of placoid scales; in the Sturiones, already a number of them have taken up the same position as that which they retain in the higher divisions. 
Others are formed in the mucous membrane of the buccal cavity, that is, in those parts of the cartilaginous arches of the mandibular and branchial skeleton which aid in forming the boundaries of this cavity. The origin of these bones may be seen, in the Amphibia, to be due to the concrescence of tooth-like structures, which are, indeed, similar to the dermal denticles, and which are also found on the covering of the buccal and branchial cavities in the Selachii.

Dentigerous plates are formed by the fusion of some of these denticles, and these afford points of support for the necessary primordial skeletal deposits, and gradually become connected with them. In some of these plates the denticles are retained; in others they disappear, or are no longer developed, so that their product - that is, the bone - is alone developed; and this, by investing or growing around the cartilage, forms a perichondrial ossification. The origin, therefore, of a large part of the osseous ce-
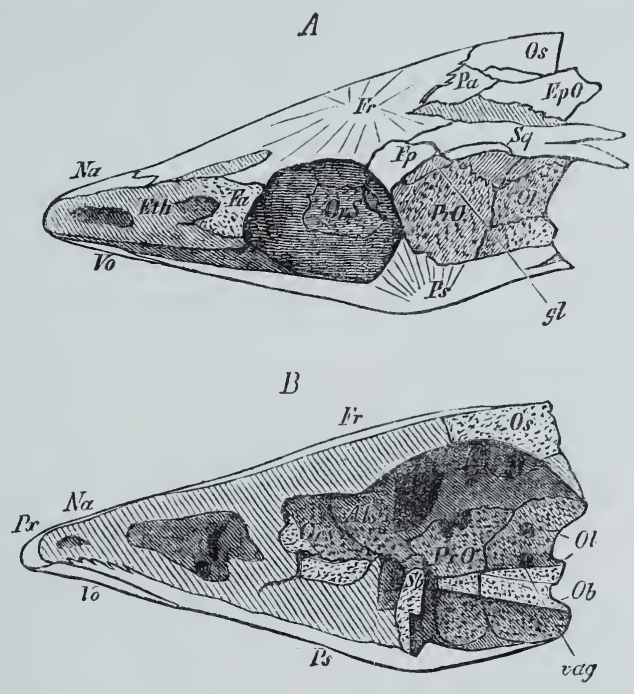

Fig. 242. Skull of Salmo salar. $A$ Side view. $B$ Vertical median section. The cartilaginous parts are hatched, while the bones derived from the primordial cranium are dotted. Ob Basi-occipital. Ol Exoccipital. Os Supra-occipital. Sq Squa. mosal. Ep O Epiotic. $\operatorname{Pr} O$ Petrosal. Sb Basi. sphenoid. Als Alisphenoid. Or S Orbito-sphenoid. Fa Prefrontal. Fp Postfrontal. Fr Frontal. $\mathrm{Na}$ Nasal. Ps Parasphenoid. Vo Vomer. Pre Pre. maxilla. gl Articular surface for the Hyoman. dibular. Eth Ethmoidal cartilage. vag Foramen for the exit of the vagus. phalic skeleton can be explained by the relations, which the ossifications of the external integument, and of the investment of the buccal cavity, have to it. The phylogeny, however, of a few of the cephalic bones is as yet unknown.

HeRTwIG, O., Ueber das Zahnsystem der Amphibien. Arch. f. mikr. Anat. Bd. XI. Supplement.

\section{$\S 343$.}

With regard to the various bones, we divide the primordial cranium into the regions which we have already distinguished. The occipital region is made up of four bones. The basi-occipital is a direct continuation of the centra of the vertebræ (Fig. 242, Ob). In its hinder portion there is a cavity which is filled by the noto- 
chord, and which corresponds to the anterior cavity of the first vertebral centrum. At its sides the exoccipitals $(O l)$ are attached to it; these always enclose the greater part of, and sometimes nearly all, the posterior foramen magnum. The supra-occipital $(\mathrm{Os})$ enters into it above; this is often distinguished by a large vertical process, which resembles the spinous processes of the vertebral column.

The next segment partly, at any rate, encloses the labyrinth, and the bones which compose it are named in accordance with this relation. The most constant, and therefore the most important, is the petrosal (pro-otic) ; this contains, or, at least, limits posteriorly, the foramen of exit of the trigeminal nerve. It extends to the base of the skull, where it may become united with its fellow of the opposite side, within the cavity of the skull. The epiotic forms a second piece; this is attached above to the exoccipitals, and generally forms a projecting process (Fig. 243). A third one, the opisthotic (inter-

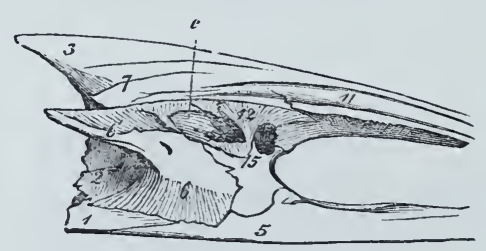

Fig. 243. Posterior portion of a cranium of Gad us (side view). 1 Basi-occipital. 2 Exoccipital. 3 Supra-occipital. 5 Parasphenoid. 6 Opisthotic. $6^{\prime}$ Squamosal. 7 Epiotic. 15 Prootic. 12 Postfrontal. 11 Frontal. $c$ Articular surface for the hyomandibular. calare), generally lies to the sides and front of the exoccipital, and varies greatly in character (Figs. 243-6). As a rule this piece has no relations to the labyrinth, with which, however, other bones, such as the exoccipital, very frequently become connected. Lastly, an external covering piece of the primordial cranium belongs to this region, and gradually becomes closely united with it. It takes part in the formation of the articulation for the hyomandibular, and often develops a process which projects backwards and outwards. This is the squamosal (Figs. 242, $A$ Sq; 243, 6).

Great differences may be seen to obtain in the development of the bones of the next portion, which depend on the size of the carity of the skull. When this extends far forwards, the walls of the primordial cranium are correspondingly more complete, while, when it is reduced in size, its walls are diminished, and are partly replaced by membranous structures. Thus, in many cases, there is a membranous interorbital septum; or bones, which are well developed in other forms, are present in a rudimentary condition.

The ossifications of this segment are the alisphenoid (sphenoïdale laterale posterius) at the sides and behind, and the orbitosphenoid (sphen. later. anter.) in front. In Amia, as in many Teleostei, the latter are separated from one another, while in other forms the pieces of either side unite at the base of the cranial cavity, and at last may fuse into one piece, or may be rudimentary. At the base of this segment there is a basi-sphenoid, which is derived from the cartilage of the primordial cranium; this is, as a rule, a bone of no great size; it is connected superiorly with the alisphenoid. When the base of the skull is traversed by a canal for the optic muscles, 
which passes obliquely backwards from the orbit, this bone forms a pillar between the canals of either side. Not unfrequently it seems to disappear altogether. On the ventral side of, and along the primordial cranium there extends the large parasphenoid (Figs. 242, Ps ; $243,5)$, which has been already seen in the Sturiones.

It is seldom that the whole of the primordial cranium is retained on the upper surface; as a rule, there are spaces in it, which are overlaid by covering bones. Nearest to the posterior primary region there are the two parietals (Fig. 244, 7); these are sometimes separated from one another by an anterior process of the supra-occipital (3). In front of them there are the frontals, which are often replaced by a united frontal (11). At the sides of this are the two postfrontals (12), which extend as far as the squamosal, and take part in the articulation of the hyomandibular.

In the ethmoidal region there is a median piece, ethmoïdale medium (16), to the sides of which the lateral ethmoids are attached (14) (frontalia anteriora. Cuvier). The latter form the base of the nasal capsules. The median portion of the ethmoids is often permanently cartilaginous. The vomer forms the covering piece of the base of the ethmoidal region; posteriorly this is united to the parasphenoid; it is paired in Lepidosteus.

\section{$\S 344$.}

The mandibular apparatus of the Selachii is not completely retained in the Ganoïdei and Teleostei, owing to the presence of bony structures in its place. It is complicated by the union of the hyomandibular with the bones formed from the palato-quadrate cartilage. The primitive relations, as they are seen in the

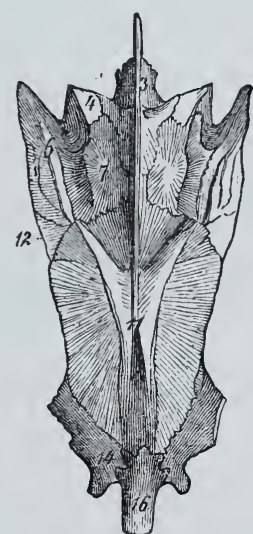

Fig. 244. Skull of a Gadus from above. 3 Supra-occipital. 4 Epiotic. 6 Squamosal. 7 Parietal. 11 Frontal. 12 Postfrontal. 14 La. teral ethmoid. $16 \mathrm{Me}$. dian ethmoid. embryonic condition, show that it has been derived from the arrangements which obtain in the Selachii. While the anterior ends of the right and left palato-quadrate bones are connected by ligament in the Selachii and Sturiones, in the rest of the Ganoïdei and in the Teleostei they are attached to the sides of the primordial cranium, and are separated from one another by the ethmoidal region, the basal surface of which enters into the formation of the boundaries of the buccal cavity.

The hyomandibular (Fig. 245, $\mathrm{Hm}$ ) is almost always a large bone, which articulates with the squamosal and postfrontal. The symplectic (Fig. 241, s) is segmented off from it; in the Selachii this is represented by a process, but in the Sturiones it is an independent piece; the lower portion of the hyoid arch is inserted at the point where the symplectic is connected with the hyomandibular. 
The quadrate $(Q)$ is segmented off from the palato-quadrate cartilage ; it carries the glenoid cavity for the mandible. The angulated ectopterygoid (Ept) is attached to the front of the quadrate; and between it and the hyomandibular there is the flattened and ordinarily quadrangular metapterygoid $(M t)$. The entopterygoid is nearer the middle line than the ectopterygoid; finally the palatine forms the most anterior end of the palato-quadrate cartilage ; it is generally movably connected with the skull.

In front of the palatine there are two other bones, which are not preformed in cartilage; the hinder one, which is generally attached to the palatine, is called the maxilla (Fig. 245, $M x$ ), and the anterior one the premaxilla $\left(P_{x}\right)$. They appear as new pieces, which henceforward play an important part. But it is exceedingly probable that they have their origin in the two upper labial cartilages of the

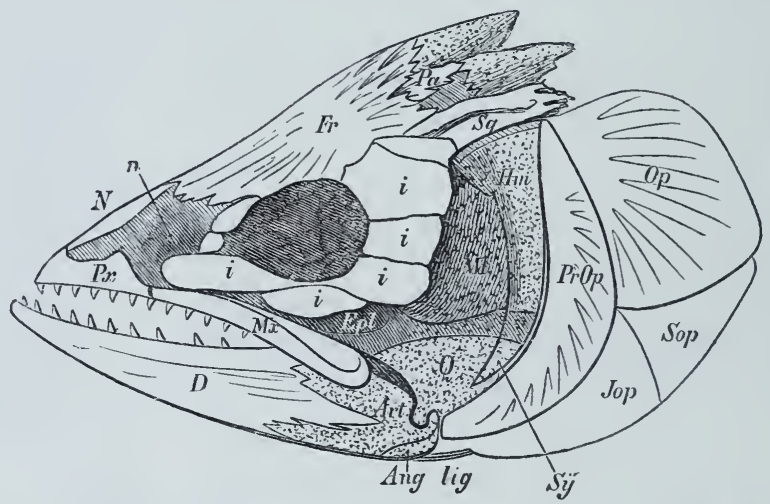

Fig. 245. Side view of the cephalic skeleton of Salmo salar (cf. Fig. 24:2, A). Fr Frontal. $N$ Nasal. $n$ Nasal pit. $P a$ Parietal. Sq. Squamosal. $i$ i $i$ infra. orbital bony ring. Hm Hyomandibular. Sy Symplectic (shown as if it were visible from the exterior). IIt Metapterygoid. Ept Ectopterygoid. Q Quadrate. Max Maxilla. Pre Premaxilla. Art Articular. Ang Angular. $D$ Dentary. Op Operculum. PrOp Preoperculum. Sop Suboperculum. Jop Interoperculum. lig Ligament.

Selachii. Sometimes they are frecly movable, and even protrusible; in other cases they are more firmly connected with the skull. This is especially the case with the premaxilla, which is frequently united firmly to the most anterior portion of the ethmoidal region. They both bound the orifice of the gape, but when the premaxillæ are increased in length, the maxillæ may be shut out from any share in it; while conversely, when the premaxillæ are shortened, the maxillæ acquire a larger share in this relation to the mouth.

In the lower jaw the cartilaginous rudiment, or Meckel's cartilage, is very completely retained. It gives rise to the dentary $(D)$, which, as it were, ensheaths the cartilage. The articular $(A r t)$ is formed from the condylar portion of the cartilage, and below it there is the angular $(A n g)$. On the inner face of the bony lower jaw a special bone is sometimes developed as a covering-piece for the cartilage ; this is the opercular. 


\section{$\S 345$.}

'I'he skeleton of the operculum is one of the most important of the skeletal parts which are connected with, although they did not primitively belong to, the mandibular apparatus. In the Selachii there are cartilaginous pieces, which are sometimes bifurcated, in the place of this bony skeleton; these are set on both segments of the hyoid arch, where they form branchial rays. This cartilage is covered in by a common membrane, as is also the bony apparatus; the membrane is adapted to this latter, and converts it into a defensive arrangement, which extends over the postjacent branchial clefts.

In the Sturiones the first to appear is the largest of these bones - the operculum; and to it, in the rest of the Ganoids, and in the Teleostei, the other bones are attached (Fig. 245). The preoperculum $(\operatorname{PrOP})$ is developed in the cartilage which connects together the hyomandibular and symplectic. It is often more closely united to the constituent parts of the suspensorium. Behind the preoperculum is the suboperculum (Sop), which is present, in addition to the operculum, in Ceratodus; and then below it there is the interoperculum $\left(J_{o p}\right)$, which is connected with the lower jaw by a ligament (lig).

Accessory bones are formed from other pieces, which are developed from parts of the dermal skeleton; the most important of these are the infraorbital bones (cf. Fig. 245, $i$ i $i$ ). They form a curved series around the lower edge of the orbit; the hindermost piece is attached to the postfrontal, and the foremost to the lateral ethmoid. Some of these bones acquire a considerable size in the Cataphracta (Trigla).

The nasals also belong to this series of bones, in consequence of their inconstant presence; there are also many other pieces that are connected with the so-called mucous canal system, and which are modifications of scales.

Vrolik, A. J., Ueber die Verknöcherung u. die Knochen des Schädels der Teleostei. Niederländ. Archiv f. Zoologie. I.-PARkER, W. K. Develops ment of the Skull in the Salmon. Philos. Transact. 1873.

\section{$\S 346$.}

In the skull of the Amphibia the primordial cranium is somes times greatly developed. It very frequently, however, loses its roof, and also its floor, owing to the formation of spaces in the cartilage.

The palato-quadrate is directly connected with the primordial cranium; it is attached posteriorly to the auditory capsule of the skull, while anteriorly it forms an arch around the orbits, and either projects freely forwards (as in the Urodela), or is connected with the cranium in the ethmoidal region. Behind, and at the sides, it carries the glenoid cavity. It presents therefore those relations which were seen in the Chimæræ, and in the Dipnoï; and there are, 
indeed, many ossifications in the cranium of the Amphibia which resemble those in the Dipnoï.

The primordial cranium gives rise to a few bones only. In the posterior primary region the exoccipitals alone are present (Fig. 246); each of these gives rise to a condyle $(c o)$. The next region of the auditory capsule presents large lateral processes, which are attached more exteriorly to the hinder portion of the palato-quadrate. The anterior portion of this segment is provided with an ossification, the prootic. This contains the anterior portion only of the labyrinth, the hinder portion of which is contained in the exoccipital; it also forms a foramen for the trigeminus. There are sometimes indica-
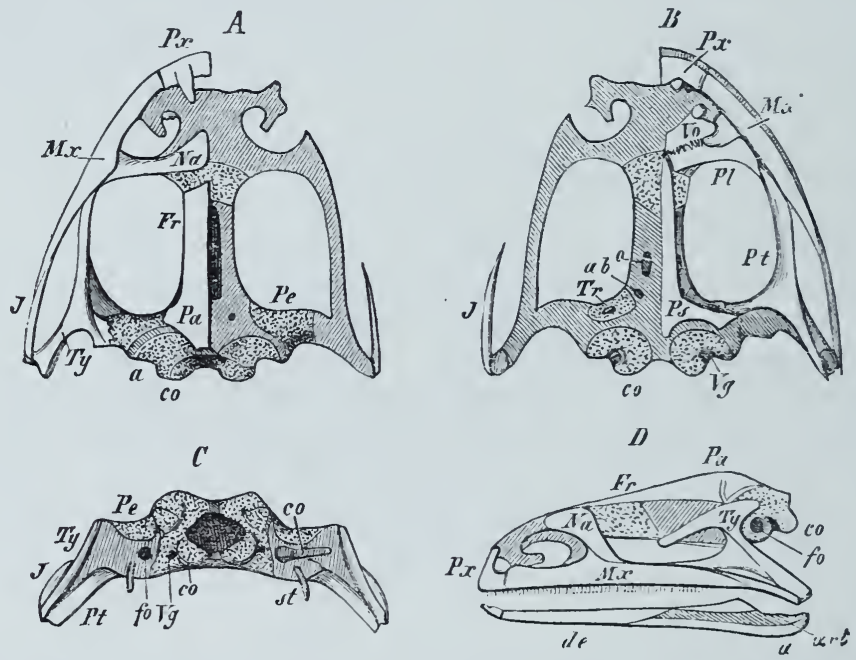

Fig. 246. Skull of the Frog. $A$ from above. $B$ from below. $C$ from behind. $D$ from the side. In $A$ and $B$ the covering bones are removed from the right half of the cranium, so that the whole of the primordial cranium and its ossifications can be seen, and, in $A$, the spaces in the roof of the cranial cavity. $\mathrm{Pa} \mathrm{Fr}$. Fronto-parietals. $\mathrm{Na}$ Nasal. Ps Parasphenoid. Ty Tympanic. Pt Pterygoid. Pl Palatine. Vo Vomer. $J$ Jugal. $M x$ Maxilla. $P_{x}$ Premaxilla. o Exoccipital. $P e$ Prootic. co Occipital condyle. Co Columella. fo Fenestra ovalis. Exits of the nerves : $O$ Optic. Tr Trigeminus. $V g$ Vagus. In the lower jaw: $d a$ Dentary. a Angular. Ait Articular.

tions of an epiotic. The fenestra ovalis is a cleft in the region of the labyrinth, which is covered over by a small piece of bone.

In the anterior portion of the orbital region there are sometimes more or less extensive ossifications. They either embrace the lateral wall only of the cranium (Siredon), or form a circular piece of bone, to which Cuvier gave the name of "girdle-bone." This bone may extend into the ethmoidal region, and even reach to the base of the nasal capsules.

The paired parietals and frontals are covering bones. In the Anura these fuse together on either side to form a fronto-parietal $(P a F r)$. In front of these, and separated from one another by the frontals, are the nasals, which we now meet with as constant 
bones for the first time. At the base of the skull is the parasphenoid $(P s)$, which still retains very much the same characters as it had in Fishes, and in front of this, and in the ethmoidal region, there is a paired bone (vo), which is regarded as the vomer.

The palato-quadrate is more simple in character than in Fishes. The whole piece sometimes remains for the most part in a cartilaginous condition. An ossification at the point where it articulates with the lower jaw corresponds to the quadrate of Fishes. In many, the palato-quadrate is divided into an anterior and a posterior portion (Triton). It is not completely united with the cranium, for there is a distinct articular surface on its lower portion, between it and the cranial capsule (Rana).

There are two covering bones on the palato-quadrate cartilage ; the upper one (Ty) is distinguished, in the Frog, by the possession of a strong process, which is directed forwards, and which probably, though by no means certainly, corresponds to the squamosal of Fishes. As it aids in supporting the tympanum, it may be called the tympanic. The lower bone is the pterygoid $(P t)$, and it extends forwards along the cartilaginous arch. Its anterior end reaches as far as the palatine, which lies transversely behind the vomer $(P l)$. In some of the Amphibia another bone is continued forwards in front of the glenoid cavity; this is the so-called jugal (quadratojugal).

The premaxillæ $\left(P_{x}\right)$ and maxilla $\left(M_{x}\right)$ appear as covering bones of the primordial cranium; relations which obtain in many Fishes lead to this condition. The maxilla varies greatly in the extent to which it is developed laterally; in the Anura it ordinarily extends as far back as the jugal. The premaxilla is connected with the primordial cranium by a process which projects forwards in the middle of the nasal region.

These maxillary bones did not primitively bound the opening of the mouth, as the presence of special cartilages (rostral and adrostral) in front of the primordial cranium of the larvæ of Anura distinctly shows.

In the lower jaw the primordial cartilage is present, as in Fishes, and bony parts are developed in connection with it, which essentially correspond to those of Fishes.

Parker, W. K., Development of the Skull in the Frog. Philos. Trans. 1871.Wiedershejm, R., Das Kopfskelet der Urodelen. Morphol. Jahrb. III.

\section{$\S 347$.}

The skulls of the Sauropsida have much in common with one another, whilst they are far removed from the skulls of the Amphibia, and from those of the Mammalia.

The primordial cranium, as a rule, has the roof incomplete, but it is much more completely ossified than in the Amphibia, while the great size of the bones which are developed in, and from, the palatoquadrate cartilage leaves but a small portion of the true cranium 
visible. The greater development of the cranial capsule in Birds is the cause of its parts being more distinctly visible than they are in Reptiles.

In the occipital region we can make out the four bones which were present already in Fishes. Of these, the basi-occipital and the exoccipitals take part in the formation of a single occipital condyle. The relation that the bones have to the foramen magnum varies a good deal. In the Chelonii the supra-occipital is continued into a

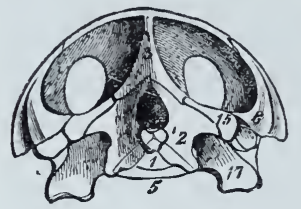

Fig. 247. Skull of a Chelonian seen from behind. 1 Basi-occipital. 2 Exoccipital. 3 Supraoccipital. 5 Basi-sphe. noid. 15 Prootic. 17 Quadrate. large crest. As in the Amphibia there is a fenestra ovalis in the osseous auditory capsule; and in addition to it there is a fenestra rotunda, which is closed by membrane. In all Birds and Reptiles the petrosal (prootic) lies in front of the exoccipital; its anterior edge is distinguished by the foramen for the third branch of the trigeminal. Another bone (opisthotic) unites with the prootic to form the boundary of the hinder portion of the fenestra ovalis; but it does not remain separate in any but the Chelonii, as in other forms it fuses with the exoccipital. In addition to these, there are several, and in Birds numerous, ossifications which are independent for a short time; these cannot be definitely regarded as similar to any distinct cranial bones in the rest of the Vertebrata. In Birds all the constituents of the auditory capsule fuse with the neighbouring bones, as well as with one another.

In the Ophidii the squamosal (Fig. 249, $C S q$ ) is a projecting bone, which carries the quadrate. In other Reptiles, and in Birds it has the same position, but is more embedded between the auditory capsule, parietals, and post-frontal, and is partly set in the roof of the tympanic cavity.

The sphenoidal segment varies in size according to the extent of the cranial cavity. A basi-sphenoid is generally present, as is also the presphenoid, which is ordinarily small ; the parasphenoid is no longer developed. Two bones, however, which are seen at the base of the temporal region in Birds, and which fuse with one another (basitemporals), may be regarded as parts of a parasphenoid. In Birds there is an alisphenoid, and also an orbito-sphenoid-in the Ratitæ, at least-which represent the side-walls of the skull. The Crocodilini, also, are provided with an alisphenoid. In most of the Saurii, however, there is an interorbital membranous septum, in which indications merely of this bone can be made out.

In the Saurii (Lacerta, Varanus, Podinema) there is a bone which extends from the parietal to the pterygoid (columella) (Fig. 248, $A$ co) ; in the Chelonii this is represented by a broad plate of bone, which passes straight down from the parietal, and which takes a share in the limitation of the cranial cavity; in the Ophidii there is a similar process, which embraces the cranial cavity, and extends on to the frontal. 
The covering bones are: the parietals, which are paired (Chelonii and Aves), or unpaired (Ophidii, Saurii, Crocodilini) (Fig. 248, $P a)$. The frontal also is unpaired in most of the Saurii, and in the Crocodilini (Fig. 248, $B F r$ ). It is paired in Lacerta and Monitor (Fig. 248, $A F r)$, and in the Ophidii, Chelonii, and Aves. In the Reptilia the postfrontals limit the posterior edge of the orbit (Figs. 248, $P f$; 249, $B C P f)$.

In the middle of the ethmoidal region there is a considerable remnant of the primordial cranium (Chelonii). The lateral ethmoids (prefrontals) bound the anterior edge of the orbit in Reptiles; in Birds they appear to be connected with the median portion of the ethmoid. The vomer is paired in the Ophidii and Saurii (Fig. 250, Vo). The nasals are almost always absent from its upper surface in the Chelonii, and in some of the Saurii. A new covering bone
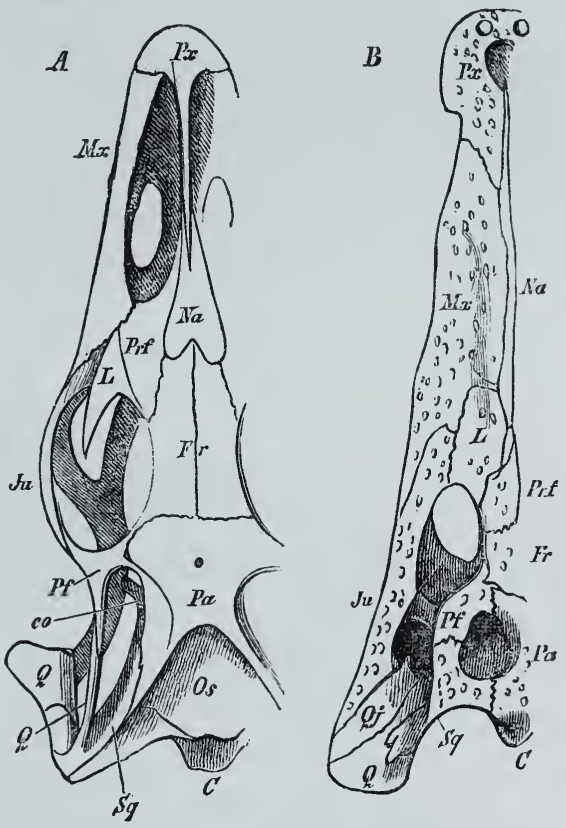

Fig. 248. Skull of Reptilia; seen from above. $A$ Monitor. $B$ Crocodile. Os Supraoccipital. C Occipital Condyle. $P a$ Parietal. Pf Postfrontal. Fr Frontal. Prf Pre-frontal. $L$ Lachrymal. $N$ Nasal. $S q$ Squamosal. Qj Quadratojugal. Ju Jugal. Q Quadrate. $M x$ Maxilla. $P x$ Premaxilla. co Columella. which is seen on the outer

face of the ethmoidal capsule is the lachrymal; it is found in most Saurii, Crocodilini, and Aves (Figs. 248, 249, L).

\section{$\S 348$.}

The anterior portion of the primitive palato-quadrate cartilage undergoes atrophy very early, so that the bones which belong to it are partly developed on the skull itself. The hinder portion of the palato-quadrate persists as the quadrate (Fig. 249, Q). The quadrate is movable in the Saurii, Ophidii, and Aves, while in the Chelonii and Crocodilini it is firmly united to the skull. The whole complex of bones, which is differentiated in the palatoquadrate cartilage, is intimately and immovably connected with the cranium, while, when the quadrate bone is movable, some, at least, of these bones are also so.

Another character is due to the development of the nasal cavity (see also $\S 413$ ). The bones, which in Fishes are placed at the sides of the base of the skull, extend to the middle 
line, so that the base of the skull is more or less shut off from taking any part in bounding the buccal cavity. The nasal cavities, which in the Amphibia lead into the buccal cavity at the very anterior edge of the skull, have their internal orifices always placed farther back in the Reptilia, owing to the union of the horizontal processes of the maxillæ, palatines, and pterygoids in the middle line, and in front of them. In this way the nasal is more completely shut off from the buccal cavity, and forms an upper cavity, the base of which is the roof of the mouth-that is, is the "hard palate." These

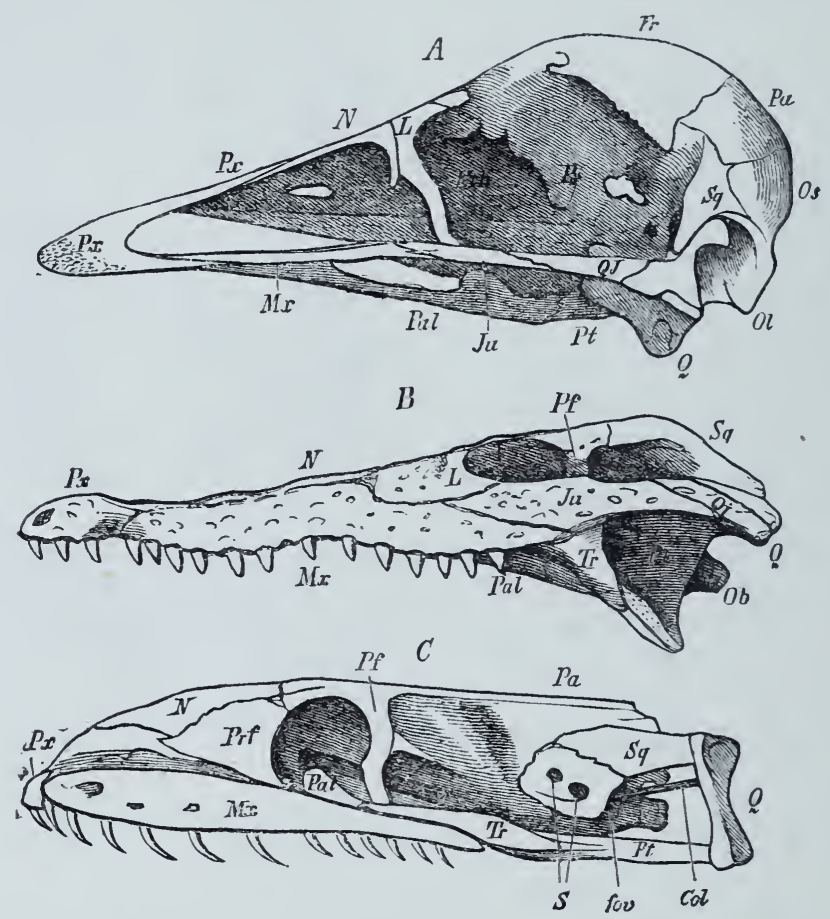

Fig. 249. Side views of Skulls. A Struthio. B Crocodilus. C Python. Ol Ex. occipital. Os Supra-occipital. Pt Pterygoid. Pal Palatine. Tr Transverse. Col Columella. fov Fenestra ovalis. $S$ Foramen for the trigeminal nerre. The other letters as in the preceding figures.

changes are least marked in the Saurii, Ophidii, and Aves, more so in the Chelonii, and most completely so in the Crocodilini.

The parts that form the suspensorium in Fishes (hyomandibular and symplectic) have undergone just the same fate as in the Amphibia; or, in other words, they have no longer any relation to the cephalic skeleton. The "columella auris" and the parts connected with it are developed from its rudiments, partly a bony, and partly a cartilaginous skeletal bit which has entered into the service of the auditory organ.

When the quadrate is movably united with the skull (Ophidii, 
Saurii, Aves), articulations, more or less well developed, appear in those parts of the maxillo-palatine apparatus which are attached to it. These are not present in the Crocodilini and Chelonii, where the quadrate is jammed between the squamosal and the bones of the auditory capsule. Sphenodon presents an intermediate arrangement; its skull is on the Saurian type, but the quadrate is firmly united with the pterygoid and squamosal.

\section{$\S 349$.}

Two sets of bones, which extend forwards, are attached to the quadrate, just as in the Amphibia. Nearer the middle line we find-in Birds, Serpents, and Lizards - the pterygoid (Fig. 250, $P t)$ articulated with the base of the cranium. The two bones are united suturally in the middle line in the Cheloniiand Crocodilini, and are also firmly attached to the base of the skull (Fig. $251, P t)$; in the latter they bound the posterior nares. In the Ophidii, Saurii, and Crocodilini, there is an external pterygoid (Os transversum, Figs. 250, $A \mathrm{Tr}$; 251, $B T r$ ) which connects the pterygoid with the maxilla; it is uncertain whether this corresponds to the ectopterygoid of fishes.

In front of the pterygoid lie the palatines $(\mathrm{Pal})$; in the Chelonii and Crocodilini they are united suturally in the middle line, but in the Ophidii, Saurii, and Aves, they are separated from one another and form the lateral boundaries of the posterior nares (Fig. $250, \mathrm{Pal})$. In the Chelonian

$\Lambda$

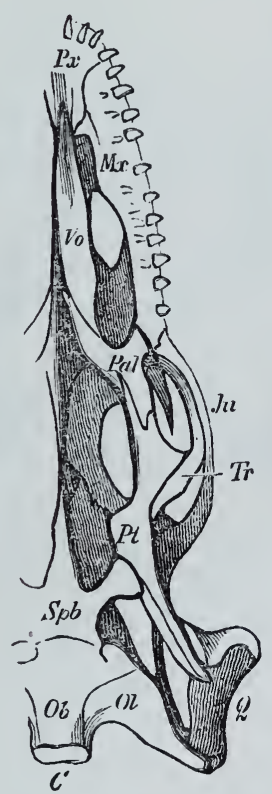

Fig. 250. View of the base of the skull. $A$ Of Monitor. $B$ Of Struthio. Ob Basi-occipital. $C$ Occipital condyle. Ol Exoccipital. Spb Basi. sphenoid. Q Quadrate. Pt Pterygoid. Tr Trans. verse. Pal Palatine. V Vomer. Qj Quadratojugal. Ju Jugal. $M x$ Maxilla. $M x^{\prime}$ Its median process. $\quad P x$ Premaxilla.

skull the vomer (Fig. 251, $A V_{0}$ ) reaches to the roof of the cavity of the mouth and lies between the palatines, while above the nasal cavity the palatines unite together at the base of the cranium. In Birds the palatines are generally long and flat bones (Fig. 250, $B$ $\mathrm{Pal}$ ), which are connected at their anterior end with a process of the maxilla $\left(M x^{\prime}\right)$, or fused with a process of the premaxilla. 
In most Saurii (and in Chelys among the Chelonii), as in the Birds, the premaxillæ are fused together; in the latter they are distinguished by the possession of a long frontal process. Their size is correlated with that of the beak, the form of which is largely

$\Lambda$

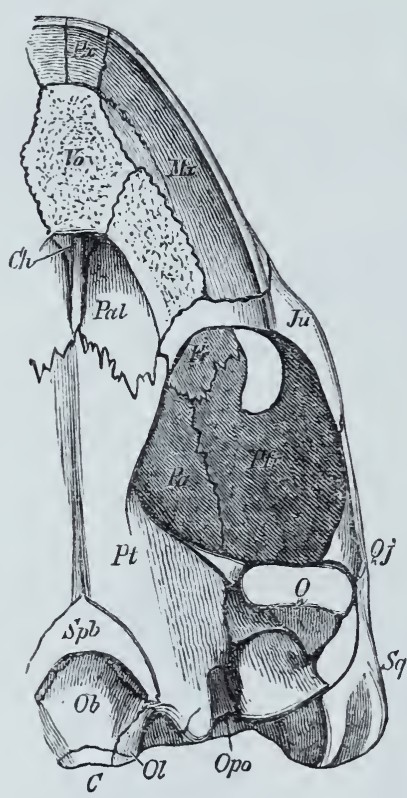

$B$

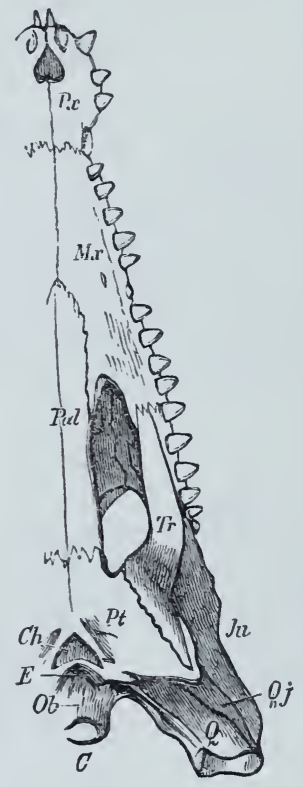

Fig. 251. View of the base of the skull. $A$ Of Chelonia. $B$ Of Crocodilus. $O b$ Basi-occipital. Ol Exoccipital. $C$ Occipital condyle. Spb Basi-sphenoid. Opo Opisthotic. Pt Pterygoid. Pal Palatine. Vo Vomer. Q Quadrate. Qj Quadratojugal. Tr Transverse. $M_{x}$ Maxilla. Px Premaxilla. $P a$ Parietal. Pfr Post.frontal. Fr. Frontal. $C h$ Posterior nares. E Eustachian tube.

due to the share that they take in it. They are rudimentary in the Ophidii (Fig. 249, $C \quad P x$ ) and small in the Chelonii. The maxilla therefore forms the greater portion of the boundary of the edge of the lower jaw (Mx); this bone is of great size in the Crocodilini and Saurii, and very large in the Ophidii, in which also it is capable of a large amount of movement.

There is a lateral series of bones attached to the quadrate. The first of these is the quadrato-jugal ; this is absent in the Ophidii. In the Saurii it is connected with the quadrate at the point where this bone is united to the skull. It is continued forwards into a second piece, which is partly connected with a post-frontal, and partly with a jugal which passes round the lower edge of the orbit. In Birds the quadrato-jugal (Fig. 250, $B Q j$ ) is a slender piece of bone, which arises from the side of the mandibular joint of the quadrate. In the Chelonii and Crocodilini it is connected with a larger portion of the quadrate, and supports the jugal, which aids in bounding the orbit.

In all cases the lower jaw is articulated to the quadrate, and is made up of the same parts as in Fishes, to which, howerer, a supraangular and a complementary bone are added on.

In the Chelonii and Ares the two dentaries fuse very early; in Birds the other bones retain indications merely of their primitive 
separation. The two halves of the jaw are movably connected together in the eurystomatous Ophidii.

PARKER, W. K., Structure and development of the skull in the Ostrich tribe. Philos. Trans. 1866.-The same, On the structure and development of the skull of the common Fowl. Phil. Trans. 1869.

\section{$\S 350$.}

In the Mammalian skull the cartilaginous primordial cranium is ordinarily developed in the basal region only, and limited to the early stages of development. That part of the skull which is derived from the cartilaginous cranium is in this group also to be distinguished from the parts which are developed from other elements, but it becomes intimately connected with them. As a capsule for the brain it is itself larger, and is enclosed by a larger number of bones. It is more distinctly divided into different segments than it is in the lower divisions, but this must be regarded as a secondary adaptation $(\$ 340)$.

In the occipital segment the lateral pieces (Fig. 252, Ol) always unite with a part of the basi-occipital (Fig. 253, Ob) to form the posterior occipital condyles, by which they bound the

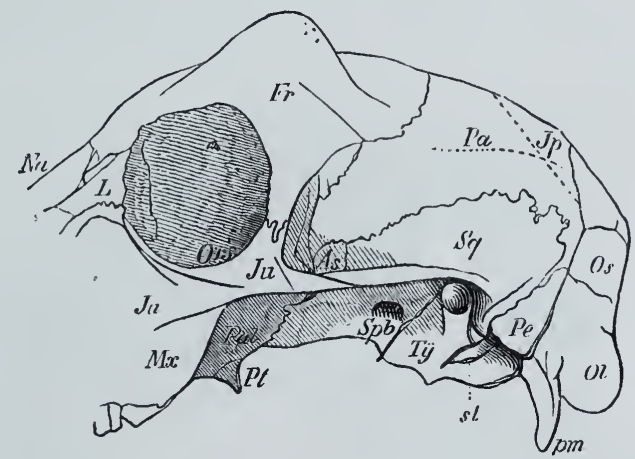

Fig. 252. Lateral view of the cerebral portion of the Skull of a Goat. Ol Exoccipital. Os Supra-occipital. $J p$ Interparietal. $P a$ Parietal. $P e$ Petrosal. Sq Squamosal. Ty Tympanic. Spb Basi-sphenoid. As Alisphenoid. Ors Orbito-sphenoid. Fr Frontal. Na Nasal. $L$ Lachrymal. Ju Jugal. Mx Maxillare superius. Pal Palatine. Pt Pterygoid. pm Para. mastoid process. $s l$ Styloid process. foramen magnum, while superiorly they enclose between them the supra-occipital $(O s)$. This latter may be shut out from the edge of the foramen magnum. The four pieces almost always fuse together, but they may remain long separate (Marsupialia). In many Mammals (several Marsupials, Ungulates, etc.) the exoccipitals send down long processes $(p m)$ (Paramastoid processes).

In the region of the auditory capsule separate ossifications in the cartilaginous portion can be seen in the earliest stages only. They form centres of ossification which partly correspond to the bones in Fishes and Reptiles; these soon fuse into a single piece, the petrosal $(P e)$, the greater part of which gets to be placed at the base of the cranium as the skull grows out laterally. T'he lateral portion of the petrosal is overlaid by other bones, which are developed from the metamorphosis of the branchial skeleton, and becomes converted into the middle wall of the tympanic cavity, in which there is a 
fenestra rotunda as well as a fenestra ovalis. The posterior portion of the petrosal, which is likewise ossified from an independent centre, is attached to the sides of the exoccipitals, and is distinguished as the mastoid portion, in consequence of its carrying the mastoid process in Man. Superiorly, the squamosal $(S q)$ is attached to the petrosal, and is sometimes fused with it to form the temporal bone, of which it then forms the "squamous portion." In some forms it is completely shut out from the cranial cavity; in others a small portion only can be seen from the inner surface of the skull (Cetacea, Ruminants). It is in the Primates only that this portion is of any great size, and this leads to the well-known arrangement which is seen in Man. A process (zygomatic process) of the squamosal which is directed forwards, takes part in the formation of the jugal arch.

In front of the temporal region is the sphenoidal region, which is made up of two perfectly developed segments. The basal

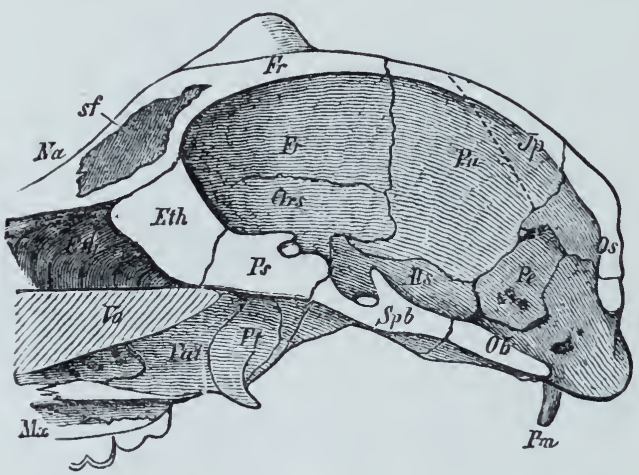

Fig. 253. Vertical median section through the same skull. Ob Basi-occipital. Ps Presphenoid. Eth Eth. moid (rertical plate of the cribriform bone, the anterior edge of which is continued into the cartilaginous par. titions between the nares, which is not seen in this figure). Eth Turbinate bones. Vo Tomer. sf Frontal sinus. The other letters as in the preceding figure. piece of the hinder one (basi-sphenoid) (Fig. 253, $S p h$ ) abuts directly on the basioccipital, and carries at its sides the alæ temporales (ali-sphenoids). The presphenoid with its alæ orbitales (orbito-sphenoid) $(P s)$ lies in front of the basi-sphenoid. The two median pieces are separate throughont life, or for a long time, in Mammals. In Man they fuse very early to form the socalled body of the sphenoid.

In the roof of the skull we again find the well-known covering pieces, which increase in extent as the cranial cavity grows larger. The parietals (Figs. 252, 253, $P a$ ) are often fused together (in the Monotremata, many Marsupialia, the Ruminantia, and Solidungula). Between them, a special bone, which marks off the supra-occipital, projects from behind forwards; this interparietal is generally, as in the Primates, fused with the supra-occipital (Figs. 252, 253, Jp), but, at times, with the parietals (Rodentia and Ruminantia).

The frontals $(F r)$ are attached to the alæ orbitales; they are always paired; they are fused in some, as in Elephas, Rhinoceros, and also in the Prosimiæ, Insectivora, Chiroptera, and Primates.

The most anterior segment of the primordial cranium presents the most important modifications. It is developed into the wall of the nasal cavity owing to the formation of various laterally and 
inwardly projecting processes. Below it, lie the skeletal parts of the maxillo-palatine apparatus, and to these a median lamella of cartilage, which forms the wall of partition of the nasal cavity, is sent down. The vomer is developed as an investing bone on this plate (Fig. 253, $V_{0}$ ). Two ethmoidal pieces are formed by the ossification of the two lateral halves of the ethmoidal cartilage, and of the lamellar processes (turbinate bones), which are given off from it. They bound a portion of the cranial cavity in front of the presphenoid, and are fenestrated to allow for the passage of the olfactory nerve. In Ornithorhynchus there are only two orifices in this region, but they are many more in all other forms, so that this portion is converted into the cribriform plate. An unpaired bone is formed by the fusion of the two lateral halves with the median piece (lamina perpendicularis) (Fig. 253, Eth).

The turbinals vary greatly in character, and by the development of multi-ramified lamellæe aid in increasing the size of the nasal cavity. The ethmoidal segment is, as a rule, covered by other bones, and especially by those which form the maxillo-palatine apparatus; and that to such an extent that no part of it at all can be seen from the outside. Except in some Edentata, it is in the Primates only that a portion of the lateral surface reaches to the median boundary of the orbit, where it forms the "lamina papyracea."

The lachrymals and the nasals form the investing bones of the outer surface of the ethmoidal region. The former $(L)$ are less constant, and often seem to pass into the neighbouring bones, so that they, as for example in the Pinnipedia, are absent as separate pieces. 'They are wanting, also, in the Delphinoidea. As in Reptiles and Birds they form part of the anterior wall of the orbit, and also appear on the facial surface of the skull, from which they extend backwards to the median wall of the orbit, in the Primates.

As to the nasals $(\mathrm{Na})$, they exhibit merely subordinate variations, which are expressed partly by degeneration (Cetacea), partly by a considerable increase in size. Their size is proportional to that of the nasal cavity, and is correlated with an elongation of the facial portion of the skull. They are small in the Primates.

\section{$\S 351$.}

The most important peculiarities in the Mammalian skull are seen in the part which is developed out of the primitive branchial skeleton. A bone which corresponds to the quadrate lies on the outer surface of the auditory capsule. It forms an auditory ossicle, the incus.

The skeletal parts, which are developed in front of the quadrate, and along the base of the skull, are intimately connected with the cranium.

The pterygoids (Fig. 253, Pt) are generally flat pieces of bone, which are placed on the inner surface of the large processes which 
are developed from the basi-sphenoid. They form the side-walls of the posterior nares, and may also limit these orifices below by uniting together in the roof of the palate (in Echidna, Dasypus, and some Cetacea). In most Mammals they are permanently distinct, as they are also, for a very long time, in the Primates, before they unite with the above-mentioned processes of the sphenoid to form the medial lamellæ of the descending pterygoid processes. The palatines generally form the inferior boundary of the posterior nares, and the hinder portion of the hard palate. The maxillæ vary in length according to the extent of the facial region, and always form the largest portions of the upper jaws. The premaxillie vary more considerably; as a rule, they also take part in the formation of the side walls of the nasal cavity. They are rudimentary, or, as compared with the maxillæ, feebly developed in many Chiroptera and Edentata. They bound the foramen incisivum. In the Apes they fuse with the maxillæ; this union takes place so early in Man that their existence was justifiably doubted for a long time.

The outer series of bones, which is present in the Sauropsida, and which extends from the quadrate to the maxilla, is reduced in Mammalia to the jugal; this bone unites the jugal process of the squamosal with the maxilla, and so forms the jugal arch. In a few forms the jugal is absent (Sorex), or, though united with the maxilla, does not reach the jugal process (Myrmecophaga, Bradypus). When it is united with a process of the frontal it gives rise to a posterior wall for the orbit, and so separates this region from the temporal fossa; there are various stages of this arrangement. This process is most complete in the Primates, where the orbital fissure represents the remains of the wide communication which exists between the orbit and the temporal fossa in other Mammals.

In the Mammalia a tympanic bone is developed on the outer face of the petrosal; this serves as a support for the tympanic membrane. It is not certain that this bone is homologous with the one of the same name which we found in the Amphibia. At first it always forms a bony incompletely-closed ring (annulus tympanicus) (Fig. 254, at), which grows out into very various forms. In the Monotremata and Marsupialia, as well as many Insectivora, etc., it is never more than a simple ring. In many forms it is never united to the petrosal; in the Cetacea it is very loosely so. In many it forms a bony capsule which is continued into the external auditory meatus. A bulla of this kind is most common in the Marsupialia, Rodentia, Feræ, and in the Artiodactyla. In some Marsupials, where the tympanic does not pass beyond the annular condition, there is an apparently similar bulla, but this is formed by an extension of the bases of the alæ temporales (Dasyurus, Petaurista, Perameles). When the tympanic is fused with the petrosal and squamosal it takes part in the formation of the temporal bone (Primates). 


\section{$\S 352$.}

Very early in development, the primitive cartilage of the lower jaw turns off from that line of differentiation which obtains in the rest of the Vertebrata. The part, which, in others, forms the articular bone is converted into one of the auditory ossicles, the malleus (Fig. $254, m)$; the Meckelian cartilage $(p)$, which is never developed beyond the cartilaginous stage, is continuous with it.

The dentary forms an investing bone on the outer surface of this cartilage. It abuts in the middle line on its fellow of the opposite side, and unites with it to form the lower jaw; this is articulated to the skull on the lower surface of the jugal process of the squamosal. It represents therefore a new formation, though the primitive one has not disappeared, but persists in other relations. Meckel's cartilage $(p)$ is retained for some time longer on the inner sur-

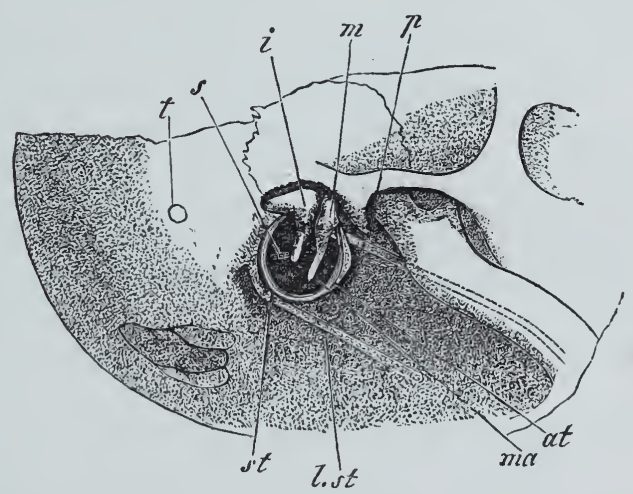

Fig. 254. Lateral view of the skull of a human foetus, with its auditory ossicles. Part of the upper wall of the tympanic cavity and the tympanic membrane have been removed. at Tympanic ring from which a piece has been removed superiorly. $m$ Mal. leus. ma Manubrium of the malleus. $p$ Meckel's process - extending along the inner side of the lower jaw. $i$ Incus. $s$ Stapes. st Styloid process. lst Stylohyoid ligament extending to the anterior cornu of the hyoid. $t$ Mastoid foramen.

face of the lower jaw,

but then disappears; the only portion of it which persists is the part which is placed within the tympanic cavity, and which extends to the Glaserian fissure, where it is ossified to form the processus folianus mallei. The early differentiation, and the, at first, relatively large size of the auditory ossicles, shows that they must be regarded as skeletal parts, which in a lower stage were much more developed in size.

The two halves of the lower jaw are permanently separate in a large number of Mammals ; in others they unite early (Perissodactyla, Chiroptera, Primates). Low morphological conditions are implied by the straight mandibles of the Monotremata, in which there is no distinct coronoid process; in some others, also, this process is merely indicated (Cetacea).

The piece which is developed from the upper portion of the primitive hyoid arch (hyomandibular of Fishes) appears to form the rudiment of a third auditory ossicle-the stapes. 


\section{Branchial Skeleton.}

\section{$\S 353$.}

A ventral system of arches is connected with the most anterior portion of the axial skeleton, and forms the organs of support for that portion of the alimentary canal which functions as a respiratory cavity. The number of arches, and the backward extension of this apparatus, depends on the size of this respiratory cavity. We meet with two very different types of these structures.

The first type is found in the Acrania (Amphioxus). In this framework there is a cartilaginous arch around the mouth-that is, in its most anterior portion; this arch is beset with cartilaginous rods which are directed forwards. The rest of the apparatus is formed of a homogeneous substance, which forms, as in Balanoglossus (cf. § 112), a complicated lattice-work. The branchial bars of either side are independent of those on the other ; that is to saj, they are not united along the ventral line.

We cannot derive the second type, which obtains in the Craniota, directly from this. In its earliest stage it is made up of cartilaginous pieces only; these do not form so large a number of arches as exist in Amphioxus, and are, while completely symmetrical as regards their arrangement, united ventrally by a copula.

In the Cyclostomata the branchial skeleton is made up of complicated cartilaginous bars, which are connected inferiorly with one another, as well as with either side of the spinal column superiorly; owing to their superficial position they may be spoken of as forming an external branchial framework. Very evident signs of this are retained by the Selachii, but in them there is another, or

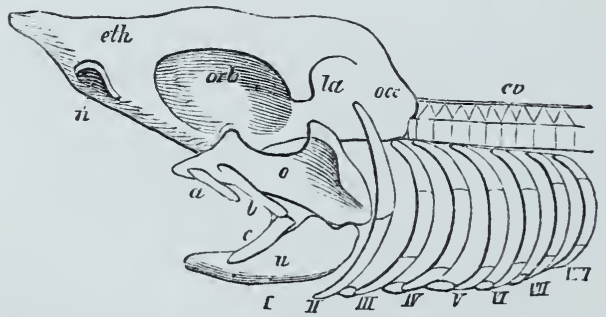

Fig. 255. Skull and branchial skeleton of a Selachian (Diagrammatic). $a b c$ Labial cartilages. I Mandibular arch. $o$ Upper, $u$ Lower portion. II Hyoid arch. III-VIII Branchial arches. internal, organ of support; and this is found in all the rest of the Vertebrata.

The various arches present indications of their primitive similarity; this disappears in consequence of the gradual change in their functional relation, which is due to a division of labour. We were obliged to speak of some of these arches in dealing with the cranium; so that now they need be but briefly considered. The first of them surrounds the entrance to the alimentary canal, and is divided into two pieces; one, superior, the palato-quadrate (Fig. 255,0 ), and the other inferior, the primitive lower jaw (11). The succeeding pairs 
of arches either retain their primitive function of being supports for the branchial arches, or undergo a number of modifications.

All these arches evidently had the same original function. Their relation to the respiratory apparatus has not disappeared in the first pair only, which has been converted into jaws; the hinder arches also have gradually lost their functional and anatomical characters ; it is, therefore, reasonable to suppose that this is but the last of a series of reductions, which first commenced on a much larger number of arches. If this is so, the branchial skeleton of the Craniota is the remnant of an apparatus, in which there were primitively a far larger number of arches. This view is supported by a comparison with Amphioxus, as well as by the considerations to which we are led by a study of the branchial apparatus and of the peripheral nervous system.

As we pass through the Fishes to the Amphibia we may note how this apparatus gradually loses its primitive relations; while in the Reptilia and all higher forms it has no relation at all to the respiratory organs.

\section{$\S 354$.}

Ail the branchial arches are united ventrally by azygos piecesthe copulæ. The various arches are always segmented into a number of pieces, which are generally movably united with one another. The upper portion of the hyoid, as above described, as well as of the mandibular arch, enters into relations with the cranium; these arches thus lose all connection with the other arches, with which the lower portion only of the hyoid arch is still connected.

The succeeding arches are either slightly connected with, or only indirectly united to, the cranium; this is effected by their being attached to the base of the skull, or, when their point of attachment is more extended, to the commencement of the vertebral column. In many Selachii the hyoid arch has the same conformation as the branchial ones (Fig. 255, II). As a rule its copula is increased in size, and affords a support for the tongue. In the Selachii and Chimæræ this arch retains its primitive function of a branchiferous portion of the skeleton. This relation disappears in the Ganoïdei and Teleostei, where this gill is rudimentary, and the rays of the upper portion, which is converted into the hyomandibular and symplectic, are represented by the opercular apparatus ( 345$)$.

The lower portion of the hyoid arch, or true hyoid, has then bony instead of cartilaginous rays (Fig. 256,I r, branchiostegal rays), and a membrane extends between them which covers over the whole of the branchial apparatus. The hyoid arch thus develops an organ of defence for the respiratory apparatus.

There are five pairs of arches connected with the respiratory apparatus; occasionally there are six or seven (Notidani). No more than five are ever found in the Osseous Fishes. While the anterior arches $(I I I I I I)$ are always provided with copula $(f g)$, 
the hinder ones $(I V V)$ are united to a single piece $(a)$, and are always degenerated, both in size and number. The last pair of all (VI), which merely consists of a single piece on either side, carries no gills; in the fifth arch also there are often gill-lamellæ on one side only; in

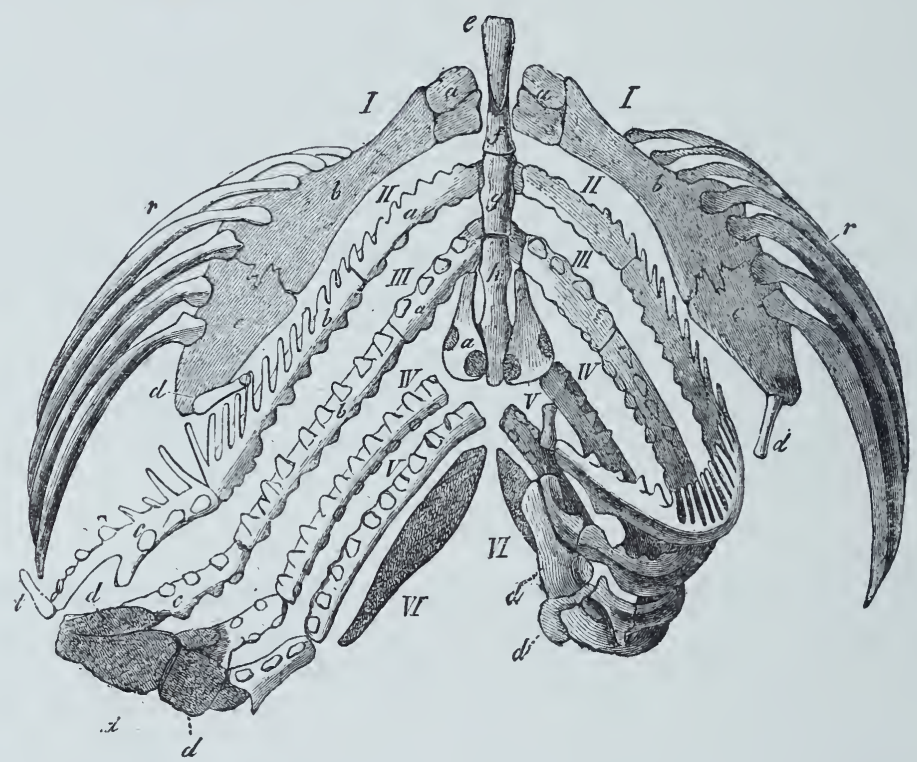

Fig. 256. Hyoid and branchial arches of Perca fluviatilis. I-VI Arches; the first $(I)$ is converted into an organ for the support of the hyoid; the next four (II-V) are branchial arches, and the last $(V I)$ forms the infra-pharyngeal bone. $a b c d$ Segments of the arches. The uppermost piece $(d)$ forms the supra-pharyngeal bones. $r$ Branchiostegal rays. $f g h$ Copulæ (after Cuvier).

the last, however, dental structures are more completely developed, so that this piece is often capable of functioning as a masticatory organ. In the Pharyngognathi the rudiments of the last arch, on either side, are fused into one piece.

We meet with other modifications of the posterior branchial arches in the Labyrinthobranchiata, and in various Clupeidæ; these are due to the conversion of various segments of the arches into the walls of spaces into which water is received.

Just as the hyoid arch of the Selachii is provided with cartilaginous appendages, so also the succeeding arches are beset with cartilaginous rays which support the walls of the branchial pouch. Even these structures are rudimentary in the Ganoïdei and Teleostei, where they form fine cartilaginous lamellæ, placed between the rows of the branchial folds.

\section{\$35̌.}

The branchial skeleton of the Amphibia is considerably reduced; such forms as undergo a metamorphosis have the gills reduced, and 
present at the same a gradual change in this apparatus. It is retained in the Perennibranchiata, and undergoes slight changes only in the Derotremata. It is made up of four or five pairs of arches ; the first of which, as in Fishes, forms a hyoid arch (Fig. $257, b)$. The succeeding arches are united to a common copula. The posterior ones do not severally extend as far as it, but are connected together on either side. In correlation with the reduction of thearches, the copula are increased in size. The only portion which remains complete

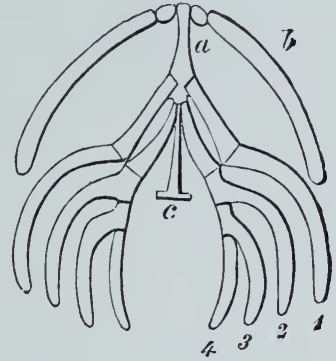

Fig. 257. Hyoid, and bran. chial arches of a larva of Salamandra maculosa. $b$ Hyoid arch. $c c^{\prime}$ Supports of the branchial arches. $d$ Appendage of the Copula.

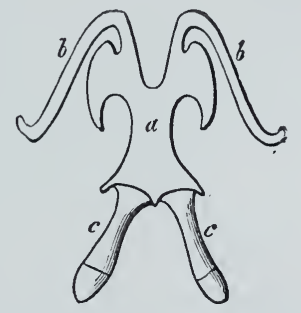

Fig. 258. Hyoid of Bufo cinereus. $a$ Copula. $b$ Cornua of the hyoid. $c$ Rem. nants of the branchial arches (after Dugès). after metamorphosis is the hyoid (Fig. 258, b). It is united with the copula (a), which is generally of some size, and which is converted into the body of the hyoid. A larger piece of the second arch is retained in the Salamandrina, and a small portion of the third arch; in the Anura, however, there is a cartilaginous plate, which is made up of all the branchial arches on either side, and which fuses with the copula into one piece. Rod-shaped pieces (columellæ), which are developed from the ends of the primitively paired plates, are attached to this (Fig. 258,c).

The changes in the branchial skeleton, which are perceptible when it changes its function, afford a striking example of the great influence which adaptation to external conditions of life exercises on the internal organisation.

$\S 356$.

The degeneration which is seen in individuals among some of the Amphibia is an inherited arrangement in the higher classes. Except those parts which enter into the composition of the auditory organ, all the parts which were developed at one time from the large branchial skeleton of Fishes, are converted into that support for the tongue, which is known as the hyoid bone. The copula forms its "body," and to this the rest of the arches are attached, under the form of "cornua." As a rule the remains of two arches-the hyoid portion of the primitive hyoid, and parts of the first branchial arch -are used for this purpose.

The simple body, which rarely consists of several pieces, is beset, in Reptiles, with portions of two or three arches; these are often very rudimentary. They are either single, or divided 
into two pieces. The arches are most numerous in the Chelonii, where there are as many as three, and next to these come the Saurii; in the Crocodilini the broad curved body of the hyoid has but a single pair of arches. In the Ophidii the apparatus is reduced to a cartilaginous remnant, and even these remnants of an arch are lost

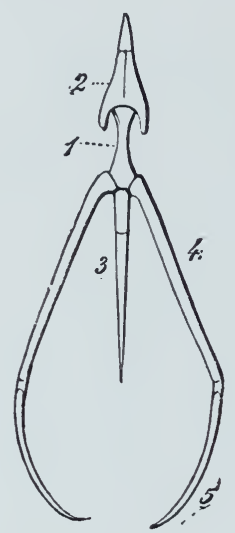

Fig. 259. Hyoid appara. tus of the domestic fowl. 1 Body of the hyoid (copula). 2 Entoglossal. 3 Hyoid process. 4 Anterior, 5 Posterior portion of the cornu of the hyoid. in various forms (Tortrix, Typhlops, etc.). Two pairs of arches can be made out in Birds. The rudimentary first arch fuses to form the so-called entoglossal bone (Fig. 259, 2), posteriorly to which lies the true body of the hyoid. The second arch, however, is well developed, and gives rise to the cornua $(45)$, which are formed of two large pieces, which generally curve backwards behind the skull, without being directly connected with it. Behind the copula there is the remnant of a second one, which forms the hyoid process (3).

In the Mammalia two arches are permanently comnected with the single body of the hyoid. The anterior cornua are the largest, and are connected with the petrosal; they are made of several (three) pieces. When the median piece is merely connected by a ligament, this portion is divided in such a way that the uppermost piece, if connected with the petrosal, as it is in the Orang and in Man, forms the styloid process of this bone; when this is the case, the remaining portion is formed by the stylo-liyoid ligament, and the rest of the arch is attached to the body of the hyoid as a small, and sometimes even unossified piece. In most Mammals, the posterior cornua, which are always formed of a single piece, are the smaller; occasionally, as in various Rodents and Edentates, they are altogether wanting. In the Primates they are larger than the remnants of the anterior cornua. They are connected with the larynx, the thyroid cartilage of which is attached to them by ligaments.

\section{Skeleton of the Appendages.}

$\S 357$.

The two pairs of appendages in the Vertebrata, however much they vary in the extent to which they are developed, have their skeleton arranged in very much the same way; this points to their being homodynamous structures. In this skeleton we may distinguish an arched piece, which lies in the trunk, and which in its lowest condition forms a band of cartilage ; according to the position 
which it occupies, it is known as the thoracic (shoulder), or pelvic (hip) girdle.

The skeleton of the free appendage is attached to the extremity of the girdle. When simplest, this is made up of cartilaginous rods (rays), which differ in their size, segmentation, and relation to one auother. One of these rays is larger than the rest, and has a number of other rays attached to its sides. I have given the name of Archipterygium to the ground-form of the skeleton, which extends from the limb-bearing girdle into the free appendage. 'The primary ray is the stem of this archipterygium, the characters of which enable us to follow out the lines of development of the skeleton of the appendage. Cartilaginous arches beset with rays form the branchial skeleton. The form of skeleton of the appendages may be compared with them; and we are led to the conclusion that it is possible that they may have been derived from such forms. In the branchial skeleton of the Selachii the cartilaginous bars are beset with simple rays (Fig. 260, $a b$ ). In many, a median one is

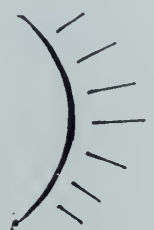

a

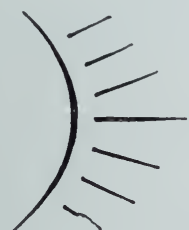

6

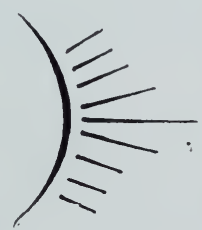

$c$

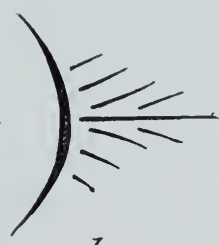

1.

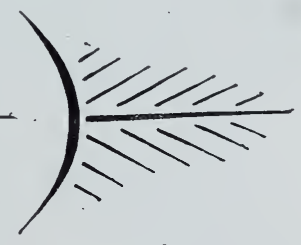

$e$

Fig. 260. Diagrams to illustrate the homodynamy of the appendicular skeleton with that of the branchiæ. $a b c d$ Branchial arches of Selachii. e Archipterygium.

developed to a greater size. As the surrounding rays become sinaller, and approach the larger one $(c)$, we get an intermediate step towards that arrangement in which the larger median ray carries a few smaller ones $(d)$. This differentiation of one ray, which is thereby raised to a higher grade, may be comnected with the primitive form of the appendicular skeleton; and, as we compare the girdle with a branchial arch, so we may compare the median ray and its secondary investment of rays with the skeleton of the free appendage.

We meet with greater difficulties when we come to examine the topographical relations of the appendages. If the comparison of the skeleton of the appendages shows that it is similar to the branchial skeleton, and that therefore it is possible to derive the appendages from branchial arches, we must further suppose that the two appendages were primitively branchial arches, which carried rays, and that they have been differentiated in a different way to the other branchial arches, and have been separated off from the branchial apparatus. The hinder one altered its position more than the anterior one, and this, of course, happened during changes which affected the rest of the organism. The anterior 
appendage has still some relations to the head, as is shown by the muscles which are supplied by cerebral nerves, while, in Fishes, it, and its arch, lie just behind the branchial arches. In this respect the hinder appendages are quite independent. They must be supposed to have travelled to a greater distance, if we are right as to the homodynamy, which a comparison of the skeletons leads us to infer. The anterior appendage has, however, clearly also undergone great changes in position; this is evident when we note how, owing to the continual increase in the number of the cervical vertebræ, it moves farther and farther back as we pass from Fishes to Birds. As there are no facts known to us which point to the formation of new vertebræ, which could only be brought about by the intercalation of new metameres of the body, this distinct change in position must be explained as due to the continual retrogression of the appendages; in other words, we are led to postulate just the same process in its case, as in that of the hind limbs. These considerations merely point to the manner in which it is possible that the appendages were developed, and there are still many questions which cannot be safely answered until a comparative examination of the muscles and nerves which belong to the appendages has been made.

Gegenbaur, C., Zur Morphol, der Gliedmassen der Wirbelthiere. Morphol. Jahrb. II.

\title{
Anterior Appendages.
}

\author{
Shoulder-Girdle.
}

$\$ 358$.

In its simplest form the shoulder-girdle is a piece of cartilage, which, in the Selachii, forms an arch on each side united to its fellow along the ventral line, and placed just behind the branchial apparatus. Owing to its attachments to the muscles of the appendages, definite sculpturing may be made out on the arch; this is most distinct in the Rays.

In the Ganoïdei the two halves of the cartilaginous arch are completely divided; a new apparatus is connected with the primary shoulder-girdle, represented by the cartilage; this non-cartilaginous part is formed of bones which primitively belonged to the integument, and in the course of its differentiation up to the Mammalia it plays an important part.

We must therefore distinguish the secondary from the primary shoulder-girdle. The latter is always cartilaginous in the Sturiones, though various bony plates of the integument are developed on it; the two lower ones I have shown to be the clavicle and infra-clavicle, and the two upper ones the supra-clavicles. In the primary thoracic cartilage wider spaces are developed from the canals which are found 
in Selachians. In the rest of the Ganoïdei and Teleostei a part alone ordinarily remains cartilaginous, and the rest is ossified, but the whole piece appears to decrease in size. As a rule two bones $(f e)$ are developed from it in the 'Teleostei, and with these, parts of the skeleton of the fin may be closely connected. The clavicle, however, which is small in the Sturiones, has increased in size (Fig. 261, c). It is connected along the ventral median line with that of the opposite side, and by the supra-clavicles $(a b)$ with

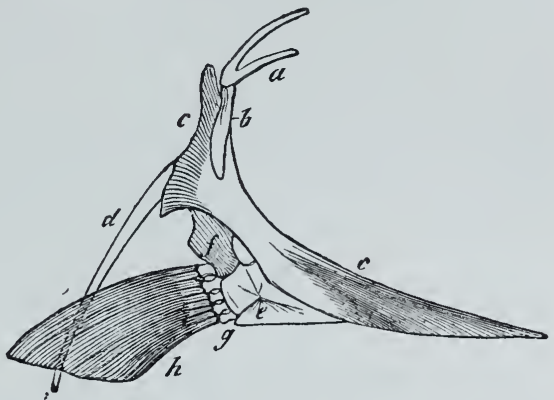

Fig. 261. Right half of the shouldergirdle, and thoracic fin of Gadus. $c$ Clavicle. $a b$ Supra-clavicles. $d$ Accessory piece. $e$ Coracoid. $f$ Scapula. $g$ Basalia of the fin. $h$ Rays of the secondary skeleton of the fin. the skull. The primary shoulder-girdle, in fact, undergoes degeneration, and forms a mere appendage to the clavicle, which becomes the chief support of the anterior extremity.

\section{§ 359.}

The clavicle, developed on the cartilaginous shoulder-girdle of Fishes, is reduced in the higher Vertebrata. The primary apparatus, however, becomes of greater importance owing to its connection with the sternum, and the greater power of movement possessed by its uppermost (dorsal) portion, which is no longer firmly connected with the axial skeleton. That region of the girdle at which the free limb is connected with it, is distinguished by the formation of a cavity which receives the articular head of the humerus, and divides the primary shouldergirdle into two parts.

The dorsal portion forms the scapula; the ventral is divided into a hinder piece, the coracoid, and an anterior piece, which is ossified from the scapula, when it is ossified-the precoracoid.

Among the Amphibia, the shouldergirdle of the Urodela forms a skeletal piece on either side; it is largely carti-

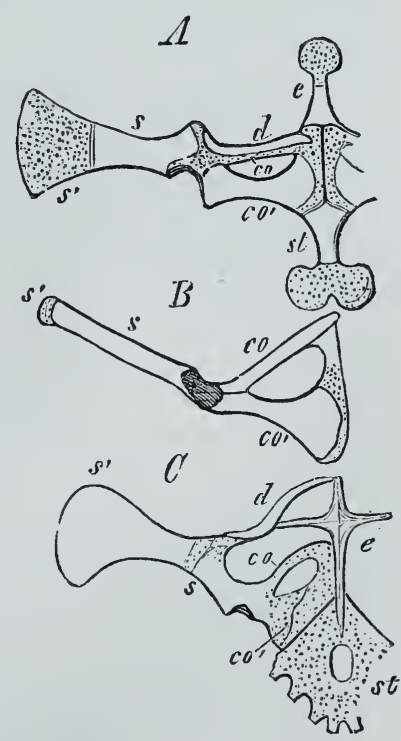

Fig. 262. Shoulder-girdle; $A$ of a Frog, $B$ of a Chelonian. $C$ of a Saurian. $s$ Scapula. $s^{\prime}$ Supra-scapula. co Precoracoid. $c o^{\prime}$ Coracoid. $d$ Clavicle. $c$ Episternum. st Sternum. The cartilaginous portions are dotted. laginous, and is only ossified in the region of the glenoid cavity. The widened dorsal end of the scapula, the supra-scapula, is almost always cartilaginous, or has an 
independent periosteal ossification. The ossification sometimes extends from the scapula on to the precoracoid. In the Anura the two ventral processes (Fig. 262, $A c o c o^{\prime}$ ) on either side of the shoulder-girdle are united by their cartilaginous ends, and may also become united in the middle line (Rana). In this case there is a foramen on either side, in the ventral portion of the shoulder-girdle. 'The coracoid $\left(c o^{\prime}\right)$ is ossified independently, while the precoracoid becomes closely related to the clavicle $(d)$.

In the Reptilia, likewise, each half of the shoulder-girdle forms a single piece, which closely resembles in form the same part in the Amphibia. The coracoid, which is generally broad, is not unfrequently fenestrated (Saurii). A process of the scapula, which is merely indicated in the Amphibia, is converted into the acromion, and unites the scapula with the clavicle (Fig. 262, $O d$ ). In the Chelonii the scapula is generally a cylindrical bone $(B s)$, which forms an angle with, and is directly continuous with, the precoracoid $\left(\begin{array}{ll}B & c o\end{array}\right)$ at the glenoid cavity. The end of this precoracoid is connected by a ligament with the cartilaginous end of the coracoid.

In the Crocodilini the precoracoid has completely disappeared, so that the scapula and coracoid alone make up the shoulder-girdle. In Birds there is a somewhat similar arrangement; the small and slightly curved scapula is united to the strong coracoid at the glenoid cavity; the coracoid itself is, as in Reptiles, attached to the plate of the sternum. The Ratite indicate their closer affinity to the Saurii by the presence of a rudiment of the precoracoid.

Among Mammals the coracoid is complete in the Monotremata only. In the rest, the only sign of it is the process (coracoid process) which is given off from the scapula, and lies in front of the glenoid cavity; it is in rare cases only that the sternal end of the coracoid persists. I have discovered it, however, in Sorex and Mus, where it forms a piece of cartilage attached to either side of the manubrium sterni. The scapular remnant of the coracoid still continues to take part in the formation of the glenoid cavity, but this share decreases as that of the scapula increases, so that at last this latter bone alone forms the support for the anterior extremity, which thereby acquires a greater power of free movement. The primitive independence of the remnant of the coracoid is implied by the presence in it of a special centre of ossification, which persists so long as it is not completely fused with the scapula.

In form, the scapula of Mammals resembles that of the Reptiles, but owing to the presence in it of new constituents it differs from the latter in some essential points. In the Monotremata there are indications of a spine, the end of which forms the acromion. In the rest of the Mammalia the lateral edge of this broad piece is developed into a larger ridge, which now, owing to the development of the median ridge also, into a projecting plate of bone, or spina scapulæ, marks off a superior and an inferior fossa. The anterior end of the spine is always developed into an acromial process. The most important of the other changes which occur in 
it is the enlargement of the base of the scapula, which obtains in the Chiroptera and Primates.

$\S 360$.

Owing to the development of the primary shoulder-girdle the secondary apparatus, which forms the clavicle (\$358), is either placed completely in the background, or used for purposes other than those which it had in Fishes. The Anura only among the Amphibia are provided with a clavicle (Fig. $262, A d$ ), which forms an investing bone for the precoracoid. It is seldom separated from the shouldergirdle, and this separation is never complete in any forms below the Reptilia $(B d)$. In them it forms a bone which connects the acromial process of the scapula with the episternum $(B c)$. In Birds the clavicle has the same relations; it is small in Dromæus, and absent in all other Ratitæ; in the Carinatæ, however, the clavicles soon nnite into an unpaired bone, the furcula, and are connected with the keel of the sternum by ligaments (Fig. 234, $f$ ).

The independent appearance of this portion of the skeleton, the primitive origin of which was that of an investing bone for a piece of cartilage, leads to a histological change in the Mammalia; the clavicie is in them largely formed from a cartilaginous rudiment, which is similar in many points to all other bones, which are preformed in cartilage. This bone, however, is retained in some Mammals only;in those, namely, in which the anterior limbs are capable of a large amount of movement. It disappears so far as to leave no signs of its presence in the Ungulata ; in other forms there are only rudiments of it which are sometimes merely formed by ligamentous bands (Carnivora).

\section{Anterior Extremity.}

$\S 361$.

All the varied forms, which the skeleton of the free appendages exhibits, may be derived from a ground-form which persists in a few cases only, and which represents the first, and consequently the lowest, stage of the skeleton of the fin-the Archipterygium. This is made up of a stem, which consists of jointed pieces of cartilage, which is articulated to the shoulder-girdle, and is beset on either side with rays, which are likewise jointed. In addition to the rays on the stem there are others which are directly attached to the limb-girdle (cf. Fig. 260, $d$ ).

Ceratodus has a fin-skeleton of this form; in it there is a stem beset with two rows of rays. But there are no rays on the shouldergirdle. This biserial investment of rays on the stem of the fin may also undergo various kinds of modifications. Among the Dipnoï, Protopterus retains the medial row of rays only, which have the form of fine rods of cartilage; in the Selachii, on the other hand, the 
lateral rays are considerably developed. The remains of the medial row are ordinarily quite small (Fig. $263, R$ ), but they are always sufficiently distinct to justify us in sup-

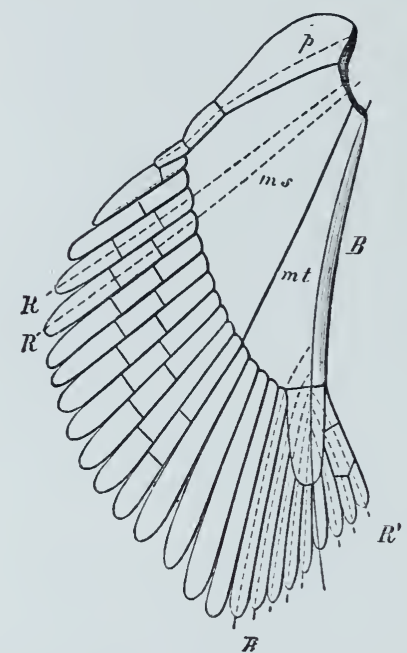

Fig. 263. Skeleton of the thoracic fin of Acanthias vulgaris. $p$ Basale of the protopterygium. $m t$ of the metapterygium. $B$ Median edge of the fin. The line drawn through $m t$ indicates the series which formed the stem of the archipterygium. The dotted lines correspond to the rays, which are mostly arranged at the sides $(R R)$, and are rudimentary only on the medial side $\left(R^{\prime}\right)$. posing that in higher forms the two sets of rays might be better developed. Rays are still attached to the stem, and are connected with the shoulder-girdle by means of larger plates $\left(\begin{array}{ll}p & m s) \text {. The }\end{array}\right.$ joints of the rays are sometimes broken up into polygonal plates, which may, further, fuse with one another; concrescence of this kind may also affect the pieces which form the base of the fin $(p \mathrm{~ms})$. By regarding the free rays, which are attached to these basal pieces, as belonging to these basal portions, we are able to divide the entire skeleton of the fin into three segments-pro-, meso-, and metapterygium.

The metapterygium ( $m t$ ) represents the stem of the archipterygium and the rays on it. The propterygium $(p)$ and the mesopterygium $(\mathrm{ms})$ are evidently derived from rays which still remain attached to the shoulder-girdle.

The peculiar form of the fin in the Ray is due to the great development of the propterygium; the arrangement in Squatina leads towards this. One ray is here converted into a support for rays, and forms, by gradually reaching: forwards, a stem for the propterygium, just as the metapterygium in the stem of the archipterygium possesses one. The Chimæræ agree in all essential points with the Sharks.

\section{$\S 362$.}

The skeleton of the thoracic fin in the Ganoïdei may be derived from a condition which is similar to that which obtains in the Shark; it is the same fin, with the peripheral parts reduced (cf. Fig. 264). In correspondence with this, a few rays only are attached to the stem of the fin $(B)$, and those which are set on the shoulder-girdle are also rudimentary. In the Teleostei the peripheral portion of the skeleton of the fin is still further reduced, and as a rule nothing remains of the primary fin-skeleton except four or five elements which are very similar to one another (Fig. 261, g); a very variable number of small and always cartilaginous pieces are attached peripherally to them. These, then, serve as supports for the 
secondary skeleton of the fin-rays $(h)$. Basal pieces can be seen in a few only, and it is difficult to refer these even to their primitive significance. The arrangements which obtain in the Ganoïdei would lead us to regard the basale of the metapterygium, and the basalia of some of the rays, as being the most constant constituents of these pieces. In consequence of their having the same function they have the same form, so that it is impossible to show that they have any connection with the primary stage, except by referring them back to the skeleton of the Ganoid fin.

In many divisions of the Teleostei these pieces undergo great changes, in addition to being diminished in number. They are, for instance, intimately attached to the shouldergirdle, and immovably connected with the parts of which it is made up (Cataphracti).

In this way we are able to make out a continuous series from the well-developed skeleton of the fin in the Selachii to that which is found in the Teleostei; the most important changes consist in the gradual reduction of smaller or

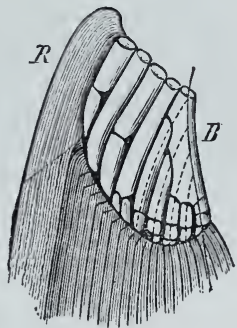

Fig. 264. Primary skeleton of the thoracic fin of Aci. penser ruthenus, after the removal of a portion of the secondary skeleton. $B$ Basale of the meta. pterygium. $R$ Bony marginal ray of the secondary skeleton of the fin, only

figured in part. larger parts. Reduction first affects the periphery, and then the base, so that the latter is the most constant portion. The decrease in size which the primary skeleton suffers is made up for by the appearance of ossifications of the integument, which consist, as in the unpaired fins, of jointed or firm bony rays, and are developed on both surfaces of the fin.

Gegenbaur, C., Untersuchungen zur vergleich. Anatomie der Wirbelthiere. II. Leipzig, 1865.

\section{$\S 363$.}

In the skeleton of the fore-limb of the higher Vertebrata we are able to recognise the stem of the archipterygium, with rays attached to one side of it; no rays are now attached to the shoulder-girdle, the stem only is so attached. The arrangement of the joints of the rays in rows set obliquely to the stem-which is just the same arrangement as that of the primitive rays-is obscured by subsequent transverse jointing, but it can be recognised without difficulty in the lower forms. This jointing gives rise to new pieces ; transverse rows of the joints of the rays, as well as the corresponding joints of the stem, being developed into longer pieces. This change is due to a change in function, in consequence of which the appendage is converted from a swimming organ into a compound system of levers.

In Ichthyosaurus among the Enaliosaurii the basale of the archipterygium is first of all differentiated from the rest of the 
appendage as a large bone, which forms a piece of about the same size as the rest of the appendage ; this may be called the humerus. In Plesiosaurus two succeeding pieces, which in Ichthyosaurus are still indifferent, are also increased in size ; these correspond to the forearm : radius and ulna; these are succeeded by two transverse rows of smaller pieces, which form a carpus, and these again by longer rows of bones, which represent the metacarpus and the phalanges of the fingers. The segmentation which affects the appendage after the stem

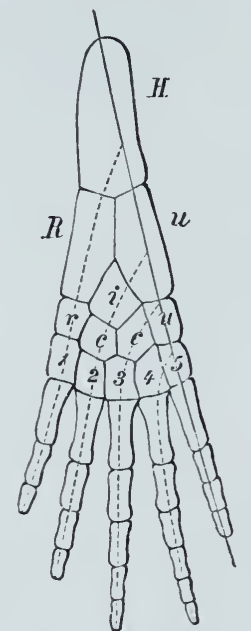

Fig. 265. Diagram of the fore-limb of an A mphi. bian. The dotted lines indicate the rays, which remain attached to the stem of the Archipterygium. and rays are broken up into several pieces, may here be seen in its different stages.

The arrangements which are presented by the Amphibia are similar in character; for although one finger is atrophied, we can fill up the void by the aid of the arrangements seen in the hinder limb, where they are complete. The stem of the archipterygium must, therefore, be sought for in a lateral series of skeletal pieces, which extends from the humerus, through the ulna to the fifth finger, and in the carpus consists of two pieces. The other skeletal pieces are arranged on these rays. One ray begins with the radius, and extends into the first finger. A second, third, and fourth begin in the carpus, and end in the second, third, and fourth fingers. The primitive carpus is therefore composed of ten pieces; five carpals carry the fingers, three are attached to the bones of the forearm; these are the radial, intermedium, and ulnare; two centralia $(c c)$ are enclosed by these two sets.

The change in the function of this appendage is connected with a rotation of the humerus on its own axis, and this rotation may be observed in the individual development of higher forms. It brings about a difference in the position of the limb as compared with that of lower forms.

\section{$\S 364$.}

A more or less complete copy of the typical form of limb derived from the archipterygium is retained in all divisions of the Vertebrata. In all there are often unmistakable traces of the characteristic relations, in opposition to which numerous deviations, due chiefly to reduction and concrescence, make their appearance. These modifications are clearly due to the varied uses to which the limbs are put, just as the complete atropliy of some parts, or even of the whole limb, are due to their being no longer required. 
In the Amphibia the two upper portions are greatly developed, but, except that the radius and ulna are fused in the Anura, they present no such striking differences as those which are seen in the carpus.

Some of the primitive carpalia disappear in the distal row; with this is generally correlated a shortening of the fingers, which are commonly limited to four; or, again, two or three carpalia may be fused together (Frogs, etc.). Concrescence may likewise be seen to affect the proximal series of carpalia.

In the Reptilia, the various portions of the skeleton of the arm are least altered in the Chelonii, which have not only nine carpal bones, but all five fingers. In the Saurii two of the three carpalia of the first row are fused together ; those of the second row are also greatly modified, and are reduced in number when any of the fingers disappear. The carpus is still more altered in the Crocodilini. The radiale has become much larger than the ulnare, and the second row of carpalia is merely represented by a few elements, which are always partly cartilaginous. The two ulnar fingers are conse-

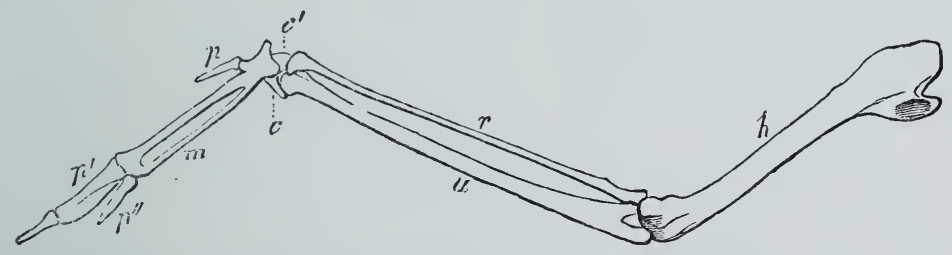

Fig. 266. Skeleton of the arm of Ciconia alba. $h$ Humerus. $u$ Ulna. $r$ Radius. $c c^{\prime}$ Carpus. $m$ Metacarpus. $p p^{\prime} p^{\prime \prime}$ Phalanges of the first three fingers.

quently shortened in comparison with the three radial ones. In the limbs of the snake-like Saurii there are all stages of reduction. The Ophdii are distinguished by the complete absence of these parts.

In birds, where the whole of the fore-limb is converted into an organ of flight, the reduction of the manus is still more marked. Two bones only (Fig. 266, $c c^{\prime}$ ) are well developed in the carpus, while a piece of cartilage, which corresponds to the second row in the carpus, soon fuses with the base of the metacarpus. Three fingers are always more or less developed in the manus; in the Saururæe these were permanently separate, but in the Ratitæ and Carinatæ the metacarpals $(m)$ of the second and third, and generally also that of the first, are fused into one piece of bone. On the third finger there is a rudiment of a fourth one.

As compared with the Saurii, the number of phalanges is reduced in Birds. In the Saurii, starting from the first finger of the radial side, which has two phalanges, we find one more in each finger as far as the fourth, which has five; but the fifth finger has not so many. In the Crocodilini this increase stops at the third; in most Birds the second finger has only two phalanges $\left(p^{\prime}\right)$, the 
first and third one only $\left(p p^{\prime \prime}\right)$; the first and second fingers rarely have an extra phalanx.

Furbringer, M., Die Knochen und Muskeln der Extremitäten bei den schlan. genartigen Sauriern. Leipzig, 1870.

\section{$\S 365$.}

The greater variation in adaptive relations to various conditions is implied by the greater variations in the structure of the skeleton of the Mammalian forelimb. Its elements somewhat resemble the lower condition, such as is seen in the Chelonii, with regard to the number of the carpal bones. Although the manus is often modified by the atrophy of certain fingers, the extremity, even in the lower divisions of the Mammalia, has very various uses. Owing to the greater power of movement possessed by the two bones of the forearm, and the connection between one of them (the radius) and the manus, the anterior extremity loses its lower function of an organ of support, and is converted into a prehensile organ. This phænomenon is seen in the Didelphia, as well as in the Monodelphia ; it is most complete in the Primates. The carpus has the three primitive pieces of the proximal row. A centrale, also, is not unfrequently present (Rodentia, Insectivora, Lemurs, Orang, and, for a short time, Man). The distal row of the carpus have the two ulnar bones fused into an uncinate (cf. Fig. 268, I II). The pisiform is a special bone, which is attached to the ulnar edge of the carpus; it

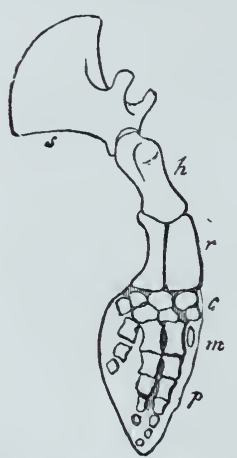

Fig. 267. Anterior extremity of a young Dolphin. $s$ Scapula. $h$ Humerus. r Ra. dius. $u$ Ulna. $c$ Car. pus. $m$ Metacarpus.

$p$ Phalanges. is very large in many forms. It is also found in the Reptilia, and may be shown to be the solitary remnant of a numerous series, which was possessed by the Enaliosaurii.

The modifications derived from this series of forms are very closely correlated with the function of these parts. When the arm is used as an organ of flight (Chiroptera), we find that its different portions are considerably elongated; and so, again, they are shortened, and various parts become very large in those numerous cases in which the arm acquires a special function, as in digging and so on; the Monotremata, many Edentata, Talpa, etc., are examples of this. Instead of this great increase in size, which is seen in various parts of the skeleton of the arm, there may be atrophy, as is the case with the fore-limb of the Cetacea. It forms a paddle, the separate parts of which have but little power of movement, and the various bones of these parts may lose all their articulations, and become united into an unjointed fin-like mass (Fig. 267).

In another series, several of the fingers are atrophied, and the 
fore-limb becomes a mere organ of support and locomotion. It is clear that this is not a primary condition from the relative position of the bones of the fore-arm, which requires us to presuppose a condition in which they were capable of pronation and supination. As the limb ceases to have more than one function, this power is lost; the radius and ulna are connected immovably, and this may lead to the atrophy of various parts of these bones, or to their more complete fusion with one another. This is the case in the Artiodactyla, where the distal end of the ulna is rudimentary in the Ruminant forms. In the Tylopoda and Solidungula this end of the ulna has quite disappeared, while the upper end is united with the radius into one bone.

The fingers may take on one of two sets of characters. In
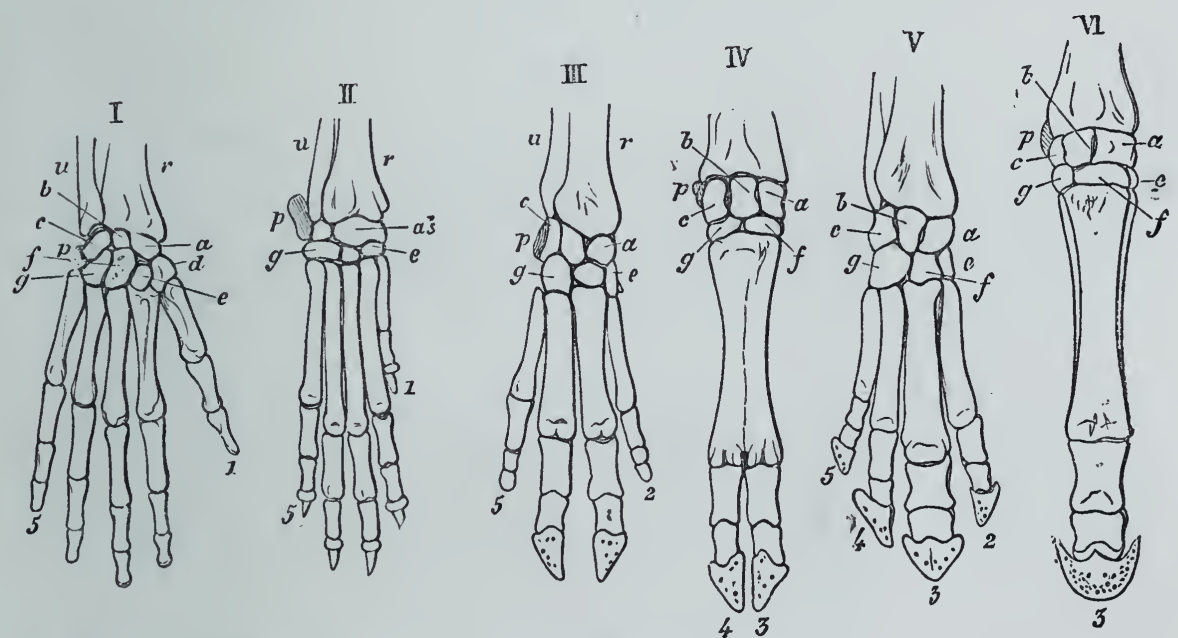

Fig. 268. Skeleton of the manus of various Mammals. I Man. II Dog. III Pig. $I V$ Ox. $V$ Tapir. VI Horse. $r$ Radius. $u$ Ulna. a Scaphoid. $b$ Lunar. $c$ Cuneiform. $d$ Trapezium. $e$ Trapezoid. $f$ Magnum. $g$ Uncinate. $p$ Pisiform.

either case the pollex is absent, and it is not functional even in the digitigrade carnivora (Fig.a 268, II). Of the remaining digits, however, the third and fourth are so greatly developed in the Artiodactyla (III IV), that the other two (2 and 5) often do not touch the ground (Suina, Moschidæ). The fifth finger is next lost, so that the third and fourth only are well-developed, and the second forms a mere appendage (Anoplotherium). The third and fourth fingers become still larger when their two metacarpals are fused together $(I V)$, while the second and fifth fingers become rudimentary (Oxen, Sheep, Deer, etc.). The Perissodactyle series also begins with the four-fingered form, but in them one finger only (the third) is markedly larger (Tapir) $(V)$. When the fifth, which is already the smallest, disappears (Palæotherium), the second and fourth are attached to the third in the form of appendages 
(Hipparion), and when the two lateral fingers are reduced to their metacarpals alone, these are attached to the large metacarpus of the third finger as mere "splint-bones" (VI), and the third finger becomes the sole support of the limb (Equus).

The number of phalanges in the different fingers is increased in the Cetacea only; all other Mammals have two in the pollex, and three in all the other fingers.

\title{
Posterior Appendages.
}

\author{
Pelvic Girdle.
}

$\S 366$.

The relations of the pelvic girdle are also correlated with differences in the functions of the extremity. The homology between the two skeletal portions is consequently more fully recognisable as the functions of the two extremities are more nearly the same, and the extent to which they are differentiated from one another less.

A single piece of cartilage forms the groundwork of the pelvic girdle. In the Selachii this is rarely enlarged in a dorsal direction. In the Ganoïdei and Teleostei the two halves of the ossified portion are connected in the middle line. They undergo considerable variations in position, for they may be placed more or less anteriorly and close to the shoulder-girdle (Pisces thoracici), or may even be united with it (Pisces jugulares).

In the Amphibia the trvo bones of the pelvis are connected with the vertebral column; at the same time they may be seen to be divided into two pieces at the point where they are connected with the femur; the dorsal one, which is attached to a transverse process (that is, to a rudimentary rib), forms the ilium; the ventral one, which is connected along the middle line with its fellow of the opposite side, is known as the ischio-pubic bone (Urodela). There is reason, however, for supposing that it merely corresponds to an ischium. This arrangement is modified in the Anura (cf. Fig. 225), for the long and slender ilia (il) are united with the ischio-pubic bones $(i s)$, which are converted into a vertical disc, and fused with one another.

The ilium of the Reptilia is greatly developed; in Chamæleo it resembles a scapula, and is continued into a process, which is comparable to a supra-scapula. In the Saurii it is elongated (Fig. 269, $J l)$; in the Crocodilini it is shorter and broader (Fig. 270, Jl). The bone is directed forwards, so that it is connected with the sacrum behind the acetabulum. In the Saurii and Chelonii the ventral portion of the pelvis is continued from the aceta- 
bulum into two divergent pieces (Fig. 269), which enclose a large opening (foramen obturatum). 'The anterior process is called the pubis $(P)$, the posterior one the ischium $\left(J_{s}\right)$. The two bones of either side are more or less connected together along the middle line, but this connection may disappear. The pelvis of the Crocodilini

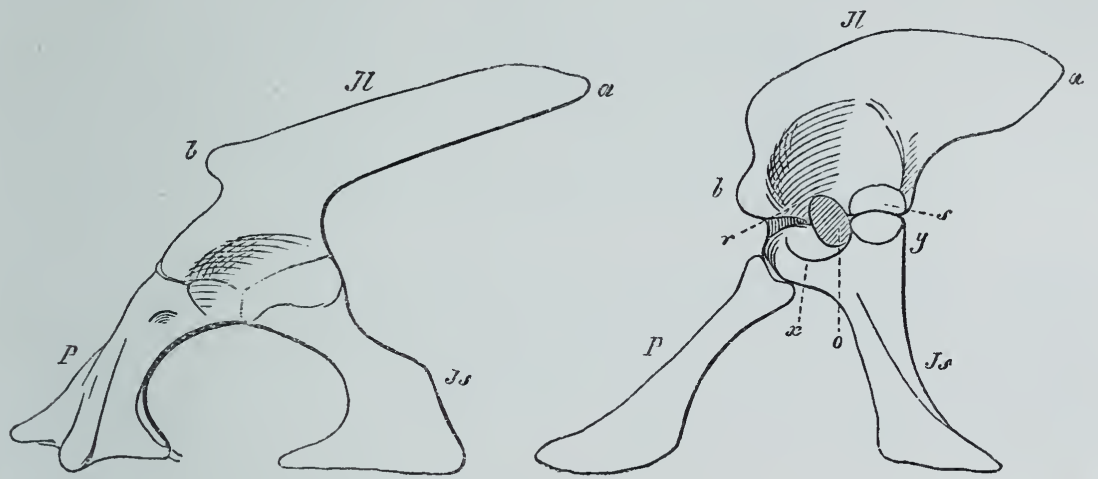

Fig. 269. View of the left side of the pelvis of Monitor. Jl Ilium. Js Ischium. $P$ Pubis. $a$ Hinder end of the ilium. $b$ Its anterior process.

Fig. 270. View of the left side of the pelvis of Alligator lucius. $x$ y Two limbs of the ischium, which unite with $r s$, two processes of the ilium, to enclose a foramen ( 0 ) at the base of the acetabulum. The other letters as in Fig. 269.

(Fig. 270) differs from this in many points, for a single bone $(J s)$ is given off ventrally from the acetabulum, and is connected by means of two processes with the ilium $\left(\begin{array}{ll}x & y\end{array}\right)$. It appears to represent an ischium only, while a bone, which takes no part in the acetabulum, but articulates with the ischium $(p)$, and converges, like its fellow, towards the anterior wall of the abdomen, represents the pubis.

The pelvis in the fossil Dinosaurii was of the same character; the ilium was distinguished by a process which was directed forwards, and of which there is an indication only in the extant Saurii and Crocodilini (b). The acetabulum was similarly incomplete, and was connected with a long ischium, which was directed obliquely backwards and downwards, and was not united with its fellow of the opposite side. A long pubis, which also ended freely, was given off from the anterior margin of the acetabulum, and ran parallel to the ischium.

This relation of parts is the same as that which characterises the Avian pelvis (Fig. 271). In them the ilium ( $J l)$ does not only extend a long way back $(a c)$, but its anterior process is converted into a broad plate $(b b)$. This extends along the lumbar region of vertebral column, and even into the thoracic region, and so presses a very large number of vertebræ into the pelvic region. The ischium $(J s)$ runs backwards from the incomplete acetabulum, and in a direction which is nearly parallel to that of the hinder portion of the ilium; the small pubis, which has a slight share in the formation 
of the acetabulum, takes the same course ; its ends project farther back than those of the ischium, and generally converge; in Struthio they

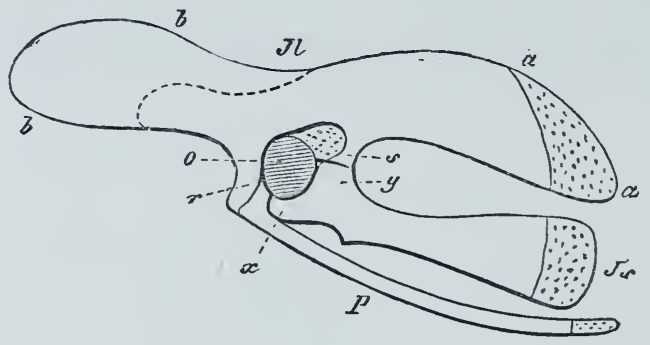

Fig. 271. View of the left side of a Bird's pelvis. The dotted portion represents that part of the three pieces of the pelvis, which extends backwards by the development of cartilage. The dotted line marks off that portion of the ilium $(b b)$ which grows forwards without the addition of any cartilage. The letters as in the preceding figures.

even form a symphysis. There are various kinds of connections between the ilium and ischium, and between these and the pubis.

The pelvis in the Mammalia is very different. The primitive connection with the sacrum is always in front of the acetabulum. The ilium, however, is directed from before backwards, and the hinder edge of the Bird's ilium corresponds to the anterior edge of the Mammal's ilium. Two different positions therefore for the ilium are derived from the Amphibia. In the Amphibia it is directed laterally and inferiorly away from its connection with the sacrum, in Reptilia and Aves obliquely forwards, and in Mammalia obliquely backwards. The ventral portion of the pelvis encloses an obturator

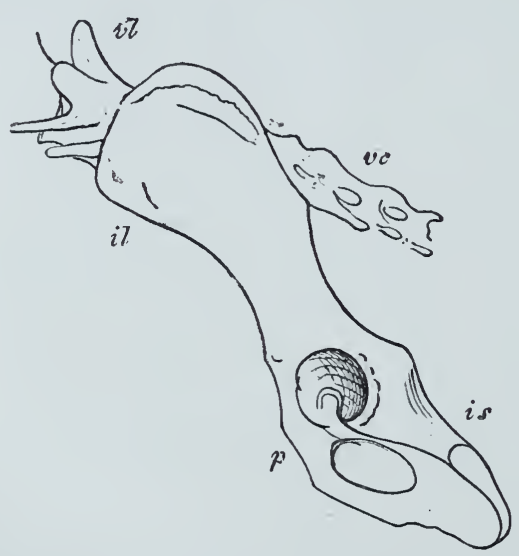

Fig. 272. View of the left side of the pelvis of a Dog. il Ilium. is Ischium. $p$ Os pubis. $v i$ Penultimate lumbar vertebra. vc Caudal vertebra. foramen, and is united ventrally with that of the other side.

The primitive pelvic cartilage gives rise to the ilium and ischium; the pubis is derived from a separate rudiment, which is united with the ischio-iliac rudiment in the acetabulum (Man). This leads us to think that the pubis is an independent piece of the skeleton, which has retained its independence in the Crocodilini. The ilium of the Mammalia is connected with a fer vertebræ. The ischium also may be united with the false sacral vertebræ (Dasypus, Bradypus). When the two ventral pieces are united at the ischio-pubic symphysis, as they are in the Marsupialia, many Rodents, Artiodactyla, and Perissodactyla, the pelvis is elongated in form. In the Insectivora and Carnivora the greater part of the symphysis is formed by 
the two pubic bones, and this is still more marked in the higher orders.

An independent adaptation, which is seen in various Mammals (Insectivora and Chiroptera), is the presence of a ligamentous connection instead of the pubic symphysis; this may be very wide in the female (Erinaceus).

When the posterior extremities are absent, the pelvic girdle also undergoes atrophy. There are rudiments of it in the Cetacea.

In the Monotremata and Marsupialia there are two bones in front of the pubes ; these marsupial bones are directed forwards ; in Thylacinus they are reduced to small rudiments in cartilage.

Gegenbaur, C., Beiträge zur Kenntniss des Beckens der Vögel. Jen. Zeitschr. VI.-Hoffmans, C. K., Beiträge zur Kenntniss des Beckens der Amphibien u. Reptilien. Niederländ. Arch. III.

\section{Posterior Extremity.}

\section{$\S 367$.}

We find just the same arrangements in the hind-limb as we have described as existing in the fore-limb. In Fishes the hind limb forms the ventral fin. In the Selachii its skeleton has the same characters as that of the thoracic fin; the most striking difference is that the rays are arranged in a simpler manner. The basale of the stem is generally greatly elongated. The joints which succeed the basal piece undergo a special metamorphosis in the male, where they are converted into copulatory organs.

The skeleton of the ventral fin in the Ganoïdei may be derived from this by supposing that there has been a peripheral reduction, very similar to that which we saw in the skeleton of the thoracic fin; and the Teleostean fin can be derived from the Ganoid. This is generally much simplified, both as regards the size and the number of its separate pieces, in consequence of the feebler development of the whole ventral fin. In both divisions the dermal skeleton takes part in increasing the surface of the ventral fin, just as it has been shown to do in the thoracic one.

When we come to compare the hinder extremity of the higher Vertebrata with the ventral fin of Fishes, we must again begin with the archipterygium, which seems to be the lowest stage of this extremity also. The segmentation of the extremity into successive pieces is a repetition of the arrangement which we met with in the skeleton of the arm. We distinguish the femur, tibia, and fibula; and lastly, in the foot, a tarsus, metatarsus, and phalanges. The four inner toes, and the parts that carry them, may be again regarded as joints of the rays which are given off from a row of bones extending from the femur, through the fibula, to the outermost toe. The tarsus is made up of ten pieces, three of which 
are attached to the leg; these are the fibulare, intermedium, and tibiale. There are two centralia; and five distal tarsalia carry the bones of the metatarsus (cf. Fig. 265).

In the Enaliosaurii the skeletal portions of the hinder extremity are an exact repetition of those of the anterior one; and even in

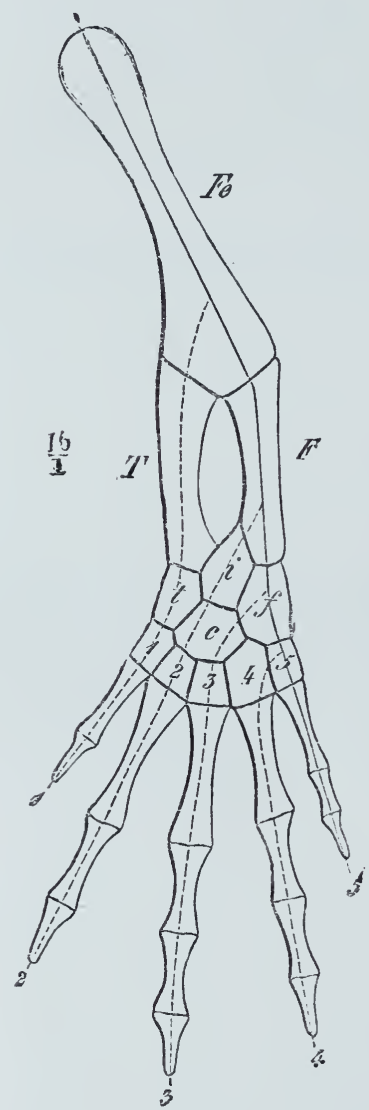

Fig. 273. Hinder extremity of a larva of Salamandra macu. losa. The dotted lines are drawn through the rays, to which the different pieces be. long. some of the Amphibia (Urodela) we meet with an arrangement which is the same in all essential points, so that we need not describe them specially. In most Urodela, all the five terminal pieces, or toes, are retained in the hind-limb; this is more distinctly like the primitive form than is the skeleton of the fore-limb. In Cryptobranchus, Menopoma, and others, the two centralia even are persistent. But in the Anura there is a very great change; the tibia and fibula are fused. In the place of the three proximal tarsal bones there are two long bones, which are, howerer, often fused at their ends; they are ordinarily known as the astragalus and calcaneum. The distal row of tarsal bones is also greatly reduced. Finally, we must note the presence of a rudiment of a sixth toe.

\section{$\S 368$.}

In the Chelonii there are unimportant modifications in the larger pieces of the extremities; in addition to this we must note the gradual concrescence of some of the bones of the tarsus, which is of great importance as explaining the skeleton of the foot in Birds, as well as in other Reptiles. An intermedium is united with a tibiale to form an astragalus; and the centrale is attached to, or even completely fused with, this bone. The fourth and fifth tarsalia similarly form a single bone, the cuboid. Oring to the formation of a single piece out of the bones of the first tarsal row, and the firm union that is effected between this piece, and the tibia and fibula, the foot gets to be articulated in a peculiar manner. It moves on an intertarsal joint. The skeleton of the Crocodile's foot is somerwat different. The tibia and fibula articulate with two bones, of which the fibulare has the greater power of movement. The larger bone, connected with the tibia, corresponds to the similar bone in the Chelonii. A piece of 
cartilage, which is more closely connected with the metatarsus, is articulated to it ; while a cuboid is articulated to the fibula. Owing to the independence of the fibula, we have here a peculiarity, which is only seen again in the Mammalia. In the Saurii, the tarsal bone developed out of four primary elements (Fig. 274, A ts) has no sigus of its constituent parts even in the embryo. It is immovably connected with the tibia and fibula, while the distal bones of the tarsus $(t i)$ are more or less connected with the metatarsus. This appears to have been most complete in the fossil Saurii (Ornithoscelida).

In these arrangements we may perceive an outline of what obtains in the foot of the Bird, which, in its embryonic condition (Fig. 274, B), presents us with those characters which are permanent in many Reptiles. The fibula $(p)$ extends to the tarsus. This is formed of two pieces of cartilage; the upper one $(t s)$ is undoubtedly homologous with the bone, which is made up of four elements in the Reptilia; the lower one $(t i)$ corresponds to the distal series of tarsal bones. The metatarsus is made up of five bones, which were primitively separate, but only four of these $(B, I-I V)$ carry toes, while the fifth is very small and completely fused with the distal portion of the tarsus. The difference between the adult and. embryonic arrangements consists in the degeneration of the fibula (Fig. $\left.275, b^{\prime}\right)$, which later on is attached to the tibia, as a small appendage $\left(b^{\prime}\right)$, and which never reaches the tarsus $(b)$. The proximal tarsal cartilage fuses with the tibia, and forms its articular condyle; the distal one unites with the single piece $(c)$, which is formed from the fusion of the three longer metatarsal bones, and in which no
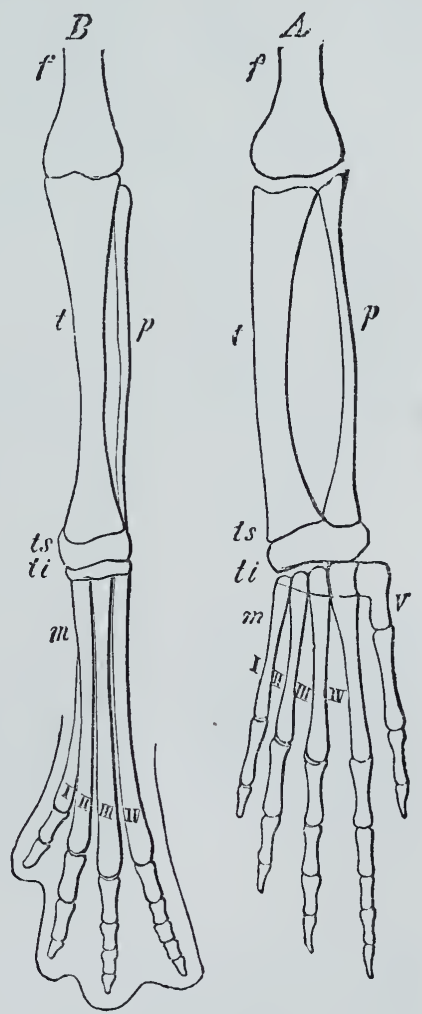

Fig. 274. Skeleton of the foot of a Reptile (Lizard) $(A)$ and a Bird $(B)$; the latter is in its embryonic condition. $f$ Femur. $t$ Tibia. $p$ Fibula. $t s$ Upper, $t i$ Lower piece of the tarsus. $m$ Metatarsus. $I-V$ Metatarsalia of the toes. permanent signs of separation can be made out except what is implied by the separate condyles at its distal end (Fig. 275, $c^{\prime}$ ). The metatarsal of the hallux remains distinct, and generally forms a small appendage of the large tarsometatarsus. The arrangements, therefore, which are seen in the foot of the Reptile are still further developed in that of the Bird, for the parts which in the former are merely united firmly together, are fused in the latter; the foot still moves on the same intertarsal joint. 
With regard to the toes, we find five to be the dominant number in the Reptilia ; it is in Birds only that they fall to four, or three, or even to two (Struthio). The phalanges of the

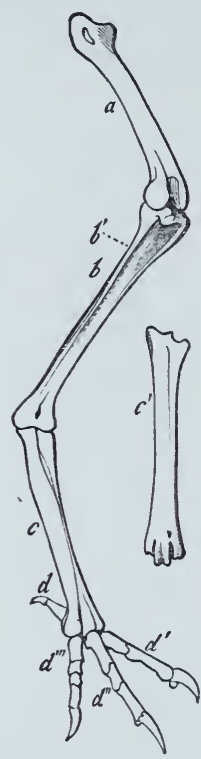

Fig. 275. Hinder ex. tremity of Buteo vulgaris. a Femur. $b$ Tibia. $b^{\prime}$ Fibula. c Tarso-metatarsus. $c^{\prime}$ The same piece isolated, and seen from in front. $d d^{\prime}$ $d^{\prime \prime} d^{\prime \prime \prime}$ Four toes. toes generally increase in number from within outwards; there are two on the hallux and five on the fourth toe. This holds for the Saurii, Crocodilini, and Aves. There are not so many in the Amphibia or Chelonii. Amongst the Reptilia the limbs are reduced in the snake-like Lizards, and in all Ophidii, among which the Peropoda only are provided with any rudiments of them at all.

Gegenbaur, C., Untersuchungen zur vergleich. Anat. I. Leipzig, 1864.

\section{$\S 369$.}

The special differentiations in the skeleton of the hind-limb of Birds and Reptiles do not resemble those which are seen in the Mammalia. As a rule it is less altered than the fore-limb. In the Perissodactyla, many Rodents, etc., the femur is distinguished by the possession of a third trochanter. The tibia is the most important bone of the leg; the fibula is often rudimentary, especially in the Ungulata. In the Artiodactyla the distal end remains; it is articulated to the tibia and to the tarsus (astragalus), and appears to enter into the composition of the latter. In some (as in Rodents and Insectivora) the tibia and fibula are complete, and are fused together.

The tarsus is the most characteristic part; it is attached by two pieces to the leg, but, as a rule, only one of these forms the anklejoint. The process on the second bone (calcaneum), of which there were indications in the Crocodilini, is still more developed. The centrale remains separate, but passes to the inner edge of the foot, where it forms the navicular. In some of the Prosimiæ it unites with the calcaneum to form a long process (Macrotarsi). Of the five distal bones the two outer ones are always replaced by the cuboid, while the three inner ones generally remain distinct (cuneiform). When the number of toes is diminished, these latter bones are often reduced; they may even fuse with the metatarsus, as in Bradypus. The cuboid also may be united to the navicular (Ruminantia).

In addition to its primitive function as an organ of support and of movement, the foot may be developed into a grasping organ; when this happens, the foot comes to resemble in many points the end of the fore-limb, or hand. But in all essential points of 
structure it is still a foot, so long as we hold to the anatomical conception of what hand and foot are, and do not put functional relations into the fore-ground; and if we do, then the proboscis of the elephant is a "hand" also.

This change in the character of the foot is seen in many Marsupials, Prosimiæ, and Primates. The chief change lies in the development of the first toe in a manner similar to that in which the thumb of the hand is developed. In Man also there are indications of the foot having once been a prehensile organ. When this function is lost, the hallux is shortened in proportion to the extent to which the whole of the sole of the foot ceases to take part in locomotion. The shorter hallux is then functionally inoperative (Digitigrade Carnivora). It disappears altogether in the Ungulata, where the limbs do nothing but aid in locomotion and support the body. The characters of the metatarsus and digits are parallel in character to those of the fore-limb in the Artio- and Perissodactyla. In the latter, the gradual conversion of the foot from a four-toed to a onetoed condition has been recognised as obtaining in the same palæontological series as that which has already been pointed out in reference to the fore-limb.

\section{Muscular System.}

$\S 370$.

The muscular system of the Vertebrata is differentiated from the mesoderm in the embryo, and is divided in a way corresponding to the metamerism of the whole body. Before the skeleton is differentiated the musculature below the integument unites with it to form a dermo-muscular tube, similar to that of segmented Invertebrata in many points, although not immediately derivable from one of those forms.

Its relations to the skeleton, and the formation of a muscular system connected with the skeleton, are therefore acquired in proportion to the extent to which they are concerned in the development of the skeleton. In Amphioxus, where the skeleton is essentially formed by the chorda dorsalis, the musculature, in the region of the trunk at any rate, has none of these relations; it is only in that portion of the body which encloses the respiratory cavity that it seems to have any relations to the visceral skeleton. The whole of the musculature is arranged in two lateral longitudinal masses, which are separated by connective tissue into dorsal and ventral masses. These longitudinal bands of muscle are separated by septa of connective tissue into a series of metameres (myocommata); and each septum serves for the origin as well as for the insertion of the fibres, which take a straight course between them. While this mass of muscle extends along the whole of the dorsal region of the body, it is modified in the ventral surface of the 
anterior portions of the body, owing to its relations to the branchial skeleton.

In the Cyclostomata also the greater part of the muscular system has no direct connection with the skeleton, for the superficial layers are here also merely connected with connective tissue, and the septa that form the metameres are to be found over the whole of the dorsal and caudal regions of the body. On the head, however, and on the visceral skeleton, we may see that some of the muscles are connected with the skeletal parts and differentiated in a special manner.

When the skeleton is formed it is necessarily connected with the musculature, since the skeletal parts grow down between the masses of muscles, following the septa of connective tissue. The primitive similarity, therefore, between the parts of the muscular system disappears, and a differentiation commences; this is implied, on the one hand, by the formation of a muscular system connected with the skeleton, and on the other by the special development of the remainder of the muscular system, which is not so connected, into a dermal musculature.

The whole of the muscular system requires, horvever, to be systematically investigated before we can know as much about it as we do about the skeleton. We must, therefore, confine ourselves in this description to a mere sketch, many details in which must be put in with great diffidence.

\section{Dermal Muscles.}

\section{$\S 371$.}

As we regard the dermal muscles as primitively forming a common complex with those which belong to the skeleton, we must distinguish from it those which belong to the integument as such.

Among the Cyclostomata some of the muscles of the trunk have no connection with the parts of the skeleton, and appear, therefore, to be essentially dermal muscles; even in the lower Gnathostomata, the greater portion of the large lateral masses of muscles on the trunk are only connected to the skeleton by the tendinous intermediate bands, which pass off from it; it has not, therefore, yet become a part of the skeletal musculature, in the sense that it forms bundles of muscles which are attached to the skeleton by their two ends of origin and insertion. This more indifferent condition enables us to understand how it is that there are no distinct dermal muscles. At the same time there are distinct layers of dermal muscles in the outer wall, at least, of the respiratory cavity in the Selachii, where they form part of a common constrictor.

In many other parts also of the body there are subcutaneous muscles, which are not connected with the large lateral muscles; the layer which runs along the lateral line in the Teleostei, and which is 
distinguished by its deeper colour, may be regarded as belonging to this system. In the Amphibia there are dermal muscles in the head, which act on the nasal orifices, and, in the Anura, in the anal region also. The muscles lying on the external nares are better developed in the Reptilia. The dermal muscles are of great functional importance in the Ophidii, as they produce a movement of the scales, which is of use in locomotion.

In Birds there are large flattened dermal muscles in various parts of the body; as in the Reptilia (Chelonii) there is a continuous layer of muscles in the neck; other dermal muscles take their origin from the skeleton, such, for example, as the musculi patagii, which pass into, and extend the membrane of the wing. The muscles which serve to move the remiges and rectrices also belong to this category.

The dermal musculature is more highly developed in the Mammalia. There is generally a large muscle below the integument of the trunk, which covers the dorsal region of the body, and is continued on to the head and neck; this is inserted by tendinous pieces into different portions of the integument, while anteriorly it is also inserted into the humerus. It is best developed in Echidna, Dasypus, and in Erinaceus, where it forms the muscle by which the body rolls itself up. In most of the Apes the large dermal muscle is distributed over the same parts as in other Mammals, but it is more distinct in its anterior portion. In the Orang and Chimpanzee this latter is represented by a muscular plate, which occupies the sides of the neck, and is continued on to the face; in Man it is reduced to the platysma myoides.

\section{Musculature of the Skeleton.}

\section{$\S 372$.}

The differentiation of the muscles, which is due to the connection between the muscular system and the skeleton, is very intimately connected with that of the skeleton, inasmuch as both parts have always reciprocal relations, owing to their being both formed for the same function. When, therefore, any portion of the skeleton is increased in size, the muscles that go to it are also increased, and, when another part undergoes atrophy, its muscles are correspondingly diminished. So, also, the greater functional independence of the muscles is bound up with a greater differentiation.

This differentiation gives rise to a number of systems of muscles, each of which can be again divided into subordinate complexes of more or less distinct muscles. The muscles of the trunk, of the cephalic skeleton, and of the appendages, may be distinguished as systems of this kind.

The above-mentioned primitive musculature gives rise to the 
muscles of the trunk, or lateral trunk-muscles. They consist of two masses of muscle which occupy the sides of the body, and extend from the head to the caudal end (m. laterales); they are separated from one another along the middle line of the dorsal and of the ventral surface. In the Myxinoidea, among the Cyclostomata, the ventral portion of these masses of muscle is distinguished by the oblique course of its fibres. It is doubtful whether this represents a new system or no. Each half is divided into a dorsal and a ventral portion, which are separated from one another along a horizontal plane drawn through the vertebral column; so that, altogether, there are four lateral muscles.

Each of the four lateral trunk-muscles is divided, in Fishes, into a number of segments by tendinous bands which correspond in number to the vertebræ (ligamenta intermuscularia); these may be

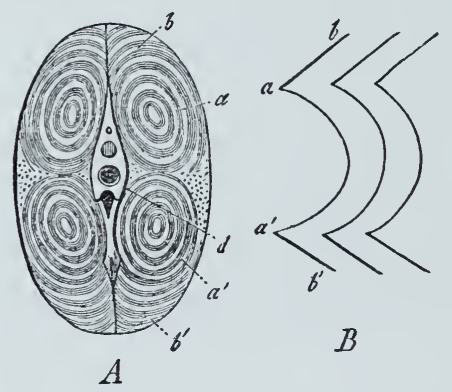

Fig. 276. A Transverse section through the caudal muscles of Scomber scomber. a Superior, $a^{\prime}$ Inferior lateral muscles. $b$ and $b^{\prime}$ Section of imperfect upper and lower investing cones. $d$ Centrum of the vertebra. $B$ Zigzag lines of the superficial ends of the Lig. intermuscularia in the tail of Scomber (after J. Müller). easily made out on the surface of the body, owing to their free edges forming distinct tendinous bands. The muscular fibres between any two intermuscular ligaments take a parallel course, and the ligaments, therefore, afford insertion as well as origin to one muscular segment. The muscles, therefore, are only indirectly connected with the skeleton. The tendinous septa first keep to one plane, but they then curve, and curve in such a way that in each dorsal muscle we can recognise a lower layer, which is made up of cones, one within the other, and with their apices directed forwards (Fig. 276, $A a$ ), and an upper one $(b)$ which is made up of parts of cones. The apices of these incomplete cones look backwards. In the ventral muscles the relations are so far reversed that the cones $\left(a^{\prime}\right)$ are placed above the incomplete cones $\left(b^{\prime}\right)$. In a vertical section through the tail of a Fish we see, therefore, two systems of concentric rings on either side, which project into one another (these are the sections of hollow cones); while above the upper ones, and below the lower ones, there are curved lines of varying length (the sections of the incomplete cones).

In the Perennibranchiata, and in the larval stages of the other Amphibia, we meet with essentially similar conditions; we meet, that is, with the same zigzag lines of the ligamenta intermuscularia, only they are not so markedly sinuous. When these ligaments take a more direct course the cones are not formed. In the adult Salamandrina the ventral portion of the lateral muscles in the trunk undergoes certain changes, and it is in the tail only that we can 
observe a symmetrical arrangement of the upper and lower halves; the persistent dorsal portion is, however, still separated by intermuscular ligaments into separate pieces, and is quite fish-like in character.

\section{$\S 373$.}

In the Amniota, other muscles are derived from the ventral portion of the lateral musculature of the trunk, but it is retained, with modifications, in the tail of the Reptilia and Mammalia, where it undergoes the same changes as the dorsal portion, which is still continued with great regularity on to the tail.

In the Saurii, it is still possible to make out a separation of the dorsal lateral muscles by the intermuscular ligarnents, but in all the rest of the Amniota they are still further differentiated, and give rise to a series of separate dorsal muscles. In the Mammalia we find them divided into a superficial and a deep portion. The former consists of the splenius, which is limited to the cervical region, and is partly inserted on to the skull, and partly on to the transverse processes of the anterior cervical vertebræ. The sacrospinalis is also one of the superficial muscles; it is broken up into a median and a lateral portion (iliocostalis and longissimus). They both contain masses of muscle which have their origin in the sacrum and ilium. As the muscle passes up to the skull, accessory masses are added on to it, which take their origin partly from the ribs, and partly from the transverse processes. The insertions of the iliocostalis and longissimus extend to the ribs, and those of the latter muscle to the transverse processes also. The deep layer is formed by the transverso-spinalis, which is formed of a system of muscles which arises from the transverse processes, and is inserted into the spinous processes; it is more or less broken up into various layers (semispinalis, multifidus).

Those parts of these muscles which reach the neck are often developed in size in proportion to the freedom of movement possessed by this region, and they may therefore be described as special muscles. The same remark applies to those ends of these muscles, which become developed into independent muscles, and extend to the skull. The trachelomastoid is the cranial portion of the longissimus, and the biventer and complexus of the semispinalis. The musculi spinales and interspinales belong to this group. The rectus capitis posticus major forms the most anterior spinalis muscle; and the rectus capitis posticus minor is the first of the interspinales.

The small muscles which move the vertical fins of Fishes must be regarded as being derived from the primitive lateral muscles of the trunk.

The intercostal muscles must be regarded as a group derived from the lateral trunk muscles. In Fishes these muscles are not 
differentiated, inasmuch as the muscles between the ribs, or their equivalents, are still portions of the lateral muscles. The ribs themselves lie in the intermuscular ligaments. In the higher divisions of the Vertebrata a more distinct differentiation obtains. These muscles are best developed in the Ophidii. The muscles which are found between the rudimentary ribs, which are fused with the vertebræ, or between the transverse processes (intertransversarii), also belong to this group of intercostal muscles. So, too, do the levatores costarum, and the muscles which lie on the inner surface of the wall of the thorax (thoracici interni), and the scaleni. The size of all these muscles varies very greatly according to the extent and power of movement of the ribs ; special retractors may be added to the elevators of the ribs, as in the Ophidii.

The broad ventral muscles must also, in all probability, be regarded as belonging to the system of intercostal muscles; these are found in those regions of the ventral wall where there are no ribs. They consist of the obliquus externus, obliquus internus, and transversus abdominis. The obliquus extermus corresponds to the intercost. externus, and the internus to the intercost. internus. The tendinous bands found in many Amphibia, and in the Saurii, must be regarded as remnants of the primitive intermuscular ligaments. The obliquus externus generally takes its origin from a large portion of the thorax; in the Reptilia it is divided into several layers.

In the Amphibia the transversus abdominis is a large muscle, as it is also in all Reptilia except the Ophidii, where it is absent. It extends as far forward as the thoracic region. In Birds it extends as far as the hinder edge of the sternum, but in Mammals it has a wider area.

The rectus abdominis appears to be the proportionately least altered portion of the primitive musculature ; its fibres retain their primitive course, and its inscriptiones tendineæ are remnants of its primitive septa. It generally extends from the sternum to the pelvis in the Amphibia, but when the sternum is shortened it is continuous with the sterno-hyoid (Amphibia).

In the Crocodilini the transverse tendinous bands are ossified, and form the so-called "abdominal ribs." The M. pyramidalis must also be regarded as part of the recti abdominis; it is found in the Salamandrina, Crocodolini, Ratitæ, and also in many Mammals. In the Monotremata and Marsupialia it is largely developed. So much so, indeed, that it nearly reaches to the sternum, and so covers the rectus; it has its origin in one edge of the marsupial bone.

\section{$\S 375$.}

The branchial skeleton of Fishes is provided with a special system of muscles, which is repeated between each of its segments. As the primary pieces of the jaw also belong to this skeleton, their muscles must be regarded as differentiations of the muscular system 
of the branchial skeleton. Part of these muscles have their origin on the skull, others belong to the different arches, while others again are arranged transversely, and act so as to approximate the arches of either side to one another. Muscles pass off from the branchial arches to the branchiostegal rays. They are well developed in the Selachii, but rudimentary in Osseous Fishes, where they appear to be converted, on the hyoid arch, into the muscles of the operculum, and of the dermal rays of the gills. The Amphibia are provided with a similar musculature during their larval stages; this is partly derived from the muscles of Fishes, and is retained by the Perennibranchiata throughout their life. When the branchial framework disappears, and the hyoid becomes more independent, part of the branchial musculature is taken up by it.

As to the muscles of the jaw, it can be shown that an adductor of the two parts of the mandibular arch in the Selachii undergoes a certain amount of differentiation into several parts, and forms the rudiment of the muscles of mastication. When the palato-quadrate, or the bones differentiated in it, are fixed to the cranium, these muscles are inserted into the lower jaw. In the Amphibia and Reptilia an inner portion of this mass of masticatory muscles is differentiated as the pterygoid, and this again may be divided into two (Pt. externus and internus) (Saurii); the differentiation of the temporal and masseter muscles is indicated by their arrangement in layers. In both classes the depression of the jaw is effected by a muscle, which forms a short but powerful belly on the hinder edge of the lower jaw. It corresponds to the posterior belly of the digastric of Mammals. The Ophidii are distinguished by an increase in the number of the muscles; in addition to adductors of the lower jaw, special muscles, which move the quadrate and the various bones of the palatine arcade, may be seen to be largely developed in the Eurystomata. In Birds there are similar muscles, which elevate the pterygoids and the quadrate, and produce the movement of the maxillary apparatus. T'he temporalis is the largest of the proper mandibular muscles, and the adductor, which is present in the lower divisions, where the two halves of the jaw are movable, is replaced by a muscle which extends transversely between the two halves of the jaw, and has a different function.

The masticatory muscles of the Mammalia are similar in number, origin, and insertion to the same muscles in Man; as a rule they are larger, but otherwise they do not differ in any points except those which are due to the form of the surfaces of origin and insertion provided by the proper bones.

\section{$\S 376$.}

Of the paired appendages, the fins of Fishes possess a number of muscles on the girdle, as well as on the free portion, but it has not been possible to compare these muscles with those of other 
Vertebrates. They are divided into those which pass to the girdle, and those which belong to the appendage itself.

When the appendages are metamorphosed, the musculature undergoes changes also; the muscles are, indeed, simplified in number, but are entrusted with more functions, in consequence of the greater freedom and independence of the skeletal parts.

As compared with the Fishes the most important change is the extension of the musculature of the shoulder-girdle and of the anterior extremity over the dorsal and ventral surface of the body; this obtains in all the higher Vertebrata. The muscles developed from the superior lateral trunk-muscles are covered over by muscles which go to the limbs, and which are substituted in Fishes by a mass of muscle which arises from the head. These are slightly differentiated in the Perennibranchiata, and more so in the Caducibranchiata; they form those muscles, which represent the cucullaris and the sterno-cleidomastoid in the higher divisions. They are supplied with nerves from the head. Other muscles, which are probably derived from the muscles of the trunk, and which pass to the appendages, partly from the back, and partly from the thorax, are added on to them.

The other muscles, which belong to the limbs themselves, are derived from the layers, which in Fishes are more similar to one another, and which cover the dorsal and ventral faces of the skeleton of the thoracic fin. The musculature undergoes great changes owing to the reduction of this fin, and to the modifications undergone by the parts that are persistent; the changes, therefore, in the anatomical characters of the musculature in the various divisions run parallel to the functional changes in the value of the appendages.

In the hinder limbs the relations of the pelvic girdle to the axial skeleton are, at first, the factors which affect the characters of the musculature. The absence of any connection between these skeletal parts is the cause of the greater independence of the pelvic girdle in Fishes; so far as this affects the musculature it is made up for by its more indifferent character. The more intimate connection between the pelvic girdle and the axial skeleton in the Amniota diminishes its powers of movement, and, consequently, the development of the muscles by which this is effected. The muscles which belong to the limb itself have their origin in the pelvic girdle, or in the skeleton of the limb; they seem, on the whole, to be divided into the same groups as those of the fore-limb, with the exception of such modifications as are due to the difference in the function of the two limbs.

\section{$\S 377$.}

The subvertebral muscles form a special group. They lie below the vertebræ and their lateral processes, so that in the thoracic region they lie within the thorax.

The musculus longus forms an anterior portion of the muscles 
below the vertebral column; this is first seen in Reptiles; it generally commences within the thoracic cavity, and extends along the neck up to the skull. It breaks up into several portions, which are distinguished as longus colli, or longus capitis, according to the point of insertion.

Another subvertebral system of muscles appears to lead to the formation of the Diaphragm. This arrangement does not obtain in Fishes, and it is doubtful whether the separate bands of muscle, which embrace the œsophagus in the Amphibia, can be looked upon as forming a rudimentary diaphragm. Among the Reptilia, the Chelonii have a muscular layer over the lamella of the peritoneum, which encloses the lungs; this takes its origin partly from the centra of the vertebræ, and partly from the rib-like transverse processes. In the Crocodilini there is no diaphragmatic muscle, for it is not possible to regard the highly-developed peritoneal musculature as being a formation of this kind, as it has its origin in the anterior wall of the pelvis. There are indications of a muscular investment of the lungs in Birds ; it is best developed in Apteryx.

It is in the Mammalia only that there is a well-developed diaphragm forming a partition between the thoracic and abdominal cavities. The oblique direction, taken by the muscle in the Reptilia and Aves, is necessarily converted into a transverse one. The muscular portions arise in part from the vertebral column, and in part from the ribs; they pass into a centrum tendineum, which is occasionally absent (Delphinus).

Humphry, G. M., Observations in Myology. Cambridge and London, 1872.Furbringer, M., Vergl. Anat. der Schultermuskeln. Jen. Zeitschr. VII. VIII. Morphol. Jahrb. II. -De Man, Vergl. myolog. u. neurolog. Stud. Leiden, 1873.-Vetter, B., Vergl. Anat. der Kiemen. u. Kiefermuskulatur der Fische. Jen. Zeitschr. VIII.

\section{Electric Organs.}

$\S 378$.

The so-called electric organs are special apparatuses which are found in a very few Fishes; they are of importance, from an anatomical point of view, in consequence of the large masses of nerves which end in them, and from a physiological point of view, in consequence of the development of electricity in them. The endings of the nerves have very much the same relations as have the ends of motor nerves in muscular fibres, while there are many points in the development of these organs which point to their having had their origin in metamorphosed muscles. There is sufficient reason, therefore, for regarding these organs as belonging to the muscular system, although we do not yet know anything of their earlier stage, in which they probably appeared as muscles. 
The Fishes which are provided with these organs belong to the genera Torpedo and Narcine among Rays, Gymnotus among Eels, and Malapterurus among the Siluroids; Mormyrus also has similar organs. There is a pseudo-electric apparatus in Raja.

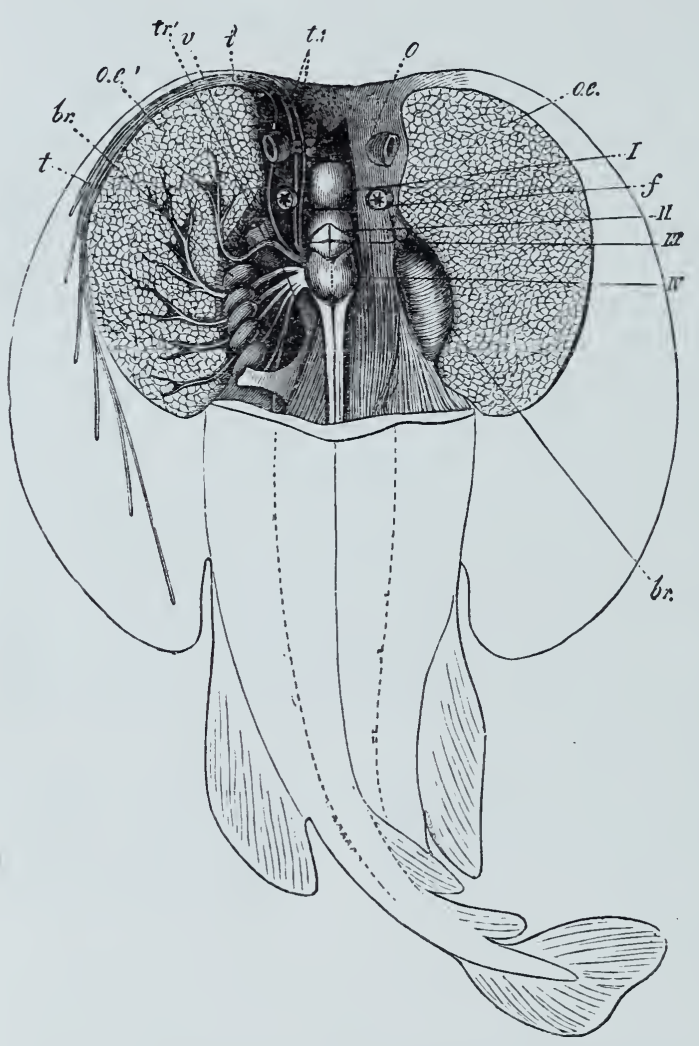

Fig. 277. A Torpedo, with the electric organs dissected out. On the right the surface only of the organ (oe) is shown. On the left side the nerre-trunks passing to the organ are dissected out, and part is followed laid open, and the brain displayed. I Fore-brain. II 'Tween-brain. III Mid-brain. IV Electric lobe. $v$ Vagus. $t r$ Trigeminal. $t r^{\prime}$ Its electric branch. $o$ Eyes. $f$ Spiracular cleft. $t$ Mucous tubes of the skin. $b r$ Branchix; on the right they are covered over by a common layer of muscles, on the left the separate branchial sacs are shown. some way into the organ. The cavity of the skull is

Although these organs differ greatly from one another in position, and in their broader anatomical details, in the different genera, they all agree in being composed of "alveoli" of various forms, which are bounded by connective tissue, and filled with a jelly-like substance. The nerves pass to one surface of these alveoli, where they form fine networks, and give rise, finally, to an " electric plate," which represents the ends of these nerves.

The relation of this plate to the whole apparatus, and its relations to the nerves, are described in the following account of what is seen in the Torpedo. The electric organ $(o e)$ is placed between the head, the branchial sacs (Fig. 277, $b r)$, and the protopterygium of the thoracic fin; it is as deep as the whole body, and is invested by a tendinous membrane, which is covered by the integument above and below. Each organ
is made up of a number of parallel prisms, which again consist of a series of elements set in rows one on the other; these are the above-mentioned alveoli. They are closely united with one another by connective tissue; they all receive inferiorly the nerves 
which pass into the prisms, and the free surface of the electric plates are directed dorsally. Five large nerve-trunks pass to the organ; these rami electrici belong to different cranial nerves, but principally to the vagus; they are distributed between the prisms.

In the other Electric Fishes these organs agree with what has been described, so far as their more minute characters are concerned, but they differ in position, and in the characters of the alveoli. In the Electric Eel, for example, the organs lie in the caudal region of the body, just below the external integument. In Malapterurus the organ extends over the whole surface of the body, just below the integument; and in the Mormyri again it is found in the tail. 'There are corresponding differences with regard to the nerves, so that we may conclude that these organs are morphologically different, notwithstanding their histological and physiological similarity.

Schultze, M., Zur Kenntniss d.elektr. Org. d.Fische.Abh. d. Naturforsch.Gesellsch. Halle, 1858.

\section{Nervous System.}

\section{$\S 379$.}

The central organs of the nervous system are placed above the axis of the spinal chord, in the canal formed by the superior system of arches of the axial skeleton. They consist of symmetricallyarranged nervous masses, which are similar in character throughout, in the Acrania only; in the Craniota they are differentiated into two large portions, the brain, and the spinal chord. Although the latter has without doubt a great similarity to the ganglionic chain of segmented Invertebrata, it is quite impossible to derive the spinal chord from it; the central nervous system of the Vertebrata is rather to be regarded as representing the superior, or cerebral, ganglia of the Invertebrata in an extremely high state of develo p ment. The earliest rudiment is derived from a differentiation of the ectoderm. The "medullary plate," which is formed in this way in the Invertebrata, does not extend along the whole

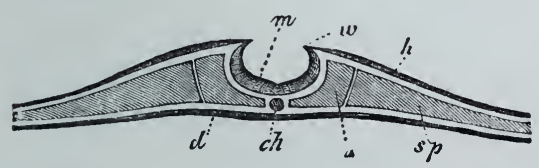

Fig. 278. Diagrammatic section through the embryo of the Fowl (end of the first day). ch Notochord. $u$ Primitive vertebræ. $s p$ Lateral plates. $m$ Medullary groove. $w$ and $h$ Epiblast. $d$ Hypoblast (after Remak).

a groove by the uprising of its edges $(w)$, which are continued into the neighbouring ectoderm (epiblast) (Fig. 278, $h$ ); this is gradually converted into a closed does so at first, it does not keep pace with the growth of the body (Ascidiæ); in the Vertebrate embryo, however, it is of very nearly the same length as the body, so that the central nervous system extends throughout the whole length of the body.

The medullary plate forms

of the rudimentary body, or if it 
tube. This gradually sinks away from the surface of the body, owing to the growth over it of the epiblast, and of parts differentiated from the mesoderm. The medullary tube, which is formed in this way, remains as a simple chord in Amphioxus, the most an-

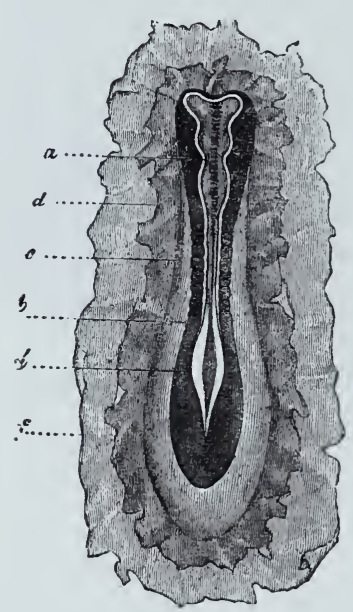

Fig. 279. Embryo of the Dog seen from behind, with the rudiments of the central nervous system, of which the medullary plate $(b)$ forms a groove open superiorly. $\quad a$ The rudiments of the three primitive cerebral vesicles. $a^{\prime}$ Sinus rhomboidalis in the lumbar region. $c$ Lateral plates, which bound the rudi. mentary body. $d$ Epiblast and mesoblast. $f$ Hypoblast (after Bischoff). terior region of which contains an enlargement of its central canal. But in the Craniota, diverticula appear in the most anterior portion before it is completely closed (Fig. 279, a) ; these form the rudiments of the brain, while the remainder of the medullary tube is equally differentiated throughout, and forms the rudiment of the spinal chord.

In addition to their position, which is always an important point in comparison, the rudiments of the nervous centre of the Vertebrata have, in common with those of various Invertebrata, certain relations to the higher sensory organs (and especially to the optic organ); and in this respect the Tunicata are those which exhibit the closest affinities. In them, as in the Vertebrata, the whole of the medullary tube is not closed in the same way; in the cerebral region, it is in connection with the exterior for a long time. In comparing the nervous system of Vertebrates with that of the Tunicata the chord, which is continued along the dorsal region, and on to the tail in the larvæ of the Ascidiæ, and in the Appendicularia $(\$ 305)$, is of importance; this, which is distinguished by its ganglia, appears to indicate the path by which the hinder portion of the central nervous system of the Vertebrata was phylogenetically developed, and gradually converted into the spinal chord. As there is such a great difference between the true central organs and this chord, that it is impossible to regard the chord as a true continuation of the central organ, or even as a portion of it, which merely differs in consequence of its position, it must be supposed that the brain, or the most anterior portion of the medullary chord in Amphioxus, represents the more primitive portion of the nervecentre; while it is also the first to appear in the embryo. The similarity between the rudimentary spinal chord and brain would then be merely a condition, which had been acquired by the Vertebrata, and which had its starting-point, phylogenetically, from a chord which was continued on from the primary nerve-centre, such as we now meet with in the Tunicata. According to this view the whole medullary tube is not derived, phylogenetically, 
from a mere elongation of a shorter nerve-centre, but from the gradual development of a nerve-chord, which primitively formed a peripheral apparatus only. The differences in the characters of the brain (exclusive of the medulla oblongata) and of the spinal chord, so far as regards the arrangement of the white and gray substance, serve to confirm this view, which is also supported by other facts.

\section{A. Central Organs of the Nervous System.}

\section{a) Brain.}

$\S 380$.

Three successive portions are developed from the rudiments of the brain (Fig. 280 a), the cavities in which communicate with one another. The last of these passes freely into the medullary tube behind it. These primitive cerebral vesicles give rise to new segments, so that we can soon distinguish five. The first is known as the Fore-brain or Prosencephalon (Fig. 280, a); the next as the Twixt-brain or Thalam encephalon $(b)$; the Mid-brain or Mesencephalon $(B C c)$ forms a third swelling; and this is succeeded by the Hind-brain or Metencephalon $(d)$, and the After-brain or Myelencephalon (e), which is directly continuous with the spinal chord, and with the metencephalon. The metencephalon forms the most anterior portion of the roof of the myelencephalon, and is not therefore as distinct as the rest of the cerebral vesicles. At first, the vesicles are placed one behind the other, and lie in the line of the longitudinal axis of the spinal chord, but they soon come to be set at an angle to one another. This is due to the unequal growth of the upper and lower portions, for the upper ones increase greatly in size. Those parts which are least developed become covered over by the growth of some of the upper parts. Between the prosencephalon and thalamencephalon the wall is thinned out, and a fissurelike portion developed (primitive cerebral
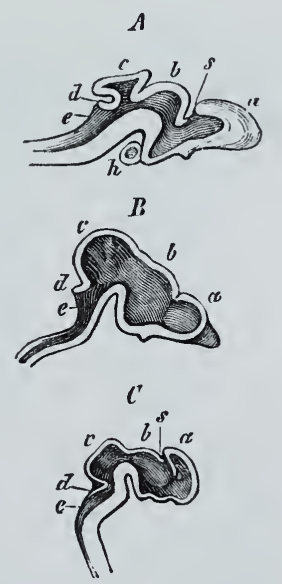

Fig. 280. Vertical and median sections through a Vertebrate brain. $A$ of a young Selachian (Heptanchus). $B$ Of the embryo of an Adder. $C$ Of the embryo of a Goat. $a$ Prosencephalon. $b$ Thalamencephalon. $c$ Mesencephalon (in $A$ it is marked by $d$ ). $d$ Meten. cephalon. e Myelencephalon. $s$ Primitive cere. bral cleft. $h$ Hypophysis. cleft, Fig. 280,s), into the interior of which a process from the envelopes of the brain is continued. This is not a true lacuna, but is merely due to the gradual thinning-out of the wall of this portion. The epiphysis (pineal gland) is developed from a part of this roof.

The lower portion of the thalamencephalon forms the floor of the 
second cerebral vesicle, and gives rise to a diverticulum, which is found in all Craniota, and is known as the infundibulum. From the lower side of the head a depression of the ectoderm grows towards this diverticulum; later on, the ingrowth becoming pinched off, forms a portion of the cerebral appendix attached to the infundibulum (hypophysis). The range of the position of the depression for the hypophysis as far forward as the entrance into the cavity of the mouth enables us to recognise in this structure an organ, which primitively did not belong to the nervous system at all, and the function of which is still a matter for speculation.

Just as the upper wall between the fore- and twixt-brains gets thinned out, so too the roof of the myelencephalon is thinned out, in such a way that no roof remains but such as is formed by the outermost vascular layer of the nerve-centre, the pia mater. The large cavity which is thus roofed over forms the fourth ventricle.

The ventricles, or cavities in the portions

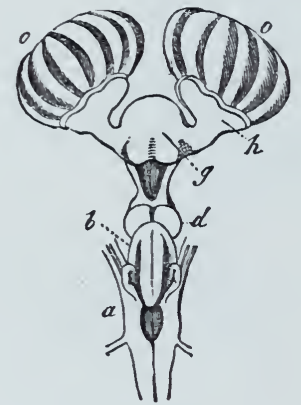

Fig. 281. Brain of a Shark (Scyllium catn. lus). $h$ Olfactory lobes. $g$ Prosencephalon. $d$ Thalam- and mes-ercephalon. $b$ Metencepha. lon. a Myelencephalon. o Nasal capsnles (after Busch). derived from the primary cerebral vesicles, are connected with one another in just the same way as the cavities of the cerebral vesicles.

The brain of the Cyclostomata is the simplest in form; among them the lowest grade is occupied by the Myxinoidea, where the various segments have very nearly the same characters.

A portion, which is developed from the fore-brain, and which gives off the olfactory nerves (bulbus or lobus olfactorius), generally forms large lobes, which, in the Selachii, are connected with the brain by a more or less long tractus olfactorius (Fig. 281, $/$ ). The ventricle of the prosencephalon is continued into them. They may also be fused with the prosencephalon, which is larger than the other divisions, in the Selachii $(g)$, and gives indications of a separation into two, four, or more paired pieces. It is large also in the Ganoïdei (Fig. 282, g), while in many Teleostei it is greatly reduced in size, as compared with the other regions of the brain.

In the Selachii the thalamencephalon is distinctly separated from the mesencephalon (Fig. 281, d), but in many Teleostei it is intimately connected with it. The anterior portion of its roof contains the above-mentioned cleft, and this portion is not unfrequently developed into an elongated tract, which forms a longitudinal commissure between it, and the prosencephalon (many Sharks and Ganoids). The remainder of the primitive roof, which contains the hinder portion of the cleft, is sometimes very large, and divided into two hemispheres; this is the case in the Selachii and many Teleostei. The floor of this segment, which surrounds the infundibulum, and forms the lobi inferiores at the base of the brain, is 
simple in the Cyclostomata, and in the Selachii presents indications merely of its divisions. It is not developed to any great size except in the Teleostei. The succeeding mesencephalon is small in the Myxinoids, but larger in Petromyzon. In the Selachii it appears to be united to the thalamencephalon, while the portion which corresponds to it, in position at any rate, is regarded as the cerebellum. This portion is very greatly developed, so much so, indeed, that it covers over those parts of the brain that lie in front of and behind it (Fig. 281, b). In the Teleostei this part of the brain is proportionately more developed, and sometimes has the form of a protuberance directed forwards or upwards. The rest of the brain, which lies behind the mesencephalon, must be regarded as one piece. It is of great importance as being the region from which most of the cerebral nerves take their origin. Its roof is unequally developed. That is to say, in the hinder, and larger, portion, it is soon atrophied, so that the internal cavity (sinus rhomboidalis), which is widened out anteriorly, is only covered over by membrane. In the Selachii and Chimæræ the edge of this sinus is thickened anteriorly (lobi nervi trigemini). It is simpler in the Ganoïdei and Teleostei. But in all Fishes it is continued mesially into a transverse lamella (Fig. 282, $b c)$, which covers in the sinus anteriorly, and has the mesencephalon projecting over it, when this portion is very
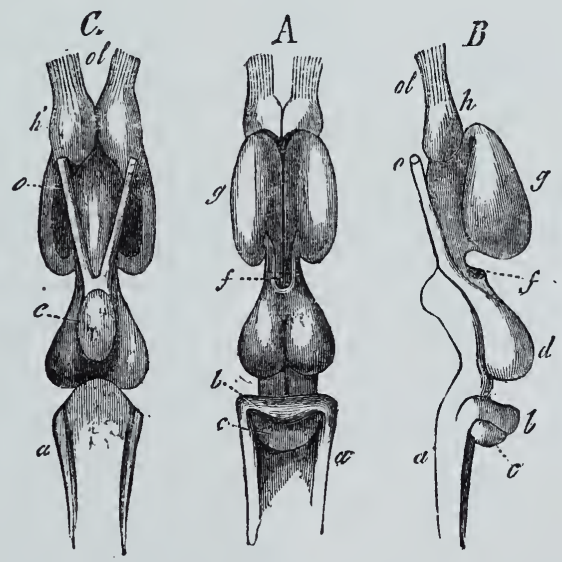

Fig. 282. Brain of Polypterus bichir. $A$ From above. $B$ From the side. $C$ From below. $h$ Lobi olfactorii. $g$ Prosencephalon. $f$ Thalamencephalon. $d$ Mesencephalon. $b c$ Metencephalon. a Myelencephalon (medulla oblongata). ol N. olfactorius. o N. opticus (after J. Müller). large. This transverse lamella appears to correspond to the cerebellum of the higher Vertebrata, while the base and sides of the sinus are formed by the myelencephalon (medulla oblongata). As we pass from the Selachii to the Teleostei we note that the medulla oblongata diminishes in size; in many Sharks it forms the largest portion of the brain. And this corresponds to a primitive stage, in which it and the mesencephalon form the largest part of the whole brain.

When it is still more developed, swellings may be observed on the sides of the fourth ventricle; these are set in series, and correspond to the points of origin of the roots of the vagus (lobi nervi vagi). The lobi electrici of the Torpedines are differentiations of this kind, which, however, unite above the narrowed ventricle (cf. Fig. 277, IV). 


\section{$\S 381$.}

The brain of the Amphibia resembles that of the Fishes in many points. The prosencephalon (Fig. 283, $b$ ) is divided into two hemispheres, and shows signs of being enlarged backwards. The cavity within it is divided into two lateral ventricles, one for each half, and these are continued forwards into the olfactory lobes $(a)$. At first, these lobes are placed at the side of the prosencephalon (b), and

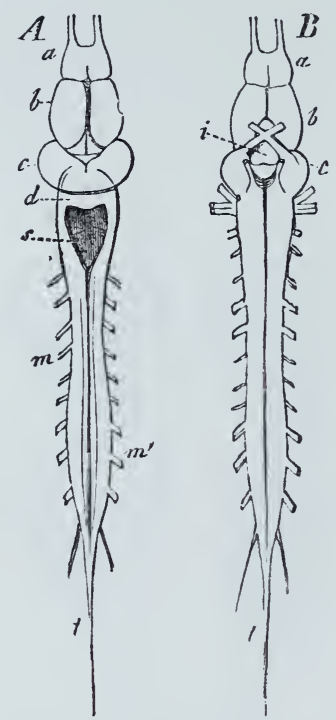

Fig. 283. Brain and spinal chord of the Frog. $A$ from above, $B$ from below. a Lobi olfactorii. $b$ Prosencephalon. $c$ Mesencephalon. dMetencephalon. e Myelen. cephalon. $s$ Fourth ven. tricle. $m$ Spinal chord. $t$ Filum terminale of the chord. are directly attached to it, but they may become closely fused with the prosencephalon, and with one another. The olfactory nerve arises on their lower surface, some way back, and near the prosencephalon. The thalamencephalon is differentiated during the larval stage from a portion which is common to it and the mesencephalon. In front of it is the primitive cerebral cleft, which is more or less continued into the thalamencephalon, and carries the epiphysis cerebri. The cleft extends anteriorly into the lateral ventricles, which are enclosed by the two hemispheres of the prosencephalon. On the lower surface of this portion there is an eminence, which corresponds to the lobi inferiores.

In the Urodela the mesencephalon remains at a certain stage, which is temporarily presented by the Anura; it is in the latter only that it becomes of any size, and is divided into two halves $(c)$. The metencephalon, however, retains its primitive form of a lamella, which bridges over the fourth ventricle $(d)$.

In the brain of the Reptilia the angulation which we already observed in Fishes has been much increased in the region of the thalam- and mes-encephalon, owing to the increased development of the upper parts; this produces a change in the relative position of the parts, which is still more marked in the higher divisions (compare the sections in Fig. 280). The prosencephalon is more largely developed, and has the form of two hemispheres, covering the thalamencephalon, and broadest behind. The olfactory lobes are attached directly to them. The lateral ventricles are very large, and communicate at the cerebral cleft with the third ventricle, which is placed between the two halves of the thalamencephalon, and is provided with a large infundibulum. The mesencephalon is divided by a groove into two hemispheres, which sometimes project very far forwards. The metencephalon varies a great deal; in the Ophidii and Saurii it 
remains at a low stage of development, and forms a small lamella, which is raised up, however, in a vertical direction; in the Chelonii (Fig. 284, A IV) and Crocodilini it is broader, and in the latter it is distinguished by the large size of its median portion.
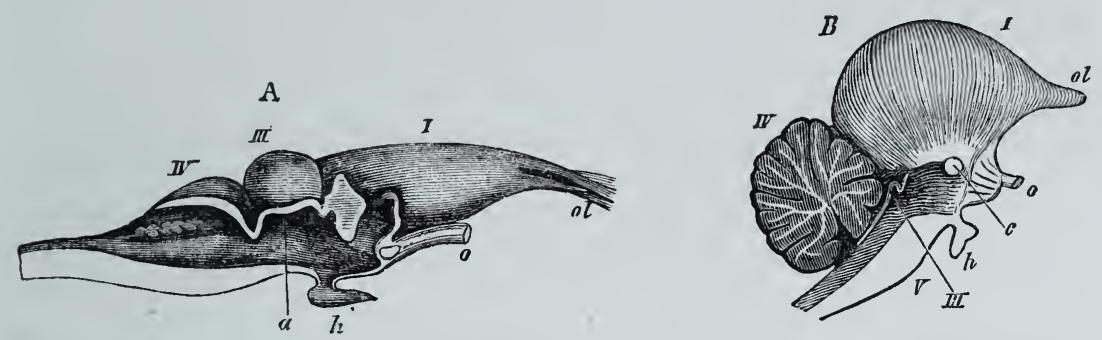

Fig. 284. Brain of a Chelonian (after Bojanus). B Of a Bird. Vertical median sections. I Prosencephalon. III Mesencephalon. IV Metencephalon. $V$ Myelen. cephalon. ol Olfactory. o Optic nerve. $h$ Hypophysis. $a$ (in $A$ ) connection between the two hemispheres of the mesencephalon. $c$ Anterior commissure.

This condition connects the Reptilia with the Aves, which are distinguished by the great proportionate size of their prosencephalon, the hemispheres of which are often greatly broadened out. They are connected by a fine anterior commissure (Fig.284, $B$ c), and enclose a ganglionic mass, which projects inwards from the side wall, and converting the primitive cavity into a narrow space which is covered over by the thin-walled roof of the hemispheres, itself forms the largest part of the prosencephalon. These masses may be observed in as low forms as the Amphibia, and in the Reptilia they are very large (Fig. 286, $A$ st). The small thalamencephalon, which is completely covered over by the hemispheres of the prosencephalon, has its roof divided. The mesencephalon, which is very large in the embryo, is divided into two pieces, which are pushed down to the sides of the brain (Fig. 285, c), and have the general
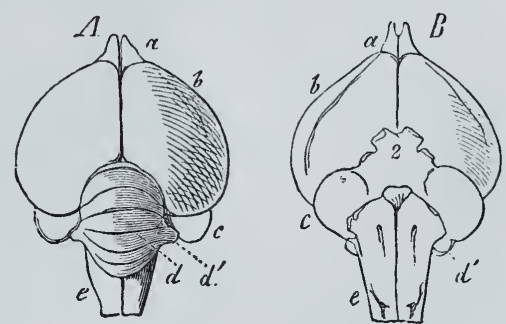

Fig. 285. Brain of the Domestic Fowl. $A$ From above, $B$ From below. a Bulbi olfactorii. $b$ Hemispheres of the prosencephalon. $c$ Mesencephalon. $d$ Metencephalon. $d^{\prime}$ Its lateral parts. e Myelencephalon (after C. G. Carus).

internal cavity continued into

them. The large median portion of the cerebellum is transversely laminated, and, owing to its size, covers over the whole of the myelencephalon.

\section{$\S 382$.}

In the Mammalia the brain closely resembles that of the lower forms in its earliest stages only (cf. Fig. 280), for, owing to its special line of differentiation, it is very different to that of Birds and 
Reptiles. The most important changes are seen in the prosencephalon, which has the olfactory lobes, which are as a rule still hollow, connected with its lower surface; these lobes are more or less covered over by the hemispheres, according to the extent to which they are developed. The prosencephalic hemispheres are always separated by a fissure, which is very deep anteriorly. They are primitively connected together by a commissure which is placed in front of the primitive cerebral cleft; through the cleft there is a passage into the cavities of the prosencephalon, or lateral ventricles. When they are more highly developed, the hinder portions of the hemispheres increase in size; the cleft, which was at first small, is laterally extended and disappear's from the surface, being completely
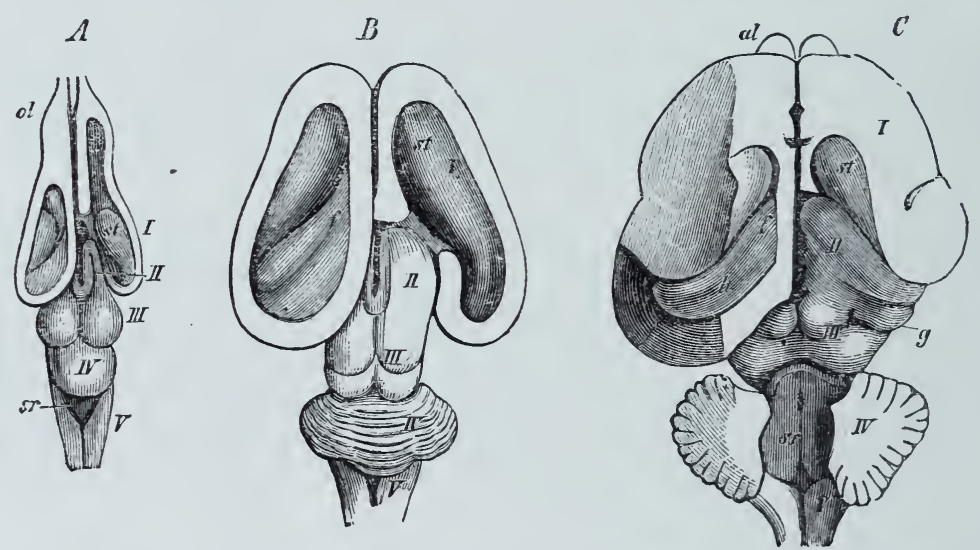

Fig. 286. Differentiation of the prosencephalon. A Brain of a Chelonian, $B$ of a fotal Calf, $C$ Of a Cat. In $A$ and $B$ the roof of the prosencephalic cavity is removed from the left side, and the fornix from the right. In $C$ the whole of the lateral and posterior portions of the right prosencephalic lobe are removed, and so much on the left as is necessary to display the upward bend of the cornu ammonis. In all the figures $I$ marks the prosencephalon. II Thalamencephalon. III Mesencephalon. IV Metencephalon. $V$ Myelencephalon. ol Olfactory bulb (shown in $A$ as communicating with the cavity of the prosencephalon). st Corpus striatum. $f$ Fornix.

$h$ Pes hippocampi major. $s r$ Rhomboidal sinus. $g$ Geniculate process.

covered over by the hinder wall of the lateral ventricles, which have grown out at the sides and behind. With this is correlated the differentiation of the primitive commissure into a system of commissures, the lowest stage of which is represented in the Monotremata and Marsupialia. The primitive commissure is first differentiated into an inferior and a superior portion; the former is the anterior commissure; the latter forms a slender bridge, which is placed above the anterior edge of the thalamencephalon, and below which there is on either side the entrance into the lateral ventricles; these last extend downwards and backwards. In their anterior region the corpus striatum forms a projection (Fig. 286, BC st), and posteriorly there is a rounded process, which is connected with the upper portion of 
the system of commissures, and which forms the posterior boundary of the margin of the cleft, which always lies above the thalamencephalon (cornu ammonis sive pes hippocampi major) $\left(C l_{l}\right)$.

The superior commissure is converted into two different, but connected, structures. The sides of one bound the entrance into the lateral ventricles superiorly, and it passes, at its sides and below, into a band which is placed on the hippocampus major. This portion (fornix) (B $(f)$ commences anteriorly by ascending columns, is somewhat broadened out over the thalamencephalon, and is continued into the posterior descending columns. Superiorly it is connected with a portion of the commissural system, the corpus callosum, which, at first continuous with it, becomes anteriorly separated from it. The backward extension of these commissures is dependent on the development of the hemispheres of the prosencephalon, which are feebly developed in Rodents, Edentates, and Insectivora. According as they increase in size, the anterior commissure is diminished in area. In the Monotremata and Didelphia it is very much so, and is converted into a thin chord placed in front of the columns of the fornix. 'The more the hemispheres of the prosencephalon are enlarged backwards, the more do they overlie the parts behind them.

In many Mammals the hemispheres are smooth, and the surface of the prosencephalon is then in a simple stage, corresponding to its embryonic characters; these may be complicated by gyri and sulci. The gyri are at first arranged regularly and symmetrically, and only become asymmetrical when they are more largely developed, as they are, for instance, in Man. But even in this case they may be divided into groups, the boundaries of which are formed by the earliest, which are in some Mammals the only, sulci present. The gyri are similar in character in their earliest stages only. When they are complicated, the arrangements differ in different divisions of the Mammalia, and serve as indications of the degree of affinity that there is between such divisions.

The thalamencephalon is divided into two masses, which lie immediately behind the corpora striata of the lateral ventricles of the prosencephalon, the thalami optici. The epiphysis is placed at the hinder end of the cleft which separates them. The cavity of this portion is reduced to a small space between the two thalami, and is continued downwards into the infundibulum, which is carried by the tuber cinereum.

The mesencephalon, which for a long time forms the largest division of the brain (Fig. 280, $C$ c), has its primitive lumen gradually converted into a narrow canal (aqueductus Sylvii), which unites the third with the fourth ventricle. Its surface is divided by shallow longitudinal and transverse grooves into four bodies (Fig. 286, $B C$ $I I I)$, in consequence of which the corpus bigeminum becomes the corpora quadrigemina. This division into four lobes is very slight in the Monotremata.

The metencephalon (cerebellum) only resembles that of the 
Fishes and Amphibia during the earlier stages of development. The simple lamella is developed into a large body, in which, as in the Crocodilini and Aves, the first part to be differentiated is the middle. In the Marsupialia, however, this forms a delicate transverse commissure for some time, while the lateral parts are developed to a greater size. Transverse lamellæ are developed in both regions, and are arranged in various groups. The median portion is always the larger in the Monotremata, and it is large in the Marsupialia, Edentata, and Chiroptera. It is not till we come to the Carnivora and Ungulata that we find the lateral parts, or "hemispheres of
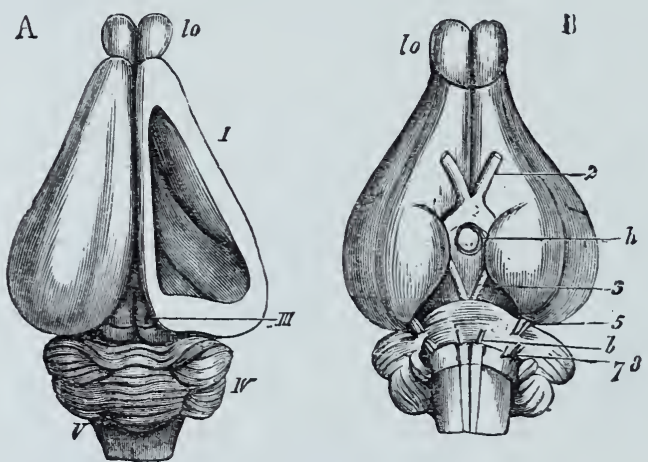

Fig. 287. Brain of the Rabbit. A From above, $B$ From below. lo Olfactory lobes. I Prosencephalon. III Mesencephalon. IV Metencephalon. $V$ Myelen. cephalon. $h$ Hypophysis. 2 Optic. 3 Oculo-motor. 5 Trigeminus. 6 Abducens. 78 Facial and auditory nerves. In $A$ the roof of the right hemisphere is removed so that we can see into the lateral ventricle, and make out the corpus striatum in front, and the fornix with the commencement of the pes hippocampi major behind. the cerebellum," developed to a greater size; in most Primates they are so much the larger, that the median portion diminishes, and is known as the "vermis."

As the prosencephalon increases in size, it gradually covers the other divisions of the brain. In many Marsupials, and in Rodents (cf. Fig: 287, A) and Insectivora, it does not reach to the corpora quadrigemina; and, in most of the other Mammalia the metencephalon is altogether or very nearly free; in the Primates, however, this portion is altogether below the posterior lobes of the prosencephalic hemispheres; in this point the anthropoid Apes most closely resemble Man. When the hemispheres of the metencephalon increase in size, a transverse commissure is developed on the lower surface of the primitive myelencephalon - the pons Varolii; this appears to unite the anterior portion of the myelencephalon more closely to the cerebellum. This commissure is feebly developed in the Monotremata and Marsupialia, and most largely in the higher Primates. 
b) Spinal Chord.

$\S 383$.

The spinal chord, which is continuous with the medulla oblongata is formed from the development of the lateral halves of the wall of the primitive medullary tube. As the lateral parts increase in size an anterior longitudinal fissure is developed. The primitive lumen of the tube is converted into the central canal.

The central apparatus of the spinal chord occupies the inner parts, and forms a gray medullary mass, which is seen, in cross section, to have the form of cornua, which pass forwards and backwards (Fig. 288, $d e$ ).

Owing to the distribution of the central apparatus in the central

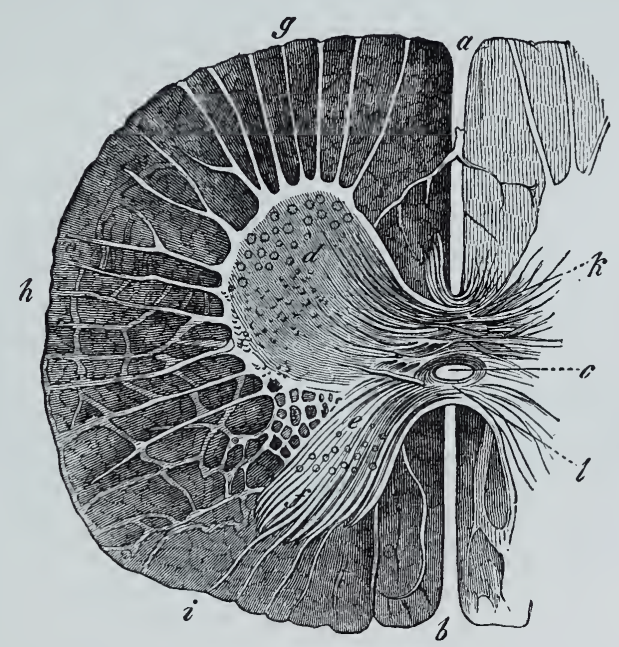

Fig. 288. Transverse section throngh the spinal chord of a Calf. a Anterior, $b$ Posterior longitudinal fissure. $c$ Central canal. $d$ Anterior, $e$ Posterior cornua. $f$ Substantia gelatinosa. $g$ Anterior column of the white substance. $h$ Lateral. $i$ Posterior column. $k$ Transverse commissures. portions of the spinal chord, that is, in the gray columns which pass off from the region of the central canal $(c)$, the white medullary mass, which consists of nerve-fibres, is chiefly placed towards the exterior, forming longitudinal columns, which are partly separated from one another by the anterior and posterior longitudinal fissures $(a b)$, and partly by the points atwhich the roots of the nerves pass out $(g h i)$. This arrangement of the white matter is a peculiarity of the spinal chord, and is also a point of difference between it and the ventral chord of the Annulata and Arthropoda, which is of great significance.

In the Cyclostomata the spinal chord forms a flat band, as it does also in Chimæra ; in most it is more cylindrical in form, and gradually diminishes in size as it passes towards the end of the spinal canal. There are often special enlargements at the points where the larger nerves are given off ; they are very strikingly

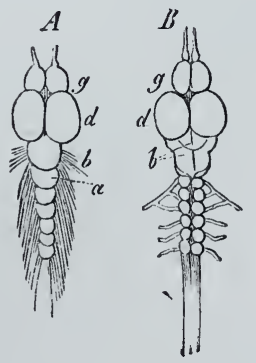

Fig. 289. Brain and spinal chord of Ortha. gosiscus mola (after Arsaky). $B$ Brain and anterior region of the spinal chord of Trigla adriatica (after Tiede. mann). 
developed in some species of Trigla (cf. Fig. 289, B), as is a smaller number of them forming the exceptionally short spinal chord of Orthagoriscus, etc. $(A)$.

As the size of the spinal chord is influenced by the masses of nerves which are given off from it, we find that in the four higher classes of the Vertebrata the great development of their extremities and the large size of the nerve-chords that go to them are correlated with the increased development of the size of the spinal chord in various regions. In this way the cervical, or thoracic, and lumbar enlargements are formed; they are very large in some cases (e.g. Chelonii and Aves). The primitive medullary cavity, which persists as the central canal, remains open in the lumbar swelling of Birds, and thus a sinus rhomboidalis is developed; this is similar to the sinus which is always found in the medulla oblongata. It is found for a short time in the embryos of Mammals also (Fig. 279, a).

As a rule the spinal chord extends through the whole of the spinal canal; but in the Amphibia (Anura) and Ares, and most markedly in many Mammalia, it is pressed more forwards, owing to the unequal development of the enclosing and enclosed parts, so that the nerves which are given off from it for the hinder parts of the body, have to run for some way in the spinal canal before they get to their orifices of egress.

c) Investments of the Central Nervous System.

$\S 384$.

As the cavity of the skull is adapted to the brain, which it encloses, this latter at first fills up the cranial cavity. The same is true of the spinal chord and spinal canal. The surface of the whole of the central nervous system is separated from the walls of its case, which are formed by the skeleton, by parts which either belong to the skeleton, or to the nervous system, or which are interstitial in character. These form the coverings of the brain and spinal chord.

The periosteal investment of this skeletal cavity develops the dura mater. In the lower divisions this membrane appears to be a mere periosteal (or perichondrial) layer; it is not much larger till we reach the Reptilia, where it first acquires the character of an independent formation. In the cranial cavity of Birds it forms a process between the hemispheres of the prosencephalon (falx cerebri), and this is found in most Mammals also, where it is accompanied by a process - the tentorium cerebelli-best developed in the higher orders, which projects between the cerebellum and the posterior lobes of the prosencephalon. In many Mammalia (Carnivora, Perissodactyla, etc.) the tentorium is ossified. The spinal portion of the dura mater is less peculiar in character. In the Mammalia it is separated from the periosteum as high up as the occipital foramen, and forms a sac, which loosely envelopes the spinal chord. 
The pia mater, part of the nervous system, is a layer of connective tissue covering the latter, in which the blood-vessels of the nervous centres run. It extends into the depressions between the different portions. It sends out convoluted vessels (retia) from the large cerebral cleft, which are connected with the roof of the cleft ; these pass into the interior of the lateral ventricles of the prosencephalon. It is continued over and forms a roof for the sinus rhomboidalis of the myelencephalon, where it often forms a vascular plexus.

The arachnoid membrane is the most variable. In those Fishes, in which the brain fills the cranial cavity, it is a thin layer of connective tissue which scarcely deserves the name of a membrane, for it is as intimately connected with the pia, as with the dura mater. When a larger space is formed between the brain and the wall of the skull, this tissue is either converted into a network filled with lymph (Squatina), or into gelatinous tissue (Scymnus), or it gives lise to fat cells (many Teleostei). In the higher Vertebrata the arachnoid is generally a delicate layer of connective tissue; in the Mammalia it is differentiated as it is in Man.

\section{B. Peripheral Nervous System.}

\section{$\S 385$.}

The nerves which are given off from the central organs are divided into spinal and cerebral nerves; in the Acrania there is no difference between these two kinds. In Amphioxus only one anterior and larger trunk is remarkable for its course, and its numerous ramifications in the anterior end of the body. It is clearly comparable to one of the cerebral nerves of the higher Vertebrata, but it must be here noted that, compared with the Craniota, the whole organisation of Amphioxus is in an indifferent condition. The other nerves of the medullary tube (with the excep= tion of those for the nasal pit and eyes) are similar in character to spinal nerves, and are remarkable for the fact that they are given off alternately from the medullary chord. The similarity in the characters of these nerves leads to the supposition that the difference between the cerebral and spinal nerves, which is seen in the Craniota, is a condition which was acquired by them, when the head was developed. The nerves of Amphioxus have no ganglia, and are formed by single roots; this, again, is a point in which they differ markedly from the Craniota. As Amphioxus has no "head," in the sense in which we speak of it in the Craniota, we cannot suppose that the nerves are separated into cephalic and spinal nerves. The most we can do is to regard the nerves which belong to the region in front of the posterior boundary of the branchial cavity, as the indifferent equivalents of the cephalic nerves of the Craniota, and the rest of the nerves behind these as spinal nerves. 


\section{a) Spimal Nerves.}

\section{$\S 386$.}

The metamerism of the vertebrate body, which is first seen in the formation of primitive vertebræ, is quite as much expressed by the characters of the spinal nerves, and by their mode of distribution. A pair of nerves corresponds to each vertebral segment. Each nerve is formed by the union of two roots which have their origin in the lateral halves of the spinal chord. The superior or sensitive root forms a ganglion, before it unites with the inferior or motor root; and the fibres thus formed mix with those of the lower root, and form the trunk of a spinal nerve. In the Selachii the inferior and superior roots pass through separate foramina in the spinal canal. As a rule, the nerves leave the spinal canal between two arches.

Each spinal nerve divides into two chief branches-a ramus dorsalis supplies the muscles and skin of the back, a ramus ventralis goes to the sides, and ventral wall of the body, and gives off a ramus visceralis to the viscera. This latter forms the connection between the so-called sympathetic, and the cerebro-spinal nervous system.

In Fishes we always find the spinal nerves at the intermuscular ligaments. They follow exactly the metamerism of the body, so long as this continues to be expressed.

The size of the nerves is correlated with the development of the parts which they supply. When the extremities are formed, their rami ventrales become very large. A number of rami ventrales of the anterior spinal nerves (cervical nerves) are then formed into a plexus (plexus brachialis), from which the nerves for the anterior extremities are given off; in the same way, the nerves for the hinder extremities are given off from a plexus (plexus lumbalis and p. sacralis), which is formed more posteriorly, either in front of, or in the pelvis. These plexuses change their position when the limbs do so (cf. pp. 473, 474).

'I'he brachial plexus of the Amphibia is formed of three or four nerves (in the Frog, the 2nd, 3rd, and 4th spinal nerves). In the Reptilia the brachial plexus is generally made up by the cervical nerves from the sixth to the ninth; in Varanus, from the seventh to the tenth; and in the Alligator the first thoracic nerve also enters the plexus. In Birds it is formed from the last cervical and first thoracic nerve, or from the 11th and 12th cervicals, or 1st and 2nd thoracic nerves. In the Mammalia, the last 3, 4, or 5 cervical nerves, and the 1st, and sometimes also the $2 \mathrm{nd}$, thoracic nerves, contribute to form the plexus.

The nerves for the hinder extremities are given off, in the Amphibia, from a plexus which is generally formed by three nerves. The anterior of the nerves thus formed is the crural; the sciatic is 
larger, and is made up from nearly all the branches that pass into the plexus; this nerve is the chief nerve of the extremity in the higher Vertebrata also.

The crural and sacral plexuses are more distinct from one another in Reptilia and Aves. In the former, four nerves generally pass into these plexuses. In Birds there are generally seven or eight nerves set apart, and most of these are for the sciatic nerve; in the Mammalia, again, it is composed of a much smaller number.

\section{b) Cerebral Nerves.}

\section{$\S 387$.}

The cerebral nerves, which by a descriptive anatomist are taken in the order of their position, are seen to break up into two very distinctly marked divisions, when examined after the comparative method. One division, the larger, contains nerves which more or less agree with, or might even be derived from, spinal nerves, while the other contains those which have not the faintest resemblance to spinal nerves.

This latter division contains two specific sensory nerves, the olfactory and the optic.

The olfactory is formed of a complex of nerve-filaments, which arise from the olfactory lobe, and are distributed in the olfactory mucous membrane. According as the olfactory lobe is near, or far from this membrane, these nerves form a trunk on either side (as in many Fishes, and in the Amphibia, Reptilia, Aves, and Monotreme Mammals), or leave the cranial cavity separately, by passing through a "lamina cribrosa" (Selachii and Mammalia).

The optic nerve, which arises from the thalam- and mes-encephalon, is formed, as is part of the eye, from a vesicle (optic vesicle), which is developed from the primitive prosencephalon; it forms the stalk of this vesicle. After the differentiation of the vesicle of the forebrain, it is connected with the thalam- and mes-encephalon. In the Cyclostomata the optic nerve of each side passes to the eye on the same side; the nerves of either side do not exhibit any union with one another excepting close to their point of origin. But in the Gnathostomata a larger portion of the optic nerve is prominent on the base of the brain, and here the fibres exhibit a crossing over from one side to the other (chiasma). The fibres which pass to this point constitute the optic tract, and form a portion of the brain, which in the Cyclostomata has not attained to the surface. The chiasma, therefore, is not a new formation, but a differentiation. In the Osseous Fishes, the fibres cross completely; the optic nerve of the right eye passes to the left, and that of the left to the right, each passing above or below the other. More rarely, one optic nerve perforates the other (Clupea), or the different bundles of nerves pass through separately. 
In the Selachii and Ganoïdei, some only cross over, and this is the arrangement which is seen in all the higher Vertebrata.

Neither of these sensory nerves have any character in which they resemble spinal nerves, nor can they be referred to metameres. They belong to that portion of the cranium which is not formed by the concrescence of vertebral segments (cf. \$ 340); and they may correspond to those nerves which we found passing to the same organs in the Invertebrata.

\section{$\S 388$.}

The second division consists of the nerves which are arranged on the type of the spinal nerves. It is sometimes possible to distinguish two roots: their dorsal branch is often very feebly developed, in correlation with the small size of the area to which it is distributed. The ventral branch is consequently the important one ; it sends nerves to the branchial arches, and the parts derived from them. The visceral branch goes to the wall of the pharynx. The nerves of this division arise at the base of the fourth ventricle, and partly, also, from its continuation in the aqueductus Sylvii. They are given off from the myelencephalon, and leave the cranial cavity by perforating the vertebral portion of the cranium $(\$ 340)$. While these relations are best seen in the cephalic nerves of the Selachii, which most resemble the primitive condition, they undergo greater changes the farther the organism has risen above this low stage, or has been differentiated in another direction.

We may note a number of special characters in the various nerves, proceeding on the supposition that the series are homodynamous with spinal nerves. Some branches of a nerve become much larger than others, which undergo degeneration; or the roots of a nerve take an independent course, and give the appearance of independent nerves. In this way a nerve is broken up; but other nerves may undergo concrescence, so that what were primitively complexes of nerves acquire the form of a single nerve.

This latter arrangement is seen in two groups of the cerebral nerves, which I have distinguished as the trigeminal and as the vagus group; the names of which are taken from that of the most important nerve in each group.

\section{$\S 389$.}

The trigeminal group supplies the anterior, and larger portion, of the head. The following nerves belong to it:

First, the trigeminus, the largest nerve of the group, which, in correspondence with the great differentiation of the regions to which it is sent, has the characters of a greatly developed spinal nerve (Fig. 290, Tr). Its dorsal branch is formed by the ophthalmic, which supplies the orbits and the ethmoidal region. A branch for the cranial cavity, which is found in the Teleostei, also belongs to the dorsal 
branch. The ramus maxillaris superior always runs on the floor of the orbit, and gives off sensory branches to the maxillary region. Its infraorbital branch is the largest, especially in the Mammalia. The R. max. sup., with the ramus maxillaris inferior, represents a ventral trunk; in the Selachii this is very clearly the nerve of the mandibular arch, and appears as the largest portion of the trigeininus. It is distributed to the muscles of the jaw, to the integument, and to a large part of the mucous membrane of the mouth (ramus lingualis). The intestinal trunk is represented by a palatine trunk of the second branch, which, in Fishes, passes directly to the palate, but in the higher Vertebrata only reaches the palate by means of its connection with a sympathetic ganglion (sphenopalatine ganglion).

The nerves of the optic muscles-the oculo-motorius and trochlearis-in origin and distribution, belong to the trigeminus, and seem to be parts which have been separated off from it. Although the statements that the nerves for the optic muscles are given off from the ophthalmic branch of the trigeminus in Lepidosteus and Lepidosiren, and that in the Salamandrina the trochlear is replaced by a branch of the same nerve, require confirmation, and whilst what really has occurred in these cases is, perhaps, that the nerves of the optic muscles have become united with the trigeminus, and not that they are completely wanting, yet the supposition that processes of segregation have been the cause of these nerves arising separately from the myelencephalon in the other Vertebrata, cannot be rejected. The fact that the ramus inferior of the trigeminus has no motor elements, and that the muscles in its area are supplied by apparently independent nerves, will always be of great importance.

The second nerve of the trigeminal group is the facialis and acusticus. The latter seems to be homologous with a purely sensory dorsal branch of a spinal nerve; its terminal area has been carried below that plane in which we must suppose it to have primitively ramified-that is, below the surface of the body; and this change has accompanied the removal of the vesicles of the labyrinth from the integument, and their passage into the interior of the wall of the skull (cf. infrà, auditory organ). This points to a dorsal branch having primitively passed upwards through the wall of the skull, and agrees with the course of the dorsal branches of the other cephalic nerves, and with the ophthalmic branch of the trigeminal.

The facialis (Fig. 290, $F a$ ) has the arrangement of a ramus ventralis belonging to the hyoid arch. It supplies the integumentary as well as the muscular portions of this segment, and is, therefore, primitively a mixed nerve. In the Teleostei it enters into connection with the trigeminal; in many Sharks, also, it is fused with it. So, also, in the anourous Amphibia it is united to the trigeminal. This union, however, is effected during their ontogenetic development. In the Urodela, as in the higher Vertebrata, it is always distinct, and in the Mammalia its sensory elements are apparently absent. In these latter its area of distribution is very great, owing to the increase in size of the facial musculature; while 
its stapedial, digastric, and stylohyoid, as also its auricular branches, belong to the primitive hyoidean area. Its visceral branch appears to be the palatine of Fishes; in the Mammalia this is represented by the petrosus superficialis major, and passes to the muscles of the velum palati through the spheno-palatine ganglion. The chorda tympani forms a connecting twig between the facial and the third branch of the trigeminal; this is found even in Fishes.

One of the nerves for the optic muscles, the abducens, must also be regarded as part of the facial nerve, as is clear from its area of origin. It supplies, as a rule, the rectus externus, and in Petromyzon the rectus inferior also. The position of the rectus externus explains how it is that it belongs to a different nervous area from that of the other optic muscles.

\section{$\S 390$.}

The first nerve of the vagus group, the glossopharyngeal, is the simplest in character. In the Selachii it is distinct, as it is also in the greater number of the Teleostei; in Chimæra, however, it leaves the cranial cavity in company with the vagus, with which nerve it is united in the Cyclostomata and in Lepidosiren. It has the same relations in the Amphibia, but in the Amniota it is ordinarily distinct.

In Fishes (many Sharks) it has a dorsal branch, which takes an upward course within the cranium, and there ramifies on the surface. The chief trunk (Fig. 290, Gp) is the ventral one; this is distributed all along the first branchial arch; it gives off a pharyngeal branch to the wall of the pharynx, which represents its visceral branch. When the first branchial arch is netamorphosed, this arrangement is so far modified that the pharyngeal branch and the lingual, which ends in the mucous membrane of the tongue, form the chief portion of the nerve.

The vagus is closely attached to the gilossopharyngeal at the point where it leaves the myelencephalon; to fully understand this nerve it is necessary to have a knowledge of its simplest characters, as they are best seen in Sharks (cf. Fig. 290). The vagus is here made up of a large number of separate roots, which have their origin in the myelencephalon, nearly as far back as the fourth ventricle; the anterior ones, which have their origin just behind the glossopharyngeal, are the larger. The posterior ones become gradually smaller and smaller than those in front of them. The last of the series are collected into a small trunk, which passes forwards, and is attached to one of the more anterior ones. The common trunk thus formed passes through the wall of the cranium obliquely backwards and outwards; on its course it gives off a small dorsal branch for the occipital region.

When it has passed out of the cranium the vagus trunk gives off a number of branchial branches, in correspondence with the number 
of branchial arches (Fig. 290). The first branchial branch $\left(b r^{f}\right)$ passes to the second branchial arch, and also gives off a fine twig to the first arch. In this point the branchial branches of the vagus

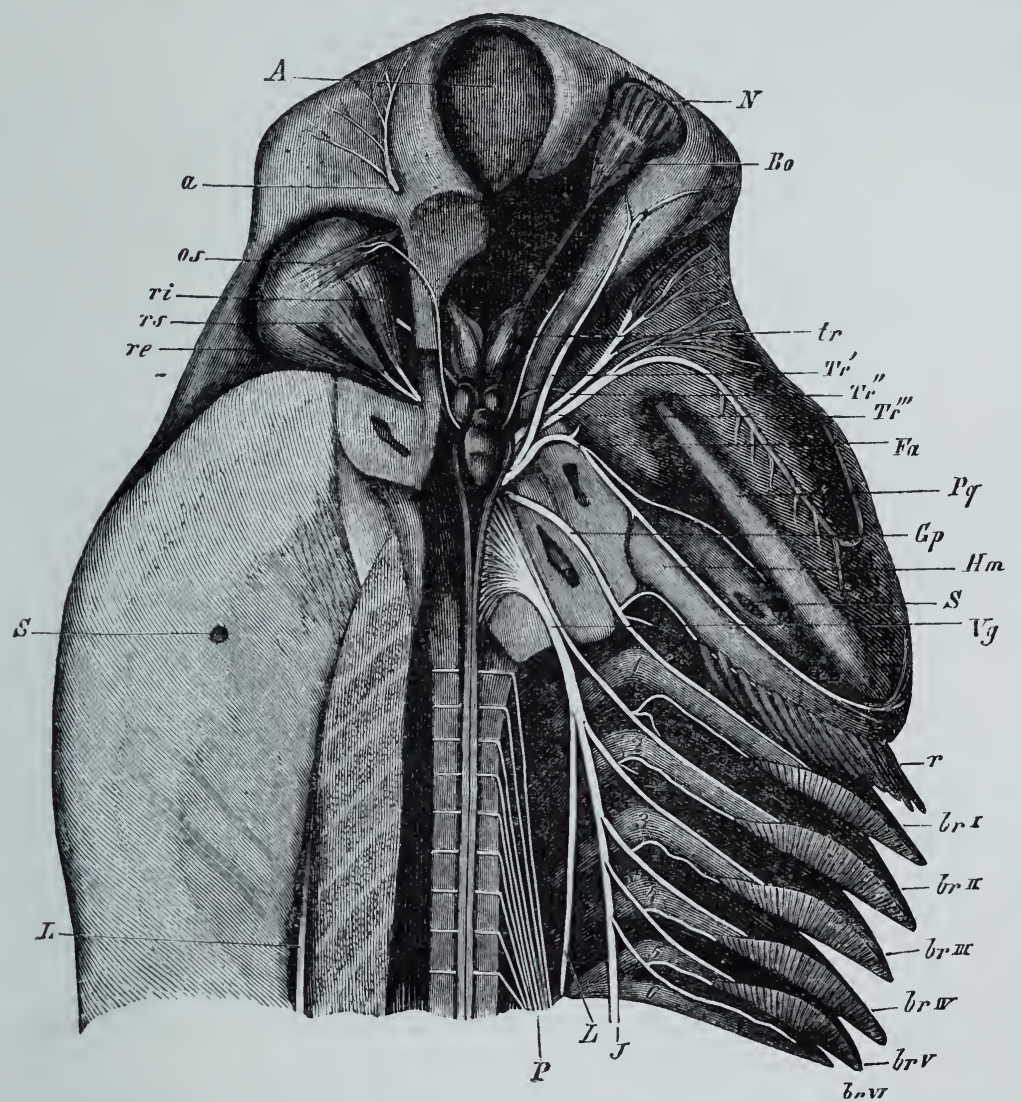

Fig. 290. Cephalic nerves of Hexanchus griseus. On the right is figured as much of the course of all the cephalic nerves as can be seen from above. The cranial cavity and spinal canal are both laid open, so that the brain and spinal chord are exposed. The right eye and its muscles is removed. On the left, the roof, only, of the orbit is removed, so that the bulb and its muscles are exposed to view. The region of the labyrinth, and of the occipital portion of the cranium, has been removed as far as the levels of the nerve-trunks, which pass out through them. $A$ Anterior fontanelle (cranial lacuna). $N$ Nasal capsule. Bo Bulbus olfactorius. $T r^{\prime}$ First branch of the trigeminal. $a$ Its terminal twig in the ethmoidal region. $T r^{\prime \prime}$ Second branch. Tr" Third branch. $t r$ Trochlearis. Fa Facialis. Gp Glossopharyngeus. $V g$ Vagus. $L$ Ramus lateralis. $J$ Ramus intestinalis. os Musc. obliq. oc. sup. $r i$ M. rectus internus. $r$ M. rectus externus. $r s$ M. rectus superior. S Spiracular cleft. $\quad P q$ Palato-quadrate. Hm Hyomandibular. $r$ Branchial rays. $1-6$ Branchial arches. $b r^{I}-b r^{V}$ Branchiæ.

resemble the glossopharyngeal and the facial, which likewise send off fine twigs to the arches next in front of them. A pharyngeal branch is given off at the point where this branchial branch divides. The vagus trunk is continued on, as an intestinal branch $(g)$, to 
the alimentary canal; it ramifies on the pharynx and stomach, and also gives off branches to the heart. Before the vagus trunk gives off the branchial branches, it sends off a large dorsal branch, which passes backwards in the dorsal region; this is the lateral branch $(L)$, which ramifies in the skin alongside the lateral line of the body, and extends as far as the tail.

While the nerve-roots, that make up the vagus trunk, leave the myelencephalon together, there are other roots, which belong to the vagus, and which arise from the myelencephalon, underneath those already mentioned; of these there are, at most, five, and, generally, only two or three filaments, each of which reaches the exterior by a special canal in the wall of the cranium. Some pass to the muscles, and some are connected with the first of the spinal nerves; they may be called the inferior roots of the vagus, while those before mentioned are the superior roots. The foramina of the lower roots lie in the same row as the foramina of the inferior roots of the spinal nerves; the foramen for the complex of superior roots is placed higher up, and is in a line with the foramina of the superior roots of the spinal nerves.

\section{$\S 391$.}

From the description that we have just given, it is clear that the whole vagus must be regarded as a complex of a large number of nerves, which are homodynamous with spinal nerves. This is indicated by the various inferior roots, which pass out separately, but much more emphatically by the distribution of the trunk, formed by the superior roots. While each branchial branch of the vagus has exactly the same characters as a ventral branch of a spinal nerve; and while, moreover, the branchial arches supplied by it must be regarded as arches, which were primitively part of the cranium ( $(340)$; and while, lastly, each of the other arches (mandibular, hyoid, and first branchial) is innervated by a nerve, in just the same way as is a metamere of the trunk by a spinal nerve; the series of the superior roots of the vagus are, on their side, the distinct equivalent of a number of separate nerves, the sum total of which must correspond to the maximum number, at any rate, of the arches supplied by them. This view of the real character of the vagus, advanced by me, is shown to be the correct one by the developmental history of these nerves, which has lately been made out in the Sharks. As there is reason for supposing that, even in the Selachii themselves, there has been a great reduction in the primitive number of the gills, inasmuch as a process of this kind, although, indeed, affecting a small number of gills only, can be observed within the limits of the group, it follows that the extension of the vagus on to a portion of the alimentary tube is explicable as due, not so much to an incursion of the nerve into a territory which was not originally within the area of its distribution, as to the modification of a region, which formerly did carry branchial 
clefts in its walls, and belonged to the pharynx, into a portion of the intestinal tract, serving exclusively for the reception of food. Nor is there anything remarkable in the existence of cardiac branches, when the fact is recognised that the heart was derived from a region which was partly within the area supplied by the vagus.

The ramus lateralis appears to be a sensory branch of the vagus, which was some time in being developed proportionately to the growth of the sensory apparatus of the lateral line, which is innervated by it.

'Taking therefore the vagus as a whole, we see that in it, just as - though to a less extent - in other nerves (e.g. the facial and trigeminal of the Amphibia), a number of nerves are united together; and these nerves show both by their origin and by their peripheral relations that they are the remnants of nerves which were primitively distinct; our view, therefore, of the characters of the vagus agrees very closely with the indications given us by the posterior portion of the cranium.

The phrnomenon of the concrescence of separate nerves is carried still farther in the vagus of the Selachii, and destroys all signs of individuality, for in most of them (all the Rays) the separate roots come nearer to one another; this arrangement is the dominant one in all other Fishes also.

In the Teleostei some of the relations of the vagus are much changed. A few filaments of the hinder roots fuse with an inferior root, and form a large nerve, which passes out from the cranium, and seems to go to the muscles of the shoulder-girdle. The relations of this nerve require more accurate investigation.

In other points the peripheral relations of the vagus are the same as those which we have described. A dorsal branch, which is present in some of the Teleostei, must be more particularly mentioned. It is united to a dorsal branch (R. recurrens) of the trigeminal, and goes to the base of the dorsal fin, receiving connecting branches from some spinal nerves as it does so.

\section{$\S 392$.}

In the Amphibia the vagus has the same relations as in Fishes, so long as the gills are present; it even gives off a lateral branch, which in the Caducibranchiata has the same fate as the branchial branches, when the gills are atrophied.

In the Amniota the only portion of the vagus which is present is the part which is developed from the anterior portion; of the series of superior roots of the Selachian vagus; the trunk, which they form, is only distributed as far as the stomach in the intestinal tract, while, owing to the absence of gills, the branchial branches have disappeared, or-and this is probably more correct-have been partly converted into pharyngeal branches. In Fishes the air-bladder, which is differentiated from the enteric tube, receives branches from the vagus, and so also the respiratory apparatus of the Amphibia and of the Amniota, which has a similar origin, also receives branches 
from the vagus ; when a larynx and laryngeal muscles are developed, some of these nerves give rise to constant branches. The cardiac relations of the vagus are retained, and, as the intestinal terminal area of the vagus becomes gradually removed from the region of the head, this branch is converted into a long nerve-trunk.

The hinder portion of the roots which belong to the vagus in the Selachii are, in the Amniota, united into a small nerve-trunk-the accessorius Willisii-which is partly connected with the vagus, and is partly distributed to the muscles of the shoulder-girdle. The rootfibres which form the nerves arise from parts which are placed much farther back than the medulla, especially in the Mammalia, where they are found between the origins of the upper and lower roots of the spinal nerves; in Man, indeed, they extend as far back as the sixth or seventh pair.

Lastly, the inferior roots of the area of the vagus form a special nerve-trunk in the Amniota ; this is the hypoglossus, which supplies the muscles of the tongue. It retains its primitive characters in so far as that it is made up of several root-fibres, which moreover pass out separately from the skull, and are arranged in pairs in the Mammalia.

The posterior complex of nerves given off from the myelencephalon is therefore the most variable in character. Probably derived from as many separate nerves as there were primitive branchial arches, it is found in its most indifferent condition in the Selachii; it separates off a hinder portion in the Teleostei, which forms a special nerve; and in the Amniota forms three different nerres-vagus, accessorius, and hypoglossus.

Gegenbadr, C., Ueber die Kopfnerren ron Hexanchus und ihr Verhältniss zur Wirbeltheorie des Schädels. Jen. Zeitschr. Bd. VI.

\section{c) Visceral Nervous System.}

$\S 393$.

After the visceral branches are given off from the cerebro-spinal nerves, they become connected together, each uniting itself to the one next behind it; they thus form a commissure, which runs along the vertebral column, and is continued to the basis cranii; this is the subvertebral chord of the visceral nervous system, or sympathetic. There are ganglia at the points where the rami viscerales of the cerebro-spinal nerves are connected with the chord; the ganglia of the sympathetic chord. From these ganglia nerres, which are made up of fibres of the sympathetic and of cerebro-spinal fibres, pass to their proper areæ of distribution. The various nerves, whether passing directly to the viscera, or first traversing the sympathetic chord, are generally collected into trunks set apart for the chief divisions of the viscera, and are known as cardiac, splanchnic, or 
other nerves. They form plexuses, which contain a large number of ganglia, and there are also separate ganglion cells, in large numbers, on the course of the sympathetic nerves.

These plexuses are distributed on the enteric tube, and on all the organs derived from it, as also on the vascular system, and urinogenitary organs.

This portion of the nervous system appears to be absent in the Acrania, and among the Cyclostomata it is not present in the Myxinoidea, where the vagus seems to supply the enteric area of the sympathetic. From the Fishes onwards this system is always present, although much modified. The fibres of the sympathetic appear to be elements, which permanently retain a lower grade of development, just as do the fibres of the cerebro-spinal nerves of the Cyclostomata.

\section{Sensory Organs.}

\section{$\S 394$.}

All the sensory organs of the Vertebrata are formed from differentiations of the integument. The kind of share which is taken by the integument differs with the quality of the organ. As in the Invertebrata, so here we can separate the sensory organs into those which preside over a specific sensation, or higher sensory organs, and those which appear to serve for the various perceptions, which are indifferent in character and may be regarded as belonging to the sense of touch.

There are many organs which cannot be reckoned among the known specific sensory organs, but which are remarkable for the high grade of their differentiation, so that their arrangements do not allow us to regard them as mere "tactile organs;" these seem to justify us in assuming the existence of specific sensory organs, other than those known to us familiarly.

Organs of this kind are most varied in character among Fishes, and as many of these arrangements are repeated in the Amphibia it is probable that they are correlated with an aquatic life. The most important organs of this kind are the following:

1. Goblet-shaped organs. Large structures embedded in the epidermal layer, and surrounded by long spindle-shaped cells; they

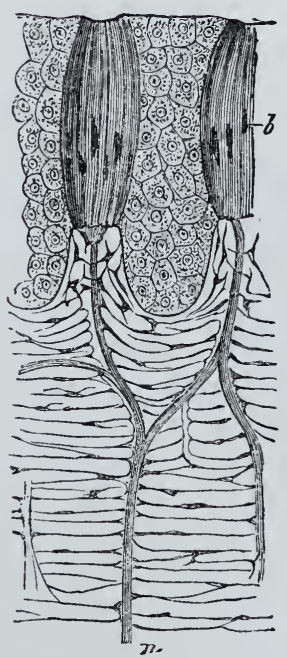

Fig. 291. Goblet-shaped organs from the mucous membrane of the palate of Tinca. $n$ Nervetrunk. $b$ Goblet (after F. E. Schulze). enclose rod-shaped end-organs of nerves; they have been observed in the skin of the Teleostei and of the Sturgeon, and appear to 
be found in the Amphibia also. They are also found on the head of Reptiles.

2. Mucous canals. A system of tubes which branches regularly in the head of Fishes, runs in the corium, and opens to the exterior by branched canals, and at definite points. Near its opening the tube contains the end-organ of a nerve-twig. A similar canal extends from the head, along the side of the body, as far as the tail. In the Ganoidei and Teleostei the nerve-endings of the system of tubes are protected, both in the head and along this lateral line, by an apparatus formed by the dermal skeleton, being embedded either in modified scales, or being embedded in passages in the larger covering bones of the head. The presence of goblet-shaped organs, or similar structures along the lateral lines of the Amphibia (larval forms and Perennibranchiata), points to a connection between these organs and the "mucous canals" of Fishes.

3. Gelatinous tubes. Thin-walled tubes of varying length, filled with a gelatinous substance, open by fine pores, and carry at the opposite end nerve-endings, which are placed in an ampullalike enlargement of varied form. These organs are found in great quantity on the head of the Selachii, where they are generally placed near the rostrum, but they are also found on more distant parts; thus, for example, in the Rays they extend as far as the pectoral fins (Fig. 277,t).

In the higher Vertebrata the nerve-endings in the integument are less complicated; as, for example, the corpuscula tactûs in the papillæ of the cutis, which are observed from the Amphibia onwards.

Owing to the modifications undergone by different parts of the body in consequence of the development in their integument of endorgans of sensory nerves, special apparatuses are developed, which function as tactile organs. The various arrangements of this kind are wonderfully different, but as they are structures which owe their origin to special adaptations, we can only mention them briefly. In Fishes these organs are frequently represented by the "beards" around the mouth, in which there are a number of goblet-shaped organs. These are found in the Sturgeons, and many of the Cyprinoids. In the Triglidæ certain rays, which are separated off from the thoracic fin, and are well supplied with nerves, function principally as tactile organs. In Birds the sense of touch is not unfrequently found in the soft tip of the beak ; this is the case in the Rails, Ducks, etc. In the Mammalia, again, we find as tactile organs stiff setiform hairs placed on the upper lip, or even above the eye; these hairs are not only greatly elongated, but are distinguished from other hairs by the large supply of nerves to their follicles. Finally, in many Mammals the limbs themselves, owing to the rich supply of nerves on their volar and plantar surfaces, and to the power of movement possessed by their terminal joints, have similar functions.

LEYDIG, Ueber Organe eines sechsten Sinnes. N.A. Acad. Leop.Carol. Vol.XXXIT. -Jobert, Les organes du toucher. Ann. sc. nat. Ser. V. Tom. XVI. 


\section{$\S 395$.}

As it is more and more impossible to form any opinion about the sense of taste, the farther the organism examined is removed from Man, it is impossible to speak with much certainty about the gustatory organs of most of the Vertebrata. We can only therefore regard the end-organs of nerves, which are placed in the mucous membrane of the mouth, as belonging in a general way to this apparatus. In Fishes, these end-organs have no specific character; they resemble rather the goblet-shaped organs which are scattered in the external integument; this is easily understood when we reflect on what is the origin of the buccal cavity. Those which are found in the palatine region are the most exactly known (cf. Fig. 291); in the Cyprinoids the mucous membrane of this region is interwoven with a large number of muscular fibres. In the Amphibia the tongue appears to be the principal seat of these structures, which are also known as "gustatory goblet-cells;" although these cells are not ordinarily found on the tongue of Reptiles and Birds, the tongue of the Mammalia is provided with them, and they are placed on the sides of the papillæ circumvallati.

\section{Olfactory Organs.}

\section{§ 396.}

In all Vertebrata the olfactory organs appear as shallow pits, placed on the head, and enabled to receive excitations from the surrounding medium by means of the rod-shaped end-organs of the olfactory nerve. The sensory organ is therefore represented by a differentiated portion of the integument. Although we cannot certainly say that these structures have in aquatic forms-Pisces and Amphibia-the same function as they certainly have in the airbreathing forms, yet we are perfectly justified in giving them the same name at any rate, for we see that they pass, and that in a continuous series, into the more complicated organs of the higher Vertebrata, which undoubtedly do serve as olfactory organs.

In the Leptocardii the olfactory pit is unpaired, as it is also in the Cyclostomata, where, however, it is converted into a deeper tube (Fig. 239, $g^{\prime}$ ), which ends blindly in Petromyzon $(g r)$, but in the Myxionoidea has the form of a canal which passes through the palate; the walls of this canal are supported by a tube of cartilaginous rings. In the Gnathostomata there are paired olfactory pits. In Fishes they remain mere pits, or are but slightly deepened. In the Selachii two processes project from the margin of a nasal orifice towards one another, and divide the primitively simple orifice into an afferent and an efferent orifice. In the Osseous Fishes this arrangement is 
carried still farther, for a bridge of integument is drawn over the pit, and the two separate openings are sometimes widely separated. Both orifices-but most commonly the anterior ones-may project forwards in the form of tubes. The investing mucous membrane sometimes forms radial, sometimes parallel folds, by which its surface may be considerably increased. The whole surface is supplied with terminal branches of the olfactory nerve. There is another kind of modification, in which the surface is so much increased towards the exterior, by the extension of the olfactory mucous membrane over a papilliform process, that the pit-like appearance is altogether destroyed.

In many Selachii, and in the Chimæræ the olfactory pit is connected with the mouth, owing to the formation of a groove (nasal groove), which passes from the pit to the angle of the mouth (Fig. 292). The groove is frequently covered over by a

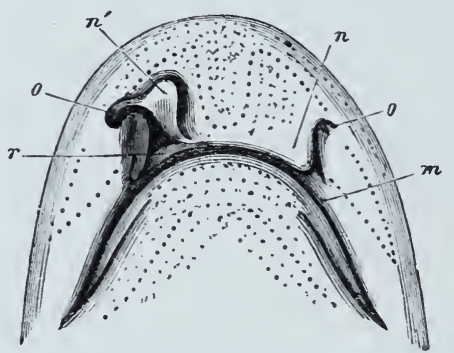

Fig. 292. Inferior surface of the head of Scyllium. $m$ Mouth. o Entrance to the nasal pit. $n$ Nasal valve in its natural position. $n^{\prime}$ Nasal valve turned back. $r$ Nasal groore. The dots represent the orifices of the mucous canals. median dermal fold, and is not unfrequently converted into a deeper canal (Rays). In this arrangement we may observe a step towards that which obtains in other Vertebrata, where the olfactory pits are only superficial in position during an early period of embryonic life. In them the arrangement which is permanent in Fishes disappears, and the processes which go on during further development cause the nasal pits to sink below the surface. This is effected by the great growth of the parts which limit the pit in front, at the sides, and in the middle line; by the growth
rds one another a canal is developed of the edges of the groove towards one another a canal is developed
which leads from the pit to the primitive cavity of the mouththat is, from without, inwards ; this canal opens behind the margin of the jaws.

We find this arrangement in the Dipnoï and Amphibia. The internal opening of the nasal canal is placed, in the former, as also in the Perennibranchiata, just within the soft margin of the mouth. In the Salamandrina and Anura it is bounded by firm parts of the jaw-skeleton.

When a nasal canal is formed, the primitive nasal pit itself is removed from the surface. The surface of the pit then becomes complicated by processes from the ethmoidal cartilage (turbinate bones). In the Amniota there are yet further modifications; owing to these, the upper part of the primitive buccal cavity is converted into a space which takes in the nasal pit, and in its upper portion the olfactory membrane is spread out. The primitive olfactory pit cannot, therefore, be any longer distinguished as a separate organ; and it 
will be best, consequently, to consider this new arrangement of the nasal carity, when we come to treat of the mouth. Glands are differentiated in the mucous membrane of the nasal cavity, which are of a relatively large size in the Amphibia, although not absent in the Mammalia. Connected with the process by which the primitive nasal pit is carried far inwards, is the development of an organ, which appears to be a part separated off from the nasal pit. This is the organ of Jacobson. It forms a tube which lies at the base of the nasal cavity, and ends blindly behind (Fig. 293, $J)$; the olfactory fibres in its walls are provided with end-organs. These organs are found in Reptiles and Mammals, and open into the buccal cavity by the ducts of Stenson.

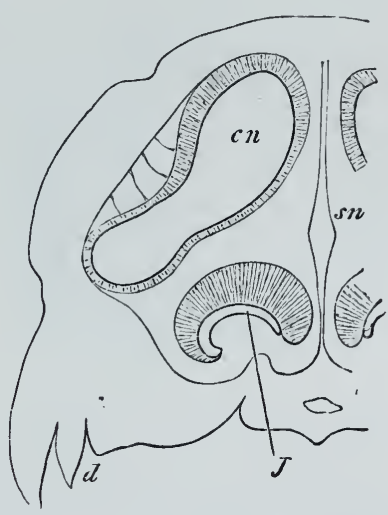

Fig. 293. Section through the nasal cavity and Jacobson's organ. sn Septum (after J. v Lacerta).

\section{Visual Organs.}

\section{$\S 397$.}

The eye in the Vertebrata appears to have essentially the same structure as in the more highly developed groups of lower animals; but the ontogeny of the organ shows that it belongs to another type, and this is also obvious from its minute structure. We cannot, therefore, connect it directly with the relatively welldeveloped stages of the eye in other animal phyla; the only indications of any connection are to be seen in the Tunicata. In the larvæ of the Ascidiæ, as in Vertebrata, the eye is not directly developed from the ectoderm, but from the anterior portion of the central nervous system. What is known as the eye in Amphioxus is of a much lower grade; it is a spot of pigment which varies in character, and is attached to the anterior end of the central nervous system.

The central nervous system chiefly, and secondarily the integument, are concerned in the constitution of the Vertebrate eye. The former gives rise to the apparatus which perceives, the latter to the apparatus which refracts, the light. The earliest rudiment of the eye is a diverticulum, which is developed from the sides of the prosencephalon (Fig. 294, $A$ a), and which has the form of a vesicle, connected by a stalk $(b)$ with the rudiments of the brain $(c)$. The "primitive optic vesicle" lies below the ectoderm; the ectoderm next gives rise to a thickening $(B)$, which pushes in the anterior wall of the vesicle towards the posterior one. Below this thickening a process of the mesoderm grows towards the optic vesicle, and puts the side-walls also of that vesicle in continuity with the epidermic thickening. The effect of these processes is to bring the anterior and posterior 
walls of the primitive optic vesicle close to one another; the whole
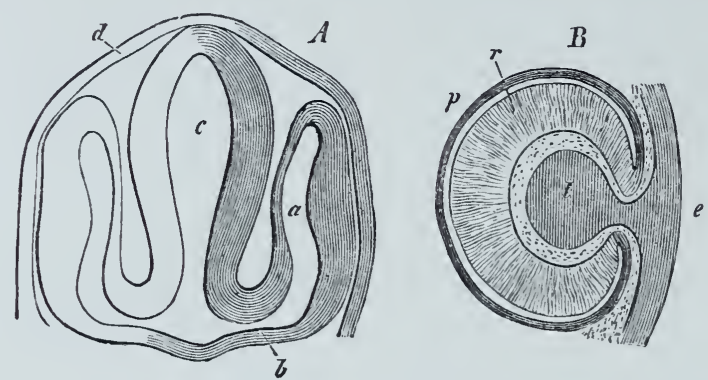

Fig. 294. A Vertical section through the rudimentary head of a Fish. $c$ Brain. a Primitive optic vesicle. $b$ Its stalk. $d$ Integumentary layer. $B$ Formation of the secondary optic vesicle. $p$ Outer, $r$ Inner layer of the primitive optic vesicle. $e$ Epidermis pushing the lens $(l)$ into the secondary optic resicle. The vitreous body is seen behind (after S. Schenk). then forms the secondary optic vesicle, and is cupshaped; the mouth of the cup is filled by the ectodermal thickening. This latter forms the rudiment of the lens $(l)$. While the stalk of the primary vesicle is being converted into the optic nerve, the tissue behind, which is enclosed by these parts, is converted into a substance, which gradually fills up the greater part of the secondary optic vesicle, and forms the vitreous body. The innermost layer of tissue around the secondary optic vesicle is converted into a vascular membrane, the choroid, while a firm fibrous layer outside it forms the sclerotic, and invests the secondary optic vesicle; this grows out anteriorly as far as the connection between the lens and

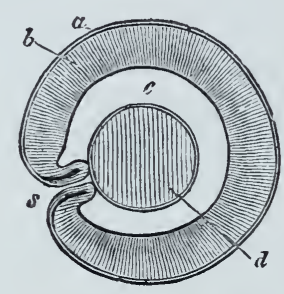

Fig. 295. Section through the secondary optic vesicle of a Fish's embryo, taken vertically to the "choroidal fissure $s . " \quad a$ Outer, $b$ Inner lamella of the optic vesicle. $c$ Vitreous body. d Lens (after S. Schenk). the ectoderm. As a result of the extension of this process, the lens becomes cut off, and a transparent portion of the sclerotic now intercalated in front of it forms the cornea, which at the same time becomes connected with the rudimentary piece of integument (conjunctiva) which lays in front of the lens.

The eye then is a rounded capsule (bulbus oculi), the investment of which (sclerotic) extends over the optic nerve, and is thence continued into the dura mater, while anteriorly it is continued into the cornea. Within this capsule is the secondary optic vesicle, which is developed from the invaginated primary one, and which is separated from the sclerotic by the choroid. The secondary vesicle, in which there is a lateral cleft owing to the ingrowth of the "vitreous body," embraces the lens anteriorly. These two, layers ( $a b)$, which pass into one another at this anterior margin, and at the lateral fissure (Fig. 295, s), are not similarly differentiated; the inner one $(b)$, which is greatly thickened at a very early stage, has its hinder portion converted into the retina, while the outer, and thin one $(a)$, forms the tapetum nigrum. When the tapetum nigrum becomes pigmented, a pale 
line can be made out on the lower and inner side of the rudimentary bulb; this extends from the optic nerve to the free anterior edge of the choroid. It corresponds to the fissure (choroidal fissure) which was formed when the rudiment of the vitreous body grew into the secondary optic vesicle $(s)$, and which must therefore affect the retina and the pigmented layer of the choroid (tapetum nigrum).

A large number of changes subsequently affect this rudiment of the eye. The anterior edge of the secondary optic vesicle grows out, together with the tissue that forms the rudimentary choroid, and gives rise to the iris, which bounds the pupil. When the process of the cutis pushes its way into the secondary optic vesicle, blood-vessels pass (in the Mammalia) into the cavity ; these are distributed in the periphery of the rudiment of the vitreous body, so that they must have a large share in the nutrition and growth of this structure. The lens, also, of the Mammalia, is invested by a vascular capsule of connective tissue, which disappears again before birth ; in some, however, it does not disappear so early.

MÜLLER, W., Die Stammesentwickelung des Auges der Wirbelthiere. Leipzig, 1875. - Kessler, L., Zur Entwickelung des Auges der Wirbelthiere. Leipzig, 1877.

\section{$\S 398$.}

As to the form of the bulb, its anterior segment is much flattened in Fishes (Fig. 296). The aquatic Amphibia have the bulb flattened anteriorly; the Ophidii and Crocodilini, among the Reptilia, are characterised by a more considerable curvature of the cornea.

In most Birds (Fig. 298) the bulb is divided into an anterior and a posterior segment; the former carries the very convex cornea. This form of eye is most marked in the Raptores, but the cornea is flattened in the Natatores and Grallatores. Among Mammals, also, the spherical form of bulb may undergo great variation in form.

The Sclerotic may be formed of various kinds of connective substances; in fact, it may be made up of connective tissue, of bony parts, or of cartilage. This latter is found in the Selachii, Chimæræ,

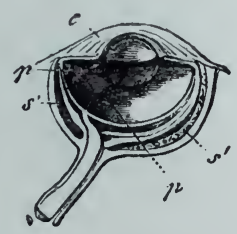

Fig. 296. Eye of $E_{S o x}$ lucius. Horizontal section. $c$ Cornea. $p$ Processus falciformis. $s^{\prime} s^{\prime}$ Ossifications in the sclerotic.

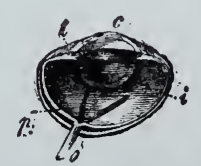

Fig. 297. Eye of Monitor. Horizontal section. $c$ Cornea. $p$ Processus falciformis.

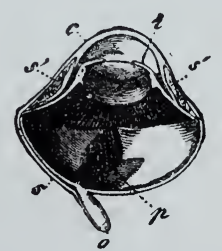

Fig. 298. Eye of Falco chrysaëtos. Horizontal section. $p$ Pecten (after W. Sömmering).

and Ganoïdei, and also in the Amphibia. These arrangements are the most varied in the Osseous Fishes. 
In the Saurii, Chelonii, and Aves, the anterior portion of the sclerotic, which abuts on the cornea, is supported (Fig. 296, $s^{\prime}$ ) by a

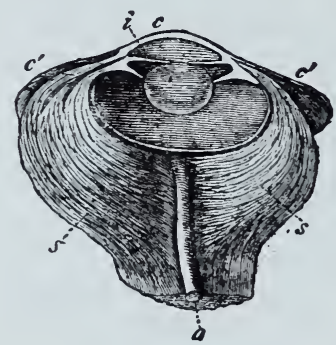

Fig. 299. Eye of Balaena mysticetus. Horizontal section (after W. Sömmer. ing). circlet of flat pieces of bone (sclerotic ring). In all Mammals except the Monotremata the sclerotic is formed of connective tissue; it is very thick in the Cetacea (Fig. 299, s).

The choroid is made up of several iayers, which, as a rule, have the same characters as in Man. Anteriorly it gives rise to the folded ciliary processes; these are feebly developed in the Selachii and Ganoïdei (Sturio), and absent in most of the Teleostei; the choroid is then continued on as the iris, which bounds by its inner margin the pupil, which varies in form.

The tapetum lucidum is a special modification of the choroid; this forms a spot of varying size, which is generally greenish or bluish in colour, and has a metallic lustre; it is sometimes produced by groups of spicular crystals placed in the cells of the tapetum (Selachii), or by a fibrous tissue (Carnivorous Mammals and Ruminants). It is owing to its presence that the eye can be seen in the dark.

A vascular plexus, which lies outside the choroid of Fishes, forms the so-called choroid gland. In the anterior portion of the choroid there is a muscular layer, which forms the ring known as the ciliary ligament. The musculature is continued hence into the iris, in which there are radial and circular fibres. In Fishes, Amphibia, and Mammals, this musculature is composed of smooth fibres; in Reptiles and Birds, of transversely striated ones.

The retina, which is placed on the choroid, extends forwards as far as the commencement of the ciliary body, where it ceases to be developed. The optic nerve is distributed, and ends, in it. The optic fibres occupy the innermost layer of the retina, which is merely separated from the vitreous body by a thin membrane. It is followed by a number of layers, of varying structure, the last and outermost of which is made up of rod-like and cone-like structures, the bacillar layer. These end-organs, which are similar to the rods of the invertebrate eye, are, therefore, turned away from the opening of the eye in the Vertebrata; the Vertebrate eye is therefore distinguished from the optic organs of the Invertebrata by a very essential point, which must not be left out of consideration when we are discussing their genetic relations.

Connected with the development of the secondary optic vesicle is the formation of a special organ, which makes its way into the vitreous body at the point at which the optic nerve passes into the retina; it has no connection with the choroid, but forms a vascular, darkly-pigmented, process. A structure of this kind is found in the eyes of many Teleostei, and is known as the processus falciformis $($ Fig.296, $p)$. Its end, which in many Fishes is distinguished by a layer 
of smooth muscular fibres, is provided with a swelling (campanula Halleri), which is attached to the hinder part of the capsule of the lens. These processes are also found, in a somewhat modified condition, in the eyes of Reptiles and Birds. In the Saurii there is a curved and thickened fold which extends to the margin of the capsule of the lens, at the side of which there may be several other folds (Fig. 297, $p$ ). This structure is feebly developed in the eye of the Crocodilini. In Birds it is remarkable for the increase in the number of its folds, and is distinguished as the "pecten" (Fig. 298, p). In many Natatores and Grallatores it reaches as far as the capsule of the lens. In the Struthiones the end of the pecten is widened out into a pouch (marsupium). In Apteryx, as in the Mammalia, it is absent. The point, at which the optic nerve enters, varies with the characters of this process, for when it is widened out at its base the nerve is placed more to the side of the eye.

With regard to the lens, the difference in its form, in accordance with the surrounding media, is a noteworthy point. In Fishes it is very large, and quite spherical, as it is also in the Amphibia, and in aquatic Mammalia; while in others, and in Birds and Reptiles, we meet with more flattened forms of lenses; the amount of flattening is, of course, very various. The internal cavity of the eye is divided into an anterior and a posterior space by the attachment of the lens to the ciliary portion of the choroid. The vitreous body fills the hinder one; the anterior one, which lies between the anterior surface of the lens and the cornea, is often but a very small portion of the whole eye. It is filled by the aqueous humour.

\section{$\S 399$.}

Accessory organs, which partly serve to move, and partly to protect the bulb, are connected with the eye. The movements of the eye are generally effected by six muscles, of which four are straight and two oblique. They are atrophied in the Myxinoïdea. In many Teleostei the straight ones are embedded in a canal at the base of the skull; this is in adaptation to their length, which again is due to the large size of the bulb. They take their origin from a point which is placed some way behind that at which the optic nerve passes out; it is in the higher divisions only that they acquire relations to this point. In the Amphibia and Reptilia there is a retractor of the bulb, in addition to the four straight muscles. This is retained also by most of the Mammalia, and breaks up into several portions (in Carnivora into four), which pass to the bulb from the point at which the optic nerve enters the orbit. In the Mammalia the superior oblique, which, like the inferior oblique, ordinarily arises from the median wall of the orbit, is altered in character. It has the same origin as the straight muscles of the eye, and the tendon of insertion passes to the bulb through a pulley, and at an angle.

Of the protective organs of the eye, the eyelids are folds of the 
integument. The inner lamella of these folds is a continuation of the conjunctiva which extends on to the bulb, and which is continuous with the integument at the margin of the lid. Eyelids of this kind are found even in Fishes. In the Selachii there are two slightly projecting and movable folds, which appear to be indications of an upper and lower eyelid; in many Sharks there is also a third fold at the anterior angle of the eye, which can be drawn over the outer surface of the bulb (nictitating membrane). In the Ganoïdei and Teleostei the immovable folds are alone present, or there may be merely indications of them; they are ordinarily distinguished as the anterior and posterior eyelids. Most commonly the integument passes at once into the cornea. In the Perennibranchiata and Derotremata there is a connection of this kind. Many Salamanders, and the majority of the anourous Amphibia, are provided with horizontal eyelids, of which the lower, and more movable one, functions as a nictitating membrane.

In the Reptilia and Aves there is an upper and a lower movable eyelid in addition to the nictitating membrane. In some Saurii (Ascalabotæ) and in the Ophidii, the eyelids are developed as an annular fold, which continues to grow until at last it forms a pellucid membrane which lies in front of the eye, and which completely separates the cornea from the external medium. 'The circular rudiment of this structure corresponds to the circular eyelid of the Chamæleons. There is a muscular apparatus for the horizontal eyelids, as well as for the nictitating membrane. Whilst the two horizontal eyelids persist in the Mammalia, the nictitating membrane undergoes degeneration. It is supported, as are the two other eyelids, by a cartilaginous lamella. It is generally reduced to a fold, which is placed at the anterior (inner) angle of the eye; in the Primates it has lost its primitive significance, and forms the plica semilunaris.

A glandular apparatus for the eyelids is first differentiated in the Amphibia and Reptilia. In Reptiles and Birds, and also in Mammals, there is a gland which opens below the nictitating membrane (Harderian gland, or gland of the nictitating membrane), which is placed at the inner angle of the orbit; it is not present in the Primates. Its secretion is different from that of the lachrymal gland.

The Lachrymal glands, which are placed at the outer angle of the eye, are first seen in Reptiles, where they are smaller than the Harderian gland; they have the same characters in Birds. They are larger in the Chelonii and Mammalia (except the Cetacea), where the lachrymal gland consists of a complex of separate glands, which are generally united into larger masses.

A special efferent duct into the nasal cavity is formed for the secretion of these glands, which is passed out below the upper eyelid. A canal of this kind, formed by an epithelial thickening on the surface of the head, is present even in the Amphibia. In the Amniota the development of the lachrymal duct is connected with that of the face. The groove, which is formed between the 
processes of the upper jaw, and the external nasal processes by the differentiation of these parts, and which leads from the region of the inner angle of the eye towards the edge of the nasal pit, sinks deeper down as these processes are developed (lachrymal groove); it now becomes grown over by their edges so as to form a canal, which, when the nasal cavity is developed, opens into it just below the inferior turbinated bones. In the Reptilia (Lacerta) it opens near the posterior nares. At the inner angle of the eye this lachrymal canal is divided into several smaller canals; there are a larger number (3-8) of such canals on the lower eyelid of the Crocodilini, but a smaller number (2) in Birds and Mammals.

\section{Auditory Organs.}

\section{$\S 400$.}

The Auditory Organ of the Vertebrata, which has been observed in all, except the Acrania, is derived from the ectoderm. During the earliest embryonic period it is laid down in the form of a thickening above the myelencephalon, which extends inwards. A superficial organ of this kind, which must have carried the endings of an integumentary nerve, must be regarded as the starting-point of the great differentiation, which commences so early. The earliest rudiment gives rise to a vesicle, which communicates with

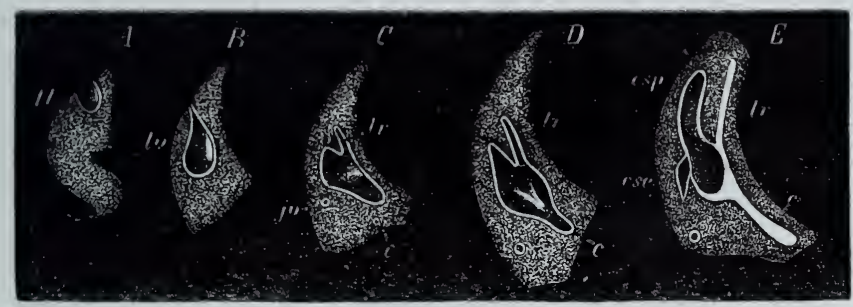

Fig. 300. Development of the labyrinth of a Fowl. Vertical sections of the rudimentary skull. $f$ l'it of the labyrinth. $l v$ Vesicle of the labyrinth. $c$ Rudiment of the cochlea. Ir Recessus labyrinthi. csp Posterior semicircular canal. cse Ex. ternal semicircular canal. jv Jugular vein (after Reissner).

the exterior, and which is gradually cut off (Fig. 300), and enclosed by the hinder lateral portion of the cartilaginous cranial capsule, when that is differentiated. The primitive otocyst is the foundation of a complicated cavitary system, in the walls of which the auditory nerve is connected with its end-organs. From this is developed the membranous labyrinth. The surrounding portions of the skull form the cartilaginous, or osseous labyrinth.

The simplest condition of the labyrinth is found in the Cyclostomata. In the Myxinoidea a tract, which remains connected at two 
points with the primitive vesicle, is differentiated from it, and forms a semicircular canal, so that the whole labyrinth has a circular form. In the Petromyzontes there are two of these canals, each of which commences with an ampulla-like enlargement, while the rest of the vesicle of the labyrinth forms the "membranous vestibule;" in this there is a special diverticulum, which is the rudiment of a new differentiation. In the Gnathostomata a third canal is developed, so that henceforward three semicircular canals open into the vestibule.

When the vesicle of the labyrinth sinks beneath the surface, its stalk-like basal piece remains open on the roof of the skull, in the Selachii, and swells out below the integument into a saccus endolymphaticus. This corresponds to the recessus labyrinthi (ductus endolymphaticus), which passes up as far as the roof of the skull in the Teleostei, and may undergo various metamorphoses. One of these metamorphoses has been regarded as leading to the growth of this portion into a tube which covers the brain (Urodela), or extends to the base of it (Anura). In the Ophidii and Saurii it reaches to the roof of the skull, being filled in the embryo with crystals of lime, and widened out. In Phyllodactylus it extends beyond the skull, and may even pass into the cervical region, being swollen out in parts. The connection between these structures and the primitive stalk of the otocyst is denied, so that the recessus labyrinthi is regarded as an independent structure. Most of its relations, however, require to be more carefully investigated. In Birds it is an open cavity $(r l)$, for a short time only; so, too, in Mammals, where later on it forms the aqueductus vestibuli. The vestibule and semicircular canals are very large in all Fishes; in the Selachii and Dipnoï they are com-

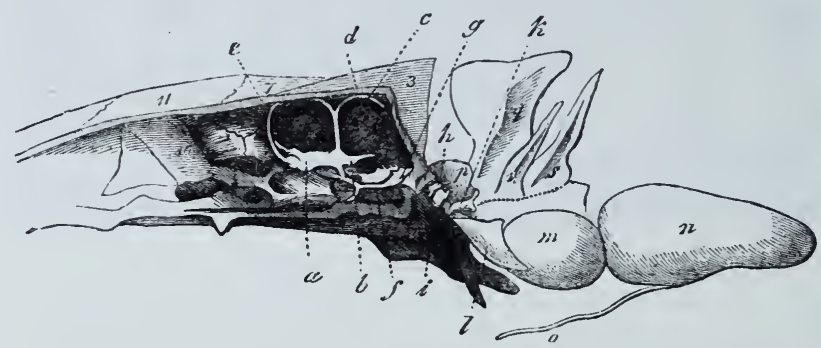

Fig. 301. Auditory organ of Cyprinus carpio. a Membranons vestibule. $b$ Ampulla of the posterior and external semicircular canal. $c$ United anterior and posterior canal. $d$ Posterior. e Anterior. $f$ Canalis sinus imparis. $g$ Sinus auditorins impar. $k$ Claustrum. $i k l$ Chain of connecting ossicles. $m n$ Air-bladder $o$ Air-duct. $p q r s$ Spinous processes of the anterior vertebræ. The numbers indicate the different bones of the skull. 1 Basi-occipital. 2 Exoccipital. 34 Supra-occipital. 6 Petrosal. 7 Parietal. 10 Alisphenoid. 11 Frontal (after E. H. Weber).

pletely surrounded by the walls of the skull, while in the Teleostei the median portion projects freely into the cranial cavity (Fig. 301). Of the three semicircular canals, two-an anterior $(e)$ and a posterior one $(d)$-are placed in the direction of two planes, which cross 
one another more or less perpendicularly; the third, and outer one, lies in a more horizontal plane, and is provided with an ampulla on its posterior limb. The two vertical canals have a common piece $(c)$ which opens into the vestibule, and ampullæe at the two other ends.

Even in Fishes the vestibule of the labyrinth is divided into several portions. An upper one is directly connected with the semicircular canals (utriculus, alveus communis), and with the subjacent sacculus. The sacculus and utriculus contain otoliths, which are constant in the same, but different for different, divisions; they are often very large. The branches of the auditory nerve pass into the end-organs which are to be found in the walls of both cavities, as well as in the ampullæ of the semicircular canals; in the ampullæ they are placed on a transverse ridge (crista acustica); in the saccules they form the maculæ acusticæ.

Of the numerous modifications which may be observed, the connections between the membranous vestibule and the air-bladder are worthy of remark; the arrangement is effected in various ways; it is simplest in some of the Percoidea, and Sparoidea, where the vestibule is continued into spaces in the skull, which are merely covered by membrane; to these spaces processes of the air-bladder are attached. The relations are more complicated in many families of the Physostomi. In the Cyprinoids the sacculus $(a)$ extends backwards, and is connected with that of the other side by a sinus impar. This gives off a membranous saccule (atrium sinus imparis) on either side, which passes to an opening on the posterior portion of the skull, which is partly closed by a small bone. This is connected by masses of ligament with a series of bony pieces $\left(i l_{i} l\right)$ of various forms, the last and largest of which is attached to the anterior end of the air-bladder $(m)$. These ossicles are modifications of ribs, and form a continuous chain between the vestibule and the air-bladder. In the Siluroidea and Clupeidea connections with the air-bladder are effected in a different manner.

\section{$\S 401$.}

In and above the Amphibia, the labyrinth is greatly diminished in size from what it is in Fishes. It is still of a relatively large size in the Amphibia, and is smallest in the Mammalia. The differences which are seen in it are partly due to the way in which the two cavities of the vestibule, the utriculus and sacculus, are connected together, and to the course taken by the semicircular canals which spring from the former. The posterior canal may sometimes be set at an angle to the external one (Birds).

There is a great difference between the portion of the labyrinth, just described, and which is very similar in all forms, and that part which is only developed as an independent structure in the higher divisions; this, which is known in Mammals as the cochlea, on account of its form, presents a continuous series of differentiations 
from the lower divisions upwards. In Fishes there is an indication of it in the form of a diverticulum, generally a small one, of the sacculus. In the Selachii it contains a number of small otoliths; in the Teleostei one larger one (asteriscus). In the Amphibia this diverticulum of the sacculus is more independent, but it is still connected as before, and is still directed backwards.

This portion, which carries the end of a branch of the auditory nerve, is still further differentiated in the Reptilia and Aves, where the diverticulum, which forms it (Fig. 300, $C D E c$ ), is a short conical piece, which is directed downwards from the median wall of the labyrinth, and converges towards its fellow of the opposite side. Its end is somewhat bent, and it forms the "lagena." Ainong Mammals this stage of the organ is seen in the Monotremata only; in the rest this stage is not the permanent one, for the organ is converted into a spirally-coiled canal. At first it is only formed by a prolongation of the sacculus, but special differentiations appear in it, and this cochlear canal, which is formed from the sacculus, is permanently connected with it by a narrower portion only (canalis reuniens, Fig. 302). The organ, which thus becomes more indepen-
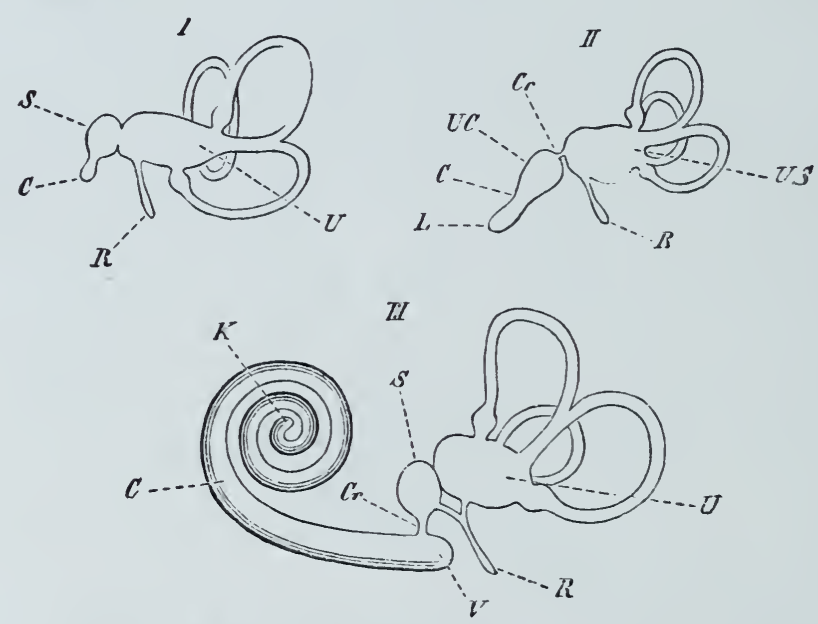

Fig. 302. Diagrams in explanation of the labyrinth. I Fish. $I I$ Bird. III Mammal. U Utriculus. S Sacculus. US Utriculus and Sacculus. $C r$ Canalis reuniens. $P$ Recessus labyrinthi. UC Commencement of the cochlea. $C$ Cochlea. $I$ Lagena. $K$ Cæcal sac at apex. $C$ Cæcal sac of the vestibulum of the cochlear canal (after Waldeyer).

dent, is surrounded on two sides of its course by lymphatic cavities, which accompany it in its coils, and pass into one another at the apex of the cochlea. One cavity is connected with the osseous vestibule, theother is shut off from it at its commencement, and is only connected with the cavity of the vestibule indirectly; that is by the communication at the apex of the cochlea. Three cavities, therefore, can be distinguished in the Mammalian cochlea; but one only, the ductus cochlearis, is connected with the vestibular labyrinth. The other two form the scalæ-the sc. vestibuli and sc. tympani. The two scalæ occupy the periphery of the coils of the ductus cochlearis, at the base of which the end-organs of the cochlear nerres (organ 
of Corti) are spread out. As the scalæ arise as spaces in the tissue which accompanies the ductus cochlearis, they are similar to the cavities between the membranous semicircular canals and their bony wall, or between the membranous and osseous vestibules, and are filled with perilymph.

In the Amphibia, and all higher forms, spaces appear in that part of the walls of the bony labyrinth which lies on the outer surface of the cranium; these in a different fashion effect a communication between it and other arrangements that are connected with the auditory organ. The fenestra ovalis, which is always closed by a plate-like piece of bone, is a hole of this kind in the osseous vestibule. A second opening, which appears first in Reptiles, and which is correlated with the further development of the cochlea (fenestra rotunda), lies in the wall of the scala tympani, and is closed by a membrane.

Both these arrangements are related to the development of external conducting organs.

Retzius, G., Anatom. Untersuch. I. Stockholm, 1872.-Hasse, C., Anatomische Studien. Leipzig, 1870-73.

\section{$\S 402$.}

Other parts are gradually added on, as accessory organs, to the auditory organ, although primitively having no relation to it. The first branchial cleft, which in the Selachii and Ganoïdei persists as the "spiracle," enters into close relation with the wall of the labyrinth in the Amphibia. As it grows over this wall it is converted into a cavity, the wider portion of which forms the tympanic carity; this is bounded in the middle line by the wall of the labyrinth; the portion which leads into the primitive buccal cavity forms the Eustachian tube. It reminds us of its primitive (spiracular) condition by at first communicating freely with the exterior. The cleft is, however, soon closed, which leads to different arrangements. In the Cœeciliæ and Urodela the cleft is closed by the superjacent muscles, so that there is no tympanic cavity. One division of the Anura (Pelobatidæ) presents the same arrangement, for in it there are only slight indications of the outgrowth of the mucous membrane of the pharynx into this cleft. In most Anura, however, that membrane does form an outgrowth, and leads into a tympanic cavity, which is closed externally by a tympanic membrane. Among the Reptilia, the Ophidii, and Amphisbænoidea have no tympanic cavity; in Chamæleo there is no tympanic membrane; but both these parts are present in all other Reptiles, and in Birds.

In the Crocodilini and Aves, the inner openings of the Eustachian tubes are united into a common canal, as is the case also in Pipa among the Amphibia.

Those parts of the visceral skeleton which are connected with the 
bony labyrinth, unite to form the apparatus of the auditory os sicles; the homologies of which have not yet been definitely made out for the different classes. The first portion is formed by an ossicle (operculum) which closes the fenestra ovalis ; in the Urodela this is either flat, or provided with a stalk-like process. Sometimes it is cartilaginous and its stalk ossified (Siredon); sometimes the reverse is the case (Menopoma). In the Cœcilix they are both ossified. The same arrangement obtains in the Ophidii (Eurystomata), where a small piece of bone (columella) reaches to the quadrate.

When there is a tympanic membrane present the columella is connected with it; for its cartilaginous end, which has often a peculiar form given to it by processes, sinks into that membrane. The lining tissue of the tympanic cavity then surrounds part of the columella, and causes this bone to appear to be more or less placed in the tympanic cavity. These arrangements are first seen in the Anura, and are still further developed in the Saurii, Chelonii, Crocodilini, and Aves. The process of the columella is in some Birds (Dromæus) connected with its plate by two limbs; in other cases it is simple, or is connected with the plate by one enlargement only.

Just the same relations are seen to obtain in the columella of the Mammalia; with this modification, however, that it is never directly attached to the tympanic membrane. It is converted into the stapes, the form of which, in the Monotremata and many Marsupials, calls to mind the columella. In the monodelphous Mammalia it is ordinarily divided into two limbs, which carry the plate. The other auditory ossicles are the incus, which is connected with the stapes, and the malleus, which is attached to the tympanic membrane by a styliform process. A connection between the tympanum and the fenestra ovalis, which was previously effected by a single bone-by the columella alone-is now effected by it, and two other bones. This "chain" of auditory ossicles is, for the most part, at any rate, placed in the tympanic cavity, for it is covered by the mucous membrane which is continued into that cavity from the pharynx, through the Eustachian tube. The tympanic cavity itself has, however, another relation, for it is principally formed by the tympanic bone, in addition to the boundaries provided by the wall of the labyrinth; this tympanic bone commences as the framework of the tympanum.

\section{$\S 403$.}

The external ear is derived from a prolongation of the edges of the first branchial cleft. In the Amphibia, Reptilia, and Aves, these parts are either altogether absent, or are only present in individual cases, where they have been dereloped in consequence of various kinds of adaptive changes. Thus, in the Crocodilini a fold of the integument forms an operculum above the tympanic membrane, and in the Owls there is a movable membranous valve. As early as the Saurii the tympanic membrane is removed some way from the surface, so that there is a short "external 
auditory meatus." The external auditory meatus in the Mammalia is different, for its deeper portion is formed by the tympanic bone. The external ear, the cartilaginous support of which is continuous with a narrow auditory meatus, is attached to this. There is no external ear in the Monotremata. The "external ear" may be much modified, either in form, or in its relations to the muscular apparatus, which moves it. In addition to the muscles, which move the whole of the external ear, and which are sometimes of much power even in Man, there are others which are placed in the cartilage of the ear itself; these are partly represented, though of course as rudimentary organs, in the human ear. This external ear is still more atrophied in aquatic Mammalia. Reduced in Otaria, it is altogether absent in the rest of the Pinnipedia, as it is also in the Sirenia and Cetacea.

\section{Alimentary Canal.}

$$
\S 404 \text {. }
$$

The alimentary or enteric canal of the Vertebrata forms a tube, which runs below the axial skeleton, and in which two chief portions can be distinguished morphologically, as well as physiologically, at a very early epoch. The most anterior portion is directly connected with the body-wall, and, as it is perforated by branchial slits, it functions as a respiratory organ, for respiratory apparatuses are developed in the vascular arches between the clefts. This portion does not, therefore, belong exclusively to the digestive organs, although it is used in the ingestion of food. It forms the respiratory cavity, at the end of which the nutrient canal, in the strict sense, commences; this is separated from the body-wall by the pleuro-peritoneal cavity. The Vertebrata have these two portions of the enteric tube in common with the Tunicata. In the Acrania the respiratory chamber of the enteric tube occupies a very large portion, which, as in the Ascidiæ, represents a large

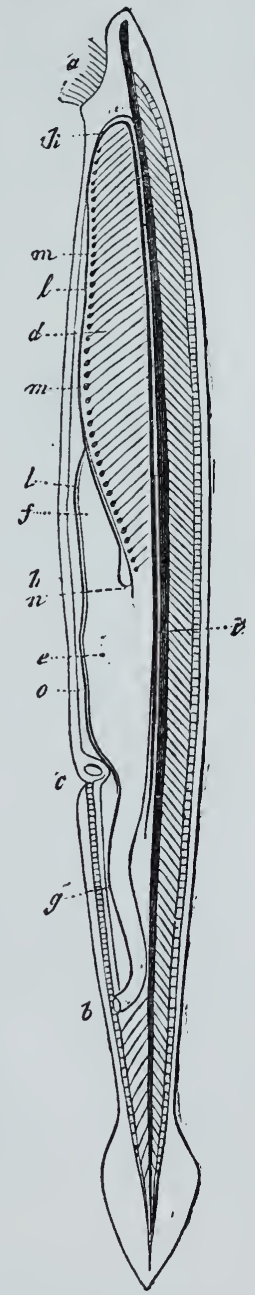

Fig. 303. Aniphioxus lanceolatus $\left(\times 2 \frac{1}{2}\right)$. a Mouth,surrounded by cirri. $b$ Anus. $c$ Abdominal pore. $d$ Branchial sac. $e$ Gastric portion of the enteron. $f$ Cæcum. $g$ Hind-gut. $h$ Cœlom. $i$ Notochord, below which is the aorta, which accompanies it for nearly its whole length. $k$ Aortic arches. $l$ Aortic heart. $m$ Enlargements of the branchial arteries. $n$ Heart of the vena cava. o Heart of the portal vein (after Quatrefages). 
part of the body. This space is gradually reduced in size in the Craniota; it still, however, retains its respiratory function, but many other organs are also differentiated in it; these are, largely, accessory organs for the ingestion of food.

Respiratory Ante-chamber (Cephalic enteron). $\S 405$.

In Amphioxus this portion is bounded, in its most anterior region, which is close to the cavity which carries the mouth, by a ciliated apparatus; there are a number of movable processes also at that point, which are directed towards the lumen of the tube, and so prevent the entrance of foreign bodies. The ante-chamber (Fig. 303, d), which occupies about two-fifths of the whole length of the body, has its walls broken through by a large number of obliquely-set clefts; these form a complicated framework, the supports of which have been already $(\$ 353)$ mentioned. The water, which is taken in by the mouth $(a)$, passes through the clefts, and so to the exterior. But as two lateral dermal folds are gradually continued ventrally over the surfaces on which the clefts are placed, and become united below, a peribranchial cavity is formed, which opens by a special pore $(c)$. It should here be remembered that there was something similar to this in the Ascidiæ (\$310). But it would not be correct to suppose that the two structures are morphologically identical. A vascular plexus is distributed in the walls of the clefts, the water that streams past effects respiration, the clefts function as branchial clefts, and the whole cavity represents functionally a branchial cavity.

There are many special points in this arrangement in Amphioxus, such as the want of symmetry in the branchial frame, and its inde-

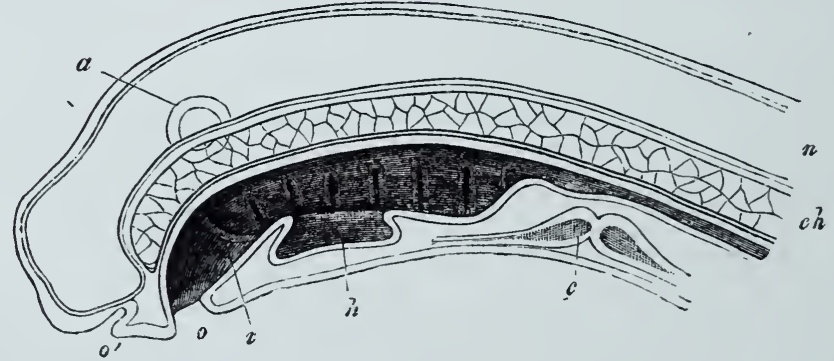

Fig. 304. Vertical median section of a larva of Petromyzon. o Mouth. $v$ Velum. $h$ Hypobranchial groove. $n$ Spinal chord. ch Notochord. a Otocyst. c Heart (after a drawing by Calberla).

pendence of the metamerism of the body; so that there is altogether a great difference between this apparatus and that of the Craniota. 
The region of the body which is occupied by the branchial cavity corresponds to a head, for the nerves which go to it arise from the myelencephalon in the Craniota. Viewed thus the branchial cavity represents a cephalic enteron. Its nutrient and respiratory significance is the cause of various differentiations in it, which are partly arrangements which are peculiar to the Vertebrata, and partly arrangements which have been inherited from a lower condition. In addition to the branchial clefts, the ventral groove (hypobranchial groove), which is developed on the ventral surface of the branchial cavity, belongs to the latter series; this has just the same relations as in the Tunicata (cf. p. 402); it is found in the larvæ of the Petromyzontes, where it forms a grooved depression, enclosed by ridge-like edges (Fig. 304, $h$ ). In Amphioxus this structure is also present. Its presence in various stages of metamorphosis in all Craniota, not only brings these forms into closer connection, but is an indication of their genetic relations to the Tunicata, which must not be forgotten (cf. § 416).

Branchiæ.

$\S 406$.

In the Craniota the branchial clefts are universally much reduced in number, as are also, in correspondence with this, the arches of the branchial skeleton. This phænomenon must be regarded as the degeneration of a primitively larger number of these structures, such as is found in Amphioxus; it is compensated for by the increased size of the surfaces which carry the respiratory vascular plexus. This increase in size is implied by the development of the gills, whereby the blood-vessels, which, in the Acrania, are distributed over a large number of arches, are limited to a less extensive region, and are therefore arranged on a smaller number of arches. The essential point in the formation of branchir in these animals is the increase of the surface which is directed towards the respired medium, and this increase may be effected by means either of lamellæ or of cylindrical processes. 'The branchial arches are provided with various forms of these organs, which enclose the well-developed respiratory vascular network. We find that in the Cyclostomata these organs have special characters, which have but little resemblance to what is found in Amphioxus; their earliest condition is most like what is seen in the Gnathostomata, for the branchial clefts are simply spaces in the body-wall (Fig. 304). They are differentiated into tubes, the median portion of which has its lumen widened, and forms a branchial pouch (Fig. 305, $b \%$. Branchial lamellæ are raised up from the wall of the branchial pouches in the form of leaf-like folds, in which the respiratory vascular plexus is spread out. Each branchial pouch is connected, by an "internal branchial duct," with the anterior 
section of the enteric tube. An external branchial duct $\left(b r^{\prime}\right)$ leads to the exterior. There are sereral variations in the characters of the two canals which spring from each branchial pouch. The inner ones either open each separately into the digestive tube (Bdellostoma, Myxine)

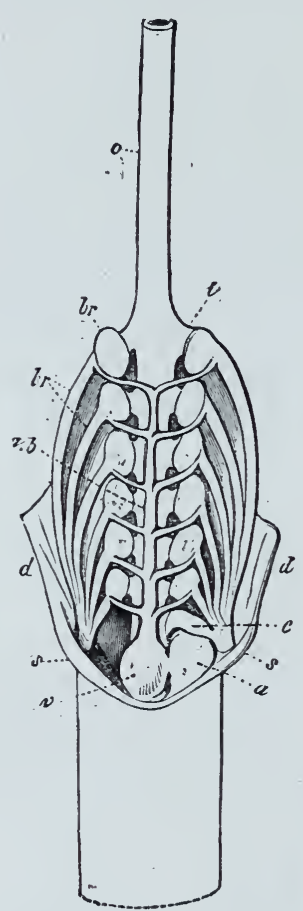

Fig. 305. Respiratory organ of Mlyxine glutinosa, seen from the ventral surface. o Esophagus. $i$ In. ner branchialducts. $b r$ Bran. chial pouches. $b r^{\prime}$ External branchial ducts, which unite on either side into a common branchial duct, which opens at $s . \quad c$ Ductus œsophagocutaneus. $a$ Auricle. $v$ Ventricle. $a b$ Branchial artery, giving off a branch to each gill. $d$ Lateral wall of the body turned outwards and backwards (after Joh. Müller).

(Fig. 305), or they all unite into a median respiratory tube which runs below the digestive tube, and being connected in front with the digestive tube carries water to each of the branchial pouches (Petromyzon). The external branchial ducts either open separately on the sides of the body (Bdellostoma, Petromyzon), or all the ducts of one side are united into a branchial pore $(s)$ which lies behind the branchial apparatus; on the left side a special canal (c), which comes from the osophagus (ductus œsophago-cutaneus), also opens into the same pore (Myxine). These different forms may be derived from one another; in the case both of the inner and the outer branchial ducts that condition should be regarded as the primitive one, in which there is a direct connection between the respiratory chamber and the surface; while, on the other hand, the formation of the respiratory tube, and the union of the external branchial ducts, is the result of a subsequent differentiation.

\section{$\S 407$.}

In Fishes, the branchial pouches are more closely related to the skeleton. The phænomena seen in them lead to the conclusion that each arch of the primitive branchial skeleton carried gills. The upper part of the first (mandibular) arch is not excluded from this; as is clear from the frequent presence of a gill in the opening, which is found in many Selachii- the socalled spiracular cleft-between the first and second arches (mandibular and hyoid arches). The spiracular canal, which represents a degenerate branchial pouch, is succeeded by the true branchial pouches, of which there are, as a rule, five; and rarely six or seven (Notidani). The wall of the first pouch is supported in front by the hyoid arch, and behind by the first (i.e. by the third primitive) branchial arch; the other pouches have just the same characters. In each of them a septum $(s)$, supported by cartilaginous rays, extends outwards from the internal branchial skeleton, and serves as the posterior wall of the 
pouch in front, and the anterior wall of the one behind. While the pouches communicate with the pharyngeal cavity by narrow orifices, which are bounded by the cartilaginous branchial arches, they also open on the side (or in Rays, on the ventral surface) of the body by just as many clefts. Rows of branchial lamellæ lie in the walls of the branchial pouches; in the embryonic condition these lamellæ develop filamentous prolongations, which form the external gills. These are also found on the spiracular cleft. The anterior wall only of the last branchial pouch is provided with a gill (Fig. 306, A).

From this arrangement we may derive what we find in the Ganoïdei, and from that what we find in the Teleostei. The spiracular gill, which has no respiratory function in the adult state of the Selachii, is the first to undergo the greatest degenerations. In some Ganoïdei, which possess a spiracle (e.g. Acipenser), the gill is converted into a Ps eudobranchia (a gill which has lost its proper branchial artery and vein); this is not found in Polypterus nor Amia. In the Osseous Fishes it also appears to be wanting, or has lost all resemblance to a gill.

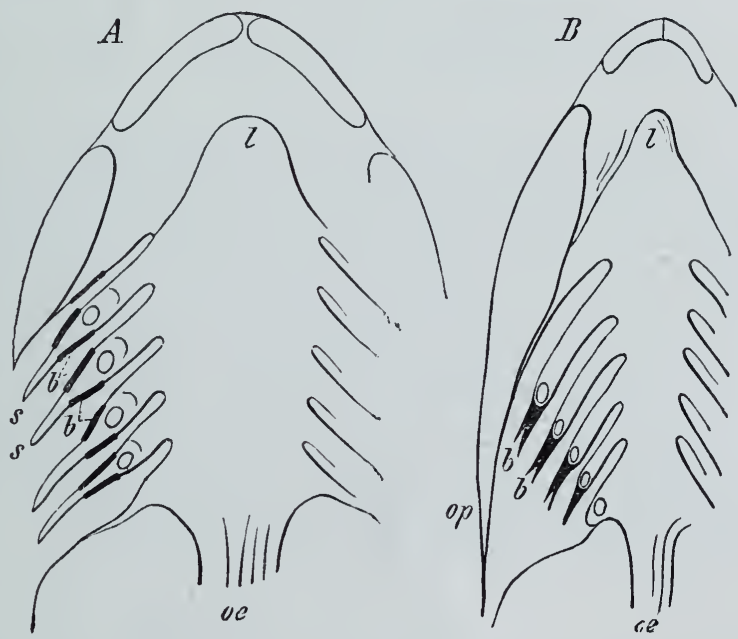

Fig. 306. Horizontal section through the branchial cavity. $A$ Of Scyllium. $B$ Of Barbus. The floor of this cavity is shown. $l$ Tongue. oe Esophagus. $s$ Septa of the branchial pouches. $b$ Gills. op Operculum. The anterior series of branchial lamellæ of the Selachii, that, namely, which is attached to the posterior face of the hyoid arch, is also found among the Ganoïdei, where it forms a respiratory op ercular gill (Acipenser, Lepidosteus). It is found also during the embryonic stages of the Teleostei, but it does not persist. It sometimes consists of a short row of lamellæ, which is attached to the operculum; sometimes it is carried to the base of the skull, and sometimes it is hidden below the mucous membrane. Even when in this condition, rudimentary cartilaginous rods may be found in it. When still more degenerated, it forms a glandular structure, which is made up of several lobules (Esox).

When all trace of the external branchial skeleton disappears, the septum which arises from each of the inner branchial arches disappears also, or is reduced to a slender fringe. Owing to this, the rows of branchial lamellæ in the Ganoïdei and Teleostei come into close relation with their respective branchial arches, and are 
consequently placed in two rows on each arch as it passes between two branchial pouches (Fig. 306, $B \mathrm{~b}$ ). The anterior row of branchial lamellæ in a Teleostean or a Ganoid corresponds, therefore, to the gill on the hinder wall of the branchial pouch of a Selachian, and the hinder row of lamellæ in a Teleostean gill corresponds to the anterior gill in the branchial pouch of a Selachian.

These relations are shown in the following diagram, in which $b$ is the indifferent stage of the rows of branchial lamellæ, $B$ is the differentiated arrangement in the various divisions. $\beta$ represents any row of branchial lamellæ, which is specially modified or reduced:

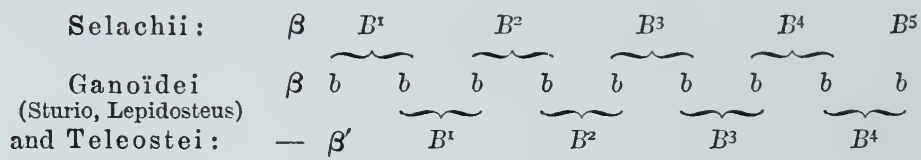

By the degeneration of the septa between the branchial pouches, the whole gill apparatus is made more compact, and no longer therefore extends back into the region of the trunk, as it does in the Selachii; it is confined to the base of the skull. Whilst in the Selachii the projecting septum $(A s)$ forms an organ of protection for the succeeding branchial pouch, a similar organ is formed in the Chimæræ, Ganoïdei, and Teleostei, from a single arch-namely, from the hyoid; the integument on this arch grows backwards and covers all the gills, and is developed, in the Ganoïdei and Teleostei, into the opercular apparatus and the branchiostegal membrane, with their various skeletal pieces ( $\$ 354)$ ( $B$ op).

\section{$\S 408$.}

In the Teleostei four arches are ordinarily beset with branchial lamellæ, the fourth arch having a single row only, or there are but three arches which carry lamellæ. When the lamellæ of the fourth arch, and the posterior row on the third arch disappear, the fourth branchial cleft is ordinarily closed. Perhaps one of the most important of the modifications which affect the lamella themselves is seen in the villous gills of the Lophobranchiata. In some divisions of the T'eleostei, the branchial arches seem to be so metamorphosed as to be able to retain the water in the branchial apparatus. The organs of the Labyrinthobranchiata are of this kind; separate branchial arches or parts of such, are modified to form coiled lamellalike processes, which give rise to a portion which is placed above the gills (Anabas, Polyacanthus). Another apparatus, which is found in various Clupeidæ, consists of a spirally-coiled tube (branchial coil), which is formed by a diverticulum of the superior pharyngeal mucous membrane. This tube is generally connected with the superior segment of the fourth branchial arch, and has processes of its skeletal parts in its walls (Heterotis, Lutodeira, Meletta, etc.). The 
arborescent processes of the branchial arches, which are placed in special prolongations of the branchial cavity, where they support a respiratory vascular plexus, also belong to this series (Heterobranchus, Clarias).

Diverticula of its investing mucous membrane have the same respiratory function as the cavity itself. Thus, in Saccobranchus, a long tube extends from the branchial cavity, on either side, as far as the lateral trunk muscles; in Amphipnous there is a similar sac behind the head, which opens just above the first branchial cleft. Both these organs contain respiratory vascular plexuses.

\section{$\S 409$.}

External gills in the form of integumentary structures were not primitively possessed by the Vertebrata, for the so-called external gills of the Selachian embryo are nothing more than filaments of the internal gills which protrude through the branchial cleft. Gills, however, may come to the surface, and take the form of tegumentary processes; such gills may be seen in the young stages of Polypterus; certain gills of Protopterus, and the gills of the Amphibia generally are of this character. In the Amphibia the gills have the appearance of two or three pairs of branched processes, which spring from as many branchial arches. In the Perennibranchiata this apparatus is permanently functional. In the rest of the Amphibia (Caducibranchiata) these external gills disappear; in the Anourous forms, where they are found for a short time only, they are replaced by shorter internal gills. A membrane which grows from before backwards covers the gills, so that there is only one efferent orifice. The orifices on either side may continue to grow out, and get nearer to one another, so as to unite into a single ventral orifice.

When the larval stage ceases, the inner and outer gills of the Derotremata and Salamander are atrophied; in the latter, as in the Anura, the branchial clefts are completely closed, but in the Derotremata a cleft is left on either side.

When the gills disappear, the branchial cavity, which constitutes the respiratory antechamber, is converted into the primitive buccal cavity, which is limited by essentially the same parts as it was before.

\section{Branchial Clefts, and Palate of the Amniota. \\ $\S 410$.}

In the Amniota, also, the arrangement which has been transmitted from their branchiferous ancestors is retained during certain stages of embryonic life, in the form of clefts in the wall of the pharynx. These branchial or visceral clefts are never more than four in number, and they appear in such a way from before 
backwards, that when the last has appeared the anterior ones have already undergone certain changes. They are all gradually atrophied, and completely disappear, except the first, part of which is converted into the middle and outer ear (cf. suprà, § 402).

The degeneration of the embryonic branchial clefts is an important point of difference between the Amniota and the Anamnia, but, in addition to this, there is a new peculiarity which is due to a differentiation of the primitive buccal cavity. This leads to the formation of the secondary nasal cavity, and of the secondary buccal cavity. The remnant of the primitive buccal cavity, which lies behind, and is not affected by this process, forms the Pharynx. The cartilaginous portion of the ethmoid, which separates the two nasal cavities, and is broad in the Amphibia, is developed in the Amniota into a thin vertical lamella (Fig. 307,e)-the internasal septum. It remains partly cartilaginous, and is partly converted into,

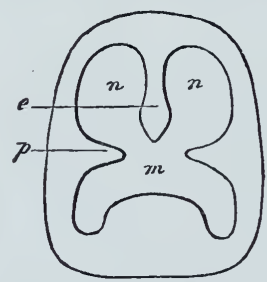

Fig. 307. Diagram of the differentiation of the primitive buccal cavity into nasal cavities $(n n)$, and a secondary buccal cavity $(\mathrm{m}) . \quad p$ Palatine plate. $c$ Internasal wall.

and develops bony structures, which were treated of under the cephalic skeleton.

A second change is brought about by horizontal ridges or processes, which are given off from the maxillary process of the first arch, and which gradually form a plate (Fig. 307, p), the palate, which divides the primitive buccal cavity into two compartments. This plate forms the floor of the upper, or nasal cavity $(n)$, and the roof of the lower one $(m)$. When the internasal septum reaches this palatine plate it separates the nasal cavity into two portions, into each of which the nasal canal now opens, while its external orifice is coincident with that of the bifid nasal cavity. The posterior orifices of the nasal cavity, the choanæ, which are separated by the palatine plate from the buccal cavity, and by the vertical internasal wall from one another, open into the pharynx.

Very various stages in the arrangement of these palatine plates may be observed. In the Ophidii, Saurii, and Aves, the process of separation is less complete, the posterior nares form a longitudinal cleft, owing to the palatine processes uniting anteriorly, but being separated from one another posteriorly. They are sometimes separate in Birds, in which case they are exceedingly small. In the Crocodilini they are placed farther back than in any other forms, while in the Mammalia they do not open into the secondary buccal cavity, but into the pharynx. This latter region is thereby-as also by the opening into it of the Eustachian tube, which is developed from the first visceral cleft-shown to be a portion of the primitive respiratory antechamber.

In Reptiles and Birds the palate is supported by pieces of the Skeleton (vide suprà); in Mammals the hinder portion is formed of soft parts, which form the "velum palatinum." 


\section{Nasal Cavity.}

$\S 411$.

While the nasal cavities are increased in length, owing to their being shut off from the buccal cavity by the palate, the increase in the size of the facial portion of the head also affects them; they increase both in length and height, and thus become large spaces. The olfactory nerve ends in their superior and posterior portion only (regio olfactoria), while the inferior and anterior portion principally serves as an "air-passage," and consequently comes into relation with the respiratory organs (regio respiratoria). The whole differentiation therefore of the nasal cavity is seen to be connected with the development of the lungs, and their increased physiological importance. The increase in the extent of the internal cavity is effected in various ways. The lateral wall of the nasal cavity, which is developed from the primordial cranium, always takes part in this process; the turbinate bones are lamellar, folded, and coiled processes of this wall.

In the Reptilia there is only one turbinate bone; this extends backwards from a cavity, which commences at the external nasal orifice, and is generally horizontal in position ; it is feebly developed in the Chelonii, and best developed in the Crocodilini. It is very varied in character in Birds. Sometimes it is simple (Columbæ), sometimes complicated by coils (Raptores), or it may be cleft into several lamellæ (Struthio). A turbinated structure is connected with the internasal septum in front of, and below this bone, and is by this connection distinguished from the turbinate bones, which are always lateral in position. This pseudo-concha separates the vestibule of the nose from the internal nasal cavity.

Above the turbinate bone, and, as a rule, at the upper blind end of the nasal cavity, there is another process which corresponds to a depression formed in the wall of the nasal cavity by an air sinus. Part of the olfactory nerve ends on this process, which is not found in the Columbidæ. In the Mammalia three turbinate bones may be distinguished. The lower one corresponds to the single bone in the Reptilia and Ares; it varies very greatly owing to the way in which its lamellæ are ramified and variously coiled, e.g. in the Carnivora (it is most complicated in Lutra and Phoca). These bones are least developed in various Marsupials (Macropus, Phascolomys), in the Apes (they are simplest in the Platyrrhini), and in Man. In the Cetacea the cavity has undergone degeneration in consequence of the loss of its olfactory function. The orifice on the upper surface of the skull leads into a vertical canal, which is divided by the internasal septum, and which can be shut off from the pharyngeal cavity by an occlusor muscle; there are no signs of any turbinate bones in it. 
$\S 412$.

There are accessory organs belonging to the nasal cavity. These are:

1) Accessory cavities of the nose. These are formed by the sinking of the mucous membrane of the nose into parts of its firm wall. They are first seen in the Crocodilini, where there is a cavity in the side walls of the nasal cavity, which communicates with it. In Birds we frequently meet with connections between the nasal cavity and the spaces in the neighbouring bones. In the Mammalia the nasal cavity communicates with a number of cavities in different bones of the skull, the most important of which are the sinus frontales. These are cavities which are placed in the frontal bone, and which are either single, or divided into smaller portions; they are very greatly developed in the Ruminantia. There are other communications with the sphenoid; these are greatly developed in the Elephant, for example, where the cavities extend through the parietal and temporal bones as far as the occipital condyles. Lastly, there are connections between the nasal cavity and the maxilla; these form the sinus maxillaris, which is developed in Marsupials and Ruminants, and very largely in the Solidungula. In Primates they are less extensive, and they are not present in most Carnivora, Edentata, or Rodentia.

2) Glands. There are larger glands connected with the nasal cavity in addition to the glandular structures which are ordinarily found on the mucous membrane of the nose. When they are more developed they may also extend outside the nasal cavity. Such nasal glands are found in the Amphibia and in the Ophidii, as also in the Saurii and Crocodilini; in the former they lie outside the upper jaw, and in the latter they are enclosed in a maxillary sinus. In Birds also there is an external nasal gland, which is sometimes placed on the frontals, and sometimes on the nasal bones. Among the Mammalia also we find a gland on the sides of the face, but it is absent in several orders.

3) Organ of Jacobson. This is a canal placed at the base of the nasal cavity; it is generally attached to the nasal septum, and communicates at the palate with the buccal, though it is shut off from the nasal, cavity; its walls, which form various kinds of processes, carry the ends of a branch of the olfactory nerve, which passes down the sides of the septum. In the Ophidii and Saurii the canal is partly enclosed by the vomer; in the Mammalia these organs are elongated, and are continued, as the ducts of Stenson, through the incisor canals, to the surface of the palate; they are best developed in the Ruminantia and Rodentia ( $\$ 396)$.

\section{Buccal Cavity.}

\section{$\S 413$.}

When the primitive antechamber of the enteric tube is divided into the nasal and buccal cavities, by the formation of a palate, a 
number of organs, which were seen in the primitive arrangement, are assigned to the buccal cavity, while other organs appear only as later developments. The teeth, the tongue, and various glandular organs belong to the former series. The soft palate, or velum palati, which is only found in Mammals, is a new organ. This muscular apparatus forms the posterior boundary of the buccal cavity, and separates it from the pharynx. The uvula is a median prolongation of the velum palati; this structure is apparently found in the Primates only.

In Reptiles and Birds the anterior and lateral boundaries of the buccal cavity are formed by the margins of the jaws, which are invested by the integument, and by the hard structures which are found on the jaws. In the Saurii and Ophidii the integument develops pad-like lips along the edge of the jaw. In all Mammals, except the Monotremata, the integument is separated from the edge of the jaws, and invests a complicated muscular layer which has its origin in them; this layer forms the ground-work of the lips, and gives them their mobility. In this way a space is developed which lies in front of the buccal cavity - the vestibulum oris. The lateral portions of this space form the cheek-pouches, and, when capable of great extension, develop into the pouch-like diverticula of many Mammals (buccal pouches of the Rodents and Apes).

\section{Organs of the Buccal Cavity.}

$\S 414$.

Of these organs those hard structures which serve for the pre. hension and comminution of food are of various kinds. Some of them are formed by the cornification of epithelial cells. The sucker-shaped mouth of the Cyclostomata (Fig. 308) is beset with horny teeth of this kind, which are also present on a tonguelike organ in these animals. In the Amphibia the edges of the jaws are provided with a similar covering; these structures, which are partly limited to the larval stages, are formed of a number of closely-set denticles (Anura); in Siren some are retained throughout life.

The larger horny coverings on the margins of the jaws of the Chelonii, Aves, and Monotremata differ somewhat from these horny denticles; they are compensatory arrangements, the presence of which is due to the absence of true teeth. Although

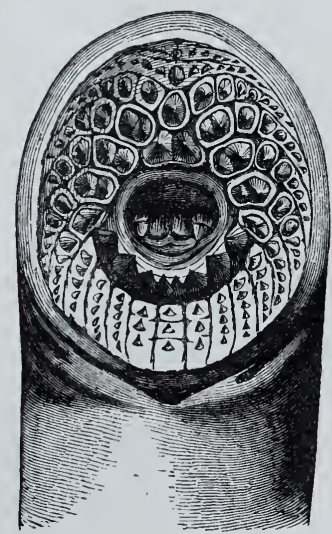

Fig. 308. Mouth of Petromyzon marinus, with its " horny teeth" (after Heckel and Kner). these structures are used for the comminution of food, they have nothing to do with true teeth ; they are purely epidermal structures, as is also the whale-bone of Whales. 
The true teeth are the product of the mucous membrane of the mouth, which is formed of connective tissue as well as of epithelium. In the Selachii their structure and mode of development is exactly the same as that of the dermal denticles, with which also they have many external points of resemblance; as, therefore, the matrix of the two is continuous, and as in many Selachii these integumentary scales are distributed over other portions of the wall of the buccal cavity, we may conclude that the teeth and scales were primitively identical. The teeth, which are developed on the edges of the jaws are, therefore, however much they are differentiated, nothing more than large structures of the same kind as those which are found in the integument. The change in them, as compared with these integumentary scales, is clearly due to adaptation to new functions, while their first appearance was contemporaneous with the differentiation of the primitive mandibular arch. The presence of these structures in the primitive buccal cavity is explicable from the fact that it was formed by an invagination from the exterior.

All teeth are developed in essentially the same way; this has been already described above (p. 423), when we were speaking of the dermal denticles of the Selachii. The dental papilla, formed of connective tissue, develops the dentine from an epithelial-like superficial layer (odontoblasts); and on this an epithelial layer deposits the enamel. When the teeth are developed on the surface, these layers are continuous with those of the surrounding mucous membrane. When the rudiment of the tooth is sunk into the mucous membrane, an epithelial thickening (enamel ridge) is developed, which grows into it; the portion of it which covers the dental papilla is separated off, and forms the enamel organ. The cement, or bony layer, is added to these two substances, and forms a third layer.

We have described above how the presence of teeth in the buccal cavity, and their deposition on the cartilaginous skeleton of its wall leads to the formation of bone $(\$ 342)$. These bones are derived from dentigerous plates, and therefore each of them may carry teeth. In the Ganoïdei and Teleostei, for instance, there are teeth on the palatines, vomer, and parasphenoid, in addition to the maxillary bones; and, also, on the hyoid and branchial arches. It is generally the last of the branchial arches reduced to a simple plate, which is distinguished by the possession of teeth (pharyngeal teeth; Fig. 256, VI). Teeth are much more common on the upper pieces of the branchial arches.

In the Amphibia there are still teeth on the palatine and vomer; more rarely on the parasphenoid; among the Reptilia, the Ophidii and Saurii alone have teeth on the palatine and pterygoid; while in the Crocodilini, as in the Mammalia, the teeth are confined to the maxillary bones. 
In the Selachii they are partly movable, and are arranged in rows of different ages. In most Fishes they retain their superficial position, and, where they are more firmly united, this is effected by their fusing with the bones which carry them. This is the case also in the Amphibia, where the earliest dental structures form their proper bones by fusing together at their bases. In the Reptilia the teeth are formed independently, like the later teeth of the Amphibia; sometimes they are mere excrescences (pleurodont Lizards); sometimes the developing teeth are sunk into their proper bones. In some of the Saurii, the teeth are attached to the edge of the jaw (acrodont Lizards). In the Geckos and Ophidii, and in all Crocodilini, the developing teeth are partly surrounded by the edges of the jaws, and are, therefore, embedded in alveoli. A similar arrangement obtains in the Mammalia. A mass of epithelium grows into the mucous membrane of the edge of the jaw, and forms a cap around a papilla, on which the rudiment of the tooth is developed; as this follicular structure is surrounded by the jaw the tooth is completely differentiated within the jaw, and only breaks through the mucous membrane as it is gradually developed; the saccule which forms it is nipped off from this mucous membrane.

The teeth vary very greatly in form, so that

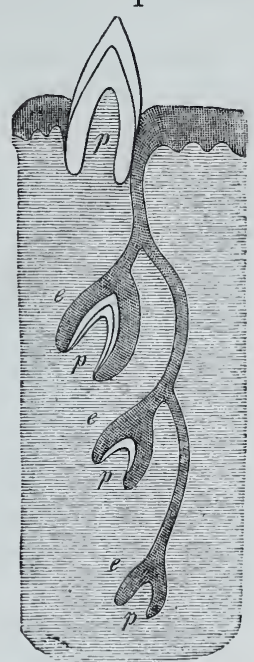

Fig. 309. Diagram of the development of teeth. A process of the epithelial layer is sunk into the mucous membrane, and forms an enamel organ (e) over each papilla $(p)$. there is every intermediate stage between broad plate-like structures, and long, fine, spicular forms; this variety of character is most common in Fishes. The teeth of the Amphibia are more similar in form; in the extant members of the group, at any rate, they are generally simply conical, or faintly notched. Among the Reptilia greater differences are seen in the Saurii, and partly too in the Ophidii, in some of which a certain number of teeth are connected with a special poison apparatus. In the Crocodilini, also, the conical form is the most common; in them the new teeth are always placed below those which are already developed, and are covered by them.

Birds have no teeth. But as fossil forms-the Odontornithes (Ichthyornis, Hesperornis)-are known in which the jaw did carry teeth, their absence in extant forms must be regarded as having been acquired within the limits of the class.

Among Mammals, the individual tooth varies very greatly, so that a single dental apparatus contains various forms of teeth. These, again, have different functions in relation to the food ingested, and vary greatly in character according to the kind of food; it is in the Delphinoidea only that the lower condition, in which all the teeth are similar, is retained; in the Balænoidea the 
teeth are merely formed in rudiment, and are atrophied while stil within the alveolar cavities.

The replacement of the worn-out, and subsequently-shed teeth, is effected in Fishes by the continual development of new teeth close to the old ones. Teeth are, therefore, developed by a process which is continued throughout the whole life of the animal, and is always being renewed. Even in the Amphibia and Reptilia we also meet with successional teeth, so that by the continual development of fresh teeth the dental apparatus is kept complete. In most Mammals this process is limited to a single change, the first (milk) dentition being replaced by a second one, in which there is a larger number of teeth (Diphyodonts). This change does not happen in the Cetacea (Monophyodonts). In the Marsupialia the diphyodont condition is in a rudimentary stage, for it is confined to one tooth only on either side of the jaw. This is the case also in several other Mammals (Elephas, Halicore), while the Rodentia would seem to belong to this series. The two series are therefore connected, and the change of teeth in the Mammalia may be regarded as a process which has been developed from a polyphyodont condition.

Toxes, CH. S., Manual of Dental Anatomy, Human and Comparatire. London 1876.

\section{$\S 415$.}

The tongue is a second organ of the buccal cavity. In Fishes it is generally a process formed by the investment of mucous membrane of the body of the hyoid. It is flat, and movably connected with the general branchial skeleton only. Like the other skeletal portions of the wall of this cavity it frequently carries a number of teeth. This organ is not provided with a special musculature below the Amphibia, where it forms a thick, and in many, a protrusible structure. It is not developed in Pipa and Dactylethra. As a rule, the anterior end alone is connected with the floor of the buccal cavity, and the posterior, and more movable portion, is drawn out into two processes. In the Reptilia there is likewise a muscular tongue, which, in the Ophidii and Saurii, can be drawn out of a special sheath. The epithelium of the tongue, which is ordinarily a delicate organ, frequently develops scales and knobs on its upper surface, while the anterior end is drawn out into two fine points (Fissilingues) (Fig. 310, z). In the Chelonii, and especially in the Crocodilini, the tongue is broad and flat. In Birds, the anterior end of the tongue is ordinarily covered by a cornified layer of epithelium, and is sometimes beset by lateral barbs (Woodpeckers), or fine setæ (Toucan); it is in the Psittacidæ only that the tongue forms a larger fleshy organ. In the Mammalia we find that the tongue is very large, owing to the greater development of its musculature, while at the same time its investing mucous membrane is provided with a number of differentiated papillæ. The function of the organ is chiefly that of aiding in the ingestion of 
food. In many Prosimii and Chiroptera, as also in the platyrrhine Apes, there is a process below the tongue which is sometimes double; this is the so-called sublingua.

The glandular organs connected with the buccal cavity are developed from its mucous membrane. When they are largely developed, and are placed outside the mucous membrane, their efferent ducts pass into it. They may therefore be regarded as greatly developed glands of the mucous membrane. Larger glands of this kind are placed between the nasal capsules, and on the palate, in the Amphibia. When they are much larger they may extend on to the skull (intermaxillary glands). The labial glands, which are set along the edges of the jaws in the Reptilia, must be mentioned (Ophidii and Saurii). 'The poison-gland of the Ophidii is a larger organ, but is merely formed by a modification of simple glands. In the Chelonii there is a pair of glands below the tongue, which are regarded as salivary glands. The Saurii also are provided with similar groups of separate glands. Larger glands of this kind, which aid in the

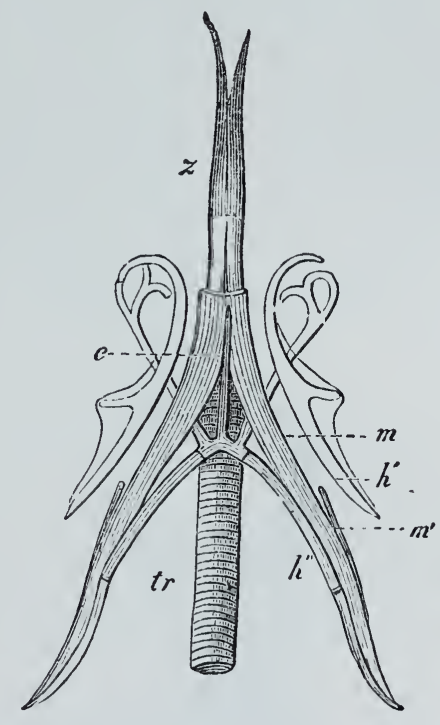

Fig. 310. Hyoid apparatus, with the tongue and trachea of Varanus. $e$ Median piece of the hyoid. $h^{\prime}$ Anterior, $h^{\prime \prime}$ Posterior cornu of the hyoid. $m m^{\prime}$ Muscles. tr Trachea. $z$ Tongue. production of a buccal fluid, are also found in different regions. They are constant in Birds and Mammals, and are distinguished as sub-maxillary, sublingual, and parotid glands. In Birds the last of these open at the angle of the mouth, but in Mammals, in the vestibulum oris. These glands are not developed in the Cetacea, and but feebly in the Pinnipedia. The three pairs are largest in Herbivora; sometimes one and sometimes another pair being the best developed.

\section{$\S 416$.}

Mention has still to be made of the hypobranchial groove and its derivates. It is an organ
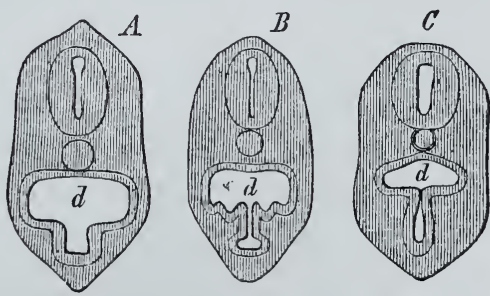

Fig. 311. Sections through the body of young larvæ of Petromyzon to show the ventral groove. $d$ Branchial cavity (after Calberla). differentiated from the primitive cephalic enteron (cf. p. 540). In Amphioxus it extends all along the branchial cavity. Among the Cyclostomata it has only been observed during the early larval condition of Petromyzon (Fig. 304, h). As it does not extend along 
the whole of the branchial cavity, it appears to have been reduced, in comparison with the same organ in the Tunicata. When the organ which functions as a tongue is differentiated, the groove undergoes still further reduction, and is converted into a canal, which is gradually cut off from the superior cavity (Fig. 311), and is at last completely separated from it. In the adult animal it is converted into a complex of follicles, covered with epithelium, which extend from the second to the fourth pair of branchial sacs. They form an organ with unknown physiological relations-the thyroid gland.

In the Gnathostomata a groove, remaining for some time, is no longer developed, but at the homologous region a process of the

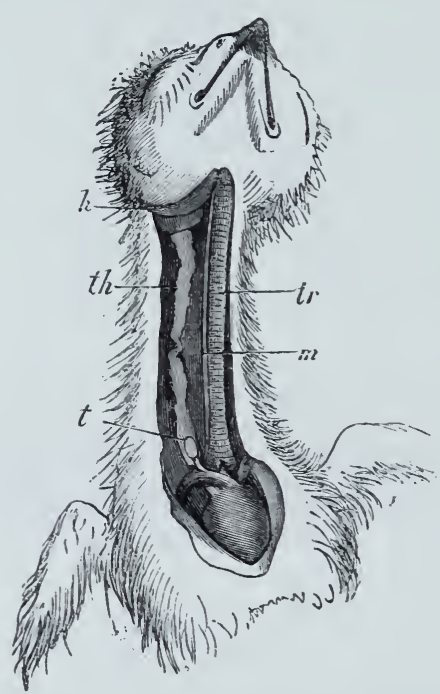

Fig. 312. Thymus $(t h)$ and thyroid $(t)$ of a mature embryo of Buteo vulgaris. tr Trachea. lumen of the cephalic enteron is nipped off, and forms an azygos follicle invested by epithelium. By a process of gradual gemmation this is broken up into a number of separate follicles, which are united together by connective tissue. In Fishes the organ is placed not far from the point at which it was formed; that is, at the anterior end of the trunk of the branchial arteries, and between it and the copula of the hyoid arch. In the Amphibia the thyroid is placed near the larynx, where it forms a paired coil (unpaired in Proteus), and is set on the inner surface of the posterior cornua of the hyoid. It is sometimes broken up into several groups. In the Reptilia it is unpaired, and lies in front of the aortic arches; in Birds, however, it is paired (Fig. 312, $t$ ), and lies near the commencement of the carotids. In both these divisions, therefore, it is removed some way from the point at which it was developed. This appears to be due to the shifting backwards of the great arterial trunks. Among Mammals it is separated into two parts in the Monotremata, many Marsupials, and various other forms; while in the rest its two lateral masses are united by a median bridge (isthmus). It always lies just below the larynx, and on the trachea.

The preservation of this organ, which lost its primitive significance even in the lower Vertebrata, throughout the long series of higher forms, is explicable from the fact that it has been inherited from what is phylogenetically a very early period; it is an arrangement, indeed, which was physiologically of great importance to the ingestion of food in the Tunicata.

MüLler, W., Die Hypobranchialrinne der Tunicaten, etc. Jen. Ztschr. Bd. VII. The same, Entw. d. Schilddrüse. Jen. Ztschr. Bd. VI. 


\section{Alimentary Canal proper (Enteron of the Trunk).}

\section{$\S 417$.}

That portion of the tractus intestinalis, which serves exclusively for the ingestion and alteration of food, commences at the hinder end of the cephalic enteron; this, which is the digestive tube in the strict sense, has an apparatus differentiated from it at its anterior boundary, which forms an air-bladder in Fishes, where it is in an indifferent condition, and a respiratory apparatus consisting of lungs and trachea in the Amphibia and all higher forms.

The most anterior portion of the digestive tube is not sharply marked off from the cephalic enteron. As they are both innervated by the vagus, there is reason for supposing that this portion was primitively derived from the respiratory portion of the primitive enteric tube after the atrophy of a large number of the hinder branchial clefts, and that therefore it corresponds to the posterior portion of the respiratory antechamber, which is so much larger in Amphioxus.

In the Craniota, not only some of the peculiar relations of the rudimentary enteron, but also later stages in the development of this tube, are due to the relations of the egg to the general rudiment of the embryo, and to an increase in the quantity of the yolk.

In the Selachii, the rudimentary enteron grows round the yolk, but it is the groove-like portion only of the general rudiment, that lying below the axial skeleton of the embryo, which is converted into the enteron; this is gradually shut off from the rest, or yolkbearing portion, which then appears as an appendage of the enteron, the yolk-sac. This, which is at first placed apparently outside the body, but which is surrounded by a continuation of the integumentary layer, is merely connected by a stalk with the enteron (external yolk-sac), and is gradually taken into the body (internal yolk-sac). As the yolk is gradually used up, the yolk-sac is atrophied. The Teleostei (and Ganoïdei) are provided with a smaller quantity of the nutrient material for the embryo, which constitutes the yolk. Owing to the larger size of the yolk of the egg in Reptiles and Birds, there is a similar contrast between the enteric canal and the yolksac, but the latter is not covered by the integument, for the parts which in the Anamnia enclose it go to form the amnion, and another of the foetal coverings of the egg. In the Mammalia also, where the material of the egg is very greatly reduced in quantity, the rudimentary enteron becomes nipped off from the embryonic bladder, which represents the yolk-sac (Fig. 319). This arrangement may be deduced from a condition which was distinguished by the possession of a large quantity of yolk-material. The want of a large quantity of yolk in the Mammalia is compensated for by the development of the foetus within the maternal organism, and the more or less close 
connection between the fotus and the uterus. A rudiment of the yolk-sac is retained as the "umbilical vesicle," which is not taken into the body-cavity, as it is not of any use in the nutrition of the embryo, but is after birth separated from the young animal with the coverings of the egg.

The divisions of the alimentary canal are the same as those in the Invertebrata ; fore-, mid-, and hind-gut.

\section{Fore-gut.}

\section{$\S 418$.}

The first portion of the alimentary canal proper is an exceedingly short piece in Amphioxus, and is placed directly in front of a diverticulum which is directed forwards, and is regarded as the liver. If we consider that the liver is always derived from the portion which is to be regarded as the mid-gut, and that it forms its anterior boundary, it follows that the fore-gut is exceedingly small in many of the Craniota also. This character is seen in the Cyclostomata, Chimæræ, and various Teleostei. The rest of the Craniota differ in this point, for their fore-gut forms a large piece, which can be divided into œsophagus, and stomach. In any case these parts appear to have been acquired by the Gnathostomata only, among the

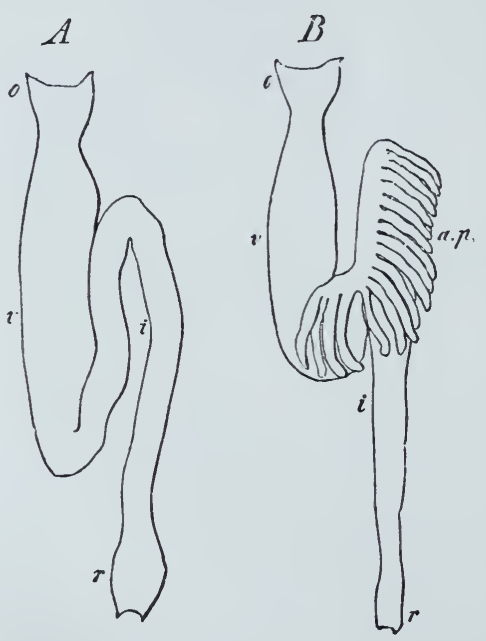

Fig. 313. Digestive canal of Fishes. $A$ Of Gobius melanostomus. $B$ Of Salmo. o Esophagus. $v$ Stomach. $i$ Mid-gut. ap Appendices pyloricæ. $r$ Hind-gut.

Vertebrata. The distribution of the vagus on their walls is of importance as bearing on their origin; and there is in consequence of this distribution reason for supposing that the tract in question has been developed from a portion which primitively belonged to the cephalic enteron. The reduction of a larger number of branchial clefts, and the conversion of a portion of the respiratory antechamber to a purely nutrient function, is in agreement with this distribution. On the other hand, the extension of this tract, and especially of the portion which represents the stomach, as well as its position in the cœlom, are due to the great quantity of food ingested. The stomach is almost always separated from the mid-gut by a fold of the enteric wall (pyloric valve).

In Fishes, the œsophagus, which is very wide and provided with longitudinal folds of the mucous membrane, generally passes at 
once into the stomach, which can only be distinguished from it by the differences in the characters of its mucous membrane. As a rule, the stomach (Fig. 313) forms a cæcal sac, which is directed backwards, and from which a narrow portion (pyloric tube) which bends forwards, can be distinguished; this leads to the mid-gut (i). This is the case in all Selachii and Ganoïdei, and in many Teleostei, while the rest vary greatly in the absence, or the great development backwards, of the cæcal sac.

Among the Amphibia we find a lower stage in Proteus, for the enteric tube, which has a perfectly straight course, has no stomachal enlargement at all. In the other Urodela, however, the stomach forms a wider portion of the enteron; and this is the case also in the Anura, where the stomach is sometimes, indeed, placed transversely (Bufo).

Among the Reptilia, the fore-gut is of a lower stage in the Ophidii and Saurii, owing to the greater width of the œsophagus and the straight course of the stomach. However, there is an arrangement in the Saurii which calls to mind the pyloric tube of the Selachii, and from this the stomach might gradually acquire a transverse position. In the Chelonii and Crocodilini the œsophagus is more sharply separated from the stomach, which in the former has a large and a small curvature, owing to the great elevation of the pyloric portion. Owing to the approximation of the cardiac end of the stomach to the pylorus, this portion is rounded in the Crocodile, and is also distinguished by a tendinous disc on each face of its muscular wall; in this point it resembles the stomach of Birds.

In the fore-gut of Birds there is a greater division of labour. The influence of adaptation to the mode of life, and here

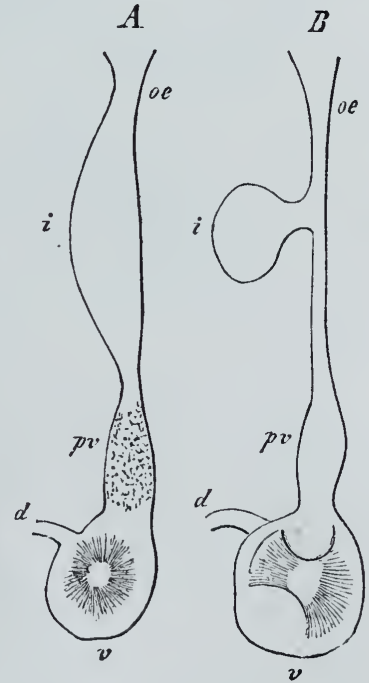

Fig. 314. $A$ Fore-gut of a Raptorial Bird (Buteo). $B$ Of a Fowl. oe Esophagus. $i$ Crop. $p v$ Glandular stomach. $v$ Muscular stomach. $d$ Duodenum. especially to the mode of nutrition, is most clearly shown by the variations in the different arrangements. The œsophagus, which is of the same length as the neck, is either of equal calibre along its whole course, or is provided with a widened portion (Fig. 314, $A$ ), or with a cæcal diverticulum $(B)$, which looks like an appendage. Portions (i) of this kind, which are characterised by modifications of the glandular organs of the mucous membrane, form a crop (ingluvies). This is best developed in carnivorous and graminivorous Birds; in the former, indeed, it generally forms a spindle-shaped enlargement, while in the latter it forms a unilateral diverticulum, which is differentiated into a cæcal appendage, in many provided with a narrow connecting piece. 
The next portion of the œsophagus, which is generally narrower, passes into the stomach, in which two divisions can be made out; the first is known as the proventriculus $(A B p r)$; its walls are greatly thickened by a glandular layer. The second portion is characterised by the great development of its muscular layer, the strength of which varies with the mode of life of the animal. Where it is greatly developed we may observe a tendinous disc on either side $(A B)$. In the Raptores, as also in many Natatores that live on animal food, the muscular layer is feebly developed. It is very strong in the graminivorous forms (Gallinæ, Anatinæ, Columbæ, Passeres). This portion, which serves for the comminution of food, aud compensates for the absence of masticatory organs, may be provided with other arrangements also which serve the same purpose; its inner surface may be covered by a firm horny layer, which is often of considerable thickness, and functions as a radula. It is produced by a glandular layer, the secretion of which passes into this firm stiff condition.

In the Mammalia the fore-gut is more completely divided, owing to the sharper delimitation of the œsophagus from the stomach, than it is in almost any other division. In many cases the shape of the stomach is of a low type. In the Phocidæ it retains its position parallel to the long axis of the body, while in other Mammals a position transverse to this axis is the common one.

We must regard a number of peculiarities, which sometimes consist in an enlargement of the internal space, at others of a differentiation of the primitively single, and, as we must suppose, uniformly functional stomach, into several portions of different function, as the results of adaptation to the material of nutrition.

The first relation is implied by the transverse position of the stomach, in consequence of which the great curvature gets to be much the larger, and, forming a swelling behind the cardiac portion, gives rise to the fundus of the stomach. This is absent in most Carnivora, but is developed in the Monotremata, Marsupialia, Rodentia, and Edentata, and is found also in most of the Primates.

When the fundus is more largely developed the stomach may be divided into several portions, but this division is not unfrequently implied by the characters of the mucous membrane only (Equus). This arrangement is carried farther by the development of a transverse constriction; thus, in many Rodents, the stomach is divided into a cardiac and a pyloric portion, to which smaller diverticula may be added on. Similar stomachs of a more complicated character may be seen in many Marsupials (Halmaturus), and in the Cetacea. The fundus is always a considerable enlargement, which, in the Cetacea, is succeeded by a number of diverticula, which are attached to the pyloric portion; these give the stomach the appearance of being made up of from four to seven spaces which communicate with one another by connecting pieces of varying width.

In the Ruminantia the complication is due to the share taken by the œesophagus, the cardiac end of which bulges out on one side and 
fuses with the stomach, of which it forms two divisions. The first has the character of an enlarged fundus, and is known as the rumen or paunch (Fig. $315, I$ ) ; it functions essentially as an organ for the reception of the large quantity of food that is ingested. Just below the cardia it is connected with the second division, the reticulum $(I I)$, which is succeeded by the psalterium (omasus); this third portion is wanting in the Tragulidæ and Tylopoda. The last portion, which is formed from the pyloric part, is attached to this ; it forms the abomasus, in the mucous membrane of which the rennet glands are placed. A groove (œsophageal groove) which leads from the œesophagus into the reticulum, and is shut off by a valvular process (Fig. 315, Bs) from the first two divisions of the stomach, represents that portion of the cesophagus which has entered into the formation of the stomach, and formed the first two portions of that organ by bulging out on one side. Thanks to its presence the food that has passed from the reti-

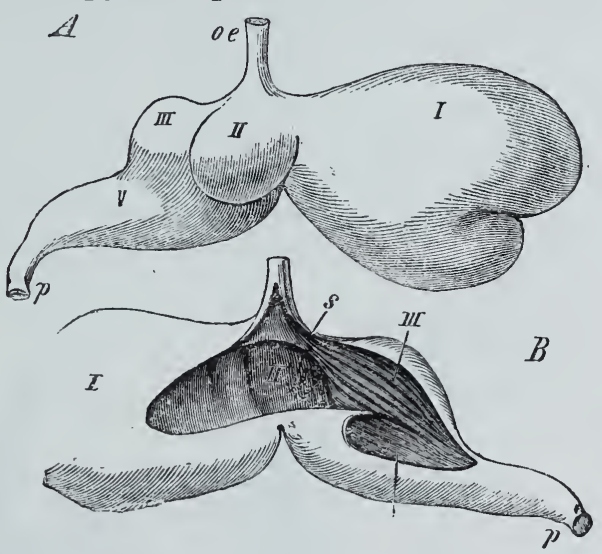

Fig. 315. Stomach of an Antelope. A From in front. $B$ Opened from behind. oe Esophagus. I Rumen. II Reticulum. III Psalterium. IV Abomasus. $\quad p$ Pylorus. $s$ Esophageal groove. culum into the osophagus, and from thence into the mouth, can be directly returned, after it has been sufficiently masticated, into the psalterium and abomasus, while, when the groove is open, the fodder passes easily into the paunch and reticulum. The influence of the food in determining the size of the various portions may be seen from the differences between the paunch and the abomasus at different periods of life. The abomasus is relatively large in the calf, while later on the paunch may be as much as ten times larger than the abomasus, and even more than that.

\section{Mid-gut.}

\section{$\S 419$.}

The mid-gut (small intestine) which is generally separated from the stomach by a circular fold, the pyloric valve, is characterised at its commencement by having glandular organs (liver and pancreas) connected with it. With regard to length it is the most variable portion of the enteric tube. It is straight in the Cyclostomata, some Teleostei, and in Chimæra. In the last it is distinguished by 
a spiral fold, which is greatly developed in the Selachii, and divides the greater part of the mid-gut into a number of more or less closely applied coils (Fig. 316, $C v s$ ). In Carcharias this fold has the form of a rolled-up sheet of paper. This spiral valve is retained in the Ganoïdei: it is reduced to almost nothing in Lepidosteus. It is not present in the Teleostei.

An enlargement may be observed at the commencement of the mid-gut of the Selachii; in the Sturiones there is a large, and externally much diverticulated glandular organ at the same point; it is divided internally into a number of spaces corresponding to the diverticula. In Lepidosteus the various portions are more sharply separated from one another, and have the appearance of groups of short cæcal tubes which beset the pyloric portion of the mid-gut, and, as in many Teleostei, form the appendices pyloricæ (Fig. 316, $A B$ ap). They beset a certain portion of the mid-gut and vary in
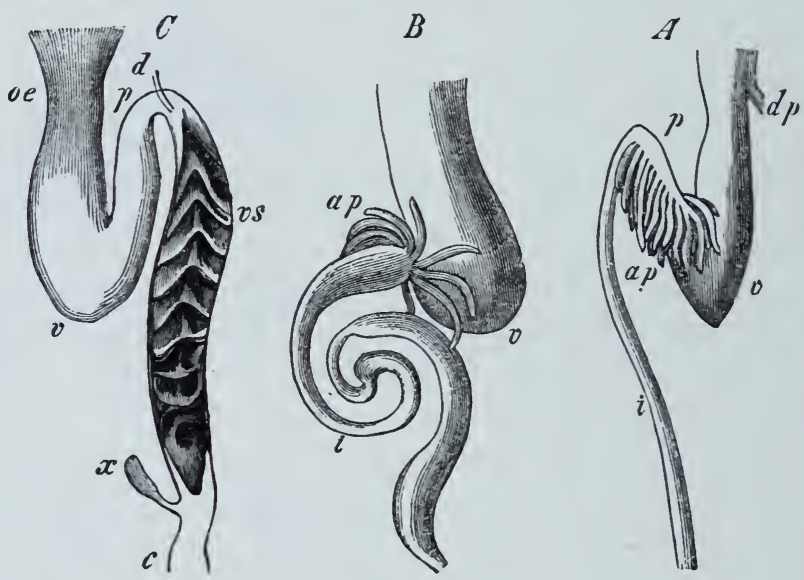

Fig. 316. Enteric canal of Fishes. $A$ Of Salmo salvelinus. $B$ Of Trachinus radiatus. $C$ Of Squatina vulgaris. oe Esophagus. $v$ Stomach. $d p$ End of the air-duct. $p$ Pylorus. ap Appendices pyloricæ. $d$ Ductus choledochus. vs Spiral valve. $i$ Mid-gut. $c$ Hind-gut, $x$ Its appendage.

number and size. They sometimes open separately into the gut, sometimes are united into larger trunks, and give rise to branched structures. They are most numerous in the Gadidæ and Scomberoïdæ. In many Fishes the different cæca are held together by connective tissue and united at a common efferent duct, in which case they have the appearance of a compact gland (Scomberoïdæ), while their affinity to the gland in the Sturgeon is implied by the frequent union of their orifices.

In many Teleostei the mid-gut is much longer than the tract of the cœlom which is given up to it, and it is then arranged in coils (Fig. 316, B i), or in several ascending and descending loops. This implies an adaptation to the cavity of the cœlom, whilst the elongation of the tract, which is always derived from a straight 
rudiment, is an adaptation to the functions required of it by the ingesta.

In the Amphibia the simple condition of the mid-gut is very rarely permanent (Proteus). It generally forms, as it does also in Reptiles, a longer tube, and, consequently, a number of coils. (Fig. 317, i.) In the Ophidii these are least, in the Chelonii they are considerably, and in the Crocodilini they are still more developed. The mid-gut is very greatly elongated in the larvæ of the anourous Amphibia, where this portion forms a long loop arranged in spiral coils. It is reduced when the mode of feeding is changed during the final stages of larval life, and this leads to an abbreviation of the length of the enteron.

The length of the mid-gut in Birds also varies very greatly according to the characters of their food. It is arranged in loops, the first of which (duodenal loop) is the best developed, and always contains the pancreas.

The mid-gut of Mammals is seen no less distinctly to vary in length according to the kind of food that is eaten; so that there are different conditions in Carnivorous and Herbivorous forms.

The surface of the mid-gut is increased by various arrangernents of its mucous membrane, as well as by its increase in length. In the lower groups there are coarser folds (spiral valve of the Selachii), but in the Amphibia and Reptilia by far the most common arrangements are fine

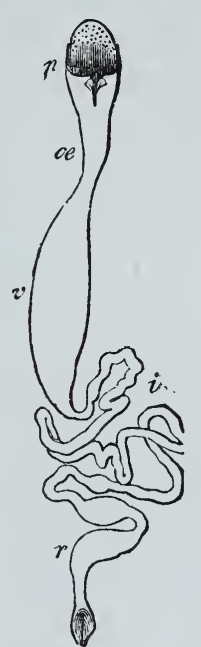

Fig. 317. Enteric canal of Menobran. chus lateralis. $p$ Commencement of the fore-gut with the Pharynx. oe Eiso. phagus. $v$ Stomach. $i$ Mid.gut. $r$ Hindgut. longitudinal folds of the mucous membrane. These obtain also in the Birds, but in them they generally form unequal elevations, and may be united by transverse lines. Fine folds arranged in zigzag* lines are seen in the Amphibia and Reptilia, and are found also on the mid-gut of Birds. In Mammals, these longitudinal folds of the mucous membrane are commonly found in the Cetacea; but in most of the other Mammalia the mucous membrane is smooth, or raised up into transverse folds, which are very generally beset with villi. When the folds are feeble we find that these villi are greatly developed in Birds also, while when the folds are present the villi are merely smaller elevations.

\section{Hind-gut.}

$\S 420$.

The end-or hind-gut is the smallest of all in the lower divisions, and is merely represented by a short and somewhat wider 
piece (Fig. 313, $r$; 316, $C$ c). In the Selachii it is provided with a special glandular appendage (Fig. 316, $C$ ). It is only in the Amphibia that, owing to its greater length and width, it becomes of some importance, but in them, as in the Reptilia, it retains its straight course in correspondence with its shortness. In consequence of this straight course it has got the name of "rectum." It is generally separated from the mid-gut by a transverse fold or valve. Many Reptiles are provided with a cæcal appendage, which, in the Ophidii is feebly, and in the Saurii is better developed. The cæca in Birds are much more independent. In this group, also, the hind-gut is short and straight (Fig. 320). The cæcum is generally paired, and is absent in a few families only (e.g. Woodpecker, Psittacus, etc.). They vary greatly in the extent to which they are developed, so that they may form short papilliform appendages, or very long tubes (Apteryx, Gallinæ, Anseres).

The hind-gut is longest in the Mammalia, where it forms the large intestine, and is distinguished, as such, from the mid-gut, or small intestine. Owing to its greater length it is arranged in coils, so that the terminal portion, only, has the straight course taken by the hind-gut of other Vertebrata. The anterior portion ordinarily forms a loop which bends from the right side of the abdominal cavity forwards, and then to the left, and then again backwards to be continued into the rectum. This loop is sometimes broken up into secondary loops.

At the boundary between it and the small intestine cæcal structures are likewise developed, but these are rarely arranged in pairs (Fig. 318, $c d$ ), and are commonly single. The size of this

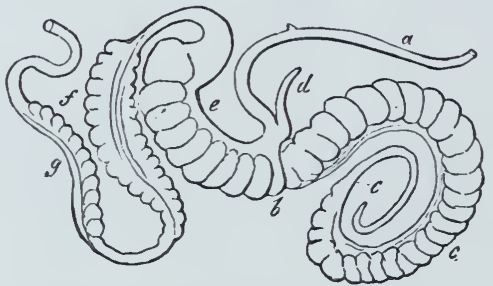

Fig. 318. Cæcum and colon of Lagomys pusillus. a Small intestine. $b$ Opening of the larger $(c)$, and of the smaller $(d)$ cæcum. e $f g$ Diverticula of the colon (after Pallas). cæcum may be shown to depend on the food. In the Carnivora it is short, and sometimes completely absent (Ursina, Mustelina): it is very large in the Herbivora, where its length is compensated for by that of the colon.

The cæcum itself may be affected by differentiations. Its terminal portion is frequently diminished in size (e.g. in various Prosimix and many Rodents) (Fig. 318,c). In various Primates, and in Man, the terminal portion, which, at first, is as wide as the rest, is not developed in proportion to it; it thus becomes more and more distinct from the other portion, which continues to grow wider, until at last it forms a mere appendage to it-the appendix vermiformis.

The hind-gut primitively opens into the same space as the urinary and generative ducts, the cloaca. This arrangement, which obtains in the Selachii, Amphibia, Reptilia, and Aves, is permanent in 
the Monotremata only, among Mammals, in the rest of which it is confined to the embryonic stage, and subsequently the hind-gut opens to the exterior by means of an anus.

\section{Organs appended to the mid.gut.}

$\S 421$.

Two large glandular organs, the liver and pancreas, are connected with the beginning of the mid-gut; they are both differentiated from the walls of the rudimentary enteron.

In Amphioxus an organ, which must be regarded as the liver, has the form of a cæcal tube (Fig. 303, f), which arises close to the commencement of the alimentary canal, and is directed forwards (Fig. 303, f). It is provided with an epithelial investment of a greenish colour. A similar condition is seen in the Craniota during the earliest stages of development, in which the rudiment of the liver has the appearance of a paired diverticulum $(f f)$ of the enteric tube, lying behind the rudimentary stomach (Fig. 319, d). It is partly formed by the epithelial layer of the rudimentary enteron (endoderm), and partly by the external layer developed from mesoderm. As Reptiles, Birds, and Mammals agree in this point, this condition must be regarded as a fundamental one, while at the same time it calls to mind the morphological characters of the hepatic organ in Amphioxus and many Invertebrata (Vermes, Mollusca).

Owing to the thickening of the splanchnopleure and its large connection with the venous portion of the vascular system, together with the simultaneous thickening of the endoderm, relations are produced, which distinguish the liver of the Craniota from that of the Acrania, as well as from that of the Invertebrata. While the first rudiment of the liver appears as a diverticulum, the later differentiations are brought about by the thickening of the endoderm, and give rise to solid chords of

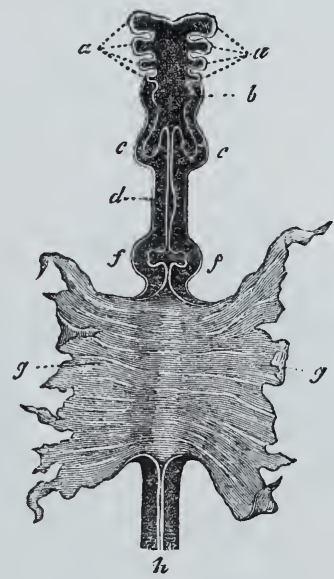

Fig. 319. Rudiment of the enteric canal and its appendages in an embryo of the $\mathrm{Dog}$, seen from the ventral surface. a Diverticula of the enteric tube towards the visceral clefts. $b$ Rudiment of the pharynx and larynx. $c$ Of the lungs. $d$ Of the stomach. $f$ Of the liver. $g$ Walls of the yolk. sac in connection with the mid-gut. $h$ Hind-gut (after Bischoff). cells which grow into the layer of mesoderm, and the vascular apparatus embedded in it; these give off new buds, and are finally connected together in a retiform manner. The parenchyma of the liver is formed by these primitively solid chords, and their secondary 
and other processes, while they give rise to the bile-ducts by the formation of intercellular passages, which run in the axes of the epithelial chords. The hepatic lobes, which are formed on either side, fuse with one another into a single organ. The two primitive diverticula, after they have formed the bile-ducts in the parenchyma of the liver, and have been continued into the network of cellular chords, form the efferent ducts of the liver.

The liver, which is thus differentiated from the enteron, forms a compact, and ordinarily, very large organ; it is embedded in a fold of the peritoneum, which extends from the anterior portion of the enteric tube to the anterior wall of the abdomen.

In Fishes, the liver generally forms a single, undivided mass, but sometimes it consists of two, or more lobes. There are two large

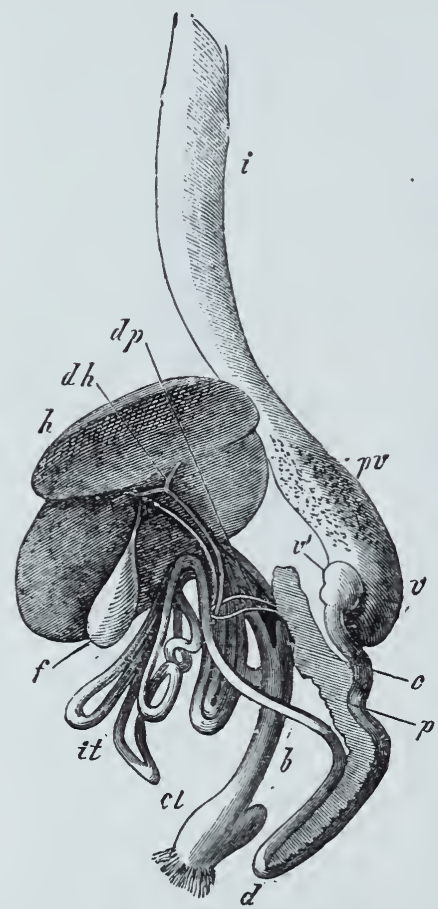

Fig. 320. Enteric canal of Ardea cinerea. $i$ Esophagus and crop. $p v$ Proventriculus. $v$ Gizzard. $v^{\prime}$ Antrum pylori. d Duodenal loop. it Mid-gut. $b$ Hind-gut. $c$ Part of the single creum. cl Cloaca and Bursa Fabricii. $h$ Liver. $d h$ Ductus hepato-en. tericus. $f$ Gall-bladder. $p$ Pancreas. dp Pancreatic duct. portions in the Amphibia; it is generally simple in the Ophidii, and is merely notched at the margin in the Saurii ; in the Crocodilini and Chelonii it is again divided into two lobes, which in the latter are widely separated from one another, and united by a slender transverse bridge. Ordinarily two lobes are, sometimes more, sometimes less, indicated in the Mammalia. In the Carnivora, Rodentia, some Marsupialia, Simire, and others we find, indeed, multilobate forms, but these may be referred to two larger primary lobes.

There are various modifications in the character of the efferent ducts (ductus hepato-enterici) in relation to their primitively double character ; for either the first condition persists, or the two ducts are gradually fused together, that is to say, the diverticulum of the enteron is converted into a single duct, or, lastly, the primitive ducts are atrophied, and secondary canals are converted into efferent ducts; in this case there is a large number of ducts (in the Saurii and Ophidii). A unilateral cæcal diverticulum, the gall-bladder (Fig. 320, $f$ ) is placed on these ducts; it has very various relations, and is by no means a constant structure.

The pancreas is developed in the same way as the liver-from a diverticulum of the wall of the enteron, which is developed behind the rudiment of the liver. The 
epithelial layer of the rudimentary enteron forms thickenings from which the glandular lobules and their efferent ducts are developed by a process of gemmation, while the pancreatic duct is derived from the first rudiment of the gland. This organ, which is never absent except in some divisions of Fishes, is always placed close to the commencement of the mid-gut, or close to the stomach; its duct is frequently united with that of the liver, or passes into the enteric canal in company with it. Not unfrequently there are two ducts (Chelonii, Crocodilini, Aves [Fig. 320], and some Mammalia), one of which is, as a rule, connected with the ductus hepato-entericus.

\section{Mesentery.}

$\S 422$.

As the enteric canal is developed the peritoneal fold, which encloses it, is developed also; it fastens the canal to the hinder wall of the abdomen. This double lamella, which contains the enteron, forms the mesentery; that portion of it which goes to the stomach is known as the mesogastrium. This does not, however, merely enclose the stomach, like the mesentery of the greater part of the mid-gut, but has its two lamellæ continued from the stomach into a double fold, which extends to the anterior wall of the abdomen, where it is again connected with the peritoneum of the abdominal wall. The liver is contained in this continuation of the mesogastrium to the anterior wall of the abdomen, so that this organ has not only a peritoneal investment, but is also connected with the enteric tube (and especially the stomach and the first part of the mid-gut), and with the ventral wall of the cœlom. As long as the enteric tube retains its primitively straight course the relations of the mesentery are simple, and peculiarities in it are only due to the partial absorption of large tracts, as is the case, for example, in Fishes. The increased size also of the liver brings about changes in the characters of the fold which passes from the stomach to the anterior wall of the abdomen ; this fold, where it forms the connection between the latter and the stomach, is known as the small omentum. That portion of it which extends to the wall of the body forms the suspensory ligament of the liver. Other changes are effected in it by its relation to the diaphragm, by the curvature of the stomach, and by the elongation of the mid-gut, which cause the mesentery to be arranged in frill-like folds. These relations are seen as early as Fishes; they are still simple in the Amphibia, and in the Ophidii and Saurii; in the Chelonii and Crocodilini they are especially modified by changes in the form and position of the stomach.

The changes of the mesogastrium are most considerable in the Mammalia. As the stomach alters its position this membrane grows out into a wide sac (bursa omentalis), which either hangs down over 
the loops of the mid-gut, as in most Mammals, or partly conceals the stomach (Ruminantia).

The mesentery of the hind-gut retains its primitive characters in Vertebrata with a short hind-gut. When that portion of the hindgut which is known as the colon increases in length, as it does in the Mammalia, the mesentery or mesocolon accompanies it, and is at the same time raised by one portion as far as the root of the mesogastrium so that the two arise close together. This gives rise to that gradual connection between the mesocolon and the posterior fold of the mesogastrium, which is seen in the Primates, and which ends with the condition seen in Man, where a portion of the colon (C. transversum) is enclosed in the hinder wall of the omental sac. At the same time the anterior and posterior walls of the omental sac grow out, so that the great omentum is formed, which consists of four lamellic of the peritoneum.

\section{Pneumatic Organs of the Enteric Tube.}

\section{$\S 423$.}

As the enteric tube is the road for everything that is taken in from the exterior, the water which serves in respiration as well as the substances that will be converted into food in the organism, so, too, the enteric tract may take in air, which is collected in special spaces which are differentiated from it, and which, therefore, represent portions of the primitive enteric tube. This ingestion of air leads us to suppose that sometimes, at any rate, the animal came to the surface of the water; indicating a not unimportant stage between those in which life was passed in water exclusively, and those in which life was also possible out of this medium.

The organs which are formed when air is taken in are known as air-bladders. It is still uncertain what is the practical use of these organs to the whole organism, but, as they are found in so many forms, they must be regarded as important parts. The arrangement of air-spaces in the body of aquatic animals must have some influence on the specific gravity of their bodies, so that there is good reason for supposing that these organs have a hydrostatic function.

A great change occurs in this character when the relations of the circulatory system are changed. The organs have a respiratory function, for the air in them exchanges its gases with those in the blood which is brought to the wall of the organ, so that the blood which passes away from it is richer in oxygen. The organ, therefore, becomes one of the respiratory organs, and is called the lung. The first point in this metamorphosis is not the change which has occurred in the blood-vascular system, but rather the commencement of an exchange of the gases in the blood of the walls of the 
organ, and the air contained in the organ itself. This must happen whenever the blood brought to the organ is less rich in oxygen than the air in the organ. The changes in the vascular system do not occur till this has happened.

The pneumatic organs of the enteric tube are divided, therefore, into two series which are functionally very different, though morphologically homologous, and each series undergoes a large number of differentiations.

\section{a) Air-Bladder.}

$\S 424$.

This organ is not found in Amphioxus, nor in the Cyclostomata. In some Sharks (Galeus, Mustelus, Acanthias) there is a diverticulum of the dorsal wall which opens into the pharynx, and which may be regarded as the rudiment of an air-bladder. All the Ganoïdei, and very many of the Teleostei, have air-bladders. If we examine the arrangements which obtain in the Ganoïdei more closely, we find that there is a single or paired sac, which is connected with the pharynx by an air-duct of varying length. This opens on to the upper wall of the fore-gut, and, generally, at the same point as that at which we find the short cæcal sac in the Selachii. In Acipenser the opening is placed very far back, and the air-bladder is connected. with the stomach. In Polypterus we find a paired air-bladder (Fig. 321, A), which opens on to the ventral wall of the œsophagus ; in Lepidosteus it is placed on the dorsal surface, and, though single externally, is divided into two longitudinal halves by the trabeculæ which traverse it, and each of these halves is again divided into smaller cellular cavities by a number of processes and bars; the inner surface is thereby greatly increased in size. In Amia, also, the cellular air-bladder is divided by a fold, and is continued anteriorly into two short cornua. In the three last-mentioned Ganoids the bladder opens into the enteron by a short and somewhat narrow air-duct which leads to a longitudinal cleft. Even in the Ganoïdei, therefore, there are great variations in the characters of the air-bladder, and their significance must be estimated in relation to the fact that this division now consists of but few living forms. It is very significant that in the various stages of the air-bladder in the Ganoildei all the essential arrangements are recognisable which are presented by this organ, either under the form of the air-bladder of the Teleostei, or the lung of the higher Vertebrata.

In one division of the Teleostei the air-duct is persistent (Physostomi); in the rest it is developed for a time only, for it disappears again after the development of the air-bladder (Physoklisti); in many, finally, the air-bladder ceases to be developed. This last character is, moreover, very variable, even within the limits of 
single genera. Thus, some species of the genus Scomber do, and others do not, possess an air-bladder.

There are great variations in the mode of connection between the air-duct and the gut. It may open at the sides, or above, and in all regions of the fore-gut from the pharynx as far as the end of the stomach. It varies very greatly in form also. In the Cyprinoids it is divided transversely into two portions which lie one behind the other, the air-duct being given off from the hinder one (cf. Fig. 301, $n n$ ). In others there are lateral diverticula, which may become simple or branched processes (Fig. 321, $B C$ a).

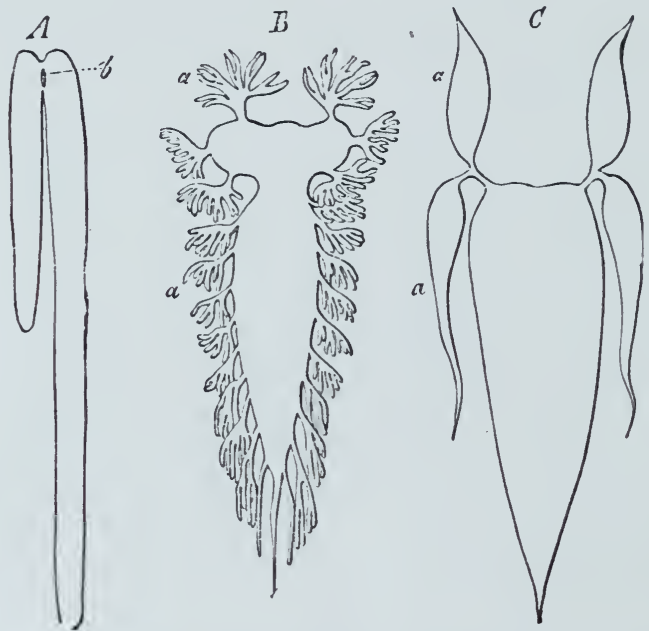

Fig. 321. Various forms of air-bladders. $A$ of Polypterus bichir (after J. Muller). $B$ Of Johnius lobatus. C Of Corvina trispinosa (after Cuvier and Valenciennes). a Appendages of the air-bladder. $b$ Its orifice.

The air-duct, which is often very narrow and long in the Physostomi, is ill adapted for the passage of air; in the Physoklisti air cannot of course be taken in in this way. In the latter, therefore, the air in the air-bladder must be regarded as secreted from the walls of the bladder, while in many Physostomi the air-duct can only serve as an occasional outlet for this air. In texture the walls of the organs resemble those of the gut, but there are at the same time a number of special differentiations which it is beyond our purpose to speak of here. The various adaptations of the air-bladder to other organs, as, for example, its comnection with the auditory organs of many Physostomi, are differentiations of this kind (cf. suprà, $\$ 100$ ).

In the Dipnoï the air-bladder is more lung-like in character. Although in its external characters the organ is just like an airbladder, yet there is an essential difference owing to the presence on it of afferent veins and efferent arteries; and, owing to this change, the air-bladder is henceforward to be regarded as a respiratory organ. In Ceratodus, where, indeed, it only occasionally functions as a lung, it is formed of a single sac, which extends along the whole of the dorsal region of the cœlom, and presents indications merely of longitudinal division; in Lepidosiren and Protopterus it is divided into two halves. 


\section{b) Lungs.}

\section{\$ 425 .}

As the pneumatic organ appended to, and differentiated from, the primitive wall of the enteron takes on its respiratory form, we meet with a gradual differentiation of that portion of it which corresponds to the ductus pneumaticus, and which gives rise to airpassages. These are the means of communication between the lung and the pharynx, and are divided into several portions which take on new functions; the most important of these is an organ for the production of voice. The lungs themselves are now paired organs. This paired condition, however, is less due to a further development of that division, which is indicated in many airbladders, or even to the completion of such division, than to the development of the organ in a manner which is adapted to its position. As it is always connected with the ventral surface of the pharynx, it is easy to see how it is that an air-carrying organ developed from this point would grow out on either side. When filled with air the two halves must be blown out into a dorsal position, the result of which must necessarily be the formation of two completely separate lungs, which are only connected together by the ventral air-passage.

We have as the first point in the differentiation of the tracliea a short wide canal which connects the two lungs with the pharynx. As this canal increases in length it develops, in its wall, cartilaginous organs of support, and is divided into two branches, which pass to the lungs. A paired and an unpaired portion can therefore be distinguished in the airpassages. The supporting organs of these canals, which are generally very short in the Amphibia, are formed by two lateral bands of cartilage (Fig. 322, $A$ a), which are continued as far as the commencement of the lungs ( $l$ ) (Proteus); in others
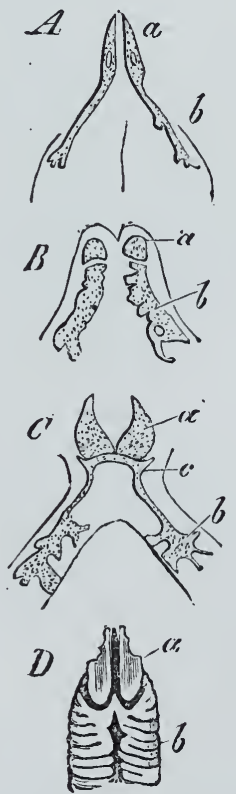

Fig. 322. Cartilage of the larynx in the Amphibia and Reptilia. A Of Proteus. $B$ Of Salamandra. $C$ of Rana. $D$ of Python. a Arytænoid cartilage. $b$ Supporting cartilage, which in $A B$ and $C$ forms the skeleton of the paired and unpaired portions of the trachea; in $D$ it is merely represented by the commencement of the unpaired portion of the trachea (after

Henle).

$(B)$ the upper ends $(a)$ of these two pieces are separated off and form the groundwork of a special portion, which is henceforward 
seen to be entrusted with the function of a vocal organ, and is known as the larynx. Part, therefore, is thus differentiated from the rest of the air-passage. The rest has very much the same characters in its unpaired portion, or trachea, and its paired or bronchial portion: the larynx varies much more considerably. In the Amphibia the two cartilages above mentioned, and known as the arytænoid cartilages $(a)$, form a support for the two folds which bound the entrance into the larynx. The change in the position of the cartilages, which is effected by muscles, opens and closes the entrance into the larynx. Functionally, therefore, they are of more importance than the more indifferent parts which have the character of supporting organs. These arytænoid cartilages rest on the anterior ends of the two longitudinal ridges of cartilage, which in other forms are connected on the ventral surface by transverse processes directed towards one another, and which thereby gave rise in many Amphibia to an unpaired portion of the supporting: framework of the larynx $(C c)$.

In the Reptilia the two longitudinal ridges are more perfectly connected transversely, but a low condition is implied by their continuous connection with the arytænoid cartilages; and this is especially the case in some of the Ophidii. In others, these cartilages are more completely separated $(D a)$. This character is even found in the Saurii, but in them the portion which carries the arytænoid cartilages is converted into a circular, and generally a closed piece. A second portion of the larynx may then be distinguished, which forms the circular cartilage, and is to be found in course of formation as early as the Amphibia $(\mathrm{Cc}$ ). In the Chelonii and Crocodilini this is more completely separated from the tracheal skeleton, and its anterior portion is considerably widened out. Not unfrequently it is possible to make out in it indications of its connection with several rings of cartilage. In Birds, this circular piece is made up of one anterior and broader, and two posterior and more delicate portions; a small piece is, further, placed on the latter and carries the arytænoid cartilages.

Lastly, in the Mammalia the large circular piece of the Reptilia is divided into two portions, for the anterior upright plate forms the thyroid cartilage, while there is a second piece, which is circular and generally very massive posteriorly (cricoid cartilage), and this carries the arytænoid cartilages on its posterior and more elevated portion.

\section{$\S 426$.}

Other parts, which serve more or less for the production of the voice, are connected with this laryngeal skeleton. Of these, the folds of mucous membrane which are placed at the sides of the entrance into the larynx are worthy of note, for they are converted by tension, and by the development of elastic tissue into vocal chords. They enclose a fissure-the glottis, which can be altered in width owing to the attachment of the rocal chords to the movable arytrnoid 
cartilages. These chords are found in most of the Anura, in the Geckos and Chamæleons among the Saurii, and in the Crocodilini. They are not found in the Ophidii.

In Birds, the vocal organ is placed in the lower portion of the trachea, and forms the so-called lower larynx. This arrangement compensates for the absence of vocal chords in the true larynx. Among Mammals the chords are atrophied in the Cetacea only; in all essential points they present just the same arrangements as in Man.

As the various cartilaginous pieces are differentiated from the primitive laryngo-tracheal muscle, muscles are separated off to move the portions which have become free. In the Reptilia these are replaced by a constrictor and a dilatator muscle, which are also present in a modified form in Birds. In the Mammalia, the musculature is complicated in comparison with the simpler arrangement found in Reptiles; this is seen partly in the number, and partly in the arrangement of the muscles. They correspond in all essential points to what obtains in Man.

A process which hangs over the entrance to the larynx from in front-the epiglottis - is merely indicated in the Reptilia by a process of the supporting cartilage; this is sometimes of no small size. It is well developed in Birds. Many Birds, however, are provided with a special epiglottis, the cartilage of which is only suturally connected with the supporting cartilage. But these arrangements never succeed in completely covering the entrance into the larynx. The cartilage of the epiglottis is more completely separated in the Mammalia, where it forms a protective apparatus during the passage of the food over the entrance to the larynx. In the Sirenia it is atrophied, while in the Cetacea it is converted into a long spout-like piece, which unites with the similarly elongated arytænoid cartilages to form a cone, which projects into the internal nares, through which the air passes in and out.

In some of the Amphibia the portion of the air-passage which commences at the larynx is more distinctly divided into the trachea and its two branches, the bronchi; these pass directly into the walls of the pulmonary sacs. The ends of the laryngo-tracheal cartilages extend into these, either in the form of finer processes (Menobranchus, Menopoma), or as broader pieces which give off lateral processes (Bufo). As the transverse processes grow towards one another at the anterior end of these ridges (cf. Fig. 322, $C b$ ), they represent the earliest cartilaginous rings. These rings are developed on the trachea of the Reptilia, which is generally long, and either are, or are not complete. In the Ophidii and Saurii a remnant of the primitive arrangement is indicated by the connection of the rings with one another by means of longitudinal ridges.

The trachea of Birds is always distinguished by its great length, and the rings, which are generally completely closed, are always more largely differentiated. The two bronchi have the same structure. We frequently meet with enlargements at various points in the trachea (Natatores), while in many Birds this tube does not keep to 
a straight course. This is the case in the Penelopidæ, various Swans, and in the Crane. In the last named a loop of the trachea is even enclosed in the sternum.

The most peculiar character is the formation of a lower larynx (Syrinx) in the Carinatæ; as a rule this is formed by the end of the trachea, and by the commencement of the bronchi. The variations

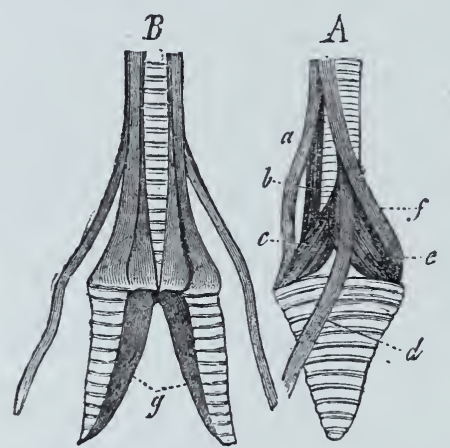

Fig. 323. Lower larynx. Muscular organ of voice of the Crow. $A$ From the side, $B$ From in front. $a-f$ Mruscles which move the lower larynx. g Membrana tympaniformis interna. in the form of this portion are due to lateral compression, or to the fusion of several rings at the end of the trachea; this latter is divided by a bony ridge (pessulus), which rises up at the angle of furcation of the bronchi and forms the tympanum. A membrane (membrana tympaniformis interna), is stretched, as if on a frame, from the median surface of either bronchus to the pessulus. The membrana tympaniformis externa is stretched between the last tracheal and the first bronchial ring, or between a pair of modified bronchial rings. In the male Anatidx we find various kinds of vesicular, and asymmetrical enlargements of the tympanum. In Singing Birds, a fold, the membrana semilunaris, projects inwards. As there are vocal membranes, which are elastic folds of the mucous membrane, on either bronchus, a double glottis is marked off. The action of a special supply of muscles varies the tension of the vocal chords, and narrows or widens the glottides. Several pairs of muscles pass into the trachea, which depress it, and relax the vocal chords. In addition to these, the Singing Birds are distinguished by the possession of a muscular apparatus, formed of five or six pairs of muscles (Fig. 323, $a-f$ ).

\section{$\S 427$.}

In, and above the Amphibia the lung's, which commence at the ends of the air-passages, form the respiratory organs of the higher Vertebrata. They do not form the sole organ quite at once, for in all Amphibia there are branchiæ either during the larval stage, or throughout life (Perennibranchiata). There is a series of similar differentiations in the anatomical characters of the lungs, as in the air-passages which lead to them. Simple sacs are gradually replaced by complicated organs, the respiratory surface of which is continually increased by the formation of smaller internal cavities.

Among the Amphibia the lungs are exactly like those of the Dipnoï; in the Perennibranchiata their internal cavity has its surface only slightly increased. In Proteus and Menobranchus they are formed of simple, but very long tubes, which are slightly 
widened out anteriorly, but always end in an enlargement. The plexiform processes in the walls of the lung of Cryptobranchus are more considerable, but they are very small in Triton. This is frequently the case also in the other Salamandrina, but in the Anura they are divided into smaller spaces by a close-set network. The lung is thereby enabled to expose a larger quantity of blood for the exchange of gas. This relation is still more complete in the Reptilia. Although many (most Saurii) have very simple lungs, yet in the Ophidii, as well as in the Crocodilini and Chelonii, each lung is divided into a number of large cavities, which again are divided into smaller ones of various kinds. In the Ophidii the lungs may be seen to be adapted to the elongated form of body and to be themselves elongated; the more or less atrophied condition of one lung is a similar adaptation. The elongation of the lung is accompanied by a peculiarity of the last portion of the lung, which is generally a good deal enlarged, gets simpler in structure and loses its respiratory function. Similar portions, which have lost their respiratory function, may be seen in the Saurii. As in the Ophidii, the most anterior portion, or that which is above the point at which they are connected with the air-passages, carries a more closely-set meshwork, while the hinder end has its internal surface less largely increased. In the Chamæleons special cæcal tubes are given off from this portion, and project far into the colom. They are rudiments of an arrangement which has other functional relations in the Birds.

In Birds conical prolongations are developed on the surface of the lung during the embryonic period, and these become connected with other organs and form cavities for the passage of air. In the adult this pneumatic apparatus is formed of membranous sacs embedded between the viscera, or of tubes which extend into parts of the skeleton. In the latter case air-cavities take the place of the medulla, which disappears, and so diminish permanently the specific gravity of the animal. In the same way, the specific gravity of the animal may be diminished at will by the blowing out of the sacs between the viscera, and this, like the other arrangement, is of assistance in flight.

As to their minute structure, the lungs of Birds have their finest cavities connected with one another. The parenchyma of the lung is spongy. In the Mammalia the lobate arrangement is continued into the smallest portions of the lungs, while larger lobes may be made out externally.

There are greater peculiarities in the position of the lungs. In the Amphibia, and in the Saurii and Ophidii, they project into the cœlom. In the Chelonii and Aves they are placed on the dorsal wall of the thorax, and are invested anteriorly by the peritoneum. In the Crocodilini each lung is placed in a serous sac, by which it is invested. The same is the case in the Mammalia, where the lungs, which are covered by a pleural investment, occupy the lateral halves of the thoracic cavity. 


\section{C'œlom.}

\section{$\S 428$.}

In all Vertebrata, just as in many of the Invertebrata, a hollow space is differentiated around the enteron of the trunk; this is effected by the cleavage of the mesoderm. It is, therefore, a space which appears in the middle germinal layer, and separates the epithelial lining of the digestive tract and the parts developed from it from the organs differentiated from the outer germ-layer. The limitation of this process of separation to the region of the trunk appears to be correlated with the formation of branchial clefts in the cephalic enteron, for this prevents any continuation forwards of the cleft, at any rate laterally. As in the Invertebrata, the cœlom forms a cavity, which is a part of the vascular system, in so far, that is, as the lymphatic portion is connected with it. The direct communication with the exterior which obtains in many Invertebrata is not completely wanting, although it is not greatly developed. It is represented by an abdominal pore which is placed near the anus, and which is generally paired; this is seen in the Cyclostoma and among Gnathostoma also, in the Selachii, Chimæræ, Ceratodus, and many Teleostei ; it is last seen in the peritoneal canals of the Crocodile. In the Chelonii there are merely indications of it. We should also note that there is a free communication between the cœlom and the excretory apparatus, for a connection with the condition of lower organisms is thereby demonstrated (vide Excretory organs).

The whole of the inner surface of the cœlom is invested by a layer of epithelium, which is greatly developed in a certain region, where it forms the germinal epithelium. The female germinal glands are differentiated from this tract. In the anterior portion of the cœlom of the lower divisions ciliated epithelium is distributed over certain regions. The epithelium of the cœlom unites with a subjacent layer of connective tissue to form a special membrane, the peritoneum, which is continued on from the walls (where it forms the parietal layer) to the parts (viscera) which are placed in, or project into the cœlom; and these parts it also covers (visceral layer).

In the Anamnia, the colom is a single cavity, as it is also in most of the Reptilia, although in the Crocodilini we may observe that an anterior portion is being separated from a hinder one. In the Mammalia this separation is complete. The diaphragm separates the hinder portion of the cœlom, or abdominal cavity, from an anterior one, which contains the two lungs, and which is divided into two lateral halves (pleural cavities), which contain the lungs, by a median wall or partition, which contains the pericardium. 


\section{Vascular System.}

$\S 429$.

The nutrient fluid of the Vertebrata moves along closed canals, which have proper walls; it is very rarely that this tract becomes lacunar in character. In this point it differs from the arrangements which obtain in the Mollusca, though it is more nearly similar to those of the Vermes. Its cavities form a system of canals-a vascular system. This portion is derived from the mesoderm, which also develops the parts that carry the vessels. The chief trunks are placed in the middle line, and give off branches in accordance with the segmentation of the body; looked at in the broadest way, the arrangement of these vessels calls to mind many arrangements which obtain in the Invertebrata. This may be still more distinctly seen in the relations of the longitudinal trunks to the respiratory portion of the enteric canal. But there is a great difference when a central organ of circulation is developed. In the Arthropoda, Mollusca, and most Vermes, this is developed from a dorsal vessel, or from part of one, but in the Vertebrata it is formed from a ventral portion. The double character of the early rudiment of the heart, such as has been made out in the higher Vertebrata (Rabbit), cannot be referred to any definite vascular organ, for none of the kind is known to us.

There are considerable differences between the circulatory centres of the nutrient fluid in the two great groups of the Vertebrata, so that we must separate the apparatus which obtains in Amphioxus from that of the Craniota. In the former, all the larger vascular trunks are contractile, and so call to mind the arrangements which obtain in the Vermes. The contents of the vascular system are driven on at various points, without one centre being at all aided by the others. The following is a description of the arrangements of these vessels. Below the respiratory portion of the enteric canal there is a longitudinal canal, which gives off branches, at regular distances, to the branchial framework; these branches are branchial arteries. They are collected into a trunk, which is placed above the gills-the aorta ; and from this they are distributed through the body. At the root of each branchial artery there is a heart-like formation, or contractile enlargement. The most anterior pair of branchial arteries passes into two arches, which are likewise contractile, and which surround the mouth; this pair is connected with the commencement of the aorta. From this vascular trunk arterial blood-vessels are distributed in the body.

Our knowledge of the vessels, which distribute the blood, is at present very slight, while the afferent vessels also require to be more accurately investigated. It seems to be certain, however, that there are contractile tracts in this portion also of the vascular 
system. In any case the whole apparatus agrees only generally with the vascular system of the Craniota, and it may be regarded as an expression of the great difference which there is between these two divisions, as shown by many other characters.

The blood-fluid of Amphioxus is colourless, and its form-elements are very small, indifferent, cells.

\section{$\S 430$.}

The Craniota possess a single organ, the heart, which effects the circulation of the nutrient fluid; they are also distinguished by a differentiation of the circulatory vessels, which is of such a kind that the nutrient fluid itself may be divided into two categories. Part of the blood which passes through the body into the tissues, and which, during the circulation, filters through them, is collected into special tracts, and is again restored to the principal stream. This fluid is the lymph, and its vessels form the lymphatic system. The vessels which pass directly from the heart, and which lead back again to it, form the blood-vascular system. The lymph-vessels which are distributed in the wall of the enteron take up the formative material, or chyle, which has been formed by the process of digestion, and carry it into the blood-stream. They make up, therefore, for the loss which the blood has suffered in consequence of the metastasis which is continually taking place in its circulation through the body. The lymphatic system is an important part of the whole vascular system. That system gains an important step by this elaboration, and, owing to its division into these two parts, is seen to be more highly developed than are the arrangements which obtain in the Acrania, and in all the Invertebrata.

The separation of the nutrient fluid into blood and lymph is accompanied by a differentiation of the form-elements of these fluids. Those of the lymph are indifferent cells, which are similar to the blood-cells of most Invertebrata. The form-elements of the blood are, in their earliest stages of development, similar in character, but are afterwards converted into coloured corpuscles of a definite form, although this form is different in different divisions. Owing to their large quantity they give the blood the appearance of being coloured, as compared with the colourless lymph.

In all points but their size the lymph-cells of the Vertebrata are similar to one another. The blood-cells, however, which are much more differentiated, differ a good deal. They are all cellular in character, so far as this depends on the nucleus, although, indeed, it is only present in Mammals during their fotal stages. As a rule, the blood-cells are flattened and discoid. In Fishes, Amphibia, Reptiles, and Birds, they are oval and biconvex, for the centre of each surface protrudes slightly. They form biconcave rounded discs in the Mammalia, but in some they are oval (e.g. Tylopoda). In size, those of the Dipnoï and Amphibia (especially Proteus, Siren, etc.) are the largest. Owing to the 
important functions of the blood-corpuscles in the economy of the Vertebrata, as carriers of gases, their number, size, and the surface which they thereby represent, is of the greatest importance. In the higher divisions the relative quantity of blood varies but little, and there is not any great difference between the relative volumes of the plasma and blood-corpuscles. With regard, however, to the distribution of the whole substance of blood-corpuscles in larger or smaller form-elements, there is a great difference between the cold and warm-blooded divisions, and, again, between the Reptilia and Amphibia; from this point of view, the latter occupy a much lower position.

Wercker, H., Zeitschr. f. rationelle Med. XX. p. 290; und Arch. f. Mikrosk. VIII.

\section{Heart and Arterial System.}

$\S 431$.

At a certain stage the heart of all Craniota is formed of a single tube. As it gradually gets longer than the space set apart for it, it is arranged in an S-shaped loop, and so takes on the form which the heart has later on. As it changes its form, it is divided into two parts. The hinder one receives the blood and sends it to the anterior one, which conducts it into the vascular arches, and so into an arterial trunk which runs along the axial skeleton, from which it is distributed in the body. The first portion of the heart is called the auricle, the second the ventricle. A special cavity, which is similar to the heart when it is first formed, encloses the auricle and ventricle; this is the pericardial cavity, and its walls form the pericardium.

We find this simple condition of the heart in Fishes. A ventricle and an auricle form the two chief portions. The latter receives blood from a sinus venosus, which is placed just behind it, and is only partly outside the pericardium. As a rule it has diverticula on either side, which grow out towards the chamber in front of them (auriculæ). The walls of the heart vary in character according to their function. The wall of the auricle is provided with a very thin muscular layer which projects inwards in a retiform manner. Its only function is to drive the blood into the ventricle. The ventricular wall has a more important function, and is consequently provided with a powerful network of muscular bands. This projects inwards, and so diminishes the size of the proper cavity of the ventricle. But this is made up for by the connection between the spaces in the network of the ventricular wall and the cavity of the ventricle itself. There are two membranous valves at the ostium atrio-ventriculare (Fig. 324, o), which prevent the blood from returning to the auricle. There are generally as many as three such pouched valves at the ostium arteriosum of the ventricle. 
The mechanical arrangements by which the stream of blood is directed are therefore, at first, essentially the same at both ostia of

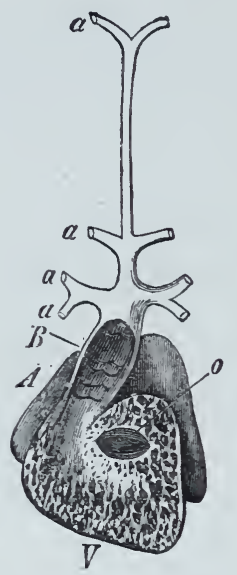

Fig. 324. Heart of Squatina vulgaris. The anterior wall of the ventricle and of the conus arteriosus is removed. A Auricle. $V$ Ventricle. B Conus arteriosus. o Ost. atrioventriculare. a Bran. chial arteries. the ventricle. The cavity of the ventricle is continued into the arterial trunk which is given off from it, and commences by an enlargement (bulbus arteriosus). That part of the ventricle which is attached to this bulb has an elongated form in the Selachii and Chimæræ, and in its wall the musculature has gained a circular arrangement. This portion of the heart is the conus arteriosus $(B)$. It is marked off from the bulb by three pouch-like valves; behind these there are a number of valves in the conus arteriosus, which are set in longitudinal rows. There is a similar arrangement in all the Ganoïdei. In the Teleostei it is rarely possible to distinguish the conus arteriosus, and, as a very general rule, there are only two valves between it and the bulbus. In Ceratodus there are rudiments of two rows of valves behind the four pouched valves that are placed on the boundary line of the conus. In Protopterus there are longitudinal folds which foreshadow a division of the cone.

The atrophy of the conus arteriosus in the Teleostei is accompanied by a development of the bulb, and the smooth muscular tissue in its walls is increased in quantity. It thus gets the appearance of an independent portion, which must not be confounded with the conus arteriosus. In all Fishes, except the Dipnoï, the heart contains venous blood only.

\section{$\S 432$.}

The trunk of the branchial artery is placed in all Fishes below the branchial framework, along the arches of which it sends out its branches. In the earlier stages of development these pass on each side directly into a longitudinal vessel which runs along the base of the skull, and from which an artery is continued forwards to the head, and especially to the brain and eye (carotis interna). Posteriorly the two longitudinal trunks (roots of the aorta) are united into an unpaired larger trunk-the aorta (cf. Fig. 325). When the branchial lamellæ are developed on the branchial arches, a vascular network is gradually developed in them by the arterial arches. When this network is well developed the arterial arches disappear. Each of them is replaced by a network of capillaries, which is supplied by a branch of the branchial artery, and which gives off an artery, or branchial vein, which takes part in the formation of 
the aorta. The blood which is brought to the gills from the stomach by the branchial arteries is venous blood, for it is returned to the heart from the systemic circulation; as it passes through the capillary network of the gills it again becomes arterial blood, and passes as such through the branchial veins into the aorta, and so to the systemic circulation.

The number of branchial arteries given off from the arterial bulb is the same as that of the functionally active gills. It is largest in the Cyclostomata and the Selachii. There are five pairs in the Ganoïdei also, while in the Osseous Fishes it is only during the embryonic stage that there is a larger number ( 6 or 7 ) of arterial arches. The two anterior ones belong to the mandibular and hyoid arches, and have either no relations to the gills, or the gill of the hyoid arches is functional for a time only (opercular gill). When the hindermost gill, which belongs to the more or less

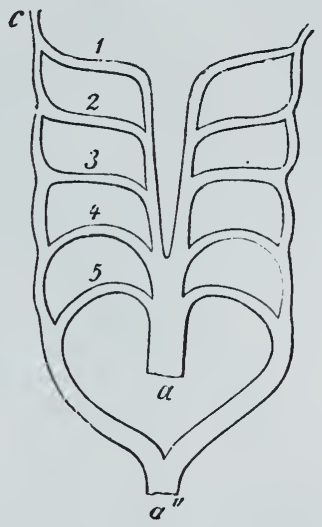

Fig. 325. Diagram of the arterial arches. 1-5. $a$ Branchial artery. $a^{\prime \prime}$ Aorta. $c$ Carotid. rudimentary last branchial arch is atrophied, the arterial arches are reduced to four, and in some cases to three pairs.

These branchial arteries are given off in very various ways. They either arise in pairs from the simple primary trunk, which ends when it has given off the last pair, or they are given off on either side of a common short trunk; this is especially well seen in the posterior branchial arteries of the Selachii, and in various Ganoids and Teleostei ; or, the primary trunk of the branchial artery divides at its origin into two equal and lateral branches, from which

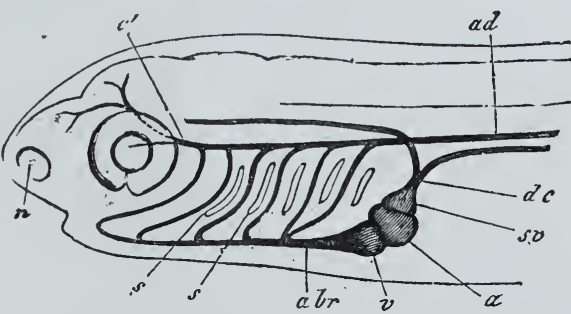

Fig. 326. Head of an embryonic Teleo. stean, with the rudiments of the vascular system (Diagrammatic). a Auricle. $v$ Ven. tricle. $a b r$ Branchial artery. $c$ Carotid. ad Aorta. $s$ Branchial clefts. $n$ Nasal pit. $s v$ Sinus venosus. $d c$ Ductus Cuvieri. the various branchial arteries are given off (e.g. Bdellostoma among the Myxinoidea).

\section{$\S 433$.}

The appearance of lungs is of the greatest importance in effecting a change in these relations, for they produce great changes in the arrangement of the large vascular trunks by taking on the function which was previously performed by the gills. This change affects also the structure of the heart; the Dipnoï afford an interesting 
example of this, for it is in them that the cavities of the heart are first divided. In Lepidosiren a network of muscular bands is continued from the wall of the auricle right through it, and forms a

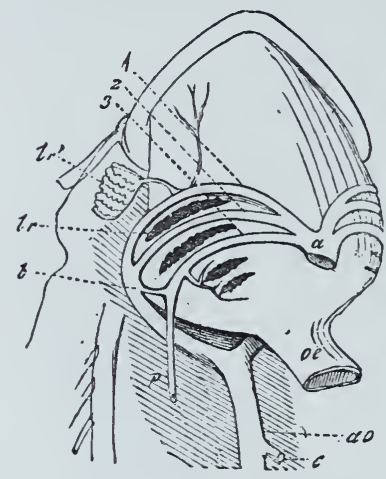

Fig. 327. Aortic arches of Lepidosiren paradoxa. a Trunk of the branchial arteries. 123 Arterial arches; the first two are united to form the aorta. $p$ Pulmonary artery. $b$ Ductus Botalli. br Branchial clefts. $\quad b r^{\prime}$ Accessory gill. ao Aorta. c Cœliac artery. oe Esophagus (after Hyrtl).

kind of partition. The auricle is thereby divided into a right and left portion, but there are many points by which these two parts communicate with one another, and they also open by a common opening into the ventricle. The venous sinus now opens into the right side of the auricle, and a pulmonary vein passes into the left side. The ventricle, also, is partly divided by muscular processes; and, in correspondence with this, the lumen of the arterial bulb is divided into two cavities, each of which gives rise to special arteries. These form three vessels which extend along the anterior branchial arches; the most anterior on either side is connected with the second one, and unites with its fellow of the opposite side to form an aorta $(a 0)$. These two vessels have no relation to the gills. The third arch gives rise to branchial arches, but it is also connected by a narrow duct $(b)$ with the root of the aorta, and it is continued on as the pulmonary artery $(p)$. This arch forms, therefore, a branchio-pulmonary artery, and the two anterior arches, as they do not give off any branchial vessels, represent aortic arches.

The circulatory apparatus of the Amphibia presents the same conditions. In most, the auricle is completely divided, but in some, as in Proteus, it is not so; in Salamandra, also, there is a hole in the septum. The systemic veins open into the right, the pulmonary into the left auricle. The ventricle is single, and there are indications merely of a partition. There are membranous pouched valves at the ostium atrio-ventriculare, which have the same character as in Fishes. A muscular arterial bulb (Fig. 328, $b a$ ) is given off from the ventricle, and its separation into two parts, which we saw commencing in Lepidosiren, is completely effected. At first it gives off as many as five pairs of arterial arches, but these are reduced to three or four. As in Fishes, a network of branchial vessels is developed leading from the heart. Owing to this the arterial arches are divided into efferent and afferent vessels; a branchial artery, and a branchial vein, between which is the capillary network. The branchial veins form the roots of the aorta.

Each branchial artery is, however, connected with its own branchial vein by a ductus arteriosus, which is a portion of the primitive aortic arch. When the lungs are developed, the last branchial artery either gives off a twig to the pulmonary artery, 
just as in Lepidosiren, or the pulmonary artery $(p)$ is continued on directly from the branchial artery.

The disappearance of the gills causes certain changes in some of the Amphibia, in this vascular apparatus, which is persistent only in the P'erennibranchiata. The direct connection (cf. Fig. 328), which already exists between the branchial arteries and veins, is developed in such a way that some of the arterial arches are continued directly from the heart into the roots of the aorta. The last arch, which already gives off the pulmonary artery, is developed into the trunk of this artery, and is either connected by small vessels only (ductus arteriosus) with the root of the aorta, or is cut off from it, and forms a separate vessel. In this way several arterial arches are connected with the root of the aorta just as in Lepidosiren, while one of the primitive vascular arches is converted into the pulmonary

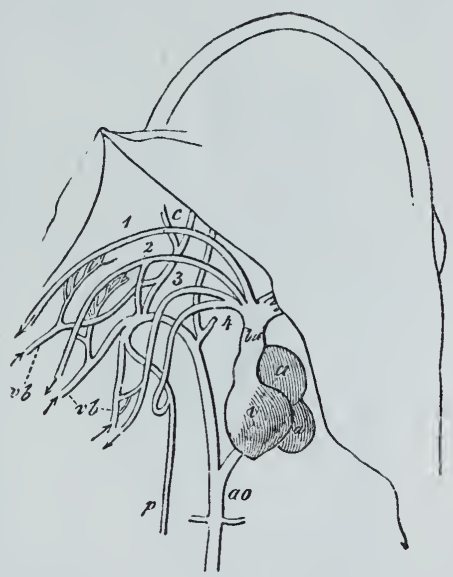

Fig. 328. Heart and arterial trunks of a larva of Triton. a Auricle. $v$ Ventricle. $b a$ Arterial bulb. 1-4. Arterial arches; the first three are the branchial arteries. $c$ Carotid. $p$ Pulmonary artery. ao Aorta. $v b$ Branchial veius (after M. Rusconi).

artery. The two kinds of blood are enabled to commingle, owing to the arrangement of the heart, and the characters of the large vascular trunks.

Brucke, E., Denkschr. d. Wicn. Acad. I.

\section{$\S 434$.}

A great step in the differentiation of the circulatory organs is seen in the Reptilia, where the heart is placed at a greater distance from the head. It is gradually moved backwards from the point at which it is formed, and is embedded in the thoracic cavity; this is its position in all the Amniota. The ventricular portion is generally elongated, but it is broad in the Chelonii (Fig. 330), and several Saurii. The two auricles are always separated from one another by a septum, as in the Amphibia; the right one receives the systemic, and the left the pulmonary veins. The former is always the larger. The strong muscular wall of the ventricle is continued into a network which diminishes its cavity, just as in Fishes and Amphibia; this is especially the case in the Ophidii, Chelonii, and Saurii. The ventricular partition is chiefly formed by this network, but some of the muscular bands are more largely developed. The right half of the ventricle contains venous, and the left arterial, blood, so that the two portions can be distinguished. The incompleteness of the separation of the two cavities is partly, at least, made up for by 
various arrangements. For example, there is a muscular process, which is able to partly shut off the cavity which gives off the

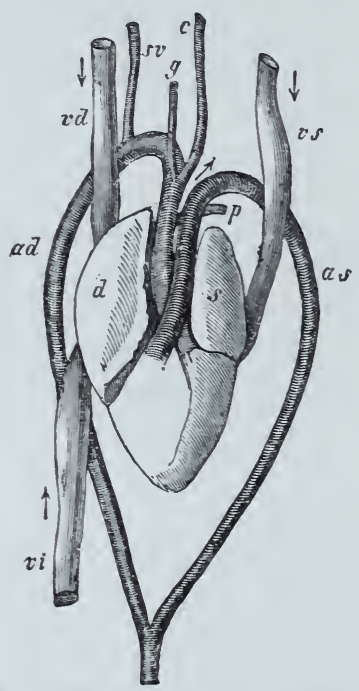

Fig. 329. Heart and arteries of an Ophidian (Boa). $d$ Right, $s$ Left auricle. $c$ Carotid. ad Right, as Left aortic arch. $p$ Pulmonary artery. branchial arteries from the rest of the ventricular cavity. The ventricle is completely divided in the Crocodilini.

The membranous valves of the ostium atrio-ventriculare are greatly developed in the right half of the heart. In the Crocodilini there is only one of these valves on the right side, and it extends along the septum of the ventricle; the other valve is replaced by a process of the lateral muscular wall of this chamber. The arterial bulb, which is single externally, is apparently given off from the right ventricle. It is, however, divided into a series of canals, which are connected with both ventricles. There are pouched valves at the root of the arteries.

Of the five primitive arterial arches, the two first have disappeared, and the rest undergo various metamorphoses in the different divisions. In the Saurii the third persists on both sides, and is connected on the right with the fourth arch, which, like the two branches of the third arch, is given off from the vessel that arises from the left ventricle. The left half of the fourth arch is connected with the third arch on its own side, and so corresponds to the right ventricle. The fifth arch on either side is partly converted into the pulmonary arteries, which primitively spring from it only, and which are given off from the trunk of the pulmonary artery in consequence of the differentation of the primitive aortic bulb. There are, therefore, two aortic arches on either side, one of which, the second on the left, conveys venous blood. It is, howerer, connected peripherally with the other arches, so that the two kinds of blood must be mixed. In the Ophidii the first pair of arches that persists in the Saurii is not generally connected with the second pair, but the continuation of this portion is converted into the internal carotid. In the Chelonii the right arterial (Fig. 330,ad), and the left venous aortic arch (as), are connected by a ductus Botalli with the pulmonary arteries, which are developed from the last pair of primitive arteries ( $p d$ ps). This disappears in the Crocodilini, so that in them a ressel, which gives off the right aortic arch and the carotids, arises from the left ventricle, while a left aortic arch and the pulmonary arteries arise from the right ventricle. The primitive connection between these vascular trunks is retained in the arterial bulb of the Crocodilini, where the foramen Panizzæ is the means of communication between 
the arterial and venous trunks. The two kinds of blood are not, however, able to commingle to any great extent.

The vascular apparatus of Birds is very closely similar to that of Reptiles, and especially of the Crocodilini. But there is this considerable advance, viz. that the arterial and venous blood are completely separated in the heart, as well as in the great arterial trunks. The auricles of the heart appear to be smaller owing to the smaller size of their anterior (ventral) portion. The musculature of the wall of the ventricle is greatly thickened, especially on the left side. The right ventricle is folded around the greater part of the left one. The atrio-ventricular valve of the right ventricle is formed by a muscular ridge (muscular valve) which projects from the wall of the ventricle, and surrounds the ostium peripherally; the second or membranous valve, which is found in the Crocodilini, is but seldom represented, even in rudiment. At the left ostium there is a valve which is connected with

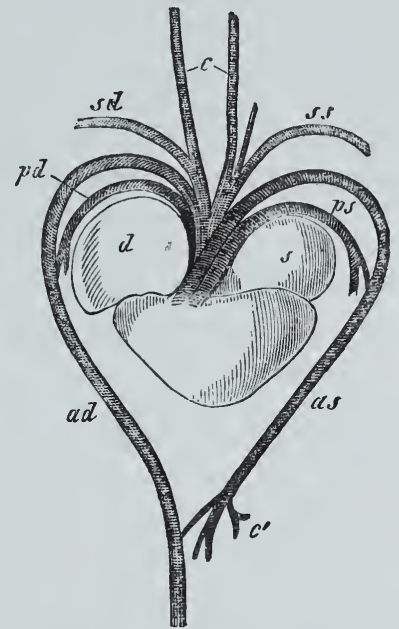

Fig. 330. Heart and arterial trunks of a Chelonian (Chelydra). The letters as in the previous figure. the wall of the ventricle by tendinous fibres. The arterial arches are reduced in much the same way as in the Reptilia, especially the Ophidii and Crocodilini. But the left aortic arch is not permanently developed. There is, therefore, merely a single right aortic arch, which arises from the left ventricle. Two arterial trunks, art. brachiocephalicæ, are given off with it; these are divided into a common carotid, and a subclavian. In some Raptores there is a permanent remnant of a left aortic arch, in the form of a ligamentous chord; this indicates the course taken by the vessel. The pulmonary artery, which is derived from the last of the primitive aortic arches, is consequently the only arterial trunk which is given off from the right ventricle.

Fritsch, G., Zur vergl. Anatomie der Amphibienherzen. Arch. f. Anat. u. Physiolog, 1869.

\section{$\S 435$.}

The heart of the Mammalia resembles that of the Birds in having its two halves completely separated; but there is a great difference in its structure, and in the arrangement of the great vessels. It is in rudiment only that there is any close similarity between either the heart or the general arterial system, which, as in Birds, is derived from a number of paired arches. During the embryonic stage the two auricles communicate; in the Marsupialia this is effected by a narrow slit, and in the placental Mammals by a 
larger space (foramen ovale). Owing to this connection, the blood which passes into the right ventricle from the umbilical vein through the vena cava inferior, is able to pass into the left ventricle, whence it is distributed through the body by the aorta. In the Monodelphia this opening is gradually closed by the forward growth of a wall, or partition, towards the left auricle (valvula foraminis ovalis); after birth, therefore, the two auricles are completely separated. 'The boundary of the primitive foramen ovale may, even later, be seen as a circular process. The most anterior (ventral) portion of each of the two auricles in the Mammalia forms a small prolongation, which has a different form on either side, the "appendages of the auricles." They correspond to the greater part of the auricles of the lower classes, for the hinder portion of the cavity of the auricles, on the right side, at any rate, is formed by a venous sinus, which in the lower forms is separated from the auricle (cf. Venous System). These appendages, therefore, in the Mammalia are the remnants of the anterior portion of the auricle.

The atrio-ventricular valves are much altered in character. In the earliest stages their place is taken by membranous folds, as in Fishes, Amphibia, and Reptilia. The ventricles have a comparatively small cavity, and their wall is formed by the same spongy muscular tissue as that which we have found in a well-dereloped condition in the above-mentioned classes. The bands gradually thicken, and part goes to form the more compact cardiac wall. That portion of this network which passes inwards and bounds the lumen of the ventricular cavity, and which is inserted into the edges of the venous ostium, is connected with these membranous valves. As the free edge of the valve ceases to grow, and as the only part which is left is that which is connected with the muscular bands, the valve becomes intimately connected with the wall of the ventricle, so that the muscular bands from the latter pass into a membrane which is given off from the ostium. This condition, which is transitory in most Mammals, is permanent in the right ventricle of the Monotremata (Ornithorhynchus). The muscular bands which pass off from the wall of the ventricle are continued into a membranous valve. In all other Mammalia this condition is replaced by another one. The muscular bands are withdrawn towards the wall of the ventricle, and so form the musculi papillares, while their more anterior portion, which passes into the valve, is represented by the chordre tendineæ. The rest of the spongy muscular network of the wall of the ventricle forms the trabeculæ carneæ. The atrio-ventricular valves and the chordæ tendineæ are, therefore, differentiations of the primitive muscular network, which was connected with the primitive valves. The similar valves in the left ventricle of the Bird's heart are formed in just the same way. The great arterial trunks in the Mammalia are differentiated from the embryonic arrangements in a somewhat different manner. The two first arches disappear completely; the third, as before, forms part of the carotid. The fourth is continuous on the right side with 
the subclavian, while on the left it gives rise to the aortic arch, from which the left subclavian springs independently. 'The carotids and the right subclavian are united to the commencement of the aorta. In the Mammalia, therefore, the chief trunk of the arterial system is formed by a left a ortic arch. The pulmonary artery is formed from the fifth arch, which is during foetal life connected with the aortic arch of the left side by a ductus arteriosus (ductus Botalli). The blood, therefore, of the superior vena cava, which passes into the right ventricle, is in this way kept away from the lungs, and is carried into the descending aorta, which till birth, therefore, conveys mixed blood. After birth the communication between the pulmonary artery and the aorta descendens disappears, and the connecting portion $(b)$ of this vessel is converted into it chord (ligamentum Botalli).

Sabatier, A., Études sur le cour et la circulation centrale dans la série des vertébrés. Montpellier, 1873.

\section{$\S 436$.}

In all Vertebrata the systemic arteries arise, in their earliest condition, from the bulbus arteriosus. In those forms that breathe by gills the system of arterial arches, which arises from the bulb (primitive aortic arches), is, as has already been remarked ( $\$ 432$ ), broken up into the vessels of the branchial circulation; and the system of systemic arteries is only derived from the efferent branchial vessels (branchial veins). The current of blood, which at first is sent to the aorta through the arterial arches, is driven into new passages when the gills are developed, so that it is only distributed to the body after taking a roundabout course, which is due to the conditions imposed by the respiratory process.

In the Myxinoidea almost all the branchial veins unite to form a subvertebral aorta, which is continued backwards as the chief artery of the body, but which is also continued forwards as the "arteria vertebralis impar." Two lateral longitudinal trunks are collected from the branchial veins in just the same way, and each trunk sends a branch anteriorly into the arteria vertebralis impar, while its other branch goes to form a carotid. The two carotids divide into an external and an internal branch, which supply the head. In Petromyzon the aorta is not continued forwards, so that the carotids, which are formed in just the same way as in the Myxinoidea, form the sole anterior arteries. In the Selachii and Chimæræ, the aorta is formed from a trunk which is developed on either side by the union of the branchial arteries. There is the same arrangement in the Ganoïdei and Teleostei. The carotids arise from the first branchial vein, or from the anterior end of the paired arterial trunk, which collects the branchial veins on either side to form the roots of the aorta, and then unites with its fellow of the opposite side to form the aorta, or enters anteriorly into a transverse anastomosis which marks off an 
arterial circulus cephalicus at the base of the skull. A special optic artery is given off from the vessels of the rudimentary gill, with which a direct branch of the first branchial vein (Selachii), or a branch of the same vessel which surrounds the body of the hyoid (Teleostei), is connected. There are numerous modifications in the mode of origin and arrangement of the different vessels, the most important of which are found in the carotid and optic arteries.

This portion of the vascular system is arranged in a similar manner in the Amphibia. In the Perennibranchiata the cephalic

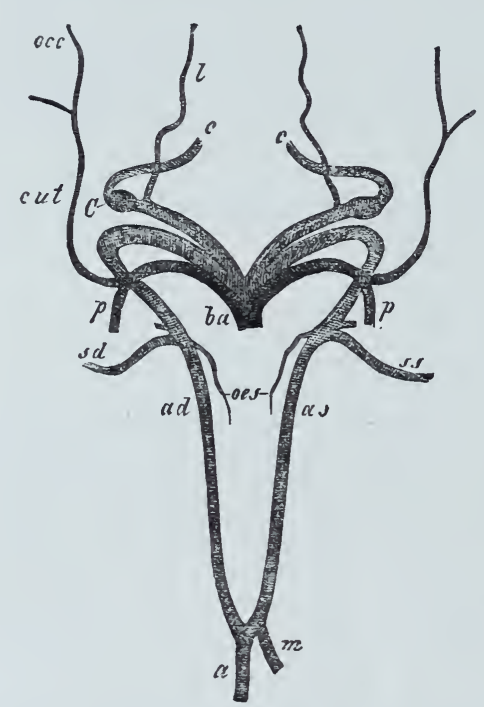

Fig. 331. Arterial system of the Frog. $b a$ Bulbus arteriosus. $c$ Carotid. $C$ Carotid gland. $l$ Lingual artery. ad Right, as Left aorta. a Aortic trunk. $m$ Visceral artery. sd Right, ss Left subclavian. oes Esophageal branches. p Pulmonary artery. cut Its cutaneous branches, occ Posterior cephalic branch. arteries arise from the anterior portion of the roots of the aorta; in the Caducibranchiata, from the first permanent aortic arch, or, they are continuations of the anterior arch itself (Fig. 331, c). In this case, an artery which goes to the tongue $(l)$ represents an external carotid. After this vessel is given off, there is a swelling $(c)$ on the carotid trunk in the Frogs and in the Salamanders; this is the so-called carotid gland. The lumen of the vessel is here traversed by a network of bands, which break it up into a number of narrower passages, just as if a capillary network had been intercalated in the course of an artery. The carotid gland appears to be derived from an arrangement of this kind, the branchial vascular network not having been completely atrophied. The next pair form the aortic arches (ad as), which converge backwards, and finally unite into an umpaired aortic trunk $(a)$. Each aortic arch gives off a subclavian ( $s d$ ss). Just before they unite, a large visceral artery $(\mathrm{m})$ is given off from the left aorta. The pulmonary artery represents a last aortic arch. Before it goes to the lungs $(p)$ it gives off a large cutaneous branch (cut), which ramifies on the back and neck as far as the posterior region of the head, and affords a distinct proof of the respiratory function of the integument.

In the earliest stages of the Amniota we meet with many similar arrangements of the arterial system. The internal carotid, which supplies the brain and the eye (Fig. 332, $A B, c^{\prime}$ ), is seen to be a forward prolongation of the roots of the aorta on either side. The external carotid is a branch of the third primitive aortic arch. If this arch loses its connection with the fourth, the two carotids are 
given off on either side of a common trunk $(C)$. They generally appear as two trunks, which pass along the sides of the neck in company with the vagus. In the Saurii they do not lose their connection with the next arterial arch, and retain, therefore, their primitive relations. In many Ophidii the right common carotid is atrophied, and may even completely disappear.
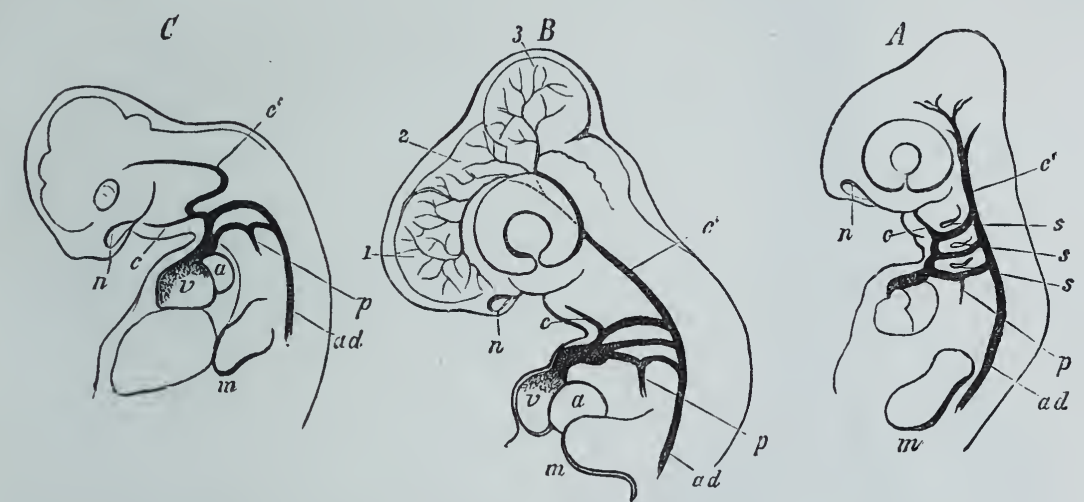

Fig. 332. Development of the great arterial vascular trunks, as seen in the embryoes, $A$ Of a Reptile (Lizard), $B$ Of a Bird (Fowl), and $C$ Of a Mammal (Pig). The two first pairs of arterial arches have disappeared in all three; in $A$ and $B$ the third, fourth, and fifth are still persistent; in $C$ the two last are alone complete, and the third is no longer connected with the fourth pair. A branch $(p)$ of the fifth forms the pulmonary artery. Its trunk from this point to the aorta forms the ductus Botalli. $c$ Carotis externa. $c^{\prime}$ Carotis interna. In $A$ and $B$ this forms the anterior prolongation of the root of the aorta, and in $C$ a common trunk with the external carotid. a Auricle. $v$ Ventricle. ad Aorta descendens. $s$ Branchial clefts. $m$ Rudiment of the fore-limbs. $n$ Nasal pit (after H. Rathke).

In Birds also this artery arises in company with a subclavian from a common trunk (art. brachiocephalica), but it leaves its primitive course, and lies in the middle line of the inferior surface of the cervical vertebræ; on the left, however, it retains its original course. In others, again, the two carotids both leave their old course, and this leads on towards that third form, in which the two closely-approximated vessels are fused together. In this case no part of the right carotid runs by itself, but a vascular trunk arises on the left side, which runs in the middle line, and which passes to the head, under the name of the primary carotid. Many Birds have this character in common with the Crocodilini. A single carotid trunk (Fig. 329, c), which obtains in the Ophidii and various Saurii, must be regarded as differing from this, although it also passes into two cephalic arteries anteriorly. This arrangement is due to the approximation of the points of origin of the two carotids on the right aortic arch. A common arterial trunk is given off from the united point of origin. Another peculiarity is the presence of an unpaired subvertebral artery, which runs forward from the right aortic arch along the vertebral column (Fig. 329, sv). 
'There are various kinds of modifications in the Mammalia, which

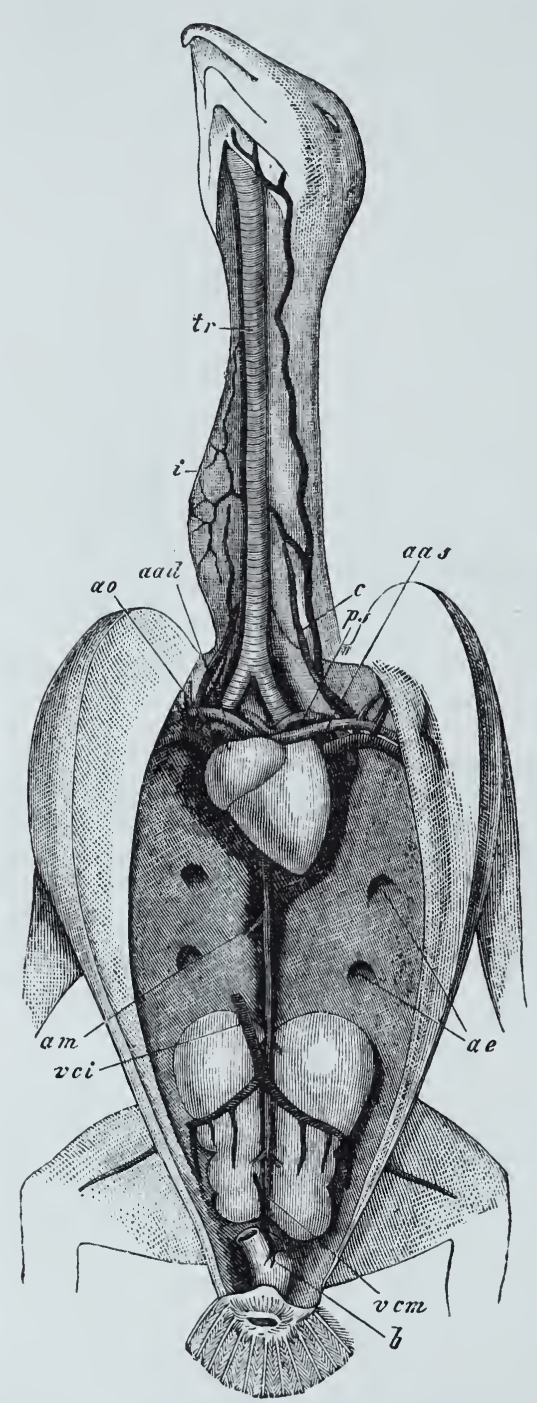

Fir. 333. Heart and great rossels of Buteo vulgaris. $\operatorname{tr}$ Trachea. $i$ Crop. ae Communication between the air-sacs and the lungs. $b$ Bursa Fabricii. ao Aortic arch. aad Art. anonyma dextra. aas Art. anonyma sinistra. ps Art. pulmonalis sinistra. $c$ Carotid. am Visceral artery. vci Com. mencement of the inferior vena cava. $v c m$ Vena coccygeo-mesenterica. in some there is a mesenteric artery also. The aorta gives off a are due to similar changes in the vascular trunk during embryonic life. Among others these specially affect the terminal branches of the carotids; the internal carotid, as in various Lizards and Birds, is not sent to the cranial cavity and sensory organs only.

The arteries of the forelimbs arise from various and very different points, so that transmission appears to be of less importance than adaptation in the development of these vessels.

The trunk of the aorta is continued along the vertebral column, without any chango in character, as far as the portion which is set apart for the caudal region-caudal artery; when the tail is shortened, it forms the median sacral artery. When the so-called cherron bones are present, the terminal portion of the aorta always lies in the canal formed by them. But in various Fishes it may be enclosed in a canal, formed by processes from the centra of the vertebræ, in the region of the trunk also; this is the caso in the Sturgeon, and in some Teleostei.

The aorta gives off arteries (arteriæ intercostales) for the metameres in regular succession, as well as the vessels which go to the viscera, and also those which go to the hindlimbs, when these are well developed. In Fishes there is ordinarily only one large arterial trunk for the viscera (A. cœliaco-mesenterica); but larger number of arteries for the renal and generative organs. As 
in the Amphibia, the Art. cœliaco-mesenterica arises from the end of the left aortic arch in the Reptilia (Saurii, Chelonii); this arch is only connected by a narrow tract with the right one; or there are several visceral arteries (some Saurii); these are especially numerous in the Ophidii, in consequence of the elongated form of their body. In the Crocodilinii, also, independent mesenteric arteries are given off from the unpaired aorta in company with the arteries from the left aortic arch.

In Birds, where the left aorta disappears, the aortic trunk is the sole vessel from which the visceral arteries are given off.

In Mammals the cœliac and superior mesenteric artery are the chief arteries of the enteric canal. In the placental forms the inferior mesenteric is also a large vessel.

The large number of renal arteries found in Fishes are found also in the Amphibia and in most Reptiles ; in Birds also there are several renal arteries, the middle one being given off from the ischiac artery. It is very rarely that there are a number of these arteries in the Mammalia.

The arteries of the hind-limbs are not direct branches of the posterior aorta until these parts are very largely developed. The two chief trunks (iliac arteries) of this region are not always the same. As is clear from their topographical relations to the pelvis, different branches may supply the area of these arteries. In the Sauropsida the ischiac are the chief trunks of the hinder extremities. In the Mammalia the chief trunks are formed by the crural artery, and there are numerous modifications in its more special characters which are of less importance.

\section{Venous System.}

\section{$\S 437$.}

The venous system of the Vertebrata exhibits no less important phænomena in the various modifications that obtain in it, as we pass from the Fishes to the Mammalia, than does the arterial portion of the circulatory system. Our knowledge is in many points as yet incomplete. The blood which returns to the heart is, in Fishes, collected into four longitudinal trunks, two anterior and two posterior. Those of either side pass into a transverse trunk (ductus Cuvieri, Fig 334, dc), which opens with that of the opposite side into a sinus (sv) which is placed behind the auricle of the heart. The anterior pair, which chiefly collects the venous blood of the head, forms the jugular veins $(j)$, which are placed above the branchial arches; the hinder pair, which receives the blood from the walls of the trunk and from the renal and generative organs, forms the cardinal veins $(c)$; an unpaired caudal vein runs below the artery in the caudal canal ; in the Cyclostomata, Selachii, and some Teleostei, this divides into two branches which are continued into the cardinal 
veins of their own side. In many Teleostei, this caudal vein is continued into the right cardinal by a large, and into the left

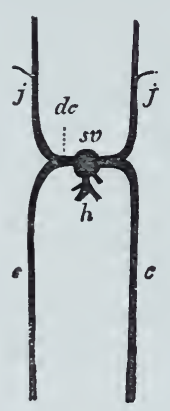

Fig. 334. Diagram of the primitive venous system. $j$ Jugular, $c$ Cardinal vein. $d c$ Ductus Cuvieri. $\quad h$ Venæ hepaticæ. sv Sinus venosus.

cardinal by a smaller branch; in this case the left cardinal vein is also smaller than the right one. This leads to the condition in which the whole of the caudal vein passes into the right cardinal; this has been observed in a number of Teleosteans.

As the caudal vein sends off branches into the kidneys, which either break up completely or partially in this organ, these branches form the venæ renales advehentes, and send their blood into the cardinal veins through the venæ revehentes. In this way the renal portal system is developed. A second vascular apparatus of similar character has its roots on the digestive canal ; its venous blood is carried to the liver by a trunk which is known as the portal vein. It is distributed in this organ, and is carried to the common venous sinus by hepatic veins, which are generally united into several trunks.

In this arrangement of the venous system in Fishes we may distinguish the paired, and ordinarily symmetrical, portion from the unpaired portion, which is solely represented by the hepatic veins.

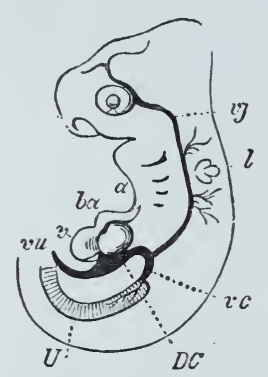

Fig. 335. Anterior portion of the venous sys. tem of an embryonic Ophidian. $v$ Ventricle. $b a$ Bulbus arteriosus. c Auricle. $D C$ Ductus Cuvieri. ve Cardinal vein. vj Jugular. vu Um. bilical rein. $U$ Primitive kidney. $l$ Rudiment of the labyrinth (after H. Rathke).

We will first follow out the former in its changes throughout the Vertebrate series, for in all of them its essential characters, at any rate, may be observed in the early stages of development, as a transmitted arrangement, and, since it is the groundwork of the embryonic venous system, it furnishes the startingpoint for all later metamorphoses.

\section{$\S 438$.}

In the Amphibia and Reptilia the venous sinus receives the two jugular veins, which have the same area of origin as in Fishes. They persist in all the higher Vertebrata, while the hinder pair-the cardinal veins (Fig. 335, vc)-have only the same characters as in Fishes, during the earliest stages of embryonic life. They are the veins of the primitive kidneys $(U)$. Their anterior portion is obliterated, while their posterior portion receives veins from other regions, and forms the venæ renales advehentes. Even before the disappearance of that part of the cardinal veins which opens into the ductus Cuvieri, four other trunks are developed in the Reptilia, which chiefly receive the intercostal veins, 
and are known as the venæ vertebrales. The anterior and posterior ones on either side are united, and open into the jugular vein of their own side. Their connection with the left jugular disappears later on, when the left vertebral veins develop transverse anastomoses, and become connected with the right ones, and, like them, open into the right jugular.

When the cardinal veins cease to be connected with the ductus Cuvieri they form prolongations of the jugular veins, which receiive the subclavians which come from the fore-limbs, and are known as the superior venæ cavæ. The vertebral veins, which collect the blood from the walls of the body, are not large after the embryonic period, and they are generally considerably atrophied. They cease to have a paired arrangement (Ophidii), and the greater part of their area is occupied by the vena cava inferior.

We meet with similar arrangements in the Birds. A pair of jugular veins, which are often unequally developed, form the chief trunks for the blood returned from the anterior parts of the body. At the base of the skull they are generally connected with one another by a transverse trunk, into which the veins from the cervical vertebral column, as well as from the head, may enter. When the left jugular is atrophied, this transverse trunk forms the vessel by which the blood is conveyed into the right jugular. The vertebral veins are now inconsiderable vessels. The jugulars unite with the veins of the anterior extremities, which form the subclavians, and the trunks thus formed are again known as the superior venæ cavæ. As these still receive the posterior vertebral veins, a portion is separated off from them, which may be seen to be derived from the transverse trunks (ductus Cuvieri), which are persistent in Fishes. These venæ cavæ, however, open separately into the right auricle, for the sinus, which is persistent in the Reptilia, here forms a portion
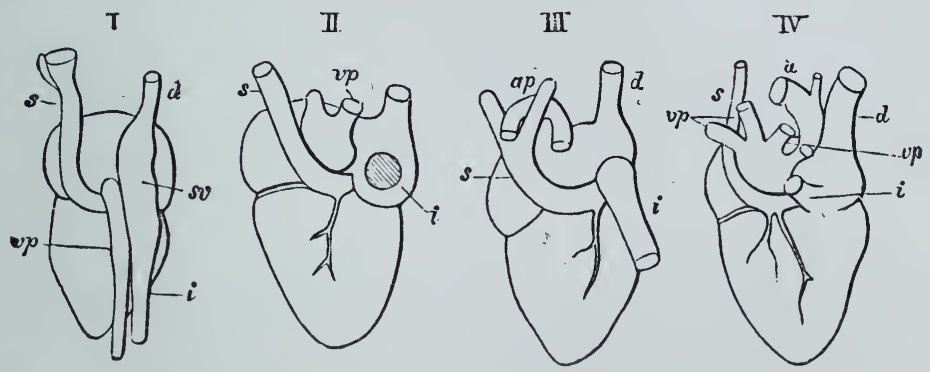

Fig. 336. Relations of the great venous trunks on the heart. I Reptile (Python). II Bird (Sarcorhamphus). III Marsupial (Halmaturus). IV Pig. They are all seen from behind. $i$ Vena cava inferior. $s$ Vena cava superior sinistra. $d$ Vena cava superior dextra. ap Pulmonary artery. a Aorta. sv Sinus venosus.

of the auricle (Fig. 336, I, sv). The vertebral veins in Birds pass along a canal which is enclosed by the ribs, by which point they are seen not to be the same vessels as the cardinal veins. 


\section{$\S 439$.}

The embryonic venous system of the Mammalia is completely similar to that of the lower Vertebrata. 'Two jugular veins (Fig. 334) receive the cardinal veins, and the common trunks on either side pass into a venous sinus, which is connected with the auricle, and, later on, forms a part of the right one. Two distinct venous trunks then open into this auricle, each of which is continued into an anterior and larger, and a posterior and smaller, trunk. When the anterior extremities are developed, the subclavian veins $(s)$ fall into the anterior ones (Fig. 337, A), and the two venous trunks thus formed are distinguished as the superior venæ cavæ.

When the system of the inferior venæ cavæ is developed, the area of the cardinal veins is diminished, for part of the blood which was col-

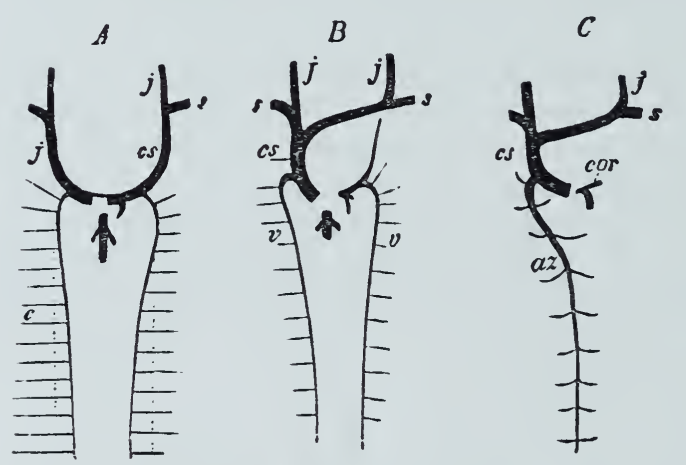

Fig. 337. Diagram of the primitive paired reins in Mammals. A The rertebral have taken the place of part of the cardinal reins, which are indicated by dotted lines. $B$ The left jugular has its lower portion atrophied, and its area is united with that of the right jugular by a transverse trunk. $C$ The left jugular vein has completely disappeared, with the exception of a rudiment on the heart. j Jugular. $s$ Subclavian. cs Vena cava superior. $c$ Cardinal vein. $v$ Vertebral vein. cor Coronary vein. az Vena azygos. lected by the cardinal veins is now carried to the inferior venæe caræ. The cardinal veins also undergo degeneration, owing to some of their roots passing into the new longitudinal trunks, which, as in the Reptilia, represent the vertebral veins, and are continued into the end of the cardinal veins, which open into the ductus Cuvieri. Owing to the decrease in the size of their area, these vertebral veins (Fig. 337, $A B, v$ ) appear to be branches of the trunks derived from the ductus Cuvieri and the jugular veins, or superior venæ cavæ. These are found in the Monotremata, Marsupialia, many Rodentia, and Insectivora. In others, part of the area of the left superior cava $(D)$ is handed over to the right one $(c s)$, owing to the development of a transverse anastomosis; the left superior vena cava is now atrophied (Rodentia, Ruminantia, Solidungula). When this arrangement is complete, the greater part of the trunk of this vein disappears, and the only part that remains is the terminal portion, which primitively formed the left ductus Cuvieri, and which is placed between the left ventricle and auricle $(C, c o r)$; the cardiac veins open into it, and it forms the sinus of the coronary vein of the heart. In Man, even, a semilunar fold separates this sinus from the true 
coronary vein, while the valvula Thebesii, which is placed at its opening into the right auricle, forms, for a long time, the valve of the left superior vena cava. The right superior cava is now the sole anterior trunk (Cetacea, Carnivora, Primates).

When the trunk of the left superior vena cava is reduced, the cardinal veins, or the vertebral veins developed in their area, undergo great changes. While in the first case they open into the vena cava of their own side $(A)$, and while in the second they pass separately into the left side of the right auricle, owing to the development of a right vena cava $(B)$; when the vascular passage which leads directly to the heart is reduced, they become connected with the right vertebral vein. The left vertebral vein is connected with the right one by transverse anastomoses, and when the connection between its upper end and the left superior cava is broken, it is converted into a vena hemiazygos, while the right one, which still retains its primitive position, becomes the vena azygos (Fig. 339). When the two superior cavæ persist, the two vertebral veins are not, in all cases, unchanged; one trunk often becomes much larger than the other, which may even be so far reduced as to disappear altogether. In this case a vena azygos receives the intercostal veins of both sides; and this sometimes opens into the left, and sometimes into the right superior vena cava, or even into the only one that is present, as, for example, in the Carnivora (Fig. 337, $C$, az).

In most Mammals the roots of the jugulars are formed of a number of veins from the external and internal cephalic regions, one of which conducts a part of the blood from the cranial cavity through the jugular foramen. It forms a small vessel only, for the greater part of this blood passes out in a canal (canalis temporalis), which is either placed between the petrosal and squamosal, or in the latter only. When the foramen jugulare is enlarged, the vein, which in other cases is a small one, increases in size, and gradually becomes the most important of all the vessels which come from the skull; in this case it forms, as it does in the Primates, the internal jugular vein. The other veins gradually unite to form the external jugular, which is the most important one in most Mammals.

\section{$\S 440$.}

The second large venous tract is very small in Fishes, for in them it is merely represented by the hepatic veins, which are united into one or more trunks, and open into the common venous sinus. When the tract of the cardinal veins is diminished in extent, a new tract is formed in connection with the hepatic veins-that of the inferior vena cava (Amphibia). This venous trunk collects blood from the kidneys, and is, therefore, the vena renalis revehens (Fig. 338, $A, c i$ ). The blood from the hinder extremities passes into an iliac vein $(A, i)$, which, in the Urodela, receives on either side a branch of the divided caudal vein. It breaks up in the liver, and forms a vena renalis advehens. A branch of the iliac vein passes to the middle 
line of the abdomen, and receives veins from the so-called urinary bladder $(A, o)$, after which it is united with its fellow of the opposite side to form a single trunk which passes to the liver, and which is therefore connected with the portal system $(a)$; this is the epigastric (abdominal) vein. The veins of the digestive canal and of the spleen are united into a portal trunk, which breaks up in the liver.

In the Reptilia also the hepatic and the efferent renal veins form an inferior vena cava $(B, c i)$, which opens into the common venous sinus below the right superior cava. But there are various modifications in the different divisions of the Reptilia, and it is in the Saurii and Ophidii only that there is any close similarity to the arrangement of the venous system which obtains in the Amphibia. The caudal vein divides into two trunks, which receive the veins from the hinder extremities in the Saurii, and form the venæe renales

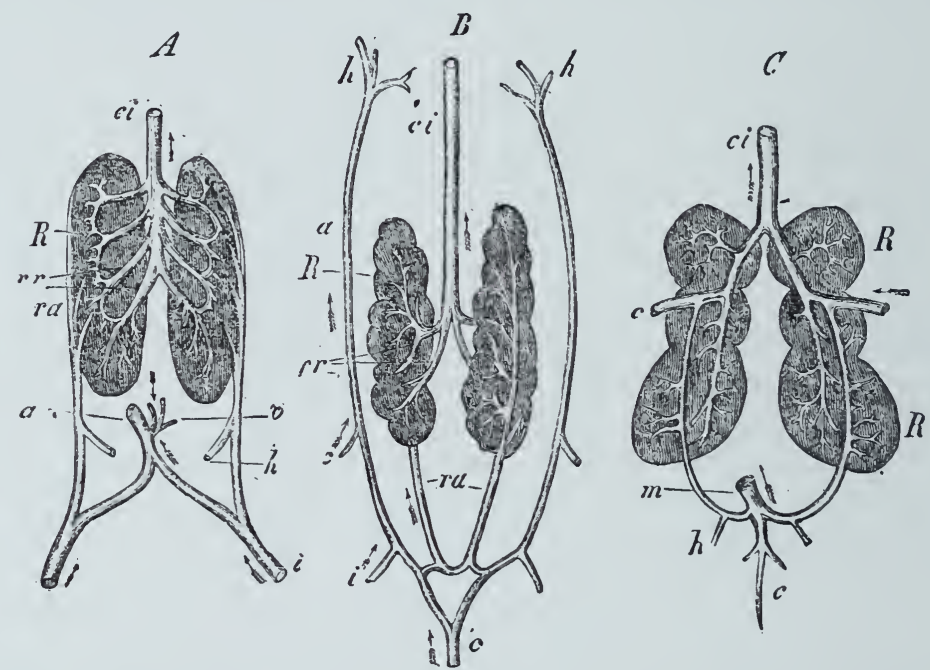

Fig. 338. Posterior portion of the venous system. A Of the Frog. $B$ Alligator. $C$ Bird. $R$ Kidneys. $c$ (azygos trunk) Caudal vein. $c$ Crural. $i$ Ischiac vein. $v$ Venæ vesicales. a Epigastric (abdominal) vein. $m$ Vena coccygeo-mesenterica. $r a$ Vena renalis advehens. $r v$ Vena renalis revehens. $c i$ Vena cava inferior. $h$ (in $A$ and $C$ ) Vena hypogastrica, (in $B$ ) End of the epigastric vein in the liver.

advehentes. The veins of the vertebral column are connected with these. Similar arrangements obtain in the Crocodilini, where the caudal vein $(B, c)$ is also divided, but this vessel then forms a transverse trunk which gives off the venæ renales advehentes $(r a)$. In all these forms the venæ renales revehentes form a trunk which runs in front of the vertebral column, and there is a renal portal system in the kidneys ; this appears to be absent in the Chelonii only.

Another venous tract in the Reptilia is represented by the ven epigastricæ sive abdominales. When the allantois is developed, a pair of veins is developed from the vascular network that accompanies it, and this primitively opens at the same point as the 
ends of the ductus Cuvieri (Rathke: Ring Snake). These umbilical veins receive veins from the abdominal wall, and are also connected with the formation of the hepatic portal circulation. In the Ophidii this umbilical vein disappears after the veins of the abdominal wall which open into it have broken up into a plexus, but in the Saurii the terminal portion of one umbilical vein persists, and unites with the abdominal veins that open into it to form an epigastric vein; this receives veins from the urinary bladder, and passes forwards to the liver.

In the Crocodilini and Chelonii the ends of the umbilical venous trunks persist, and form, as they are continued into the veins of the abdominal wall, a part of the epigastric veins. Like the single veins in the Amphibia and Saurii they also go to the liver, and, in the Crocodilini, they are connected with branches of the portal vein. In the Chelonii, those of either side unite into a transverse trunk, which receives the various venæ intestinales, which are not, in them, united into a portal venous trunk. In both cases they are distributed in the liver, and belong therefore to the portal venous system. In the Crocodilini, as in the Chelonii, the epigastric veins $(B, a)$ are given off from the two branches of the caudal vein (c), and receive the crurals $(c)$; they also receive the ischiac veins more anteriorly. But, since in the Crocodilini, the venæ renales advehentes also arise from the caudal vein and its connection with the ischiac vein, part of the venous blood from the hinder portion of the body is carried into the renal portal circulation, and the rest into that of the liver. But in the Chelonii, where there are no advehent renal veins, all the blood from the hinder end of the body is carried into the liver, for the vertebral veins, in these forms, also open into the epigastric veins.

\section{$\S 441$.}

Several of the veins which are found in the Reptilia are not permanent structures in Birds. The inferior vena cava (Fig. 338, $C, c i$ ) is indeed still made up of two trunks from the kidneys, but these receive the veins of the hind-limbs $(c)$, and might from their size be taken to be the continuation of these veins. Two hypogastric veins $(h)$ are connected with these trunks in addition to the vessels which arise from the kidneys. They are united by a transverse anastomosis at the root of the sacrum; this anastomosis receives the caudal veins $(c)$ from behind, and gives off in front a vena coccygeo-mesenterica $(m)$, which goes to the mesenteric vein. This vena coccygeo-mesenterica is a wide trunk in the Crocodilini also, where it anastomoses with the transverse trunk, which unites the two branches of the caudal vein; part of the venous blood from the tail or hinder extremities is conducted away from the renal portal circulation by it.

In the Mammalia there are no indications whatever of a renal portal system. The umbilical and omphalo-mesenteric veins have the same relations as in Reptiles, though there are several variations 
in some, even of the larger, trunks. The inferior vena cava (Fig. $339, c i)$, which collects the blood from the kidneys and generative glands, is developed very early; it accompanies the united umbilical veins, and, when the right one disappears, it receives the left. After the cardinal veins $(c)$ disappear, the veins of the pelvis $(h y)$ are connected with the end of the trunk of the vena cava, as are also the veins of the hinder extremities (il), and the caudals. At the time when the umbilical is the largest of the veins, the inferior cava appears to be merely a branch of it. Where the umbilical vein

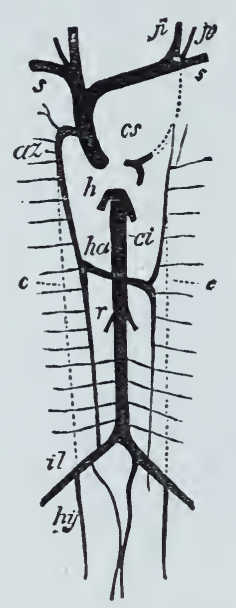

Fig. 339. Diagram of the chief trunks of the venous system of Man. cs Vena cava superior. $s$ Vena sub. clavia. je Jugularis ex. terna. ji Jugularis in. terna. $a z$ Vena azygos. ha Vena hemiazygos. $c$ Indication of the car. dinal veins. ci Vena cava inf. $h$ Venæ hepa. ticæ. $r$ Venæ renales. il Vena iliaca. hy Vena hypogastrica. enters the liver, the hepatic vessels are formed, while at the same time similar branches from the liver pass to the point where the umbilical unites with the vena cava inferior; these form the hepatic veins. As the blood which is returned to the heart from the umbilical veins passes through the liver, that portion of them which lies between the afferent and efferent veins is atrophied, and forms the ductus venosus Arantii. That portion of the omphalomesenteric vein which receives the mesenteric veins is then converted into the trunk of the portal vein, while the hepatic branches of the umbilical form the branches of the portal vein after the obliteration of the ductus Arantii. The inferior vena cava is thus converted into the chief hinder trunk, into which open the veins of the pelvis, of the hinder extremities, of the renal and generative organs, while the veins of the digestive canal and spleen form the portal vein.

\section{$\S 442$.}

The blood-vessels are ordinarily distributed in the body by the gradual branching. of the different trunks, until at last the finest branches of the arteries and veins give rise to the capillary system, which connects the two kinds of blood-vessels with one another. To say nothing of the various special arrangements in certain organs, a somewhat different method of distribution obtains in the bloodvascular apparatus of several regions of the body. A vein or artery suddenly breaks up into a tuft of fine branches, which either do, or do not anastomose, and which lose themselves in the capillary system, or are again soon collected into one trunk. This distribution of the vessels has been long known as a rete mirabile. Its function is clearly to slacken the blood-current, and to increase the surface of the walls of the vessels, so that there must be a change in the amount of nutrient fluid diffused by osmosis. If from this rete a vascular 
trunk is given off similar to the one that was broken up, it is called bipolar or amphicentric; if the rete remains broken up, then it is known as a diffuse, unipolar, or monocentric rete mirabile. Sometimes arteries only, sometimes veins only (rete mirabile simplex), sometimes both kinds of vessels are united with one another (rete mirabile geminum seu conjugatum) to form the rete.

Arterial retia are found in the pseudobranchia, in the choroid of the eye of Fishes, and, in very various forms, on the air-bladder. In Birds and Mammals, retia are not unfrequently found in the area of the carotids and their branches. They are very common on the limbs of the Mammalia (Monotremata, Edentata). In the area of the visceral arteries there are both arterial and venous retia; thus, in the Pig, the mesenteric artery forms an arterial rete. Arterial retia are very common on the terminal branches of the renal arteries, where they form the Malpighian glomeruli, from which, as we all know, another artery is given off to be distributed in the capillaries on the urinary tubules (cf. Fig. 343, B).

\section{Lymphatic System.}

\section{$\S 443$.}

The presence of a system of canals connected with the bloodvascular system-in which the nutrient fluid, which has passed out from the capillary portion of the hæmal system, is conveyed again to the blood stream as lymph, after having filtered through the tissues-is an arrangement which is peculiar to the organisation of the Craniota. It appears to be correlated with a high development of the body, for it is wanting in Amphioxus, and in embryological development it only begins to appear at a relatively late period, and not until the blood-vascular system has been differentiated into its arterial and venous portion, and is in full function. That portion of the lymphatic system which has its root on the digestive canal is of especial importance, for it receives the chyle, or nutrient material, which has been prepared from the chyme by the process of digestion, and conveys it to the blood-vessels.

In addition to the function of conveying the lymph, this system of canals has yet another duty, which complicates its anatomical relations. The points at which the form-elements of the lymphatic fluid, or lymph-cells, are developed, are embedded in its vessels; these lymph-cells are carried to the blood, and are gradually converted into its form-elements.

In the lower divisions of the Vertebrata this lymphatic system has not much independence, for its vessels are chiefly formed of wide spaces, which enclose other organs, and especially arteries. The sheath of connective tissue of the artery also encloses the lymphatic vessel. The veins also may be surrounded by wide lymph-spaces; 
thus, for example, the abdominal vein of the Salamander is enclosed in a lymphatic vessel.

There are, however, other vessels in the lower divisions besides those which accompany the blood-vessels - in the skin, or on

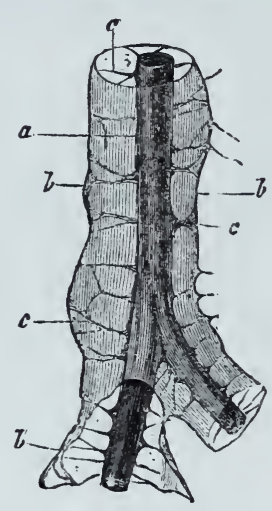

Fig. 340. Portion of the Aorta of a Chelonian (Chelydia) surrounded by a lymphatic space, $a$ Aorta. $b$ Outer wall of the lymph space, which is removed at $b^{\prime}$ so as to expose the blood. vessel. $c$ Trabeculæ. portions even of the digestive canal, or other viscera. Peripherally, the lymphatic vessels anastomose largely, and form capillary networks or similar spaces. These gradually give off wider spaces, either in the form of canals, or of sinuses with irregular boundaries, the place of which is taken, in the higher Vertebrata, by vessels allied in structure to veins.

Although we may note that, as we pass from the lower to the higher Vertebrata, there is a gradual differentiation from spaces, similar to those of the lacunar system of the Invertebrata, to a definitely developed canalicular system, so that the interstitial nature of the lymphatic ducts is well marked at the periphery only; nevertheless, in the colom we have an arrangement which indicates the origin of the lymphatic vessels from a lower condition-for the cœlom is a lymphatic cavity. In this point the cœlom of the Vertebrata resembles closely that of the Invertebrata. The communications between the cœlom and the pericardial cavity, which obtain in various Fishes (Sturio, Selachii), are indications of the same thing; as are also the pleural cavities of the Mammalia, which are merely differentiations of the general cœlom.

\section{$\S 444$.}

In Fishes the chief trunks have the form of $1 \mathrm{ymph}-\sin u s e s$. There are generally two pairs of them, or one unpaired one is placed below the vertebral column. The unpaired trunk divides into two branches. Smaller simuses, and narrower canals, form the lymphatic vessels which are collected into these trunks. They are generally connected with the venous system at two points. A cranial lymphatic sinus opens into the jugular vein of its own side, and two sinuses, which receive lateral trunks, are connected by a transverse anastomosis with the caudal vein near the last caudal vertebra.

The subvertebral lymphatic cavity of the Amphibia forms a portion of the system which is about the same size as a very large subcutaneous series of lymphatic cavities, which are present in these animals; in the anourous Amphibia especially, this latter series extends over a large portion of the surface of the body. The lymphatic vessels of the digestive canal (chyle-ressels) open into it, as do those of the other viscera, while it is connected also with the lymphatics of the 
extremities. In the Reptilia the subcutaneous lymphatic cavities are more varied and numerous, and the system is more intimately related to the arteries; the lymphatic vessels are sometimes wide spaces (Fig. 340), which surround the arteries and are traversed by trabeculæ; sometimes they form plexuses which accompany these vessels. When their trabeculæ are more largely developed, the lymphatic cavity is broken up into several anastomosing canals. The space which surrounds the aorta is broken up, in the Crocodilini and Chelonii, into two trunks which surround the veins of the anterior extremity; and lymphatic vessels from the head, neck, and extremities open into them. The lymphatic trunks of Birds have the same characters, but, in them, both the large trunk in front of the aorta (thoracic duct), and the small vessels are more independent. As in the Reptilia, the thoracic duct opens into the superior venæ cavæ (venæ brachiocephalicæ). At the commencement of the tail the lymphatic system is also connected with the ischiac veins, or with the afferent renals, in which point they resemble the Amphibia and Reptilia.

In the Mammalia the walls of the lymphatic system are still more differentiated, although it often happens that in them also the sheath of the arteries bounds the course of part of the lymphatic current. Where they do not accompany the blood-vessels they form frequent anastomoses, or wide-meshed plexuses, and are distinguished by valves, as are the same parts in Birds. The lymphatic vessels of the hinder extremities, as well as the chyle-ducts, unite into a chief trunk in the abdomen, which is rarely paired, and the origin of which is frequently distinguished by a considerable enlargement (cisterna chyli). Thence they are continued into a thoracic duct, which opens into the commencement of the left brachio-cephalic vein; the trunks of the lymphatics of the anterior parts of the body (of the head and anterior extremities), and of the wall of the thorax, open into, and on either side of the same vein.

The lymphatic trunks are generally widened out near their opening into the veins, and the wall of these enlargements is distinguished by its muscular investment; it executes rhythmic contractions. These arrangements are known as lymphatic hearts. They have been occasionally observed on the caudal sinus of Fishes, but they are more accurately known in the Amphibia (Ranidæ) and Reptilia (Chelonii); in the former they are found on both the anterior and posterior openings into the veins, but in the urodelous Amphibia and in the Reptilia only the posterior lymphatic hearts have been made out. These latter are found also in the Ratitæ (Struthio, Casuarius), and some Natatores, but in other Birds they have no muscular investment, and form mere 
vesicular enlargements. Finally, in the Mammalia such structures do not seem to be developed.

\section{$\S 445$.}

The apparatuses that produce the lymph-cells are simple in Fishes, where they are placed in the course of the various lymphatics; the cells are produced in the meshes of reticular connective tissue. Where more largely developed, this arrangement gives rise to local enlargements, which accompany the arteries, in consequence of the relation between these vessels and the lymphatics. This arrangement obtains even in the higher Vertebrata, although the cells are not always developed in the sheaths of the arteries. Follicular enlargements are formed beneath the mucous membrane of the enteric canal, the lymphatics of which are connected with these cell-forming regions. They are either scattered, or variously grouped together (closed glandular follicles). At the commencement of the wall of the enteron these structures form the tonsils already mentioned; in different parts of the mucous membrane of the mid-gut they are placed closer to one another, and form the so-called Peyerian Glands, which are present in the Reptilia, but are only found to any great extent in the Mammalia.

When a number of these lymphatic follicles are united together they form larger structures, lymphatic glands, which are also placed on the course of the lymphatic vessels. In Fishes, Amphibia, and Reptilia there are not, so far as we know, any true lymphatic glands. In Birds, also, they seem to be confined to the neck; it is in the Mammalia only that they are generally present, and in them they are found in other parts of the body, as well as in the chyliferous portion of the lymphatic system of the mesentery. In some Mammals (e.g. Phoca, Canis, Delphinus) the mesenteric glands are united into a single mass, the so-called pancreas Aselli.

The Spleen is also one of the organs that form lymph-cells; in its histological structure it only differs from the lymphatic glands by the fact that the cells formed in it pass directly into the bloodvessels. Essentially it is formed of a fine lacunar system interposed between the efferent and afferent blood-vessels; this forms the greater part of the so-called parenchyma of the spleen.

The spleen is found in all Vertebrata save Amphioxus, and is always placed in the region of the stomach, and generally close to the fundus. It forms an elongated or rounded organ of a darkred colour, which is sometimes, as in various Selachii, broken up into a number of smaller lobules, some of which are, in other cases, converted into secondary splcens.

An organ which is very generally present, and which resembles the lymphatic glands in various points of structure, cannot be 
passed over, although its relations to the lymphatic system are still very uncertain; this is the thymus. This is an organ which is also made up of glandular follicles, and which is divided into larger and smaller lobes; its smallest vesicles are filled with cells. In the Selachii this organ is placed on the branchial sacs, and between them and the dorsal muscles. In the Sturgeon, and some Teleostei, the similar follicles that are found on the superior hinder boundary of the branchial cavity are regarded as the same organ. In the Amphibia the thymus is a small swelling, placed behind the angle of the lower jaw. It has the same characters in the Reptilia. In the Ophidii and Chelonii it is placed on the carotid, and above the heart. In the Crocodilini, as in Birds (Fig. 312, th), it extends from the pericardium to the lower jaw. The lower portion is the larger in the Mammalia, so that it rarely passes beyond the thoracic cavity. In all cases it is best developed in early life, after which it undergoes atrophy, and it is very rarely that it retains its earlier size in the adult stage (Pinnipedia).

In the higher divisions of the Vertebrata there is an organ which lies in front of the kidneys and on either side of the body; it is consequently called the sup ra-r enal gland, but we have no information at all as to its function. In the Anamnia these structures are replaced by the investment of the sympathetic ganglia by means of a cortical layer made up of cell-containing tubes ; these form yellowish or whitish bodies, and are scattered over a larger portion of the body, whereas in the Amniota they form a mass on either side, while nerve-elements can be made out in their medullary substance. The relatively large size of this organ during fotal life is a noteworthy point. The function of these organs cannot be regarded as at all definitely known; nor are we aided in our inquiry by classing it as one of the "blood-vascular glands" - a term which is altogether obscure, and consequently objectionable.

\section{Excretory Organs.}

\section{$\S 447$.}

The arrangements which are found subserving the purpose of excretory organs among the Invertebrata obtain also, in their most essential relations, in the Vertebrata, and are therefore indications of the affinity between the vertebrate phylum and lower forms, which in other morphological details are very remote. Organs of this kind have as yet been vainly sought for in Amphioxus; but in all the Craniota they are found to exist, and to be formed on the same type. The type has been obliterated by gradual differentiation, but it is revealed by the study of individual development. The simplest stage is represented by a canal which runs in the dorsal wall of the colom, and opens to the exterior posteriorly, and in the 
region of the anus, while anteriorly it opens into the cœlom by an internal orifice. Although it is clear that this arrangement has much in common with the excretory organs of the Vermes, yet the peculiarity must not be overlooked, that although the vertebrate body is a metameric one, this archinephric duct is not a metamerised organ; it is not therefore completely homologous with the metameric looped canals of the Annulate Vermes. It must consequently be derived from a still lower condition, that is, from one in which the organism was not divided into metameres; so that it represents, as does also the unsegmented chorda dorsalis, one of the, phylogenetically, oldest organs of the Vertebrate body.

This archinephric duct has been observed to be derived from the

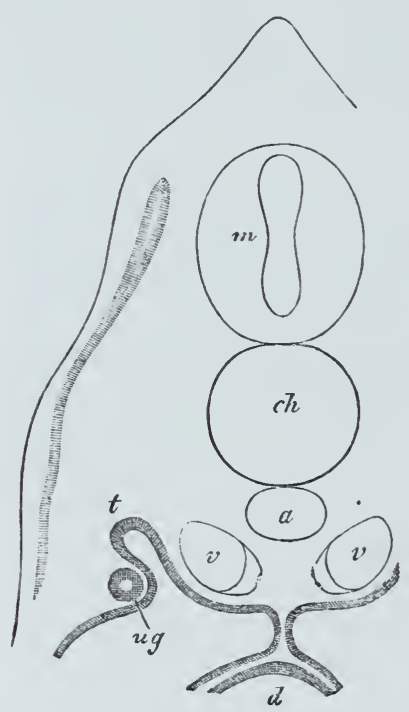

Fig. 342. Section of an Embryo of Pristiurus. ug Archinephric duct. $t$ Rudiment of a funnel-shaped organ. $d$ Enteron. m Medullary tube. ch Notochord. a Aorta. $v$ Veins. mesoderm; in its rudimentary condition it has the form of a solid chord of cells, or is differentiated as a groove from the epithelium of the peritoneal cavity (Teleostei). The rudiments of the canals (Fig. $342, t$ ) are derived from the same parts ; these, either permanently, or for a time only, open by an infundibular orifice into the abdominal cavity, while they are also connected with the archinephric duct (Selachii, Amphibia). They develop coiled glands on their course, and so form the secreting portion of the primitive kidneys. At definite points a coil of arterial vessels (glomerulus) pushes its way into a dilatation of these metamerically arranged canals, and gives rise to a Malpighian body, lying in a capsular enlargement. This last arrangement obtains in all forms of the renal organ, however much it may be modified in various members of the vertebrate group.

The fundamental form of this primitive kidney must be regarded as being a longitudinal canal, which receives transverse canaliculi, which open by ciliated infundibula into the abdominal cavity; this is the form which the rudimentary apparatus really has in the Selachii. The connection with the colom, the epithelial investment of which always gives rise to a large portion of this system, allows us to compare it with the excretory organs of many Vermes, and points to those distant forms in which these organs are the sole cavitary organs that are developed from the mesoderm (Platyhelminthes). The metameric arrangement of the open transverse canals is due to the general metamerism of the vertebrate organism. It must not, therefore, be regarded as the same as that of the looped canals of the Annelids, or even as derived from it, for those canals open to the exterior on the 
surface of the metameres themselves ( $\$ 145)$, and not into a longitudinal canal. This canal is the part which, by being the first part to appear in the Vertebrata, defines the type of the whole apparatus.

But, just as in a large number of Invertebrata the excretory organs partly lose their function, and serve as efferent ducts for the generative products, so too among the Vertebrata do we meet with a process of this kind, by which great changes are effected in the primitive excretory apparatus. It loses, and that often very early in life, its primitive arrangement. And when this is not seen in the embryo, it must be regarded as due to new, acquired, relations.

\section{$\S 448$.}

In the Cyclostomata, Teleostei, and Amphibia, a special portion of the primitive kidney appears at the most anterior end of the archinephric duct, which deserves especial mention, as it does not only appear earlier than the rest of the primitive kidney, but is generally separated by sume distance from it. This portion is made up of a small number of canaliculi, which commence by ciliated infundibula, and are generally set in a coil. There may be only one canaliculus. A Malpighian body may sometimes be observed on the canaliculi. In the Amphibia this pronephron undergoes atrophy, while in the Amniota it does not seern to be even rudimentarily developed. It persists, however, in the Cyclostomata, where it is provided with a tuft of ciliated infundibula, which project into the abdominal cavity.

Among the Cyclostomata the primitive kidney is seen at its simplest in Bdellostoma. An elongated canal (Fig. $343, A B, a)$ gives off short transverse canaliculi at various points $(b)$; the blind end of which $(c)$ is constricted off, and encloses a glomerulus $(B)$. The transverse canaliculi form the secretory apparatus (urinary canaliculi); the archi-
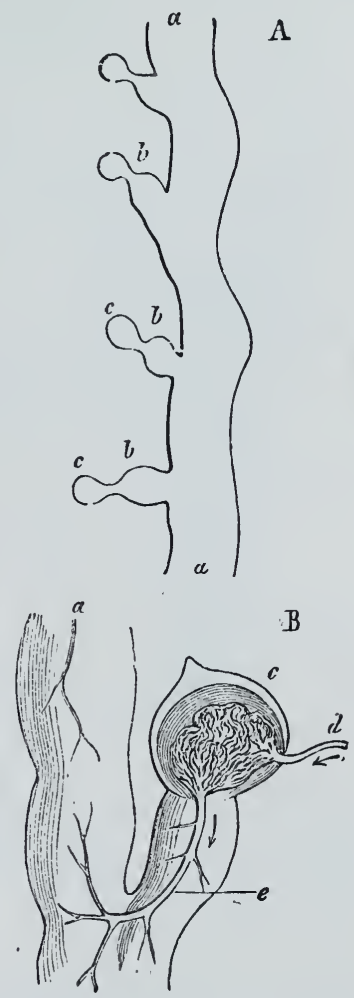

Fig. 343. A Portion of the kidney of Bdellostoma. $a$ Urinary duct. $b$ Urinary canaliculi. $c$ Terminal capsule. $B$ Portion of the same more highly magnified. ac As before. In $c$ there is a glomerulus. $d$ Afferent, $e$ Efferent artery (after J. Müller).

nephric duct is the collecting tube, and functions as the ureter. In the Myxinoidea and Petromyzontes, the kidneys, which are set along the posterior third of the cœlom, are larger, but the urinary canaliculi have exactly the same relations. In both forms the ureter takes a lateral course to the abdominal pore; but in the 
Petromyzontes it is first connected with the one on the opposite side, to form an unpaired and wider portion. We do not yet know its relation to the metameric ciliated infundibula.

In the Selachii the primitive arrangement is limited to the early stages of development. The primitive kidney extends along the dorsal wall of the cœlom, and is made up of separate canaliculi, which commence by ciliated infundibula (Fig. 344, i), which open

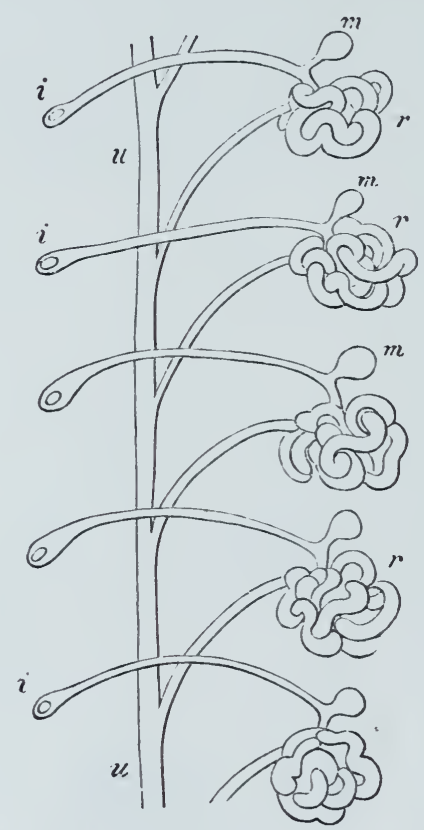

Fig. 34t. Portion of the kidney of an Embryo of Acanthias (dia. gram). $i$ Ciliated funnel. $m$ Mal. pighian body. $\because$ Renal lobules. $u$ Archinephric duct. into the abdominal cavity. Each canal, after having broken up so as to enclose a glomerulus $(m)$, is continued on into the archinephric duct. These canals increase in length, so that each of them forms a coiled lobule $(r)$; each kidney, therefore, is composed of a series of these coils, which are collected together into the archinephric duct $(u)$. This duct opens into the cloaca. Changes may occur in the glandular portion of these kidneys, as well as in their efferent ducts. The anterior portion, which is made up of a number of lobules, does not undergo any very great development, as does part of the hinder portion. This, which is made up of a varying, but large number of primitive lobules (13-14 in Acanthias), is converted into a larger organ, the canaliculi in which may be seen to increase in number by budding off new ones. This portion retains its renal function, while the anterior part is atrophied, and, in the male, enters into connection with the generative gland. The ciliated funnels (nephrostomata) are retained in some Sharks only; they disappear in all the Rays, and many of the Sharks. Where they are retained they are reduced in number.

Of the changes which obtain in the primary archinephric duct the most important is its division into two parts. This commences at its anterior end, and extends backwards, so that there come to be two canals. One commences at the anterior abdominal orifice of the primary duct, and has no further relations to the kidney. This is the Mullerian duct. The other canal retains its connection with the primitive kidney, and forms the secondary archinephric duct. But even this portion may undergo certain changes, inasmuch as in the male it is converted into the seminal duct. The efferent ducts from the posterior portion of the kidney are then collected into a common ureter, which opens into the sinus urogenitalis, into 
which, however, several ureters may open separately. In the females, also, the efferent ducts from the anterior and aborted portion of the primitive kidney are connected with the ureter.

In the Ganoïdei and Teleostei the kidneys have the same position. The primitive kidney appears to be considerably increased in size, while the efferent ducts are not so completely differentiated as in the Selachii, where they were the cause of much complication ; in the Ganoïdei, however, the presence of a nephrostome, with a wide abdominal orifice on the efferent duct, speaks to the commencement of the process by which the Mullerian duct is differentiated; the ureter, therefore, no longer corresponds to the archinephric duct.

In the Teleostei the secondary portion of the gland first appears on the anterior division of the archinephric duct, and forms that portion which, in many, extends as far as the head (head-kidney). The hinder portion, which is developed later, becomes connected with this. The whole forms a compact glandular organ, which is covered by the peritoneum, and extends along the vertebral column; it varies in size in different regions. Its differentiation into lobes is generally implied by the greater development of certain regions. The efferent ducts (Fig. 345, $u$ ) either pass along the anterior surface, or more to the sides; they generally unite into an unpaired portion, which opens behind, or below the generative orifice. The ducts are widened at different points, either in the common, or in the separate portions; these structures function as " urinary bladders," but they have no morphological connection with the urinary bladder of the higher Vertebrata.

The renal organs of the Amphibia have many points in common with those of the

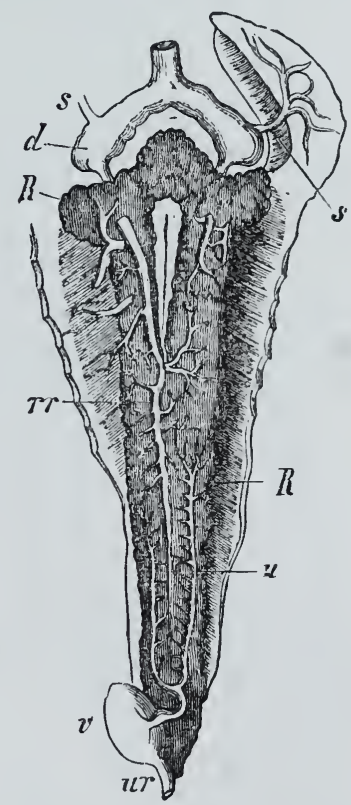

Fig. 345. Kidneys of Salmo fario. $R$ Kidneys. $u$ Ureter. $v$ Vesicular enlargement. $u r$ Its efferent duct. $r r$ Venæ renales revehentes. $d$ Ductus Cuvieri. $s$ Vena subclavia (after Hyrtl). Selachii. The rudimentary ducts are always provided with functionally active nephrostomata. The primary ureters form lobules by becoming arranged in coils. In the Cociliæ they are all of much the same size, but in the Urodela and Anura the hinder ones are increased in size and number, so that this portion becomes much larger than the anterior part. The nephrostomata, also, are greatly increased in number in this region, and are persistent. In the Urodela the anterior portion of the kidneys receives the efferent ducts of the testes, while in the Cocilizo and Anura different parts of the kidneys are connected with these organs. The primary archinephric duct is differentiated so as to give rise 
to a Mullerian, and a secondary archinephric duct (Fig. 348). The latter serves as the efferent duct of the kidney, or ureter, in the Cociliæ, Urodela, and female Anura, while in the males of many of these latter the primary archinephric duct appears to retain its original function. They open independently into the cloaca.

MIÜlleR, W., Das Urogenitalsystem der Cyclostomen. Jen. Zeitschr. IX.SEMPER, C., Das Urogenitalsystem der Plagiostomen. Arbeiten aus dem zool. Institut zu Würzburg, II.-SpexGeL, J. W., Das Urogenitalsystem der Amphibien. Ibid. III.

[BaLfour, F. M., A Monograph of the Development of the Elasmobranch Fishes. London, 1878.]

\section{$\S 449$.}

The primitive kidney is likewise developed in the Amniota. For some time in development it extends through the cœlom, and

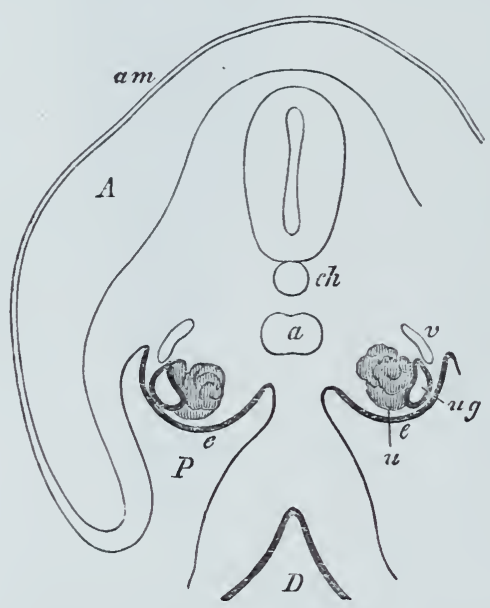

Fig. 346. Section through the embyro of a Bird (Fowl). A Amniotic carity. am Amnion. ch Notochord. a Aorta. $v$ Cardinal veins. $u$ Primitive kidney. ug Archinephric duct. e Germinal epithelium. $P$ Pleuroperitoneal cavity

$D$ Enteric groove. projects into it from the dorsal wall of this cavity. The archinephric duct is again (Fig. 346, $u$ g) the first part to be developed. The urinary canaliculi $(u)$, which form the glandular portion of the organ, open into it.

The hinder portion of the primitive kidney, which has always the same function, is well developed even in the Selachii, but still more so in some of the Amphibia; this is effected by the increase in the number of the urinary canals, and by the formation of special efferent ducts. These processes indicate prophetically the relations of these parts in the Amniota. In the Reptilia the additional portion of the urinary canals is directly connected with the hinder portion of the primitive kidney (Lacerta), but it is not connected with it to form the same, but a new organ-the permanent kidney. For a long time it is present in company with the primitive kidney, but it has its own ducts (ureters), and it takes on the function of the primitive kidney, in proportion as the latter is atrophied, or converted to the purposes of the generative system. In Birds the rudiment of the permanent kidney appears to be formed independently; and this is still more the case in the Mammalia. We see, therefore, that the so-called permanent kidney of the Amniota is at first an organ which is connected with and forms part of the primitive kidney, and that it is gradually separated from 
it both in space and time. No rudiment of the nephrostomata has been observed, nor is the archinephric duct divided as in the Anamnia; the Mullerian duct has a separate rudiment.

The kidneys of Reptiles and Birds somewhat resemble those of Fishes in their size and position. They are placed far back and close to the cloaca; in the Snakes only are they placed farther forwards, while at the same time they are longer. They vary very greatly in form, in consequence of the development of lobes. In Birds they are placed in depressions between the transverse processes of the sacral vertebræ, and are generally divided into three portions, which are sometimes connected with one another, and which may vary greatly in size. The ureters (Fig. 349, $u$ ) are generally placed on the inner edge of the kidney, and receive at various points larger urinary canals (Ophidii, Chelonii); or these canals are enclosed in the parenchyma of the kidney, and do not leave the organ except at its termination (Saurii, Crocodilini). In Birds a large part of the canal is outsido the kidney. In all cases they open separately into the cloaca, or into a sinus urogenitalis, into which the genital ducts also open.

The kidneys of the Mammalia vary in several points, and especially as to the characters of the orifice of the ureters, after the differentiation of the rudiment which is known as the "renal canal."

The kidneys, which are developed at the blind end of the " urinary canal," are, after they are differentiated, placed behind the primitive kidneys. At first they appear to have a smooth surface, which becomes uneven when the glandular parenchyma is developed into separate lobes. In either lobe the urinary canaliculi are united together at a papilliform process, with which the common efferent duct of the lobe is connected. It forms the pyramid, and a number of these unite to form the pelvis of the kidney, from which the ureter is given off. The permanently distinct lobes are very numerous (about 200) in the Cetacea. There is a smaller number in the Pinnipedia. In many Carnivora, also, the lobes are separate (Ursus, Lutra), while in others they are fused. This gives a knobbed appearance to the surface of the kidney (e.g. in Hyæna, Bos, Elephas). In others there is a condition of this kind for some time, but when the cortical substance of the lobes is completely fused, the surface of the kidney becomes smooth, although the grooves that remain indicate its primitive division into lobes. Within the organs, however, the division is more or less completely retained, and the number of primitive lobes is implied by the greater or less extent to which the papillæ are fused together. This fusion, further, may affect some, or all the lobes, so that the number of renal papillæ may be much reduced; at last, indeed, they may all unite into one (Marsupialia, Edentata, Rodentia, several Carnivora and Primates).

The ureters formed from the renal canals, after they are separated from the archinephric duct, primitively pass into that portion of the allantois which runs in the abdominal cavity of 
the embryo, and is connected with the primitive cavity of the pelvic portion of the enteron (urachus). This is gradually converted into a fusiform widened organ-the urinary bladder, while the continuation of the urachus into the umbilicus, and from thence into the umbilical chord, is obliterated. The former portion forms the ligamentum vesico-umbilicale medium. The primitive (fusiform) form of the urinary bladder is retained in some Mammals (Seals), while in others it is gradually modified, and with these modifications there are correlated differences in the way in which the ureters open. Thus in many Rodents they open high up on the posterior wall of the bladder (Fig. 354, $C, u$ ).

The other characters of the efferent ducts are common to them and the generative apparatus, with which, therefore, they will be treated.

\section{Generative Organs.}

$\S 450$.

In the Vertebrata, the reproductive organs are shared by different individuals; the separation of the sexes is the rule, although there are various exceptions to it in the class Pisces. In the higher divisions also there are various arrangements which are indications of hermaphroditism. But it seems to me that the point which is of real importance in this matter is the reproductive material, and that the characters of the efferent ducts are of no importance, for they were not primitively part of the generative apparatus.

Our knowledge of the earliest development of the male generative matter is not quite definite, but we know certainly that the female elements are derived from the epithelial layer which invests the abdominal cavity. In this there are points of likeness between the Vertebrata, and the Vermes among the Invertebrata. In Amphioxus follicular structures, covered by a layer of epithelium, and forming diverticula of it, are developed at various points in the colom, or in the cavities connected with it; these structures are the germ-glands. The ova are formed in them, between indifferent and flattened cells, which form the stroma of the organ. In this character Amphioxus is very different to the Craniota, where the germ-glands are always developed at a sharply defined and less extensive region. The epithelial investment of the abdominal cavity retains its primitive character along a tract which corresponds to the rudiment of the primitive kidney longer than it does in other regions; and this epithelial layer may be distinguished as the germinal epithelium (Fig. $346, e$ ). At the side of the mesentery in this region there is an elevation of varying length, which is formed by a thickening of the connective tissue-the genital ridge. The epithelium dips into this, and forms the rudiments of the ova. Of a group of cells which grows inwards, one 
cell becomes an egg, while the rest form a cellular layer around itthe follicular epithelium, which unites with the surrounding connective tissue to form the ovarian follicle. Each invagination of the germinal epithelium either forms a single follicle, as in the Anamnia (Selachii), or these groups of cells grow out and form the rudiments of a number of follicles, as in the Craniota.

The cells of the ovarian follicle that are set around the egg generally remain indifferent, and aid in the nutrition of the egg as well as in the formation of the yolk-sac, which surrounds it. The egg itself, and the cells of the follicle which surround it, undergo more or less considerable modifications. When the egg and the follicle increase equally in size, the follicle-cells form a simple epithelial layer, as in Fishes, Amphibia, Reptiles, and Birds. But in the Mammalia they multiply while the egg-cell remains relatively small, and for a long time they fill up by far the greater part of the follicle. As this follicle grows a cavity is gradually formed in its interior which is filled with fluid; this causes the cellular layer of the follicle to be extended around its wall (membrana granulosa), while at one point, which is somewhat thickened, it encloses the egg.

The changes which obtain in the egg-cell relate to the yolk, and they are accompanied by an increase in the size of the egg. This may be seen in the Teleostei, where the granules of the yolk often undergo great metamorphoses. The same happens to the eggs of the Amphibia. In the Selachii, Reptilia, and Aves the yolkgranules are greatly increased in number, and are specially differentiated. Owing to the number present the ripe egg is of a considerable size.

The region invested by the germinal epithelium is the point at which the male germ-glands are also developed, but it seems that this epithelium does not take any direct share in the formation of the testes. The earliest differentiation of the glandular tubes (seminal canals), which make up the testes, has not yet been observed; the view that they are formed from a portion of the primitive kidneys is beset by the difficulty of their having no relationship of any kind with these organs.

The form-elements of the sperm are developed by the differentiation of the epithelium of the seminal canals. In all Vertebrata these are movable filaments which are given off from a thicker portion of varying form-the so-called head. This head is discoidal or elliptical, as in many Mammals and Fishes, or elongated, as in the Selachii, Amphibia, and Aves. In the latter it is frequently coiled in a corkscrew fashion. The seminal filament of some Amphibia (S alamandrina and Toads) is distinguished by an undulating membrane.

\section{$\S 451$.}

The germ-glands are developed from the structures known as genital ridges. Sometimes more and sometimes less of this ridge is converted into the ovary or testis. The simplest condition is seen 
in the Cyclostomata. The ovaries of the Petromyzontes have the form of paired lamellæ, which extend along the cœlom, and are thrown into a large number of folds, in which the ora are developed. The testes are similar in character. In the Myxinoidea the germglands are unpaired, and arise from the right side of the mesentery. Both sets of generative products are passed into the cœlom, whence they reach the exterior through the abdominal pore.

The ovaries of some Teleostei have almost the same characters; thus, in the Salmonidæ the eggs are passed into the abdominal cavity, and are eracuated through the abdominal pore. The same is the case in Læmargus borealis among the Selachii, where the ovaries contain much smaller eggs, and are themselves much larger. In the rest of the Fishes there are efferent ducts in both sexes, which are largely-perhaps always-due to the differentiations which affect the primitive kidneys (cf. $\S 448$ ).

In this relation the Ganoiddei are of a low grade, for their germ-glands have no direct ducts, and their products are passed into the cœlom. In both sexes the products escape by an apparatus which is homologous with the Mullerian duct, consisting of a canal of varying length, and provided with an infundibular orifice, which is attached to the ureter (secondary archinephric duct); this takes up the generative products. This fact must be regarded as one of special importance, for we learn from it that the Mullerian duct may be turned to use in the male. The presence of this duct in both sexes leads to a correct apprehension of the real facts of the case, and renders it unnecessary to regard the presence of the rudiments of these organs in the male as due to a primitive hermaphroditism, which, cannot be shown to have obtained at the required stage of development.

Two different arrangements can be derived from that which is dominant in the Ganoidei. One is seen in the majority of the

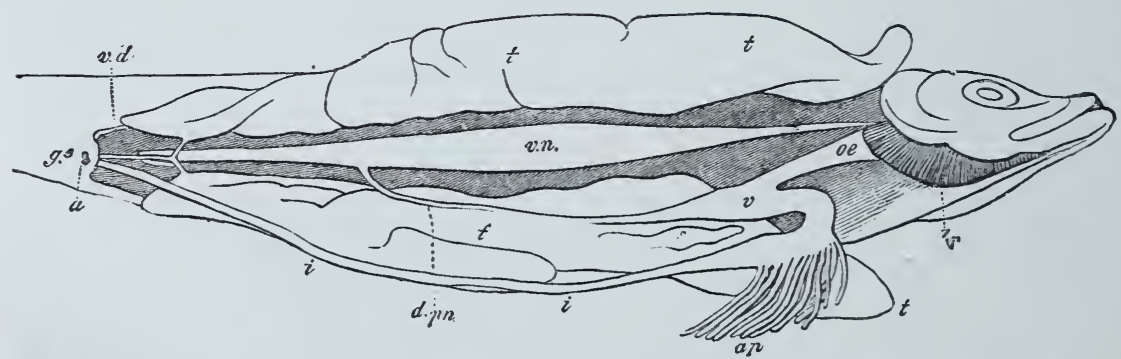

Fig. 317. Generative organs and enteric canal of Clupea harengus. oe Esophagus. Stomach. ap Appendices pyloricæ. $i$ Enteron. a Anus. $v n$ Air bladder. pn Air duct. $s$ Spleen. $t t$ Testes. $v d$ Their efferent duct. $g$ Genital pore. br Branchiæ (after Brandt).

Teleostei, and the other in the Selachii, and from thence in the Amphibia and all Amniota.

The male organs in all Teleostei and the female organs in all 
except those above mentioned, have the tubular form. The germinal region is often limited to one portion of the tube, whence it extends more or less considerably, according to the degree to which its products are devcloped. The lateral efferent ducts of these genital tubes (Fig. 347, $t$ ), are united into a common duct, which opens by the genital pore. In this arrangement the germ-gland is not ordinarily represented by the whole apparatus, but by the germinal region only, which projects on the inner wall of the tube, and is often lobed or branched. The germinal region is probably invested by the Mullerian duct, which is converted into a tubular form, but this has still to be established by embryological observation.

In a number of Teleostei hermaphrodite arrangements have been observed, a testicular as well as an ovarian tube being developed; this is best known in species of the genus Serranus.

\section{$\S 452$.}

In the Selachii the arrangement which obtains in the female Ganoïdei is retained and further developed. The germ-glands are generally developed on a certain portion only of the genital ridge, while the rest of the organ has its stroma increased in thickness, and converted into a special tissue (epigonal organ). As a rule, the ovaries are paired, and lie some way forward. In many the left one is rudimentary (Mustelus, Galeus, Scyllium, Pristiurus, Carcharias). The long oviducts, which are developed from the Mullerian ducts, unite with their fused abdominal mouths to form a wide infundibular opening; this is correlated with the great size of the eggs which it has to take up. The hinder end of each oviduct is differentiated into a portion which is distinguished by its greater width, and often by its thicker walls; this generally functions as a uterus, and opens into the cloaca. In the Selachii, as well as in the Chimæræ, a glandular portion is differentiated close to the abdominal end of the oviduct. The generative organs of these two groups, and of the Dipnoï, agree in the most essential points.

In these divisions the male organs are generally represented by small testes, the ducts of which are connected with the anterior portion of the excretory organs, so that this portion of the primitive kidney, with its efferent ducts, is adapted to the service of the generative apparatus. After several coils the vas deferens passes to the cloaca; in Chimæra it first unites with its fellow of the opposite side; it generally opens with the ureter into a sinus urogenitalis, which opens by a papilliform process into the cloaca. Part of the Mullerian duct remains connected with the infundibular ostium, at the same point as that at which it is found in the female. At the hinder end also a portion remains connected with the cloaca, in the males. The Mullerian duct in Chimæra is retained in the same way. In the males of the Selachii and Chimæræ certain parts of the posterior appendages are converted into copulatory organs (p. 487). 
The generative apparatus of the Amphibia is very similar to that of the Selachii. The ovaries (Fig. 348, $A, o v$ ) form lamellæ, which project into the abdominal cavity, and vary in size according to the number of the eggrs which are being developed. In the Urodela they enclose a cavity, which is broken up into several spaces in the Anura. The Mullerian duct forms the oviduct (od), which com-

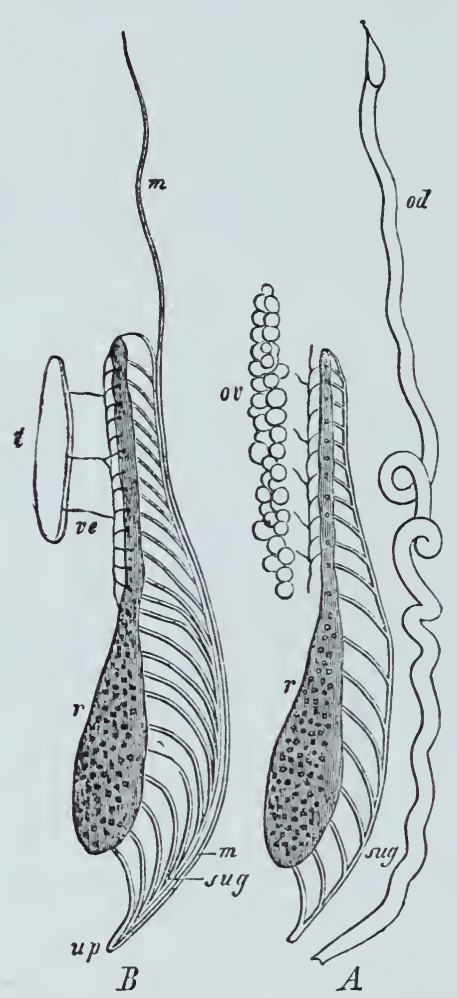

Fig. 348. Urogenital system of the $\Lambda$ mphibia (Triton). Diagrammatic. $A$ Female. $B$ Male. $r$ Kidneys; on the surface of which the nephrostomata are indicated. sug Ureter. od Oviduct. $m$ Mullerian duct. ve Efferent duct of the testes. $t$ Testes. ov Orary. $u$ Uro. genital orifice (partly after Spengel). placed between these two organs. The canals, however, which pass into the kidneys from the longitudinal collecting duct, traverse the kidneys, without being connected with the Malpighian corpuscles, and open directly into the ureter. Bufo is the sole exception; in it there is a connection between the vasa efferentia and the Malpighian corpuscles. In the Urodela the anterior portion of the kidney (genital portion) is connected with the generative organs. Transverse canals $(v e)$, are given off from a collecting duct, which is 
placed in, or on the testes $(B, t)$; these pass through the mesorchium to a longitudinal canal, from which canals are again given off, and these pass into the so-called renal portion. The sperm, therefore, passes through a certain portion only of the kidney, and only passes to the common ureter by the ducts which are given off from this portion; this ureter is developed from the secondary archinephric duct. In proportion as this portion of the primitive kidney is freed of renal secretion it is converted to the uses of the generative apparatus, so that the two secretions are not commingled except in the ureter.

In the males, the Mullerian duct remains free anteriorly, but it is generally closely connected with the secondary archinephric duct. It is either complete $(m)$, and even has a colomic ostium, or parts only are canalicular, and the rest is converted into a solid chord at various points. This is most commonly the case in the Anura, but in Bufo it is very well developed. In the Cœciliæ the hinder portion has its walls provided with welldereloped glands, in consequence of which this portion is still functional.

In many Anura (Bufo) there is a peculiar large organ containing ova-like cells on the testis; this was formerly regarded as a rudimentary ovary. We do not know what function it has, any more than we know that of the so-called fatty bodies which are found attached to the anterior end of the germinal gland of the Anura.

Since the generative organs open into the cloaca this organ functions as part of the generative system. In the female Urodela (Salamandra) the cloacal glands take up the sperm, and function as receptacula seminis. In the Cociliæ the cloaca of the male can be everted, and serves as a copulatory organ.

Semper, C., Urogenitalsystem der Selachier.-SPenget, Urogenitalsystem der Amphibien, l.c.

\section{$\S 453$.}

The generative apparatus of the Sauropsida resembles that of the Amphibia in the more important points, and has, therefore, some of their arrangements more highly developed. The ovaries are racemose organs, which are placed in front of, or at the sides of, the vertebral column, and form large organs, which vary in size according to the extent to which the eggs, which are very large in this division, are developed. In the Ophidii the ovaries are placed at different levels. The right one is the larger, and is generally placed in front of the left one. In Birds the right ovary is atrophied. In the embryo it is as large as the left one, but while the left is developed it remains at a lower stage, and may at last disappear completely. Rudiments of it are found in the diurnal Raptores.

'The oviducts are again developed from the Mullerian ducts, and when fully developed are large, and ordinarily coiled canals which 
commence by a wide abdominal mouth. The mucous membrane which invests them is set in a number of longitudinal folds; the lower portion, in addition to the greater thickness of its muscular wall, is distinguished from the other and longer portion by the larger size of its folds and villi; this is especially the case in Birds. This differentiation of the oviduct corresponds to the different function of the different parts; the longer and more anterior portion secretes the albumen, and the thick-walled terminal part forms the shell. This portion is connected by a short and narrower piece with the cloaca. In correlation with the atrophy of the right ovary the oviduct of the same side is also atrophied in Birds; not unfrequently, however, remnants of it are found near the cloaca. While the oviducts open by one orifice in the Ophidii and Saurii, as well as in Birds, in the Chelonii they open into the neck of the so-called urinary bladder; this foreshadows the relation which is the typical one among the Mammalia. In many Ophidii a diverticulum of the posterior wall of

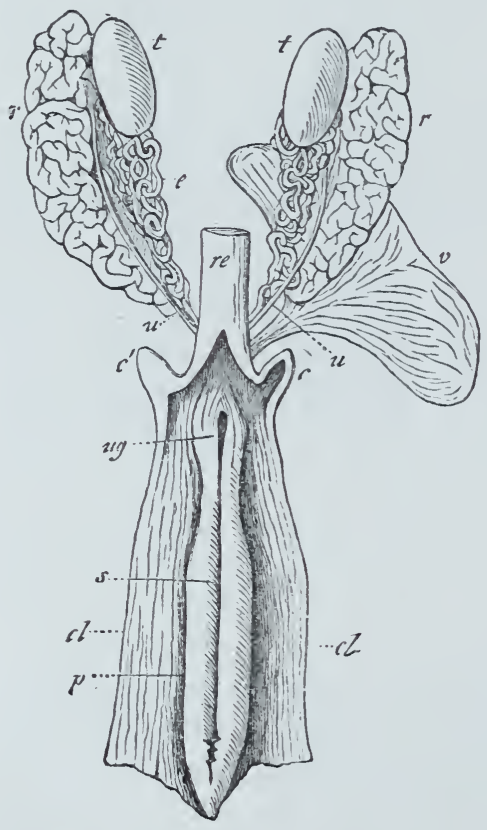

Fig. 319. Urinary and generative organs of a Chelonian (Chelydra serpentina). $v$ Kidney. $u$ Ureter. $v$ Bladder. $t$ Testes. $e$ Secondary testes and ras deferens. $u g$ Opening of the urogenital sinus into the cloaca. cl Cloaca, opened from behind. $p$ Penis. $s$ Groove of the penis. re Hind gut. $c c^{\prime}$ Cloacal cæcal sacs. the cloaca receires the openings of the oviducts. A remnant of the primitive kidney is retained behind the ovaries (this has been observed in the Saurii and Aves).

The testes, which are generally oval, are attached to the vertebral column by a fold of the mesenteries; this is either effected in front of, or between the kidneys. Their size is closely correlated with their physiological activity; this is especially the case in Birds. In the Ophidii they are arranged in the same way as the ovaries. The vasa efferentia pass to a parorchis, which generally consists of a few canals only, and from which a vas deferens extends to the cloaca. In the Crocodilini it is straight, in the Ophidii, Saurii, and Aves it is arranged in a number of smaller coils, while in the Chelonii (Fig. 349, e) it forms a complex of coils. In many Saurii and Aves, as well as in the Crocodilini, its hinder portion is widened out.

In the Saurii the vasa deferentia still unite with the ureters

to open into the cloaca; in the Chelonii they open into a sinus urogenitalis, which is formed by the neck of the urinary bladder. 
Sometimes each spermatic duct opens on a papilliform process (Saurii, Aves).

A rudiment of the Mullerian duct may sometimes be seen in the form of a filament passing forwards from the anterior end of the secondary kidney (Saurii), while further remnants of the anterior portion of the primitive kidney which are not converted into the secondary testis may be recognised.

\section{$\S 454$.}

In the Mammalia the generative apparatus undergoes great metamorphoses, owing to the further development of various portions of the efferent ducts and the formation of a number of accessory organs. In the female apparatus these are largely correlated with the relations that obtain between the embryo and the maternal organism. As this is least marked in the Monotremata, they undergo the least amount of modification, and have therefore direct relations to the lower divisions of the Vertebrata, and especially to the Sauropsida. The oviducts (Fig. 350, $t$ ) open separately into a

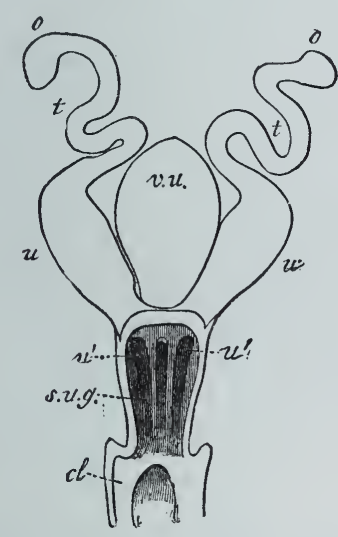

Fig. 350. Female generative organs of Ornithorhynchus. o End of the oviduct and ovary. $t$ Oviduct. $u$ Uterus. $u^{\prime}$ Point at which the orifice of the uterus projects upwards, close below the opening of the ureter. $v u$ Urinary bladder. sug Sinus urogenitalis. cl Cloaca.

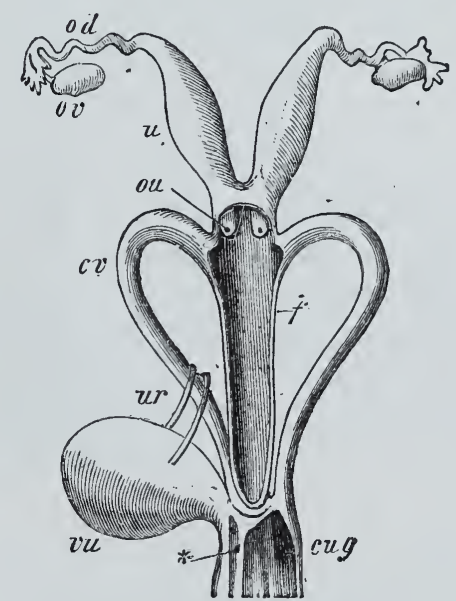

Fig. 351. Female generative organs of Halmaturus. ov Ovary. od Oviduct. $u$ Uterus. $c v$ Vaginal canals. cug Sinus urogenitalis. vu Urinary bladder. ur Ureter, * Opening of the bladder.

sinus urogenitalis, which communicates with the cloaca $(c l)$. The lower end of the oviduct, which is distinguished by the greater thickness of its muscular wall, forms a uterus $(u)$; but this merely corresponds to the structures which likewise function as a uterus in many Anamnia and Sauropsida.

In the Marsupialia the efferent ducts of the female are connected together on the outer surface, and each of them gives rise to an oviduct and uterus, as well as to a new portion, or vagina, which 
opens into the sinus urogenitalis. The upper portion, which commences by a very wide cœlomic orifice, forms an oviduct (Fig 3j1, odl), while the next and thicker-walled portion forms a uterus $(u)$. Each of the two uteri open by a papilliform process into a portion, which from the exterior appears to be common to them both, and which is formed by the union of the two Mullerian ducts. A curved vagina is given off from this on either side (Didelphys), or the commencement of the tube is replaced by a cæcal vaginal sac which is pushed out backwards, and is usually, though not always, divided internally by a median partition; from this sac the distinct "vaginal canals" $(c v)$ pass in a curved direction to the urogenital sinus (cug) (Halmaturus).

In the monodelphous Mammalia the archinephric ducts are united with the Mullerian ducts to form a common chord (genital chord). The connection between the two Mullerian ducts, which is well marked in Halmaturus, is effected in them at about the middle point of the duct, and thus they become connected during embryonic life. A portion of these ducts have their cavities fused, while they are separate in front of, and behind this point; this is an indication of the common sac, which gives off the vaginal canals in the Marsupialia. But in the Monodelphia the lumina are fused as far as the end of the genital chord, and so form a single canal (genital canal) which opens into the sinus urogenitalis. 'There are, therefore, two canals, which are separated from one another at their commencement, but which unite into an unpaired portion of varying length ; these canals are derived from the Mullerian ducts, which are separate in the early stage of the embryo. The parts, which are distinguishable even in the Marsupialia, are due to the differences in the extent to which the walls of the different parts are differentiated, and the modifications in them are essentially due to the greater or less extent of the two tubes. The uterus undergoes a number of changes, most of which are due to adaptations to its relations to the fœtus. Two completely separated
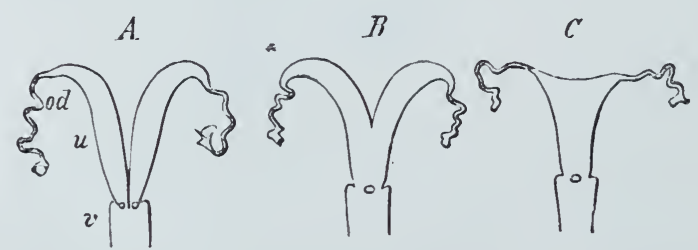

Fig. 352. Various forms of the uterus A $B$ C. $u$ Uterus. od Oviduct. $v$ Vagina. uteri open into a vagina in many Rodentia(Lepus, Sciurus, Hydrochœrus, etc.), and in Orycteropus (Fig. $352, A$ ). In other Rodentia the two uteri are only united for a short distance into a common opening into the vagina (e.g. Cavia, Cœlogenys, Mus). This leads to the arrangements seen in the uterus of the Insectivora, Carnivora, Cetacea, and Ungulata, where a single uterus is continued into two separate cornua $(B)$, which are continued into the oviducts. When the common portion of the uterus is elongated, the cornua are shortened; this is the case in the Chiroptera and Prosimiæ; in the Simiæ, as in Man, there is a single uterus $(C)$, which receives an oviduct on either side. 
The cornua of the uterus, and the common uterus itself, vary very greatly in length; so, too, does the vagina, the mucous membrane of which may be variously modified. In many Rodents (Lagostomus) a certain portion retains its original double nature. Its opening into the urogenital sinus is sometimes distinguished by a temporary fold of mucous membrane, which is known as the hymen. 'This has been observed in the Ruminantia, Carnivora, etc.; but it is in the Simiæ only that it has the same relations as in Man. The primitive Mullerian duct, which only served for the passage of the generative products, is therefore differentiated into three parts, owing to the great physiological changes that happen to it; and of these parts the first, or Fallopian tube, alone retains its primitive relations.

The ovaries, which are generally small, vary greatly according to the relation that obtains between the follicles and the stroma of the ovary. In a large number of Mammals they are racemose in form. They seldom retain their primitive position, and generally travel towards the pelvic basin, or, with their oviducts, are completely enclosed in it. They are always in close relation to the oviduct, or rather to its infundibular colomic mouth, for a process of the margin of the ostium extends to the ovary. The mesenteric folds (ligamenta uteri lata), which support the ovaries and oviducts, not unfrequently unite with the pouch that encloses the ovary to form the mouth of the oviduct (as in the Carnivora).

Remnants of the primitive kidneys and their ducts, which are enclosed with them in the genital chord, are retained at the sides of the uterus, or in the folds of the peritoneum, which connect the ovaries with the uterus. The so-called canals of Gartner are formed by remnants of the archinephric ducts, which accompany the uteri in Echidna, and open into the urogenital sinus; in other forms, portions only of these canals persist. A rudiment of the primitive kidney, which is placed near the ovaries, is known as the parovarium.

\section{$\S 455$.}

In the male generative apparatus of the Mammalia the testes have, at first, the same position as the ovaries-that is, they are placed at the inner edge of the primitive kidneys. A chord extends from the archinephric duct to the inguinal region of the abdominal wall $(n)$. The primitive kidneys are partly united with the testes, and there form the parorchids (epididymes). As in the female, the archinephric duct unites with the Mullerian duct to form a genital chord, which passes to the urogenital sinus, developed from the lowest portion of the allantois. It forms the vas deferens; the Mullerian duct is atrophied, its terminal portion only being, as a rule, converted into a permanent organ, corresponding to a sinus genitalis, the so-called uterus masculinus; this generally opens into the urogenital canal between the orifices of the seminal ducts. 
The apparatus thus formed is variously modified in different parts. The testes do not retain their primitive position anteriorly to the kidneys in any Mammals except the Monotremata. In the Cetacea, Hyrax, Elephas, and various Edentata, they are placed a little to the side of, or below the kidneys. In others they are found in

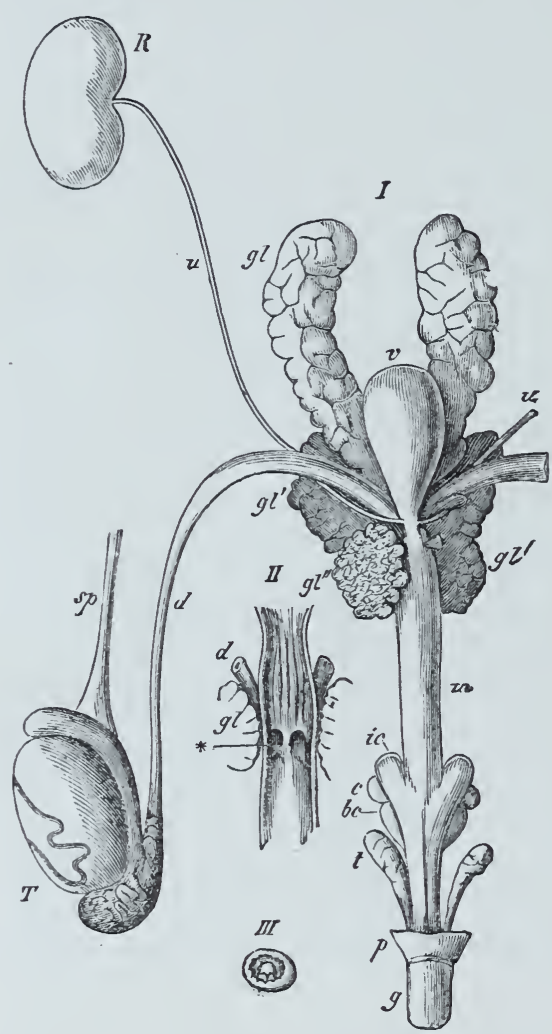

Fig. 353. I Urinary and generative organs of Cricetus $\mathrm{vulgaris.} R$ Kidneys. $u$ Ureter. $v$ Urinary bladder. $T$ Testes. $S p$ Vasa spermatica. \&l Vas deferens. $g l$ Vesiculæ seminales. $g l^{\prime} g l^{\prime \prime}$ Prostatic glands. $m$ Muscular portion of the urogenital sinus. ic Corpus cavernosum penis. bc Corp. cav. urethræ. $c$ Cowper's glands. $t$ Tyson's glands. $p$ Prepuce. $g$ Glans penis. II Neck of the bladder, and commencement of the urogenital sinus, opened in front. * Opening of the ductus ejaculatorii. III Glans penis seen from in front.

The primitive urogenital orifice. Monotremata and Marsupialia, Carnivora and Cetacea. In the rest it gives rise to glandular structures, which are known as "vesiculæ seminales," as the sperm may be collected in them $(g l)$. These organs are greatly developed in the Insectivora and many Rodents; 
in the former they are often broken up into several large lobes, while in the latter they are distinguished by their length and by the diverticula which are found on them. The terminal portion also of the vas deferens often has a glandular structure.

Besides the seminal ducts, the short terminal portion of which receives the vesiculæ seminales, and is known as the ductus ejaculatorius, rudiments of the Mullerian ducts open into the urogenital sinus in many Mammals. They either consist of a single or of a paired diverticulum, which corresponds to a rudimentary sinus genitalis of the female, or, rather, to its vaginal portion, so that it is not very exact to call it a uterus masculinus. Part of it sometimes forms a portion of the male genital sinus, for the seminal ducts open into it. These organs are largest in the Rodentia (Fig. 354, g), although, indeed, they are not altogether wanting in other forms; in Man they are represented by the prostatic vesicle.

Lastly, the urogenital canal is provided with yet another set of glandular organs, the prostatic glands. These may be of a considerable size, and form paired lobate structures (Rodentia, Elephas, Insectivora) (Fig. 353, $\left.g l^{\prime} g l^{\prime \prime}\right)$, or they are formed of a number of smaller tubes, which are connected by layers of smooth muscular fibres to a mass which is attached to the wall of the urogenital canal. By the further development of the musculature, which is found on these glands in other forms also, the prostate is converted into a circular body.

\section{$\S 456$.}

In the lower divisions the efferent ducts of the urinary and generative apparatus unite with the terminal portion of the enteric canal to open into the cavity which has already (p. 562) been called the "cloaca;" but it is doubtful whether this should be regarded as the primitive condition, for we might take the arrangement which obtains
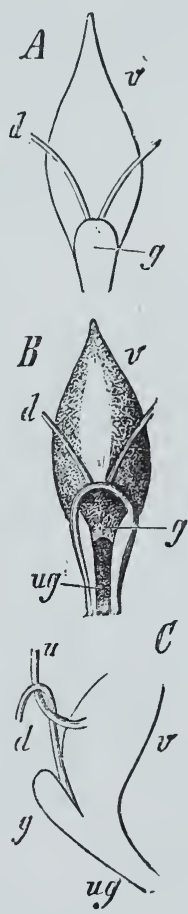

Fig. 354. Urogenital canal and urinary bladder of Lepus Cuniculus. A From behind. $B$ The posterior wall of the uterus masculinus is laid open. $C$ Side view. $v$ Bladder. $u$ Ureter. $d$ Seminal duct. $g$ Sinus genitalis. ug Urogenital canal. in the Cyclostomata, Ganoildei, and Teleostei, to be such, where the urogenital organs and the tractus intestinalis open separately. In them, the anus is in front of the urogenital orifices, although, and especially in the Ganoidei, there is a depression into which both these orifices open ; this depression is an early indication of a cloaca. The cloaca is well developed in the Selachii, and the orifices of the urogenital apparatus, which lie, in other forms, behind the anus, are there placed on the dorsal wall of the cloaca. 
This relation is henceforward the common one; in the Amphibia, Reptilia, and Aves, there is a cloaca of pretty much the same kind; in Birds it is provided with a diverticulum, the bursa Fabricii (Fig. 333, 6), which is attached to its hinder wall. The cloaca must be regarded as being inherited by all the Mammalia, although it is in the Monotremata only that it persists without any great modification; in the rest it undergoes considerable changes. The most important of these is the share which it takes in the differentiation of a copulatory organ, and which was faintly indicated in the Amphibia; these changes end by giving rise to a urogenital orifice distinct from the anus. The allantois is one of the most important of the organs which are differentiated from the cloaca; it is dereloped from the anterior wall of the cloaca, that is, from the part of the primitive cavity of the hind-gut that represents it. In Lepidosiren and in the Amphibia this organ forms a body which springs from the anterior wall of the cloaca by a short stalk; in the latter it is continued into two anteriorly placed diverticula; it lies freely in the cœlom. It is known as the urinary bladder, and seems indeed to function as such, although the ureters open some way from it. Blood-vessels are distributed on its thin wall; the arteries come from the pelvic vessels, and the veins pass to the portal vein.

In the Amniota this organ is very greatly developed during the embryonic stages, and becomes a large sac which grows out far beyond the embryo, and is provided with a large number of vessels; it envelops the embryo, already covered by the amnion. In Reptiles and Birds it gradually atrophies as the abdominal wall is closed in, and disappears altogether, In the Saurii and Chelonii only the portion of the allantois within the abdominal cavity is retained; in them it is widened out into a sac, which is provided with diverticula on either side (Fig. $349, v$ ).

In the Mammalia this organ has different relations to the developing organism. As in the Reptilia and Aves it grows out into a resicle, which communicates with the cavity of the hind-gut by a stalk which runs inside the umbilical chord. That portion of the chord, which passes into the cœlom (urachus) is partly converted into a ligament(Lig.vesico-umbilicale medium), partly into the urinary bladder, and partly iuto a sin us urogenitalis, where the orifices of the generative ducts pass into it. In the Monotremata and Marsupialia the peripheral portion appears to have the same relations as in the Sauropsida, while in other Mammals it aids in the formation of the "chorion," which is connected by villous elevations with the mucous membrane of the uterus. When these vascular villi of the chorion are further developed, the fotal blood passing along the vessels of the allantois, acquires a distribution in the peripheral regions of the sac. This effects exchanges with the blood which is distributed in the mucous membrane of the uterus. As it becomes more intimately connected with the uterine mucous membrane a placenta is dereloped; this varies greatly in character according to the way 
in which, and the extent to which, the chorion is connected with the mucous membrane of the uterus, and according to the modifications undergone by the latter organ.

\section{$\S 457$.}

The copulatory organs form another series of parts formed by the differentiation of the wall of the cloaca. In the Selachii, indeed, organs which did not belong to the generative apparatusparts of the hinder appendages-are used as organs of copulation and modified accordingly, but new organs begin to be differentiated, which in the Amphibia are faintly indicated by the presence of a papilla which projects into the cloaca. These belong to one of two typical forms; in one the organs are connected with the posterio:, and in the other with the anterior wall of the cloaca.

One of them is dominant in the Saurii and Ophidii. The copnlatory organs first appear as external appendages, placed just behind the cloaca; later on these are invaginated in a tubular fashion (Fig. 355, p), and are only protruded during copulation. When protruded, each of these organs is continued into two more or less blunt ends, which vary in form. At the sides there is a groove, which is continued on from the cloaca, and which has a spiral course posteriorly, and is then directed towards the middle line; this serves to convey the sperm. The largest of the muscles supplied to it are the retractors, which are inserted into the blind end of the tubes. Glands open near the root of the tubes $(g l)$.

The second form contains a number of different structures, all of which, however, proceed from the anterior wall of the cloaca, and must be regarded as modifications of one and the same arrangement. One form of these organs is found in most of the Ratitæ, and

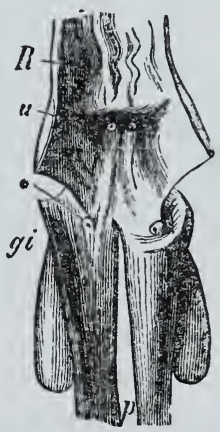

Fig. 355. Cloaca of Python, opened from in front. $R$ Hind. gut. $u$ Orifices of thie ureters. $g l$ Glands, which open at *. $p$ Penial tubes, ono of which is laid open longitudinally. in the Penelopidx and Natatores (Anser). It consists of a tube which is supported by two fibrous bodies, and which, when protruded, forms a groove which leads from the cloaca. The terminal portion of the organ is retracted by an elastic ligament.

Another form is seen in the Chelonii and Crocodilini, and in Struthio; this is distinguished. from the previous one by the fact that it is not protrusible. This organ is likewise supported by two fibrous bodies, which are intimately connected together, and covered by mucous membrane (Fig. $349, p)$. On the dorsal surface there is a groove between the two bodies $(s)$, which appear to be invested by cavernous tissue, at the root in the Crocodilini and Chelonii, and along the whole length in Struthio. As this tissue is more abundant at the anterior end of the fibrous bodies (in Struthio it is formed from the continuation forward of a third elastic body, which is 
placed below the two fibrous ones) it forms an erectile welt, which represents a penis. Special muscles, which are inserted into the fibrous bodies, act as retractors of the penis; in Struthio this organ is provided with special elevator muscles, and is hidden in a diverticulum of the cloaca.

The copulatory organs of the Mammalia also belong to the second type; those of the Monotremata differ markedly from the organs in other Mammals. Their copulatory organs consist of a short penis, which is formed of two erectile bodies, and which lies in a pouch which opens into the cloaca. By means of a muscle this can be approximated to the urogenital canals, and so takes up the sperm through an orifice which is placed at its root, near the opening of the urogenital sinus. Owing to the special mode by which a portion of the wall of the cloaca is differentiated, this organ comes to be exclusively related to the generative apparatus, while the urine passes out through the cloaca.

When the cloacal aperture is differentiated into two orifices, the copulatory organ becomes more closely related to the urogenital sinus. During the embryonic stage a fold begins to be raised up around the cloacal orifice, and a process is developed on the anterior wall of the cloaca, which carries on its posterior surface a groove which leads to the opening of the urogenital canal. As the embryo continues to grow, the cloaca becomes shallower, and the wall of partition between the orifice of the hind-gut and the urogenital canal, which is formed from the lower end of the urachus, becomes more distinct. At last the orifices which were formerly placed on the floor of the cloaca come to the surface. The anterior fissure at the base of the genital protuberance forms the opening of the urogenital sinus, while the hinder orifice forms the anus. In many Mammalia the two orifices are always close to one another, and may even be enclosed by the same fold of integument; in the female sex the two orifices are ordinarily close together. This is most markedly the case in the Marsupialia (where there is even a common sphincter for the anus and urogenital orifice) and Rodentia; in which forms, indeed, it obtains in the males also.

\section{$\S 458$.}

The urogenital sinus is developed to a different extent in the two sexes, and this is due to the difference in their functions. In the male the urogenital sinus and genital protuberance grow out into a narrower, but ordinarily long canal (the so-called urethra), with the walls of which erectile organs are connected. They form the penis. In the female there are parts which are similar to, though less largely developed than, this organ and its erectile bodies; they form the clitoris, an organ which corresponds to the penis.

The erectile organs of the Marsupialia are formed of two bodies 
which are derived from the genital protuberance and surround the urogenital canal; in some they are divided at their free end (Fig. 356, $a b$ ) and form the glans penis. The urogenital canal is continued on to each half in the form of a groove $(s)$, and these grooves may unite together to form a canal. In others (Halmaturus) these erectile bodies are connected with two others, with which they unite to form a cylindrical penis, and bound the urogenital canal. The firstmentioned erectile bodies generally fuse very early in other Mammalia to form a corpus cavernosum urethræ which surrounds the urogenital canal (urethra), and of which the most anterior end, which varies greatly in form, forms the glans penis. The two other erectile bodies (corpora cavernosa penis), which in the Marsupialia are not firmly connected with the pelvis, are connected with the ischium; they pass above the corpus cavernosum urethræ, but do not extend into the wall of the urogenital canal. In most Mammals the penis thus formed extends for-

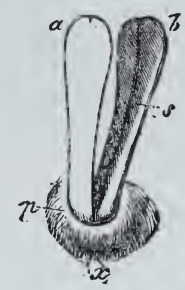

Fig. 356. Divided penis of Didelphys philander. $\quad a b$ Halves of the glans. $s$ Groove on its inner surface. $x$ Region of the anus which is placed behind the orifice of the prepuce (after Otto). wards from the symphysis pubis along the median line of the abdomen, and ends at a varying distance from the umbilicus; in others (Chiroptera, Primates), it is free and hangs down from the symphysis pubis. In either case, the integument covers it and forms a fold in front of, and around the glans - the prepuce.

In the female, the genital protuberance is never developed to the same extent as in the male; it forms the clitoris, which carries on its lower surface the opening of the urogenital sinus, which is bounded by lateral folds. The clitoris is generally more largely developed in the embryo than in the adult, as it projects from the pubic fissure and is afterwards withdrawn into it. In some Apes, however (Ateles), the clitoris continues to be developed and become an organ of some size. Two erectile bodies (corpora cavernosa urethræ) lie in the walls of the urogenital sinus and surround it as far as the clitoris, at the base of which there is another pair of erectile bodies. The end of the clitoris is generally provided with a gland, and is also covered by a prepuce. Sometimes this organ is provided with special muscles, which are mostly differentiated, as are also those of the erectile bodies, from a common occludor of the cloaca, such as is seen in the Marsupialia. In addition to these, many Mammals have muscles which raise, or retract the penis.

Glandular organs open into the urogenital sinus of both sexes. There are others besides the prostatic glands already mentioned (p. 619); there may be one or more, or as many as four pairs (Marsupialia); they lie at the root of the penis (Fig. 353, c). 
Cowper's glands are connected with the portion which is enclosed by the erectile bodies. These are not always present (Cetacea, Carnivora). In the female they open into the vestibulum vaginæe (glands of Duverney or Bartholin). The glands of the prepuce ('Tyson's glands) are developed into large organs in many Mammals, and especially in the Rodentia, among which they are best dereloped in Castor (Fig. $353, t$ ). 


\section{IN DEX.}

Acalephæ-Tentacles, 101; Ectoderm, 103; Urticating capsules, 163; Skeleton, 106; Nervous system, 108; Gastric filaments, 118; Sexual organs, 119.

Acanthias-Fin, 477; Kidneys, 603.

Acanthocephali-Integument, 136; Muscular system, 143; Muscular fibres, 144 ; Nervous system, 147 ; Enteron, 159; Lemnisci, 174; Generative organs, 176.

A canthometridæ-Skeleton, 82.

Acanthopteri-Urostyle, 431.

Acarina-Metameres, 237; Cerebral ganglia, 256; Cæca, 269; Hind-gut, 270 ; Malpighian vessels, 276 ; Germ. glands, 298.

Acera-Gauglia, 348.

Acervulinæ-Supporting organs, 81.

Acheta-Testis, 304.

Achetida-Auditory organ, 262.

Acineta-Figure, 88.

Acipenser-Dermal bones, 425; Ribs, 439; Cartilaginous cranium, 450; Dermal denticles, 450; Thoracic fin, 479; Spiracular cleft, 543; Pseudobran. chia, 543 ; Air-bladder, 547.

Acopa-Form of body, 390 ; Gemmation in, 391; Ganglia, 397; Branchial sac, 399; Branchial slits, 402; Sexual organs, 407.

Acrania-Auditory organs, 533; Respiratory cavity, 541.

Acridida-Auditory organ, 262.

Acrodont Lizards-Teeth, 557.

Acrocladia-Spines, 206.

Actæon-Excretory organ, 377.

Actinosphærium-Figure, 84.

Adder-Epigastric veins, 595.

Aginidæ-Tentacles, 102, 107 ; Marginal vesicle, 110; Gastrovascular system, 115; Generative organs, 122.

Eginopsis--Tentacles, 102.
Aolidia-Ganglia, 349 ; Liver, 362 ; Vessels, 372 ; Hermaphrodite glands, 382.

Aquoridæ-Generative organs, 122.

Aschna-Tracheal gills, 290.

Alciopidæ-Visual organs, 155; Auditory organs, 156; Excretory organs, 176.

Alcyonaria-Generative organs, 122.

Alcyonella-Nervous system, 146.

Alcyonidæ-Digestive persons, 117.

Alcyonium-Skeleton, 106; Figure of, 116.

Alepas-Mantle, 236.

Alligator-Pelvis, 485; Spinal nerres, 514.

Alveolina-Shells, 81.

Amia-Sphenoids, 452.

Ammocotes-Vertebral column, 426; Notochord, 426.

Ammonites-Shell, 333.

Ammothoë-Digestive organs, 269.

Amniota-Muscles of paired appendages, 498; Glossopharyngeal, 518; Vagus, 521 ; Nasal canal, 526.

Amoba-Figure of, 78; Supporting organs, 81 ; Contractile vesicle, 86.

Amœbidæ-Protoplasm of, 76 .

Amphibia-Anterior appendages, 414; Limbs, 415 ; Corium, 417 ; Epidermis, 418; Tegumentary glands, 420 ; Der. mal bones, 425 ; Vertebral column, 429; Vertebræ, 432; Atlas, 437; Sternum, 442 ; Dermal denticles, 450 ; Skull, 450; Jugal, 457; Branchial skeleton, 469; Branchial arches, 470; Hyoid, 471; Shoulder-girdle, 475; Fore-limb, 480; Radius, 480; Ulna, 480; Carpalia, 480; Pelvicgirdle, 484; Hind-limb, 488; Toes, 490 ; Dermal muscles, 493; Trunk muscles, 494; Intercostal muscles, 496; Muscles of the branchial skele. ton, 497; Masticatory muscles, 497 ; 
Brain, 506; Spinal nerves, 514; Brachial plexus, 514; Olfactory nerve, 515; Glossopharyngeal, 518; Vagus, 521; Goblet-shaped organs, 524; Lateral line, 524; Gustatory organs, 525; Olfactory organs, 525; Nasal canal, 526; Optic bulb; 529; Sclerotic, 529; Optic muscles, 531; Eyelids, 532; Lachrymal ducts, 532; Labyrinth, 535; Eustachian tube, 537; External ear, 538; External gills, 545; Pharynx, 546; Nasal glands, 548; Teeth, 550; Tongue, 552; Thyroid gland, 554; Stomach, 557; Mid-gut, 561; Hind-gut, 562 ; Cloaca, 562 ; Liver, 564; Mesentery, 565 ; Lungs, 569, 572; Blood-corpuscles, 576 ; Heart, 580 ; Branchial arteries, 580; Pulmonary arteries, 581; Renal arteries, 589; Venous sinuses, 591; Inferior venæ cavæ, 592 ; Lymph sinuses, 598; Lymphatic hearts, 599; Glands, 600 ; Archinephric duct, 602; Kidney, 603; Pronephridion, 603; Mullerian duct, 604, 612; Ureter, 604; Ova, 609; Sperm, 609; Generative organs, 612 ; Oviduct, 612 ; Allantois, 621 ; Urinary bladder, 621; Copulatory organs, 621.

Amphigl ena-Auditory organs, 156.

Amphinoma-Appendages, 136 ; Nerre trunks, 149.

Amphioxus-Nerve fibres, 32 ; Branchial skeleton, 142; Trunk, 413; Epidermis, 418; Notochord, 427; Vertebral column, 428; Cephalic skeleton, 442 ; Cranium, 447 ; Branchial ske. leton, 468; Muscular system, 491; Medullary tube, 502; Peripheral nervous system, 513; Eje, 527; Figure of, 539; Respiratory cavity, 540; Branchial cavity, 540; Branchiæ, 541; Hypobranchial groove, 553; Enteron, 555; Fore-gut, 556; Liver, 556 ; Hearts, 575 .

Amphipnous-Branchial lamella, 545.

Amphipoda - Branchiæ, 241; Ventral chord, 255 ; Heart, 280.

Amphistoma-Visual organ, 154; Shell gland, 183.

Amphiuma-Vertebræ, 433.

Anamnia-Epidermis, 418; Supra-renal gland, 601 .

Anatidæ-Stomach, 558 ; Syrinx, 572.

Animals-Cells in, 15; Classification of, 66 ; Phyla of, 69.

and Plants-Boundary between, 68,75 .

Anisobranchiata-Branchiæ, 337.

Annelides-Excretory organ, 46; Vascular system, 50; Parapodia, 135; Cilia, 137 ; Rod-like bodies, 140; Tubular glands 141; Muscular system, 143
Nervous system, 149; Visceral nerves, 151: Tactile setæ, 151; Visual organs, 155; Auditory organs, 156; Hindgut, 164; Cœlom, 166; Blood-vessels, 171.

Annulata-Musculature, 42 ; Nervous system, 42; Excretory organs, 42; Homology in, 64; Metamerism in, 130; Integument, 136; Setæ, 140; Esophageal ring, 148; Eyes, 154; Alimentary canal, 162; Cœlom, 165; Vascular system, 167 ; Excretory organs, 176; Spermatophores of, 191.

Anodonta-Branchiæ, 336; Muscles, 342 ; Nervous system, 346; Heart, 370; Organs of Bojanus, 370 ; Renal ducts, 376 ; Genital canal, 380.

Anomia - Foot, 321; Branchiæ, 336

Visceral ganglia, 346.

Anoplotherium-Manus, 483.

Anseres-Cæca, 562.

Ant-Salivary glands, 23.

Antedon-Pinnulæ of, 195.

Antelope-Stomach, 558.

Anthozoa-Form of, 99; Tentacles, 102 ; Skeleton, 106; Muscular system, 108; Gastric system, 110; Generative organs, 122.

Antipatharia-Generative organs, 123.

Antipathidæ-Skeleton, 106.

Anura-Ribs, 439; Maxilla, 457; Branchial arches, 471; Shoulder-girdle, 476 ; Pelvic-girdle, 484 ; Hind-limb, 488; Sixth toe, 488 ; Dermal muscles, 493; Mesencephalon, 505; Facial nerve, 517 ; Ductus endolymphaticus, 534; Columella, 538; External gills, 545 ; Horny teeth, 549; Kidneys, 604 ; Testes, 612 ; Fatty bodies, 613.

Apes-Dermal muscles, 493; Turbinate bones, 547; Buccal pouches, 549; Sublingua, 553; Clitoris, 623.

Aphides-Wax-secreting organs, 250; Salivary glands, 274 ; Pseudova, 302. Aphroditaceæ-Elytra, 135; Maxillary apparatus, 162 ; Mfid-gut, 162.

Aphrodite-Origin of nerves, 151; Alimentary canal, 162 ; Liver, 165.

Aphroditidæ-Setæ, 140.

Aplysia-Ganglia, 348; Vessels, 372 Copulatory organ, 384 .

Appendicularia-Tail, 394; Axial organ, 394; Branchial sac, 399; Sexual organs, 407.

Aptera-Gnathites, 245; Ventral chord, 258; Malpighian vessels, 276; Tracheæ, 288.

Apteryx-Diaphragm, 499; Accessorius Willisii, 522.

Apus-Shell, 235; Ventral chord, 254; Cæca, 268; Salivary glands, 274; Generative organs, 290.

Arachnida-Form of Body, 237; Appendages, 244; Spines, 249; Ventral 
ganglia, 256; Visceral nerrous system, 259 ; Eye, 265 ; Enteric canal, 269; Salivary glands, 273; Liver, 275; Malpighian vessels, 276; Circulatory system, 281 ; Tracheæ, 290 ; Germ glands, 292; Generative organs, 296; Seminal elements, 304.

Aranea-Gnathites, 244; Cæca, 269 ; Hind-gut, 270; Salivary glands, 273; Hepatic tubes, 278; Circulatory system, 281; Tracheæ, 290; Copulatory organs, 299.

Arca-Byssus gland, 329 ; Visceral ganglia, 346; Visual organs, 353.

Arcella-Supporting organs, 81; Tests, 83 ; Contractile vesicles, 86.

Archegosauria-Dermal bones, 425 .

Ardea-Enteric canal, 565.

Arenicola-Appendages, 136; Auditory organs, 156; Fore-gut, 163; Hind. gut, 163; Vascular system, 169; Central organs of blood-vascular system, 169.

Argiope-Figure of larva of, 307.

Argonauta - Hectocotylised arm, 327; Shell, 335 ; Liver, 366.

Argulus-Cæca, 268.

Argyroneta-Tracheæ, 290.

Aricia-Dermal glands, 141 .

Artemia-Eye, 265 ; Heart, 280.

Arthropoda-Connective tissue, 24 ; Tests, 38; Muscles, 40; Nervous system, 42 ; Vascular system, 50; Homodynamy in, 64; General review, 228; Classification, 229 ; Bibliography, 232; Appendages, 237; Integument, 248; Muscular system, 252 ; Cephalic ganglion, 252 ; Ventral ganglionic chain, 252; Esophageal ring, 252; Peripheral nervous system, 252; Visceral nervous system, 259 ; Visual organs, 263; Alimentary canal, 267; Salivary glands, 273; Cœlom of, 278; Fat bodies, 278; Vascular system, 279; Dorsal trunk, 279 ; Venous ostia, 279; Pericardial sinus, 279; Blood-fluid, 280; Generative organs, 291; Germ glands, 292.

Arthrostraca-Auditory vesicles, 261; Salivary glands, 274.

Artiodactyla-Dorso-lumbar vertebræ, 437 ; Fore-limb, 483 ; Pelvis, 486.

Ascalabotæ-Eyelid, 532.

Ascaris-Muscular fibres, 144; Female organs, 184.

Ascidiæ-Form of body, 390; Muscular system, 395; Median dorsal nervechord, 396; Peripheral nerves, 397 ; Branchial sac, 400; Heart, 401; Languets, 401; Branchial slits of, 402 ; Ventral groove, 403; Enteron, 404; Sexual organs, 407.

Ascones-Gastrula of, 93.

Asellus-Renal concretions, 278.
Asiphonia-Respiratory cavity, 319.

Aspidisca-Carapace, 83.

Aspidogaster-Pharynx, 158.

Astacus-Respiration in, 269.

Asteracanthion-Arms, 195, 199; Am. bulacral feet, 195.

Asterida-Nervous system, 208; Visual organs, 210; Alimentary canal, 213; Colom, 217; Blood-vessels, 218; Generative organs, 226.

Asteriscus-Arms, 195, 199 ; Alimentary canal, 213.

Asteroidæ-Rays of, 195; Ambulacral plates, 202; Paxillæ, 203; Dermal skeleton, 205; Internal skeleton, 205; Spines, 206; Pedicellariæ, 206; Muscular system, 207 ; Nervous sys. tem, 208; Interradial crea, 215 ; Stone-canal, 221; Generative organs, 224.

Astræidæ-Skeleton, 106.

Astropecten-Alimentary canal, 213; Interradial cæca, 215; Stone-canal, 221.

Ateles-Clitoris of, 623 .

Atlanta-Shell, 323, 332; Foot, 324; Excretory organ, 378; Receptaculum seminis, 385.

Aulacostomum-Mid-gut, 162.

Aurelia-Disc of, 24; Figure of, 98; Discophora form, 99; Muscular system, 108; Gastrovascular system, 115.

Aves-Corium, 417; Scales, 418; Claws, 419; Feathers, 419; Vertebral column, 429; Centra of vertebræ, 433; Sacrum, 434; Caudal vertebræ, 434; Cervical vertebræ, 434; Axis, 437; Odontoid process, 437; Ribs, 440; Covering bones of skull, 459; Lachrymal, 459; Palato-quadrate, 459; Pterygoids, 461; Palatines, 461; Premaxillæ, 462; Dentaries, 462; Shoulder-girdle, 476; Clavicles, 477; Phalanges, 471 ; Pelvis, 486 ; Tarsus, 488; Brain, 505 ; Spinal chord, 512 ; Olfactory nerve, 515; Gustatory organs, 525; Olfactory organs, 525; Sclerotic, 530; Eyelids, 532; Lagena, 536; Tympanic cavity, 537 ; Colu. mella, 538; External ear, 538 ; Cloaca, 562, 620; Pancreatic ducts, 565; Larynx, 570; Lower larynx, 571; Epiglottis, 571; Ova, 609; Sperm, 609 ; Bursa Fabricii, 620.

Avicula-Byssus gland, 329 ; Branchiæ, 336.

Balanida-Mantle, 236; Branchiæ, 243 ; Dermal skeleton, 249; Cement glands, 286 ; Hermaphroditism in, 293.

Balanoglossus-Enteric branchiæ, 163; Vascular system, 170; Ventral groove, 402. 
Bdellostoma-Respiratory organs, 542 ; Branchial arteries, 579 ; Kidney, 603. Bees-Stomach, 262; Parthenogenesis in, 302.

Belemnites-Shell, 334; Phragmocone, 334; Pro-ostracum, 334.

Beroe-Generative organs, 123.

Beroïdæ-Gastrovascular system, 117.

Birds-Fore-limbs, 417 ; Tegumentary glands, 420 ; Sternum, 442; Squamosal, 458; Foot, 489; Toes, 490 ; Dermal muscles, 493 ; Intercostal muscles, 496; Masticatory muscles, 497; Dura mater, 512 ; Falx cerebri, 512; Brachial plexus, 514; Sacral plexus, 514; Crural plexus, 515; Optic bulb, 529; Pecten, 531; Lens, 531; Lachrymal glands, 532 ; Lachrymal ducts, 533 ; Ductus endolympha. ticus, 534; Labyrinth, 535; Palate, 546; Turbinate bones, 547; Nasal cavities, 548; Nasal glands, 548; Buccal cavity, 549; Teeth, 551; Tongue, 552; Buccal glands, 553; Thyroid gland, 554; Stomach, 557; Crop, 557; Proventriculus, 558; Mid-gut, 561; Hind-gut, 562 ; Cæca, 562; Liver, 563 ; Lungs, 573 ; Heart, 583; Arterial arches, 583 ; Carotids, 587; Visceral arteries, 589; Vertebral veins, 591; Renal veins, 594; Inferior vena cava, 595; Arterial retia, 597; Lymph sinuses, 599; Lymphatic glands, 600; Thymus, 600 ; Renal organs, 604; Ova, 609; Oraries, 613; Oviducts, 614; Testes, 614.

Boa-Heart, 582.

Bohadschia-Cuvierian organs, 216.

Bombinator-Vertebræ, 432.

Bombyx-Nervous system, 259.

Bonellia-Cerebral mass, 148; Alimentary canal, 161; Vascular system, 170; Excretory organs, 174.

Borlasia-Blood-corpuscles, 172.

Bothryocephalus - Male organs, 181 ; Female organs, 182.

Botryllus-Colonies, 391.

Bowerbankia-Alimentary canal, 160.

Brachiata-Arms, 196.

Brachionus-Excretory organ, 174.

Brachiopoda-General review, 306; Clas. sification, 306 ; Bibliography, 307; Form of body, 307; Mantle-folds, 307; Shells, 307; Tentacles, 308; Integument, 308 ; Arms, 309 ; Gills, 309 ; Muscular system, 309; Nervous system, 310 ; Sensory organs, 310 ; Alimentary canal, 310 ; Ileoparietal band, 311; Excretory organs, 312; Generative organs, 313.

Brachycephalus-Dermal bones, 425.

Brachyura-Branchiæ,243; Ventralchord, 254 ; Heart, 281; Male organs, 295.
Bradypus-Cervical vertebræ, 436; Jugal, 466 ; Pelvis, 486 ; Cuneiform, 490.

Branchiata-Appendages, 238; Nervous system, 255; Heart, 280; Germ gland, 292.

Branchiobdella-Vascular system, 168; Excretory organs, 176; Generative organs, 189.

Branchiomma-Visual organs, 156.

Branchiopoda-Shell, 235; Gnathites, 239; Branchiæ, 242; Dermal skele. ton, 249; Cæca, 268; Liver, 274; Germ glands, 294.

Branchipus-Larva, 234; Eyes, 265 ; Germ glands, 294.

Brisinga-Rays, 196 ; Anus, 203 ; Cœlom, 216 ; Generative organs, 225.

Bryozoa-Form of body, 129; Gemmation, 132 ; Tentacles, 134, 136; Cilia, 138 ; Cells, 139; Muscular system, 143; Nervous system, 148; Tactile setæ, 152; Alimentary canal, 161; Liver, 165; Cœlom, 166 ; Generative organs, 179.

Buccinum-Siphon, 322 ; Crop, 361 ; Penis, 386.

Bufo-Branchial arches, 471; Stomach, 557 ; Testes, 612.

Bugs-Stomach, 272 ; Cement glands, 303.

Bulla-Copulatory organs, 384 .

Bullæa-Copulatory organs, 384.

Bursaria-Contractile vesicles, 86 .

Buteo-Thoracic vertebræ, 441; Sternum, 443; Hind-limb, 490; Thyroid gland, 554; Thymus, 555; Stomach, 557; Heart and vessels, 588.

Caducibranchiata-Muscles of appendages, 498; Vagus, 522; External gills, 545 ; Cephalic arteries, 586.

Calcispongiæ-Skeleton, 105; Amphidiscs, 105 : Gastric system, 112.

Calf-Prosencephalon, 508; Spinal chord 511.

Callianiridæ-Tentacular organ, 103.

Calveria-Pedicellariæ, 206.

Calycozoa-Organisation of, 99.

Calymnidæ-Tentacular organs, 103.

Calyptræa-Branchiæ, 338.

Camelidæ-Testes, 618.

Campanularia-Tentacles, 93, 101 ; Cormi of, 93 ; Tests, 104.

Campanulariæ-Buds of, 95.

Campodea-Feet, 246; Tracheæ, 288.

Canis-Pancreas Aselli, 600.

Capitella-Nervous system, 149 ; Vascular system, 170.

Caprella-Heart, 280.

Carabidæ-Ventral chord, 254; stomach, 262: Ovary, 301.

Carcharias-Spiral valve, 560; Ovaries 611.

Carchesinæ-Stalk, 83. 
Carcinus-Nervous system, 254.

Cardium-Nervous system, 345 ; Cœlom, 367.

Caridina-Male organs, 295.

Carinaria-Shell, 325, 332; Cutis, 328; Branchiæ, 338; Commissures, 348; Excretory organs, 377.

Carinatx-Fore-limbs, 417; Ploughshare bone, 435; Sternum, 443; Clavicle, 477 ; Furcula, 477 ; Syrinx, 572.

Carmarina-Generative organs, 122.

Carnivora-Nipples, 422 ; Dorso-lumbar vertebræ, 437 ; Tympanic bone, 466 ; Clavicle, 477; Symphysis pubis, 486 ; Cerebellum, 510; Tentorium cerebelli, 512; Tapetum, 530; Optic muscles, 531 ; Turbinate bones, 547 ; Cæcum, 562; Liver, 564; Venæ cavæ, 593; Renal organs, 605; Uterus, 615 ; Hymen, 616 ; Ovaries, 617 ; Vas deferens, 618; Cowper's glands, 624 .

Caryophyllæi-Segmentation of, 129.

Cassiopeia-Discophora form of, 99.

Cassis-Proboscis, 361; Salivary glands, 364.

Castor-Tyson's glands, 624 .

Casuarius-Lymphatic hearts, 599.

Cat-Prosencephalon, 508.

Catallacta-Cells of, 19.

Cataphracta-Infraorbital bones, 455 ; Thoracic fin, 479.

Caterpillar-Spines, 250; Enteron, 271; Tracher, 287.

Cavia-Uter'us, 615.

Cecidomyiæ-Parthenogenesis in, 302.

Centrophorus-Dermal denticles, 423.

Cephra-Discophora form of, 99.

Cephalopoda-Cartilage cells, 26, 27 ; Head, 324; Tentacles, 326; Suckers, 327 ; Chromatophores, 328 ; Integumentary glands, 329; Shell, 332 ; Respiratory organs, 340; Internal skeleton, 341; Muscles, 343 ; Ner. vous system, 350 ; Buccal ganglia, 351 ; Olfactory organs, 353; Eye, 354; Auditory organs, 356; Alimen. tary canal, 358; Jaws, 360 ; Radula, 360 ; Pharynx, 362 ; Salivary glands, 364; Liver, 366; Ink-bag, 367 ; Cœlom, 367 ; Heart, 373 ; Excretory organs, 379; Nidamental glands, 387; Generative arms, 387; Spermato. phores, 387.

Cerambicidæ-Ventral chord, 258.

Ceratodus-Operculum, 455; Fin, 477; Abdominal pore, 574; Conus arteriosus, 578.

Ceratophrys-Dermal bones, 425.

Cercarim-Stylets, 141.

Cereanthus-Colonies, 100; Pore, 117; Generative organs, 123.

Cervus-Sternum, 443.

Cestoda-Form of body, 129 ; Segmenta. tion, 130; Cystic form, 130; Cysticercus form, 131; Coenurus, 131; Echinococcus, 131; Cilia, 137; Aciculi, 140; Suckers, 143 ; Pigment spots, 154; Enteron, 159; Excretory organ, 172; Generative organs, 180; Genital pore, 184.

Cetacea-Nipples, 422; Vertebral column, 435 ; Ribs, 441 ; Nasals, 465 ; Pterygoids, 466; Fore-limbs, 472; Symphysis pubis, 484; Sclerotic, 530; Lachrymal glands, 537; Auditory meatus, 539; Milk teeth, 549; Buccal glands, 553 ; Stomach, 558 ; Midgut, 561 ; Venæ cavæ, 593 ; Renal organ, 605 ; Uterus, 615 ; Testes, 618; Vas deferens, 618; Cowper's gland, 624 .

Chætoderma-Ventral surface, 130 ; Nervous system, 152 ; Enteric branchiæ, 164.

Chætognathi - Muscular system, 142; Muscular fibres, 145; Lateral line, 143 ; Alimentary canal, 160 ; Generative organs, 185 .

Chætopoda - Tentacles, 133, 134; Parapodia, 134; External Branchiæ, 135 ; Cilia, 138; Dermal glands, 141; Longitudinal nerve-trunks, 149 ; Visceral nerves, 151; Tactile setæ, 152 ; Visual organs, 155 ; Muscular stomach, 162 ; Fore-gut, 163; Vascular system, 169; Excretory organ, 178; Generative organs, 189.

Chama-Respiratory cavity, 320 ; Renal ducts, 376 ; Genital canal, 381.

Chamæleon-Episternum, 444; Ilium, 484; Eyelids, 532 ; Tympanic membrane 538.

Charybdea-Marginal vesicles, 110.

Chelonii-Scales, 418; Dermal bones, 425 ; Plastron, 426 ; Processes of vertebræ, 433 ; Candal vertebræ, 434; Ribs, 439; Columella, 458; Coveringbones of skull, 459; Etbmoid, 459; Palato-quadrate, 459 ; Dentaries, 462 ; Hyoid, 472 ; Shoulder-girdle, 475; Fore-limb, 481; Toes, 490; Metencephalon, 507; Spinal chord, 511 ; Sclerotic, 530; Lachrymal glands, 532; Turbinate bones, 547; Horny teeth, 550 ; Tongue, 552 ; Stomach, 557 ; Hind-gut, 561; Liver, 564; Mesentery, 565; Larynx, 570; Lungs, 573; Abdominal pore, 574; Heart, 581; Arterial arches, 582 ; Arteria intercostales, 589 ; Renal veins, 594 ; Iymph sinuses, 599; Lymph hearts, 599; Thymus, 600; Testes, 614; Allautois, 670 ; Copulatory organs, 621.

Chelydra-Aortic arches, 583; Lymphatic system, 598 ; Generative organs, 616. Chelys-Premaxillæ, 462.

Cherreulius-Mantle, 394. 
Chiastoneura-Nervous system, 348; Commissures, 348.

Chilodon-Pharynx, 85.

Chilopoda-Tracheæ, 288.

Chimæra-Vertebral column, 428; Caudal region, 431; Vertebræ, 439; Hуоmandibular, 450; Fin, 478; Spinal chord, 510; Branchial lamella, 544; Fore-gut, 556; Mid-gut, 559; $\mathrm{Ab}$. dominal pore, 574; Conus arteriosus, 578 ; Aorta, 585 ; Oviducts, 611 ; Copulatory organ, 611.

Chimpanzee-Dermal muscles, 483.

Chirodotæ-Alimentary canal, 214; Lungs, 215 ; Excretory organs, 224.

Chiroptera-Nipples, 422 ; Dorso-lumbar vertebræ, 437; Premaxillæ, 460; Centrale, 482; Symphysis pubis, 484; Cerebellum, 510; Sublingua, 553; Uterus, 615; Testes, 618; Penis, 622.

Chiton-Spicules, 330 ; Nervous system, 344.

Chreseis-Tentacles, 326; Shell, 332 ; Excretory organs, 377.

Chrysomitra-Generative organs, 97.

Cicadæ-Organs of roice, 250; Hind. gut, 273; Malpigh hian vessels, 277.

Ciconia-Carpus, 481.

Cicindelæ-Stomach, 272.

Cidaris-Skeleton, 201, 205; Spines, 206 ; Stone-canal, 222.

Ciliata-Cilia, 77 ; Mouth, 84, Peristoma, 85 ; Pharynx, 85 ; Nucleolus, 89.

Cirratulus-Ganglia, 150.

Cirripedia-Mantle, 326; Cirri, 239 ; Branchiæ, 243 ; Dermal skeleton, 249 ; Optic nerve, 253; Ventral chord, 253; Eyes, 264; Enteron, 269; Liver, 274; Cement-glands, 285; Herma. phroditism in, 293; Seminal ele. ments, 304.

Cladocera-Shell, 235 ; Eye, 265; Heart, 280 ; Germ-glands, 294.

Cladolabes-Polian vesicle, 223.

Cladonema-Tentacles, 101.

Clarias-Branchial coil, 545.

Clepsine-Ganglia, 150; Mid.gut, 162; Vessels, 167 ; Excretory organ, 177; Generative organs, 187.

Clio-Respiratory organs, 339 ; Nerrous system, 349.

Clupea-Optic nerve, 515; Branchial coil, 545 ; Generative system, 610.

Clupeidæ-Vertebræ, 439; Labyrinth, 533. Clubiona-Tracheæ, 291.

Clypeastrida-Skeleton, 204; Mastica. tory apparatus, 206, 214; Stone. canal, 222.

Coccidæ-Pseudova, 302.

Coleoptera-Thorax, 237 ; Gnathites, 245; Elytra, 247; Wings, 248; Dermal Skeleton, 249; Spinning vessels, 250; Ventral chord, 254; Audi. tory organ, 262; Stomach, 272; Mid.gut, 272 ; Salivary glands, 273; Nervus recurrens, 259; Malpighian vessels, 277; Tracheæ, 288; Ovary, 302 ; Bursa copulatrix, 303; Testis, 304 ; Copulatory organs, 305 .

Coleps-Carapace, 83.

Collembola-Gnathites, 245 ; Ventral chord, 258; Excretory organ, 276; Tracheæ, 288.

Collidæ-Skeleton, 82.

Columbæ-Stomach, 558.

Cœciliæ-Dermal bones, 424; Vertebræ, 433 ; Eustachian tube, 537 ; Auditory ossicles, 538; Kidneys, 601; Ureter, 604; Testes, 612 ; Cloaca, 613.

Cœlenterata-Classification, 90,91 ; Mesoderm, 90; Cœnenchyma, 93; Biblio. graphy, 91; Form of, 91; Gastrula, 92 ; Hydranth, 93 ; Sensory organs, 109; Alimentary canal, 111; Ova, 124; Seminal filaments, 124.

Cœlogenys-Uterus, 615.

Comatula-Centro-dorsal, 204; Pinnulæ, 204; Nervous system, 209; Alimen. tary canal, 213; Blood-vessels, 218; Generative organs, 226.

Conchifera-Shell-gland, 331.

Conus-Proboscis, 361.

Convoluta-Auditory organ, 156.

Copelata-Form of body, 390; Mantle, 393 ; House, 393 ; Nervous system, 396; Auditory organ, 398 ; Branchial sac, 300 ; Enteron, 403 ; Heart, 404; Sexual organs, 406.

Copepoda-Appendages, 238; Gnathites, 239; Optic nerves, 253; Ventral chord, 254; Eye, 226; Cæca, 268 ; Enteron, 269; Excretory organs, 276 ; Heart, 280; Germ-glands, 293.

Corallium-Skeleton, 106; Generative organs, 123.

Cordylophora-Tentacles, 93.

Corethra_Stigmata, 289.

Coronula-Nervous system, 254; Liver, 274.

Corvina-Air-bladder, 468.

Corycaida-Ventral chord, 254; Eyes, 265 ; Germ-glands, 293.

Corynactis-Urticating capsule, 104.

Coryne-Tentacles, 93; Buds, 95.

Crabro-Stomach, 272.

Crane-Trachea, 572.

Craniota-Cranium, 427; Branchial skele. ton, 427, 445, 468; Appendicular skeleton, 428; Vertebral column, 428; Cephalic skeleton, 445; Skull, 445,447 ; Nervous system, 501; Medullary tube, 502; Peripheral nerves, 513; Glossopharyngeal, 518; Visceral nerves, 523; Respiratory cavity, 541; Branchiæ, 555; Eso. phagus, 556; Stomach, 556; Liver, 563 ; Heart, 576, 577 Lymphatic 
hearts, 576, 598; Blood-vascular system, 576; Excretory organs, 601 ; Archinephric duct, 602.

Crania-Crea, 311.

Craspedosoma-Generative organs, 299.

Crayfish-Green-gland, 286; Male organs, 295.

Crepidula-Branchiæ, 338.

Cricetus-Generative organs, 618.

Crinoida-Pinnulæ, 196; Arms, 199; Tentacles, 200 ; Dermal skeleton, 203 ; Muscular system, 207 ; Alimentary canal, 213; Cœlom, 217; Bloodvessels, 218; Stone-canal, 222 ; Excretory organs, 224.

Crocodilini-Centra of vertebræ, 433 ; Processes of vertebræ, 433; Caudal vertebræ, 434; Axis, 437; Ribs, 439; Sternum, 442 ; Episternum, 444; Covering-bones of skull, 459; Lachrymal, 459; Palato-quadrate, 459 ; Pterygoids, 461 ; Palatines, 461; Maxilla, 462; Hyoid, 472; Shoulder-girdle, 476; Carpus, 481; Phalanges, 481; Ilium, 484; Pelvis, 485 ; Tarsus, 488 ; Toes, 490 ; Cal. caneum, 490; Abdominal ribs, 496 ; Diaphragm, 499, 574; Metencephalon, 507; Optic bulb, 529; External ear, 538; Turbinate bone, 547; Nasal glands, 548; Teeth, 550; Stomach, 557; Mid-gut, 561; Liver, 564; Mesentery, 565 ; Larynx, 570; Lungs, 573; Abdominal pore, 574; Cœlom, 574; Heart, 582; Arterial arches, 582 ; Carotids, 587; Arteriæ inter. costales, 589; Renal veins, 594; Lymph sinuses, 599; Thymus, 600 ; Testes, 614; Copulatory organs, 621. Crow-Syrinx, 572.

Crustacea-Blood-corpuscles, 29 ; Dermal branchiæ, 46; Form of body, 234; Nauplius stage, 235; Head, 235 ; Cephalothorax, 235 ; Appendages, 238 ; Branchiæ, 240 ; Integu. ment, 248; Spines or setæ, 249; Muscular system, 251; Optic nerves, 253 ; Visceral nervous system, 254 ; Tactile rods, 260 ; Olfactory organs, 261; Simple eyes, 265; Compound eyes, 265; Faceted eye, 267; Alimentary canal, 267; Masticatory stomach, 268; Salivary glands, 273; Liver, 275 ; Excretory organs, 276, 285 ; Fat bodies, 278; Copulatory organs, 299 ; Seminal elements, 305 .

Cryptobranchus-Hind-limb, 488; Lungs, 573.

Cryptochiton-Spicules, 330.

Cunina.-Cartilage cells, 26 ; Gelatinous disc, 107 ; Marginal vesicles, 110.

Curculionidæ-Ventral chord, 254.

Cuvieria-Integument, 207.
Cyanea-Marginal filaments, 102.

Cyclas-Byssus gland, 329 ; Auditory organ, 356.

Cyclifera-Bony plates, 424.

Cyclomyaria-Chorda, 394; Axial organ, 394; Auditory organ, 398; Enteron, 404; Gill, 406.

Cyclops-Nauplius stage, 234; Appen. dages, 238.

Cyclostoma-Lung, 339.

Cyclostomata-Nerve fibres, 33 ; Vertebral column, 428; Vertebræ, 438; Skull, 447 ; Branchial skeleton, 468 ; Muscles, 492 ; Brain, 504; Spinal chord, 510; Optic nerves, 515 ; Glossopharyngeal, 518; Horny teeth, 542; Hypobranchial groove, 553; Fore-gut, 556; Mid-gut, 559 ; Branchial artery, 579 ; Caudal vein, 579 ; Kidney, 603 ; Cloaca, 619.

Cydippidæ-Axes, 101; Grappling lines, 103 ; Gastric system, 117.

Cymbulia-Hermaphrodite glands, 382.

Cymbulidæ-Foot, 324 .

Cynips-Stomach, 272.

Cynthia-Muscular system, 395.

Cyprinoids-Caudal region, 431; Vertebræ, 439 ; Beards, 525; Gustatory organ, 525; Auditory organ, 534; Sacculus, 534 .

Cythera-Nervous system, 345; Auditory vesicle, 357 .

Dactylethra-Tongue, 552.

Dactylogyrus-Visual organs, 154.

Daphnia-Figure of, 268; Cæca, 268 ; Respiration in, 269.

Daphnida-Branchiæ, 243.

Dasypus-Pterygoids, 466 ; Pelvis, 486 ; Dermal muscles, 493.

Dasyurus-Tympanic bone, 466.

Decapoda-Cephalothorax, 235 ; Appendages, 239; Mandibles, 239; Maxillæ, 239 ; Maxillipeds, 239 ; Locomotor appendages, 239; Feet, 240 ; Bran. chiæ, 242; Ventral chord, 254; Auditory vesicles, 261; Masticatory stomach, 268; Liver, 274; Heart, 281; Male organ, 295 ; Seminal elements, 305 .

Delphinoidea - Teeth 551; Pancreas Aselli, 600.

Dentalium-Auditory vesicle, 357.

Derostomum-Auditory organ, 156.

Derotremata - Branchial arches, 471 ; Eyelids, 529; External gills, 545.

Desmosticha-Ambulacra, 197; Integument, 204.

Dibranchiata-Funnel, 325; Arms, 326 ; Internal skeleton, 342; Nervous system, 350; Eye, 355; Salivary glands, 364; Liver, 366; Ink-bag, 369; Auricles; 369; Veins, 374; 
Branchial heart, 374; Excretory organs, 379.

Dictocyrta-Shell, 83.

Dicyema-Organism of, 68,69 .

Didelphia-Nipples, 422; Manus, 482.

Didelphys-Vagina, 621; Erectile bodies, 623.

Didemnum-Colonies, 391.

Difflugia-Supporting organs, 81 .

Digitigrada-Hallux, 491.

Dimyaria-Internal skeleton, 342; Mouth, 359.

Dinosaurii-Pelvis, 485.

Diphyidæ-Nectocalyces, 96 ; Generative organs, 121.

Diplopoda-Nervous system, 255.

Dipnoï-Vertebral column, 428; Caudal region, 431; Nasal canal, 521; Vesti. bule, 534; Air-bladder, 568; Blood. corpuscles, 576; Conus arteriosus, 578 ; Oviduct, 611.

Diptera-Thorax, 237; Setæ, 246; Labrum, 246; Wings, 248; Halteres, 248; Ventral chord, 259; Auditory organ, 262 ; Stomach, 272; Mid-gut, 272; Salivary glands, 274; Malpighian vessels, 277 ; Tracheal vessels, 289 ; Ovary, 302 ; Cement glands, 303.

Discophora-Marginal filaments, 102 ; Gelatinous disc, 107; Muscular system, 108; Marginal vesicles, 110; Gastro-vascular system, 114; Gastric filaments, 116; Generative organs, 122.

Distoma-Enteron, 158; Excretory organs, 174; Shell-gland, 183.

Dog-Manus, 483 ; Pelvis, 486.

Doliolum-Larvæ, 390 ; Sensory organs, 397; Branchial slits, 402; Ventral groove, 403; Sexual organ, 404.

Dolium-Siphon, 322 ; Proboscis, 361; Salivary glands, 364 ; Penis, 385.

Dolphin-Fore-limb, 482.

Dorididæ-Branchiæ, 338.

Doridopsis-Liver, 366.

Doris-Branchiæ, 338; Arms, 362; Salivary gland, 364; Liver, 365 ; Ves. sels, 372; Receptaculum seminis, 381.

Dromæus-Auricle, 477 ; Columella, 538.

Dysdera-Tracheæ, 291.

Dyssycus-Gastric system, 112.

Dytiscus-Visual organ, 263; Stomach, 272 ; Hind-gut, 273.

Ecardines-Shell, 308; Vascular system, 312 ; Generative organs, 313.

Echidna - Pterygoids, 466; Dermal muscles, 493 ; Canals of Gartner, 617.

Echinida - Masticatory organs, 214; Stone-canal, 222.

Echinocucumis - Integument, 207; "Lungs," 216.
Echinoderes-Nervous system, 146 ; Ex. cretory organ, 174.

Echinoderma - General review, 192 ; Classification, 193; Bibliography, 194; General form, 194; Larvæ, 191; Muscular system, 207 ; Nervous sys. tem, 208; Sensory organs, 210; Alimentary canal, 211 ; Vessels, 217 ; Water vessels, 219; Stone-canal, 220; Excretory organs, 224; Generative organs, 224.

Echinoïda-Dermal branchiæ, 200 ; Cal. careous skeleton, 202; Dermal skeleton, 205; Internal skeleton, 205; Pedicellariæ, 206; Masticatory apparatus, 206, 214; Spines, 206; Muscular system, 207; Nervous sys. tem, 209; Blood-ressels, 218 ; Stonecanal, 222 ; Generative organs, 225.

Echinorhynchus-Aciculi, 140; Generative organs, 186.

Echinothurida-Dermal skeleton, 205 ; Masticatory organs, 214.

Echinus-Figure, 204; Pedicellariæ, 206; Nervous system, 209; Blood-vessels, 218 ; Stone-canal, 222 ; Generative organs, 226.

Echiurus-Ganglion cells, 148; Alimen. tary canal, 161; Vascular system, 171 ; Excretory organs, 175.

Edentata-Scales, 4.18; Carapace, 426; Sacral vertebræ, 436; Episternum, 444; Turbinate bones, 465; Premaxillæ, 466; Centrale, 482 ; Cerebral hemispheres, 509; Cerebellum, 510; Stomach, 558; Arterial retia, 597 ; Renal organs, 605 ; Testes, 618.

Eledone-Suckers, 327 ; Copulatory or. gans, 328; Salivary glands, 364 .

Elephas-Nipples, 422; Milk teeth, 552; Renal organs, 605 ; Prostatic glands, 619.

Elysia-Branchiæ, 339.

Enaliosaurii-Hind-limb, 488.

Enchytrœus-Central ganglia, 149.

Enteropneusti-Branchial skeleton, 142 ; Alimentary canal, 398.

Entomostraca - Nauplius stage, 235 ; Branchix, 243; Eye, 264; Masticatory stomach, 268; Liver, 275 ; Fat bodies, 278; Excretory organ, 285; Shell-gland, 286.

Epeira-Eye, 266; Tracheæ, 291.

Ephemera-Tracheal gills, 290.

Ephemerida-Salivary glands, 274; Ecdysis, 289; Tracheal gills, 290 ; Ovi. duct, 302.

Epistylis-Stalk, 83.

Equus-Digits, 484; Stomach, 556.

Erinaceus-Dermal muscles, 493.

Ervilia-Pharynx, 85.

Esox-Vertebræ, 430; Cartilaginous cra. nium, 480; Eye, 529; Opercular gill 543. 
Eucopida-Marginal vesicles, 110.

Eudendriuın-Tentacles, 93, 101 ; Cormi, 94; Figure of, 94; Tests, 104.

Eunice - Appendages, 136; Vascular system, 169; Generative organs, 190.

Euniceidæ-Longitudinal nerve-trunks, 149.

Euphausia-Eye, 264.

Euplectella-Skeleton, 106.

Euplotes-Carapace, 83.

Euryalida - Arms, 196 ; Integument, 203.

Eurylepta-Alimentary canal, 158.

Eurystomata - Auditory organ, 538; Columella, 538 .

Fabricia-Auditory organs, 156.

Falco-Eye, 529.

Fibrospongiæ-Skeleton, 106.

Firolidæ-Kidney, 377.

Fishes - Corium, 417 ; Tegumentary glands, 420; Vertebral column, 429; Articular processes, 437; Ribs, 438 ; Hind-limb, 487 ; Intercostal muscles, 496; Muscles of branchial skeleton, 497 ; Do. of paired appendages, 498 ; Electric organs, 500; Cerebellum, 505 ; Medulla oblongata, 505; Arachnoid, 513; Spinal nerves, 514; Olfactory nerve, 515; Vagus, 517; Visceral nervous system, 523 ; Beards, 524; Olfactory organs, 525; Eye, 528; Optic bulb, 529; Lens, 531; Eyelids, 532; Labyrinth, 535; Branchial pouches, 542; Enteron, 555 ; Esophagus, 556 ; Liver, 564; Mesentery, 565; Air-bladder, 567; Heart, 577; Bulbus arteriosus, 578; Branchial artery, 578; Carotids, 578; Arterial arches, 579; Aorta, 579; Arteriæ intercostales, 589; Venous system, 590; Ductus Cuvieri, 590 ; Jugular veins, 590; Cardinal do., 590; Caudal do., 590; Portal system, 590; Renal portal system, 590; Inferior venæ cavæ, 593; Lymph sinuses, 598; Lymphatic hearts, 599 ; Lymphatic glands, 600 ; Ova, 609; Sperm, 609; Efferent ducts, 610 .

Fissurella-Branchiæ, 337 ; Heart, 371 ; Excretory organ, 377; Generative glands, 385.

Flagellata-Protoplasm, 76.

Flea-Ovary, 301.

Flies-Nervous system, 257.

Flustra-Hind-gut, 161.

Foraminifera-Protoplasm, 76; Shells, 81.

Forficulidæ-Elytra, 247.

Formica-Masticatory stomach, 272.

Fowl-Hyoid, 472; Brain, 507 ; Auditory organ, 533; Stomach, 557; Development of arteries, 587.
Frog-Vertebral column, 4.33; Brain, 505; Branchial plexus, 514; Cephalic arteries, 586 ; Arterial system, 586; Renal veins, 594.

Fungiæ-Colonies, 100; Skeleton, 106.

Gadus-Skull, 452; Shoulder-girdle, 475. Galeodea-Nervous system, 256; Ganglia, 256 ; Digestive organs, 270; Lungs, 291; Generative organs, 297.

Galeus-Ovaries, 610.

Gallinæ-Cæca, 562.

Gammarus-Heart, 280.

Ganoïdei-Vertebral column, 429 ; Transverse processes, 431; Fin rays, 432 ; Ribs, 439; Cartilaginous cranium, 450; Mandibular apparatus, 453; Operculum, 455; Shoulder-girdle, 4.75; Thoracic fin, 478; Pelvic-girdle, 484; Ventral fin, 487; Olfactory lobes, 504; Thalamencephalon, 504; Sinus rhomboidalis, 505; Medulla oblongata, 505; Optic nerves, 517 ; Ciliary processes, 530; Spiracle, 537; Spiracular cleft, 543; Teeth, 550; Enteron, 555; Spiral valve, 560; Air-bladder, 567 ; Conus arteriosus, 578; Aorta, 585; Kidneys, 601; Mullerian ducts, 610; Cloaca, 619.

Gasteropoda-Velum, 319; Foot,321,323 ; Mantle, 322 ; Siphon, 322 ; Tentacles, 326,352 ; Integumentary glands, 329 ; Shell, 332; Operculum, 335; Branchiæ, 337 ; Internal skeleton, 341 ; Corebral ganglia, 347 ; Buccal ganglia, 351 ; Olfactory organs, 352 ; Visual organs, 353; Auditory organs, 356; Alimentary canal, 358; Jaws, 360; Circulatory centres, 368, Auricles, 369, 373 ; Heart, 371 ; Vessels, 371 ; Blood-fluid, 375; Excretory organ, 377 ; Generative organs, 381 ; Lfferent ducts, 382 .

Gasterostomum-Pharynx, 158.

Geckotidæ-Teeth, 551.

Geophilus-Nervous system, 225.

Gephyrea-Incomplete metamerism, 310 ; Respiration in, 136; Integument, 136; Cilia, 137 ; Dermal glands, 141 ; Cerebral mass, 148; Alimentary canal, 161; Vascular system, 170; Excretory organs, 174; Generative organs, 189.

Gerardia-Generative organs, 122.

Geryonidæ-Nervous system, 109 ; Marginal vesicles, 110 .

Glomeris-Enteric canal, 271; Trachex, 288.

Glycera-Visceral nerves, 151; Vascular system, 170.

Glyceridæ-Blood-corpuscles, 172 .

Gnathostoma-Nerve-fibres, 33; Cranium, 447 ; Lower jaws, 448 ; Palato-quadrate, 448; Dermal muscles, 492; 
Optic nerves, 515; Semicircular canals, 534; Hypobranchial groove, 554.

Goat-Skull, 463.

Gordiacea-Alimentary canal, 160 ; Ex. cretory organs, 173.

Gordius-Alimentary canal, 160; Excretory organs, 173; Generative organs, 185 .

Grallatores-Optic bulb, 529; Pecten, 531.

Gregarinæ-Sensory organs, 42; Form of, 76 ; Cuticular layer, 78 ; "Head," 80; Nutrition in, 83; Figure of, 87 ; Reproduction in, 87 ; Pseudonavicellæ, 87.

Gymnolæmata-Cells, 139.

Gymnophiona-Ribs, 439.

Gymnosomata-Cephaloconi of, 326; Respiratory organs, 339.

Gymnotus-Electric organs, 500.

Haliotis - Epipodium, 324; Branchiæ, 337; Ganglionic chain, 347 ; Heart, 371 ; Excretory organs, 377; Germ. gland, 385.

Halisarca-Ectoderm, 103; Sexnal organs, 119.

Halmaturus-Nipples, 422; Veins, 591; Erectile bodies, 623 .

Harpa-Siphon, 323; Proboscis, 361 ; Penis, 386.

Hedessa-Cæca, 268.

Helicinæ-Shell, 332 ; Lung, 339 ; Spermatophores, 383 ; Receptaculum seminis, 383 .

Heliozoa-Protoplasm, 76.

Helix-Tentacles, 326; Hæmal spaces, 373; Hermaphrodite glands, 382 ; Generative apparatus, 383.

Hemiptera-Setæ, 246; Wings, 248; Ventral chord, 259; Stomach, 272; Mid-gut, 272; Malpighian vessels, 277; Generative organs, 302 ; Testis, 304 .

Hermella-Tentacles, 134; Appendages, 136; Ganglia, 150 ; Vascular system, 170.

Heterakis-Alimentary canal, 159.

Heteropoda-Gelatinous tissue, 25; Shell, 322, 332; Foot, 324; Cutis, 328; Branchiæ, 338; Muscular system, 342 ; Commissures, 348; Olfactory organ, 352; Eye, 354; Auditory resicles, 357; Radula, 360; Crop, 361; Excretory organs, 378; Recep. taculum seminis, 385 ; Penis, 385.

Heterotricha-Cilia, 79 .

Hippolyta-Auditory vesicles, 261.

Hirudinea-Respiration in, 136; Integument, 136; Dermal glands, 141; Muscular system, 142; Muscular fibres, 144; Nervous system, 149; Ventral ganglia, 150; Visceral nerves
151 ; Cup-shaped organs, 153 ; Visual organs, 154; Fore-gut, 162 ; Enteric glands, 164; Vascular system, 167; Excretory organs, 177; Generative organs, 186; Generative products, 190, Spermatophores, 191.

Hirudo-Vascular system, 167 ; Excretory organs, 177; Generative organs, 187.

Histiotheutis-Eye, 355.

Hœmopis-Mid-gut, 162 ; Generative organs, 187 ; Generative products, 190.

Holothuria-Alimentary canal, 214; Polian vesicles, 223.

Holothuriæ-Integument, 207.

Holothuroida-Ambulacra, 197; Tentacles, 200; Calcareous skeleton, 202; In. tegtument, 206; Internal skeleton, 207 ; Muscular system, 208; Nervous system, 210; Sensory organs, 210; Alimentary canal, 211, 214; " Lungs," 215; Cœlom, 217; Blood-ressels, 218; Stone-canal, 221; Polian vesicles, 222 ; Water-ressels, 223 ; Excretory organs, 224; Generative organs, 226.

Holotricha-Cilia, 79.

Homarus-Male organs, 295.

Hyæna-Kidney, 605.

Hyaleidæ-Foot, 324; Respiratory organs, 339; Hermaphrodite glands, 382.

Hyalonema-Skeleton, 106.

Hydra-Neuromuscular cells, 30 ; Cormi, 94.

Hydræ-Generative buds, 120.

Hydractinia-Tentacles, 93, 101 ; Buds, 95; Polymorphism in, 95; Tests, 104.

Hydriformes-Muscular system, 108.

Hydrobius-Generative organs, 303.

Hydrochærus-Uterus, 615.

Hydroida-Musculature, 39; Tentacles, 93; Medusæ, 93; Buds, 95; Tests, 104; Supporting lamella, 107; Nervous system, 109 ; Gastrovascular system, 114; Ova, 124.

Hydroid Polyps-Flagellate cells, 21.

Hydromedusæ-Tentacles, 101; Muscular system, 108; Marginal vesicles, 110 ; Gastric system, 115; Pigmented investment of stomach, 118; Separate sexes, 120.

Hydrophilus-Development, 245.

Hymenoptera-Gnathites, 246; Linguæ, 246; Paraglossæ, 246; Wings, 248; Dermal skeleton, 259; Wax organs, 250 ; Spinning vessels, 250; Ventral chord, 259; Stomach, 272; Malpighian vessels, 277 ; Tracheal vesicles, 289 ; Generative organs, 302 ; Testis, 304.

Hyperida-Optic nerves, 253; Auditory vesicles, 261.

Hypotricha-Cilia, 79.

Hyrax-Dorso-lumbar vertebræ, 436 ; Testes, 618. 
Ichneumonida-Mid-gut, 272 ; Cementglands, 303.

Ichthyornis-Teeth, 551.

Infusoria-Sensory organs, 42 ; Alimen. tary canal, 47; Protoplasm, 77; Nucleus, 77; Cuticular layer, 78; Figure of, 79; Skeleton, 83 ; Contractile vacuoles, 85; Conjugation, 89.

Insecta-Alimentary canal, 48; Head, 235 ; Metameres, 237; Thorax, 237; Gnathites, 244; Mouth organs, 245; Feet, 245; Mandibles, 245; Maxillæ, 245 ; Feet, 246; Dorsal appendages, 247 ; Wings, 247 ; Integument, 248 ; Spines, 250; Spinning vessels, 250; Nervous system, 257 ; Ganglia, 258; Visceral nerves, 259; Nervus recurrens, 259; Nervi transversi accessorii, 260; Tactile rods, 260; Olfactory organs, 261; Auditory organs, 261; Simple eye, 265; Compound eyes, 266; Faceted eye, 267; Enteric canal, 270; Malpighian vessels, 271, 273, 276 ; Hind-gut, 273 ; Salivary glands, 274; Subneural cavity, 279; Blood-cells, 280; Alæ cordis, 283; Circulatory organs, 283 ; Aorta, 283; Ecdysis, 289; Germ-glands, 292 ; Generative organs, 300, 304; Par. thenogesis, 302; Pseudova, 302; Seminal elements, 305 ; Copulatory organ, 305.

Insectivora-Centrale, 482; Symphysis pubis, 486; Fibula, 490; Cerebral hemispheres, 509; Venæ cavæ, 592 ; Testes, 618; Vesiculæ seminales, 618 ; Prostatic vesicles, 619.

Isopoda-Branchiæ, 241; Ventral chord, 255; Compound eyes, 267 ; Chyle intestine, 268; Liver, 275; Heart, 280 ; Female organs, 294; Seminal elements, 305 .

Ixodes-Generative organs, 298.

Janthina-Branchiæ, 338.

Julus-Salivary glands, 274; Malpighian vessels, 276; Renal concretions, 278 ; Circulating system, 284; Generative organs, 299.

Labyrinthobranchiata-Branchial arches, 470.

Læmargus-Ovaries, 610.

Lacerta-Columella, 458; Frontals, 458.

Lagomys-Cæcum, 562.

Lagostomus-Uterus, 616.

Lamellibranchiata-Velum, 318; Diagram of, 319 ; Foot, 321 ; Tentacles, 326 ; Integumentary glands, 329 ; Byssus gland, 329; Shell, 331; Hinge of shell, 332 ; Branchiæe, 336; Muscles, 342 ; Nervous system, 345 ; Visceral nerves, 351 ; Tentacles, 352 ; Visual organs, 353; Auditory vesicles, 357; Alimentary canal, 358; Stomach, 359; Hind-gut, 359; Liver, 364; Cœlom, 367 ; Circulatory centres, 368; Auricles, 369, 373 ; Vessels, 372 ; Formelements of blood, 375; Organs of Bojanus, 376; Generative organs, 380 .

Lamellicorniæ-Ventral chord, 258; Tracheal vesicles, 289.

Lemur--Dorso-lumbar vertebræ, 436.

Lepadidæ-Dermal skeleton, 249; Ven. tral chord, 255 ; "Cement glands," 285 ; Hermaphroditism in, 291.

Lepidoptera-Thorax,237; Mouth organs, 246; Feet, 246; Wings, 248; Spinning vessels, 250; Nervus recurrens, 259; Malpighian vessels, 277; Tracheal vesicles, 289; Ovary, 302 ; Cement. glands, 303 ; Bursa copulatrix, 303.

Lepidosiren-Vagus, 517 ; Air-bladder, 568; Heart, 580; Aortic arches, 580 ; Allantois, 620 .

Lepidosteus-Opercular gill, 543; Spiral valve, 560.

Lepisma-Ventral chord, 258.

Lepus-Uterus, 617; Urogenital canal, 619.

Leptocardii-Notochord, 426; Olfactory organs, 525; Vertebræ, 438.

Leucifer-Auditory vesicles, 261.

Libellulidæ-Tracheal gills, 273; Salivary glands, 274; Respiration in, 290.

Limax-Tentacles, 326; Byssus gland, 329 ; Shell, 332 ; Lung, 339.

Limicolæ-Excretory organs, 177; Generative organs, 185.

Limnadia-Shell, 235; Respiration in, 269 ; Liver, 274; Ovary, 294.

Limnadiaceæ-Branchiæ, 241.

Limnetis-Branchiæ, 241.

Lineus-Proboscis, 141.

Linguatulidæ-Tracheæ, 291.

Lingula-Gill, 308; Stalk, 308; Alimentary canal, 311 ; Cæca, 311; Vascular system, 312.

Lisarmatidæ-“Lungs," 215 ; Excretory organ, 312.

Littorina-Denticles, 360 ; Stomach, 362; Penis, 386.

Lizard-Foot, 489 ; Arteries, 587.

Lizzia-Tentacles, 101.

Locustidæ-Auditory organs, 262.

Lœmodipoda-Compound eyes, 267.

Loliginidæ-Fins, 325 ; Arms, 326 ; Calamus, 335 ; Respiratory organs, 340 ; Eye, 355; Pharynx, 361, 362; Digestive organs, 363 ; Liver, 366 ; Ink-bag, 367 ; Circulatory centres, 368; Arteries, 374; Oviduct, 386; Pouches of Needham, 387.

Loligopsis-Eye, 355 ; Cæca, 363.

Lophobranchii-Carapace, 424; Ribs 438 ; Vertebræ, 439. 
Lophopus-Cells, 139.

Lottia-Branchiæ, 337.

Loxosoma-Tentacles, 134.

Lucernaria-Marginal filaments, 102 ; Gastrovascular system, 112.

Luciæ-Colonies, 391.

Lucina-Nervous system, 345.

Luidia-Alimentary canal, 213.

Lumbricidæ-Ganglia, 150 ; Visceral nerves, 151 ; Excretory organs, 177 ; Generative organs, 185.

Iumbriculus-Vascular system, 169.

Lumbricus-Muscular stomach, 162 ; Vascular system, 167; Excretory organs, 178.

Lutra-Testes, 618.

Lutraria-Ganglia, 346.

Lycosa-Circulatory system, 281; Tracheæ, 291.

Lymnæidæ-Lung, 339.

Lysidice-Masillary apparatus, 162.

Lymnæus-Crop, 361.

Macgillivraya-Telum, 321.

Macropus-Turbinate bones, 547.

Macrotarsi-Calcaneum, 490.

Macrostoma-Generative organs, 180.

Macrura-Ventral chord, 254; Heart, 281 ; Male organs, 295.

Mactra-Siphons, 320; Ganglia, 345, 346 ; Tentacles, 352 ; Cœlom, 367 ; Genital canal, 381.

Madrepores-Skeleton, 106.

Madreporinæ-Generative organs, 122.

Malacostraca-Development, 234; Nauplins, 235; Appendages, 239; Ventral chord, 253; Auditory resicles, 261; Cæca, 268.

Malapterurus-Electric organs, 500

Malleus-Byssus gland, 329.

Mallophagus-Generative organs, 303.

Mammalia-Stomach, 9; Blood-corpuscles, 29; Corium, 417; Hoofs, 419; Hairs, 420; Anal glands, 421; Mammary glands, 421; Vertebral column, 435; Processes of vertebi: 436 ; Cervical rertebræ, 436 ; Caudal vertebrx, 436; Odontoid process, 437 ; Ribs, 441; Cervical ribs, 441 ; Sternum, 442 ; Clavicles, 442 ; Xiphoid process, 442 ; Episternum, 444 ; Primordial cranium, 463; Tympanic bone, 466; Meckel's cartilage, 467; Glaserian fissure, 467; Stapes, 467; Hyoid, 472; Coracoid, 476; Scapula, 476; Manus, 482; Pelvis, 485; Hind-limb, 490; Dermal muscles, 493 ; Dorsal muscles, 495; Intercostal muscles, 496 ; M. pyramidalis, 496; Masticatory muscles, 497; Diaphragm, 499, 574; Corpus striatum, 508; Cornu ammonis, 508; Fornix, 509; Cerebral hemispheres, 509 ; Gyri, 509; Sulci, 509; Tha- lami optici, 509; Epiphysis, 509; Mesencephalon, 509; Aquxductus Sylvii, 509; Corpora quadrigemina, 509; Cerebellum, 510 ; Pons Varolii, 510; Spinal chord, 512; Falx cerebri, 512; Tentorium cerebelli, 512; Arachnoid, 513; Brachial plexus, 514; Crural plexuses, 515; Sacral plexuses, 515; Olfactory nerve, 515: Facial nerve, 517; Accessorius Willisii, 522 ; Tactile organs, 524; Gustatory organs, 525; Nasal canal, 527 ; Organ of Jacobson, 547, 528; Stenonian ducts, 527, 548; Eye, 528; Lens, 529; Optic bulb, 529; Sclerotic, 530; Optic muscles, 531; Eyelids, 532; Harderian gland, 532 ; Lachry. mal glands, 532; Lachrymal ducts, 533 ; Ductus endolymphaticus, 534; Labyrinth, 535; Cochlea, 536; Columella, 538; Stapes, 538; Auditory meatus, 539; Palate, 546; Enstachian tube, 546; Pharynx, 546; Velum palatinum, 546, 549; Nasal cavity, 547; Turbinate bones, 547; Teeth, 550; Milk teeth, 552 ; Tongue, 552; Buccal glands, 553; Thyroid gland, 554; Stomach, 558 ; Mid.gut, 561; Hind-gut, 562; Cæcum, 562 ; Cloaca, 563, 620; Liver, 563, 561; Mesentery, 565; Larynx, 570; Epiglottis, 571; Lungs, 573; Colom, 574; Blood-corpuscles, 576; Heart 583; Arterial system, 583; Auricles, 583; Atrio-ventricular valve, 584; Aortic arches, 581; Ligamentum Botalli, 585; Aorta, 588; Ischiac arteries, 589; Vertebral veins, 592 ; Vena azygos, 593; Jugulars, 593; Inferior vena cava, 595; arterial retia, 597; Pleural cavity, 598; Lymph sinuses, 599; Peyerian glands, 600; Lymphatic glands, 600; Thymus, 600; Renal organs, 605; Urachus, 606; Urinary bladder, 606; Ora, 609; Sperm, 609; Generative organs, 615; Ovaries, 617; Male organs, 617; Mullerian duct, 617 ; Placenta, 620; Chorion, 620; Prepuce, 624; Clitoris, 624; Cowper's glands, 624 .

Man-Mastoid, 464; Hyoid, 472; Centrale, 482 ; Manus, 483; Pelvis, 486; Dermal muscles, 494; Masticatory muscles, 497 ; Choroid, 530; Auditory meatus, 539; Turbinate bones, 547; Cæcum, 562; Appendix vermiformis, 562; Venous system, 596; Uterus, 615; Hymen, 616; Prostatic vesicle, 619.

Manatee-Cervical rertebræ, 436.

Marsupialia-Nipples, 422 ; Odontoid processes, 437 ; Episternum, 444 ; Paramastoid, 463; Pelvis, 486; Marsupial 
bones, 487; Hallux, 491; M. pyra. midalis, 496 ; Cerebral commissures, 508; Cerebellum, 510; Stapes, 538; Turbinate bones, 547 ; Sinus maxil. laris, 548; Milk teeth, 552 ; Thyroid gland, 554; Stomach, 558; Liver, 564; Auricles, 583; Venæ cavæ, 592; Renal organs, 605; Oviducts, 615; Copulatory organs, 622; Erectile bodies, 623; Scrotum, 618; Vas deferens, 618; Testes, 618.

Medusæ-Gelatinous tissue, 25 ; Cartilage cells, 26 ; Cartilaginous skeleton, 38 ; Nervous system, 109.

Melolontha-Mid-gut, 272; Heart, 283 ; Generative organs, 304.

Menobranchus-Vertebræ, 432; Enteric canal, 561 ; Trachea, 571.

Menopoma-Vertebræ, 432 ; Hind-limb, 488 ; Trachea, 571.

Mermis-Excretory organ, 174; Generative organs, 185.

Mesostomum-Nervous system, 145 ; Visual organs, 154.

Microstomeæ-Generative organs, 183.

Mites-Renal concretions, 278.

Mnemidæ-Axes, 101.

Mœandrina-Colonies, 100.

Molgula-Nervous system, 395; Sexual organs, 407.

Molpadia_"Lungs" 216 ; Cuvierian organs, 216; Polian vesicles, 223.

Mollusca-Connective tissue, 24; Cartilaginous skeleton, 38 ; Dermal branchiæ, 46 ; General review, 315 ; Classification, 316 ; Bibliography, 317 ; Form of body, 318; Velum, 318 ; Appendages, 325 ; Integument, 328 ; Cilia, 328; Shell, 330; Branchiæ, 335 ; Nervous system, 343; Sensory organs, 351 ; Tentacles, 352 ; Visual organs, 353; Auditory organs, 358 ; Alimentary canal, 358; Pharynx, 359; Radula, 360 ; Liver, 364; Pancreatic gland, 356 ; Cœlom, 367 ; Vascular system, 368 ; Blood-fluid, 375 ; Excretory organs, 46, 375 ; Generative organs, 380.

Monitor-Frontals, 459; Pelvis, 485; Eye, 529.

Monodelphia-Stapes, 538; Genital canal, 615 ; Scrotum, 618.

Monotremata-Mammary glands, 421 ; Odontoid process, 437 ; Episternum, 444; Coronoid process, 467; Coracoid, 476; Scapula, 476 ; Marsupial bones, 487 ; M. pyramidalis, 496 ; Cerebral commissures, 508 ; Cerebellum, 510 ; Olfactory nerve, 515; Eye, 530; Lagena, 536; Stapes, 538 ; Auditory meatus, 539; Horny teeth, 549; Thyroid gland, 554; Stomach, 558; Cloaca, 563, 620 ; Foramen ovale, 584; Venæ cavæ, 592; Oviducts, 615 ;
Testes, 618 ; Vas deferens, 618; Copulatory organs, 622 .

Mormyrus-Electric organs, 500.

Moschidæ-Manus, 483.

Muelleria-Cuvierian organs, 216.

Murex-Siphon, 322 ; Dermal glands, 329 ;

Proboscis, 361; Anal glands, 362.

Mus-Uterus, 615.

Mustelina-Cæcum, 562.

Mustelus-Ovaries, 611.

Musca-Proboscis, 260; Malpighian ves. sels, 277.

Muscidæ-Heart, 283.

Mya-Ganglia, 346.

Mygalida-Lungs, 291.

Myrianida - Appendages, 136; Visua organs, 135.

Myriapoda-Head, 235; Form of, 237 ; Gnathites, 244; Integument, 248 ; Nervous system, 255; Tactile rods, 260 ; Eyes, 265 ; Enteric canal, 260; Salivary glands, 274; Malpighian vessels, 276; Renal concretions, 278 ; Circulatory system, 283; Tracher, 280; Germ-glands, 292 ; Generative organs, 299; Seminal elements, 305.

Mysis-Branchiæ, 241 ; Auditory vesicles, 261; Heart, 281; Female organs, 294; Seminal elements, 305.

Mytilidæ-Respiratory cavity, 320.

Mytilus-Byssus gland, 329; Branchix, 336 ; Genital canal, 381.

Myrmecophaga-Jugal, 466.

Myxinoidea - Auditory capsules, 448 ; Trunk muscles, 494; Brain, 504; Visceral nervous system, 523 ; Laby. rinth, 533; Systemic arteries, 585; Carotids, 585 ; Kidney, 603 ; Ovaries, 610.

Myzostoma-Intestinal tube, 163.

Naiades-Byssus gland, 329 ; Auditory vesicles, 359 .

Narcine-Electric organs, 500.

Nassula-Pharynx, 85.

Natatores-Anal glands, 420; Optic bulb; 529; Pecten, 531; Stomacl, 558; Trachea, 571; Lymphatic hearts, 599 ; Copulatory organs, 621.

Nauplius-A ppendages, 238; Eyes, 264.

Nautilus - Funnel, 325 ; Copulatory organs, 327 ; Shell, 333 ; Respiratory organs, 340 ; Cephalic cartilage, 342 ; Nervous system, 350, 351; Eye, 354; Otocyst, 357 ; Pharynx, 362 ; Cæca, 363 ; Circulatory centres, 368 ; Branchial veins, 373 ; Excretory organs, 379 ; Oviduct, 386.

Nemathelminthes-Muscular system, 142 ; Muscular fibres, 144; Nervous system, 147; Alimentary canal, 15\%; Genital products, 190.

Nematodes - Muscular system, 142 ; 
Lateral line, 143; Tactile papillæ, 153; Generative organs, 184; Seminal elements, 191.

Nemertes-Proboscis, 141; Visual organs, 154.

Nemertina-Incomplete metamerism, 130; Proboscis, 140, 141 ; Nervous system, 147; Tactile setæ, 152 ; Cephalic pits, 153 ; Visual organs, 154; Alimentary canal, 157.

Neomenia-Nervous system, 152; Enteric branchiæ, 164.

Neophanta-Visual organs, 155.

Nepa-Respiratory tube, 289.

Nephelis-Vascular system, 167 ; Excretory organs, 177; Generative pro. ducts, 190.

Nephropneusta - Shell, 323 ; Nervous system, 349 ; Jaws, 360 ; Excretory organs, 378 .

Nerita-Heart, 371.

Neritacea-Branchiæ, 338.

Neritina-Heart, 371.

Neuroptera-Thorax,237; Gnathites, 242 ; Enteric canal, 272; Ovary, 302 ; Male organs, 304 .

Nodosaridæ-Shells, 81.

Notidani-Branchial arches, 469 ; Clefts, 492.

Notommata-Excretory organs, 175.

Nudibranchiata-Branchiæ, 338; Tenta. cles, 352 ; Intestine, 362 ; Vessels, 372.

Numida-Sternum, 443.

Oceanidæ-Marginal vesicles, 110.

Octactiniæ-Tentacles, 100; Transverse axes, 100 ; Tentacles, 102 ; Gastric system, 117.

Octopoda - Tentacles, 327 ; Internal skeleton, 340; Muscles, 343; Oto. cysts, 357; Pharynx, 362; Liver, 366 ; Oviduct, 386.

Octopus-M[antle, 325 ; Copulatory organs, 327 ; Eye, 355; Salivary glands, 363 ; Circulatory centres, 368 ; Figure, 373.

Odontornithes-Teeth, 551.

Erstedia-Nervous system, 147; Auditory organ, 156

Oikopleura-" House," 393.

Oletera-Ovaries, 296.

Olynthus-Gastrula, 93; Pore canals, 112.

Ommatoplea-Nervous system, 147.

Onchidium-Lung, 339.

Oniscus-Liver, 275 ; Generative organs, $294,295$.

Onychoteuthis-Suckers, 327.

Ophidiaster-Generative organs, 225.

Ophioderma-Generative organs, 224.

Ophidii-Scales, 418; Centra, 433; Processes of vertebræ, 433 ; Caudal ver. tebræ, 434; Axis, 437; Ribs, 440 ;
Squamosal, 458; Columella, 458 ; Covering-bones of skull, 459; Vomer, 459; Palato-quadrate, 459; Quadrate, 460; Pterygoids, 461; Palatines, 461 ; Maxilla, 462; Hyoid, 472; Fore-limb, 481; Toes, 489; Dermal muscles, 493 ; Intercostal do., 496; Masticatory do., 497 ; Brain, 507; Optic bulb, 529; Ductus endolymphaticus, 534; Tympanic cavity, 537; Palate, 546; Nasal glands, 548; Organ of Jacobson, 548; Teeth, 550, 552 ; Labial glands, 553; Stomach, 557 ; Mid-gut, 561; Hind-gut, 562; Liver, 564 ; Mesentery, 565 ; Larynx, 570; Lungs, 573 ; Heart, 581; Caro. tids, 587; Epigastric veins, 595; Ovaries, 613; Testes, 614; Copulatory organs, 621 .

Ophiothrix-Figure, 202.

Ophiura-Anus, 199 ; Generative organs, 225.

Ophiurida-Larva, 195; Rays, 196; Anus, 199; Calcareous skeleton of larva, 202; Integument, 203; Alimentary canal, 212; Stone-canal, 222 ; Generative organs, 224.

Ophrydiæ-Anal opening, 85.

Opilionea-Visceral nerves, 259.

Opilionida-Eyes, 265; Cæca, 270; Hepatic tbues, 275; Malpighian vessels, 276; Circulatory system, 284, Tracheæ, 291 ; Germinal glands, 297.

Opisthobranchiata-Shell, 323; Tentacles, 326 ; Urticating cells, 329 ; Rhinophor, 352 ; Visual organs, 354 ; Jaws, 360 ; Salivary glands, 364; Heart, 371; Vessels, 373 ; Circulation in, 373 ; Excretory organs, 377 ; Uterus, 383.

Orang-Centrale, 482.

Orchesella-Ventral chord, 258.

Oreaster-Arms, 195.

Ornithorhynchus-Ethmoid, 465; Atrio. ventricular valve, 584 .

Ornithoscelida-Tarsus, 489.

Orthagoriscus-Spinal chord, 511.

Orthoceratites-Shell, 332.

Orthoptera - Thorax, 237 ; Gnathites, 245; Dermal skeleton, 249; Ventral chord, 258; Nervus recurrens, 259 ; Auditory vesicle, 262; Tympanum, 262 ; Masticatory stomach, 272 ; In. gluvies, 272; Mid-gut, 272; Hindgut, 273; Salivary glands, 274, Ovary, 302; Testis, 304; Seminal elements, 305 .

Orycteropus-Uterus, 616.

Ostracion-Carapace, 424.

Ostracoda-Appendages, 238 ; Gnathites, 239 ; Branchiæ, 243; Dermal skele. ton, 249 ; Seminal elements, 305.

Ostrea-Muscles, 343 ; Genital canal, 381. Owls-External ear, 538. 
Ox-Manus, 483.

Oxyuris-Alimentary cxnal, 159.

Pagurus-Ventralchord,254; Male organs, 295.

Palinurus-Ventral chord, 254; Liver,275.

Palæotherium-Manus, 483.

Paludicella-Alimentary canal, 169.

Paludina-Nervous system, 348; Figure, 371 ; Albuminiparous gland, 385 ; Penis, 386.

Pandora-Generative organs, 381.

Panorpa-Masticatory stomach, 272.

Paradoxurus - Dorso-lumbar vertebrae, 437.

Paramœcium-Trichocyst, 79; Pharynx, 85 ; Figure, 85 ; Contractile vesicles 86.

Passeres-Stomach, 558.

Patella-Branchiæ, 337; Radula, 360; Excretory organs, 377 ; Germ-glands, 385.

Pecten-Byssus gland, 329 ; Branchiæ, 337; Muscles, 343; Ganglia, 346; Tentacles, 352; Visual organs, 353 ; Organ of Bojanus, 376 ; Generative organs, 381.

Pectinaria-Branchial tentacles, 135.

Pedicellina - Tentacles, 134; Nervous system, 146.

Pelagia-Larvæ, 99; Marginal filaments, 102 ; Marginal vesicles, 110.

Pelobates - Vertebræ, 432 ; Tympanic cavity, 537.

Penelopidæ-Copulatory organs, 621.

Pennaria-Buds, 95.

Pennatulida-Skeleton, 106; Generative organs, 123,

Pentacrinus-Ambulacral groove, 204.

Pentacta-Cuvierian organs, 216.

Pentastomum-Generative organs, 298.

Perameles-Tympanic bone, 466.

Perennibranchiata - Branchial arches, 471; Muscles of branchial skeleton, 497; Eyelids, 532; External gills, 545; Lungs, 572 ; Cephalic arteries, 586.

Peripatus-Form of, 237; Appendages, 238, 244 ; Spinning glands, 250; Muscular system, 251; Nervous do., 255 ; Enteric canal, 270; Salivary glands, 273 ; Circulatory system, 283 ; Tracheæ, 286.

Perisossodactyla-Nipples, 422; Dorsolumbar vertebræ, 436; Manus, 483 ; Pelvis, 486; Third trochanter, 490.

Peritricha-Cilia, 79.

Perlida-Tracheal gilis, 247, 289; Ecdysis, 289.

Petalosticha-Ambulacra, 199.

Petaurista-Tympanic bone, 466.

Petromyzon-Auditory capsule, 448; Olfactory organ, 525 ; Ear, 533 ; Respiratory organs, 542 ; Aorta, 585.
Phalangium-Generative organs, 298.

Phallusia-Mantle, 394.

Pharyngobranchiata-Branchial arches, 470 .

Phascolomys-Turbinate bones, 547.

Phascolosoma-Alimentary canal, 161.

Pheruseidr-Branchial tentacles, 135.

Phoca-Turbinate bones, 547; Stomach, 558; Pancreas Aselli, 600.

Pholas-Nervous system, 345.

Phronima-Optic nerves, 253; Heart, 280.

Phryganida-Tracheal gills, 247, 273.

Phrynida - Gnathites, 244; Nervous system, 256; Eyes, 265; Enteric canal, 269 ; Lungs, 291.

Phylactolæma-Muscular system, 144.

Phyllodoce-Visceral nerves; 187; Pro. boscis, 163.

Phyllopoda - Shell, 235; Appendages, 238 ; Ventral chord, 254; Eyes, 265 ; Liver, 275 ; Heart, 280.

Phyllosoma-Liver, 275 ; Germ-glands, 294.

Physemaria-Ectoderm, 103.

Physoklisti-Air-bladder, 567.

Physophoridæ-Nectocalyces, 96 ; Airsac, 97 ; Generative organs, 121.

Physostomi-Caudal region, 431; Ribs, 438 ; Air-bladder, 567.

Pinna-Visceral ganglia, 346; Visual organ, 353; Renal ducts, 376; Genital canal, 381 .

Pinnipedia - Auditory meatus, 539 ; Buccal glands, 553; Thymus, 600 ; Renal organs, 605.

Pipa-Sacrum, 433; Atlas, 437 ; Eustachian tube, 537 ; Tongue, 551.

Piscicola-Vascular system, 167 ; Generative organs, 184.

Placentalia-Foramen ovale, 584.

Placophora-Groove, 318; foot, 321 ; Tentacles, 326 ; Spicules, 330 ; Branchiæ, 336, 337; Nervous system, 344 ; Liver, 365 ; Auricles, 369 ; Excretory organs, 375 ; Generative organs, 380 .

Planaria-Tentacles, 132; Liver, 165; Cœlom, 165; Excretory organs, 177 ; Genital pore, 184 .

Planorbidæ-Lung, 339 ; Crop, 361.

Planorbis-Blood-fluid, 375.

Platyhelminthes-Form, 129; Mouth,129; Integument, 136; Dermal glands, 141 ; Muscular system, 142 ; Muscular fibres, 144; Nervous system, 145; Sexual organs, 184; Alimentary canal, 157; Liver, 165; Excretory organs, 175; Generative organs, 179.

Platyrhini-Turbinate bones, 547.

Plectognathi-Bony plates, 424.

Pleione-Origin of nerves, 151.

Pleurobranchus-Jaws, 360; Salivary glands, 36t; Vessels, 372 ; Recepta. culum seminis, 384 . 
Pleurodonts-Teeth, 5 5̣̂1.

Pleuroma-Generative organs, 293.

Pleurophyllidia-Branchiæ, 338.

Plumatella-Alimentary canal, 160.

Plumularia-Colonies, 89.

Pneumodermon-Respiratory organs, 339; Olfactory organs, 352 ; Liver, 366 ; Hermaphrodite glands, 382 ; Copulatory organ, 385.

Pœcilopoda-Derelopment, 234; Abdomen, 235 ; Branchiæ, 242 ; Liver, 275 ; Circulatory system, 282 ; Generative organs, 296.

Podinema-Columella, 458.

Podophthalma-Eye-stalk, 267.

Podophrya-Nucleus, 89.

Polyacanthus-Branchial lamellæ, 544 .

Polycera-Vessels, 372.

Polydesmus-Renal concretions, 278.

Polygordius-Cilia, 138; Muscular sys. tem, 142; Cephalic pits, 153.

Polymorphina-Shells, 81.

Polypterus - Vertebral column, 429 ; Ribs, 438; Brain, 505; External gills, 545 .

Polyzoa-Skeleton, 82.

Pontolimax-Branchiæ, 339.

Porifera-Ectoderm, 103; Skeleton, 105; Sexual organs, 119.

Porpita-Air-sac, 97.

Priapulus-Alimentary canal, 161.

Primates-Nipples, 422; Episternum, 444; Interparietal, 464; Turbinate bones, 465 ; Tympanic, 466; Hyoid, 472; Manus, 482; Hallux, 491; Cerebellum, 510; Pons Varolii, 510; Uvula, 549; Stomach, 558; Venæ caræ, 592 ; Renal organs, 605; Penis, 623.

Pristiurus-Ovaries, 64.

Procyon-Dorso-lumbar vertebra, 437.

Proporus-Auditory organ, 156.

Prorhynchus-Alimentary canal, 157.

Prorodon - Contractile bands, 80 ; Pharynx, 85.

Prosimii - Nipples, 422; Cæca, 562, Uterus, 616.

Prosobranchiata-Epipodium, 324; Cere. bral ganglia, 347 ; Ommatophor, 359 ; Jaws, 360 ; Crop, 361 ; Salivary glands, 364; Germ-glands, 385.

Proteus-Vertebræ, 432; Thyroid gland, 554; Stomach, 557; Mid-gut, 561; Larynx, 570; Lungs, 572; Blood. corpuscles, 577 ; Auricle, 580.

Protista - Cilia, 39; Conjugation, 52 ; Spores, 87.

Protopterus-Fin, 477; External gills, 545 ; Air-bladders, 568 ; Conus arteriosus, 578 .

Protozoa-Review of, 75; Bibliography, 77 ; Supporting organs, 81; Respira. tion in, 85; Sensory organs, 86.

Protracheata-Enteric canal, 269; Tra. cheæ, 286; Generative organs, 296.
Protula-Tentacles, 133.

Pseudoneuroptera--Thorax,239; Gnathites, 245; Feet, 246; Ventral chord, 258;

Enteron, 272; Nalpighian ressels, 277 ; Tracheal system, 289.

Psittacidæ-Tongue, 551; Cæca, 562.

Psolus-Ambulacra, 199.

Pteraster-Arms, 196.

Pterocera-Siphon, 323.

Pteropoda-Velum, 322; Mantle, 322; Foot, 323, 324; Shell, 332, Muscles, 343 ; Nerrous system, 349 ; Buccal ganglia, 351 ; Tentacles, 352 ; Olfactory organs, 352 ; Auditory resicles, 357 ; Alimentary canal, 358; Radula, 360 ; Stomach, 362; Liver, 366; Heart, 371; Vessels, 372 ; Generative organs, 381 ; Hermaphrodite glands, 383 ; Receptaculum seminis, 381; Copulatory organ, 385.

Pterotrachea-Shell, 323; Sensory organs, 354; Ommatophor, 359; Receptaculum seminis, 385 .

Pterygota-Gnathites, 245; Ventral chord, 258 ; Tracheæ, 288.

Pulex-Ventral chord, 259.

Pulmonata-Shell, 332 ; Salivary glands, 364; Excretory organs, 377 ; Hermaphrodite glands, 383 .

Pupipara-Ventral chord, 259.

Purpura - Dermal glands, 329; Anal glands, 362.

Pycnogonida-Nervous system, 256; Eyes, 266; Cæca, 269; Circulatory system, 284; Tracheæ, 291; Generative organs, 299.

Python-Skull, 460; Larynx, 569; Veins, 591 ; Copulatory organs, 621.

Pyrosoma-Stolo prolifer, 396; Luminous organs, 294; Muscular system, 395; Nerrous system, 396 ; Langnets, 401; Branchial slits, 402; Sexual organs, 407.

Pyrula-Cœlom, 367.

Radiolaria-Central capsule, 76,82 ; Shells, 81; Skeleton, 82; Figure, 82 ; Spores, 87.

Rana-Sternum, 442 ; Palato-quadrate, 457; Shoulder-girdle, 476; Larynx, 569.

Raptores-Optic bulb, 529; Turbinate bone, 547 ; Stomach, 558.

Ratitæ-Sternum, 443; Shoulder-girdle, 476; Precoracoid, 476; Clavicle, 477; M. pyramidalis, 496; Lymphatic hearts, 599; Copulatory organs, 621.

Rays-Dermal denticles, 424; Articular processes, 437; Hyomandibular, 449; Shoulder-girdle, 474; Protopterygium, 478; Electric organs, 500; Vagus, 521; Gelatinous tubes, 524; Nasal groore, 526; Branchial clefts, 543. 
Renilla-Pore, 117.

Reptilia-Vertical fringe, 415; Corium, 417; Scales, 417 ; Dermal bones, 425 ; Vertebral column, 429; Atlas, 437; Axis, 437 ; Ribs, 440 ; Sternum, 443 ; Episternum, 444; Squamosal, 458; Postfrontals, 459; Nasal cavities, 460; Hard palate, 460; Branchial skeleton, 469; Hyoid, 471; Shouldergirdle, 476; Ilium, 484; Fore-limb, 481 ; Toes, 490 ; Dermal muscles, 493; Intercostal muscles, 495 ; Masticatory muscles, 497 ; Brachial plexus, 514; Crural plexus, 515; Sacral plexus, 515; Goblet-shaped organs, 524; Gustatory organ, 525; Lens, 531; Optic muscles, 531; Eyelids, 532 ; Lachrymal glands, 532 ; La. chrymal ducts, 533; Lagena, 536; Tympanic cavity, 537; External ear, 538; Buccal cavity, 549; Teeth, 550; Tongue, 552; Labial glands, 553; Thyroid gland, 554; Enteron, 555; Stomach, 597; Hind-gut, 562 ; Cloaca, 563; Epiglottis, 571; Trachea, 571; Lungs, 573; Blood-corpuscles, 576; Heart, 587; Jugular veins, 590; Venæ vertebrales, 591; Cardinal veins, 591; Superior venæ cavæ, 591; Ductus Cuvieri, 591; Renal veins, 594; Lymphatic hearts, 599; Peyerian glands, 600; Thymus, 600; Renal organs, 609; Egg, 609; Yolk, 609; Cloaca, 620.

Rhizocephala-Mantle, 236; Eyes, 264; Enteric canal, 269.

Rhizocrinus-Figure, 197.

Rhizopoda-Sensory organs, 42 ; Aliment. ary canal, 47 ; Protoplasm, 76 ; Psendopodia, 78 ; Spores, 87.

Rhombifera-Bony plates, 424.

Rhynconella-Excretory organs, 313.

Rodentia-Nipples, 422; Episternum, 444; Interparietals, 464; Hyoid, 472; Centrale, 482; Pelvis, 486; Fibula, 490; Cerebral hemispheres, 509 ; Buccal pouches, 549; Teeth, 552; Stomach, 558; Cæcum, 562 ; Liver, 564; Venæ cavæ, 592; Renal organs, 605; Uterus, 616; Testes, 618; Vesiculæ seminales, 618; Prostatic glands, 619 ; Copulatory organs, 622 ; Tyson's glands, 624 .

Rotalia-Figure, 78.

Rotatoria-Segmentation, 129 ; Cilia, 138; Wheel organ, 138; Dermal carapace, 139; Tubes, 132 ; Nervous system, 146; Tactile setæ, 152 ; Visual organs, 154; Alimentary canal, 160; Glandular organs of enteron, 174; Cœlom, 166; Excretory organs, 174; Generative organs, 190.

Ruminantia--Interparietal, 464; Cuboid, 490 ; Tapetum, 530 ; Accessory cavities of nose, 548; Sinus maxillary, 548; Stenonian ducts, 548; Sto. mach, 559.

Saccobranchus-Branchial tubes, 545.

Sænuris-Vascular system, 168.

Sagitta-Nervous system, 149; Eyes, 151.

Salamandrina-Vertebræ, 432, 433; Hind. limb, 488; Trunk muscles, 494; M. pyramidales, 496; Lungs, 573; Heart, 581; Lymph spaces, 598; Vagus, 517; Eyelids, 532; External gills, 545.

Salmo-Notochord, 427; Cartilaginous cranium, 450; Mandibular apparatus, 454; Enteron, 556; Kidneys, 604; Ovaries, 610.

Salpa-Stolo prolifer, 391; Asexual form, 392 ; Sexual form, 392 ; Muscular system, 395; Nervous system, 395 ; Sen. sory organs, 397; Ciliated groove, 397; Visual organs, 397 ; Ventral groove, 402 ; Enteron, 404; Nucleus, 404 ; Heart, 405 ; Sexual organs, 407.

Saltatoria-Thorax, 237.

Saphenia-Tentacles, 102.

Sapphirina-Female organs, 294.

Sarcoptes-Tracheæ, 291.

Sarcorhamphus-Veins, 591.

Sarsiadæ-Gastrovascular system, 115.

Saurii-Centra of rertebræ, 433; Pro. cesses of do., 433 ; Candal do., 434 , Ribs, 438; Sternum, 442 ; Columella, 458, 538; Vomer, 459 ; Lachrymal; 459 ; Pterygoids, 461 ; Palatines, 461 ? Maxilla, 462; Shoulder-girdle, 476 ; Carpus, 481 ; Ilium, 484 ; Pubis, 485; Tarsus, 489; Intercostal muscles, 496 ; Masticatory do., 497; Brain, 507; Sclerotic, 530; Eyelids, 532; Ductus endolymphaticus, 534; External ear, 538 ; Nasal glands, 548; Organ of Jacobson, 548; Teeth, 550; Tongue, 552 : Labial glands, 553 ; Hind-gut, 562 ; Liver, 564; Mesentery, 565; Lungs, 573; Heart, 581; Arterial arches, 582; Carotids, 507 ; Arteriæ intercostales, 589; Epigastric veins, 595; Oviducts, 618; Testes, 614; Vasa deferentia, 614; Mullerian duct, 615 ; Allantois, 620 ; Copulatory organs, 621 .

Sauropsida-Vertebral column, 433 ; Skull, 457 ; Ischiac arteries, 589.

Scaphopoda-Appendages, 326 ; Nervous system, 347; Visual organs, 353; Auditory vesicles, 357 ; Alimentary canal, 358.

Schizopoda-Branchiæ, 241; Heart, 281; Female organs, 294; Male organs, 295 ; Seminal elements, 305.

Schizostomeæ-Alimentary canal, 157.

Scincoidea-Dermal bones, 425.

Scinrus-Uterus, 616.

Scoleina-Dermal glands, 141; Nerve 
trunks, 149; Ganglia, 150; Vascular system, 168; Excretory organs, 176.

Scolopendra-Salivaryglands, 274; Liver, 274; Malpighian vessels, 276; Circulatory system, 284; Generative organs, 299.

Scomber-Trunk muscles, 494; Appendices pyloricæ, 560 .

Scorpionea-Metameres, 237; Cheliceræ, 244; Integument, 218; Poison glands, 250 ; Nervous system, 256; Eye, 265; Enteric canal, 269; Hind-gut, 270; Salivary glands, 274; Hepatic tubes, 275 ; MIalpighian vessels, 276 ; Circulatory system, 281; Lungs, 291; Generatire organs, 297.

Scutigera-Tracheæ, 288.

Scyllæa-Branchiæ, 338; Stomach, 362 ; Vessels, 372 ; Excretory organs, 377 . Scyllium-Brain, 501; Olfactory organs, 525; Branchial carity, 543 ; Ovaries, 611.

Scymnus-Arachnoid, 513.

Scyphostoma-Gastrorascular system, 116.

Segestria-Trachex, 291; Ovaries, 297.

Selachii-Cartilage cells, 26; Anterior appendages, 414; Dermal denticles, 423; Vertebral column, 429 ; Transrerse processes, 431 ; Fin-rays, 432 ; Ribs, 439; Cranium, 447; Palatoquadrate, 448; Hyoid, 448; Mandibular apparatus, 453 ; Branchial skeleton, 468; Hyoid arch, 469; Copulæ of hyoid, 469; Archipterygium, 473; Anterior appendages, 474; Shoulder-girdle, 474; Fin, 477; Pelvic-girdle, 484; Ventral fin, 487; Dermal muscles, 494; Muscles of branchial skeleton, 497; Olfac. tory lobes, 504; Thalamencephalon, 504; Mesencephalon, 505; Sinus rhomboidalis, 505; Medulla oblon. gata, 505 ; Optic nerve, 515 ; Cephalic nerres, 516; Vagus, 517; Facial nerve, 517; Glossopharyngeal, 518; Gelatinous tubes, 524; Olfactory organs, 525; Sclerotic, 529; Ciliary processes, 530; Tapetum, 530; Eyelids, 532; Vestibule, 531; Otoliths, 536; Spiracle, 537; Branchial pouches, 542 ; Spiracular cleft, 542 ; Branchial lamellæ, 544; Teeth, 550; Enteron, 555; Stomach, 557; Spiral valve, 560; Hind-gut, 562; Air-bladder, 567; Abdominal pore, 574; Conus arteriosus, 578; Bran. chial arteries, 579; Aorta, 585; Spleen, 600; Thymus, 600 ; Archinephric duct, 602; Kidney, 603 ; Nephrostomata, 603; Mullerian duct, 603; Eggs, 609; Yolk, 6c9 ; Sperm, 6с9; Oraries, 610, 611; Germinal glands, 611; Epigonal Organ, 611 Copulatory organs, 611; Cloaca, 619.

Sepia-Mantle, 325 ; Copulatory organs, 327 ; Respiratory organs, 310 ; Iuternal skeleton, 341 ; Buccal ganglia, 351; Eye, 355; Auditory plate, 357; Cæca, 363; Liver, 366; Pouches of Needham, 357.

Sepiadæ-Fins, 325 ; Arms, 326; Os sepiæ, 334; Arteries, 374 .

Sepiola-Liver, 366.

Sergestes-Auditory vesicles, 261.

Serpula-Tentacles, 133; Stalk of operculum, 134; Ganglionic chain, 149.

Serranus-Hermaphrodite arrangements, 611.

Sertularia-Colonies, 93; Tests, 161.

Shad-Beard, 524.

Shark-Skin, 423; Dermal denticles, 423 ; Caudal region, 431.

Sialida-Salivary glands, 274 .

Siredon-Vertebræ, 432 ; Anditory ossicles, 538.

Siphoniata-Siphons, 320.

Siphonophora-Division of labour in, 95 ; Colonies, 95; Nectocalyces, 96; Figure, 96; Nutritive persons, 93; Protective persons, 97 ; Tentacular persons, 97 ; Generative persons, 97 ; Cilia, 101: Muscular system, 108; Gastric system, 114; Pigmented investment of stomach, 118; Separation of sexes, 120; Generative products, 121.

Silurus-Lymph sinuses, 599.

Simiæ-Liver, 561; Uterus, 616; Hymen 616 ,

Singing Birds-Syrinx, 572.

Siphonostoma - Tentacles, 133, 135 ; Degeneration in, 236; Ovaries, 293.

Sipunculus-Cerebral mass, 14S; Gan. glion cells, 148; Alimentary canal, 161, 162; Vascular system, 171; Excretory organs, 176.

Siren-Blood-corpuscles, 576.

Sirenia-Nipples, 422 ; Caudal vertebræ, 436.

Smynthurus-Tracher, 288.

Solaster-Arms, 196; Alimentary canal, 213.

Solen-Ganglia, 345.

Solenogastres - Ventral surface, 130 ; Aciculi, 139; Nerrous system, 151; Groove, 318.

Solidungula - Fore-limb, 483 ; Tenæ cavæ, 592.

Sorex-Jugal, 466; Coracoid, 476.

Spatangidæ-Semitæ, 201; Dermal skeleton, 205; Spines, 205; Muscular system, 207; Blood-vessels, 21s; Water-vascular ring, 220; Stone. canal, 222; Generative organs, 226. 
Sphærodol'um--Integument, 138.

Sphinz-Digestive organs, 270.

Sphenodon-Ribs, 440; Quadrate, 461.

Spiders-Chelieeræ, 244; Spines or setæ, 250 ; Spinning glands, 250; Nervous system, 256; Cerebral ganglia, 256; Eye, 265 ; Digestive organs, 269.

Spio-Dermal glands, 141.

Spirochona-Carapace, 83.

Spirorbis-Stalk of opereulum, 134.

Spirostomum - Contractile bands, 80 ; Nucleolus, 87.

Spirula-Shell, 334 .

Spondylus-Shell, 336.

Spongir-Gastrula, 92 ; Tentaeles, 101 ; Integument, 103 ; Alimentary canal, 111; Gastrie system, 112; Lipogastria, 113; Sexual organs, 119 ; Ova, 124.

Spongilla-Amphidises, 106.

Squatina-Protopterygium, 478; Arachnoid, 513 ; Enteric canal, 560 ; Heart, 578.

Squilla-Branchiæ, 241; Nervous system, 253.

Stauridium-Tentacles, 93

Stentor-Contractile bands, 30; Shells, 83 ; Anal opening, 85.

Stephanoceros-Wheel organ, 138.

Sternaspis-Respiration in, 136; Cerebral mass, 148; Ganglion cells, 148 ; Vascular system, 171; Excretory organs, 176.

Stomapoda-Liver, 275; Heart, 281.

Strepsiptera-Wings, 248; Male organs, 304.

Strombus-Siphon, 323.

Struthiones-Sacrum, 435; Skull, 460 ; Toes, 490 ; Pecten, 530; Marsupium, 531; Turbinate bones, 547; Lym. phatic hearts, 599; Copulatory organs, 621.

Sturiones-Dermal bones, 425; Vertebral column, 430; Ribs, 439; Cartilaginous cranium, 450; Mandibular apparatus, 453; Operculum, 455; Goblet-shaped organs, 523; Ciliary proeesses, 530; Branchial lamellæ, 544 ; Thymus, 600.

Suina-Nipples, 422; Manus, 483.

Suetoria-Pseudopodia, 83.

Swans-Traehea, 572.

Sycaltis-Skeleton, 105.

Sycones-Ectoderm, 103; Gastric sys. tem, 113.

Syllidæ-Parapodia, 134; Visual organs, 155.

Synaptro-Ambulacra, 198; Tentacles, 200 ; Calcareous anehor, 206 ; Integument, 206; Internal skeleton, 207; Museular system, 208; Alimentary canal, 214; Figure of, 223 ; Polian vesicles, 223; Water-vessels, 223 ; Hermaphrodite organs, 226.
Syncoryne-Tentacles, 93; Figure of, 93. Syncorynidæ-Cormi, 94.

Tænia-Segmentation, 129; Cystic form, 131; Aciculi, 140; Female organs, 182.

Tamoya-Marginal filaments, 102.

Tanais-Auditory vesicles, 261.

Tapir-Manus, 482.

Tardigrada-Eye,266; Generative organs, 298.

Talpa-Manus, 482.

Teleosaurii-Dermal bones, 425.

Tegeneria-Traeher, 291.

Teleostei-Bony plates, 424; Vertebral column, 430; Transverse proeesses, 431 ; Caudal region, 431; Fin rays, 432 ; Artieular processes, 437 ; Ribs, 439 ; Cranium (cartilaginous) 450 ; Sphenoids, 452; Mandibular apparatus, 453; Operculum, 455; Branchial arehes, 469; Shoulder-girdle, 474; Thoracic fin, 478; Pelvic. girdle, 484; Ventral fin, 487 ; Dermal museles, 492; Muscles of branchial skeleton, 497; Olfactory lobes, 504; Thalameneephalon, 504; Mesencephalon, 505; Sinus rhomboidalis, 505; Optie nerves, 515; Faeial nerves, 517; Glossopharyngeal, 518; Vagus, 521; Goblet-shaped organs, 523 ; Mucous canals, 524; Lateral line, 524; Olfaetory organs, 525; Selerotic, 529; Optie museles, 531; Eyelids, $: 532$; Duetus endolymphatieus, 534; Asteriseus, 536 ; Branchial clefts, 543; Spiraeular cleft, 543 ; Branehial lamellæ, 544; Teeth, 550 ; Enteron, 555 ; Foregut, 556 ; Stomach, 557; Midgut, 559 ; Air-bladder, 567 ; Bulbus arteriosus, 578; Branchial arteries, 579 ; Aorta, 585 ; Thymus, 600; Kidney, 603,604; Yolk, 609; Ovaries, 610 ; Cloaca, 619.

Tenthrediner-Feet, 216; Cement glands, 303.

Terebella-Tentacles, 133; Branchial do., 135 ; Vasenlar system, 169.

Terebratula-Circlet of cilia, 307 ; Museular system, 307 ; Nervous system, 310 ; Exeretory organs, 313.

Termes-Nervous system, 257.

Termites-Malpighian vessels, 277.

Testieardines-Stalk, 308 ; Shell, 308 ; Skeleton of arms, 308 ; Alimentary canal, 311 ; Generative organs, 313.

Tetraetinia-Tentaeles, 99.

Tetrarhynehus-Acieuli, 140.

Thalassema-Excretory organs, 176.

Thaliadæ-Stolo prolifer, 391 .

Thaumantias - Gastrovascular system, 115. 
Thecidium-Metameres, 307 ; Generative organs, 313.

Thecomedusæ-Organisation, 98 ; Scy. phostoma form, 98; Strobila, 99; Ephyra form, 99; Discophora of, 99.

Thecosomata-Velum, 318, 321 ; Head, 324.

Thelyphonus-Nervous system 256 .

Thomisus-Trachea, 291.

Thoracostraca-Optic nerves, 253 ; Ventral chord, 251; Heart, 281; Female organs, 291.

Thylacinus-Marsupial bones, 407.

Thysanopoda-Branchix, 212.

Thysanozoon-Tentacles, 133; Alimentary cana!, 158.

Thysanura-Gnathites, 245; Feet, 246; Ventral chord, 258; Eye, 265; Enteric canal, 272 ; Trachex, 288.

Tinca-Goblet-shaped organs, 523.

Tintinnus-Shells, 83.

Tipulidø-Eye, 267.

Toads-Sperm, 669.

Tomopteris-Generative organs, 189.

Torpedo-Electric organs, 500, 505.

Tortrix-Hyoid, 472 .

Toucan-Tongue, 552.

Tracheata-Derelopment, 234; Appen. dages, 238, 243; Antennæ, 244; Ner. rous system, 255 ; Salivary glands, 273 ; Liver, 275 ; Malpighian vessels, 276, 286; Fat body, 278; Renal concretions, 278 ; Circnlatory system, 282 ; Tracheae, 286.

Trachelius-Contractile vesicles, 86 .

Trachynema-Tentacles, 102, 107 ; Mar. ginal vesicles, 110.

Tragulida-Stomach, 559.

Trematoda-Sporocyst, 131 ; Cercarix, 131; Cilia, 137 ; Aciculi, 139; Stylets, 141 ; Dermal glands, 141 ; Suckers, 143 ; Nervous system, 146; Visceral nerres, 151; Visual organs, 153 ; Alimentary canal, 157; Glandular organ of enteron, 164; Liver, 165 ; Excretory organs, 173; Generative organs, 179 ; Pore, 183.

Tremoctopus-Hectocotylised arms, 327.

Tricocephalis-Muscular system, 143.

Trionyx-Plastron, 426.

Tristoma-Excretory organs, 173.

Triton-Lungs, 573 ; Heart, 581.

Tubicolæ-Tube, 134; Setæ, 140 ; Visual organs, 155; Hind-gut, 163 ; Vascular system, 169.

Tubifex-Generative organs, 189 ; Spermatophores, 191.

Tubipora--Skeleton, 106.

Tubularia-Buds, 95 ; Tentacles, 101 ; Tests, 104; Supporting lamella, 107; Pigmented investment of stomach, 108.

Tunicata-General review, 388; Biblio. graphy, 389 ; Classification, 389 ;
Form of body, 390 ; Integument, 393 ; Mantle, 393 ; Skeleton, 391 ; Muscular system, 394 ; Nervous sys. tem, 395 ; Sensory organs, 397 ; Ali. mentary canal, 393 ; Ventral groove, 402 ; Sexual organs, 406.

Turbellaria-Mouth, 129; Tentacles, 132; Epidermis, 137 ; Rod-like bodlies, 1.10 ; Muscular system, 112 ; Nervous system, 146 ; Visceral nerves, 151 ; Tactile setæ, 152 ; Visual organs, 153 ; Auditory organs, 156; Alimentary canal, 157; Glandular organs of en. teron, 164; Excretory organs, 173; Generative organs, 179 ; Pore, 181.

Tylopoda-Fore-limb, 483; Stomach, 559 ; Blood-corpuscles, 576.

Ungulata - Nipples, 422 ; Vertebral column, 435 ; Paramastoids, 463 ; Clavicle, 477 ; Fibula, 494; Hallux, 491 ; Cerebellum, 510 ; Uterus, 616.

Unio-Muscles, 312 ; Nerrous system, 345 ; Efferent renal ducts, 376, Geni. tal canal, 381.

Urodela-Vertebræ, 432, 4.33 ; Shoulder. girdle, 475; Pelvic-girdle, 484; Hind. limb, 488; Brain, 505; Ductus en. dolymphaticus, 531; Auditory ossicles, 538; Stomach, 537 ; Iliac vein, 593 ; Lymphatic hearts, 599 ; Kidneys, 601; Ureter, 601; Cloaca, 613.

Uromastix-Sternum, 442.

Ursina-Cæcum, 562.

Ursus-Renal organs, 605 .

Vaginicola-Shells, 83.

Varanus-Columella,458; Brachial plexus, 514 ; Tongue, 552.

Vegetable Kingdom-Cells, 15 ; Tissues, 21.

Velella-Generative persons, 97 ; Air-sac, 98 ; Hepatic canals, 118 ; Generative products, 121.

Vermes-Gastrula, 35; Dermal branchiæ, 36 ; Excretory organs, 46 ; Alimen. tary canal, 47,48 ; Respiratory or. gans of the enteron, 49; Vascular system, 50; Gemmation in, 61 ; Ho. mology in, 64; Classification, 125 ; Bibliography, 127; Form of body 128; Segmentation, 129 ; Muscular system, 142 ; Visceral organs, 153; Auditory organs, 156 ; Alimentary canal, 156 ; Colom, 165 ; Generative products, 190.

Vertebrata-Branchial clefts, 7; Lungs, 10; Connective tissue, 24; Osseous tissue, 28; Blood-corpuscles, 29; Cartilaginous skeleton, 38; МLuscu. lature, 40; Excretory organs, 47; Respiratory organs of the enteron, 48 ; Homology in, 61:; General 
review, 409; Classification, 409 ; Bib. liography, 412 ; Form of body, 413 ; Head, 113 ; Appendages, 414 ; Limbs, 415; Integument, 417; Ectoderm, 417 ; Dermal papillæ, 417 ; Dermal bones, 425 ; Internal skeleton, 426 ; Ribs, 438; Thoracic-girdle, 473; Pelvic-girdle, 473 ; Archipterygium, 473 ; Fore-limb, 479 ; Muscular sys. tem, 491; Nervous system, 501 ; Brain, 503; Pineal gland, 503 ; Infundibulum, 501; Pia mater, 513; Arachnoid, 513; Spinal nerves, 514; Vagus, 516; Olfactory organs, 52.; Eye, 527 ; Iris, 530 ; Fenestra ovalis, 537 ; F. rotunda, 537 ; Tympanic cavity, 537 ; Auditory ossicles, 538; Euteric canal, 539; Gall bladder, 561; Pancreas, 565 ; Cœlom, 574; Vascnlar system, 575; Systemic arteries, 585; Spleen, 600 ; Excre. tory organs, 601; Generative organs,
606; Germinal epithelium, 606; Iale germinal glands, 609 ; Sperm, 6.99 ; Genital ridges, 610.

Virgularia-Generative organs, 123.

Viverra-Testes, 618.

Voluta-Proboscis, 361.

Volvocineæ-Cells, 19.

Vortex-Generative organs, 180.

Vorticellinæ-Stalk, 83 ; Anal opening, 83 ; Nucleus, 89.

Vultur-Cervical vertebric, 431.

Waldheimia-Nervous system, 310 ; Vascular system, 382.

Wasps-Mid-gut, 272 ; Parthenogenesis, 302.

Whales-Whalebone, 549.

Woodpeckers - Tongue, 552; Cæcum, 562.

Zoothamnium-Contractile bands, 80 . 


